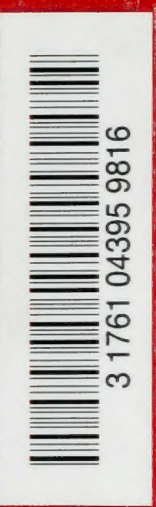

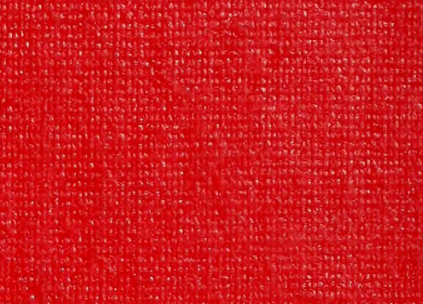









\section{BRITISH HUSBANDRY;}

EXHIBITING

THE FARMING PRACTICE

IN

\section{VARIOUS PARTS OF THE UNITED KINGDOM.}

VOLUME THE SECOND.

PUBLISHED UNDER THE SUPERINTENDENCE OF THE SOCIETY FOR THE DIFFUSION OF USEFUL KNOWLEDGE.

\section{O N D O N :}

BALDWIN AND CRADOCK, PATERNOSTER-ROW. 

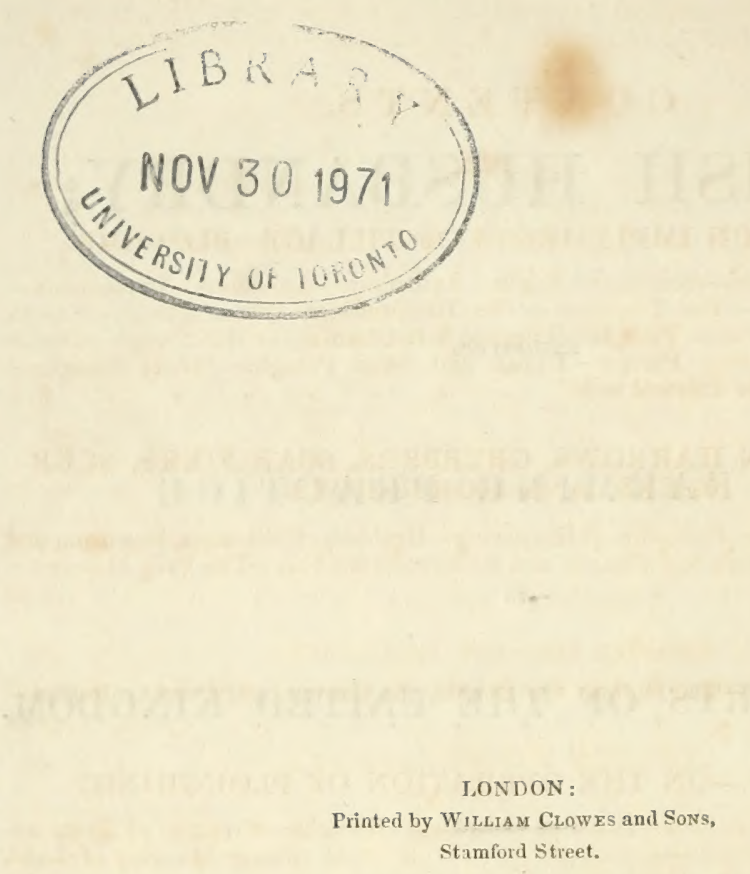

$$
\begin{aligned}
& 5 \\
& 511 \\
& 38 \\
& v .2
\end{aligned}
$$




\section{O N T E N T S.}

\section{Chapter I.-ON IMPLEMENTS OF TILlaGE-PLOUGHS.}

Object and operations-Scotch and English Agriculture-Labourers-ImplementsThe Swing Plough - The Turnwrest - The Hertfordshire Wheel Plough-Small's Plough-Ransome's Patent Ploughs-Imperial Self-Cleaning, or Rid-Plough-Double Furrow Plough-Ribbing Plough-Trench and Skim Ploughs-Wheel PloughsOpinion of Ploughs for different soils . . . . . . . . p. 1

\section{Chapter II.-ON HARROWS, GRUBBERS, SCARIFIERS, SCUF FLERS, AND HORSE-HOES.}

Harrows and Drags-Operation of Harrowing-Grubbers, Cultivators, Scarifiers, aud Tormentors-Finlayson's Self-Cleaner and Kirkwood's Grubber-The Drill HarrowsHorse Hoes

\section{Chapter III.-ON ROLlers.}

The Solid Cylinder-The Hollow, the Double, the Convex, and Concave RollersThe Spiky Roller

\section{Chapter IV.-ON THE OPERATiON OF PLOUGHiNG.}

Management-Ploughmen--Taste in ploughing-Draught-Strength of Team required on heavy land-Modes of operation-Formation of ridges-Quantity of Land ploughed in a day's work-Depth of ploughing on various soils . . . p. 38

\section{Chapter V.-ON Fallowing.}

Bastard Fallow-Fallow Crops-Summer Fallow-Proofs of the necessity of Fallowing-Modes of conducting the operation-Different ploughings to effect a clean Fallow - Seed-furrow-Winter Fallow-Greg's system and profits-Beatson's plan-His total abolition of Fallows ; description of his Scarifier; his course of cultivation; expenses and difference in favour of the new method . . . . . . . . p.54

\section{Chapter VI,-ON SOWING BROADCAST, DRILLING, AND DIBBLING.}

Tull's experiments and result-Broadcasting, with a Machine to effect it-DrillingArguments in favour of and against it-Operation of, and Drill Implements-Dibbling - Experiments on the different modes of putting in the Seed . . . p.71

\section{Chapter VII.-ON THE ROTATION OF CROPS.}

Its importance, and judgment required to arrange these judiciously-Old culture of Corn-Modern culture of Corn and Roots-General principles of the different coursesTriennial rotation-Alternate rotations-Norfolk system-Plans of bringing Farms into a regular succession of green and corn crops-Double crops-Flemish mode-District rotations: on chalk; on sandy loams; on cold heavy clays ; on thin clays; on strong dry land of a fertile nature-System in the Fens-Hill-moss Moorland; Bent-moss; Flow-moss-Summary . . . . . . . . P. 90

\section{Chapter VIII.-ON SOILS AND COURSES OF CROPPING.'}

Classification of Soils-Crops for deep strong lands; for rich clay loams; for inferior clays and loams; for light, calcareous, and gravelly soils; for sandy land--Courses 
on the uplands--Produce, charges, and profits upon a good Wheat and Bean Soil ; and also upon a good Turnip and Barley Soil-Effects of Cropping-Vegetative power and exhaustion of the Soil, according to the calculations of Von Thaër ? - p.112

\section{ChAPTER IX.-ON THE GROWTH OF CORN.}

Chemical composition of Plants-Weight of the different species of Grain-SeedTime of -owing, and depth at which Corn should be sown-Proper quantity of SeedEffects of change-Quality and preservation of Grain-Power of germination-Product and vegetation-Blooming-Tillering-Ripeness-Average of Meal and Household Bread produced by different kinds of Corn . . . . . . p.127

\section{Chapter X.-ON Wheat.}

Species-Winter and Spring Wheat-Soil-Cultivation-Pressing Machine-Manure-Application of Lime-Seeds-Sowing of Winter Wheat-Quantity per acre -Depth-Spring tillage--Soils-Operations of Weeding--Practice of feeding it off -Previous crops--Spring Wheat-Season for Sowing-Produce and qualities-FlourExperiment upon-Seed Wheat-Judgment of the Sample-Foreign WheatDiseases: Mildew, Rust, Blight, Smut-Wheat Fly-Steeping-Experiments on and Machines for cleaning

\section{Chapter Xi.-ON RYE.}

Rye Bread-Varieties of the grain-Soils most appropriate to its growth-Cultivation-Seed-Period of flowering-Product and analysis of its properties . p.166

\section{Chapter XII.-ON BARLEY.}

Species-Summer and Winter Barley-Bere or Bigg-Rammel-Soil-Cultivation - Seed and sowing-Mode of operation with Clover and Grass Seeds-Product of the Chevalier sort-Analysis of qualities of various kinds-Straw-Hummelling p.171

\section{Chapter XIII.-ON OATS.}

Species-Common feed, Poland, Dutch, Potato, and Hopetoun Oats-Experiments to ascertain the difference of their quality-Skinless, Tartarian, and Naked Oats-Pilcorn and Pilez-Soil and cultivation-Seed and sowing-Produce and analysis of their properties-Mealing process-Quantity and quality of Straw-Table of the comparative value of Oats per barrel in Ireland and per quarter in England . . P. 177

\section{Chapter XiV.-ON HarVest. :}

Season of its commencement-Indications of the ripeness of Grain-Experiments respecting different periods of reaping Barley-Operation of reaping-Forming of the sheaves-Mowing of Corn--Cradle Scythe-Hainault Scythe-Reaping MachinesStooking-Stacking-Square and round Stacks-Thatching-Skeleton Barns-Stackyards-Operation of thrashing by the Flail-Thrashing Mill-Portable Thrashing Machine-Wages of thrashing-W innowing Machines-Marketing-Measurement of Grain-Expenses of reaping, thrashing, and marketing-Barley Hummelling-Kiln drying-Russian mode of drying in the sheaf-Gleaning . . . p.185

\section{Chapter XV.-On BEANS, PEASE, AND VETChES.}

Bean soil-Different sorts of Beans-Their cultivation-When grown after CornWhen grown preparatory to Wheat-Period of sowing-Comparative advantages of drilling, dibbling, and sowing broadcast-Dibbling Machine-Harvesting and stacking of Beans-Produce and diseases of the Plant-Weight and quantity of nutritive matter -Pease-Grey hog Pease and White boiling Pease-Different species of the latterOperation of podding-Appropriate soil and culture-Application of Manure-Seed and produce-Indications of ripeness-Reaping-Analysis of their nutritive propertiesVetches-Winter and Spring Tares-White Vetch-Soil-Seed-Nutritive qualityCommon Lentil, or Dill 


\section{Chapter XVI.-ON BUCK-WHEAT.}

Soil-m Cuiture-Seed-Product and uses of the Grain . - . . p. 222

\section{Chapter XVII.-ON INDIAN CORN.}

Origin--Growth and appenance-Appropriate soil-Modes and time of sowingTransplantation-Culture-Harvesting--Husking-Corn-cribs--Shelling-Produce of the Crop-Opinion regarding the success of its cultivation in this country - p. 225

\section{Chapter XVIII.-ON TURNIPS.}

Introduction of-Appropriate Soil-Species; White Globe, or Norfolk; Alerdeen Yellow; Ruta Baga, or Swedish-Time of Sowing-Quantity and quality of SeedCulture-Application of Manure-Drilling-Hoeing-Produce-Weight and nutritive qualities of the different kinds-Diseases-Storing-Grubbing-Application-Turnip Slicing Machines-Value of an Acre of Turnips . . . ? . p. 231

\section{Chapter XiX.-ON RAPE.}

Soil-Seed-Culture-Growth-Application . . . . . P. 249

\section{Chapter XX.-ON MANGEL-WÜRTZEL.}

Soil and Culture-Growth and period of maturity-Produce and application-Comparison of its nutritive qualities with Swedish Turnips-Experiments on its feeding properties-Report of the Doncaster Association . . . . . p. 251

\section{Chapter XXI.-ON CABBAGES.}

Soil and Culture-Seed and Season-Mode of Planting-Quantity of nutritive matter-Comparison between the feeding qualities of Cabbages and Swedes . 1. 255

\section{Chapter XXII.-ON POTATOES.}

Battatas-Introduction of Potatoes-Description and produce of different rarietiesSoil and Culture-Time and modes of P'lanting-American Plan-Lazy-bed fashionKxperiments by the London IIorticultural Society-Morde of spreading the DungGrowth of Lankman's Potatoe-Seed-Sets and Tubers-Produce from whole Tubers, and from single eyes of fine varieties-Experiments on the depth and distance of planting-Plucking off the Blossoms-Cutting the Haulm-The Curl-Recent failure of the Crops, and various opinions of its cause-Storing, Produce, Value, Application, and Aualysis of Nutritive Matter

\section{Chapter XXIII.-ON CARROTS AND PARSNIPS.}

Introduction of the Carrot-Soil best adapted to it-Application of Manure-Seed, and Time of Sowing-Culture-Species-Haulm-Storage-Cultivation of the Parsnip -Soil-Seer-Produce, Value, and Appropriation-Production of Sced-Comparative fattening qualities of various Root Crops . . . . . . p. 283

\section{Chapter XXIV.-ON ARTIFICTAL GRASSES.}

Clovers; White and Red; Cow-grass; Trefoil and Melilot; Scarlet Trefoil, or Crimson Clover-Soils-Time and Mode of Sowing-Treatment of the Crop-Harresting-Hay-Growth and produce of the Seed of the different species-Difficulty of Thrashing-Perennial and Annual Rye.grass; Italian; Pacey's and Stickey's-Value for feeding-Produce when grown for Hay and Seed-Cultivation of Winter and Spring 
Tares-Times and Mamner of Sowing both for Seed and Soiling-Produce and Valte of both sorts - Growth and Value of Chiccory and Corn Spurrey . • p. 292

\section{Chapter XXV.-ON ARTIFICIAL GRASSES (contimued).}

Sainfoin-Appropriate Soil-Culture-Nanagement in the making of the IayProduce of Seed-1'ractice when broken up-Ciltivation of Lucerne-Preparation of the Land-AIode of Sowing-Treatment in the subsequent years of its growth-Application of the Crop-Comparison of its nutritive properties with White and Red Clover and Sainfoin-Its effect on Cow's Milk . . . . . p. 304

\section{Chapter XXVI.-ON THE SEED OF COLE, MUSTARD, AND POPPY.}

Fen Culture of Cole-Flemish Process-Harvest and Produce-Haulm Ashes-White and Brown Mustard-Soils-Seed and Sowing-Mode of Thrashing-Produce-Oilcake-Soil and Caltivation of Poppy-Process of the Harvest-Experiments on the produce and value of the Crop . . . . . . . P. 312

\section{Chapter XXVil.-ON FLAX AND HEMP.}

Flax Seed-Soil and Growth-Treatment of the Crop-Process of Water-rettingImproved mode of Watering-Dew-retting-Breaking, Scutching, and Ileckling for Tarn-Rippling for Seed-Irish Flax Barn-Linseed Oil-Male and Female HempSoil and Cultivation-Seed-IIarvesting of the Fibre and Seed-Expense and ProfitOn the growth of Hemp in Ireland . . . . . . p. 316

\section{Chapter XXVIII.-ON TEASEL-CORIANDER AND CARRAWAY - CUMIIIN, ANISE, CANARY, RADISH AND SPINACII SEEDS-PHYSICAL PLANTS.}

(nultivation of Teasel-Duscription of the Plant-Mode of gathering-Manarement together with Coriander and Carraway-Produce and application of the Crops-Grawth of Cummin and Anise-Culture of Canary Seed-IJarvest-Produce and Value of the Ifaulm-Kentish mode of growing Radish and Spinach Seed-Plysical and Aromatic Plants; Camomile; Liquorice; and Peppermint-Soils, Modes, and CultivationPlantations of Lavender-Red and Damask Roses-Culinary Rhubarb . P. 326

\section{Chapter XXIX.-ON MADDER, WOAD, AND WELD.}

Description of Madde - S Soil-Dutch mode of planting-English manner-C Culture and Manufacture-Growth of Woad on grass and on arable Land-Flemish mode of Culture-Manufacture and Value of the Crop-Soil, Cultivation, and Value of IVeld -Observation on these Crops . . . . . . . p. 332

\section{Chapter XXX.-ON HOPS.}

Introduction and Species-Fit Soil and Situation-Preparation of the Land-Time and mode of planting - First, second, and third Year's management - Poling Cost and manner of placing the Poles and tying the Bine-Picking, Oastage, and Bagging-Produce and expenses in various parts of Kent, in Worcestershire and Herefordshire-Duty - Propagation by Seed - Subsequent Culture-Training horizontailyCasualties and Diseases

\section{Chapter XXXI.-ON ORCHARDS-CYDBR AND PERRY.}

Ancient plantation of Orehards-Soil and Situation-Preparation of the GrumdPeriod and modes of planting-Proper $A$ ge of the l'lants-Pruning-Grafting-Node 
of raising Plants from Seed-Age of maturity-Species generally used for CyderCropping-Pommage-Cyder Press-Pulp-Qualities of the Juice-Manufacture of Cyder-Stumming-Fining-Time of arrival at perfection-Cocagee and Sure-Qualities necessary in the Pear when intended for Perry-Extraordinary Produce-Tree at Home Lacey-Table Varieties; the celebrated St. Germain; the Bon Chretien; and the Pera de Mantega-Fabrication of Perry-Imitation of Champagne Winc-Nixed Fruits; Cherries; Damsons ; Filberts

\section{Chapter XXXII.-ON THE FEEDING OF NEAT CATTLE- GRAZING-STRAW.}

Choice of Stock-Difierent Breeds-Spayed Heifers and Free-Martins-Practice of Grazing on rough Pasture and on rich Land-Age and increase of Weight-Stocking of the Ground-Objects of the Straw Yard-Warmth and Protection-On giving Hay and Turnips along with Straw to Store Cattle, and in breeding Farms : p. 368

\section{Chafter XXXIII.-ON THE FEEDING OF NEAT CATTLE (crmti- nued)-SUMMER SOILING.}

Advantages of Soiling-Objections to it-Conduct of Food; Yards; Tethering; and Hurdling-Experiments on the comparison between soiling and grazing - p. 372

\section{Chapter XXXIV.-ON THE FEEDING OF NEAT CATTLE (conti- mued)-WINTER STALL FEEDING.}

On Houses-Sheds and close Stalls-Sort of Beasts to be fatterl-Shape of the OxSkill in handling-Position of the flesh on the Carcass-I isposition to fat-Mard feeders-Age and breed-Produce of beef-Management in stalling-Proportion of foodTrials of different breeds upon oil-eake, turnijss, and hay-Comparative experiments on steamed and raw food-Most profitable size of the animal for feeding . p. 380

\section{Chapter XXXV.-ON FAT CATTLE.}

Butcher's Profit-Fifth Quarter-Offal, Hide, and Tallow-Description and judgment on the points of an Ox-Live and Ilead Weight--Measurement-Renton's and M'Dermot's Tables, and Cary's Gauge for ascertaining the Weight-Stewart and Ainslie's Tables-Douglas's mode of calculation-Accuracy of the Eye and TouchMode of Sale, and Estimates of Value in Smithfield Market . . . p.391

\section{Chapter XXXVI.-ON Milch COWS.}

Dairy Farms-Their advantages, and essential points in their estahlishment-Breed of Cows: Yorkshire; Long-horns; Suffolk polled; Devons; Kyloes; Alderney, and Kerry-Mixed Races-Form and qualifications of a perfect Cow-Temper-StockManagement-Construction of the Cow-house-Effect of food-On pasture, and when taken up-IVhen suffered to run dry-Time and act of milking-Choice of ServantsCramp's Cow-Quantity and quality of the milk of different breeds-Arerage product of milk, butter, and cheese

\section{Chapter XXXVII.-ON THE DAIRY-MAKING OF BUTTER AND CHEESE.}

Construction of the Dairy-house-Plans-Materials of the roof and flours-UtensilsCheese-presses-Churns-Superintendence of the Dairy-Management of the MilkAnalysis of its component parts-Mocle of ascertaining them-Maling of butter from cream alone, and from the whole milk-Corstorphine and clouted Cream; how madeMode pursued in making Epping Butter-Operation of Churning-Temperature of the Milk-house-Washing of the Butter-Operation of Salting-Pyety, or pinsuwed, Butter-Packing-Choice of Casks-Irish Butter trale-Manufucture of whole-milk Cheese -Remnet-Temperature of the Milk-Formation of the Curd; its pressure, and mode 
of salting and drying-Quality of Salt-Cheese-lofts-Racks, and machine for drying -Different modes of making, and value of Cheshire, Gloucester, Cheddar and IViltshire, Stilton, and Dunlop Cheese-Skim-milk Cheese-Cream Cheese-New CheeseForeirn Cheese: Gouda, Eidain, and Friczland, Parmesan, and manner of making it Account of the Saxony Cheese made of Potatoes . . . . . p. 404

\section{Chapter XXXVIII.-ON CALVES-THEIR REARING AS STOCK, AND FOR VEAL.}

Cows : their period of groing with Calf, and time of parturition-Abortion-Dropping of the Calf-Rearing of Calves, when meant to be brouglit up for Stock-Process of Weaning-Subsequent treatment-When fattened for Veal-Business of Sucklingr-Operation by hand-Age at which the Calf should be killed-Experiment on the weights-Expenses and profit on different weights-Modes of feediug around Lomilon; in the district of Strathaven; and at Abbey-holm in Cumberland-Dairy Estimates on the production of Veal; and on the manufacture of Butter and Cheese - p.438

\section{Chapter XXXIX.-ON SHEEP.}

Synopsis of the breeds-Age-Technical names-Procreation-Number of Ewes to be put to a Ram-Blossoming-Teasers-Description of a Tup-Period of Gest:tion-Yeaning-Management at that time-Lambs : their Castration and DockingGrass-Lamb, when in season-House-Lamb-London mode of rearing and preparing them for Market-Lamb-house--Lambs bred for stock-Time of Teaning-Management when separated from the Ewes-Feeding of Sheep-On dry groun-On wet land-With turnips-Pasturing on the Wolds and Downs-Folding-Formation of the Fold--Value of the Manure-Standing Folds--Sheep-cutes - Cost of building - Stells -Advantages and disadvantages of moveable and standing Folds-Shearing-W Washdykes-Operation of Washing-Clipping the Wool-Salving-Receipt for the Materials, and mode of laying it on-Shepherds--The Shepherd's Dog-Wool; combing and carding-Pile, staple, and felting property-Quantities of the different kinds of ITool in the fleece, and their assortment-Saxon and Spanish Wools-Trade of Ramletting-Tup-Masters-Rules for crossing the breeds of Sheep-Carcase-Quality of the flesh of different breeds-Age and food, as influencing the flavour of the meatIV eight of the several joints-Fatting-Experiments on the use of Sugar-Coiling Grazing-Breeding in-and-in-Cros,ing--Improvement of the form-ConstitutionDisposition to fatten-Diseases: Flies; Sturdy ; Sheep-lice; Scab; Sheep-pox; Foutrot; Red-water; Braxy; Blowd; Flux; Pining ; Rot; Hunger-rot; Blindness; Hoving ; Slipping of the Lamb; Sore Teats-Signs of Health . . . p. 453

\section{Chapter XL.-ON GOATS AND DEER.}

Formation of the Goat--Flesh of the Kid-Goat's Whey-Deer . . p.50t

\section{Chapter XLT.-ON SWINE.}

Breels : the Chinese; Rulyewick; Hampshire; Berkshire; Essex; Half-blacks ; Suffolk and Norfolk; Shropshire; Woburn; Dishley-Procreation-Progeny-Choice of a Buar-Farrowing-Rearing-Sucklers-Porkers-Store-pigs-Mule of ringingProduce-Number of Pigs maintained on arable and dairy Farms-IIanagement when for family use-Fatting-Time required-Quantity and quality of food-Inerease of flesh_Experiments on Rice and steamed fool-Bacon and Ham : cutting up; salting ; and drying-Smoke-houves-Modes of curing: in Trestmoreland; in Hamplite ; and in Wiltshire-Brawn-The Piggery-Plans, and management in different StyesDiseases: Surteit; Eruptions of the Skin; Murrain; Measles . . 1.506

\section{Chapter XliI.-ON POULTRY AND RABBITS.}

Fowls-Ducklings-Necessity of warmth - Turkeys-Dueks and Geese-Rahhit;, in a domestic and wild state-Warrens-Stock per acre-Food and produceSpecies, and assortment of the Skins-Capital, expenses, and profit, on establishing a Warren 


\section{Chapter XLIII.-ON FARM FENCES.}

II the planting and ditching-Different moles of operation-Value of Whitethom - Advantages of raising a heaithy aud quickly-effective Fence-Proper hejght-Cutting and re-maling Ifedres-1'runing and pleashing-Gapping-IFedge-row Timber-Lopjing-Dry Stune $\mathrm{VI}$ alls-Gates, their mode of hanging and fastening-Swing-gates-Stiles-Cage-wickets

\section{Chapter XLIV.-ON VERMIN : INSECTS-BIRDS: ANIMALS.}

Various species of Insects, and modes of destroying them--Earthworms-BirdsRooks; Owls; and Wood-Pigeons-Doubts whether they are more destructive or advantameous-Animals-Rats and Mice; best mode of destroying them, and keeping the barns clear-Field-mice-Moles-Difference of opinion respecting the effect of their operations upon the land-MIudes of destroying their nests; and of driving them outHedge-hogs . . . . . . . . . . I. 547

Chapter Xat.-ON COTTAGE ECONOMIY-COTTAGE GARDENS -BEES-ALLOTMENTS OF LAND-SPIDE IIUSBATDRY.

Old English fure of Cottagers-Change of System-The Cortager's Garden and its Cultivation-Nanagement of Bees-Allotments of Land-Dutch Pauper ColoniesSpade Husbandry: Expense of, and Crops produced by p. 554

\section{Chapter XLVI.-THE FARMER'S GARDEN.}

Growth of Asparagus-Sea-kale-Cauliflower-Brocoli-Dwarf Kidney-beansArtichoke-Celery-Endive-Red-beet-Winter-spinach-Cucumbers-Hardy Fruits - Saving of Seerls; and the quantities required for sowing different quantities of land -Culture of Potatoes, and prevention of their failure ? . . . p. 572

\section{APPENDIX.}

\section{No. I.-Abstracts from Acts of Parlianent relating to Husbannry.}

Right of Toting-Tithe Commutation Act-Peor Rates-Finclosure of Fields-Turnpike Fuads-Cirriages - Tolls-Duty on Horses and Dogrs-Cattle-Man-TrapsGame Laws-Duties on Corm . . . • . • . . P. 579

\section{No. II.-LandLord AND Tenant.}

Modes of Tenancy-Agrements-Entry, and Notice to Quit-Payment of RentScotch right of Hypothec-Distress, Rejlevy, and Ejectment-Seizure of Farming Stock

No. III.-On Weights and Measures.

Avoirdupois Weight - Wool Staplers'-Flour Factors'-Cheesemongers'-Hay and Straw-Sales by Tale and by Bulk-Scotch Measures-Imperial Standard-Liquid and Lineal Measures-Long Measure-Square Measure-English, Scotch, Irish and Customary Acres - Superficial Contents and Cultivation of Great Britain-Solid Measure.

\section{No. IV.-Remarks on the TVeather.}

The Barometer; Hygrometer; Thermometers of Péaumu, Fahrenheit, aud Rutherford-Causes of Dew-Phases of the Moon-Divisions of the Year . . p.592

$$
\text { No. V.-TVEEDS. }
$$

Glossary of the Common Sorts and Soil in which they generally grow • . p.594 


\section{ERRA'TA.}

\section{ADDIT IONAL IN VOL. I.}

At p. 132, n. for "francaises," road francaise.

147,1 . 3, for " $10 \mathrm{lbs}$ and $12 \mathrm{lbs}$," read $8 \mathrm{lbs}$, and $10 \mathrm{lbs}$.

$158,1.48$, for "£3 $6 s 6 d$." read $£ 26 s .6 d$; and at the lust line,

for " $£ 8210$ s. $6 d$." read $£ 8110$ s. 6 ( 6 .

153, 1. 9, after the word "inches," add in.

205, 1. 20, for "Ruet," read Ruck.

395, 1. 23, for "Mr. Hawden, in Kincardineshire," read Mr.

Hawden, of Lawhead, in East Lothian.

439, 1. 22, for "marble," read marl.

\section{VOL. II.}

At p. 27, 1. 19, for "Grey," read Greg.

$95,1.28$, for "fallowed," read followe d.

$119,1.18$, fur " to secure," read fruilless.

$\left.\begin{array}{l}\text { 163, 1. 34, and } \\ 402,1.30 \text {, }\end{array}\right\}$ for "Bralley," read Bradby.

178,1 . 1, for "field-oat," read feed-oat.

$250,1.51$, for " soil "read oil.

279 , in $n$. at foot, for 390 , read 590 .

280, 1. 1, for "ring," read gathering.

313, 1. 33, for "faulness," read foulness.

$340,1.18$, for "rigour," read vigour.

$419,1,1$, omit the following passage regurliug the branding of butter, as the Act of Parliament has been repealcd.

458, 1. 46, for "Gimners," read Gimmers.

465, 1. 10, for "Billingsby," read Billingsley.

501 , in $n$. at foot, for "Quar. Jour. o Agric. v. xi. p. 712," read Quart

Journ, of Agric., vol, ii. p. 712. 


\section{COMMIT'TEE.}

Chairman-The Right Hon. LORD BROUGHAM, F.R.S., Memb. Nat. Inst. of France. Vice Chairman-JOIIN WOUD, Es

Treasurer-WILLIAM TOOKE, Esq., F.R.S.

W. Allen, Esq, F.R. \& R.A.S. T. F.Ellis, Esq., A.MI, F.R.A.S.|Thomas Henry Lister, Esq. W. Roget, Sec. R.S., F.R.A.S. Capt. Beaufort, R.N., F.R. \& John Elintson, M.D., F.R.S. James Loch, Esq., M.P., Edward IRomillv, Esq., A.M. R.A.S. IIydrographer to the George Evans, Esq, M.P.
A Thomaliralty.

G. Burrows, M.D. I. L. Goldener, Esq. and J. Wh Lubbock, Es.

Peter Staftord Carev, Esq.A.M.| R.A.S. F.R. R.A., and L.S.S. M.A., Sir M.A. Shee, P.R.A., F.R.S. William Coulson, Edq. A. R. Gompertz, Esr., F.R. and Sir Frederick Maulen, K.C.H. John Abel Smith, Esq. M.P. R. D. Craig, Fsq. H. R.A.S. H. T. Dela Beche, Esq. F.R.S. G. B. Greenough, Esq., F.R. A. T. Malkin, Esq., A.M. James Manning, Esq. Dr. A. T. Thomson, F.L.S. T.T. Dela Beche, Esm., F.R.S. and L.S

Samuel Duckworth, Esq., M.P. Rouland Hill, Esq., F.R.4.S.

Samuel Duckworth, Esq., M.P. Rou land Hill, Esq., F.R.A.S.
B. F. Dunpa, Esq., A.Mf. The Right Rev, the Bishop of Bizht Hon. Sir

The Right Rev, the Bishop of Bart., M.P.
Durluam, D.D.
David Jardine, Fsq., A.M. The Right Hon. Viscount Henry B. Ker, Esq. Ehrington, M.P. W. Ehrington, M.P. Wiscount Henry B. Ker, Esq.
Sir Henry Ellis, Prin. Lib. J.T. Leader, Esq., M.P., M.
Brit. Mus. James Manning, Esq. Herman IIerivale, Esq. Thomas Vardon, Esq, A.M., F.A.S.
J. Whishe, Esq., H. Warmourh, Esq.
J. Whishaw, Esq., A.M., F.R.S. Sir W.M. Wolesworth, Bart.M.P. J. Whishaw, Esq., A.M., F.R.S. R.J.Murchison, Esq., F.R.S., F.li.A.S. The lkight Hon, Lord Nugent. Thomas Wyse, Esq., M.P.

\section{LOCAL COMIITTEES.}

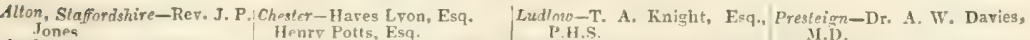

Anglesan-Rev, F, Williams. Rev. IV. Johnson.

Chichester-John Forbes, MI.D. FR.S.

Arthurer, C. C. Dendw, Fsq.

Barnetanle-1. F. Kingston. Fsq.

William Grihble, Est.

Relfrel-D-. Drummond Rilston-Rer. W. Teigh.
Birmingham-I. Corrie, Esq., Denhi-t -John Madocks, Esq. F. R.S. Chairman.

Pail M. Tames. Fsn., Treas Bridnori- ITm. Foreter, Esq. James Williams, Esq.

Bristn'-J. N. Sandere, Esq̨. Chairman.

J. Rexnolis, Ean., Treas.

J. B. Estlin, Fen F.L.S., Sec Calcutha-Sir B, H. Malkin.

James Youn $=$, Far

C. II Cameron, Fíq.

Cambridge-Rev. James Bowstead, M.A.

Whit rid.e.

Corfu-Tohn Cramford, Esq. Peirides. Thomas Evans, Esq. Den by-Toseph Strutt, E Edward Strutt, Esq.. M.P. Dermnert and StonehouseJohn Cole, E=n.

- Norman, Esa.

Lt.-Col C. Hamilton Smitl, F.R.S.

Dublin-T. Drummond, Esq t? E., F.R A S.

Edinburgh - Sir C. Bell F.R.S.L, and E. Etrutia-Jos. Wedmwond, Esq.
Rev, "rof. Hensiow, M.A.. Expter-J. Trrrell. Esq. John Milford. Es7. (Conter.) Corganshire-Dr. Malkin. F.L.S.

Rev. Jotin Londge, M.A. lev. Geo, Peacnck, M.A. F.R.

G'asgono-K. Finlay, Esq. M.A., F.R.A.S, and G.S." Alexander IIcGrigor, Esq. liev. Prof Sedgwick, M.A.

Tiev. C.Thirlsall. M.A. Guernsey-F. C. Lukis, Esq. Canterbury-Jobn Brent, Esq., Hull-J, C. Parker, Esn.
Alderman. William Masters, Esq.

Can'on-Wm. Jarúline, Esq., Leamington Spa-Dr. London,

Lecits-J. Marshall, Esq. Rev, C. Bridgman, \} Lexces-J. W. Woollgar, Esq. Rev. C. Gutzlaff, Sacs Limerick-Wm. Smith O'Brien, J.R. Morrison, Esq., Isq. M.'

Cardiran-Rey. J. Blackwell, Liverpool Local AssociationM.A. [F.R.S.E. V. WV Cocal Association-. rlisle-Thomas Rarnes, M.D.,

Carnarion-R. A. Poole, Esq. William Roberts, Esq. mian.

J. Mun,

J. Mulleneux, Esq.,
Rev. Dr. Sbenherd.

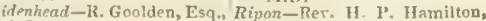
F.L.S. M.A. F.R.S and G S. Iaidstone-Clement T. Smyib, Rev. H. Ewart, if A.

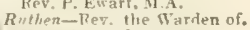

John Case, Esq.

Halmesbury-B. C. Thomas, Ryde. Isle of Wight-Sir Rd Esq.

Simeon. Bt., Mi.P.

f. W. Wnod, Esq., Chairmnn. Shefteld-J. H. A brahams, Esq. Beni. Hevrrod, Esq., Treas. Whentmo Mnllet-

T. W. Wiustanley, Esq., Hon. G. F. Burrounhs, Es?.

Sec. Philins. Bart., M.P. $\quad$ M P
Sir G. Phry-K, A.Slaney, Esq.

Genj. Gott, Esq. South Petherton-

asham-Rey, Georige Wad- J. Nicholetts, Fsq. dington, M.A. St Ascaph-Rev, Geo Sirong.

Iterthyr Tydv l-J. J. Guest, Stocknort II. Marsland, Esq.,

Winchinhampton-John G. Henry Coppnck, Esy. See. Ball, Es?. Siynth $\%$, Nere Sinith Wales-

Jonmoulh - J. H. Moggridge, William M. Manning, Esq. Es?

Teath-John Rowland, Esq, Thristock-liev. W. Evin

Nercastle Rev T. Turner. John Rindle. Esq.

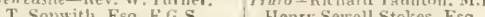

Netonot, Iste of Hight-Abr. Tunbridye Wells-Dr. Yeats.

Clarke, Esq.

T. Cooke, Jun., Eaq. Utlozeter - Robert Blurton, R. G. Kirktratrick, Esq. Esq.

velonort I'agmell -J. Millar, Warcick-Dr. Lonolly. Esq. $\quad$ The liev, Wr. Field, (Leam.) Nentown. Montgomeryshire - Naterford-Sir John Newport, W. P'ugh, Esq. Bart.

Forrich-Richard Bacon, Esq. Wolverhampton-J. Pearson, rscit, Esser - Dr. Corbett, Esq.

F.R. W orceste-Dr. Hastings, MI.D. forl-Dr. Daubeny, F.R.S., C.II. llebb, Esq. F'rof. of Chem. Hrexhm-Thomas Edgworth, Rev. Prof. Howell.

Rer, John Jordan, B.A. E. W. Head, Esq., M.A.

Pesth, Hungary-

Count Szechenyi. Varmouth-C. E. Humbold. mouth $\rightarrow$ H. Woollcombe, Esq., M.P.

Esq., F.R.S., Ch. $\quad$ Dawson Turner, Esq.

E. Moore, M.D., F.L.S., Sec. Hork-Rev. J. Kenrick, M.A.

G. Wightwick, Esq.

THOMAS COATES, Esq., Secretary, No. 59, Lincoln's Inn Fielus. 



\section{BRITISH HUSBANDRY.}

\section{Chapter I.}

\section{ON IMPLEMENTS OF TILLAGE-PLOUGHS.}

TiLlage, of which we have now to treat, embraces that portion of rural economy which consists in the production of corn, pulse, and every species of root and green crops which are obtained by the cultivation of the soil, independently of natural grass, and requires more practical knowledge of agriculture than those subjects which we have already discussed; for, although manure is, perhaps, the most important in a scientific sense, and blunders are not unfrequently committed by persons who are ignorant of its various properties, yet that knowledge can be obtained through reading, and may therefore be considered as chiefly theoretical. That which the land produces without labour is only trifling in comparison: it is by industry that the greater part of those sensual enjoyments are obtained which are procurable by wealth, and it is only by a continuation of that industry that their possession can be secured. Even the capital which is necessary to the development of our efforts can only be acquired by labour, and it is the amount and quality of that labour which determine the value of the thing produced. It must, however, be applied to some definite object, and the primary one is the soil; for, it being from thence that our subsistence is extracted, it is the original source to which all our powers of exertion in every human art are due. Political cconomists, however, so too far in attributing national wealth solely to labour, for it is evident that the soil alone contributes a considerable part in its natural productions. When cultivated, also, its products, if it be fertile, will be more valuable than if it be sterile; and if an equal number of inhabitants be located upon a given quantity of land, those upon the former will reach to afluence sooner than upon that of the latter description.

The operations of tillage are, it is well known, chiefly performed by means of the plough, the harrow, and the roller, though various other instrumentsto be licreafter noticed-are also used for pulverizing the soil and cleaning it from weeds; as well as a variety of machines for drilling and sowing the seed. From the impulse given to agriculture during the late war, the art of husbandry was carried much nearer to perfection; the construction of most of those implements partook of the improvement, and many new inventions are daily added to the stock. Much has, in this respect, been owing to the Scotch, both as farmers and as mechanics. The agriculture of Scotland was, until the middle of the last century, far inferior to that of England; but the soil of a large portion of the southern districts is of a very fine description, and, unlimited capital having been latterly applied to its cultivation, large tracts fell into the hands of men of extensive fortune, as well as of superior education, who adopted the profession of husbandry as a science, and thus had the means of pushing improvement farther than the generality of those English farmers who were already settled in the homes and habits of their forefathers. 'The encouragement thus

VOL. II. 
afforded to mechanism also necessarily occasioned the employment of the best artists, and there can be no doubt that the generality of farming imflements are constructed with more care, and formed upon surer mathematical principles, than those in common use throughout the greater part of Engrland.

That many of these new and improved implements have much lessened the labour of the farmers by whom they lave been employed, is certainly true; but it must also be admitted that the rage for jnmovation has been carried to such a length, that numberless new-fangled machines lave been invented with little else to recommend them than mere novelty, while they lave been in most instances so very expensive as to deter farmers who have thus experienced their uselessness from adopting anything new. This, added to the notable failure of several theoretic experiments, will, in great measure, account for that dogged arersion to what is called improvement which is attributed to most of them; and it is hardly to be wondered at that men who are now so severely pinched by the circunstances of the times, and the immense loss which has fallen upon farming capital, should look not only cautiously, but even distrustfully, upon everything which seems like clange of system. A strong objection is also to be found in the prejudices of the workmen; for, when they have become accustomed to an implement, it is found no casy task to dive them to the adoption of a new one. Of this no farmer who has tried the experiment can be ignorant: an instance is mentioned in the Stafford Survey, of one who moved out of Leicestershire, and took some of the ploughs and ploughmen of that county along with lim. "Good work was made so long as those men stayed; but when they left, they might as well have taken the ploughs along with them, for the Staffurdshire men could not plough with them." Arthur Toung relates a similar anecdote regarding lis own practice; for, when he went into Hertfordshire, he carried with him the same ploughs which he had on his farm in Suffolk, and for three or four years was obliged to set all his plonghmen from that county, as no Hertfordshire man would liold them; nor was the difficulty overcome until a couple of the natives were bribed to try them, after which they made better work than the common ones of the country.

\section{PIOUGHS.}

The common plough of many counties is, however, an exception; for, except on soils of a peculiarly deep or flinty nature, which require the strength and weight of very powerful teams, it has been generally superseded by some of an improved construction; but before we advert to any of lhese, it may be as well to describe the several parts of the suing plough, as it is called, in contradistinction to those which are mounted upon wheels.

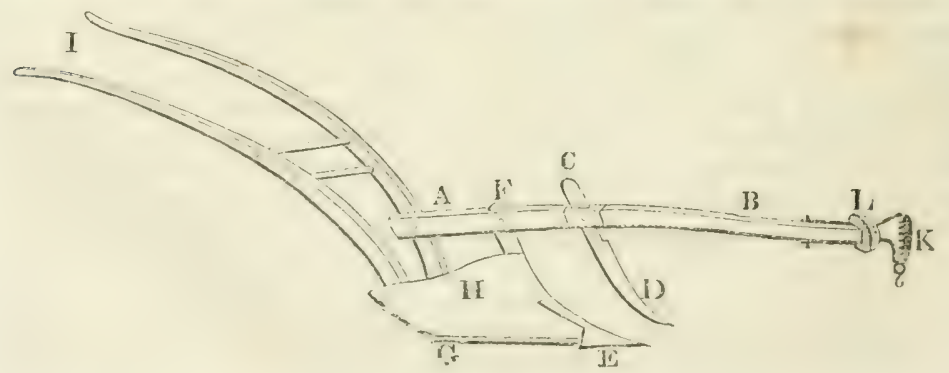


$A B$ is the beam; $C D$, the couiter; $E$, the sock, or share, which is made with a fin or feather, whereby the firm earth at the bottom of the furrow is cut more completely than was practicable by the old plough, the share of which was merely pointed. $F$ is the head, or sheath; G, the sole, or chep; and the heel, or fout, which in some ploughs is a continuation of one of the stilts. 'The mould-board is at $\mathrm{H}$; at $\mathrm{I}$ are the stilts; and at the end of the beam, at $K$, there $i$ an irom crank, called judifferently by the names of "copse," "cat-head," "bridle," or "muzzle," which is bolted through it in different ways to suit the various opinions of the makers, so as both to elevate or depress it, and to move it to different sides; but is gencrally fastencl with a moveable crescent, as at L.

The uses of these several parts are, that the beam serves as a line of connexion for the reception of the coulter, the head, and the foot of the implement, and thus combines its different members in one furm basis. It must, therefore, be male of considerable strength, and the mortises for their seception should be placed nearly in the centre of its breadth. The couller can be moved up or down through the mortise in the beam, as circumstances nay require, and is formed of iron, like the blade of a kinife, the front being edged with steel, and shapened so as to facilitate the cutting of the sward and the breaking up of leys, or land abounding with reeds. The share, which is attached to the sole and sheath, penetrates and tums up the soil under the coulter to such a breadth and depth as it may be judred cxpedient to stir it; and the mould-boctrd, which is inclined in a slanting direction to the right, lays the furrow over to that side. The sheath, when the implement is partly consiructed of wood, is defended by thin plates of iron, to cause it to move smoothly along the land side without allowing loose carth to fall in and encumber its comree. A thick piece of cast iron is also put upon the lower part to protect it as being the most subject to friction, and thus forms the sole; and, according to the best rules of construction, the point of the share, point of the fin, and heel of the land sile, shoukd all tonch a level surface. Whatever kind of share be used, the rake or sweep made by the head should also correspond with that formed by the coulter; for the one is intended to follow the other as closely as yossible, and, if the two parts are not parallel, a partial action is given to the instrument.

The muzzle, to which the harness of the team is hooked, is notched, so as to admit of altering the position of the plough, and this regulates the draught, either by giving it more or less land, and fixing the depth at which it is to be worked; and the stilts are the handles by which the ploughman holds and guides it during the operation of plotighing. When a pair of stilts are used, one usually forms part of the foot, and the other is firmiy riveted to the moukl-board, both being conbined by cross pieces, and thus adding considerably to the firmness of the instrumcit. In Norfolk, however, and latterly in other counties, it is nuch the custum to employ only one fixed stilt, attached to the beam, as the ploughman is thus prevented from bearing his weight upon them and increasing the draught; and, in stony land, there is the further objection that accidents sometimes happen to the ploughmen by their recciving severe blows from resistance oftered to the plough when walking between the stilts. A loose handle, called a plough-staff, is, however, generally attached for occasional use, when necessary, to aid the motion of the implement, on which it acts as a lever, and is furnished with a hook to clear the mould-board and coulter from weeds.

Formerly the plough was a cumbrous machine, made with little regard to the principles of mechanism; for it was considered that an 
instrument for executing so coarse an operation as that of turning over the crround, and which was committed to the hands of ignorant peasants, required but little nicety of form. The work was, therefure, in most cases imperfectly done, aud the expense of working such defective implements was an evil of serious magnitude, for they were almost invariably drawn by a power at least equal to that of four horses; whereas now, on the improved plans, they can in most cases be manarged with a pair. In some places, however, the ancient form is still continued, though with such various modifications of its different parts in order to suit the nature of the soils and the notions which are entertained of its efficiency, that a jarticular account of them would be tedious; we therefore confine our description to that one so well known and still so grenerally used in the county of Kent, here delineated, under the name of the

TURNIVIEST PLOUGH.

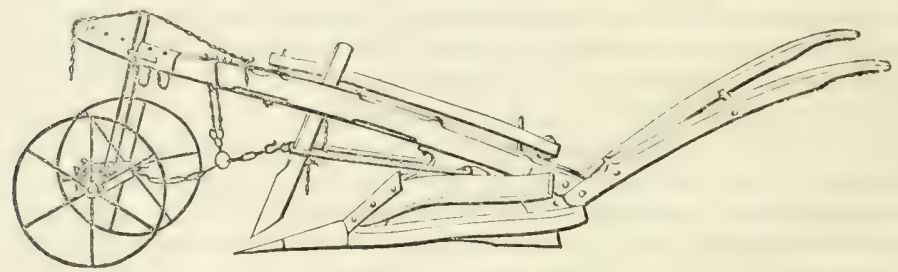

It consists of a beam of oak, 10 feet long, by 5 inches deep and 4 broad, behind which is a foot 5 inches by $3 \frac{1}{2}$, and $3 \frac{1}{3}$ feet long, on the top of which the stilts, or handles, are placed; the foot is tenoned to the end of the beam, and mortised at the bottom to the end of the chep. Through the beam, at 2 feet 5 inches distance from the foot, is a sheath of oak 7 inches wide by $1 \frac{1}{2}$ thick, which is mortised into the chep, or sole, in an oblique direction, so that the point of the share is 22 inches distant from the beam. The chep, to which the share is fixed, is 5 feet long, 4 inches wide, and 5 deep. The share is of hammered iron, weighs about 32 pounds, is 20 inches long, and from $4 \frac{1}{2}$ to 7 inches wide at the point.

The upper end of the beam rests on a carriage with two wheels, 3 feet 2 inclies ligh. On the axle-tree is a gallows, on which is a sliding bolster to let up and down. Through the centre of the axle is a clasp iron, to which is fixed a strong chain, called a tow, that comes over the beam so fised, as, by means of notches (or a pin called a chick), to let the whole plough out a greater length from the axle, thereby letting it down to a greater depth*.

* An extraordinarv $i$ ustance of its strength and power upon stubborn leys is mentioned hy Mr. Malcolm in his' Account of the Agriculture of Surrey,' in which he says " that in breaking up the side of a very sharp hill full of large holes, inequalities, and irregularities, having strong roots of furze, fern, sallow, and stones of six or eight inches thick, it took eight horses or twelve oxen to perform it. The soil was a good loam in some places, in others a clay, but the whole on a substratum of blue clay. It required two able men to hold the plough; one, and sometimes two, to bear upon the beam, in order to keep the plough in the ground ; and two drivers. Yet with this force, and notwithstanding the continual sudden jerks which the plough received by the inequalities of the soil, every part of the tackle stood well, and nothing broke. It was very apparent that in this rough work the oxen were far preferable to the horses: the former, by the slowness and regularity of their motion, avoided the small holes, and did not tumble about the larger ones; the horses, on the contrary, from their impetuosity, were always falling, beiug scarcely recovered from one hole before they found themselves in another, which matde them fret and jerk-injuring themselves, destroying their harness, and causing the plough to gro in all manner of airections. It was not the fault of the plongh ; for cvery 
Such is the description given of this plough by Mr. Boys, in his Survey of Kent, and it differs but little except in being more ponderous and mo:c clumsily formed by country ploughwrights than the implement depicted in the preceding page.

Alogether it is certainly very heavy, and from its construction muat ise made very stout, as otherwise either the beam or chep will break with the force of four strong horses, when it comes suddenly against a rock, the root of a tree, or any unusually stiff part of the land. It is, however, remarkable for going well amongr flints, which abound in some yarts of Iient; the soil may also be turned up with it to a very great depth, and laid quite flat without any kind of inter-furrows being left open; for it is worked with moveable mould-boards, which can be shifted to the left or right side, and can, consequently, turn a furrow to either. By removing its share and coulter, and substituting a broad share-perhaps 20 inches wide-it can also be made to act as a scufler, or as a paring-plough. These certainly are no small advantages in a dry soil; and it is, indecl, so greneral a favourite throughout Kent, that many of the improved ploughs which have been recently introduced have been laid aside. Mr. Boys says decidediy, "that it is, for all sorts of soils, and all required depths of plounhing, the best he has ever seen tried:" an opinion which we have known to be confirmed by many very extensive and intelligent farmers.

On light soils three horses are found to be sufficient, and an acre and an haif is not uncommonly ploughed by it in the day's work; but in that part of the county called the Weald, it is necessary, in consequence of the tena. city of the soil, to make the ploughs much stouter, and to use, in some instances, six horses, the plough being there drawn by a large iron lint, which comes from the axle of the carriage round the heel. We recollect, indeed, when visiting that district some years ago, to have secn as many as six very powerful Sussex oxen, yolied in pairs, brought to a dead stand still in breaking up a clover ley, and not able to move until the plough was set in a more shallow furrow: very little more than half an acre is there the common work of the day.

The great Hertfordshire whel-plough is also an implement of ncarly similar form, except that it is furnished with a deep mould-board, and is it great favourite among the farmers of that county for the operation of breaking up strong finty fallows in a dry season, for which diflicult work it is well calculated. "Here, however," as has been observed by Arthu" Ioung, " its merit ends; for all other work it is a heavy, ill-formed, and ill-going plough. The faults are numerous: heavier than necessary for every other work, they are all so pilched that the ploughman universally walks on the unploughed land, resting nearly all his weight on the handle., his body moving in an angle of forty-five regrees with the horizon. The share, joint, and fin (which latter is placed on very backward, to cnable the point to work among the stones) are at unequal levels; three or foul inches of the furrow next the unploughed land are cut three inches diejer than the rest of it; so that when the stirred moulds are turned away, in order to examine the unploughed land beneath, it is found all in grooves and ridges: worse work can scarcely be imagined, while the surface is left apparently very well and neatly ploughed *." It is easy, indeed, to inagine what unnecessary toil must be occasioned to both men and horses by such

sort that could be procured for several miles round was tried against the tumwrest without the least chance of success, but the greater part of them were broken by the exertion, and not one of them did the work so well."-vol. i. p. 194.

* Survey of Hertfordshire, p. 36. See also Batchelor's Bedfordshire, p. 163. 
unvicldy construction, as well as by the weight of the plough; the shares of which weighed formerly as much as 60 to $70 \mathrm{lbs}$, though now reduced to somewhat less than 50 .

On this it has, however, been not improperly remarked, "that yersons accustomed to a light-land country too frequently pronounce hastily on the power required, as well as the nature of the instrument most proper for working with effect in stronger soils, and with which they are in a great measure unacquainted. The surface of a clay loam is often observed, after it has been acted upon by the winter's frost, to be loose, tender, and friable ; a decision immediately takes place on the obstinacy and folly of the farmer for applying a power unnecessarily expensive to the tillage of his land: whereas, would these rentlemen condescend to examine the subsoil of such a field, or talie hold of what they term an unwicldy apparatus, that may be at work unon it, they would soon discover that it is not the loose surface of one or two inches in deptl that the plough has to contend with, but that the macline must pass through a subsoil of tough, strong, and perhaps stony Ioam, and that little short of the power employed would be able to effect the purpose*. Weight, as well as strength, is, indeerl, in such cases so necessary, that we have lately seen a lad sitting upon the end of the beam of a licrlit swing plough, to keep it down in the furrow, while ploughing a deep clay; when made to get down, the implement occasionally twitched out of the oround on its meeting with any extraordinary impediment, and the ploughman declared that, unless thus weighterl, he could not otherwise keep it steadr. That improvements may be made upon these ancient ploughs is froved by daily experience, but it also clcarly demonstrates that those principles should not be altogether departed from, the utility of which has been established by the practice of ages. In all tough and stubborn clays which, on their breaking up, are meant to be ploughed clean, and to a full depth, machines of strength and steadiness of draught are indispensable; though, in the subsequent ploughings of such land, lighter implements, requiring less force, are generally made use of.

\section{SMALL'S PLOUGH.}

The first improvement of much note was that of a light swing plough invented upwards of a century ago in Forkshire, whence it obtained the name of the Rotherham plough, and is very generally employed throughout that county, and many other parts of England. Its extreme length is $r$ feet $t$ inches, and the weight of the wood and ironwork $1 \frac{1}{4}$ cwt. It differs also greatly from all the common ploughs of ancient construction, in being more slightly as well as more neatly constructed, and having the coulter and share formed and placed so as to raise, and then gradually turn over the new cut furrow much cleancr, and with less resistance, than the others.

This plongh was the only one worked with a pair of horses abreast, until the vear 1764, when Mr. James Small, a scotch mechanic and farmer in lerwickshire, improved upon it by inventing the iron mould-board, or mouldiron, which turns the furrows cleaner than that of wood; then the land-side jlates, sheath and head, were made of cast-iron, and eventually the whole machine has been formed of metal. He thus-alihough no mathematician -made such progress in perfecting the construction upon sound mechanical principles, that his swing ploughs are now very universally employed with a pair of horses and whip reins, without a driver, and on an average of soils plough an acre a day with ease.

* Vancouver's Hampshire, p. 91. 
Its chief merit consists in the fore-part being formed in such a slender wedge-like manner, as to cut the plit from the land with the least possible resistance, while the thin feather-sock under-cuts the furrow-slice with equal ease. The gradual curvature of the mould-board from the point of the sock to the heel of the wrest also lessens the friction in elevating and turning the furrow-slices, and thus renders the operation of ploughing so much less difficult than it formerly was, that it was proved by an experiment made before the Dalkeith Farming Society, that Mr. Small's plough, in tearing up an old ley, was drawn by a force of from 9 to $10 \mathrm{cwt}$., while the old Scotch plough required one equal to $16 \mathrm{cwt}$ to perform the same work * ; it has also been successful in similar competitions in many parts of both England and Ireland, as well as in Scotland, which it is here unnecessary to detail. It, however, fell short of the perfection aimed at, and lias since undergone many modifications, almost every ploughwright having his own peculiar cast of some alteration. That by Wilkie is certainly a material improvement, as it turns up the furrow with a bold shoulder, like that for which the Scotch plough was so much valued: its shape, like that of a welltapered wedge, turning over the soil with very moderate powers; and its broad-winged share leaving nothing unstirred.
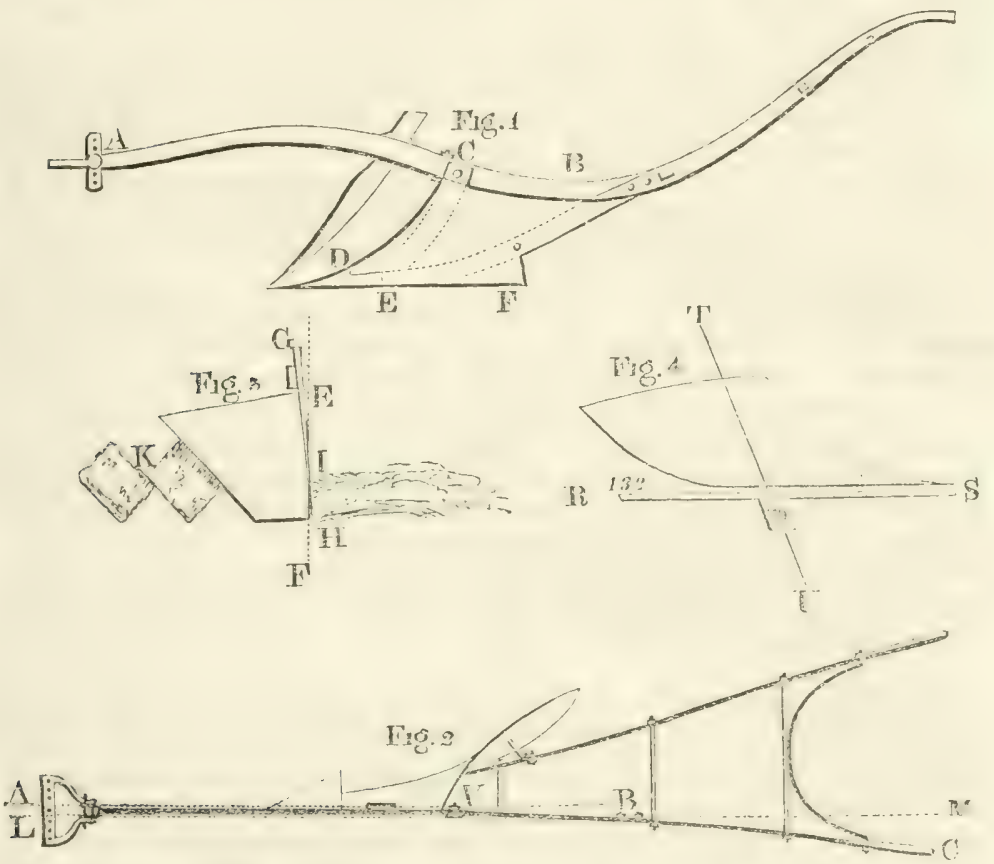

This plough,-which, although partly altered, still goes by Small's name, - of which fig. 1 is a profile, as described by Mr. Willie, although entirely of iron, weighs less than a hundred and a half. $A$, the beam, is $6 \frac{1}{2}$ feet longr, and $2 \frac{1}{2}$ inches deep, by 1 inch thick: at the coulter side it is $\tilde{3}_{2}^{1}$ inches deep and 13 inch thick. The sheath is fixed to the beam by two screw-bolts; one going up through the beam at $\mathrm{C}$, the other

* Gen. Rep. of Scotland: Append. vol, i., p. 354. 
at $\mathrm{V}$, fig. 2. The stilts are 2 inches deep, and $\frac{\mathrm{I}}{2}$ an inch thick, and connected by slight iron rods. D is the sock; and L F two screw-bolts by which the head is fixed upon the plough.

"The beam is wrouglit quite straight on the land-side, and tapered towards both ends on the furrow-side, as represented by the dotted lines $A B$, in fig. 2. By forming it in this manner the mortise for the coulter is placed more to the right of the land-side plane, than if the taper were taken off the land-side. The advantage of this position will be understood by consulting fig. 3, which is a cross section of the plough; the dotted line E F being the land-side edge of the sheath G II; the coulter intersecting the land-side plane at $I$, about six or seven inches above the sole, that being the depth of an ordinary furrow. The plough, in that case, has no twist, nor projecting parts on the land-side to give resistance to its motion, except the point of the coulter itself, standing lialf an incls to the left of that of the sock at II, which is necessary for clearing the land-side of the plough, and also to give a greater depth and boldness to the shoulder of furrow at $K$; the furrow having, in that case, the advantage of being cut, and of standing, when turned over, from ten to fifteen degrees above the square; which position of the furrow has the important advantage of perfectly covering the seed.

"The left handle at $\mathrm{C}$ stands seven or eight inches to the left of the land-side plane, as may be seen by the dotted line L M, in fig. 2: which position, together with that of the coulter, as at G H, fig. 3, gives ever: pos. sible ardvantage in cutting the furrow with a bold shoulder. The plutighman is thereby permitted to walk with ease in the bottom, and betwixt the handles, leeping the land-side plane of the plough perpendicular to the horizon; whereas those whose left handle is in a straight line with the landside plane of the plough, must be laid over to the land-side above, to enable the ploughman to walk in the furrow."

This position of the left-liand stilt is indeed one of the principal improvements which it possesses; for another advantage, on which Mir. Willie insists, that-" where the plough is laid over to the land-side above, the point of the coulter is turned more towards the furrow, and from the land-side; of course the shoulder of the furrow which it cuts is not so bold and full; the friction of it is increased; the mould-board is kept too far from the furrow slice, and on that account does not set it in a proper position"-llas been disputed, and appears subject to much doubt*.

"The form of the seed-furrow mould-board-as designated in fig. 4-is curved upon a scale divided into 132 degrees, marked in divisions of 10 degrees each, as at $R$ S, and its twist is delineated by the distance at which

* MIr. Wilkie supports his statement regarding the position of the stilts, by laying it down as a fixed principle, that the land-side of the plough ought to stand perpendicular, and that all its other parts should be so constructed as to preserve that position while at work, so far as circumstances will admit; and he proves that it is only practicable to preserve that position by placing the left handle seven or eight inches to the left of the land-side plane. Thus - "Let the ploughman stand in the bottom of the furrow, immediately behind the plough, the land-side of which is standing perpendicular; raise a prerpeudicular line from the edge of the furrow where the land-side of the plough has just passed, and it will strike the man about 5 inches to the left of the centre of his body. Now, let him extend his arms to their natural position, and it will be found they reach to 12 or 14 inches on each side of the centre. According to this rule, then. I regulate the position of the left handle-from the centre to the land-side plane is 5 inches, and from the land-side to the outside of the handle 7 inches, makng 12 inches in the whole. The right handle may be placed about 30 inches from the left, as convenience or fancy may dictate."-Furm. Mag., vol. xii., p. 341. See also vol. xiii., pp. 73, 148, 303, and 444; and the Append, to the Rep. of Dumfriesshire, No, xxi. 
those degrees are placed from each other; the divisions being large towards the point of the sock, but becoming gradually closer until No. 90, where it is understood the furrow has gotten perpendicular, after which the curve is regularly diminished. As the twist is regulated by a common level, the blade must stand at an angle of 110 degrees from the square of the stock, when applied to the sole of the mould-board, as at T U." We shall not stop to inquire whether this is upon true mathematical principles, for mouldboards must be made of different sizes and construction, to suit the nature of the land on which the plough is to be worked. The great difticulty in the construction of a plough is that of adapting it to all soils, in all seasons, and to all depths. If the soil break up in whole furrow, every inch of depth requires, in strictness, a separate plough, or a separate mode of regulation; and this curve in the mould-board, admitting of no such regulation, forms a serious objection to its use. If the semi-arch, or hollow of the hind-part of the mould-board be raised sufficiently high to turn a thick furrow completely, it is of little use in turning a thin one; on the contrary, if it be brought down sufficiently low to turn a shallow one properly, it is impossible to turn a leep one with it in a workmanlike maner*: thus one that is calculated for ploughing a free and loose soil, and for working a fallow completely, is by no means adapted for making neat work on lea land, or in a clay soil. Every farmer should therefore have two sorts of ploughs -one for fallow, and another for seed-furrow work; or should have two mould-boards fitted for the plough, which may be changed at pleasure by the ploughman, if it be made of iron. Those, however, whose pursuits and wishes for information may lead them to further inquiry upon the subject, we refer to the ingenions reatise of Mr. Bailey of Chillingham-which may be found in the Northumberland Report-as well as to the writings of Messrs. Moore, Arbuthnot, Brown of Markle, Williamson, Finlayson, and Professor Low + .

Previously to Small's time, it seemed, indeed, as if men of science were cither insensible to the defects of the ploughs then in use, or imagined that they were incapable of being formed upon more correct principles; for their construction was generally left to ignorant mechanics, who were not alone unskilled in any calculation of their powers, but also interested by their prejudices in opposing all innovation upon that which they had been previously tanght. However rude the operation may be, it yet requires very considerable judgment in its execution; and the implement is one which, though apparently simple, is still so complex in its powers of application to the soil, that it affords ample scope to the ingenuity of the most learned mathematician. The art of its construction is indeed only to be attained by experience; for the difficulty of fixing its principles, and reducing them to such a regular theory as may suit all cases, appears insurmountable. Like every other operation in tillage, the instrument, as well as the process of its use, must vary according to circumstances, as no plough las yet been, nor probably ever will be constructed, superior to all others in every season, and on every variety of soil and situation. Although the principles of construction may be the same, yet the form must be partially

* See Marshall's Rural Economy of Yorkshire, vol. i., p. 258.

+ An improvement of the mould-board has been also suggested by Mr. Jefferson, formerly President of the United States of America, and formed upon the idea, that, as the bottom cut of the furrow is, or ought to be, perfectly flat, the breast, which comes in direct contact with it, should be flat also. On trial by the Board of Agriculture, it was however found liable to this objection-that from the concavity, or flatness in the fore-part of the breast, the loose earth of the furrow was apt to rest upon it,-Essr.x Rep., vol, i., p. 138. 
varied according to the particular nature of the soil, the purposes, and the local habits of different districts, and there is no one plough, whatever may be its general merits, which is equally suitable to every kind of land; it should therefore be the study of mechanics to adopt such forms of the different parts, whether of wrought or cast iron, as may adapt the plough to different sorts of ground when fixed to one frame.

By the frame we mean that part of the plough forming the land-side and nose-piece upon which every other part may be fitted and screw-bolted. This would admit breasts or mould-boards of various shapes, to suit the land on which they are intended to be cmployed; and these breasts might be set out to different widths, so as to open a wider or a narrower furrow, and might be removed and replaced by the ploughman when required. This improved facility has indeed been sticcessfully applied by those eminent makers, the II essrs. Ransome, of Ipswich, to both wheel and swing-ploughs of every description; the variation of the beams and whoels making the difference of applying them to their work. They for some time made all their ploughs with iron beams and handles; but it was found, in practice, that they did not answer so well as those of wood, for they twigged-that is, vibrated, in their work-and occasionally bent, so that the ploughman did not feel them so steady in the liand, and they were also more difficult to repair. The fashion of iron plonglis has indeed gone so far out of use in this part of the country, though still general in the North, that in a late ploughing match, which we attended a short time since, in the county of Surrey, out of forty plouglis of various kinds, which were brought to the ground, there was not one formed wholly of iron.

To this they have also adied another very material improvement, which has obtained the approbation of the Bath and West of England Agricultural Society, and which consists in tempring cast-iron plough-shares to an even hard surface, from 1-16th to $1-5$ sh of an inch on the under side, similar to a thin layer of steel. Ploughshares tempered in this way wear to a thin cutting edge while at work; for the upper surface, retaining its oricrinal softness, wears away faster than the lower one, and thus leaves a sharp bevelled edge of the hardened metal, thimer and sharper than can be produced by any other method of tempering. On this subject we can speak confidently, for we have compared shares of their composition with those of other manufacturers, and have found them superior; but their general use by other ploughturights is not thus prevented, as we understand they are sold by Messrs. Ransome independently of the ploughs.

\section{RANSOME'S PATENT PLOUGHS.}

One of their ploughe, which is now in very general use throughout the milland countiss, as one of the many improvements upon the principles adopted by Wilkie, Veitch*, and other modern manufacturers, and can be drawn either by a pair of horses abreast, or with three or mure in line, is a compact tool, of the following description-as representing both sides of

* A description of AIr. Teitch's plough may be found in the Edinburgh Encyclopxdia: and also, with an encravin , in the fiouth volume of the Transactions of the IIighland Society, in which it is favourably spoken of. It is longer than Small's plough, and has, on that account, been preferred in some parts of England; but the principles upon which its suppostd imporements are demonstrated, have been combated by $\mathrm{Ir}_{\mathrm{r}}$. Bitiley. of Chillingham, who clams the merit of the invention as due to his own treatise.See Currespondence between the parties, in the Farmer's Magazine, vol. xii. pp. 182, 504 , and 511 . 
the implement-which we have lately had frequent occasion "of seeing at work with great advantage, on both heavy land and light soils.
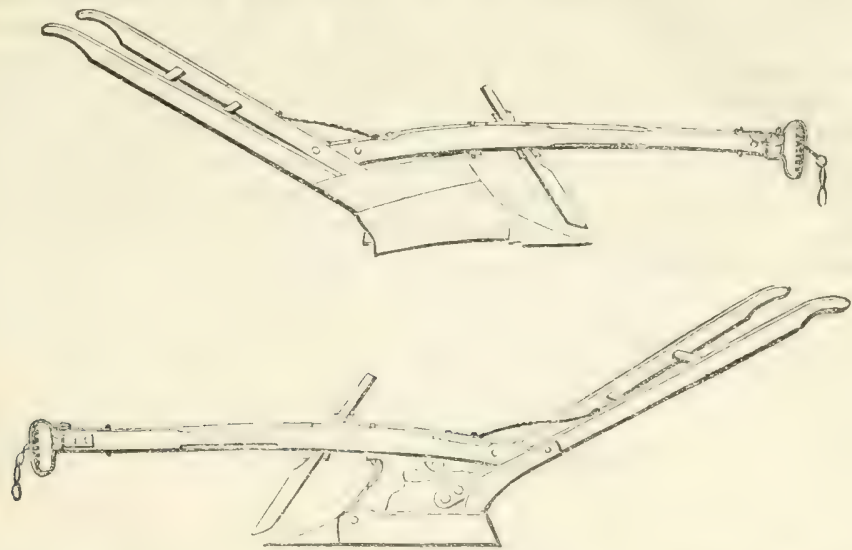

In stating this, however, as our opinion, it is only candid to say, that in two county ploughing matches which we have recently witnessed, in which there were employed a grent variety of ploughs by diferent makers, some drawn by horses in pairs, or three in a line, and others by oxen double-yoked and in harness, the work was in general so equally performed, that it was very diflicult for the judges to assign any peculial merit to the construction of the implement. On trials of this kind it should, however, be remarkad, that the machine which is commonly used for measuring the draught is made on the principle of the graduated spring steel-yard, with an index, the vibrations of which are taken upon an average, as the inequalities of hardness in the ground, and the irregular pulls of the horses, seldom allow it to remain stationary. The relative pace of the teams must also not be disregarded; for if one pair of horses walk after the rate of two, and the other three miles in an hour, the conclusions, which refer only to the index of the steel-yard, may be directly contrary to the truth. Another source of error may be found in the different degrees of skill in the ploughmen, which, in the opinion of many, is more conspicuous than the different merits of the ploughs; and,indeed, so much of the utility of the instrument depends upon the strength of the team and the dexterity of the ploughman, that it cannot have escaped observation that the same individual ploughs have in numberless instances repeatedly won, and as repeatedly lost, the prizes at the agricultural meetings. The relative resistance of the various depths and widths of soil, both at the bottom of the furrow and at the surface, is also a necessary, and, indeed, should be a primary object of investigation. It need scarcely be observed that the soil should also be in a medium state of dryness, and as free from stones and irregularities as possible. The experiments should also be made on different sorts of land, in order to ascertain the powers of the various forms of construction as working tools; whereas, in general a piece of tender ley is marked out for the ground of competition. Perlajs, also, in trials of this kind, a one-furrow wheel-plough would be the most eligihle, as it might he set at various depths and widths, and depend but little on the exertion of the ploughman*.

We have been partly called to make these remarlis by the observations

* See remarks on some trials in Bedfordshire-Batchelor's Survey, p. 168. 
which we made at the ploughing matches to which we have just alluded, in one of which a heavy Kentish turnwrest performed its work quite as well as the lightest swing-ploughs in the field, though the soil was gravelly, and the furrow hardly more than four inches in depth. On a lieavy band farm, on which we are residing, we however see very different results; for there Scotch iron ploughs have been buried in the furrows, when it was found necessary to plough the ground to a certain depth, and they have been thrown aside for every kind of heavy work. For clay-land we have, indeed, found another of Ransome's far better andinted to the soil than that which we have already described, as it worlis freer, and carries a deeper furrow with more ease.

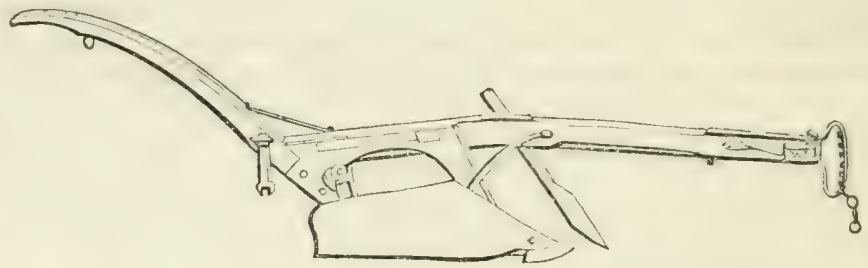

It however requires a powerful team; and, if it has a fault, it requires to be higher in the beam for soils of that description; for, when the gromb is ploughed deep, it breaks up in large clods, which gather under it and choke the muzzle : though we must admit, that if the beam be mounted too high, it occasions an increase of draught, and the plough will not so easily cut a flat furrow, nor go close at heel. This, indeed, we have seen correcterl in one from the manufactory of Jefferis and Co., of Guildford Strect, Suuthwark, upon nearly the same plan in every other respect, except that the muzzle is fixed laterally to the end of the beam, with a pivot to elevate or depress it-instead of being placed perpendicularly, as on Ransome's-with a serew to alter its position to the land or furrow-side, in order to regulate the line of draught: the effect is, however, quite similar.

There are various modes of regulating the pitch of the plough. Thus it may be made to go deeper by lowering the back-bands, or increasing the distance of the horses, by setting the muzzle higher up in the index of the beam, and by slanting or giving the coulter a greater rake forwards; and the reverse will make it go shallower. It can also be constructed with a regulating lever, which may be attached to any of the foot and wheel-plouglis now in use, and can be used occasionally, or otherwise, as circumstances may require. The side motion may be thus altered so as to make the plough take a broader slice, or, as it is commonly called, "to give her more or less land :"by putting the hook of the traces into the notches of the muzzle more towards the unploughed ground, you take land from the plough; but by shifting it to the furrow side, you give it land: it ought, therefore, to be made about eight inches in length, and may be fixed either to the sides of the beam, or to the top and bottom, as here described.
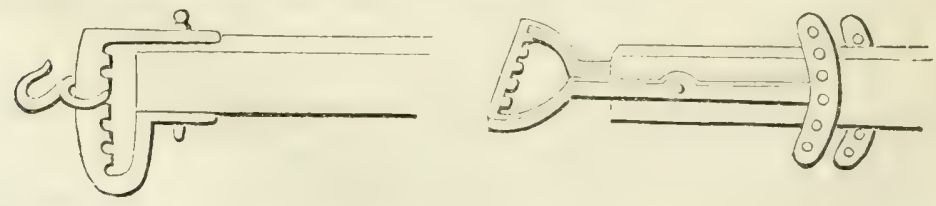

* See observations on Draught, in Chapter IV. 
The ploughman may also give more brealth by pressing the stilts towards the right ; though it is a bad plan, and requires too much exertion to be continued throughout a day's work. If the line of draught be not then true, the error will be discovered hy more earth being thrown up on one than the other side; or, as labourers say of the plough, by its "not riding fair," which it will not do if it does not move forward in a straight direction : in which case it will be proper to give the muzzle a cast in favour of whichever side may have too much or too little hold of the soil.

It would lead us too far to delineate the numberless ploughs which are used in different districts, and we have therefore no intention of mentioning those made by other eminent manufacturers, - although many of them are well worthy of attention,-yet it would be injustice to withhold the description of one of those invented by the late Mr. John Finlayson, of Muirkirk, which has received strong marks of approbation from many intelligent farmers in different counties of England and Scotland, and is called the

IMPERIAL SELF-CLEANING, OR RID-PLOUGH.

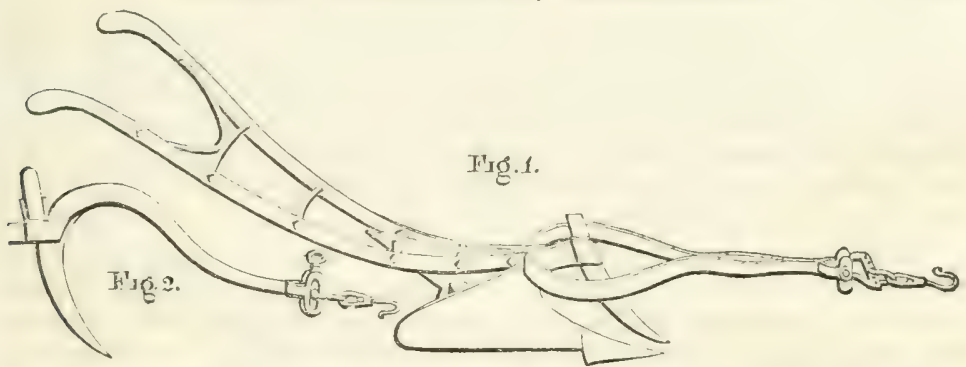

It is formed entirely of malleable and cast iron, the mould-boards and plates being of the latter; and it is stated in Mr. Finlarson's account of its merits, that, " besides being guided with less exertion, and making better work, more may be performed by it in a given time, and with the same power, on any soil, and the double or triple on wet, rough, foul lands." Indeed on rugged, uncultivated ground, it certainly has been shown to have a decided advantage, when used in several instances in competition with Small's or indeed any other of the common swing-ploughs with which it has been tried*.

"The coulter and beam, as in $f \mathrm{ig}$. 1 , furm a semicircle of about 22 inches diameter, with the concavity towards the muzzle; i. e. taking the coulter, from within 6 or $S$ inches of the point, for so many degrees, and the heam, from where it is inserted to about twenty inches forward, for the remainder.

* In a trial made in presence of the Dalkeith Farming Society, upon a field of clover ley, along with a very well-constructed plough on Small's plan, the work of the rid-plough was fuind far superior to the other. A second trial was then made upon very rough, peaty meadow-pround: when the rid-plough went nine rounds, laying out the furrow in the most perfect manner, while the other plough only went two, and that not without the assistance of a man to clear the rubbish.

The Carrick Farmers' Society have also certifiel "that in ploughing rough, coarse land, or cross-cutting fallow, they consider it greatly superior to any other construction of plough they have ever had an opportunity of seeing at work." It was also tried in Berkshire, in competition with various other ploughs, with similar suecess, "ploughing up heath and furze with as much apparent ease as if it had been ploughing a stubble-field; while the common ploughs left scarcely a vestige of tillage." An acre of stubble-ground has also been ploughed with it, in good style, at Sillwood Park, Sunninghill, in three hours and twenty-eight minutes, with a pair of small horses; and another of heath, at 'Titness Park, with the same cattle, in thre hours and forty minutes. 
The other half of the boam makes a regular round-turn, downward and forward, to the muzzle." It will, however, be observed in fig. 2, that another form of the beam has also been adopted by Mr. F. "The coulter, from above the cutting part, has a triangular piece of plate-iron welded on its front, the edges of the two longest angles of which are turned back till they join both sides of it. It swells gradually till it reaches the beam, a few inches of which it cosers, and when the coulter is let down, prevents a niche being opened between the two. The mortise for the coulter is often chamfered a little to the furrow-side, to give the plough what is commonly called a 'good cut;' and it is brought pretty near the one receiving the sheath, that the coulter may have a proper ralse forward, without unduly lengthening the head and share, or sole, which, for ordinary purposes, is about 33 inches."

"The front edge of the different mould-boards malies a circular sweep, from the share to that part of the beam which receives the coulter."

"The share, in place of having a hose, or sheath, for receiving the head, has itself a tang, or maundrel, that goes into the head; which arain is entirely covered by a cast-metal sole, or shoe. It is made about 12 inches long for a free-working earthy soil, from its point to the point of the mouldboard, with a straight-edged feather, or fin, going of at an angle of 30 degrees. As the lamd approaches to moss, the feather is made longer and more acute; and for cleau fibrous moss it is made 30 or 34 inches long, at an angle conforming to the spread of the mould-board-of 20 degrees with the land-side, and fitted with a moveable pin, standing vertically, for cutting out the two first furrow-slices of every ridge."

"There are other particulars-such as an expeditious mode of shifting the draught in the index of the muzzle; a share for stony land; the sizes and sets of the different mould-boards, \&c., which need not be here enumerated; but its cleaning ciualities may be said to consist chiefly in its freedom from choking. It is well known that the cleansing of the plough from vegetable matter, with which it becomes entangled, frequently occasions great loss of time to the plourhman, and has been one great bar to the cultivation of heath-land; and even on ground otherwise clean, but having a rough stubble, or foggaye, or upon which long dung or sea-ware has been laid, it often happens that half the day is spent in stoppages to clear the plough, while the draught is grently increased, and the work badly executerl. Now, from the curve formed by the beam and coulter, there is no resting-place for vegetable matter getting jammed between them; and, from the inclinel position of the latter, it must be obvious that any matter which it does not sever in going forward, will glide more realily upon its edge, and keep a letter hold of the ground, than when standing nearly vertical. The curve in the front edge of the monld-board produces similar effects; and, in consequence of the upper half being longer, and forming a more acute angle than any other of the same length and angle, below, earth does not accumulate on it, as on the bosum of every mould-board that has a sudden turm, or lies flat over the upper part of the sheath, when working any pulverized soil in a medium degree of moisture." We have extracted this description from the copious account of its properties furnished by Mr. F., whose tracts upon that and other agricultural subjects are well wortlyy of perusal; but not having had an opportunity of personally witnessing its powers, we can oniy recommend it to trial, without further vouching for its merits*.

* The testimonials in its favour may be found in the Appendix to his 'British Farmer, or Ploughman's Guide.' 


\section{THE DOUBLE-FURROW PLOUGH,}

represented below, as made by Messrs. Ransome, is much used upon sandy lands, and particularly on farms where ox-teams are employed; it is an implement of such ancient date that it is mentioned by Walter Blythe, who wrote during the protectorate of Oliver Cromwell, but was not extensively brought into use until it was recommended by some improvements of the late Lord Somerville, whom many persons have viewed as its original inventor. That eminent farmer, the late Mr. Billingsley, of SheptonMallet-of whose work with it we have given an account in our vicw of the comparative merits of horses and oxen as beasts of draught - says, "that some may doubt the possibility of making the double plough so generally useful; but he can truly say, that he never yet found an instance where it could not be worked to advantage; and it is well known that, in the various trials made under the auspices of the Bath Society, on lands of the most dificult nature, the double plough has always gamed the prize $\uparrow . "$ It has, indeed, been recommended by judges, when drawn by four horses, and put in competition with a Norfolk wheel plough-a light Carlisle swing plough-and a common Wiltshire plough-each drawin by a pair, "as the best and the cheapest for general use:" the decision also stating, "that the double-coulter plough had been preferred for the general purpuses of husbandry, laying the furrow more flat than the others, and consequently more new surface to the influence of the elements, and preventingr more completely the growth of grass and weeds between the furrows $t . "$

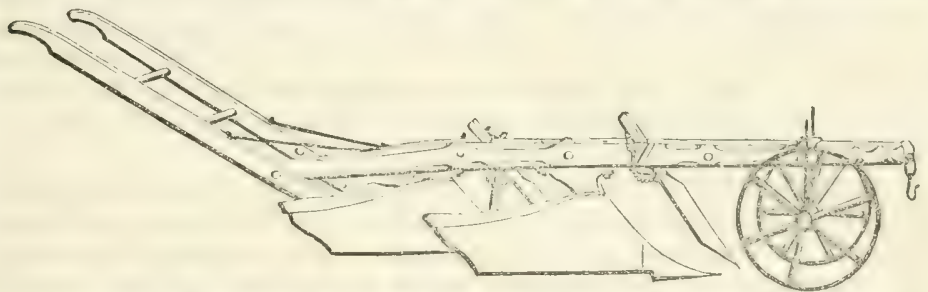

Although on light soils it may be used with considerable profit, if the ground be tolerably level, yet if the surface be very uneven, it works to disadvantage, nor can it be properly employed on land that has not been previously broken up; and it can be of very little use where it is the custom to raise the crown of the ridge considerably above the furrow, since it cannot perform the operations of "gathering or cleaning." Lesides this objection, arising from its confined application, many farmers who have given it a fair trial maintain that it does not do its work well, even where the soil and the surface are favourable to its operations; for it malses the furrow-slices "ride," that is to say, they are laid up by it too close and too narrow; or, more properly speaking, in a field ploughed by the double: plough, the two slices which it makes have more the appearance of one slice, with a very narrow seam between them, while every alternate slice, being made by a new furrow, is at a proper distance, and gives a sufficient seam. When driven with a team at length, it is also frequently necessary for the horse which is nearest to the plough, to draw it a yard or more at the ends of the land, without the aid of the other horses, which, with a double plough, is a considerable inconvenience: and as two doublehorse teams will perform as much, or more work than four horses with a

* Vol. i., chap. viii.

$\dagger$ Survey of Somersetshire, p. 309.

‡ Bath Soc. Papers, Introd, to vol, iv., p. 25, and vol, v., art. 48, y. 471. 
double plough, there does not appear to them any sufficiently powerful reason to recommend it to general use *.

It thus certainly does not perform such neat work as a single-furrow plougl, nor can it plough so deep as is done by the two-horse system; though it is evident that, from its construction, it must take a firmer hold of the ground than a swing-plough, and consequently, not being so ensily thrown out, can be managed with less difliculty. On the slow work performed by ox-teams, a double yoke will thus be found to get over a great deal of ground with comparative ease; and were horses harnessed three abreast, they would probably be found to do their work with as much facility, and certainly with more equality of labuur, than if driven four in a line.

A three-furrow plough was also invented some years ago by the Rev. Dr. Cartwright, to whom the silver medal of the Society of Arts was in consequence granted $t$. In the description which is given of it he observes-that "any man who considers the action of the common plough will find a very material part of the labour in ploughing arises from the friction of the land-side of the furrow, and of the other against the bottom. In a single plough a certain length and width are required in those parts of it, to make it go steady; and even then the effect would be imperfectly obtained did not the ploughman assist by the leverage, or lifting, of the handles of the plough. Hence it is clear that the less disposition any plough has to follow the draught in a straight line, the greater is the libour of working it, because the ploughing in that case requires a greater power of leverage to keep it steady. On the contrary, when two, three, or more ploughs are combined, they serve to stearly each other, and require comparatively very little power of the lever to keep them in a straight lins. Under these circumstances, neither the first nor the second plough has any sole or land-side whatever, and even the third does not require so much of either as a single plough."

He then calculates the saving of power from this consideration alone, as equal to at least one plough; and attributes a further saving to the lightness and compactness of the implement. The construction is certainly simple; consisting of three straight beams of different lengths mounted upon a pair of wheels, and comnecter together by cross pieces. The coulter and share are both in one piece, and fixed before each other, each turning a furrow of nine inches broad; and, when the land is either too strong, or too foul, to work all three, one of them can be removed. Notwithstanding these presumed advantages, we, however, do not find that this jlough has been brought into general use; though others with even mine shares have been emploved, and when drawn with six liorses have been found to get over eight acres a-day with great success + .

THE RIBBING PLOUGH.

The advantages lerived from deep and effectual hoeing are now universally admitted by all intelligent cultivators: this, however, can only be done where the growing crop has been sown in straight lines. Good drills for corn are expensive in the construction, and often not attainable by the small farmer; but the ribbing plough is within the reach of all, and will be found admirably calculated for every description of grain that may be

* Stevenson's Survey of Surrey. p. 122 ; Batchelor's Redfurdshire, p. 164 ; and Loch's Account of the Improvements on the Estates of the Marquess of Stafford, p. 187.

$\frac{i}{f}$ Se vol. xxi. of the 'lransuctions of the Society; in which there is an engraving of the implement, with full directions for its construction.

* See the description of one used by the late Morris 13irkleck, at Guildford, in Malcoln's Survey of Surrev, \&c., vol, i., p. 203. 
sown after a fallow. It was first employed in the Lothians, but was intro. duced, from Mr. Morton's manufactory of agricultural implements at Leith, Ly Mr. Charles Howard, into the East Riding of Yorkshire many years ago, and it is now much used in that and several other districts.

When the land has been preparcd to receive the crop by a summer or winter fallow, it is slichtly harrowed, to break the plough seam. Lines are then formed by the small share of this plough, drawn by one horse, at such intervals and such a depth as may be required. The seed is sown imme. diately afterwards broadcast; it falls chiefly into these lines, and the little which remains lodged upon the intermediate ridges is, of course, drawn into them by the harrows. The grain is thus deposited at a regular depth, springing up in a straight line, and, though rather broaler, or more spread, than when drilled, is equally capable of being horse or hand-hoed. When the land is in good order, one horse will rib about three acres a day at in. tervals twelve inches apart, which is not an uncommon distance for wheat, and nine inches for oats and barley.

With this implement the neatest work is made by leaving the finished work always on the left hand, and turning the fresh mould on the old ploughed land, which is exactly the reverse of the common mode of ploughing, as by this means the crumbling mould is prevented from falling into the last-made furrow. The width of the furrow may be regulated by the expanding iron mould-board, in the same manner as the strike-furrow or double-breasted plough, which is in common use, for landing up and earthing plants sown in ridges, as well as for opening water-furrows; but it will be seen that the narrower that is kept, the closer will the grain rise in the furrow.

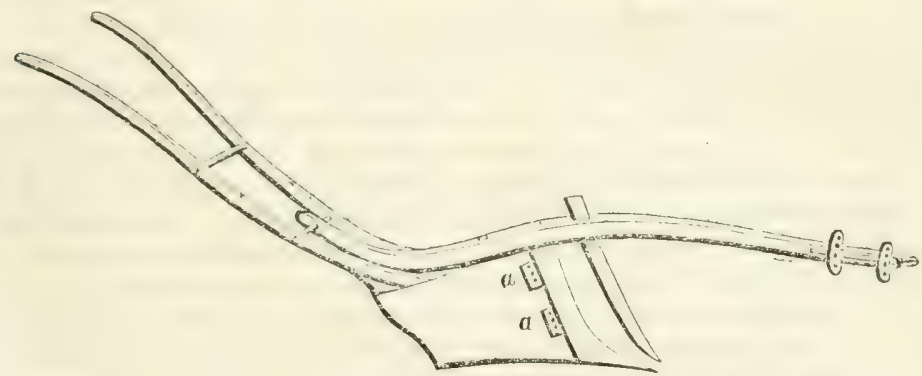

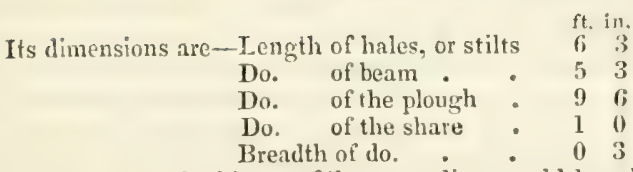

$a, a$ the hinges of the expanding mould-board.

TRENCH AND SKIM PLOUGHS.

The trench plough is intended for cutting stubbles without either turning the turf or making a furrow. This instrument has two shares placed behind each other, the first at ahout one-third of the length of the beam, the other at the same distance from the first. Each of these shares is supported by two iron bars, or coulters, of iron about $2 \frac{1}{2}$ inches wide, and three-quarters of an inch thick, which pass through the beam, and are let up and down in the same manner as any commun coulter: the fronts of these are also brought to an edge in order that they may cut thcir way effectually through the 
ground, and are fastened to the shares by nuts and screws; the front share having its broad ide to the left, and the hind one to the right: thus forming, as it were, a double plough, with two shares, but without any mould-board. The beam is a straight piece of oak, about six inches wide at the handles, but gradually reduced to about four inches at the point, and the stilts are set on at each side of the beam at about six inches from the end. Its mode of operating is thus performed:-it cuts under the surface from three to four or more inches deep, by which operation the stalls of root-weeds are divided below, while the seed-weeds are left untouched above; so that no part is turned over: and as the under stratum is only loosened, neither the seeds nor the weeds are buried by this process". Thie trench plough is, lowever, used more renerally for the purpose of cutting the land two or more slices deep. "The first cuts off the weeds, and deposits them at a considerable depth; the next raises a slice of clean mould from below the soil which produced the last crop, fresh and full of heart, which it lays upon the vegetable rubbish turned down by the former, in such a thickness as to smother the weeds, and decompose them into nurture for the next crop. This operation will, indeed, in every soil require a strong team; but one such plonghing is sufficient for almost any crop, and the slices being laid one over another, the land will generally harrow well $\dagger$. It certainly thus saves the labour of raking, picking, and burning weeds; and if these advantages can be rained hy that one process, it must be allowed to be greatly in its favour, and we believe it is much employed by market-gardeners. But it is to be observed that these men are constantly stirring the ground for the pruluction of more than one crop in the same year, and therefore are enabled to keep their land in a state of cleamess, which prevents the growth of weeds in a manner that cannot be accomplished by the farmer ; and they also invariably work upon a soil of deep staple, which gives the roots of those crops which they usualiy produce full room for the utmost range of their productive powers: while in the common mode of farming, seed-weeds, if not buried to a great depth, would, at certain seasons, be apt to shoot again with increased vigour, instead of being decomposed. Although it hiis been highly recommended by the eminent agriculturist whom we have qunted, and is used to a certain cxtent by some very intelligent men, we yet think that it demands both judgment and caution in its execution.

The skim-plough-or shim, as it is in some places called-as described underneath, was originally invented by the late Mr. Ducket, formerly of Wher, in surrey, with the intention of burying the sod of clover and sainfoin leys, or turf, especially in sandy soils; or, in ordinary cases, it can be Insed on stubbles for the purjose of burying weeds. The coulter is so formedby having a wing or small iron mould-board firmly serewed to it at any fixed height-as that in its progress through the ground it will not only pare off the surface to any depth that is required, and tum it over, but the slice thus cut off is also immediately buried by the common share of the plough; the work thus has at one ploughing the appearance of a fallow, and harrows nearly as well. In this manner it can be added to any common plough, and is now much employed, with various modifications, one of which consists of a very small joloughshare fixed below the beam in front of the conliter, and another of a double skim, which can be either added to the plough or removed at pleasure ${ }_{*}$. It requires, however, some additional

* Malcoln's Survey of Surrey, vol. i. p. 199.

+ Middleton's Survey of Middlesex, 2nd ed.p. 105.

† See vol, i. chap, svi, and plate, p. 350. 
strength, and is generally used with wheels; on the employment of which, on ploughs of any description, a few observations may not be considered misplaced.

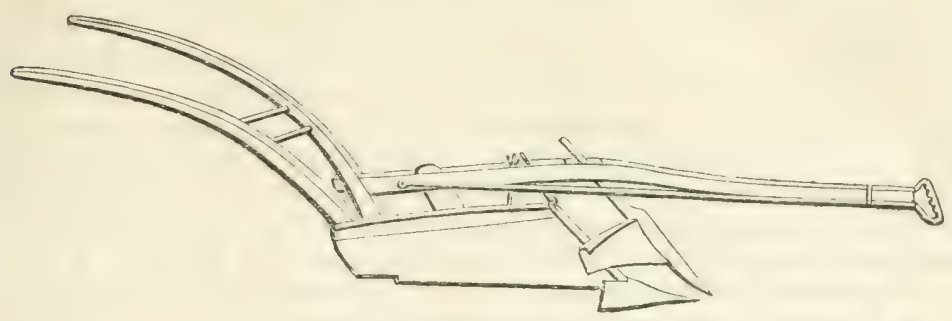

WHEEL-PLOUGHS.

All the theoretic writers on ploughing, and certainly also some practical men of great experience, object to wheel-ploughs as expensive, cumbersome, clogging with dirt, and occasioning mmecessary friction and conserquent increase of draught, as well as being a nursery for bad ploughmen, as they can be managed with much less dexterity than a swing-plough, and can only make equal work on land that is quite smooth. The advocates of wheelploughs, however-which are very generally employed on heavy land throughout the midland and southern counties of England-contend that, on strong soils, they work steadier, and are less apt to be thrown out than swing ploughs; that by pitching the plough a little deeper, and setting it so as to prevent its drawing too deep, the wheels are found to be so sufficient a guide as scarcely to require any one to hold, except in cases of difficulty or on turning at the end of a furrow; and that the supposition that a wheel increases the draught of a plough is practically erroneous, inasmuch as, by regulating its depth, the draught is rendered more steady and uniform.

The theory regarding the increase of draught, by the addition of one or more wheels, is doubtless correct; but practically, it may be observed, that it is usual to fix a heavy iron wheel to malt-mills, and other machinery, which are to be worked by hand labour: the object of which is to regulate, but by no means to diminish, the quantity of labour required. Now, if regularity of force is found most favourable to the muscular exertion of a mian, it may surely be presumed to be equally so to that of a horse; and so far as our own observation has extended, or been confirmed by that of other persons with whom we have consulted, we have seen no difference between the distress of horses performing a day's work upon light land, with swingploughs, and those with wheels ; whereas, on a stubborn suil, it has evidently occasioned more labour to the ploughman, and apparently also to the cattle, when done with a swing-plough. With respect to the ease with which the work is performed, when the plough is set upon wheels, being "a nursery for bad ploughmen," it may be true that good plougimen will never be numerous, where their ignorance or unshilfulness can be so easily remedied: but it is no part of the duty of farmers to educate their plourhmen : they must take them as they find them, gencrally from the same village, and frequently without choice respecting the difference of their capability; and if, independently of any other reason arising out of the nature of the land, they can get the work equally, or nearly as well performed, by an indifferent ploughman with a wheel-plough, as by a good one with a swing-plough, we think few men will hesitate about using the former. 
IIeavy, stubborn land, when hardened by the sun, and stony soils, are very apt to throw the ploughs out of the ground, thus rendering it impossible, notwithstanding all the care and skill of the workman, to form the furrows of one uniform depth, and therefore seem to require instruments of a peculiar construction. A plough may, indeed, be prevented from being thrown out, by giving the point of the share a dip below the line of the furrow, or by a greater length of the mould-board, and a greater weight and length correspondent to that in the other parts of the plough. If the point of the share, however, has a dip given to it, it has a tendency to draw the plough too much down, and experience has shown that no method has been found so effectual, notwithstanding the objections made to them, as the use of a wheel, or wheels; for if attention be paid to the state of the land, so as to ascertain whether they sink more or less into the surface, the depth at which it ought to be worked can thus always be accurately regulated *. Their utility is indeed so generally admitted in many counties, in the ploughing of a hard fallow, that those famers who are unprovided with them to their fallow-ploughs frequently make use of an iron foot, which, having a flat bottom, slides over the ground, and produces nearly the same effect. One we, however, think preferable to a pair, both for simplicity of construction and use. When a frair are useal, that which goes in the furrow must be larger in diameter than that which goes upon the land, and thus, whenever the implement has no receding furrow to work along, the wheels must be brought to a level to prevent the plough from being cast to one side, which can only be rectified by causing the furrow wheel to be fixed upon a bar sliding vertically through the right end of the axle; thus occasioning frequent delay and application to the blacksmith. As the tendency of the point of the share towards the unploughed ground is, in common ploughs, resisted by the land-side of the neck, it has been recommended to place the wheel in a diagonal position, by which it resists both the horizontal and the vertical tendency to deviate from its proper place, as manifested in the following design of a plough, which was found superior, in lightness of dranght, to both Norfolk and Northumberland swing-ploughs, in competition with which it was tried at one of the Woburn Sheep Shearingst. In saying

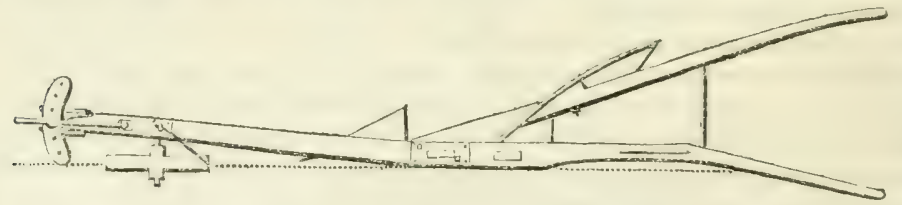

this, however, we must beg not to be understood as recommending wheclplongrlss on free and light soils, since, on such land, there can be no

* "In the operation of working wheel-ploughs, with inproperly turned plates, or mould-boards, it will frequently happen, from the resistance produced against the plough by stones, the tenacity or compression of the earth, that they are obliged to be let down below the corresponding line of level, so materially necessary to the equal bearing between the pitch of the plough, and to the inclination which is thus given to the point of the share downwards, and which onght always to be, as nearly as possible, in a line drawn parallel to that of the draught, and with the breastwork which forms the fulcrum for the beam to rest upon. When a plough is so constructed, and set to work, that it bears unequally in these points, the end of the share will be rooting or dragging, with its point downwards, kicking up, and sideways at the heel, and rendering it utterly impossible to plough the ground clean, or in anywise to lay the work uniform, or even in a folerable manner; notwithstanding, an excessive and unnecessary degree of labour is thereby pro.
duced to the plourhman and horses."-Essex Rep, vol, i. p. 140.

+ See the Bedford Survey, p. 165, and the Am, of Agri., vol. xl. p. 502. 
necessity for them, unless either the plough be ill-made, or the ploughman ill-qualified for his work.

We have thus given a succinct, and we trust an accurate account of the ploughs most generally employed throughout the Lnited Kingdom, though we are conscious that there are many others-as, for instance, the Beverstone, the Norfolk, and Suffolk, together with one much used in Rutlandshire and the neighbouring counties, as well as several under different denominations-which also merit description, if our limits would permit. Most of them have been adopted by the experience of farmers, arising from their knowledge of the peculiarities of their soil ; and as every man of intelligence conceives that the implement is one which he can improve in a manner to render it more suitable to his own land, the number of those which have thus been brought into vogue can occasion no surprise; neither ought we to wonder if, when found to answer the intended purpose, and put into the hands of workmen who have been brought up to their use, both masters and servants should entertain prejudices, even though unfounded, in their favour.

It is a very commonly received opinion, that every lind of soil requires a different kind of plough; and this idea is so firmly established, that if the use of a pluugh be recummended to the farmers of one district, more simple, and requiring less iabour than their own, the answer generally is " that it may suit that country, but will not answer in theirs." Whatever truth there may be in the remark when applied to particular cases, it cannot however be generally correct; for if we take into consideration the different soils of the lingdom, both as they exist in themselves, and as they are operated upon by climate, we shall probably find that only two difierent principles of construction should prevail in the ploughs that might be most advantageously used for general purposes, in Great Britain ; and could an altera-. tion to that effect be brought about, it would be to the full as desirable as a uniform standard of weights and measures.

The different degrees of tenacity in strong soils do not seem to require any other variety in the construction of the plough than a difference of strength in the materials, to meet the greater exertion of the teams in tillace. Deep stiff land, both from its stubborn nature, which occasions it to clog, and from its generally requiring to be deeper ploughed than loose friable soils, may require a greater power of beam and a differently formed mould-board, as well as a stronger plough, than a light dry soil. It may also be necessary to elevate the beam to a greater height*; and it may likewise be proper, in some cases, to work it with wheels; but on all

* " In many modern ploughs, the beam receives a considerable curvature, which, at the same time, diminishes its strength and adds to its cost. It this curvature is intended to give more room for weeds which may accumulate under the beam, this purpose is effected only by an inconvenient length of coulter, which makes it unsteady ; and if the coulter be fastened by a hook, \&c., the space that was rained by the elevation of the beam in the middle is again taken away by the hook and screw, which of course, to say nothing of the expense, renders the whole scheme abortive. The apparatus of iron bars and chains, which is sometimes made to pass several inches below a crooked beam, appears to me at best to answer no other good purpose than that of showing the most proper position of the beam itself.'-Batchelor's Survcy of Bedfordshire, p. 166. There is much truth in this; and it has accordingly been justly remarked by Captain Williamson, 'that much ink has been expended by the several advocates for various forms of the beam; a matter which surely need not have excited controversy, if it had but been consilered that all lines of draught are direct lines, and that the straightest beam is the shortest, the lightest, and the strongest. The beam should, therefore, have no curve whatever, but. proceed in a straight line from the muzzle to the foot, and that too in a paraliel line with the sole, or chep." -Agric, Mcchanism, p. 149. 
those soils which distinctively go under the name of "turnip land" there can be no doubt that a short swing-plough, such as those we have describedor perhaps still better if made with a perfectly straight beam, and held by a skilful and attentive ploughman-will perform the work effectually. Respecting the mode of performing it, we shall add a few words in a future chapter, when we have disposed of what we have further to say on the subject of agricultural implements.

\section{Chapter II.}

\section{ON HARROTS, GRUBBERS, SCARIFIERS, SCUFFLERS, AND} HORSE-HOES.

Tie next operation after those of the plough is that of harrowing, which is performed on soils of every description for the purpose of pulverizing. them, by reducing the clods made by the furrows upon stubburn land, of tearing up the couch and other weeds upon light ground, and of thus preparing both for sowing; and afterwards for covering the seeds. It therefore requires instruments of different size and strength, and as the objects for which they are employed, though nearly similar, yet vary in letail, it is manifest that they should also differ in form ; but they have nevertheless been both made and worked without any material alteration upon the same principle on which they have been used for ages.

\section{HARROW}

The frame upon which the harrow is constructed, though generally formed of wood, has, in some instances, been lately made of iron; though it is very questionable whether that can be justly called an improvement, for although durable while in good order, yet, if broken, it is more difficult to repair, as well as more expensive. It is composed of cross-bars, the strongest of which are called "bulls;" into which strong iron teeth, or tines, of a werge-like form, tapering to a point, are generally fixed in a straight downward direction, and are pressed into the earth by the weiglit of the machine. The tceth are made of diflerent lengths, according to the strength required for the performance of the work, and are, in tools of the small kind, from 7 to $S$ inches long; but, in the larger, they are frequently

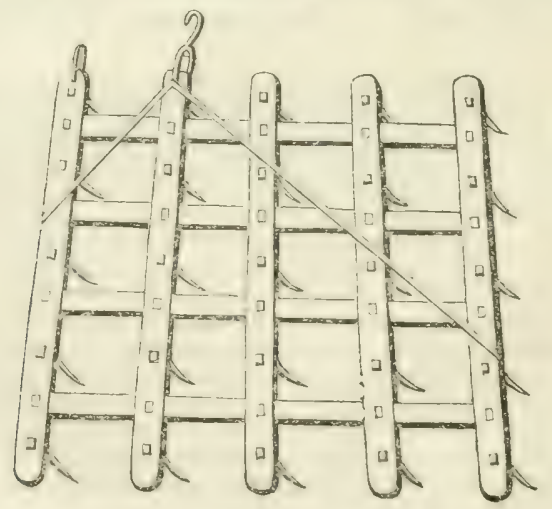


11 inches in length from the under surface, and proportionably strong, five being firmly mortised and passed through a plate of iron into each bull; those in the front being also, in some cases, shorter, with a gractual increase of length to those at the back. The bulls consist of four, and sometimes five, very stout bars, chiefly made of ash, being generally not less than 3 to 4 inches square, placed lengthwise, and four, of a smaller description, across: thus containing 20 to 25 teeth. The small ones are commonly about $3 \frac{1}{2}$ feet wide in the front, and 4 at the back; but the large ones, here represented, are intended for heavy work, and are usually termed "drags"-in some places "brakes;" not unfrequently made as wide as 6 feet in the front, with a bar of iron screwed on in a diagonal direction to keep the whole secure.

The drags, even when used singly, are generally worked by a pair of horses; but when employed, as they commonly are, upon rough ground, and two together, the labour is usually found sufficiently heavy for four; and, on tuming over the immense clods upon the summer fallows of heavy clay land, as many as six horses or oxen are frequently necessary. A boy usually drives, and a man walks behind to clear the harrows of weeds and lift them when they get encumbered; for which purpose he usually carries a motched stick, or else attaches a rope to them, for handles are not used. 'They are thus often nearly as effectual as a ploughing; for, in such a summer, for instance, as that of 1534 , the land could not, during a great part of the season, be properly said to be ploughed: the clods migrit indeed be moved about; but the work, so far as it could be performed in such weather, would be better and more expeditiously done by the drag. It is, in fact, a very powerful tool, requiring great strength and considerable weight to do efficient execution, but generally doing work in proportion, particularly when it can be worked at a quick rate, in which case the clods are much more effectually broken than when the teams are driven at a slow place.

The perpendicular position of the teeth, however, evidently does not fit those heavy implements for working to a proper deptl with effect, and therefore some farmers, judiciously observing that those straight tines do not take firm hold of the ground, set them with a slight rake forwards, and made in the form represented above; but this is sellom done with the lesser harrows, as it would render them more apt to collect the weeds between them and the frame, instead of merely tearing them out of the ground, and would thus impede the forward motion of the implement. It has indeed been recommended, as a preferable plan, to form the tines in something like the shape of plough coulters*; and also to mount the harrow at each comer upon low wheels, with axles set upon a pivot, so as to fix them cither higher or lower, as the nature of the ground and the depth to which it ought to be stirred may require; but the teeth, if thus constructed, would form an angle with the frame-work, and the use of wheels would render the implement more like a grubber than a harrow.

The small harrow's are always worked either in pairs, or sometimes three together, so connected by links as to allow them sulficient play to yield to the curvel form of the ridges, and thus they perform the work very completely. The links by which they are coupled are formed in various ways: either by sliding iron hinges, or by pins bolted through the adjoining bulls, and joined by pivots, which, while allowing them full liberty to work, yet keep them effectually asunder, and prevent them from entangling or getting foul of each other. The most common method, however, is to comnect

* See an Essay on Drags and Harrows, Art. No. 11I, in the papers of the Bath and West of England Society, vol. $x$. 
them by a cross-bar, called a "way-tree," attached at each cnd to the bulls by short chains, and fastened by moreable pins, as at $\mathrm{A}$ in the annexed cut.

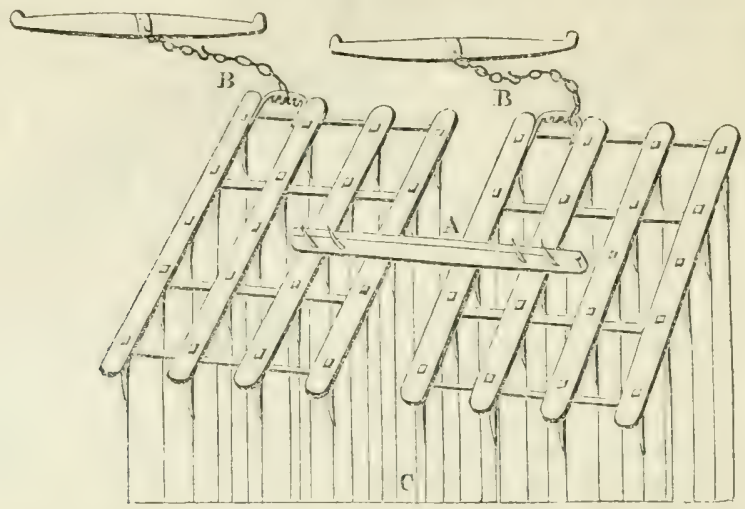

The swingle-trees by which the cattle are harnessed are, it will appear, hooked into a plate, or to a chain, placed between the bulls on the left, admitting the line of draught to be shifted as occasion may require, as at $\mathrm{BB}$, so that the harrows are drawn in a diagonal direction. The teeth of the harrows, it will therefore be observed by the lines drawn from them at $\mathrm{C}$, cut the ground at irregular distances, and in some places follow the exact track of each other; thus stirring the earth unequally. This indeed arises more from the mode of draught than from the form of the implement, and it has therefore been frequently proposed by writers on the subject to alter the method of dratcht, so as to allow the cattle to draw in a direct line abreast; by which means they aftirm that less force would be required to be exerted by the team, and the work would be executed with greater uniformity. On this, however, it may be remarked, that the difference of draught can only be trifling; that the uniformity of the work is, in almost all cases, nearly ensured by the operation being repeated in a different direction; and that the irregular motion of the harrow, occasioned by its being drawn sideways, and thus being shaken and tossed about, is favourable to the perfect execution of the work: for thus, in cleaning the land, the clods of earth are more effectually broken and cleared of weeds, than they could be were the motion in a direct line; and in seeding, the object is evidently not so much to penetrate the soil as to cause the teeth to stir as much of the surface as possible, and thus to cover the corn. Most farmers, therefore, continue the common mode of draught, using the implement, though of different sizes, in the same way, both for pulverizing the ground, cleaning it from weeds, and again for covering the seed; and generally find those several objects to be attained with tolerable correctness.

The seed-harrows, though seldom of a lighter construction than the former, are yet by some persons made with small tines, not more than four inches in length, and occasionally with wooden teeth, especially for sandy or other very light land, in order to prevent the seed from being too deeply buried beneath the soil when covering it after the sowing; an object which is certainly of great moment, and merits the cautious attention of every farmer, as seed, if sown too far below the surface of the earth, will not regetate to perfection. If the frame of the harrow be as light as the necessary dergree of strength will admit, it will necessarily ride high ; but if required to worli deeper, that object may be attained by tying a heavy 
log or two of wood upon it, to sink the tines, which otherwise do not gene. rally penetrate farther than one-half, or at most two-thirds of their deptl: It is also an object of some importance, in covering the sced, to avoid the treading of the cattle when harrowing it in, with which view it is by no means uncommon to join three, or even more harrows together, so as to cover the entire breadth of the ridge. They are then fastened by chains to a long pole cxtending their whole wilth, to the extremities of which the swingle-trees are hooked; so that the horses, being harnessed to each end, walk in the furrows, thus:-

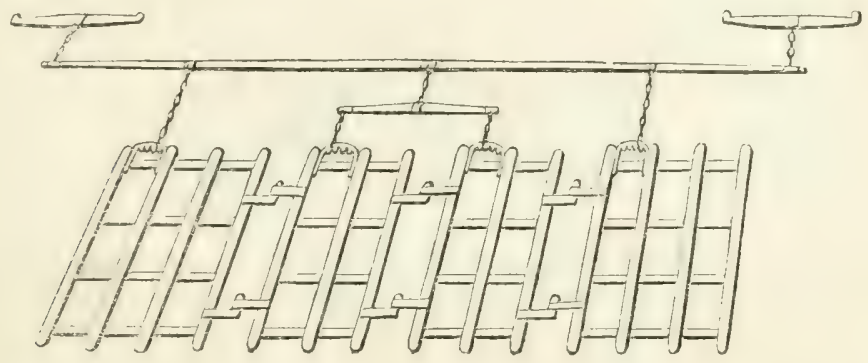

The operation of harrowing, it will be readily perceived, is one of great importance, and should be executed when the soil is in a proper state, between wet and dry, and according to the nature of the land; for, if too wet, it will often do more harm than good, and if too dry, it will, on tenacious land, have very little effect. In a climate like that of this country, where the opportunities for many of the processes of husbandry are so transient and precarious, it must indeed occur to every farmer, that this is one that should never be neglected, and that, particularly at seed-time, he should always possess the power of putting the crop into the ground within the shortest possible space of time. It has indeed been well observerl by Arthur Joung, "that the most ticklish business in tillage is putting in a spring com crop on lands tending to tenacity, or in any degree binding. Tou walk over a field soon after ploughing, or after rain, and you find the adhesion of the soil under your feet too great to allow it to break readily. You come acrain one or two days after, and find it in exact temper; if at that moment it is not harrowed by means of as many harrows as you have pairs of horses, the right time is lost. Let a drying north-east wind blow, and in two days the temper of the soil is gone; every bit that now moves is a clod, and the effect of the harrows upon them is nearly lost."

Many other harrows have been constructed, and occasionally brought into use upon nearly similar principles, though in various shapes and with a different arrangement of the bars and mode of draught; but their object having generally been to ensure a more equal distribution of the work of the teeth, which we have already seen is not always sought after by practical men, those which we have already described still maintain their ground. There can, however, be no doubt that although the implement is particularly well adapted for covering the seed, and also materially assists in pulverizing the soil, yet in the operation of cleaning the land it is very apt to become choked with weeds, and does not in general enter sufficiently into the ground to be attended with the desired effect; the consequence of which is, that the work is in many cases very imperfectly performed. This, indeed, has been so universally admitted by those farmers who are alive to the necessity of eradicating every species of weed previous to the admis- 
sion of a fresh crop, that efforts were long ago made to supply the deficiency by the introduction of implements of superior power, and accordingly numberless tools have been invented with that intent, under the various names of cultivators, scufflers, scarifiers, extirpators, lormentors, and grubbers; which, although thus differing in name, have all however but one object, and we shall therefore consider them collectively under the latter denomination*.

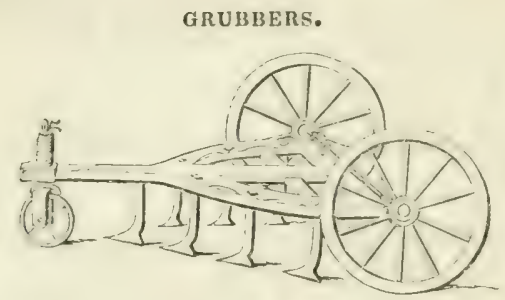

Although these implements may be generally spoken of under one head, yet their mode of operation is, in many respects, as different as their form ; for some act with small shares, others with coulters, or tines, like those of heavy harrows, but inclined forward, and a few with skims for cutting the surface, each varying in some point of construction, and claiming for their inventor the merit of superiority over all others, although there is seldom much difference to be perceived in the work when equally well performed. The ease and expedition with which it is said to be executed by them are also usually much exaggerated; for although we have known assurances to be given of "their being easily able to knock off" an acre within the hour without distressing the cattlet," yet, when the land was stirred to only a moderate depth, we have never seen half that amount well done, and they require a powerful team, some being of such great power as not to be easily worked with so few as four horses. The most common mode of construction is however that of a triangular form, as described above, with several rows of either coulters or shares, made of different sizes, as the nature of the ground may require, and capable of being moved in the mortises of the beams according to circumstances; the frame being usually mounted upon one wheel in front and two behind, with moveable axles, as represented above. In many cases, however, two wheels are used in the front, and in others, the hind ones are altogether dispensed with; but a pair should always be used in front, when intended to be applied to the cleaning or stirring of the land on which a crop is growing, as they ensure the regular passage of the instrument between the rows without injury to the roots; and if made with one wheel, if the ground be hilly, the implement is continually verging towards the lower side. The wheels may be raised or lowered either with holes and pins, or by means of rings with grooves to admit of the height being altered; and in all instruments of this kind a very considerable degree of strength is necessary.

* Among these we may enumerate that of Mr. Lester. whose cultivalorobtained a medal from the Society of Arts, and has been improved upon by General Beatson and Messieurs Emerson and Wir ; Haywarl's exlirpator, or scalp-plough; M'J)ougall's, Cook's, and Parkinson's scarifiers; Plenty's senfler; Dutgeon's and Kirkwood's grublers; Wilkir's parallel adjusting brake; Finlayson's self.cleansing harrow; and Morton's revolving harrow, the teeth of which are fixed upon an axle, round which they revolve like the spukes of a carriage-wheel; besides those old well-known instruments called the odyet and the Kentish nialget.

t Suffolk Report, p. 36 . 
The grubber, indeed, usually follows the harrow for the purpose of stirring and cleaning the land still more effectually than that has been already done; and in light lands, the object may, in most cases, be accomplished without the further use of the plough, and certainly with far less expense. In working lime or compost into the soil, it is also essentially useful; for, by passing it once along and again across the rilges, they are thoroughly mixed, without being buried too deep in the ground, as frequently happens when the plough is employed for that purpose. Another important advantage is, that it may be employed with great power on all lands ploughed in the winter, and to be sown in spring ; for, by passing it once or twice over the field, the soil may be effectually loosenerl to any moderate depth; and, in the hurry of a catching season, barley and oats may thus be got into the ground with far greater expedition than by the common mode. In the preparation for turnips it is likewise equally efficacious; it must, therefore, in such cases, occasion a vast saving of animal labour, as well as time; and in all the farming operations in which it is employed has been generally found eminently useful. When well worked, it indeed affords such a fine tilth, that even on strong soils it has been used on a great scale; and in two wellknown instances of the farms belonging to Mr. Grey, of Coles, in Hertfordshire, and that of General Beatson, of Knowle, in Sussex, it has, for many years, been made to nearly supersede the use of the plough. The implement used at Coles is of nearly the same kind as that represented at the head of this article, while that employed at Knowle Farm is of a much lighter description, yet they each appear to perform the work with nearly equal effect; but as we shall in a future chapter have occasion to refer to the mode of cultivation pursued on those farms, it is here unnecessary to describe them.

Although coming under the denomination of the self-cleaning harrowthough more properly a grubber-an implement of great power has been lately invented by Mr. Finlayson, to whom we have already alluded. It is made wholly of iron, in this form-

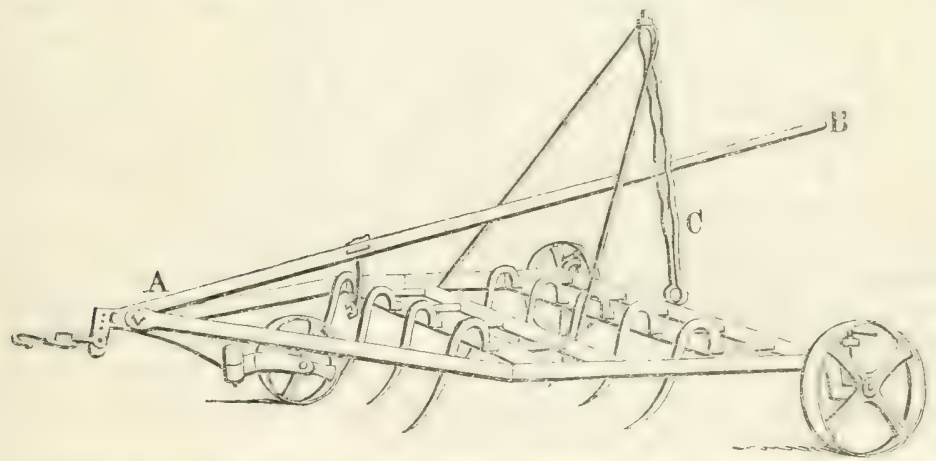

consisting of parallel sides, with two sets of transverse bars, into the hindmost of which there are five, and in the foremost four curved teeth, fived in a quincunx mamer, and the frame moves upon three wheels, one of which is in front, and the other two behind. A little before the foremost teeth the frime is made to tend to the same point at $\mathbf{A}$; where it is attached by a bolt to a spring, forming the point of draught, and a lever, B, by which the depth at which the instrument is to be worked is regulated. This lever passes through an upright bar, $\mathrm{C}$, which is supported by the side poles, and contains notches for it to rest upon. It acts upon the draught 
by either raising or lowering the forepart of the frame, which is done by either elevating or depressing it in the notehed bar; for if it be raised, the frame is thereby lowered, and the teeth are consequently forced deeper into the ground: or, by the coistrary motion, the opposite effect is produced. When lowered to the bottom of the upriglit bar, it will be without any efiect unon the tecth; but when raised to the uppermost notch, they will then penetrate eight or nine inches into the soil. The forewheel runs always on the surface, but the hindwheels can be either raised or lowered by means of screws upon the axle, and thus it can be lifted out of the ground and turned at the end of a field with the greatest facility. The space covered by the working part of the implement is $5 \frac{1}{2}$ feet, and the length of the teeth, which are made extremely strong, is 29 inches; the weight of the machine is not much more than $4 \frac{1}{2} \mathrm{cwt}$; and the cost at the manufactory is $10 \mathrm{l} .10 \mathrm{~s}$.

It may be readily conceived that an instrument of this form, when worked to the deptl of which it is capable, must not only have a very powerful effect in stirring tenacious soils, but must also be far superior to the common harrow, or indeed to the plough in tearing up and extirpating root-weeds; which, by the curved shape of the tines, are thus brought up to the surface. When used to a great depth, it however requires a very powerful team ; for, although stated by the maker as being suitable to the strength of two horses, we have this week seen it at work upon moderately tenacious land, which had been already ploughed, and which it was only stirring to the depth of five inches, yet occasioning quite sufficient labour to three. He also says, that, "from the curved form of the tines, it naturally follows that all obstructions, by way of vegetable matter-whether stubble or couchthat they may cncounter in their progress, is rolled up to the face of the tines, when it loses its liold, and is thrown off; and that the instrument thus always relieves itself from being choked, however wet or foul the land." In which latter statement, however, we cannot altogether agree; for it required the assistance of an additional man, beside the driver, to clear it, and it was occasionally necessary to stop the team to perform that operation. Still, it must be admitted that it turned up a vast mass of weeds, and went over about six acres of ground in a very complete style, within the day's work. The mode by which it can be adjusted to the depth of work is also a great advantage; for it is so simple in principle, that it can be regulated without difficulty by the dullest farm servant.

There is also another machine, by the same maker, of nearly the same form and principle, but of a lighter description, and with fewer teeth, which is therefore suitable for more tender soils, though it is not so generally used as the former. It has, however, been employed with considerable advantage as a drill harrow, or horse-hoe, for working between the rows of root crops, and it can be regulated to work at any width or depth required, so as to suit the breadth of the drill.

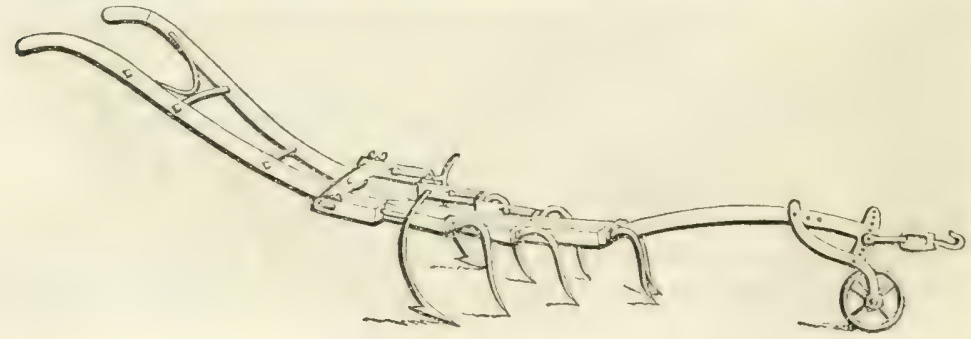


An alteration has, however, been recently male in the construction of Finlayson's harrow, which has been brought into very general notice, under the name of Kirkwood's Grubber; and is, by many persons, considered as a material improvement. It is also made entirely of irom, of the weight of about $4 \mathrm{cwt}$, and, with its tines or coulters, which are 2 inches broad, and stand 15 inches below the bars, covers a breadth of 4 feet 4 inches, in the following manner-

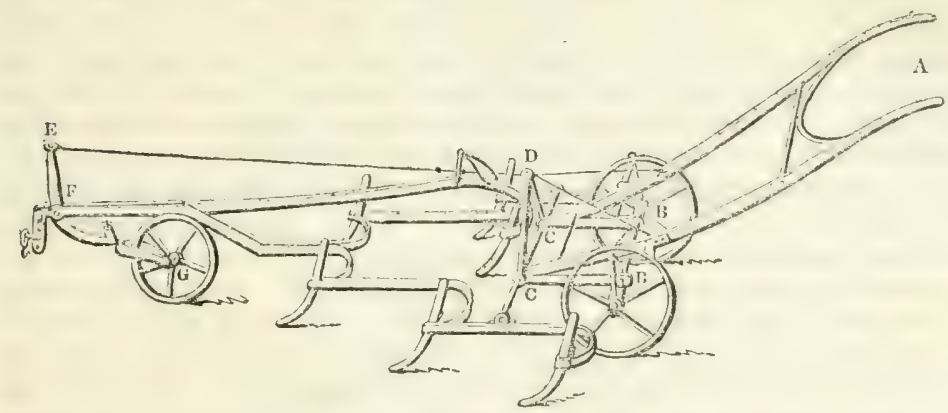

From the description given of it in the Transactions of the Highland Society*, it will be observed that the pressure on the gruiding handles at $\mathbf{A}$ turns the whole handle round the axle of the hind wheels, BB, as round a fulcrum, so that the handle then becomes a lever, on the shorter extremity of which the frame of the teeth rests. It is evident, therefore, that, by bearing on the handle which forms the long end of the lever, the shorter end must be raised, and along with it the hinder part of the teeth of the frame, and of course the teeth also. But there is still another contrivance by which the force is made to act at the same time on the fore part of the frame, and to raise it likewise. This is done by the long rod, DE, which is attached at the extremity to a fulcrum, D, raised on the handle frame, and at the other to the one end of a bent lever, EFG, which turns on the axle of the fore wheel as a centre, and, at an intermediate point, carries the fore end of the teeth frame. While the liandle therefore is depressed, and raises the hinder part of this frame, it at the same time pulls the rod, turns the front lever round the axle of the fore-wheel, and by this means elevates the teeth before as well as behind.

The whole operation is simple, ingenious, and effective; and although it does not cover the same breadth of ground, yet the draught is lighter. than that of the other grubber, and thus it can move at a quicker rate. Another peculiar advantage is, that the whole body of the instrument can be raised out of the ground at pleasure, by the driver's merely bearing with his weight upon the handles; which is extremely ronvenient both at turning the corners of the ridges, or meeting any sudden obstruction in the ground.

A very ingenious implement, upon the same principle of elevating or depressing it by means of the handles, and thus regulating its depin of work, has also been invented by Mr. Wilkie, and is highly spoken of; but not having seen it, we are unable to describe it so accurately as we could wish, and we therefore abstain from any particular mention of it.

From what we have already sail of these instruments generally, we think that no doubt can be entertained of their utility ; and their effect may be further improved by adding a small light harrow, formed with only a couple

* N. S. Vol, II., plate No. II, and p. 132. 
of bars, and fixed behind the grubber with iron arms hanging on pivots, to fallow for the collection of the weeds. They may also be employed in preparing land in certain cases for the seed, without the necessity of a repetition of the operation of ploughing; though, in saying that, we by nomeans wish to be understood as recommending the disuse of the plough, for the range of the application of the grubber is obviously limited. Arthur Toung, in his directions for wheat after beans, states, "that it will be more adrantageous to trust to the scarifiers than the plough;" and again, speaking of wheat after tares, on a light soil, he says, "the land should not be ploughed at all, but left to consolidate at bottom, to become firm for the roots of the wheat to fix in, and the surface worked with the scarifiers just sufficient to keep it clear of all weeds, and in that state drill the wheat, without any ploughing *; and this was not only his own practice, but has been adopted by many eminent farmers. Thus, land on which crops of pulse and roots have been cultivated under the drill system, and which has consequently been kept clean by horse-hoeing, may, in almost ail cases, be sown with corn without any necessity for a second ploughing; and, if the soil be light and dry, it may be prepared for the seed solely by the use of the grubber. In this case, however, it is generally expedient to go twice uver the ground: setting the teeth, in the first instance, at a less depth than in the second operation; and if the land requires much stirring, it should be worked at right angles; afterwards collecting the rubbish by the operation of the common harrow, either to be carried to the dungheap, or burned, and the ashes spread upon the field,

\section{HORSE-HOES.}

The operation of horse-hoeing, though in most respects similar to those of the grubber, yet is distinctively confined by farmers to that of earthing up crops, and cleaning land which has been already cultivated upon the drill system; and being mercly intended to act between the rows of the different crops as they stand upon the ground, is executed with implements of a lichter description, and drawn invariably by only one horse. 'The necessity of using extreme caution in keeping the horse exactly between the rows, also requires a steady animal to be employed along with the driver, and the implement should never be confided to any other than an experienced and very careful workman. They are made of several different forms : sometimes, when the object is simply that of cleaning the land from surface weeds, in that of the paring instrument represented in the first volume of this workt; but more commonly in the triangular shape, resembling the grubber already depicted at the commencement of the account of that instrmment, though so slightly constructed, that we have seen them drawn by a donkey when carrying three flat shares, in the following fashion-

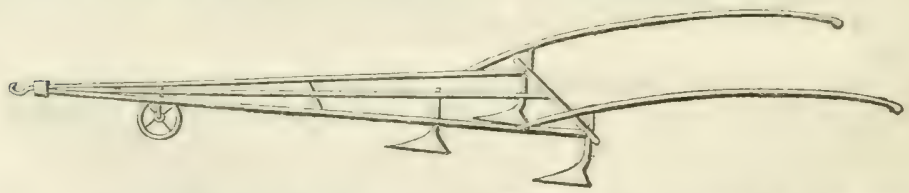

the two outer bars being of iron, but fixed with hinges to the centre piece, and having a cross-bar, which admits of their being either widened or narrowed as the wirth of the rows may require. In this manner it performs very fair work, particularly if the crop be in such narrow drills as to

$$
\text { * Farmer's Calendar, p. } 457 .
$$$$
\text { f See Chap. XVI. p. } 345 \text { and } 346.1
$$ 
occasion any fear of their being trodden on by a larger animal, but the hand-hoe may, in most cases, be also necessary for cutting up the weeds between the plants, which no horse-hoe can reach without injuring the crop.

Although, upon the same principle of operation, the most common form, however, and perhaps the most effective, is that resembling the Flemish binot*, though better known as the expanding horse-hoe, the frame of which is like that of a small plough, without either mould-board or coulter, but liaving two other shares, placed, one at each side, in a bar screwed to the: back part of the beam, but jointed in the centre, so as to adjust the work to the precise breadth of the drills: thus AA are the stilts, $B$ the beam, $C^{\prime} C$ the arms to which the side shares are affixed, D the centre share, and E the wheel, though it is sometimes used without a wheel, and at others with two.

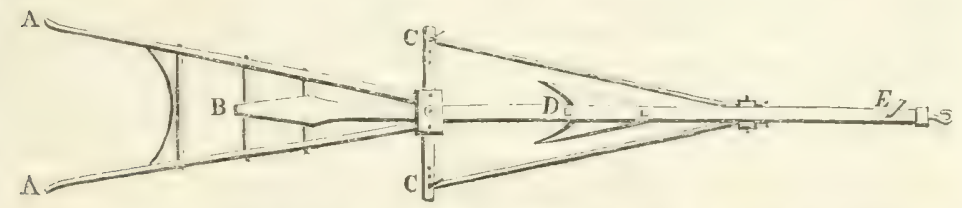

It must be observed, that the bar above-mentioned being moveable, the shares can be removed altogether, or replaced by coulters, and when the object is to eartl up the plants, a double wrought iron mouk-board, with hinges and comnected by a sliding bar, can be added, which converts it into a double-breasted plungh of this appearance; but these expanding

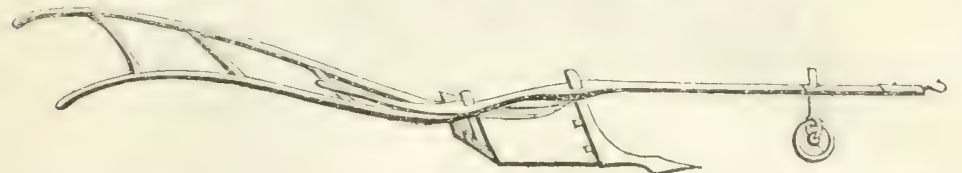

horse-hoes, with mould-boards, have been found inefficient in their operation upon hinding soils when they arrive at more than twelve or fifteen inclues wide; and three small hoes, set to the same width, or even a number of harrow times, bent considerably forwards, have been found preferable, particularly if the ground be infested with couch + . The cost of the whole machine, which is made entirely of iron, complete in all its parts, is $5 l .10 \mathrm{~s}$.

Such are the usual modes of horse-hoemg between the drills; but it is evident that they must be of sufficient width to allow of the amimal by which the implement is drawn, to step without injury to the plants; and, as com is generally sown too close to admit of that, the operation is commonly performed by the hand-hoe, which requires no particular description. As grain, however, when sown upon land which is tolerably dry, and lias got high above the ground, will seldom sustain much injury from the moderate tread of cattle, a machine has been invented by Mr. Wilson, of Traprain, in East Lothian, for cleansing the ground between the rows of white corn crops.

* This instrument is extensirely used in the tillage of the Netherlands, and has been much improved since its introduction into this country. A full account of it. together with an engraving, may be found in the Appendix to Sir John Sinclain's Code of Agriv culture.

† Bedford Report, p. 192. 
The experiments made by Mr. Wilson have been carrical on with one horse, guided by a boy, while the implement is held by a inan; and, from his calculation, it appears that about five acres a day could be clressed, at the expense of $5 s .6 d$. to $7 s$, or at $1 s .1 d$. to $1 s .5 d$. per acre; which is certainly very far under the average expense of hand-hoeing; but he admits that its greatest advantages will be found in situations where a sulficient number of hands cannot be procured to meet the exigences of the season when circumstances render expedition indispensable. In this view of it we agree ; and we therefore annex the description given of it in the Transactions of the IIighland Society*; though we think it much too complex, and are of opinion that it might be materially simplified, without lessening its effi. ciency.

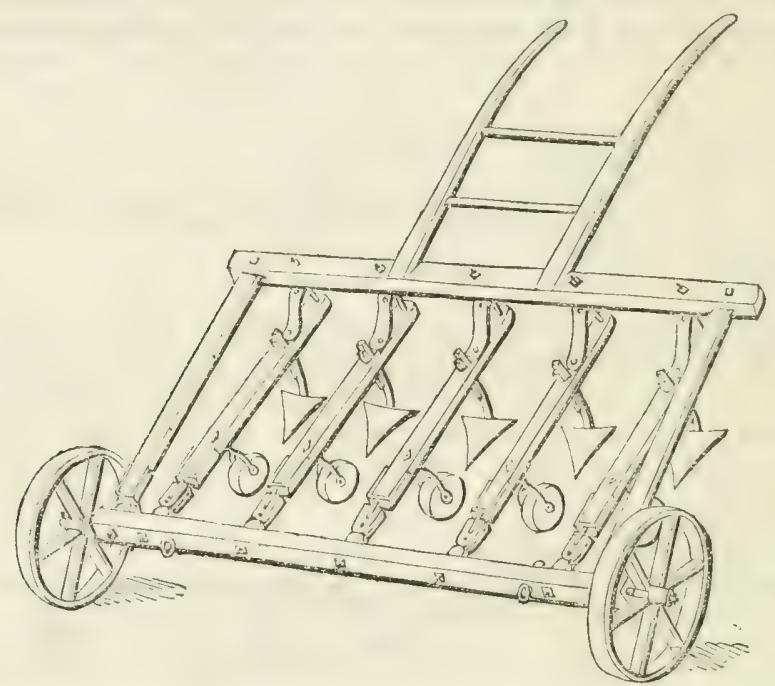

* "It consists of an outer frame of timber, measuring $4 \frac{1}{2}$ fect in breadth by $3 \frac{1}{2}$ in length, and supported in the fore-part by two wheels of about 20 inches in heirht, the axle's of which are bolted to the lower side of the front bar, and to this bar also the draught is applied. The two side bars are attached to the front by a hook-and-eye joint, allowing a considerable freedom of motion in every direction; they are also atfached to the hind bar by a mortise-joint and loose tenon, admitting of motion in a horizontal direction only. The five coulter-bars are attached to the front bar by a species of double hinge, having one joint moving vertically, and the other in a horizontal direction, giving a certain degree of universal motion along with the side bars. The hind end of the coulter-bars are connected to the back bar of the frame by means of a bolt, which is fixed in the coulter-bar, but is made to slide easily in the corresponding holes in the hind bar, the coulter-bars being thereby allowed to rise and fall in the hind end. A slight spring is attached to each of the coulter-bars, and is adjusted to act against the lower side of the hind bar, thereby lieeping the coulters at their greatest depression. The coulters are fitted to a mortise at about two-thirds of the length from the fore end of the bars, and are fixed by wedges at the required height. In front of each coulter a wheel of about six inches diameter is attached to each bar, serving to regulate the depth to which the coulters are to work. To the hind bar are also attacherl a pair of stilts or handles, adapted to the height of the man who directs the implement.

"Although the implement is shown with goose-feet coulters, any other form may be substituted-as plain teeth-to act as a harrow for pulverizing or for covering in grass seerls; or they may be so formed as to earth up the crop if reguired."-N. S. Fol. III, 1. 382. 


\section{Chapter III.}

ON ROLLERS.

THE roluer, which is used both to hreak the clods turned up by the plough, as also to compress the earth and smooth the surface of the ground, although an instrument to be seen on every farm, and one of a very important description, is yet rarely found of proper proportions, or mounted in such a way as to conduce either to its efficiency of draught or operation; and, of all the other implements in common use among farmers, appears to be the least attended to. It is of various dimensions-generally formed as a cylinder of wood, occasionally spiked with iron, or encircled with large rings of metal to assist in crushing the clods: it is, however, not unfrequentiy made of granite, and in many cases it is formed of a hollow tube of castiron. It is also, in some instances, formed convex, and in others concave, and in a few it has been fashioned of an octagon shape; but each is furnished with shafts for either one or two horses, according to the weight of the machine and the power required to draw it. 'That which is the most commonly used, however, is the solid cylinder made of oak or ash, in this form,-

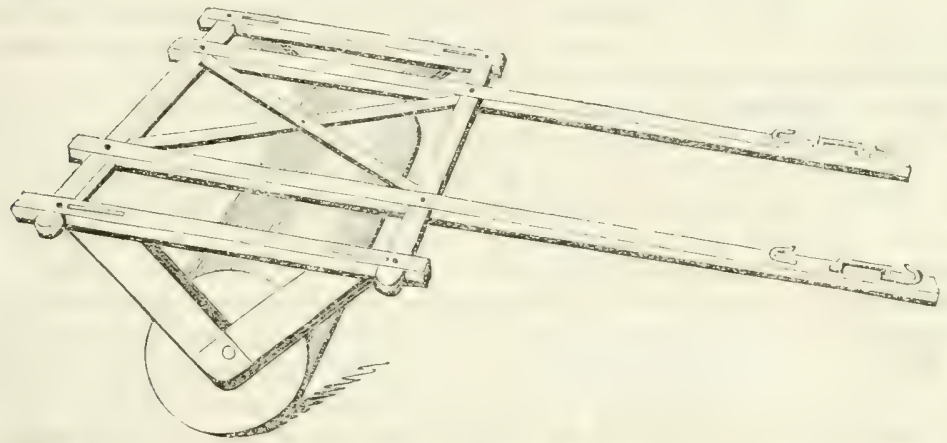

The dimensions vary from 5 to $7 \frac{1}{2}$ feet in breadth, the diameter from 16 to 20 inches, and the weiglst from 10 to $15 \mathrm{cwt}$; but sometimes it is made 9 feet in length and 20 to 30 inches in diameter, in which case it is worlied with double shafts, and if much weighted is occasionally drawn by four horses, and even by six oxen. Though all constructed upon the same principle, yet, as their size and weight are materially diflerent, it is evident that they should be employed for different sorts of work; yet there is not one farmer in ten who has more than one, and this he uses upon every occasion where a roller may be necessary. It is often also nothing better than a rugged log of wood, not formed on anything like an accurate circle, and having its two gudgeons-by which it is hung-driven in without any regard to their being exactly placed in the centre of the cylinder or axis: thus not only occasioning much irregularity, and consequently great increase of friction on the action of the machine, but also never failing to loosen the gudgeons, each of which is in turn wedged up with nails, or whatever the labourer can find at hand, until being at length too much widened for future use, a job is created for the blacksmith*.

The frame, it will be perceived, is not alone clumsily strong and heavy, but is usually mounted so far above the cylinder as to be nearly on a level with

VoL. II.

* Williamson's Agricultural Mechanism, p. 239. 
the horses' shoulders, thus being contrary to the correct principles of draught, which require that when the muscular exertion of an animal is necessary in drawing a load over rough ground, it should be in an inclined direction from the joint of draught upwards to the shoulder. It has no doubt, however, heen originally formed in this mamer for the convenience of mounting a box upon it for the carriage of any rubbish that may be picked of grassland, as well as for adding to the weight where that may be thought necessary, or for placing a seat upon it for the driver. 'To the collection of rubbish when grass is to be rolled, there can be no objection; nor much to the indulgence of the driver with a seat; but, if merely intended for the increase of weight, the object may be more effectually answered by hanging it to the axle withinside of the hollow crlincier hereafter described: for, if placed upon the frame, it adds more to the draught than in the latter mamner, without equally increasing the pressure upon the land; as the weight rests upon the gudgreons of the axle, in consequence of which the friction is far greater than in rollers of equal weight, if constructed upon proper principles. The mode of mounting them, as delineated in the frame of the convex roller, hereafter described, is therefore preferable.

In some cases, indeed, the horses' traces are applied directly to the axle without any frame, in the manner depicted in the description of the concave roller: this saves expense, bnt the instrument is less true in its revolutions, and is therefore subject to drar in a manner which may be injurious to grass, or to seeds which require rolling.

A very general notion prevails among farmers, that rollers of equal weight are more effectual when made long than short: it is, therefore, not unusual to see the diameter of the cylinder lessened, so as to bring them to the same weight, though made of greater length; in the idea that, as they will thus go over a greater extent of cround in the same time, more work will be equally well performed. In this, however, there is a double error: first, because the same weight, resting upon a greater length of ground, has more points to sustain it, and therefore the weight which jresses upon each is lessened; wherefore, althongh more ground will be gone over, yet the work upon it will be less efrectually performed; secondly, by lessening the diameter of the cylinder, the power requisite for draught is increased; for it has been proved that the same strength which is required to draw a roller of half a ton weight over a height of two inches, when the diameter is one foot, will sufice to draw rollers of 15 and $18 \frac{1}{2}$ cwt. when their diameters are respeclively two and three feet* If; however, the weirhts of the respective instruments be equal, that of the smallest diameter will make the deepest impression upon the ground; but, on leavy arable land, it will be more subject to drag the soil before it. It follows, therefore, that large rollers are so much more easily drawn, that their weight mav be increased to an extent suficient to comterbalance any probable adrantage in eliciency which may be possessed by those of smaller diameter.

\section{HOLIOW ROLLERS.}

This increase of diameter-were the cylinder sulid-wouhi, however, necessarily occasion cither too great an increase of weight, or else too great a climinution of length; to avoid which hollow iron rollers are much employed upon grentlenen's parks and other grass-lands in which it important to preserve great smoothness of surface. 'They also possess the advantarge of allowing their heaviness to be increased, when necessary, by hanging

* Quart, Journ, of Agric. N. S. vol, i. p. 705. 
weights to their axis. The expense of these instruments, though now less, in consequence of the fall in the price of iron, than it was some years aro, is, however, so very considerable, that one of them is mentioned, in the vorfolk Report, as being in use in the park at Holkham, which cost $60 l$.; it was cast at the Carron foundry, is five feet six inches in diameter, as well as length, and is drawn by four horses.

Hollow wooden rollers can, however, be constructed with much less cost, by simply getting three broad wheels of the proposed diameter, placed one in the centre and the other two at each end, and through the naves of which an iron axle is to be fixed, which runs the whole of the intended length and fastens them together. Epon these, strong planks, cut very narrow, and bevelled at the edves, are to be firmly nailed lengthwise until the roiler is completely covered; it may then be mounted in the usual manner, and the weights may be hung in the same way as in the iron roller. We havo lately seen one of these, made by a farmer and one of his men out of the wheels, stripped of their tire, and shafts of a wom-out three-wheeled tumbril, which perfectly answers the intended purpose; and the whole cost of which, for smith's work and plank, exclusive of the old materials, did not amount to much more than twenty shillings.

These hollow rollers are also sometimes made of an octagon shape, which do heavy work better than those which are round, because each turn brings the flat surface with more force to the ground; but they require greater strength to draw them. Mr. Malculm mentions having seen one of them at work which weighed at)out a ton and a quarter, and executed the operation so well, that it was comstantly borrowed by the neighbouring farmers: it was five feet in diameter, cut into as many sides as there were spolves in the wheel, and the axletree was sixteen inches in diameter*

\section{DOUBLE ROLLERS,}

in the form of the common cylinder, divided into two pieces, distant about an inch from each other, and moving each on its own axis, are sometimes used, both for the convenience which they afford in turning, and for laying a more cqual pressure upon ridgy land, and in these respects are certainly to be recommended. Long rollers, when turned short at the extremity of a field, having only the same direction at one end, necessarily twist whatever is under the centre; for the motion round the axis is nearly lost, and, by that means, the implement, instead of rollingr round in the manner it does when drawn straight forward, comes round in the same way as if it had no axis; thus frequently tearing up young grass and displacing seeds in a mamer that is not only always unsightly, but often prejudicial; whereas, by being shortened by their division, they re. volve in different directions, and that inconvenience is avoided. In rolling land which has been ploughed into ridges, it is also evident that, when the operation is performed lengthwise, the longer the roller is, the more unequal will be its pressure upon the crown, or upon the sides of the ridge. It is true that land should, in most cases, be ploughed across the ridges; but in water mcadows, which are sometimes slightly raised in the centre of the panes, and in arabic land that is intersected by very deep furrows, that cannot be done.

\section{CONVEX ROLLERS}

are therefore occasionally employed to meet the latter difficulty, by rolling, without crossing, the furrows of arable land. The centre part, or belly,

* Survey of Surrey and the Neighbouring Counties, vol, i. p. 217. 
being drawn lengthwise between the ridges, thus press into the furrow, and partly, though not always effectually, accomplish the object; for the power of this roller is chieffy exerted under its greatest diameter, where the soil, being in general the most crumbly, is the least in want of its influence. We have lately seen it in the hands of a very intelligent farmer, who occupies land of such a heavy nature as to require it to be thrown into very raised ridges, and he uses it attached to the front of a double roller, between which it acts in the following manner, the length, or breadth, of the entire ma. chine being equal to cover a twelve-bout lani-six on each sile,-but, although drawn by horses, they are attached in single shafts to avoid poaching the land, and thus walk in the furrow.

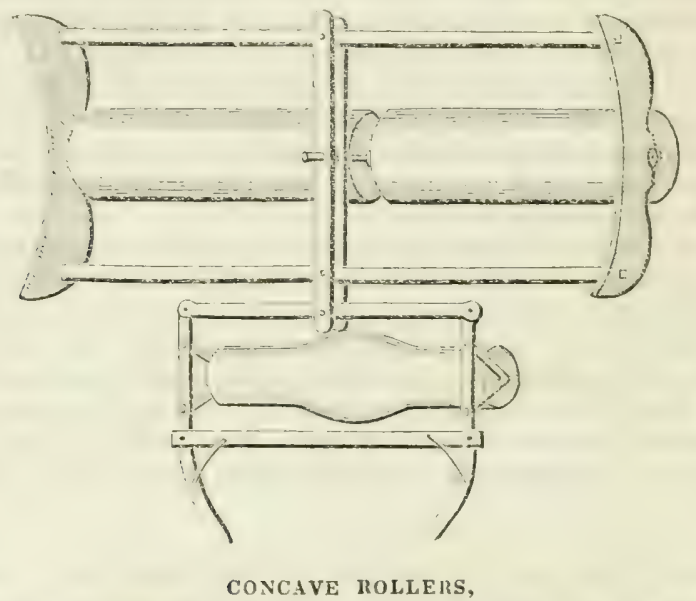

of the same description as that shetched underneath, are, on the contrary, used chiefly for the purpose of equally compressing the crowns of the ridges, in which form they are certainly effectual when employed upon land which is laid into very narrow ridges of a convex shape; but they must be then marie the full width of the ridge, as they are evidently useless upon level ground.

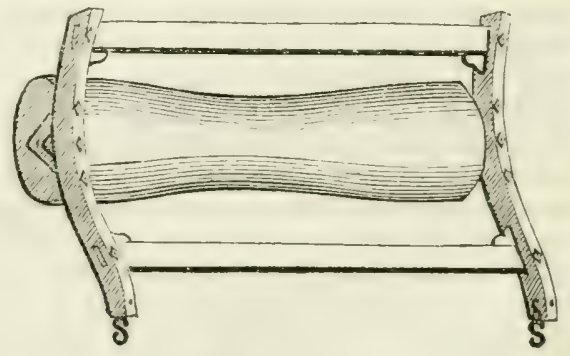

SPIKY ROLLERS

are not unfrequently employed to crush the clods upon tenacious soils which break up in large limps, and, if the land be dry, they are good instruments; but if it be moist, they clod up with the earth between the spikes with which they are studded, and thus become of less use than those which are plain. To remedy this defect, however, a great im- 
provement has been made by constructing them of double cylinclers, placed so close together as to admit of the spikes intersecting each other, so that they clear the dirt as the roller turns. When thus made, they are very powerful, for their weight is such as to break the hardest clump of dry clay, and the intersection of the spikes between each other grinds it almost to powder. When made in the common form, of a single wooden cylinder, the spikes are about three inches in length, and nearly the thickness of the thumb at the base, though somewhat pointed at the other extremity, and driven into the wood in the quincunx order. It is in this form a most efficient implement; but if made duuble, then it is almost umnecessary to say that the spikes must be placed in regular rows, each about nine inches apart. A very complete one, which is described in the Leicester Report as being in use on Lord Moira's land, consists of two rollers, each about nine inches diameter, armed with eight rows of spikes fixed in a frame, and mounted upon wheels about $3 \frac{1}{2}$ feet high, with double shafts fixed on the frame. There is also an upright post windlass and power of pullies fixed upon the frame to raise or lower the roller at pleasure. This part of the machine we, however, consider unnecessary; for the object of raising the machine for the purpose of carriage upon the roads might be attained by the more simple plan of wheels upon moveable cranks; we therefore merely insert a representation of the mode of arranging the spikes, without noticing the frame.

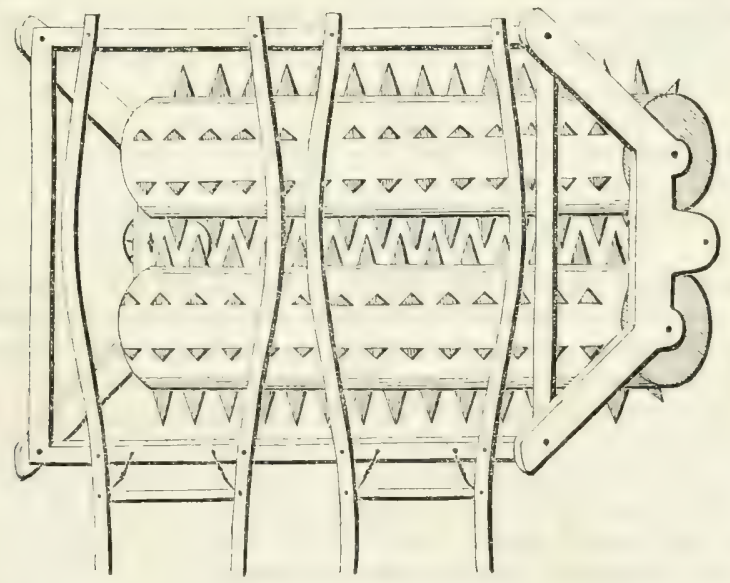

The spikes, thus working in and in, mutually cleanse each other, and if made with spiked cast-iron wheels, hooped round the cylinder, they could be more correctly formed than when the spikes are driven into the timber. When applied to fallows, it is very efficient on land which is so stiff as to bear the clods of the common one; and although we agree with Marshall, "that one effectual crush by a plain roller is worth a dozen partial indentures by a spiky one*" yet when the ground is too hard to submit to its pressure, the spikes will then be found to add greatly to its power.

In some cases, rollers are made to act in nearly the same manner as those with spikes, by having solid wedge-like rings of cast iron, with a hole through them to receive a stout wooden axis. These wheels are each $2 \frac{1}{2}$ inches thick, and are made moveable, so that they can be placed at different distances. They are sometimes 3 feet in diameter, and have been loaded so 
heavily as to be sufficient work for six oxen: they thus crush the elods with great effect; but they are more commonly used under the name of drillrollers*, as they are occasionally entployed without weights in broad-casting, to ensure a more regular distribution of the seed.

From this it is evident that famers ought to be provided with rollers of different length and diameter, or, if they have only one, that it should be so constructed as that its weight may be eitler increased or diminished to suit the purposes to which it is to be applied. In the opinion of many intelligent persons, they should not be less than about 16 , nor more than 30 inches in diameter; and the best length, if in one piece, is 5 feet, but if double, from 6 to 7 . Their importince is indeed too apparent to need illustration; for, without them, the process of tillage upon strong land cannot be completely executed, and light soils are by their operation rendered more compact and solid. Rolling is essential to most summer crops after the sced is sown, and particularly to wheat in the spring, for the winter frost frequently throws the roots out of the ground, but the pressure of the roller fastens them in the soil, strengthens the stems, and occasions them to tiller. It is, however, improper to roll wheat at seed-time, for it is better that the surface of the ground be left rather uneven during the winter, as the water passes through it more freely than if it be compressed, and the plants are more effectually preserved dry.

A roller of the common size will go over about six acres in a day; wherefore, upon large farms, more than one slould be employed to suard against delay in bad weatler, for the operation should never be performed when the land is wet.

\section{Chapter IV.}

\section{ON THE OPERATION OF PLOUGHING.}

Ir has been justly observed by Marshall, that to set a plough conrectly is, perhaps, one of the most diflicult lessons of plonghmanship. One field is of rich, mellow land, ploughable whith a pair of horses and whip reins, while another is a gluey clay that is hardly manageable with four, and different parts of the same field may often require separate modes of plougling. The plough, even if the same be used, must therefore be set in dilierent ways, both in the distance of the coulter from the breast, the fixing of the mouldboard, and the placing of the swingle-trees in the muzzle of the beam, so as to suit the various depths to which the land is to be ploughed and the breadth of the furrows, aceording to the tenacity or to the friability of the soil. All this requires different kinds of management, which can neither be conceived hy theory nor described by writing, and can only be learned by practice; it would therefore be a useless waste of labour were we to offer any directions on that portion of the subject. It has indeed been shrewdly remarked, that "voluminous works have been written upon agriculture by able, scientific nen in varions ages of the world; and the theory of the art may be said to be well understood, particularly in what are called the improved districts of these kingdoms. The practical is, however, but imperfectly understomb by any others than the operative labourers; one man, by dint of experience, obtains a complete knowledge how to set a plough to go steady, and how to hold it straight; another, how to drill or to sow broadcast; a third, how to reap, mow, and stack; others, how to cut an under-dratin in a proper direction to cary of the water; some, to hedge

* See the Reposts of Leicestersh., n. 58; Derbysh., vol, ii. n. 46; Oxfordsh., p. 77; and Norfolk, p. 58. 
and ditch; in short, most farm-labourers acquire superior information upon some one branch of the art; but infortunately, when these men die, their knowledge dies with them; others, young and inexperienced, succeed them, and it follows that they unnecessarily toil their own bones, and waste their employer's property, before they acquire sutticient experience to cxecute the work they take in hand in the most expeditious and correct manner. The farmer's time and attention are occupied in superintending and directing the general operations of his farm, the rotation of crops, application of manures, selection of stock, and other important concerns. He knows when his labourers are doing their work well, and he finds fault when he sees they are in error* :" to experienced and skilful husbandmen, therefore, little need be said regarding any part of the operation; but there are many persons in the occupation of land, who, not having been regularly bred to the business of farming, are not sufficiently acquainted with the details of tillage to be able to direct what is proper to be done, or to correct the errors comnitted by their servants, over whom they necessarily lose every proper degree of ascendency, and to such persons, a few observations may not be considered misplaced.

\section{PLOUGHMEN}

are not unfrequently engaged more from the appearance of their strength, than from any real knowledge of their competency to do justice to the work; a necessity, indeed, which is often imposed upon falmers by a view to the employment of the inhabitants of their parish. Tet no craft can be learned without due practice; and it has been justly remarked, that it is harder to become a good ploughman than to learn the common practice of most handicraft trades. A man who can handle the implement with dexterity and judgment, and who is, besides, careful of his team, must, therefore, be consideresl a valuable servant; but it camnot have escaped the observation of most masters, that such men are more rare than may be generally imagined.

The first care of a young beginner, who has been brought up as a driver and assistant in the farm-stable, and is thus, to a certain degree, acquainted not only with the management of the team, but also with the handling of the tool, should be to make the ridges straight, in which he will be much assisted if, instead of depending wholly on the accuracy of his sight, hewhen ploughing with a pair of horses-puts a cross-bar between the chechs of the brilles, so as to keep them precisely at the same distance from each other, and then setting up a pole at the end of the furrow, exactly measured to the same line as that from which he starts, fixes his eye steadily upon it, and carries the plough in a direction precisely to that point. But although this is a very important object, yet the finishing of the ridge neatly must depend on the exactness with which he holds to the depth of the furrows; for, if they be not all cut regulariy, by the plough being held in one position, they will be left with a rough surface, and a mane of grass or weeds on the edge of each plot: whereas, if ploughed in sung plits of a moderate width and regular depth, scarcely any space will be left between them, and the ridge will be thus completed with less labour, as well as in a more workmanlike manner. An idea is very generally entertained that the position in which the furrow sods are laid depends on the form of the mould-board; but although this is partially the case, it depends more on the breadth and depth of the furrow. To prove this would lead us into a dissertation which could interest but few of our readers, and we must therefore refer to those writers on the subject whom we have alrealy quoted; merely remark-

* Blaikie, on the Conversion of Arable Land into Pasture, \&c., p. 38. 
ing, that it may be commonly calculated that ploughs of the ordinary form will lay the furrow on a proper angle if the land be ploughed in about the proportion of three to two: that is, if a furrow of nine inches in breadth be carried to six inches in depth. It must, however, be admitted, that the width and depth of the furrows are not always thus regulated in practice, and that, although the principle of the rule is correet, it may yet be partially deviated from without much inconvenienee.

Bad ploughmen are remarkable for leaning on the left stilt, thus giving the bottom of the furrow a slope towards the land side, which they justify by saying, "that it eases the horses, and keeps the loose soil from tumbling in over the mould-board." Of this there can indeed be no doubt; but a portion of the land is thus left unstirred, or only ploughed to the depth of perhaps two or three inches, and consequently a portion of the tillage is imperfectly perfurmed. This partly arises from the breadth of the furrow-slice being greater than that of the feather of the sock, and partly from the plough being set with too much land; to counteract which it is necessary to press upon the left handle, and thus to raise the feather of the sock above the level of the heel of the plough. This may, indeed, be in some measure remedied by allowing the point of the share to penetrate about half an inch deeper into the ground, so as to prevent the plough from going too wide; but it should never be allowed to lean farther to the left than is necessary to effect that purpose. There is not, perhaps, one ploughman in twenty who can "land up" well, and fewer still who can do it neatly: they begin and end with their plit too deep, which, instead of laying the ridges convex, leaves them hog-troughed and lank-sided: that is, with a hollow place on each side. If broad-cast ridges are of unequal breadth, bent, or zigzag, the work cannot be uniform; and with crooked drills there is a loss of ground, an unequal distribution of manure, and the hoeings cannot be effectually performed *.

As the perfection of good ploughing can only be attained by practice, it must be evident that nothing like a system can be framed for the operation upon every diversity of soil; but the following rules, which are partly those of the late Mr. Finlayson, who was himself an expert practical ploughman, may be laid down as worthy of being observed by every man who means to become a proficient in the performance of his work.

1st. The horses should be harnessed as near to the plough as they can be placed without impeding the freedom of their step; for the closer they are to the point of draught, the less exertion will be required to overcome the resistance.

2nd. When ploughing with a pair abreast, the most forward and powerful horse should be worked in the furrow; but if the team be harnessed in line, ard there be any difference in the height of the cattle, the tallest should be put foremost if he be in every other respect equal to the other's.

3rd. When at work, they should be kept going at as regular and good a pace as the nature of the work will permit; for they are thus more manageable, and the draught easier than when slow. By due attention to this, the heavy soil will also cling less to the coulter, and the land will be found to work more freely.

4 th. The breadth and depth of the furrow being ascertained, the plough should be held upright, bearing equally all along on a straight sole, and be made to move forward in a regular line, without swerving

Sce the Reports of Bedfordshire, p. 278; Bucks, 8.106 ; and Surrey, p. 152 ; Marshall's Minutes of Agriculture, digest, p. 66 ; Finlay'son's Complete Ploughman, p. 44; and Williamson's Agric. Mechanism, p. 173. 
to either side. The elge of the coulter should also be set directly forward, so that the land side of it may run on a parallel line with the land side of the head, and in such a position as that their slant, or sweep, may exactly correspond.

5th. The ploughman should walk with his body as nearly as possible upright, without leaning on the stilts, and without using force to any part, further than may be absolutely necessary to keep the implement steadily in a direct line. He should also be sparing of his voice, and of correction to the team: of the former, because too much cheering and ordering only confuses the cattle ; and of the latter, because punishment, when often repeated, at length ceases to have due effect, and thus leads to unnecessary beating.

There is, in fact, a certain degree of taste in ploughing, as well as in every thing else, - a kind of tact, which is difficult to be taught, and hardly to be acquired except by a sort of instinct. Many a capital rough-rider is still but an indifferent horseman, because he has a heavy hand on his horse's mouth; and many a good seaman is still but an indifferent steersman, because he feels the helm imperfectly. You see the one pulling at a horse's head, straining him out of his paces, fretting and teasing him; yet with another, who knows how to play with his mouth, he will ride light in hand and pleasantly. So the seaman who can humour the helm makes good and easy steerage; while another toils and sweats, throws the vessel up in the wind, and makes her roll. So likewise the ploughinan who tills the ground with dexterity, never presses upon the plough without necessity - a mere touch, or a glance of the eye, tells him when she is going wrong, and a slight turn of the hand sets her instantly right; whereas a clumsy fellow, without feeling in his palms or readiness of perception, is continually either throwing the plough out, or she is riding upon heel or point, straining the team, tiring himself, and altogether making bad work.

\section{PLOUGHING:}

On the subject of dranght, it may be observed, that when horses are properly harnessed to the plough, their traces will be in a direct line from the point of draught at the shoulder to the point of the share passing through the regulating notch of the muzzle. It is therefore proper to ascertain the animal's height, in order that the muzzle may be fixed accordingly; but as his shoulder is not so far from the ground when he is pulling as when he is in a state of rest, an allowance must be made for the difference. 'Thus if a line be drawn from $A$ at the share of the plough to $B$, and then a perpendicular line from $B$ to $C$ at the horse's shoulter, an angle is formed: then, if another perpendicular line be drawn from $\Lambda$ to $a$, and measured upon the same scale, it will give the height of the bean from the ground, at the ilepth at which it is to be ploughed.

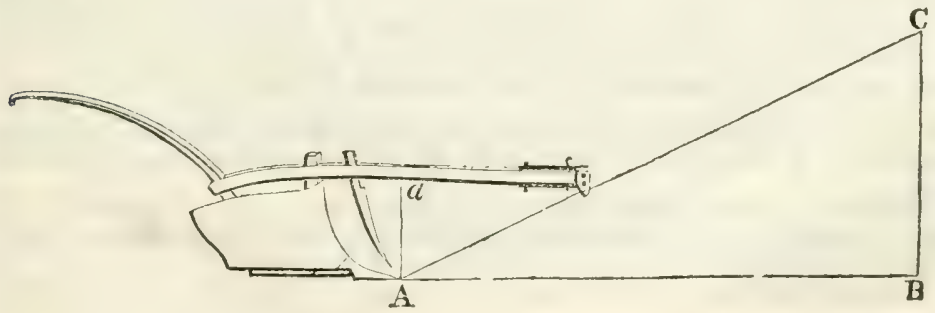


It follows, therefore, that the more the beam is raised in height, the longer it must be made, and consequently the traces of horses must be lengthened: this however, lessens thir power; for it is sulficiently well known, without entering into any mathematical proof upon the subject, that the farther the animal is placed from his work, the less effectual will be his exertions*.

It has, indeed, been calculated that when two horses of equal strength are attached to a plough, drawing both from the same point, but one before the other-if the one next to the plough draws by a line perpendicular to the line of his shoulders, and the foremost draws by a line which forms an angle of $30^{\circ}$ with the inclination of his shoulders, the cflect of the foremost horse is but one-half of the effect of that behind; and if another be added, and the angle already mentionel be about $15^{\circ}$, the power of that horse upon the plough will only equal one-fourth of the first: " so that the principal part of the dranght lies upon that horse which is nearest to the plough ; but that is sometimes transferred from one to another through the team, whilst the driver, walking by their side, now and then alarms a sluggish one or two with his whip, and then the plough runs along with the ploughman almost crazed at the tail to keep it in the groundt."

The foregoing calculation, although perhaps correct in point of theory, is however not true to the same extent in practice; yet there can be no manner of doubt that ploughing with a pair of any cattle abreast, instead of in line, is a great saving of power, and the work is thus generally better performed : for, when placed in line, the beam must be turned somewhat to the furrow in order to give the plough land; the cattle, therefore, draw in a different direction from the share and coulter, which occasions an increase of resistance, and of unsteadincss in the implement. Ploughing, either with a pair, or, if great strength be required, with three abreast, ought therefore to be adopted on all soils and under all circumstances that adnit of it; but, in saying this, we by no means agree in the sweeping assertion made by some writers, "that plough-work may be exccuted upon every lind of land by two good horses, if they are properly maintainedt," for our own experience and observation in many parts of this country liave proved to us that it is unfomded. We have already stated so much on the subject in different parts of this work, that it may be almost superfluous to add anything in corroboration of our opinion; but we beg leave to mention one instance, out of numberless others which could be adduced, of a neighlour of our's-a gentleman lately retired from the army-who has settled upon a farm of that heary species of clay which is usually ploughed with four.

Being convinced by all he had read that the farmers around him were

* In confirmation of this, an experiment is related by the Rev. St. John Priest in the Buckinghamshire Report, as made upon his own farm, on ploughing a wheat stuible with a Norfolk plough and two horses. "The furrow was $9 \frac{1}{2}$ inches wide, and $3 \frac{3}{4}$ deep. When the length of the horses' traces was 10 feet 4 inches from the point of the share to the point upon their shoulders from whence they were drawing, the force exerted upon the point of draught of the plough, or the power of their draught, was only $2 \frac{1}{15} \mathrm{cwt}$; but when the traces were lengrthened to 15 fect 6 inches, the force exerted to draw the plough was $3 \frac{1}{2}$ cwt." - p. 105.

It is almost umecessary to add that, when oxen are worked in yokes, a similar calculation should be made from the shate of the plough to the bearing point of the yole chain.

† Bucke. Rep., pp. 105 and 110 . See also the Treatise on Draught, in the History of the Horse, published in the Farmer's Series by the Suciety for the Diffision of Useful Kuww. ledge, pp. 418 and 424.

Brown of Markle, on Rural Affairs, vol, i., p. 231. 
wrong, he, last year, got two plonghmen out of the Lothians, to show his southern friends how the work should be performed. They were both expert men, and were put to work in the spring with a couple of Scotch ploughs and a pair each of certainly as fine and as highly-fed cart-horses as any in the kingdom. The land being then in good condition, and having only to effect the tillage of ground which had been already broken up in the winter, all went on to admiration until the soil became baked by the summer's sun, and then the ploughs could make no impression upon it. At length, however, autumn brought its rains, and after the weather had again become fair, and the gromb in apparent good order, they were set on to break a clover ley; but there they stuck fast. The soil, which is very deep, and requires de'e ploughing, is in fact of such a tough nature, that, when at all wet, it resists the action of the plough and adheres to it like glue; while the cattle stick in it in like mamner, and intependently of thus poaching the land, they cannot obtain a firm footing until they get at something like hard ground in the furrow. In answer to this it lias indeed been said, "that if the ground is in such a situation as not to bear a horse on the unploughed part, it is unfit for labouring, and ought not to be touched ;" but such land is seldum in any other state during the winter or the early part of the spring; and upon the farm where we are now writing, we have under our eye a large tract of ground which always requires four, and frequently five strong Lincolnshire horses to break it up, for shallow ploughing will not suit it, and the strain of draught upon the team is necessarily in proportion to the depth at which it is worked*. The Lothian men were at last obliged to use four in line and a stronger plough; but, not being accustomed to that mammer of ploughing, they made but indiflerent work, and, being laughed at by their fellow-labourers, they returned home in disgust.

Few farmers are so fond of their teams as to throw away money in the keep of an unnecessary number of horses for the performance of their work, and it cammot be presumed that many of them are ignorant of the manner in which that work can be best and most economically executed: we may, therefore, safely leave to their own judgment the decision of the question of expediency regarding the modes which they deem the most jroper. It should however be observed, that many of them use three, and sometimes even four, horses upon land of which a great part is so light as hardly to require more than one; but that is occisioned by circmmstances which render it unavoidable. There are several clayland farms of so heavy a nature as to require very powerful teams, but which are interspersed with portions of sand and gravelly soil, sometimes even in the same field; adted to this, there is still in many parts of England much common-field land, the divisions of which are in general extremely narrow, and in some plices so steep that they are necessarily ploughed up and down hill t : the farmer therefore is in some measure obligred to have at all times in the field strength enough for each kind of work, and even if not thus compelled in all cases, does not always fund it convenient to make any difference in his usual mode of tillage. As to the saving of a driver, more has been said about it by economists than the matter is worth: any boy is sufficiently capable; lis warges are a mere trifle; it is the only way of teaching him his business as a carter and

* The strain of draught upon a plourh is generally calculated acenrling to the square of whatever portion is under ground: thus when only three inches are burici, the pressure will be only 9 ; but if the furrow be carried to the depth of 6 inches, it will be 36 . - Williamson's Ágric. Mech., p. 161.

† See the Wiltshire Report, p. 36. 
ploughman; and if not employed in some shape, lie must bo brought up in idleness at the expense of the parish.

Having said thus much upon the handling of the plough, we have only a few words to add regarding the common modes of operation, which we shall state thus succinctly, because every workman who knows his business must be supposed capable of performing them.

The ground, when not intended to be laid down to permanent grass-in which case it is usually laid flat, and gripped where necessary by cross surface drains-is laid up in ridges, or lands, as they are sometimes called, of greater or less breadth, according as the ground may be more dry, or retentive of moisture. The brearth and form of the ridge have long been subjects of controversy, but it is evident that they must depend both upon the nature of the soil, and the mode of culture to which it is subjected. The most common width, on land of ordinary quality and cultivation, is 15 or 18 feet, each being intersected by a deep furrow; and they are formed in a slightly convex slape, with the intention of draining the superabundant water from the surface. This, however, being less necessary on dry ground than on that which lies wet, the ridges are in that case much broader, and laid proportionably level : thus, for instance, in many parts of the county of Kent, entire fields may be seen without either ridges or inter-furrows, for the soil admitting a quick filtration does not need them; or, if furrows are thought necessary, they are opened with a turn-wrest plough after the corn has been harrowed in, and the crops when in ear exhibits one uniform appearance*; but cold heavy land that retains the rain, is commonly laid up in sharp narrow ridges, having the crown raised some inches higher than the sides, and the inter-furrow sunk below the surface for the purpose of drainage. This, although occasioning the sacrifice of some portion of the ground, and in many instances carried farther than is prudent, is yet, to a certain extent, absolutely necessary on soils of the latter description: for, if the ridges be not raised in the centre, so as to throw off the rain, they will probably absorb a much greater quantity of water than may be useful for the purposes of vegetation; and the seed sown upon the land will be chilled: so, also, if the inter-furrows be not made sufficiently deep, they will not carry ofi the superfluous inoisture, and if it be allowed to stagnate in the furrows, it will saturate the land on each side of them and occasion mischief to the crop. The necessity thus occasioned of deep ploughing, also renders the soil porous and tends to drain it; but wherever water is allowed to stand upon loose mould it forms a puddle, which is reduced to a crust as soon as the moisture is dried up, and a caked surface on a tenacious soil is productive of certain injury to the crops. Therefore, although the practice of thus forming the ridges has been objected to, as parching the summits during the summer's drought, and wasting the soil by the deep inter-furrows being rendered sterilet-yet that is a choice of evils, of which the latter is the least: it should however, of course, be exercised with judgment, and care should be taken to secure a suflicient fall for the water. In "gathering up," or rounding narrow ridges, perhaps a rise

* Boys's Survey of Kent, p. 64. On dry turnip soils it is usual to plough the land into ridges of 30 to 36 feet-called in the North band-win ridges, because reaped by a band of shearers served by one binder. In finishing off this kind of soil, more especially before laying it down to grass, it is customary to cast up a narrow ridgelet, or single-bout drill, between the broad ridges, which diects the eye and hand of the sower; and when the field is harrowed to cover in the seed, all the ridlyelets are obliterated, or nearly so, leaving no bare unproductive portions, and giving a more beautiful lawn-like appearance to the field when afterwards under pasture. Gen. Rep. of Scotland, vol. i., p. 349.

† See Marshall's Min. of Agric., Digest, p. 66 ; and Rep. of the E. R. of Yorkshire, p. 109. 
of two to three or four inches per yard in width will be found suflicient; but in many places they are carried considerably higher*

In forming the ridges from land which has not been already tilled, the ploughman measures the brearth at which each is intended to be laid; setting them out from the headlands, which are ridges ruming across the top and bottom of the field to allow of space for turning the plough when it arrives at the endt; but, if the slope of the ground and the direction of the fences will allow it, ruming them north and south, as the corn will ripen more equally than when they are in the direction of east and west. He then plants some poles along the line of the ridge at different distances, to

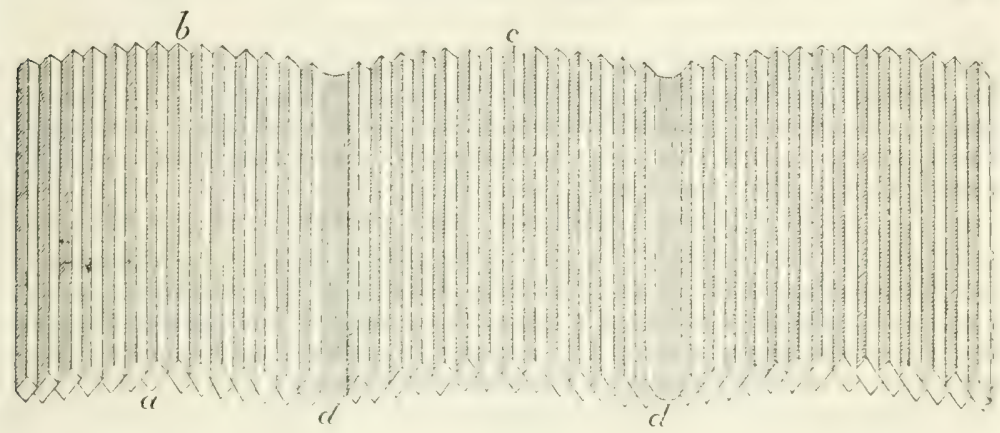

serve as guiles in striking a straight inter-furrow on each side to make the boundary; having done which, he proceeds to plough from the centre of the ridge. Thus, supposing the headland to adjoin each end of the fence, and the inter-furrows between the ridges to be represented, as in the preceding cut, by the letters $d d$, the ploughman enters the implement at $a$, thus laying the slice of the plough-furrow, or plit, to the right; and when he arrives at the end of the ridre, he then either returns by the upposite side of the same line-as at $b$-so as to lay the furrow in a contrary direction, and thus proceeds ploughing round until the entire ridge is completedt. Or, what is more usual - as allowing greater room for turning the tean, after ploughing the first furrow - he goes to $c$ in the second ridge; and when he has ploughed the furrow, he then returns to the first; thus altemately ploughing each half ridge until both are finished.

In this manner, the furrow-slices being laid upon each other towards the centre, the ridge thus acquires a certain degree of convexity; but when it

*In some parts of Gloncestershire the ridges are from $2 \frac{1}{2}$ to 3 feet high, and in others even still higher; but the ridges are also of a great breacth, - from 15 to 20 , though more generally 8 yards, and were probably thus constucted at a period when drainage was hut imperfectly understood. See Marshall's Gloucestershire, vol. i. p. 75 ; and Rudge's Survey, p. 105. In Essex wet land is laid on the two-bout ridge, or four-furrow work; but on the strong land, in the maritime district, stitches of eight furrows are general. Essex Rep., vol.i., p. 199.

+ As these headlands are necessarily much trodden on by the cattle during the operation of ploughing, they require to be afterwards particularly well worked, so as to destroy the tenacity thus occasioned in the ground. In some places they are constantly left in grass, which affords a firm footing to the teams, and imparts a very neat appearance to the fields: it also induces sportsmen, when in pursuit of their grame, to avoid riding over the crops.

$\ddagger$ Before the ridge is thus taken $u p$, it is, however, essentially necessary to form a furrow in that precise place by ploughing it outward; otherwise, either the centre of the land or ridge must remain unplonghed, or it would he raised to an unsightly height above the remainder of the land, from the first two furrows being united. 
becomes necessary to give it a greater curvature, it must be ploughed again, with furrows of less widh than the forner, but deeper at the centre, and gradually decreasing in depth towards the slirts, which operation is termed gathering.

It is sometimes desirable to throw two ridges into one; which is performed by causing the plough to enter at the inter-furrow, and to lay the successive plits of the whole ridge in the same direction, doing the same in an opposite direction with the adjoining ridge; but the inter-furrow which lies between the two ridges unavoidably leaves a shoulder, or hollow place, of more or less width according to the exuertness of the ploughman, in the centre of the crown, which ilefect can only be completely relieved by reploughing. This, lowever, may be partly prevented by using two ploughs carrying different mould-boarls, then striking deep narrow furrows on the insides, and shallow wide furrows on the outside; or, if only one be used, it should have a monld-board considerably wider set than is required for light soils, in order that the furrow may be close cut below and duly turned over*. This operation is called casting.

It is also frequently found expedient, in the process of summer fallowing, to alter the form of the ridge by putting the centre in the place of the interfurrow; which is done by commencing at the sile of the furrow, laying the first plit towards it, and then returning by another bout on the opposite sile and laying that plit againet it, and is distinguished as croun ond furrow. Or, sumetimes the ridges are divided by cutting them at the centre, thus forming an additional interiurow, and ploughing from each in the common way, so as to form new ridues of half the former width, which is linown as slitting or cleaving.

In summer fallowing, the entire field is likewise generally ploughed in a direction across that of the former ridges; but, as the intention is merely to stir the land more effectually and afterwards to replace the ridges in their old position by a subsequent ploughing, these ridges are usually made of greater breadth, and the sime neatness is not requisite as in any of the former operations : it is, not unaptly, denominated cross-ploughing.

Another mode of cleansing leys and stubbles consists in ploughling alternate furrows, and layiner each plit upon the adjoining umploughed land; by which means, althongh only one-talt of the soil is stirred, it wears the appearance of a complcte fillow, and the surfaces of the sods being turned against each other, the grass and weeds are rotted. It is therefore much practised in many places as a saving of labour; but, as it only partially exposes the soil to the atmosphere, it is not to be recommended. However, when thought expedient, the furrow-slices should be cut somewhat broaler than the balks, or muloughed ground on which they are turned; for, if laid in plits of the same breadth, and the land be at all clayey, they remain solid and unporous: whereas, by leaving the balk something marrower, and letting the edge of the plit overhang it, the inverted sod is shivered into pieces, and more effectually exposed to the wenther. It is indifferently named balking; or, more commonly, raftering.

In whatever mole these various operations are performed, it is further necessary, when they have been completed and the land is ready for the reception of the seed, to draw deep furrows through the headlands, in order to allow a free passage for the water which may be collected from the ridges to flow into the adjoining ditch. These, and indeed all the interfurrows between the ridges, shouli alsu be deepened and levelled, if neces-

* See Marshall's View of Agric., Digest, P. 79 ; and Brown of Markle, on Rural Affairs, vol. i. p. 219, 
sary, by the spade; and if any slope in the field lies in a different direction to that of the ridges, a deep furrow should also be cut in the direction of the slope, so as to form a chamnel for the escape of the water, by what is called gripping.

The quantity of land ploughed in a day's work necessarily depends on so many circumstances-comprising the nature of the soil, the season, the strength and agility of the teams, and the breadth and depth of the furrows, that no jrecise calculation can be formed; and we refer to what we have already said on the subject in our observations on animal labour * The ordinary estimate is generally an acre a day; but, taking into consideration the various impediments to which the work is exposed, perhaps three roods are nearer to the average.

Independently of the nature of the soil, the irregularity of the fences occasions in many instances a great waste of labour by causing frequent short turnings, and the narrowness of many strips of land held in common fields also prevents them from being cross-plonghed; thus depriving them of the benefit of being exposed, as they ought, to the influence of the sun and air. Added to which, there is the frequent occurrence in old inclosures of ridges, ploughed time out of mind in a variety of uncouth shapes, placed without regard to the boundaries of the fields, and divided by such deep inter-furrows, that improvement is almost impossible by any other means than laying them first quite level, which every furmer finds to be attended with very considerable trouble and expenset. "It can, incied, be only done, if the land be at all wet, by previous unler dranage, and a gradual course of careful tillage; for, if the crowns of the ridges be hastily levelled, the most fritie portion of the soil is thus buried in the furrows, while a sterile subsoil is turned up, and the attempt, if made in one vear, would certainly prove abortive. It should, moreorer, never be attempted but in a year of clean fallow.

In some places also, as we have alrearly stated, the partition of the land in common fields requires them to be plonghed up and doun hill. Exclu.sive of the great increase of labour thus occasioned, the cattle never work equally ; for those which are sluggish hang back upon the rise, while those which possess more spirit heat ani fatigue themselves by over-exertion. It al:o occasions the further inconvenience of causing mich of the manure, and some of the ricliest particles of the soil, to be swept down from the upper part through the furrows by the rains. In order to avoid this, whenever the size and livision of the ground acimits of its being ploughed in any other direction, the ridges should be carried diagronally across the ascent, so as both to allow the fall of the water to be as gentle as possible, and also to ease the toil of the team. The latter object will be best effected by sloping the rilges upwarls from the right, instead of from the left hand: for, in the former case, when the horses are mounting the hill, the plough casts the furrow downwards; whereas, in the litter, when they are ascending, it is turned upwards, and thus greatly increases their labour. The turnwrest plough is also a good implement to use for this purpose; and, in steep ascents, the grubher may likewise be cmployed with good cfect.

To prevent, as far as possible, the upper parts of a hilly field from being deprived of a portion of the manure, attentive furmers usually lay on a larger quantity in proportion to the rise of the ascent. In some places,

* See vol. i. chap. vii. p. 152, and chap. viii. p. 188.

$\dagger$ For a detailed acconnt of the mode of effecting this, sec pp. 13 and 14 of Scoreby, in No. V. of the Select Farms published by the Society tur the Diffusion of Useful Kuowledge. 
where it is very steep, the ground is ploughed directly across; thus forming terraces, by which means much of the labour of the cattle is spared; but land thus situated is more generally left under permanent grass.

Such are the chief processes by which the land is commonly tilled; for although there are many others-to some of which we shall hereafter have occasion to allurle-which are employed by speculative or scientific farmers, these are the means generally adopted throughout the United Kingdom. In the operation of ploughing, the Scotch labourers have acquired the character of being neater workmen than the generality of their southern neighbours; but the soil of the Lothians, from which district they have chiefly attained their reputation, though strong, is of a mellow description of loam, which admits of being easier worked than much of the heavy clays in many parts of England. In Ireland, very considerable improvement has been lately effected in tillage by some spirited farmers and country gentlemen; and there can be no doubt that, when the farmers generally acquire that capital to which their present exertions seem to entitle them, it will gradually reach a high degree of perfection. We learn, however, from the most recent of the Irish county surveys, published within these few years, under the authority of the Dublin Society, that, except on the lands of wealthy individuals, it still remains in a very imperfect state. "The common plough of the small farmer is constructed on a bad principle, if principle at all there be to guide the workman, since for the most part it is put together by guess, and whether the instrument works well or ill is a matter of chance. Upwards of twenty years ago it was remarked by Mir. Wakefield, in his Statistical Accomt of freland, that if it was necessary to plough deeper than the instrument from its original set would permit, there existed no means of altering it; so that an extra person was employed to press upon the beam, and most ploughs were accompanied by an attendant with a spade to turn back the earth, which, after the plough had alvanced, would otherwise revert to its former bed, and the shovelling of the trenches was always practised, whether the plough or the spade had been employed. Ploughing was, indeed, merely marking the land with the furrow, and even this was executed so bally, that he ascribed the produce of corn much more to the spade than to the plough*".

These observations the reporter considers strictly applicable to a great portion of the country at the present day; though, as already stated, examples may every where be foumd on the lands of the principal gentry of excellent tillage, with Scotch ploughs of the most approved construction, drawn by a pair of horses and driven by the ploughman. Much has indeed been done by the distribution of ploughs, and by ploughing matches established in many places by the late Farming Society of Ireland; for, although it is so difficult to counteract the prejudice attached to old habits, especialiy in farming, that the old plough continues in use to the present day, yet many of those who attended carried home useful hints to act upon. The ploughman saw that the instrument was held level, instead of having one stilt kept almost to the ground; yet though held and worked in a manner so different from his, the ploughing was nevertheless well performed, and with less labour both to the workman and his team. He therefore changed his mode of holding, and the ploughwright also made a little alteration in the fashion of his cumbrous machine; by which means the furrow slice is now more correctly cut, the whole surface more uniformly turned, and this 
change in the mode of performing the work has had a very perceptible good effect upon the improvement of the crops*.

From what we have thus stated, it must be apparent that, as every lind of soil has its appropriate qualities, each requires a peculiar mode of tillage. Ploughing, which is the chief operation, ought therefore to be executed according to the nature of the land, and not performed upon any one invariable principle. On strong clays and loams, and other soils of a deep and rich staple, the plough should go to a considerable depth; whereas, on thin clays and barren sands, the benefit of deep ploughing is very questionable, especially when incumbent on a sterile subsoil : on each description of land distinct modes of operation and implements of different construction are therefore absolutely necessary. On wet retentive clays, too, where the ridges must be considerably raised, in order to preserve the ground in something like a dry condition, and in all cases where the gathering up of the land is particularly desirable, greater care is requisite than in the management of light soils, which technically admit of "crown and furrow" ploughing, and the ploughman who is capable of performing it with accuracy is invaluable. In short, heavy land necessarily occasions heavy work; and the farmer who is not provided with proper implements, teams of sufficient strength, and servants of capability, can never cultivate such land with advantage.

Very opposite opinions are lowever entertained by many farmers regarding the proper depth of ploughing; and, as the application of deep or shallow tillage to various soils respectively has not been ascertained upon any settled principle, we shall here add a few observations on the subject; in doing which we have, however, to acknowledge much obligation to that eminent agriculturist Von Thaër, whose great practical experience and deep science, added to the candour with which his remarks are imparted, stamp an inestimable value upon his works on Husbandry.

\section{DEPTH OF PLOUGHING。}

The proper lepth of ploughing must necessarily depend upon the nature of the soils; but although every intelligent husbandman must be aware of the superiority of those of a deep staple over those which are shallow, yet, in discussing the propriety of the extent to which the operation may be safely carried, it should be borne in mind that there is a wide difference between the effects of ploughing deeply into land, the vegetative stratum of which is of nearly equal fertility throughout, and that of augmenting a shallow surface of fertile soil by mixing it up with a subsoil of inferior quality.

The depth to which the roots of plants penetrate into the earth in search of sustenance, varies as much, according to the properties of the soil, as does the nature of the plants themselves. There are many, the roots of which are found at fifteen to twenty, and even thirty feet under ground-sainfoin

* "The sharp-witted Irish are sensible to ridicule; and the small farmer, formerly content with the vile old plough and its miserable accompaniments, upon receiving one of iron, with a handsome set of painted swingle-trees, thought it 'too bad,' as he would express himself, 'to have it drawn in the ould way;' and in downright shame would strain a point to make a hempen back-band, horse-skin collar, and winkered bridle supplant those articles in former use, of fragile and temporary manufacture from the flail. He would, moreover, try 'to swap the ould mare for one of a stronger cut;' and gaining something better in the horse way, would perhaps be disposed, by a patch of clover or vetches, to provide something better for the animal's support." See an article on the Objects and Effects of the late Farming Society of Ireland, in the Quart. Journ. of Agric. N. S. No. xxii, ; also, on the Present State of Irish Agriculture in No, xxi, of the same work. 
and lucerne, for instance; even red clover will strike down to nearly three fect if the soil be a fertile loam; and some of our commonest vegetables, if it be friable or sandy, push their tap-roots to about the same depth. But as the land is chiefly destined for the production of grain, its value is rather to be considered according to its capability for the growth of corn than for that of any particular species of green crop.

It may be observed by the mere naked eye, that if the land be of a free nature, the roots of wheat will penetrate as far as eight inches into the earth; and when sown on the crowns of ridges, they have been found in rich soils at the depth of twelve; though that probably arose from the greater influence of the atmosphere, for they do not reach to the same depth on level gromul. The seed is usually sown at about two inches deep; consequently, the roots may be ten inches long. We may, therefore, assume the dejth of twelve inches as the utmost veretative limit of corn land.

If the plants lie close torether, they are disposed to penetrate more deeply into the earth than when they are wide apart; for it may be remarked that the roots of corn avoid each other, and push their strongest shoots into those spots where they have most room to spread; but if the seed be thickly sown, they are then furced to strike their roots perpendicularly, instead of laterally, into the ground. Still, however, this must depend upon the mature of the land; for if the subsoil be sterile or tenacious, the roots then cither meeting opjosition, or not fuling nourishment, must again seck the upper stratum. There, they become matted together, and, each contending for the share of nutriment of which it has been deprived, the weakest are often seen to perish; which in a great measure accounts for that partial failure which frequently attends corn crops at the very lieight of the season. Provided the soil be open and tolerably fertile, the nearer its depth approaches to that which we have stated to be its vegetative limit, the greater number of plants may it therefore be supposed capable of furnishing with support"; and, if attentively observed, a material difference will be found between the nutritive properties of land of a deep staple, and that which is more shallow, although the quality of both, when tried in equal quantities, may be the samet. When deep, it also possesses the great advantage of suffering less either by extreme heat or by wet, than when thin: and the corn being supported by a greater deptl of root, which probably affords

*A remarkable instance of this is mentioned in the Bedford Report, in which it is stated "that a road having been made across the ends of some ridged lands in the parish of Ridgemount, the cultivated soil of the riciges was cast into the hollows. The fielil was afterwarks sown with barley, and the scattered coms which grew where the soil was accumulated in the ancient furrows formed a remarkable contrast with the rest of the field. The proiluce of the barley crop was little more than three quarters per acre, while that which grew on the same poor soil, accumulated, perhaps, a foot deep, was remarkably luxuriant, many of the corns producing from ten to twenty stalks, and the ears large in proportion." An effect which the reporter ascribes to no other cause than the dipth of the soil. - p. 277.

$\dagger$ Von Thaër calculates this difference in proportionate degrees in land which contains a regetative stratum of soil of four, six, eight, and twelve inches in depth ; provided, of course, that it he all of equal cuality. If, therefore, each seed were to produce a plant. it would follow that ground which contains eight inches depth of fertile mould, might be sown with double the ruautity of that which consists of only four inches. He, however, admits that this principle cannot be carried to that extent, because the action of the atmosphere must erer afford such a superiurity to the surface, that a cubic foot of moule!, if divided into two square feet, will always produce a grenter numler of plants than if the seed were sown upon one foot superficial; but he assumes the value of the land to be increased in the proportion of 8 per cent. for every inch of mould beyond the depth of six to ten inches, and to be diminished, in the same proportion, from six to three inches in soils of a thinner staple.-Principes líaisonne's d'Agriculture, rol, iii., p. 138. s. 735. 
proportionate strength to the lower part of the stem, maintains its freshness with greater vigour in times of drought, and is less subject to be laid by storms of wind or rain. It is almost umnecessary to add, that depth of soil is equally advantageous to every crop, the roots of which go deeper into the soil than those of corn, though its progressive increase in value is not so great as that which lies nearer to the surface.

In order to maintain the properties of a deep soil in a perfect staie, it is, however, expedient to plough it from time to time to the full depth of the vegetative stratum ; so that, being completely worked by frequent turnings, it may be freely exposed to the influence of the atmosphere. If that be not done, or only superficially performed, the ground will, by slow degrees, become gradually impoverished; for, besides being deprived of the benefit of exposure to the air, a crust of hard and almost impenetrable matter will, in some soils, be formed immediately below that portion upon which the plough was usually made to act, thus constituting a barrier between the upper surface and the subsoil, which is well known to farmers under the appellation of the moor-band, or pan. It is not, however, necessary to repeat the operation, even on heavy land, more than, perhaps, once in six or seven years, especially if the intermediate ploughings under the common course of cultivation be made at various depths; for nothing tends more to the formation of the pan than the constant repetition of the process at the same depth. Thus it will not escape obscrvation, that one great advantage of the alternate system of husbandry arises from the distinct nature of the different roots which are cultivated, and the consequent necessity of ploughing at various depths, so as to afford them room to seek for sustenance. On tenacious clays, which, being confined to the growth of grain, are usually flloughed to the same depth, it also forms a strong reason for the adoption of the summer fallow.

The maintenance of the properties of the soil in its original state is, however, a very different mode of procedure from that required by an endeavour to restore it to perfection; for there is much land of a deep and naturally mellow staple, the lower strata of which have been rendered sterile by shallow ploughing, and can only be progressively removated by gradual degrees; or, according to the practice of those farmers who " like to go decp enough to chip up the dead soil now and then, and show it a little upon the surface after all is finished*." Many instances have, indeed, been recorded in which the subsoil, when brought to the surface and exposed for some time, has proved extremely fertile; but in all those cases, the earth, when chemically analyzed, has been found to contain a considerable portion of carbon, and unless afterwards sustained by proportionate quantities of manure, its good qualities have been speedily exhausted. It cannot, therefore, be deemed generally expedient to bring up a greater quantity than at the most two inches of the virgin earth, even supposing it to be naturally of the best average quality. The operation should be performed before the winter, and the soil should be incorporated by the means of a summer fallow; observing, however, that its main object being to expose this fresh substance to the atmosphere, it should be left as long as possible on the surface. The manure should be also applied at the same time, and, instead of being mixed up with the soil, should be spread uniformly over this layer of new earth, which thus imbibes its juices during the whole of the wet season, and, notwithstanding all the objections which have been urged against the evaporation of dung $\dagger$, never fails to produce a

* Survey of Essex, vol, i., p. 198.

+ On the subject of evaporation, see vol, i., chap. x., Pl. 239 and 2-18. If the land E 2 
striking effect on its amelioration. In this manner, M. Thaër assures us that he has successively, during a series of years, effected the gradual and complete mixture of different layers of soil in one season; that he has thereby not only angmented the vegetative stratum of his soil, but has experienced a very sensible improvement in his crops; and that, after the close of a rotation, he has repeated the experiment with equal success. To which we may add, that many farmers who have followed the same plan, have uniformly found it attended with similar results; though others, who have not taken the precaution of going leisurely to work, or who have not been sufficiently aware of the nature of the subsoil, have, in some instances, done considerable injury to their land*.

There are, no doubt, many deep soils naturally of an equal quality throughout, but which, consisting of tenacious clay, or till $\dagger$, of various kinds, would, if brought up to the surface, impoverish the ground, and bring in their train a long list of noxious weeds, were they not corrected by the process of perlıaps more than one summer fallow, together with an application of both lime and dung; and it is also true, that even if these substances can be readily obtained, yet the expense, even if repaid by the land, may not suit the farmer's pocket. Under ordinary circumstances, indeed, large operations of that nature, which are intended to effect a permanent improvement in the soil, cannot be considered as coming within the scope of common farming, and can only be undertaken by the owner of the land, or on terms which will secure the tenant in the return of his outlay; for it should be clearly understood that in no case can it be effected without either the as. sistance of extraneous manure, or by the sacrifice of a portion of that which properly belongs to the usual cultivation of the farm, and might, therefore, probably be more advantageously applied to the support of the soil already under tillage.

Neither should poor soils be ploughed deep; for the regetative mould with which the surface of land of that description is covered, having been formed by the decomposition of the scanty herbage which grew upon it when in a state of nature, is proportionably thin, and the subsoil on which it reposes consisting generally of sterile materials, it will be found bad policy to mix them with the upper stratum, which, though perhaps not very productive, yet requires the whole power of the small portion of manure which it furnishes to preserve it in tolerable condition.. This, more especially, if it be intended to return the land to grass; for the sward seldom penetrates farther than about two inches into the ground, and its value, therefore, depends chiefly upon the fecundity of the surface upon which it rests. Thus the generality of those farmers who are situated upon wolds

lies upon such a slope as may cause the dung to be swept off by the rains, then it slould, however, be buried by a very slight ploughing.

* See the Reports of Eissex, vol. i., p. 197 ; Bedford, p. 276 ; Bucks, p. 128 ; Stevenson's Surrey, pp. 148 and 176; Malcolm's Surrey, vol. i., p. 268; Kent's Norfulk, p. 37 ; Gen. Rep. of Scotland, vol. i., 11). 347 and 419 ; and Loch's Improvements on the estates of the Marquess of Stafford.

+ In Scotland, "till" is understood to imply, as a subsoil, a mixture of clay with sand and gravel, devoid of vergetable matter, and impervious to water. In Ireland, this deseription of soil is called "Lackleagh," and the pan which it forms is there broken up with a plough of immense weight and strength, called a " miner," which acts without a mould-board, and is followed in the furrow by another plough of the common power. See vol. i., chap. xxix., p. 465.

\ "The Norfolk farmers, fenerally possessing a thin light soil, with a poor and barren sulisoil, prefer shallow ploughing at all times, and argue that it is easier to keep a small quantity of soil in good heart than a greater quanlity, which would be formed by deep ploughing." - Dumfries liep., App. p. 578. 
and downs, carefully avoid ploughing below the top-soil : for when there is a vein of rubbly chalk, or of small broken flints, immediately under the topsoil, they look upon it as " the dross of the land," and that, if ploughed up, it is quite poison to the field

If the land be of a sandy nature, the ploughing of which has been alivays carried to one depth, and that a pan has thus been formed underneath, in that case, also, it is injudicious to break it up; for, independently of the labour of the operation, the surface may have been much ameliorated by good culture, and the hard crust which lies at the bottom both secures the manure from being carried down below that part which is actually tilled, and also prevents the escape of humidity from the upper stratum. Another layer of sand, indeed, not unfrequently exists under the pan; for that species of crust is often found where land has been much marled; but although it might be desirable to have it at a greater depth, yet its removal is a work of extreme difficulty, and, on such soils, will rarely repay the expense. On sand veins where there is a great depth of soil, and in which no obstruction of this kind is found, it is however not unusual to plough very deep, and to have a second plough following in the furrow of the first, so as to throw up new soil, and to bury that which is supposed to be exhausted: as is the case in many parts of Devonshiref.

In fine, it will, in most cases, be found inexpedient to add to the stratum already under tillage by any other means than those which, little by little, may gradually tend to increase the depth of vegetative mould by the mixture of small quantities of the virgin earth of the subsoil with the surface; but if a more extensive operation be contemplated, then the following questions may be not unaptly put before coming to a conclusion.

lst, What return may be expected from land, the subsoil of which has not yet been submitted to the plough?

2ndly, What change will be occasioned, either by the increase or diminution of the tenacity, or of the friability of the land, by the admixture of the subsoil with the surface?

3rdly, What quantity of manure will be required beyond that already disposable upon the farm? and

4 thly, What will be the entire cost?

To solve which, the earth should either he submitted to a chemical analysis, or its qualitics ascertained by spreading some of it, to a certain depth, in a garden, and sowing seeds upon it. The probable improvement in the soil may thus be ascertained, and the supposed increase in the future crops, when compared with the expenditure, will determine the propriety of the experiment.

We have not hitherto touched upon the subject of the actual depth to which ploughing is commonly carried, nor is it necessary to say much regarding it; for it of course depends more upon the state of the land and the judgment of the farmer than upon any general rule. The usual operation is generally confined to from four to five, and rarely exceeds seven inches; though, in the hundreds of Essex, and some other districts where the soil is rich and deep, it is sometimes carried as far as nine. An opinion is indeed entertained that, if carried decper, more manure is necessary to ensure a crop; and it will be readily admitted that, were this idea well founded, it

* "Many instances are shown where land of this kind, ploughed too deep upwards of twenty years ago, has not yet recovered its former goodness; and, to keep the top-soil as deep as possible, the best farmers will not permit the surface-flints to be picked off for the roads, lest it should make the land both lighter and thinner." -Davis's Survey of IIilshire, 1. 52 . 
would form a strong argument in favour of moderate depths. It is, lowever, only justly applicable to land of which the subsoil is inferior to the surface; for when this is of the same quality with the cultivated part, not only can no injury arise from gradually incorporating it with the tilth, but benefit will be derived by all plants which penetrate far into the ground, as they naturally absorl their nutriment both from the soil and the manure with which it is mixed. It must, however, be admitted, that when ground is sudlenly ploughed to an unusual depth, it increases the difficulty of destroying weenls. This is particularly observable in thin stapled clays, on which they spring up as if indigenous to the soil; it is thereiore obvious that no benefit can be derived from ploughing such land deeper than common, unless it be done in the manner we have already pointed out.

\section{Chapter V.}

\section{ON FALLOWING.}

FArLows are commonly distinguished into two kinds: the wintr, or green, and the bare summer fallow; the ground being, in both cases, usually ploughed immediately after harvest, and, in the former, sown during the spring with some species of root or leguminous crop; but in the latter, left unsown until the following autum, by which time it is generally prepared for wheat. Rye and winter tares are, however, not unfrequently sown in the autum as a preparation for wheat, and being fed off early in the spring, the land is ploughed and dressed for the corn crop during the remainder of the summer, and is then said to receive a bastard fallow.

Before the alternate return of green crops on light land was joined to the recurrence of summer fallow upon strong soils, there was also a system much practised under the terms autumn and spring fallous. The former was employed to clean the stubble land intended for wheat; and as at that time wheat frequently succeeded oats, the stubble was made as clean and fit for the wheat as it could be at that time and period of the year. The latter was generally used as a jreparation for barlev; and, indeed, in many places, the barley crop is still considered as the cleaning or fallowing crop. The consequence, however, of both these methods was, cither that the land was incompletely cleared for the reception of the wheat, or that the sowing of the barley was deferred beyond the proper time; but at present, both spring and autumn fallowing are almost exploded upon light soils, and the land is senerally cleaned for spring crops by the process of hoeing, or, upon heary land, by that of the summer fallow*.

The crops under a green fallow-which are also called fullow cropsconsist of every species of root and vegetable usually produced by field culture, provided they be not allowed to perfect their seels; for, in that case, they exhaust, instead of improviner, the soil. Thus the various sorts of turnips, rape, cabbages, carrots, parsnips, bect, and potatoes, though producing large quantities of food for both man and beast, yet returning it again to the land in the shape of manure, are all, when consumed in that immature state, of an ameliorating nature; but when permitted to ripen, and to seed, have a contrary effect. Clovers, also, partake of the same properties when either fed down or made into hay for the supply of cattle; but if allowed to stand for seed, they are said to rob the land. Perhaps the only

* See Stevenson's Survey of Surrey, chap, vii, sect. 2. 
exception to the remark is beans, which, though probably drawing more nourishment from the soil than they return in the shape of food, yet are generally viewed as a fallow crop, and are constantly sown under that denomination on those heavy soils which are found appropriate to their production: properly speaking, however, they cannot be considered in that light unless when sown in drills, or dibbled at wide distances which admit of their being horse-hoed, and thus keeping the land clean.

To the introduction of these crops into our farming system the improved state of agriculture in this country is mainly due, and there can be little doubt that the extension of that system will still further tend to its improvement. Their culture will, however, be separately treated under their distinct heads, to which we refer our observations, our present object being confined to an account of

\section{SUMMER FALLOW.}

The practice of summer fallow dates in England from the earliest cultivation of the land. It was introduced into this island by the ancient Romans, of whose farming it formed a part of the constant course, and who conducted it in the same mamner as at present; and it has been continued up to this day on most of the heavy soils throughout the kinglom, though its object was, in former times, rather to allow the ground time to rest from the production of corn crops-then the only ones grown-than to clean it by tillage.

It is, however, a singular fact in the history of Scotch agriculture, that although fallowing in the present mode, for the purpose of cleaning the soil, had been long prevalent in England, and, consequently, could not have been unknown in Scotland-particularly after the comnexion which took place between the two nations in the time of James VI.--yet it does not seem to have passed the northern horder before the close of the seventeenth century; for in a book entitled 'Husbandric Anatomized,' which was published in Edinburgh in 1697, and is supposed to be the oldest known treatise on Scottish rural ecomomy, we fund the fallowing of land mentioned in a manner which shows that it was not then known in that comntry, and it is recommended by the author, who had been an officer of the Cameronian regiment, serving in King William's wars, as a practice with which he had become acyuainted in Flanders. We learn, indeed, from other accounts that the first person who actually set the example of fallowing land upon his estate, in Scotland, by preparing it for a crop of corn with frequent ploughings and harrowings, was either Thomas, sixth Ear] of IIaddington, or, as some say, it was introduced by one John Walker, tenant at Beanston, in East Lothian, who was the chief of a family long distinguished in that district as superior farmers, at the commencement of the last century. From the success attending the experiment, the practice then rapidly spread over the country, and we are told, in 'Miaxwell's Collections,' that it afterwards became customary upon the strong soils every fifth or sisth year, but was not general until about the year 17:-1. Nir. Walker is also understood to have been the first person in Scotland who sowed wheat upon what was then termed out-field land,-or that which lies far from the homestead, and is commonly of indifferent quality, - which, previous to the introduction of fallow, could not have been effected with profit, and under the old system was only practicable upon in-field or crop grounds*

* It was formerly proposed to erect a pillar to the memory of Mr. Walker, with an inscription detailing these circumstances; but, although they eminently merit record, the intention has not been carried into effect. See the Farm, Mag., vol, i. p, 161, and the East Lothian Rep., p. 92. 
; The necessity of fallowing, when viewed merely as a means of cleansing the ground from weeds, and bringing it to a fit state for the reception of the seed, depends entirely upon the state in which the land may be found after a previous course of tillage; and therefore, upon some soils, and under different kinds of manargement, the repetition of the practice is more or less frequently required than upon others. No really practical man will deny that whenever the soil has become foul, it can be cleaned in no other way than by frequently stirring it during the spring and summer, for the purpose of bringing the seeds of weeds to vegetate, and then destroying them. On light, free soils, having a gravelly subsoil, this can indeed be done by means of green crops, drilled at intervals of sufficient width to admit of the operation of the horse-hoe; for they can be worked while those crops are growing, and on such land they are, therefore, generally sown after corn, under that system which has obtained the name of "alternate husbandry." But when the soil is of a more stubborn and tenacious quality, incumbent upon a clayey bottom, it is both naturally more subject to become foul than land of a drier and more friable species, and being, from its nature, not adapted to the production of green crops, the farmer has no other resource, when it gets into that state, than to plough and harrow it repeatedly, and thus to effectually cleanse it through the operation of a naked summer fallow.

This, however, has been denied; and aithough the operation of preparing the land for a course of crops must be admitted to be the foundation of all good husbandry, and therefore might be presumed to be clearly understood by every farmer, yet it is not a little remarkable that the most oppo. site opinions are entertained regarding the necessity or advantages of fallowing, and that the question, though of the very highest importance to agriculture, both in a general sense and as it regards the profits of cultivation, and treated of by many distinguished writers of the present day, has still been left undetermined. It would, indeed, appear that much of this indecision has arisen with persons who have reasoned upon the subject without sufficient acquaintance with farming to enable them to judge of its effects with accuracy. Thus some argue against the practice, because, as nature does not seem to require any pause of this kind, but intends the earth to produce uninterrupted crops in every year, the management of the ground should be governed by that supreme law : forgetting, however, that nature does not discriminate between the value of the crops, and that weeds are produced as bountifully as grain. Others contend, that the loss of a whole year is a waste of the national resources; and even men of business, who are not easily led away by such crude premises, yet insist that a year's rent, taxes, and expenses, thus drawn out of the farmer's pocliet without retum, can only arise from slovenly mismanagement. These, indeed, represent green crops as acting like a spell upon the coffers of famers, and, being men of figures, they run up such an account of the profits attendant upon them, in opjosition to fallows, and state the difference so clearly in pounds, shillings, and pence, that the result is quite astounding, and, so far as calculations go, there is no reason to doubt their accuracy ; yet many of those who have adopted their plans have suffered severely in their pocket*. At the head of these authors stands the opinion of a celebrated chemist, who says decidedly, as the sum of a treatise upon the subject, " that it is scarcely possible to imagine a single instance of a cultivated soil, which can be supposed to remain fallow for a single year with 
alrantage to the farmer ". While another learned professor, who upholds the sistem, yet ascribes its whole value to the effects of solar light upon the soil; which, if true, would tend to prove that land must be rendered more productive in proportion as it is exposed to the ravs of the sum, though every farmer knows that, when impenetrably shaded by a dense crop of clover, it afterwards produces the weightiest crop of comt.

Leaving, however, the specious arguments of theorists for the sound practical proofs of experienced men, we ret find much prejudice amongst them, occasioned by their taking a limited view of the subject without sufficient attention to the climate, as well as the soil of the district which they mean to describe; for, although the nature of all soils, of any particular description, may be the same in every county, yet the superiority of the climate in one may enable the farmer to work his land more frequently and more effectually, and thereby to kecp it more clean than it can be maintained in anotlier where the seasons are dissimilar: which in a great measure accounts for the difference of opinion prevailing among the agriculturists of the North, and many of those in the South, of the Enited Kingdom. Among the former, the more intelligent class in general contend that summer fallowing is indispensable on wet, strong clays; for that having, in various instances, endeavoured to avoid it, the land, after a certain lapse of time, invariably became foul, and they were compelled to have recourse to it in order to render the soil clean; that some of it is subject, after two or three crops, to run together, and is of such an untoward nature that all the art and industry of man cannot keep it in good order for any considerable length of time without the intervention of a summer fallow to render it mellow; and that it is the most certain remedy, the speediest, and, in the end, the cheapesti.. Many of the latter, however, insist, on the contrary, that land, to the full as strong as any to be found in Scotland, can be cropped continually by the mere process of carefully drilling their crops, without ever recurring to the operation of a naked fallow. Here, then, the question is at issue upon two points-namely, can clays be licpt constantly clean, or, if they chance to become foul, can they be made clean without the intervention of a summer fallow? And this may, perhaps, be decided by a brief statement of the practice adopted by both parties for the attainment of their object.

That of the south country famer consists in sowing winter tares, beans, and clover altermately with corn; for of course we do not allude to suils which admit of the tumin husbandry. Iis crops come so early of

* Sir Humphry Dary, Agric. Chem., Lecture the First.

i See a 'Treatise on the Scientific Practice of Fallowing;' \&c., by James Reunie, A. MI., Prof, of Zoology, King's Coll., London; Quart. Journ. of A gric., No. XXX., and also the answer to it in the following Number, by James Main, author of Vegetable Thysiology, \&c. Sce. The influence of light and heat upon regetable productions is well linown, but their effect upon the soil is lint very imperfectly uiderstoor ; and it can only be supposed from analogy that certain combinations and decompositions take place by means of their agency, which are nerer performed by cold and darkness.

T. See Boys' Survey of Kent, p. 6, and also Stevenson's Surrey, p. 17i, in which it is stated, that "when the clays of the weralkl," which lie in a flat and unventilated situation, "get thoroughly wet in September or Octuler, there is very little chance of their leeing in a fit condition to suw with wheat that year. On such suits, therefore, the command of a complete season seems necessary to work them f roperly, and get the seed into a dry bed." The remark may, indeed, be apjlied to land of the same kind, similarly situated, in any part of the kingdom, and is supported by many of the reports on the state of the southern counties; and in the General Report of Scotland it is said that "it may be foretold of every farmer on a strong soil, in such a climate as that of the north, that his afluence and prosperity will always be in proportion to the excellent state of his fallows." -Vol. i., p. 424 . 
the ground, that he is enabled to dress the land in time for tares, and thus he obtains the benefit of a bastard fallow, while, luring the growth of the bean crop, the sunimer generally allows of the effective operation of the horse-hoe; and by these means he, in common seasons, gains nearly all the advantages of a complete summer fallow. In the north, however, these objects cannot always be accomplished; for the crops come off so much later than in the south, that corn cannot generally be reaped sufficiently early to admit of the land being properly dressed and sown with tares, and the bastard fallow is consequently lost. Then the summers are frequently so cold and dripping, as to render every eflort inadequate to the destruction of weeds, which spring up with rapidity after every shower, and thus defy the operation of the hoe. Indeed, those which propagate their species by suckers from the roots, are invigorated by the process, which, by affording a fresh supply of air and openness to the soil, gives freedom to the shoots which are left in possession of the ground; and no one who is acquainted with the practice of tillage will calculate upon their eradication merely by the effects of hoeing.

These facts prove incontestably that climate, in conjunction with soil, and not the soil alone, should be taken into consideration in every discussion regarding the expediency of summer fallows, and that the continuance of the practice camnot be justly attributed to either stubbormmess or ignorance. But supposing that, in despite of drilling, horse and hand-hoeing, the land becomes foul, as it will in any county if the season prove cold and moist, it may then be asked, Can it afteruards be made clean withoul a thorough summer fallow? And to this we venture to assert, that the answer of almost every farmer who has to contend with a wet and heavy soil will be decidedly given in the negative*. In saying this, however, we are far from assuming that fallowing should be universally adopted: that it may be warded of during a certain number of rears by the expedients of the improved husbandry cannot be doubted; but that it can be blotted out of the system is, we assume, more than can be supported upon any tenable ground. Thercfore let the natural catses which render it necessary be duly considered, and, if these be found suficient, let it then be ellectually performed, without allowing any speculative theory to interfere with the sound dictates of practical experience.

The practice, indeed, althcugh formerly universal, has been long wearing partially out, and has been very generally abandoned upon soils of a nature to achmit of alternate cropis of com and artificial grasses, or roots

* "If we had no wet weather and no winter months, then constant cropping would certainly be practicable, and an extra stuck of men and horses would be all that was recuired; but in the British climate little or no work can be done from Autumn to the lst of April, which can tend to materially clear the ground of root-weeds. The sole object of plunghinc. in winter is to rot the stubble, and prepare a bed for spring seeds; and with erery degree of attention it is often, from necessity; performed unler such unfavourable circumstances as to hasten a return of summer fallow sooner than could be wished.

"In a word, upon all clay soils, and, generaliy speaking, ujon soils of every c'escription incumbent upon a wet bottom, the best time of cleaning them is in the summer montlis; and in this opinion we are decidedity supported by the respectable author of the Staffordshire Survey. That genleman, in his repurt to the Luard of Agriculture, says, - Fallowing for wheat on cold, wet, strong land's, and on all such as are unfit for turnins, is alisuiutely necessary; and he who attempts to manage such land without fallowing, will have reason to repent his mistake. Mixed soils, which are too wet for tumips, have a particular propensity to the production of root grasses. Summer fallow, therefore, becumes absulutely necessary; and every attempt to (rop' without it, for any length of time, on such land, has terminated to its injury, and to the loss of the occupier." Brown of Markle, vol, i, p. 209. 
under the drill husbandry; but on strong clays it has becn continued in most places time out of mind, under nearly the same system of manarement as that of our forefathers*. The common idea, that the soil acyuires an increase of fertility by being exposed to the influenee of the atmosphere, and allowed to rest without production, although still entertained by some persons $\dagger$, has also been generally relinquished by most intelligent men, who see in it no other benefit than what may be obtained from a cessation of the growth of exhausting crops, and perhaps some advantage arising from the decomposition of weeds, instead of allowing them to arrive at maturity; though it certainly affords a superior tilth for com than what can be obtained by a fillow crop. But as no crop that is exhausting can be considered as a fallow crop, the sole object is to clean the land whenever it becomes foul; therefore, whenever that happens, without reference to the period which may have expired since the operation was last performed, they determine upon the application of a bare summer fallow.

That summer fallows are frequently carried to an unnecessary extent, cammot be denied; but it should be recollected, that incoming tenants very commonly find the land left in such a state that there is no other mode of cleansing it ; nor can it be forgotten, that they have been established for ages by the usage of common-ficld, and that the covenants of leases upon many large estates still bind the tenant to fallow regularly at stated periods. Iis this, indeed, some proprietors liave acted upon the same principle as that laid down in the laws of the Jews by Moses, who ordered that the tribes of Israel, when led out of Egrpt, should give the land a juhilec every seventh year ${ }_{t}^{+}$; and others act upon a system of still closer restriction. These leases lave, indeed, been, in most instances, copied from those which vere drawn up long before the husbandry of the country had reached its present improvement: the bulk of farmers were at that period comparatively ignorant of the art of agriculture; the system of alternate green and corn erops was unkmown, and linitation was necessary, in order to guard the interest of the landlord, and prevent the gromnd from being run out of heart by over cropping. Absurd clauses of restraint have thus, in several instances, been continued to the injury of both parties, and doubtless should be, in most cases, exploded. Restrictions nuay, however, be still considered prudent to a certain extent; for though no intelligent and conscientious farmer should be bound by such fetiers as comprel him to any particular course of crops, until within two or three years of the expiration of his lease, yet his landlord should have the power of enforeing them in case of mismanagement.

There are various modes of conducting the operation of a clean fallow: its continuance and the number of plunghings reyuisite depend on the

* Thus, on comsulting that original writer, I:llis of Great Craddesten, who published an octavo volume in 1745, under the title of 'The Chiltern Vale Farming explained, but little alteration can be perceived to have been made at his time in the Viale of IVhiteIorse, where uninclosed, from that which he recommends.-Berks Rep., p. 161 .

$\dagger$ "So far as my experience goes, sooner or later the land will be reduced to that state of weakness and imbecility in which no manure, no hasty stimulus, or green crop, will enable it to sustain and perfect a beneficial crop of grain. Manure and green crops, it is true, will produce a crop of straw, but fur want of that regetalle aliment, which cin only be restored by fallowing and rest, the crops of grain will ever fall short in that farina which is the life and soul of all white crops." - Brown, Orig. Rep. of Derbysh. p. 29.

"Every good Wiltshire farmer will say, that, upon the downs, two years" rest for wheat is equal to the best coat of dung. Dung may give the quantity, but rest must give the quality." -Dayies' Wilts Rep., p. 57.

${ }_{+}^{+}$"And six years shalt thou sow thy land, and gather the fruits thereof: but the seventh year thou shalt let it rest and lie still."-Exodus, chap, xxiii, v. 10, 11. 
season, the nature of the soil, the tilth and cleanliness of the land, and the kind of weeds to be destroyed. "If the spring season be found insufficient to effect it, take the summer, and even the autumn, the winter, and the ensuing spring, rather than crop an under-worked fallow, which is but little superior to a single ploughing. One stirring towards the close is frequently more valuable than two or three at the outset; and, to begin $九$ fullow without continuing it until its intention be fully accomplished, is throuing away labour unprofitably*." The most usual method is, however, commonly as follows.

The first winter-furrow or fallow-ploughing is usually given as soon as the hurry of harvest and wheat-seed time is over; for, if deferred too long, and the weather become wet, the land may be rendered altogether unfit for the operation, or at least so heavy as to be attended with considerable additional labour, as well as being less beneficial to the soil; though a moderate fall of rain is desirable before the commencement of the process, in order to bring the ground into that flexible condition which is necessary to the easy performance of the work. It is, indeed, only on dry, sandy, or light soils, that ploughing can be carried on with any advantage in bad weather, and a remarkable difference may be observed in favour of the crops upon fields which have been ploughed when tolerably free from wet, or in a state between moist and dry. The process must, however, depend upon the senson; but, provided that be favourable, and the land be properly laid up so as to receive the full benefit of the winter's frost, the exact period at which it is performed is not very material, though it slould, if possible, be always completed before Christmas. If the land can be easily laid dry, it is not uncommonly worked in crown and furrow, and is usually ploughed nearly with a square furrow-slice, by which means it is left rough, and as much as possible exposed to the atmosphere. It is, therefore, neither rolled nor harrowed; and the ridges should be formed of a size suitable to the nature of the land, but in all cases carefully gathered up and interfurrowed, so that they may be laid high and dryt: the field should also be cross-gripped, to prevent the stagnation of water, and in that state it is generally allowed to lie until the following spring.

Some farmers, however, let their land lie under stubble during the whole of the winter, in the opinion that the soil, if of a cold nature, becomes chilled and perished by the wet; and that, if left unploughed until the spring, it is kept much drier than if the surface had been moved, as it throws off the rain more completely when firm and unbroken. After the more indispensable operations of wheat-seed time are finished, it also very commonly happens that the state of the weather will not permit their clay-lands to be ploughed up dry; but that, by leaving them unploughed during the winter, and giving them the first furrow in the spring, they allege, that the ground turns up in large clods, by the exposure of which to the sun during summer, the root-weeds are killed in the easiest and most effectual manner; whereas, if the land had been ploughed before winter, it would at the second ploughing turn up like mortar, and never thoroughly recover its proper state + . But to this it may be answered, that although the land may lie drier under stubble than that which has been ploughed during the winter, yet this advantage may be considered comparatively trifling, when the beneficial effects of the frost upon clay land are taken

- * Marshall's Rur. Econ. of Yorkshire, vol. i. p. 339.

+ "On strong good lands, apt to be wet in winter, no ridges are so proper as what are termed ten-furrowed or five-bout ridges." - Norfolk Husbandry, p. 72.

t Bedford Rep., p. 330. Surrey do., p. 172. 
into account; and it is not improbable that the practice has rather arisen from the neglect of drainage than from any other real necessity.

Some difference of opinion also prevails regarding the proper depth of the furrow-slice to be taken under this first ploughing, and the angle which the furrow-slice ought to form with the horizon. The most general practice is to go the full depth to which the plough is usually carried; for it is thought that if summer fallows are not ploughed deep enough when they receive the first furrow, it will afterwards be found impossible, in a dry season, to go to a sufficient depth. There are farmers, however, who contend that, although attended with difliculty and hard labour, it yet is far from impracticable, and who, therefore, plough shallow at first, and after clearing the soil thus turned up, go to the utmost depth they intend with the next furrow*. When land is much covered with surface weeds, and that it can be ploughed early in the season, this light ploughing may be judicious; for it encourages the growth of annuals, and if the weather prove moist, many of the seeds which would have been buried by a deep ploughing may spring up, and be destroyed by harrowing, or by an after ploughing. The remark will also hold good upon the breaking. up of grass leys, the sward of which, if buried deep, will not rot, and, therefore, should be ploughed very superficially; but, when the winter ploughing has been late in the year, it must be evident that the more root-weeds are exposed to the air, the more easily will they be detached from the soil, and destroyed during the ensuing faliow process; and that the deeper the furrow, the greater will be the benefit which the gromnd will receive from the mellowing influence of the frost. In adhesive soils, the plough should, therefore, be set narrower and deeper than in the common practice, and the furrow-slice should be well tumed over, or laid as nearly as possible at an angle of forty-five degrees with the horizon; for, if completely turned upside down, or laid too flat, land of that sort will lie water-sogged during the whole of the winter + .

"The second ploughing is given some time in April, if the season permits, though more generally in May, soon after the spring sowing has passed, and not until the ground is in a proper temper between wet and dry; but if the land be much subject to annual weeds, it is highly expedient to run the harrows across it some time previously, in order to encourage their growth. When the soil is very heavy, the ridges are again ploughed end-long; and, if formerly gathered up high and narrow, they are now cleaved. 'The third commonly takes place in the month of June, and is performed at right angles, or as nearly so as the form of the fields will permit ; thus cutting across the former ridges, from which it has obtained the name of the cross-furrow. Sometimes, however, if the land be tolerably dry, and in such condition that five turnings are deemed sufficient, the cross-furrow is given at the second ploughing; but, in either case, it is intended to go deep, and turn up the ground in large lumps, which, though wearing an uncouth appearance, yet expose a large surface to the sun and air, and are thus well adapted to further the progress of the fallow process.

The rollers, drags, and harrows, are shortly afterwards set vigorously to work, to crush the clods, tear up and extract the root-weeds, which should be invariably gathered into heaps by women and children, and either carried off the ground or burned: the former is, however, of the two, far the most desirable plan. Double turns of the harrows shoul, indeed, be

* General Report of Scotland, vol. ic p. 347. Kent's Norfolk, p. 41. + See Blaikie on the Conversion of Arable Land into Pasture. 
repeatedly given in opposite directions, to secure, as far as possible, the eradication of the weeds, and no labour should be spared on the efficient performance of these latter operations. The time when they are to be performed must, however, depend upon the weather; for, at this season, the atmosphere has a powerful effect both upon the soil and vegetation: if rain falls, they camnot be too soon executed, for the land being thus brought into a tolerably fine state, the weeds will spring promptly up in readiness for the next turning; but, if the weather continue lry, it will promote the decomposition of those root-weeds which lave been already loosened from the soil, and assist their extraction. The harrowing need not, therefore, be hurried; yet care should also be taken not to defer it too long, as tenacious land would in that case become so baked by the sun, as to render the efforts of those implements wholly ineffectual.

The fourth ploughing should be completed some time in the month of July, the earlier the better, or at furthest not later than the begiming of August, so as to come between the hay and corn harvests; and the land should be ridged up, or gathered, in the original direction, in order to aroid the conseguences of heary rains, which on clay land should always be guarder against as much as possible. The ridges should also be this time harrowed lengthwise, as cross-harrowing would bring the root-weeds into the furrows, where they would immediately strike root, if not picked off. Some farmers, however, nerrlect that precaution, and lay the land in large divisions without regarling the ridges; while others not unfrequently plough it in a direction diagonal to the former ridges, so as to cut them in a different point from either of the former ploughings; but it is a dangerous plan, for if the weather happen to be wet, the land will become sour, and so poached in working, as to be nearly ummanageable. Instead of this fourth ploughing, lowever, the grubbers may be emploved; and if they, togrether with the roller, drags, and harrows, he assiduously used, the rootweeds will be completely eradicated by their successive operation; the ammuals will be either brought to maturity and destroyed, or their seeds, together with numerous tribes of insects, will be exposed to be devoured by bircls, and the soil will be brought into, jerhaps, a finer tilth than can be effected by the plough.

Some persons, indeed, object to the use of these implements in the fallow process, alleging that frequent ploughing is all that is necessary to destroy root-weeds by the baking of the clods in the sun and wind: certainly there are some lands with which it will not agree to pulverize them too much, as by so doing the seed is often thrown out of the ground in winter time. Such suils, howerer, are more friable than those of the stubborn nature of which we have been treating, and it has been justly remarked, as having been ascertined by experience, "that frequent turning over the ground, though absolutely necessary while the fallow process is going on, can never eradicate couch-giass or other root-weeds. In all clav-soils the ground turns up in lumps or clods, which the severest drought will not penetrate so sufiiciently as to kill the included roots. When the land is again ploughed, these lumps are simply turned over, and no more, and the action of the plough serves in no legrec to reduce them, or at least very imperceptibly. It may be added, that these lumps likewise contain innumerable secls, which camnot vegetate unless brought under the influence of the sum and air near the surface. The diligent use of the roller and harrows, followed by careful hand-picking, is, therefore, indispensably necessary to the perfection of the process"." That of the grubber will also be found essen-

* Gen, Rep, of Scotland, vol, i., p. 421. 
tially useful after the second or third ploughings; for when these have been accomplished, it not only brealis the clods more effectually than the plough, lut it also possesses the decided advantage of executing the work with a degree of promptitude which enables the farmer to seize upon any favourable moment for its completion.

If these operations have been all eficiently performed, the land may then be supposed to be in a fit state for the reception of the manurewhether dung or compost; but if it be not yet perfectly clean, they must be repeated until it becomes so; and indeed another stirring of the land, either with the grubbers or the plough, will amply repay the expense, by the improvement which it will occasion in the working of the soil, and the production of the future course of crops. In the maritime district of the Hundreds of Essex, for instance, which are much infested with a species of weed called black-grass, the process is seldom thought to have been well completed until after eight ploughings, and some farmers are linown to give as many as nine or ten, and even to the extent of twelve. In that county it is, however, the common practice to apply the fallow to the barley crop ${ }^{*}$.

The fifth p!oughing, or that which preceles the seed-furrow, is therefore, the time for laying on the manure. As it is important to the success of the future crop to apply it during the continuance of fine weather, so that the soil may receive the full benefit of the reciprocal action of the earth and manure while forced by the heat and occasional showers which may be expected to fall at that censon, the operation should not be too long delayed; and as it is peculiarly necessary to get it upon the land while the fallow is in a dry state, no time should be lost in spreading it. It is laid on under a shallow furrow, and in farms properly managed, there is generally strength of teams suflicient to carry out the dung and complete the ploughing without delay; which is a great advantage, for when allowed to lie long uncovered, it may be supposed to lose some portion of its fertilizing properties $\dagger$. The mode of procedure is as follows.

The manure should be carried to the ground in single horse carts, which poach the land less than those which carry a heavy load, and is carted along the ridges, where it is thrown out by a man standing in the cart into heaps of equal size, and set at equal distances, more or less apart, according

* Essex Report, vol. i., p. 195. The following is an account of the manner of performing it, furnished by Lord Western.

"To make a grood fallow, we give the first ploughing in November, February, or quite to the end of March, after barley sowing; if in November, the stitches are laid a little round, to be water shot, and afterwards well water-furrowed. The land is grenerally ploughed first into stitches of eight or twelve nine-inch furrows, then crossed and ploughed in different ways very often during the summer, turning up every time a different surface to the sun and air. Before harvest, it is got up on four-furrow ridges; afier harvest it is immediately ploughed again, and if the weather admits, twice, leaving it upon the ridge for the winter: the later the last ploughing the better, to prevent the black-grass from getting up; after which it is never water-furrowed. By these means we get upon it much earlier in the spring than we could otherwise do, and when we flough fo: barley, these stiff, tenacious lands break down into the finest tilth it is possible to conceive.

"Upon the whole, we give not less than six, seven, or eight ploughings. In ploughing stitches for wheat, or anything else, much attention is paid to turn the furrows well, draw them straight, size them alike, and lap them with such regularity on to each other, that the harrows cannot fail to lay hold of them all. The shutting up furrow, in particular, is drawn straight, handsomely turned, clean swept out, and at the same time the space between stitch and stitch not left too wide."

+ On this subject see, however, the remarks in vol. i, chap. X.g pp. 238 and 240 ; chap. xxvi., p. 431; and the T'able, p. 437. 
to the quantity which is intended for the land. Some persons are so particular as to mark the places at which the lieaps are to be laid, by running furrows at regular intervals along and across the field, by which means the cross-cut indicates the spot; but this is generally found to be unnecessary, as, when once the distance at which any two heaps are to be placed is pointed out, an experienced workman can measure both the ground and the manure with sufficient accuracy by his eye. A sufficient number of labourers must also be employed to spread the manure with regularity and expedition, as it should not be allowed to settle in heaps: three or four of these follow each cart, and spread out the dung with light three-pronged forks; but women, if properly superintended, can do the work equally well. The plough follows close after the spreaders, and covers the dung; the ridge being formed according to the custom of the country, though at the same time opening the furrows to leave a clean course for the water, and the land, being then well gripped, is allowed to remain in that state until the seed-furrow, by which time it may be supposed that the dung will be partly decomposed and embodied with the soil.

- If lime is to be spread, men only must, however, be employed:-a moderately calm and perfectly dry day should be chosen for the purpose, not only to prevent the waste of its finer particles by their being blown upon the fences and the adjoining land, but also to prevent injury to the jeople and cattle employed in the operation. There is no doubt but the earlier in the season the lime can be applied, after the land has become so far broken as to prevent its falling directly to the sole, or pan, the more favourable will be the result. The application of it in its quick and powdery state greatly promotes the destruction of weeds, and all the slimycoated insect tribe; renders the land more friable in the working, and becomes intimately mixed with the soil by the further operations of the fallow, the whole process of which it greatly facilitates. As soon as the lime is spread, it should then be immediately harrowed lightly in whilst dry, as rain will soon convert it into a viscous and adhesive pulp**. Either lime or compost, if thoroughly decomposed, may indeed be effectually applied by the aid of the grubber, but the spreading of stable dung camot be effected without the use of the plough; for it camot be properly buried and covered by the grubber.

The sixth, and generally the last plougling, is that of the seed-furrow, which is usually completed during the month of October; though sometimes as early as the middle or latter end of September: the period depending not only upon the state of the land, but also upon that of the weather. It is very desirable not to delay it upon wet soils, as many disadvantages may attend a late seed-time; but the lateness of the previous harvest may impede the preparation of the land, or the work may be prevented by drought. If grain be sown when the land is perfectly dry, it will not sprout; so that, if showers do not fall, much of it may be spoiled, to the serious injury of the future crop. No precise rule can, therefore, be laid down upon the subject, and it not unfrequently happens that farmers are compelled to defer their sowing until late in the winter. Too much harrowing is not proper for wheat; but when once sown, the inter-furrows should be opened by the ribbing-plough, and at the ends of the ridges

* Sec vol. i., chap. xii., pp. 256 and 296. Chalk, when applied to a fallow, is, however, usually laid either upon the stubble, or upon a ley intended to be broken up, and is carried to the land before the commencement of winter, in orter that the lumps may be broken by the frost. - Ib., p, 279. 
they should be carefully cleaned out with the spade, so as to allow a free passage for the water into the cross-grips and ditches. Thus close the labours of the summer fallow; and if conducted in the manner described, the land will probably carry crops of grain and pulse for a number of years, without any necessity for a repetition of the process. When the operation has been well performed, the ground will also be undoubtedly rendered both more productive as well as more easy to be worked; and when it may again require to be fallowed, it will demand fewer ploughings than those stated as necessary for foul land.

\section{WINTER HALLOW.}

The labours of winter fallowing, when intended for the preparation of spring crops, though conducted nearly in the same manner as those already detailed, are necessarily more confined in their operation: seldom, indeed, exceeding three ploughings, and not unfrequently consisting of only two; for it is a prevalent opinion that light soils-particularly if they be of a sandy nature-are frequently much injured by being overworked; and it is clear that they neither require, nor will bear, to be more pulverized than may be absolutely necessary for the destruction of weeds and the production of a good seed-bed. If the land, however, be of a firmer nature, and especially if it partake of the qualities of loam, the best practice is to give three ploughings, the last of which is preceded by the effectual operation of the grubber; for although strong loams with a good bottom, having been once thoroughly cleaned by a well-wrought summer fallow, may afterwards be kept in order by drilled crops, yet to maintain them in that condition requires the most constant attention and the most careful management, or they will again inevitably become foul.

The first of these ploughings should be given as soon as possible after harrest, and if it can be effected early in the autumn, then a second with a deeper furrow can be given in the course of the winter : the grubber follows when the weeds have begum to sprout in the spring, and the last ploughing is the seed-furrow.

It is thus that the sowings of the spring crops should be prepared; but on many farms both time and strength are wanting, and the tenants are obliged to content themselves with two ploughings, of which the first is not unfrequently very imperfect. Oats being a common crop on poor land, and being put into the ground early, are very commonly sown in this manner; but this species of culture is evidently insufficient for the destruction of weeds, or for the due amalgamation of the manure with the soil ; so that the dung is sometimes found in the following year in hard lumps, like turf, which are not broken up without much difficulty, and it consequently cannot afford nourishment to the ground until the following crop. Those who trust to dung for the production of good crops of com, should also consider, that unless the ground be previously relieved from the load of weeds with which it is encumbered, they can never expect it to produce the same benefit that it would if it had been brought into proper order.

It has been not unaptly remarked by a man of known experience in husbandry, that the science of agriculture is nothing more than an endeavour to discover and cure nature's defects; and the grand outlines of it are-" how to make heavy land lighter, and light land heavier; cold land hotter, and hot land colder. He that knows these secrets is a farmer, and he that does not know them is no farmer*." From want of attending to these general 
ideas, many absurd doctrines have however been propagated concerning it, and in no instance more than in that of fallowing land, which it has been very common to condemn in the gross, as a mere waste of labour and loss of crops. If by fallowing be meant repeated summer ploughings, it follows that on some lands it is proper, and on others totally wrong. It can never be right to make land lighter that is too light already; but there are few instances where strong heavy land can be pulverized too much. It may be replied that this pulverization may be as well effected under crops as in a state of fallow. On some soils it may; on others it cannot. In veins of rich sand, it has been found to be totally improper: such land will not bear a summer fallow; for it can never be made too close, if it can be kept clean; and on such a soil, this can be done better with a crop than without one *.

With regard to the system of fallowing in the northern districts of the kingdom, it is considered by most intelligent farmers as not generally suited to the more tenacious clays of the southern counties of England. The fallow-land on such soils, they contend, should not be broken up before it is dry in April or May; for if ploughed in autumn, and if the winter prove open, with much rain, it retains the moisture, becomes soured, and requires much trouble to get it dried for cleaning in spring. The treading of the cattle on such land, while ploughing, also does much mischief, for the impression of their feet will hold water when taking up the last two furrows of a ridge, and will, in a great measure, prevent the proper turning over of the furrow slice. In wet seasons the horses should, therefore, be always driven in the furrow. In some situations it is, no doubt, an easy matter to lay aside fallows, and to raise wheat after green crops; but we are borne out by the opinion of practical agriculturists of great eminence, in saying, that no man, who is generally acquainted with the soil and climate of Great Britain, will attempt to explode their use altogether. The essential points of tillage-besides preserving the land in heart with manure-consist in keeping it dry by drainage, and clearing it of weeds: if fallowed steadily, with perseverance and judgment, they are sure to succeed; but if negligently or imperfectly performed, no land, however cheaply rented, can leave a living profit to the farmer.

Having thus stated our opinion on the expediency of fallowing, we should be wanting in candour if we neglected to advert more particularly than by general mention of its opponents, to the opposite practice pursued for some years past, on heavy land, by the late Thomas Greg, Esq. and General Beatson : the details of which, although already before the public, are so pertinent to the subject of this chapter, that a further account of it cannot, we think, be deemed misplaced.

GREG'S SYSTEM.

The farm of Coles, near Buntingford, in Hertfordshire, consists of $\mathbf{2 4 0}$ acres of arable land, which is described as " a very tenacious clay, in some places mixed up with calcareous earth, which causes it to bind at top after heavy rain;" and was formerly worked nearly under a three-course system of summer fallow, white corn, and pulse, or clover. Turnips were seldom sown, as the difficulty of feeding or carting them off was found to be injurious to the succeeding crop; and, consequently, only a small flock of 80 ewes or 140 wethers was kept, which was constantly folded during the

* In common fields, where the course is two crops and a fallow, or, as in some, three crops and a fallow, the report says that " on such soils, the fallowed year fills the land with nore weeds than can be got rid of in the succeeding round of crops." - Surv. of Wilts., p. 56. 
summer. Upon this, and the observations regarding the disadvantages attending the similar plans of his neighbours, it is unnecessary that we should here offer any remark, for we know that they have been, in many instances improved, and our more immediate object is to state the system afterwards adopted by Mr. Greg, and since followed by his nephew, during upwards of twenty years.

Having, as he tells us, "established in his mind as a general principle, that fertility was to be derived from pulverizing the soil, clearing it from water, and keeping it clean, he proceeded to inquire how those objects were to be obtained at the least expense; and he found that the best method to promote them was to reverse the whole system of the former cultivation." Accordingly, instead of ploughing four or five times only in summer and spring, and fallowing every third year, he formed the determination " to plough only once for a crop ; to plough only in winter; never to fallow the land in summer; to practise the row-culture, and to use the horse-hoe." The mode in which he carried his plan into execution was as follows.

He divided the farm as nearly as possible into six equal parts, which are cultivated in a six-course shift, consisting of turnips; barley or oats; clover, standing two years; pease or beans, upon the ley; and lastly, wheat. The ground is marked out by a drill into ridges of five and a half feet in width, intersected by furrows of ten inches wide; thus leaving only fifty-six inches for each land, which is worked by a Suffolk swing plough, formed upon a construction to cut a perfect trench of seven inches deep, and requiring four bouts to complete the ridge, which is made sufficiently convex to describe an inclined plane of three inches from the crown to each furrow*. Thus water is prevented from remaining upon the land intended to be cropped, by being drawn into the ten-inch furrow, which is carried two inches deeper; the horses never tread but in a furrow; and by the soundness of this ploughing Mr. G. states, that "when effected in the autumn or before Christmas, a perfect friability is obtained in the tilth by the influence of the frost during the winter, and the surface water may be as effectually got rid of as by under-draining."

As suon as the harvest is completed, the wheat-stubbles are haulmed, and the lands are marked out and ploughed one bout: dung is then ploughed in to the amount of ten loads per acre, and three bushels of winter tares with a bushel and a half of winter barley are sown, to precede turnips, to the extent of about half the ground intended for that crop, which, in common seasons, it does not impede, as the tares are cut upon a moist furrow for the turnip sowing.

The tare sowing being finished, the bean and pea stubbles are prepared for wheat; which is a difficult operation on heavy land, when the object is to get the seed early into the ground. The labour which they require from the plough, roll, and harrow, was so great as to induce Mr. Greg to use a powerful grubber, or scarifier, of a form which covers an entire land; and it performed so well that he has since continued to use it instead of the plough, as he found that he could thus sow forty acres of wheat in a very few days, regardless of weather, and at a sixth part of the expense.

Having sown the wheat, the remainder of the land intended for turnips is ploughed and dunged. The ploughing is also performed for pease and beans; and it is desirable that these operations should be completed before

* Thus the first bouts are plourrhed only four inches decf, which bouts are turned into one, forming eight inches of soil in the centre of the lands. The second bout to be ploughed up to it, should be five inches deep; the thind sis. and the fourth nearly eight inches. See the Frontispiece to Mr. Greg's Pamphlet descriptive of the jrocess. 
Christmas. As soon as the season turns, the land which was ley, and intended for beans and pease, is scarified; and when the growing weather commences, the beans are drilled at fifteen inches for the convenience of horse-hoeing. The pease are next dirilled; but as these, by failing over, preclude the possibility of hoeing them more than twice, they are sown at intervals of twelve inches.

As the ground is cleared of turnips, it is ploughed into lands. In the spring, the barley is drilled in rows of eight inches-not leaving any space for furrow-and the clover and rye-crrass is sown up, and then across the lands.

As soon in May as the weather permits, and the sun is sufficiently powerful to kill weeds, the scarifier is set to work, succeeded by a strong harrow; and having by these operations obtained cleanliness, the first favourable weather is made use of to sow Swedish turnips; or, should they fail, they are succeeded by white turnips, and in the event of a further miscarriage, coleseed is sown. With these, and the assistance of about ten loads of clover, and ten weeks' run on pasture in bad weather, 500 sheep are now kept on the farm, but lie enclosed at night in a spacious and welllittered yard. 'The fodder produced by straw and clover hay supports from forty to fifty head of cattle, and nine working horses are kept, which are soiled during the entire summer: thus so large a quantity of dung is made that no manure is purchased.

In this manner 200 acres are ploughed between harvest and Christmas, besides the cartage of dung and other odd jobs on the farm; but this is easily performed with the aid of the grubber, and the land being entirely ploughed in the winter, there is only the sowing of Lent corn to execute in the spring: the horses are therefore put upon green food, by which a considerable saving is made in the consumption of corn. Many other details of management are given in Mr. Greg's pamphlet, which is brief and well worthy of attention, but which we refrain from enumerating, as we only meant to call attention to the extraordinary statement which it contains of such a system of culture having been so successfully pursued upon land of that nature, as to yield an average, during six years, of the following crops, namely :-

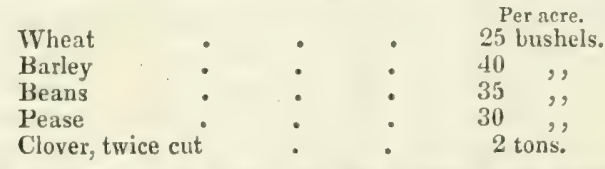

thus, after the deduction of rent and the interest of $2500 l$. capital, presenting, upon an average of six years, a profit of $671 \mathrm{l} .3 \mathrm{~s}$, or $2 l$. $15 \mathrm{~s} .11 \mathrm{~d}$. per acre, and a result in favour of his mode of cultivation of no less than an annual difference amounting to $638 \mathrm{l}$. $13 \mathrm{~s}$.

Of the accuracy of the minute account thus furnished by Mr. Greg, we have no reason to doubt, though we confess ourselves somewhat sceptical regarding the justice of the conclusions which he has drawn respecting the superiority of his own plans over those of his neighbours; for every man, however high his honour and impartiality, is yet unconsciously biassed in favour of any pursuit of his own, and no farmer could live upon the profit which he has assumed as that of cultivation under the old plan. On a subject of such vital importance to agriculture as that of the fallow system, we indeed deemed it prudent to apply to the present Mr. Greg for

* It may, however, be observed, that at the period when Mr. Greg's account was published, wheat sold at $9 \mathrm{~s}$, the bushel, or 50 per cent. more than it does at the present period; whilst the cost of labour, including the increase of the poor's-rate, is scarcely reduced. 
further information, which he readily afforded; and, from recent personal communication and correspondence, we are assured by him, "that his uncle's system is still pursued upon his farm with the best effect; as is evinced by the clean condition of the land, the heavy crops produced, and the quantities of stock maintained. The only alteration of importance made in his mode of cultivation subsequent to the publication of his pamphlet, was the substitution of a seven years' course, in place of that of six years, by which he obtained two crops of wheat-one on the clover ley, and another after the beans and pease. The annual course of cropping in the several years now, therefore, stands thus-
1. Turnips.
2. Barley.
3 and 4. Clover.
5. Wheat.
6. Beans and Pease.
7. Wheat.

No material alteration has been made in the implements; nor was any fallow permitted so long as the late Mr. Greg's health allowed his superintendence of the farm; but the bailiff now occasionally fallows a field of the heaviest land: this, however, is only resorted to when the land sown with turnips has not been prepared in time for the barley crop, and only averages about 16 acres a year out of $250 . "$

\section{BEATSON'S SYSTEM.}

Knowle Farm, in the neighbourhood of Tunbridge Wells, which was a few years ago in the occupation of the late General Beatson, contains about 300 acres of land, of which 112 are arable, and is described as abounding with clay, and retentive of surface moisture, but when dried by the summer heat, it becomes as hard as a brick, and impervious to the plough, unless with a great power of animal exertion, particularly as the general mode is to plough deep. The established rotation in that part of Kent and the neighbouring portion of Sussex is fallow, wheat, and oats, with occasionally clover and rye-grass; and the husbandry appears to have remained unaltered for many ages, with the single exception of substituting lime for manure instead of marl. Upon this system the farm was managed during the General's absence while Governor of the Island of St. Helena; and finding on his return, in the year 1813, "that he had no cause to boast of its profit, he resolved to trace the whole progress of the operations, from the commencement of the fallow to the close of the rotation;" the result of which was, that " having made a series of experiments to which he devoted his attention during five years, he determined upon the total abolition of fallows*.

* His experiments were extended to various objects besides the working of the land; particularly to the combinations of different kinds of manure, and the burning of clay (for which, see our vol. i., chaps. 16 and 17); but our' present extracts only extend to the subject of fallowing, the charges of which he states to have amounted to $16 \%$ per acre, thus :-

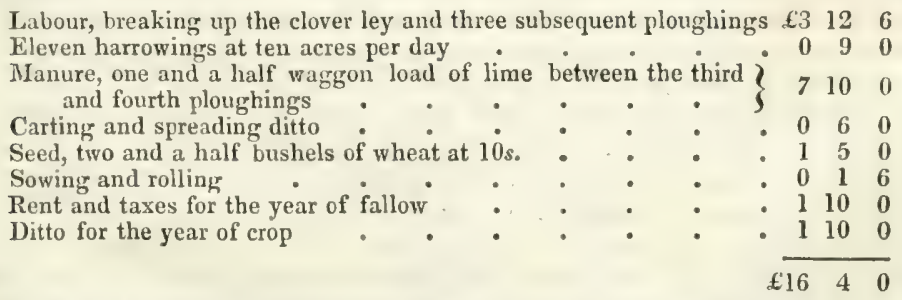

See his 'New System of Cultivation,' together with the Supplement, containing plates of several machines invented by him; some of which well merit atteution. 
In order to effect this, he adopted several new implements, chiefly of his own invention, for a description of which we must refer to his 'New System of Cultivation,' as we have only seen the scarifier in use. This is of a light construction, and certainly performs well ; though, upon land such as that described by the General, it is worked by a pair of horses, and sometimes more, instead of one. It is made by Weir, of Oxford-street, and Morton, of Leith, for about $\mathbf{J} 0 l$, and, with the exception of the crossbars, is formed entirely of iron, in this fashion-

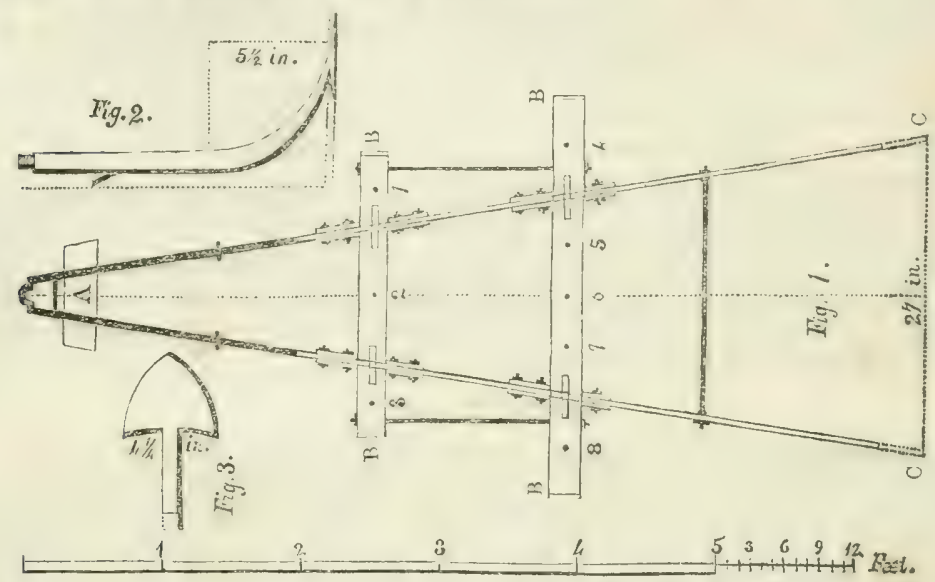

The frame, however, can be made of wood by any country carpenter. It is drawn upon one wheel, fixed at A, in fig. 1. The tines, or shares, which are moveable-and consequently may be made of various shapes to suit the nature of the proposed work, though figures 2 and 3 represent the elevation and lower part of the latter-are mortised into the bars at the holes marked 1 to 8 ; that opposite to No. 6 beingr not generally used as a hoe, but occasionally added when only one bar of the machine is employed; as for the purpose of a stubble rake. The bars are at the letters $B$, and the stilts at $C$.

He conceived that the grand source of all the heavy expenses of the old method might be traced to the fallow itself, and to the mode of preparing it- "by bringing up immense slags with the plough, by reversing the soil, and thus burying the seeds of weeds that had fallen on the surface, hy which a foundation is laid for all the subsequent laborious and expensive operations." To avoid these, he therefore thought it necessary to proceed in a different manner--" to only break and crumble the surface soil to any depth that may be required; to burn and destroy the weeds; after which he would have the land in a fine and clean state of pulverization, and in readiness for receiving the seed, without losing a year's rent and taxes; and all this at a mere trifle of expense when compared with that which is incurred by a fallow."

In pursuance of this, he reduced the ploughing to a single operation at the depth of four inches. The chief use, indeed, which he made of the plough was to open furrows at twenty-seven inches apart, which was performed by a cuuple of horses at the rate of three acres per dar, and was merely intended to prepare the land for the scarifiers, "which, by passing twice across these furrows, loosen all the stubble and roots of weeds, which are afterwards, with a small portion of the soil, placed in heaps and burned." By these means, together with the more frequent repetition of the horse- 
hoeing, and the introduction of the row culture, the General assures us "that his lands were rendered much cleaner, and yielded better crops than they did formerly, after all the heavy expenses of lime and fallows*." He indeed, states, that these operations produced the effect of pulverization to the depth of six or seven inches, and their expense was-

Five searifyings, with a single-horse implement, at $1 s .8 d$. per acre $\begin{array}{ccc}s_{1} & d . \\ 8 & 4\end{array}$

Two harrowings, at $10 \frac{1}{2} d$. . . . . . 1 . 9 s. $d_{\text {。 }}$

that the whole charge of cultivation, under a four-course system upon this plan, including rent, was-

\begin{tabular}{|c|c|c|c|c|c|}
\hline \multirow{2}{*}{\multicolumn{2}{|c|}{$\begin{array}{l}\text { Tares, beans, pease, \&c., per acre } \\
\text { Wheat }\end{array}$}} & & & & $\begin{array}{cc}\mathfrak{L}_{0} & s . \\
\mathbf{5} & 0\end{array}$ \\
\hline & & & & & 50 \\
\hline Dats and barley . & - , , & & $\theta$ & . & 313 \\
\hline lover and rye grass & - , , & & - & - & 215 \\
\hline
\end{tabular}

thus only amounting to a trifle more than that of the fallow upon the former plan; that land cultivated upon his farm in this manner has yielded $\mathbf{4 6 0}$ sheaves of wheat per acre, whilst the average produce of the other fields did not exceed 360 ; and that the difference in favour of the new method amounts, upon an average-when wheat is at $10 \mathrm{~s}$. the bushel-to $350 \mathrm{l}$. per annum upon the cultivation of 100 acres.

On the cultivation of these farms we have no observation to offer, except that they both merit attention. That, belonging to Mr. Greg, although described as a tenacious clay, yet must be land of a superior quality, or it could not support the course of crops under which it has been cultivated; it lias now, however, been continued under the same system for a number of years with distinguished success; and without offering any opinion upon the jlan, we conceive that similar experiments, upon a moderate scale, are fairly entitled to a trial. Regarding Knowle Farm, we have no information since the decease of its late proprietor; but the account which he has left, though inconsistent with the established practice of farming, and appearing, in some respects, not likely to be advantageousiy continued, yet contains lints which perhaps might be carried into effect without impropriety. Whether really deserving of imitation, or not, we at least do not consider vurselves entitled to withhold them from our readers, in a work professing to give an account of the practice of the United Kingdom.

\section{Chapter VI.}

\section{ON SOIVING BROAD.CAST, DRILLING, AND DIBBLING.}

In ancient times the only mode of sowing corn which was customary in this country, was that termed broad-cast; although we leam that in China,

* On this subject he also observes, that " those who plough deep, and bury the seeds of weeds by the first plunghing, are not aware that, hy this outset of their fulluw, they lay the foundation of a great deal of labour and mischief, and bring upon themselves the alisolute necessity of a fallow, as the only means of eradicating the progeny of those seeds which they have inadvertently deposited deep in the soil. Now, if the whole of the stubble and its roots, with a small portion of the surface suil, which must contain those sceds, be collecter and destroyed by fire, it is reasonal, le to suppose that lands might be as effectually cleared of weeds in this manner, as by a summer fallow; besides they would have the benefit of a considerable portion of ashes. Perhaps, also, this mode of burning might have a tendency to prevent the disoriters of smut and blingt; disorders with which my crops have not been in the smallest degree affected."-2d edit. p.90. 
the sowing of grain in rows has been practised for ages; and in Spain, an instrument, called the sembrador, was invented about the year 1650 , for the purposes of drilling. Our field-culture was indeed, at that time, nearly confined to the production of corn, for root-crops were rarely grown except in gardens; and although the planting of such crops in drills must have been well understood by gardeners, yet it did not attract the attention of farmers until the commencement of the last century, when the celebrated Jethro Tull, of Berkshire, began his experiments on drilling grain, an account of which he published in 1731 .

Like all enthusiasts, Tull was apt to be deceived by theoretic notions, which failed in practice, and the principle on which he acted was, therefore, for a long time, viewed with distrust. His system supposes " that land adapted for the production of any particular species of grain might always be sown with the same grain, without manure;" for it assumes "the use of dung to consist chiefly in dissolving the terrestrial matter which affords nutriment to the mouths of vegetable plants," and this he contended could be more effectually done by the sole means of tillage. He, therefore, deemed it necessary not only to repeatedly plough the land intended for the production of a crop, but, in order to secure its perfect pulverization and cleanness, he introduced the plan of sowing the seed in drills, which were lept clear of weeds by the operation of the horse-hoe; and had he confined his improvement to that practical object, without speculating upon the theory regarding the food of plants, he would have deservedly acquired a high rank among the friends of agriculture. Conceiving, however, that the earth was of itself sufficient to bring the roots to perfection, and that the same species might be annually sown upon the same land, he proceeded upon the plan of sowing the seed at wide distances, and the following season again sowing the interval which had been previously left unoccupied between the drills; thus cropping the ground every successive year in alternate spaces.

In the culture of wheat, he began with ridges six feet broad; but after trying various numbers of rows upon the ridge, he at length determined upon two, with an intervening space of ten inches, also reducing the ridge to four feet and a half; and he only allowed three pecks of seed to the acre. The first hoeing was performed by turning a furrow from the drill, soon after the plant had shot forth in the beginning of winter; the second was in spring, by which the earth was returned to the roots; and those which followed were governed by the condition of the land and the state of the weather. Thus, he observes, " my field, whereon is now the thirteenth crop of wheat, has shown that the rows may successfully stand upon any part of the ground. The ridges of this field were, for the twelfth crop, changed from six feet to four feet six inches. In order for this alteration, the ridges were ploughed down, and then the next ridges were laid out the same way as the former, but one foot six inches narrower, and the double rows drilled on their tops; whereby, of consequence, there must be some rows standing on every part of the ground, both on the former partitions, and on every part of the intervals. Notwithstanding this, there was no manner of difference in the goodness of the rows, and the whole field was in every part of it equal, and the best, I believe, that ever grew on it *."

In the cultivation of turnips, his practice was more correct, and differs but little from the present management, except that the seed was mixed " half new and half old," and deposited at different depths, in order to se.

* Horse-hoeing Husbandry, p. 424. 
cure the plants from the ravages of the fly, by occasioning them to sprout at various periods. Although drilling seems to have been partially known in England previous to the publication of Tull's book, and that handhocing was generally practised, yet his system"of culture claims the merit of originality, and entitles him to be considered as the father of the drill and horse-hoeing husbandry*, which has tended more than any other improvement, since the introduction of green crops, to the advancement of our agriculture. It is, however, painful to add, that the loss attendant upon his experiments led him to end his days in the Flect Prison.

\section{BROAD-CASTING.}

In the broad-cast mode it is surprising with what extreme precision an expert sower regulates the prescribed quantity of seed to the acre, and distributes it over the ground in measured casts, his step and hand methodically keeping pace with each other; whereas an inexperienced man sows it irregularly over the field, and every throw that he makes may be distinctly traced at harvest by the seed not being equally spread, some spots being covered too thick, and others lying comparatively bare. The waste thus occasioned is not only in itself injurious, but it also occasions an unequal and consequently imperfect crop of corn, and is likewise mischievous to the vegetation of grass-seeds sown after it, which require a very equal distribution, or large patches will be left uncovered + .

It is hardly necessary to observe that all sowers in Scotland and Ireland use a sheet slung over the right shoulder, for the purpose of carrying and distributing the seed; whereas, in some parts of England they employ a basket suspended round the neck, and held by the left arm thrust through the handle; which, if not the most convenient, certainly has the advantage of occasioning the sower to measure the handfuls more accurately than when the seed is condensed at the bottom of the sheet. He passes up and down along the ridge, and walks upon the crown, unless the ground is in a very rough state; in which case he goes in the inter-furrow, so that each cast is made across half the ridge, and thus covers an entire land. If, lowever, the ridge be more than twelve, or, at the most fifteen feet in width, he should make three casts in order to ensure its being completely covered. The foot and hand accompany each other, a cast of seed being thrown from the right with each step. Sume sowers are, however, so expert, that they throw the seed right and left from both hands, the basket being strapped before them; but this is not to be recommended, for there are very few men who possess equal power in both arms, and if the seed be not cast in precisely the same measure, both as to distance and quaritity, the crop will not come up regularly. The harrows follow, giving the necessary number of turns requisite to cover the seed; and thus the process of sowing is completed.

The same method is adopted in the sowing of the grasses; but the equal

* "The shallow horse-hoeing used in some places betwixt the rows where the intervals are very narrow - as sixteen or eighteen inches," he calls " but an imitation of the handhoe, or a succedanerm to it, and can neither supply the use of dung nor of fallow, and may be properly called scratch-hoeing."

+ "This egregious fault is peculiarly observable in mixed rye-grass and clovers, which require very small pinches of seed to each throw, and, when not equally distributed by a judicious twirl of the hand in throwing them out, a third, or even more, of the land is often left unprovided with plants, and the bare intervals are apt to grow up full of weeds. On ridges of eighteen feet, where rye-grass and clover are sown, three casts are necessary, as the seed of the rye-grass, in particular, in very calm weather, does not spread abroad sufficiently, on account of its lightness, with two casts."-Gen. Rep. of Ścotland, vol. i. p. 505 . 
distribution of their seeds is much more difficult than those of corn, and it is therefore advisable to go twice over the land, each time with only half the entire quantity to be sown, as in this manner a better chance is afforded of avoiding blank spaces. In fine weather, and upon land over which he can step with ease, a steady labourer will sow perhaps an acre and a half within the hour, or about ten acres in an ordinary day's work; but there is perhaps no farming operation about which more circumspection is required, and therefore great care should be used in the choice of an experienced person every way capable of performing it with due precision.

To obviate the inconvenience of sowing corn broad-cast by hand, a machine has been invented by which the work is performed with as much accuracy as with the drill; and the greater equality of the distance at which the seeds are deposited, necessarily adds to the goodness of the crop. It is formed in the shape of a narrow oblong box, which contains the seed, and is usually made from fifteen to eighteen feet long, or the breadth of an ordinary ridge in length. When of this size, it is mounted upon three wheels-two behind and one in front; the horse by which it is dirawn and the fore-wheel moving in the inter-furrow, so that the box extends over the half of each adjoining ridge. The length of the machine should thus be equal to the breadth of the ridges; but they can be easily adapted to each other if the land be laid in narrower stitches; for, in that case, the dropping of the corn may be prevented to any extent at each end of the seedbox, by which means its action can be shortened to the requisite length. Some makers, indeed, construct it of only eleven feet and a half in length, which renders it so light that it can then be worked by the hand: it is, in that case, suspended upon only one wheel, which runs either upon the crown of the ridge, or in the furrow, and the labourer pushes it before him.

It acts by an axle communicating with the hind-wheels upon revolving spindles and short brushes placed lengthwise in the seed-box, which force the com through lines of holes perforated through the box; and the size of the holes can be adjusted to that of the seed by slides. Instead of passing into tubes, as in the drill machine, the seed falls at once upon the ground, as if broad-cast ; but in every other respect it nearly resembles that instrument. The annexed is a perspective view of it as employed for work; but a description of its machinery would not enable a common artisan to make it.

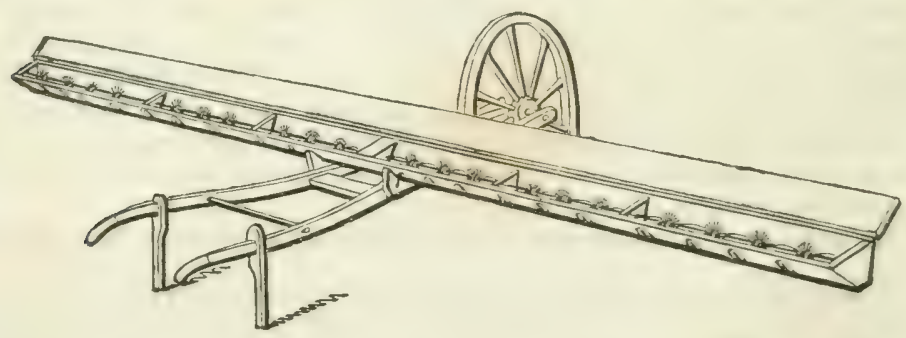

Besides the sowing of corn, it is equally well adapted to the sowing of the grasses, which, indeed, from the minuteness of their seeds, and their easy dispersion by the wind, require still greater care, and it certainly disperses them with much regularity, without being at all dependent upon the skill of the sower : a vast advantage this, if it be considered that the farmer has no other means of detecting an error in the sowing than by the subsequent appearance of the crop, when it can be no longer rectified. It is 
said, by a late writer on agriculture, "that a man and a horse, with a machine of sixteen feet in length, and the assistance required to bring forward the seeds and empty them into the seed-box, will sow from twentyfive to thirty acres in a day *." If so, we can only say that it must be a good day's work. The machine, however, is not very generally employed, for it requires close attention to secure the regular dropping of the seed, which sometimes clogs the holes of the seed-box, particularly in the sowing of wheat, if it be not well dried after being steeped and spread with lime. The cost in London is also 12l. 12s. for those of the smallest size.

\section{DRILLING,}

Like all other innovations, was only slowly adopted; and it is not a little remarkable that, unless by a few gentlemen-farmers, it is even at this day scarcely practised in the south of England for the cultivation of turnips, although it is to that crop that it is perhaps the most essentially useful. In the north, however, and throughout Scotland, it is the only mode employed in the culture of that root; and as it is there superiorly managed, and carried to a greater extent than in other places, we shall hereafter devote a separate chapter to the subject. For other root crops, and indeed for the sowing of corn, especially wheat, and pulse of every kind, it is also becoming more general, and the breadth of land now sown with drilled grain is, in every part of the United Kingdom, very considerable. The advantages arising from the practice are generally considered to consist in these, namely-

That it is a more perfect and economical mode of sowing grain than when broad-cast, for the seed is deposited with greater exactness and regularity, in regard to depth and proportion of quantity, than can be effected by the most expert seedsman; it is also thus more eyually covered, a less quantity is consequently requisite, and it is better secured from the depredation of birds.

That it admits of a more effectual use of manure, by bringing it into immediate contact with the seed, as well as a more sparing use of it, as it may be laid in the drills without intermixture with the rest of the soil.

That the admission of air between the rows is materially beneficial to the growth of all crops, and so much increase the strength of the straw of white corn, that it is much less apt to be lodged than when sown broad-cast + ; that the grain is also of more equal quality; and that the operation of reaping corn can be more expeditiously performed when it is grown in drills than when broad-cast.

That, if the land be hand-weeded, less damage will be done by the weeder's feet in passing between the drills, than when the crop is scattered over every part of the ground. In the early stages of its growth, weeds are also more easily distinguished from the sown plants,

* Low's Elements of Practical Agriculture, p. 109:

† "The grain being placed at an equal and proper depth, the coronal roots are after. wards formed at such a distance below the surface of the field as best suits with the nature of the plant and the peculiarities of the soil, and which, in most cases, is from an inch and a half to two inches below the settled surface of the field. The plants being thus seated, grow strong and vigorous, and which, if necessarily prostrated by heavy storms, having such excellent foot-hold, the crop soon resumes an erect posture, and in that state continues until it is ready to be shorn." - Vancover's Survey of Hampshire, p. 215. 
and consequently the weeding may be done by very inexperienced jersons; while, broad-cast, it can only be entrusted to those who are very careful.

That, by its admitting the use of the various implements used for horse-hoeing, it affords the opportunity for cleansing the ground from weeds, of earthing up the plants and pulverizing the soil during a great portion of the growth of leguminous crops, and thus not only assists the plants in their progress to maturity, but confers on light soils nearly all the benefits of a summer-fallow.

In opposition to this, however, it has been contended-

'That, in regard to the argument in favour of the preference to be given to drilling over the sowing of grain broad-cast-because the seed is better covered, more equally distributed, and deposited at a more regular depth-it may be observed, that if harrows be used of a construction appropriate to the nature of the soil, the seed will be sufficiently covered, and deposited at any given depth with much regularity; that some latitude should also be allowed in this respect, as the superincumbent soil is not always of equal weight, nor in an equal state of pulverization, and that the saving of seed is nearly balanced by the difference of expense between the two modes of operation.

That if dung be laid in drills, although it may thus be more effectually applied to that crop, it will be less effectual to those which succeed; and that the sparing use of it is of no real advantage to the land, which can only be benefited in proportion to the quantity employed.

That, although the admission of air is beneficial to the growth of all leguminous crops and to pulse, which carry their seeds from the bottom of the stalk to the top, yet doubts may be entertained whether the influence of the atmosphere be equally necessary to white corn, the ears of which are so placed as to be always accessible to the air in whatever position the plants may stand. That it may also be doubted whether their being crowded together in rows, instead of allowing them to spread over the whole surface of the land, and thus to draw their nourishment equally from every portion of the soil, may not rather be considered as a disadvantage. That although drilled crops may be less subject to be laid by storms of rain than those which are sown broad-cast, yet the latter suffer less from the wind * and that, if the grain be more equal in the sample, yet if the drills be wide apart, the general quality is inferior to the broad-cast. That, although there may be some slight advantage in the reaping, yet the chief labour is in the binding, and depends upon the bulk of the crop.

That in regard to hand-weeding corn, the operation is useless if it be intended to clean the land from root-weeds, and seldom necessary

* "Drilled grain crops, from wanting an equable and general support among their own stems, owing to the distance between the rows, are more liable than those sown broad-cast to suffer injury from heavy rains and strong blasts of wind, by which their stems are apt to be broken down irregularly and interlaced among each other, which is, in Scotland, termed linee-shackled. Owing to the same circumstance, they are more liable to be root-fallen or wind-waved, their coronal roots becoming disengaged from the soil by a circular motion or waving, from the irregular and changing impulse of the wind-a circumstance which seldom or never happens to a regular and close crop." Gen. Rep. of Scotland, vol, i. p. 369. "It seems pretty well ascertained that, while crops are standing, the broad-cast will suffer less from wind, the rows operating upon one another like skutchers." - Sinclair's Code of Agric. 3d ed. Append. p. 64. 
for the destruction of seed-weeds; for, if the soil be in proper order, they may, in most years, be kept down by broad-cast crops covering the whole of the ground; and that, if the crops be not weeded at all, the drilling will have no other effect than to render the ground foul by filling the intervals with trumpery.

That driliing is not applicable to tough old sward, or to leys of more than three years' standing, unless they have been brought into an unusually fine state of tillage; neither is it appropriate to stony soils; nor is it well calculated for strong clays; for the process requires the land to be reduced to a state of fineness, which is attended with extraordinary labour and expense, and which is impracticable in an unfavourable season; or, if attempted, and perhaps nearly completed, and the soil ready for the seed, the fall of a few hours of heavy rain may render it so lumpish as to prevent the operation.

From this it will be perceived that strong objections are entertained to the drilling of corn upon all soils, and, upon strong land, they are certainly entitled to considerable weight. It must, however, be confessed that the advantages arising from the regular distribution of the seed by the drillmachine are very great, when the soil and weather admit of the operation being well effected; but to secure that, the land must be, in every respect, in a fit state, or the coulters of the drill will clog with dirt, and the seed be ill covered. To attempt it, therefore, under other circumstances, would be imprudent, for it is not often that it can be conveniently delayed. The autumn sowing of wheat is, for instance, a matter not of choice but of necessity; and when large tracts are to be sown at the precarious season at the close of autumn, it frequently happens that the land cannot be got into proper order to admit of the slow process of the drill. The system of broad-casting still, therefore, very generally prevails; and although it in all cases occasions a certain waste of seed, and is, in many instances, indifferently executed, yet, generally speaking, the crops do not appear to suffer.

Drilling wheat may, however, be judiciously resorted to when grassseeds are to be afterwards sown amongst it; for, although there may be little danger of weeds, yet the opportunity afforded by the drill for hoeing in the spring is ever desirable, as the seeds do not always succeed well on winter-sown wheat, when merely harrowed in ; and there can be no doubt that they will thrive better after the soil has been loosened and pulverized by the hoe. It may also be adopted with advantage-if the soil be not too strong a clay, nor too wet-wherever the land is infested with weeds ; for, although the operation of hoeing will not destroy root-iveeds, vet it will tend to keep them down, and lessen the injury which the crop might otherwise sustain from them. Even when the land is so stiff as to require a summer fallow, it may likewise be very properly used on the fallow, when dung is applied; for, if the dung be not well rotted, it is never quite free from the seeds of weeds, the plants of which may be got rid of by hoeing in the spring.

Yet it must be observed that the operation of hoeing, although incontrovertibly advantageous in the destruction of weeds, is yet thought to be prejudicial to the wheat, and indeed to corn crops generally; for, as it cuts the roots, it occasions the plants to stool or tiller very much by means of suckers; and when this happens, it has been generally found that the grain has proved coarse. Neither is it considered advisable to earth them up, if the land be rich; though, if poor, it may prevent them from 
becoming root-fallen. Another objection has also been urged with much weight against the drilling of white crops-"That as their roots seek their pasture more by spreading than by penetrating to any great depth, of course the less they are crowded the better they will thrive; and the plants in the rows are much more crowded than they would have been had they been sown broad-cast;" and therefore "there is no necessity for the seed being put into the ground with such interspaces *."

That great objects are to be attained hy drilling all kinds of pulse and vegetable crops, there can be no manner of doubt; for, by the opportunity which it affords of horse-hoeing between the rows, it certainly can, under skilful management, be made to supersede the use of bate fallows upon all light soils $t$. Doubts may, however, be entertained, from the reasuns which we have already stated, whether it is equally advantageous to corn crops; and heavy clays present strong objections, particularly to the drilling of wheat: nor does such land require hoeing like sandy soils, in which the seeds of weeds are sure to vegetate. That it will assist in keeping the land clean must be readily admitted, as well as that it will leave it in fine order for the sowing of grass-seeds, which latter point is unquestionably of great importance; yet the practice must still be necessarily governed by circumstances, and can neither be held out as a universal rule for imitation, nor should any man be censured for ignorance who refuses to implicitly adopt it. It is difficult to determine liow far the influence of particular seasons may affect experiments in particular years, and it has been justly observed by a writer whom we have more than once quoted, that it is this influence, and not the want of observation in farmers, that has hitherto prevented, and ever will prevent, agriculture from being reduced to one inva-

* Stevenson's Survey of Surrey, p. 158. See also vol, ii. of this work, p. 50.

中 Upon the subject of a general adoption of the drill husbandry, which has been recommended as a substitute for fallows, we should feel that we were not doing justice to a proposition of such imprortance, if we refrained from quoting the opinion of that intelligent farmer, Brown of Markle, who thus expresses himself min hreatise on Rural Affairs:- "In the first place, let any person reflect on the condition of all land incumbent on a retentive subsoil, in an average of years, when spring seeds are sown. Land of this description, which has been ploughed in winter, will, in the spring season, when ploughed a second time, turn up raw and wet, even when the surface is tolerably dry, and in a workable state. The new turned-up furrow is tough and waxy in the first instance, but afterwards, especially if a hot sun prevails, the surface becomes hard like a brick, even before the bottom or subsoil is free of the superabundant moisture there accumulated. U'nder these circumstances, the processes of drilling cannot be executed upon clay soils with the slightest prospect of advantage. It is even difficult to form drills for beans in many seasons, though this grain is generally drilled at wide intervals. To drill wheat, barley, or oats upon clay soils in the spring months, may be regarded as a measure physically impos. sible. Such grains must necessarily be drilled with narrow intervals-say nine or twelve inches; and though this is practicable on light soils during dry weather, it cannot, with the slightest prospect of success, be executed upon those of a different description. The correctness or justice of these arguments will not be questioned by any person versant in the culture of heavy lands, or by those who have paid attention to the difficulties of performing seed-work upon them in a majority of cases.

"In the second place, were we to allow, for argument's sake, that the drilling of grain was a practicable measure upon wet lands, still it remains to be inquired how such soils can be cleaned in a satisfactory manner, when the intervals betwixt the drills must necessarily be narrow and confined, admitting little more than a narrow hand-hoe, which never can extirpate root-weeds, or free the rround of its original inhabitants. Were a horse-hoe to be used, the surface would break up in lumps or massy pieces, to the destruction of the grain plants, whilst, alter all, a hoeing of a deepness sufficient to destroy the weeds could not be given. If the interval were wide enough to allow a horse-hoe room for work, the produce of the crop would be proportionably curtailed, while the quality of the grain would be materially injured from a continuance of tillering, till a very late period." - Vol. i. p. 205. 
riable system. What is right one year, and even for years together, may another year be wrong; and that farmer who happens to suffer severely by pursuing a right system in a wrong year, is shy of it for ever after; especially if he has suffered by deviating from any old mote to which a popular opinion has been long attached; for, in this case, he not only suffers the loss of his property, but he is sure to be laughed at by all his neighbours, and even by his own labourers *" Added to this, time and labour at all times, and especially during the seed process, are very important considerations, independent of expense: the establishment of men and horses is the heaviest charge on farming, and should be economically proportioned to the business of the whole year; but drilling demands more than double the time of broal-cast sowing, and in precarious seasons may consequently require a greater strength of hands and cattle than may be convenient.

\section{DRILL IMPLEMENTS.}

The operation of drilling requires that the land should be brought into a fine state of tilth, and the greatest attention must be paid to the execution of the work, for the nearer the mode of culture approaches to that adopted by market-gardeners, the closer will it be found to arrive at perfection. After the usual ploughings have been given, the most customary plan is to pulverize the top soil by the means of the grubber-used crosswise if necessary; the ground being thus equally tilled and cleansed from weeds by subsequent harrowing, is regularly cast into ridges and furrowed, and is afterwards rolled down in a direction with the inclination of the field; though, in very dry and light soils, the land is sometimes quite level. The rows are then accurately marked out at various distances, according to the nature and quantity of the seed intended to be sown-as will of course be stated under the separate heads of the different crops intended for cultivation-but much care is requisite to keep the machine in a straight direction, or the drills will run into each other in some places, and leave corresponding gaps in others. On rich land it is thought a good practice to run the drills from north to south, because the rays of the sun, when in its greatest altitude, striking directly between the rows, have a powerful effect in promoting vegetation, and, especially on wheat crops, it has been found to assist in preventing mildew. But it also tends to absorb the moisture too suddenly from the earth; and, we should conceive, that in most cases - and more particularly in light soils-it is more advisable to draw them east and west.

A great variety of implements have been invented since the practice came into vogue for the purpose of facilitating the process; but we only deem it necessary to enumerate a few of them, as they all act upon nearly the same principle, and those which are only used for one particular purpose will be described under that head.

The drills which were at first used were constructed upon very simple principles, consisting only of a hopper, containing a funnel of tin with a small hole in the bottom, through which the seed passed, and a coulter to mark the drill. The hopper was mounted upon a small frame supported by a single wheel, and was wrought by hand, being pushed along the furrow by the persun who sowed the seed, whence the implements obtained the name of drill-barrows. They are made either with a single hopper, or 
not unfrequently with two seed-boxes, one on each side of the wheel, in this fashion-

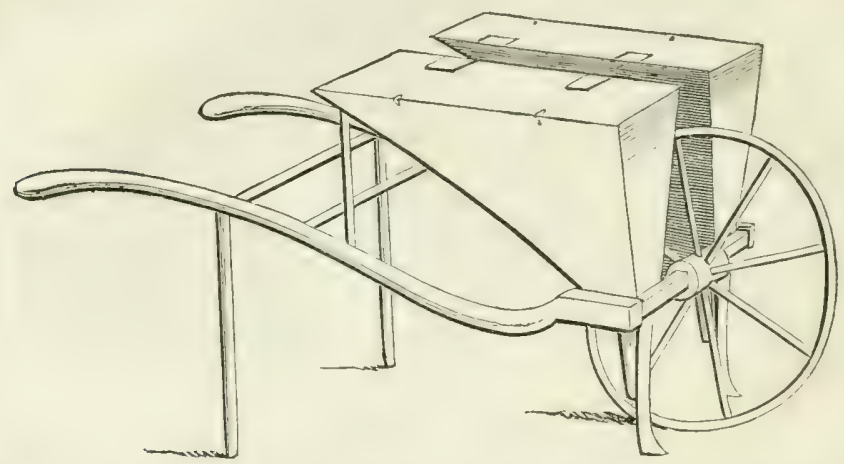

They were, however, very imperfect; for if the orifice of the fummel was only wide enough to exactly admit the seed, it was apt to clog, thus occasioning large spaces to be left blank; and, if made sufficiently wide to prevent this inconventence, it then frequently sowed too much. To remedy this defect, Mr. Bailey, of Chillingham, some years agro made one of these implements upon an improved plan, which has been since much arlopted for the sowing of beans and turnips-as will be more particularly noticed when we treat of that culture - though larger ones, sowing two or three drills at a time, and drawn by a horse, are now more generally used-particularly for turnips-and are certainly better calculated for that husbandry than small ones, as they not only save much time at seasons when it is precious, but they also bury the seed with greater exactness, and are therefore more certain to ensure vegetation. They, however, are found useful by small farmers, as a boy with a barrow can follow the plough and deposit the seed at the bottom of the furrow; or, sometimes the barrow-when used with a single hopper, and without its coulter-is fixed between the stilts of a double-mould board, or ridge-plough, or indeed of any common plough that is not worked too deep, and the seed is sown as it goes along, and either covered by the next furrow slice, or brushed over by the harrows. It thus forms a cheap and simple apparatus, which renders it highly eligible in moderate concerns, as well as to persons of moderate means, as it may be used in the culture of any crop, and can be made to sow four or five acres a day in clay-lands, and in seasons when the drill will not act.

The machines now commonly employed for drilling the different species of grain, are similar in principle to those generally known as Cooke's drill, though with several minor improvements upon the original invention, and are mounted upon a frame drawn by horses, containing an oblong box or hopper for the reception of the seed and part of the machinery. When ready for work, the back part of the implement is of the following appearance; having iron coulters moveable to various distances to form the drills, which are cut in grooves, of about two inches wide to any deptl, being regulated by weights held by small chains, which are supported by a rack-wheel, wound up by a screw, and having tin funnels communicating with the hopper for the purpose of depositing the seed. The bar, to each end of which the horses are harnessed, being at the front, does not 
appear in the drawing; but when the machine is so light as to be drawn by one horse, shafts are affixed to its centre.

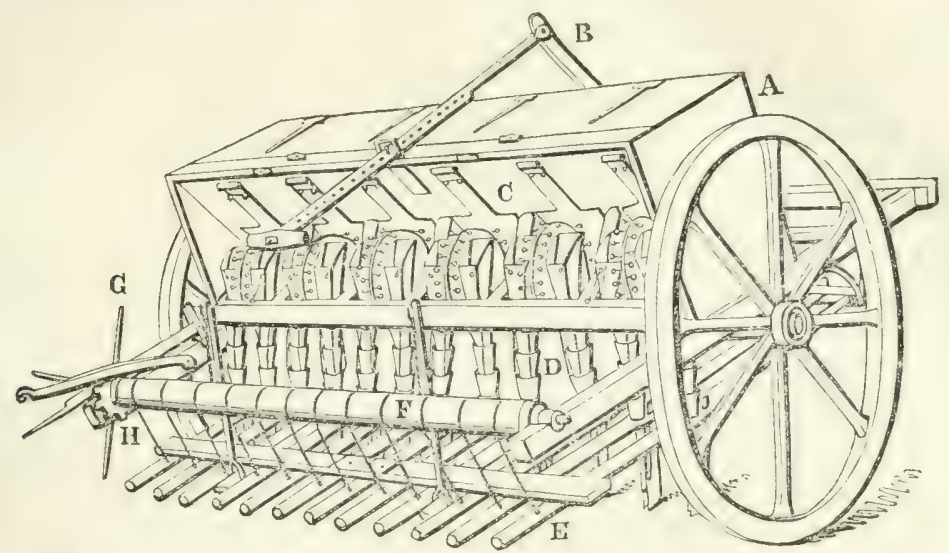

The inside part of the machinery by which the quantity of seed is regulated, consists of a small iron axle, which passes through the lower part of the box, and is moved round by means of cogs comnected with the wheels. Upon the axle are fixed either small brass fluted cylinders, or else cups or spoons, projecting more or less over the mouths of the funnels, so as to deliver a larger or smaller quantity of seed as mav be required. For the smaller seeds, teeth with stiff brushes, are placed at different distances from each other, and can be moved upon the axle to wider or narrower intervals, so as to suit those at which the drills are to be made, and as they revolve among the seeds, a certain portion of them is forced through holes in the bottom of the seed-box, and thence through the fumels into the drills. The opening into these tubes can be enlarged or diminished at pleasure by a sliding plate fixed upon them by a screw for regulating the quantity of seed; a measure which, it may be observed, is sometimes necessary, when the land varies considerably in quality, or in the inclination of the ground, and therefore requires the management of a very careful and experienced workman. 'The annexed references, pointing to the various parts of the implement, will afford a sufficient idea of its manner of work; for, as it can only be constructed by a regular manufacturer, we think it unnecessary to give further drawings of the machinery, and more minute descriptions may be found in the works to which we refer at foot $*$

A The hopper for the reception of the seed.

I A lever by which the hopper can be inclined so as to increase or diminish the supply of seed.

C The wheels and cuns by which the seed is moved round and delivered into the funnels.

D The funnels for distributing the seed connected with the coulters for forming the drills at different depths.

E The weights which press upon the coulters.

F The rack-wheel which regulates the pressure of the weights.

$G$ The winch by which the rack-wheel is turned.

H Cogged wheels which act upon the rack-wheel and axle.

* See an Essay on the Construction of the Plough, App. p. 39 ; Bailey's Survey of Northumberland, p. 46 ; Brown of Markle, Treat. on Rural Affairs, vol. i. chap. VI. Sect. 5 ; aud Low's Pract. Elem. of Agric., p. 103. The foregoing contain descriptive plates; and in Rigby's Holkham there may be found various remarks on the manuer of working the drill, and some improvements on it made hy, Mr. Frost, of Siham, T.86. 
The drill represented in the preceding page, is, it will be perceived, of a large size,-being $8 \frac{1}{2}$ feet between the wheels, - and is that most generally approved for the sowing of corn, cutting the rows at intervals of eight to twelve inches, either on ridges expressly ploughed to their breidth, which are sown at one bout going up half the land, and returning down the other half: thus sowing six, seven, or eight rows at once, according as the drills are previously adjusted, and cutting them an inch and a half, or two inches wide, and about two inches in depth. Machines are however constructed of various dimensions, and there is one now very much in use which covers an entire ridge of ten feet, and thus sows it at once. It is drawn by a double set of horses, which being hooked on with whipple trees to each end of the frame, each move in the interfurrow on each side of the ridge, and the wheels also ruming in the furrow, the land is thus sown without being poached by the treading of the cattle. It requires, however, not less than two pair of horses, with a driver to each pair, and we have seen it worked on clay-land by six horses, three on each side.

The usual work with an implement of this size is from eight to ten acres a day ; for the land must be in very superior order to admit of twelve being sown. They cost about forty guineas ; or the price of one of the first-mentioned description is $35 l$., exclusive of the charges of packing and conveyance, and the machinery is besides very subject to get out of order. There are, however, persons to be found in most parts of every county who let them out for hire, generally at the rate of two shillings the acre, the farmer finding his uwn cattle, and also feeding the man who conducts the work. The management, indeed, requires considerable intelligence, and very marked attention; and this circumstance, together with the cost of the implement, or else the uncertainty of being able to get it on lire at the moment when it may be wanted, forms a strong objection to the more general adoption of the drill husbandry on corn-land.

An implement called a drill-roller, though employed in broad-casting, was also formerly much employed in many parts of Norfolk, but has since been partly superseded by the adoption of the regular machines. It is a cylinder of iron, about seven feet long, around which are cutting wheels of cast-iron, that turn, each independently of the others, around the common cylinder, weighing from a ton to a ton and a half, and occasions heavy work to four horses. The cutting wheels, being moveable, may be fixed at any distance, and, by passing over a fresh-ploughed layer, the soil is cut into littlo chammels; the seed being then sown broad-cast, the land is afterwards bush-hatrowed, and the corn is thus deposited at an equal repth. On heavy soils it is, however, not found to answer; but "for breaking clods in dry weather," Arthur Young says "no tool he ever beheld comes near to it*." The land-presser also acts much in a similar manner upon light soils, and will be more particularly described in the chapter on the cultivation of wheat.

\section{DIBBLING,}

Or selling corn grain by grain, is a practice which was not introduced until some time in the midale of the last century, and though now much adopted in most parts of the kingdom for the planting of beans, is only generally used for corn in Suffolls and Norfolk, and the lighter parts of Essex, in which counties it is practised to such considerable extent, that there are farmers who thus sow from 100 to 300 acres yearly. As comjared with drilling, it is indeed found to be cheaper; and, when skilfully

N Norfolk Report, p. 58. 
and carefully performed, it is there considered by many experienced persons as decidedly superior. The price paid for dibbling is $7 s .6 d$. per acre, including the charge of the dibbler and his assistants ; and the cost of drilling, including all expenses, may be from $5 s$. $6 d$. to $6 s$. : but the usual quantity of seed sown by the former method is only six or seven pecks per acre, whereas, upon the drill system, nine or ten pecks would be required upon the same land, all other circumstances coinciding.

Through the kindness of Lord Suffield,-who considerately keeps a portion of his home-farm for the purpose of making experiments for the information of the neighbours, - we learn from Mr. Henry Smith, his Lordship's steward, that "the land in that district is usually sown with wheat after clover, or after grass of two or more years, and is prepared for dibbling by one plougling, unless in a fuul state, when the general practice is to slip furrow, or half plough it first; by repeated harrowings all the weeds are thus brought to the surface, and when killed by the sun or drought, are ploughed under the next furrow with the manure. A heavy roller is then applied, when the land is in a proper state for the operations of the dibbler, who, with three children, are reckoned a set. An expert dibbler will hole half an acre a day: with two irons he goes backwards down the first furrow, and returns upon the third, making two holes in an oblique direction, three inches apart on each furrow; the children then drop three or four grains of seed into each hole, which is afterwards covered by means of a bush harrow, and the work is thus completed."

Such is the usual process for wheat; but the distance of the holes, and the number of seeds, of course vary on different crops. The operation is usually performed with irons, of a conical shape, somewhat resembling the following, with which a hole of about two inches deep is punched on the middle of the flag, or turned up earth of every furrow.

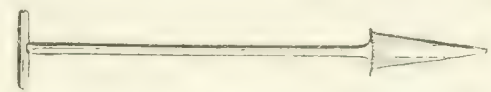

Drill, and spiked rollers, and an implement called "the self-souing dibble," have also been employed for the same purpose, with a view to the economy of both time and money; but they have not been found to answer so well as the common practice. The success of the sowing, as well as the saving of seed, depends, indeed, very much upon the care and attention of the droppers; for if not closely watched, some will put in too many, and others none. It is, therefore, unadvisable to allow many of them to work together, as they thus distract each other, and the dibblers, by being placed in distant parts of the field, have a better opportunity of observing them. If a careful man, he can do this with perfect ease and certainty, for the droppers follow him with their faces towards his, and consequently going forwards. Women or children are generally employed, and we have just seen a four-acre field of beans very completely dibbled with a man and a couple of boys under twelve years of age, within the week.

When correctly executed, there can be no manner of doubt that it not only possesses the important advantage of saving at least a bushel of seed per acre, but it also sets out the corn at equal distances as well as depth, which must, it is presumed, give more equal nourishment than can be ensured by either the drill or the broad-cast systems. We accordingly learn, from various accomits, that the plants are found to be more vigorous in their growth, and the straw stronger in the stem, and less subject to be root-fallen, when dibbled, than when sown in eiber of the other modes. 
The grain, both of wheat and barley, is also said to be of better quality, being more free from dross, and the kernel larger; and that of wheat to weigh more by 10 or $12 \mathrm{lbs}$. in the quarter. Respecting the difference in the quantity of produce, the accuunts vary; some stating it as yielding an excess of ten to twelve bushels of wheat over any other method*, and others reducing it to three, while many insist that there is no perceptible difference + . We have heard of barley being part dibbled, and part sown broad-cast, on the same field, tilled and manured in the same manner, and the former producing twelve bushels per acre more than the latter; and Mr. Henry Smith tells us, that his own crop of last year was half-drilled and half-dibbled, and the latter yielded two bushels per acre more than the former.

It must, however, be admitted, that it is only properly adapted to loose friable land, and is best calculated for clover leys, the pulverization of which is unfavourable to the culture of wheat, but can never be made to answer on wet tenacious clays; for the dibble forms a cup which holds water in wet weather, and when that is evaporated, the earth around becomes so hard as to be impenetrable to the roots of grain, and the seed yerishes. Were not this the case, another objection arises in the difficulty of properly covering the seed by filling the holes in such soils, though harrowed ever so often + .

This mode of culture, though so nearly allied to drilling, yet differs from it in two particulars: as, first separate holes are made to permit the insertion of the seeds; and secondly, they are set in bunches of several grains each, which is supposed to be favourable to their growth, though opinions differ on its merits. Thus, by some farmers, four, five, six, and seven grains of wheat, and two, three, or four beans, are dropped into each hole: it is, indeed, thought by many, that crowding the roots of corn promotes their growth in a young state, and it is a common observation, that young vegetables of every kind, when growing near together, draw each other up: though it is also supposed, that they are proportionably deficient in strength and thickness, as their height exceeds that of single plants. The experiment stated at the close of this chapter would, however, show, that dibbling does not require the quantity of seed generally thought necessary to ensure a crop; and, it must be admitted, that " further than the voice of general opinion, there is no proof that the beneficial effects of crowding the roots extend to the period of maturity. But when people gain better crops than the broad-cast, by dibbling four or five corns in a hole, and that without any assistance from the hoe, it affords a strong presumption that such is the case $\S$."

The observations which have been made in respect to drilling will apply, in many respects, with increased force to dibbling, as being a much slower operation, the tediousness of which may occasion the possible risk of losing a favourable season. The chief argment usually urged in its favour is the saving of seed, and that, in a national point of view, is certainly important; but farmers naturally look only to their own interest, and, at the present price of corn, it is of little weight. 'The consideration of the additional employment which it would aflord to every class of the labouring peasantry-men, women, and children-is, however, an object which must

* Kent's Surv, of Norfolk, App. p. 211.

$\dagger$ Arthur Young's Surv. of Norfolk, p. 280.

t See the Essex Rep. vol. i. p. 272 ; Middlesex ditto, 2 nd edit. 1. 178 ; and Sinclai:"s

Code of Agric., 3rd edit. p. 121.

$\S$ Bedford Rep. p. 300. 
come home to the breast of every man of right feeling, and, in that sense, it is doubtless deserving of encouragement. To this we venture to add Marshall's recommendation of the transplantation, not only of many rootcrops, but also, under certain circumstances, of wheat. In cases, for instance, of the partial failure of a crop, which is sometimes known to grow luxuriantly in some spots, and to be bare in others, the plants in those parts of the field where they are too thick, may be thinned, and those taken out dibbled into places where they are deficient. This should be done in the early part of April ; though it has been known to succeed when performed so late as from the 5tl to the 12th of May*. Whether the operation might not be carried further with advantage on peculiar soils, and in particular seasons, is also a hint which may be found worthy of attention.

\section{EXPERIMENTS.}

As in every innovation upon the established practices of agriculture, it may be imagined that men, however well-informed, experienced, and candid they may be, yet differ in opinion regarding the relative superiority of the broad-cast and drill husbandry. It may, however, be presumed that the opposite conclusions which they have drawn, have generally arisen from no other cause than the peculiarity of the soil on which experiments have been tried; for where the sole objects in view are truth and utility, it cannot but be supposed that the opinions both for and against either practice have been well founded, and that, in equally eligible situations, both practices are proper and advisable to be pursued: a truth which can in no instance be better exemplified than in what occurred upon an experimental farm established several years ago by the South Hants Agricultural Society, which, after a trial of five years, upon 200 acres of arable land, one-half exclusively employed in the drill, and the other in the broadcast husbandiry, was then given up without any definitive decision upon their respective merits.

The Surveyor of Hampshire has, however, given some details of the separate practice of intelligent persons in that county, which lead to the conclusion that drilling is the preferable system of the two. Thus in a course of husbandry, on the broad-cast plan, in a five-shift rotation, upon the strong arable lands in the chalk district, begimning with a thorough winter and summer fallow for wheat, he states the abstract of the gross expense and returns as having been as follows :-

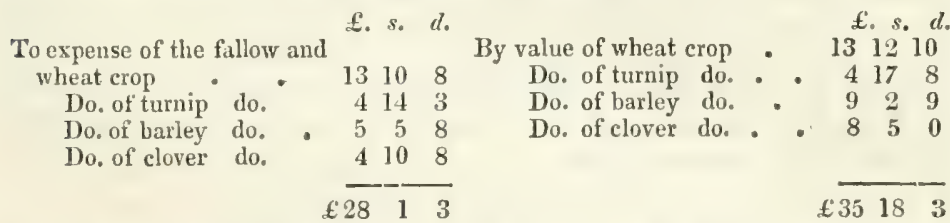

leaving a balance of $7 l .17 \mathrm{~s}$, or a net annual profit, including the interest of capital, of $1 l .15 \mathrm{~s} .5 \mathrm{~d}$. per annum; rent, tithe, and parochial assessments being then $25 s$. per acre; wheat selling at $8 s .6 d$., and barley at $4 s .3 d$. per bushel.

The drill system of a gentleman, also in the chalk district, but whose land is chiefly of a rubbly nature, and of a thin staple, but much deepened and improved by scarifying, is thus described. He begins, as early as possible after wheat-sowing, to plough a last year's stubble, or a ley, to a 
good full pitch, which done, the ground remains in that state till some time before Candlemas, when the next operation is to upridge it, crosswise to the direction in which it was last ploughed; and it thus lies until the end of April or the beginning of May. The ridges are then split down with a strong duuble-breasted plough; and in order to pulverize the top and submould, they are scarified crosswise, completely harrowed, and laid into three-feet ridges. The furrows are drest with a compost of dung and mould, of 25 to 30 one-horse cart-loads, lıolding about 20 bushels each, per acre, and the ridges are arain split down with the double-breasted plough. The manure in the furrows being thus covered, the ridges are now rolled with a roller, which, taking two ridges at a time, reduces their tops to an even and uniform bearing. Turnips are then drilled upon the ridges; the plants are set out upon the rows with a hand-hoe, and the intervals are horse-hoed as often as occasion may require.

As soon as the field is cleared, which is alwars before Candlemas, the first operation is to split down the turnip-ridges, and the land is left in that state until near the time for sowing barley, when the next tilth which it undergoes is that of closely scarifying it diagonally and crosswise, until it is in a fit condition for the reception of the seed; which, after the necessary harrowing and rolling, is then drilled-in nine inches apart, requiring about two bushels of seed per acre, and vielding an average produce, taken from the crops of the last three years, of 45 bushels 3 pecks, of $511 \mathrm{lb}$. 20z. each, per acre; the weight of the broad-cast barley being 50lb. $120 z$.

Previous to the last tillage operation which is given to the barley, and a month or six weeks after it has been sown, ten pounds of red, and two pounds of yellow clover are sown broad-cast per acre; which, with the aid of a dressing of peat or turf ashes, applied in the spring of the year, will yield an average produce of 25 cwt. of hay, per acre, from the first swath. The after-grass is then fed down as close as possible, and the ley is broken up with a skim coulter plough, which cuts the furrow-slice on the land-side to a thin feather-edge, leaving on the furrow-side a small comb or balk: the feather-edge slice is cast down to the bottom of the furrow, and the small balk, with the great body of the slice, is taken up a good full jitch, and whelmed completely under. The field is then rolled smooth and close down, and after harrowing to obtain a sufficiency of loose mould for the drill to work in, the wheat is put in nine inches a-part; the seed required is about five peclis ${ }^{*}$, and the average produce is 24 bushels per acre, weighing each $6 \mathrm{llb}$. 2oz.; the weight of the broad-cast wheat being $61 \mathrm{lb}$. per bushel.

Under this management, calculating prices as in the foregoing account, and rent, tithe, and assessments as nearly the same, the average of the charges and the returns were-

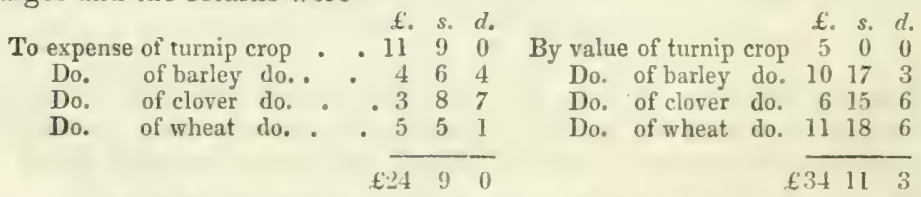

thus leaving a balance, in four years, of $10 l$. 2s. $3 d$, oz a net average profit of 21. $10 s .63 d$. per anmum, leaving about 15 s. per acre annual gain beyond what was made under the broal-cast system. 'The sam report, imleed, furnishes several otier statements nearly th the same efiect, and containing the spe-

* The statement says "two bushels :" but the sum charged in the account is only for five picks. 
cific items of every charge*, of which our limits only allow us to give the above abstracts; and there are various authentic accounts spread throughout the County Surveys, and other works of equal authority, most of which are corroborative of the superiority of the drill over the broad-cast husbandry, on those soils on which it can be conveniently managed. It is well known to be universally practised by the tenantry on the extensive estates of Mr. Coke, and of many other men of large property on the light soils of Norfolk; and Sir John Sinclair mentions an instance of 150 Scotcl acres of light loam, much subject to annual weeds, having been drilled with wheat by Mr. Brodie, of Scoughall, in East Lothian, the produce of which was 42 Winchester bushels per acre, while that sown broad-cast, though weighing about a pound more in the bushel, was only 35, and the grassseeds sown with both crops answered best upon that which was drilled + . It is indeed evident that grass-seeds are frequently injured by small weeds which cannot be got at when the ground is covered by a broad-cast crop; whereas, if drilled, they can be easily extirpated by the operation of hoeing:

A very satisfactory comparison between the different systems of drill and broad-cast has been also recorded by the Rev. Mr. Radcliff, in the Report delivered by him to the Farming Society of Ireland, upon the agriculture of Eastern and Western Flanders, in which it is stated, that some land of equal quality, which had been manured the preceding year for a crop of winter-barley, was sown with beans-one half broad-cast, the other drilled in rows at two feet distance, and hand-hoed. The following season both moieties were again sown in the usual way with winter-barley, broad-cast; the grain of which was much finer on the piece that had becn drilled than on the other, and the ground itself much freer from weeds and more easily worked. The produce of the respective crops, when reduced into English measure, was, as nearly as it is necessary to calculate-

\begin{tabular}{ccccc} 
& SEED. & \multicolumn{3}{c}{ PRODUCE. } \\
& Bushels. & Qrs. & Bush. & Pks. \\
BEANs, broad-cast & $3 \frac{3}{4}$ & 3 & 1 & 3 \\
BARLEY, broad-cast & $2 \frac{1}{2}$ & 3 & 7 & 2 \\
,, drilled & - & 6 & 0 & 0 \\
Barilled & - & 8 & 7 & 0
\end{tabular}

Thus, not only was there a saving of seed which would probably equal the extra expense of drilling the beans, but a considerable increase of produce was also obtained; the tilth also appears to have been so improved by the drill method, that the succeeding crop of barley was nearly one-half larger than on the land on which it was sown broad-cast: but the difference of seed is not stated.

On examining the various accounts of these and other experiments upon drilling, the soil will, in most cases, be found light and dry, or at least

* See Vancouver's Surv. of Hampsh., chap. vii. sect. in comparison of the drill and broal-cast husbandry, p. 210; and the Norfolk Report, by Youncr, in which a comparative expleriment on drilled and broad-cast wheat is stated to have produced five bushels more per acre than the latter-p. 289.

$\dagger$ Farmer's Mag., vol. xxii. p. 171. Sir John also mentions a communication from Mr. Burroughs, which states that "on a field sown in drills 12 inches apart, which received one hoeing in spring, a more productive crop, and far more valuable grain, was raised than on a broad.cast crop which had received three times the quantity of dung. The manure for the drilled crop of wheat was applied in drills made by the plough, the seed sown, and then harrowed down."-Code of Agric., 3d edit., p. 375. 
sound land, on which the difficulties in the management are not great; but wet, strong, tenacious soils, that are apt to poach if not tilled with much care and attention, are not easy to manage, and have been thought peculiarly unfavourable to the practice. Those who have had much to do with heavy soils, and consequently know that they can only be ploughed in the spring, when the land is dry on the surface, must be sensible that very favourable weather must occur, or the farmer will be exposed to great inconvenience; for, if turned up when wet, it dries into hard compact clods, and much time may be lost before a tool can stir on it. To make use of the original friable surface-which once lost is not to be easily regained-and thereby secure not only a favourable matrix for the seed, but also an early sowing, is an object which cannot be valued too highly, and which appears to have been attained on some large clay farms in Suffolk, of a very wet and adhesive nature, by putting in the crops upon. a stale furrow, with the drill, and without any spring ploughing.

The management consists in giving the necessary degree of preparation to the land intended for spring crops, and ploughing it into the usual form before the winter frosts. The most customary ridges are five feet three inches broad-for one movement of the drill machine, though some farmers make stitches of nine or ten feet, for a double bout; but, in either case, the horses go only in the furrow. Whatever the preparation by tillage-whether many ploughings in a fallow, two or three after tares, or one after beans-nothing is left wanting in the spring but to scuftle and harrow, and then drill in the seed. The only difficulty is on the turnip land, which should be cleared as early as possible for the chance of late frosts, to obtain a friable surface, which, once gained, is never ploughed again, but, when in the right temper, is scuffled and drilled. Some farmers, indeed, have found their account, when the weather has been favourable, in only scarifying and scufling, and then harrowing the turnips. But season must govern the practice; which, however, proves the great importance attached to the avoidance of spring ploughing on strong land*.

It is a trite observation, "that the cleaner any land is kept, the less care is requisite to continue it so ;" to which remark we have only to add, that if crops are preserved from weeds in their infancy-which is the period of their greatest danger-their produce will be increased; and that no method can be recommended as more certain to attain those objects on soils which admit of the alternate growth of corn and roots than the operations of the drill and horse-hoeing husbandry.

Regarding the process of dibbling, an experiment is recorded in the Essex Report + as having been made upon a clover ley, which was ploughed in the month of October into four-ridge stitches. Two acres of it were rolled, and wheat dibbled in-one acre with about three pecks of the common white rivets, and the other with two pecks of small American red wheat. The remainder of the field, containing three acres, was sown broadcast, in separate pieces, with seeds of similar quality. During the winter, the broad-cast had vastly the advantage, while the dibbled, on the contrary, wore an indifferent appearance; and both continued in the same state until March, during which month and that of April, the dibbled wheat was

* A very interesting account of the practice and experiments-though too long for extraction here-may be found in the Appendix to the third edition of Arthur Young's Survey of Suffolk.

t Vol, i. p. 273. 
twice hand-hoed. It now soon got the start of its broad-cast neighbour, and kept it ever after: its blade was scarcely like the common blade of wheat, but broad and vigorous ; and when it shot into ear, the superiority in both linds was striking, each ear containing, on an average, a fourth part greater number of grains than the undibbled, and being more uniform in their growth.

When thrashed, after harvest, the broad-cast wheat produced exactly four quarters the acre, each bushel weighing 60lbs.; while the dibbled small wheat yielded five quarters of $6 \mathrm{llbs}$. the bushel, and the rivets four quarters, seven bushels; but the latter were slightly damaged by mildew, and not quite so heavy as the other. Taking the expenses and profits of the former at the present prices, the account would stand thus :-

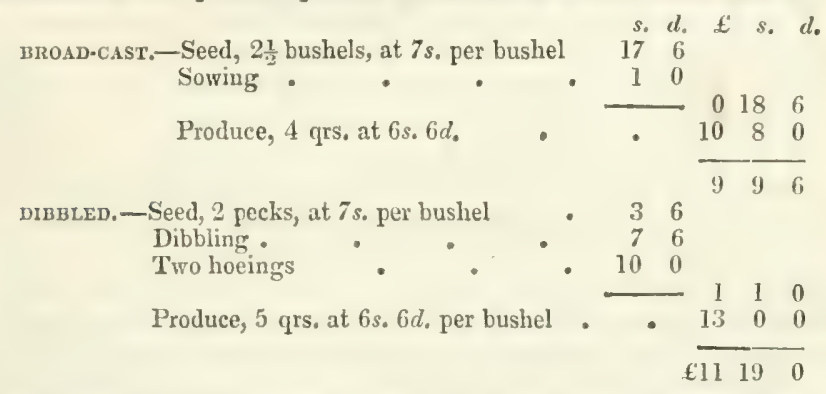

Thus leaving a difference in favour of the dibbled wheat of no less than 2l. $9 s .6$., exclusive of any estimate of its superior quality. Other experiments, however, have not turned out so much in favour of dibbling; and perhaps the result of this is rather to be attributed to the hoeing than to any other circumstance.

In addition to what has been already stated in a former chapter, on the process of hoeing, we distinctly recommend horse-hoeing in preference to hand-hoeing every crop that is drilled at sufficient distance within the rows to admit of its operation. The latter implement may undoubtedly be used with advantage, and ought never to be neglected, except when the former camot be employed; but it is not so efficient as the horse-hoe,which, besides its greater power, has the decided advantage of going over several acres in a day, while a single labourer must be a good workman who can eficctually hoe half an acre; and it frequently happens that hoeing, in any way, can only be executed with advantage during a very few days in the spring. Among the many implements which have been lately brought into notice for horse-hoeing drilled green crops, there arc three which are strongly recommended, and engravings of which may be seen in the 'Transactions of the Highland Society*;' and a correct description of Plenty's Scarifier may be found in the 'Penny Cyclopiedia,' under the head of 'Arable Land.'.

Mr. Blaikie's inverted horse-hoe is also an instrument of general utility in the different operations of cleaning land; and, besides being procurable at moderate cost, possesses the great advantage that it may be worked with perfect safety between the rows of plants in their infant state + .

Mr. Wilkie's, which we formerly alluded to, we have since seen at

* N. S. vol. iii. p. 80 .

$\dagger$ See Blaikie on the Management of Farm-Yard Manure, in which there is a cut, and a detailed description of the implement. 
work, and having found it a very efficient tool, we here annex a drawing of it.

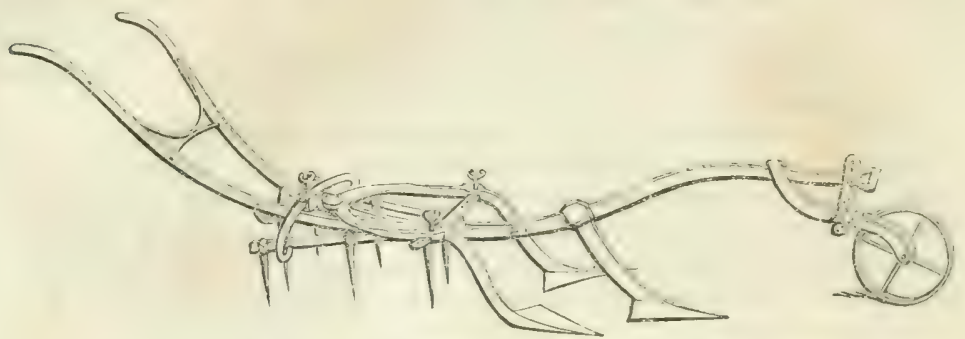

\section{Chapter Vil.}

\section{ON THE ROTATION OF CROPS.}

Ir has been observed by Lord Kames, "that no branch of lusbandry requires more sagacity and skill than a proper rotation of crops, so as to keep the ground always in heart, and yet to draw from it the greatest possible profit*." Indeed no one, who understands the subject, can donbt that it is one of the very last importance, and so prominent a place does it holk in the art of agriculture, that no better criterion can be found on which to estimate the merits of a farmer, than the course of cropping which lie has adopted. Although an adequate degree of knowledge concerning it be seemingly of very easy attainment, and it is very generally thought to be comprised in the rule laid down by his lordship,-- "that it should consist of alternate crops, culmiferous and leguminous,"-yet in fact no small degree of judgment and experience are necessary to arrange the plant and to adapt it to all the varieties of climate, soil, and situation; for although it may be an apparently easy matter to follow up an alternate course of white and green crops, yet very little observation will convince any practical man that the general rule may be strictly observed, and still the system of cropping may be very defective. It is therefore not to be wondered at that many different opinions should be entertained, though all perhaps very much founded upon truth: what being suitable to one case being totally inapplicable to another, and no general rule being of such a nature that it may not be in some instances judiciously altered, according to the judgment and discrimination of the occupant of the land.

Nore stress hav, in fact, been laid upon a systematic succession of crops than seems requisite. The great art of cultivation consists in the maintenance of the land, at least in sound condition and without impoverishing it, if it canmot be enriched; and it is essentially necessary that the amount of labour, as well as the quantity of manure employed upon the ground, should be duly alpportioned to its quality. The main object of all rotations should therefore be to establish such a series of crops as, by preventing the too frequent recurrence of any one of those which are considered exhausting, shall guard against the dissipation or loss of those component parts, or gualities of the soil, which seem peculiarly adapted to the growth

"Gentleman Farmer, 6thedit., p. 166. 
of each, and in the abundance of which consists its fertility. The precise nature of those qualities, or rather the causes which influence their peculiar effect on plants of different species, has not been ascertained; indeed has been only vaguely conjectured; and all the researches of chemical science on the subject have ended in proving little more than what was already known by experience,-namely, that certain plants can only be grown with advantage on certain soils; and they can only rarely be continued without evident diminution of their amount*

The land, to use the farmer's phrase, "grows tired" of a repetition of the same crops, and refuses to reproduce them in their former abundance, though it will yield an ample return of others of a different species + . From this it has been conjectured, that some particles of the soil are adapted to the nourishment of one kind of vegetable, and others to another: though, from their effect being more perceptible on the production of grain than of leguminous plants, it has also been presumed that the exhausting properties of the former are partly to be attributed to the greater exposure of the ground to the influence of the sun when bearing them; and that the meliorating consequences of the latter arise, in a great measure, from their shade: as well as that the large-leaved vegetables derive a considerable portion of their sustenance from the atmosphere, while corn seems to draw its support entirely from the earth. The tap-rooted vegetables, too, penetrating deeper into the soil than those which are grown nearer to the surface, derive their nutriment from a greater extent of earth, and may therefore be presumed to be less cxhausting to that portion of it which is most appropriate to the production of corn.

Whether these hypotheses be well or ill-founded, experience-that surest guide in farming operations-lias demonstrated that land, in the common course of tillage, cannot be kept in heart without a frequent change of crops. An exception to that rule may, indeed, be found in the vicinity of large towns which afford the command of unlimited quantities of manure, which, to some crops, furnish a succession of that nutritive substance, or vegetative mould \pm , which supplies the want of peculiar matter necessary to

* Although making this remark upon the application of chemisty, we are, however, by no means insensible to the value of chemical research upon agriculture, as well as upon every other subject to which it is at all applicable: thus De Candolle's 'Theory of the Rotation of Crops,' though still only a vague idea, yet holds out a field for research which, if followed up, as it doubtless will be by the chemists and naturalists of every country, bids fair to solve the problem of the principle of regetation.

t It is not, in fact, solely by exhausting the soil that certain plants deteriorate, if planted in the same ground year after year; for, were this the case, manure would renovate the ground; but it fails to do so, and thus if peas or wheat, for example, be grown repeatedly on a piece of land, the farmer may manure to whatever extent he chooses, his crops will dwindle and become poorer and poores. This is remarkably the case in the Isle of Thanet, where, to use a local term, if the land be "overpea'd" it becomes, as it were, poisoned, and if the peas be again planted, though they rise from the soil, they sown turn yellow, are "foxed," and produce nothing of a crop.-Tower's Domestic Gardener, p. 397.

This, however, does not apply to all crops indiscriminately; for potatoes, for instance, are known to thrive year after year in the same ground if well manured: and there is a remarkable instance related in Young's 'Annals of Agriculture,' of an experiment on the estate of the Duke of Grafton, in Northamptonshire, on some cold land of which beans and wheat were sown alternately during eight successive years, being; only - slightly dunged every third year, and yet continued to produce equally good crops throughout. It is worthy of remark, that the crops of wheat which fullowed the dunged beans, were finer than those for which the wheat was dunged.

$\ddagger$ This is known to chemists by the names of humin, humic acid, and humus, and is considered as that portion of the soil on which the ferrility of the land entirely depends, as with the exception of water-it is the only substance which furnishes nutriment to prants, 
their growth; and virgin soils, which have been kept under pasture, are generally capable of producing a series of similar crops during a number of years. Under such circumstances, the whole art reduces itself to the choice of those crops which the land will produce with the greatest profit in its actual state, and little fear need be entertained of its exhaustion; but these are incidents of a nature which do not belong to the ordinary range of cultivation, and which therefore do not fall within the scope of our observations.

The necessity of supplying the land, when under tillage, with a certain quantity of animal manure, has taught all farmers the expediency of combining the production of grain with the support of a certain number of cattle; by which they are enabled to draw from each all the advantages of which they are susceptible. Upon that point there is but one opinion; but regarding the reciprocal relation of each to the other, or the relative quantity of land which ought to be applied to each, much difference prevails. The various systems of cultivation may be divided into two classes: the one, chiefly confined to the culture of corn; and the other, comprehending the alternate culture of corn and roots. Under the former, it is absolutely necessary to retain a certain portion of meadow and jasture for the maintenance of the stock; while under the latter, the same object can be effected without the aid of natural grass. The mode of cropping must therefore differ on each, and must depend upon the nature of the land;

It is formed from the putrefaction of regetable and animal matter, and is described as "a dark, unctuous, pliable substance, nearly uniform in its appearance. It is a compound of oxygen, hydrogen, carbon, and nitrogen, which-with the exception of nitroyen, which is found only in some substances-are the elements of all animal and vegetable substances: it is the result of the slow decomposition of organic matter in the earth, and is found in the greatest abundance in rich garlen-mould, or old neglected dunghills. It varies somewhat in its qualities and composition according to the substances from which it has been formed, and the circumstances attending therr decay. It is the product of organic power, such as cannot be compounded chemically:" - See the Penny Cyclopædia, under the article 'Arable Land.'

Sir Humphry Davy also thus alludes to it in the following passage of his Fourth Agricultural Lecture:- "In most of the blark and brown vegetable muulds, the earths seem to be in combination with a peculiar extractive matter affurded during the decomposition of vegetables. This is sluwly taken up, or extracted from the earths by water, and appears to constitute a prime cause of fertility in the soil."-Quarto, p. 170.

It may be fairly presumed that the ruantity of this humes must be necessarily diminished by the growth of vegetables in proportion to the sap which they extract from the soil, and that its diminution must be equal to the amount of nutritive matter which they contain. This is indeed proved by experience in the case of corn; for wheat is known to exhaust the land more than rye ; rye more than barley; and barley more than oats; and experiments which have been carefully made by analysis of those species of grain show that, when of ordinary quality, they respectively contain the following proportions per cent., of gluten, starch, and mucilage, of which this nutritive substance is composed, namely-

$$
\begin{array}{ll}
\text { Wheat, } 78 \text { per cent. } & \text { Barley, } 65 \text { per cent. } \\
\text { Rye, } 70 \text {, } & \text { Oats, } 58 \text {, }
\end{array}
$$

Thus, having regard also to some differences in the nutritive value of the straw-

\begin{tabular}{|c|c|c|c|c|c|}
\hline 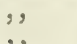 & Rye & , , & 55 & , , & $38 \frac{1}{2}$ \\
\hline , & $\begin{array}{l}\text { Barley } \\
\text { Oats }\end{array}$ &, & $\begin{array}{l}46 \\
34\end{array}$ & $\therefore$ & $\begin{array}{l}30 \frac{1}{2} \\
20\end{array}$ \\
\hline
\end{tabular}

$A$ bushel of Wheat, weighing 59 lbs., would absorb about 46 lbs. of nutritive matter.

Supposing this view of the subject to be correct, the result of these experiments would show that the different grain-crops exhaust the soil in the relative proportions above stated: and admitting season and culture to be the same, we must therefore conclude that if the qualities of humus are in every respect equally effectual in promoting vegeta. tion, its effects will be visible in the crops; consequently, that if any one of them be unusually abundant, the succeeding one must be proportionably deficient.-See Thiler, 'Principes Raisonnés d'Agric.,' tom, i. p. 254. 
but as the sole view of the farmer is to obtain from it the greatest amount of net product of which it is capable, that end, under proper management, is, in either case, equally attainable.

Under the old system of farming, the land was cropped with corn until it was partly exhausted, and was then left to recruit itself under natural pasture; but after the introduction of turnips, and the culture of other roots as field-crops, the alternation of grain and vegretables was adopted from Flanders, where it is the invariable method to carry an alternate crop for man and beast; and it was found that the land was both preserved from the baneful effects of over-cropping, did not demand such frequent periods of rest, and, through the means thus obtained of feeding live-stock, could be more abundanty supplied with manure. Experience, however, proved that although this change has laid the foundation of our agricultural prosperity, yet it had only made one stride towards its completion; for it was found that the succession of green crops to corm, still left undecided the serious question, whether the particular crop selected was the most proper to follow that by which it was preceded? Various trials were therefore made, and are still continued, in order to ascertain the best succession of crops on different soils*; and these several series are termed the rotation of crops.

In cultivating the ground, two objects ought always to be kept in view : first, to obtain from it the greatest quantity of the most valuable produce; secondly, that this produce be obtained in such a manner as mayleast exhaust the earth; or, in other words, that the care of the farmer, while prompted by regard to his immediate benefit, be so guided by discretion as not to dry up the sources of future and more lasting advantage.

The general principles upon which every separate rotation should be

* Particular attention has been lately attractel to the investigation of this subject by the writings of some foreign naturalists, whose experiments on vegetable physiology have led them to the conclusion that the roots of plants contain secretions, formed by their sap, which being thrown off in the nature of excrement, render the land unfit for the re-production of similar crops ; in like manner as no animal can subsist upon its own freces. When the soil has become much impregnated with the exudations of any particular vegetable, they are therefore supposed to poison it, so far as to be injurious to the future vegetation of the same species of plant; and the land is then said to be overcropped, though they may assist the nourishment of other plants.

Many curious experiments have been tried to prove the truth of the hypothesis; the most interesting of which, to farmers, are those upon beans, potatoes, and grain.

The bean exists in pure water, which continues nearly clear, though it assumes a yellow tince, occasioned, as it was found, by the discharge from the plant of a gummy matter and a little carbonate of lime. Fresh plants of beans did not live well in it : but in order to ascertain whether this was occasioned by the presence of the exuded matter, plants of wheat were placed in the water, and they throve perfectly; the colour of the water became lighter, the sediment was reduced, and it was evident that the plants of wheat had absorbed a portion of the matter discharged by the beans : from which it muy be inferred, that the experiment justifies the practice of sowing wheat after beans.

The potato also puts forth its leaves in pure water, which becomes scarcely discoloured, leaves but little sediment, affords but a slight taste, and leads to the supposition, that it is one of those plants whose roots secrete little of a decided character : this experiment, therefore, goes far to prove the truth of the well-known fact, that the potato, unless aided by a large quantity of manure, is a bad preparative for corn.

Wheat, rye, and barley do not live well in pure water: that in which they vegetated was without colour, smell, or taste; but contained some salts, alkali, and a very small portion of gum. As gummy matter appears-as in the experiment on beans-to be a good preparation for wheat, and grain seems to afford but little of that substance, this pruves that corn-crops should not snceed each other, and the experiment shou's the neces. sity of preparing the land by the cullure of green crops.-See the experiments of M. Macaire as stated in the 'Transactions of the 'Sucieté de Phys, et d'Hist., Nat. de Genève,' vol. v.; and the ' Edinburgh New Phil. Journ.' No.'xxviii.; also the 'Field Naturalist,' No. ix.; and various Papers on the subject in Nos, xxi., xxiii., and xxiv., of the 'Quart. Journ, of Agric.' 
arranged, whatever may be the nature of the soil on which it is undertaken, after the land has been first-as a primary consideration-completely drained and brought into a state of perfect cleanness, should embrace the following rules:-

To avoid the immediate succession of similar crops-particularly if they be of an exhausting nature-and to throw their return as far distant from each other as circumstances will admit.

To grow intermediate crops of artificial grass and roots, if the nature of the soil permit, in each year between the crops of corn.

On soils which admit of the alternate culture, to prefer those green crops which afford the largest prospect of food for live-stock, and which allow of the horse-hoeing husbandry for cleansing the land: and,-

On heavy soils, or land of any kind which requires rest from cropping, never to lay it down to grass until it is clean of weeds.

Bearing these principles in mind, the following courses of cropping have been found in most cases to be the best adajted for maintaining particular soils in a state of cleamness and fertility, as well as ensuring an economical disposition of the farm labour.

\section{TRIENNIAL, ROTATION.}

The mode of cropping, which was anciently practised throughout Europe, was confined to-

\section{Fallow-2. Winter Corm-3. Lent Corn,}

which thus occasioning the constant recurrence of a three-course shift, is called the triennial system. When farms contained, as formerly, a large proportion of out-pasture and meadow, a sufficient quantity of manure was made by the stock in the straw-yards during winter to afford a tolerable dressing to the fallow, perhaps every third, or, at least, every sixth year; and thus the land, if naturally good, was maintained in fair condition, and fine crops of grain were produced. It is still partially continued on common-lands of every description; which, being held under the old tenure of joint tenancy, cannot be cultivated in any other mamner, as the tenants in common have the right of pasturage after the crops are off the ground: there are also in some districts strong clays of so wet and heavy a nature, that they only admit of the occasional introduction of an intermediate crop of beans or clover; and the covenants in many leases require a fallow previous to a crop of wheat.

This has been much decried by theorists, who, looking to nothing but what they call the "national benefits of increased produce from the soil," and being unacquainted both with the difficulties and the expenses attendant upon its cultivation, as well as regardless of the result, in point of profit or loss to the farmer, call loully for a changre of system. To cnable us, however, to judge soundly of the propriety of any alteration, even supposing the land to be of a quality which renders it practicable, it is necessary to maturely weirh not only the nature of the soil, but also the farmer's means of working it ; for we cannot lose sight of the well-known fact that the large capitals which were lately employed in husbandry, have been very materially reduced, and that men are now very generally compelled to forego many of the advantages which they formerly derived from the pursuit of more spirited plans.

First, then, the system is economical; for all its operations are of the most simple kind, requiring nothing but the most unexpensive implements and teams of oxen.

Secondly, it demands less constant attention to the mamagement of 
the land than when alternate crops are continually grown; for the repetition of the fallow affords ample time for the preparation of the ensuing crop of wheat, or the clover leaves the land to rest.

Thirdly, the labour upon the land is equally divided throughout the seasons; and notwithstanding the loss of a year's rent, occasioned by the fallow, the following crop of wheat is usually so superior as in a great degree to make up for it.

These are considerations, both in point of personal ease and pecuniary means, which call upon the farmer to look carefully to the expediency of any change, however it may seem warranted by appearances; for it will assuredly dip deep into his pocket, and if that be not so well filled as to meet the full measure of expense, he had better not put his hand into it. His calculations must be left to himself; for he alone can julge of the circumstances attending his situation, and, if he be a man of business, he can be at no loss to make them with accuracy. It must, however, be admitted, that to conduct it with anything like energy, there should be a large proportion of mearlow; the whole of the hay and straw should be consumed in the yards and stables; and some beasts should also be stall-fed, with the assistance of oil-cake, to aflord a sufficient supply of manure: for there is this danger attendant upon such a plan, that if not well supported from the commencement, it must necessarily decline, as the production of manure depends in its greatest measure upon the straw, and if the corn crops fail during one year, they will also suffer proportionably during the next.

Besides being customary on clay-lands, the triennial system is extensively fallowed and found very productive in East Kent, on some naturally poor soils, as well as on deep sandy loam in the Isle of Thanet, where it is known as the Kentish round tilth, which consists of-

\section{Barley-Beans-Wheat,}

the process of managing which is as follows-

The wheat gratten, as the stubble is there called, is raked off, or spuddled, and the land ploughed five or six inches deep as soon as possible after harvest; is cross-ploughed when the land is tolerably dry in the spring, and again ploughed two or three times, according to the cleanness of the ground. The barley is either drilled in, at the rate of two and a half or three bushels, or sown broad-cast, four bushels per acre, some time in April ; and between the times of ploughing, collections of mould, farm-yard dung, and sea-weed, which is there met in great abundance, are found in convenient situations in the fields; are turned over in the autumn, and in the frosty weather of winter are laid upon the barley-stubble preparatory to the planting of the beans. The beans are drilled eighteen or twenty inches apart, at the rate of three and a half or four bushels per acre; the furrows are then harrowed and rolled down smooth; the land is repeatedly horse and hand-hoed, kept completely clean, and at the last hoeing the beans are earthed up. As soon as the beans are harvested the land is scuffled with the broad-share, and made perfectly clean by harrowing and burning the weeds, after which the wheat is either drilled at the rate of three bushels, or sown broad-cast from that to three and a half, and, in some cases, four bushels per acre.

Under this system clover is sometimes substituted for beans, and the manure is then laid upon the wheat-stubble preparatory to the crop of barley; but it is a manifest improvement to sow the barley upon a fallow, well dunged, during the previous autumn, by which means, as the land will then be thoroughly clean, the grain can be sown earlier than when it is necessary 
to allow time for three spring ploughings, and no preparation is equal to a barley fallow for a crop of clover.*

\section{ALTERNATE ROTATIONS.}

The alternation of green and white crops-which is now sufficiently understood to form the leading principle in every judicious rotation in which it is practicable, - is necessarily varied in the details of practice, according to the numerous circumstances of time, place, and soil, by which it must be governed; but the most prominent feature of all the courses upon those light soils which are usually termed "turnip land," is that commonly known as the Norfolk system : which consists of four shifts, comprising-

\section{Turnips-2. Barley-3. Clover-4. Wheat-}

has been continuer, with very little variety, during a long series of years, upon many large farms in that county, and is now very grenerally adopted upon similar land throughout many parts of the United Kingdom. There indeed can be no doubt that light land may be maintained by it in a state of perfect cleanness, and that, when managed with capital and spirit, it may be rendered more profitable to the farmer than any other; but so far as the production of grain is concerned, it is clear that wheat of a finer quality, and heavier crops, are obtained from a bare summer fallow.

This course, short and simple as it is, contains the just elements of good husbandry; but it must be understood as requiring the whole produce of the cultivated grasses and green crops to be consumed upon the farm, and consequently to need the assistance of a flock of sheep. Its commencement is founded upon the cleansing of the ground; and there is no crop with which we are acquainted which, upon dry soils, presents so many advantages as the culture of turnips; for the same ends are as effectually gained by it as by a naked fallow upon heavier lands, while, independently of its own particular value, its beneficial effects extend the whole period of the rotation. The crop necessarily requires a complete tilth; this-particularly if it be drilled and horse-lioed -brings the earth into a state of superior mellowness for the reception of the barley and seeds; and the clover, broken up by one plonghing, leares a sound bed for the production of the wheat.

To this indeed it has been objected, that the most valuable crop in the course is thereby placed at the farthest distance from the meliorating influence of the turnips, the application of the manure applied to which, it is thought, must be in a great measure exhausted before the wheat is sown. The argument is certainly not without weight; but it should at the same time be recollected, that it would be no easy matter, either by disposing the crops already enumerated in a different order, or by introducing others in addition, to arrange a plan of cultivation which might on the whole prove more advantageous. It is obvious, that if turnips are to enter into the course, they cannot be eaten on the ground in time to admit of the suwing of winter wheat; and it is chiefly when fed off the land that we consider them of superior value. Other green crops might, it is true, be removed sufficiently early in the autumn for the sowing of wheat; but on the land to which our remarks apply there are but few cases in which they could be so profitably planted. Potatoes, for instance, cannot be generally cultivated to any great extent; beans are adapted rather to heavy than light soils; and peas, besides being very precarious, are found of little avail in cleansing the ground. Independently of the high place which

* Boys's Surv, of Kent, 2nd edit, chap. viii, sect. 3. 
turnips hold as a fallow crop, are also the important purposes which it serves in the feeding of stock, and the consequent improvement which the manure thus raised occasions in the condition of the land.

It must however be admitted, that this course, although forming the best foundation for an established plan, where the soil and state of the farm will allow it, may yet be conveniently departed from on particular occasions; and so rigid an adherence to system as would exclude all regard to circumstances which demand alteration, would indicate but little acquaintance with the casualties of rural life. The soil, indeed, delights so much in variety, that it sickens of the frequent growth of some vegetables; thus, although clover is generally found to succeed better after barley than after any other crop, yet, if too frequently repeated, it sometimes fails, and must then be replaced by some other green crop, which may occasion the necessity of altering the entire of the following rotation. The farmer must, therefore, consider himself at liberty to make whatever selection he may deem most eligible or convenient; only avoiding the immediate recurrence of any two crops of grain.

On very light land it would also be deceiving one's self to imagine that crops of corn can be grown, even in alternate succession, or that an uninterrupted course of tillage can be advantageously continued during at long period; and it is undouhtedly necessary that such soils should be occasionally invigorated by being laid down to pasture. The ordinary course is, indeed, sometimes extended to a five-course shift, by leaving the land for two years under clover; which, in that case, is generally sown with a mixture of rye-grass ; and rye, or oats, are sometimes substituted for wheat. A smaller portion of the farm is thus required to be ploughed and manured, and the sheep are regularly folded upon the ground; but, although advantages are thus scemingly gained, yet the course is not so profitable as the former, when that can be carried into effect, for the clover leaves but a poor return during the second year, and the land, when continued under grass, engenders worms and weeds, which are injurious to the future crop of wheat. A better plan is, therefore, to extend the course, by continuing the two next successive crops-namely, turnips and barley, or oats - and then, instead of sowing this second crop of barley alone, as it intended to be followed by wheat, to lay the land down with pasture-grasses. The land will thus be brought into a state for the feeding of stock during such a number of years as may be deemed sufficient to accomplish its renovation: it may then be again broken up with a crop of oats, after which the former courses may be again pursued.

Or the course may be extended beneficially thus :-
1 Turnips.
2 Barley.
5 Turnips.
3 Red clover.
4 Wheat.
6 Barley.
7 and 8 Pasture.
9 Oats.

The grass-seeds sown for the two years' pasture should consist of sixteen pounds white clover, three pounds trefoil, two pounds rib-grass, and two quarts of perennial rye-grass ; entirely omitting red clover at this period,

* We have lately witnessed an iustance of a crop of clover, which showed evident signs of failure, having been brought to produce au abundance of green food for soiling, by running the harrows over it, and partially sowing it with about a bushel of tares; the cattle seemed to relish it more than the clover which had been grown unmixed, and we doubt not that it would have made excellent hay. It was bush-harrowed and rolled after the seed was sown; and were the same plan adopted for all clover that is allowed to stant for the second year, it probably would be found to add largely to the pasturu while the additional expense would amount to a mere trifle. 
to prevent its too frequent recurrence. This is a favourite rotation with many of the best farmers on the Yorkshire Wolds, who vary it occasionally by sowing winter tares in lieu of the red clover, and alternate the turnips between the common sorts and the Swedes.

In Norfolk, as well as on the sandy and dry light loams of other districts, the management is, however, confined by some landlords to a sixcourse shift of cropping, namely:- Wheat the first year; barley or oats, without clover, the second; turnips the third; barley or oats, with clover, the fourth; clover, mown for hay, the fifth; and the sixth, grazed till Midsummer, when it is again broken up for wheat*. This last rotation has, indeed, become more general during the last few years; for those who practised the Norfolk system on thin light soils, have found their crops grow so much worse as to be compelled to adopt it. We learn that it has even been tried with good eflect, not only upon turnip-soils, but upon strong clayey lands; substituting naked fallows, or beans drilled at thirty inches, instead of turnips; and those who have pursued it on such land find, that after two or three years' clover and grass-seeds, fed off with sheep, the land will grow good crops of oats, which they could never gret it to do under the old plan + . It appears to us, however, to be more exhausting than the four-shift course; and that, on such light soils, two successive grain-crops ought not to be allowed.

On entry to a farm it not unfrequently happens that the tenant finds it has been conducted in a manner wholly different from either of the rotations above mentioned, or indeed, from any plan accordant with the alternate system of husbandry, and has been entirely thrown out of condition by bad management. There is great difficulty attendant upon any change of cultivation, upon so extensive a scale as to bring the land immediately under a different course from that in which it bas been already managed: the regular succession of some of the usual crops must be sacrificed, additional labour may be occasioned, and present expense increased; but if it be an object to adopt the four-course shift, perhaps the following mode will be found as short and simple, as under most circumstances, can be suggested. Thus-

Supposing land of any definite extent, and of the fair average quality of dry turnip-soils, to be found the greater part in a foul state, occasioned by the production of successive crops of corn, and the remainder to be under rough pasture not worth keeping in grass. The course which we should recommend would be-to divide the arable into three parts, with a portion of pasture equal to one-third of their quantity, thus forming the whole into four divisions, as nearly equal as the size of the fields will permit: to break up the pasture, No. I, for a crop of oats, slightly pared and burnt, if the weeds and herbage be sufficiently rank to admit of the operation without injuring the staple of the soil; for this grain will uniformly yield a greater return than any other till the surface of the land be completely reduced and the natural grasses destroyed.

* Kent's Survey of Norfolk, p. 33. Mr. Kent, however, rather recommends wheat, vetches, buck, turnips, larley, clover: which he conceives "would keep the turnips and clover-crops at such a distance, that there would be no fear of their success; and as the buck-wheat inight be considered as a neutral crop, the altemate advantage would not, in fact, he lost in its grood effect. By means of the vetches too, more stock might also be kept on very light land than from the six-course shift; and, where a flock is kept, it can never be employed so well as in feeding upon this sort of light land, as som as the wheat or rye be sown, especially if the sowing be upon one ploughing; in which case it is lust to begin rather early, and sow by degrees as many ridges each time as the breadth of the fold will cover."

$\dagger$ Bailey and Culley's Surv. of Northumberland, 3d edit. p. 70. 
No. 2 should be naked fallow, succeeding the foulest of the previous corn crops, and dunged or limed, as means may be found for manuring it: on No. 3, the common four-course shift may be entered at once; and at No. 4, it may also be continued; except that, as the turnips are there omitted, the following crop of barley must be succeeded by a cleansing crop, which, if the land will admit it, should be beans: if not, peas at wide intervals, and both horse-hoed. The divisions will then stand thus:--

$$
1.204 .
$$

First Year. - Oats, or Cole. Fallow. Turnips. Barley, or Oats.

Second Year.-Turnips. Wheat. Barley. Beans, or Peas.

Third Year.-Barley. Turnips. Clover. Wheat.

And, in this order, the whole of the land may be brought under the fourcourse system within the space of three years. If the entry should be at Lady-day or Whitsuntide, or if it should be thought more advisable to commence with a green crop than with oats, then the pasture land, No. 1, may be sown with cole to be fed off; and a spring crop of corn may be substituted for the whent in No.2. As there probably will not be sufficient dung upon the farm, even with the assistance of the fold, to manure both the fallow and the turnips, we should, in that case, strongly advise the farmer not to hesitate in using a liberal quantity of bone-dust for the latter: at all events, no pains should be spared to bring both that crop, and the condition of the land upon which it is grown, to the highest state of perfection.

In the breaking up of the land in No. 1, although turnips are set down to follow oats, yet, if the land be not burned, we should recommend a clean fallow in preference. Every one who is acquainted with old grassland, must be aware of the difficulty which attends the working of it in the second year; and if the ground be at all heavy, it cannot be brought into a proper state of cultivation until that radical measure, of paring and burning, be resorted to. The wire-worm and the grub also abound in grass-fields, and there is no other way of extirpating those destructive insects. The course can then be carried on until it may be deemed advisable to rest it from the operation of the plough, when it should be carefully laid down with those grass-seeds which are known to be most appropriate to the soil, and an equal portion of the remaining pasture may be broken up.

Or again - supposing the farm to consist of 300 acres, of which 30 are occupied by the homestead, orchard, and paddocks; one-third of the remainder to be retained in pasture ; and the rest to be brought into a regular course of cultivation : the following may be found a judicious mode of dividing the crops, so as to prevent their too frequent repetition. Thus-

1. $\{15$ acres of bare fallow, dressed with lime or compost.

- 15 do. turnips, well dunged.

2. $\{15$ do. wheat after the fallow.

- 15 do. barley after the turnips.

3. $\{15$ do. clover, to stand only one year, for mowing, after wheat.

$\{15$ do. perennial grass-seeds, to be laid down for pasture, after barley.

4. $\{15$ do. oats, after the clover.

- 15 do. oats, after an equal quantity of the old pasture-land to be broken up.

5. 30 do. drilled beans and pease, or any rout-crops, well manured and horse-hoed, after the oats.

6. 30 do. wheat to follow.

By which means the whole of the rough pasture will be brought under the 
plough during the course of the rotation, while an equal quantity of the arable will be rested, and the other half will undergo a different course of cropping during the next shift.

\section{DOUBIE CROPS.}

The winter, or half-crops, have been omitted, as the object was to show, as clearly as possible, the order in which the crops that occupy the ground the greater part of the year should succeed each other. The usual half-crops are, winter-tares, rye for sheep food, or stubble-turnips, and are generally sown on the land that is intended for summer-turnips. A more varied and extended course is, however, not unfrequently carried on with considerable advantage; of which we select an instance of a large farm situated upon a soil of very moderate powers in the county of Surrey, but conducted with the greatest vigilance and exertion, as well as with abilities far above the common level. The course there pursued was-

1. Wheat, after sainfoin, or fallowed.

2. Tares, cut or fed off very early, and immediately ploughed up for turnips.

3. Barley, lightly ploughed in with rye-grass, and trefoil harrowed in.

4. The grass early fed off by ewes and lambs, and afterwards dunged.

5. Wheat upon the ley.

6. Grey peas, or early beans.

7. Rye for feed, folded for turnips, which are also fed off in the winter.

8. Barley, with clover.

9. Clover for hay, and pasture.

10. Ditto for pasture and seed.

11. Late wheat upon the ley.

12. Rape harrowed in, and fed off soon enough for oats, or barley, with sainfoin.

Thus fifteen crops-three of them wheat-were obtained within twelve years, and the land was kept in such good heart by those which were fed off and folded, that the produce was strikingly abundant: it was also maintained in such a state of cleanness by the several hoed crops, that the course was closed with sainfoin in the best possible condition*.

The practice of double-cropping light lands is indeed pretty generally carried on in some of our southern counties, in regard to winter-tares, turnips, and rape, and no doubt might be in many places advantageously extended +; it can, however, be only applied to white turnips, for the tares would not be off the ground early enough for Swedes, and the land should, besides, be in a very high state of cultivation, or it cannot be got in proper condition for the second crop. Rape, if sown immediately after a corn crop is cut-as in the course above stated-can be raised at a trifling expense, will afford a good bite to sheep as winter feed, and will improve the land for a succeeding crop of oats; but when intended as a crop to stand for seed, it can only be grown with advantage upon fen-lands, or upon deep loams. Stubble-turnips, though sown without manure, and consequently

* Malcolm's Surv. of Surrey, vol. iii. p. 404.

t Stevenson's Surrey, 1. 199; and Vancouver's Surv. of Hampshire, p. 171. On the South Downs the practice is to sow $2 \frac{1}{2}$ bushels of tares, and half a gallon of rape; or winter and summer-tares are sown on the same field, at different periods, about a month intervening between each, by which means successive crogs are ensured during the whole summer.-Sussex Rep. p. $10 j$. 
of diminutive size, will yet, in some seasons, produce a fair crop of succulent food for lambs in the trying season of spring; and spurry, if mo: $e$ generally known, would be found a useful article for cattle during the winter. In Flanders, the spurry is extensively grown, upon a slight ploughing after wheat, and is there universally employed in the feeding of the dairy cows. The farmers of that country are, indeed, famous for other double-crops, as for instance-carrots after flax; turnips after wheat, rye, and oats; and green corn before flax, or potatoes. Sometimes they even procure three crops in one year: first, a crop of corn to be cut green ; then flax, with which carrots are sown; or after the flax is pulled, turnips, spurry, or buck-wheat; and in that mamner, the cattle being soiled, they malie prodigious quantities of manure, by which they are enabled to raise a large produce from soils of such a light sandy nature, that, until thus improved, they were nearly sterile*.

The Flemings have, however, been farmers time out of mind, and there is not a cultivated acre, the properties of which are not matter of notoriety. According to those properties, the most suitable succession of crops, and the most profitable application of manure, have been long invariably adopted from the tried results of acknowledged practice in different districts. To follow their rotations, indiscriminately, in this country, camnot indeed be recommended; but it has been justly remarked, that if we could be brought to imitate them, generally, by the adoption of some kind of rotation system, governed by our experience of soil and circumstances, a valuable improvement might thus be effected in British agriculture + .

While on the subject of the different rotations which are apparently followed in conformity with the alternate system of cropping light lands, it may be proper to notice that two or more green crops are frequently grown in succession during two following years; so that no general rule separates them by a white crop in the same manner as a regular course. Except in the vicinity of large towns, which afford a profitable demand for such produce, or in cases where the state of the land may render two drilled and horse-hoed crops advisable to assist in cleamsing it, and thus preventing the necessity of a fallow, it however appears to us that the propriety, or rather the economy, of the practice, may be justly doubted. A farmer who takes more than one crop of corn in succession, from liglit land, is blameable, because he, to a certain extent, exhausts it; but if he unnecessarily takes two green crops in following years, he suffers a loss; for, when consumed upon the ground, they are rarely found to pay so well as corn. In defence of the practice it is indeed argued-" that a succession of green crops will keep the land clean and in good condition:" but to this it may be answered-that if the land be carefully tilled at proper scasons, a green crop every other year on light lands, and the occasional introduction of a complete summer fallow on those which are strong, will

* After a crop of cole-seed, or hemp, spurry, carrots, oi turnips are grown; and after wheat, a crop of carrots, sown with the wheat, or spurry, or turnips, sown after the crop, is reaped. Carrots are also sown with rye; or turnips, or spurry, after the crop is cut down. It is howerer thought indispensable to vary the crops, and never to sow the same for two years in succession.-Vanderstraeten's Flemish Husbandry, p. 42. We learn also from Mr. Radeliff, that upon the inferior sands in some parts of lilanders, after a crop of rye has been taken, rape and turnips are both sown in succession. The rape, which is transplanted from seed-beils, sown in August, is dibbled after three ploughings, with about a ton and a-half of lime, and 52 hhds. of urine per acre: after the rape is harvested the turnips are sown upon one ploughing-with 10 tons of farmyard manure per English acre.-Rep. on the Agric, of Flanders, p. 100.

+ Ibid. p. 58. 
effectually maintain the ground in good order; and, if it be poor, green crops are more likely to increase than diminish an evil which can only be remedied by laying the land down for a series of years to pasture. Inferior soils, indeed, though naturally improved by being converted to grass, are not calculated for the production of root crops, and experience proves that it is easier to raise a heavy crop of wheat than a good crop of turnips upon poor land. Besides, even supposing the land to be kept in that high state, it may vet be justly asked, what object of real value is thereby grained, if advantage be not taken of it to raise those crous which will best pay the farmer? It is undoubtedly wrong to exhaust the soil by over-cropping; but it certainly must be considered as equilly injudicious not to crop the land up to what it will bear without being injured*.

These light turnip-lands, it must however be remarked, contain such a great variety of soils, that no rotation of crops can be expected to reign uniformly. Nor, even were the quality of the land the same, would farmers adopt a similarity of practice, and to notice the endless changes of method made by individuals would fill a volume; whereas, a work of this kind must evidently be confined to an account of only the most proninent features of the prevailing systems of cultivation. The nature of such soils, howerer, generally partakes of chalk, sand, or loam, and the most usual rotations in those districts are the following.

\section{DISTRIC' ROTATIONS.}

The chalky districts generally consist of separate layers of either hazelloams, strong red clay, or thin flinty clay, of different degrees of depth, and incumbent upon a subsoil of chalk; but when consisting, as they do in many parts, mostly of pure chalk, but very little profit can attend their culture, and they are commonly left for sheep-ivalks: thus forming what in the south of England we call "downs," and in the north "wolds;" large flocks being kept for the purpose of folding on the arable land, and much of it being farmed under the common Norfolk system. The variations are-

On the chalky loams-

1. Turnips, dunged, and fed off.

2. Beans.

3. Barley, or oats, with seeds.

4. Cluver.

5. Wheat.

6. Tares, fed off.

7. Oats.

8. Cole, or rye, for spring feed.

But if the land be of a stronger quality, the course is then altered for-

1. Fallow, dunged.

2. Wheat.

3. Oats.

5. Turnips, fed off.

6. Barley.

4. Beans.

7. Clover.

8. Oats, or in some cases, wheat ;

and two crops of corn are thus taken successively: in both cases the land is, however, frequently laid down with sainfoin.

On the strong species of red clay the usual rotation is-

1. Fallow, folded or dunged.

2. Wheat.

3. Peas.

4. Tares, generally fed off, but sometimes left for seed.

5. Oats, if the tares are left for seed; wheat, if folded off.

When peas are taken, the land is skim-ploughed, trench-ploughed, and

* See Stevenson's Survey of Surrey, chap. vii. sect. 3. 
sometimes rafter-ploughed in the autumn; then ploughed again in the spring, and very generally sown broad-cast. Beans are, however, frequently planted on the strongest portion of the soil, and are then more commonly drilled, and followed by oats.

On the cold thin clay and flinty chalk, the common courses are-

1. Fallow, folded.

2. Wheat.

3. Peas, upon one ploughing.

4. Tares, cole, or turnips, fed off.

5. Oats, or barley.

6 and 7. Clover; or, in some cases, sainfoin left for five, six, or seven years; and then

S. Wheat, pared and burnt, for turnips.

The preceding are some of the most usual rotations on the different soils incumbent on chalk, and where the upper soil is sufficiently light and open to admit of turnips, they may be considered as generally calculated to preserve the land in proper condition, and to secure tolerable crops. A farm, of the average quality of such land, under this mamagement, and with a proportionate quantity of ground in sainfoin, must, with any thing like proper attention, be carried on with little difficulty or libour at all seasons. The sainfoin should, however, be in a larger proportion to the size of the farm than appears in the statement above given; for it requires a long period to intervene before it can be grown with advantage a second time, and it consequently cannot be made to enter into every course* neither can peas be repeated, with much probability of obtaining a fair crop, more than once in ten or twelve years. On the strong and comparatively deep red clay, which is not unfrequently found at the bottom of the chalk-hills, wheat might perhaps be taken, with safety and advantage, more frequently; and, as its greater depth and soundness fit it better for beans than the thinner clays, tares, beans, and clover, might be with great propriety alternately sown between the wheat-crops, with the occasional substitution of a fallow instead of clover. On those chalkyclays where flints abound, it would, however, be difficult to suggest any advantageous alteration in the mode of cropping, for the nature of the land is there an almost insurmountable impediment to the use of the horse-hoe.

The dry and sandy-loams, being everywhere peculiarly adanted to al. termate rotations of white and green crops, are very generally cultivated with little variation under that system; though some farmers occasionally "cross-crop" their land for the purpose of pocketing the imme-

* See the Reports of Select Farms in North Hampshire, and on the Gloucestershire Hills, Nos. I. and IV., published in the Farmer's Series, by the Suciety for the Diffusion of Useful Knowledge.

On the extensive farm of Beverston, in the calcareous range of the Cotswold Hills in Gloucestershire, a great part of the arable land is under a course of turnips, - barley, or oats, - clover, trefoil, and rye-grass, every two years, being mown the first, and pastured the second; then wheat, and lastly oats, succeerled by winter-retches ferl off in the spring by sheep, and then sown for turnips. On this Mr. Hayward, the very intelligent farmer who holds the land, says, "The last crop of this course, it may be supposed, ought not to have been taken; but having had clover for two successive years before the crop of wheat, he not only thinks himself entitled to a crop of corn after the wheat, but believes he gets better crops by this rotation, upon such poor thin land, than he could gret by having the crop of wheat after the first year's clover, or by putting a green crop of any description between the wheat and the oats, or barley. Sometimes, however, the crop of wheat is taken after one year's clover; and if the land is firm enough for wheat, it succeeds very well: indeed, we have seen it succeed much better than after the second year's clover; upon land given to land-grass." 
liate profit upon an additional crop of corn. There is also an exception to the principles of good farming, which not uncommonly prevails upon the loams, by wheat being taken from them instead of barley, when the turnips can be eaten off by the end of November: in which case the rotation usually stands thus-

\section{Turnips, 2. Wheat. 3. Clover. 4. Peas, 5. Wheat.}

Heavy clays of a cold nature, when not under the common-field system, or restricted by leases to one particular mode of cultivation, are commonly worked under courses which commence with summer fallow, very generally dressed with lime, and continued with crops of wheat, alternately with beans and clover; the principle being-that a fallow, when once given effectually, will enable the farmer to omit it in the second return of ihe course: thus improving upon the old system of fallow every third year. On the stiff wet clays of the wealds of Kent, Surrey, and Sussex, and, indeed, not unfrequently in many other parts of the kingdom, a crops of oats still succeeds that of wheat; the land is then laid down with clover and rye-grass, or trefoil, for two or three years, when it is again broken up; but is in that time frequently becomes covered with weeds, or grasses, which are indigenous to the soil. More is, in fact, lost by keeping such soils two or three years in grass, than can possibly he made or saved by the stock which they will maintain; for the sown grasses begin to leave the land even in the second year; they should therefore be either broken up earlier, or, if it be thought proper to keep them more than one year in pasture, they should be suffered to lie till the grasses which are natural to them take place of those which are sown*.

The years for growing these several crops are, however, frequently reversed; and barley is never sown on land of this adhesive descrip)tion. It seems, indeed, to be a general principle among the farmers of the south of England, that a clover ley is in all cases the best preparation for a crop of wheat; while, in the north, and throughout Scotland, clover is more commonly sown with wheat and followed by oats: both from the latter being almost invariably found to produce a large return after clover, and from the wheat being better placed in the immediate succession of the fallow.

On thin clays, oats are, indeed, throughout the north, a more prevailing crop than wheat; and on the hilly districts of that moist climate, they are more profitable to the tenant, as well as less exhausting to the soil. "The general course in extensive districts of that country is, therefore-

\section{Turnips, well dunged. 2. Oats. 3. Clover. 4. Oats.}

which has succeeded completely; and, in such situations, it may be doubtful whether oats would not be generally found the best crop of the two after the ley; for, if the clover crop be good, the succeeding oat crop seldom fails. In most clays of the ordinary description, however, the last crop of oats should be sown with pasture-grasses, and left for a few years, ur until they begin to engender moss. It is, indeed, frequently necessary to sow down this kind of land with pasture grasses: this should, however, be done when it is free from weeds, and in good condition; and if the pasture can be manured the second, or at the farthest the third year, it will renovate the grass, and leave it in capital order for being again broken up ; whereas,

* Sussex Report, p. 78; Surrey do., p. 195. During the high prices of corn many farmers, indeed, adopted a course, upon light lands, consisting of turnips, harley, wheat and then barley or uats, with grass-seeds; and this ruinous jractice has not jet been entirely discontinued. Surv, of Cornwall, p. 57. 
if allowed to become exhausted, it will make no return of any value, and will rather impoverish the soil than improve it*

On strong land, of a dry and not too tenacious quality, such as that in the higher part of the Carse of Gowrie-which is, perhaps, as regularly managed as any district of similar extent in Great Britain + - $t h_{1}$; most general routine is-
1. Fallow.
4. Barley.
2. Wheat.
5. Clover.
3. Beans.
6. Oats, or sometimes wheat.

In less favoured parts of Scolland, beans however will not ripen in some scasons: being, therefore, a precarious crop, they are seldom planted, and potato-oats are generally sown in preference.

A favourite rotation on strong land in some of the hundreds of Essex is

1. Summer failow, limed.

2. Barley.

3. Clover ; first fed, and afterwards kept for seed.
4. Wheat.

5. Beans, dunged.

6. Wheat.

7. Oats.

For it is an universal rule in that county, never to put in wheat on a fallow. Although two successive crops of white corn are justly objected to upon the best principles of cultivation, yet upon land of this nature both wheat and oats are frequently taken, either before or after each other, withuut any material detriment to the soil.

On the fertile loams of the Rochford Hundred, turnips, oats, and clover, followed by wheat, oats, beans, and then wheat again, are succcssively taken, and produce abundant crops. We visited one of these farms a few years ago, and found the land, which had been cropped in nearly the same manner for a great length of time, not only in a state of admirable cleanness, but were assured that the last crop of wheat was equal to the first. Oats are, however, in this district found so much better than barley, that they not only produce more profitably, but wheat, following clover sown with oats, is in general found to yield near eight bushels an acre more than that which succeeds barley. Rape-seed, coriander, mustards (both white and brown), and carraway, are likewise occasionally grown to a considerable extent ${ }^{*}$. The cultivation of which will be duly noticed in their proper order.

* See the Survey of Mid-Lothian, p. 88; and the Reports of Select Farms, published in the Farmer's Series, viz., No. II., Kyle, in Ayrshire, and Netherby, in Cumberland; and INo. III., Strathnaver, in the county of Sutherland.

+ We, however, cammot he! p noticing the exaggerated account of farming in this district, given in the Survey of Perthshire, as extracted, under the head of "Expense and Profit," from the letails of a farmer who is there held up as a model of correctness, and who is represented as laving regularly realized a profit of more than four pounds per acre on lind held at a rent of three pounds. The charges upon which this statement are founded are, in fact, all lower than the actual expense of cultivation, and the prices of produce higher than they were during the years upon which the account is founded; and such mistaken views are highly injurious, as they tend to impress sujerficial enquirers with a belief that husbandry is a much better business than it really is.

\$ The course of cropping in the temperate lands of this hundred is thus described:"First, through summer and winter fallow for oats or barley, with which sow $16 \mathrm{lbs}$. of red clover, or, in lieu thereof, $6 \mathrm{lbs}$. of white Dutch clover, and $10 \mathrm{lbs}$. of trefoil per acre. Clover ley sown with wheat, the etches of which are dunged and sown with beans, that are kept well hoed through the summer, and again sown with wheat. After this, a crop of oats or barley is sometimes taken; but most generally the wheat stubbles are haulmed, sown with winter tares for spr ung food, then dunged and prepared for cole-seed, which is fed off and succeeded by wheat. The stubbles of this crop are dunged and sown 
On land of peculiar excellence in Mersea Island, on the Essex coast, great crops of wheat and beans have been long taken alternately; and an instance is mentioned in the County Peport of their having been not only sown for thirty-six years with equal success, but that plan of husbandry having been for some time changed, the farmer complained "that he had not been paid by it so well as by that incessant cropping *." Sir John Sinclair, however, adds another, of a farmer who followed this plan during fourteen years, near a town, from which he probably obtained large quantities of manure, and who had in that period grown four crops of potatoes, three of beans, and seven of wheat; but he found that although the quantity of produce had not diminisher, yet the quality of both the wheat and beans had degenerated + .

It may indeed be observed, that all the successful deviations from the alternate system, arising from the pore frequent growth of corn than grecn crops, or the too frequent repetition of any one species, have been either upon rich alluvial earth, or fomnded upon the application of lime to virgin soils, the puwers of which have been forced into action by the stimulus which it has occasioned; or else, by large quantities of putrescent manure laid upon land of a deep and naturally strong staple. But, unless under extraordinary circumstances of fertility in the earth, and management in its cultivation, there must be a period beyond which such deviations cannot be advantageously continued; for, when lime has been applied in any considerable quantity, and a course of severe cropping has been afterwards pursued, the land, unless largely supplied with dung, will become exhausted; and dung, if too jrofusely applied, will injure the quality of the corn, and produce crops more abundant in straw than grain. Thus wheat, though sown alternately with turnips and clover, and pro. fusely ntanured with both dung and sea-weed, and the turnips eaten off by sheep, yet could not be grown with success, every other year, for any great length of time $f$; and we learn that many parts of the highly-cultivated district of the Lothians have been so much exhausted by the too frequent use of lime, that the crops have lately failed, and much of the land has consequently been laid down to pasture.

Alternate crops of potatoes, wheat, and clover, have indeed been successively grown upon a large scale during a long series of years, by some farmers in the neighbourhood of London, and other large cities, where extraneous manure can be readily oltained, and which ensure a sale for the potatoes. There, however, the wheat is a crop of secondary value in comparison with the potatoes, and therefore the deterioration of its quality may not be an object of much interest; but where that is regarded, a better course is that followed in the vicinity of Elinburgh and Glasgow; which is -

\section{Potatoes, 2. Wheat. 3. Clover. 4. Oats.}

Though in this country, oats or barley would be made to precede clover, and the latter to be succeeded by wheat; which is not an uncommon rota-

with beans, which are agrain followed by wheat; after which the land is fallowed in course, or sown with turnips, if its condition will permit. The whole is then esteemed clean, good farming, and when the hean-land is not too loose and mellow, is always sure to produce excellent wheat." - Kissex Rep., vol, ii. p. 228

* Surv. of Essex, vol. i. p. 219. See also the Lincoln Report, p. 322 ; and a note in the last chapter of this work, p. 91 .

$\dagger$ Cude of Agric, 3d edit. p. 448.

\pm Ibid., p. 450 . 
tion among dairymen whose farms are situated upon stiff and heavy land *: though it is an old Scotch saying, that-

"He who sows wheat after bear,

Had need of muckle gear."

Or, he should be a rich man who sows wheat after barley. Mr. Middleton, indeed, urges the propriety of growing potatoes after tares or early peas are off, in the same year, and then alternately following those crops with wheat, as a practice which might be beneficially extended to every part of South Britain t: but he forgets to tell us how the manure is to be procured for their production; and, although he instances the fortunes which have been made by some of those men, yet they are to be looked upon more in the light of market-gardeners than husbandmen, in the common acceptation of the term.

Upon rich alluvial soils, when recently broken up, no rotation which it is possible to continue for a great length of time can be said to prevail, for the first object is to repay the expense of drainage and embanking. Thus, upon newly broken up marshes in some parts of Lincolnshire, the tenants are allowed to take three crops of white corn; then potatoes; and after that, two crops of grain to one of potatoes; or two courses of flax, rape, and potatoes, followed by crops of flax and wheat. Thus on warpland along the course of the rivers Ouse and Humber, in Yorkshire, the common course is, the first vear to plough up and sow rape to be fed off : the second, rape-seed for a crop; and during the four following years, crops of wheat and oats; or potatoes, wheat, and beans are tatien alternately ${ }_{\ddagger}$. It is, however, evident that such a mode of cultivation camnot be long continued, and therefore the usual rotation, when the land has been brought under a settled course, is either-

1. Rape, fed off.

2. Oats.

3. Beans.

4. Wheat.

5. Clover, either repeated with the exception of the rape,

or followed by-

1. Oats, on the clover ley. 3. Rape.

2. Wheat.

4. Oats.

In the Fens of Lincoln, Huntingdon, and Cambridgeshire, which are usually incumbent either upon a clay soil or upon peat, no system is generally pursued, except that of beginning with paring and burning; which, upon land encumbered with coarse herbage, is certainly an excellent practice, though its repetition may be thought injudicious $\delta$. Oats and cole,-or rape, as they are indifferently called,-are the only produce till the first luxuriance of the soil is in some degree abated; but, when the land begins to acquire more consistence, wheat, and crops of cole and ryegrass, which are allowed to stand for seed, are frequently taken, according to the peculiar properties of the soil : the fens, being of various degrees of

* Survey of Middlesex, 3d edit. pp. 252 and 188.

† Stevenson's Lancashire, p. 258. Holland's Cheshire, p. 131.

+ Reports of the East Riding, p. 119, and of Lincolnshire, pp. 126, 127, and 322.

$\$$ On this, however, it is the opinion of a man of the most extensive experience as a fen farmer, that the quality of cole for feeding sheep is not only not deteriorated by the sward being a second time burned, but that, in no other way can it be procured of equal groviness; and that, when grown upon very oll land, it is apt to kill the sheep put upon ir._Cambridgesh. Rep., p. 109.

Many instances may, indeed, be pointed out where the soil has been improved by having been pared and burmed several times; but then the land must be covered with a rank sward, aud the operation be very carefully performed, or it certainly will cause material injury.-Ibid. p, 112. 
richness, and both cole and rye-grass, when allowed to ripen their seeds, being very exhausting crops. The system which is recommended as the best to be generally adopted upon such lands, when of an average quality, is however-after paring and burning-to sow cole, to be fed off with sheep only; then oats twice, on one earth each time. Then muck for cole on two earths, or three, according to circumstances, feeding off the crop, and sow oats or spring-wheat with seeds, to be laid down for four, five, or six years, and even longer, as they may be found to stand.

It is a common practice not to lay the land down to grass until the third crop of oats, after the first breaking up; but three successive crops of corn on soil of a middling, or indeed of any quality, are too much. The mly proper period for laying down land to grass, is, when it is in good lieart, has been well worked, and thoroughly cleaned from weeds : this, in ordinary cases, points out the propriety of sowing the grass seeds with the first crop after a fallow; but in the fens, if laid down with the first crop, the ground is not brought into sufficient tilth to secure a farourable crowth to the seeds, and as the pasture is there an object of the first importance, two crops should, therefore, be taken; but no more*

Peaty soils, though generally distinguished by the different characters of "hill-moss," "bent-moss," and "flow-moss," according to the situations in which they are found, either on mountains, gentle acclivities, or vales; yet being all formed by the accumulation of partly decomposed aquatic plants, or herbage saturated with water, partake more or less of the nature of the materials of which they are composed, and, when brought into a state of cultivation, are generally found to produce earth of a light and porous kind, the properties of which are also governed in some measure by the soils upon which they rest, and with which they sometimes become intermixed.

Hill-moss moorland is found in wide districts of different parts of the Lnitel Kinglom, and is known under the different names of "heath" and "moss;" the former being covered with a sound, short, and sweet, though indifferent turf, and the latter-which is found chiefly in the north and west of Scotland,-being of a wet and peor quality, is chiefly left in a state of nature: all that can be done for its improvement being to open chanmels for carrying off the superfluous water, and liming it, by which means it may be converted into dry pasture for sheep. In every case where that is practicable, at such an expense as will repay the charge, it ought not lowever to be neglected; for, if not drained, the flocks pastured upon such land become infected with the rot, and many thousands of sheep are thus annually destroyed $t$.

The bent-moss of the moors, or heath, is always covered with a close sward of coarse herbage, and lying upon declivities which frequently afford the convenience of draining it at a moderate cost, it can be, in many cases, reclaimed and brought under the plough with considerable ease. The mode of operation is much the same as that practised on the fens; but the ap1.ication of large quantities of hot lime is absolutely necessarv. The method of cultivation is also nearly similar, except that cole is generally omitted, oats being more commonly used for the two following crops after brealing up, and rye being not unfrequently sown. This land, when brought to a state of sufficient consistence, and particularly if it be mixed

* See the Surveys of Lincolnsh. and Cambridgesh., chaps. iv. sects. 3; that of Hunting. diunsh., chap. vii. sect. $\frac{1}{4}$; the Farmer's Magazine, vol. v. p. 290, and vol. i. chap. xxiv. 1. 466 of this work.

† See the Surveys of Dumfriesh, and Ayrsh, chaps, xi. sects, 2. 
with sand, is capable of growing every species of green crop, and we may therefore refer on that subject to the rotations on light soils. $\mathrm{O}_{\mathrm{i}}$ moss of that nature, however, short courses are considered the best: the first to consist of only two crops, to reduce the earth completely, and after that, two, or at most three years' pasture. The reasons assigned for which are these:-If laboured more than two seasons together, after the first breaking up, the soil gets too dry, loose-or deaf, as the country people call it-heaves, throws out the corn, and produces the dead-nettle and other weeds; and if allowed to be more than two years or so in pasture, the rich grasses die, and the coarse herbage rises; but if only one, or at most two crops of grain are taken in each rotation, the soil is still kejt in a soft, moist state, and the grasses, by being so often manured, contintio sweet, succulent, and vigorous. The first crop of outs is seldom weiglity ; the second is however better; and during the after rotations, the grain is generally found to be of good quality *.

Flow-moss constitutes that species of soil which in Ireland is termel $\mathrm{bog}$, the first step towards the improvement of which is the drainage of the land; for nothing can be effectually done towards bringing it into cultivation, until the surface be relieved of stagnant water. Of that we have already treated t; and when brought into a state to bear the tread of cattle and the operation of the plough, its management is the same as that of bent-moss; except that, being more tender, it is better to convert it as soon as possible into permanent pasture.

We have thus stated nearly all the settled rotations most commonly uractised on the various soils of Lngland and Scotland; but we lave not adverted to Ireland; because in that country-with the exception of some men of fortune and a few wealthy farmers, who cultivate their land wit! spirit; with that, also, of the baronies of Forth and Bargy, in the county of Wexfordt; and of a small portion of the province of Ulster, where the Scottish mode prevails-nothing like what may be properly termed a system of husbandry, upon any fixed plan, is linown. We have looked through all the county surveys, and have read attentively most of the publications which have appeared of late years respecting the agriculture of the island; but

* Survey of Ayrshire, p. 346.

+ See rol, i. chap. xxvii. 1. 445, and chap. xxx.; also the Survey of Ayrsh. and Dumfriesh., chap, xi. sect. 3.

$\ddagger$ The soil of this district is, for the most part, a retentive clay, except near the seacoast, where it becomes a siliceous loam, to which the long and uninterrupted application of sea-weed and calcareous sand has, in a grent measure, contributed; and the chief merit of its agriculture seems to be the systematic rotation which is uniformly observed, and which, although with some individuals it may occasionally vary, is nevertheless in general as follows :-

1st. Wheat upon land-either lea or stubble as may happen-marled at the rate of 1000 load, of 5 cwt. each per Irish acre.

2nd. Barley or oats, but chiefly barley.

3rd. Drilled potatoes with dung, or beans sown broad-cast with composts of earth, seasand, dung, or sea-weed.

4th. Barley with red clover and rye-grass.

5th. Clover fed off, or mowed; or the second growth saved for seed.

6 th. Wheat, upon which they chiefly rely as their surest crop.

In some cases they feed off the clover very early, and marl the lea previously to sowing the wheat, but often sow it without marl, and apply dung to the wheat stubble for potatues, or manure it with a compost for beans, and so proceed to barley and clover arain. Notwithstanding this scourging course, yet, we are told, the fertility of the soil, with the abundance of manure, produces such abundant crops that, in districts near the sea, seldom less than twenty barrels of barley, of 16 stone each, are grown on the Irish, or twelve and a half per English acre, and the others in proportion.-See Observatiuns on the Baronies in the Quart, Jour. of Agric. for March, 1833, No. XX. 
both from these, and from information personally received from persons well acquainted with it, the cultivation is described as being generally confined to the continued production of potatoes and grain, with some patches of flax; and the land, after being thus rendererl foul and exhausted, is suffered to recruit itself as best it may, by being left for a series of years under natural pasture.

Although possessing a more genial climate, and more favoured in soil than almost any other part of the Cnited Kingdom, consequently better adapted to the growth of every crop which can be produced in this quarter of Europe; and although, from the redundancy of the population, labour is below its value, yet the produce of the land is inferior. It is, indeed, extensively under the plough, and, from the large exportations of corn and cattle, it might be supposel that its husbandry must be flourishing; but, when compared with that of this country, it is in a very backward state. This, however, camnot be entirely attributed to the farmers, who are neither wanting in industry nor shrewdness; but their holdings are almost invariably small, they are deficient in capital, and they meet with but little encourage-ment from their landlords. The great estates are chiefly in the hands of absentees, who generally entrust the managrement to attorneys, who are more careful of the collection of the rent than the cultivation of the land; though, were the latter better attended to, both might be materially improved.

\section{SUMMARY.}

The various rotations which we have enumerated are generally confined, it will be observed, to single courses; but, in repeating them, although the same corn may in most cases be again grown, yet the green crops should be frequently changed, for they admit a greater variety, and, even supposing the land to be in every respect well cultivated, it tires of some of them more readily than it does of grain. Thus turnips and clover, when reiterated, are often known to fail, and although the Norfolk system, which is generally described as consisting of a repetition of the same seeds, has been recommended as constituting the best principles of cropping, yet a change is sometimes necessary; therefore, were those crops sown only once in eight years instead of four, with the substitution on very light soils, of rye for one of the crops of wheat, it would, no doubt, be found more advantageous.

The situation of the land, in point of aspect and elevation, must also be considered, as well as the nature of the soil; for climate is a point of the first importance in the choice of all crops. 'Thus although, generally speaking, the south of England has the advantage in respect of climate, yet finer oats and weightier crops of turnips are grown in the north: from the usual lateness of the season, too, wheat is there not only sown when the winter is far advanced, but frequently in the spring, and generally along with clover; whereas, in the south, wheat is usually got in before the close of autumn, and clover almost always fullows barley or oats. Lucerne, which forms a valuable grass, during several vears, on our light and sandy soils, camnot be grown with advantage in the cold of Scotland. Sainfoin, which is extensively grown on the chalky soils of this country, is also there unknown; and, doubtless from the deficiency which there exists of that peculiar quality of land, their permanent meadows are inferior to ours; while in Ireland, both from the land lying generally upon a limestome bottom, and from the mildness as well as humidity of the climate, the pasture is in most places greatly superior. 
Although the principles upon which the alternate system of husbandry rest are perfectly clear, yet the specific succession of the particular crops is an object of considerable perplexity, and, until ascertained by the experience of their effect upon different soils, must occasion no small degree of anxiety and embarrassment; for it should combine the various considerations which regard the best cultivation of the soil, both as it respects the preservation of its fertility, for the production of the present and succeeding crops, the state of the markets, and the reparment of the farmer's cost and toil. It has indeed been justly observed by a good judge of soils, "that no general principles can be laid down respecting the comparative merit of the different systems of cultivation, and the various systems of crops adopted to different districts, unless the nature of the land, and the physical circumstimces to which it is exposed, are fully known. Stiff coherent soils are those most benefited by minute division and aëration; and in the drill system of husbandry, these effects are produced to the greatest extent; but still the labour and expense connected with its application in certain districts may not be compensated for by the advantages produced. Moist climates are best fitted for raising the artificial grasses, oats, and broadleaved crops; stiff aluminous soils, in general, are most adapted for wheat crops, and calcareous soils produce excellent sainfoin and clover"." The course of cropping may, therefore, be said to require a considerable share of judgment; but, if once established, "it remedies all confusion, distinguishes and arranges the season and the appropriate mode of work, economizes labour, reciprocates the improvement of both soil and stock, and forwardis the interest of both landlord and tenant.$+ "$

In laving down a plan of management, it is often found impracticable to have the whole of the land subjected to the same rotation. Sume parts of almost every farm of moderate estent vary in the nature of the soil : part, perhaps, being elay, gravel, or sand, some good, some indifferent, and the remainder poor; each must, therefore, be worked in the manner most suitable to its respective properties; but were they all of equal quality, still, to follow the same course regularly-though seemingly very practicable on paper-is not always in the power of man. Unfavourable seasons, the state of markets, and other contingencies, sufficientiy obvious, will sometimes defeat his most earnest endeavours, and force him to have recourse to measures which are contrary to his better reason. Added to this, farmers have frequently to struggle with the difficulties of inadequate capital, which places them under a strong and tempting necessity to prefer a small present advantage to a greater but more distant benefit, which must be purchased at an immediate expense, which they can little afford to defray; and thus compels them to use means for rendering their land productive for the time, though eventually injurious to them. Men of judgment as well as capital, when left to the exercise of their own discretion, will indeed be at no loss to accommodate themselves to such exigencies; for the general managrement of a farm ought to be considered rather as a rule, the principles of which are never to be entirely lost sight of, than as one which should be always servilely obeyed. It seems, however, to be the opinion of some proprietors of estates, that a proper course of cropping may be laid down for the tenant and entered in his lease; but the idea is wrong in principle, and in point of practice can hardly fail to disappoint the hopes of those who entertain it, as being inapplicable to circumstances which are in

* Sir Humphry Davy's Lectures on Agricuitural Chemistry.

+ Radeliff, on the Agric. of E. and W. Flanders, 1. 58. 
their nature liable to vary. No landholder, or his agent for him, can be supposed capable of arranging fixed rules with such accuracy as to meet incidents so numerous, uncertain, and remote; nor can any such plan ba carried properly into execution, unless it coincide with the interest as well as the skill and means of the tenant. The general principle of the rotations which are deemed most suitable to the land, may indeed be agreed upon, and a watchful eye may be kept upon his proceedings; but, if he be a man of intelligence and integrity, they should be liberally construed; for the interest of both parties is the same, and if that of one be considered in preference to the other, both will surely suffer.

In the following chapter we purpose giving a cursory view of the component parts of some soils, and their adaptation to various rotations, which we have extracted from the works of eminent agriculturists, as being, in our opinion, the most able summary of cultivation with which we are acquainted; for the plans there recommended are founded upon acute observation resting upon mature experience; and, although the part which we have taken from Von Thaër is calculated for the north of Germany, yet neither the climate nor the husbandry are materially different from our own: we therefore trust that it will be found to throw some additional light on the obscurity in which the subject of vegetation is still shrouded.

In doing this, however, we have omitted all further notice, than the slight mention which we have already made, of the interesting experiments recorded by De Candolle and Macaire upon the excretory powers of plants ; for although they seem to have ascertained that matter is exuded from the roots, the nature of which varies according to the families of the vegetables that produce them; and the facts which they have stated will tend, if confirmed, to enlarge our knowledge of the principles of vegetation, and to simplify the regulation of the course of cropping; yet the discussion of the subject, in its present crude shape, belongs more properly to naturalists than to farmers.

\section{Chapter VIII.}

\section{ON SOILS AND COURSES OF CROPPING.}

The notation of crops, or, in other words, those species of plants and modes of culture which best maintain the soil in a state of fertility, and return the most abundant produce, has long been a disputed question among naturalists, and probably will remain so; for the inquiry has been founded upon experiments made upon soils, and under climates and circumstances of a variable nature. We have numerous accounts of prodigious returns from peculiar modes of cultivation; but there are many reasons why all trials which have been only casually made, and not followed up through a series of years in the ordinary method of field-tillage, should be received with distrust.

First, they are generally made with a view to establish some peculiar theory, or to try the effect of some novel idea, which, being a favourite speculation, is pursued with all the bias of partiality, and recorded with a degree of favour from which the most candid statenients are rarely exempt.

Secondly, they are usually conducted on a small scale, on ground peculiarly adipted to the purpose, and attended to with a degree of care that 
cannot be extended to an entire farm; and, after all this, when the produce is reaped and accurately weighed or measured, the result is calculated according to the exact proportion between the size of the plot on which the experiment was tried, and the square contents of an acre; though every farmer knows that a material portion of every crop is wasted in the harvesting, and that-what with that loss, and the space occupied by headlands and fences-a ten-acre field will hardly yield more than the produce of eight acres estimated in the manner stated.

Thirdly, even supposing those objections to be obviated-the experiment to be conducted without prejudice, and managed by an intelligent husbandman on the common field plan-still there are so many circumstances of season, soil, and culture, which operate differently at difierent times, without any assignable cause, that what prospers this year fails the next, and what succeeds with one man does not thrive with another.

Calculations of produce are thus often very fallacious; and however correct in one situation, are frequently altogether inapplicable to others. Nothing but a series of experiments, and an average of results during many successive years, ought therefore to be considered as decisive of any new practice. Even if found productive, the question may then be fairly asked, at what expense was the produce obtained? And the farmer will find it peculiarly prudent to look with attention to both sides of the account. The only safe guide is, therefore, to be sought in the practical effects of long continued experience; but when these coincide with the theories of naturalists, they may be safely regarded, if not as unerring rules, at least as being entitled to a considerable share of confidence.

\section{SOILS.}

The amnexed table has been given by Von 'Thaër' as a classification of soils, which we deem entitled to particular attention, as in some degree governing the course of crops ; for, although the real value of every rotation depends, in a great measure, "pon the manner in which its several processes are executed; yet, abstractedly speaking, some courses must be viewed as better than others, because the crops may be more suitable to the peculiar qualities of the land on which they are to be grown. The last column in the table comprises an analysis of the comparative value of the different component parts, and is the result of many years' careful examination of the soils, as well as of the proceeds of the crops, after the deduction of labour and seed.

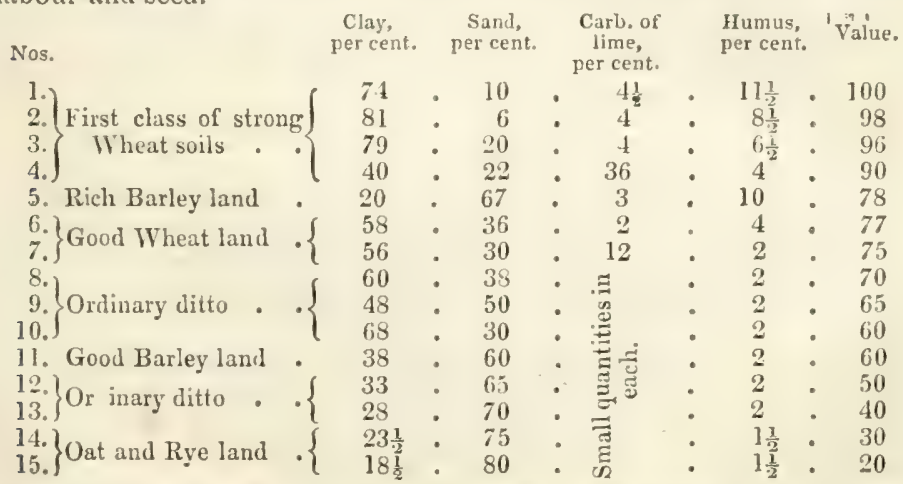

Nos. 1, 2, and 3 are alluvial soils of the richest quality ; and, from the large portion which they contain of vegetative mould, or humus, and the 
intimate state of mixture in which it is found, they are not so stiff as the quantity of clay which they contain would seem to indicate.

No. 4 is a fine clay loam, such as is found in many parts of the United Kingdom; and, although what may be called strong land, is yet of a texture which renders it easy to be worked, and consequently not difficult to be kept in heart if properly managed.

No. 5 contains such large proportions of sand and humus mixed with the clay, as to peculiarly adapt it to the growth of barley and green crops; it is consequently evidently suitable to the altermate system of husbandry.

Nos. 6 and 7 are both good soils-the quantity of carbonate of lime in No. 7, compensating, in a great degree, for the smaller portion of humus; but necessarily requiring the addition of a proportionate quantity of dung to supply that deficiency.

Nos. 9 to 13, although soils of the fair averarre quality of common tillage land, yet, from the want of carbunate of lime, require the addition of lime or marl.

Nos. 14 and 15 are evidently light sands, which, though sometimes brought into cultivation, and even rendered productive through the application of manure and judicious management, yet can rarely be kept in heart without the aid of a folding flock; and land of a poorer quality should be invariably kept in sheep pasture.

Throughout the entire linglom there are but few districts the soil of which contains more than perhaps five jer cent. of humus; but if the staple of the land be loamy, even two per cent. will render it fit for the production of grain. The friability of its texture is, indeed, an object of the first importance, for it occasions a great saving of labour and expense; and if the ground be tolerably well managed, and dunged, it can be always maintained in good heart*

The analysis which has been thus made of those various soils, it should, however, be observed, extends only through the surface to the depth of six inches, without noticing the subsoil, which may greatly affect their value; for, however rich they may be, if that be either too porous, or too retentive, they may be rendered, in certain seasons, unproductive. If, however, they contain a layer of from nine inches to a foot of good earth, the subsoil may then be considered of little consequence; though a limestone bottom will always command a preference. The exposure, with regard to the sun, and the situation of the land, in respect to its shelter from cold winds, are also, as we have already had occasion to observe, objects of such extreme importance, that they may make a difference in point of climate equal to several degrees of latitude; they should, therefore, never be lost sight of in estimating the value of the ground.

\section{COURSES.}

The rotations which have been recommended in the Annual Report of Scotland, as well as by Brown of Markle, both upon his own experience and that of many other eminent farmers, and which are very generally adopted by men of intelligence, on soils of different descriptions, are some

* By loamy land is to be understood any of the earths combined with decayed animal or vegetable natter. Thus, it is termed "clayey," or "sandy loam," when the greatest proportion of the earth consists of either clay or sand: rich black and brown clayey loams being' according to the proportions in which sand and clay are mixed with the vergetative mould ; the clay being, however, greatly divided, and in smaller quantity than the sand. Light sandy loams are varieties of the same nature. Sir H. Davy's Lect. on Agric, Chem. App. lx. 
of them, as follows $*$ - It being borne in mind that he considers the basis. of each to be either a bare summer fallow, or a fallow on which drilled turnips are cultivated, and its conclusion to be with the crop taken in the year preceding a return of fallow, or turnips ; when, of course, a new rotation commences, unless the land should be then laid down to grass.

No. I.

Being intended for deep strong lands, for which it is alone calculated, begins with a summer fallow, and is carried on alternately with-

\section{Wheat; and 2. Beans,}

or the occasional substitution of clover and rye-grass for the latter. On this plan, provided the bean crop be drilled and attentively horse-hoed, as well as abundantly manured, Mr. Brown is of opinion that the same course may go on for any length of time, while the land remains clean; and will probably turn out one of the most profitable rotations that can be exercised. Of that, indeed, there can be no doubt, so long as the ground will support such cropping; but in reference to what has been stated on this subject in a former Chapter, we think it a dangerous system to be continually pursued, and that an alternate change of barley or oats for wheat would be found more advantageous.

Every soil which is intermediate between absolute clay and sand, or gravel, has obtained the name of loam; and as loamy clays may be rankerl, so far as regards the course of crops, under the head of the best description of clay soils, they may be cropued under nearly the same principles as those applied to light ground, from which they differ only in their degrees of quality. Rich free loan is unquestionably the most profitable description of land, as it is of easy cultivation, and almost uniformly produces excellent crops. The minutice of its management depend, however, much on the nature of the subsoil: if that be retentive, the surface will require to be cleaned from root-weeds once in a rotation of six or eight years, by means of a summer fallow; whereas, if it be incumbent upon a porous bottom, an effectual cleausing may be given liy a drilled crop of turnips.

\section{No. II.}

Lpon rich clay loams, of a less strong quality than the former, or where it may not be deemed advisable to carry the first rotation into execution, the following may be practised; according to which the labour will be more equally divided, and the different species of grain being alternately sown, will be found a safer course: thus-

1. Fallow, or Turnips, dunged.

2. Wheat.

5. Clover and Rye-Grass.

6. Oats or Wheat.

3. Beans, drilled and horse-hoed.

4. Barley.

7. Beans, drilled and horse-hoed.

s. Wheat.

This rotation Mr. Brown states as excellently calculated to ensure an abundant return throughout the whole of it, "provided dung is administered upon the clover stubble; without which supply the rotation would be criptled, and inferior crops of course produced in the concluding years." On the necessity of a second application of dung during the course, we entirely agree with Mr. Brown; but not in his opinion of the propriety of laying it upon the clover stubble, particularly if that crop be followed by oats. The clover, we think, would be more beneficially improved by a dressing of ashes, or gypsum; and the second coat of dung better applied

* See the General Report of Scotland, rol. i. part 3; aud Brown's Treatise on Agriculture and Rural Affairs, vol, i, chap. viii. 
to a second crop of beans. Thus we should, in preference, advise whent instead of barley at No. 4 ; then beans, dunged; and followed by barley, clover, and oats; or, perhaps still better, the Essex course of allowing barley and clover to be the crops immediately succeeding the fallow. Many intelligent farmers, indeed, are of opinion that it would be more profitable to stop at the sixth crop, and to make it wheat instead of either barley or oats; thus recommencing the course with a fallow, or with turnips. Mr. Russell, of Kenilworth, who has lately written on the subject, however, recommends the following course upon good wheat and bean land, namely-

1. Wheat on a Clover ley.

5. Wheat.

2. Tares.

6. Tares.

3. Barley.

4. Beans.

7. Barley.

8. Clover.

It will, however, not escape observation, that he excludes the fallow; that he puts tares in preference to turnips, both on the presumption that it is equal in profit to a crop of common turnips, and the subsequent barley produces a better crop after the grass than after the turnips *; for clover is almost sure to succeed after a well-manarged fallow; but, if it fails, so, in a great measure,will the following crops.

No. III.

Is calculated for clays and loams of an inferior description to those already treated of ; and consists of-

1. Fallow, with dung.

2. Wheat.

3. Clover and Rye-Grass.
4. Oats.

5. Beans, drilled and horse-hoed.

6. Wheat.

According to this rotation the rules of good husbandry are strictly adliered to ; it is, indeed, one of the best courses that can be levised for soils of that description; and if the land be well drained, as well as carefully tilled, it will produce excellent crops, and may be kept without the recurrence of a fallow for some time longer than the rotation above stated, but the land must be, in either case, well dunged.

Instead of this course, however, another rotation is sometimes followed; which beginning, as in the former, with a bare fallow and wheat, is then carried on with grass for two years, after which oats and wheat are successively taken. This system of cross-cropping, though contrary to the general principles of good husbandry, may perhaps be justifiable in practice on strong soils and in certain climates: its supporters maintain that beans are an uncertain crop, and cultivated at considerable expense, and that in no other way can corn be grown in equal quantity, and of equal ralue, at so little cost. On cold thin lands it may also be not unfrequently found tire surest mode of repaying the charges of cultivation.

\section{No. IV.}

Light, calcareous, or gravelly soils, are very generally managed according to the Norfolk system, which has been already described; but it may be doubted whether its continuance for any great length of time is advisable, as both turnips and clover are found to fall off when repeated so often as once in four years. Perhaps, therefore, the course might be improved by extending it, as we have formerly remarked, to eight years, either under the following rotation, as advised by . Mr. Brown; for instance, to sow the seeds for pasture in the second year, and keep the ground three years under grass : thus, -

\footnotetext{
* Treatise on Practical and Chemical Agriculture, p. 92, 103, and 317.
} 
1. 'Turnips:

2. Barley.

3. Seeds, under pasture during three years.
4. Oats.

5. Drilled beans and peas.

6. Wheat.

Or that recommended by Mr. Russell, which consists of-
1. Wheat on a clover ley.
2. Sivedish turnips.
5. Barley alone.
3. Barley and stubble-turnips.
4. Peas and ditto.
6. Tares and brush-turnips.
7. Barley, as above.
8. Clover.

by means of which the land would be kept fresh, and in good condition; while the management, besides being agreeable to the soil, would considerably reduce the labour of tillage.

No. V.

Sandy soils, however, though well adapted to the above system, are not favourable to the growth of wheat, unless they contain a large portion of clay, or receive dressings of alluvial compost, marl, or some substances which will impart to them that body and strength which they do not naturally possess. Barley, oats, and rye-the latter especially-are however sure crops on such land; and if rye were more generally sown upon it, instead of wheat, it would be, in most cases, found to return a better profit.

The courses adopted by Von Thaër on the royal farm of Moëgelin, in Prussia, on sandy loam of the quality of good barley land, are:-
1. Hoed root crops dunged.
2. Barley.
3. Clover, for tivo or three
4. years, but broken
๖. up early.
6. Cole-seed.
7. Wheat.

8. Peas and tares.

9. Rye.

10. Tares cut green, or drilled and dunged beans and turnips.

11. Barley, or rye.

12. Rye, or oats.

The culture of cole-seed should, however, not be attempted unless there be abundance of manure; without which, a green crop should be substituted for No. 6. In these rotations he gives the preference to winter corn, as their produce is more certain, and they not only furnish a greater quantity of straw, but the sowing is prepared with greater ease : but if that cannot be accomplished, then spring corn is sown. When drilled beans are planted, turnips are sown after the last hoeing, and are followed by barley: but in some instances tares are sown and cut green, in which case they are followed by rye, and then oats.

On poor sands he recommends-
1. Buck Wheat.
2. Rye.
3. Hoed root crops, dunged.
4. Oats.
5. Spurry.
6. Rye.

The land to be then laid down with seeds for at least three years in pasture*.

No. VI.

Is adapted to cold thin clays, and the course may be shortened or lengthcned according to circumstances, but should never extend further, in point of ploughing, than when dung can be given to the fallow break; for this is the key-stone of the whole, and, if it be neglected, the land will become exhausted, and the rotation rendered unequal to the expense of cultivation. Soils of this description do not improve much in grass, unless laid down for

* Principes raisonnés d'Agric. $2 d$ Edit. tom. i. § 395. 
a very long time in permanent pasture; but if an additional quantity of manure cannot be procured, and beyond that which is usually made upon such farms, they must be refreshed in that way, although the produce may in the mean time be of comparatively small value. The following rotation has been recommended :-
1. Fallow, dunged.
2. Wheat.
4. Pasture.
3. Seeds.
5. Ditto.
6. Oats.

though, if a sufficient dressing of dung or compost could be laid on onec in five instead of six years, we have no doubt that the land would be more improved, and a better return be obtained from the crops, were it broken up after lying two years, instead of three, in grass.

The feregoing courses are chiefly intended for the general run of lowland soils, without reference to the fens-to the peculiar management of which we have already alverted; or to that of peat-earth, which, when brought into cultivation, may be considered under the greneral denomination of light land. In upland districts, however, where the soil is dry, and the climate not too severe for the production of wheat, Mr. Brown recommends the following rotation:-

\section{No. VII.}

1. Turnips, drilled and dunged.

2. Barley, with red clover and rye-gras.

3. Grass, to be used in soiling, or for hay.

4. Wheat.

The land to be ploughed, if possible, by the end of September for wheat, and to receive a slight dressing of dung if it can be procured; though, according to our opinion, we should prefer applying the dung to the following crop of peas; for which two furrows, one before winter, and the other at seed-lime, may be given.

If, however, the situation be so elevated as to render the growth of wheat hazardous, a system has been successfully practised by other eminent farmers which consists in
1. Turnips.
2. Bear, or bigg.
3. Clover.
4. Red oats.

5. Peas.

6. Barley, with seeds.

7,8 , and 9 , Pasture.

10. Oats.

being the species best calculated for such high land. Instead of the tworowed barley, the fourorowed, or that inferior sort called bear, or bigg, should always be preferred in unfavourable climates: but then in elevations so great as to cause other crops to fail, rye has been known to prosper *; or, if the land lies at a great distance from the dunghill, a crop of cole can be taken, and fed off, previous to the turnips, and the clover may be mixed with other seeds, and allowed to stand for two or more years.

As many parts of the upland districts are, however, too wet for turnips, a different course, he conceives, may be successfully practised in places ineligible for the turnip husbandry: as thus-

No. VIII.

1. Fallow, with dung.

2. Wheat early sown.

3. Clover and Rye-grass, for hay

4. Oats. or soiling.

5. Tares, or any of the varieties of

grey peas, with a dressing of dung or compost.

6. Barley, with seeds.

7, S, and 9. Pasture.

10. Oats.

* Sinclair's Code of Agric., 3rd, edit. p. 461. 
In all these humid and elevated situations, tillage is however a dangerous speculation, and can only be considered advisable in so far as it conduces to the production of turnips and hay, to create manure, with a view to the permanent improvement of the soil for pasture; for the crops of grain, which necessarily form part of the rotation, can never be depended on for profit, any further than as they are useful as litter and forder for the cattle.

The most eligible rotation for every kind of soil may thus be generally ascertained by reference to the local circumstances of the district in which it is situated, and may be either long or short in a direct proportion to the richness of the soil on which it is to be executed; and, although we have ouly enumerated a few of the grain crops which are commonly cultivated, yet they may be all inciscriminately crown, provided they be not too frequently repeated, nor allowed to jerfect their seeds. We have, indeed, lately seen an account of the cultivation of a farm in the nortl of Germany, comprising nearly two thousand acres of tillage land, which is rent. larly arranged under a double course of twelve years, each ending with three years pasturage, yet bearing different crops during each, so as to present only one rotation of twenty-four years*; and Mr. Maxwell of Fletton, in Huntinglonshire, an experienced farmer, who furnished a report of the agriculture of the county, recommends the following course as a general basis of management to be adopted upon every kind of soil, except the fens; also stating, in corroboration, some extraordinary instances of its success upon strong land.

\section{No. IX.}

1st. Clean deep winter and spring ploughing for a vegetable crop of whatever kind is best suited to the soil; as turnips, maugel-wurzel, tares, or cole-seed: this to be hoed, but not to stand for seed.

2nd. A crop of white corn, of whatever kind, is best suited to the soil: and with this, clover-20lbs. of seed per acre t.

3rd. Clover, either grazed or mown, but not to stand for seed.

4th. Beans, sheep-fed, and hoed.

5th. A crop of white corn suited to the soil.

Thus, instead of cole-seeri, any other vegetable; instead of barley any other white corn; instead of clover, any other grass-seed; instead of beans, any other pulse or ameliorating crop ; and instead of barley again, any other species of corn may be used, while a small portion of either division may be set a jart for supplying such seeds as, from their exhausting nature, are not admitted for general cultivation $\ddagger$.

* The rotation is as follows :-
1. Cole-seed, well dunged.
2. Autumn sown grain.
3. Putatoes, dunged,
4. Barley.
5. Clover, mown.
6. Ditto, Ditto.
7. Autumn sown grain.
8. Peas and Tares.
$\begin{array}{ll}\text { 9. Autumn sown grain. } & \text { 21. Autumn sown grain, } \\ \text { 10, 11, and 12. Clover and pasture. } & 22,23 \text {, and 21. Clover and pasture. }\end{array}$
13. Oats.
14. Beans well dunged.
15. Autumn sown grain.
16. Clover, mown.
17. Autumn sown grain。
18. Tares cut green, and turnips, dunged.
19. Spring corn.
20. Peas.
21. Autumn sown grain.
22, 23, and 24. Clover and pasture.

The autumn sown grain is either wheat or rye.

$\dagger$ In Flanders, where red clover is very extensively grown, and the seed largely exported, the quantity of seed sown is only 6lhs. [er English acre-Dadcliff's Agric. of E. and W. Flanilers, p. 61. Here the usual proportion is almost 12lbs, on dry. soils, and from 14 to 18 on strong loam or clay; though different quautities are sown when intended to produce seed, hay, or pasture.

$\ddagger$ Parkinson's Survey of Huntingdonshire, p. 108. 
These rotations, it will be seen, are all calculated for land that is already under the plough; but where it is in an uncultivated state, it is evident that none of these can be successfully practised; therefore, in addition to what we have said in the preceding chapter on the subject of fen-lands, the following measures may be pursued:-

First, If the ground be not pared and burned, to break it up with a deep furrow during the winter, or early in the spring months; sparing neither harrow nor roller, and using the grubber sufficiently in the first year, so as to rot and destroy the roots and the surface grasses.

Secondly, Upon the summer fallow, to lay a dressing of five or six chaldrons of lime, plougher in as hot as possible; and in the following spring, to commence whatever course may afterwards be deemed prudent with a crop of oats, followed by turnips, dunged.

If the land be naturally dry, or well drained, and of a good staple, this mode of procedure will bring it into a state for the regular continuation of the alternate husbandry; provided it be sufficiently light to carry turnips to be eaten upon the ground. If it has been pared and burned, the lime may be dispensed with, as the ashes will afford a good dressing, and two crops of oats are not unfrequently taken in succession, without a fallow, in order to completely rot out the matted grass roots. The soil should however be of good quality, and should be supported by the regular application of dung. To this it may be also added, that although farmers well know "Mluck to be the mother of the meal-chest," yet if the ground be not worked well, with judgment, and at proper seasons, the object of successful cultivation cannot be attained by the mere intermixture of green crops with those of grain.

The alternate system of cropping being founded upon the basis of providing food for both man and beast, may be carried on either by allowing a portion of the land to rest during each course for more than one year, under natural or artificial grasses, upon which the stock are pastured, while another part-which had been previously laid down-is again broken up; or, by annually changing the crops of grain and roots, and soiling the cattle in the yards. The former is best adapted to light soils and large holdings, upon which flocks of sheep are kept, whereas, on smaller farms, and stronger land, the latter is found to be the most profitable plan; but it absorbs proportionably more capital, and demands a greater share of attention: this, however, shall be more particularly adverted to when we treat of soiling.

The great object of the latter being to produce large quantities of dung, nearly the whole of the straw should be converted into litter, by which, and the consumption of the green crops by the stock, such an amount of farmyard manure may be raised as will not only preserve the land in good heart, but ensure its gradual improvement. Either system may however be profitably adopted upon land capable of growing good crops of turnips. This is perhaps the only crop which, upon such soils, can neither be overploughed nor overdunged. On very light land it may indeed be thought that ploughing may be carried so far as to render it too tender, and consequently hurtful to the after-crops: but on soils of that nature, the turnips should always be eaten off by sheep upon the ground, and their treading will bring it to a proper consistence.

\section{EXPENSES.}

The produce, charges, and surplus balance of profits upon farming, depend 
upon so many circumstances of an uncertain nature, that no statement, lowever accurately represented, can be regarded in any other light than as an approximation to the trutls. The experience of farmers, their constitutional energies and intelligence, vary as much as their persons; and, although these are important to the success of the business, yet their effect cannot be estimated. Neither can details, which apply specifically to particular cases, be assumed as applicable to the general course of husbandry. We, therefore, regard the numerous accounts which have been published rather with curiosity than confidence; and for that reason, we decline assuming any of those which have been transmitted to us by private individuals, or which have been inserted in the county reports; but, as the subject of labour and expenses may interest many persons who are only partially acquainted with agriculture, we transcribe the following brief calculations, founded upon the rotations Nos. II. and IV. which we have taken from Mr. Russell's late work, and which, allowing for differences in the market prices of corn, come as near the truth as most of those which we have examined: with this observation, however, - that he has rum into the general error of agricultural writers, in making no allowance for unfavourable seasons, or for losses which neither caution nor judgment can avert, and has thus placed the gross profits of the farmer at a rate considerably above par.

\section{EIGHTY ACRES OF GOOD WHFAT AND BEAN SORL.}

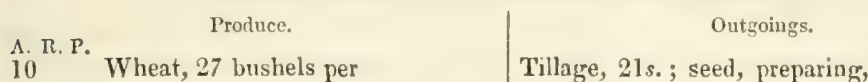
acre, at $60 \mathrm{~s}$. per qr. $£ 10150$ Tares, at $105 \mathrm{~s}$, per acre $52 \quad 10 \quad 0$

Barley, 40 bushels per acre, at $32 \mathrm{~s}$. per yr.

$80 \quad 0 \quad 0$

Beans, 30 bushels per acre, at 40 s. per qr.

$\begin{array}{lll}75 & 0 & 0\end{array}$

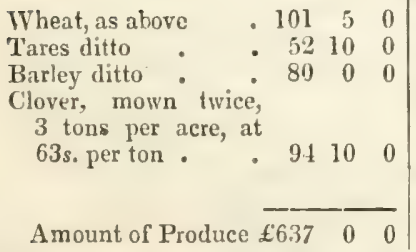
and sowing, 23s.; reaping and harvesting, 16s. $6 d$.; stacking, thrashing, and marketiug, 22s.6d. per acre. $£ 41 \quad 150$

Tillage, 21 s.; seed and drilling, 18s.; soiling, 5s. per acre. 22000 Preparing and carting dung from two crops of straw

Tillage, equal to five ploughings, $70 \mathrm{~s}$; seed, \&c. $16 \mathrm{~s}$; harvesting, 12s. $6 d_{\text {. }}$; taking in, thrashing, and marketing, $20 s$. per acre - .

Tillage, $21 s$, ; seed, $16 s .6 d_{\text {s; }}$ planting and hoeing, 16 s.; harvesting, 16s.; taking in, thrashing, and marketing, $15 s$. per acre - $\quad$ • $42 \quad 5 \quad 0$

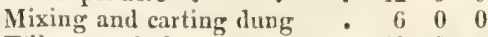
Tillage, as before $\quad$ : 41150 Ditto, ditto - . $\quad 2200$

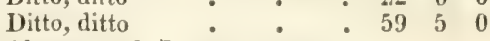
Clover seed, 7s. 6d. per acre; harvesting 30 tons of hay at 7s. $6 d$. per ton; purchased manure, 35\%"

$\frac{50}{50} \quad 0 \quad 0$

The cost of wear and tear being included in the charges for labour,

* The kind of manure used is not stated; were it gypsum or ashes, it could be obtained at much less expense, ard would probably be found at least as effectual, if not more so in its improvement of the clover, as the application of dung, see Vol. I. p. 323, and 336 . But the clover seed is under the usual charge, and the cost of harvesting hay far below it. 
would leave a balance, exclusive of profit on the feeding of stock, of $286 l$. 15s. for the discharge of rent, tithes, and parochial assessments. This may appear large; but, upon land of that description, these charges may be fairly estimated at full forty shillings per acre, and the residue would only afford a living profit to the farmer, including the interest of his capital, even were the prices of wheat to be maintained, and were he secured against accidents, or the chance of bad crops.

\section{PIFTY ACRES OF GOOD TURNIP AND BARLEY SOIL.}

\section{Produce.}

61 Wheat, 27 bushels per acre, at $60 \mathrm{~s}$, per qur.

Rye or stubble turnips, $\begin{array}{lllll}33 s \text {. per acre } & \text { • } & 10 & 6 & 3\end{array}$ 61 Siwedes, at 100 s. per

61 Barley, 40 bushels per acre, at $32 \mathrm{~s}$. per qr.

Rye or stubble turnips, at $33 s$. per acre

61 Peas 28 bushels per acre, at 40 s. per qr.

Brush turnips, at 35 s. per acre . .

61 Barley, 40 bushels at 40 s. jer yr.

61 Tares, at 105 s. per acre

Turnips at $70 s$, per acre

61 Barley as above ..

61 Clover, mown twice, 52 civt. per acre, at 63 s. per to

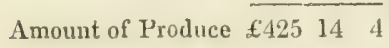

\section{Outgoings.}

'Tillage, 18s.; seed and drilling, 23s. $6 l_{\text {. }}$; reaping, harvesting, \&c. 16s. 6d.; stacking, thrashing, and marketing, IIs. prer alre. food, or rye, or stubble turmips, at 19s. per acre

Fallow forturnips, $60 \mathrm{~s}$. ; mixing and carting dung, $12 s$; seed, sowing, and hoeing, 14s. per acre Tilage, equal to three plough-

Tillage, equal to three plough-
iners, $36 s$; seed and drilling, 16 s.; harvesting, 12s. 6d.; thrashing and marketing, 20s. . . . .

Tillage, seed, and sowing of stubble turnips, or rye, $19 \mathrm{~s}$.

Tillage, $36 s$; mixing and carting dung, 12s.; seeding and drilling, 18s.; hoeing, 10s. $6 \%$.; harvesting, 12s.; thrashing, 12s. per acre .

Tillage, seed, and sowing of turnips, 19s. .

Tillage, as before

$\begin{array}{lll}5 & 18 & 9\end{array}$

$\begin{array}{lll}26 & 8 & 1 \frac{1}{2}\end{array}$

Tillage of tares, $18 \mathrm{~s}$.; seed and sowing, $18 s$.; soiling, $5 \mathrm{~s}$. perace : : $36 s^{\circ}$; seed $12 \quad 16 \quad 3$

Tillage for turnips, $36 s_{0}$; seed,
drilling and hoeing, $10 \mathrm{~s} .6 l_{\bullet}$; carting dung, 12s, per acre $1857 \frac{1}{2}$ Tillage, as before $\quad . \quad 26 \quad 8 \quad 1 \frac{1}{2}$ Clover seed, $7 s .6 d$. per acre; harvesting 16 tons 5 cwt, at

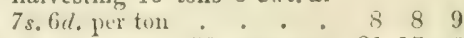
Prepared manure, 79 s. per acre $21 \quad 17 \quad 6$ 'Total outgoings $\overline{£ 241 \quad 17 \quad 6}$

\section{EFFECTS OF CROPPING:}

It can hardly be doubted, that the land is in certain regular degrees impoverished in proportion to the crops which are grown upon it: thus, after a fine crop of wheat, an abundant one of spring corn is seldom obtained; and in the triennial rotation of grain and fallow, a good spring crop is rarely succeeded by a heavy production of winter corn. On this, it has been suggested by Von Thaër, that, were this law of nature maturely inquired into, it might lead to measures by which abundant crops might be produced in years which, in the ordinary course of cultivation, might be expected to 
yield bad ones; for it would teach us to reserve manure, and to make arrangements regarding future crops better adapted for times succeeding those which had been previously prolific, by which means a judicious farmer might overcome the difficultis attendant upon an untoward season. We have already adverted to some observations which he and other naturalists have made upon the exhanstion of the carth occasioned by the growth of grain * and, in order to further elucidate what he has said in support of the above remark, we shall add some brief extracts from the copious reasoning, and the numerous tables, which he has furnished regarding the effects of various rotations upon different soils.

The vegetative power is supposed to be in proportion to the quantity of humus, or mould, which is contained in the soil, and its consumption has been found to be regulated according to the amount of nutritive matter furnished by the crops which are grown upon it. The degrees of exhaustion thus occasioned, have only been fixed by naturalists with any degree of certainty in so far as regards the usual species of cultivated grain and pulse; for, as to the other products of the earth, although they duubtless have similar effects when frequently repeated, yet those which consist of vegetable roots and grasses, and which are drawn from the land before they inave perfected their seerl, are nevertheless-whether from the influence attributed to their shade upon the soil, from sustenance drawn from the air and water, or from other causes with which we are unacquainted-only viewed as ameliorating crops, and have therefore been assimilated to the application of tare fallows. Corn-crops are however respectively considered to exhaust in proportions which render the production of about $4 \frac{1}{2}$ bushels of wheat equal to that of 6 bushels of rye, $8 \frac{1}{2}$ of barley, and 12 of oats.

In certain seasons, however, one species of grain, though more exhausting than another, may yet produce the largest crop; but it will comparatively impoverish the ground, and the succeeding crops will be proportionably deficient. If we estimate the produce of each crop according to the quantity of sap which it contains, regard must be had to several accessary circumstances; thus, besides the temperature of the air, which we can neither foresee nor regulate, a precarious crop or a species of culture which has prepared the soil in a particular manner, will have peculiar effects upon the vegetative powers of the earth, and its absorption by the attractive strength of the grain. Accordingr to all the experiments which have been hitherto made, there is however reason to suppose, that upon a soil of moderate fertility, an average crop of wheat impoverishes the land to the extent of 40 per cent., while one of rye only produces that effect so far as 30. Although barley is more exhausting than oats, yet, upon strong land, in a less perfect state of culture, the latter produces proportionably larger crops, consequently absorbs more nutriment; and, for this reason, they may be both stated at 25 per cent.

The exhaustion occasioned by these crops is proportionately repaired, and the land is restored to its former nutritive jowers in three ways; namely-

By the application of putrescent manure ; accorling to its quantity and quality.

By the ground being left a certain time under pasture; according to the number of stock which it can support.

By the operation of a summer fallow, according to the manner in which it is performed.

The soil is never so utterly impoverished by cropping, as not to be still capable of producing something. This productive faculty composes what

* See the note marked + in the last chapter, at p. 91. 
may be termed its nalural fecundity; which, although existing in various proportions accurding to its original fertility, yet, when capable of producing five bushels of rye per acre, besides the seed, may be supposed equal to 40 degrees: its full value being estimated at 100. Now, from various experiments which have been made upon a large scale, it is supposed that the application of about eight tons per acre, of well fermented farm-yard manure, of average quality, are equal in their effects to 50 degrees of nutritive matter; and that a bare summer fallow-not only by the influence of its working on the land, but also by producing the decomposition of the weeds which it destroys-is equivalent to 10 degrees; thus bringing the soil round to its former state, and rendering it again fit for the production of further crops.

Independently of the fertilising properties which the fallow may be supposed to draw from the atmosphere, the richer the land may be, the greater will be the quantity of weeds which it will produce. Their decomposition will consequently add proportionably to the amelioration of the soil ; so that if its original fecundity be 40 degrees, and that the fallow adds 10 , it will be increased to 11 when the former reaches 50 , and to 12 when carried to 60 .

The calculations of Von Thaër are made upon the weights and measures of Berlin; by which, if the Journal of land be divided into 1000 parts, 1586 constitute an English acre; and he assumes five loads, each of 20 centners Prussian, or $18 \frac{1}{2}$ cwt. British weight, as the quantity of manure applied to the Journal. If the scheffel be also divided into 1000 parts, 645 are equal to the Winchester bushel* ; and the absorption of nutritive matter by the crops of grain is presumed to be-

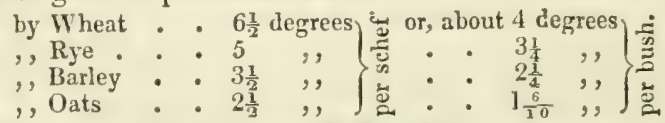

These estimates, however, although founded upon extensive experience, heing yet to be considered as partly hypothetical, and only intenderl to give a general idea of the subject, we do not deem it necessary to reduce them to our standard, and therefore state them as they there stand. In order to demonstrate the proportion of exhaustion occasioned by the growth of particular crops, he has furnished detailed accounts of several rotations, from which we extract those of the triennial and alternate systems; by which the state of the soil will be seen at the close of each course, supposing the land to be of the original fecundity already mentioned, and to produce, on an average, the following crops per Journal.

TRIENNIAL SYSTEM.

Crops and Manure.

Fallow
$6 \frac{4}{y 0}$ loads of manure
Rye, 6 scheff.
Barley, 6 do.
Fallow
Rye, $3 \frac{1}{2}$ scheff.
Oats, 4 do.
Fallow, light folded
Rye, 4 scheff.
Barley, 3 do.

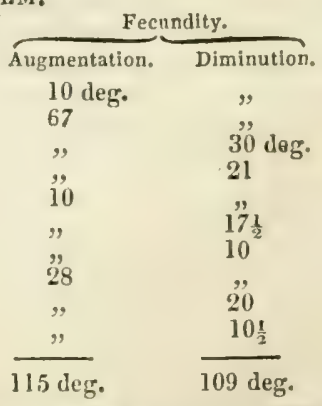

$*$ The centner is $=103 \frac{2}{3}$ lbs. English. The Dantzig scheffel is $=1552$ Winchester bushels, and the last of 60 scheffels $=11$ qre. 3 bush.; but wheat is shipped by the last of $56 \frac{1}{2}$ scheffels. 
By which course the land would gain six degrees of fertility in nine years, provided the manure was of the average quality produced chiefly by the dung of well-fed eattle; but being essentially composed of straw, it probably would not occasion any amendment.

ALTERNATE SYSTEM.

Crops and Manure.

9 loads of dung

Potatoes *, 80 scheff.

Barley, 9 do.

Peas

$3 \frac{3}{4}$ loads of manure

Rye, 8 scheff.

Clover, mown

Pasture

Oats, 11 scheff:

\begin{tabular}{|c|c|c|c|}
\hline & & Fecu & ity. \\
\hline & & Augmentation. & Diminution. \\
\hline • & - & 90 deg. & , \\
\hline • & - & 10 & 30 deg. \\
\hline • & - & , & $31 \frac{1}{2}$ \\
\hline - & - & "̈ & $10^{-}$ \\
\hline • & - & $37 \frac{1}{2}$ & Mg \\
\hline • & $\bullet$ & מִּני & 40 \\
\hline - & - & 12 & $"$ \\
\hline - & $\bullet$ & 20 & 271 \\
\hline " & • & 9 & $21 \frac{1}{2}$ \\
\hline & & $169 \frac{1}{2}$ deg. & 139 deg. \\
\hline
\end{tabular}

This course would therefore augment the fertility of the ground by 30 ? degrees within eight years, besides producing crops of superior value; $\tilde{a}$ result which is entirely due to the additional quantity, as well as the superior quality of the dung made by cattle fed upon roots and clover; and which, if extended to a few more years in pasture, with the advantage of either a folding crop or the adoption of soiling, would in time bring the land into a state fit for the production of wheat. There can, indeed, be no doubt that it must be progressively improved by the production of good crops; for the better they are, the more dung will be furnished by the stock. Thus if clover grown upon land of 60 degrees of fecundity will ameliorate it to the extent of 10 degrees, if sown on a soil equal to 80 degrees its effect will be increased to 14 , and so on in proportion. This will be rendered still more apparent by the following summary of four different rotations actually carried into effect, and each consisting of 120 journals, or $=76 \cdot 1 \cdot 6 \frac{4}{5}$ acres English, and bearing the crops here mentioned, after deduction of the seed.

$$
\text { No: } 1 .
$$

Course of Crops.

Fallow, dunged

Rye

Barley

Oats

Clover once mown

Ditto, pastured during two years together with 170 journals of extra meadow and sheep pasture
Product per Journ.

$$
\begin{aligned}
& 82 \\
& 8 \frac{1}{2} \text { scheff. } \\
& 8 \frac{1}{2} \\
& 8 \\
& 14 \text { cent. }
\end{aligned}
$$

39

12 scheff.

10
10
5
20 cent.

15

* The augmentation of fertility is here added, because of the culture bestowed upon the potatoes as a fallow crop; its diminution is carried to the account of the production of the crop, which is supposed to require a quantity of manure equal to 30 degrees for its support. 
No. 3.

Course of Crops.

Potatoes

Barley

Clover

Oats

Peas

Rye

Tares

Rye

Meadow dunged

Besides 100 journals sheep pasture

\section{No. 4.}

Oats upon pasture ley

Fallow, folded and sown both before and after winter and Rye

Peas

Rye

Potatoes

Barley

Clover, mown

Ditto, pastured with sheep during two years

Meadow ( 150 journ., ) dunged
Produce per Journ.

87 scheff.

12

24 cent.

14 scheff.

6

10

20 cent.

9 scheff.

15 cent.

s

14 scheff.

20 cent.

10 scheff.

6

9

87

12

24 cent.

$15^{\prime \prime}$

The produce of these various crops, both in fodder and manure as well as in grain, and the profit gained by the feeding of stock, was then summed up, aud being calculated according to the price of grain, was reduced to scheffels of rre, from which were deducted the charges of cultivation, thus affording a parallel between the diflerent courses, as follows *

\begin{tabular}{|c|c|c|c|c|c|c|}
\hline & $\begin{array}{c}\text { Product of } \\
\text { Straw. }\end{array}$ & $\begin{array}{c}\text { Product of } \\
\text { fodder reduced } \\
\text { in weight to an } \\
\text { estimate in hay. }\end{array}$ & Manure. & $\begin{array}{l}\text { Profit on } \\
\text { Cattle. }\end{array}$ & $\begin{array}{l}\text { Profit of } \\
\text { Grain. }\end{array}$ & $\begin{array}{c}\text { Net } \\
\text { Balance. }\end{array}$ \\
\hline Nos. & Centners. & Centners. & Centners. & Scheff. & Scheti. & s shefr. \\
\hline 1 & 4173 & 2936 & 14219 & 992 & 1948 & 1869 \\
\hline 2 & 6464 & 4650 & 22228 & 1651 & 2958 & 3028 \\
\hline 3 & 7916 & 9120 & 29272 & 2430 & 2960 & 3458 \\
\hline 4 & 10973 & 12315 & 41791 & 3178 & 4323 & 5188 \\
\hline
\end{tabular}

In following up any of these rotations, there are some fundamental principles to be observed from which no prudent farmer should depart; and in order to be able to put them into execution, it is almost unnecessary to say, that he should be perfectly master of the lnown theory as well as the practical details of his business. On the latter point, especially, his jrofits will be found mainly to depend; for, unless he observe a strict and judicious degree of economy in the application of his means, he will assuredly become the dupe of his ignorance. If he has not already acquired the proper experience, he should therefore seek, either by observation on the practice of others, or rather by small comparative experiments of his own, to ascertain what are the most suitable crops to be grown upon his land, as well as the best mode of their disposal; and he will do well to con-

* These tables may be found in the quarto Atlas of Ton Thiler's works on agriculture, numbered respectively $3,6,7$, and 9. The quantities of land were in some places larger than here stated, but the produce has been in each proportionably reduced, and the cost of culture has been omitted, as being irrelevant to our charges. 
fine his system to as broad and simple a scale as possible. This we deem the more necessary to impress upon him, because both time and altention are sensibly wasted by passing from one species of work to another; and a great variety of crops, though interesting to the naturalist in the progress of their cultivation, may yet, if unguardedly adopted, lead him astray from the main object of his pursuit-which should be solely that of doing justice to himself and his landlord.

When he has finally arranged his plan, he should not be induced to depart from it hastily by any representation of extraordinary advantages to be obtained by novel modes of cultivation, unless clearly satisfed of their evident utility. A prudent man, who wishes to steer clear of difficulties, will indeed see, that however apparently profitable they may appear to others, yet to him they may prove the reverse ; for, beside the various circumstances which may operate differently, there is the material consideration of the employment of capital. Its liberal expenditure will no doubt be, in most cases, proportionably repaid, if it be directed with judgment; but it is essential that the nature of the work on which it is engaged be well understood, and that all the charges of management, as well as the probable product, be rigorously examined: a plan, it may be observed, which, if more regularly followed, both by farmers and writers upon agriculture, might prevent many egregious mistakes regarding the profit and loss of husbandry. Whatever may be the system which he adopts, it should be founded upon the basis of securing a sufficiency of manure to carry it into complete effect; for, if he sows a crop upon land which is not in proper heart to carry it to the highest pitch of average product, he will assuredly be a loser.

\section{Chapter IX.}

\section{ON THE GROWTH OF CORN.}

THE plants of the description termed cereal grasses that are most commonly grown in this country are, wheat, rye, barley and oats; which partake of one common character, and admit of great similarity of culture. 'I'u these -which are used as bread-corn-we might add maize, buck-wheat, and millet, which are also extensively employed for that purpose throughout many parts of Eurupe and America, though here they are chiefly given to poultry; and maize, though long known in our gardens, is a plant of only recent introduction to our climate as a field-crop.

\section{COMPOSITION.}

The chemical composition of plants has, within these few years, been elucidated by numberless experiments, both in this and other countries. The subject is, however, too extensive to be minutely treated of in this work; we shall, therefore, merely quote a brief extract from the observations of Sir Humphry Davy, "that the compounds in vegetables really nutritive are very few : farina, or the pure matter of starch, gluten, sugar, vegetable jelly, oil, and extract. Of these the most nutritive is gluten, which approaches nearest in its nature to animal matter, and which is the substance that gives to wheat its superiority over every other grain. The next in order as to nourishing power is oil, then sugar, then starch, and last of all, gelatinous and extractive matters. Sugar and farina, or starch, 
are, however, very sinilar in composition, and are capable of being converted into each other by a simple chemical process. All the varieties of substances found in plants, are produced from the sap, and the sap of plants is derived from water, or from the fluicis of the suil, and it is attended by, or combined with, principles derived from the atmosphere *." They vary, however, in quantity and proportion, not only in the different kinds of grain, but also in those of the same species : the temperature of the season, the nature of the soil and manure, the degree of maturity which the crop has attained, and the weather at harvest, all give rise to distinct degrees of quality; and this occasions corn to contain more nutritive properties in some years than in others.

The weight of the different species of grain varies according to the quality; but the nutritive properties, though approximating to the weight, yet do not exactly accord with it. The common weights are-

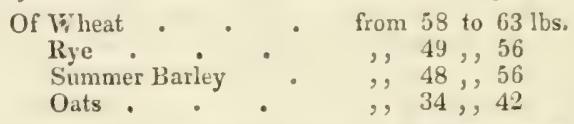

It has been lately much recommended to sell corn generally by weightas at present customary at Liverpool, and some other markets - and a Bill las been brought into Parliament to enforce the practice. This has given rise to much discussion, and we doubt whether, if the measure be carried, it will have any further effect than occasioning some temporary inconvenience to those farmers and dealers who adopt the plan of sale by measure; for every one must estimate the grain by its general qualities, and no man who understands his business will judge either by weight or measure alone.

It has been calculated by distillers, that certain weights of the various kinds of corn of average quality, produce the following quantities of proof spirits ; nanely-

\begin{tabular}{|c|c|c|c|c|c|}
\hline \multicolumn{6}{|r|}{ Quantity of Spir } \\
\hline 53 lbs. & & Wheat & & . & 11 Pints. \\
\hline 50 & & - Rye & • & & - 9, \\
\hline 42 & & Barley & & - & 7,9 \\
\hline 30 & • & Malt & - & & 6, \\
\hline 28 & & Oats & & - & $3 \frac{1}{2}$, \\
\hline
\end{tabular}

This proves that the saccharine matter extracted from different species of grain is not exactly in proportion to their respective weights; but experience has shown that the increase is nearly in the same proportion in those of the same kind. We have, however, already seen, that their nutritive properties, however, do not depend wholly on the quantities which they contain of saccharine matter.

\section{SEED.}

Seed of every kind should attain full maturity ere it be sown; for, althongh that which has not arrived at perfection may produce sound crops, when favoured by soil and season, yet there is always considerable risk in employing it. It was formerly, indeed, maintained by the late Sir Joseph Banks, that stunted seed not having lost the faculty of germination, might be sown with entire safety, and the opinion of that eminent naturalist, hacked by that of some other writers, brought it for a short time into use. It is, however, evident that, - as the farina, or flour, contained in the corn constitutes the food of the embryo plant, until its roots are grown suficiently large to absorb their own nourishment,-the vege- 
tative powers must be increased in proportion to its quality : the nourishment can never be so abundant when the parent stock is lean and shrivelled, as when sound, healthy, and vigorous; and there can be little doubt that the plumpest grains are the most eligible. The universal experience of farmers has, therefore, convinced them that the employment of inferior seed is a dangerous kind of false economy, and the finest qualities of each species are now generally chosen for the land to which they are most appropriate *.

Instances of repeated occurrence might, in fact, be stated, in which, through the selection of seed from the finest ears of corn, crops of superior value have been raised + ; and to that, as well as to the importation of the best qualities of foreign grain, is chiefly to be attributed the improvement which is perceptible in those in which that care las been taken. Were farmers, therefore, to pay some additional attention to the growth of seed for their own use-by choosing out a favourable spot of land, hoeing and earthing-up the plants until they attained a perfect state of maturity, and sowing only the finest portion of the crop-they probably would thus find advantages in the practice which would more than recompense them for their additional trouble.

There is a particular period at which each species of seed ought to be soun, in orler to bring the plants to a perfect state of ripeness. 'I'his, however, depends so much upon soil and season, that it camot be fixed by any general rule, and the farmer can only be governed by the state of the weather and the forwardness of his work; for, whatever may be his experience, his judgment may be deceived in the choice of time. The condition of the land is, in fact, the best guide; for, if it be in a mellow state, between drought and moisture, the seed may be put in with confidence. Some kinds, lowever, prefer a dry and warm soil ; others, that which is more humid and tenacious : thus barley, rye, and buck-wheat, succeed best on the former; and wheat and oats on the latter. It has, indeed, heen remarked that a certain state of the atmosphere-with which all countrymen are well acquainted-is favourable to sowing. Some farmers place great confidence in the full and change of the moon; others, recommend barley to be sown late in the evening, and not to be covered in until the following morning, in order that it may have the influence of the dew : but if this have any good effects upon its vegetation, warm weather should be chosen; for a white frost may have a contrary effect.

The depth at which the seed of corn should be sown is a matter of some nicety. When a seed which is fully ripe is exposed in a certain state of humidity to a degree of heat much above the freezing point + , it absorlus

* A method has been pointed out by the Irighland Society, by which any farmer may satisfy himself regarling the sufficiency of the grain which he intends to use for seed: that is, by making trial of small quantities of it in a warm exposure, or in flower-pots, a considerable time before the season of sowing, numbering the grains employed in the experiment, and examining how many of them produce healthy plants; and this, it is said, must prove infitlible for the purnose in view, when properly conducterl. On this, however, it must be observed, that the experiment may become fallacious, from the circumstance that grain is frequently found to germinate, although the plants never arrive at maturity. A feeble plant will also sometimes live in a very rich soil, that would perish in a poor one : instead, therefore, of depositing the seed in the earth usually employed in flowerpots, it should be deposited in mould dug from the field in which it is intended to be sown.

$\dagger$ Thus hedge-wheat, which is well known as a productive variety, was produced from the seed of a single plant of extraordinary size, found casually growing under a herlye.

\pm Vegetative life commences under every degree of heat between the freezing point and $150^{\circ}$ of Fahrenheit, and is arrested when the temperature is below it, or at $32^{\circ}$. Maine's Veg. Pliysiol. p. 17.

VOL. II. 
moisture through the pores of its outer covering; in consequence of which it swells with such force, that beans and peas, during this process, have been made to split hollow balls of considerable strength, which had been filled with them, and even to raise a weight of nearly 200 lbs. If the seed should have met with any accident to destroy its vital powers, it decays and becomes putrid. The same effect takes place if it be alive, unless it have the benefit of air; but if the seed be good, and the presence of both air and the requisite degree of heat and moisture be secured, the nutritive matter contained within it soon becomes fluid, milky in its appearance, as well as sweetish to the taste, and is carried off by the sap-vessels, first towards the root, and then to the stem of the plant. Wheat, for instance, has two sets of roots; one springing immediately from the seed, and another from the stalk. The latter, which are called coronal roots, may always be observed to form themselves immediately below the surface of the soil, whether the grain be sown deep or superficial; which is probably occasioned by the facility afforded by this position to the spreading of the roots, and their absorption of nutriment from that portion of the ground which is usually the richest. They are therefore subject to be chilled by an early frost; and, if the seed be not sown at a proper medium depth, the seminal roots may also be exposed to similar attacks: which forms a strong reason for early sowing *.

If the grain be too deeply buried, germination is impeded, and may be altogether prevented; while, if sown too shallow, sufficient moisture is not left in the surface to afford nourishment to the roots of the plant. The depth at which it ought to be placed must, therefore, be regulated by the nature of the soil. If stiff, more moderate covering should be used than if it be light and porous: wheat, barley and oats, also require more than rye or buck-wheat; but, except in some few instances, from $1 \frac{1}{3}$ to 3 inches is, in every case, the lowest to which it should be carried. Some farmers adopt the plan of sowing the seed at different depths, for the purpose of insuring the growth of some portion of it. With regard to autumn sowing, this only causes the unnecessary sacrifice of an additional quantity ; but in the sowing of spring corn, it has the injurious effect of preventing the crop from becoming ripe at the same time. To promote equal veretation, it is therefore desirable that all the seeds should be placerl at an uniform depth.

With regard to the proper quantity of each species of seed, the practice depends upon whether it be sown broad-cast, drilled, or dibbled, and also in some measure upon the nature and condition of the land; but, as this will be considered under their different heads, we need now only remark, that, generally speaking, wheat is usually sown broad-cast at the rate of three-barley at that of four-and oats to the extent of four or five bushels, or even more, per acre $\uparrow$.

If the seed could be uniformly spread at a regular denth,-if it were not subject to destruction by birds, field-mice, and insects, as well as injured by being in some cases buried beyond the influence of the air, and in others too much exposed to the atmosphere, and that every grain might be pre-

* See Macricar's View of Vegretable Economy, Part I., Quart. Journ. of Agric. N. S., vol. i. Hunter's Georgical Essays, No. V.; and a Paper in No. VI. of the British Farmer's Magazine.

+ It has been calculated that wheat, barley, and oats, of good quality, when sown to that extent, contain the following number of grains, namely-

3 Bushels of Wheat, weighing $61 \frac{1}{2}$ lbs. per bushel $\quad 2,685,900$
4 Do. Barley , $53 \frac{1}{4}, 2,508,280$

5 Do. Oats $, 42^{4},, \quad: 3,534,720$ 
sumed to produce a shoot-such quantities might be deemed excessive, for not one-fourth of them could find space for their roots. But as in the ordinary mode of sowing, such precision cannot be expected, nor can the germination of every seed, though duly deposited in the earth, be relied on, experience has shown the sum already stated to be necessary, and the experiments of those who have tried lesser quantities have not been generally successful. The just amount must, however, depend upon the goodness of the seed, the expertness of the sower, the state of the soil both in respect to manure, fertility, and the condition into which it has been brought by tillage; and lastly, by the carliness of the sowing. The latter point is, indeed, of such importance-particularly in autumm sowing-that some sorts of rye will produce a crop, if sown in July, with half the quantity of seed that is found necessary in October. Savings may, therefore, unquestionably be made; and the seed be further diminished by more careful attention to the different operations of sowing.

Among the general run of farmers there is a far greater disposition to sow profusely than to lessen the quantity of seed. This chiefly arises from their fear of throwing away the chance of a crop by not sowing enough ; and the land is, therefore, very generally sown with more seed than it requires. 'The practice has also been advocated, though upon another principle, which we shall hereafter notice, by Mr. Colie, of Norfolk. Thick sowing certainly gives a finer appearance to a crop of corn, in the early period of its growth, than when the seed has been put in thin; but when thus crowded, the shoots exhaust each other, acquire a yellow, dusky tinge, and, if the weather be at all unfavourable, many of them perish: it may also be seen that the most vacant spots are precisely those on which the plants stood the thickest*.

Another motive for thick sowing exists in the idea that the growth of weeds is thereby prevented. It not improbably, however, has a contrary effect: for, if the corn be sown at proper distances, the spreading of the roots occasions them to interweave among each other, and form a issue which may choke the weeds; but, if sown too thick, they strike downwards and do not prevent the weeds from springing. On this point we have the cvidence of Von Thäer, who says, that, on some marsh-land upon the hanks of the Oder, it is customary to sow oats at the extraordinary rate of nearly eight bushels per acre, with the intention of keeping down the rank. ness of weeds with which the ground is much infested. Yet it has not that effect; while he, who sows only half the quantity, finds his land quite as clean as that of his neighbours. He, indeed, states that the finest crops of any corn which he has ever seen contained only five or six plants to the foot square + .

* See chapter iv. p. 50, of this volume. In addition to what is there said, we ourselves witnessed a field in which the plants of the wheat were so thinned by the grub during the spring of 1834, that there was some intention of ploughing it up. It was, however, allowed to stand, and they tillered to such an extent, that the crop was much finer than upon any other part of the same extensive farm.

A remarkable instance of a similar nature has also been mentioned in a recent number of the Quarterly Journal of Agriculture, in which it is stated that two-thirds of a promising crop of potatoe-oats were destroyed by the grub. The remaining shoots, however, stooled out so luxuriantly, that "so many as 14 stems were counted from one root; some of the heads contained upwards of 500 grains, and some of the straws were nearly as thick as the little finger. The strength of the straw made the reaping of it a difficult task; and the crop yielded, with all the unfivourable appearance in which it was left by the grub, 60 bushels per imperial acre of very fine corn: the land was, however, a deep hazel-loam of first-rate quality." - No. XXII. p. 541.

† Principes Raisonnés d'Agric., 2de ed., tom, iv. 967, p. 27. 
It is commonly imagined that a change of seed is necessary, and therefore farmers generally choose what they purchase out of a different district from that in which they reside. There may be some truth in the opinion; for unquestionably a change has frequently proved beneficial to the succeeding crop, though it is contradicted by many men of sound judgment, who hold that the only advantage thus obtained is by the effect of superior quality being generally selected; and that, by paying equal attention to the choice of seed of their own growth, they lave never found any necessity for purchasing elsewhere. It seems, however, as if it were an inscrutable law of nature that all seeds, to maintain the vigour of their growth, require a change of soil; and, therefore, those who adopt that plan appear to us to act wisely, and cannot, we think, be justly accused of prejudice. There is also another circumstance which may render it advisable, and which, though not generally adverted to, may have added strength to the idea: it is, that grain, however carently it may be screened, can rarely be found unmixed with the seeds of weeds; which, if grown on land of one kind, will not prosper on ground of a different nature: thus, wheat grown in the fens is generally very full of weeds, which will seldom grow upon the high-lands, and are consequentiy not much to be dreaded *

There are, indeed, many peculiarities in grain of the same species, and a change may sometimes be hit upon which will prove alvantageous; or instances may be found of a kind which was originally suitable to the soil, having from unknown causes degenerated. In such case, doubtless a change should be made without hesitation; and provided a sort be chosen which is alizpted to the land, and in every respect perfect, it cannot prove injurious. 'The nature of the soil upon which the seed is to be sown should however be taken into consideration, and it is generally thought advisable to procure it from land of an inferior quality, as well as from a drier, or a warmer climate : for strong lands, from poor light soils, or from the fens; and for friable loams, from stiff clays. Thus a shrivelled sample of wheat from a poor gravel has produced a beautiful crop when sown unon an adhesive clay, and other instances of a similar nature are too numerous to be recorded t; but attention should always be paid to the colour, as well as the weight of the grain.

\section{QUALITY AND PRESERVATION.}

It has been assumed as an axiom, regarding the quality of corn, that "the more severe the climate, the thicker is the skin of the seed t." Jrom this, however, as a universal law, we must dissent, as perhaps some of the thimest skimned wheat grown in all Europe is produced in the northern parts of Poland; but the observation will hold good if applied to humid soils and climates. Thus the Scotch and Irish wheats are gencrally foumd to be more productive of bran than those of the same species crrown in the midland and southern counties of England; and it is alleged by distillers, that in parcels of English and Scotch, or Irish barley, of equal weights, the former will produce more spirits of equal strength: the difference arising from the thickness of the husk.

It is extremely important to the preservation of grain to guard it carefully from damp and heating; for although, if thus injured, it may not be entirely deprived of the power of germination, and the young shoots from it may show an early appearance of vigour, yet, at a more advanced stage of their growth, they fall off, become weak, and produce crops which are in

\footnotetext{
* Drew's Norfulk Husbandry, p. 129. + Marshall's Minutes of Agric., p. 87.
} † Macvicar's View of Vegetable Economy, Part I. 
many respects defective. From experiments made in the year 1817, by a special commission appointed by the French govermment, to inquire into the state of the crops, and to determine in what degree grain which has been in different proportions deteriorated might be made available as seed, it was found-that grain which has suffered a commencement of germination rises only in the proportion of one-half of the seed employed; if strongly germinated, in the proportion of one-third; and if fired or moulded, of not more than one-fiftl.

This is particularly observable in oats imported from the Baltic, which are invariably shipped in bulk, and after a long passage are frequently thus dimaged. The wheat from Dantzig is generally shipped in bags, which have some effect in preventing it from heating: it is, besides, previously screened with extreme care, in the warehouses of that immense granary, and, being of the former year's growth, it is consequently in a dry state; but from other ports much is exported in very different order. Caution is therefore requisite in the purchase of foreign corn for seed; for although it may have been cooled by exposure to the air, and thus appear undamaged, yet, if sown, it may disappoint the expectation of the grower*. When intender for consumption as food, it may be preserved with safcty for a great length of time, by completely drying it, and depriving it of all communication with the air; and in this manner wheat has been kept for many years at Malta, and other places, in rocks hollowed into deep conical caves, and hermetically sealed at the top. In this country, when injured by wet, it is kiln-dried, in the manner stated under the liead of 'Harvest;' but although it may be thus rendered fit for mealing, it yet can never be safely used for seed.

Some sceds retain the power of germination for a long time, if gathered when ripe, and preserved with care. Others lose it after the first year. That of wheat has been known to vegetate when five years old, and rye at three; and all the best species of corn can be grown when not more than two years old; but, in general, wheat should not be sown when more than one year old, and rye, barley, and oats are always preferred when of the last harvest. Seeds of recent growth also germinate more freely than those which are comparatively old; but it has been observed that they afford no superior advantage in point of crop, and are even thought to occasion an increase of straw rather than of corn. If kept long in the granary, their sources of regetation are more weakened than when held in the stacks; and therefore, when intended for seed, grain should never be threshed until the time of sowing.

\section{PRODUCT AND VEGETATION.}

Endeavours have been made to ascertain the product of grain on different soils, and in various countries. The results from which these averages are drawn are, however, extremely deceitful, and although fumishing ideas for the calculations of political economists, yet contain no data in which a farmer can place confidence, for the crops vary every year, and if any given series of rears be taken, the averages will be invariably found unequal. Circumstances however occur during the vegetation of corm, which deserve

* Many cargoes have been so heated on long voyages, when shipped in bulk, as to render the pick-axe necessary to get the wheat out of the ship's hold, and in several instances the whole has been thrown overboard by permission of the Board of Customs, or burnt for manure, as it would have endangered the safety of the warchouses, from the intense heat which it engenders; for eren spreading out heated corn on a floor three or fuur inches thick will not cool it. 
attention, as affecting the state of the crop, and enabling men of experi. ence to form some probable estimate of its produce.

Thus, it is thought advantageous when Autumn-sown grain remains a proportionately long time in the ground without sprouting-provided always that this be not occasioned by unusual drought, - for the roots are thus found to acquire strength; and it has been observed, that, under a favourable temperature of the air, the braiding of the seed has been thee days later on deep land than on a superficial soil. For spring corn, it is on the contrary best that it should sprout quickly, to enable it to get ahead of the annual weeds.

It is considered a good sign when the shoots rise in a uniform manner; for any inequality tends to mark some future deficiency in the crop. This, as we have already observed in our remarks upon the depth of sowing, - is more to be dreaded in spring corn than in that which is sown in autumn; for the plants of the latter acquire an apparent legree of equal growth, while the former always maintain their disparity.

The young plants should be of a dark-coloured hue, and the first leaves which are found should be short, thick, not sharply pointed, but firm and elastic. Those of wheat, approaching to a linge of brown; rye, brown, drawing towards red; and those of all spring corn, of a deep green, without any intermixture of yellow: which latter colour always indicates some disease, from which the plants rarely recover.

When these first leaves have unfolded themselves, numerous lateral shoots spring from the coronal root, in the manner called "tillering," and from their strength and abundance a judgment may be formed regarding the future crop. It is, however, neither desirable that they should rise promptly in height, nor that they should droop through weakness: they should first spread over the ground, and when they have covered it, they should then rise with a vigorous stem; but the more gradually this is effected the better. 'The strength of the stem, especially in the lower part, is quite as important as their height, for it is only when they are proportionally strong that they will carry large ears, and the knots of the joints should be thick and brown. When the ears are formed, and have become in bloom, their tops should present a nearly equal appearance throughout, and, when viewed by casting the eye across the field, the crop should seem level in height; for when the stems rise at different heights it is a bad sign.

The time of blooming is also a critical moment. If the weather continue long wet, veretation proceeds imperfectly; but if the month of June be dry and warm, with only occasional showers of rain, it is desirable. This is also a time when there is the greatest danger of the crop being laid. If this happen before the blooming be completed, through the effects of storm, it occasions no great mischief, for the plants soon recover their original position; but it is very prejudicial if it be caused by their apparent weight and richmess, for that is frequently the conseguence of weakiess in the stem, and a general failure in the yuality of the plant, occasioned, not uncommonly, by injudicious application of dung and insuflicient tillage.

The autumn sowings reach the winter season at various stages of their growth; but although their progress may be cheched by severe cold, it is never entirely destroyed, and vegetation is renewed with increased vigum when the weather becomes open. Thus, in very long continued frosts, which have penetrated far deeper into the earth than the roots, the plants have tillered with extraordinary luxuriance in the ensuingr spring, and the sharpest winters have gencrally been productive of the most abundant crops. Those winters which are in this comtry termed mild, and which 
constantly occasion alternate changes of weather, are far more dangerous to vegetation, especially upon wet soils.

A heavy fall of snow, particularly if the ground be in firm order, is always favourable, and the plants continue to grow under its cover. The period of thaw is, however, attended with serious risk; for if there be not a sufficient fall from the land, the seed' may be materially injured by the lodgment of the water, and this camnot be prevented by under-ground drainage, as the snow will have melted before the thaw penetrates into the earth, and consequently the water cannot reach the drains. It is still more dangerous when the thaw is only gradual, with sunny days and frosty nights; for the ground being thawed during the day, sinks from the roots, which thus become exposed to the frost of the night, and the most vigorous plants find great difficulty in resisting these alternate changes of weather. The danger, too, is alivays greatest if the soil be of a porous nature.

The effects of frost, and other injuries upon grain which is approaching to a state of ripeness, are such as in many cases to deprive it of the power of bringing corn to maturity when used as seed, although it may germinate when sown. It is often very difficult to ascertain the degree of damage thus incurred by common inspection; and farmers who purchase their seed are thus frequently deceived. If, however, the seeds be closely examined, the hulls, or coats of their kernels, if the grain be internally damaged, will be found to present the following appearance. The outer rind, which encloses the mealy substance of the kernel, is marked by a scar, through which the sap-vessels enter; and, upon stripping off this sheath, a hollow groove is observable, running lengthwise through the inner rind, from the root end of the grain to the point. If the grain be sound, and fit for seed, this groove will not be discoloured; but if damaged, it will be found nearly black at the lower end, and more shrunk into the body of the kernel than in healthy seed. The kernels, if unsound, are also easily divided in the line of the groove, and upon being laid open, the sooty colour will be seen to cover the root end. 'This designates rottenness; whereas, if they are healthy, they are always tough, smooth, and bright in colour, and it is difficult to split them.

Similar, or nearly similar bad effects to those that attend using seed which has suffered from frost, are found to arise from using corn that has partially sprumg in the ear from wet weather. The grain of wheat, lowever, if sound, is plump and full, of a peculiarly healthy colour, well known to farmers and dealers, and in the case of oats it is commonly free of chaff. Barley, if tainted, loses somewhat of its usual colour, and becomes paler, with black ends in some of the grains. These indications will certainly go far in guiding the judgment to a correct opinion; but neither weight nor colour are sure proofs of soundness, and perliaps the safest test is the character of the seller.

If it be wished to examine the grain in the stack, it will be proper to take a few ears from each of a considerable number of sheaves, to have all the grain in these ears carefully beaten out, and then to take a part promiscuously for inspection. If the corn be discovered to be only partially injured (some grains being quite sound), it then becomes a question of economical calculation whether it should be sown or not. The most prudent course would certainly be to reject it. Circumstances may, however, render that inexperlient, or the grain may be only so slightly spoiled, as not to b: unfit for use; in which case it should be sown thicker in proportion to the extent of the injury : thus, if five grains in fifty be damaged, ten pecks should be sown for nine. 
$\Lambda$ question has been for some time agitated, regarding the slate of ripeness in which corn should be reaped; and it has been recommended, as a general rule for practice, to cut down the crops before the uppermost grain can be shaken out:- a period which can only be accurately accertained by close examination of the state of the straw, respecting which some remarks will be found under the head of harvest. The advantages which are supposed to arise from cutting it down when it has arrived at that state of maturity consist in a presumed improvement in its quality, and in the evident saving of all loss by shelling out; for, if perfectly ripe, and the weather be dry, the grains will be in danger of falling from the husks, cither by the least a gitation of the wind, or by the act of reaping, and the ears are daily plundered by myriads of birds, which destroy greater quantities of corn than is generally imagined. There is, therefore, an evident waste both in the ficld and in the stack-yard; and, in an unsettlerl season, the early-cut com may be safely havested, while the latter still stands out exposed to the injuries of the weather.

These are, indeed, motives of such weight in point of cconomy, that many farmers have been induced to cut down their crops while almost in a green state. It is, lowever, necessary to discriminate between the ripeness of the straw and that of the grain; for, in some seasons the straw dies upwards; under which circumstances, a field may appear to the eye to be completely fit for the sickle, when in reality the grain is imperfectly consolidated, and perlaps not much removed from a milky state. 'Though it is obvious that, under such circumstances, no further benefit can be conveyed from the root-as nourishment is withheld the moment that the roots die; yet it does not follow that grain so circumstanced should be immediately cut, because, after that operation is performed, it is in a great measure necessarily deprived of every benefit from the sun and air, both of which have greater influence in bringing it to maturity, so long as it remains on foot, than when cut down, whether laid on the gromel or bound up in sheaves. The state of the weather at the time also deserves notice; for, in moist, or even variable weather, every kind of grain, when cut pre maturely, is more exposed to damage than when completely ripened *.

The sample is certainly superior to that of corn which is allowed to become what is termed "dead ripe $+; "$ and it has been also said that secd corn in that state of miturity, " if it gencrates at all, grenerates feebly.$+ "$ On this assertion we, however, cntertain doubts; for it is not easy to perceive how any deterioration can be occasioned by allowing it to stand in tise field, exposed to the sum and breezes, and to arrive at a state of perfection, more than if it were threshed out and laid up in a well-aired granary. An advantage is, however, undoubtedly gained in the quality of the straw, which makes much better provender when cut down in a succulent state. This is evident in all hot seasons: thus the straw of the crops of 1834 is so britle, that it breaks under the flail, and has been found nearly worthless when given as food to store cattle.

* Brown of Markle, vol, ii. p. 34.

+ In addition to numberless proofs in support of this, which have been brought forward in this country, a parier on the sulject has appeared in a perjodical work entitled the "American Farmer," published at Baltimore, in which it is said, that "the true and satisfactory cause of the inferiority of the flour manufactured to the northwarl of Pennsyl. visuia arises from suffering the wheat to remain too long in the earth before harsesting it, after it has arrived at a state of pure and perfect maturity."

+ Quart. Journ, of Agricult., N. S. vol, iv. p. 540. 
Taking these things into consideration, it seems the most prunent plan to have the corn cut before it is fuliy ripe: but in this a medium course should be alopted; for although grain, if allowed to become too ripe, assumes a dull dusky hue in the sample, yet, if not ripened enough, it shrivels in the drying. Under both circumstances, it is found to want that brightness of colour and plumpness of kernel which constitute its benuty, and are characteristic of its value; nor, in either state, does it contain that quantity of meal which it is capable of yielding when reaped at a proper state of ripeness. The subject, however, chiefly applies to wheat.

The corn, of each species, proluces, when ripe, nearly the following quantities of meal, or household flour, and bread, per bushcl: namely, -

Wheat, if weighing 60lbs., of Flour 48lbs. of Bread G4lbs.

$\begin{array}{lllllll}\text { Rye, } & \text { do. } & 54 & \text { do. } & 42 & \text { do. } & 56 \\ \text { Barley, } & \text { do. } & 48 & \text { do. } & 37 \text { t } & \text { do. } & 50 \\ \text { Oats, } & \text { do. } & 40 & \text { do. } & 221 & \text { do. } & 30\end{array}$

\section{DISEASES.}

Nuch has been written, to very little purpose, regarding the discases of curn. Farmers have generally not sufficient science, and men of science not sufficicut practical experience of the phenomena presented in the various stages of growth, to enable them to judge accurately of the causes, or the means of curc. Thus they reason upon circumstances, assumed as matter of fact, which have no real existence; palpably coniradict each other; and advance sucls conflicting opinions, that the subject appears wrapped in greater mystery than when first investigated. This is particularly the case with regard to wheat; which, although well known to be subject to maladies that indiscriminately attack and spare land of the same nature, and in the same or different states of preparation, have been attributed by men of the highest eminence as chemists, botanists, and naturalists, to a variety of causes, each of which has been in turn refuted. Thus

Jethro Tull ascribes smut to moisture.

Duhamel says it is an infectious powder.

Dr. Inales deemed it to be occasioned by bruises; and, on further experiment, by damp weather during the flowering.

Spallanzani asserts that it is occasioned by the want of due impregnation ; and his opinion is supported by the memoirs of the Bath Agricultural Society, as well as by Dr. Darwin.

Linniens, Walker, Somerville, and many other naturalists, suppose it to be occasioned by the inoculation of an insect.

Sir IIumphry Davy conceives that it is produced by a fungous excrescence in the grain ; but this is contradicted by Bauer, who has ascertained it to be a morbid swelling of the ear, not at all connected with the growth of a fungus.

Dr. Home, in his principles of vegctation, imagines it to arise from an over-abundance of juices; and Chalmers ascribes it, in his Cyclopedia, to fatness in the soil: but Lisle attributes it, in his observations unon husbandry, to a total want of sap in the plant.

Prevost, Jussieu, and many others, presume it to arisc from infection: in which Vauquelin and Fourcroy partly agree, although their analysis dues not prove it.

Bradley, and the author of the New Farmer's Calendar, call it blight; and several other writers confound it with mildew.

We abstain, therefore, from all comment on so pcrplexing a subject, and shall confine our observations to those brief notices which will be found under the separate heads of the different species. 


\section{Chapter $\mathrm{X}$.}

\section{ON WHEAT'}

OF all those kinds of grain which are cultivated in this country for breadcorn, wheat takes the first rank, and almost numberiess varieties are grown under denominations given to the numerous species in consequence of some existing shade of difference in their qualities, or from local circumstances, arising either from the place of their growth, or the name of the grower. Thus we have the "beariled" and the "downless," the "golden-ear," the "velvet-ear," the "egg-shell," and " hedge-wheat;" the "Essex-dun," the "Kentish-yellow," the "white and red Essex," and the "Mungoswells ;" the "Burwell-red," "Hunter's," and the "Georgian" wheats: the history of which, and of the numerous other sorts which have been introduced into cultivation, if abstracted from the accounts which have been published of their properties, would form a moderate volume.

\section{SPECIES.}

Some of an apparently peculiar nature have also been introduced from foreign countries; as the "Egyptian," the "Polish," the "siberian," the "Zealand," and the "Talavera wheat," and additions are being constantly made to the stock from various parts of the world; but, although differing in the proportions which they contain of nutritive matter, as well as in some particulars comnected with their growth, yet they have all sprung from one origin, and being composed of similar elements they are applied to the same purpose. Botanists, indeed, class some of them as distinct species: thus, for instance, the Eryptian wheat produces several ears from the same stem, which is not the case with any other sort ; but, when repeatedly sown upon poor land, its supernumerary ears become gradually diminished, and it at length loses all appearance of varicty. In like manner, other kinds of wheat, produced in soils and climates more favourable to vegetation than our own, have flourished when first grown in this country, and have appeared to become habituated to our atmosphere, yet in a series of years have degenerated; while other sorts, imported from a more northern climate, or taken from land of an inferior quality, have, on the contrary, been improved.

The same circumstance occurs with respect to those species generally distinguished as Winter and Spring uhcat; for although they seem, from their time of growth, to be of a diflerent nature, yet one can be at jleasure transformed into the other by the common means of culture. Thus, if winter-wheat be sown in the month of Februaly, or the bergimning of March, a portion of it will ripen, though the lateral shoots will be weals, and the crop will only be moderate. If, however, the seed thus produced be sown the next spring, it will throw out stronger stems, will tiller with more luxuriance, and, if the operation be repeated in the following year, it will then be found converted into the nature of summer-wheat. If, on the contrary, spring-wheat be sown in the month of October, and the next winter prove severe, the crop will perish; or can only be saved if it be completely covered by a heavy fall of snow. Should the weather continue mild, the seed will then, however, produce a tolerable crop, which will ripen earlice than autumn-wheat; the seed obtained from it will in the following year take longer to rijen than that of the former senson; it will also tiller better, and partake so much more of the nature of the winter species, that, if sown in the month of May, it will not produce a crop. 'Thus, also, how- 
ever early the true winter-wheat may be sown in autumn, it will not produce stems in the same year; but the real spring-wheat will do so if sown any time before midsummer.

The various species of wheat are indeed frequently found to aiter their character, through the difference occasioned by change of soil and climate. To point out such marked distinctions as would make each variety accurately known, by merely mentioning their names, would therefore be a hopeless task, for the shades of difference are in most cases so small, that one might easily be mistaken for another. There are, however, two distinctions which run through every species of winter, or Lammas wheat, and these consist in the colour of the grain, which is either white or red; that of the straw not always partaking of the difference, but being sometimes lightly coloured when the corn is nearly brown. There are also two general varieties of these, the grain of which may be classed as the thick and thin husked, or the "downy-chaffed," and the "smooth-chaffed.

The red wheat, or thick-skinned quality, is usually grown upon the strongest clay land, and degenerates slightly when sown upon soils of a lighter description. It is of various shades of a reddish brown, or deepyellow tinge, and generally retains the same hue, whaterer may be the quality of the ground on which it is produced. The outer husk is coarse in proportion to the humidity of the soil, and consequently lowers the value of the grain ; which, accordingly, bears a price in the market of from twelve to fifteen, and sometimes twenty per cent. less than fine qualities of the white. It is, however, so hardy, and so much better adapted to ensure the production of a crop on wet and adhesive soils, that it is very generally sown on land of that description. On fine loams, or soils of that kind which are usually considered as good barley-land, the white or smoothchaffed wheat is, however, preferred, from the thinness of the husk rendering it more profitable to the miller.

The downy-chaffed variety was formerly in the greatest repute; both from its generally producing the whitest and the finest flour, and from the grain in consequence of the shortness of the straw, and the closeness of the ear, being less liable to be shaken ou by the wind at harvest-time, than the smooth-chaffed tribes: qualities which jeculiarly adapt it to land of so rich a nature as to endanger the lodging of the crop. In consequence, however, as it is supposed, of the husk retaining the dew and moisture longer than the other sort, it was found more liable to be affected by mildew, and to be in other respects so much more tender, that it has fallen much into disuse, and the thin-chafted wheats are now more generally cultivated, particularly throughout Scotland*.

The diflerent species of wheat which are the most profitable to the farmer, must therefore depend upon the nature of the soil upon which it can be best produced; for aithough the white Lammas will always command the best price, yet it is more uncertain in its produce than the red, and land

* A variety of this species was first cultivated, alout thirty years ago, on Coldingham MIvor, in Berwickshire, by MIr. Hunter, of Tynefield, in East Lothian, from which it has since taken its name, and is now so extensively sown in the neighbouring districts as to be considered the standard of the thin-skinned Scotch species.

Some red wheat of extraordinary productiveness has also been introduced within these three years from Mark-lane into East Lothian, of which the following description is given in the catalogue furnished in the third Report of Drummond's Agricultural Museum at Stirling: namely, "Chaff without down; joints close-set; quality of grain superfine; straw ten inches shorter than that of the Lammas red; tillers woll ; is said to be more productive of flour than any other variety, and to bake as well as the best Dantzic wheat, while it has yielded to the growers, in 18:32. from seventy to eighty imperial bushels per Scoteh acre. It also, in the following year, continued to yield uncommonly well, and is liked by the bakers." $-\mathrm{P}, 50$. 
which has produced indifferently under one species, will frequently be found to yield abundant crops when sown with the other. It has even been observed, that land which is equally appropriate to either lind will produce better crops if the species be alternately changed; that mixed wheat produces the heaviest grain, and that a mixture of flour will make the best bread; which goes far to prove, that it is not altogether that the land is exhausted by a crop when it refuses to grow it a second time with vigour, but that it requires a change of plant to excite its vegetative powers*.

SOIL.

Good whent land ought always to possess a certain degree of consistence, and consequently, the larger the proportion of clay, and the less sand which it contains, the better; for although light soils, composed chiefly of sand and gravel, will often produce wheat of good quality, yet heavy loams, and strong clays, always yield that which is the weightiest in the bushel, and the most productive in the crop. If, along with a small quantity of sand, it have about fifteen per cent. of lime, it may be classed among soils of the best quality for the production of the crop; provided it also contain a sufficient portion of nutritive humus, or mould. Soils of that description are generally of a dark-brown colour, and work freely, in consequence of the mixture of lime, which prevents them from being too adhesive; but in many districts they are much encumbered with large flints, which, though found to diminish their fertility, not yet entirely prevent the use of the drill, and very much impede the employment of the horse-hoe.

Sandy soils are unfavourable to the growth of wheat; for they are deficient in that degree of firmness which is necessary to support the roots of the plants. It is, therefore, a crop which should never be sown on such land; or, if grown, it should only be upon one ploughing of a clover-ley, and when afterwards folded by a flock of sheep. Although it is true that numberless instances may be cited, in the experience of every farmer, of sandy soils, which contain a due proportion of mould, producing tolerable wheat, yet no dependence can be placed on them ; for the crop will fail, if attacked either by a severe frost in winter, or by the drought of a very hot summer. findeed, were it not for the prejudice existing against the use of rye, which in this country throws it much out of cultivation, it would form a much more appropriate crop than wheat upon dry, sandy, and porous land.

\section{CULTIVATION.}

Wheat, although forming one of the crops in every rotation upon good land under the alternate system, yet, upon strong soils, is generally sown upon a summer fallow; though, not unfrequently, a bastard fallow is substituted, by taking a previous crop of tares either cut green for soiling, or fed off by sheep. Thie bastard fallow certainly has the advantage of producing an early crop of spring feed, and upon land that is easily worked, and not too foul, the ground may generally be got in order for the autumn sowing; but if the soil be very adhesive, or much infested with root-weeds, no other plan than a bare fallow can effectually answer the purpose : indeed as the surface-soil becones more closely bound by the growth of wheat than of any other grain, too much efiort camnot be used to pulverize it when it is tenacious. We liave already so fully stated our opinion on this subject, that we have nothing further to add, except to remind the farmer, that the expense will not be wholly thrown away; for, on such land, the

* Burrounhis, on the Cultivation of white Crops, p. 21.-Gulden Farmer, p. 75Von Thaër, Principes Raisonnés d'Agric. 2nde kdit., tom, iv. p. 92. 
crop of wheat will, by that process, be both more productive in quantity, as well as better in quality, than under any other which he can pursue.

Notwithstanding what we have said in favour of fallowing, we are yet aware that in many instances finer crops of wheat have been produced after drilled crops than after a bare fallow: this, however, may have been occasioned by the temperature of the air-as in a wet season-being unfavourable to the working of the fallow, while it has been propitious to the growth of the green crops, by the culture of which the land has been got into a good state of preparation for the wheat; and is no proof of the system being injudicious on stifl soils, though it may be dispensed with upon friable loams.

There is also much land of so light a nature that, although appropriate to the growth of wheat, a bare fallow--unless rendered absulutcly necessary by the foulness of the ground-had better be avoided; as it deprives the soil of its necessary degree of firmmess, and better crops will generally be obtained upon one ploughing after a clover-ley.

The ley should, in this case, be broken up at lcast a month before the sowing of the wheat; both that time should be allowed for some decomposition of the sward: and chiefly that the land may be allowed to settle. It is also decmed essential by many persons that the second crop of clover should he ploughed-in, or at least that the ley should be ploughed up with a good head of grass upon it, in order to give the land the benefit of the green manure; but farmers generally meter the immediate profit of the clover to any prospective advantage to be obtained by such mode of its apilication. The best mode of performing the operation is with the trenclsplough; the furrows being formed narrow, and turned well over, as the complete inversion of the sod is essential to its perfection. Or the grassy elge of the furrow-slice can be cut away by a skim-coulter plough; which, if the work be neatly performed, will completely bury the surface of the sward, and will thus both improve the closeness and the cleammess of the tilth. The seed is then sown either broad-cast or by the drill unon this stale furrow, and either buried by a strong harrowing, or by any of the grubbers commonly in use, and the land is afterwards heavily rolled. Greater accuracy is, however, necessary in the performance of this one ploughing, than when many stirrings are given, and the operation is only usual upon a one year's ley; for clover which has lain two years upon the ground, and more particularly if it has been pastured, is generally found to require three ploughings to get rid of the weeds.

Unless the land be uncommonly clean when the clover is sown, a two years' ley will indeed be not only foul, but will be frequently found infested with the wire-worm, grubs, and slugs, which commit great ravages on the succeeding crop of wheat; and it is, therefore, generally thought necessary to stir the land more than once, both to get rid of the weerls by raking and burning, and to disturb the propagation of those insects. On this, however, it has been thonght worthy of consideration, by a well-known and eminent agriculturist, "whether the disturbing the compactness of the furrows, which a rest of one or two years under artificial yrasses has imparted to them, he not a drawback to the system, greater than any benefit that can be expected to arise. It by no means follows that those who prefer depositing their wheat upon a single ploughing are at all insensible to the value of a frequent stirring of the soil, but they consider that it is then misapplied; they consider there are other crops for which repeated preparatory ploughings are as beneficial as they are worse than useless for ley land; and that a soil should be brought into such a state by well-manged previous crops, 
as not to require at that period any additional tillage for the extrication of the weeds *?"

There is much sound sense in the above observation; for the more light soils are stirred, the more is their compactness and solidity impaired, and the less are they thereby rendered adequate to the production of a strong and healthy crop of wheat. The soil may, indeed, be arrain partially restored to its former denseness, but it cannot be so perfectly consolidated by the means of rolling, as it had been by the previous state of rest in which it had been left. The treading of sheep is a plan very generally resorted to for giving a firm bottom to wheat lands; and florks are folded in dry weather, upon young wheat, previous to the frost setting in, and afterwarls early in the spring, with very beneficial effect. In Norfolk, intieed, they are not unfrequently fed upon the wheats, with turnips carted from the other fields and spread upon the land.

\section{PRESSING MACHINE.}

An ingenious mode of compressing light soils has, however, been long practised by some farmers in the use of a machine called the "LandPresser," or "Furrow-Slice Compressor," which being drawn over land recently ploughed, leaves it as if the work had been executed by a drill sowing machine, pressing down the grips, or channels left by the common ploughs, laving the furrows flat at the bottom, compact, regular, and in a proper state to receive the seed wihout any further preparation, so that no hollow places may remain in which it can be buried too deep.

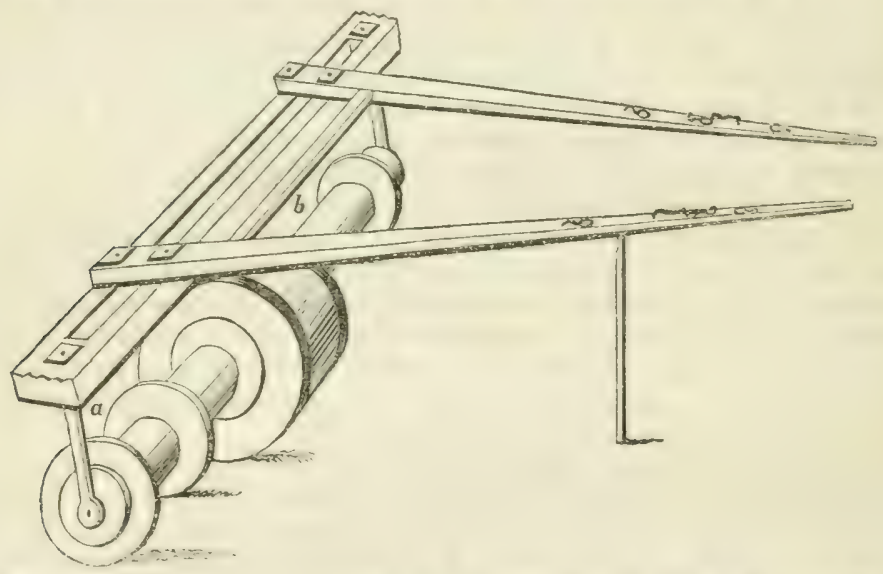

Ahove is the represcntation of an implement of this kind, for which a premium was a few years ago awarded by the Bath and West of England Acrricultural Society to Mr. Charles Adains. It is worked by one liorse, which walks in the furrow, and follows two ploughs. The small wheels repuesent three cast-jron rollers, which are fastened in an oak shaft, or roller. The larger ones, marlied a, weigh from $2 \frac{1}{2} \mathrm{cwt}$. to $3 \mathrm{cwt}$. each, are from 2 feet 9 inches to 3 feet in height, and act between the furrow-ilices. The small wheel, marked $b$, worlis on the unploughed land, excent where the implement is fullowing the two last furrows; wh:n, if properly adjusted, it will run between the two last furrows, on

* Wehb Hall's Prize Lssay on the growth of Wheat: Trans, of the Bath and West of England Soc.-Vol, xv, Art, xiv. p. 132. 
the other side. The large wheel, or roller, which is made by screwing planks or wedges between the ironed bonds, works in the furrow, and both prevents the machine from going out of the proper line of draught, as well as forms a proper space for the next furrow-slice, as it can be easily adjusted to any breadth of furrow, by shifting the wedges on the large roller. The cost is from $5 l$. to $6 l$, according to the weight of the rollers*.

Au improvement has since been made on this machine, by screwing two forked cleavers to the back part of the frame, immediately behind the pressers, to scrape off any earth that may adhere to them during their action; and the draught is regulated by a chain passed through pulleys placed between the shafts, behind the horse, and fastened to the draughthooks of his collar $†$.

The utility of this instrument is very conspicuous wherever it becomes essential to the important object in good farming of making light land heavy; for additional weight can, if necessary, be laid upon the frame, so as to suit the state of the tilth; and it has been invariably found that the soil, when thus compressel, has lost its turfy appearance, has been rendered firm under foot, and the plants of wheat liave grown more strong and healthy than where it is has not been used. The seed may be sown broadcast, or by afixing a sowing-box to the implement. The whole process of pressing and sowing can indeed be effected at the same time, as nothing further than a double turn of the harrows is requisite to complete the operation, and the seed comes up so evenly, that a considerable saving may be made in the quantity. Drilling and pressing have thus been long practised in Berlshire, where a machine of the following description was many years ago invented by Mr. Berriman of Speen+.

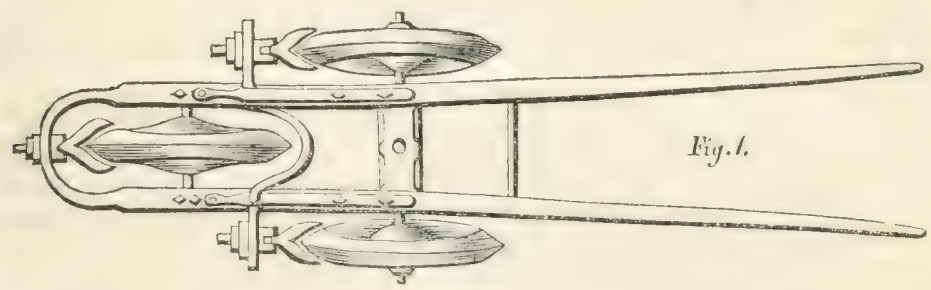

It consists of very heavy cast-iron wheels, with angular edges, set on at fixed distances, as represented in the bind's eye view at fig. 1; though by extending the axle the whecls and scrapers could easily be set at difierent

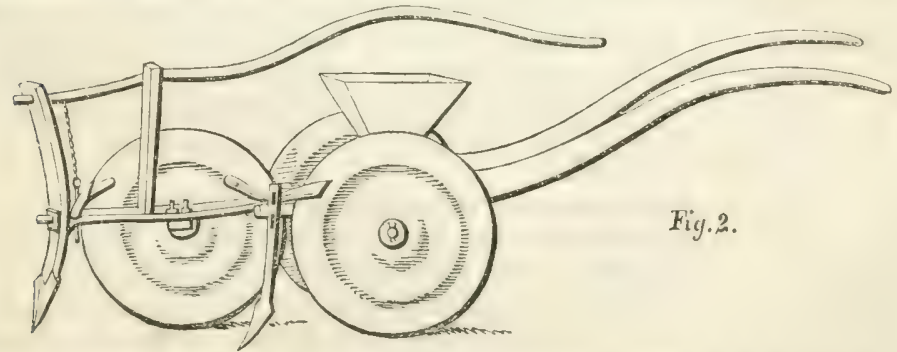

* Trausactions of the Bath and West of England Society, Vol. xv. Art, iv. + Quart. Jour. of Agric. N. S. vol. iv. p. 546.

\$. Survey of Berkshire, p. 123. 
distances, so as to suit the furrows in various linds of work. IV hen used also as a drill, the seed-box and coulters are added as in fig: 2, which represents the machine when in action.

\section{MANURE.}

Soils of an ordinary quality, when fallowed for wheat, are very commonly dunged in the course of the summer; but in those of a richer nature, or those which have been liept in heart by the previous application of manure to other crops, it is dispensed with; and in no case is it ever applied by any farmer who understands his business, at the time of sowing. Experience has, indeed, shown that its application is very generally rather productive of an increase of straw than corn; that the crop is thus subject to be lodged; and the wheat has been found inferior in weight, as well as more subject to smut than when it has been avoided. An attentive and experienced observer can, indeed, discriminate by the bearded appearance of one end of the grain, whether it has been forced by putrescent manure, or has been spontaneously grown upon a free and well-tilled soil. Intelligent men, therefore, endeavour so to arrange their alternation of crops, as that the wheat shall come in before it can have exhausted the power of the dung; and, when it does not follow clover, they, if possible, make it succeed a drilled crop, either potatoes, mangel-wurzel, or peas; or more generally beans. When, however, the state of the land is such as that a bare fillow cannot be avoided; that it is too poor to afford the promise of a crop without the aid of manure; that the refuse of fish, blubber, rape-dust, or any of the extraneous manures of a putrescent or nutritive quality cannot be procured, and that, consequently, farm-yard dung must be employed, it should then be previously mixed with the scrapings of roads and ditches, the headlands of fields, or earth of some kind to form it into a compost, in which state, when completely decomposed, it may be laid upon the land with safety.

Chemistry, with all its powers of analyzation, has hitherto alded so little to our knowledge of the food of plants, that our acquaintance with the subject has been chiefly derived from experience; and we are still at a loss for a scientific account of the mixture and application of composts ; though it may be reasonably supposed that, as different plants require different species of nourishment, a selection might be advantageously male of the matters most appropriate to that object. Thus it has been conjectured that wheat, containing more of the substance called gluten than exists in any other species of grain, would be peculiarly benefited by the application of animal manures, as ajproaching nearer to its nature than those composed of vegetables; and some experiments have tended to prove that the idea is correct. In our present ignorance of the subject, however, farmers can do nothing better than to collect any animal and vegetable refuse which fall within their reach, and incorporate them together for the formation of composts, which will in all cases be found more suitable than raw dung to the growth of wheat.

There can be no doubt that application of lime is beneficial to the production of wheat, as improving the quality of the grain, and correcting the adiesiveness of the sirong clays upon which it is usually grown. 'The nature of the soil, as well as the amount and period of its former employment, should, however, be attentively examined, before any further use of it be allowed; for, if applied in a causlic slate, it acts so powerfully as a stimulant, that if the land be not supported by an equivalent application of putrescent manure, it will speedily become exhausted. If mixed in com- 
posts of dung, or other putrescent matter, it also prevents their fermentation, and it should, therefore, never be laid upon land in which those manures are not already decomposed; but it materially assists in the destruction of weeds and insects, and, therefore, may be employed with manifest advantage when discreetly used. Mild lime-or lime in an effete state -is, on the contrary, of a totally opposite character; may be used with perfect safety in the formation of composts, and is not injurious when laid upon the land after the application of dung. It also binds sands and gravels, while it opens clays. In whichever state, whether effete or caustic, the lime be applied to a wheat fallow, it should, however, be intimately combined with the surface soil previous to the sowing of the sced.

\section{SEED AND SOWING.}

The time of sowing winter wheat must depend both upon the state of the land, as well as the season, and it is not always in the farmer's power to choose the moment which he would prefer; for if the corn be suwn after another crop, that crop must first be removed, and even if it be sown upon a fallow, the operations of a late harvest, or the state of the weather, may interfere. It is, however, generally recommended to put it into the ground as early as may be convenient in autumn, and, on strong soils, it is not unfrequently sown in the latter end of September, though more usually in the course of October or the begimning of November. Wheat sown early on friable loams is, however, apt to exhaust its vegetative powers so much, as to grow weak and languid at that period when vigour is most requisite, and on poor soils, the plants being so rapidly deprived of their vegretative properties, become foxy. The seed is, therefore, not generally sown before the 10 th of October; and if the land be then either so wet as to impede the working of the cattle, or so dry as to risk the vegetation of the seed, the operation is sometimes delaved until Norember or December. When sown after turnips, wheat is, indeed, sown by many farmers throughout the winter, according as the land is cleared, until the middle of March*; but, when deferred until that period, the seed chosen should be of the true spring species. Perhaps it may be safely assumed as a general rule, that strong Janis should be sown from the middle of September to the latter end of October; and those which are thin and shallow, probably from the latter part of October to the middle of November. Early sowing is, however, on most soils, adrantageous; for late-sown wheats are more apt to become mildewed; and even should they escape that danger, yet, if the season prove unkind, they will not ripen so soon by some days, as com which is sown earlier, and every experienced farmer well knows the inportance to be attached to this fact in a fickle harvest.

The quantily of seed per acre must necessarily depend upon the mammer in which it is sown-whether broad-cast, drilled or dibbled; on the various modes of performing which operations we have already said all that we deem necessary, and, as to their results, we have only to add, that so far

* An instance is mentioned in the Survey of Northumberland, of many hundred acres being sown in Glendale-ward so late as the begiminy of April, which were all well harvested, and produced, on an average, 24 bushels per acre of excellent grain, in many cases superior to that sown in autumn, which is rather singular, as it is generally thought that wheat sown so late does not produce grain so well perfected as that which is sown earlier--C'Third Edit., p. 75. Juring a very wet season, Mr. Brown, of Markle, also delayed his sowing until after the 19th of February: he then sowed 145 acres of Essex White, and Egyptian Red Wheat; and although the harvest was about ten days later than usual, his crops yielded from 24 to 40 bushels per acre, of a first-rate sample.-Middlesex Rep., 2nd Edit., p. 211.

Vox, II. 
as our own experience goes, we have seen equally fuce crops of wheat produced in either way. In the first, it is usually at the rate of $2 \frac{1}{2}$ to 3 and sometimes $3 \frac{1}{2}$ bushels ; but if drilled or dibbled, it must be governed by the distance at which the rows and the holes are placed: thus it is drilled at various intervals, from six to twelve inclies apart, and generally dibbled at about eight inches by three. Various experiments have been tried to ascertain the effect of different distances in the production of the crop, and the result has shown the best space at which wheat can be drilled to be from seven to eight inches*. From 2 to $2 \frac{1}{2}$ bushels are thus sown; though some farmers limit the quantity to 6 or $\mathrm{S}$ pecks: when dibbled, from 5 to 7 pecks are considered sufficient. This, however, must also be partly regulated both by the nature of the soil and of the seerl, as well as the time of sowing ; for if both the corn and the land be of superior quality, less seed will serve than if they be of a bad kind; and whatever may be their quality, the quantity of seed must be somewhat increased in proportion to the lateness of the season at which the grain is sown.

In the remarks which we have made upon the growth of corn, we have stated it as our opinion, that farmers generally err on the side of sowing too profusely. The practice, however, has been still further extended by the recommendation of $\mathrm{Mr}$. Colie, of Norfolk, on whose estates wheat is now usually sown at the rate of full four bushels per acre. The reasons upon which it is founded, are stated by Mr. Blaikie, his land-steward, as having partly arisen from accidental circumstances, but to have been adopted as a general rule, after repeated trials, upon a very extended jractice; the result of which has shown that carly and thick sowing is productive of an increase in the exops, and is also a preventive of mildew. The experiments $\uparrow$ recorded by Mr. Blaikie are no doubt strongly corroborative

* Mr. Burroughs mentions his having sown wheat in drills at eighteen inches distant, which in the following March were horse-hoed, and then landed; in April they were again hoed and landed, which causer the plants to tiller so much that they nearly filled the space between the drill: the heads grew extremely large, but loose and open, and the crop in many respects proved unprofitable. In the following year he hoed the drills in the same manner, but did not land them, and found that the quality of the grain was very superior. Still, however, he observed the straw to be too luxuriant, in consequence of the intervals between the drills being too wide; in a third experiment, he therefore narrowed them, and found the produce more and of better ciuality. - On the Cullication of White Crops, p. 12.

+ They are as fullows:- "The first was in a thirty-acre brake, or field of wheat: the seed was sown iu the autumn upon a stale furrow, after a one-year's clover ley; the soil a kind of sandy loam, incumbent upon a chalky clay sub-soil. Two drills started at the same time in the middle of this field; one drill worked towards the west and the other towards the east, the rows ranging from north to sonth. Both the drill-men received the same directions, and one deposited the usual quantity of seerl, fuur bushels to the acre; but the other, being a young practitioner, ganged his drill wrong, and deposited only three bushels to the acre. The result was, that the four-bushel seeding escaped the mildew, and was a very fine crop: the three-bushel seeding was mildewed, and was the only thin wheat, and the only mildew worthy of notice ujon the Holkham furms.

"The other experiment was in a thirty-five-acre field of wheat, sown in the same manner, after a clover layer of one year: the soil a lighter sandy loam than the former; the sub-soil similar. A new drill was put to work upon this field, and the man had instructions to sow four bushels of seed to the acre; hut the liacksmith having gauged the drill improperly, the labourer went on with it as put into his hands, and deposited five bushels to the acre. The mistake was discovered. and corrected, after the first day's work; and the remainder of the field was finished at the rate of fuur bushels to the acre. This accidental experiment was exhibited at the following sheep-shearing, and was subse. quently viewed hy many practical men, who gave it as their decided opinion, that the five-bushel seeding was the best crop in the field. 'The whole was, however, extraor-

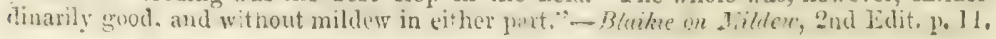


of the benefit to be derived from the plan : the best proof of its efficacy is, however, certainly to be found in the success with which it has been attended; though this we imagine to be chiefly owing to the judicious rotation of crops, the proper application of manures, and the general superiority of management uncier which his farms are conducted. It is also to be observed, that the greater part of his land is of a very poor and sandy nature.

It must be evident that, supposing the seed to be, in all cases, equally good, and the season equally propitious to its growth, yet the quantity to be used should be in a great measure governed by the state and quality of the land upon which it is to be sown. Now the farms which Mr. Colie has under cultivation being in general light and thin, as well as lying in a very exposed situation, mainy of the plants may naturally be expected to perish from want of nourishment and protection. On such soils, and in such situations, it may, therefore, be the mest prudent course to fill the land with plants at seeding time; but judging from all that we have seen and read of the prejudicial effects of thick sowing upon good land, we cannot but consider it as injudicious, both as a waste of seed, and a disadvantage to the crop. On this, indeed, in adlition to what we have alrealy said on the subject in a former chapter, it has been justly observed by Mir. Burroughs, "that soils naturally rich, or such as are highly improved by cultivation and manure, will require much less seed than those in an unfertile state; on the contrary, the lighter and the less cultivated the soil, the greater will be the quantity of seed which it requires. The reason is obvious; plants tiller more in xich and strong tiran in unfertile soils, and, therefore, occupy respectively a greater space : they are less liable to be killed on good soils in the winter months, and every plant generally comes to maturity; the straw also becomes more luxuriant, and consequently requires a greater circulation of air to preserve it in health and vigour; whereas the plants on light soils will but weakly and partially tiller; therefore this deficiency of stock on the land must be supplied by producing a greater number of plants; and as the straw will not be so liable to rot or mildew on light soils, little dancer need be apprebended on such from growing too tisick a crop*.

The grain may be sown at the depth of from two to three inches upon a clay soil; and upon land which is more dry and porous, even at four; for it will thus obtain a frmer loold of the earth, and will be more out of the reacil of that extreme drombt to winch those soils are frequently exposed. It may, therefore, on such land be safely put in upon a superficial ploughing, when not sown upon a clover ley. These are, however, the extreme depths. On every soil, the nature of the land, as well as its condition, should also be vell understood, before the depth can be properly determined; for if it be a heavy, wet clay, the seed should be sown proportionately nearer to the surface, than if it consists of a friable loam; and if it has been brought into complete order by the operation of a summer-fallow, it will necessarily be in a state of opemess which will allow of the seed being placed deeper.

\section{SPRING TIILLAGE.}

Although wheat generally supports the rigours of the winter, yet the uncertainty of the ten perature in our northem climate is such as sometimes to expose it to hazard. On the return of suring it is, therefore, occasionally found in such a sickly state as to afford no prospect of a crop : this particularly occurs on poor soils, lying in a low situation, which have 
not been sufficiently drained; and it has thus become necessary to break up the land, in order to re-sow it with Lent corm. This, however, should not be hastily done; for after a few days of warm weather, the plants are firequently seen to shoot forth, and tiller with great luxuriance. Springwheat has also been dibbled among the crops, and both species have ripened at nearly the same time, with such little difference in the quality, as to render the plan advisable.

Strong adhesive clays also frequently become hide-bound after a wet winter; in which case many farmers resort to the practice of harrowing the ground in spring, in order to loosen the surface, and others invariably resort to it when the crops are thin, with a view to encourage them to shoot out and fill up the vacancies: it has, however, been objected to by some intelligent men, as occasioning mildew*, and others would dread the destruction of their crops by the tearing up the roots of the plants. The practice is therefore by no means general in this country, and evidently can only be carried into execution with propriety on lavid which has been sown broad-cast; but, throughout many parts of Germany and Poland-with which countries the writer of this is intimately acquainted, and in which he had during three years the occasional management, though only as an amateur, of a fine arable farm, consisting of 900 acres-it is universal. There, on the first return of fine weather, the harrows are immediately passed freely over the wheat; so freely, indeed, that the whole field wears the appearance of laving been newly sown, for the plants aypear buried under the soil thus freshly stirred, and an aniple top-dressing is thereby given to the crop. The crust formed upon the surface of the soil is thus broken, and the cromd is rendered more pervious to the coronal root of the plants, which in a week or ten days spread and tiller with great strength. The operation is performed upon every kind of soil, but of course with harrows of a weight proportionate to the tenacity of the land, and not heary enough to tear up many of the roots, though if a large quantity be not destroyed it is considered immaterial; and any farmer who omits harrowing is thought umpardonably negligent. It should be executed when the crop begins to re-vegetate; which necessarily depends on the climate and the state of the season: here it usually occurs, in good soils, some time in February, and in those of a poorer kind, rather later. Attention is requisite to this ; for if the work be done while the plants are in an inactire state, they may be rotted, and if when they are too forward, their growth would be checked. It must also be observed, that it should never be resorted to when the crop is root-fillen; for, in that case, the roller, and not the harrow, should be passed over the soil $\dagger$.

Light soils are, in this country, now so generally drilled for whent, that harrowing them would be injudicious, because the implement would strip the land too much in some places, and in others would leave the plants untouched; nor are soils of that description subject to become hide-bound, as are heavy clays, and, if very light, the stirring of the earth about their roots might have the effect of weakening their hold upon the ground. Land of that description is, however, not uncommonly sown with clover in the spring, and to the successful growth of that crop, the deposit of the seed upon a fresh surface is essential. It should, therefore, be looed, instead of larrowed, and by passing the hoes carefully between the rows of the drills, the earth will not be stirred about the plants so as to injure their roots.

"Blaikie on Mildew, 2nd Edit. p. 9.

$\uparrow$ See Von Thaër; Princ. Rais. d'Agric., 2nde Edit, tom, iv. $\$ 978, \S 1014$. 
Most farmers, however, object to the use of the horse-hoe in the performance of the operation upun wheat; both from the great difficulty of so correctly guiding the implement as to prevent it, in some places, from cuiting up the roots, and from the injury lone by the treading of the horse. The latter objection is, indeed, not of much moment, for the work cannot be performed when the weather is wet; there is, therefore, but little danger of poaching; or, if the weight of the animal be thought too great, a donkey may be employed. It is, however, more usually performed with the hand-hoe; but the process is tedious, and very generally indifferently executed, for a great portion of the ground is left untouched, and many of the plants are always carelessly struck out. He have seen this partially-though certainly not effectually-lemedied by the use of a hoe of the following description; which being drawn regularly through the drills by a labourer, is more under his command than when drawn by a horse, besides its avoiding much of the poaching ; and, if he uses it carefully, no injury can be done to the plants. It can however be only used upon land of the lightest kind, and not too much encumbered with weeds, or the labour might prove too secure, unless the number of hoes were diminished; and it is, of course, only applicable to drills at the same distance.

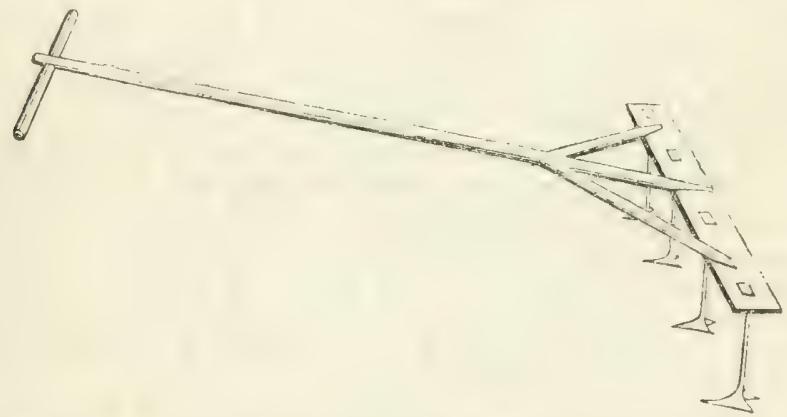

By these operations-of harrowing and hoeing - the land is also in some measure cleansed of weeds, and they are not unfrequently allowed to supersede that of hand-weeding. This, however, is a most injudicious saving; for, in no other way than by plucking the weeds by hand, ean they be got out from spots where they are close to the plants of wheat, and where they are consequently the most injurious to the crop. The process should, therefore, never be neglected, whatever may be the state of the land; for it never can be so thoroughly clean as that weers may not be found in the spring, and the farmer who does not take every means in his power to eradicate them will in the long-run find himself a loser by his economy.

When the temperature of the season has occasioned the vegetation to be very forwarl, the winter-sown crops of wheat are, however, subject, on rich land, to become what farmers term winter-pioud; or sprout with such luxuriance in the early part of the spring, that the vigour of the plants is exhausted by the sudden growth of a great mass of straw, and the grain becomes proportionately unproductive. From the greater height to which the straw thus rises, and as well the weakness of its stems, the crop is also more in danger of being lorlgerl by storms, and is found to be generally more liable to mildew; it is, therefore, in such cases, very commonly eaten down by sheep, or, by some farmers, it is occasionally, though very rarely, mown. The progress of vegetation is thus checked, and the apparent danger is 
avoided, but it requires great circumspection and practical experience to ascertain when it may be proper to adopt it; for, although the temperature of the season may have pushed the plants too forward, yet a change may immediately take place, and their having been thus weakened, will throw them too much back.

If the plants tiller with such strength as to grow in thick tufts which cross each other, or that they appear likely to become lodger, then it may be safely adopted; otherwise, it is better to leave the crop to its chance, without meddling with it, and in no case should it be attempted unless the vegetation be extremely rank. The practice of feeding, though by far the most general, has yet been objected to, because the sheep prefer the tender blade in the lieart of the plant, which ought to be preserved, and reject the coarse blades which ought to loe taken off; but if arlopted, such a number should be put upon the ground at once, as will eat the wheat down to the required distance promptly, and, when removed, they should not be again returned to the land. If mown, the work should be very carefully performed, at such a distance from the surface of the land as not to touch the heart of the plants*.

\section{SUCCESSION OF CROPS.}

Universal experience has proved, that to sow wheat a second time upon the same land without an intermediate crop, is exhausting to the soil, and, if pursued for any length of time, eventually unprofitable to the farmer; for, although it was pursued by Tull, and has been upheld by a few other writers, yet his plan consisted in sowing the corn at such wide intervals that only lualf of the grouind was really cropped, and the remainder was left in a state of fallow. It is, howerer, a curious fact, that the white and brown species of wheat, if sown alternately, produce better crops than if the same sort be sown in the following years. It is, indeed, injudicious to sow wheat upon the stubble of any corn crop, and it can only be justified by peculiar circumstances; but, when expedient, it is always found to succeed better after oats than after barley.

Clover has been called "the mother of wheat," and the ley is justly a favourite preparation for the crop; but it onght not to be repeated more than once in eight years, and it should then only be sown when the ground is in such a state of cleamness, and so well manured, as to ensure the pro. duction of an abundant sward of grass; for, if this be not obtained, the wheat will invariahly be found proportionately deficient. On good lands, which do not require a second year in grass, it will also be generally found most acivisable to break it up after the first year's crops have been taken off; in which case, the wheat may be sown, as we have alrealy ubserved, upon a single ploughing, and thus a very considerable expense will be saved in the tillage of the ground.

Much difference of opinion prevails upon the subject of cutting both crops, or only cutting the first, and allowing the second to he pastured: the latter is the most generally practised, and consequently the most approved, but so many instances have been stated of superior crops of wheat having been obtained after both the crops of clover had been mown, that it is very difficult to draw any positive conclusion regarding the superiority of either system. When the crops of clover have been very good, the experiments are grenerally in favour of allowing both to stand for the scythe; and we believe that the superiority of that plan will be found to consist in

* See Blaikie on Mildew, 2nd Edit, p. 19 ; and Von Thaër, Princ. Rais, d'Agric., Inte Edit., tom, ir. \$ 1016. 
the greater denseness of the sward, whether the increased vegetative porer which is thus imparted to the wheat be occasioned either by the shade aflorded to the land, or by tise nutriment derived from the atmosphere. It should, however, be clearly understood, that the second crop, though mown, is not to be allowed to stand for seed; for the ground will be thus considerably exhausted, and the season generally too far advanced to be favourable to the sowing of the wheat.

Tares, though highly recommended by some writers, have grone much out as preliminary to whent-particularly in the north; for they are found to be very productive of the crub, and render the land otlierwise so foul, that they can only be sown with arivantage if fed off, or cut carly lor soiled cattle, so as to form a bastard fallow.

The other crops which usually precede wheat, muler the aiternate system, are those which, being drilled, are susceptible of lieeping the land in a clean condition by the operation of the horse-hoe-such as peas, beans, potatoes, mangel-wurzel, turnips, and cole. All these, with the single exception of potatoes, are, however, not unfrequently sown broad-cast-by sone farmers, throurh slovenliness; and, on some land, in consequence of the flinty nature of the soil preventing the empioyment of the proper implements. The three latter, bemg also rearded as winter-fa!low crops, are more grenerally prenaratory to the sowing of Lent corn; or, if succeecied by wheat, it is most commonly of the spring-species. It is unnecessary that we should add anything to what has been already said on the expediency of drilling pulse and root crops as a preparation for wheat; particularly as they will be each treated of separately: but in regard to the land wo which the three first are best adapted, it should be observed-that peas suceecl best on calcareous soils; beans, on those approaching to strong clay; and potatoes, on those of any light and friable nature.

The main object of all these crops is to manure them lieavily, in preparation for the wheat; with the exception of turnips, they are, therefore, very generally dressed with long dumg, the slow decomposition of which alluws it to retain a material portion of its strength for the protuction of the com. Peas, too, are not tinferguently limed, and are then found to get the lind in fine condition; but they must not be repeated during several years, or they will fail. Beans are the most usual crop upon clays and strong loams, and the facility with which the land can be worked when they are drilled at proper distances, renders them a very superior introduction for wheat; though, in some seasons, its sowing is comsiderably impeded by the lateness of their harvest. Heavy crops of winter-wheat have in many instances been reaped after potatoes; yet in other cases this has failed : there is, therefore, a great discordance of opmion respecting the propriety of planting potatoes in that order of rotation, and many experiments show that any of the species of Lent corn succeed better than wheat, when sown after them. This doubtless arises in a great measure from the lateness of the period at which they are usually removed from the ground, as well as from the carly sureading of their haulm preventing the working of the horse-lioe; for, althongh no doubt can be entertained that they are an exhausting crop, yet they are generally planted with such an abundance of dumg, that the lind camnot be impoverished hy their growth, though it may be biought into a better state by spring-tillagge.

It frequenily happens that winter-wheat is so much injured, either by insects or the inclemency of the weather, as to thin the plants to an extent which has occasioned a material deficiency in the crops. 'This may, however, be in a great measure remedied by either transplanting tufted 
shoots from spots where they may have grown too thick*; or, if that should be deemed too troublesome, by dibbling spring-wheat into the vacant spaces. We have seen many instances of this being successfully performed ; and one in which it occurred upon a drilled crop, which in the latter end of April showed very little appearance of producing more than a return of the seed. The drills were at nine inches apart; and about a bushel and a-half an acre of Talavera wheat was redrilled in the intervals. Both species ripened at nearly the same time; so nearly, indeer, that by allowing the spring-wheat to stand a few days longer than would have been thought prudent if it had been sown alone, and cutting the Lammas wheat rather earlier than usual, a trifling loss was only incurred upon either, and their joint produce was very abundant.

\section{SPRING-WHEAT}

Is frequently mixed by the dealers with the red Lammas winter species; for, although somewhat smaller in the grain and lighter in the bushel than the latter, yet the colour is the same, the flour is equally good, and their general appearance is so much alike, that it is difficult, if not impossible, to discern the difference. The time of ripening is however different; and when sown in the autumm, it has been so frequently injured by the frost, that the seed camnot be depended upon for the production of a winter crop. Although too tender to bear the frost of a severe winter, it is yet as quick in its progress to maturity as any species of lent corn, and many farmers sow it instead of barley-particularly on lands on which the good malting species of the latter grain camot be grown; for they have found, when the usual difference of price existed between wheat and barley, that a crop of the former was generally more profitable. It is also frequently sown when winter crops cannot be got off the ground in time, or when the season is unfavourable to a winter sowing; and after potatoes, it has been found to succeed better than the winter kinds. It should, however, be borne in mind that it is more exhausting; so much more so, indeed, that winter-wheat, if sown in the usual alternate succession, two years afterwards, will not produce so good a crop as when succeeding either barley or oats.

Spring-wheat does not appear to have been formerly known in England as a distinct species, for it is not mentioned under that denomination in any of the older books on lusbandrv. It has however been long extensively cultivated in the southern countries of Europe, and is there distinguished by names which mark the difference between it and winter corm*. Of these

* The transplantation of corn has been much ridiculed by practical men; and, on a large scale, it certainly would be attended with considerable trouble, though little more than that of dibbling, which was formerly decried as impracticable, and is now not uncommonly performed on some extensive farms. The great increase which takes place in the transplantation of wheat depends upon the circumstance that each layer thrown out in tillering, may be divided and treated as a separate plant; so that a very small quantity of seed will serve to sow an acre. Thus, some roots, lately transplanted by Mr. Lance, of Lewisham, contain each. after being diviled, 40 to 50 straws, with ears that are from 6 to 7 inches long. The account published in the 58 th volume of the "Philusuphical Transictions" shows, that by an experiment on transplantation made by Mr. Miller, of Cambridge, one grain of wheat fruduced $3 \frac{3}{4}$ pecks of corn, weighing $47 \mathrm{lb} .7 \mathrm{oz}$; and a recent statement by a cottager-mentioned in the "New Farmer's Marazine" - rives nearly similar results.

The saving in seed might thus go far to meet the expense of additional labour: an advantage in point of time would be gained in the sowing of land covered with rumips to he eaten off by sheep; and, alove all, some additional employment would thus be affordcd to a number of hanis now dependent unon parish subsistence.

+ Thus in France it is called "Bled de Mars;" in Germany, "Sommer Waitzen," from the season in which it is sown; and in Portugal, it is also linown as "Trigo de tres nezes," or three-months' wheat. 
a species, now well known as Tularera wheat, was brought from Spain during the late war by an officer serving with our army in the Peninsula, and is largely crrown, particularly on the light soil of Norfolk and some of our southern countries. It succeesls when sown either in the autumn or in spring; but having been found rather too delicate for our winters, it has been more generally propagated with a view to spring-sowing. It has very long straw, long ears, with a fine, clear, thin-skinned grain, and being very prolific, it has become a very general ficvourite, although it has somewhat degenerated in the cold of our climate: a defect, however, which can be easily remedied by an importation of the seed from its parent country. It can be grown-like all the spring species-on all wheat lands; but does not require so strong soil as the winter kind: the land should, however, be equally rich, though not too dry, and the soil should be brought into as perfectly clean a state of tillage; the best way too of preparing it being by a crop of turnips eaten upon the ground.

The season for sowing every kind of spring-wheat is from the latter end of March until the beginning of May; but the middle of April is preferable, as both avoiding the chance of white-frosts, and ensuring a greater prospect of gentle showers for the promotion of vegetation. The quantity of seed must be larger than that commonly employed in autumn; and, if sown broad-cast, is usually from three to three and a-half bushels per acre, when put in alone; but if clover be also sown, then two bushels and a-half, or nime pecks are considered sufficient; the crop, however, being in that case smaller. This, however, must not be assumed as an argument in favour of sowing a large quantity of seed; for the diminution of the whent is occasioned by the simultaneous growth of the clover.

In warm summers, spring-wheat comes to perfection about a fortnight earlier than the common wirter species; but it is rather inferior in productiveness, although the difficulty of getting winter crops off the ground frequently compels the farmer to substitute spring for autumn wheat, rather than clear his land prematurely, and thus disarrange his winter economy : indeed, if the land be in a wet state, it will be found more prudent to sow this species in the spring, when mellowed by the frost, than Lammas wheat in the month of December. Its tenderness has however thrown it a good deal out of use; for, if the season be not of equal temperature, it is apt to fail: either much cold, wet, or heat, heing prejudicial to its growth. It might be supposed from this, that the genial climate of Ireland would suit it better than that of England; but the summers are there said not to be sufficiently ardent to mature the crop before the commencement of the autumnal frosts; though perhaps a better reason for its not being cultivated in that country arises from the general inattention of the Irish farmers to the growth of green crops.

Mr. Burroughs, however, tells us that in order to give a fair trial to its growth in Ireland, the Dublin Society, some years ago, offered premiums to persons who would grow it, and report on the result. Fortythree persons tried it in different proportions-limself among the numberand the reports given by the different individuals were six to one against the probability of being ever able to establish its cultivation, with a certainty of insuring an adequate remuneration to the farmer*.

\section{PRODUCE AND QUALITIES.}

The farm produce of wheat varies so much upon different kinds of land, and is so much governed by climate and mode of cultivation, that it is

* On the Cultivation of White Crops, p. 24. 
difficult to form any acreable estimate of the amount. On soils of average quality, in ordinary seasons, and under the common course of management, it may, however, be fairly calculated at three quarters, or perhaps 28 bushels per Imperial acre. To produce the latter quantity, circumstances must, however, be favourable, and anything beyond that may be considered large; though, on some land, four to five quarters are not unusual, and we have lately heard of a crop in Warwiclshire amounting to rather more than 60 bushels per acre, of clean screened corn of superior quality. The weight may average $60 \mathrm{lbs}$. per bushel.

The straw is generally reckoned to be about double the weight of the grain; an acre, producing three quarters of wheat of the ordinary quality, may therefore be presumed to yield about $26 \mathrm{cwt}$.

The use to which the grain is applied is almost exclusively that of food in its various preparations, and cliefiy in that of bread, though a considerable quantity-but generally of an inferior or damaged lind-is employed in the manufacture of starch. This preference is due not only to the sujeriority of its nutritive properties, but also to their peculiar nature; for " more water is consolidated in bread made from barley, and still more in that from oats; but the gluten in wheat, being in a much larger quantity than in any other grain, seems to form a combination with the starch and water, which renders it more digestible than any other*."

From a careful analysis made by Sir Humpliry Davy, it was found that the quantities of nutritive matter afforded by 1000 parts of wheat of different qualities, were-

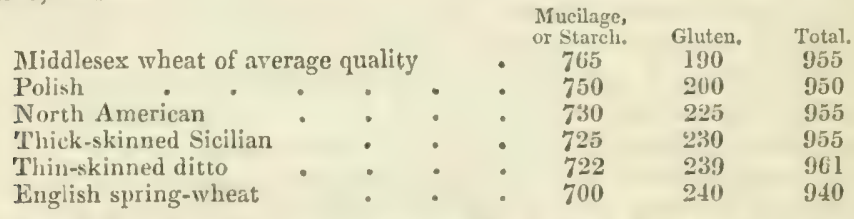

From which it has been concluder by Sir Joseph B̉anks, - " that bread made of the flour of spring-wheat is more nutritivus than that from winterwheat, because spring-wheat contains a larger quantity of gluten, or halfanimalized matter; and also, that a miller ought not to deduct from the price of spring-wheat more than 2 per cent. on the money price of winterwheat of the same weight, as the excess of insoluble matter, or bran, is no more than 2 per cent. when compared with good English wheatt." This, although the reasoning of a man of ability, however, only serves as an additional proof that mere scientific linowledge, when unaccompanied by practical experience, can never be relied upon by farmers; for it is well known that the qualities of all grain rary accurding to the soil upon which it is grown, and the manure with which it is nourished, and that wheat when dressed with stable-dung, or particularly if folded with sheep, contains the largest portion of gluten: any generai rule of this lind can therefore be only regarded as an unsupported theory.

\section{FLOUIR.}

The flour of wheat which is cut before it is quite ripe is whiter than that which is allowed to come to maturity, and bears a higher price in the markets. The grain which is intended for the miller should therefore be reajed before it has reached its perfect gowth; hut that which is meant

* Lectures on Agricultural Chemistry, lect. iii., p. 121.

+ Communications to the Board of Agriculture, vol, v., art. viii., p. 281. 
for seed should be alloweci to stand until the last moment at which it can be cut with safety. The corn is ground into meal of various degrees of fineness; and a bushel of $60 \mathrm{lbs}$. ' weight generally yields, when dressed, about the following quantities, namely:-

\begin{tabular}{|c|c|c|c|c|c|}
\hline Fine flour & - & - & & - & $25 \frac{1}{3} \mathrm{lbs}$. \\
\hline Household ditto & & - & - & - & $22 \frac{1}{2} \mathrm{lbs}$. \\
\hline Pollards & - & - & - & - & $8 \mathrm{lbs}$. \\
\hline Bran . & - & . & & . & 3 lbs. \\
\hline
\end{tabular}

A bushel of wheat, therefore, averages $48 \mathrm{lbs}$. of both kinds of flour, of that sort called "seconds," which is alone used for making bread throughout the greater part of England; and a sack of marketable flonr inust by law weigh $280 \mathrm{lbs}$. These products must, however, vary accoriling to the quality of the grain, as some will produce more or less bran, as the lousk may he more or less thick; and the baliers admit they can make two or three more quartern loaves than the usual quantity, from one sack of flour, when it is the genuine produce of good wheat. 'Thus it was found upon a comparative trial between English and Scotch wheat, of apparently equal quality, that there was a difference in favour of the former of no less that 13 libs. of bread upon $2 \frac{1}{2}$ cwts. of flour*.

\section{SEED WHEAT.}

We have already observed, in our remarks unon the growth of corn, that most experienced famers prefer a change of seed to that wrown by themselves. This is, indeed, more generally considered desimale with regrard to wheat than any other species of grain, and some men look unon it as so important, that they will make almost any sacrifice raiher than not have a different kind from that produced on their own land. The change, however, is not always successful; for it frequently lapplens that a fine sample of wheat produces an inferior crop, and good grain is grown from seed of indifferent quality. This must, however, be in most cases attributed rather to the soil and season than to the sort of corn, and it will ever be found the most prudent course to choose the best qualities for seed.

In order to judge correctly of the sample, it should be retained a minute or two in the closed hand, and then passed gently through it, to ascertain if the grain be plump, hard, dry, and smooth, with a certain sense of mellow fulness in the feel; for, if it handles rough, and does not slip rearlily through the fingers, it will be found thick-slimed, damp, and unprofitable to the milier. Of

* The experiment was upon a Winchester quarter of each kint, ground and baked into bread at the Rotherhithe mills, near London, and the result was as follows :-

\section{SCOTCH.}

Weight $60 \frac{1}{2}$ lb. per bushel



Waste $11 \mathrm{lb}$.
ENGLISH.

Weight $60 \mathrm{lb}$, per bushel.

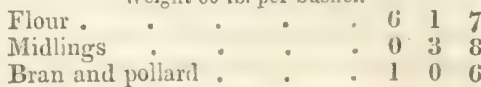

IVaste $11 \mathrm{lb}$

From the above trial it will, however, be seen, that while the Scotch wheat, weirhing 60t lis. per bushel, yielded only I bushel of bran or pollard, the English, weighing 60 lbs., gave 6 lbs. more of bran and pollarl, which is contrary to the general estimate of their properties, the English wheat being supposed to always yield the greatest cuantity of flour. The bread of each was of equal quality.

As to the rreater quantity of bread produced by an equal weight of Encrlish flour, the cause appears to be, that the English flour is more absorbent than the Scotch, and therefore, requires more water to bring the dough to the same consistency for being baked into bread; and the physical quality is supposect to be oceasioned by the climate of England affirding greater strength of sunshine during the ripening of the com.See the Farmer's Mag., vol, xxii., p. 2. 
whatever species it may he, it should also be carefully examined, to see that it be of a bright and healthy appearance, the grains of nearly equal size, and unmixed with the seeds of weeds, and smutty or sprouted corn. The smell should likewise be noted; for the least taint of its sweetness is a sure sign of its having been either damp or heated. The weight can be guessed within a pound or two in the bushel, by being poised in the hand by any one accustomed to examine samples; but aceuracy of jurgment can only be attained by long experience. The quality of any one species can thus be correctly estimated by any one who is in the habit of attending a corn-market; but large quantities are imported from abroad, and also marle up for sale by the dealers of this country in a mixed state.

The foreign wheat imported into this country comes principally from the Baltic, and the finest qualities produced in any part of the world are grown in the Polish provinees of Volhynia and Sandomir. It is of a surprising degree of whiteness: the grain is fine, hard, and thin-skinned; and, in consequence of the small quantity which it contains of bran, it vields a larger yortion of flour than the finest specimens of the celebrated Kentish or Essex species. It is, however, generally mixed with red-whest, and is known in our markets under the denominations of "good," and "fine high mixed""red-mised," and some more ordinary qualities; the first weighing from 61 to $64 \mathrm{lbs}$. * and the latter from 56 to 5 Slbs. per bush., running in the proportions of one-third white to two-thirds red; and the price varying from $10 s$. to $15 \mathrm{~s}$. per quarter less than the superior articles, in proportion to the quantity of red. 'This mixture, however, although not rejected by the millerswho frequently find the use of a portion of it indispensably necessary to the grinding of the new corn-yet renders it unfit for the use of the farmer as seed; for the different qualities could neither be advantageously grown on the same land, nor would they ripen at the same time. The wheat which is shipped in large quantities from Odessa, on the Black Sea, and which in scarce years sometimes finds its way to this country, it would be also dangerous to use as seed, for the length of the voyage generally occasions it to become heated. Some fine species have lately been imported from the Cape of Good Hope and from Van Diemen's Land; but it was found, when sown on one of the finest farms in Bedfordshire, that it would not grow; and it is said-though we know not with what truth-that "scarcely any wheat is ever known to vegetate in this country that had crossed the Line, unless particular care be taken to preserve it from the effects of the atmospheret."

\section{DISEASES OF WHEAT}

That all plants are liable to disease is generally admitted; but wheat is more subject to various distempers than any otlier species of grain, each proceeding from some unknown cause hostile to its growth, either in the soil, the atmosphere, the encroachment of parasitic plants, or the attacks of the numerous tribes of insects with which the air and earth abound. It is thus difficult to ascertain the cause of the maladies with which it is affected, for they are often of a complicated nature, presenting different symptoms according to the season, though they are well known under the names of 'Smut,' and 'Rust,' or 'Mildew ;' all, however, whaterer may be their origin, being usually comprehended under the common term of 'Blight.' Sir Joseph Banks thus takes it for granted, that blight, rust, and mildew are one and the same; though most farmers, and among them Sir John

* We recollect having, in one instance, seen it of the weight of rather more than $66 \mathrm{lbs}$. but it more commonly averages $63 \mathrm{lbs}$. per bushel.

+ Dimsdale on the Foreign Corn Trade. 
Sinclair, lave considered them as separate diseases, brought on at different periods, and occasioned by different causes*. The late Presidents of the Royal Society and the Board of Agriculture are therefore, on this point, at variance. The numerous treatises which have been written by ingenious men upon these disorders, have, indeed, in many instances, either neglected their proper distinction, or confounded them with each other; or else have applied cause to effect, and have so perplexed the subject that it is far easier to apply the facts which they have stated to every-day practice, than to attempt any abstract definition of their respective theories.

It may be assumed as a principle, that the immediate cause of every distemper which attacks the plants of wheat may be ascribed to the state of the season, combined with the circumstances of soil, situation, and seed. It is indeed not easy to class them; but the great body of farmers look upon rust, miklew, and blight as distinct disorders, arising solely from the influence of the atmosphere.

Mildew they view as a disease which affects the ear, though, as it is called the spotted distemper, it is apparently more injurious to the straw, and is brought on by causes somewhat similar to those which occasion blight, though at a more advanced period of the season. It usually first attaclis the leaf, and then the straw, just at the time the corn is blooming; and when it comes on immediately after the first appearance of the ear, the straw will also be aflected ; but if the grain be fully formed, then it is but slirhtly liscoloured. It has been supposed to arise either from a dewy kind of reddish gum, which attaches itself to the ear ; or from a prasitic plant, or fungous excrescence, which attaclis the wheat under peculiar circumstances of air, warmth, and moisture, and to be especially occasioned by damp, hazy, easterly winds. The following quotation would indicate it to be white:-

- Shield the young harvest from devouring blight,

The smut's dark poison, and the mildew white ;'

but it generally appears first of a dingy red, afterwards succeeded by darkbrown spots, which appear on the stallis from the first or second husk upwards, rough to the touch, increasing in size, and at last appearing livid, or approaching to black. It hardly ever appears until the period of ripening, but when it has fairly struck a crop, it then effectually prevents both corn and straw from maling any further progress, the wholc plant apparently going every day backward, till existence in a manner ceases. The damage thus occasioned depends, however, in a great measure upon the time at which the attack takes place, for if early in the season, the crop will be hardly worth reaping; but if the grain be well advanced, the loss is then considerably diminished.

Rust, like mildew, also seizes on the leaves and straw; a kind of clust gathers on them, which increases with the disease, and withholds the current of the sap until the plant is, in a great measure, exhausted: bence the grain is imperfectly filled, and the crop, of course, generally deteriorated. It is thus described in a small volume lately published by a person of great practical experience, whose work contains much interesting information on the subject of which it treats-

"Rust first appears on the leaves of strong growing plants of wheat early in May, but is not seen to fix itself injuriously on the culms till about

* See A Short Account of the Cause of the Disease in Corn, called by Farmers the Blight, the Mildew, and the Rust, by Sir Joseph Banks, Bart.; and Result of an Inquiry in to the Nature and Causes of the Blight, the Rust, and the Mildew, by Sir John Sinclair, Bart., M.P. 
the 20 th of July. Should the weather, at this last-mentioned period, be dry, and at a ligh temperature, no ill efiects take place; but should the weather then set in wet, chilling the air, and checking the exhalations from the ground, the straw is immediately struck, and suddenly changes from a bright yellow to a dingy liue, a certain sign that the blight, as it is commonly called, has seized the crop. About this time the grain is just arrivinc at perfection; if the attack takes place before this is effected, it never fills; if afterwards, less damage is sustained : the straw may be injured, but not the grain *."

This description accords very closely with that of mildew; but whether considered separately, or viewed as one and the same disorder, appraring at different periods of the plant's growth, both may be justly ascribed to an unhealthy state of the atmosphere. Farmers are, indeed, generally satisfied, that not only the extent, but the very appearance of blinht, rust, and mildew are entirely governed by the seasons; and that with respect to wheat, the kind sown, whether thin or thick-chaffecl, has a considerable effect in lessening or increasing their influence. It rarely lappens that they are felt in dry, warm seasons, except in confined enclosures or marshy ground, where the evening dews stagnate, and fogs are renerated; while, on the other hand, in every moist season, whether cold or warn, bliglit, mildew, rust, and gum on the ear, are always more or less prevalent. Thus, in looking over a blighted field of wheat, we may ubserve that he luwest and richest parts, or where the crop is thick unon the ground, are more affected than those which stand higher.

Some writers have imagined that the fungus is the effect, not the cause, of the malady : ti:e evil, as they contend, proceeding from exuberant growth and a surcharged state of the sap-vessels, and atvise the farmer to dress the gromil moderately, in order to save his crop. Were the stunted plants never spared, their opinion might have some weight; but in a blighted year, every plant, from the smallest to the largest, suffers. Thin-chaffed wheats are also much less injured than those which are thick-chafferl, which circumstance is in direct opposition to the doctrine of disease being brought on by parasitical plants, or fungi.

The same species of fungus, or one nearly aliied to it, is frequently seen on the common barherry bush, colt's-foet, and other plants; and the discase is thus commonly supposed to be communicated to wheat. Sir Joseph Banks indeed tells us, "that the vilige of Rollesby, in Norfolk, where barberries abound, and wheat seldom snceerds, is called by the opprobrious appellation of ' Hildew Rollesby.' A langh has been thus raised against olit-fashivined farmers, who eradicate barberry from their hedges; there is, however, more propriety in the prectice than superficial observers are disposed to allow; for as the ova float in the air, there is no means of checking their attacks: and although it is said to have been lately ascertained by Nir. Remnic, that the fumgus is a distinct species from that observed on the barberry, yet no harm can accrue from the precaution $\uparrow$.

Blight is accounted for, as being brought on the corn by the effects of very sudden changes of the weather, as during a hot sun and a still air, when the atmosplere is suddenly over-charged and obscured with fog or nist; or when, after a hot day anl night, the sun in the succeeding day has nut been suliciently clear and hright to dispel the vapuours which have

* Illustrations of Vegetable Physiology, by James Main, A.L.S., p. 284.

+ Main's Vergetalle Physiology, 1). 2S5; Farm. Mag., vol. vi. p. 225; Dumfries Rep, p. 186. See also an Account of the Barberry-bush Fungus, with Descriptive Plates, in the Devonshire Survey, p. 438. 
arisen, in consequence of the extraordinary exhalations from vecretation, occasioned by the increased heat. It may thus be produced by combined causes in different states of the air; or it mav arise in corn, if, when unslieathing its ear, it slıould happen to rain, and to be succeeded by a frosty night, for the grain will then be certainly injured in the part which is left uncovered; or if, when the corn is in blossom, and the farina just ready to scatter, much cold, driving rain should happen, one side of the ear may have all the farma washed away, or otherwise injured, while the other side may be only partially so. Even a dry frost will injure it, though not so materially; but any sudden check in the progress of vegetation, whilst the grain is forming, will probably exhibit a blighted or disordered car. The plants assume a pale emaciated appearance, the straw becomes spotted very thick with black or grey spots, looks mouldy, and the grain becomes lean and shrivelled ${ }^{*}$.

In the present state of botanical knowledge, as it regards the diseases of corn, it is out of our power to offer any remely for injuries arising from such various and uncertain causes; but as they seem to be chiefly occasioned by a close state of the atmosphere, they may be partiy guarded against by preserving as free a circulation as possible of air among the plants, by keeping the fences as low as the security of the crops will permit; and especialiy increasing its current, by drilling the grain instearl of sowing it broad-cast. It las also been fomd, - notwithstanding what has been said regarding the production of fungus, - that wheat which has been recently dunged has been found more sul.ject to rust and mildew, than that which has been sown unon a clean fallow; it may, therefore, be advisable to check the too rapid growth of the sap, by moderating the cxcess of manure, as strength and solidity will be thus imparted to the straw

Smut is a disease almost peculiar to the grain of wheat, the husls of which, instead of containing healthy farina, is filled with a black, stinling porder, rendering it unfit for the baker, and in many cases, utterly mimsaleable. Though clistinctiy known as 'Smut, it is yct often confounded, both by farmers and naturalists, under the different names of 'Smui-ball,' 'Pejperbrand,' Collibrand,' 'Brand-bladders,' 'Dust-brand,' and numy others ; but it is actually occasioned by two different species of very minute suistance, resenbling seeds, the one composed oi hard grains, like those of pepper, and the other of soft bladderst. It thus exhibits itself in two ways: in one, the lusk busts, and the black powder is mostly dispersed by the wind and rain; and in the other, the husk remains entire, is cut, carried, and thrashed with the buils of the crop. In that state smut-balls camnot be separated from the corn, and when in large quantity their offensive smell materially affects the flour.

It is grenerally supposed to be a fungous production; but its historv is ohacure, and it has not been ascertained whether it arises frum a parasitic plant, or is the work of an insect. That it is not a radical distemper appears to he armitted, else all the culms and ears from the same seed would be equally afiected, which is seldom the case, whole plants, and even the same ears, being only partially smutted, which would not happen did the disorder originate in the root $\delta$.

The real nature of the disease has hitherto eluded the researches of the

* Malcolm's Comp. of Mad. Husb., vol. ii. p. $245,252$.

t See A Prize Essay on the Growth of Wheat, hy George Webb IIall, Esq., in the Papers of the Bath Agric. Soc., volo xv., art. I4.

\pm Papers of the Bath Arric. Soc., rol, xv., art. 1 .

$\$$ See Main's Vegetable Physiology. 
most scientific inquirers; among the most recent of these, Sir Humphry Davy says, that it is occasioned by the animalcula of insects depositing their eggs, and recommends lime to be laid upon the land as an effectual preventive. Mr. Bauer, of Kew, seems to have succeeded in proving both the existence of the fungous excrescence, and that of grain worms * ; and the Rev. Mr. Hoblyn declares, 'that the disorder is not so likely to be occasioned by worms, by animalcula, or by fungus, as by the contami. nating principle of the putrid oily gluten affecting the chemical composition of the grain $t^{\prime}$ ' The experiments in support of these opinions go far to favour the presumption that they are all right: the existence of the fungus seems to be established; and that regarding animalcula shows that-

On a careful examination of a crop of very fine wheat, the seed of which was known to be perfectly free from any appearance of smut, some grains were, however, perceived to contain indications of being affected with the disease; the interior of the lernels having changed colour, though in several shades, varying from light-brown to deep black. The kernels were in every case found to contain a small puncture in the husk, and the change of colour was observed to have commenced in each instance precisely at the spot where the puncture appeared. It was therefore natural to conclude, that it was the work of an insect; and this was proved by eggs having been discovered, which, after a few days, became converted into maggots of a small species. It should, however, be observed that grains were also punctured which did not produce maggots.

An instance is also mentioned of smut-balls having been actually hatched into life, from one of which were produced near one thousand animalcula ${ }_{+}^{+}$. That this disease is contagious, is stated by Mr. Batuer to be suliciently proved by the fact, that it can be at pleasure inoculated on the soundest seed-corn. The infection, however, is not so generally nor so readily communicated as the discases occasioned by the fungi of the smutballs or dust-brand, a few infected ears of which are capable of contaminating the whole contents of a barn.

Whether these worms were of the same species as those described by Mr. Baver, or those deposited by the uheat-fy, we are unable to determine, and think it here unnecessary to inquire. The latter insect has in some vears committed extensive devastation on wheat crops; it is of a deep orange colour, closely resembling a midge, and generally appears when the plant comes into ear, continuing its ravages in great numbers for two or three weeks, and sometimes longer. Its labours commence about sun-set, and it deposits its eggs in the blossom of the plant, where compact clusters, amounting to from six to fourtecn in each, are laid embedded in a gummy sulistance, and from the moment the egors are thus laid vegetation ceases.

Of all the injuiries to which wheat is liable, there are perhaps none which are more to be dreaded than those alising from insects, which invariably commit great devastation, and in some seasons spread their ravages to a very alarming extent. Of these the various tribes of the beetle species-whether known under the names of 'wheat-fly,

* This paper is published in the Philosophical Transactions of 1823, but may be found, together with other very interesting treatises on similar subjects, accompanied by plates, in vol. ii. of the Penny Mag.

$t$ Prize Essay on the Diseases of Wheat, in the Papers of the Bath Agricultural Society, vol. xiv.

$\ddagger$ British Farm, Mag., No, VI. 
'Hessian,' and 'American weevil,' or any of their numerous varieties-are the most dangerous; but although learnedly treated of by naturalists, they have furnished no means of either prevention or cure. Receipts without number have indeed been published, with a view to ensure their destruction, but of these not one has proved efficacious*. The only modes which can be safely relied upon, are therefore those of preserving a free circulation of air in the granary where the corn is deposited, and frequently screening it, so as to stir the heaps, and impede the growth of the larva; and these, being within the reach of every farmer, should never be neglected.

\section{STEEPING.}

Wheat requires more care in the choice and preparation of the seed than any other species of grain : not only, therefore, should great attention be paid to its quality, but it should be completely screened and sifted, in order to free it as far as possible from the seeds of weeds; and it should be afterwards steeped, to destroy the larvæ of insects and the germ of smut and other diseases to which it is subject. Although this has been very universally practised, and a great variety of steeps have been invented, yet their infallibility has never been decisively proved; for many of those farmers who have been the most constant and regular steepers have nevertheless reaped smutty crops. This, however, should not decry their use, which is merely to purify the seed, and extends no further; for it cannot prevent any second infection.

Among the various washes which have been recommended, those most generally used are either stale urine, or a brine made of salt and water sufficiently strong to buoy up an egg. In either of these the corn is steeped, in tubs contrining enough of the liquid to be a few inches higher, and to allow it to be well stirred, so as to bring the light grains to the surface, from whence they are skimmed off solong as they continue to rise; or, it is put into baskets having two handles each, which are immersed in the tubs, and can thus be conveniently taken out and placed over an empty tub to drain off the liquid. The seed is then left for three or four hours in the chamber-lye, or for full six hours if in the pickle; after which the liquor is drawn off, and the wheat is spread thinly on the floor of the barn or granary, where it is well sprinkled with sifted quick-lime fresh from the kiln, which has been recently slaked with a small quantity of the liquor. About half a peck of lime is sufficient for a bushel of wheat, and it should be carefully stirred, that every grain may receive a portion; but if the seed is to be drilled, it should be passed through a coarse sieve, after being limed, to facilitate its passage through the machine. The grain will thus be quickly dried, but it should not be left longer than six hours in the heap; after which it should be spread open and used the following day. Some caution is requisite in the use of the lime; for if not properly slaked, so great a degree of heat might be raised as to destroy its

* The weevil deposits its egus, or larva, probably during various periods of the summer and autumn, and the progeny are brought to life in the course of the following year. In hot climates, the devastations which they occasion are so serious, that whole carroes of wheat are frequently devoured by them; and, in the granaries of Lisbon, we have seen them swept by bushels from the walls. There, they are supposed to have an antipathy to the odour of craw-fish, which are hung over the corn as preventives; hut evidently with little or no effect. In Bavaria, and other parts of Germany, green hemp, if sfread upon the grain, is said to attract the insect in such numbers, that they desert it : the smell of elder is, on the contrary, found objectionable; and fresh hay is thought to have a powerful effect in deterring then.

The most effectual method of destroying the weevil is, however, by the operation of kiln-drying: for which see the Chapter on "Harvest." 
vegretative power. Doubts have indeed been raised whether the lime is in any way serviceable; and it is certain that it is not used in addition to those solutions of copperas which are employed as steeps on the continent. Dry lime, in powder, would certainly have no effect; but in a modified state of moisture the steep is imbibed by the lime, and, as they mutually saturate each other, the mixture thus probabl; acquires a corrosive power which operates more effectually upon the smut than the brine would if applied alone*: thus, in a series of experiments recorded in Young's Annals of Agriculture +, it was found that a steep of lime-water alone, in which wheat was immersed during four-and-twenty lours, proved powerfully preventive of disease, while the guod effects of unmixed brine were very inconsiderable; we therefore see no objection to the use of lime, for, if properly employed, it can do no harm.

Of the two steeps above mentioned, that of urine is the most eficient, but it should be neither too fresh nor too stale; for it is ineffectual in the one case, and injurious in the other: its strength also differs according to the nature of the food from which it is extracted, and is more powerful when produced by human beings than animals; it is also in the most perfect state when disengaging itself from the ammonia ${ }_{+}$. The seed should be sown as soon as it is dry; for if suffered to continue longer than a day or two in sacks, or large heaps, it is apt to heat and lose its powers of vegetation. Among the numerous other steeps which have been tried, that of sulphate of copper, or blue vitriol-a preparation of which is much used as a wash for wheat in Flanders, and some parts of France and Switzerland-has been highly commended by Sir John Sinclair as a preventive of smut, and has been in many instances successfully employed for that purpose in this country. The modes of using it are ciescribed to be as follows :-

Into eight quarts of boiling water, put one pound of blue vitriol; and while it is quite hot, three bushels of wheat are wetted with five quarts of the liquid; at the end of three hours the remaining three quarts are added, and the wheat is suffered to remain three hours longer in the solution. The whole should be stirred three or four times during the six hours, and the light grains skimmed off. Then add a suffeient guantity of slihed lime to perfectly dry it. Or,

After dissolving five pounds of the sulphate of copper in hot water; then add as much cold water as mas be suffi:ient to corer three bushels of wheat. After beingr iepeatedly. stirred and cleared of light grains, it is suffered to remain in the liguid five or six hours: but it has remained in some instances from twelve to twenty-four hours without any bad effect being experienced.

After the first two or thee bays - of three bushels each - have passed through this liquid, one pound of the sulphate should be added for each succeding bag, until ten or twelve bags have been used, when a fresh quantity should be prepared.

Either of these modes may be used with safety, and a probable certainty of success. Sir John says that a gentleman who employed the latter upon his recommendation, and who had suffered so severely by smut during four preceding years, that he had considerable breadths of wheat rendered absolutely unsaleable by the ravages of the disease, had thirty-three acres of wheat sown in the autumn, and nine acres of Talavera wheat sown in the spring, the seed of which was steeper in the solution, and crops were obtained from both entirely free from disease, and various experiments of a similar nature have been attended with nearly equal advantage. He adds, indeed- "tluat lis

* See a prize essay "On the Diseases of Wheat" in vol. xiv. of the Bath and IVest of England Agric. Soc.. by the Rev. Rubert Iloblyn; and sume remarks on it in vol. xv. of the same work.

+ Vol. xli. p. 12. See also the Survey of Bedfordshire, p. 367.

t See vol, i, chap. x. p. 229. 
friend found his crops in the course of his further experiments to be free from contagion, exactly in proportion to the quantity of sulphate which he used*;" and remarks to nearly the same effect have been made in some foreign treatises upon the same subject + . This, however, appears to us to be rather incautiously stated; for other experiments have been made, from which it would appear that although blue vitriol may be an antidote against smut, yet, if the solution be made much stronger than that already described, it has the effect of greatly injuring the vegetation of the seed+.

A portion of the sulphate has however been used with good effect to give additional strength to brine, and to chamber-lye: two pounds of it being used to as much urine as will wet twelve bushels of wheat $\delta$. It has indeed been satisfactorily proved, by numberless experiments, that a steep either of brine made of a sufficient strength, or chamber-lye, but more especially if either be mixed with a moderate solution of copperas, is efficacious in preventing smut, even if the seed-corn were previously smutty. Various other preparations of vitriol, nitre, sulphur, and arsenic, have been also tried, in some instances with goorl effect; and a solution of lib. of arsenic in thirty gallons of water, has been recommended as destructive of insects and field-mice. When marle of only that strength, it however has not the desired effect; and if made much stronger, it has been thought that its poisonous qualities might prove dangerous: the subject however appears to merit inquiry.

It is stated in the Northumberland Report, that Messieurs Culley, who grow annually from 400 to 600 acres of wheat, have had only one instance of smut in a practice of more than forty years, and this was when the seed was not steeped. In experiments tried by Mr. Bailey of Chillingham on seed, in which were a few balls of smut-one-third of which was steeped in chamber-lye and limed; one third steeped in chamber lye, dried and not limed; and the remainder sown without either steeping or liningr; the result was that the seed which was pickled and limed, as well as that which was pickled and not limed, was nearly free from smut; but that which was unpickled had smutty ears in abundance $\|$.

Two experiments are recortied by Mr. Blaikie as having been made by him at Lord Chesterfield's farm of Bradley-hall in Derbyshire:-

The first was on a peck of very smutty wheat, one-half of which was sown in the state in which it was boright, and the other half washed as clean as possible in three waters, and then steeped during two hours in brine strong enough to carry a new-laid egg, and dashed over with lime: the result was, that two-thirds of the wheat grown from the unwashed seed was smutty; while that produced by the limed seed was a full crop, without a single ear of smut.

The second was made upon some very fine wheat perfectly free from smut. A quart of this was washed in three waters in order to secure its thorough cleanliness; it was then put for two days into a bagr in which there was some of the black dust of smutty grain; and the result was, that a large proportion of wheat thus sown was smutty, while

* Code of Agric. 3rd edition, App. p. 82.

† See "Instruction pratique sur la Carie, ou Pourrides Blés," par M. Bénédict Prévost de Montauban; id. par M. Charles Lullin de Genève; et Mémoire de M. P. A. Fontanel de Montauban.

t See some experiments to that effect in the Farmers' Mag. rol. xxii. p. 411.

$\oint$ Surv. of Derbysh. vol. ii. p. 116.

II 3rd edit. Surv. of Northumberland, note, p. 73. 
out of twenty acres sown with the same grain - not inoculatednot one smutty ear was found*.

Mr. Samuel Taylor, jun., of Ditchingham near Bungay, also rubbed a number of grains of wheat with the powder from some smutty ears, having first moistened them to make the powder adhere : one half of those were then washed, wetted with chamber-lye, and limed. A similar quantity of dry wheat was then procured; the whole being dibbled, each parcel by itself. The produce of the infected wheat was above three-fourths smut: the same infected wheat steeped and limed, perfectly sound: the dry wheat, uninfected, all sound $\dagger$.

Similar experiments have been repeatedly made, and generally found in favour of the pickled seed when properly made; for although some have proved unsuccessful, yet that probably was occasioned either by the inefficiency of the operation, or by contagion having been again imparted by a communication with diseased grain. It is also not impossible that some of the infected grains may not be thoroughly penctrated by the solution, and thus the disease may be in some cases partially continued. The seed should be in a perfectly dry state when immersed in the pickle; and it should not therefore be previously washed, unless it be aftenwards carefully dried. The following table, compiled by the same gentleman from the results, exhibits in a striking manner the infectious nature and degrees of smut upon different sorts of wheat, as well as the beneficial effects of steeping.

I. Wheat when merely put, without any preparation, into a bag which lad held smutted wheat :-

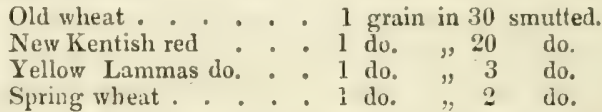

II. Wheat when moistened and rubbed with smut powder :-

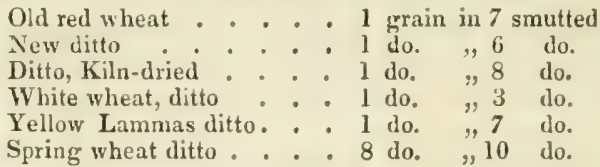

III. Treated as in the last experiment, but steeped previous to sowing in a solution of blue vitriol and chamber-lye :-

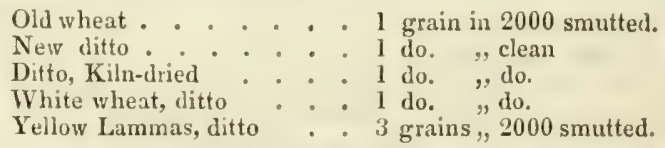

Some steeps, combined with oil and the drainings of dumghils, have been lighly praised as stimulating the germination of the seed; but experiments which have been carefully made to ascertain the results, have proved that all stimulation, thus umaturally produced, occasions a counteracting effect on the future growth of the plant. It may also be observed, that the notion of any stimulus being given to the germination of corn by the mere application of any substance-independently of its power as a liquid steep-must be fallaciuus; for the shoot receives its first nourishment from the grain it:elf, and when it begins to seek nutriment by means of its roots, these are

Farmers' Mag. vol. ix. p. 404.

† Papers of the Bath aud W. of England A gric. Soc. vol, xv. art. i. See also in that paper numerous experiments to the same effect. 
already too much grown in the earth to obtain it from any substance immediately surrounding the spot in which the seed is placed, and not amalgamated with the soil. The practice is, indeed, not uncommon among gardeners, with a view to accelerate the growth and afford an increased degree of vigour to the young sprouts; and it is true that the mere immersion of the grain for a long time in pure water will have the effect of hastening the growth of the seed. If rain follows immediately, the intention may succeed: it is, therefore, not injudiciously resorted to by gardeners, who sow only small quantities of seed, and can either choose their time, or re-sow if it fails; but it is a dangerous plan for farmers who work upon a large scale, for if dry weather continue, the seed will probably perish.

From what has been thus stated of the manifest good effects of steeping wheat, there can be no doubt that it is an operation which should never be omitted.

Machines for cleaning wheat have atso been used upon the principle of the fanner ; and one has been lately invented by Mr. 'Tuxford, which is said to rid it of all the impurities and refuse which may have been left after the usual operation of winnowing. It is described as acting by the means of sieves, which may be attached either to a thrashing mill, or to any moving power equal to much less strength than that of a horse; and it is said that one man can attend upon twelve sieves, each cleaning the corn at the rate of one quarter per hour. It is, however, attended with this material objection : that the setting up of a machine of the smallest size, containing three sieves, and occupying a space of nine feet by seven, costs no less than sixty-five guineas*

Unless other means be taken to guard against infection, the farmer can, however, never be secure against the communication of the contagion, even after all these operations have been carefully performed. It has been truly observed, that many farmers liave felt themselves disappointed in not having their wheat crops clear of smut, after being at the trouble and expense of changing the seed, as well as brining it: not being aware of the infectious nature of the disease, and that the very means they were taking to clean the seed was perhaps the cause of its being inoculated with smut; for, after the process of pickling is performed, the grain is generally spread on the barn floor to dry, and probably on the same floor on which smutty wheat had been previously thrashed; or perhaps it is put into sacks which held infected corn. Farmers have, indeed, been lknown to sow the same wheat for a series of years, with very little preparation, and yet to have no smut in their crops; while others who have carefully cleansed their seed from every appearance of infection, have had their crops destroyed. This is generally attributed to the soil, or the state of the atmosphere; but it is moreprobably owing to want of attention to the contagious nature of the powder, and its being allowed to adhere to sacks or barns with which it had been infected, and which, therefore, should be frequently and carefully cleansed. It attaches itself also to the straw and chatf, and is thus probably carried from the barn or stable door when the dung is taken green to the fields without being properly turned and fermented. The infection may, indeed, be communicated by the wind from other fields, and in various ways which cannot be guarded against ; but it is fool-hardy to run any risk that can be avoided, and no one who is duly sensible that the disease may be checked, if not wholly eradicated, by careful attention, should hesitate to employ all those

* See the New Farm. Mag. for August, 1832, which contains a full description of the machine, with a plate. Also the Bedfordshire liep., for the plate of an implement called "the Smut Hachine," 
means of prevention which may be in his power. The barn in which corn has been either stored or thrashed should, therefore, be thoroughly aired, and every corner swept : if, also, the walls of the interior were well washed with strong lime-water, the precaution would not be improper; and sacks which have held infected grain should be immersed in a similar solution.

\section{Chapter XI.}

ON RYE.

Rye comes next to wheat in its qualities, as bread-corn, and is used for that purpose, as the food of the peasantry, throughout the entire of the northern part of the continent of Europe. It contains a greater proportion of nutritive matter than either barley or oats, and the husk possesses an aromatic and slightly aciduous flavour, which renders it agreeable to the palate. The bran should not, therefore, be entirely separated from the flour; for if the grain be ground fine and divested of the husk, the bread will be deprived of much of its pleasant taste*.

\section{RYE-BREAD}

Is, consequently, most generally made of coarse flour; which, together with its dark colour, has probably given rise to much of the prejudice which prevails against its use in this country. It is here even thought to be unwholesome +; though formerly it was so extensively used, that it fed nearly one-seventh of the nation +; and certainly the working classes of Germany, who use no other kind of bread, are as strong and healthy a race of people as any in the world: it is now, however, only eaten by our labouring population in some parts of the north of England and Wales. After being leavened until it gains a considerable degree of acidity, it is made into loaves, and either baked in a large brick oven, or made into cakes of an inch and a half or two inches thick, called "sour calies:" these arc cooked upon a round flat iron, which is supported over the fire by short feet, and is well known throughout Ireland as the primitive mode of baking, under the name of "sriddle." The bread is very firm and solid, retaining its moisture or juiciness longer than any other species; and, though dark coloured, is very nutritious.

In some farmers' families household bread is made of a mixture of onethird rye and two-thirds wheaten flour; which makes a siveeter bread than that made solely of wheat, and is preferred, by those who are in the liabit of using it, to any other $\S$; it is sown for that purpose along with wheat, under the common appellation of "meslin."

* If, however, the bran of either rye or wheat be boiled in the water which is used in making up the dough of bread, it will impart much of the peculiar sweetness of which the grain of both species is partly deprived by the loss of the husk.

t It is also said to be pernicious, as producing gangrene; and it is true that the grain is in some cases refused by cattle : but this is occasioned by a peculiar disease, termerl ergot.

+ It has been caicnlated hy Mr. Smith, in his tracts on the corn-trade, that the popnlation of England and Wales amounted in 1760 to about $6,000,000$; of whom he supposes that

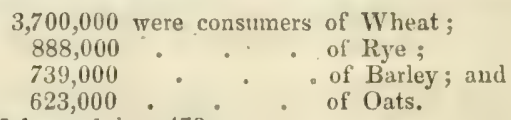

$\oint$ Survey of South Wales, vol. i. p. 479. 
RYE-CORN.

A similar objection seems to reign against the employment of rye in this country as horse-corn; for it is never given to cattle in England, though it is much used as provender in many parts of North America; and throughout Holland the post-boys may be seen baiting their horses with rye-bread, and then cutting a substantial slice for themselves from the same loaf. This prejudice is deeply to be regretted; for the grain thrives upon poor sands, upon which wheat woukd scarcely return the seed; while its weight of corn is nearly as great as that grown upon good land, and it is produced at far less expense. It also yields a larger quantity of straw, and the finest kind of ardent spirit is distilled from it in Holland; it mav, therefore, be justly considered as a precious gift of Providence to the inhabitants of sterile soils.

The varieties of rye are fewer than those of wheat; but there is a winter and a spring species, partaking of the same peculiarities in their growth, and also occasioned solely by the same difference in the mode of culture and the season of sowing as we have stated to produce similar varieties in wheats *: that sown in the autumn takes longer to vegetate, tillers more abundantly, and gives larger crops, than that sown in the spring. The latter is, however, rarely grown by the farmers of this country; though on the Continent it is found to succeed well after potatoes, which cannot be conveniently got off the ground in time for the sowing of the autumn species. It must, in that case, be sown, at latest, by the end of March; for, if the sowing be protracted much beyond that period, it has been, in many instances, known to fail. It is smaller in the grain, and the product is less; but the lusk is thinner, and the flour is peculiarly fine.

The chief distinction in the sorts usually grown here, lies between the white and dusky species: the grain of the latter being smaller, and the meal of inferior quality. The most hardy kind is produced in the Russian provinces on the shores of the Baltic: its vegetation is slower, and it ripens later than any other; but it stands the changes of weather better, is more productive, and less subject to shed the grain. It should, therefore, be preferred for seed when it can be obtained; but the chief portion of that iniported into England is grown in Poland. This species must, however, be sown much earlier than any other, and the quantity of seed should not be more than about two-thirds of that commonly used for the ordinary kinds. As it tillers slower than other sorts, farmers often imagine that they have not sown enough of seed, for it sometimes appears very scanty, even in the midlle of May; but towards the middle of $J$ une it surpasses every other variety known + .

\section{SOIL.}

The Soils which are the most appropriate to the grouth of rye are those which contain the greatest proportion of sand; and it is the only corn which can be grown upon land which is composed of a mixture of more than 85 per cent. of sand with other earths + . Those soils, however, which contain a less portion of sand, are preferable; for, although it will grow upon ground of the poorest description, yet the produce will be more

* See the paragraph upon Winter and Spring wheat, p. 138.

+ Von Thaër says, that he has sown it so early as the middle of Juue, without its having shown any appearance of rising during that year: and that, if sown so late as October, it tillers very feebly.-Principes Raisonnés d'Agric., 2 de ed. tom. iv. p. 123.

$\mp$ See the Table in page 113 . 
abundant upon good land, provided it be not of a clayey nature. It also admits of a greater degree of that property which is termed "acidity of soil," than is favourable to the growth of wheat or barley; and it may, therefore, be grown upon heaths, which naturally produce nothing but scanty herbage*

\section{CUlitivation.}

Rye, being usually sown upon light soils, obtains less attention in its production than wheat : it also suffers less by being sown upon the stubble of another corn crop; or even upon its own; and it is, therefore, not unusual to grow it two years successively: the spring species being sometimes made to succeed that sown in autumn. Some farmers even repeat it a third and fourth year; but this exhausts the land to a degree which can hardly be repaid by any manure produced by the crops. Thus, upon many of the hilly manors of North and South Wales, which were enclused during the late war, the ground was pared and burned, and being repeatedly sown with rye, was afterwards thrown open without any further care: the consequence of which has been the destruction of the grass which the land naturally produced, and which, not being capable of renovation during a long series of years, has occasioned the utter ruin of a large extent of valuable sheep-walk.

On well-managed farms, in bleak situations unfit for the production of wheat, the ground has, however, been broken up with such advantage, that 36 bushels, and upwards, of rye, are stated as not an uncommon return; and men of much experience assert that they never had so much profit from wheat crops on lowland gravelly loams, as from rye crops on fresh wastes of some elevation. It has also been sown among late turnips, which have been fed off, and the rye, being allowed to stand, has produced good crops.

On half-reduced fallows, or ground where the farmer cannot expect a good crop of wheat, he sows meslin, or mingled corn, generally in the proportion of one-fourth, though sometimes of one-half of rye to wheat: the object being to guard against a thin and weedy crop of the latter grain ; and it has been remarked, that "when wheat and rye are grown mixed in this manner, the grains of each are larger and more perfect than when grown singly, without any admixture.$+ "$ It is also said, that they are more free from disease. The rye is well known to be the hardier plant of the two, less subject to the attacks of insects, rust, or smut, and when both sown together, they ripen at nearly the same time; but it must not be sown on land which has been limed, for it is an extraordinary fact, that lime is injurious to its growth. It has, indeed, been asserted, upon the authority of the Survey of South Wales, "that were meslin sown on a pared and burned turbary, or on any other coarse soil-a grain of rye for every grain of wheat-by harvest-time the rye would probably be found to compose full three-fourths of the crop: while, on old limed ground, the case would be reversed."

It further states, - " that on cutting a new road on the side of a steep declivity, the materials dug up, consisting chiefly of 'shale,' mouldering readily in the air, were thrown underneath upon part of a fallow for rye, which was sown the September following, over the whole piece. The lower part had been treated with lime; the upper pared and burned, and on part of it the shale had been spread. In May and June following, the crops were very different. 'The limed crop had a fair appearance; but

* Von Thaër, Principes Raisonnés d'Agric., 2de ed, tom, iv. \$ 1035.

+ Survey of Northumberland, 3d edition, p. 80. 
that which had been covered with shale was so uncommonly luxuriant is to raise the curiosity of passengers. It had tillered so abundantly that 28 perfect stems were numbered on one root. The shaled part had the disadvantage of the other, in being exposed to a higher climate, and in a full northern aspect*." This-as many extensive hills in some parts of Wales consist entirely of friable shale-might prove a valuable hint to the farmers situated in those districts.

No accurate comparisons have been made to ascertain in what degree wheat, when sown on light soils, is rendered more productive in quantity and superior in quality, by the addition of a small quantity of rye being sown with it; but, that such is the fact is fully proved by the continual occurrence of parts of fields being sown with a little rye, and other parts with wheat alone, where the land is of the same description. On these light soils it is evident that the shade and shelter afforded by adding about one-thirtieth part of rye to a given quantity of wheat, frequently protects the latter from the mildew, and renders the sample much finer. If to twentynine parts of a bushel of wheat one-thirtieth part of rye was added, it might probably lessen its value about one-fifteenth part-say sixpence per bushel; but a sample of corn of this description, if fine in quality of grain, would be worth as much money as a sample of inferior wheat ; besides which, upon light soils, that are not properly adapted for wheat, the protection given by its taller growth not unfrequently increases its produce by two bushels per acre.

In most other respects the cullivation of rye nearly resembles that of wheat; the chief difference being, that it requires less tillage-three ploughings being considered sufficient for a fallow. It, however, profits equally by careful hoeing, as well as by harrowing in the spring; provirled that, in the latter case, the roots be found to have taken strong hold of the ground, and that the operation be performed with light wooden harrows: the land should also be afterwards rolled. It is largely grown throughout the whole of the North of Europe, and in the sandy soils of Flanders. The mode is there, to commence by one good ploughing and harrowing; then to spread a coat of farm-yard manure upon the surface of the land and plough in the dung; the seed is harrowed in at the rate of a bushel and a quarter, or in some places not more than a bushel per acre; yet the average produce is there rated at four quarters and two bushels + .

\section{THE SEED}

Is generally put into the ground a full month earlier than wheat. It may indeed be sown with advantage in the latter end of July ${ }_{+}^{+}$; but the usual time of sowing is some time in August, and seldom later than the beginning of September; as the crop suffers materially in produce by late sowing, and the quantity of seed must be increased. Two bushels are found enough in the first instance; $2 \frac{1}{2}$ being requisite in the latter, or near 3, if the period be longer deferred. The seed must also be more lightly covered than that of wheat; for, if it be deeply put into the ground, and the land be at all tenacious, it may be prevented from germinating : it should, therefore, never be ploughed under.

It does not require so much care as that of wheat; nor is it.customary to steep it. The precaution, however, can do no harm, for it is not

* Rep. of Sonth Wales, vol, ii. p. 115; of North Wales, p. 193.

+ Raclift's Rep. of Agric. of E. and SW. Flanders, p. 66.

I It has been sown in Bedfordshire so early as the $20 \mathrm{th}$ of $\mathrm{July}$, and fed in autumn with sheep and cows; and is said to have produced remarkably good butter. Bedtord. Rep., p. 387. 
quite free from disease; and, at all events, grain should never be sown unless it be in a perfect state of soundness and maturity. The disease to which it is peculiarly subject, is called the ergot. It is of the same nature, in some degree, as smut, and is a great drawback on the value of the corn. Farmers on the Continent attribute its prevalence to a certain moist state of the atmosphere at seed-time; and an acute observer in this country thinks that it is sometimes occasioned by late frosts, which occasionally affect the plants so as to cause a malformation of the grain. The general opinion is, however, that it is really a fungus, which, seating itself within the husk, changes the meal into grey powder** It chiefly prevails in damp places, or in wet seasons, and is an excrescence projecting from the ear of a dark violet colour: if taken in a fresh state, it is highly injurious to animals; and, if mixed in large quantities in the flour of which bread is made, it has been known to have the most pernicious and even fatal consequencest.

The period of flowering is more decisive of the prospect to be entertained regarding the success of rye than any other corn; and, until it be passed, no opinion can be correctly formed upon the subject. A white frost attacking it whilst it is in flower may so injure it as to destroy the crop, though it frequently only suffers in the borders of the fields, or in parts much exposed to the influence of the wind; in which case, it is sometimes seen that only that side of the ear on winich it has blown is affected. A rainy seasor is also prejudicial; for, if not accompanied with frequent brealis of dry and sumny weather, the anthers do not come to perfection, the ears lose their colour, and it is not improbable that the ergot is thus engendered.

The ripening of the grain is earlier than that of wheat; and is denoted by the straw losing somewhat of its bright yellow colour, becoming paler, and when the knots of the stem no longer continue green. The corn then sheds easily from the ear, and should he reaped and carried without delay. When allowed to stand until very ripe before it is cut, a small shower of rain will also occasion it to sprout.

\section{PRODUCT AND QUALITY.}

The product of Rye is nearly the same as that of moderate crops of wheat, but seldom anounts to those which are very large; about three quarters per acre being considered the average: the weight is from 50 lbs, to 56 lbs. per Winchester bushel. The quantity of straw is, however, greater, and not unusually vields as much as nearly two tons per acre. It grows to a greater height than that of wheat, and though thinner in the stem, is stronger; but being hard and wiry, is not esteemed for fodder, and the chief use made of it is for thatch. It is also in great request by brick-makers, who not uncommonly buy it at the rate of three pounds per load $\ddagger$.

Some of the grain obtained from Yorkshire was found, according to an analysis made by Sir Humphry Davy, to contain the following proportions of nutritive matter in 1000 parts of vegetable substance, namely :-

645 parts of mucilage ;

38 do. of saccharine matter; and

109 do. of albumen, or gluten.

* Main's Illustrations of Vegetable Physiology, p. 286.

† There is an account in the "Philosophical Transactions," No. 130, of a dreadful malarly which raged at Sologne, in France, among those who lived upon rye-bread. It is probable, however, that the effect was dednced from a wrong cause; for we know, frum long experience, that its qualities are far from being insalubrious : it is aperient and cooling, and is, therefore, to some constitutions, more wholesome than any other species.

\$ Five pounds per load have been paid for it ly brick-makers in the neighbourhood of London, during the winter of 1834-5. 
Ryc is frequently sown and cut green for early sheep-feed without any intention of obtaining a crop of grain ; though, if not too late or too close fed, both objects may be attained. It is extremely useful to farmers who kcep breeding-flocks, as it comes forward earlier than tares, and affords a good bite at a season when fresh food is so scarce as to put them to every kind of shift to carry on their stock. It is, also, not uncommonly sown along with winter-tares: but, unless the latter can be got off the ground very early, it is a bad plan; for when the rye begins to spindle, the stem becomes tough, and the sheep then rejecting it, a great part of the crop is wasted: it should, therefore, be fed off quickly.

\section{Chapter XII.}

\section{ON BARLEY.}

UNDER the general name of Barley is inclucied all grain that is commonly used for malting; though there are two distinct kinds, the one being the lent-corn, usually sown in spring; and the other, a winter species, known under the denomination of Bere or Bigg.

SPECIES.

The different species are distinguished by botanists as "two-rowed," "four-rowed," and "six-rowed barley ;" though properly speaking, there is no species to which the term "four-rowed" ought to be applied *; and the many varieties which exist are designated by farmers as "sprat, or hattledore barley," "rath-ripe, or hotspur, and naked barley ;" "Egyptian, Moldavian, Thanet, Chevalier, and Annat barley †:" with a number of other sorts, named either from the places of their original growth, or from the persons by whom they were bronght into notice, and distinguished chiefly by the productiveness or qualities of their grain, and their habit of late and early ripening.

Of the different species of Summer Barley, the only varieties which we deen it necessary to notice, are the common two-rowed, or late; the naked; and the rath-ripe, or early: the two former requiring a stronger soil than the latter, beingr also sown earlier, and grenerally producing better crous. The grain of the first species-which is the sort must generally cultivated-is larger than the other two, and should not be sown later than the beginning of April, as it does not sulfer materially from the frost hy which it may be

" Barley is termed "two-rowed," or "six-rowed," according to the number of its fertile florets. In two-rowed barley, one row of florets on each of the two sides of the spike is fertile, and consequently one row of seeds on each side is perfected. In sixrowed barley, three rows on each side of the spike are fertile, and consequently three rows on each side are perfected. In this sense only it is termed six-rowed barley; but there is no species known to us in which only two rows on each side of the spike are fertile. Slightly examined, indeed, six-rowed barleys frequently present the appearance of four rows; but this is in appearance only, for such barleys have always the three rows on each side perfect. In poor soils and unfavourable situations, two of the rows run much into each other, and this has, perhaps, given rise to the mistake; but the two rows which thus run into each other in appearance, are on the opposite sides of the rachis." -Low's Flem. of Pract. Agric., p. 241.

+ This latter species has been produced from a few ears casually picked from a field in the Carse of Gowrie, during the harvest of 1830 . It has been sown along with the common and the chevalier barley, and has heen found superior to either, both in bulk of straw, weight, and quality of grain; but has not been sufficirntly diffused to warrant a decisive opinion regarding its merits; and it appears to require a rich soil. 
attacked at that season. Its leaves are sometimes discoloured either by the action of frost, or the continuance of very dry weather; but if the ground be moist during the development of the ear, the crop will not suffer. If, however, the leaves turn yellow through excess of humidity, then it will probably be spoiled.

The naked barley is of the six-rowed species, and the ears losing the beard with which the common kind are furnished, and separating naturally from the husk as if they had been shelled at a mill, gives the grain the bare appearance from which it has derived its name. It is highly esteemed on the continent, where it is known as blé de Jérusalem, as having been originally obtained from that neighbourhood; or, more generally, from its great productiveness, as orge céleste. It unites, indeed, very recommendable qualities, being hardy in its growth, strong in the stem, tillering with great vigour, and producing abundant crops of very superior grain. It is also well adapted to the making of pearl barley; and the quality of the straw is better than that of any other lind: it, however, requires to be sown earlier than any other sort, and only succeeds if grown in a very rich and well-tilled soil*. There is also a two-rowed species, which in most respects resembles it, but is considered rather inferior.

The rath-ripe, though better adapted to light soils, is yet so delicate as to be sometimes destroyed by a slight frost, and is easily affected by any sudden change of temperature; it is, therefore, not generally sown until the beginning or the middle of May; and it has been frequently put into the ground so late as from the 10 th to the 20 th of $\mathbf{J u n e . ~ I t ~ r i p e n s ~ s o ~}$ early, that in warm summers, it is usually reary to be cut within nine or ten weeks from the time of sowing; and in the forward soils of our southern counties, it is frequently reaped within a still shorter period. These advantages of late sowing and early ripening are of such importance, that notwithstanding its delicacy and the comparative smallness of its produce, it is very generally cultivated.

Winter Barley, Bere, or Bigg, is six-rowed, containing in the proportion of at least three to two more grains in the ear than the two-rowed species+-which usually holds only twenty grains - and requiring different treatment in its growth and cultivation from the summer kinds, has been looked upon by botanists as a different sort; but it is, in fact, only a variety of the same species. It is the hardiest of all grain, and is remarkable for witlstanding the inclemency of winter, and ripening at a much earlier period than the common barley; on which account it is largely grown in some of the exposed parts of Scotland for the crop, and in England it is also grown by many farmers in the view of obtaining early spring feed for their flocks, in the same manner as rye. When thus fed off while green, the produce, if afterwards allowed to stand for a crop, is generally found to be very large, and it is peculiarly suited to rich alluvial soils which have been pared and burned; but the grain is not only much smaller, but in every

* Von Thaër states that it is considered in Germany as in every respect equal, both in weight and quality, to rye; and its nutritive properties have been found, upon analysis, to the even superior.-Prisc. Rais. d'Agric., 2nde ed. tom. iv. 1050.-In some parts of France it is said to equal the weight of the best wheats; and its products in mountainous countries is there calculated at 24 to 1.-Bullet. des Sciences Agric., No. IV.

+ The respective number of grains contained in a pound weight of good English barley, and biggr of three different cutalities, have been found to he nearly as follows, viz.-

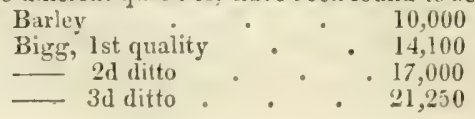


respect inferior to the summer species *. Although commonly sown upon poor land, and comparatively not so well cultivated, it yet properly requires a strong soil, and in every respect the same treatment as wheat.

A mixture of bigg and two-rowed barley is commonly cultivated in some parts of Scotland under the name of "Rammel," or "Blandered-Bere." When these two kinds are grown in separate fields and mixed together, the seed will not at first spring equally, nor can the grain be malted by the same process; but after they have been for some time sown in a mixed state, there is at last no perceptible difference in these respects, and the quality of the corn seems to hold a middle place between barley and beret.

SOIL.

Barley, of every species, requires a rich, friable, and mellow soil, which retains a moderate quantity of moisture, but without approaching to that which may be denominated wet: as, for instance, land which contains from 50 to 65 per cent. of sand, and the remainder chiefly clay; though it may be grown with advantage in ground containing a still larger portion of sand, provided the season prove rainy; but if the weather continue dry, the crop will suffer in proportion, and therefore upon such land, its growth camnot be deemerl secure. It, however, succeeds well upon a more clayey soil, provided its tenacity be tempered by such a proportion of mould as may bring it under the class of good wheat land +: or, if the clay contains a certain portion of chalk mixed with the sand, it will also be found favourable to the growth of barley, as the chalk neutralizes all acidity, and thus checks a quality in the soil which is peculiarly prejudicial to the grain. For poor, tenacious, and cold land, it is wholly unfit, and except upon genial soils, its growth ought not to be attempted.

\section{CULTIVATION.}

Barley requires that the land upon which it is sown should be perfectly well worked, and brought to a state of clean and friable tillage. If sown, as it frequently is, upon a wheat stubble, it should have at least three ploughings; but if, after spring crops, which have been drilled, and the ground well pulverized and cleaned by horse-hoeing, one is sufficient. It, indeed, usually succeeds turnips, and is perhaps never so generally productive as when it follows that crop; for it loves a good tilth, and flourishes upon land that has been well tilled, and heavily manured. The turnips should, however, be fed off upon the land by sheep; and the "teathe," as the improvement thus afforded to the land both by the excrements and the contact of their bodies is termed in Norfolk-should be "sealed," or ploughed in, as close to the flocks as possible $\$$. If, however, this be not

* Its produce on the Polders, or Carse lands, of the Netherlands is stated by Mr. Radcliff to equal ten quarters per English acre. Rep. on the Agric. of E. and W. Flanders, p. 15.

$\dagger$ Gen. Rep. of Scotland, vol. i. p. 496.

+ See the 'liable in chap. viii. p. 113.

$\$$ Drew's Norfolk Husbandry, p. 101. "The effects of barley after turnips are, however, very different upon different soils. If the soil be light, dry, and tender, and admits of the turnips being fed off' by sheep, it generally proves an ameliorating crop, and the subsequent crop is rather increased than diminished. But if the soil be wet, tough, or heavy, it is very pernicious and exhausting, and the following crop is generally thereby lessened from twelve bushels to two quarters per acre. Whence this arises is not altogether certain; but is probably owing to the two following causes : first, it being, for the most part, impossible to feed them off with any kind of enttle upon the ground, there is no lung left upion it; and secondly, the soil is so cut and torn up by the carts, and trampled upon by the horses in carrying off the turnips, that it can seldom be properly prepared to receive the barley." - Essex. Rep. vol, i. p. 228. 
done, and that the land be at all exhausted, it should be renorated with a good dressing of well fermented spit-dung, in a complete state of decomposition; for it is a delicate plant, and the nutriment furnished to it should be rich and easily absorbed.

Although more commonly sown after tumips than in any other way, yet in Essex, it is very generally sown upon a fallow; as fallowing for wheat is there considered barbarous management. This is, indeed, more liaboriously performed than in any other county, and the autumn ploughings much exceed those which are usually bestowed*; but, on strong land, much spring tillage is considered inexpedient, and the best crops are produced by perfect summer fallowing, and then putting in the seed upon one ploughing; or, still better, by the operation of the grubber. The land, after being ridged up, is therefore left in that state until spring, and when dry, it receives the seed-furrow; as it is a general rule always to sow barley upon a fresh-stirred soil. When it follows turnips, the land stroukl be ploughed into ridges as soon as they can be got off the ground, and a second ploughing should be given for the seed-furrow. 'I'en-furrowed rilges are those most generally employed, but as the land is sometimes laid down at the same time to permanent pasture, broader ridges are then commonly used; and if only suwn with clover, to stand for one crop, it is hardly necessary to say that a smouth and well levelled surface will not only temi to the good appearance of the field, but will much facilitate the operation of the mower.

\section{SEED AND SOWING.}

Barley is not exposed to any disease, except partially to smut, and from that it rarely suffers mischief. The ears which are attacked are those which sprout first, and the whole field then seems to be covered with it; but these are succeeded by healthy ears, and no sooner do they apjear, than the former are dislodged, and scarcely a trace of it is left. Steeping the seed is, therefore, rarely practised, but the operation, when perfurmed, assists in cleaning it from the small light seeds of grass and weeds with which it commonly abounds; and it should, at all events, be carcfuily screened. If steeped during twenty-four hours in soft water, it will also cause the grain to germinate at the same time; and this, especially if it be sown at a late period, is of more importance than may be generally imagined, as it is otherwise apt to ripen unequally.

The month of April is the time most commonly chosen for souing summer barley; though it may generally be put into the ground with advantage until towards the middle of May, and good (rops have been frequently reaped which were not sown until the beginning of June. Discrimination should, however, be used in regard to the species of grain, as well as the nature of the soil on which it is to be sown; for if the latter be warm and rich, the sowing may be deferred longer than if it is cold and poor, and attention should be paid to the qualities which we have already stated as distinguishing some peculiar kinds of barley. On strong lands, which have been got into good tilth, the first or second week in April is early enough; but those which are light and dry may-especially in the southern counties-be sown in the latter end of March. Cold springy soils, or such as have not been sufficiently drained, should, however, be not touched until later, as a wet spring would starve the crop; but, whatever may be the property of the ground, the time of sowing must still be chiefly governed by the temperature of the season.

* See Chap. v, and Essex Rep., vol. i. p. 195. 
Early sowing is generally recommended; and, in fact, ilie funest samples of every kind of grain are usually thus produced. Barlev, if put soon into the ground, and if the weather prove favourable, casts better, and usually produces better crops than if sown late. Thus, upon strong lands, it is apt to run to straw when not sown in grood time, and this, occasioning it to lodge, discolours the grain; while, tyrom light lands, there will be little straw and less corn, and both will be of very inferior quality . Opposed to this, however, is the risk of early frosts ; which, if they happen with much severity, will so far check vegetation as to render the crop very inferior. On this subject we have the evidence of Mr. Burroughs, who" "having been an advocate for early sowing, under any circumstances whatever, gave directions to have his general crop of barley sown early in April, but deferred the sowing of two acres until the first week in May $t$. The result of the experiment was as follows :-

The early sown crop looked very promising until eariy in May, when, after a few nights frost, it changed its colour, many of the collateral shoots died away, and the crop produced but indifierently. On the contrary, that part of the field sown in May was not checked, but grew luxuriantly, and produced considerably more than the former to the acre. The field was a light loamy sand, with a subsoil of cold yellow clay.

Upon a second experiment it was found that, notwithstanding the early sown crop was not injured by the frost, yet it was not so productive as that which was sown three weeks later.

The quantity of seed usually sown is about four bushels per acre, of all kinds; but the ordinary species of two-rowed barley, if allowed room enough, tillers strongly, and if sown too thick, the plants become weak; it consequently requires less seed than most other sorts: the land also requires different quantities of the same species of seed according to its prcperties: thus, if rich, ten pecks may be sufficient; if adhesive, twelve are requisite; and if very light, from fourteen to sixteen are necessary, when sown broad-cast.

The mode of souing may be either by the drill, or broad-cast ; the former is, however, to be preferred; both as being well adapted to the light soils upon which barley is generally grown, as ensuring a more equal deposition of the seed, and as affurding a surer method of distributing any grass-seels, which may he put in afterwards. When drilled, the most usual distance for the rows is eight inches for fine light soils, and nine for those which are stronirer : the quantity of seed may also be full two pecks less than when broad-cast. The land then gets a single turn of the harrows; and just a sullicient number of harrowings to cover the seed when sown broad-cast. It is afterwards rolled; but when clover is to be sown, this latter operation is deferred until the seeds and barley are both in the ground.

The sowing of clover and grass-sceds is sometimes effected simultaneously with that of barley; but it is generally considered better to let the barley plants first get above the ground, and then harrow the clover in with very light wooden harrows. The rolling then takes place along the ridges; and if the land be very dry and open, the seeds are frequently sown without being harrowed in, as they should be only slightly covered by the soil, and they will, in such case, be sufficiently buried by the roller. If, however, heavy rain should occur after the sowing of the barley has been completed, the land should be lightly harrowed as soon as it is dry,

* Drew's Norfolk Husbandry, p. 103.

$f$ On the Cultivation and Harvesting of White Crops. 
in order to break the crust which will have been thus formed, and through which the young sprouts would find difficulty in forcing their way. It is also useful even if rain should not have fallen, as it aids the subsequent sowing of the clover. This, however, is rather a dangerous operation, for the plants, if already sprouted, are extremely brittle, and easily broken; it should, therefore, if possible, be always performed before they rise. If done afterwards, very light wooden-teethed harrows should be used; and the work should not be commencerl until late in the evening, as the stems of the plants will be then more pliable than during the heat of the day.

The water-furrows and drains should then be cleaned out, and this closes the field-operations until the ensuing harvest, unless weeds should appear, in which case they should be immediately pulled up by hand, without regard to the expense; for, if not extirpated at once, they will obtain firn possession of the land, as the succeerling crop of clover will prevent their being destroyed by the plough.

\section{PRODUCT AND QUALITY}

depend not only on the soil, but vary so much in the different kinds, that in an experiment made in 1833, uncier the sanction of the Last Lothian Agricultural Society, upon the chevalier and a common sort of barley, both sown on a light gravelly soil, the produce of each per imperial acre was-

Chevalier, 65 B. 2 P. of grain. weighing $56 \frac{3}{4}$ lbs. per bush. : and of straw 4251 lbs.
Common, $61,2,2$, ditto
ditto
$54 \frac{3}{4} 1$ bs. ", ditto
3733 ,

The seed was sown after a crop of turnips and mangel-wurzel, only one third of which appears to have been eaten upon the ground; yet barley may be grenerally considered to yield only an average product upon soils well adapted to its growth of about four to five quarters per imperial acre; though crops of much larger amount are continually grown in favourable seasons upon rich and well cultivated land. The weight of the good qualities of the common two-rowed species may also be averaged, when grown upon such soils, at about 53lbs. per bushel, while that of bigg is frequently under $40 \mathrm{lbs}$, and rarely exceeds $46 \mathrm{lbs}$. per bushel. The nutritive properties of the former have been estimated, according to an analysis made by Sir Humphry Dary, as containing in 100 parts,

79 per cent. of mucilage, or starch;

7 ditto of saccharine matter; and

6 ditto of gluten or albumen.

This applies, however, only to some grown in Norfolk, a county which is celebrated for the production of the finest species, and is given without reference to any distinction in quality. An extraordinary difference, however, exists in the various kinds, and particularly between those grown in the midland and southern counties of England and in Scotland, as well as Ireland. The weight of the former is generally perhaps from three to four pounds leavier than the latter; but, even when less weighty, it yields more meal, as well as more pot and pearl barley, and is far superior in its malting properties, and its consequent production of distilled spirits.

Thus, upon a fair experiment made '.- wann wal quantities of Norfolk barley and some produced in one of the most fertile and best cultivated Scottish counties, both malted by the same person, it was found that, although the Scotch weighed about 3 lbs. per boll more than the Norfolk, yet the latter yielded two gallons more of spirits.

So likewise upon an average of several scientific experiments, made some years ago by order of the House of Commons, and some learned observations afterwards made upon them, by Dr. Skene Smith, for the 
purpose of ascertaining the relative qualities of malt made from English and Scotch barley and bigg, it would appear, so far as reliance could be placed upon the usual qualities of the different growths, and the accuracy of the process of malting, that a Winchester quarter of each grain, when malted, would produce the following quantities of proof spirits, viz.,

English barley about $21 \frac{1}{5}$ gallons,

Scotch do. do. $19 \frac{1}{4}$ do.

Scotch bigg do. $16 \frac{1}{4}$ do.

From these and other trials of a similar nature, it apneared, that the English barley was about 11 per cent. superior to the Scotch, and full 2 per cent. better than Scotch big.g*. The same disparity would probably have been found between the general qualities of Irish and English barleys, and doubtless a like difference would have occurred had the barleys been brewer ; for both in brewing and distilling it is the quantity of saccharine matter contained in the grain which furnishes a criterion for ascertaining its comparative value, and the finest-flavoured ale brewert in Edinburgh is made from Norfolk barley. The climate of Ireland and Scotland is more humid than that of England, and it is apparent, that grain ripened and harvested in the early part of autumn, and under the influence of a hot sun, must be superior to such as may have been harvested, even upon the same farm, three or four weeks later, when the air is damp and the heat less intense. Every farmer knows that this inferiority is frequently occasioned by artverse changes in the weather, and barley suffers more than any other grain from the effects of wet: it is, therefore, unnecessary to impress the advantages of an early harvest upon their attention.

The straw is lighter than that of wheat, and is chiefly used as litter; for although the awns are sometimes boiled and given to cattle, yet the stem is so deficient in nutriment, that it is seldom employed as fodder. The peculiar operation used for dressing the grain may be found in the chapter on Harvest, under the head of "Hummelling."

\section{Chapter XIII.}

ON OATS.

THE Oat, as if intended by nature to supply the deficiencies occasioned by the climate of the north in the growth of wheat and barley, thrives better in Scotland than in any part of England. It, indeed, appears to be indigenous to the cold latitudes; for it is there found in a wild state as a most troublesome weed, while it degenerates in the southern countries, and is not reared in the warmer parts of Europe. Of all corn crops it is that which is the most easily produced: it is, therefore, grown upon almost all kinds of ground, but is especially profitable upon fresh land and crude moory soils. Its varieties are quite as numerous as those of any other sort of grain ; and, in like manner, although designated by numberless different names, as possessing some distinct qualities, they are yet all derived from one parent stock. These may, however, be comprised under the separate heads of the " common feed-oat;" the "Poland;" the "Dutch ;" the "Potato;" and the "Tartarian oat."

* See the Farmer's Mag. rol. v. pp. 72, 342; rol, viii. p. 495. 


\section{SPECIES.}

OF these, the Common Field-Oat is the lind most gencrally grown, and is the most certain in its product upon poor exposed soils. The grain is of different shades: one species being almost black; while another is dum, approaching to red; and a third is nearly white. The corn of the first is very small, but it is the most hardy, and, as it ripens carly, it is very commonly sown throughout the Highlands; though it produces but little fodder, and that of indifferent quality. 'The red species is also an early oat, well adapted tó peaty soils, and very proper for hilly districts, as not being easily shaken out by the wind. It derives its name from its colour, which when ripe is reddish, and it is a valuable acquisition to the northern uplands, in which, before the introrluction of this seed, the crops of other sorts not unfrequently failed; though now they are harvested nearly as soon, and in as good order as in the lowlands*. A variety of it, known as the "Peebles oat," from being originally produced in that county, has the smallest grain known, though, from the very thin skin, it meals weil, and is liked by the millers; and the straw, though scanty, makes tolerable fodder. There is also a very hardy sort called the "Dun-oat," from its dirty grey colour: the quantity of straw is, like the red, small; but it yields well, and the thimness of its husk renders it very productive of meal. The white lind, though more delicate and requiring the land to be in better condition than the other two, is yet of finer quality. Of this, the "Blainslie," the "Kildrummie, and the "Halkerton," are in much request, and a very productive variety of the latter has been introduced from Angus-shire, where it is highly esteemed for the quality of its straw and grain; but it is rather later in ripening, which partially militates against its adoption in places where the harvest is frequently backwards $t$.

The species called Poland Oats, which probably came originally from that comtry, and of which many varieties have been since brought into notice, has long been cultivated in England, and is still highly valued as a very productive and early lind; " linown by the grain being remarkably large, plump, round, well filled, and not in the least tailed; a bushel generally weighing 46 lbs. f;" the husk, however, is thick, and it is best suited to a rich or marshy soil.

The Dutch Oats, though equally early, and hardier than the former, and frequently grown upon light dry lands, were, however, originally produced in the rich alluvial soil of East Friezeland, from whence large quantities are ammually imported into this country. From this also a variety has been introduced called "Church's," which, besides yielding fine grain, produces a large quantity of straw, with stems so stiff as to be little liable to lodge, and consequently favourable to the growing of grass-seeds.

Since the introduction of the Potato-Oat, the use of the two latter spe-. cies has been somewhat circumscribed, it being consiclered, in every respect, as of superior value where the suil is rich and properly cultivated; it tillers and stocks ivell, and appears to do best with thin sowing. It was accidentally discovered in Cumberland, in $17 S S \oint$, and has since spread over all the North of England and the South of Scotland, where it is now almost

* East J othian Survey, p. 126.

+ What commonly go by the name of Angus oats, differ from the Halkerton oats, which is a large oat, and produces a tall coarse straw ; the other is a neat plump grain, and produces a fine straw, not so apt to lodge as the Blainslie, and about a week lunger in ripening. Mid-Lothian Survey, p. 103.

+ Northumberland Rep., 3rd edit, p. 83.

See the Farmer's Mag., vol. xiv. p. 167. 
the only kind sown on many of the best farms. It seems to be nearly allicd to the Dutch oat; but the grain is plumper, better formed, and thinner skinned; the number of bushels per acre greater, and more productive in meal; it also ripens early. It is said to have lately rather degenerated; but its superiority has been confirmed by a recent trial made between it and a celebrated new species, known as the "Georgian Oat," which has been reported to the Highland Society as follows:-

The experiment was made at Preston, upon two English acres of equal land: the quantity sown of potato oats being four bushels, and of the Georgian six bushels per acre. The appearance of the latter was by far the most luxuriant during the summer till the end of July, when the potato shot out considerably longer in the straw; but the Georgian was reaped ten days, and might have been a fortnight, earlier. Both were carefully cut down and stacked till the month of March, when they were thrashed, and the quantity produced was nearly equal; the potato oats yielding 69 and the Georgian 68 bushels. The value, however, showed a material difference-

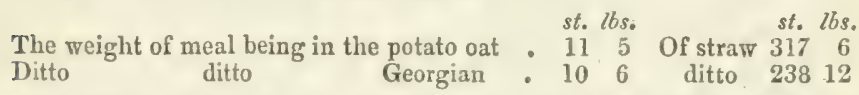

which, when reduced into money, amounts to upwards of $\mathscr{L} 2$ per acre in favour of the potato: thus-

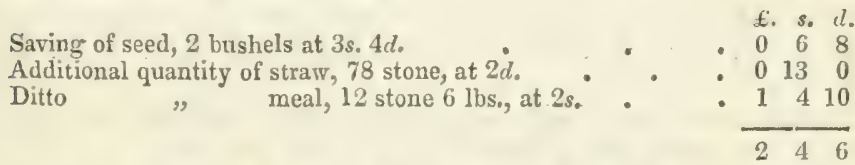

It will thus be seen that the bulk of both crops was very great, but the only advantage gained by the Georgian consisted in their earlier ripening.

A variety of the potato species has, within these few years, been broucht forward in East Lothian, and having been strongly recommended by Mr. Shirreff, who introduced it-and to whom the silver medal of the Highland Society has been awarded-as well as by several persons who have used it, has been already extensively cultivated. Some experiments made in comparison with the Angus and other Scotch onts, show it to possess a decided superiority ; and, although derived immediately from the potato stock, it now claims a separate title, under the appellation of the Hopetoun Oat.

One of these trials was made upon a field of twenty-two Scotch acres, of a rather heavy soil, and four varieties were sown on the 19th and 20 th of March: namely, the Hopetoum, the grey Angus, the Potato, and the early Angus; all of which sprouted well, but the IIopetoun was strongest, and througliout exhibited a more healthy and vigorous appearance. Two portions were then laid off ; one of the Hopetoun, the other of the grey Angus, where the land seemed equal, and were buth cut by the sime reapers. The result was as follows :-

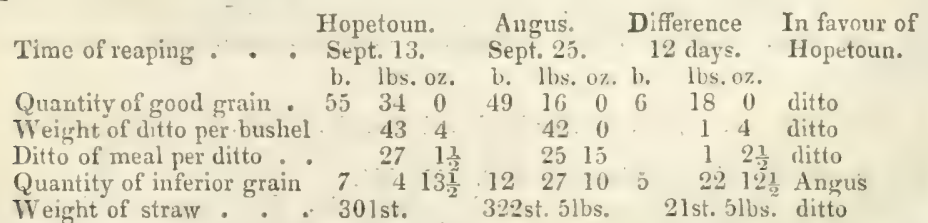

Complaints have, however, been made of its not answering the description 
given of it; and a distinguished agriculturist, who is a member of the Highland Society, states that, on trying it in the same field with the potato-oat, it was found later in ripening, and decidedly inferior both in quality and produce. When examined before the Society, it was found neither so large nor plump as the original species, and the weight of both was-

Of the Hopetoun Oat, $45 \frac{1}{2}$ lbs. per imperial bushel;

Of the Potato Oat, 48 lbs. ditto*.

It is, however, evident that all may not have been equally successful in their neriments, and the trials have fully established the value of the stock, by whatever name it may be distinguished, provided it be sown upon good strong land. One great disadvantage attending the Hopetoum arises, however, from the lateness of its ripening; which appears to be further established by a communication from the land steward of Mr. Dun, of Duntocher, in Dumbartonshire, who states that it was sown in 1832 and 1833 , with a very prolific and early oat from Berwickshire; that the difference was not mate. rial in the first year, either as to grain or straw, but the Hopetom was rather the latest; in the second it was considerably bulkier and stronger, but still proved about a week later.

Among the numerous different sorts which are grown, we understand that a stimless oat has been lately brought from a remote district of China, which possesses the extraordinary advantages of being not only free of liusk, but of containing far more farinaceous matter than any of the known linds, while its produce has amounted to 26 barrels of 14 stone each, to the Irish acre. It was, also, not sown until the 4 th of May, 1830, and was reaped early in August + .

The species more peculiarly adapted to poor soils are the Tartarian, the black, and the naked oat, or Pilcorn and Pilez, all of which are hardy and generally prolific: of these the Tartarian is the only sort that may be considered as a distinct kiad; the others being only varieties of the common ficld oat. It is good horse-corn, and has been known to produce as much as 80 bushels to the acre; but its strong reedy straw renders it of but little value as fodder. The black oat is a profitable species on exhausted lands or wet and starved clays, and on upland soils, well managed, good samples weigh about 41 lbs. per bushel: it is also much used for green food when sown wilh tares. The naked oat, or, as it is not uncommonly termed, "Pilcorn," from the corolla being detached from the seed, as in the case of wheat, is thought to be not improbably the only surviving branch of our primitive com, if not the only bread corn of our remoter ancestors; but its only recommendation is its hardiness in producing a moderate crop where no other grain can be expected to grow $f$.

Tiie "Pilez," as it is provincially called in Corinwall, to the western parts of which its culture seems to be confined, is probably a variety of the Pilcorn, as it grows something like the oat, but the straw is much finer, and almost as good as hay. The grain is small, being only about the size of a shelled oat, but weighs as heavy as wheat jer bushel, and is excellent for feeding poultry and pigs. It is generally the farewell crop on ground that has been exhausted by preceding crops of potatoes, wheat, and oats $\S$.

$\Rightarrow$ Prize Ess. and Trans. of the Highland Soc., N. S., vol, ii, p. 356, and vol. iii. p. 48 .

+ New Farmer's Mag. for August, 1832. No. XI.

\$ See the Report of Northumberland, 3rd edit., p. 84. Burroughs on White Crops, p. 32 ; and the Surveys of South Wales, vol, i, p. 474; and North Wales, p. 166.

§ Survey of Cornwall, p. 66. 


\section{SOIL AND CULTIVATION.}

Although oats may be grown upon any kind of soil that is not too dry, and are in this country seldom sown upon land that will produce barley, it is yet a mistake to suppose that they may not be advantageously cultivated upon good soils, and it is now generally admitted that in many parts of Ireland, and throughout the north of Scotland, they form a more certain as well as a more profitable crop than wheat; for they support unfavourable seasons better; and although they suffer, like all other grain, from bad weather, they yet recover more readily when it changes. When land is broken up from rough pasture, they form the most usual crop, and are even frequently sown upon one ploughing, as they will flourish before the soil can be brought into a fit state for the regular courses of husbandry by the decomposition of the sward; and, for the same reason, the crop is very commonly repeated. They will also be always found the best crop to follow clover which has stood two years, and which it may be convenient to feed until the close of autumn: or, even should it not be requisite to feed it after the summer, yet-unless the land be peculiarly adapted to wheat-it may be found a better practice to break up the clover late in the season, to leave the land in that state until early in the spring; and then to sow oats under furrow, slightly harrowed, and afterwards to harrow them once again when they have sprouted.

Oats are very rarely sown after a summer fallow, though they frequently precede it. However, when the plan is adopted, the land is ridged up during the winter, and generally receives a seed furrow in the spring; though this is frequently dispensed with, and the seed is put in either with the grubber or the harrow.

The crop is rarely dunged for; and, in the triemnial system, upon soils of a nature so heavy as not to admit of the alternate husbandry on which corn crops necessarily follow each other, wheat is generally made to precede oats: the better practice would, however, be to invert the plan,- to sow the oats first, and to dress with long dung, by which the crop will be much benefitted, and the field will be brought into fine order for the production of the succeeding wheat.

When sown upon the stubble of a previous corn crop, the ground should get three ploughings; for, if only two be given, the land becomes frequently so infested with weeds, that, extraordinary as it may appear, it is often found more foul than when only ploughed once.

Oats are sometimes sown with clover; but the practice is not so common as it is with barley, for they shade the ground more than the latter, and are, therefore, apt to smother the young grass seeds. This in a great measure prevents the necessity of sowing them with the drill; and, as newly broken up ground is, besides, seldom in a fit state to allow of the operation being performed in that mamner, they are mostly sown broadcast : there can, however, be little doubt that when drilling, and the more careful management with which the process is continued, can be conveniently carried into execution, the crops will be materially benefitted.*

Oats are very commonly sown along with tares, in order to be cut green for cattle; and in some instances the crop is allowed to stand until ripe; but

* In the Survey of Devonshire an instance is mentioned of a crop of white oats, grown upon a sound grey loam, and highly manured, having yielded the extraordinary quantity of 115 bushels jer acre, weighing about 35lbs. The seed was drilled at 11 i inches : the land twice scarified, horse-hoed, and the rows afterwards moulded up. A part of the same field of like quality, and equally manured, was at the same time sown broad-cast and harrowed in: the yroduce at the rate of 88 bushels per acre-p, 180. 
in such cases the grain is not generally thrashed, and the straw is cut for manger-meat, in which state it forms a very substantial food for cart-horses. They may in this manner be also mixed with peas; and we learn that when they have been sown with barley, on soils that were neither too clayey nor too sandy, their joint produce has always exceeded that of either species of grain when sown separately*.

\section{SEED AND SOWING.}

The seed of the different varieties should be adapted to the kind of soil upon which it is to be sown; the Poland and Potato species require good land; the Tartarian, the black, and the red oat, are best adapted to mountainous districts and late climates; and the other sorts may be grown upon the generality of soils throughout the United Kingdom. It is, however, thought advantageous to procure the seed, if possible, from inferior ground; or, if it cannot be obtained from a poorer soil than that on which it is intended to be sown, then the best mode is to sow heavy, good seed, and particularly that produced in the fens $\dagger$. It is also of extreme importance that it should be in a state of perfect ripeness when cut; and, also, that it should not be afterwards allowed to become heated. The latter defect may be generally detected by a bad smell which the grain acquires by fermenting in the heap; the sure consequence of which will be that, although it may sprout when sown, the plants will grow up weak, and will be apt to fail at the time of blooming. It is only partially subject to a disease called "sedge-root, or tulip-root," resembling smut +; and it is never steeped.

The quantity usually sown varies with the species. The amount is much larger than that of any other sort of corn ; seldom being under four, and often as much as from six to seven bushels per acre: an old Cornish farmer, indeed, on being asked how much he would recommend, advised to "sow

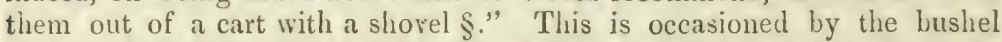
containing fewer grains, and neither being so uniform, nor so certain in their germination; nor does the seed tiller so profusely as other corn. The potato-oat requires the smallest portion, both hecause it tillers well, and has not an awn, or tail, on which account a measure contains many more grains than any other of a good hind; the Poland, on the contrary, not plinting so well, requires more seed $\|$. Much must also depend upon the richmess of the soil, and the equality of the depth at which the seed is placed; for it should neither be sown too deep nor too shallow; and, as it is not easy to hit the appropriate medium, it is generally considered the safer plan to sow a good round quantity.

The usual time of sowing is in the months of March and April: from the

* Von Thaër, Prin. Rais. d'Agric. 2nde edit, tom. iv. p. 218.

† Drew's Norfolk Husbandry, p. 119.

+ "This disease, which is, in some places, called "seying," makes its appearance, in ordinary seasons, towards the middle or the latter end of June, and is known by its soon changing the natural colour of the spront into that of a dark luxuriant green; but the plants soon become pale and dwarfish, and at length decay without coming into ear, leaving the place, for several yards around, an entire blank, or nearly so. At the bottom of the stock, a multiplicity of small white sickly-looking fibres, resembling ruots, are generally found warped together, and in most of the diseased plants the seed is attached to a single root, the only one at the plant, and from half an inch to an inch and a half from its main junction with the stock."-Farm. Mag, vol. xxi. p. 32.

The persoin from whose account this is extracted, ascribes the malady to the ground being ploughed or harrowed while covered with snow: On the correctness of which wo have no means of forming an opinion.

\$ Survey of Cornwall, p. 66.

if Brown's Rural Affuirs, vol, ii, p.49. Gen, Rep, of Scotland, vol, i. p. 501. 
beginning to the middle of the former is, however, generally considered as the best period; for, if deferred so long as until the latter end of April, the yield will be found deficient. If, indeed, the seed can be conveniently got into the ground in the close of February, it will be still better; for early-sown oats are almost invariably found to produce the largest quantity of grain : late-sown, of straw. In wet, low ground, this may not be always practicable; and in parts of the country where the spring is usually dry, it may not be necessary; but in much of the western coast of England and Scotland, as well as throughout the whole of Ireland, the climate is humid, and the month of March is commonly so wet, that the land is frequently not in a condition to be sown until May*. It should also be observed, tirat oats require more moisture in the soil than any other kind of corn, and that it is important to have the grain formed before the commencement of the parching droughts of summer; in proof of which it will no doubt be recollecteri, that while the crops of wheat were most abundant in the years 1826 and 1834 - which were the driest within the memory of manthose of oats were deficient, the grain smaller in size, thicker in the husk, and with a longer awn than is usual in moist seasons.

The autumnal sowing of outs is practised by some persons, and the autumn crops on the sandy loams of the counties of Wicklow and Wexford are seldom known to fail if sown early in October; but on poor sands, or on cold clays, they probably would not succeed. The autumn-sown crop has the advantage of being ripe three weeks at least before that sown in spring; which is certainly a very material point; but it interferes with the sowing of wheat, and occasions the inconvenience of crowding the other farm work. No particular species of this grain has been hitherto discovered as being peculiarly suitable to autumnal sowing; but it is said that the potato-oat, when sown in that season, has been found uniformly unproductive. It was sown, however, on poor land; but the gentleman by whom the account is riven conceives that, had he tried the Tartarian or the red oat, he might have been more successful. There can, indeed, be little doubt that were the seed of any hardy kind which had been raised in the winter, to be sown in the following autumn, the species would become gradually naturalized to the climate, in the same manner as occurs with winter and spring wheat + .

\section{PRODUCE AND QUALITY.}

The climate of Ireland and Scotland is not only more favourable than in most parts of England to the production of oats-as being of a more cold and moist nature-but the grain there gets at least an equal share of good soil and attentive cultivation; whereas, in this country, it is very generally grown upon the poorest land, and the tillage is very much neglected. To which it may be added, that oats are there generally taken as the first crop after grass, and the best both in quantity and quality are thus alwars obtained. The produce and the quality thus differ so greatly with the nature of the soil and the mode of manarement, as well as with the varieties of the different species cultivated, that in some situations 25 bushels per acre are thought a fair crop; while in others five quarters are considered as only an average; and from six to eight, and, in many places, even ten, are not unfrequently raised, of weights varying from 34 to $48 \mathrm{lbs}$. per bushel + .

* Lambert's observations on the Rural Affairs of Ireland, p. 3.

t Burroughs on White Crops, p. 34.

† Gen. Rep. of Scotland, vol, i. p. 509. Brown on Rural Affairs, vol, ii. pp. $48,50$. Surv, of Essex, vol, i. pp. 349, 350. 
The average produce upon medium soils throughout the United Kingdom cannot, however, be estimated at more than four quarters per acre; nor the weight than, at the most, 40 lbs. per bushel.

According to the analysis made by Sir Humphry Davy, the whole quantity of nutritive matter contained in 1000 parts of a bushel of Scotch oats, which probably weighed at least $4 \%$ lbs., was 743 , containing

641 parts of mucilage;

15 of saccharine matter; and

87 of gluten.

These analytical tables, it should, however, be observed, can only be received as affording a general idea of the comparative qualities of different kinds of grain; and the most usual mode of estimating the value of any particular species is by grinding the corn of the same kind, but different weights, into flour, and ascertaining the quantity which it will produce of meal, as shown regarding oats, in the first volume of this work *

In the mealing process, the oats, after being previously dried on a kiln, are made to pass through the mill-stones, to divest them of their coarser husks or "shealings," before being ground. The kernels are then named "grits" or "groats;" and are next ground over again into a coarse rough meal, varying in its fineness according to the custom of different districts. This is afterwards either baked upon a heated iron into thin flat cakes; or made up with water, usually boiled into a thick consistence, and is eaten either with skimmed milk, butter, molasses, or ale. It is thus very generally used as the common brealifast and supper of the greater portion of the peasantry of the northern parts of England, Scotland, and Ireland, and forms a very nutritive and healthy food + .

The quantity and the quality of oat straw produced per acre differ, of course, according to the nature of the soil on which the crop is grown. The quantity generally bears a proportion between that of wheat and barley; or, upon an average, about a load of straw may be expected from every twelve bushels of grain; but the weight depends greatly on the species grown. The quality also varies in the different sorts more than in any of the other kinds of corn; and this, as it is universally used as fodder for store cattle, is an object of such importance as, in some cases, to occasion a preference being given to the growth of oats, the grain of which is known to be of an inferior species. Thus the kinds known as the common feedoat have a strong stem, which affords a large quantity of good fodder, while the Tartarian or Siberian, though very productive of grain, yet yields straw of a nature so coarse as to be only fit for litter. The produce of the Polands is small; the red, the potato, and the Hopetoun, yield straw which is too reedy to be relished by cattle; while the late Angus species is both more abundant and superior in quality.

There is, however, much difficulty in ascertaining the merits of the straw of the different varieties, in consequence of the many contingencies to which they are exposed. Some kinds-such for instance as the Polish and the Georgian-grow rapidly in the early part of the season, and soon attain the moderate height to which they arrive; while the Potato, the Dutch, and early Angus, though also short, are yet later in attaining their growth; and others-as the Hopetoun and the late Angus-which are taller than the former, are also still longer in reacling their full extent. Thus, when the

* Chap. vii p. 146.

† Surveys of Lancashire, p. 333; of Northumberland, p. 85; Gen. Rep. of Scotland, vol. i. p. 513 ; of East Lothian, p. 12; Mid-Lothian, P. 105 ; and Surveys of Ireland, passim. 
early part of the summer proves wet, and is followed by drought, the shortstrawed species have an advantage over the other kinds, as they arrive at maturity before the dry weather commences; but, when the early part of the summer is first dry, and then followed by rain, the long-strawed sorts are benefitted by the moisture ; and when the season is throughout wet, the smaller species frequently obtain the advantage in point of grain, over the larger oats, in consequence of the straw of the latter becoming too luxuriant*.

The straw of oats is generally preferred, as fodder, to that of wheat; for when given to cattle, they are found to like it better if well saved, and if the quality be good. It is also thought to be more nutritive; but that, we imagine, arises from the difficulty which sometimes occurs in cleanly thrashing the grain, in consequence of the irregularity with which it ripens. It is, indeed, not unfrequently given by farmers to their working horses unthrashed, and cut into manger-meat without any hay ; but it is a practice by which nothing more is gained than the mere saving of the expense of thrashing, while it prevents any just estimate of the proper quantity of corn to be consumed + .

\section{Table showing the Comparative Value of Oats, per Barrel in Ireland, and per Quarter in England.}

\begin{tabular}{|c|c|c|c|c|c|c|c|c|c|c|}
\hline \multirow{2}{*}{$\begin{array}{c}\text { Cost per } \\
\text { Barrel } \\
\text { of } \\
196 \mathrm{tbs} .\end{array}$} & \multicolumn{10}{|c|}{ Weight per Imperial Bushel. } \\
\hline & $34 \mathrm{tbs}$ & $35 \mathrm{tbs}$ & & $36 \mathrm{tbs}$. & $37 \mathrm{Hbs}$. & $38 \mathrm{tbs}$. & $39 \mathrm{fbs}$. & $40 \mathrm{lbs}$. & $41 \mathrm{ttss}$. & $42 \mathrm{lbs}$. \\
\hline s. $d$. & s. $\quad d$ & s. a & d. & s. $\quad d$. & s. $d$. & s. $\quad i$. & s. $\quad d$. & s. $d$. & s. $\quad d$. & \\
\hline 100 & 1310 & 14 & & 148 & 151 & 156 & 1511 & 164 & 168 & 171 \\
\hline 103 & 143 & 14 & 8 & 151 & 155 & 1510 & 163 & 168 & 171 & 176 \\
\hline 106 & 146 & 15 & & 155 & 1510 & 163 & 168 & 171 & 176 & 1711 \\
\hline 109 & 1411 & 15 & & 159 & 162 & 168 & 171 & 176 & 1711 & 184 \\
\hline 110 & 153 & 15 & 8 & 161 & 167 & 170 & 176 & 1711 & 184 & 189 \\
\hline 113 & 157 & 16 & & 165 & 1611 & 175 & 1711 & 184 & 189 & 192 \\
\hline i1 6 & 1511 & 16 & & 1610 & 174 & 1710 & $18 \quad 3$ & 189 & 192 & 19 \\
\hline 119 & 164 & 16 & 9 & 173 & 178 & $\begin{array}{ll}18 & 2\end{array}$ & 188 & 192 & 198 & 201 \\
\hline 120 & 167 & 17 & 1 & 177 & $18 \mathrm{l}$ & 187 & 191 & 197 & $20 \quad 1$ & 206 \\
\hline 123 & 170 & 17 & 6 & 1711 & 185 & 190 & 196 & $20 \quad 0$ & $20 \quad 6$ & 2011 \\
\hline 126 & 174 & 171 & 10 & 184 & $18 \quad 10$ & 194 & $19 \quad 10$ & $20 \quad 5$ & 2011 & 214 \\
\hline 129 & 178 & 18 & 3 & 18.8 & 192 & $\begin{array}{ll}19 & 9\end{array}$ & 203 & 2010 & 214 & 219 \\
\hline 130 & 18 & 18 & 7 & $\begin{array}{ll}19 & 1\end{array}$ & 197 & $\begin{array}{ll}20 & 2\end{array}$ & 208 & $21 \quad 2$ & 219 & 222 \\
\hline 133 & 18 & $18 \mathrm{l}$ & & 195 & 1911 & 206 & $21 \quad 1$ & 217 & $22 \quad 2$ & 227 \\
\hline 136 & 18 & 19 & 3 & $19 \quad 10$ & 204 & $\begin{array}{lll}20 & 11\end{array}$ & 215 & 220 & 227 & 231 \\
\hline 139 & 191 & 19 & 8 & 202 & $\begin{array}{ll}20 & 9\end{array}$ & 213 & $21 \quad 10$ & 225 & 230 & 237 \\
\hline 140 & 195 & 20 & 0 & 206 & 211 & 218 & $22 \quad 3$ & 2210 & 235 & 240 \\
\hline
\end{tabular}

\section{Chapter XIV,}

\section{ON HARVEST.}

Although the term Harvest properly applies to the gathering-in and storing of every species of vegetable which is carried off the ground, yet we think it may be more aptly attached to those crops which are commonly

F * Transactions of the Highland Society, N. S. yol, ii, p. 358.

† See vol, i. chap. vii, pp, 132, 144. 
used in this country as bread-corn, and which are reaped and thrashed in a nearly similar manner. These are cut down either by mowing with the scythe, or reaping with the sickle; for although machines have been invented for the performance of the operation, they are far from being generally employed. The grain is then either stacked, or housed in barns, and is afterwards thrashed out either by the manual labour of the flail, ox by means of the thrashing-mill; and after being winnowed, in order to divest the kernels of their husks, it is either ground into flour of various degrees of fineness, or malted, according to the various uses to which the different species are applied.

\section{RIPENESS.}

The commencement of harvest is necessarily regulated by the state of the weather, and varies in different seasons, even when the weather is favourable, from the middle of July to the end of August; while in some years, and in exposed situations, it is still later. It is, therefore, an object of importance to the farmer to ascertain the exact time when it may be begun : for lie must employ extra hands to perform the work; and, as it only lasts during a comparatively short period, they receive high wages, and are maintained at heavy cost. It is also attended with the most anxious solicitude; for it is a business which cannot be for a moment neglected, and the man who wishes to get it rightly managed, must superintend it, without intermission, from the dawn of day until its final close. He should previously get rid of all other work, and make every preparation for the due performance of this : the barns should be thoroughly swept out, both roof, walls, and floors; the stack-frames repaired, and every tool should be in complete condition. The straw-bands should be in readiness for tying the sheaves, as well as the ropes for securing the stacks; and arrangements should be made in the house for the regular supply of whatever is to be furnished to the labourers, so that every unnecessary delay may be avoided. The strictest order should also be maintained; but the work will never be well performed unless it be conducted with perfect good temper. Fortumately, however, the crops do not usually ripen at the same precise period: that of rye being the earliest, and wheat about a fortnight later; some of the early species of oats and barley come in between the rye and wheat; but barley more generally comes afterwards, followed by some of the later kinds of oats. Grain, if not reaped until the straw is wholly yellow, will be more than ripe; as the ears generally, except in late seasons, ripen before the entire of the straw, and it is observable that the first reaped usually affords the fairest sample.

The indications of ripeness are few and simple. When the straw exhibits a bright golden colour from the bottom of the stem nearly to the ear ; or, when the ear begins to bend gently, the corn may be cut. But-as the whole crop will not be equally ripe at the same time-if, on walking through the field, and selecting the greenest heads, the kernels can be separated from the chaff when rubbed through the hands, it is a sure sign that the grain is then out of its milky state, and may be reaped with safety; for although the straw may be green to some distaince downwards from the ear, yet if it be quite yellow from the bottom upwards, the grain then wants no further nourishment from the earth, and, if properly harvested, it will not shrink. These tokens will be found to sufficiently indicate the ripeness of wheat, barley, and oats; but that of rye arises, as we have already stated, from the straw losing some of its golden hue, and becoming paler.

Without adverting to the unsettled point, regarding the quality of wheat 
being really better when cut in a partly green state, it is, however, evident that - not only is the sample superior in the eye of the miller, when it is not quite ripe, but that, if left until that moment ere the harvest be commenced, it becomes over-ripe before the harvest can be completed. A man who has a large farm, and is likely to be short of hands, should therefore begin the work of harvest much earlier than might be necessary, were his extent of land and number of labourers such as to enable him to secure his crops without delay; for if the weather continue dry, the grain will frequently shed to the amount of perhaps from two to five bushels per acre. This is a loss to which all corn is more or less subject if allowed to stand until completely ripe: barley, however, in a less degree; and oats, particularly of the early species, in a greater than the others. Oats are, indeed, in that respect, difficult to be treated; for they ripen so unequally, that a portion of the crop which is ready for cutting, will shed freely, either with the wind or in harvesting; while that which is unripe cannot, without great difficulty, be thrashed out of the straw. The grain which is first ripe is, however, always the best; and what remains unthrashed is not to be considered as lost, for it can be used with good effect in the straw-yard : the crop should, therefore, always be cut before any part of it is completely ripe; and the further advantage will be thereby gained of securing the straw in a more succulent and palatable state for the cattle.

The following experiment, though on a minute scale, respecting the reaping of barley at various periods in different states of ripeness, may throw some light on the subject as it regards every species of grain.

The quantity was equally divided into six parcels: the first of which was cut when the ear was pretty well filled, but soft and quite green; the stem and hose also green, and the leaves only partially decayed. The other lots were severally allowed to remain until the dates hereafter mentioned; the sixth being-both in the awns, ear, stem and hose - completely straw-coloured, and in that state of ripeness which was thought sufficient for the reaping of the entire field. The parcels were then subjected to various tests, by measure and by weight, with the view of determining their comparative value; and the result appeared to warrant the following statement of their relative proportions, assuming 100 as the test of perfection.

No. 1. Cut on the 11 th of August, was as 40

2. Do. 18th do. as 59

3. Do. 25th do. as 77

4. Do. Ist of September as 89

5. Do, 6th as 91

6. Do. 11th as 95

A greater difference, it will be observed, occurred between Nos. 5 and 6 than in a similar space of time between Nos. 4 and 5 ; which may be attributed partly to an improvement in the weather, which had become more favourable in the latter week, and partly to the accelerated progress towards maturity which it is supposed crops acquire in the latter stage of ripening. If, however, in that period, high winds or rain had ensued, the consequence might have been to turn the scale in favour of No. 5, and to aflord an instance of the danger of delay in reaping when the crop is in a state closely approaching to maturity*.

The harvesting of barley is, indeed, even in the best of seasons, a very troublesome business, requiring more care than any other white crop, and 
in bad years it is often found difficult to save it; for, in consequence of lateness and inequality in the time of sowing, it is very subject, when growi on cold lands, to be caught in dripping weather. Let the state of the weather be what it may, yet, owing to the brittleness of the straw, the corm must however be cut down after it has reached a certain period; as, when suffered to stand longer, much loss is sustained by the breaking of the heads. It is, therefore, reaped before the grain is sufficiently hardened to be put in stack without long exposure in the fields; and if put up sooner it is apt to heat. Thus it often happens that it is much discoloured and injurcd before the clover, which is usually mixed with it, can be carried without danger; and as its malting qualities are in a great measure estimated by the brightness of the sample, it is of the utmost importance to secure it as early as the season will admit.

\section{IFAPING.}

The operation of cutting down the corn is performed, as we have already stated, in various ways, and although some of these are considered as improvements upon the old method, they should yet be cautiously adopted: indeed never, unless the farmer be himself perfectly master of the new mode, and has at least some good hands who understand it ; for it changes much of the usual course of work, and every innovation is more or less destructive of that good humour which it is necessary to encourage among the labourers if it is to be well performed. To carry it on in a regular manner, they should be divided into separate gangs, arranged according to their ability, so that cach band may possess, as nearly as possible, equal powers; for, if this be not attended to, it will be found that the weak, or inexperienced, will hinder the work of the expert and able-bodied. As the extra people employed frequently come from a distance, it may, indeed, be at first not easy to class them, but one day's observation of the manner in which they perform the work will enable the overseer to place them accurately.

The reapers are usually placed in bands of six each, many of them being women, headed by the most experienced man among them; and these are followed by a man - as a bandster-to tie up the sheaves, and place them upright, in stooks of twelve each, upon the middle of each alternate ridge. The implement commonly used by the reapers is a light tool with a short handle, and a semicircular blade, called a "sickle." In ancient times, the blade was always serrated in the manner of a saw; but it has been lately made with a stronger and broader blade, with a smooth edge divested of teeth, and is termed a "cutting-hook," in which form it is said to cut the grain more neatly, and closer to the ground; but the sharpening of the iustrument occasions much loss of time, and, in the hands of inexperienced persons, is often imperfectly done. In using either tool, the reaper takes a handful of the corn in his left hand, and cuts through the straw with the instrument in his right; then places it upon a straw-band laid upon the ground, and, when enough is thus laid together to form the sheaf, it is bound up by the bandster. This is a much more expeditious and a neater method than that used in some places-of allowing each reaper to bind his own sheaf; for the sheaves are thus made up with greater accuracy, and time is not wasted in twisting straw-bands; an operation which ought always to be performed at previous leisure, and should never be allowed to interfere with harvest work.

On the subject of forming the sheaves, some judicious observations have 
been lately made by Mr. Howard, of Melbourne, in his report of Scoreby, to the following effect $\%$. That when reaping is done by the acre, it is very often imperfectly performed, in consequence of the persons employed, who are generally strangers, wishing to make the most of the job; to effect which they take as large a grasp as possible near to the top of the corn; the consequence of which is, that it must be cut ligh and irregularly, and much waste is thus occasioned. Added to this, to save trouble in the making of bands, as much is compressed into one sheaf as it can possibly contain, and the band is consequently drawn as tight as it will bear. If the weather be favourable, a large and tight-bound sheaf will require to stand two days Ionger than a small one before it is in a condition to stack; or, if the weather be wet, it must be opened and spread to the sun and air, and this not only occasions much trouble, but is not unfrequently the cause of the crop becoming sprouted through damp. In shearing by the acre, the interests of the farmer and the labourer are thus in all respects at variance; and Mr. Howard, therefore, gets his corn cut by the stook. The straw being considerably thicker near the root than at the top, it is the interest of the shearer to cut it low, which ensures its being cut clean and of equal height; and as the sheaves are paid for by number, he of course will not make them too large; thus his interests are combined with those of his employer, as the better he performs his work, the more money he will earn. If he attempt to make the sheaves too small, this may be guarded against by the simple expedient of using a light gauge, in the form of a prong, embracing a sheaf of the proper size, or 33 inches in circumference at the band. Another, and very great advantage, arises from the facility which this plan affords of paying off all the persons employed according to the quantity of work which they perform, whether individually or by the gang; and from the convenience of removing labourers from one field to another, without the trouble of measuring the work which they have done in it.

The sickle was formerly the only implement employed for the reaping of wheat, the scythe being then only applied to oats, and in some instances barley. Of late years, however, it has come very much into use, and seems to be gaining ground for the cutting down of every kind of corn; except the crop happens to be very much laid, or the ground very rough and uneven. It is certainly an efficient tool when well managed; but it reduces the employment of women; and, even among men, the operation of mowing not only requires a degree of expertness which is not necessary in reaping, but is also the hardest of all agricultural labour.

The operation of mowing corn may be performed by the common scythe, and that is the sort most generally used; but it ought to be more carefully constructed than one fur common purposes. The blade should be of well-tempered steel, strengthened by a plate of iron along the back; it should also be more than four feet in length, and fixed into a handle of the lightest and the toughest wood, less curved than those in common use. This will act with accuracy when the crops are heavy, for the corn will then fill regularly over, but when they are light, it should be furnished with a cradle attached to the lower part of the handle, for the purpose of gathering the stems and laying down the swathe evenly. The cradle should be made of three light and slim rods of the finest ash, of unequal length, the nipuermost about two feet long, but gradually diminishing, and fastened to an upricht stem strengthened by two upright rods which supjort them at equal distances, each about four inches in height, in this form-

* See Reports of Select Farms in the Lirbary of Useful Kinowledge : Farmer's Scries, No. 25. 


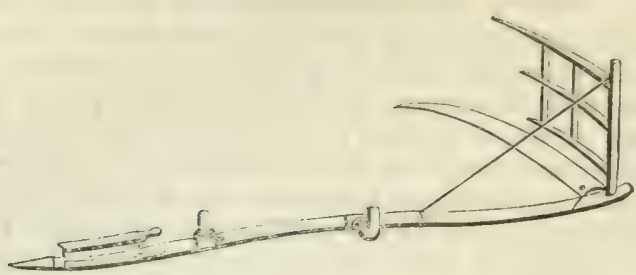

The wind should be in the back, or on the right hand of the mower, so as to keep the corn a little from him; but at whatever side of the field the work is to commence, a breadth of corn should be cut down equal to the whole band of mowers, and the better practice is to cut it diagonally across the ridges, from right to left, or to that side which will cast it from, instead of towards, the standing corn. Two mowers are generally put upon a ridge, each followed by a woman, to make bands, take up the corn, and place it in the hands of the bandster, who is followed by another woman to rake up the straggling ears; and, when the crop is good, the mowers will keep these women and the bandsters so hard at work, that the bands should be always previously prepared; for the sweep of a good scythe-man is from 7 to 8 feet, and when the corn stands fair, his forward cut is from 12 to 15 inches. The com is cut closer to the ground by the scythe than it is commonly done by the sickle, and it occasions less waste by shedding, when the grain is over-ripe. Another advantage is, that the straw is not crushed as done with the hand in reaping with the sickle; and, being thus less compressed, the grain is more open to the action of the air, consequently dries more readily, and becomes sooner ready for the stack. 'The sickle, however, lays the swathes more regularly; and the scythe cannot be efficiently employed upon crops that are much laid and entangled, nor upon gromnd that is not per. fectly even.

Two good scythe-men, with their attendants, will mow, bind, and stook, upon an average, ahout three imperial acres of all the corn crops within a working day of ten hours; which is something more than double the work which can be performed with the sickle. A band of thrce mowers, three gatherers, three bandsters, and one raker, is, however, the most economical number that can be employed; as one girl can rake up the refuse corn for the whole of them*.

An instrument called the Hainault Scythe has, within these few years, been introduced from Flanders, where it is generally used for the reaping of corn, and has attracted so much attention, that two young farmers were brouglit over from that comtry in 1825 , by the Highland Society, for the purpose of instructing our labourers in its use. 'They exhibited the mode of working with it in many of the northern counties, and details of the result having been submitted to a committee of the Society, the report stated"that their inquiries warranted thom in assuming the saving to be from onefourth to one-third of the ordinary expense of reaping by the sickle." This was upon an average of different trials; one of the most unfavourable of which assumed that a labourer with the seythe will cut a quantity equal to that cut by two good reapers with the sickle in the same timet. Rating

* Trans. of the Highland Soc., N. S., vol. iv. p. 192.

f See the Transactions of the Highland Soc., N. S, vol. i, p. 247; and The Farmer's Mag, vol, xxvi, p, 339. 
harvest labour at three shillings per day, the comparative expense of the two modes will stand thus :-

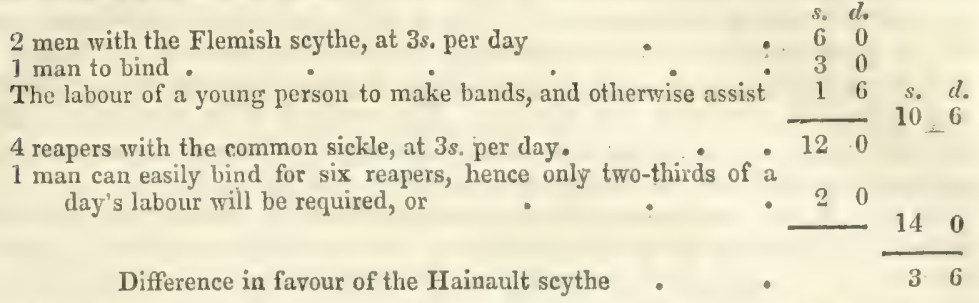

It thus appears that only about the same amount of work can be performed with it than with the common scythe; but the labour with that implement is much more severe, and the wages are consequently higher: it may therefore be considered a useful improvement both in point of economy and expedition, particularly in the cutting down of weighty crops. Although continually used by women in Flanders, it is however much heavier than the sickle, and cannot be employed to advantage where stones or rugged substances abound upon the surface of the ground; and these, added to the weightier objections ever made against innovations upon the common modes of performing farming operations, have hitherto prevented it from being brought into general use.

The tool, as here described, is used in both hands:-

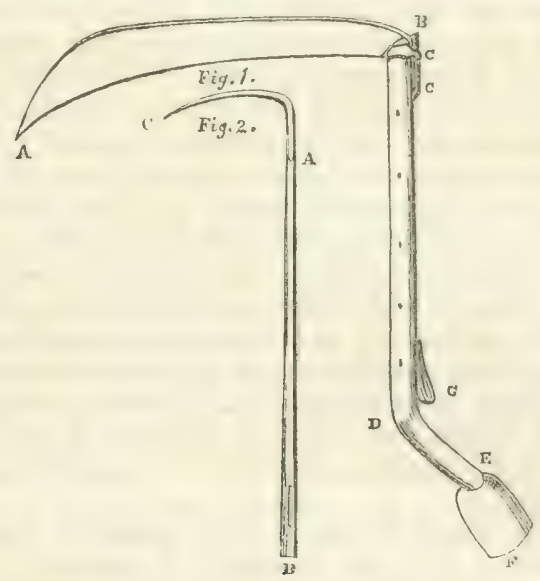

Fig. 1 represents the blarle of the scythe, which is 21 inches long from $A$ to $B$, and $2 \frac{7}{8}$ inches broad; the back is a quarter of an inch thick. It is fixed into the handle by wedges at C. C. The length of the handle from B to D is 17 inches; and from thence to $\mathrm{E}$ it is $5 \frac{1}{2}$ inches. The part marked $\mathrm{E} \mathrm{F}$ is 4 inches long by $3 \frac{1}{8}$ wide, serving as a balance to help the workman, and at $\mathrm{G}$ is a small leatheru strap in which he inserts his forefinger.

Fir. 2 is called the honk. The workman uses it with his left hand to gather in the quantity of corn he intends to cut; to support it when he is cutting, and to lay it afterwards behind him. This, which is of wood, has a handle of a square shape, $3 \frac{\pi}{2}$ feet long from A to B; with an iron hook abont 10 inches long from A to C fantened to the end of it. The small opening above $B$ is for the purpose of inserting the blade, so as to prevent the workman from being hurt when carrying the implement. 
The operation takes place by pressing the back of the crook against the standing corn in the direction of the wind, and by cutting close to the ground with a free swing-less by force than by the impetus of the scythe, till, in three or more strokes, according to the thickness of the crop, a sufficiency is secured to form a sheaf, which is caught by the inverted hook and placed in readiness for binding. The work is thus performed with extreme neatness, and such an additional length of straw is gained as to occasion almost the total disappearance of the stubble *.

In this age of invention, several Reaping Machines have been made for the cutting down of corn without manual labour, and have been brought forward with exaggerated accounts of the advantages to be derived from their use ; though it is evident that-exclusive of their great cost-they must be confined, in point of economy, to the difference of expense between working with a pair of horses and a driver, or with men and women as mere reapers; and that the saving of time extends solely to the cutting down of the corn, as it must be bound, dried, and carried in the same manner as at present. That many of them, particularly Smith's of Deanston, Gladstone's, Bell's, and Mann's, possess considerable merit, we do not mean to deny; but in the complicated processes of gathering the corn, and depositing it in regular order after it is cut, they have all, in some degree, failed. We do not, therefore, deem it necessary to enter into any detail regarding their mode of operation, but refer our readers to those publications in which they have been described + .

Grain should not be cut when it is wet; for, although the oat dries more readily than any other species, and suffers less from the rain, yet wheat and barley both sprout very rapidly when put together in a damp state, particularly if the weather be warm. The reaping of them should not, therefore, be commenced until after the sun has exhaled the dew ; but when a fieid of oats becomes ripe at the same moment with any other kind of corn, it is a good plan to reap it in the early part of the dewy mornings, and then to go to the wheat or barley during the dry period of the day. If the weather hold good, the wheat will be ready to be stacked in four or five days; the barley and oats in ten or twelve-according as they are more or less mingled with clover, which must be completely withered before the corn can be stacked; or otherwise the danger will be incurred of its becoming heated and mow-burnt, to the certain injury of the grain, and the probable risk of firing the rick. Barley is very subject to this; and its tenderness is such, that the least tendency to heat materially deteriorates its value: it demands, therefore, even greater attention during harvest than wheat.

The corn should always be cut as low as possible; for otherwise many straggling ears will be left upon the stubble, by which either much of the most succulent part of the straw will be lost, or the stubble must be mown and carried to the straw-yard for litter. When taken from the swathe, it should also be carefully laid in the bands; for, in lifting a sheaf with a fork, the hold is obtained at the band, and if that be not so tied as to balance the sheaf when it is to be raised in the cart, or on the stack, it occasions much additional labour. The sheaves should be firmly bound tugether; but not so tightly as entirely to exclude access to the air, for the more freely they are exposed to the atmosphere, the sooner will they be in a fit state to be carried. When bound into sheaves, these are generally put to-

* Radeliff's Agriculture of East and West Flanders, p. 125.

$\dagger$ See the Farmer's Mag., vol. vii. p. 273, and xvii. p. 10. Quart. Journ, of Agric., N. S. vol, iv. pp. 84 and 250 , in which are plates descriptive of the machinery. 
gether in stooks, or shocks of twelve each, resting upon their butts, and placed in opposite rows with the ears leaning acrainst each other, but the bodies so inclined as to be quite separate, and thus to admit the wind to blow through them. Wheat, being usually sufficiently dry in the course of a few days, is commonly allowed to stand in that manner, if there be a prospect of the weather continuing fair ; but, as barley and oats remain Jonger upon the ground, and showers may be expected, only ten of the sheaves are allowed to stand together, and are covered by the two others, which are placed lengthwise upon the ears, with the butt-ends against each other, and the ear-ends drawn a little down, so as to allow any rain that may fall to shoot over those which are upright.

Such is the usual mode of stooking; but many others are employed: thus in some places six or eight sheaves are set up together in a circular form, without any hooding ; while in others they are set up singly, or "gaited ;" in which case the sheaf is tied close under the ears, and the butt-end, being struck hard against the ground, is sufficiently distended to form a base upon which it can rest. In fields where wet is much dreaded, the crop is gatliered up into small ricks of a cart-loar, or less, as a preparation for the stackyard; and in many parts both barley and oats are forked from the swathes into cocks, and the grain is carried to the barns loose*. Indeed, as the cultivated grasses are frequently grown with these crops, it is sometimes necessary to leave them for a few days in swathe, and if foul weather should not be apprehended, the operation of binding them into sheaves is then omitted; but the practice may be justly considered as wasteful and slovenly.

In Sweden, it is very generally the practice to secure the crops from being damaged by standing on the ground when cut, by fastening the sheaves in an elevated position to stakes; which is thus performed. The stakes are about 8 feet long, $1 \frac{1}{2}$ inch in diameter at the top, and 4 inches at the bottom: the ends being pointed at the top, to allow of their being passed through the sheaf; and at the bottom, to facilitate their being fixed in the earth. They are set up at harvest, at the same distance as is common in stooking the crop, and the sheaves are put on by raising the first sheaf up to the top of the stake, and passing it with the root-ends downwards to the ground; the stake being kept as nearly as possible in the middle of this sheaf, which stands around it in an upright position. 'The second sheaf is then put upon the stake in an inclined position, with the ears sloping a little downwards, and in that manner it is pressed down to the first sheaf; thus forming a transverse covering to it. All the other sheaves are threaded on the stake in a similar way; keeping them above one another until the stake is filled: their root-ends being pointed in such a direction as will afford them the greatest benefit of sunshine for drying the grass which they contain $t$.

The plan has, we understand, been partially adopted in Scotland; and in those parts where timber is cheal, and the climate variable, we think it might be advantageously pursued; for it will protect seven-eighths of the crops from rain with very little additional labour, and the stakes, when carefully collected and laid aside, will last for twenty or thirty years.

\section{STACKING.}

In carrying the corn to the stack-yard, four-wheeled waggons are preferable to carts; and are, therefore, generally used for that purpose. Of

* Surveys of WVilts, p. 69 ; Suffulk, p. 75 ; Leicester, p. 108 ; Essex, vol. i. p. 49 ; Hants, p. 224. Marshall's Rur. Econ, of Yorkshire, vol. i. p. 392.

1. See the Quart. Journ, of Agric, N. S., vol, iii. p. 636 . 
these, the Cornish wain is the most simple, the lightest, and the handiest implement for the carriage of corn in the sheaf; but farmers are all attached to the particular form which is usual in the district which they inhabit, and it is, therefore, little known beyond the precincts of the West of England *. 'The persons engaged in this business form a band quite separate from those empluyed on the harvest-work, and are generally selected from among the most careful of the farm-servants. One man forks the corn from the field into the waggon, two carters drive the waggons and lift the sheaves to the stack, while an experienced workman builds it, and another assists in trimming it; a woman also sometimes rakes up any corn which may have dropped from the stooks, though this is not unfrequently left for the gleaners.

We have but little to add to what we have already said in a former volume on the subject of stacking hay + , except that the ricks should be protected until they are thatched, by awnings spread over them and supported by poles temporarily fixed in the naves of a couple of cart-wheels ; the rick-cloth being suspended upon a cross-spar, which is moved up and down by pulleys, to raise or lower it as occasion may require.

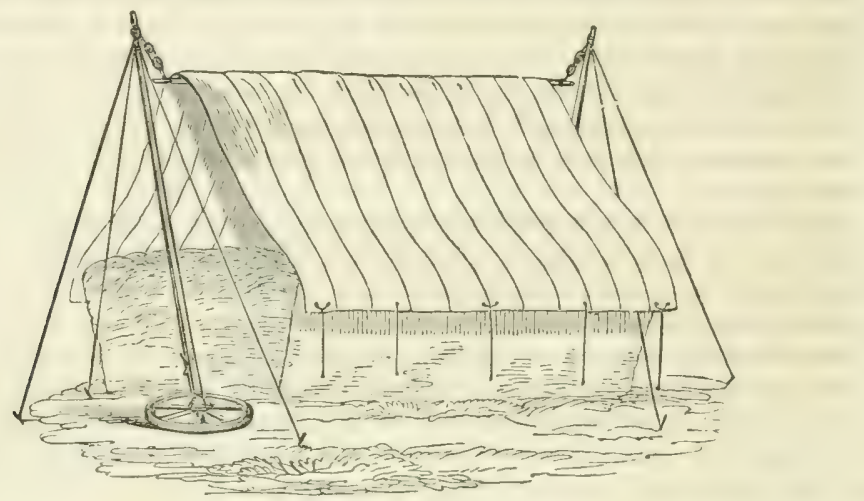

The cost may be altogether about $\mathfrak{L} 20$; for the expense of cloths is-

10 yards by $8, \mathfrak{L} 9.9 s . ; 12$ yards by $10, \mathfrak{2} 14.14 s$.

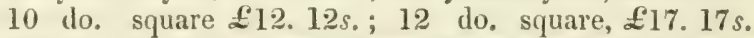

and that of the poles and pulleys is from five to six guimeas the set.

The stack, instead of being built upon the ground in the manner usually adopted for hay, should be erected upon "staddles," or stone pillars of a conical form and about two feet in height. These are topped with circular caps which project over the posts, and nine being ranged in rows of three each, a stout frame of timber is laid over them upon which the stack is built. The coping of the stone caps effectually prevents the access of rats and mice, which otherwise lorge in the corn, of which they consume large quantities, besides injuring it by the offensiveness of their odour; and the air, being admitted under the frame, tends to keep the straw dry and free from becoming mouldy. The sheaves are then laid with the ear-ends inwards, and the butts gradually projecting until they reach the eaves, which thus overhang the base and protect the rick from rain. These oblong stacks require less time and labour in the building and thatching, and are, therefore, more economical than any other construction; but those of a
* See a description of the Wain, in vol, i, p. 153.
$\dagger$ Chapter xxxii. p. 496. 
round form, if of small size, admit of a more free circulation of air, and are, therefore, preferable for the preservation of corn.

In building round stacks, a sheaf is first placed upright on its butt end, as nearly as possible in the centre; around which other sheares are placed circularly-likewise upright-but with a small inclination of the tops inwards, until the bottom of the rick is nearly filled. The stacke: then places an outside laver of sheaves on their sides, with the ear-ends inwarcis, but leaning obliquely upwards against the upright sheaves; and, pressing them together with considerable force, he thus continues to lay on rows, with their butts all outwards, till he has raised the outside of the rick to nearly the height of the standing sheaves. He then fills up the whole of the stack, in nearly the same manner-the corn-ends of the sheaves inwards, with a regular slope downwards and outwards to their butts; the centre of the rick being always kept somewhat higher and less compressed than the outer layers: an attention to the proper sloping of the sheaves which is necessary from the foundation, but particularly so at the "intake" of the "heart-gang,"-as the inner layer is called* ; that part being alsays left more open. When that is done, he sets on the "easing-gang," which is an outside circular row of sheaves, having the butts projecting a few inches beyond the body of the rick, and forming the eaves; after which every successive row of outside sheaves is placed gradually more inwards until the roof is drawn to a narrow circle, when two or three sheaves are placed upright in the centre, which they fill completely up. He then firmly binds these topmost sheaves by two or three turns of the middle of a straw-rope, and the assistants fasten the two ends on opposite sides, so as to secure the crown from the effects of the winds.

These circular ricks are from twelve to fifteen or twenty feet in diameter, and generally about fifteen feet in height to the eaves. Barley, however, should never be put together in such large stacks as wheat; and the smaller stacks are frequently built upon frames of the form in Fig. 1, with cast-iron starldles of a foot in diameter, a set of which can be had at many foumderies for a sum not exceeding fifty shillings-

Fig. 1.

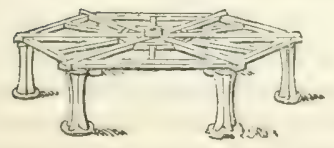

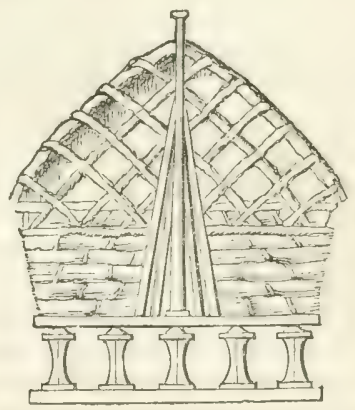

To this frame an addition is not uncommonly made of an upright, triangular woorlen stand, in Sicotland termed a "Boss," which is fixed in the centre, in the manner represented in the annexed section of the stack; and if the corn be in a damp state, it assists in the prevention of its becoming heated.

It will be readily imagined that none but a careful fellow can be safely intrusted with the building of any of these stacks; and after that, they must also be securely thatched. For this purpose, well drawn rye or

* Gen. Rep. of Scotlaud, vol, i. p. 391. 
wheat straw, of the toughest kind, and with the ears cut off, should be prepared in equal lengths*; which, since the introduction of thrashing mills, is a more difficult matter than formerly, and the only remedy, when the straw is short or broken, is to thicken the covering.

"In laying on the thatch, the thatcher stands upon a ladder resting on the sloping conical roof of the rick, as near as he can to the eaves, and lays on the thatch in handsful, from sheaves placed within his reach. Ife thrusts the inner end of his handful of thatch, gathered into a neck or wisp, into the butts of the sheaves, and spreads out the lower end like a fan, overhanging the eaves; then covering as much of these as he can reach at arm's length, he works upwards, causing each successive handful to overlap that immediately below; and he thus covers the roof in triangular portions, till he has gone round the whole backwards, that he may avoid treading on his work. When he has reached the top of all, he lays a considerable thickness of short straw upon the crown, over which a straw-rope is carried perpen. dicularly, dividing the roof into two equal sections. He then covers this topping of short straw with long thatch drawn to a point at the summit, and ties the upper end of this with a small straw-rope into a peak; giving this last part the appearance of an umbrella."

"With the aid of two assistants on the ground, while he remains aloft on the ladder, so as to be able to reach the top with his hands, the thatcher now places a number of well-twisted ropes of tough oat-straw to lace down the cover and secure the thatch. These obliquely embrace the roof in the lozenge fashion, and are fixed below, either to the butts of the sheaves, or to a beit-rope firmly bound round the body below the eaves t". If the ropes slacken, as they necessarily will do when the stack is compressed by its weight, care must be used to retie them; and in this manner the corn may be secured under the severest tempests: neatness in the building is, however, necessary to the security of the stack, and if this be neglected, it may be endangered.

An improvement upon these corn-ricks has been made by the construction of Skeleton Barns, as shown in the following page. Upon a timber platform, raised upon capped stones, the frame of a perfect barn is erectedends, sides, and roof; and this is either substantially thatched, or tiled. The advantages of this over the common ricks consist in the saving of the annual expense of thatching, and the superior security which it affords from the contingencies of bad weather at the time of harvest; for the corn may be deposited there in such quantities as may be most convenient, and all is perfectly safe. The barn may likewise be finally filled up to the top, or not, as occasion may require, the grain being at all events equally secure, and the side wings may be either occasionally used as shelter under which the waggons may be unloaded, or constantly as cart-sherls; or else, if the platform be laid on, the wings may be filled up with corn. The cost of erection is trifling in comparison with the convenience which such a building affords; for the frame may be made with rough timber, and the thatch, being free from the attacks of vermin, has been known to last, when well put on, upivards of thirty years + .

* The corn should not, in that case, be thrashed in the straw. "Ear-pitching" is the provincial term for this management, and the sheaves thus prepared are called "reedsheaves." They make a more durable covering than any other kind of straw; and the thatch is of superior neatness. The circumference of the sheaf at the band should be six feet, the weight 56lbs. ; and a dozen sheaves will cover a square of 100 feet. Survey of Somersetshire, 3rd edit. N.p. 98.

† Gen. Rep. of Scotlanil, vol. i. p. 394.

† Surver of Essex, vo?, i. . 48. 


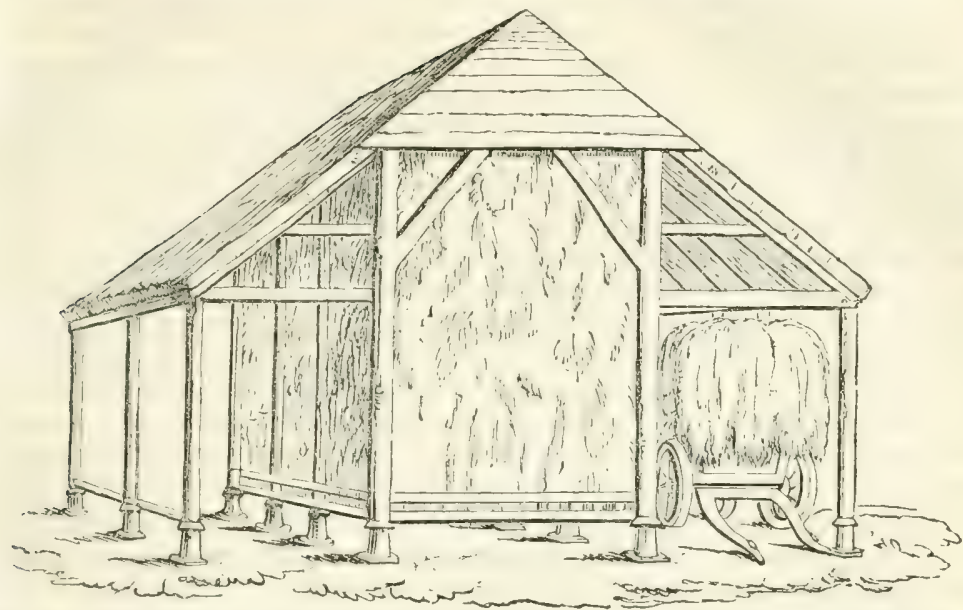

The Stack-yards throughout England are very generally arranged with grreater attention to order and neatness than in Scotland, and any deficiency in that respect is there looked upon as distinctive of slovenly farming. In Ireland, however, the stacks are described in a recently published agricultural report, "as commonly made very small, and carelessly put up; for they are generally laid upon the bare earth, without any bottom to protect them, and if wet weather comes on and continues long, much of what lies below perishes by attracting moisture; the frail structure is likewise liable to be swayed by the wind, and the tops and sides losing their original form, and being no longer capable of throwing off the rain, still more damage cnsues : to which is to be added, the depredations of vermin on all stacks thus exposed. As for Barns, in the English acceptation of the tcrm, they are literally unknown in the small farms of the sister lingdom. The floor of some out-house, perhaps, or even that of the family room, may be used for thrashing; but a vast proportion of the grain is beaten out in the open air, very commonly near the road side, when there happens to be a dry spot*."

\section{THRASHING.}

The operation of thrashing was formerly performed wholly by the flail, which is an implement too well known to require any particular description; though it may be remarked that-whether from the men being stronger, or the corn being not so well got in-the tool used by the labourers of the north of England is heavier than that employed in the southern counties. The labour is unremittingly severe, and if not very carefully performed, a portion of the grain is left in the straw; it also proceeds slowly, at a moment when dispatch may be necessary, and these ohjections, rather than any material saving of expense in the mode of performing the work, have given rise to the invention of the thrashing mill. Notwithstanding the acknowledged advantages of this machine to great farmers, it vet deprives the labourer of a large portion of the wages which he used to earn at a perivi of the year when little other employment could be obtained, and by an occupation carried on under shelter during the inclement season; therefore, however we may regret the outrages to which it has led, we camnot be surprised at its having roused the anger of an untutored and a starving peasantry, who are not aware that their own interests and their master's are inseparable. 
The origin of the Thrashing Machine is due to Scotland, where, nearly a century ago, the first attempt was made to construct one diriven by a water-whecl, which put in motion a number of flails of a similar kind with those used in thrashing by hand; it fell, however, into disuse, and it was not until about fifty years since that the invention was brought to nearly its present state of perfection by an ingenious mechanic, named Antrew Meikle. Some few improvements have been since introduced by other artisans, to simplify the labour, as well as to augment the quantity of work; and the unthrashed corn is now male to pass between two revolving rollers, while it is acted upon by a set of scutchers, or beaters, placed lengthwise upon a large cylinder, or drum, which moves with great velocity upon a horizontal axle. The power reguired to thrash the grain out of the straw is, indeed, so great, that the drum is reguired to revolve with the speed of two thousand five hundred feet in a minute; which necessarily demands the impetus of such considerable force, that the machinery must be worked by horses, by water, or by steam. It must, therefore, be solidly executed with the best materials, or it will constantly require repair, and this occasions the necessity of erecting fixed machinery, the expense of which is so great as to prevent its employment on any other than large farms-for the cost of a horse-mill varies from $100 \mathrm{l}$. to $150 \mathrm{l}$; and this, if the power used be either steam or water, will be increased by the cost of the engine in the one case, and the erection of the dam and sluices in the other.

Many Hand-machines have been invented, yet the labour required to move them is so great that the men employed in driving them must be relieved at intervals; therefore they possess no advantage in point of economy over the flail, and are consequently but little used. We have, infleed, lately seen the drawing -as at foot-of a Portable Thrashing Machine, made by Messieurs Ransome, of Ipswich, which is coming much into use, and can be efliciently worked by three or four horses. The cost is only from fifty to sixty guineas; and, as it admits of removal in a common cart, it can be used by different farmers at a moderate charge.
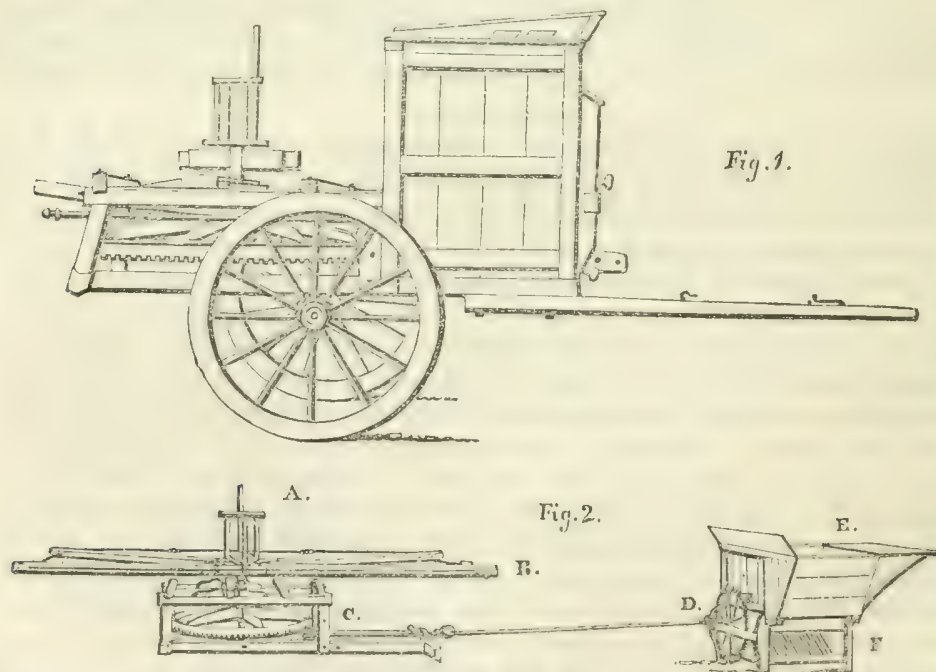

Fig. 2 .

F.

Fig. 1 shows the implement when packed for removal; and fig. 2 when taken out for work, The thrashing part $\mathrm{D} \mathrm{E} \mathrm{F}$ (called the "barn-work") stands within the barn, 
and is connected by the shafts D C with the "horse-work" standing in the yard. The horses are attached to the ends of the levers, and are driven by a lad seated on the centre at A, stepling with perfect ease over the connecting shaft D, which turus with a very rapid motion, acquired by the great difference in diameter between the horsewheel and the pinion on the shaft $\mathrm{C}$, which shafts are connected by an universal joint, and a further velocity is acquired by the introduction of a pair of wheels on the barn-work, one of which works on the suindle attached to the shaft D, and the other on the axis of the beaters, which are four in number, and thrash out the corn with astonishing rapidity. The corn is put into the machine at $\mathrm{E}$, and the straw driven out over the grating at $\mathrm{F}$. The quantity thrashed will in great measure depend on the nature and quality of the grain, and the grood or ill management of those engaged in the work; but, under favourable circumstances, the machine will thrash seven coombs of wheat per hour *

This will afford a sufficient idea of the implement, but we deem it unnecessary to give a detailed description of the machinery of any of these mills, as they can only be built by regular millwrights: we shall therefore confine ourselves to a brief account of their usual management, with this remark-that but few of them are erected upon correct principles; nor do farmers possess sulficient mechanical knowlerge to enable them to judge of their imperfections.

A part of the barn is commonly appropriated to that portion of the fixed machinery by which the operations of thrashing and winnowing are performed. The preferable power is decidedly that of either water or steam - the former as the most economical, and the later the most steady ; for, whatever be the nature of the moving power of the mill, it should be made to act with an equal force throughout the whole process. The most generally employed is, however, that of active horses, working a large wheel, in the manner of a mill, placed under a covered shed outside the barn, but communicating with the machine. The number of these is never less than two, nor more than six, but is usually four, and they should be cattle of much activity as well as strength; for the perfect execution of the work requires that they should be driven with speed. The unthrashed straw is placed on a floor immediately above the machinery ; underneath is an apartment for the reception of the corn, after it has passed through the machine, and adjoining is the straw-barn: there is also an apartment called a "dressingr-barn," in which the grain is winnowed and prepared for market.

In commencing the operation of thrashing with the mill, it has been properly remarked, "that the power should be at first applied with caution ; for a great and rapid force is apt to strain some weaker part of the machinery, and even if all the horses start strongly off at first, the coursewheel may be heard to creak + . The full velocity of the mill should, therefore, be gradually produced before any corn is put in, and, in feeding it, one sheaf at a time, spread evenly on the board, is quite suflicient for those of ordinary power; for, if its speed be impeded, much of the corn will remain untouched. Oats, in a damp state, are the most apt to prosluce this retarding effect; but it is also occasioned by wheat, if the straw be tough and long." Regularity of motion is also essential to the perfect frecing of the straw from its grain; and, whatever may be the nature of the moving power of the machine, it should be made to act with equal force throughout the work, or much waste may be occasioned by supplying it unequally. Thus the sheaves should be steadily furnished in due proportion to the strength employed; for which, indeed, no rule can be given,

* Survey of Essex, vol, i. p. 48.

+ See a very detailed and intelligent article, in the Quarterly Joumal of Agriculture, on Barn Management, N. S. vol, iii. 
but a short experience will enable any careful man who has the charge of the business to make himself master of the practice.

In order to perform the work completely, with a four-horse machine, nine persons should be employed in the following manner:-

1st. A person accustomed to horses to drive them steadily at one uniform pace of about three miles per hour.

2nd. $\Lambda$ man (or two boys) on the stack, to bring the sheaves to a woman who unties them.

3rd. A woman to untie the sheaves, and lay them handy for the fourth person, in order that he may feed the machine fast enough.

4 th. An active man, as feeder, to put the corn into the machine.

5th. A man with a rake, to take the straw and such colder ${ }^{*}$ as comes from the machine $t$.

6th. A man with a fork to take the straw, shake the corn from it, and carry the straw away as far as the door.

7th. A person with a rake to pull the chaff-colder from under the machine and dispose the same about the floor; so that he can with the rake separate most of the colder from the chaff and corn, putting the colder to the door, and the chaff and corn to the

Sth and 9 th Persons. A woman and bov, one of whom should sift the chaff and corn through a coarse sieve, which is filled by the other. What remains in the sieve is put into a large "skep," or basket, and most commonly emptied, when required, outside the barn door. It should, however, be carried to the straw-yard, and given as forlder to the store cattle, to whom it will be found very acceptable so long as it is fresli.

These nine persons, if well arranged, will keep the barn in tolerable order, and the machine constantly going for eight hours; when, if it be worked by horses of moderate strength, they will generally have thrashed about twenty quarters, or one hundred and sixty bushels of wheat. If a stronger power be employed, more grain will be thrasher, as well as winnowerl-for winnowing machines are now very generally attached to the mill; but the quantity thrashed by the mill should not be estimated so much by the number of quarters of corn which have been thrashed, as by the quantity of straw, for it is that alone which retards its operation: thus supposing the same quantity of straw to yield more corn in one year than in another, the time consumed in thrashing by the machine would be the same. Some farmers dispense with a portion of these hands, employing only one to fetch and loosen the sheaves, and one to riddle the grain; but although the work may be thus performed, it is generally executed in a slovenly mamner.

The thrashing of grain by the flail was formerly paid for, in most parts of the north, by the allowance of a certain portion of it to the thrasher, and one twenty-fifth part was then supposed to be a fair remunerating price for his labour; but now he would hardly be content with less than oneeighteenth. The work is, however, at present almost universally paid for in daily wages, or else at a rate per bushel according to the different species of corn. If to this be added the wages of the persons employed on the machine,

* Short pieces of straw and ear-ends are usually called "colder."

t A moveable skreen should be placed between the man's face and the rake, to protect him from the grain, which is thruwn agamst the rake with extraordinary velucity by the scutchers of the drum. 
together with the maintenance of the horses, the original cost, and the wear and tear of a mill, it will be found that no great saving can be made by the firmer of expenditure in money; but the saving of corn, by its more effectual separation from the straw, and the avoidance of all pilfering by servants, and depredations by vermin during the nights when it lies upon the barn-floor, has been supposed to amount in most cases to not less than one twentieth of the crop, when thrashed by a machine. To which also must be added the advantage which it affords of getting the corn ready for market at any moment, whenever either prices or convenience may render it expedient.

Winnowing machines, or fanners, are attached to most thrashing mills, as they are now constructed, and they are a necessary appendage to every homestead, either in that form or separately; as there is, perhaps, no single fact which so decisively attaches the character of slovenimess to a farmer as that of his presenting ill-cleaned grain at market. The principle of construction is in all the same : the hand-winnower contains four ohlong boards, termed "fanners," crossing each other diagonally at equal distances through the whole breadth of the machine, and fixed upon an axle which is driven round by a wheel turned by hand; so that a strong current of air is produced by the fanners, and the corn which falls from the top through the hopper is thus cleared of the chaff which is blown through the opening. The grain is sifted, in falling, through sieves, moved by cranks, in the same manner as the fanners; and, after passing through them, it is received on a floor perforated with small holes to clear it from dust and sand.

When these machines are not connected with the mill, they are made so light as to be easily moveable, and the side on which the axis, of one of the most simple construction, is fixed, appears thus :-

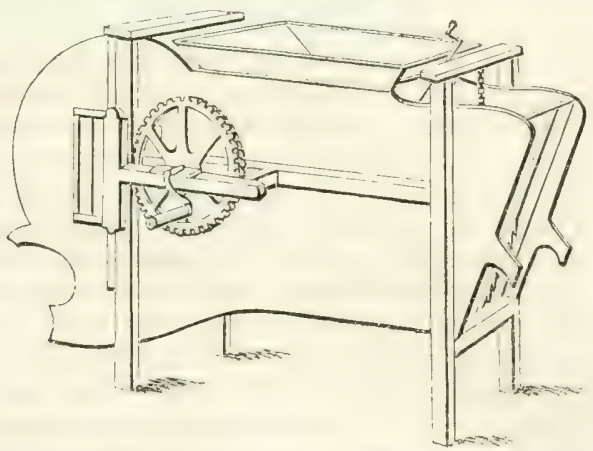

It is framed of wood, and stands upon a space six feet by two feet four inches, and is about four feet high. At the circular end of the machine in the interior there is a fan-blower or winnower, which, being put in rapid motion ty the wheel and pinion where the crank is attached, occasions a strong blast of wind. Beneath the hopper are two sieves horizontally placed at two inches apart, which are also put in motion shaking from side to side by turning the crank. Upon these the corn falls from the hopper, and while in motion, and the corn falling through them, the blast from the fan-blower separates the chaff and other rublish from it, blowing them out some war behind the machiue. The chobs or grains of wheat in the calyx, and other substances larger than the corn, pass over the sieves into a skep placed by a spont on the side of the machine. The corn then falls upon a fixed skreen placed in the centre of the machine, and laid in a slopiug direction towards the ground; from this it works off;, and on to a moving skreen, placed also in a sloping position, and at such an angle as at the time best suits the purpose. This skreen, shaking from side to side, completes the ciressing, the very thin grains falling through the skreens.-There are five sieves belonging to the machine, of different sized meshes, which are used fur the various sorts of corn, and two fine sieves for the purpose of dressing clover-seeds, \&c. When the machine is used for clover-seeds, the two sliding 
shutters on each side must be closed in order to take off part of the force of the wind from the fan-blower, (which would otherwise be strong enough to blow out the seeds, ) the two fine sieves put into the sieve frame, and the skreens covered with the board to prevent the seed falling through, in order that the fan may agrain act upon the seed while it runs down the bo:ard.- The corn and seeds, to be perfectly well dressed, should be twice passed through the machine.

Their interior construction, however, admits of various modifications, and they are in many instances very imperfectly formed by country carpenters. Our readers may, however, find a very minute description of the best machinery, together with plates, both in Low's Elements of Agriculture, and in the third volume of the Quarterly Journal of Acrriculture; to which we refer, without further detail, as the machines can only be made by practised artisans. Messrs. Weir, of London, have made some valuable improvements on them; and we have lately seen one made by Mr. Cooch, of Harlston, near Northampton, - who is also a practical farmer-which is much approved of in that part of the country, and appears to possess considerable merit. The cost of that depicted above is ten guineas; but they vary, according to workmanship, from that to twenty; and we are informed that those in common use throughout the north of England, though very perfect in their operation, yet cost only about five pounds.

\section{MARKETING.}

It is well known to corn-dealers that all grain shrinks when held for any great length of time in the warehouse; and although it may weigh something more per bushel, yet this additional value gained in quality by no means repays the loss in measure. Farmers do not generally advert to this, because they seldom thrash out their corn until they mean to send it to market; but there can be little doubt that a considerable difference may in most cases be found to exist between the measurement of grain immediately after harvest, and during the following spring and summer montis; of which the following instance is related in the Mid-Lothian Survey :-

"The farmer by whom the trial was made, took every third stook from a field containing 120 stooks of oats; and having immediately thrashed them, the 40 stooks produced 8 bolls, and 6 pecks of good grain, with 2 pecks of refuse. The 80 stooks remaining-which at the same rate should have produced 16 bolls 3 firlots-were stacked, and not thrashed until the following April, when the produce was only 10 bolls 3 firlots of good crrain, and 6 pecks of refuse : being a loss of about 6 in 17, or nearly 35 per cent. in measure, with an increase of only 2 lbs. per boll in weight ; and this without any loss by vermin."

In measuring grain for market, it has been acutely remarked by a late writer, "that there is an art, of which those who do not know it would never conjecture the importance. It is found by experience, that when a bushel is filled at once, it can be filled with a less quantity than when it is filled by degrees with small quantities: wherefore, the bushel should not be heaped "1) high above its rim; nor should the grain be forcibly poured into it; nor should a commotion be allowed on the barn-floor-particularly if it is a wooden onc-when the corn is being measured. There is no trick or unfairness in a farmer taking these advantages when in his power; for, if he takes the ordinary means to fill the measure, he is not obliged to press or tramp the grain into it. The difference in the measurement of grain, like the shrinkage of it in the stack, must however depend, we presume, very much upon the quality of the grain and the state in which it has been harvested: grood, plump, dry grain nust both measure its quantity more certainly, and shrink less, than ill-filled hungry stull"." 
According to the regulations of some markets, the corn intended for sale must be carried there and pitched at the market-house, where the whole quantity is exposed to the purchaser; but it is more generally sold by sample. The former mode is the fairest, as it precludes all dispute between buyer and seller respecting the quality; but is attended with the serious inconvenience to the farmer, of compelling him to carry his corn to a certain point, which may be far distant from the residence of the purchaser to whom it is to be delivered; and in case it be not sold, his must be at the further expense of either carrying it back, or storing it at the market. The sale by sample puts him, on the other hand, to no further trouble than a ride to the neighbouring town; but it not unfrequently leads to cavils respecting the quality; for the farmer-though without any intention of passing off an inferior article-yet always puts the best in his pocket, and this, after being handled by several persons, becomes brighter and better in appearance than that which remains in the sack: thus, sometimes griving the buyer apparent reason to complain; while occasionally, also, objections are unfairly made when a fall has suddenly taken place in the price.

With regard to the expense of reaping, inrashing, and marketing, the following account of the charges attending an acre of wheat in the listrict by which London is surrounded, may afford a general idea of the cost on other crops, namely,-

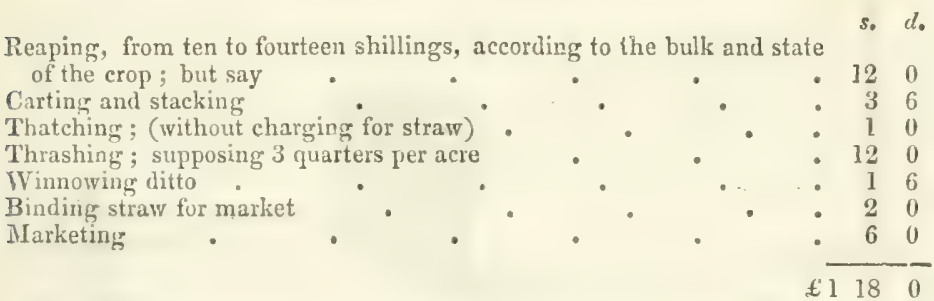

To these charges must also be added the expense of a supper, which, in former times, was always given at the close of the work, by the farmer to his labourers, in joyous celebration of the harvest-home! A custom originating in the religious and hospitable feelings of our forefathers, as evincing their sense of thankftilness to the Almighty for the blessing with which he had crowned their labours, as well as the kindliness of the master towards his servants; but the practice is now fast wearing away with the fastidiousness of modern mamers, and is more generally commuted for by the gift of a couple of shillings.

The expenses on an acre of oats or barley will be a little less; unless the crop is very bulky or lodged, in which case it will be quite as much. So much, however, depends upon the state of the crop-wet or dry harvest- good or bad to thrash and winnow-distance from market-and actual wagres of labour-as to make it difficult to say to what the expense may really amount; it must also differ in various parts of the country. Even after the harvest is completed, the season still has an efiect on the pre. paration of the corn for market; for, if fine, the grain will be casily separated from the straw, but in damp and sumless weather it separates very reluctantly from the chaff. Nothing but repeated thrashings will clear the grain of wheat from the chaff; and it is well known that farmers have such difficulty in clearing barley from the awns, that they are obliged to have recourse to various tedious, and, in many cases, expensive means to effect it, especially when it is of poor quality. 


\section{BARLEY IIUMMELLING.}

The process to which this more particularly alludes is in some places called "fattering," and many machines have been invented to effect it. Some of these liave been attached to the thrashing machine, and are simultancously driven by the same power; they act as scutchers, with arms fixed to a vertical spindle enclosed within a cylinder, and the corn being put in at the top of the cylinder, which is kept in rapid motion, the awns are broken off by their impulse*. They do not, however, answer the purpose so completely as could be wished; for if the straw be damp, or mixed with clover and grasses, it is apt to choke up the cylinder, by adhering to it as it turns round. WTe therefore annex a description of one lately invented by Messrs. Grant, of Granton in Aberdecnshire, which has been brought before the Highland Society, and will probably be found to perform the work more perfectly than it lias been hitherto done, as it is certified to have given satisfaction to several persons by whom it has been userl; and, being moveable, it can be put aside when other corn comes to be thrashed.

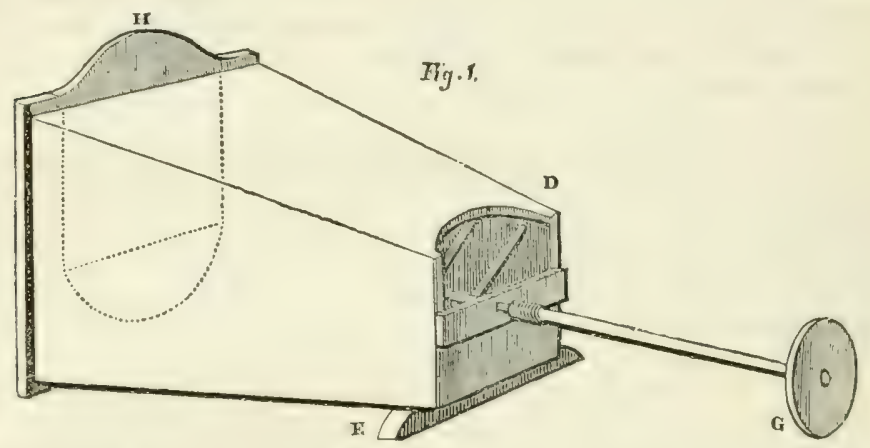

This machine, when viewed externally-as in fig. l-consists of a nearly square box, thirty inches on the side at the base, twenty inches at the smaller end, and four feet in length. In the interior of the box, the two lower angles are filled up with wood, so as to form the half of a conical surface; while the two upper angles are left void, but their sufface is thickly studded with iron spikes.

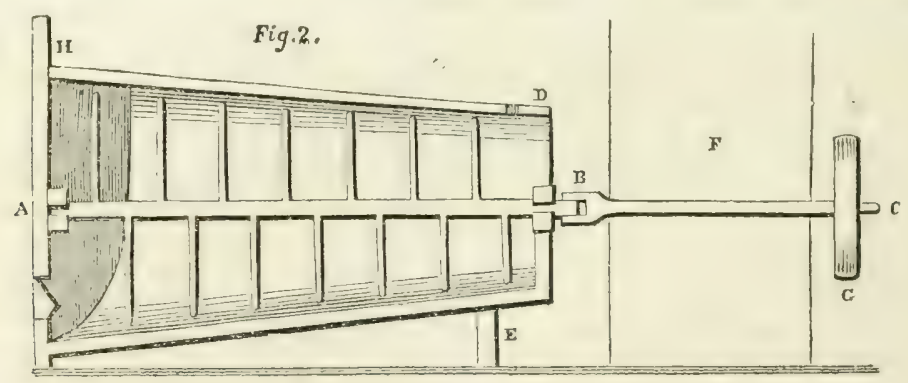

Fig. 2 is a longitulinal section of the machine, through which an iron axle or shaft passes, as at A aud $\mathrm{B}$; and is supported on bearings at each end, formed in the bars

* See Nos. 46 and 50 of the Farmer's Magazine; in which there are descriptive plates: also the Appendix, No. VII. to Sir John Sinclair's Scottish Husbandry. 
crossing the ends of the box. The shaft is armed with two rows of blunt iron beaters, seven in each row, all lying in one plane; the beaters on the one side being placed alternately with those of the other. When the hummeller is in the working position, the opening $\mathrm{D}$ ), in the smaller end, is brought under the corn-spout of the thrashing-mill fanners, supported on the foot $\mathrm{E}$, to bring the axis to a horizontal line. The vertical lines, bounding the space $\mathbf{F}$, represent a transverse section of the fanners, and an addition to the main shaft coupled to it as at $B$, and extending to C. The pulley $G$, giving motion to the beaters, is driven by a strap from the fan-shaft, producing a velocity of about 400 revolutions in a minnte. The grain received from the spout of the fanners, is thus violently aritated as it nasses through the revolving beaters, and the lower side of the box having a considerable inclination, the grain is impelled during the process towards the lower orifice, through which it is finally discharged. The size of the opening being regulated by means of the slide II in $f g . \mathrm{l}$, which also serves to retain the barley within the box until divested of its awn, while it prevents it from being seattered about by the force of the operation.

Other modes are, however, used which are still more simple; as, for instance,-after the barley is thrashed, the cover of the drum is taken off, and another cover is jut on, lined with tin, having small holes, about three-sixteenth parts of an inch wide, perforated in the manner of a grater. The grain is laid on the floor near the thrashing table, and a bushel or two at a time is laid close to the rollers; an experienced man being employed to direct the barley between them. The thrashing machine is then set to worl, and with the assistance of a couple of young people to lift the corn on the rollers, the whole quantity may be passed through in ten or fifteen minutes. Or, -

A bit of notched stick, lined on one side with a thin plate of jron, and just the length of the rollers, is fixed by a screw bolt at each end to the inside of the cover of the drum, about the middle of it, so as to be about one-eighth of an inch from the arms of the drum as it goes round. Two minutes are sufficient to put it on when its operation is wanted, which is when putting through the barley a second time, and it rubs off the awns completely *.

When the thrashing mill or other machines are not in use, the method commonly employed is to lay the barley upon the barn floor, and to free it from the awns by repeated strokes of a square instrument, consisting of a set of parallel iron plates, or small bars fixed in a frame, either in the manner of a gridiron, or divided like the chequers of a draught-board, and fixed to a stout handle, by which it is worked in the same mode as a pavier's rammer.

\section{KILN-DRYING。}

Corn is frequently harvested in a damp state, and either sprouts in the sheaf, or, if not immediately thrashed out and kiln-dried, becomes mouldy and unfit for use. The straw is also rendered unfit for any other purpose than litter, and in bad seasons serious damage is sustained by the farmer. The mode of preserving thrashed corn by the process of kiln-drying is well known to be effected by laying the grain to the depth of three or four inches upon a raised and tiled floor, heated by coke, wood-embers, or peat, and allowed to remain there, with frequent turnings, for about four-and-twenty hours, more or less, according to the state of dampness in which it may be found. Great precaution is however requisite in heating the liln to such a degree as will effectually secure the drying of the corn, as well as the destruction of the weevil, which, if the grais be infected with those insects, will appear in great numbers; and care must also be used in stirring the grain so regularly as to prevent it from being scorched. 
In Russia, and many other parts of the north of Europe, the plan is very generally adopted of kiln-drying the corn and straw in the sheaf, previons to the sticking ; and it appears to have been attended with such remarkable success, that if initated in this country, there can be no doubt of its being found advantageous in many exposed parts, where the farmers suffer greatly from the lateness of the harvest. The kiln used for that purpose is a simpile and cheaply-erected structure, the walls of which are 8 feet high and 15 feet square within. At this height there are two strong crossbeams to support the small timbers laid over them as ribs; the corn stands in sheaves above these ribs, closely set up-the band-ends of the sheaves down, and the corn or grain-ends up: the walls then risc above the ribs about 5 or 6 fect more, the kiln being closed by a simple ceiling of crossjoists, covered with turf. The floor contains about 25 stooks of corn, or 300 sheaves, which are put on of an evening-after the woorl which is usually emploved to heat the kiln has been burned to charcoal-and the straw is generally found in a dry state on the following morning. It may then be stacked with perfect safety; for all danger of its becoming either heated or mouldy is thereby prevented, and it is in the best possible condition for the flail.* The construction of the old British round kiln might be very easily adapted to the same purpose by raising the walls of the floor to a competent height ahore the ribs; but it would evidently require to be much larger when intended to dry both straw and grain. The expense of such a biilding would however be very trifling; for the frame could be put together of rough materials, and the walls might be filled in with clay or turf, while any ordinary roof will serve as a covering; and, in countries where peat is plenty, the charge of firing would not amount to much.

\section{GLEANING.}

A custom has existed, time immemorial, in this country, of allowing the neighbouring poor to gather the scattered ears of corn which are left upon the ground after the grain is harvested, and when that is peaceably conducted, and not attempted until the entire crop has been carried from the fields, no rational objection can be opposed to it; but although it should evidently rest upon the permission of the occupier of the land, it has yet been assumed as a right to which the peasantry are entitled. In pursuance of this, numbers of young and old, strmgers as well as neighbours, often enter the inclosures in defiance of the farmer, while the sheaves are still upon the gromd-generally indeed before they are bound; and they not unfrequently resort to violence if opposed. The work of harvest is thus not only much impeder, but the practice holds out temptations to pilfering from the sheares, and habits are acquired by children, which they extend during the winter to the plunder of turnips, potatoes, and other roots from the fields. Regulations lave indeed been promulgated on the subject by magistrates, and, were they strictly enforced, the evil might be remedied; but it seems equally absurd in principle, as unjust berween man and man, to afford the apparent guarantee of the law to such a pretended right as that of entering a farmer's property for the ostensible purpose of gleaning. It is, in fact, clearly a trespass, which he ought to repel, by force if necessary, until every slieaf be cleared; then, doubtless, every man of common humanity ought to throw open his fields to the poor; but it should be entirely left to his own discretion.

* See the Farm. Mag., vol, xviii, p. 385, in which are engravings of the eleration and sections of the kiln. 


\section{Chapter XV.}

\section{ON BEANS, PEASE, AND VETCHES.}

Altinugir buck-wheat, Indian corm, and millet should, in a botanical arrangement, more properly follow those suecies of corn of which we have just treated, yet we give these pulse precelence, as being more generally cultivated under our common system of farming ; and we omit the mention of kilney-beans, chick-peas, and lupines, though belonging to the same class of vergetables, and extensively grown ois the continent, because they are only known in this country as garden plants.

Both peas and beans were formerly more commonly arown by farmers than at present; for, under the old method, they formed almost the only intermediate crops between those of white com, and the grain was also much used by the peasantry for bread; but since the more general introduction of clover, turmips, and the other artificial grasses and roots into our alternate system, and the universal adoption of wheaten bread, they have been much superseded by other green erops. They are also objected to, as being so dependent upon the state of the weather as to be extremely precarious, and as promoting the growth of weeds when not carefully managed. In favourable seasons they are, however, very valuable, both in recard to the grain and fodder which they produce, and beans still hold their ground on tenacious soils, when drilled as a fallow crop; while vetches are extensively grown, as green food, under the common name of tares, but the grain is seldom used for any other purpose than seed.

\section{BEANS}

Require the same kind of soil as that which best suits wheat, and are therefore very generally sown as a preparatory crop upon heavy clays. Botanists only notice one species; there are, however, many varieties cultivated by gardeners, but those grown as field crops are confined to the long-podeled common liorse-bean, or " large tick," the sceds of which are flat, broad, and of a brownish colour; and the "small tick," which is nearly round, hard, and almost black, but is the most esteemed in the stable, and bears the highest price in the market. The "large ticks" are, however, crenerally preferred by farmers, on strong lands, both as covering the gromul better, and becoming ripe nearly a fortnight sooner than the smaller sort: they also yield heavy crops, and although their coarseness reduces the price, yet the quantity more than compensates for the difference. There are also the "Mazigan," which though not usually a pruductive kind, yet of which, in some instances, great crops have been obtained, and which was formerly the most early sort in cultivation; and the "Heligoland," which has been more lately introduced as a rival variety, and has been found very early and productive. They are all much used as food for cattle, partictilarly for draught horses, being very heartening to those which have to perform hard work; but they should be given in moderate quantities, as they are of a heating quality, and they should not be used until they are fuli a year old. They are then usually broken in a mill, and either mixed with oats and bran, or with cut straw and hay, and for other cattle and pigs they are commonly ground into coarse meal. If the millers be not much belied, it is indeed said, that, when ground fine and properly boulted, the flour is not unfrequently mixed with that of wheat. 
The cultivation of beans was formerly conducted entirely on the broadcast plan, as practised under the commion-field system, and there are yet many farmers who still pursue that method; but they, in that case, usually take the crop after wheat or barley, and without dunging the land*; whereas the most judicious mode is to take it previously, in the place of a fallow, and to lay the manure in the ground either during the winter or at the time of sowing the seed. Beans are, indeed, considered as an exhausting crop, and, as their produce is lighly nutritive, there can be little doubt that they accordingly rob the soil; but the plant is tap-rooted, and thus seeking its sources of nourishment at a greater depth in the earth than corn crops, which spread their roots upon its surface, it is not improbable that they may draw sustenance from parts where it would otherwise lie dormant. Whatever may be the truth of this, it is a well-known fact that large crops of wheat are constantly grown after beans, and that they have been successfully grown for years together alternately. 'Throughout the Isle of Thanet, barley, beans, and wheat is the common system, and among numberless experiments in proof, by several agriculturists, we select the following accounts of produce per acre, in three different courses of six crops each, upon a cold and wet soil; taken from a series of 36 rotations, tried by Arthur Young t:-

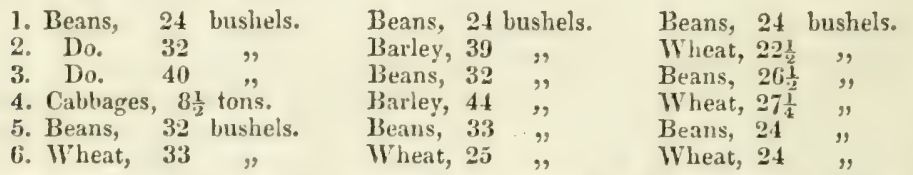

When beans are grown after corn, the land seldom gets more than one deep ploughing, and is generally laid into narrow ridges in the same direction as the former; being well water-furrowed to keep the ground as dry as possible during the winter, and remaining in that state until the following spring, when it is sometimes cross-ploughed, though the seed is not unfrequently sown upon a stale furrow. It is, indeed, not an unconmon practice to break the stubble up in the spring, and sow the beans after the plough; the seed being then put in as deep as possible with the drag and harrows, and the furrows commonly closed a good full pitch with the double mould-board plough. They are then hand-hoed and examined from time to time in order to keep down weeds; but this mode of culture is often attended with ineffectual expense and the produce is rarely abundant $\neq$. 'I'he object in view being also rather the production of the crop than the cleansing of the land, the seed, even if not sown broad-cast, is usually deposited in much closer rows than when meant as a fallow crop, and consequently cannot be followed by the operation of the horse-hoe.

When grown preparatory to wheat, the ground should be ploughed as soon as it can be conveniently got ready in the autumn, and should be treated in every respect as if intended to furm a summer-fallow : it should, however, be stirred mure deeply than when merely intended as a preparation for corn crops, in order to loosen the earth and afford sufficient room

* In Leicestershire, Lincoln, and many other Fnglish counties, the corn stubble is frequently ploughed up, and the beans sown broal-cast upon one ploughing, and merely harrowed in, without being ever rolled. Rep. of Leicestsh., p. 112.

+ See Nos. x., xi., xii. in the 132nd and 133rd Nos of the Annals of Agric. : also some instances in Chap. VII. of the present volume, on the rotation of crops, $\mathrm{pp} .91$ and 93: Burroughs on the Cultivation of Green Crops, 2nd edit., p. 34: Essex Rep., vol.i., p. 363: and Pictet sur les Assolemens, pp. 114, 132, 162.

† Drew's Norfolk Husbandry, p. J14. Surv. Hampsh., p. 170. 
for the roots of the plant to search for nourishment. The manure should be long-dung; and it should be ploughed under during the winter, or as early as the season will permit in the following spring. The latter is the most convenient, because it allows time for procuring the dung from the stables and the clearance of the straw-yard; iout many farmers prefer burying it as soon as it can be got into the ground; while others oliject to the employment of fresh dung, as encouraring slugs and vermin. Upon this it may, however, be observed that, if the beans be sown at wide distances, the land may be lept clean by the operations of the hoe and the scuffler during the growth of the crop; and that the long-dung, while imparting powerful support to the beans, will become completely decomposed and amalgamated with the soil, which will be thus brought into the best possible state for the production of the wheat.

Some farmers grive the winter-ploughing across the lands, with the intention of confining the culture to one ploughing in the spring: on heavy clays it is, however, an injudicious plan, for the ground can be laid drier, and thus be more mellowed by the winter's frosts when plonghed end-long. Although spring ploughing for corn crops is now much gone out of fishion, yet on strong soils, on which beans are sown chiefly as the saving of a fillow, two spring ploughings are found highly advantageous. The winter furrow should therefore be ploughed at length, and the lamd gathered into one-bout ridges before Christmas; but the second furrow should be across the first, as soon as the ground is iry enough to undergo the operation. 'This being done, the field should be left untouched until it is suthiciently dry, when a cast of the harrows may become necessary in order to reduce the surface, and the seed-furrow may be then given. This, though certainly the most approved mode, yet in some years, and upon wet soils, cannot be practised, and one spring ploughing can then only be given: the land, indeed, will not be left in an equally good condition, and the hoeing will be attended with greater difficulty, but these are inconveniences which nust be submitted to in adverse seasons, unless seed-time be put so far back as to render the after-crop unproductive**

The period of sowing must of course depend in a great measure upon the condition of the land, as well as the state of the weather, and whether the seed is of a good and early sortt. The bean ripens slowly, and should, therefore, be put into the ground early in the spring: it is indeed generally sown during February, and sometimes even in the beginming of Jinuary; but March is not too late, and late sowing has been in many instances attended with success ${ }_{+}$. The quantity must in some degree be governed, if sown broad-cast, by the sort of seed; the large tick being generally used at the rate of four, and the small at that of three and a half bushels per acre. When drilled, three bushels of the former are sufficient; and two, if dibbled. In the latter case, indeed, a still smaller quantity may suffice, for it las been clearly shown that, in rood soils, and favourable seasons, one bean is enough in each hole. It should, however, be observed

* See Brown, of Markle, on Rural Affairs, vol. ii, p. 59.

+ 'To judge properly of these, and to ascertain whether they be old or new, we are told by an eminent seedsman, that they should be bitten across, so as to make them crack or split in a longitudinal direction; if they split readily, and the bean appears Jry and huvey, lut nut flowery, it is a sure sign that they are either old, or have heated in the mow, or have been kin-dried; on the contrary, those which are fresh and sound will require a great deal of trouble to separate, and they will be almost as readily bit asunder in any other direction as down the middle. When old, they should be sown 15 or 20 day's forwarder than new ones.

+ Von 'Thaër says that he has found late sowing very advantageous. l'rinc. Rais. d'Agrie, 2nde ed., tom. iv., 1. 197. 
that, if sown so thin as not to completely close the rows, and thus sharle the land after clearing, the weeds will get ahead after that process is finished, and therefore, unless the land be very rich, the safest plan is to allow an abundance of seed. They are never steeped, as upon trying the effect it has been found more injurious than serviceable; and they are usually sown at the depth of abont four inches, or according to the tenacity of the soil. The seed is then covered by going once over it with a light harrow; or, what is better, by a double mould-board plough running up between the rows and making a hollow interval between them, which keeps them dry in wet weather*.

In about a fortnight after the land has been sown, or as soon as the beans are appearing above ground, it may be cross-harrowed; for although this may appear a rude operation, it is yet found to check the growth of the weeds and to promote the vegetation of the plants without doing any injury to the crop, as even should the tops be torn off, they will again push forward; but nothing short of frequent and effectual hoeing can keep the soil in a fit state of tilth for the due perfection of the crop. When hand-weeding is resorted to, it is frequently performed only once when the beans get about half a foot high; though, if properly performed, it should be repeated when they come into blossom, and indeed whenever a weed is to be seen, and the hoe should never cease going until the plants completely shade the ground, or the labourer cannot go among them without doing material damage. The expense should never be spared; for, be it what it may, it will be amply repaid by the benefit which the crop will receive from the stirring, earthing up, and the destruction of weeds. Much of the charge may, however, be saved, if the seed be sown in rows at such distances as will admit of horse-hoeing.

The comparative advantages of drilling, dibbling, and sowing broadcast, have been already so fully discussed, that it seems unnecessary to add anything to what is there stated in support of the superiority of the two former methods + ; we shall therefore merely remark-that they afford a decided superiority of produce, for they thus throw out pods from the bottom of the stem, which they rarely do when pressed close together, and the crop is thus found to be more free from insects and disease. The rows should be, if possible, placed east and west, as they will afford some shelter to the young plants from the cold March winds; and when handhoed, the best way to do it is to employ two men closely following each other in different drills, the one drawing the soil from the north or east to the plant, and the other from the south or west, according as the situation of the field may have caused them to have been laid; by which means the plants are maintained erect without being broken by the weight of the soil on either side f.

We here also insert the cut of a machine which has been used for dibbling beans, with great regularity as well as economy of seed. The wheels, by which the process of perforating the holes is performed, being moveable, may be separated to any prescribed distance; but the implement must be weighted in proportion to the toughness of the soil, or it will not dibble the land to a sufficient depth. The operation may be thus managed more neatly, and with more expedition, than in the common way; but is, of course, exposed to the same objection of its forming a chamber for the reception of the seed, which, in stiff clays, prevents the water from

* Bailey and Culley's Survey of Northumberland. 3rd edition, p. 8

+ See chapter vi. of the present volume.

† Malcolm's Compo of Mlod. Ilusb., vol, ii, p. 356. 
sinking through the earth, and thus, in wet seasons, sometimes occasions the grain to rot*.

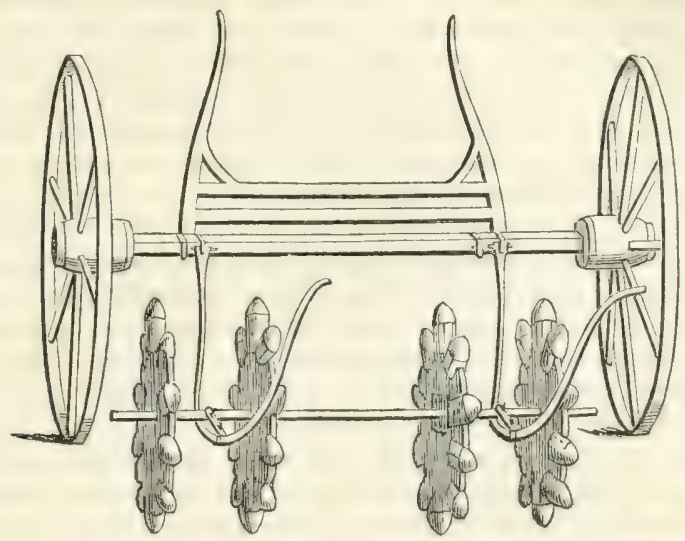

$\Lambda$ very considerable difference of opinion prevails among farmers, who adopt both the drill and dibble, regarding the distance at which the rows should be placed; some sowing them from 9 or 10 to 15 inches apart, and others in double rows, within 4 inches of each other, though separated by an intermediate space of eighteen inches. Those who practise the horsehoeing system, however, more generally sow them in sirgle lines at full 27 inches, and sometimes 3 feet wide, thus allowing ample room for a lorse to work within the rows; and throughout their growth scuflers, or skims, with shares of various sizes, are constantly kept at work between them. These tools are usually combined with a double mould-board plough, which is made to expand at pleasure for the purpose of earthing up, which is usually done when the plants are about 18 inches high; but although this generally finishes the operations of horse-hoeing, yet it is sometimes necessary to go through the field again with the hand-hoes. By these means the stems are sheltered, the vegetation of the plants is greatly strengthened, and if the implements be skilfully and sedulously applied, the value of the crop, as well as the cleanness of the stubble, will both afford sure proof of the farmer's care and judgment; and, on good soils, a clean bean-stubble is little, if at all, inferior to a clover ley for wheat $\uparrow$.

* We also expected to have been alle to insert the representation of a dibble drill upon the lever principle, which has been lately advertised by Mr. Bayldon, of Barnsley; but we did not receive an answer to our application for a description of it until too late to insert an engraving of it in this Number; though it apparently possesses considerable merit.

This, indeed, is not the first instance by many of much greater inattention to our requests by eminent agriculturists, who profess to have the interests of lusbandry at heart; and, if they treat the subject with carelessness, we have no right to complain of the jealousy and neglect of which common farmers are generally accused.

+ In treating of the cleaning process, that recommended by Brown of Markle is as follows :- "A bout ten or twelve days after the young plants have appeared above the surface, enter with the scraper and loosen any weeds that may have vegetated. At this time the wings or cutters of the implement ought to be particularly sharp, so that the scraper may not run too deep, and throw the earth upon the plants. In about ten days after the ground is scraped, according to the state of the weather and other circumstances, use the small swing plough to lay the earth away from the sides of the 
We learn, indeed, from an experienced practical farmer in Norfolk, that beans sown in drills at 12 inclees, and dibbled one hole on a furrow 4 inches between the holes, at the rate of 3 , or, if very large, 3 - bushels per acre, produced the most abundant crops he has ever seen; and he instances those of a neighbour, who grows every year 50 acres in that manner, and whole crops have averaged 6 , and in one year 7 quarter's per acre*. In opposition to this, he however admits, that if beans stand too thick on the lind they will be drawn up to a great height, but will not pod kindly; which is corroborated by the following experiment:-

A field of light free loam which had carried a crop of oats the preceding year, and had been dunged previous to the seed-furrow, was divided into three parts, two of which were drilled in single rows at intervals of 27 and 15 inches, and the third in double rows, standing close together within the same space as the first division. They were all harrowed before coming up, carefully hand-hoed, and fmally baulsed up with a double mould-board plough.

The crop at harvest was good, but when cut the stalk was smaller, and the pods not so well filled in the second division, at 18 inches, as in the first at 27 ; which yielded at the rate of upwards of four bushels per acre more than the former. That in double rows only returned the same quantity as the first, notwihstanding its containing several more drills; nor was the land so perfectly cleaned 7 .

From which we may conclude, that not only is there an evident saving of seed, but that a fiee admission of air is materially beneficial to every plant which-like the pods of beans-carries its fruit on the stem from the root upwards. The greater facility which it affords of cleansing the land is also an object of such importince, that it should ever be the primary consideration; and we conceive that horse-hoeing at any less distance than from 24 to 30 inches between the drills is a perfect mockery.

Where this is not fully acted upon, it is the practice of some farmers to turn a flock of sheep upon the land so soon as the beans are four or five inches high, as they will eat down the weeds which may have sprung up among the plants without consuming any of the latter, and with very little injury by treading. 'The taste of the bean is indeed so much disliked by sheep, that they may be seen feeding between the rows without nibbling a single plant; and if they should bite off some of the heads when in flower, it is supposed that the plant would be thereby benefited, as it is the constant custom of gardeners to lop them off, in order to set the blossoms and accelerate their maturity; without which precaution they are found apt to fill off at particular seasons, and to become unproductive ${ }_{+}^{+}$. The sheep should, however, be turned in upon an empty stomach, and they should be only suffered to remain in the field during the day, lest they should injure the crop by lying down upon it; but the practice mat be occasionaliy re-

rows; and in doing so, go as near to the plants as possible, taking care at the same time not to loosen their roots. If any weeds stand in the rows, pull them out with the hand ; afterwards earth up the plants with the small swing plough, or run the scraper in the intervals, as may seem expedient. Both ways are eligible; but when properly earthed-up, some people are of opinion that the plants are nourished by the operation; while others think that the benefit gained in that way is counterbalanced by the extra trouble of cutting the crop; the bottom being necessarily more unequal than when the scraper is employed to conclude the cleaning process." - On Rural Affuirs, vol. ii. p. 62 .

Drew's Norfolk Husbandry, p. 114.

+ Farm. Mag., vol. i. p. 155. See also the Reports of Northumberland, 3rd edit. pp. 86 and 88 ; Surrey, p. 236.

\$ Survey of Bedfordshire, p. 403; Malcolm's Sussex and Surey, rol. ii. p. 359. 
peated, until the plants become so hich as to endanger their being broken. The plan is certinly, to some extent, beneficial to the crop both by the manure ileposited upon it, and by the destruction of a portion of the weeds; but it can be only regarded as a poor substitute for the use of the hoe.

Althongli the culture of beans contributes greatly to the profitable management of heavy soils, by enabling the farmer to occasionally dispense with the recurrence of a maked fallow, yet their growth is attended with the scrious inconvenience of being so slow, that the harvest is sellom completed until the latter end of September, or not unfrequently until late in October. This prevents all further preparation for the sowing of wheat than merely that of one ploughing; and if the bean stubble be foul, or the season be unpropitious, the seed cannot be got into the ground either in proper time, or without great additional labour. 'The getting in of the crop is also attended with extraordinary delay, in consequence of the great length of time which they take in drying; and in humid climates-like, for instance, that of Ireland-they are therefore rarely grown. Mr. Parkinson, indeed, who farmed largely in that country, says that the crops when cut were sometimes rotted by the wet, and that, "taking one season with another, he does not there think them worth cultivation*." It is therefore almost unncessary to say that, when grown as a fallow crop, the earliest species should be sown.

The harvesting of beans is always an object of anxiety, in consequence of the danger to be apprehended from the changes of weather which may be expecter to occur about that season, and the serious injury to which the crop may be thus exposed by wet before it can be got into a fit state for carriage. Loss may be also sustained in hot weather by the sherding of the pods, if the crop be allowed to stand upon the ground until fully ripe; and cire must also be taken in the binding up, stooking of the sheaves, and stacking, in order to secure the whole produce. The period of ripening is also, in many cases, unequal; for, in late seasons, the plant sometimes takes on a second growth, and the cutting must be determined by that portion which is first ready. Indeed, although if not tolerably well ripened, the pods will shrink, and their quality will be impaired, yet, if allowed to become fully ripe, an equal loss may arise from the scattering of the grain: therefore, when the greater part of the porls become nearly black, or when the eye of the bean is thoroughly blacked, and the skin has acquired a yellowish, leather-like colour and appearance, not a moment should be lost in submitting the crop to the sickle; for, even if the produce be thus diminished, yet advantages will be gained in the superior quality of the haulm, the greater probability of the continuance of good weather for carrying the crop in good condition, and the certainty of an earlier seed-time for the wheat.

Beans are commonly "bagged" close to the ground-which is done by an instrument much in the form of a sickle, only wider in the blade and sharp as a scythe, or they are cut with the sickle; though sometimes they are mown. Some farmers who occupy small holdings, if the haulm be short-as is the case with the Mazagan bean-in some cases pull them up by the roots; which ccrtainly leaves the ground in a better tilth than when they are cut, but it requires a number of hands, and can only be done when the land is

* On the Management of a Farm in Ireland, p. 54. Mr. Lambert, however, who is a resident in the country, and has published Otservations on the Rural Affairs of Ireland, ascriles the neglect of beans to the superior value and utility of the potatoe, which seems to have there set aside the desire of cultivating any other leguminous of green crop. p. 122. 
loose and friable: great care is also necessary in cleaning the roots from the earth before the crop is thrashed. They are then left in the swathe for a few days, if the weather be dry, but are occasionally turned, and are afterwards bound into very small sheaves, either with ropes of straw or yarn, or else with hop-bine, or with haulm twisted from peas, with which they are occasionally intermixed. These ropes are laid upon the ground behind the reapers, who place the sheaves upon them with the heads all one way, for the convenience of the bandsters, who then set up in a little standing five or six together, but in such manner as to allow them the full benefit of the air; and if heavy rain should not intervene, they may be ready for the stack within ten days or a fortnight, according to the strength of the air and sunshine to which they have been exposed. Still greater delay is however occasioned in moist seasons, from the extreme difficulty of drying the haulm, and it has been therefore recommended to carry the crop off the field immediately, and stook it in some other place, so as to ensure the sowing of the wheat in due season*. If the sheaves be piled in the manner of a small, round stack, and so placed as to allow the air to pass through them, they may however be kept during a long time in the field without incurring material danger.

When carried to the rick-yard, the beans are stacked in the same manner as wheat, and when the boss is placed in the centre of the rick-as described in the preceding chapter-they may be considered out of further danger from mould or heating, even if they should not be yet perfectly dry. It is, indeed, so difficult to exhale their succulence, that they require a greater degree of care than any other kind of grain, and therefore stacks are sometimes constructed for them with divisions in the body of the rick, composed of cross-rails at separate heights, which prevent the pressure of any greater weight than the load contained in each division; and these separate portions being thus kept a little apart from each other, admit the air freely between the sheaves. We have seen an estimate of the charge of an oblong frame of this kind, the dimensions of which are, in length of the square 30 feet, and 5 feet round at each end; being thus 40 feet at the base, and about 44 feet long by 18 feet in height at the eaves. The cost, including the charge of the staddles, was calculated at less than 12l., and would probably last during many years. The annexed section will afford an idea of its construction.

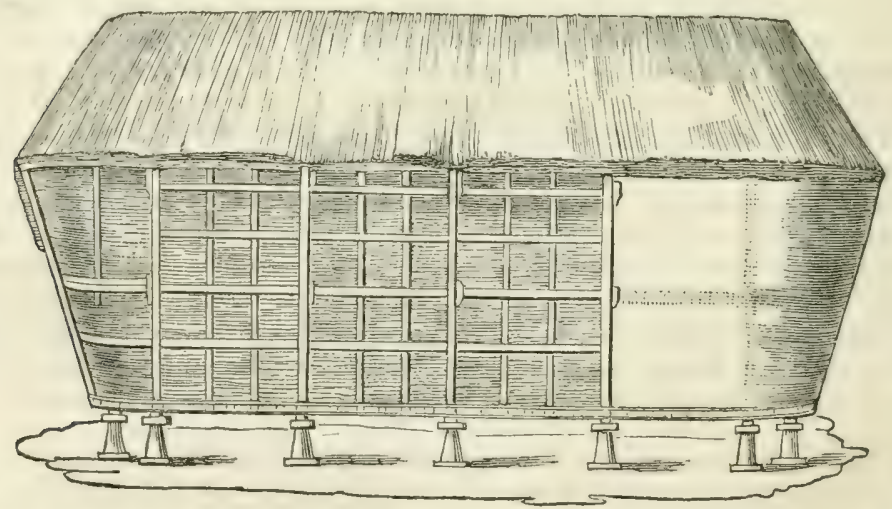

* Gen. Rep. of Scotland, vol. i. p.523; Sinclair's Code of Husbandry, 3rd edit. p. $399^{\text {; }}$ Berwickshire Rep., p. 257. 
The thrashing is usually performed with the flail, though it may be also done by the mill, and the mode of dressing is the same as in the common process with other grain; but, in riddling, the light grains and broken sliells are carefully separated. The first is, however, the plan generally preferred by those farmers who give the liaulm as food to their working horses, as its succulence, when thus gradually thrashed, is better prescrved than when a large quantity is prepared together. The haulm, when not allowed to stand too long, and when harvested in good condition, is a hearty kind of winter food; but, if not grot up in perfect order, it is only fit for litter in the straw-yard. It must also be observed, that whatever may be the advantages, in point of the produce of grain, by sowing beans in rows, yet, in regard to the quality of the haulm, there is a loss; for the stem becomes more hard and woody at the bottom, and more of the leaves fall off than when suwn broad-cast: its feeding properties are thus rendered inferior, and the value of the crop should, therefore, be always estimaterl with reference to the mode of culture. It has been known to yield two tons per acre; and we have heard of the crop, if cut when green, being found very profitable in the feeding of pigs.

The produce of beans depends more upon the state of the soil and weather than any species of white com, and is so exceedingly various, that while six quarters per acre are not an uncommon crop on rich alluvial soils, twenty bushels are considered a fair average on those of an inferior quality; and, even on the best land, the crop, notwithstanding the most arduous attention to its cultivation, will sometimes turn out a complete failure; for it is exposed to injuries which arise wholly from the temperature of the season.

The diseases to which the plant is subject somewhat resemble those of rust or mildew : the first showing itself on the leaves, the points of which become brown, but it extends over the whole leaf until it becomes black, and finishes by destroying the plant itself. The mildew, or, as it is sometimes termed, the "honey-dew," manifests itself generally about the middle of May upon the tops, and is accompanied by swarms of a black insect, known to entomologists as the aphis, and in some parts of the country called the "bean-dolphin," or the "collier." It is something in the form of a louse, and spreads over the whole stem, to which it ciings until the entire juice is consumed, and the vegetation of the fruit is prevented; but so little is known either of its propagation, or its habits, that no means have been discovered to prevent its ravages*. Endeavours have indeed been made to destroy the insect with the smolie of sulphur, but it was ineffectual, unless of such strength as to destroy the plant; and the only linown remedy is to nip off the buds, which is done by a person walking through the rows with a sword-blade, or a small scythe set in a handle, with which he cuts off the heads of the plants: it is said that not only is the progress of the aphis thus arrested, but that the blossoming of the bean-which is the crisis of its future growth-is thus much invigorated + .

It has indeed been recommended to watch the course of the flowering, and, whenever ants are seen about the plants, then to immediately have the buds cut off without loss of time, as they commonly precede the appearance

*An interesting treatise on the subject of "British Plant-Lice,"-to which class of insects the aphis belongs-has just been published by Professor Rennie; from which they would appear to be propagated without pairing! Whether this singular deviation fiom the common laws of nature can be established by future proof, remains to be seen; but according to some calculations made upon nine generations, proceeding, in the same season, from one mother, the births would amount to the incredible number of $350,970,489,000,000,000$. See the Quart. Journ. of Agric., No. xxx.

† Gen. Rep. of Scutland, vol. i. p. 523. Malcolm's Mad, Husb, vol, ii. p. 359 , 
of the aphis, and in a couple of days great mischief may thus be avoided*. The buds should be carried off the ground; and therefore, perhaps a better method than that of cutting the heads is to employ women and children to pinch them off and deposit them in a bag, or in an apron tied round them, until they collect suficient to fill a cart. This may appear a tedious and expensive process, but if sufficient jersons be employed, and they pinch with both hands, the work will be more expeditiously performed, and with less cost than may be generally imagined; but were both the charge and labour doubled, they should not be grudged, as they may be the saving of the crop.

The weight of the common bean is generally about $64 \mathrm{lbs}$. per bushel, and the nutritive matter which it contains has been estimated by Sir Humphry Davy as consisting of 570 parts in 1000 , containing-

426 parts of mucilage; and

103 ", of gluten.

PEASE.

Although many varieties of peas have been producer by gardeners for the use of the table, and several different sorts are grown for the same purpose by farmers who reside in the neighbourhood of great towns, yct those commonly known as field-crops, and sold in a dried state as grain, are of only two species, and are usually distinguished as the "grey or hog-pea," and the "white or boiling pea ;" though the former are of two sorts, and the latter of numberless kinds, named, like many other vegetable productions, either after the persons who have brought them into notice, or the places in which they have been first cultivated.

The grey hog-pease differ in their habits of ripening, one being earlier than the other, and, admitting of being put later into the ground, are known as cold and hot seed; the former ripening late, and requiring to be sown early, while the latter comes sooner to maturity, and may be suwn so late as the end of April. The cold seed produces the most haulm, and it is frequently sown along with beans, under the name of "polts" and "blendings," in the proportion of perhaps one-fourth, as their upriglit stallis afford support to the young plants, and assist in the inciease of shade imparted to the ground; but when grown separately, the produce of grain is uncertitin, while the hot-seed, which is of a dwarf species, seldom fails to yield a decent crop. The seeds of both sorts are small, and of various shades, of a dingy colour; but the blossoms are invariably purple.

The white boiling-pease-known severally as the "early Charlton," the "golden hotspur," the marrowfat," and the "Prussian biue"-are those kinds which, when allowed to stand until ripe, are used in families, as well as largely in the navy, as split-peas. They are of a larger size than the grey kind, come earlier to maturity, and usually bear white flowers. They indeed partake so much of the garden species, that they are largely grown by regular husbandmen with the intent of being podded and sent to market in a green state.

The operation of podding is performed by poor persons of every descrip. tion, but chiefly women, who come from different parts of the country about the time of haymaking, and pick them by the bushel. The scene, though not very conducive to the morality of the peasantry, is yet one of bustle and cheerfulness; but is attended with the inconvenience of bringing

* Von Thaër, Princ. Rais. d'Agric, 2de edit. tom. iv. p. 200. De Crud, Econ. de l'Agric., 4to, p. 238. 
a multitude of paupers around the premises, who sleep either in the barns and out-houses, or under rude tents fixed in the lanes, like gipsies, and their fuel is invariably purloined from the hedges: together, not unfrequently, with any other article that may fall conveniently into their clutches. The number of persons employed is of course in proportion to the state of the crop, and abuut ten acres will generally afford employment to near forty people*. As the pods are gathered the moment that any portion of the fruit is fit for the table, the peas are grenerally picked twice over, and the haulm is immediately cut up and removed if possible to an adjoining field, where it is cured, and the ground is then usually prepared for turnips. At times, however, large quantities are necessarily left to stand for seed; this indeed is esteemed a loss, as they are less profitable in that state than while green, but those which come late to maturity are hardly worth the expense of carriage.

The carts are loaded, and sent off at various hours proportioned to the distance from market, so as to deliver their load to the salesman between three and five o'clock in the morning. When the crop succeeds, both in produce and in earliness, no species of farming cultivation can equal the profit; but so great and immediate is the fall of price within a day or two after favourable weather, that nothing can be more variable than the market returns, and these early species can never be grown with any prospect of success, except the land is of a light and loamy nature, and has a warm exposure. The expenses of picking and carrying to market, and the salesman's charges, can only be supported by the high rate at which they are usually sold during a short period of the very earliest part of the season: farmers who prefer security to speculation, therefore, very generally make a previous agreement with a dealer for a fixed sum per acre, clear of all cxpenses - varying usually from $6 l$. to $S l$. or $9 l$, according to the appearance of the crop, and binding lim to take it off by a certain day; leaving, however, the haulm upon the ground.

The soil to which pease are the most appropriate partakes of sand and loam mixed with calcareous particlcs, and of a nature neither too much exposed to a cold humidity, nor to drought; but they are also linown to succeed in some seasons on strong clays, and on clayey sands, when the latter are not too dry. The grey species are best adajted to the stronger soils; the white to the drier and the lighter ones: in all cases, however, a mixture of calcareous soil is so highly favourable to their growth, that shell-marl, or lime, is ever found to forward a crop more than any other kind of mineral manure, though it is said to communicate a degree of hardness to the grain which renders it unfit for boilingt. It is, however, worthy of observation, that when dung sapplied, that of sheep or horses has been found to impart a better flivour to the pea, and to render the lusk thinner than when that of hogs or oxen has been used. Although the quality of their nutritive properties is nearly similar to those of beans, vet it will be thus seen that they not only require a different species of soil; but that, so far from admitting the frequent repetition of a bean crop, it is

* Middlesex Rep., 2 nd edit. p. 249.

i A considerable mystery seems to hang about the boiling properties of peas ; for good hoilers have been known upon sandy and gravelly soils, which have afterwards produced a hard, indissoluble pea from the same seed, and many instances might be related of their being apparently dependent upon accident. (Surv. of Hants, p. 167.) Some opinions refer this peculiarity to the seasons, though it occurs every year on some land; others conceive it to arise from the time of putting in the seed, or to its leing citlier new or too old; but we are not acquainted with any series of experiments on the subject upon which reliance can be placed. 
the general opinion of farmers that they will not succeed oftener than once in eight or ten, and in some cases twelve years*.

The propriety of applying farm-yard manure to the soil is also a subject of dispute; and fresh dung is generally objected to, as occasioning the crop to run to haulm, unless it be ploughed into the land previous to the winter. The latter objection is no doubt founded upon practical experience, and perhaps the application of dung in any shape may be inexpedient if the soil be unusually rich. 'This, however, is only rarely the case, and, su far as our own observation extends, we have ever found that a moderate coat of spit-dung, when laid upon land of a middling quality, has improved the crop both in grain and haulm: but, even were the produce of grain thereby somewhat diminished, yet, if the haulm be increased, a material point is gained both in its value as food for cattle, and by its fertilizing influence upon the future com crop; for it is well known that, if it be deficient, the soil will be left covered with weeds.

A startling assertion is, indeed, made on this subject in the works of that eminent agriculturist Von Thaër, whose opinion is entilled to the greatest deference; to the effect-" that repented comparative experiments, made during several years, have proved to him that dung, whether rotten or fresh and long, when spread upon the land after the sowing of the peas, is not only more advantageous to the crop then in the ground, but is also more favourable to the growth of the succeeding corn than if it had been buried by the plough." He, indeed, confines that opinion to sandy loam (glaise sablonneuse), as he had not extended the trials to strong clays ; but on soils of that nature, he adds, - "that experience had demonstrated its correctness in a manner so striking as to destroy all those theoretic principles by which it might seem to be contradicted + ."

The cullure of pease is nearly similar to that of beans; except that they are more generally sown broad-cast; and, when drilled, are not usually placed at such wide distances between the rows: they are, therefore, more frequently cleaned with the hand-hoe than the scufler. When sown bruad-cast, they are hoed out to nearly equal distances and hand-weeded; or sometimes harrowed in the manner of beans; the object being rather to loosen the ground about their roots preparatory to a future loeing than to destroy the weeds. However well the operation may be performed, it is still inferior to the process of drilling: of which an instance is recorded in the Berwickshire Report, where it is stated,_- "that the pease in a field of seven acres, after being drilled, were carefully hoed and weeded, and produced an abundant crop of both grain and haulm; but the headlands, which were sown broad-cast, produced a wilderness of weeds, and the crop was a mere nullity."

When drilled, the rows should be laid east and west, in the same manner as beans; and the hoer ought to take particular care at the first hoeing not to draw the soil from the rows, but rather to them, so as to form a skreen for the tender sprouts. It is, indeed, a good practice to leave the back part of the drills, that are exposed to the north or the east, standing in the form of a rilge, which will thus afford protection to the young plants, and their growth will be much forwarded. As soon as tisey begin to run, they should be again clean hand-hoed and weeded, laid up against the ridge facing the sun, and well earthed up ${ }_{+}^{+}$. They will then soun spread across the rows, and their stems will interweave amongst each other, so as to overshadow the land and cause the weeds to decay.

* Reports of Bedfordsh., p. 398; Herts, p. 98.

+ Principes Raisonnés d'Agriculture, 2de edit., tom. iv., p. 182.

† Malcolm's Survey of Surrey and Sussex, vol, ii., p. 368 . 
Regarding seed and produce, no aricle of farm growth is subject to greater fluctuation in point of price, or is charged for more extravagantly when sold for seed, than favourite sorts of peas; for new kinds are being constantly raised, and, when they turn out well, every grower is anxious to try them: thus, during the last season, we have known $16 s$. the bushel charged by the seedsman for a favourite species which he had actually bought in the previous year of the same farmer at $6 s$. The choice of seed is, however, a matter which must be left to the character of the seller; for unless the buyer grows it himself, he has no other guide than its reputation. The period of sowing varies with the nature of the pea ; the late kinds being commonly put into the ground so early as the beginning of January, and continued during the two following months. Sometimes, indeed, they are sown even in November, and covered over with loose straw to protect them during the winter; while the early sorts are frequently not sown until the beginning of April, or even in May; but the crop is subject to become more or less productive and thick in the husk according to soil and climate. Early sowing is, however, thought to guard against mildew; and cautious farmers-particularly if they wish to pod the crop-often adopt the plan of sowing the land at three or four different periods, - ten or fifteen days between each.

The quantity of seed usually sown broad-cast is about three bushels per acre; but when drilled, it of course depends upon the distance between the rows. Much less than what is generally sown would no doubt fill the land with plants, if the coming up of all the seed could be relied on ; but such havoc is committed by field-mice, birds, and slugs, that perhaps more than one-half is destroyed. Steeping the seed is never used as a preventive agrainst disease; but that which is old, or that is sown late in the season, is somelimes soaked for a few hours in water to forward its germination. The diseases to which the plants are subject are nearly similar to those of beans; and they are also exposed to injury from various species of beetles, and other insects, which deposit their eggs in the young pods, as well as to the destruction of the seed by vermin.

Nothing can be more uncertain than the produce of the "grain, which seems entirely dependent upon circumstances regarding the state of the atmosphere at the time of blossoming, and with the precise nature of which we are unacquainted. Even when that is successfully passed, the crop may be so burnt up, by the want of showers, during a dry summer, as to be scarcely worth gathering. In dripping seasons-which are favourable to the growth - so much as 84 bushels of the Marlborough grey pea have been got from a single acre, though on good soils, and in favourable seasons, 30 bushels are considered a fair crop; but on land of a less fertile quality, 20 bushels, or perhaps three yuarters per acre may be about the average. The haulm, however, makes up in a great measure for this deficiency, for it is not only in general abundant, but very nutritive, and much relished by cattle. It is commonly given as rack-meat to cartliorses, instead of hay, and sheep are extremely fond of it ; so much so, indeed, that it is by no means uncommon for farmers, who keep large flocks, to grow pease chicfly with a view to it as winter food in pinching seasons: the seed being in that case generally sown broad-cast, both to preserve the succulence of the haum, and to save the trouble of the drill culture. They are also not unfrequently grown on many farms in conjunction with tares, as green food for soiling.

The early kinds of peas are commonly ready for the barn about the middle of July, though, of whatever species, the crop may be considered 
ready for harvesting whenever the lower pods are ripe; for, were those which are not yet full to be waited for until they became in a perfect state, the better part of it might be lost. They should, therefore, be reaped without further delay; unless, indeed, it should clearly appear, that only a small number of the first blown pods had ripened, and the blossoming of those of later growth should give evident signs of their producing a larger quantity. This, however, seldom occurs; and although it often happens that the upuer part of the stem contains pods which are green, and its tops are still in flower, yet the crop sliould not be allowed to stand any longer : the hatum will be all the better, and the umripe pods will add materially to its value.

The reaping is performed by "the hook," by which the haulm is pulled towards the reaper; for it is so much matted in its growth that it is difficult to cut it. A tool is however sometimes used for that purpose called the "pease-make," which is part of an old scythe fixed in a handle; though frequently it is cut with the sickle, and in some instances pulled up by hand. It is then left for some days in the swathe to dry, and is afterwards rolled up and bound into small bundles, or "wads," and in due time carried to the barn or stack without being set up in shocks: indeed, the crop is more usually put loose into the barn than into ricks, and in that case the operation of wadding is unnecessary. The process of drying is however one which requires great care; for the hauln should be occasionally turned over, and, if that be not cautiously done, much of the grain may in hot weather be shed: the better way is, therefore, instead of turning it with hooks, as usually done, to lift it gently with the hands. In case of alternate showers and sunshine, the weather will then indeed have such an effect in opening the pods, that a great portion of the crop will be inevitably lost if it be exposed; so soon, therefore, as the liaulm is a little withered, the swathes should be gathered into rather large cocks, and left there while the rain continues, without any other change of position than merely lifting them slightly in different places with the handle of the rake.

The application of the crop of the builing pea is almost confincd to the Nayy, and to family consumption, while that of the grey sort is chiefly emploved in fattening of pirgs, the flesh of which it is futund to render firmer and better flavoured than when they are fed on beans; though the amount of their nutritive properties-according to the analysis of Sir IImphry Davy-is, it will be seen, nerrly the same : namely, 571 , or in 1000 parts-

501 parts of mucilage ;

22 ", of saccharine matter; and

35 , of gluten.

\section{VETCHES,}

Of vetches there are several varieties, although only two are generally distinguished by farmers-as " the winter and spring tare" - which are more frequently grown as green food for the soiling of cattle, or made into hay while in i state of blossom, than allowed to ripen their seed. They have incleed acquired habits of so difierent a nature, by being sown at those sea. sons of the year, that they are considered distinct; but they are in fact of the same species, and their seed-which is nearly black, and bears a purple blossom-differs so little in appearance, that $j$ is difficult to tell one kind from the other. The winter sort is put into the eround any time during the autumn, from August to the latter end of October, and the spring kind from Match to May : it is indeed, not imfrequently sown after the green winter tares have been cut. We must, however, reserve our observations upon 
them, as forage, until we treat of that subject, and now consider them merely as pulse grown for seed.

When intended for that purpose, we have to notice another species known as the "white vetch," both the seeds and flowers of which are white, and the grain is said to be both more succulent, as well as to become earlier ripe than the two former: it is also better adapted to light soils, and stands the winter extremely well: but it is not generally cultivated. The most usual mode of growing vetches for their grain is to sow them as a spring crop; and their management is in every respect so similar to that of the field pea, that any detail would be a mere repetition of what has been there stated. It may, however, be mentioned, that they are more frequently sown broad-cast, and that beans are also sometimes intermixed with the vetches to support them, like sticks to garden pease; the produce being easily separable by proper riddles after it has been thrashed. It should also be observed, that the winter species, after being cut before the blossoming has been completed, is then sometimes left for seed, though the crop is rarely su large as that produced in the other mode, and there is not periaps a fortnight's difference between their time of ripening. Most farmers recommend early spring sowing, but we have known many instances in which those sown about the begimning, and even towards the middle of Nay, have tumed out the best. If the temperature be cold, vegetation is stopped, and then the plant is attacked by an insect which gnaws its buds, and, unless the soil be rich, prevents it from again sprouting into flower; whereas late sowing generally prevents that injury.

Tise soil required for the common species of velch should be clayey: when it contains more than about sixty per cent. or three parts in five of sand, the plant will rarely succeed unless the land be wot and well manured, or very moist. Although this will be found correct when the crop is to be cut green, yet when meant to stand for seed, a drier soil should be preferred, and the land will produce better if it be limed; for it is known by experience, that a rich loamy soil occasions the plants to throw out a sticcession of blossoms, which cause the grain to ripen unequally*. It does not, however, require very rich land; thuugh it should be aliways well dressed and in clean condition.

The seed is rather smaller than that of the grey pea; and, as when grown for seed, it should be sown more sparingly than when intended for soilingr, 2 bushels per acre are sufficient. The crop is reaped, carried, staclied, and thrashed in the same manner as pease, and it is supposed that the grain contains nearly the same nutritive properties; but it is seldom used for any other purpose than seed. The quality of the haulm has been vaunted by some writers, and cattle, when starving, may eat it; it is however so tough, as to be fit for little else than litter. The produce fuctuates extremely both in quantity and price; for it varies from two quarters to five per acre, and from $5 \mathrm{~s}$. to $12 \mathrm{~s}$. per bushelt: last year and this spring the price, near London, has been about $6 s$.

The commonlentil is of the same family as the vetch, which it in many respects resembles, and is said to he the most nutritive leguminous plant with which we are acquainted +. It is largcly grown throughout the south of Lurope, and has been repeatedly trieci in sonte parts of England, in which it has been thought of rather fivourably, and is there generally linown

* Gen. Rep. of Scotland, vol, i. p. 550.

+ Both Messrs. Middleton and Malcolm say that they have known $5 \frac{1}{2}$ quarters grown on an acre, and the price at a guinea the bushel. Middlesex Rep., 2nd edit., p. 257; Surv, of Sussex and Surrey, vol. ii, p. 382.

+ Von Thaër, Princ. Rais, d'Agric., 2de ed., fom. iv. \$ 1096. 
under the name of "Dill." It can be grown without difficulty as a spring crop, and thrives best in light, dry, sandy soils, on which it is generally sown broal-cast, sometimes in March or April, at the rate of about a bushel and a-half to the acre. It is more delicate than the vetch; and, when given in a green state, is considered peculiarly beneficial to ewes with suckling lambs: it is indeed so acceptable to all kinds of cattle, that when the crop is allowed to ripen, the haulm is still eaten with avidity; and the grain is given as food-generally ground and mixed with barley or potatoes-to fatten pigs*. The produce of grain is however not very abundant; and the haulm, though good in quality, is yet so deficient in quantity, that the cultivation has been abandoned, except upon land which is too poor and thin to admit of tares.

\section{Chapter XVI.}

\section{ON BUCK-WHEAT.}

Altноugh buck-wheat belongs strictly to the same class of plants as those termed cereal grasses, and its grain partakes of all the essential properties of those already describedt; yet, in consequence of its greater deficiency in the quality of gluten, it is not so generally employed in this country as bread-corn, though used for that purpose in many parts of the continent.

It is supposed to have been introduced into Europe from Syria in the time of the Crusades, in allusion to which the French call it ble sarrazin; but we have no account of its cultivation in this country earlier than the latter part of the sixteenth century. The species liere commonly cultivated wears in its growth more the appearance of tufted grass than standing corn, its stem being full of knots, and rising only to the height of about two to three feet: the blossoms are white, tinged with red, and the external cont of the grain is of a dark brown colour, hard, small, and triangular; but the flour is white. It is an annual plant, and the seed is always sown in spring. There is, however, another sort, known as the "Tartarian" or "Siberian buck-wheat," which withstands the winter, and is said to bear a crop in the second year; but the accounts given of its produce are not favourablet.

The soil best suited to buck-wheat is a dry, sandy, and calcareous loam, or moory soils, well adapted to the growth of rye : it is however seldom grown upon any other than the poorest land; but provided that be light, and unmixed with clay, heavy crops are frequently produced. It is grown either for soiling or for its grain, and is not unfrequently ploughed-in while in a green state as manure $\S$. In either case, the land however rarely gets the benefit of any dressing; or, if dunged, it is done very sparingly; as, unless the crop is to be cut green, it is found to lessen the product of grain, and

* Surveys of Berksh. p. 230; Oxfordsh. p. 162; Surrey, p. 239.

+ See Chapters ix. x. xi, xii. and xiii.

\$ We understand that a variety of the buck-wheat has been for some years grown in Wurtemberg under the name of Blé sauvage ditalie, which suffers less from the atmosphere than the common sort, is more productive, and the grain of which affurds a more white and savoury meal : the culture being in every respect similar.-Bull. des Sciences, Agric. Avril, 1831 .

Sce Young's Reports of Suffolk, p.82, and Norfolk, 318: also the observations in the latier part of vol, i. chap. xx.p. 408 ; in addition to which, it should be remarked that buckwheat, or any green crop, will, on retentive soils. prove useful, but, on light soils, it renders the land too porous and puffy fur the production of a fulluwing crop of wheat. 
the best dressings that can be applied are said to be those made from the different preparations of peat*

The culture being late in the spring, it generally gets two ploughings; but the seed is usually sown broad-cast; no further attention being paid to the crop, which is left to Providence, without any care being bestowed upon its weeding. When intended to be ploughed-in, this is admissible ; but the same negligence is commonly practised when the crop is meant for grain, the excuse being, "that it will not repay the trouble;" though, if worth growing, it should assuredly be kept clean, were it only for the benefit of the future crops. The land intended for the growth of buck-wheat should, indeed, be highly worked and well cleansed from weeds; its root not striking in depth more than two inches into the soil, whilst the spread of its numerous small fibres will entirely fill a circle of five or six inches in diameter. It is one of the greatest advantages in the cultivation of this crop, that the late period at which it may be sown affords ample time for such a preparation as is requisite to its success. The rapidity of its growth, its numerous branches, and the great shade afforded by its heartshaped leaves-which, though variable in size, are often more than three inches in breadth-has a great tendency to keep down annual weeds, and to render the soil extremely mellow: this is also further increased by the falling of the leaves before the crop is taken from the ground. In this country it is sown the most frequently either after turnips which have been eaten off late, or after tares and rye which have been soiled: in Flanders, however, it enters regularly into all the rotations upon light, sandy loams, and Von 'Thä̈r mentions his having sown it in July with rye; then having cut the buck-wheat, while green, and left the rye to stand for corn during the following year, as a plan which had perfectly succeerled $t$.

The plant retains so much of its eastern origin, that it is destroyed by the slicrhtest frost: in this climate it is therefore never sown until the end of May, and it may be deferred until the middle or even the latter part of June, for it is of such rapid growth that crops sown in the beginning of July have been reaped by the second week in September. It is however hazardous to sow it later than Midsummer, for it may be attacked by the white frosts of autumn before it ripens; and, even should it escape that danger, the prorluce will not be so large as when the seed is put earlier into the ground +. If the crop is to be soiled, two bushels of seeri will not be too much; but if meant to stand for the production of corn, the quantity should not exceed five or six pecks. The seed, if sown broad-cast, is harrowed in: some farmers afterwards roll it; but many think that operation had better be omitted.

The success of the crop depends, indeed, very much upon chance; for the seed requires dry weather immediately after it is sown; but when the plant has pushed forth its third leaf, rain is necessary to forward its blossoming, and when in flower, it may be destroyed by a strong easterly wind, while it must have hot sunshine to ripen it. A week more or less in the sowing may thus occasion a sensible difference in the produce, for if the season prove hot and dry, it may be too late, and if sown before the last weck in May, the occasional frosts in that month may effectually kill

* See vol. i., chap. xxii.

+ Princ. Rais, d'Agric, 2 de edit. tom. iv. p. 215.

I In Pennsylvania, and throughout the middle and eastern states of America, buckwheat is sown broad-cast upon once ploughing the wheat or rye-stubbles immediately after harvest; which is there generally by the 1st of August.-Vancouver's Hampsh. p. 172 . 
almost every plant; the first week in Jume is therefore the most usual time, but many farmers choose two or three separate preriods. It remains during a long time in blossom; the grain therefore ripens so unequally, that the latest portion will somctimes be the most productive; but when. ever any part is ready for the scythe, the whole should be immediately cut. Indeed, if showers occur with hot weather while the crop is in the swathe, a great share of the unripe seed will come to perfection, and the plant has the advantage of sustaining the rain better than any other grain-crop : it is therefore generally left upon the ground while the others are secured.

The product in grain nay be thus supposed to be uncertain, and can hardly be rated at more than from $2 \frac{1}{4}$ to 4 quarters per acre. The purpose to which it is applied is chiefly for seed; though it is frequently grown in gentlemen's preserves for the support of the pheasants, and, when given to poultry, is thought to impart a peculiarly fine flavour to their flesh. It is also sometimes coarsely ground and used along with potatoes in the fattening of pigs, which thrive well upon it, and it is occasionally-though very rarely-bruised for horses; in which application it is said that two bushels will afford as much nutriment as three of oats*: the weight is, however, nearly the same, being about $46 \mathrm{lbs}$. jer bushel. Cows fed wilh it are found to vield a large increase of milk, and the butter that is made from it is very sweet and pleasant-flavoured $t$. Pigs are also very fond of it. A prejudice is, however, grenerally entertained arrainst it, from an idea that cattle dislike it when it is blossom; and it is said that sheep when fed with it, in that state, stagger and tumble about as if drunk + . In Hollimb and Germany, it forms, besides these uses, a considerable portion of the food of the boors; and in North America, after being bolted and dressed into fine meal, the flour is made into cakes, which are regarded as a great delicacy $\$$. In those countries btick-wheat is, indeed, looked upon as an important comcrop; and we camnot help thinking that its cultivation upon our poor sandy soils might be advantageously extended along with that of rye, and confined, upon such land, to their production in a rotation with vegetabie crops, to the exclusion of every other species of grain.

Buck-wheat, it is thought, may also be advantageously sown in a small proportion with clover, lucerne, or sainfoin; but we should fear its beingr rather prejudicial to the growth of those crops, and they are not fit for those moory soils which best argree with it. The hay made from it is likewise said to be very nutritive; but, unless it be cut early, it is harsh, and not relished by cattle: it is also tedious in the making, and it is, therefore, seldom used in that state; or, if made, it should be eaten before Christmas. The crop when allowed to stand for corn is ustally mown, and thrashed out in the usual mamner; but the straw, though also praised as food, is more generally used as litter.

* Stevenson's Survey of Surrey, p. 241 ; Malcolm's ditto, vol. iii. p. 87. The latter gentleman indeed says, that if it be given to horses more abundantly, or if they be fed upon it without intermission, it is apt to bring on humours in the blood; it should therefure be chiefly used ou fatiguing journeys or in wet weather.

+ Surveys of Perth, p. 190; and Malcolm's Sussex and Surrey, vol. iii. p. 88.

\$ Young's Survey of Sussex, 1. 128.

$\$$ The mode of making them is, - to mix up the flour over night with yest and water: this being kept warm during the night near the woul-embers rises by morning to a light and frothy consistence, ur batter: small portions of which are spread upon a flit iron, ur zriddle, suspended over the fire, or placed upon a trivet, which is slightly rubbed oser with hog's lard between the baking of each cake. The bread is very light, and the cakes, whel resemble our crumpets, are caten hot with butter, and are almost universally served up to every breakfust table. Vancouver's IIampsh., p. 174. 


\section{Cinapter XVit.}

\section{ON INDIAN CORN.}

Tre plants, known as Indian-corn, Rice, and Millet, though largely used as bread-corn throughout the East, and in many parts of the south of Lurope and America, have been yet thought too tender to be brought to perfection by means of our common system of husbandry in this country, and have been only cultivated as rarities in our gardens. We should therefure pass them over in a work devoted to British agriculture, were it not that some experiments marle within these few years upon a large scale by the late Mr. Cobbett, and the accounts published by him of the cultivation of Indian-corn, as a field-crop, have justly attracted so much attention in the farming world, as to induce us to extract some of the leading particulars of his details, together with some further information on the subject, which we have gleaned from other sources, as well as from our own observation.

\section{INDIAN CORN}

Is not indigenous in any part of Europe, but has acquired the name of Blé de Turquie, by which it is generally known throughout the continent, from its being presumed to have been introduced from the Levant by the Mahometans after their conquest of Constantinople. Some strong reasons have indeed been adduced from the Scriptures by Mr. Cobbett, in support of his supposition that it came originally from Syria; though it is not improbable that he may lave confounded it with miliet, which plant it in many particulars resembles. Whether they be well-grounded, or not, it is however certain that it was found in its native state in the West Indies by Columbus; for it is particularly mentioned by Pedro Martyr-who was a contemporary of his, and who published an account of the first voyage immediately after its completion-as being commonly grown in the island of IIayti, and there called Mä̈s; or, as now termed, Maize.

The plant springs upwards with a tall and strongly-jointed stem, the summit of which is crowned with "a tassel" of numerous variegatted flowers containing the pollen, and performing the functions of a male; while the blossoms on the lower part of the stalk throw out long pendulous filaments, called "silks," which are impregnated by the pollen falling from the tassel. These female flowers produce from 2 to 5 ears-or, as they are usually termed, "cobs"-varying from 6 to 10 inches in length, and from $\frac{3}{4}$ of an inch to double that size in dianeter; around which the corn is closely piled in rows, betring frequently more than 500 grains : differing, however, in size, from that of a small tick-bean to that of a flattened pea; and in colour, from a bright orange to a very pale yellow, stained in some instances with dark red, but producing a farma much resembling that of wheat.

The interior of these cobs is a pithy substance enveloped within delicate but strong folds of a silky covering, termed the husk, which serves as a complete protection to the grain; and the leaves, though not numerous, are very broad. In tropical countries it grows to a great size, and the varieties are perhaps as numerous as those of wheat; though, in America, they are solely distinguished as the "yellow, or golden," and the "white, or flint-corn"-meaning the early and the late species. Either kind, however, requires the full heat of our warmest summers, and botanists describe the plant as being incapable of being regularly brought to perfection in any country of Europe farther north than the loth degree of north latitude. 
The seeds of the sort which has been introduced into England under the name of "Cobbett's Corn" were however obtained from the province of Artois, in France, which lies nearly parallel to our southern counties, but the plant is a dwarf species, the height of which does not exceed four feet on good land; neither does it usually produce more than three cobbs, nor the ear attain the length, or produce an equal number of grains, though the corn is of the full size of the maize grown in a warm climate. 'The fibres of the root spread equally around to the extent of full six inches in diameter, and take a firm hold of the ground.

Fig. 1, in the annexed cut, is a drawing of the plant in a state of maturity; and fig. 2, represents a cobb, or ear of corn, when stripped of its husk.
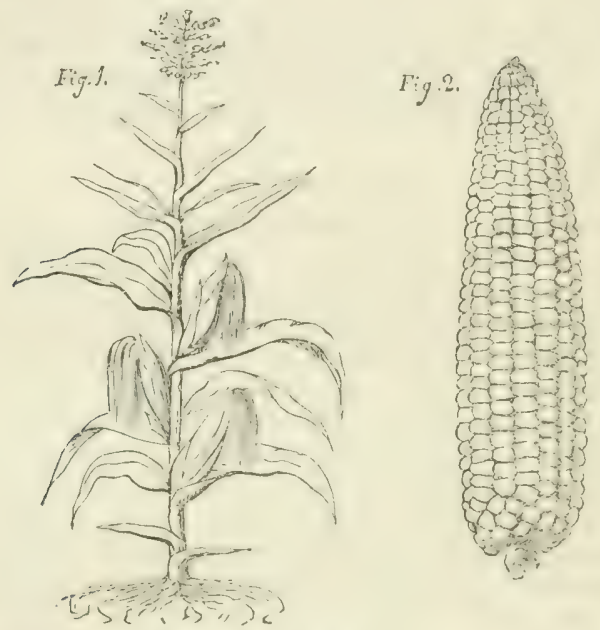

The soil best suited to Indian corn is a friable loam, and it is found in foreign countries to succeed better on light lands than in those which are clayey. Last year, however, we have known a large crop of it grown upon heavy clay; but the land was dug, and it is almost unnecessary to add, that it was by that operation rendered more pervious than it could have been by common ploughing. Cobbett indeed says, "that a tolerably good crop would certainly succeed, in numerous cases, on land much too poor to yield a crop of wheat, or even a grood crop of barley, oats, or rye ; "yet in that assertion we think he is mistaken, for other accounts, from countries where it is grown extensively, and under climates more favourable than this, say, that it requires an abundance of manure, and on poor land does not pay the expense of cultivation.**

Cobbett describes two modes of sownng: one, by dibbling in hills four feet every way apart-" which may be formed by ploughing longways and crossways ;" the other by drilling the corn in rows at the same distance.

In the first method, if the land be not jreviously dunged, the manure may be laid in the hills, which are marked out by the plough, and at every crossing a hole is made with the hoe, about six inches in diameter, around which five or six seeds are placed, at the depth of less than two inches, and the earth is drawn upon them by the hoe; taking care that it be fjec of clods, and merely pressed down by the back of the hoe, or lightly trodden. This

* De Crud. "Economic de l'Agric.," 4to, p. 303. 
morle is therefore nearly similar to that of dibuling. The planting in hills, lie however only recommends with reference to the larger species of corm, which is, in this climate, of hazardous growth; though, in warm countries, it is grown in conjunction with root crops planted between the rows: in which mamner we have seen maize and pumplins extensively cultivater together in Portugal and many parts of Spain.

In the second node,- or that which he adopted in the growth of the dwarf species,-he sowed the seed in rows at three feet apart, and at eight inches from each plant; but he advises the rows to be at the distance of five fect, and to sow the seeds as near to each other as six inches. In botin these directions, we however conceive that he is wrong; and, in saying this, we speak not only from our own experience, but also from the writings and the information which we have obtained from others. We know, indeed, from our own observation, during a long residence in the Peninsula, that the distance at which maize is planted rarely exceeds three feet between the rows, unless intermediate crops be grown, and the plants are never less than a foot apart from each other. Such is also stated to be the usual mode of husbandry throughout the best cultivated parts of France and Italy*; and, to come nearer home, we have lately gone over a couple of acres of Indian corn, - grown by Mr. Tallis, of Hornsey, who has for some years paid considerable attention to its cultivation, - on examining which we have found the best plants in his crops to stand at full twelve inches and three feet apart: added to which, we learn from Mr. Cobbett's bailiff, that the corn on Normandy farm, which is at six inches, stands much too close together; and we are persuaded that the crop, of six acres, though represented as very fine, will yet suffer from want of room between the plants. There camot, indeed, be any doubt that a plant, growing to the size which it attains, besides bearing its chief produce on the lower part of the stem, should have air and room to expand, and that an intermediate space of full three feet should be allowed between the rows for the operation of the hoe; but more we deem unnecessary, if not perhaps prejudicial, by depriving the ground of shade, and exhausting it by the exhalation of the sun.

The time of souing is stated to be from the 15th to the 20th of April, and may be continued to any time in the month of May; but the plant is so very susceptible of injury from slight frosts, that we should consider the carlier period as a dangerous experiment. It is evident that the chances of a favourable moment must be governed by the weather, and we should conceive that, in this climate, the safer mode would be to sow the secd in beds at different times early in the spring, and to set the plants out in the first week or by the midlie of the month of June. Time would be thus given for the transplantation after the sowing of the usual Lent crops had been completed; the additional expense would be trifling; all chance of failure by frost would be aroided; and that of loss, occasioned by the constant depredations of birls, wire-worm and slugs, would be of no account, as of course sced enough would be sown to secure a sufliciency of plants for filling the ground. Although we have no experiments upon the subject on which we can rely, except those made by ourselves in a garden, yet we know from these that the shoots will bear transplanting without injury, if not with advantage, and that they should be allowed to acquire considerable strength before they are taken up.

*De Crud. (Econ. de l'Agric. 4to. p. 303) descrilies the cultivation as leing usually with alternate rows of potatoes, or of the beans called haricots; and Simonde (de l'A gric. Toseane, 1. 79) says that when sown singly, it is always at two feet distance between the rows, and one foot between each plant, 
The act of inansplantation should however be carefully performed by a man who is well acquainted with the use of a dibble, or such a setting-stick as is employed for planting out cabbages. The shoots should be cautiously heaved out of the bed with a spade, and the earth which adheres to the roots should not be shaken off. 'T'hey should be laid in a shallow baslset, slightly covered to shade them from the sun and wind, and should be immediately carried to the planter, who ought to reject such as appear weak or damaged. The holes in which they are placed should neither be too large nor too deep, and care should be taken to fix the roots well in the ground, filling up the vacant spaces which surround them with loose mould: a lad should also be allowed to attend with a donkey and a light water-cart to wet the earth in the holes around the plants, and if this be repeated every second or third day until they strike, or show an appeaiance of vigour, it will ensure the success of the operation.

\section{MANAGEMENT.}

With regard to culture, it is almost unnecessary to insist on the absolute necessity of the land being previously well manured, and brought into a perfect state of cleanness and friability; after which, Cobbett's mode of management is nearly as follows:- When the plants have got to the height of three inches, the first hoeing is given, the effect of which is so apparent, "that if a row be left untonched, you would, at any distance that would allow you to distinguish one row from another, be able to tell at one look the one which had been neglected: and a man who could not, or would not, go over two acres a day at this hoeing, would be unfit to go into a field." "When they have reached a few inches higher, the ground is ploughed to a good depth, and to within two or three inches of the stems of the plants, and they are earthed-up to support and furnish them with fresh food.

About the middle of July, or perhaps earlier, the plant will be about a foot and a.half, or two feet high, and then a second ploughing is given with a double turn-stirring the soil to within six inches of the roots, and then turning it so as to earth-up the plants: on which subject Mr. Cobbett says, " that no fears need be entertained regarding any injury to be thus done to the corn by the tearing of the roots, for the Americans perform the operation in a violent manner, tearing up the ground and tumbling it against the plants when the tassel is nearly at its full height and the silks appearing from the ears: one rule is, indeed, invariable; that is, that if the coin be not ploughed at all, there will (upon ordinary land at least) be no crop."

After the last ploughing, a final earthing-up takes place; which is per. formed with a large hoedrawing from the midule of the rows earth sufficient to hill up the stalks of the plants to the height of 6 or 7 inches above the level of the ground whereon they stand; but before that is done, the plants must be suckered: as thus-Each plant sends forth from the bottom of the stalk, just where it meets the ground, 1, 2, 3, 4, or more suckers, and these must be taken off by pressing the fingers strongly downwards into the soil and pinching them clean out of their sockets, or they will start again; for although they may throw out cobs, yet they weaken the plant. They amount to but little in bulk; but, as they are excellent food for cows, they will pay for the labour.

Towards the beginning of September, the plants are topped: which is rendered necessary when the tassel has performed its functions by shedding its pollen upon the female flowers beneath. The operation takes place so soon as the tassel is completely dead and withered, when the grains of corn at the top of the cobs are sufficiently hard to resist a strong pressure 
of the thumb-nail; and is performed by cutting off the top with a sharp knife, at perlaps two-thirds of its height, or some short distance above the lateral cobs which spring from the stem. The workman then strips off the blades as near the stem as he can-thus leaving the cobs to ripen divested of leaves - and lays the whole down in convenient little parcels between the rows, where they are left for a few days to dry, and are then tied up into small sheaves, and stacked in the usual manner.

These tops and blades will, of course, be of greater or less value, according to the weather in which they have been harvested; but are not liable to any inconvenience to which hay is not subject. Mr. Cobbett estimates them higher than he does hay; for he says "that an acre of tops and blades will give more nutriment; that they are excellent when cut into chaff, and are even given to race-horses, and other highly-prized cattle." That they are nutritious we doubt not, for we know that they are greatly relished by all kinds of stock when in a rreen state; but we cannot admit that they afford equal nutriment to hay, when dried, and we have never seen them griven in that state to horses in any part of the European continent.

The harvesting of the ears does not take place until some time in November, when the grain is quite hard; and there is no occasion for hurry, as the cob being enveloped by its husk, the corn is secure both from the effects of rain and shedding by being over-ripe, and dry weather may be waited for, even should it not occur until later in the year. The operation is performed by passing along the rows and stripping the cobs by the hand downwards off the stems, instead of twisting them off; then throwing them either into an accompanying waggon, or carrying them of in panniers to the barn-floor, where they are husked: the stems when thus stripped being pulled out of the groumd.

The operation of husling may be done by women and children-who are seated round a large heap with a basket before them, stripping off the husks, which they throw behind them, and throwing the ears into the basket; but in America, it is performed by the neighbouring families, who assemble together at each other's houses to enjoy a "liusking frolic," of which Mr. Cubbett gives a very humorous description. The cobs are then sorted, and put away into the "corn-crib," which is a little granary, formed of open rails and fixel upon staddles, two or three feet broad at the bottom, but projecting upwards and outwards until it reaches the height of perhaps seven or eight feet: something in this form-

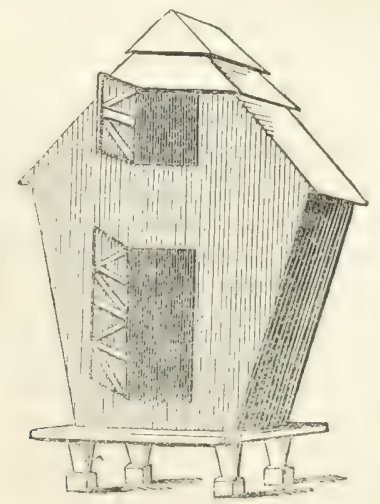


The mode of shelling the corn, or separating it from the pith of the cob, is performed in America and many parts of Europe by fixing a strong piece of iron, with a sharpish edre, across a tub, and scraping the cob lengthwise across it with hoth hands until the corn falls into it. The pith is then thrown aside, and we are told that a man with stout wrists will in this manner shell from 20 to 30 bushels a-day; but we know, that it can be effectually shelled, by putting the ears into a sack, and thrashing them with a short flail or a stick. The grain, however, requires considerable care to guard it from any kind of damp after it is shelled, for it is subject to heat; it should not therefore be spread upon the floor more than 15 inches thick, for the purpose of drying; neither should it be left long in the granary without turning; and, if further danger be apprehended, it should be kiln-dried.

\section{SUMMARY.}

Colbett estimates the produce of the crop at 100 bushels, and we have heard of several instances of experimental crops in this country having reached from 70 to 90 bushels per imperial acre. This must, however, have been upon rich soils, well manured, and under superior cultivation; for he acknowledges that he has only rarely heard, in America, of so much as 60 to 70 bushels, and he admits that the average in Pennsylvania is only from 20 to 30 bushels per acre. In New South Wales the ordinary crops raised by English farmers upon forest land, or those of ordinary quality, only averagge about 40 bushels per acre; though extraordinary crops are sometimes grown upon alluvial land." We linow also, that in the south of Lurope, on favourable soils, and with every advantage of climate, 60 bushels is considered a fair produce; and, reasoning from analogy, we cannot suppose that on medium soils, however sedulously cultivated, it can in this country ever be expected to return an average excecding perhaps the produce of a good crop of beans.

The crop, however, has other advantages; namely-it is neither subject to smut, blight, nor mildew, and it can be harvested with safety at any period of the year. The grain weighs perhaps 60 lbs. to the hushel, with very little chaff, and is extremely mitritive: more so, we should suppose from its effects on animals, than either rye, barley or onts, and perhaps nearly equal to wheat ; $\uparrow$ though from its deficiency in gluten, it cannot be made into bread without an admixture of wheaten flour. 'The straw is more valuable than that of any other grain crop: the husk is universally used, wherever the grain is generally known, as the stufing for mattresses; $;$ and the pith of ihe cobs, when completely dried, forms, along with the stalks, good firing.

It may also be strictly called a fallow crop; for althongh we are persuaded that it must rob the ground of a portion of its sap, equal to the nutriment which is found in the grain, yet, if that be supplied by a proportionate dressing of putrescent manure, the land will not be exhausted. The cul-

* Indian corn is in New South Wales dibbled into holes formed with a hoe about 3 feet a-part every way; and, at a proper stage of the growth, it is hilled up with a considerable quantity of earth.-Atkinson's Account of the State of Agric. in N. S. W, p. 44.

t Ifors are, in the sonth of Europe, almost invariably fattened upon Indian corn, and the pork has a much finer flavour than any we have ever tasted in England. In the West Indies, too, our saddle-horses were in good condition with an allowance of half a peck of bruized maize; while, in this country, we have never been able to keep them in working order with less than double that quantity.

† The mattresses are not quilted, as when stuffed with hair, flocks, or straw, but the husk is placed loose in the covering, the centre of which is slit open, so as to allow the chambermaid to put in leer hand and spread it evenly. 
ture also, as directed by Cobbett, though certainly expensive, is yet such as completely to replace a summer fallow, and it is used in the best cultivated parts of Italy as a preparatory crop for wheat. It is there, however, and in many other parts of the continent, sown along with intermediate crops which are planted between the rows; but, from what we have here seen of the practice, we cannot recommend it, for it is destructive of the tillage upon which the cleanness of the land and the success of both that and the future crops mainly depends. In southern climates, these double crops are, indeed, off the ground so early as not to interfere materially with the cultivation of the Indian corn; and in this country, perhaps carly potatoes, or podded peas, might be grown in like manner: but, in that case, the corn should be sown in rows at a wider distance apart. Trials should be made of it, upon a small scale, in various times and modes of sowing, so as to ascertain the best season and the most profitable mode of culture; for, if it can be counted upon as forming one among a regular rotation of crops, it must prove a very valuable acquisition to our husbandry.

In point of climate, we think the experiments which have been already made leave no rloubt that the plant, when attentively cultivated, may be brought to succed in our southern and midland counties-particularly when the seed shall have been naturalized by being repeatedly grown in this country: but we have no idea that it can ever be extended very far north; and we question whether the humility of Ireland will not prevent it from being advantageously introduced into that island.

\section{Chapter XVIJI.}

\section{ON TURNIPS.}

IT would have been perhaps more consistent with due order in the arrangement of a work on husbandry, to have commenced with the cultivation of turnips, - as the foundation of our alternate system of tillage, - rather than with the growth of corn; and, hard we undertaken to direct the practical management of any one farm, we should have adopted that plan. It should, however, be borne in mind, that, although we furnish the best details with which we are acquainted regarding the culture of crops, yei we only profess to treat of them separately; leaving our readers to judge for themselves, of the best course of cropling, according to the peculiar nature of their land.

The introduction into this country of turnips as field-crops-which is supposed to have taken place in the county of Norfolk, nearly two centuries ago, and which afterwards spread gradually over the kingdom, las contributed more than any other improvement in rural economy to the advancement of our agriculture. Before their adoption, it was, indeed, diflicult to manarge light soils to any advantage; for they were soon exlansted by the repeated production of corn, and as no regular rotation of green crops was then known, they were necessarily thrown for a series of years into pasture to recruit. It was also so difficult to support the stock ilyon a farm throughout the winter, that the fatting of cattle for market during that season could only be effected with hay, and it was, therefore, customary among country families, to slaughter a number proportionate to

* Pictet, sur l'Italie, 2 de edit., ก. 398. 
their wants, for the purpose of salting them down for winter consumption. This, indeed, saved the expense of feeding; but, besides abridging the comforts of the table, it prevented the production of manure for the culture of the land, which thus became impoverished, and it was not until the admission of turnips into our farming system, that those inconveniences were remedied. They are now, however, universally grown for the common food of sheep and oxen, upon all soils to which they are adapted; and lands so poor as to be formerly worthless under the plough, have now been rendered productive by the application of the dressings which they furnish.

\section{SOIL AND SPECIES.}

The soil best adapted to turnips is of a dry-bottomed free nature, of some depth and fertility; but, although distinctively termed "turnipland," it yet comprises every species of earth which can be profitalily used for any arable purpose, provided it be light, dry, and friable: consequently exclusive of heavy clays. It must, however, be understood, that although the common root can be grown on the poorest sands and gravels, yet there are some species which require stronger soils-even rich, free loams; and they all demand very careful culture, with an abundant supply of manure. The plant delights in a cool, temperate, and moist climate, and therefore thrives better in those districts upon the borders of England and Scotland, than in our southern counties : its culture is also more general in those parts; and as-like every thing else-it is best understood where most practised, it is chiefly with reference to the management there adopted that our remarks will be directed.

There are several kinds of this valuable root, and new varieties are constantly produced; but, although distinguished by different appellationsas "the white, the green, the purple, and the red-topped ;" "the tankard," which, though large, is porous and unsubstantial; "the Norfolk," and "the globe" - and varying in appearance from the globular form to the flatcopped and the oval, or pear-shaped, they may yet be generally clissed under the heads of the white and yellow species, and the Swedish.

The white globe, or Norfolk turmip, was the first known, and is the most commonly cultivated; particulariy as being the best suited to those light soils which are generally deroted to the feeding of sheep, as producing the heaviest crops, and as coming the most early into use. It grows to the largest size, partly out of the ground, and is very sweet; I it it is tender, and should be consumed before Christmas, while its leaves are green and its bulbs large and succulent, otherwise it grows pithy, and rarely survives the frost, or else runs to seed, and thus becomes useless as food. Of this, however, there are other sorts, the roots of which, being more deeply inserted in the ground, better support the severity of winter: of these there is a small, hard kind,-called the "stone-turnip,"-which stands the weather better than most others, and being not alone equally sweet, but of a finer grain and of greater specific gravity, is very generally grown in order to secure a succession of feed; but it does not attain so large a size.

The Aberdecn yellnw is an intermediate species-between the "globe" and the "Swede" - of a hardier nature than the former, and is of considerably slower growth: it is therefore sown earlier than the first kind, and also remains much longer in the ground; which brings it into use after the other sorts have been consumed. It bears large crops, and, though not so large as the Norfolk, is fully as nutritious as any of the white lind, but is not so 
much relished by cattle, and requires a rather stronger soil. Of this also there are many varieties: the colour of some being red, approaching to purple, and in others green, diverging to various shades, until gradually sinking to nearly white; and attentive farmers mix them, in the same field, from having observed that the animals fed upon them are partial to this change of food ${ }^{*}$.

The ruta baga, or Swedish turnip-which was of comparatively late introduction into this country-is by much the hardiest species known; the weather, however severe, being found to have very little effect upon it when all the others are entirely destroyed by the alternate changes of frost and thaw. The true sort has yellow flesh and is without a sten; but it is ajut to degenerate either by the flesh becoming white, or by the crown running up into a stem of more or less length. Its roots are more solid + , and its leaves more palatable than those of the common turnip. It has also the superior advantage of retaining its nutritive properties until much later in the spring, and is therefore highly valued as affording a certain support to live-stock at that pinching season which intervenes between the consumption of the common turnip and the growth of the young grass. The crops which it yields are, indeed, not so large as those produced by the white and yellow species, but the nourish. ment obtained from an equal weight of the root is so much "greater, that not only does a much smaller quantity satisfy the appetite + , but cattle fed upon it thrive in condition; whereas, if supported solely upon white turnips, they will only hold their own. It, however, requires a larger proportion of manure, and a much better soil than any other kind of turnip); for, upon land of ordinary quality, whatever quantity of dung may be applied, it becomes tough and fibrous, and will not grow to any considerable size: it also comes later to maturity, and should, therefore, be sown carlier than any other kind. In every other respect the management is the same; except that it has the peculiar property of bearing to be transplanted, and therefore admits of filling up those blank spaces which often occur. This operation may be performed at any time during the entire of the month of June, until the commencement of July; but, when applied to entire crops, the plan has been seldom found to succeed, as the bulbs show a disposition to shoot earlier than when left in the ground on which they were originally sown, and the advantage of spring feeding is thereby lost.

* See the Farmer's Series in the Library of Useful Knowledge, Report of Strathnaver Farm, No. XVIII. p. 73.

$\dagger$ On weighing a Winchester bushel each of the Swedish and common turnip, the former was found to weigh $88 \mathrm{lbs}$., and the latter only $60 \mathrm{lbs}$. (Dickson's Surv. of Lancashire, p. 359) a statement which has been in many instances confirmed by other experiments.

I. On the 16th of March four tups, of the New Leicester breed, were put up in one pen, and eight draught ewes in another, to be fed on common turnips, freed from their tops and fibrous roots.

In eight days the 4 tups ate $1003 \mathrm{lbs}$, or on an average $31 \frac{3}{4} \mathrm{lbs}$. each per day.

$$
\text { " } 8 \text { ewes } 895 \mathrm{lbs} ., \text {, } 15 \frac{1}{2} \text { do. }
$$

The whole were then put on Swedish turnips, sliced, weighed, and given regularly three times a day; of which

in eight days, the 4 tups ate $553 \mathrm{lbs}$, or on an average $17 \frac{1}{4} \mathrm{lbs}$. each per day.

$$
8 \text { ewes } 544 \mathrm{lbs} \text {. } \quad 8 \frac{1}{2} \text { do. }
$$

The experiment should, however, have been begun in November, and continued luring the winter, as it is well known that common turnips grow lighter and less succulent in the spring months than they are in the earlier part of the winter; and it is therefore probable that the diffirence would have been less at that season.-Northumberland Rep. 3rd edit, p. 105. 


\section{SEED AND SOWING.}

The time of souing these different species, it will be thus secn, varies according to their quality; those which are the most nutritive requiring the longest period in coming to perfection, and consequently to be the earliest put into the ground. First, therefore, the swedes are sown as early as possible from the becrimning of April, throughout May, and should never be deferred beyond the latter end of that month, or the crop, mless unusually favoured by a dripping season, will assuredly suffer in weight: then the yellow sorts, some short time afterwards; and lastly, the white, at any time from about the middle of May until the close of Jume. Sometimes, indeed, the sowing is not completed until July, and the common Norfolk species are not unfrequently put in upon one ploughing-as "brushturnips" - after the corn-crops have been reaped. These late sowings, however, yield but little*; yet, as they produce a delicate root for early dropled lambs, they are not uncommonly used by farmers who have large flocks.

These periods, it should also be observed, are entirely dependent upon the state of the land and weather, as well as the ability to get the ground realy in due season for bringing forward the crops. If the soil be of a cold nature, and slow in communicating its vegetable powers, the sowing should be proportionately early ; but if rich, and well manured, it may, in that case, be later. Still, however, there is an advantage in early sowing, which arises from the suil being more moist, and the heat less intense; which forwards the plants, and subjects them to less injury from insects. In contradiction to this, however, it is frequently found that if the seed be sown tou early, the plants are apt, in warm seasons, to run up their fluweriner-stems befure winter, to the great injury of a feeding flock. The third or the fourth week in May is therefore the time most generally adopted; but in every instance the sowing should be deferred until the ground is in a perfect state of dryness-though unaccompanied by drought, -and sufficiently moist to ensure vegetation. Alany farmers, indeed, sow their turnips-particularly those of the white species-at stated times, in diflerent portions, each at the distance of perhajes a week or fortuight from the other; by which means they come successively intu rotation for the hoe, and partially also into use for consumption.

The quantity of seed usually employed seldom exceeds from one to two pints-or about one pound when drilled, or a pound and a half to two poums when sown broal-cast; though some famers use considerably more as a precation against the attacks of the flyt; in the view that, if the plants be numerous, probably sufficient will be spared by the insect to form a crop, and, if it should not appear, they can then be hoed out.

The quality of the seed is matter of considerable importance, for much of a siourious kind is commonly sold in the shops, and though charged by

* Mr. Malcolm gives an extract from his diary regarding the sowing of the "tankardturnip,"-a large varicty of the white species,-irom which it appears, that lie sowed it during three following years : in the first, he commencerl on the 14 th of $J$ une, and continued sowing it every day as fast as three plunghs could get the ground ready, until the 14th of July; and in the two following years, he began on the 14th of May, and sowed every other day for two months. The result was, that those which were sown in May, were, in the month of January following, worth two acres of those sown in July.Surv. of Surrey, vol. ii. p. 402.

$\dagger$ It has been remarked that the fly attacks the turnip plants more voraciously unon chalky soils than upon any other; and therefore, upon such land, a greater portion of seed is necessary. Sulfulk Report, 3rl edit. 1. 97.-Mr. Colie of Ilolkham invariably sows 3lbs, on an acre, and his crops are seldom if ever injured by the fly. 
the dealers at exorbitant prices, ret serious losses are thus frequently incurred by farmers; for not only do the jobbers commonly mix a certain portion of rape, or of old seel, that will not grow along with that which is grood, but even when no fraud is intended, the sceds of the different species of turnip are constantly mistaken by the most experienced persons, and there is no certain rule for judging between them. Some observations, indeed, will be found at foot, which have been made by a very experienced seedsman*; but we should recommend every farmer who is desirous of procuring the best kinds, and of continuing the growth of any valuable species of turnip of which he may be in possession, to save the seed for himself.

The best mode of doing this, is to collect from the neighbours small samples of seeds which bear a good reputation, and sow them at various times in different lots, keeping notes of the periods of sowing and state of the weather, so as to ascertain as nearly as possible the time for putting them into the ground, and that of their coming to perfection. 'Thus, when they have arrived at the proper period, the finest roots should be carefully selected, and separately planted out in a crood soil previously dug deep, and set in rows two feet and a half asunder, and fourteen to eigliteen inches apart. They should also be placed at a considerable distanee from each other, in order to prevent any admixture at the flowering season, and this to avoid the chance of growing a bastard stock; which will occur if the firina of one species be carricd to the other, either by the wind or by bees. In July or August following, the seed will be ripe, and the haum is stacked and thrashed out in the usual manner, but it should not be left until dead ripe, or the birds will destroy a large guantity. The trouble of selecting the roots and transplanting them is indeed too often neglected, although seed raised in this manner is the only kind that can really be depended upon; and when sold, always commands a superior price: it should, however, be

* "In looking at various samples of turnip-seed, it often happens that they are found to be of three, four, or five colours-green, green and yellow, green and red, green and black; and all these may come from the same ground, in the same season, mixed together. In cleaning them for sale, they are so much heavier than chaff, or any other extraneous body that may be found among them, that they will not separate in the best winnowing machine, or with the fairest wind that ever blew; and which, indeed, is the best way of cleaning them in fine weather. Now, upon compressing and examining the green and yellow seed, it will be found to contain but a small quantity of vegetable oil, and must be considered as unripe seed; it will however grow, but, like every other immature thing, after vegelating it feels the want of a sufficient borly, and comes to little or nothing. The red seed would be the true vegetative seed, if it had not been either heated in the mow, or put into damp bags when thrashed, or before it was thoroughly dry; but this has changed its dark, clear, black skin, for that of a dingy red, and if more closely examined, it will be found to smell and taste musty. In conzpressing it, the oil will be tound less clear, and not so strong as in the black seed, and, however slight the degree of heat that may have caused this clange of colour, that its power of vegetation is partly destroyed.

"The black full-bodied seed-although we may suspect it to be the siftings of the rape, yet if we can depend on the grower or seedsman - is the kind of seed we ought to choose; but I would advise every man to procure his seed at least a month or two hefore he wants it, and sow a duzen grains promiscuously in a small pot placed in a shady spot; sowing also the same number every other day for ten days in similar pots, flaced in different situations. By observing them he will see how many of them grow upon au average, and he may safely then apportion. his seed from a pint to a quart, or four pints, or even more per acre, according as he finds the seed to wrow. This is an infallible gruide, and has saved me a crop of turnips two or three times; for I have often known the seison and the fly charged with the loss of a crop, when, in reality, it was unly caused ly defect in the seed,"-NIalcolm's Coml. of Mlod, Husb, rol, ii. p. 400 . 
occasionally crosscd from other soils, as the plant is said to degenerate after the second year. The produce, being subject to many casualties, is variable, but an average crop may be reckoned at about twenty bushels, or half a ton per acre*

\section{CULTURE.}

Turnips are regarded by all farmers as the most complete failow crop which can be grown, and are therefore generally introduced upon light soils into that part of every rotation which closes one course, and is intended to commence another. The land must, with this intention, be cleansed from every species of weed, without regard to labour, and should be lieavily manured with dung, and also limed if necessary, as thereby affording the best chance of securing an abundant crop, and thus through its consumption by the stock, still further increasing the fertility of the soil.

Upon the introduction of the turnip into our field-system of cultivettion, the seed was of course sown broad-cast; but although that mode was universally abandoned throughout the northern counties upon the adoption of the drill, it has ret been very generally continued in the midland and sonthern districts. Whe reason assigned for this does not, huwever, arise from any generil preference to the latter method in those places where it is followed, but is justified both upon the principle of its liaving been the immemorial habit in which the labourers are the most expert, and that the land is, in many widely extended tracts, so much encumbered with flints, as well as of such a compact and cloddy nature, as to prevent the due application of the drill and horse-loeing implements $t$. The latter fact of course opposes a decided objection to the practice, and the force of custom must be allowed to have its weight in other places; for, as to the performance of the work by hand-lroeing, the plants may be every where seen regularly hoed out to nearly equal distances with the greatest accuracy. Comparisons between the produce obtained from the two modes have, indeed, often shown them to be nearly alilie; but the object in view is both to clean the ground, as well as to prepare a crop of winter food for cattle, and the true criterion for determining their merits, appears to us to depend xpon the management of the land. On loamy soils, which admit the operation of the horse-hoe, drilling certainly affords the most effectual means of cleamsing the ground; but on such land as that to which we have alluded, it has been abandoned after repeated trials by intelligent and umprejudiced practical men, and it may therefore be justly doubted, whether those great advantages which are in every case ascribed to the drill system would, in such instances, follow any alteration of the established plan.

Whichsoever system be adopted, the stubbles of the land intended for turnips should be ploughed to a good depth, inmediately after harvest, or as soon as possible after wheat-seeding is over. The upper drains and water-furrows should be drawn and opened as soon as the ploughing is finished, and the ground be laid dry, in as complete a manner as if it were under crop). Perhaps on soils of a strong nature, or exposed to lie wet during winter, no mode is more proper than those lands terned "ten-furrowed," or "five-bout ridges ;" and if carefully laid up in this manner, the

* Northumberland Rep. 3rd Edit. p. 97.

+ See No. I. of Reports of Select Farms, in the Library of Useful Knowledge, j. 9. and the Surveys of Surrey, p. 249. Berks, p. 222. Herts, p. 104. Oxfordshire, J.172. Young's Norfulk, p. 225. 
ground will have all the benefit of the frosts, which will tend to make if work kindly in the following spring: though on drier soils, broader work may be used with safety. It may lie in this state until the close of the oatseeding, when a second ploughing is given, usually in an opposite direction to the first; but if the weather prove dry, and the tcams slould be at leisure at any interval between those periods, another stirring mav be given with good effect. After these ploughings, the land should be repeatedly crossharrowed with the heavy break and common harrow, and rolled, in order to free it from every species of rubbish, and to bring it into a complete state of pulverization; the root-weeds should be carefully ralied up, and either carried to the dumg-hill, or burned upon the land, and nothing should be left undone to bring the ground into a state of perfect tilth, - that is to say, clean, dry, free from clods, finely reduced, and wearing the appearance, to both the eye and touch, of kindly mellowness. If the land be very clean, three earths may be sullicient to bring it into condition; but four are more frequent,- the second and third not quite so deep as the first and fourth,and if foul, a fifth ploughing is sometimes necessary: after which the seed process usually commences.

In the application of Manure, if lime be used, it will of course have been mixed with the soil during these operations; and if the land be intended to be sown broal-cast, the dung must also be previously laid on. Many farmers, indeed, who adopt the drill, yet lay it on before giving the winter furrow, both from conceiving that the soil is thus more equally meliorated for the cultivation of the subsequent crops during the course, and from its also saving much time and trouble during the hurry of the turnip season: considerations which certainly merit attention, and which may not be found unadvisable where there is an abundance of manure. Most men have however to wait for its production in their yards during the winter; and, as the growth of a good crop of turnips is the main point in the commencement of the course, the most generally approved method of applying the muck is in drills, immediately before sowing, in the following manner.

The land, being levelled by the former ploughings, is divided into straight lined ridgelets, either by the operation of the double mould-board plough, or by that of the common swing plongh: the most usual distance being twenty-seven inches; and a consilerable alvantage arises from preserving this distance, as it admits of the horse walking in the centre furrow whilst the wheels run in those on each side, consequently the land is not poached by putting on the manure, or in drawing of the turnips. However, much difference of opinion prevails, for many intelligent farmers use drills of only half that breadth: assigning as reasons-that although tumips growm in willer rows, reach to a larger size, yet the crops are not superior in weight; that they are more liable to injury from frost; and likewise, after Christmas, less nutritive than those of moderate size**

Be this as it may, and still adverting to the wide drills nencrally adopted throughout the north, the manure is then carted on, and tumed in, after the last ploughing; the quality being ahways duly fermented spit-duner of the richest lind, and the quantity as large as can be affurded,-say, from twelve to twenty tons per acre, according to the state of the land and species of tumips: Swedes requiring more than any other. The loaded dung-cart goes between the rows, so that the whecls run in each of the furrows of the

* See Letters and Remarks on the Subject in the Farmers' Magazine, vol. iv. 
two adjoining drills on each side. The muck is then drawn out by the driver into small heapss at recular distances, and is followed by women and children, who spread it with forks. It is thus laid into the loollow drills, and the ridgelets are then immediately split open by the passage of a plowgh through the centre of each; by which means the manure is completely corered, and a bed is formed for the reception of the seed.

It should, however, be observed, that as the land for the grain crop, which follows turnips-whether barley, oats, or wheat-seldom gets more than one ploughing ${ }^{*}$, it is customary to lay off the drills obliquely, in a direction somewhat different from that in which the ridges are to be afterwards drawn for the com ; as thus a more equal distribution of the manure is secured to the soil, for the nourishment of the ensuing crops. Neither is the land always raised into small ridges for the reception of the dung; for the seed is frequently sown in drills drawn through the soil after it his been mixed with the manure, and laid quite flat.

In thus stating the application of manure, we have only adverted to the use of lime and dung, though ashes, rape-dust, oil, and sea-weed, together with many other kinds, both natural and composite, are frequently employed, and latterly bone-dust has come into such general favour for the cultivation of turnips, as to nearly supersede every purcliased species of a putrescent nature. On this, lowever, we have already dilated so fully in our first volume, that we deem it unnecessary to jursue the subject any further; but we particularly refer our readers to the details in the 19 th Chapter.

The next operation is that of sowing the seed. This ouglit to be done as soon as possible, while the earth is fresh and moist, after being newly turned up, and when not done broad-cast, is usually performed by means of one or other of the numerous machines already mentioned $t$, which all act upon the same principle. When diried, some farmers, however, still put it in by hand, from a small implement formed like a pepperbox, with holes at one end, fastened to the end of a walking-stick $\ddagger$, and followed by a man with a rake to cover the seed; the crowns of the drills being in either case flatted down by a light roller, and the seed sown in recrular rows, at the depth of about an inch and a half, along the mildle of the tops, directly over the manure. A common mode is also by the hand-drill, which follows the roller, to which it is attached by a rope, and the seed is sown upon one-bout ridges in the following manner. The roller, though of course wide enough to cover two ridges at once, yet, on the first turn, "takes but one ridge with one of its ends; and, in returning, while it rolls this a second time with the same end, rolls a secund ridge the first time with the other; which again, in returning, it rolls a second time, along with a third ridge the first time. In this way it goes twice over the ground: the drill depositing the seed between the first and second sowings §."

The more general practice is, however, to sow two drills at once. The frame-work of this machine is supported by two separate concave rollers, which work together, and are formed to embrace each drill, in the manner

* Berwicksh. Rep. p. 271. In Norfolk, however, barley when accompanied with clover is generally sown upon a third ploughing. Kent's Norfolk, p. 45.

+ See Chapter vi.

+ Surv. of Mid-Lothian, p. 111.

Thepurt of a Gloucestershire Irill farm, in the "Farmer"s Series, No. XXI." See also the plate in the Northumberland Report, and in Brown of Markle's Treatise on litural Aftairs. 
depicted in the unterneath back viet, in which $A$ A are the rollers, B $B$ the hopners, and C C the coulters, and its weight ensures a regularity of sowing hardly to be gained by those of a less size.

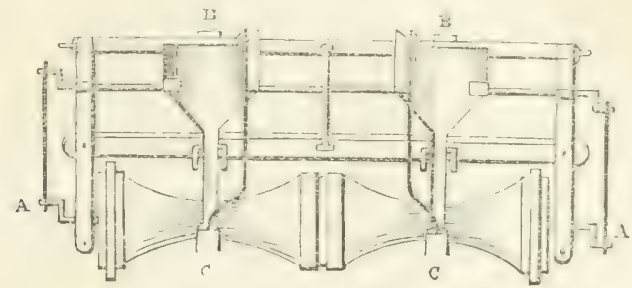

There are, however, various deviations from this construction; the rollel being more frequently in the common form, and without any division in the centre. Sometimes, also, light rollers are made to follow the drills for the purpose of covering the seed, when the land is friable and sandy; this, however, is not always thought necessary, as the tracks made by the coulters are then found to answer the purpose, though we think it ought never to be dispensed with: in some machines, indeed, a small rake with two or three teeth is fixed behind the coulter, and thus mixes the seed with the soil.

After the sowing has been completed, the plants generally make their appearance within about ten days or a fortnight, according to the soil and the temperature of the air; and if the weather prove showery they will grow into what is termed "rough-leaf," when they are a couple of inches high. The process of horse-hoeing then commences, by rumning a small single-horse plough, or one of the many implements already described in the second chapter of this volume, up and down between the rows, as near as it can be done without injury to the crop; or at about three inches distance from the plants; so as to cut up any weeds that may have sprung, and to turn of a shallow portion of earth from the turnips. Within two or three days afterwards, this is succeeded by the operation of hand-hoeing, which is performed by the labourer going along the siles of the drills with a gardener's hoe, having a blade of about eight inches in breadth.

With this he stands opposite the rows, and at one stroke across the ridge he cuts out the plants at regular distances, leaving then standing singly, with a vacant space of at least nine or ten inches between each; thus thinning them sufficiently, and leaving room for the roots to grow to a proper size. To see this process for the first time, one would suppose the crop totally destroyed, but the weeders soon acquire great esperience in executing it, and a turnip-plant, with ever so little hold of the ground, soon recovers its vigour. When intended for consumption early in the season, the turnips are, indeed, frequently thinned out so as to stand at the distance of full a foot from each other, for they increase in bulk partly in proportion to the distance at which they stand; but when meant to be kept for spring use, it is thought that they should be left thicker, in order that their growth being thereby somewhat checked, and the bulbs thus ren. dered more solid, they may run less risk of being injured by the winter's frost.

Within a fortnight or three weeks after this lias been performerl, the weeds will again shoot forth, and the same work must be repeated, with still greater care; as the hand-hoers should then trim the earth around every single plant, and attentively cut out any other sprouts which have 
escaped their previous notice; for if they grow two or more together, they draw up long and weakly, and do not swell properly at the root. This, generally speaking, terminates the operations of horse and hand-hoeing; as the plants will have now got broad leaves overshadowing the groma for some distance on each side of the drills, and preventing the weeds from growing: but if the weather prove untusually moist and warm, the weeds may get so fast ahead as to render a third hand-hoeing necessary, and neither this, nor the further expense of getting women and children to weed out wild mustard, charlock, ketlock, or any other rubbish which may afterwards spring up, should ever be grudged.

I.astly, after the last hand-loeing, and when the turnips have begun to bottle, a double-mould hoard plough is sometimes passed down the rows for the purpose of ridging them and earthing-up the plants. This, however, is an operation which is not always performed, as the intention is chiefly to lay the turnips dry during the wet months of winter, and the bulbs are found not to swell with much luxuriance if heavily earthel. On land which lies dry enough to be fed off by sheep, the ridging of it uy is also exposed to the further objection of occasioning accilents, by causing the animals to be lost by falling upon their backs into the furrows, without being able to recover themselves; but in spring frosts it shields the turnips, and supplies the place of their natural covering of leaves.

\section{PRODUCE AND QUALITY.}

In the ordinary course of cultivation, turnips are usually sown previous to barley and clover, but the common sorts are not unfrequently fed off during the autumm, and the land is then generally sown with winter wheat; though sometimes, when the crop has not been got off in time, spring wheat is substituted. 'Thus, according to a statement drawn up by sir John Sinclair and Mr. Curwen, of the practice which they witnessed on the farm of the late Mr. Rennie of Phantassic, the rotation of crops upon two fields of a good gravelly loam, then under Swedes and common turnips, was as follows :-

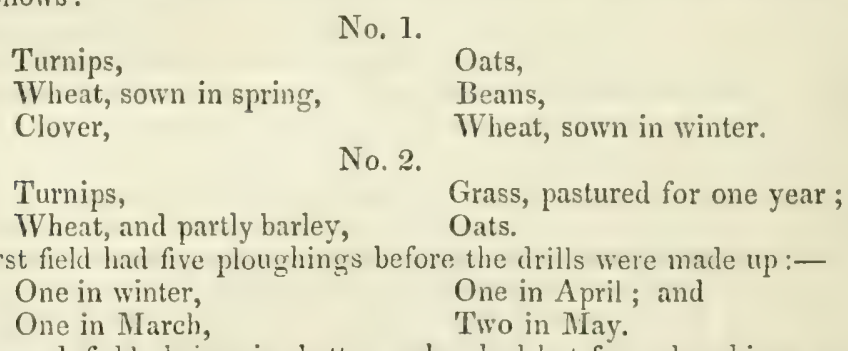

The second field, being in better order, had but four ploughings, only one being given in May; but, besides these operations, the plough was made use of when the stitches were formed, and agrain when the dungr was covered previous to the seed being sown. The Swedes were dumged at the rate of 1:, and the common turnips at 10 tons the English acre; the sowing of the former being in the two last days of May, and the three first of June; and the latter, between the 10 th and 22 nd of Junc. The produce of the Swedes was estimated at 30 , and the common sort at 40 tons per acre; but this amount was partly attributed to the fields having been formerly limed, at the rate of 300 bushels per Scots acre-though so far back as sixteen years ago .

* Farmer's Mag., vol, xvi. p. 430. 
Much larcer products of roots, exclusive of tops, have, indeci, been in many instances obtained: thus in a report from the Artrossan Society, in Ayrshire, it is stated that the weights grown upon an Imperial acre, without any peculiar mode of cultivation, were

Of common white turnip $\quad: \quad 060$ tons 0 civt.
, Swedes $\quad-\quad 46,7^{*}$ ditto.

and it appears, from a paper published last year, by the Kilsyth Farmers' Society, that in a competition between the tumip crops in that parish, the greatest weights of the species there grown, were-

Jones's Yellow $\quad$ - 42 tons 8 cwt.
Aberdeen, or Green-top Yellow
$\begin{aligned} & \text { Dale's Hybrid } \\ & \text { - }\end{aligned} \quad 35,17$ ditto.

They were sown in drills, at the usual distance of two fect to two feet three inches, and hoed out from eight and a half, to about ten inches apart; but the statement is deficient in not having mentioned cither the soil, the date of sowing, or the quantity and quality of the manure.

It should however be observed, that on assuming weights for any veretable crop, though an idea approaching to the truth may be gained, yet no correct estimate can be obtained by any other morle than weighing the entire produce; for whatever may be the regularity of its appearance, the roots are, partly at least, below ground and difier extremely in size + . Ailded to this also, the quantity of manure should be in every instance stated; for as that forms a main portion of the expense, so wiil it also largely affect the calculation of the farmer's profit.

Regarding the comparative weights and sizes respectively atiained by various sorts of tumips, it has been found, "after repeated trials, that sound green-top yellows, of seven or eight pounds weight, gave, as their highest average, thirty-two cubic inches per pound; which may be assumed as a medium standard of solidity or density, and being compared with the actual weight, will afford a just criterion of their value, and a correct idea of their sizes +." Upon this principle we learn, from the third Report of Drummond's Agricultural Nuseum at Stirling, llat single turnips of the different species, grown during the last few years upon various soils, and there exhibited, were therefore selected and proved to be as follows :-

\begin{tabular}{|c|c|c|c|c|c|c|}
\hline & $\begin{array}{l}\text { Weight } \\
\text { er Standard. }\end{array}$ & Actu & & $\begin{array}{l}\text { ight } \\
\text { andard. A }\end{array}$ & Ac & \\
\hline $\begin{array}{c}\text { Swedish } \\
\text { ditto }\end{array}$ & $\begin{array}{l}\text { - } 111 \mathrm{~b} .20 \mathrm{z} . \\
\text {. } 105\end{array}$ & $\begin{array}{l}131 b .12 \\
138\end{array}$ & $\begin{array}{l}\text { White Globe } \\
\text { ditto }\end{array}$ & $\begin{array}{l}\text { 20lb. } 8 \text { oz. } \\
190\end{array}$ & $\begin{array}{l}151 \mathrm{~b} \\
15\end{array}$ & $\begin{array}{l}0.80 z . \\
0\end{array}$ \\
\hline op Yellow & 160 & 15 & Red-top White & 168 & 13 & 0 \\
\hline & 0 & 0 & & 148 & 12 & 0 \\
\hline tto & - 120 & 10 & hite & 120 & 11 & 0 \\
\hline & 118 & 12 & & 11 & 0 & 8 \\
\hline Tybrid & 1312 & 13 & d. & 160 & 14 & 0 \\
\hline & 128 & 11 & Purple ditto & 1210 & 11 & \\
\hline
\end{tabular}

From the above test the superiority of the Swedish turnip appears to be very decided; and, contrary to other sorts, greater size indicates also greater solidity, which agrees with the products which it has been found to afford

* See the 'Farmer's Magazine,' rol. xv.'p. 169. and vol. xvi. p. 157.

+ Thus in turnips, for instance, diameters, will acculut for the very different quantities of live-stock, of similar weights and kinds, that crops apparently much alike to the eye, are observed to feed.

+ Thus-" By dividing the number of cubic inches contained in any bulb by 32 , (the remainder being halved for 16 ths or ounces, ) the quotient grives the number of pounds and ounces that should be of the standard solidity : the actual weight of the bulb will indicate the solidity, which will either be at, or above, or below the standard-as the numbers of the weight are equal, or greater, or less than the numbers of the size." 
of nutritive matter-being full 6 per cent, and in the larcest roots, nearly 7 per cent. of their whole weight; while the white varieties have afforded 4 per cent., and in the largest roots, only $3 \frac{1}{2}$ per cent. of their whole weiglit. This nearly accords with the experiments of Sir Humphry Davy; which showed that Sivedish turnips yielded $6 \%$, and common turnips only 42 parts of nutritive matter, in 1000 parts of vegetable substance contained in the roots: hence, one pound of Swedes should be equal to one and a half of the same weight.

The quantity of nutritive matter, and the average produce on a good soil, per acre, are also calculated, in the same publication as follows:namely-

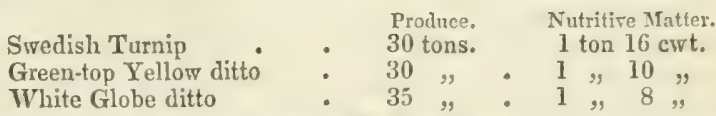

The relative portion of nutriment afforded by each of the following species has, however, been estimated by the late Mr. George Sinclair, as amounting, in 64 drachms, to-

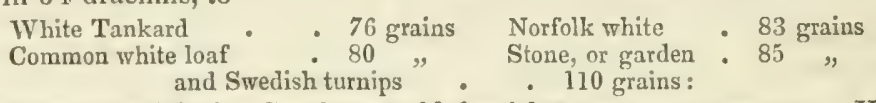

according to which the Swedes would furnish 1 cwt. 1 qr. per ton. While the largest roots of that species produeed 110 grains, the small ones however only yielder 99 ; and the common white loaf varicty, measuring 7 inches in diameter, gave only $72 \frac{1}{2}$ grains, while the same quantity of a root measuring only 4 inches, afforded $80^{*}$.

\section{DISEASES.}

Turnips are subject to many injuries, arising from mildew, rotting with frost, and the destruction of insects. Among the distempers to which they are liable are those termed the "anbury," and "fingers and toes ;" the former appearing soon after the formation of the apple in the shape of larce uncouth tumours, which form themselves below the bulbs; and in the latter, the root, instead of swelling, runs into those small divisions from which it takes its name, a lare portion of the surface usually wanting the natural skin, being tinged of a dirty blue colour, and finally becoming rotten. They are indeed frequently confounded together, and are generally attributed to internal disease, cilher owing to some defect in the seed, or in the quality of the soil and manure, or telse to the time of sowing; though it would appear, from more recent investiration, that they arise from insects, the excrescences being thought to be the nidus of one species, and the destruction of the root being caused by anothert. Farmers generally suppose them to be occasioned by the too frequent cultivation of tumips on the same land; but the malady has been found to exist in ground which had been newly broken up t, and no mode of either prevention or remedy is known.

Another malady, called the "black canlier," arises from a species of slug, or caterpillar, which commits great devastations among the tumips when the plants are in rough-leaf and have partly formed their tops. The

* See the Hortus Gramineus Wobernensis, and the 'Complete Grazier,' 6th edit,, p. 533.

t See several treatises on the sulject in the Transactions of the Highland Society, vols. viii., and N.S. ii.: as well as, "Ohservations on the 1)istase in 'Jumil s temed Fingers and Toes," by William Spence, F.L.S.

$\ddagger$ Lincoln Report, 2nù edit. p. 161. 
most usual mode of destroying these worms is by heary rolling during the night, as it is then they come out to feed; but it is very far from being cfiectual in rough and stony soils. Ducks may, however, be successfully employed for the purpose of devouring them, and so certain is this expedient, when the flock is sufficiently large, that a field of thirty-three acres was effectually cleared within fivedays by 400 , which were turned upon the farm of MIr. Colie at Holkham*. Much injury has been latterly experienced from an attack of mildew on all the varieties of turnip, when halfgrown, but the Swedish and yellow turnips have suffered most frequently and severely from it.

The most destructive enemy which the crops have to encounter is, however, a small winged insect, well known to all farmers under the common name of the "turnip-fly." It makes its appearance as soon as the tender seed-leaves of the plant burst forth, and frequently swarms in such numbers as to completely strip them, but disappears the moment the growth of the rout converts the buds into rough-leaf. This usually happens within a few days after the seed-leaves have first sprouted, and within that short space of time it commits such serious mischief as sometimes to require the ground to be resown.

Numberless expedients besides rolling have been resorted to, with a view to check this evil, among which top-rtressings of soot and quicklime have been the most strongly recommended; and, from some experiments made by the Earl of Thanet, and published in Mr. Greg's account of his farm in Hertfordshire, on the application of the latter, great expectations were formed of its success. The mode of applying it is in powder strewed along the drills and dusted over the leaves the moment the tumips come up, the quantity employed being usually six or eight bushels jer acre; but a much larger amount may be used without injury to the plants, and may be considered useful as aftording a dressing to the land. It should, however, be laid on when the weather is quite dry, as a shower of rain will slake it. Its effects have accorlingly been extensively tried, and in many instances found useful: but the accounts are so contradictory that the advantages which have been found to result may be not unreasonably ascribed to some favoumble circumstances in the weather; and we have lately learned from the nephew of Mir. Greg, that the plan was, on his farm, found ineffectual.

Sir Humphry Davy records an experiment made with a composition consisting of three parts of soot and one part of yuicklime, slaked with urine, about four bushels of which, per acre, were let to fail into the drills along with the turnip-seeds; the result of which was, that the rows to which the compost was applied were scarcely touched by the fly, while those adjoining to them were eaten away t. On this, however, it may be observed, that although the volatile alkali of these mixtures may be offensive to the insects, yet they also afford a highly stimulating food to the plant, which mav thus be jut speedily out of danger; for the most weak and sickly sprouts are always the first to be attacked. The sowing of radish seed intermixed with turnips has also been successfully tried as a preventive of the fly $\neq$. The burning of weeds and damp straw have also been found

* Complete Grazier, 6th edit. p. 517.

† Lectures on Agricultural Chemistry, 4to. p. 320.

t The Cures gold merlal of the London Society of Arts has also been awarted to Mr. Poppy, for an experiment made on his own farm, in which four acres were sown in drills with the ustul yrantity of siwedes intended to stand for a crop, and half a peck peracre of cummon turnips in alteruate rows for the purpose of attracting the fly. The result 
efficacious; and various steeps have been adopted for the seed *; but no remedy has been hitherto found to completely answer the intended purpose, and it is not unfair to presume that, when crops have been thus apparently saved, their preservation ought rather to be attributed to causes beyond our control. Such expedients may not indeed be productive of harm; but we should rather place confidence in a good soil, with an abundance of well-rotted dung, a full quantity of seed, and careful culture, to secure the rapid growth of the plants, than in any other means of furcing vegetation.

\section{STORING.}

Turnips are not unfrequently grown on land which is too wet in the winter to allow of their being fed off the ground by sheep; and it is also in many cases expedient to draw a portion of them--perhaps every second or third ridge-for the support of cattle in the yards. To eflect this, and also to guard against the danger w which they are exposed in severe weatler from the frost, it has become a very prevalent custom among famers, whor hold land in bleak and exposed situations, to store up considerable quantities in the month of November and the beginning of December, or later according to the climate and the state of the crop. Like most other operations in husbandry, there are several methorls of storing; some carrying off the entire crop, or only a part, and either stacking them in the field, or placing them in pits, or under sheds, and others cutting off the tops and tap-roots, while many consider the latter operation unnecessary; but the most usual practice is-to "top and tail" the best roots, preserving only the sound buibs, and to give the tops to the store stock while fresh and newly cut; by which means a considerable portion of food is saved, which is otherwise either lost, or ploughed down for manure. They are then carted to some dry spot near the feeding sheds, and piled upon the surfice of the ground in narrow ridges, about five feet wide at the base; and tapering graduaily to the form of a wedge; which is afterwards well thatched with fern, straw, or rushes, and secured with ropes. Careful men also hed them upon refuse straw, or stubble, to secure them from the wet; and, when the frost becomes intense, it is not a bad precaution to cover them also with long stable dung. Attention should also be paid to the operation of topping and tailing; for, if any great portion of either be left, the turnips are liable to vegretate; and if the bulb be wounded, it is apt to rot. It is indeel impossible in practice to always hit the precise point where they should be cut: the safer way is therefore to leave a part of the top and root, rather than incur the risk of injuring the bulb; as it is wiser to run the hazard of a slight vegetation than of entire putrefactiont. 'Tlie process is in some cases performed in the field before the turnips are carted liome, and the tops and tails are then ploughed into the land as a partial dressing for the future crop.

was, that the thinly sown Swedish turnips were not sensibly injured by the fly, while the thickly sown common ones were quite black with swarms of the insect. After the Swedes hal got into rough-leaf, the rows of common turnip were ploughed up, and the field being managed in the usual way, turned out a good crop, whilst all the other turnips in the parish failed,-Trans, of the Soc, of Arts, vol, xiv.

*Among these we learn from the Annals of Agriculture, (vol, xiv.) that train-oil has been used; and in the Survey of Essex an instance is mentioned of an eminent farmer who never lost a crop of turnips; "owing," as he said, "to his having steeped the seel the night before sowing, in water mixed with black brimstone powlered."--Vol. i.1. 371 .

+ See Two Essays on the Storing of Turnips, for which a premium was offered hy Sir G. S. Mackenzie, Bart, Farmer's Mag. vol. vi. 
Turnips thus stored at Hallowtide have been found perfectly sound in the following April, and it is the only certain mode of securing a supply on farms situated as those to which we have alluded. 'They however lose a portion of their nutritive juices; and become so much less patatable to cattle than those taken fresh from the ground, that if a root in the most perfect apparent state of preservation be taken from the heap, and offered to a cow together with one newly drawn from the field, she will invariably preter the latter. Unless, therefore, in those northern districts where the frosts set in early, and are often severe as well as lasting, most farmers who have land sufficiently dry to allow of the tumips being carted without poaching the ground, content themselves with only drawing such a quanlity at a time as may suffice for the consumption of their stall-fed stock for two or three weeks and placing the heaps without further care under sheds. They may thus remain during that time in safety; for, if frost-bitten, they may be thawed by being left a very few hours under water; but if liept longer they will decay; and if exposed to the heat of the cattle-sheds, they will become putrid.

There are some, however, who adopt a less troublesome and equally chiccual mode for the preservation of turnips, which are sown in drills, and meant to stand throughout the winter. They are drawn, by the "grubber" described below, in the nonths of November and December, and the bulbs are placed, with the top entire, and only a small portion of the root cut off, in the centre furrow, upright, making one furrow contain the plants which grow upon two or three ridges, and taking care that the bottom of each plant shall touch the ground. The soil is then ploughed up to them, covering the outer edges; and if the weather be very severe, a little straw or any species of litter is thrown over them. Those that remain unconsumed in February are then removed to the quarter where they will be required for consumption, and placed close as possible to each other Ipon grass land, having then the remainder of the root cut off. This plan lats the advantage of preserving the turnips in the best order, alike free from cxhaustion by growth, and from decay by too much exposure, as well as preventing the attacks of wood-pigeons and crows, which commence their depredations at the season when their usual food begins to fail. The soil is also less deteriorated by this mode, than it would be by allowing the plant to remain in the land*.

The process of grubbing is indeed seldom omitted even when the turnips are fed off upon the gromel, and is jerformed with a two-pronged forls of this form:-

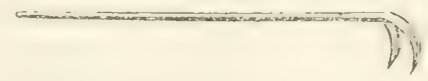

with which one man will grub up in a day as much as will serve seven or cight score of Sumthdown sheep; or, if the land is dry, the turnip may be turned by the plough $\uparrow$.

* Library of Useful Knowledge ; Reports of Select Farms, No. 1. p. 9.

+ Rigby's Hollham, 3rd edit. p. 11. "In deep snows, too, the turnips may be uncovered by the use of an instrument called a snow sledge, which is simply formed of two or three deal boards, from seven to nine feet long, and ten or tweive inches deep, set upon their edges in the form of an equilateral trangle, and strongly sceured together at the angles, at one of which is fastened a hook or eye to hang the horses to. Being thus drawn along the ground it forces the snow into a ridge, and a strip of turnips is liid bare without receiving any material damage."-Nalcolm's Surv. of Surrey and Sussex, vol, ii. p. 415. 
The expenses of topping and tailing, thatching and storing turnips in the manner first described, are indeed considerable; and if to this: be added the injury sustained by raising them from the ground, it may be cloulded whether they do not exceed the amount of the average loss occasioned by frost; for it seldom happens that a whole crop is destroyed, and in some years the weather is so favourable that the plants escape entirely unhunt. The practice, when abstractedly considered, mity therefore be viswed as dis. advantageous; but, on wet soils, it is mavojdible, while on uyland famm it is rendered justifiable, by its guarding arrainst the uncertainties of climate; and, if regarded with reference to the husbandly of land thus situated, it may at least be deemed a necessary evil.

\section{APPLICATION.}

The most usual mode of consuming turnips is by eating them off upon the ground with sheep, though larere quantities are also carried ofl the land for the support of store cattle and cows; in which latter case they are almost invariably topped, tailed, and sliced. Although when fed ofï by sheep, they are usually allowed to remain whole, yet the horrots frequentiy lose many of their young teeth from eating turnips when they become tough in the spring; and, from the same cause, the older sheep become crones before their natural time, to the serious detriment of the flock. To prevent these inconveniences, the roots are commonly cut into pieces by an instrument called the "turnip-chopper," consisting of a handle with an iron head, formed as if the blades of two gardener's hoes were placed at right angres the mamer of a cross. The bulb being placed upon the ground is thus divided into four equal parts by a single stroke, perpendicularly griven by the workman; and the other end is usually furnished with a fork, which is useful both in adding force to the blow, and in tearing the roots out of the earth.

It is an efficient tool in the hand of an expert man, but it repuires considerable strength of arm as well as constant exirtion, and therefore various implements have been invented for the purpose of expediting the work and lessening the labour. These are generally constructed upon the principle of containing a hopper for the reception of the turnizs, and knives fixed in a wheel, which is worked by a winch-handle, and cuts the routs as it revolves: but some are formed, in a more simple mamer, with upright slicers, which receive the roots from an inclined trough, and are wrought with the hand by a lever*. They are, howerel, so well known, that we deem it unnecessary to describe them more minutely; but we insert in the following jage cuts of a turnip-slicing cart, with the drawings of which we have been lately firoured by Messis. Ransome of Ipswich, and we transcribe the description from an account fumished to us by a juactical farmer, at whose suggestion the implement was constructer, with the intention of cutting the roots upon the ground.

When the tops of the turnip'; are fed off, the bottoms, or bulbs, are picked up and put into the cart. The horse moviner forward, the turnips are cut in a few minutes either into slices or smaller picces on the place where they were grown, or any other part of the field: the pieces are scattered behind the cart, and not a vestige will be left uncaten by the flock on dry

* See plates of improved specimens of these in the Quart. Journ. of Agric., N.S., vol. iii. p. 127; and in the Transactions of the Highland Society, N.S., vol. iv. p.51.The former is made by Mr. Morton of Lidinburrh, and the latter by Mr. Baird of Shotts. 
feeding land where no troughs are required. The teeth of the sheep are thus preserved, and crones may be fattened upon tumips so cut which they could not otherwise eat. On dirty land, troughs are used with great advantage for feeding fat sheep with cut turnips; the practice, indeed, is increasing every winter, and the slicing cart is adapted for that purpose, as the turnips may be cut by hand directly from the cart into troughs, or skeps, with a winch, or crank, on the slicing wheel, when the cart is not moving forward

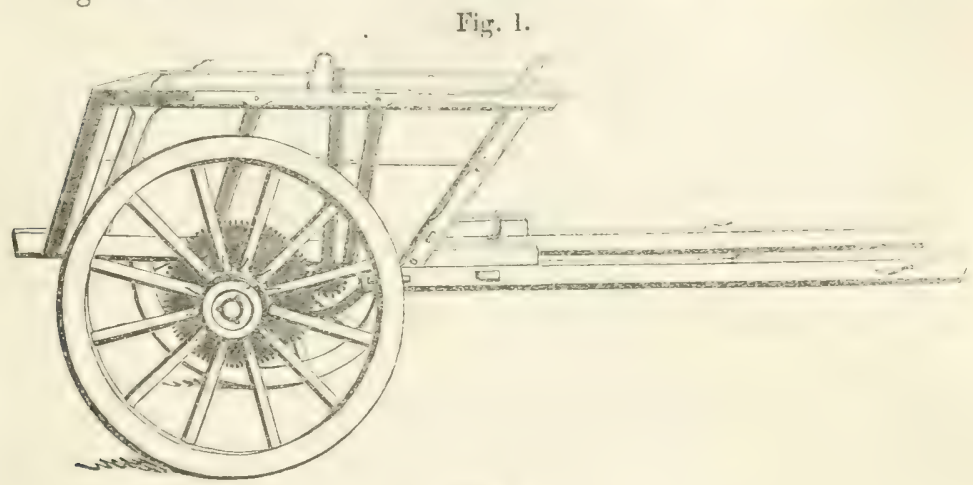

Fig.: 2.

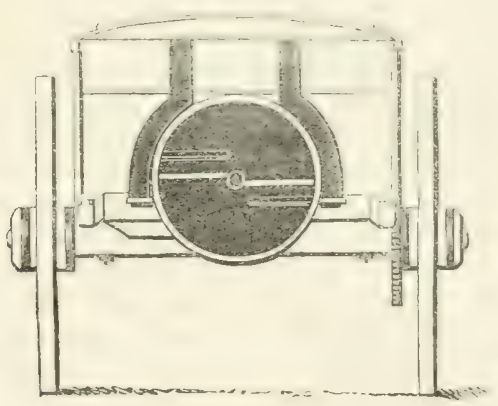

Fig. 1 presents a side-view of a common three-quarter loal Suffolk cart, with the fist large corr wheel of the slicer attached to the back part of the nave of the cort-wheel. The upright lever $\mathrm{B}$, moving on a fulcrum (a lolt in the cart cill), brings the small cogwheel into gear with the large cog-wheel on the nave: a small wedge at the top of the lever, passing through the rail of the cart, keeps the cong-wheels either in geur, when the slicer is worked, or out of gear when not required to be turned by the horse. The axle of the smaller curr-wheel (passing through the earl of the upright lever which is its only wear at that eni) goes under the cart and half-way across, where, at a right angle, it meets, and, by a small pair of bevil wheels, is connected with the axle of the slicing wheel, with its two knives, shown in the back view of the cart, Fig. 2. When this slicing wheel is jut in motion (by the horse moving furward and the cors-wheels, before described, put into gear), it turns with great velocity, and the turnips roll to the linises from a sloning false bottom. When the cart is wanted for common agricultural jurposes, this false bottom and the slicing wheel are remored, aud easily replaced when required.

The necessary slicing apparatus may be placed upon an old cart for 3l. 13 s. $6 \mathrm{~d}$; ; but if an entire strong-made cart of this description be made new to accompany a flock of sheep, it is desirable to have the rails, or reaves, of the cart projecting over the horse's back and supported from the 
shafts for carrying lurdes. The cost of such a cart with the cutting apparatus would be from fourteen to seventecn guineas; and we think it would be found eminently serviceable to large flock-masters.

The Barrow turnip-slicer, described at foot, is an implement used for the same purpose, and also constructed by Messrs. Ransome.

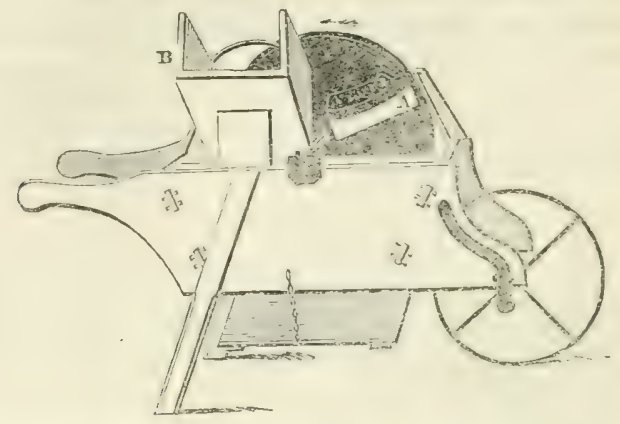

It consists of a that steel knife, A, placed upon a vertical iron wheel, which is turned by a crank, as shown in the accompanying cut. 'The turnips are put into the hopper, at $\mathrm{B}$, which stands in a slanting direction towards the wheel. By turning the crank the knife passes across the lower end of the hopper, and thus cuts the turnips into slices of about half an inch thick, which fall through into a skep, or basket, placed beneath. When it is requisite to cut them into smaller pieces, a set of small knives are introduced so as to stand crosswise before the edge of the flat knife, and thus, in passing the hopper, cut each slice into strips of abont one inch wide. These knives are fixed upon a small plate, and may be inserted or removed at pleasure.

If wanted for in-door work, these cutters are generally fitted up on a stout wooden stand of sufficient height to admit of a basket being placed underneath to receive the turnips; but if to be used out of doors, we prefer placing them upon a barrow with a moveable bottom; and the barrow is wheeled from heap to heap or from trough to trough, instcad of removing the turnips. They are certainiy very handy tools, for a man and a boy, working one of them at moderate sjeed, will cut with ease one bushel per minute; they are, therefore, coming much into use, and their cost is only three guineas:

The calue of an acre of turmips is, in consequence of the differences in soil and scasons, so very various, and fluctuates so much according to the demand and modes of application, that nothing decisive can be stated on the subject. When drawn and carried off the land for town consumption in the neighbourhood of large cities, they are sometimes extravagantly dear, and are not unfrequently contracted for by market-men and cowlicepers at from eight to ten guineas, and even still larger sums. Hardly any price can, lowever, be said to compensate for the loss of the manure; for, eren if dung be carried back, that will not supply the waste of urine of which unusually large quantities are roided by animals which are fed on turnips.

It is by no means uncommon among flock-masters in upland districts, or holding land which runs bare of pasture, to feed a portion of their sheep ul. on the turnips of their neighbours in the lowlands; and, upon the other hand, farmers in the marshes frequently send theirs to the ground of men who live on the downs and wolds, or upon dry turnip soils. The turnips are in such cases eilier paid for by the acre, or the sheep are boarded by the score at a fixed price per week - the agreements, of course, 
varying according to the quantity of food and the demand-from three to five and, in some cases, cight shillings per score; and the calculation upon the general run of turnip crops, mon land of the ordinary quality in the midland districts, is, that an acre will feed from 14 to 16 score Southdown wedlers during one week; or at the rate of about 16 lbs. each day per head. The crojs in the climate of the north, and upon rich loams, are of course larger; but viewing the ordinary course of farming throughout the greater part of the kingdom, the average vilue of an acre of turnips can hardly be estimated at more than five pounds. This, when considered singly, cannot be viewed as a source of direct profit to the grower; for, were rent, manure, and the extraordinarily expensive culture, which is requisite to the proiluction of a good crop, to be all taken into consideration, perhaps double that sum would not more than repay him. But if its beneficial consequences be regrarded, as standing in place of a naked fallow; as producing an abundant supply of succulcnt food for the winter and spring support of sheep and cattle; and as augmenting, by its increase of fertility, the means of procuring heavy crops of grain and hay, the turnip culture must be considered the main stay of all good husbandry on those soils to which it is adapted *."

\section{Chapter XIX.}

\section{ON RAPE}

ON soils which are too adhesive for the growth of turnips, this plant is very frepuently cultivated for the purpose of alfording food for sheep, and perbaps there is no other upon which they fatten with greater rapidity, provided the soil be of sufficient luxuriance to give full vigour to the plant. It may indeed be grown for this purpose with advantage upon every rlescrip)tion of land as an occasiunal crop, particularly upon pasture land, when broken-up; and it is found valuable upon the best turnip soils, as a change, to prevent the too fiequent repetition of turnips. Thus we find, in the report recently published of "Farining at Wauldby, upon the Wolds, in the Sist-Riding of Yorkshire," A portion of the falluwed lands lave for the last few years been sown with rape, solely for the use of the sheep: it is cultivated in drills twelve inches apart, but not thinned in the rows, thoush well hoed between them. 'The quantity of seed used is 4lb. per acre. To aflord a regular succession of food, it is sown at three different periods, between the middle of May and the end of June; and the sheep are put upon it three months after it has been sown. A considerable quantity of the very best food is thus raised, to come in at at period when it is most wanted, viz., when the freshness of the clover and grass leys is on the decline, and before the turnips are fully grown. It is considered equally valuable for the lambs, when taken from the ewes, and for the shearing wether sheep, eighteen months old, which are at that periud nearly fat, and require food of the most nutritious quality. The land is afterwards sown with wheat, and the crop is usually considerably heavier than it is after turnips, or after any other preparation of WVold land for wheat.t"

linpe is, however, more peculiarly partial to alluvial, or rich clay lands; and it is upon these, especially aftor having been long in grass, that it grows whih the greatest lusuriance. In the fens of Lincuhshire and Cambridge,

* Survey of Berwickshire, p. 279.

$\dagger$ Library of Useful Knowledge, Select Farms. No.64. 
where it has been chiefly cuitivated for seed, upon their first beiner broken up, the produce has been immense, aflording from five to six quarters per acre; although upon sinilur soils, which have been long in cultivation, half that quantity of seed is esteemed a very fair cropl. "The viguour of the plant when grown for sheep will be found to vary in the lii.e froportion. Few plants are less liable to failure in consefuence of the variations of weather, or the attacks of the insect tribe, than this; if therefore the land be in good heart, and the cultivation be attended to, the grower will be rarely disappointed: but if it be joor, the produce is of little value, being small in quantity, and very inferior as an article of food. A luxuriant plant of rape, with a thick stem, is what the sheep are most partial to, and upon which they fatten with the greatest rapidity. Perhaps the most nutriment is contained in the abundant pith with which the stalk abounds, for the sheep evidently prefer this to the leaves. When grown upon weak land, or when allowed to stand too thickly upon the ground, and thereby deprived of its full proportion of nutriment, and of a fice circulation of air, the stem is small, composed of hard woody fibres, and the leaves become red or mildewed. In this state it is neither relished by sheep, nor are they benefited by consuming it.

Perhaps the most usual mamner of cultivating rape is to sow it broadcast upon a fallow, at the rate of three quarts of seed per acre, early in the month of June, and then leave it to its fate; neitler bestowing the pains of hoeingr nor hand-weeding upon it. 'This is however giving place to a better mode, and we have seen several intelligent farmers, in various parts of the kingdom, adopt the use of the drill and horse-hoe; setting the rows at various distances, from twelve to twenty-seven inches apart, and thiming out the plants from six to twelve inches asunder in the rows. By this means the crop acquires great vigour, and the purpose of the fallow is made eflectual, by the destruction of weeds. But however valuable this plant may be found ats food for sheep in the months of August, September, and October, it must bes admitted that the quantity of food per acre, in proportion to that which is afforded by turnips, cannot be rated at above two thirds even in those months; and that it must be consumed immediately upon acpuiring its fuil state of vigour, as decay of leaf will commence soon after, and when sown at that early period, it would be cut off by the winter frosts. It is indecd frequently sown in August, as spring food for ewes and lambs; but its produce is then very light, the plants being at that period disposed to run into flower, rather than to put out any strong stem or leaf. In this latter vicw it is certainly inferior to some of the open broccolis; especially in the opinion of Mr. Howard, of Nelbourne near Tork, to that species of broccoli known by the gardeners under the name of Jerusalem Kiale. II has cultivated this plant upon a small scale for sheep, and forms a very high opinion of its value in April and May.

It may not be improper to remark, that although rape and cole, are often used synonymously, they are two very distinct plants; and that although equal in value for the production of secd, the former ought only to be cultivated as food for sheep: its habit being in the first instance to form a strong upright stem; whilst the latter spreals into leaf during the autumn, and only shoots into stem during the following spring, coming into flower even whilst the stem is forming." We shall however retum to the subject in a future article, on seeds for the production of soil.

* Strickland's View of the East-Riding of Yorkshire, I. 1-10 ; Rep. of IIuntingdunsh., p. 136 ; and of Cambridgesh., p. 144. 


\section{Chapter XX.}

\section{ON MANGEL-WURTZEL.}

Tus plant which we generally call mangel-wätzel, though more proper!y termed mangold-üïrel, was brought to us from (iemany, and introduced into this country as a field-crop, about fifty sears aigo, by the late Dr. Lettsom, under the title of "the ruot of scarcity;" though the garden species was previously well known here by the English mane of is beetroot." They are both, however, merely varietics of the same class of tinproots: the latter being of a bright crimsun, and sweeter to the taste than the mangel-wurtzel, which is of various shades of colour, though generally of a yellowish white, sometimes internally streaked faintly with pale red; and it is remarkable that, although the seed be taken from one plant, yet it will produce roots of dissimilar appearance. It grows also much larger than the garden beet; and yields crops of such great weight and suceulence, that its cultivation may be justly viewed as a valuable link in the chain of our soiling system.

Of this latter hind-which is in this country exclusively grown for cittle -the flesh-coloured is preferred, as afiording the largest produce; though the whitish species of a moderate size has been found in the French manufactories, where sugar is made from it, to contain a greater portion of saccharine matter: It also comprises two varieties; the one striling its routs deeply into the earth, while the other grows partly above it. A writer in whose opinion we place great confidence, attributes this difference, however, partly to the nature or preparation of the soil, for he found, on dividing some seed of one species with a neighbour, that his roots struck well into the ground, while a material portion of the length of those grown by his friend appeared upon its surface. His land, however, was ploughed to a depth of ten inches, ame that of his friend only superficially $t_{\text {. }}$

\section{SOII. AND CULTURE.}

MIangel-wurtzel can be grown with advantage upon the generality of those soils which are moderately moist; but, if too wet, howerer rich the land may naturally be, although the roots will grow to a great size, they will become watery as well as hollow in the interior, and will soon rot when taken out of the ground. On sands, the ruots remain small, unless a considerable quantity of rain should fall duriner its vegetation; and on chalks, a profitable product is very duubtful. The most appropriate soil is, therefore, of a deep loamy, or clayer nature-though not too tenacious; and in land of that description, the plants will arrive at their full size and consistence; the crops frequently raching the weight of full fifty tous, and in some instances even more, per acre. The upper soil should, lowever, be of considerable deptl-the deeper indeed the better-and if at all shallow, the seed should be sown upon raised ridges.

* The produce of 19 tons of beet-root is stated by M. Crespel Dellisse, of Arras, who has a large manufactory of sugar in that neighbourhood, as leaving

$$
\text { Of unrefined muscovado sugar - } \quad \begin{gathered}
110 \mathrm{lbs} \text {. } \\
\text { " molasses }
\end{gathered}
$$

besides about 6 evt. of compressed pulp, which is used for the feeding of cattle.

In other accounts, "the quantity of the finest white refined sugar is calculated at $2 \mathrm{lbs} .12 \mathrm{oz}$. 16 dwts. from $100 \mathrm{lbs}$. weight of roots: and the whole quantity ammally made was estimated according to a report presented in 18:2 to a Cummitte appointed to inquire into the manufactures and commerce of France, as amounting to nearly 5000 tons of muscovado sugax."'-Enquêle sur les Sucres.

† Von Thäer, Princ. Rais. d'Agric, 2nde edit, tome i., p. 35. 
The culture is so nearly similar to that of turnips, that very little further detail of the process is necessary. The land should be equally well worked, but ploughed deeper, and the supply of manure should be at least as liberal as for Swedes; but we should rather recommend its being well mixed with the soil, instead of being deposited in the drills. Long dung may thus be employed with good effect, as tending to keep the ground open, and green crops, when ploughed in decp, have also been used with advantage*. In thimning out the plants also, greater care is necessary than in performing that operation on turnips ; for two or three of them generally spring from each grain of the mangel-wurtzel, and adhere so closely together, that it is extremely difincult to separate them with the hoe without injury to the root which is meant to be preserved. The better way is, therefore, to employ women to follow the hoers, and draw out the superfluous plants, which is easily done, when they are about the thickness of a crow's quill, and before they have entwined their roots together; for if two grow together, neither will come to perfection.

The plant requires rather a warmer and a drier climate than that of our northern counties; and as it is very susceptible of injury through frost, the seed is seldom put into the ground sooner than from the latter end of $A$ pril to the middle of May : on cold suils, carlier than on those which are warm, and according to the state of the land, and the appearance of the weather. In this country the seed is rarely steeped, but it is advantageous to steep it for thirty-six hours before it is sown, which is found to promote its speedy vegretation; on the continent, it is occasionally wetted with dungwater three or four days before being used.

The seed is small, flat, and light, and is not unfrequently sown by hand broadcast; the plants being afterwards singled out to about fifteen inches distance from each oiner. The most usual, and perhaps the best mode of sowing the seed is, however, to put it in with a dibble upon ridges twentyseven inches aprart; each seed being deposited $l \frac{1}{2}$ inch in depth, and twelve inches distant in line. When any vacancies occur they are filled up by transplanting the largest of the supernumerary plants; though, through an ill-judged economy of soil, we occasionally see the plants crowded within less than two feet apart.

The objections which exist against the transplantation of Swedish turnips, as well as the trouble with which the operation is attended, have probably prevented it from being adopted by English farmers, in the growth of mangel-wurtzel; but in France and Germany, where the root is extensively grown, it is by no means unusual; and upon ordinary soils, the practice is recommended t. As, however, the process checks the proyress of regetation the plants should be prepared early, upon warn beds, and should not be set out until their roots have attained about the size of a goose's quill; when it may be done in the manner stated in the last chapter.

The leaves of the plants become broad and very sueculent in the month of August, and some persons strip them off about that time for the supply of their cattle. Sufficient trials liave not been made to ascertain how far this practice may be injurious to the growth of the roots; but, if done early in the season, and to any great extent, there can, we think, be no doubt that it must materially impede their vegetation. The operation is also troublesome, for only the side leaves should be removed, and those upon

* De Crud. Econ. de l'Agric., 4to. p. 288.

† De Crud. Econ. de l'Agric, 4to. p. 187. Von Thäer, Princ, Rais. d'Agric., 2nde edit, tome iv., p. 360 . 
the crown left standing, and a vast quantity is required to satisfy the appetite: it therefore may be doubted whether the advantage gained by it may not be more than counterbalaneed by the habour and the consequent loss of weight in the roots. The leaves, however, are relished by cows, and produce an abundant flow of mils without any bad flavour to the butter, though its richness may be doubted. Wie, indeed, last autumm saw a small dairy mainly supported by them for upwards of two months, without any apparent diminution of the average size of roots which had not been stripped: they were, however, only gradually stripped, nor were they left quite bare.

The crop arrives at maturity some time from the latter cur of September to the begimning or middle of October, and it should be taken up before the commencement of frost. It should be also often loulied over previously, as some of the plants are frequently found ruming to seed, and should in that case be immediately drawn, and given to the stock. Being a tap. rooted plant of large size, and heavy acreable produce, this operation is one of considerable labour, and, if the land be stiff, it must be loosened around the roots ere they can be drawn out of the crround: in general, however, they can be pulled up with tolerable ease; and, if not deeply buried in the soil, they may be turned up with the plough. They are then topped, tailed, and stored in the same mamner as tumips, and may be liept nearly as long as Swedes; but after frosts, the stacks should be examined, and repacked if they show any appearance of sweating.

\section{PRODUCE AND APPLICATION.}

In the account to which we have alluded in a former chapter of an estimate of the value of various regetable productions, lately issued from Drummond's Agricultural Miseum, both the acreable product and the quantity of nutritive matter furnished by mangel-wurtzel, are stated to be precisely the same as those which are found in Swedish turnips. The comparative produce of the two crops is, however, rated in the Report of the Doncaster Association, to be about one-fifth in favour of mangel-wurtzel ; but, although according to the analysis of vegetable matter made at $\$$ oburn by the late George Sinclair it was proved to contain more nutriment than common turnips, it was yet shown to be inferior to Sivedes*.

This latter specimen accords with some details of its feeding properties given in the Transactions of the Highland Society, as well as some other statements which have been published, but is in opjosition to an experiment marle under the direction of Earl Spencer; the following particulars of

* According to the Report of Mr. Andrew Howden, of Lawhead, Last Lothian, on the feeding of cattle (breed not stated) with potatoes, Swedish tumips and mangel-wurtzel, as stated in the Transactions of the Highland Society, the girth of a pair each, was

1832.

January 30

April 30 .

\section{Lot. No. 1.}

Potatoes.

. $10 \mathrm{ft}, 8 \mathrm{in}$.

. $] 1 \mathrm{ft} .6 \mathrm{in}$.

Lot Nu. 3.

Mangel-wurtzel.

Swedish Turnips.

10 fis 5 ins.

$11 \mathrm{ft} .3 \mathrm{in}$.

$10 \mathrm{ft}, 4 \mathrm{in}$.

$11 \mathrm{ft} .2 \mathrm{in}$.

The opinion of the dealers was also slightly-(from $7 s$, to $10 s$, a-head) -in favour of the quality of those fed on Sivedes, in preference to mangel-wurtzel. N. S., vol. iii.

An experiment is also mentioned in the 'Farmer's Magazine,' of three cows having been put up to feed solely on carrots, cabbages, and mangel-ivurtzel, the result of which was, that those on carrots and cabbages soon took to fattening, and their improvement was every day visible; while the one fed on the root of scarcity was inclined to scour, and made no progress.

Another trial was then made with moderate quantities of the same food on milch cows, but the mangel-wurtzel was found neither to increase the quantity of the milk, or to improve the flavour of the butter, though both objects were attained by the cabbages and carrots, - Vol, xvi. p. 32 
which have heen just sent to us, in answer to some inquiries on the subject, which we lately took the liberty of addressing to his lordship.

"At Christmas I put two Durham steers to feeding, the one upon Swedish turnips, the other upon mangel-wurtzel. I ascertained the weight of the steers by measurement. First month.

"No. 1 consumed 1624 lbs. of Swedish turnips, and increaserl in weight $34 \mathrm{lbs}$.

"No. 2 consumed 1848 lbs. of mangel-wurtzel, and increased in weight $52 \mathrm{lbs}$. Second month :

"No. I consumed 1884 lbs. of mangel-wurtzel, and increased in weight $32 \mathrm{lbs}$.

"No. 2 consumed 1950 lbs. of Swedish turnips, and increased in weight $14 \mathrm{lbs}$.

Third month :

"No. I consumed 1792 lbs of mangel-wurztel, and increased in weight $50 \mathrm{lbs}$.

"No. 2 consumed 1792lbs of mangel-wurtzel, and increased in weight $31 \mathrm{lbs}$.

" The year following I tricd an experiment of a similar nature upon an individual beast, and the result appearing to be the same, I pursued it only for two months, and I do not at present know the exact particulars.

"The comparison between the increments of the two animals during the two first montlss should not only be observed, but the fact that when No. 2 was changed from mangel-wurtzel to Swedish turnips, in the second month, his growth was nearly stopped; but as soon as he was again put to mangel-wurtzel, in the third month, he began to increase again."

The result of a trial so much in contradiction to those to which we have already alluded, camnot fail to strike practical men as very extraordinary, and must be admitted as a strong additional jroof of the caution which should be observed in drawing positive conclusions from isolated facts; we therefore trust the subject will nut be allowed to drop until further comparative experiments shall have been marle. We should, however, observe, that although foreign writers on agriculture place a high value upon the nutritious properties of mangel-wurtzel-assuming them to be, in comparison with those of meadow hay, in the proportion of 10 to 46 ; yet they do not, in any instance with which we are acquainted, advise its being given without a mixture of hay, straw, or other roots; nor in the proportion of more than two-thirds of the entire quantity of food. Combined with potatoes either steamed or raw, it is said to have a particularly fine effect upon the milk of cows.

With regard to the effect of its growth upon the future crops of a rotation, they even consider it so far exhausting, as that the succeeding corn should have a slight dressing : of course in proportion to that previously given to the mangel-wurtzcl. Among English farmers it would appear, from the answers made to the inquiries of the Doncaster Committee, that opinions are upon that subject divided: six representing it as detrimental; two stating the proportion of deficiency to be onc-fifth; and eight describing it as not injurious*.

The Committee have devoted considerable attention to the investigation,

* Fie port of the Committee of the Doncaster Agricultural Association, on the adrantage of mangel-wurtzel as a fallow crop. $1830, \mathrm{p} .3$, 
and "considering that there must be in every farm some land not kind to turnips; that with the same quantity of manure, a larger bulk of produce can lie grown; and that for all land a change of fallow crop is desirable; they strongly recommend the introduction of it into every arable farm in the country, and sum up its advantages as these :-

"It is more sure to plant; being very little liable to the fly, or grub.

"It will produce more weight.

"It is off the land earlier.

"It is better spring food.

"It is useful as a change of fallow crop when the land is tired of turnips; and can be grown on soils on which they cannot be raised."

On the other hand, in favour of Swedish turnips it may be said :-

"That the weeding and singling out are less expensive.

"That there is rather more time allowed for fallowing in the spring.

"That the succeeding crop is better than after mangel-wurtzel ; and,

"That perhaps cattle feed best on Swedish turnips when they are used alone."

It must however be remarked, that these two last evidences in favour of lice Swedes are not fully proved, and only partially supported.

\section{Chapter XXI.}

\section{ON CABBAGES.}

TuE varieties of the cablonge tribe which are grown in our gardens, though all derived from the same stocl; are extremely numerous; but those which are cultivated as fiele-crops, are chiefly used for the support of cattle, and are usually confined to the "Iork," the large "Scoteh," "the ox-head," and the "drum-head," which commonly produce heads of 10 to $20 \mathrm{lbs}$, and not unfrequently arrive to upwards of $30 \mathrm{lbs}$. weight.

These large field-cabbares are those which are generally considered as the best suited to farm-culture, and are therefore those most commonly jianted; lut the specins lnown as the "sugar-loaf cabbage," and socalled from its pointed form, thouls rarely excecding from 5 to 7 lbs., may yet be in many cases found more arvisable, for it can be grown on land of more ordinary yuality than the other kinds; it is hardier in constitution, more sulid and nutritive, and the inferiority of its weight may be in a great derree made up by the smallness of its size allowing of the plants being set cluser together. There is also the "turnip-rooted cabbage," or "kohl Rhabi," a hardy variety, and a species called " Borecule," which are not aflected by the frost, and are emincutly serviceable in the feeling of sheep; and the latter will produce a fresh crop within a few wecks after its leaves have been cut down.

SOIL AND CULTURE.

The cabbage requires a deep clayey soil of the richest nature, and in a moist or marshy situation; the land should also be heavily manured with spit-dung, or compost of the best quality; which should be incorporated with the soil; and if the plants he afterwards dressed with the drainings of the stable it will add to the value of the crop*. On land of

* "The market-warleners generally apply cow-dung to cabbages, when it can be got in suficient gliantfy; if not, a rich compost is used, and at least one-half more manure is given to cabbages than to turnips."-Stevenson's Surrey, p. 270. 
that description, or on wet and sandy loams thus enriched, it yields an abundant produce of very valuable food, and will therefore be found more advantageous than turnips; but it is an exhausting and expensive cropl, much exposed to hazard from drought and insects, and requiring a warm though moist season: for which reasons it is not generally grown, and seldom upon a very large scale *, thourg it has the convenience of being easily come at in frost and snow. With respect to the exhaustion which it occasions to the soil, this has however been attributed, perhaps not unjustly, to the fact of its being a common practice when the crop is taken, to cut the head from the stem at a considerable distance from the ground, leaving the root in the earth, which throws out sprouts, and thus draws the land when the effect of the growth of the plant ought to lave entirely ceased t. But when the entire cabbage has been drawn, or the roots have been turned out of the ground by the plough, the succeeding crops of corn have been very abundant $\neq$.

The mode of culture nearly resembles that of turnips; but the plougriings are so much deeper, that one plough is fiequently made to follow another in the same furrow, and the land being heavy as well as generally wet, is more generally laid in ridges, commonly of four furrows eacli. When laid flat, the manure is commonly ploughed in previous to the last ploughing; but when laid into ridges, the muck is usually spread in the furrows between the bouts, and the ridges, being divided by the plough, are turned upon the dung immediately before planting.

The seed may be either sown in drills - the shoots heing afterwards singled out to the requisite distance, and the roots allowed to stand for a crop; or, it may be sown in a nursery, and the shoots be afterwards transplanted: the latter is the plan most usually adopted. The ground intended for this purpose should be rich, well sheltered, and dry, in order that the seeds may vegetate lindly and equally. The beds are commonly made about four feet broad; and, if the goodness of the seed can be depended on, it is sown rather thin, and covered lightly with well pulverized earth. About a quarter of a pound of seed will be found sulficient to produce plants enough for an acre.

The proper season for sowing depends upon the time when it is intended to make use of the crop. If meant for consunution in the latter end of autumn, the seed should be sown at Michaelmas, or rather sooner, and the shoots will be ready for transplanting early in the followingr spring; but if intended to stand during the winter, it should he sown in March, and the shoots may be planted out in all June. Most farmers indeed plant a succession, perhaps from April until the end of Jume, so as to afford a constant supply of fresh and perfect plants ere they begin to run to seed: April and May are the most usual montlss.

The mode of plunting out is the same as that already lescribed in the transplantation of turnips; but, in consequence of the greater length of the plants, and their long and slender tap-roots, requires still greater care. The operation should therefore never be conficled to any other than an experienced and careful man who has been accustomed to gardening: the roots should be dipped in a paste made of marly clay and cowodungr united with

* Arthur Young indeed says, "that he has seen from 40 to 70 acres of cabbages on a farm, every year, for sercral years;" (Survey of Suffolk, 3rd edit. 1). 115.) but such instances are extremely rare.

t Report of Suffolk, 3rd edit, p. 119. Cambridge, p. 119. Essex, vol. i. p. 375.

†. Young's Norfolk, p. 321. 
the drainings from the stable, and the hole in which the plant is to be dibbled should be saturated with water; the earth closely pressed around the plant, and again well wetted. Notwithstanding all this care the plants will droop for a while, and if the weather remain dry, they must be again plentifully watered; or, according as the season may prove more or less kind, many of them will die, and the gaps must be filled up from plants still remaining in the beds. The distance between the plants, singly, is commonly the same as that between the rows,-namely $2 \frac{1}{2}$ to 3 feet; but the latter is certainly the smallest space which should be allowed to the larger species, which will thus bear 4800 plants per acre.

In order that the roots may have as much as possible equal benefit from the air, some persons place the plants in the second and fourth drills, half-way between those which are in the first and third drills; but we doubt whether any real advantage is thereby gained, and it prevents the ground from being wrought by the horse-hoe, both along and across the ridges. This is a material inconvenience, not only in the cleansing of the land, but in the earthing up of the plants, which should be done at least twice with the double mould-board plough :- the first time about three or four weeks after the cabbages are planted, and the second as soon after the first as they appear to require it, which is commonly in about a month. When this mode of planting is adopted, the land should therefore be laid flat, and it may then be worked diagonally, tlus :-

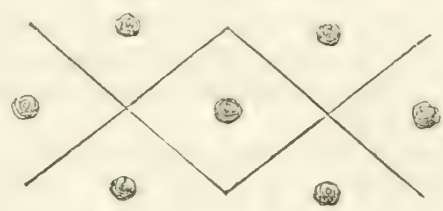

PRODUCE AND VALUE.

It is no uncommon thing to raise single cabbages that weigh $40 \mathrm{llss}$., and if we suppose the roots upon an acre to weigh on an average 20 lbs. cach, and one to be planted on every square yard, the produce would amount to forty-three tons: it is indeed not uncommonly calculated to average thirty; but we believe that few crops, except under peculiarly fivourable circumstances, reach to that extent. They are greatly esteemed by those farmers who have land capable of growing them, as they are not only highly convenient as a substitute for turnips during frostv weather, but also afford an admirable change of food, as they are much relished by cattle, and are found very nutritious when used with the addition of sound hay, either for stall-feeding or for the dairy. Hogs prefer them to turuips; and they are excellent for rearing calves and toothless crones. An acre of good cabbages is thus considered by many men as worth two of turnips, and is very generally thought equal to one and a half*. We do not, however, know of anv accurate comparisons having been made between the two crops; but consilering the superiority of the land usually devoted to the culture of cabbages, and the greater quantity of manure commonly employed, it may be fairly assumed that the presumption is just, so far as regards the difference of nutritive properties between common turnips and cabbages; though in Swedes it can only exist when supported

* See the Reports of Suffolk, 3rd edit. p. 120. Surrey, p. 272. Essex, vol.i. p. 378. Cambridge, p. 109. Dickson's Laucashire, p. 363. Young's Norfolk, p. 321. 
by superiority of weight. Thus, according to Mr. Sinclair's experimentsNutritive matter. Fibre.

7000 grains, or $1 \mathrm{lb}$. of the drumhead cabbage, contains 430

Early York

Purple borecole . . . 448

Turnip-rooted cabbage . . 251

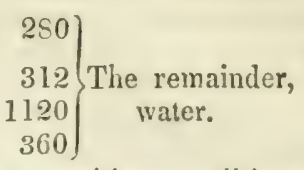

and upon an analysis of the respective averace nutritive qualities of each species of root, cabbages were generally found superior to common turnips in the proportion of $107 \frac{1}{2}$ to 80 , and inferior to Swedes in that of $117 \frac{1}{3}$ to 110 . It is, however, the opinion of an experienced firmer that the culture, considering the greater consumption of manure, and the superior mature of the requisite soil, does not atiord advantages to be compared with the scourge which it occasions to the land*.

With the exception of the turnip-rooted cablage and borecole, which are fed off by sheep, cabbagres are never eaten as they stand on the ground; but are more generally carted off the land, and either given daily fresh in the yards, or spread upon grass-land, and in this way they are in use from the latter end of autumn until the close of the month of March. If, however, they show any disposition to run to seed, they should be drawn, and either laid close tonether on a piece of dry pasture, or stacked in the mamner of Swedish turnips. Borecole is of two kinds, both green and purple, and is commonly known as "Scotch kale." It is capable of enduring the severest frost, and affords the best food after its flavour has been thus mellowed. Sheep and cattle are extremely fond of it, but it requires a good soil, perhaps rather moist, and superior to those on which turnips are generally sownt.

Woburn perennial liale, which has been lately introduced, appears far superior, in point of its amount of produce, to either the green or purple borecole, and requires less manure. It is planted about the begimning of April, from cuttings taken from the stem of the old plants, and cut down to six or seven inches in length. They are then placed in rows, each from twenty inches to two feet apart, three parts of their length being inserted into the ground, and must be watered until they are firmly rooted. The soil should be of an intermediate kind, between wet and dry. It is equal to the best winter greens; affords abundant crops during several years, without the aid of further manure, or any other labour than that of keeping the ground free from weeds, and may therefore be justly considered a valuable acquisition to every farmer who keeps store-stockt.

The turnip-rooted cabbage is distinguished by its irregularly-shaped root and the swelling of the stalk in the upper part, which forms a kind of round fleshy head at the end of the stem, on which the leaves are produced. This root is the part employed for culinary and other edible purposes, and the comparative thimness of the leaves is a constant feature of its character. It is cultivated in the gardens of Germany under the name of kohl Rabi, and has been introduced into England under that denomination $\$$. It indeed much resenbles the Swedish turmip, for which it is frequently used as a sub-

* Brown of Markle, vol. ii. p. 124.

† Dumfries Rep., p. 227.

Trans. of the Hort. Soc, vol. v. art. 40. MIr. Sinclair says that it flourished during cinht years successively at Woburn Abbey, and that the produce on ten square yards was $144 \mathrm{lbs}$.

$\$$ De Canilolle, Mem, on the different species of cabbage. Trans. of the ILort, Soc. vol.v, art. 1 . 
stitute, and is much relished by sleep; but it is hard and lifficult to bo fed off, and not being superior in its nutritive properties, it las therefore fillen much into disuse. It requires to be som at the same early season, bears transplanting, and is never injured by the severity of the weather; retaining its freshness until the latter end of Mlay*. Its root has a hard thick rind, with solid internal substance, and it continues edible after its sced-stalls has attained considerable height. Its root, however, does not rive an equal weight of crop; and, instead of a solid bulb, usually consists of a number of branches having such hold of the earth that they cannot be pulled up by hand: it is therefore found necessary to turn over the drills with a plough and then shake out the rootst.

These different species, as well as rape, appear to be hybrid products of the cabbage and turnip, which both, indeed, belong to the same genusproduced by means of cultivation; and the various kinds which have been disseminated throughout Europe have become so confused in nomenclature, that it has become difficult to state their properties with any great degree of precision, or to draw any certain inferences to guide us in their use.

\section{Chapter XXII.}

\section{ON POTATOES.}

Tue supet potato-which is a native of both the Fast and the Tiest Indies - was very early introducerl into many parts of Europe, and is the species spoken of by all those ancient writers who allude to it muler the name of battatas. It is of a pale brown colour, somewhat in the form of a pear, but larger, and is the kind mentioned by whispeare $t$, in whose time it was commonly sold in our markets, to which it was prohahly brought from Spain, as it obtained the appellation of "Spanish sweet jotato ;" but the root which is the object of our present inquiry was brought either from Chili or Peru, in which countries it is indigenous, or from some part of North America, in the course of the sixteenth century. It is supjosed to have been carried into Ireland by Sir Walter Raleigh, on his return from America, in 1586 ; but the first mention made of it by any Euglish botanist is by Gerarl, who says, in lis Herbal, that some specimens were sent from Virginia, which he planted in his garlen near London in 1597.

Although then considered a great delicacy, and supposed to be possessed of extraordinary powers of invigoration, it yet fell into disrepute, and its progress, even in gardens, was so extremely slow, that a long period elapsed (re it was adopted into our field-culture. Notwithstanding there may be some truth, in a national point of view, in the severe observations of the late Mr. Cobbett upon the pauperising efiect which the ereat extension of its growth and its habitual use has occasioned among the peasantry of Ireland, yet there can be no doubt that, in this country, where it is only used in addition to other food, it comes materially in aill of the substantial

* Suffolk Report, ird edit. p. 101. Cheshire ditto, p. 162.

+ Farmer's Magazine, vol. vi. p. 344 .

+ "Let the skye rain potatoes, and hail kissing-comfits."-Mienry Wires of Windsor, act v. scene 5 .

\$ The roots are described by Heriot, who accompanied Ralcigh, "as round, some as large as walnuts, others much larger: they grow in damp soil, many hianging together: as if fixed on ropes. 'They are good food either boiled or roasted, and the plant is called by the Indians 'openawk." -De Bry's Coll. of Voyages, vol, i. p. 17. This, however, would apply equally to the Jerusalem artichoke. 
comforts of the working classes, and cannot be looked upon in any other light than as a special boon of Providence. Indeed, whether considered as the food of man or beast, it may be regarded in every sense as being, after corn, the most valuable of our vesretable productions; and if to this be added the facility with which it is reared in every part of the kingrdom, it certainly forms a most important branch of our rural industry.

\section{SPECIES AND SOIL.}

The potato plant grows with a large quantity of coarse spongy haulm, which generally rises to the height of about two feet, but in some species is higher, and in others more diminutive, and usually bears white or purplish flowers. The root, or tuber-which is the edible part-grows under ground, of irregular form and size; but the haulm carries a small fruit, called the "apple," about the size and appearance of a green plum, which turns nearly black when ripe, and continins many seeds from which the plant can be propagated. These, if sown, produce generally different kinds of potatoes; and as experiments are daily made for the jurpose of procuring new sorts, their names and descriptions are numberless. The process is, however, very tedious, as well as the quality uncertain, and as crops of approved species can be immediately grown by planting the tubers, that is the plan always adopted by farmers. It has been supposed that every portion of a potato will grow into a plant, but this is a mistake; for only those spots, or "eyes," which appear upon the root will vegretate; and it is, therefure, either planted whole or divided into cuttings. It is to be observed, that wherever the terms "seed," or "sowingr," occur in this account of the culture of the potato, it is meant to designate the sets, or tubers; and not the real seed gathered from the apples.

Those most commonly cultivated as field-crops, are-various kincis of the "kidney potato," which requires a good soil, and will hardly grow in poor land. 'The " Cumberland and the Lancashire white," the "purple-streaked," which are early, grow large, and produce abundantly. The "Scotch dun," the " pink-eyes," the "golden dun," or "coppernose," which, though not very productive, are very early, and highly esteemed for the table. The "Perthshire red," which is an excellent late potato, extensively cultivated in that county, and the only kind shipped from thence for the London market; being a good keeper, fine flavoured, and generally prolific. The "Wellington;" the "cluster;" the "champion;" the "ox-noble ;" and both the white and red "Surinam" produce the largest quantities of nutritive matter for the support of cattle; though the ox-noble is apt to grow hollow, and is very coarse food, upon which the beasts thrive but slowly: there is also the "Yam," which may be grown upon poor soils, but is entirely unfit for human food. The "Munster," the "Connaught cups," the red and white " apple," the red-nosed and the white "kidney," are favourite sorts in Ireland, being very productive, mealy, and pleasant to the palate; ${ }^{*}$ but when intended for the supply of the town-markets, the taste of the consumers must be considered as governing the price, and thereby regulating the profit of their growth. The following are table-varicties of six superior qualities, of the produce of which, upon twenty square yards planted in the second week of Harch, under garden culture, an account has been lately presented to the Highland Societyi: namely-

* A large species, which is described as yellow, mealy, and good, has also been latelyhrought from the Continent, and cultivated in the garden of the Loudon Horticultural Society, under the name of "la divergente."

$t$ Trans. of the Soc. N. S, vol, iv. p. 49. 


\begin{tabular}{|c|c|c|c|c|c|}
\hline \multirow{2}{*}{\multicolumn{2}{|c|}{ North of Ireland pink kidney }} & \multicolumn{3}{|c|}{$\begin{array}{l}\text { Per Drill. } \\
\text { bush. pks. }\end{array}$} & \multirow{2}{*}{$\begin{array}{c}\text { Per Acre. } \\
\text { bush. } \\
363\end{array}$} \\
\hline & & & & 2 & \\
\hline Dryden's white kidney & & . & $: 1$ & 1 & $302 \frac{1}{2}$ \\
\hline American early white & & . & & 0 & $242^{2}$ \\
\hline Ash-leaf round white & & . & & 2 & 363 \\
\hline London particular & & . & & 2 & 363 \\
\hline rince of Wales. & . & . & - 2 & 0 & 484 \\
\hline
\end{tabular}

The qualities are, indeed, so various as almost to defy description. One list, lately published by Mr. Ilowden of Lawhead, Last Lothian, contains an enumeration of experiments upon no less than one hundred and thirty different species, procured from the Continent, as well as from various parts of the Inited Kingdom: some being of early and other's of late growth; white, pale, yellow, or reddish; dense and waxy, or large and mealy; and varying in produce from 77 to 493 bushels per acre, as well as in quantity of starch, or nutritive matter, from 140 z. to 2 lbs. per stone. Forty of the most productive of these varieties were planted in 1834 in separate drills, each 120 yards long and $2 \%$ inches wide: the manure being decomposed farm-yard dung, at the rate of 16 tons per acre; and the land a free soil, about 100 feet above the level of the sea. Each variety occupied one drill, and whole potatoes were planted; the seed was covered by a single mouldboard plough, and the crops were kept clean and earthed up with the hando hoe. The varieties were divided into carly and late species, and the result of the estimated produce per acre of one half of the most productive, which we select, was as follows:-

\section{EARIIY SPECIES,}

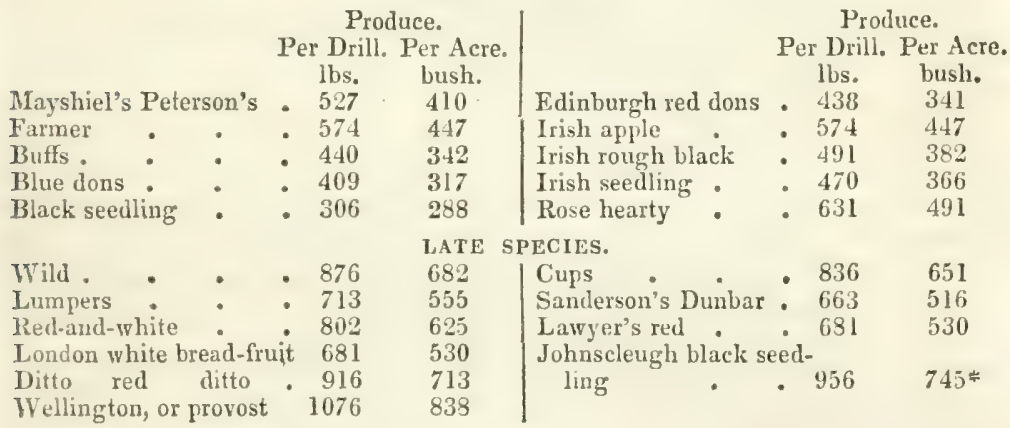

* Trans. of the High. Soc. N. S. No. xxx. p. 85. Added to these and to the various other accounts furnished by persons in this country, we have before us a report published by Professor Candolle, and submitted to the Agricultural Society of Geneva, of comparative experiments made upon 154 difierent vaieties collected from many parts of the Continent and America, as well as from the United Kingdom, many of which are well deserving of attention.

An account has also appeared in a foreign journal, entitled "Le Cultivateur," for January, 1835, of a very extraordinary species which has only latterly appeared in Switzerland, and is thus described by Prince Charles de Rohan, from whom it has been called the "Rohan Potato":-

Three tubers chosen at random weighed each $13 \mathrm{lb} .7 \mathrm{oz} ., 11 \mathrm{lb} .9 \mathrm{oz} .$, and $9 \mathrm{lb} .13 \mathrm{cz}$; and a small tuber, having only four eyes, weighing, when planted, a few grains less than half an ounce, produced $48 \frac{1}{4} \mathrm{lbs}$. It is, however, not the largest tubers which succeed the best as seed. 'The mode of planting is described as by the spade; the earth being dug to the depth of 20 inches, and the distance between the holes four feet, two or three eyes being dibbled into each. The plants should be earthed up frequently, for the stems reach to six or seven feet in height. It is a late species, but very farinaceous and of excellent flavour, and should not be taken up until abont Martinmas, after the stalks have withered. We believe the only nurseryman from whom they can be at present obtained, is M. Jacquemont Bonnefont, at Annoney, in the Ardèche. 
Distinc' sorts are also not unfrequently known to different persons under the same denomination *. Even experienced pracical men grenerally satisfy themselves with ascertaining the properties of the root, without infuiry regarding the haulm, the blossom, the time and depth of planting, the soil in which it has been reared, and other peculiarities which merit accurate discrimination as tending to establish the species best calculated for particular soils, and the neglect of which has, in many instances, been attended with serious loss.

The potato will grow upon aimost any kind of soil, provided it be not too wet and clayey; but light, dry, and friable loams, or sards of tolerable consistence, are the most appropriate. Reclaimed bogs and peat land, when well drained, produce large crops; and some of the finest qualities are srown on alluvial soils, and in the warped land in the neighbourhood of the IIumbert. Grubbed wood-land is also favourable to its growth, and the planting of potatoes will probably be found the most profitable mole of brin ing it into immediate cultivation; indeed, as much as 560 bushels per acre have been thus obtained, but the land was manured with twenty waggon-loads of dung $\uparrow^{\circ}$. A sward, or first ley, is, however, the mo, desirahle; and it is for this purpose the common morle of breaking I1) crass-land in Irelam, where it is frequently let to the peasantry at extrasagant rents, in what are there termed "Con-acres $\$$," and yelds crops of stiperior cyuality. The climate, inceed, is there more genial to the growth of esculents than that of England, and the soil is generally so much richer, that in no other country has the culture of the potato been carried to such perfection.

The produce of four eyes cut from the cluster species, and planted in four different kinds of soil, was-

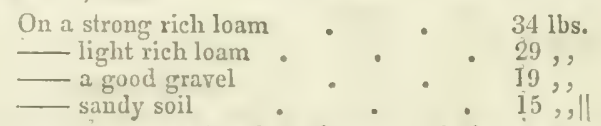

But, although some idea may be this formed of the probable produce, yet no defintive conclusion can be dratwa from this experiment regrardine the crops to be olftaine fivm the land, for other surts might liave been better adipted to the soils: they must be all heavily manured, and good sands are especially favourable to the growth of the larger roots.

Various opinions are cntertained regarding the effects of the crop upon the land, some farmers asserting that it is a sreat impoverisher of the soil, liurtful to the future crops of com, and rumous to grass, and in many leases the cultivation has been restricted. Others, however, contend that it is friendly to com, and not injurions to grans ; observations have also hecn made on the continent tending to prove that it is not exhausting, and others of a mature totally opiosite* . On this, however, we must oliserve,

* See Scot's Farmer, vol. i. p. 329, orig. 4to. Rep. of W. Lothian, p. 26; E. Lothian, p. 81; Perth, p. 40. Dickson's Survo of Lancashire, 1. 371; E. R. of Yorksh. p. 150; N. R. of Yorksh. p. 149 ; Cambridge, p. 134; Holland's Cheshire, pp. 145-148; Curnwrall, p. 76; Burroughs on Green Crops, 2nd edit. p. 45; Drew's Norfulk Husbandry, p. 151; Brown of Mirkle, Treat. on Rur. Aff. vol. ii. p. 82.

† Lincolnshire Rep., 2nd edit. p. 321. It is, however, remarkable that potatoes do not succeed on the "Corkass land," or alluvial soil found in those parts of Ireland bordered by the Shannon, which consists of a very rich blue clay.-Young's Irish Tour, vol. i. p. 324 .

A Annals of Agric., vol.x. p. 324.

\& Young's Irish Tour, vol. i. pp. 176, 284, 289, 297, 305, 339.

i) Rep. of the Board of Agric. p. 11.

G Marshall's Rur. Econ. of Yorkshire, vol, ii. p. 64.

* Pamentier, Traité sur les Pommes de Tere, 11. 164. De Crud, Econ, de I'Agrie., 4to. 1. 285, Von Thä̈r, Agric, Auglais, tom, iii, p. 237. 
that, considering the great quantity of nutritive matter which it extracts from the earth, it cannot-reasoning analogicallv-be viewed in any other light than as a very serious drain upon the vegetative powers of the land; and numberless experiments have shown that, when planted a second time without manure, the crop has been sensibly diminished*. There can, however, be little doubt, that if the produce be consumed by stall-fed cattle, and the dung be laid upon the land, it will then be in a proportionate degree renovated, as well as improved by the friable state in which it will be left by the labour bestowed upon the crop. When this is done, or when extraneous manure can be obtained, it is therefore generally viewed as a fallow crop, in consequence of the attention necessarily paid to its culture, and is not unusually made to precede wheat, which is then sown upon one ploughing. With some persons this, indeed, is a favourite practice; but we believe the cxperience of most farmers proves that the land is never left in so clean a state after potatoes as after turnips $\dagger$.

\section{CULTURE.}

Potatoes are cultivated both by the sprade and, in the common course of farming, by the plough. In the latter method, the land, if tenacious, should be ploughed early in the autumn; though, if light, the operation had better be deferred until after the winter rains, and shumbl be prepared previous to the planting, in all respects in the same manner as if intended to be sown with turnips, 'The sets are laid in rows either by the drill or dibble; but the manure, which is in that case well decomposed, should, in this, consist of long stable dung, laid on at the same time, unless the soil be very light and sandy : but when previously worked into the ground, then spit-dung should be employed. The quantity should also be at least as much; and indeer, in many instances, more is used. It has, however, been remarked that, although a large quantity of dung increases the produce of the crop, yet, when intended for table consumption, it injures the quality. It is thought of considerable utility, when the young plants appear above ground, to plough the earth from the rows, and then spread slacked lime evenly over the whole surface to the amount of about sixty bushels to the acre, then, after a few lays, returning the earth to the roots and moulding them up ${ }_{t}^{+}$: it is, however, impossible to draw accurate conclusions from the effects of various linds of manure when applied to soils of different natures. Many sorts of manure and composts lave been tried and found effective in proportion to their respective powers of aflording untriment; but dung is found superior to every other kind, though of course its powers may be increased by other mixtures $\$$.

The time of planting is usually during the month of April; and, in cold

* Rep. of the Board of Agric. p. 45.

T At Aveley, and o:her paits borlering on the Thames, the wheat-stublies are very commonily sown for rye and spring food, after which the land is ploughed for potatues; which are then said to be highly ameliorating: Essex Rep. vol. i. p. 396; but the situation admits of procuring manure from London by water-carriage. In Micldlesex, they generally precede wheat. Rep. 2d Ed.p. 252; also in Mid-Lothian, p. 59; and Tweeddale, p. 21. In the Isle of Man, in good land, the rotation is, however, potatoes, barley, clover, p. 30 ; and in Lancashire, a plan has been suecessfully followed in the planting potatoes and turnips during a succession of years, $\mathrm{p} .31$.

+ Dickson's Lancash., p. 373.

\$ See vol. i. chaps. x. and xi. p. 267. One of the comparative experiments made by Arthur Young showed the acreable produce of fotatoes was increased in the following proportions:-

By 160 bush. of lime worked into the land, from 400 bush. to 480 bush.

By 480 galls. of urine Added to the dung-heap, 400 , , 520, , Aun, of Agric, vol, ix. p. 692. 
seasons and exposed situations, is sometimes protracted until the beginning of May, in order to avoid the chilling winds and night frosts which check the vegetation of the plants, and may even destroy them. This may, however, be in a great measure guarled against by using a sufficiency of manure as well as by planting at a proper depth; and an incontrovertible argument in favour of early sowing, is the security which it affords of bringing the crop to maturity before the setting in of the wet season and the destructive blasts of the autumnal equinox. which, by occasioning the premature decay of the haulm, prevents the root from coming to perfection, and deprives it of its mealy quality. So much, however, depends upon the temperature of the season, that crops put down in the first week of June sometimes succeed better than those planted before; and it is no uncommon practice anong market-gardeners to take off an early crop of young potatoes, gathered for table consumption, previous to sowing the one intended for winter use*.

It has been recommended upon high authority, as will be seen by the experiments of the Horticultural Society, to plant the sets at the latest ir the early part of March, at the depth of eight inches; thus securing them from the frost, and allowing time enough for the roots to become perfectly ripe. But, although it must be admitted that potatoes thus grown are more farinaceous than those which are planted late, yet it must be remembered that a vast quintity of land, on which potatoes are reared throughout the kingdom, does not allow of that mode of cultivation: the sort of potato, the soil, the manure, and the climate, are therefore points which should be carefully taken into consideration, and may in many cases impede its execution.

The mode of planting practised by a great number of extensive marketgardeners and farmers in the county of Essex, from which the London market is chiefly supplied, is to dibble in the sets; and a plan which is followed by men engaged in such large undertakings is certainly entitled to every degree of attention. They generally mark off the land in drills, at distances of eighteen to thirty inches wide-two feet being, however, the most usual-and lay it either flat or in ridges, according to the nature of the soil. Thus, in Sorkshire, and other parts of the north, the ground is ploughed into one-bout ridges, and the "sets," or cuttings from the potatoes to be planted, are placed in heaps or baskets, and laid by women and children in the furrows; the manure is laid at the same time, and the ridge is covered with earth by the plough dividing it, and making a fresh one over the potatoes. As soon as the plants make their appearance above ground the ridges are harrowed down, and are suffered

* This is frequently done by planting the sets in a dry soil, with a southern exposure, at or before Christmas, and covering them with fern or straw to gruard them from the frost. In planting early potatoes, some people depusit the seed-potatoes in horse-dung, until they sprout to a moderate length, afterwards taking them carefully out, and dibbling them in at narrow distances, with a round stick, either entire or cut into sets, one by one, rubbing off all the shoots but one, and scarcely suffering the top of the young shoot to appear. Others cut the sets, and place them on the floor of a garret or out. house, where a current of air can be occasionally admitted, with sufficient light to assist in strengthening them; taking care not to have more than three layers upon each other, and that the air be only admitted in mild weather: they should also be covered with chaff, which should be carefully removed at intervals to admit the air, the oftener the better when free from frost. Another method is to spread some dry warm turf-mould under them, and cover them with a woollen rug for a few weeks, occasionally removing it. In either case they may be planted out in February or March, to seeure a succession, when the shoots are ahout two inches long, and covered over with a couple of inches of mould, mixed, if possible, with ashes. 
to remain in that state about a week, when the weeds will again begin to appear; the ridges are then earthed up, and in a week or two as much of the earth from the sides of them is ploughed down as can be done without leaving the roots too bare. After this the tops of the ridges are carefully hand-hoed, and the earth which was ploughed from the ridges is again turned to them: if afterwards weeds grow up, they are again hand-hoed, after which the earth is drawn up to the top of the ridges. The plants having by this time got to a considerable size, soon overcome all weeds, and consequently require no further attention till the time of taking up * I $^{*}$ has, however, been justly observed, that " On all very dry sands, and in a dry cimate, the land should be laid quite flat, and the plants should be loved by hand, as the only means of preserving the ground sufficiently moist to promote the growth of the crop; but in every situation where there is no danger of the land being toodry, and on all thin soils, one-bout ridges liave the advantage over every other methodt."

Another mode-which it seems has been borrowed from America-is to plant the sets in banks. "The field is marked out in shallow drills, at about two feet and a half distance, by the double mould-board plough, and is then marked out transversely at the same distance; thus intersecting the surface at right angles, and the dung is laid at the time of planting. The holes for the reception of the sets are formed by a labourer at the intersections marked by the plough, in such a mamner as to leave them flat at the bottom, about ten or twelve inches diameter, and three or four inches deep. This being done, the manure is divided into the holes as evenly as possible, taking care that the particles be well separated by women and chiliren breaking it with their hands. Four cuts are then to be laid in each hole, within four inches of the edge, and about six or seven inclses distance, and covered by levelling the soil into the holes with shovels, which finishes the work until the plants appear above ground; after which only a small quantity of earth is thrown lightly on the banks, just sufficient to refresh the surface, and if the land be foul with weeds, it should be hand-hoed before the banks are dressed. The last earthing should not be performed until the crop is in full blossom, when it must get another light dressing."

Mr. Burroughs, from whom we have extracted the above account, says, that " many who have heard of this culture, but who have not practised it, imagine that its chief object is to afford a great quantity of earth being thrown up to the plants; but so far from this being the scientific principle of the system, that should the sets be deposited too deep, or the banks be landed too high, the crop would prove very unproductive." He adds also, from his own experience, that "the banks being left quite flat at the top when finished, the crop proved much more productive than the adjoining drills sown with an equal quantity of manure, and the potatoes were of better quality+." On which we shall only remark, that it is contrary to the chistomary practice, and opposed by several experiments to which we shall hereafter call attention.

The mode almost universally practised throughout Ireland, in some parts of Lancashire, Cheshire, and the Isle of Mian, as wcll as in many parts of the western Islands of Scotland, is that known as the lazy-bodfashion, which consists in planting the sets in beds, of a few feet in width, covered from trenches formed with the spade. The manure is spread upon the land when ready for planting, and the beds are formed of various widths-

* Middleton's Surv, of Middlesex, 2d edit. p. 251.

+ Surv, of the N. R. of Yorkshire, p. 152; Lancashire, p. 371.

† Burroughs on Green Crops, 2nd edit. 1.51. 
from threc to five and seven feet; the trenches being dung to the width of eighteen inches to three feet, and to the depth of one foot to twenty inches *. according to the nature of the soil. The sets are then placed in drills upon the dung at nine or ten inches apart, and at various distances between the rows, and covered with soil from the trenches. About three weeks afterwards, or as soon as the sprouts begin to appear, the beds are covered equally with two or three inches of earth dug also from the trenches. The operation is not only beneficial to the potato plants by giving an addition of fresh mould, but has also a tendency to check the growth of, and indeed in a great measure to destroy, the couch and other weeds which make their appearance upon the land; and it is repeated, so far as eartling-up the plants, without covering them after they are in bloom.

This plan is disapproved in most parts of England, where it is generally viewed as one proof of the wretched state of farming in Ireland, as it is there carried into exccution wholly with the spade; while in this country, the land is always prepared for planting with the plought. It is not, however, clear that the parties who have adopted it are wrong ; for it may he peculiarly well alapterl to certain soils and situations, and it has been justy observed by an Irish gentleman of great experience in acgricultural allairs, that independently of its being forced upon the small tenantry, whose holdings are not sufticient to afiurd the expense of teans, and who have little other employment than manual labour- "it is, beyond dispute, the best method on all hilly, rough, rocky, mountain, moss, or bor land; and the potato is lrier also when sown in this way, there being in fact a drain on each sile; and this is one reacon why potatoes are generally better and more mealy in Ireland than in England: and it must not be forgotten, that although the potato likes a moist climate, it also likes a dry, unadhesive soil, where it can freely send forth its roots+.",

There can, indeed, be no doubt that the land is better worked up and pulverized by the spade than by the plough; and that the lazy-bed culture is equally as productive as that by the common mole, seems to have been proved by an experiment made by Mr. Drummond, of the botanic garden at Cork, and recorded by the London Horticultural Society $\oint$.

IIe laid out a plut of ground sixty-five fect wide by fifty-six long, and divided it lengthways into three equal parts, which were treated in the following mamer; the cuttings being from the red-apple potato, which is there much esteemed.

1. The sets were planted in the beds of the first plot, about nine inches asunder, but not too deep; the manure was afterwards spread on the surface of the land, and covered with earth dug from the alleys. When the plants made their appenance they were earthed a second time; and, as they arlvanced, a third time, where the depth of the earth below the beds allowed of it: which is all the care requisite until they are fit to be dug up.

* In the comnty of Inildare the beds are seven feet wide, the trenches three feet and a half wide by eighteen to twenty inches deep, in order to bring up some of the limestone gravel; but, generally speaking, the widths throughout Ireland are about five feet by two.--Young's Irish Tour, vol. i. p. 7. At Frodsham, in Cheshire, which is celebrated for the superiority of its potato culture, the beds are three to fuur feet wide, with deep intermediate trenches.-Chesh. Rep. p. 141.

+ Thus in Cornwall the farmers plant seven successive furrows, then miss two; which two furrows are hacked and well broken, and spread over the ridge when the potatoes appear above ground.-Cornish Rep. p. 70.

I ambert on the Rur. Aff: of Ireland, p. 127

$\$$ Trans, of the Soc, vol, iii, art, 24. 
2. The other two parts were planted in drills; one plot having ten single drills two feet asunder; and the other having eight double drills; that is, a drill on each side of a line, so as to have two rows about six inches asunder. The treatment of the two drills was preciscly the same, and the quantity of manure in each of the lhree plots was the same; but the number of sets was in each different. The produce of each was as follows:

\section{No, of Sets.}

Produce.

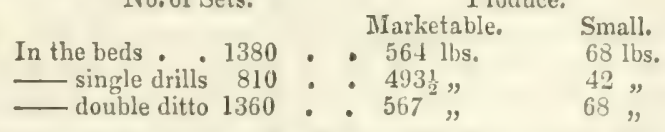

Regarding the mode of spreading the dung-whether above or under the sets-some difference of opinion prevails; for although the latter is the method usually adopted, and it may be rationally supposed that, as the roots shoot rather laterally than upwards, it is from the manure placel underneath that they extract their nutriment, yet it is supposed by many persons that, if the land be light and dry, it answers better to lay the mawure in furrows above the plants; and some farmers seem to think the manner of its application immaterial*. Accordiner to an experiment made with the utmost degree of attention, under the direction of the Board of $A$ griculture, the former, however, appeared to have the advantage of five to four in its favour: the produce of an equal weight of sets, and quantity of manure, being - when laid over the dung, $105 \mathrm{lbs} .4 \mathrm{oz}$.

\section{Under the dung, $84,3,$, ,}

Such are the ordinary modes of culture usually adopted throughout most parts of the Lnited Kingdom; but a novel plan, which deriates in some essential particulars from those generally employed, has lately been brought into notice by Mr. Kinight, the very intelligent President of the II orticultural Society ; and, as the very interesting ascount which has been published by the Society may yet be unknown to many of our readers, we here transcribe it, and beg earnestly to call it to the serious attention of every farmer who is engaged in that branch of husbandry.

"The experiments were malle upon different varieties of potatoes; but as the results were in all cases nearly the same, I think that I shall most readily cause the practice I recommend to be understood, by describing minutely the treatment of a single variety only, which I received from the Horticultural Society, under the name of 'Lankman's Potato:" a tall sort raised some years since in Flanders.

"The soil in which I proposed to plant being very shallow, and lying upon a rock, I collected it with a plough into ligh ridues of fuur feet wide, to give it an artificial depth. A deep furrow was then made along the centre and highest part of each ridge; and in the bottom of this, whole potatues, the lirhtest of which ciid not weirh less than four ounces, were deposited, at only six inches distance from the centre of one to the centre of another. Manure, in the ordinary quantity, was then introduced, and mould was athed, suffcient to cover the potatoes rather more depply than is generally done.

"The stems of potatocs, as of other plants, rise prerpenlicularly under the influence of their unerring guile, gravitation, so long as they continue to be concealed beneath the soil ; but as soon as they rise above it, they are, to a considerable extent, under the control of another agent, lighit.

* Rep. of Cornwall, p. 73 ; Dickson's Lancash. p. 371.

+ Trans. of the Buard, p. 30 . 
Each inclines in whatever direction it receives the greatest quantity of that fluid, and consequently each avoids, and appears to shun, the shade of every contiguous plant. The old tubers being large, and, under the mode of culture recommended, rather deeply buried in the ground, the young plants in the early part of the summer never suffer from want of moisture; and, being abundantly nourished, they soon extend themselves in every direction till they meet those of contiguous rows, which they do not overshadow on account of the width of the intervals.

"The stems being abundantly fed, owing to the size of the old tubers, rise from the ground with great strength and luxuriance, support well sheir foliage, and a larger breadth of this is thus, I think, exposed to the light during the whole season, than under any other mode of culture which $x$ have seen; and, as the plants acquire a very large size early in the summer, the tubers, of even very late varietics, arrive at a state of perfect maturity early in the autumn.

"Having found my crops of potatoes to be in the last thrce years, during which alone I have accurately adopted the mode of culture above described, much greater than they had ever previously been, as well as of excellent quality, I was led to ascertain the amount in weight which an acre of ground, such as I have described-the soil of which was naturally poor and shallow-would produce. A colony of rabbits had, however, in the last year done a good deal of damage, and pheasants had eaten many of the tubers which the rabbits hat exposed to view; but the remaining produce per acre excceded 539 bushels of 82 lbs. each*: 2libs. being allowed in every bushel on account of a very small ciuantity of earth which adhered to them."

"The preceding experiments wcre made with a large and productive variety of potato only; but $I$ am much inclined to think that $I$ have raised, and shall raise in the present year, nearly as large a produce per acre of a very well-known small early variety-the 'ash-leaved lidney potato.' Of this I selected in the present spring the largest tubers which I could cause to be produced in the last year; and I have planted them nearly in contact with each other in the rows, and with intervals, on acconint of the shortness of their stems, of only two feet between the rows. The plants at present display an unusual degree of strength and vigour of growth, arising from the very large size-for that variety-of the planted tuhers; and as large a breadth of foliage is exposed to the light by the small, as could be exposed by a large variety; for I lave always found the amount of the produce, under any given external circumstance, to be regulated by the extent of foliage which was exposed to light ; and I have uniformly found that to obtain crops of potatoes of great weight and excelIence, the period of planting should never be later than the beginning of March.†"

The produce of this small early variety, in fact, very considerably exceeded that of the large one first mentioned-being per acre 665 bushels of 82 lbs. - and both so far exceeded the ordinary crops which had been previously raised, except in very rare cases, that doubts were entertained of the exactness of Mrr. Knight's conclusions, and experiments, it will be seen, were made in the garden of the Society in order to obtain further evidence.

* The London salesmen allow 70lbs, as the weight of a bushel of potatoes; but it varjes, according to the size and quality of dificent sjecies, from 68 libs. to 72 lbs. per bushel.

+ Transactions of the Horticult. Soc, vol. vii. 
SEED.

It has been ascertained by repeated trials that cvery variety of the potato, when propacated during a series either by cuttings from the root or by the whole tubers, is subject to degenerate: in some, the quality remaining good, after the produce in quantity has become defective, whilst with others, it disappear's with the vigour of the plant. In order to obviate this inconvenience, and to preserve those species which are known to be valuable, farmers occasionally raise them from the seed contained in the apples which grow upon the stalk; for which purpose, a few large ripe apples should be chosen from a healthy plant, and be carefully preserved apart, in some dry sand, during the winter.

The seed is then picked out, and usually sown in rich garden ground in the month of April ; but it is more expedient to sow it in a hot-bed early in March, to expose the shoots gradualiy to the open air, and to plant them out in a bed of rich earth in the middle of May. In the month of October, these seedlings will produce tubers, the largest of which are to be gathered, and planted out, in the following spring, at a few inches distance from each other. When they rise about two inches above ground, they should be covered with two or three inches of mould, and managed in the same manner as if grown from the old potato.

When arriving at maturity, they should be continually and carefully examined, to discover those which appear the earliest in coming to perfection; which will be denoted by the decay of the haulm. These should be taken up, and in like mamer those of a later growth; but those which show extraordinary vigour should be selected, and the produce of each sort being again sown in the ensuing year, a correct judgment may be formed of the respective properties of each. The process is thus so slow and troublesome, that it tiklies three years to bring any new variety to maturity, and a fourth before the real properties of the root can be correctly ascertained. The prorluct will also sometimes disappoint the expectations of the grower; for although, generally speaking, the major portion of the crop will be found of the same quality as the original stock, yet it occasionally fails, and new varieties are constantly produced, differing in both colour, flavour, size, and the periods of ripening. Thus, it appears from an experiment lately made in the garien of the London Horticultural Society, upon twenty-five seedling varieties reared by Mr. Knight, that the estimated produce varied from one to upwards of eighteen tons per acre; all of different qualities. The best produced from a single tuber twenty-seven limge and seven small roots, the argregate weight of which upon an acre of ground it was supposed would equal 17 toms $9 \frac{1}{2} \mathrm{cwt}$, and being both large, solit, mealy, white, well-flavourerl, and in substance and appearance much resembling the white yam, has been named by the Socicty, after Mr. Innightis's country-seat, the " Downton yam potato."

\section{SETS AND TUBERS.}

We have already stated that in the common course of ficld-husbandry, potatoes are invariabiy planted for a crop either by depositing the entire root, or cuttings from it, called "sets." No objection appears indeed to have been commonly entertained against the planting of the whole root, or "tuber"-except on the score of cconomy, though probably no other advantage will be thereby gained, for every tuber contains many beads, or "eyes"-from each of which a shoot will spring, and by sowing these 
separately, or in cuttings containing two or more together, a saving is made in the quantity ; but an extraordinary opinion is entertained regard. ing the best method of performing this operation.

Among numberiess experiments which have been made with a view to compare the produce of plantations of different sizes of whole tubers, and sets from different sizes of cuttings, those of entire potatoes reported to the Bath Society were generally stated to be superior. Dr. Anderson found that the crop was in some measure proportionate to the weights of the seis; and that it was more profitable to plant small potatoes than small cuttings. Others, however, found that the difference in acreable produce, between large and small, cut or uncut potatoes for sets, was quite immaterial; but that the saving in the quantity sown, was so much in favour of the cuttings as to require only twenty bushels, while whole potatoes consumed thirty-seven bushels per acre*. I tet, according to a report made to the Dublin Society of Agriculture, it was stated, on comparison with sets cut from reasonably large and small tubers, that the produce in favour of the former was as 84 to $64 \uparrow$; and another well informed gentle. man states, "he has uniformly found in all his experience that large sets of potatoes made a more productive return than small ones. And upon trial, both in garden and field, he has repeatedly found that planting whole putatoes, even though large, very much increases the crop. In this way, however, they reyuire to be planted thinner, as the stems, being stronger and more luxuriant, occupy more space+."

To set this point at rest, five plots of ground of equal size, and as nearly as possible of equal quality, were also lately selected by the London Ilorticultural society for the growth of five different varieties: one half of which being planted with whole tubers, and the other with sets containing but one eye each; and, being placed at equal distances-eighteen inches apart-the result was as follows:-

\begin{tabular}{|c|c|c|c|c|c|c|c|}
\hline \multirow[b]{2}{*}{ Species. } & \multicolumn{7}{|c|}{ Weight when taken up. } \\
\hline & & $\begin{array}{l}\text { Who } \\
\text { tons. }\end{array}$ & $\begin{array}{l}\text { le Tic } \\
\text { civt. }\end{array}$ & $\begin{array}{l}\text { abers. } \\
\text { lb. }\end{array}$ & $\begin{array}{l}\text { Sing } \\
\text { tons. }\end{array}$ & & $\begin{array}{l}\text { yes. } \\
\text { lb. }\end{array}$ \\
\hline nly & & 17 & 10 & 4 & 18 & 19 & 82 \\
\hline Shat & . & - 20 & $1 i$ & 26 & 20 & 0 & 4 \\
\hline ed kidney & . & - 18 & 7 & 71 & 17 & 12 & 49 \\
\hline d Scotch & . & - 22 & 15 & 83 & 20 & 2 & 7 \\
\hline pion. & . & - 23 & 14 & 0 & 24 & 9 & 18 \\
\hline
\end{tabular}

The whole tuhers appeared above ground, in each instance, three or four days earlier than the sets, and the haulm became somewhat longer; but the experiment shows that, although the total amount thus estimated to have been obtained is,

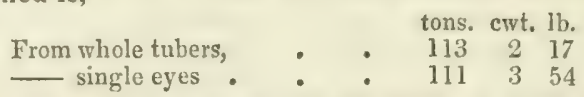

thus giving an apparent difference in five acres of about two tons, yet it was hardly more than the difference between the weight of the tubers and the sets originally planted§.

* Bath Soc. Papers, vol. i. p. 28; vol. iv. pp. 14, 20, 22 ; vol.v. pp. 127, 234 ; rol.vi. p. 207.

t Rep. to the Dublin Soc. p. 109.

† Farmer's Mag, vol, iii. p. 215.

Trans. of the Hort. Soc., $2 d$ Ser. vol. i. art. 55. In some recent experiments reported to the Highland Society, on the planting of cuttings fom large tubers and small potatoes, both cut and uncut, it was found that the produce from the large tubers was in every instance superior. Trans, of the Soc. N. S. vol, iv. p. 306. "The cuts are never made into mure than four fieces for flants, and that through the nose, or must remete end from the parent stem." No, xxix. p. 49. 
These and other trials, indeed, afford presumptive evidence that sets cut from full grown, healthy tubers, are as productive as the whole root; for although it is recorded as the opinion of the President of the Societr, founded upon a great variety of experiments carried on cluring a long series of years, "that the heaviest crops of potatocs, and those most profitable to the grower, will in most soils and spa-ons be obtained from tuhers of considerable weight, and will be found least sulject to decay in wet and cold springs; he, however, thinks it extremely probahle that, when the soil is very dry, so as to preclude all grounds of fear of the cuttings decaying, more regular and better rows of plants might be ohtained from sinele eves placed at short distances, with a moderately large portion of the matter of the tuber, than the whole tubers."

It may also be ohserved that the eres or beads, - which appear like spots upon the skin of the potato-are of different kinds; those at one end being more prolific than the other; yet, when planted in sets, some farmers cut off both encis, only making use of the part in the middle, while others cut it longitudinally-from "nose to tail"-and set both balves indiscriminately; and many scoop out the eres and plant them singly. The stems which spring from that end of the potato into which the filire which connected it with the mother plant, and from which the potato itcelf is grown, germinate but feebly, and do not attain the same size as those which are found upon the other end, which may be seen by looking at potatoes in the spring, when they bergin to bud: those which spring from the top end, having far greater vigour and luxuriance than those which spring from the root end *

It is indeed a point which deserves very serious attention; and, as the other portions of the potato can always be used for other purposes, it should never be neglected. Instead, however, of sowing single eyes, we should rather recommend the use of the entire cluster of buds which will be found on the top-end, or nose, of the tubert.

\section{PLANTING.}

We must further remark, that, whether planterl whole or in sets, the roots should not be taken up, when intended for that purpose, before the haulm is withered; so as to allow the roots to reach a state of perfect maturity. 'This, indeed, merits peculiar attention; for it has been stated in the report of a county celebrated for the growth of potatoes, that they

* The upper vr nose end, although the most watery part of the potato, should be preferred, as the roots produced from it have been found to become sooner ripe, and to be of better quality than those grown from the bottom.-Farm. Mag. vol. xviji. p. 27.

"In every field of potatoes which I have ever seen, where the cuttings for seed were taken from both ends indiscriminately, some of the stems grow with much mure vigour than others; which proceeds, I believe, in nine cases out of ten, from planting weak sets cut from the root end of the potato."-Ayton's Surv. of Ayrsh. n. 280. Mr. Knight says that " the buds which vegetate from the lower sides of the tubers produce feeble stems." - Trans. of the Hort. Soc.

+ Instruments have been made for scooping out the eyes for the purpose of saving a portion of the pulp for ordinary consumption; and three potatoes, weighing $10 \frac{1}{2}$ oz. have weighed $3 \mathrm{oz}$. after twenty scoops have been extracted from the three. A considerable saving is thus made in the quantity to be planted; but, according to an experiment made by the Rev. Charles Findlater, Surveyor of Peebleshire. upon sconpings and cuttings planted in alternate drills, the whole of which came to perfection, the plants from the scoopings were dwarfish, and the prodnce only half the weight of the cuttings.Narm. Magr. vol. iii. p. 103. In other trials, however, little difference has been observed; but in sume they have entirely failed. The latter circumstance may, not improbably, be attributed to the eye cut from the scoop not being enveloped in a sufficiency of pulp to preserve it from decay in certain soils and seasons, ere it has time to vegetate. 
should be gathered before they are quite ripe*. We believe, however, it will be found that they cannot be too ripe; for it cannot but have occurred to the observation of every farmer who has paid attention to the subject, that those stray roots which are unavoidably left in the ground when the crop is cleared, invariably produce the most vigorous shoots in the ensuing year. The reason of which is thus explained by Mr. Andrews, who, in is3!, received a premium from the Royal Dublin Society, for the best essay on the failure of potato crops.

"The potato lying in the ground during the winter becomes perfectly matured, retains its juices, is preserved from fermentation, and germinates at the natural season; and we have found that the plant proceeding from it is luxuriant and healthy. Lnder our treatment the tuber is taken up immature; it has, therefore, a greater tendency to fermentation, from its juices being more crude. It is heaped up in large quantities, in close houses or pits, and these large heaps increase the tendency to fermentation. The time of planting is protracted beyond the natural period of germination, and the tubers become exhausted by germinating in the pits; and when at last committed to the ground, they are frequently planted in mould which has become dried up, and not unfrequently placed in manure which is also dry and withered, from improper separation or neglect; and are sometimes put into the ground so rough and ill prepared, that the air is freely admitted to the seed, to dry up any moisture that may remain. If seed be perfectly sound and uninjured, it may be able to surmount the obstacles which improper managrement at the time of planting opposes to its germination; but when injured and its powers of germination weakened, it may fail to overcome them," which views are strictly in accordance with the observations of the majority of the numerous communications which have been recently made on the subject to the Highland Society, in consequence of a medal offered by them for the best treatise on the subject $†$.

In order also to ascertain the effect of planting the tubers and sets at different distances, a great number of experiments were made by the London Horticultural Society, with several rarieties, upon pieces of ground of various measurement : thus -

1. A plot of ground was divided into squares of four feet, in the centre of each of which was planted a whole tuber, a single eye, a set containing three eyes, and the whole surface of a tuber pared off so as to leave the eyes safe, but to remove the centre-a practice which is not uncommon in Scuiland and Ireland. The result of which proved to be, in thirteen cases out of sixteen, in favour of the single eyes as compared with tubers; in nine cases out of sixteen, in favour of single eyes as compared with sets containing three eyes; and, in ten cases out of sixteen, in favour of single eyes as compared with parings.

2. Eight different whole tubers were planted in a row eight feet long and two feet distance from each other, and the result showed, "that in those varieties which were of very strong growth, producing large plants, the first plan, at wide distances, was the best ; but, when the varieties were weak or of a dwarfish kind, the lesser distances were the most productive. Thus it may be assumed that in every case the difference will be in proportion to the vigour or debility of the variety."

It being, however, thouglit desirable to repeat the comparison of whole

* Dicksou and Stevenson's Rep. of Lancashire, p. 371.

+ See Trans. of the Soc. N. S. Nos. xxix. and xxx. 
tubers and sets, for the purpose of further illustrating the advantages and disadvantages of cluse and distant cropping, by trials with the varieties commonly in cultivation among those who supply the London markets, a quantity of early Champion potatoes was purchased by the Society in the beginning of 1834, and a piece of ground, on which no potatoes had been previously grown, was selected for the purpose. No manure was emploved, nor was the soil by any means in a fertile state for garden ground. 'ithe following accomt of the experiment was drawn up by br. Lindley, who, as one of the secretaries to the institution, attended to its management.

"The ground was divided into four equal parts. In one of these the rows of potatoes were as much as two and a half feet apart; in another, two feet; in a third, one foot and a half; and in the fourth, only six inches. IJ alf of each division was planted with whole tulers, and half with sets cut to a single eye. The whole were committed to the ground on the 27 th of Fehruary; both the tubers and sets being in every case six inches apart in the rows, and nine inches deep."

"On the 24 th of $A$ pril the points of the potatoes had reached the surface of the soil, and the next day about three inches of soil were drawn over them, for the purpose of protecting them from sround frosts, which in low and flat places, like the Society's garlen, are still prevalent at that time of the year. By the 2 nd of May the whole surface of the ground, in the division where the rows were only six inches apart, was a mass of entangled stems. By the 20th of the munth, the stems in the division where the rows were one foot and a half apart had nearly covered the ground; and in a week after, those in the two feet division were in the same state; but the ground was not covered during the whole season, where the rows were two feet and a half apart.

"The shoots. from the whole tubers were, in all cascs, much stronger than those from the single eycs, but they began to be prostrated in the six-inch division, on the 29th of May, and the whole of them, in all the divisions, were in the same state by the 2 \%th of June; while the stems from the single eyes continued erect till they hean to tum yellow and wither, in the end of August. This will probably account for the superiority of sets over whole tubers: could the crop be protected from winds, and the stems of the tubers prevented from breaking, I have no doubt that tubers would yield the largest ciop) but their very vigour makes them brittle, and onee broken, they are no longer able to perform tlieir functions perfectly.

"The greatest length to which the stems attaincd was two feet: the principal part of them attained that length, but many did not exceed one foot and a half; and those in the division where the rows were at that distance were the most uniform in their appearance. The important inferences to be drawn from this were afterwards shown by the result."

"On the 26th of September the whole crop was taken up, freed from mould, and weighed. Where the rows were only six inches apart, a number of new potatoes were partially decayed, and a very litrge proportion was too small to be fit for use. The most uniform size was obtained from the division where the rows were two fect apart. The result of the experiment was as follows:- 


\begin{tabular}{|c|c|c|c|c|c|c|}
\hline $\begin{array}{r}\text { Distan } \\
\text { between }\end{array}$ & & Sets. & & & $\begin{array}{l}\text { Weight of sced } \\
\text { required per acre. }\end{array}$ & $\begin{array}{l}\text { Estimated protuce per } \\
\text { acre, deducting the } \\
\text { weight planted. }\end{array}$ \\
\hline $\begin{array}{l}\text { ft. } \\
2\end{array}$ & \{ & $\begin{array}{l}\text { Whole tubers } \\
\text { Single eyes }\end{array}$ & - & - & $\begin{array}{l}\text { Ilos. } \\
6,497 \\
1,470\end{array}$ & $\begin{array}{ccc}\text { tons. cwts. } & \text { lbs. } \\
18 & 8 & 4 \\
15 & 19 & 82\end{array}$ \\
\hline 2 & \{ & $\begin{array}{l}\text { Whole tubers } \\
\text { Single eyes }\end{array}$ & $\dot{.}$ & • & $\begin{array}{l}7,426 \\
1,794\end{array}$ & $\begin{array}{lll}16 & 8 & 46 \\
24 & 0 & 87\end{array}$ \\
\hline 1 & I & $\begin{array}{l}\text { Whole tuhers } \\
\text { Single eyes }\end{array}$ & : & : & $\begin{array}{r}11,764 \\
2,055\end{array}$ & $\begin{array}{rrr}21 & 4 & 72 \\
22 & 16 & 102\end{array}$ \\
\hline 0 & \{ & $\begin{array}{l}\text { Whole tubers } \\
\text { Single eyes }\end{array}$ & : & : & $\begin{array}{r}32,065 \\
5,008\end{array}$ & $\begin{array}{llc}16 & 77 & 91 \\
16 & 17 & 110 *\end{array}$ \\
\hline
\end{tabular}

"I think this result the most interesting that we have yet olotained, for" it not only reduces to something like a demonstration the superiority of sets over tubers, but it shows that the crop will be greater where the distance between the rows is most in accordance with the average height of the potato stems; and that if we take the minimum height-which in this variety is one foot and a half-although the crop may be the most promising while growing, it will in reality be smaller than when the branches are less dense. Thus, the most uniform crop of stems in this experiment was in the division where the ruws were one foot and a lialf apart; but the crop in that division was less by 1 ton 3 cwt. $97 \mathrm{llos}$, than where the rows were two fect apart- hat is, equal to the average height of the stems.

"For the salie of contrasting; the produce thus obtained with the crop in the fielda of those who cultivate potatoes for the market, I caused the weight in some neighbouriner fields to be ascertained by the same men who weighed the potatoes which were the subject of the foregoing experiment.

"A field of Iorkshire Sliaw potatoes, belonging to Nirs. Nedley of Acton, was found to yield at the rate of 14 tons 1 cwt. 26los. per acre: about 27 bushels of sets per acre being planted; which, allowing $68 \mathrm{lbs}$. as the weight of the bushel, will give a clear return of only 13 tons 4 civt. 94 lbs. per acre net. A crop of long kidney potatoes, in a field of hir. Jessop, a tenant of the Dulie of Devonshire, at Sutton Court, rielded a produce of 12 tons 4 cwt. $8.1 \mathrm{lbs}$, or only 11 tons S cwt. 40 lbs. net.

"The rows were from twenty-two to twenty. four inches apart, and the sets at uncertain distances in the rows, varying from six to nine inches; but, in the last case, they were not planted more than five inclies deep, including the subsergent earthing-up by the plowgh; and in the first, not more than eirht inches. Moreover, Mrs. Medley's crop was not planted till the latter end of May; and Mr. Jessop's some time in the beginning of April.

"It is not difficult to account for the small amount of produce obtained in hoth these cases, as comprared with what was yielded in the Socicty's garden; and they are the more interesting, because, so far as distance between the rows went, that point was attended to. The society's potatoes were planted on the 27 th of February, at the depth of nime inclses, and were subsequently carthed up thrce inches more; so that, on the whole,

* The yuantity of seed, and the estimated produce per acre, were calculated upon the quantities sown and gathered, which are stated in the original table. 
they were buried a foot below the surface of the soil. Nr. Jessop's notatoes were only five inches deep, and were not planted till the beginning of A pril; consequently, he lost seven inches in depth: a most important fact, and about five weeks of the growing season. Mrs. Medley, on the other hand, did not lose more than four inches of soil, her potatoes having been buried eight inches deep; but she lost nearly three months of the growing season. It is, however, worthy of remark, that notwithstanding this great disadrantage, her crop exceeded that of Mr. Jessop liy 1 ton 16 cwt. 54 lis. net; from which it may be cuncluder that the greater depth at which hers were buried, more than compensated for the loss of time in planting**.

" MIr. Knight also planied in his garden some tubers of a varicty of potato of very early habits, but possessing more vigour of growth than is usually seen in such varieties. The soil in which they were planted was in good condition, but not richer than the soils of gardens usually are, and the manure which it received consisted chiefly of decayed oak leaves. The tubers were planted nine inches in the soil, and the monld was afterwards raised three inches higher in ridges, to guard the young plants from frost. The produce was at the rate of 3.1 tons 9 ewt. per acre; and Mr. Knight is of opinion that still larger crops may be obtainedt."

From the foregoing facts it has been assmmed by the Socjety, "that, in oriler to acquire the greatest possible weicrlit of potatees yer acre, it is necessary that large, loavy, sound umbers should be emplored; and that the space allowed for the growth of each plant should be as nearly as possible such as it would naturally occupy if suffered to syeread freely on all soils without interuption; that this sace will vary according to the habits of particular varieties, and can only be letermined by accurate experinents; and that too much, and too little room, are alike injurious to moductiveness. Finally, that it is quite practicable to double the crops that are usually obtained."

In the previous part of these observations we cordially agree, and we think it very probable that crops may be increased by attention to the rules there laid down. Fe however doubt the possibility of their being doubled in the orimary course of culture hy any known improvement in its process; for the farmers in the neichbourhood of large towns, which afford both high prices for the proluce and abunlant supplies of manure, and who are thus not wanting in the incentives to good cultivation, have not yet found means to raise their prorluce to anything like that cxtent. The instances stated would indeed lead to that conclusion: but it must be recollected that they are garden cixperiments; and men who work upon a large scale justly look upon such trials with some degree of scepticism.

\section{MANAGEMINT AND DISEASES OF THE PLANTS.}

It has been asserted, on the authority of Sir Jolm Sinclair, that the plucking of the flowers chsures a grecter weight of crop); and Mr. Inight says, "it has been proved by many other persune, as well as by himself, that if the 1,70 s.soms of a potato piant be picked ofi as soon as they become visible, the guantity of potatoes will be considerably increased +." 'This, lowever, is contrarlicted by the Rev. Mir. Camploll, who carefully deprived alternate rows of sets cut from large roots, planted 12 inches asunder, in drills $2 \frac{1}{2}$ feet wide, of

* Trans. of the Hort. Soc., 2nd Ser. vol, i. art. 64.

$\dagger$ Trans. of the Hort. Suc., 2nd Ser. vol, i. p. 417. 
their blossoms from their first appearance, and found, when the crop was taken ui, not the least difierence in the weight * and lis statement is corrobo. rated by a similar experiment recorded by the Rev. Mir. Findlater, and mads with all the accuracy which might be expected from so able an agriculturist; the result of which was a difference of nearly 38 to 37 in favour of the produce of the untouched rows; or,

$$
\begin{aligned}
& \text { Weight, when plucked } \quad: \quad 2140 \text { stone } \\
& \text { Ditto, unplucked }
\end{aligned} \quad \begin{aligned}
& \text { Dutch weight } \\
& 2200 \text { ditto }
\end{aligned} \text { per Scotch acre } \uparrow \text {. }
$$

In opposition to this, however, is the report of an experiment male by the Honourable James Murray, of Aberdona, to whom the honorary silver medal of the Ilighland Society was last year adjudged as being the best account of effects produced by the plucking of the flowers.

The soil consisted of two acres of a shallow loam, upun a bed of what is generally called " till;" and the variety of the potato sown was one which had been brought some years argo from Ireland, where it is known as the "red potato." The whole field was divided into parts of three drills eaclı; and, having explained what was to be done, to a few children from nine to twelve years of age, previous to the appearance of any blossoms, they never allowed a day to elapse without looking after their charge, and no sooner had a blossom begun to appear upon No. 1, than it was immediately plucked off. The other two drills of each part remained untouclied until the blossoms upon No. 2 seemed to be fully expanded, when they were also plucked off; while No. 3 was allowed to rijen its fruit. The whole crop was carefully gathered in distinct parcels, and when the whole was carried off the following was the result:-

\section{Drills on No. 1 contained 30 bolls 2 bushels.}

$\begin{array}{lllllll}" & 2 & & 27 & & 3 & \end{array}$

Thus affording a superior produce by having the flowers phacked off; aml that according to the period at which it had been donet. The difference, however, probably depends upon the season; for in some years a considerable grain has been thus obtained, while in others it has amounted to a mere trifle $\$$.

Some other trials, however, made by .Ir. Findlater, showed an advantage to arise from the pullimy of the apples-the produce of the drills, from which they had been tatien on the :Sth of August and the 9 th of Septemher, and those which were left untouched, being at the rate of about 51 to 44 in favour of the former, or

Weight, when plucked, 6 tons $7 \frac{1}{4}$ cwt. (English weight and

Do. unplucked, $\left.5,19 \frac{3}{4},\right\}$ acre $\|$.

Culting off the haulm or straw at difierent stages of its growth has been found to occision a deficiency in the crop in exact proportion to the earliness of its cutting. It was also prejudicial to the quality of the root; for the tubers continue to improve till the stems begin to decay, but become stationary the moment they are deprived of it, and consequently do not arrive to perfection in either size or nutritive property. Thus, by 'an experiment tried by Dr. Anderson upon plants from which the haum was cut from the 2nd of August to the 5th of September, the loss in produce

* Farm. Mag. vol. x. p. 480. † Ib. vol. xi. p. 16.

+ Transactions of the Highland Society, N. S., vol, iv. p. 236.

I Ibid. vol. v. p. 87.

if Farmer's Hag., vol. xii, p. 564. 
from the August cutting appeared to be very great, and that from the September cutting was calculated at not less than 93 bushels per acre *.

This is a point which very decidedly calls for attention; for many persons are in the habit of regularly cutting the stems for their cows, which they eat with great avidity, and, like any green food, occasions an increase in the flow of mills: some farmers also turn their sheep in about Michaelmas to feed of the haulm. When deferred to this period, or not until about the time when the stems show symptoms of decay, it probably will do no harm to the roots, and an addition will thus be made to the value of the crop; but if done earlier we apprehend there can be no doubt that it will occasion serious injury + .

The chicf disease to which the jotato is liable is called the curl, which is known from the leaves of the stem lecoming shrivelled, when the roots are found in a state of prartial decay, and finally turn rotten. Much speculation has arisen in the farming world to discover its origin and cure. In a treatise which has obtained the gold medal of the Caledonian Horticultural Society, it is supposed to be occasioned by the use of over-ripe tubers as plants; and it is presumed that the disease would be banished entirely were they constantly taken from districts in elevated situationst. It is also said that potatoes grown on peaty soils are free from curl, and composts with peat have been therefore recommended as manure. By some writers it has been ascribed to the plants of particular species becoming tired of . the soil; by others it lias been supposed that it may be communicated by the sets, in the same manner as unsound sced $\$$; and not a few impute it to a small insect, which adheres to and preys upon the stem, and the attacks of which may, it is thought, be prevented by dressings of quick-lime, soot, and tobacco-water $\|$.

By the Bath Agricultural Society it is referred to the following causes: Frost either before or after the sets are planted; planting sets cut out of large unripe potatoes; planting too near the surface, or on uld worn-out ground;--and the best modes of prevention appear to consist in cutting the sets from mirdle-sized potatoes, that were fully ripe before, and had been kept dry after they were taken out of the ground, and in planting them deep in fresh earth with a mixture of quick-lime का.

The theories which have been thus elicited have, however, in various instances been contradicted by the result of further experiments; and we have yet to learn to what the malady is to be attributed, or how it is to be

* Bath Agr. Soc. Papers, vol. iv. p. 43. Another trial of a similar nature showed also a great deficiency in the crop, which was also ill ripened and of bad quality where the ridgres were cut, but where left untouched were excellent.-Farm. Mag. vol. ii. p. 412. And in a further experiment, reported by Sir A. Grant to the Board of Agriculture, "the cutting of the stems in every instance completely failed." - Ib., vol. iii. p. 145.

+ A French writer of eminence says that the clustered sort of potato may be cut in September without injury, but that any other sort would suffer by it materially.-Parmentier, sur les Pommes de Terre, p. 115.

+ Dickson on the Curl in Potatoes.-Trans, of the Cal. Soc., No. i. p. 58.

5 Thus, in one of the series of experiments made by the Rev. Mr. Camplell, to which wc have already alluded, from eight potatoes, the product of a curled one, three came up curled, and that in a farm where it was, at that time, a matter of difficulty to find a curled plant upon which to make the trial.-Farm. Mag., vol. vii.p. 132.

|| "In proof of its being occasioned by an insect, and conseguently not hereditary in the tuliers, ridgres manured alternately with quick-lime, scot, and dunir, have been found guite free from curl on the two former, but on those with the dung there were some hun. dreds of diseased plants."-Farm. Mag., vol. iii. p. 151; vol. xi. p. 61 .

G) See papers on the suliject contained in a selection from those of the Bath Soc., in 2 vols, vol. ii. II) $402-406$. 
remedied. It probalyly arises more from the temperature of the seasons in diflerent years than from any cause which can be controlled by management; but we conceive that it mat be in some degree guarded against by occasionally changing the species grown unon the soil, and by always paying close attention to the quality of the roots from which the sets are to be cut, and choosing them from those of the most perfect growth. Plants which come "1p curled shonld be treated like weeds, and hoed out, which evinces the propriety of retaining more than one eye in every set, so as to allow of cutting them out without creating blanks.

Independenty, however, of the curl, and of every linown species of disease, a very extraordinary failure has talien place within the last three years in the crops of potatoss, extending in some places over entire districts, and in others partially confined to portions of the same field. Various essays have been recently published with a view to ascertain the canse, all written with intelligence, and some assigning facts and inferences which may perlings at length lead to its discovery, and then, it may be rresumed, to its prevention or remedy. Some of them, indeed, appear to carry such weight of evilence that they might almost be assumed to be conchuse diul they not vary from each other, and did they not all leave in some degree unaccomted for, the singular circumstance of the sime land, tilled and manured in the same mamer, planted with the same root, and som and grathered at the same time, still failing in one part and being productive in another, as well as the injury being only of late occurrence. 'Thus, Mr. Andrews, to whose essay we have already alluded, ascribes it "to our altered system of cultivation, in defering the tine of planting to a later period, and taking up the crop in an immature state."

MIessicurs Macdonald, of Huntly, to whom a premium was awarled by the Uighland Society, attribute it "to the healing of the secd; and to that cause alone:" for, "in April and Nay of the years 1932 and 1933 , in which nearly half of their crops failed, the heat of the sum was great, accompanied by frequent shuwers; which, combined with the common plan of cutting seed-potatues two or three wecks before planting, and the exudation of the natural sap from the cuts, occnsioned the heaps to be lieated to such an extent as for the most part to destroy the vegretative principle." This, they say, was in some measure proved by the circumstance that "the cuttings taken from the outside of the heaps, having undergone little or no heating, froduced healthicr plants than those taken from the centre*:" and also "that in 1.1 acres planted in 1834 , and put into the ground the same day on which the sets were cut, there was no deficiency whatever."

But although this may be one cause, it has yei been properly ubserved by Mr. 'Towers, "that the potato is extrenely susceptible of injury from two very ojposite agents-heat and cold $t^{\prime}$ ' Thus, on collecting the remains of a fine crop into separate heaps to dry and harden, the thermometer fell during the evening to two or three degrees below the freezing point, and alihough the frost only lasted two or three hours, yet those pareels which had been exposed to it were foum in the following March to have the slin soft, the nuass pulpy and loose, and resembling the same injury as if it had been scalded.

Miessicurs Walker and Himler, of Liverpoul, who obtained the silver

* Potatoes, the tubers of 'which were planted whole, were however found to escane better tham cattings : probably, from their being better defended by their slind frum the danger of being rotted by the effects of heating.

† Quart, Journ. of Agric, N. S., No, xsx, p. 233. 
medal of the Society, accord with many other of the writers who have stated their views, in the opinion that the failures have been chicfly owing to "the mildness of the winters, in conjunction with improper management." For, say they,- "Although farmers are generally unwilling to admit that their mode of manarement has at all contributed to produce the injury, yet they cannot avoid acknowlelging that a few years acro their potato-crops were much less extensive than now,-that they then planted earlier, - that they deferred digging them until the tops were entirely dead and broken down, - that they allowed them to remain in the frelds awhile, in small covered heaps to dry and season before they stured them,-and that, in springs, they deposited them in moderate quantities, in dry and airy buildiners, or in narrow pits, covering them well, when in pits, with straw, dried fern, or dead tops, as well as with soil, and alluwing sulficient ventilation." While of late, the planting they state to be performed at a later, and the diggring up at an earlier period; while the storing is generally cffected in a liurry, in large roomy pits containing upwards of two tons in the length of a yard, covered with a heavy cuating of earth, with little or no straw, and without any means of ventilation.

In corroboration of which he says, "that some of the purple sets which were planted on a piece of ground without any dung, all sprung up very well; yet where sround, which had been dunged, was planted with potatoes of both the purple and a white lind, without recrard to whether the skimny or the cut side was placed next the dung-the white sprung well, while the purple proved defective *."

A Correspondent in the (Quarterly Journal of Acriculture, however, conceives it to have arisen from "the cut sets having been planted with their surface placed upon the duny, which had become, as it were, glued to it." For, on digging up some ridges planted in trenclies, at Lisburn, in Ireland, with a purple kind of potato, in order to ascertain the cause of large blanks which appeared, - it was found that all the sets which had been laid with their skinny sides upon the dung were sound, and putting forth vigorous shoots; while those of which the cut surfaces were laid in contact with the dung were uniformly rotten. And on mentioning the fact to a friend, who hud three fields of potatoes, one half of which had failed, the injury was discovered, on examination, to have arisen in the same manner.

These conflicting testimonies would tend to show that it was rot the action of the dum by which the potatoes were affected, and would lead us to imagine that the decay of the purple sets was rather to be attributed to some constitutional decay, were it not evilent that they were uninjured on that side which was protected by the skin. Tie are, therefore, still in the dark regarding the origin of the failures which have occurred, aml it is thus jussible that none of the theories which we have stated may touch the real cause; for it is obvious, that under the same circumstances it hats appeared at one time to be in tlie seed, at another in the soil, then in the manure, or in the weather; and it is also evident that among the numerous varieties of potatoes which are cultivated throughout the Cnited Lingdom, without regard to their peculiar moperties, some must be more concenial than others to particular soils and situations, and therefore more or less exposed to injury, without our being able to discover in what the difference consists. Iet curlainly no harm can arise from paying strict attention to all that we have already stated,-to the choice of mature and 
well-grown tubers, - to early and deep planting,-late gathering-, and attentive storing.

We should have here done with the subject, were it not of such importance that every fact which bears in the remotest degree upon it should be stated; and the following instance, as related in the Irish Farmer's Magazine for January, 1835, shows one among the many ways in which the root may be affected:-

"A gentleman who holds a farm of 150 acres, planted in the usual manner 34 acres with potatoes in the year 1832. The result was, a complete failure and total loss of the crop. This induced him to try several experiments upon the root, all of which failed, except the following:- $-\mathrm{He}$ took six potatoes, and divided them into 20 cuts; he then got a large basin of water, into which he put a cupful of salt and a piece of blue-stone about the size of a wahnut. Into this basin he put 10 of the cuts, and let them remain there one night, leaving the others untouched. On the 10 cuts, which were not immersed in the basin, he could distinctly perceive, by a a very strong microscope, many small white particles like eggs; but those cuts which were immersed presented no such appearance. This discovery urged him to follow up the examination attentively, and every day, for a short period, he continued to watch the appearance of the aforesaid matter. The result was, that those white globular particles were animalcula; for, in a few days, they became quite visible to the naked eve in the form of little worms, or maggots. The cuts that had been stceped never showed the least appearance of any such thiner, and they retained their solidity and firmmess when the other ten were completely decayed and rotten. Still, unwilling to believe without further proof, he tried the experiment five or six times, and planted them, distinctly marking a division between those cuts which were steeped and those which were not; the consequence of which was, the almost total failure of the one kind and the complete success of the other."

This, indeed, accords with other instances, which go far to prove that the failure of crops has been occasioned by the use of cuts from tubers which have generated maggots and have turned rotten, thus manifesting some constitutional malady; and quicklime powdered over the cuts has been recommended as a preventive. This, however, has been thought by some as a dangerous experiment, which, if not cautiously used, might destroy the vegetative principle; but this, we conceive, might be avoiderl, and the object equally well attained by dipping the sets into a paste made of gypsum and water, which, though perfectly immocent, will form a conting impenetrable to the external air, and therefore preventive of the generation of insects. From whatever cause the failure of sets may arise, it is, however, evident that whole tubers, if in a sound state, or if left in the ground during the winter, never fail to spring; which proves that the skin can resist injuries which are fatal to the uncovered pulp; and that "wherever maggots have been found, they have been a consequence and not a cause of the partial or entire decay of the sets."

\section{STORING.}

The cheapest mode of taking up potatoes is by means of the double mould-board plongh; but the single plough is infinitely preferable, for, if fut in at the full depth, it turns the potatoes fairly over without cutting them, while the double plough, even in the hands of the best workman, bruises them considerably. Siany persons, however, prefer having them dug up with a pronged furk, or "grapes," both as more completely clearing 
the land and giving it a more effectual dressing. The expense of digging varies in different counties; being in some places only from $1 \mathrm{~d}$. to ? $\mathrm{d}$., and in the neighbourhood of London from $3 d$. to $4 d$. per bushel; but in whatever way the operation may be performed, the crop is gathered in baskets by women and grown children, who usually place it in sacks, each containing three bushels, and eight of them will commonly pick up an acre in the course of a day. The cost is thus readily ascertained; and a grood male labourer is generally able to get up and fill, without the assistance of a partner, about 12 to 15 bushels in a day.

When taken up by the plough, the double mould-board is used with a spread to the extent of the drills, and is run through the centre of the space between them; then returning through the next row but one, so as to allow time to the pickers, and not to throw loose earth on the unpicked drills, and coming back in the row between the two.

When dug up, the fork should have four prongs, each about an inch broad by fifteen inches long, with an inch between each prong, to allow of the mould being separated from the roots; and the ends of the prongs should be rounded, so as to prevent them from cutting the potatoes.

When grown in small quantities, and used for family consumption, they are commonly placed in moderate heaps under cover of some outhouse, where they may be preserved from frost: to secure which, they should be placed upon a bedding of straw, with which they should also be well cuvered. Upon large farms, however, that plan is found inconvenient, and the mode usually adopted is to store them in narrow pits of an indefinite length and not more than two feet deep; which should also be bedeled with straw or dry ferm, if the earth be damp; though we have leard it objected, that if any thing be laid below the potatoes, it subjects them to decay, and injures the flavour of the roots. They are then filled uj) into ridges and thatched in the same manner as tumips; after which they are covered with a thick coating of clay, closely compacted with the spade; on which, if some ashes be sifted, the mixture, or if the covering be coated with turf, it will effectually resist the frost; but, if frost-bitten, they should not be thrown away, as they may then be converted into flour, as well as if they were sound*.

In addition to this it has been justly remarked, " that not only should the crops be thoroughly ripe before they are dug, but that, after having been allowed to dry and season on the surface of the land, they should be so stored as to be liejt dry, cool, and free from untimely vegretation, whether lept in cellars, potato-houses, or pits. Expedients for effecting this will readily suggest themselves; but in the case of pits, it is recommended, when puttiug in the potatoes, to stick up narrow wicker-work funnels, at regular distances along the centre, thus leaving cavities or chimneys, from the bottom of the heaps, for the escape of steam. When lieaped up, the potatoes should remain a while uncovered, except with dead tops, dried fern, or straw, with the addition of tarpatilin covers all night and when raining, until sufficiently seasoned, when they may be finally covered with earth; sods being laid over the chimneys at night and during rain or frost, to prevent injury $\dagger^{\prime \prime}$

* In the severe winters peculiar to the climate of Russia, the potato is preserved from frost in the following manner: A building is erected over a pit sunk about two feet in the grovnd, the walls and roof of which are formed of turf, and the whole inside is lined with planks of wood. The door, which is very small, and placed on a sheltered side, is covered with bundles of straw, to prevent the ingress of air, and the interior is divided into square divisions reaching along the sides, into which the potatoes are piled, without any straw, in heaps reaching nearly to the roof.-Farm. Mar. vol, xx, 1. 4j3.

+ Transactions of the Ifighland Suciety, N. S., No. xxix. p. 37. 


\section{pRodUCE VALUE, AND APPLICATION.}

The potato growers about London usually reckon upon crops of eight to ten tous per acre; and the ox-noble and other large sorts will occasionally yield as much as twelve tons. 'This, it must however be observed, can only be calculated upon in situations which command an unlimited supply of dung, and on soils which have been ameliorated by a long course of careful cultivation. The accounts given of produce are indeed so various, that no specific estimate can be formed without classing the difierent varietics of species, soil, and management; but, gencrally speaking, we should be rather inclined to assume the average of crops on land of fair ordinary quality, and under the common system of farming, as not anounting to more than from six to eight tons, or about 200 to 250 bushels. The ton is usually calculated at 32 bushels of $70 \mathrm{lbs}$. each, which is the exact weight; but heaped measure will sometimes yield more; and in some places $\mathbf{3} 6$ bushels are reckoned to the ton.

It has been found by chemists, that in every 100 parts of the potato there are about 70 parts of water and 30 of potato-meal, which is a powder of a rather prevish colour, retaining slightly the taste of the raw tuber. This shows that the quantity of water contained in the roots when raw is much greater thim usually supposed, and proves the propriety of steaming them for the use of cattle. Aecorling to an analysis made by Sir IImphry Davy*, the avoirdupois pound of ro00 grains of the black kidmey potato contained-

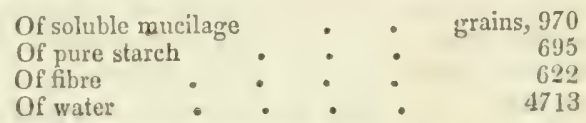

and the quantity of mutritive matter contained in 64 drachms of the following species, was -

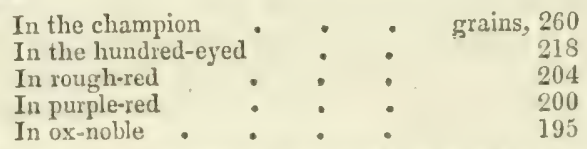

Although failures have recently occurred to a wide extent in the crops of potatues, yet there is perhaps no vegetable of which the produce has been usually considered so certain; and it has the advantage of being most abundant in those wet seasons which render corn crops so defective. It usually, indeed, bears a certain ratio to the price of wheat, as 4 lbs. of a good quality contain as much nutritive matier as $1 \mathrm{lb}$. of wheat, and the demand is such, as always to leave a fair profit to the grower in the vicinity of large towns; but the cost of manure and the expenses of culture are so high, that unless in such situations, or in those which possess advantage of water-carriage to a market, and which therefore do not confine its consumption to the tenants of the homestead, it can seldom repay its cultivation on a large scale. In most cases, however, it will be fumd advantageous to grow a quantity proportionate to the number of bullocks or other lirge stock maintaincel upon the farm; for it frequently hajpens that, when liept on turnijs, the crojs fail through circumstances which camot be foreseen, and considerable expense is then incurred, by the necessity of laving recourse to the hay-stacli, or to oil-cake, to licep the cattle in condition. The loss thus occasioned may be saved by the growth of potatoes; and, even if

* See Sinclair's Hort, Gram, Woburn. j. 409. 
not wanted in the straw-yarl, a certain quanlity will always be found useful when steamed, as affording a most hearty foul to pigs, worling horses, and stall-fed cattle*, on which some further remarlis will occur under the lead of "Soiling:"

It is perhaps not generally known, that the meal mude from potatoes, if mixed with that of wheat, renders bread more light, palatible and digestible, than when manufactured from wheaten flour alone. Most bakers, there. fore, use a small portion of it, with a view to improve the quality; and it is for that purpose largely manufactured in the neighbourhood of Paris, where the excellence of the bread is very remarliable. 'To obtain the powder, the potatoes should be washed clean, accurately peeled, grated with a coarse grater, and the pulp washed repeatedly through a hiar sieve, after being each time allowed to settle; after which, when the water is found to pass quite pure, and without sediment, the pulp should be spread upon a cloth to dry very gradually, and then pounded or ground down into flour. When manufactured upon a large scale, the trouble of peeling may be avoided. The dried pulp may be ground and boulted in a common corm. mill.

It is thus obtained in different proportions, according to the goodness of the potato, from one-fourth to one-fifth of the weight of the rout $t$, and in nowise difierent from the starch made from grain. It indech answers many domestic purposes, for it makes all sorts of pastry of a superior quality than when formed from wheat alone. It also possesses the advantage of retaining its qualities for a great number of years, without the least deterioration; and if the flour be not used, breal can be made with a mixture of potatoes, by choosing the most mealy, which, when boiled and peeled, are beaten and rolled smooth on a table with a rolling-pin, then lineaded with one-fourth, or one-third, of wheaten flour.

\section{Chapter XXiti.}

\section{ON CARROTS AND PARSNIPS.}

Tine cultivation of these roots-which are both tap-rooted plints of about the same size, and almost similar properties-is conducted in nearly the same manner, but they require a very different soil.

THE CARROT

appears to have been brought into this island before tumips; and thourh dhought to be indigenous to our soil, there is yet reason to sumpose that we are indebted for its introduction as a garlen-plant to some liemings who settled about Sandwich, in Kent, in the reign of (jucen Lizibeth; though, so little was its culture attended to, that we find it imported fiom Holland in the latter end of the sixteenth century ${ }_{+}$. The original object was the supply of the London market; but the husbandry having spread into other districts, a greater quantity was raised than could be used for family consmution, and trials having been made mon it as food for cattle, the result proved its utility to be very aprarent when given to

* See the Article on Steam, vol i. ch. vii. p. 129.

$\dagger$ It was found; by experiments made for the Society of Arts at Geneva, that $1000 \mathrm{lbs}$. of ore quality yielded $214 \mathrm{lbs}$. of flour, while the same quantity of another species, better chosen, produced 270 lus.-Rapports de Prof. de Candolle, p. 12 and 13, and de MI. André de Beaumont, p. 10.

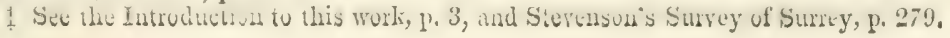


all kinds of cattle, but more particularly to horses, whose coats it strikingly improves within a very short time *

The soil which is best adapted to carrots is a loose, sandy, friable loam, of considerable depth, and they are accordingly verv extensively grown in the sandlings of Suffolk and Surrey; but, althongh so valuable for the nourishment of stock, their culture on this kind of land, of which there are extensive tracts throughout the kingdom, is by no means so general as might be supposed. It is indeed thought that they will thrive equally well upon a poor soft sand as upon one of a superior quality, and, from experiments made in Scotland, it is also thought that they will flourish upon peaty soilst, the richness of the ground not being considered so material as its depth and freedom from stones; and farmers are almost so universally of that opinion, that the crop is very commonly produced without manure; it is, indeed, viewed as so little exhausting to the soil, that succeeding crops of barley have, in no single instance, been found in any way deficient when they have been raised after turnips, one-third of which have been fed off upon the land + .

The objection to the use of manure also exists in its being found, when coming into contact with the roots, to cause them to divide, thus forming two prongs, and becoming so deformed and ill-flavoured, as to render them unsaleable for table use. The richness of the ground occasioned by the manure also has a tendency to make them run to seed, or at least frequently causes them to become hard and sticky, and of course unfit for the market, and its application has therefore been strongly condemned by many eminent writers $\$$. This, indeed, may be founded on fact with regard to long dung; but with respect to other surts, their opinion should be received with caution; for the market-gardeners, though generally preferring to sow carrots without dumg after a crop which has been previously manured, yet sometimes use decomposed manure; and we find Arthur Foung recommending it in such strong terms that he sars, "If you would command your crops of this root, you should manure the land with 25 or 30 loads of dung per acre, pretty rotten, ploughed in, and then cover the seed hy harrowing. The dung neither injures the taste of the carrot, makes them grow deformed, nor causes the canker. A farmer's object is to produce as great a quantity as possible from every acre, which must undoubtedly be accomplished by manure \|." Although we think he goes umnecessarily far in the amount of the manure, we are yet persuaded that lie is, to a certain extent, right, provided it be perfectly decomposed spitdinig, and previously well incorporated with the gromend, and that the object be rather the production of crops for the use of the homestead than of the table; in confirmation of which we have seen a statement of the cultivation of carrots upon soil of precisely the same nature, one-half sown unmanured, and the other manured, both after potatoes, the result of which wasUnmanured.

Manured.

Roots, 9 tons per acre.

Tops 4 do.

Roots, 12 tons per acre.

Tops, 5 do.

the quality of both being, in both cases, precisely similar **.

* See vol. i. chap. vii. pp. 125 and 128.

+ Sinclair, on Scottish Husbandry, vol. i. p. 303.

I Farm. Mag., vol. xv. p. 483.

ii Farmer's Calendar, p. 71 .

6. Limir clung lias been also nsed with grood effect, when laid upon the land after the sect has been sown, and then raked off when the ground requires hoein - - Von Thaër, Princ, Rais, d'Agric., 2nd edit. tom. iv. p. 386.

** Farm, Mag, vol, xv, 1. 7 . 
The Seen of the carrot is extremely light, and clings so close together with forked hairs which connect it, that it cannot be separated without great dificulty, for which reason it is generally mixed with sand previous to its being sown; it is indeed so extremely difficult to separate them, as to form a great objection to their being sown in drills; for the plants come up in tufts with blank spaces, and the seed is therefore generally sown broad-cast, and afterwards hoed out. They are also so very slow in spronting, that the weeds usually make their appearance first, and their foliage is so slender that it is difficult to hoe out the weeds without injuring them. It is therefore a good plan to have the seed and sand well mixed together, and laid in heaps in a warm place-though screened from the rays of the sum,-which should be wetted with drainings from the stable, and turned over daily. 'This, if rone ten days or a furtnight before the sowing, will occasion the seed to germinate, and the plants will be thus so forward as to be easily distinguished when the land requires hoeing. It is also a good precaution to cover these heaps with a little cinders in order to prevent the upper part from becoming dry.

'The quantily of' seed depends in a great measure upon its goolness, for there is no complaint more general among farmers than that of its quality; we shall therefore state, at the end of this chapter, the manner in which that and parsnip seed is raised. If it can be depended upon, four lbs. per acre are thought to be sufficient; but if there is any reason to doubt its being perfectly sound, one-half more, or at least five libs. per acre are sown. Some persons, indeed, use double that quantity; and if the land be very dry, rather more seed is necessary than when moist: it should not be sown deep, nor should it be more than very lightly harrowed.

The time of sowing is determined partly by the weather, and partly by the desire to have the crops coming forwarl at difierent seasons. If they can be got in well, the sonner they are sown the more productive will they be, and the better the quality of the root. From the middle of February to the end of March is considerel the best season in our southern counties, though in the morth it is not unfrequently deferred until the middle or even the latter end of April *.

The culture, when following corn, is generally by three ploughings: the first in September or October-at which time, if manure be applied, it should be laid upon the land-and the second in November; or the second in the lechming of February, and the third at seed-time. It is, however, by no motus unusual, on lands of this light nature, to sow them after turnips; and a sucession of turnips, potatoes, and carrots has been found very beneficial to the farmer's interest, as well as to the ground, when the crops have been either entirely eaten offं, or replaced by a proportionate quantity of manure $\uparrow$. When succeeding turnips the ground is usually once trench-ploughed as soon as the land is clear, then harrowed fine, and the seed immediately sown; but if after potatoes, the land is then laid up in the same manner as after corn, and the crop being a robbing one, the ground should rcceive a moderate portion of spit-dung or compost; a dressing of ashes might perhaps, however, be substituted with good effect, if the land be not already too light, though market-gardeners say it injures the quality of the potato. In whatever manner the tillage may be varied, either to suit the previous crop, the nature of the soil, or the particular views of the farmer, the great ohject should never be disrecgarded-of having the soil completely pulverized, and ploughed to the depth of twenty inches or two feet. edit.

* Stevenson's Rep. of Surrey, 1. 283; Essex Survey, vol. ii. p. 2; Suffolk ditto, 3rd + Bath Papers, vol, i. p. 233. 
Alout a fortight after the first appearance of the carrots-which will not be in less than six or seven weeks, and even longer if the weather should prove dry-they should be thinned ont to the distance of about six inches, ind the limd should be well hoed, to clear it of weeds. In about a fortnight afterwards, when the roots have acquired some strength, a very ligrht harrowing is generally given; and within another fortnight a second thimning-out takes place, by which the plants are set at nine to twelve inclies. Sometimes, indeed, another harrowing is given a few days after the second hoeing; and this, if it be done with care, and particularly if the surface of the land has been caked by rain, is of essential service. Some persons, however, do not use the harrows, conceiving that they must do harm; while those, on the contrary, who do use them say-and they speak from evidence of the fact-that the drawing of the soil to the plants is rery advantageous. A four or five-inch loe is used for the first and second hoeing; and at a later period the weeds should be still further checked by the operation of a nine-inch hoe, which sets the plants out to the distance of twelve to sistecn inches; but some persons use hoes of seven inches, which set out the plants from nine to twelve. Careful farmers, indeed, frequently also hand-weed their crops; and all these operations, recuiring great nicety and attention, should never be confided to any other than very experienced labourers, for the haulm of the corrot is so very trifling, and lics so very close to the ground, that it has very little chance anonge weeds, which, if suffered to grow up, will soon clolse its regetation, even if it had the start of them.

The species are all of the same original nature, differing only in colour and flavour as the sorts hase been rendered by cultivation more appropriate to the field or garden, and are usuaily clistinguished as the "early lom" and the "long oranere." The first, which is much grown in the neighbourhood of great towns for the use of the table, is of a bright red, the root short and rather thick, terminating in a fine thread. Its growth is rapil, and its top small, con-equently it may stand closer upon the ground than any of the other varieties; and when sown, it ought to be at the end of February or the begiming of March, and is ustally in a fit state to be taken up about the close of July. The long orance, which is chiefly cultivated in the sandlings of Suffolk, at Sandwich in Kent, and near Bigroleswarle in Bedfordshire, from which places it is also sometimes named is of a pale rellow, bearing roots of a lirger size, though coarser, and of inferior fiavour, but extensively grown by farmers, on account of its great produce, for the use of cattle.

In addition to these, there is also the "Altrincham carrot," so called from a place in Cheshire, where it was originally produced, in the neighbourhood of which it is rery highly estecmed, and is now cultivated upon the greatest scale as being the most jroductive, both in root and haulm, of any species known. It grows to the cxtent of about two inches above the ground, of a green colour; the root attains a very great size, and is of a briglit orange, with a small dark coloured rind *.

'The haulm of the carrot is so delicate and slender, that it is by no means unfrequently mown for the use of cows, who are said to relish it extremcly. We have even heard of its having been made into hay, of which so much as three or four tons per acre has been produced. Thie effects on the crop, like the efiects of mowing the haulm of potatoes, are so differently and oppositely stated, that it is hazardous to draw any general conclusion either for or against the practice. It has, however, been thought probable,

*Trans, of the Hort, Soc, vol, iv, Art. 55. 
"that the mowing of the top of carrots will not injure the roots much, as it evidently depends for its sustenance upon the soil, and little, if at all, upon the atmosphere ;" the chiferent eflects attributed to the practice may therefore result from the different states of the crop and of the weather*

The storage does not often occasion much inconvenience, for the carrots bear the cold so well, that farmers sometimes keep them in the ground during a great part of the winter, taking them up oniy as they are wanted; but it is a slovenly practice, and they should be taken up whenever they are evidently done growing, or at the latest, some time in November.

In the neighbourhood of towns, wher: large quantities of roots are constmmed, they are talien Ly, bunchell, and washed for the market in the following manner: Two men, a woman, and a boy, are employed. One man takes up the roots with a pronged fork, the other bunches them; and the boy carries them to the woman who washes them, and from the buncher to the cart. In order that the bincher and the washer may perform their several tasks with convenience, two holes are dug in the field, sufficiently deep and wide to permit the persons in them to move round, and to stand nearly breast high, and so near, that the person in the one can reach to the other. The buncher lias a board placed before him, un the surface of the fiehl; and the washer a large tub before her, in which she washes them carefuly, and then hands them over to the man, who, after cutting of the haulm and the ends of the roots, ties them up in bunches on her board. The charge near London is paid by the load of 40 bushels, at rates varying from ten to twelve shillings to the labourers; the man who digs them getting four to five slitlings for himself and hoy, and the buncher from six to seven shillings for himself and partner, which, added to the cost of carriage, renders the expense very consicuable; but when only intended for the use of cattle, the cost of washing and bunching is of course not incurred.

Then stored for preservaion during the rinter, difierent modes are practised, according to the conveniences possessed by the farmer. Whose who have a suficicut range of outhuildings, lay clean dry straw on the floor, on which they pack the carrots, the hamim being first cut off, to the height of five or six feet, and straw is again laid close upon the top of the heap. Others, who have not that room, make jits with a trench round them, in some iry sandy spot, at the bottom of which they place a considerable quantity of pea or wheat straw, and then alternate lavers of carrots fliced cluse to each other in an upright position, with a layer upon them of sand and straw, carried up in the form of a ridge, and upon the top of the last layer of straw, a layer of earth well pressed down with the spadet. A better mode is, however, instead of pits, to pile the roots upon an earthen bank, in short spaces, raised a little above the level of the soil ; upon this to spread a layer of straw, in which they are to be placed two or three deep, with successive intervening strata of straw; the tops being turned "ipwaints, and their ends folding over the centre, while the smaller roots are topperl and thrown in between. The stacking may be continued in the ridge-form to the heiglit of about four feet, which should then be well thatched. Another line should then be commenced, leaving room for one to pasis hetween; and the intervals are filled up with lerm, or any dry stuff, stacked down, or fastened with hurdles $\begin{aligned} & \dagger \\ & \text {. }\end{aligned}$

* Stevenson's Rep. of Surrey, p. 284.

† Stevenson's Surrey, p. 287. Malcolm's ditto, vol, ii. p. 481.

$\$$ Complete Grazier, 6ih edit. p. 526. 
THE PARSNIP

closely resem?les the appearance of the carrot in all but its colour, which is of a pale yellow, and there is but one distinctive species, with very few varieties. The common root grows to the length of perhaps 30 inclies, and from three to fuur inches in diameter at the shoulder; being thus somewhat larger than the carrot. The crown is short and narrow, contracting gradually from the shoulder, which is generally below the surface of the earth, and the leaves are strong and numerous, generally growing to the height of about two feet.

One of these varieties is grown in the islands of Guernsey and Jersey ; in the latter of which particularly, it is universally cultivated, both for the food of cattle and for culinary purposes. It grows to a larger size than the common kind, as it sometimes reaches the length of more than three feet; and the seeds evidently produced roots of a superior quality. Another kind has also been recently introduced by the Horticultural Society, which is much the earliest of its kind, but, if left too long in the ground, is apt to decay in the crown. It is not, however, so productive as the common suecies; and, although of superior flavour, and therefore advantageous as a garden plant, it is not well adapted to farm culture*.

The soil should be a good clean hazle loam, or a strong deep mellow loam upon a dry, gravelly, or sandy bottom, or even a simdy soil if not sharp and hungry; but strong clays, unless well opened by liming and ashes, as well as by deep ploughing and perfect drainage, or strong shallow loams, with a chalky bottom, or abounding with flint or sharp gravel stones, are improper.

Whe prepuration of these soils should be effected in the same manner as for carrot; but the stronger and more tenacious the under stratum, the deeper should be the ploughing, as no pains must be spared to bring the land into a perfect state of complete pulverization. A potato fallow, provided the ground be marle loose enough underneath, is among the best preparations for parsuips that can be made, because the dung will have been therehy thoroughly intermixed with the soil by the taking up of the roots, and by the subseguent ploughings. "In Jersey, where the parsnip husbandry is most successfully prosecuted, the soil is either dug with a sparte after the first ploughing, or stirred with two ploughs of difierent shapes following cach other, as the soll for this purpose must be stirred from the bottom, and a plough is made for this sole purpose, which will go to a depth of 15 inches. 'This operation is performed in January or Feturuary; the ground thus tilled is then coarsely harrowed, and beans are dibbled by women in pairs five feet asunder, after which the parsnip seed is sown broad-cast, and the whole is finely harrowed. In May the gromd is carefully wecded hoth by hand and with a small weeding fork, and the crop is thimmed like turnips. The beans are pulled the moment they are ripe; and towards the close of September the parsuips are taken up, or only gradually removed, as they are wanted for cattle + ." The cultivation, in conjunction with beans, las, however, of late greatly fallen off, as it occasions much additional dilliculty in weeding the land, an operation which is there invariably performed by women.

The seed is very light, and not so tenacious as that of the carrot. When fresh and good, full ripened and free from any semblance of dark brown or black among it, it should be sown thin, and upon a light harrowing, in the

* Trans. of the IIort. Cult. Soc. vol. vi. Art. 26.

† Complete Grazier, 6 th edit. p. 527. 
quantity of from four to six lbs. the acre, according as the season is wet or dry, or the soil strong or light.

The time of sowiny is usually frum the first week in February to the first week in March; though it is sometimes deferred until the beginning of April. It has, however, been properly observed, "that seasons have a great effect upon the period of sowing, and so has the soil, for it may be so wet in February and March, that few soils could be got in condition much before April ; as a general rule, however, all cold soils and exposed situittions should be sown as early as possible, while the more genial ones may be left until later*",

Mr. Malcolm, however, adds, "that he has known it sown in the middle of October, with very superior success;" and it is not an uncommon opinion, that " in order to render the cultivation of this root advantageous to the furmer, the seed should be sown in the autumn, immeriately after it has come to perfection; by which means the plants will appear early in the following spring, and will get strong before the weeds can injure them; for frosts never affect the seeds, nor do the young plants ever materially suffer through the severity of the seasons + ."

The cullure is not materially different from that for carrots; the ground should be harrowed as soon as the rough leaves appear so high as to be distinguished from weeds, and the hoeings should be performed in the same mammer as there described, though the superior strength of the soil may demand a little more attention. The plants will thus stand at 16 inches asunder, giving 393,290 roots for a full crop ; but the haulm is never mown for cattle.

The storuge is effected in precisely the same manner as the carrot.

\section{PRODUCE, VALUE, AND APPROPRIATIUN.}

The produce of currots upon appropriate soils in good heart, though without any extraneous addition of manure, is commonly very large: so much as 900 bushels per acre have been known, and four bushels per rod have been frequently grown; but these may be considered as unusually great crops, and the average has been generally estimated at full 400 bushels, or ten loads of 40 bushels each-equal to about 12 tons per acre + .

'That of yorsnips is still greater, and instances frequently occur of their amounting, on rich and well-manured land, to 30 tons, and the average produce has been rated as high as 24 tons per acre $\$$. They are both peculiarly exempt from accilents arising either from insects or from the weather; and being thus in some measure independent of the seasons, their product may be more generally relied upon than that of any other root crops.

In point of value, of four varieties of the carrot, that known as the "long red" is the most productive of nutritive matter: the parsnip, however, contains a far larger portion of saccharine matter; and as we well know that this quality, besides being grateful to the palate of animals, is greatly conducive to their fattening, it is somewhat singular that the root has not been more universally brought into use $\|$. Indeed, although

* Malcolm's Surrey, vol. ii. p. 493.

+ Papers of the Bath Agric. Soc, vol, iv, Art. 22.

t. Hissex Rep. vol. ii. pp. 3, 5, \& 11 .

Malcolm's Surrey, vol. ii. p. 495.

II It thus yields by distillation a spirit so pure and good, as to approach nearly to the 
partially used for that purpose on some farms, it is only in the island of Jersey that it forms a regular part of the field-system.

They are there given in a raw state to hogs and horned cattle; the flesh of the former they are said to render delicately white, and the benefit derived by the latter $\mathrm{is}$, in the opinion of many graziers, nearly equal to that obtained from oil-cake in point of the weight of flesh, and so superior in the flavour, that in the island it always commands the highest price. Cows fed upon them during the winter-months are stated to produce a greater quantity of milk and butter than when fed upon potatoes, and of flavour and colour equal to that of the most luxuriant grasses*; but they soon become cloyed with the parsnips alone, and the leaves must not be used, as they impart a disagreeable taste. When griven to horses they are however thought to render them dull, and even to injure their sight $t$ : the former effect may indeed, if lomg persevered in, arise from their cloying the appetite; but of the latter, we know of no positive proof which can be relied on.

When carrots are given to horses, they, on the contrary, are found so heartening a food, that in the sandlings of Sufolk, where they are very extensively grown, the working catile are fed upon them throughout the winter, without any oats, and with but little hav, and yet are invariably in even more healthy condition than when they have corn. 'The quantity given is from four bushels to seven each horse per week; it is, however, more usually unlimited, and chopined into the manger with corn-chafi; but probably the superiority of condition is rather to be ascribed to the evident reason that carrots are very generally given to the farm-horses in as laree quantities as they will eat, while oats are invarialyly portioned out in a mammer very far short of plenty t. In grentlemen's stables a moderate quantity of raw carrots is also well known to improve the horses' coats, and is also thought to have a good effect upon their wind. They impart a rich colour as well as a fune flavour to the butter when given along with hay to cows; and they are highly beneficial to wemling calves, as well as all young beasts and store cattle.

SEED.

The seed of both carrots and parsnips requires two years before it can be brought to maturity; and that of the carrot demands two sorts of soil to bring it to perfection; namely-rich sand to raise the roots in the first year, and strong loam for the production of the seed in the second.

The preparation of the soil for this second planting is thus conducted: - soon after Michaelmas the roots are dug up; the tops are cut off to the

best brandy, of which a large manufactory was some years ago established in France, the pulp of the parsnip being afterwards used for the feuling of cattle, We: believe that an attempt of the same kiml has heen lately male in the Island of Guern. sey; and it is remarkable, that so long ago as the time of Oliver Cromwell, a patent was taken out for distilling carrots and parsnips. See Houghtun's Collectiuns, vol. ii. p. 161.

* In the Island of Jersey, about $35 \mathrm{lbs}$ of parsnips are given daily to the dairy cows, with hay, and the cream is more abundant than from an equal quantity of milk from cows differently fed-7 quarls producing as much as 17 oz, of hutter,-Quayle's fren. View of the Norman Isles. Mr. Inglis, in his Account of the Channel Islands, also arrees in the fine flavour which they impart to the beef-Vol.i. p. 187. See also Le IIardy on the Culture of Parsnips, Agric. Mag., No. xxxiii.

$f$ See the Observations of MI. Le Brigaut, in "Corps d"Observ. de la Suc. d'Agric. d" Commerce \& des Arts, établie par les Etats de Bretague," p. 141.

+ Young's Survey of Suffolk, 3rd edit., Y. 135. 
length of an inch, and they are then packed in barns with straw, taking care that they are dry enough, and allowing air to have access to them, yet carefully excluding the frost. In the following rear, when the season has become dry, in February or March, a fresh piece of rich land, if to be had on the farm, is chosen for the second planting; or if that cannot be procured, a piece of deep soil well manured with dung and earth, and deeply ploughed, so as to allow full room for the penetration of the roots.

The modes of planting are thus-the top-end of the roots is cut off, and four or five are planted together, within the complass of a square foot, at the distance of a yard from each other: the roots are then thrust into holes of a proper size and depth, made by a wooden dibble cased with iron at the tip; the same individual making the hole with one hand, and putting in the plant with the other, while a boy going before lim lays lown the proper number at the spaces intended for each hill or cluster. They are hoed four or five times in the course of the summer, berimning as soon as the plants are above ground, and the clusters are moulded and rounded ip into hills after the third hocing; the hill being regularly cularged and heightened by a fourth, and sometimes a fifth, hoeing. Some persons however plant them singly, three feet from carrot to carrot, and two feet in a row, in a quincunx position. In Torkshire they are usually dibbled in rows of 30 inches distance, and plantel separately at 20 inches apart; for if too much space be allowed, the stems are apt to be broken down by rain and wini before the seed is ripened. The routs are not there cut at the time of planting; but in all cases those of a moderate size are considered the best, and the hoeings are very necessary both to keep the land clean and to encourage the vigour of the stem.

The gathering of seed is generally performed by women, who go between every row, and with a knife or scissors cut off the heads which appear to be ripe, and which they drop carefully into a bacr or basket suspended round their neck. The ripening is very irregular in point of time, and this operation must therefore be frequently repeater ; sometimes four or five times. The heads are then carried to the barn and laid up in packs, or wool-bags, until they are thrasher for market. The thrashing must be performed three or four times over before the seed can be completely separated from the husk; and in the interval between each thrashing respectively, it is sifted through sieves, every one finer in succession to the last and final thrashing. After all, there is, however, some offal or refuse remining, which, though not sufficiently clean for the market, yet answers the farmer's purpose for his own sowing.

The seed of parsnips separates with more freedom, and therefore may be prepared for market with less trouble. It may also be grown upon the same soil as that upon which the roots have stood, and, indeed, it is a plant of so much more hardy a nature than the carrot, that it is freyuently leit. to stand out in gardens to the second year without removal. 'This, however, even if danger were not to be apprehended from the climate, is a plan which camnot be conveniently practised in field-culture, as of course the best roots should be selected for planting, and were they allowed to stand when the remainder of the crop is taken up, they would occupy the gromul unnecessarily.

'The quantity of roots, of either kind, when planted for seed, is usually about 80 bushels per acre, and the produce may be estimated at from

6 to 10 cwt. per acre of carrot,

8 to 12, ditto of parsnip.

The price is very various at difierent seasons: in the latter end of the 
year 1835 three guineas per cwt. was the highest that could be obtained for either sort; yet the London seedsmen charge, by retail, as much as 3s. $6 d$. per $1 \mathrm{~b}$.

From what we have thus stated regarding the production of root-crops, it will be seen that they may be advantageously grown for the support of livestock upon every kind of soil except the wet and heavy clays. It is, therefore, evidently in the power of almost every farmer to produce a certain quantity of one or the other species, if not of all ; and if to their intrinsic value be added the improvement upon the land by the application of the dung resulting from their consumption by cattle, we camnot too strenuously urge upon their attention their still further increase, particularly upon soils of a poor description, and under the present depressed prices of corn. Their comparative fattening qualities may be ranked in the following order' namely-
1 Potatoes,
2 Parsnips,
3 Carrots,
4 Swedish turnips,
5 Kohl-rabi,

6 Cabbages,
7 Mangel wírtzel,
8 Yellow turnip,
9 Rape,
10 Common tumip.

\section{Cinpter XXiv.}

ON ARTIFICIAL GRASSES.

\section{CLOVERS-RYE-GRASS-TARES-CHICCORY AND CORN-SPURREY.}

Having thus stated the chief details concerning the culture of those roots which are commonly employed for forage, we now come to the production of those crops termed artificial grasses, which are used for the same purpose, and, whether consumed green, or in the form of hay, not only enable the farmer to support his cattle, by soiling them without the aid of natural grass, but also, by the manure thus raised, to maintain more live-stock upon an arable farm, besides the product of the grain, than upon an equal quantity of land solely under pasture. Many persons are, indeed, not aware that since the adoption of this mixed species of husbandry, some of our light soils, which previously were incapable of yielding wheat, have been made to produce crops of corn equal in value to the feesimple of the land while it was in pasture.

\section{CLOVERS.}

Botanists enumerate a great many varieties of pea-blossomed plants under the names of "trefoil" and "melilot," but they all belong to the same class, and the species chiefly cultivated in this country are those commonly known as the "white and red clover," and the trefoil termed "cow or marle-grass," which, as well as rye-grass, we have already enumerated among our natural grasses*; but as they have been only mentioned as forming part of those species which are devoted to permanent pasture, whereas they enter into our regular course of tillage crops, we think it right to add some account of their cultivation.

Most of them are indigenous to our climate, and the white clover is commonly found in calcareous soils, where its oily seeds will lie dormant for

$$
\text { * See rol. i. chap, xxxiii, pp. 514, } 515 .
$$


ages; so that it frequently springs up, as it were, naturally, if they be turned up, or if rough lime be allowed to lie for any great lengtli of time upon the land. It is a peremial plant, and lies so close to the ground that it has also acpuired the name of " creeping clover ;" but the great luxuriance of its gruwth, its nutritive quality, and the sweetness of its flavour, render it the most valuable of our natural grasses, and, when cultivated, it is invariably mixed with the seeds intended for laying down permanent pasture.

The broad-leaved red clover-so called from the appearance of its leaves and blossom, - although also a native species, is more generally grown from seed imported from the continent than from that grown in England. 'The knowledge of its culture was, indeed, as we bave elsewhere mentioned*, at first derived from Flanders, and, laving been introduced to our agriculture as the "great clover," was found so valuable, that within ten years afterwards it was not only well known in this country, but liad even made its way to Ireland. It was, indeed, the first great step to the improvement of our tillage system, for its abundant produce is not alone profitable to the farmer, but land which has been exhausted by the growth of corn is greatly restored to fertility by the shade, smother, and decomposition of weeds occasioned by a weighty crop of this lind; and red clover-if its general adaptation to our soil and climate are taken into consideration-is in those resuects recrarled as superior to any other of our artificial grasses. It is, however, only a biennial plant, which does not arrive at perfect maturity until the year after it is somn, and dying in the next, it is generally broken up after the crops of that year have been taken off; though, on poor land, it is not unfrequently allowed to stand the second year in pasture, in oriler to afford rest and consistence to the ground.

The con-grass is also a native plant of luxuriant growth, though not equal to the former; but as it continues in tolerable perfection until the third year, and is, indeed, looked upon as a peremial, it is not unfrequently sown along with the red clover, when it is meant to leave the land for two or three years in pasture. There is likewise a species of scarlet trefoil, known as "crimson clover," which has been introduced within the last few years and seems to be gaining reputation, of which cattle are very fond, and which produces abundant crops. It is an amnual plant, sown in autumn, and is said to thrive best upon dry land, when sown upon a corn-stubble which has been mercly scarified, without having been regularly ploughed $t$. Its growth is peculiarly rapid in the months of April and May; and the crop may be mown in time to be succeeded by turnips: it is also very useful for the purpose of filling up vacancies in fields sown with red-clover, when such liave occurred from the lodging of corn or other causes.

Cluver will grow upon almost any kind of soil, from the most sandy-if combined with a sufficient portion of vegetable mould to afford it some degree of tenacity-to the stiffest clay; but on marly land it appears as if at home. The white is, however, better adapted to light calcareous land, and to permanent pasture, than the broad-leaved red species, which is more calcu-

* See the Introduction, p. 25.

$f$ " I have never known a single instance of failure when the seed was committed to an unploughed surface. In the beginning of 1833 I sowed some upon a strong and rich soil upon a chalk bottom; the seedlings came up well, but in the course of the winter all perished. In September Colonel Beach sowed a few acres in a field of a similar nature, and here again the crop was a fitlure. At a distance of 200 yards, upon soil precisely similar, and in the same season, a stiff unpluughed wheat stubble produced as fine a crop as could have been clesired; the fumer had previously pursued the same plan and has realized large produce,"-British Farm. Dlag., A pril, 1835. 
lated for strong soils than those which are very friable, and indeed thrives with great luxuriance upon the heaviest clays. When sown upon light sandy soils, the ground should be ploughed deep, to secure the roots from the effects of drought; but on those of a more adhesive nature less care is necessary, and provided the land be perfectly clean and well furnished with manure, it is very commonly made to enter every fourth year into the rotation of crops, though it is sometimes found to tire of that frequent repetition, and once in six, or even eight years, is certainly better *

Rel clover is, however, the only species grown in the common courses of cropping; for wheat sown upon white clover leys has been found much inferior to that which, on the same soil, has followed the red lind. It is never sown alone; for not only is it very slightly productive in the very first year, but the protection of some other plant is favourable to it in the early stages of its growth, provided it be taken early of the gromad: it is therefore more generally sown along with lent-com,--in the common rotaion of tumips, barley, clover, wheat,-than with any other crop'; though, upon strong land, it is not unfrequently put into the ground in the spring, after winter wheat, and fine crops are thus frequently grown. The farmers in the north, indeed, generally prefer oats to follow rather than to precele clover; but in our southern and midland counties clover is considered the very best preparation-except a clean fallow-for a crop of wheat. It may also be sown with great advantage upon good land, along with flax to be pulled green; and on light soils the product has been foumd greater after buck-wheat-whetler cut for soiling or allowed to stand mtil ripe-than after oats + .

The quantily of seed is usually about 10 to $14 \mathrm{lbs}$. per acre on light friable soil, when sown with barley, and from 12 to 16 or $18 \mathrm{lbs}$. with wheat or oats, upon cold clays; poor soils requiring more seed than those in a high state of tilth and fertility. Some farmers also mix with it ahout a peck of rye-grass, from an idea that a small mixture of this seel, which produces an early plant, serves both as a nurse to the young clover and improves the quality and quantity of the crop) the propuriety of the practice has, however, been much questioned +, unless when the crop is intended to stand for a second year, in which case it is also expedient to sow about 4 his. of white clover along with it, instead of that quantity of the red.

The time of sowing depends both upon the nature of the land, the state of the weather, and the kind of crop which it accompanies. Being however uniformly sown in the spring, the seed-when sown among oats upon the stronger soils-is usually put into the ground during the course of the month of March. When following wheat, it is lightly harrowed in before the young crop lats get very far a-head; or, if it be winter-proud, an excellent plan is then to feed it down with shcep, and when the land has been harrowed, to sow the clover and afterwards roll it. Among barley grown after turnips, the month of April to the beginning of May is the usual season; and if the barley has been drilled, the sowing is sometimes protracted until a fortnight after the seed has been put into the ground, or until the plants have taken root, after which the clover is sown and bushharrowed and rolled, which answers the double purpose of covering the seed and fixing the roots of the barley more firnly; which, especially on

* Survey of Essex, vol. ii.p. 17. See also Russell's;'Treatise on Practical and Chemical Agriculture, chap. $x$.

† Von Thaër, Princ. Rais. d'Agric, 2nde edit. tom. iv. § 1304.

\pm Northumberland Rep.p 112 
light soils, is of great scrvice. If, however, the barley be sown broad-cast, the clover then follows the last harrowing, and the whole is afterwards rolled down, by which means an umnecessinry trampling of the ground by the horses is avoided.

The difference in the times of sowing must necessarily occasion, in many instances, a corresponding difference in the growth of the seed; but when the period can be chosen, if the weather be favourable, the earlier it is put into the ground the better, for it will then probably escape the danger to which it may be exposed by drought and by the ravages of insects. It is always sown broad-cast, and unless the operation be intrusted to a very expert sower, of whose care there can be no dunbt, the better way is to divide the seed into two parcels-sowing the one lengthways and the other across-for it is of the utmost importance to lave it equally spread over the land. It will not bear deep sowing, but it should be lightly covered with fine mould; the land should therefore be previously harrowed twice in a place, with the finest-tined implements, so as to reduce the clods and cluse up the holes into which the seeds might otherwise fall, and it should afterwards be bush-harrowed or lightly rolled: in light soils both may be proper, but on strong or stony land the rolling may be omitted.

The success of clocer depends greatly on the state of the weather, both when sown and afterwarts when standing for a crop. In the former case it recpuires gentle showers to bring the plants forward and root them in the ground; and, in the latter, it may be destroyed by a severe frost, or burnt up for want of rain in the following spring and summer. Upon strong land these dangers will probably, however, be avoided, if the soil be bronght into a fine state of friable titth by its careful preparation for the jreviuus com crop; for as the roots strilie downwards to a considerable depth, they will be thus rendered secure from the effects of any frost that does not jenetrate very far into the earth, and its temperate coolness and moisture may also guard it from the drought occasioned by a hot summer; but on shallow soils the crop sometimes turns to nothing.

In order as far as possible to jrevent this, some farmers spread the land in the commencenent of the winter with stable.dung, which-if it can be spared-doubtless afiords a protcction against the frost, but has been found to render the piants too terider in the spring; on light lands, however, whether manure be laid on or not, it is a good precaution to roll them heavily as soon after the com-harest as they may be well soaked with rain; and, on those of a more allesive description, the harrows should be used early' in the spring. 'Top)dressings of lime, soaper's-waste, and various other mattors, of which we have already treated, have also been recommended; but none will be found more effectual than Dutch ashes and gypsum, the latter of which we do not hesitate to advise as being more powerful in its immediate efiects upon the crop, as well as cheaper, than any other appilication*. Notwitlstanding all these precautions, and under the very best syotem of management, it will however sometimes happen that the crop partially fails, or proves patchy; in which case no better plan can be pursued than to harrow in tare seed upon the vacant spots, whether they occur in the autumm or in spring; for in cither case recourse may be had to the sjring or winter seed, which may be sown at the rate of two bushels to the acre, or in that proportion, in such quantity as the deficiency may seem to require. The seed may also be dibbled in holes made at four inches apart + ; or scarlet clover may be applied as a substitute, with perhaps

* See vol. i. chaps. xiv. and xv.

+ Buckinghamshire Rep. p. 201. 
still better success, if sown in the month of September, and harrowed in over the stubble.

The trealment of the crop depends more upon the purpose to which it is to be applicd, than upon the nature of the soil; for in the neighbourhood of great tums it is very generally made into hay, and in more remote situations it is partially fed off, and sometimes allowed to stand for seed. The practice on making it into hay has been already detailed , and if mown early, the nearer it is cut to the ground the better; but if it has stood too long the bottoms of the stalks will be dry, tough, and maked. It uften hajpens that in favourable seasons, and on grood land, the clover attains a considerable heirht before the commencement of the corn-harvest, and if that be cut close to the ground it is cured along with the straw, and thus adds considerably to the value of the forage. At other times it is found to have scarcely more than made its appearance before harvest, and then the corn should not be cut so close as to injure the young ylants, for when it is off the ground, if the weather be warm and showery, they will sometimes sprout to a head, which affords a good bite to cattle; and in that case the crop is fed lightly off with sheep-stock, which should be early removed from the field, or otherwise they would eat into the heart of the plant and destroy it.

In the following year, it always produces two, and sometimes three cuttings, in the course of the season; but on the mode of consuming these much difference of opinion prevails. Some farmers turn their stock upon it as carly in the spring as it will afford a bite, and eat it off until some time in May, at which time it is cleared off for a crop of lay, or they not unfrequently let it stand for seed: others take two consecutive crops of hay, or a first crop of hay and then seed; but the more general mode is to mow it once and feed off the second crop. When intended for seed, the first crop is, however, more gcnerally fed off in the spring, and the stock taken off at an early period of the summer; or, if cut, it is mown before it comes into blossom, so as to allow full tine for the plant to afterwards arrive at perfection, and to ensure the reaping of the seed-crop before the arrival of the autumnal rains.

If allowed to stand a second vear, the whole growth is then almost invariably pastured, by gradually eating the crop off in diflerent enclosures, or penning the cattle ufon small patches, and removing the hurdles as it is consumed; by which means they have it fresh, and the ground-particularly if it be of a light and spongy nature-is more materially improved by their droppings and treading, when thus confined, than when they are allowed to range over the land without restriction. On some soils, when intermixed with white clover and cow-grass, a tolerable crop is sometimes found even in the third year, and the ley is then not brolien up until that is eaten off. On widely extended farms, where store-cattle are chiefly kept, and where it may be an object to rest the land and lessen the expense of tillage, the plan may be advisable; but in all other cases it certainly is objectionable, both as filling the ground with weeds, and hardly ever affording an adequate return. 'The ley should, therefore, always be ploughed at the farthest after the second year: many farmers, indeed, plougl the crop in while in a state of succulence, as a dressing for the land + .

When the crop is to be preserved for seed, it is highly important that it should be got off the ground as early as possible, for it is late in rijen-

* See vol, i. ch. xxii. p. 493.

$\dagger$ The mode adopted is-" After the first mowing of the crop they let the clower run up 10 or: 12 inches high; it is then rolled, the field ploughed in large sets, and left 
ing, and if wet weather occurs at the time of reaping, the crop may be spoiled. An early harvest is also attended with the advantages of bringing the seed to greater perfection; in consequence of which it is thrashed with much less trouble and expense, the sample is better coloured, and the straw makes better fodder. The greatest quantity and the best quality of seed is uniformly obtained from crops of moderate bulk, and is therefore more generally to be found upon land of ordinary strength than upon the richer loams. The crop seldom attains maturity until some time in the month of Seplember; and, indeed, in many places, is sometimes not off the ground until the middle of October. This shows the necessity of using every means of forwarding the growth; in which view the first crop should be only pastured with sheep, or other light stock, until the begiming, or not later than the middle of May, when they should be withdrawn, and the field closely shut up until the time of ripening. The crop thus obtained is termed "Haiden-seed," and is generally found to be more plump and strong than that produced by clover which has been already mown for hay; though the latter is cleaner from the seed of weeds, and, if cut early, nearly equally productive.

The period when it may be considered ready to cut down is a point which rejuires some judgment, but may be ascertained by attending to the followjug observations. "As the crop approaches to maturity, it changes its verdant appearance to a light brown. After having gradually assumed that complexion, the leaves begin to grow hard and shrivelled; and at last, when the crop may be considered ripe, they drop off, leaving a bare stem with a witherel flower nodding on the top. These may be considered the common external indications of maturity, and, to a farmer of experience, are often suflicient to regulate his judgment; but to a person of limited practice, a more minute examimation is absolutely necessary : that is, by inspectiner the seed itself.

"He should be aware that it is chiefly its having fully ripened under a favourable sun that gives the seed that rich glossy purple colour, by which its superior excellence is ascertained. This change of colour from a bright yellow to a deep purple is a sure criterion by which a grower may determine when his crop is fit for the scythe; and, as the seed assumes this colour gradually, he ought to watch the change very carefully, by plucking the flowers from time to time, and rubbing them very hard in his liand between the finger and thumb till the seed appears; which requires some perseverance-for it is very difficult to extract it from the "glumes," or cups. When the crop is rich, the flower feels rather firm when pressed, as being better charged with secd; and, on the contrary, soft and spongy, when less full or worse furnished. Although a strict examination of the seed thus rubbed out in the hand be the surest method of proving the maturity of the crop, yet its jurecise degree of richness camnot be exactly ascertained by that simple test alone; fur the most sagacious labourers take a long time in proving the crop, before they can with safety enter into a contract for thrashing it*."

In harvesting, the crop is cut down with scythes in the ordinary way of mowing ; and, as the seed is contained within the flower, cvery precaution

in this state for six or eight weeks, during which time the roots and leares are partly dissolved into vegetable manure; the land is then harrowed and plonghed a second time, and a small quantity of slaked lime spread on the surface, and harrowed immediately; to promote putrefaction. It is then left to undergo a second process of fermentation before the seed-wheat is deposited, which is generally done about a month afterwards," - Burroughs on Green Crops, p. 8.

* Farm, Mag., vol, xix, p. 271. 
should be used to prevent it from breaking off the already dry and brittle stem: in order, therefore, to lessen the risk, the operation should be performed as early as possible in the morning, before the night-dew is exhaled. When that is done, the swathes should not be disturbed by either fork or rake until it may be necessary to form them, within a day or two, into very small cocks, which may be gently turned once or twice before carting them home. Should the weather be favourable, this may be done within from six to ten days from the time of cutting; but, if it prove fickle, it is impossible to say how long the process may require, for the crop must be thoroughly dry before it is carried, as it is almost impossible to extract the seeds from husks which are moist.

The seed always undergoes two successive thrashings-the first merely taking the heads, or flowers, from the stem; which is, in some places, termed "heading," and in others " cobbing," and the chaft thus removed is styled " the col)." This is laid upon a large wooden frame, consisting of oaken planks two or more inches thick, and is smartly and repeatedly beaten with flails till the chaff is literally reduced to powder, before the seed in which it is wrapt up can be made to separate from it. When thus forced from its outer husk, the next operation is with the fan, to clean it from the dust, which can only be partially effected at once, for repeated thrashing and famning are absolutely necessary -in some cases so often as four or five times before it can be brought into a perfectly clean state, quite free from dross and inner husk, and saleable in the market; though, when intended for home use, farmers do not talie that trouble. Frosty weather is the best for the operation, but at whatever season it may be performed, the expense of thrashing clover is thus very great; seldom less than $4 s$. or $5 s$. a bushel, and sometimes a rast deal nore: in some wet years it has cost as mueh as from a guinea and a half to two guineas*.

The lateness of harvest, and the consequent uncertainty of the weather at that period of the year, necessarily prevents the saving of the seed to any creat extent in our northem districts, and has thus rendered the farmer's ilmost entirely dependent for their supplies upon the seedsmen, who import it largely from the Continent. 'This has given rise to frauds, which, although exposed some years ago before a C'ommittee of the llouse of Commons, are ret continually pactised in its preparation for sale. The dealers call it "docioring ;" which, with white clover-seed, is done by first wetting it slightly, and then drying it with the fumes of burning sulphur: the colour of the red sort is improved by shaling it in a sack with a small quantity of indigo ; lut some use a preparation of logwood, tinctured with a little copperas, and sometimes with verdigris. Did the effect of this merely enhance the price by improving the appearance of the seed, the mischief thus done miglit not be very serious; but it in some cases destroys the vegretative power, and in all gives to that which is old and worthless the semblance of the best. Serious losses are thus constantly incurred by the sowing of imperfect seed; the risk of which imposes the necessity of at least increasing the quantity, if it does rot occasion the failure of the crop, and should, therefore, induce every farmer-or at least some one in every district, whose integrity may be relied on-to grow it for the use of himself and neighbourst.

* Essex Rep., vol, ii. p. 23.

+ In Flanders, for instance, where the farmers invariably grow seed for their own consumption, the quantity usually sown is only about $6 \mathrm{lbs}$. to the Engrish acre.-Radeliff's Agriculture of $\mathrm{E}$. and W. Flanders, $\mathrm{g} .60$. 
The produce of seed is infinitely various: in some seasons it has not exceeded two, in others five, some extensive crops have exceeded eight, and a few have reached so high as ten, twclve, and even fourteen bushels; but the average cannot be assumed at more on most soils than, at the most, six bushels per acre: averaging in weight about $64 \mathrm{lbs}$. each. Although the white and red species differ considerably in appearance, and require different soils, yet, when grown upon land which is suitable to each, thev are nearly equally productive. In price, however, the white has the advantage, in conseguense of its being less cultivated than the red as a separate crop. The actual price in the London Marlet, for new English seed, is-

White, or Dutch Clover, 75s, to 82s.: superf. 85s, to 90s. per cwt.

Red, or broad-leaved ditto, $68 \mathrm{~s}$. to $75 \mathrm{~s}$ : : do. $80 \mathrm{~s}$. to $82 \mathrm{~s}$. ,

of Hay, the produce of the first cut, upon land of good ordinary quality, may in general be estimated at about one and a half to two loals, and that of the second at perhaps half the quantity : both, of course, depending greatly upon the state of the season.

\section{ON RYE-GRASS,}

we have little to add to the remarks which we have already made upon the cultivation of clover. The perennial species lasts from three to six years, and may be grown under common management upon almost any kind of soil that is not too tenacious; it is therefore always sown along with the grasses intended for permanent pasture. There is, however, a kind called "Amual Rye-Grass"-though it is in fact a biemnial plant-which is more productive than the perennial sort in the year after being suwn, and therefore is preferred when the land is to be broken up after producing a crop of hay; but it is extremely diffcult to ascertain, by the seed, the difference which exists between them, and thus frequent mistakes are made in their growth. The Italian species-which we noticed in our arcount of natural grasses-has been found to support its peremmial character. It has been sown with barley without hurting the crop; and, by suwing a little thimner, it is found not to cholse the clover in the first crop, while its quick growth enables it to keep a-head of it in the second, producing lony little inferior to the first. At the last meeting of the Edinburgh $\Lambda$ gricultural socicty, it was stated by Professor Traill, that it was found in the experimental garden established by the Duke of Buccleuch, to arrive at maturity much earlier than any other perennial rye-grass, and to produce nearly double tho quantity. 'The experiments made upon it, inciecd, show that it is decidedly relished by cattle in pasture; and in hay-having been submitted to horses along with that of the common ryc-grass, in separate bundles,-it was uniformly preferred. When allowed to ripen the seed, it is also said to give double that of the rye.grass, and the nutritive substance extracted from the herbage affords more saccharine matter than that of the celebrated sort known as "Pacey's*." Sticliey's Rye-grass has also been highly extolled in the East Riding of Yorkshire as very superior to all other varieties for forming a closely-matted sward, continuing its verdure through all seasons, and being less liable to run into bents.t

Although the common species has been longer cultivated in England than any other lind of grass, and is still extensively grown, there are yet various opinions regarding its value, and it is considered to have an cxhausting effect upon the soil. There can indeed be no doubt of this, if it be

* See the Third Report of Drummond's Agric. Mus, at Stirling, p. 65.

r Farmer's series, in the Library of Useful Knowledge : farming at Ridgemont, p. 146. 
allowed to perfect its seed; and if wheat be sown upon a clover or a ryegrass lev, the product will be found less upon the latter: it is also thought to render the land foul; and peas have been recommended as better adapted to follow it than wheat. * It is however an excellent grass both for pasture and hay, as all sorts of cattle are fond of it, and horses fed with it are said to preserve their wind better upon it than upon any other kind of hay . Its chief value, however, consists in the quickness of its growth, which furnishes a very early support to sheep in the spring, and it can be grown upon upland soils where clover will afford but a trifling prodtice; in such circumstances, therefore, its use is great and indispensable, and it is sown at the rate of 2 or $2 \frac{1}{2}$ bushels to the acre. In soils suflicicntly rich to produce a sward from mixed seeds, it is, however, more commonly grown in a molerate proportion along with clover and trefoil than alone; for the first cut ensures a good crop of hay, while, if retained for a second vear, it affords a great increase of pasture; and no other mode has been found so effectual for producing a full growth of rich herbage. When either fed or made into hay, it should however be taken early off the ground, as the stems soon become wiry and run into "bents," which are then rejected by the cattlef.

The produce of both hay and seeds varies so much from soil and season, that it is impossible to state, with any decree of certainty, what may be the average amount of either per acre. The first shoot is almost invariably pastured by ewes and lambs; after which, if it be not wholly fed off by other cattle, that part of the crop designed for mowing is shut up about the middle of May, and may be generally considered to yield-

If made into hay, from $1 \frac{1}{2}$ to 2 loads, or,

If allowed to stand for seed, 40 bushels $\}$ per acre.

The sced is, however, thrashed without difficulty; but the market-prices vary so much, according to the different sorts in request, that it is dificult to state them accurately.

TARES,

or, as they are also called, Vetches, are, according to botanists, confined to one species; but there are several varieties-linown as the "white tare," the "strangle vetch," which abounds in chalky and sandy soil ; the "tufted" and the " wood-vetch;" besides the " broad-leaved vetchling" or " everlasting tare," which, although chiefly raised in gardens for the sake of the flowers, and not noticed by tarmers, has yet been strongly recommended as affording large crops of superior quality: added to which, a new variety of a hardy kind has been imported from Flanders; but those commonly cultivated are only the "winter and spring tare," the names of which have been rather acquired by their time of ripening and being more or less hardy than by any essential difference in their quality.

Although the seed of the tare-which somewhat resembles that of the pea-is very nutritive, and is frequently given with advantage to pigs, yet the chief use to which the plant is applied is that of soiling; for, although the hay makes hearty food for working cattle, it yet is of a coarse nature, and attended with considerable difficulty in the getting of it into stack. The

* Young's Norfolk, p. 267 ; ditto Surv. of Essex, vol. ii. p. 25 ; ditto of Oxfordsh. p. 190.

+ Berksh. Rep., p. 284. "It is also thought to afford greater nourishment than trice the quantity of common hay."-Bath Soc. Papers, vol, ix, p. 148.

\ Bedfordsh, Rep., p. 432. 
common tare is an annual plant, indigenous to our climate, and extremely hardy. There are, however, many varieties which are perennial : the winter lind-which is sown early in the autumn-supports the severest frost, and being one of the first and most productive grasses in the spring, is the sort most generally grown; but requires a strong and rather rich soil. Both species may however be said "to suit every situation, and to flourish in all the variety of soils in this country*",

IVinter Tares are sown either as a bastard fallow prior to wheat, or upon a wheat stubble, with the intention of being either soiled and followed in the ensuing summer by turnips, or left to stand for a crop of hay, or seed. In the former case, the dung is laid upon the land previous to the sowing of the tares; but in the latter, it is not put into the ground until the sowing of the turnips, which are sown upon one ploughing, while the soil is in a moist state, immediately after the tares are fed off. They are usually sown broad-cast, when meant to be soiled, and most commonly upon one ploughing; though the land ought to be in clean condition, and it is unquestionably wrong to grow them when it is any other state. The time of seeding is from the middle of August till the latter end of October; but if the land be poor, or the situation exposed, the earlier the better; and, in all cases it is advisable to sow at different periods-early, medium, and late, about a month between each-in order to secure a succession of feed at different seasons. The seed is never steeped, though perhajs, in very dry seasons, the precaution might not be unadvisable.

The quantity of seed, on ordinary soils, is about $2 \frac{1}{2}$ bushels per acre ; but those which are moderately rich, and early sown, require rather less seed than those which are poor and late. Along with the secd it is also customary to sow a small portion of rye, oats, or winter barley; which, coming forward equally early, add greatly to the burden of the green forage. Winter beans are also sometimes sown for the purpose of supporting the tares, and preventing them from lodging on the ground.

Spring Tares may be sown at any time during the months of March and April ; they are, indeed, sown in succession even so late as the end of June, and are not unfrequently made to follow a crop of winter tares. On the South Downs, "for instance, "it is a conmmon practice to sow forward winter tares, which are fed off late in the spring with ewes and lambs; they then plow and sow summer tares and rape-2! bushels of tares and $\frac{1}{2}$ a gallon of rape-and this they feed off with their lambs in time to plough once for wheat + ." This, when a summer fallow can be dispensed with, certainly turns the land to good account; for it is made to support the utmost possible number of sheep which can be maintained upon such soils, while the stock yields an ample supply of manure, and their treading puts it into a state of sufficient firmness for the reception of the wheat.

When sufficient time is allowed for the preparation of the land, by early spring sowing, it should if possible be ploughed before winter; then crossploughed, well harrowed, and again ploughed for the reception of the seed; which, in short, should not be put into the ground until it is in a state of perfect cleanness : it is afterwards rolled, and treated in every respect like clover. Spring tares produce rather a lighter crop than those grown in winter, and as they come into use a full fortnight later, they are more subject to risk from a dry summer; there is, however, no difference in their application, or value per ton.

When sown for seed, both species should be drilled and managed in the

* Middlesex Rep., 2nd edit., p. 253.

$\uparrow$ Sussex Rep., p. 105. 
same mammer as a crop of peas. If a few beans be dibbled in the rows, they will also aflurd support to the plants; for, although the crops be reaped togrether, the grain may be easily separated by the riddle. When ripe enough, they are generally mowed, or hooked, and laid into rows and turned over two or three times, according as the weather may suit for carrying home and stacking them; but rain occasions great havock, for the necessity of frequently turning and drying them opens the pods, by which half the crop is sometimes lost. The thrashing is performed in the common mamer by the flail ; but the seed usually contains a considerable portion of weeds, which if not carefully extracted will render the land very foul. The difference between the seeds of the winter and spring species is hardly to be discemed: to judge of their aure and quality, scedsmen bite them asunder, ant, if they separate readily, if they are very hard, and the colour of the skin dark, they are considered as not new. Old seed will frequently not vecretate; and, when the price rises, frauls are commonly practised by dealers, who mix them : to guard against which, as also to secure the certainty of discrimination between the different sorts, farmers should always grow sufficient for their own consumption. The haulm, though sometimes used as foracre for cattle, is exceedingly tough, and is in many cases emplused merely for litter. The hay is made in the same manner as that of clover.

The application of tares, of cither species, is, when used green, by penning sheep, and in some cases logg, upon them; but for the larger cattle, they are ahmost invariably cut and served to them in their stalls, by which a vast yuantity of manure is made, as they are highly relished by every species of stock. 'They are also very nutritions, and agree well with ewes and lambs when they come into islossom; but, for horses, they are best when the pod is formed; and although, when given to cows, they enrich the quality of the milk, yet dairy-women complain that they render it ropy. Like all kinds of very succulent food, they should be at first cautiously given, or the cattle may become blown, or "hoved;" and sheep should not be turned upon them until the dew is exhaled.

The average value of the crop is not easily ascertained. Some of the county survers stating that an acre of grood tares will afford plenty of nourishing food for 200 to 220 sheep a weel: , and others reducing that calculation to $100+$ : perhaps, therefore, on the generality of soils under common management, the medium may be taken; or, supposing nime score, at 4rl. per head, that would bring the folding with sheep to $60 \mathrm{~s}$. per acre: when soiled, it has however heen found that an acre will support a large number of cattle, and will thus amount to considerably more + ; and it has been estimated that an acre of green tares will produce 12 tons, which, when made into hay, will yield full three loads $\$$ : but this we must presume to be far beyond the arerage quantity, which certainly cannot be assumed as much more than about half that amount. Of seed we have heard of crops cxceeding 40 bushels per acre, and prices varying from $5 \mathrm{~s}$. to $21 \mathrm{~s}$. per lushel |f ; but the present prices, and the average produce may, we believe, be more correctly stated at-

$$
\left.\begin{array}{l}
\text { Winter tares } 4 \text { s. } 6 \mathrm{~d} \text {., and } 22 \text { bushels } \\
\text { Spring tares 5s. } 6 \mathrm{~d} \text {. , } 18 \text { ditto }
\end{array}\right\} \text { per acre } 9 \text {. }
$$

* Sterenson's Surrey, p, $2 \mathrm{~S}$.

+ Bedfordsh. Rep., p. 407.

light acres have kept 12 horses and five cows for three months without any other food.-Sussex Rep., p. 104.

\$ Middlesex Rep., 2nd edit., p. 257 ; Berksh. ditto, p. 218.

II Ibid.; and Malcolm's Surrey, vol. ii. p. 382.

af Hampsh. Rep., p. 171 . 
CHICCORY,

Or. Succory, is a common English perennial weed, which some years ango was much brought into notice by the late Arthur Toung, "as a forage plant; he having grown it extensively on his own farm, and having stated, in the "Annals of Agriculture,' that "on various sorts of soil, there is no plant to rival it." * It has, indeed, been long extensively cultivated in many parts of the European Continent as a substitute for clover ; and during the late war, when the Berlin and Milan decrees prevented the importation of colonial articles, the dried roots were gromat, and used throughout Germany instead of coffee. 'The only use made of it in this comtry-and that to a very limited degree-is as a field-pliant for green forage. It grows in the form of lettuce, producing blue flowers; and being a hardy plant, which withstands the greatest severity of winter, it comes very early forward for the support of cwes and lanbs, to which stock it is chiefly appropriated, though in nutritive properties we inagine it is rather deficient.

It may be grown in almost any soil, however poor, provided it be dry and open, and yields several cuttings during the season; but the most appropriate soil is a deep sandy luam. It may also be either sown broadcast, along with Lent corn, at the rate of 12 or $14 \mathrm{lbs}$. to the acre, or drilled; and the seed is sometimes mixed with 4 or 5 lbs. of trefuil. It, however, reyuires deep ploughing; and as judicious culure in the early stages of its growth is necessary to secure its future luxuriance, the following is recommended by an inteligent agriculturist, who has lately grown it, as the best mode of culture:-

"Let the seeds be sown in a bed early in March, and when the leaves are shout three inches in length, which they will be by the begimning of May; let them be planted out in well pulverized ground, in rows nine inches apart, and six inches from plant to plant in the rows. The weeds must be bept down by hoeing in the first season, but no further trouble is necessary for seven or eight years, till the plants begin to show symptoms of decay, when they may be thrown out, and the ground cleared of the roots, and laid under a fresh plantation.

"If allowed to stand too close, the root is small, and the stem short and wire; but, zonler the above treament, even in ordinary soils, and with little manure, the stems will rise to six or seven feet in height, and they will be so close as to admit of being covered with foliage, the stem varying from three-eighths to five-eighths of an inch in diameter near the ground, and being replete with a rich nutritive milky juice. The succulence is retained in full virgour till the flower-buls begin to appear, when the stems should be cut near the ground, and cirrier to the byre, where, even at the first trial, they are devoured with avidity-indeed, no plant cultivated in this country will bring the cow-feeder nearly an equal return with the chiccory; and it should hold a prominent place in the garden of every cottager in the kingdom, whether to be used as coffee by the family, or as green food by the cow or pig." +

I'o this, however, we must add, on the authority of Von Thä̈r, that it is

* "In an experiment made by the late Duke of Bedford, it was sown on a fallow in Angust, and fed about Michaelmas five sheep an acre for a month. In the following year it lept six sheep an acre from the second week in April until Michaelmas. Another piece, sown upon one ploughing, kept ten sheep an acre in the first year, from the second week in April to July 2.2; and then seven per acre to the end of October; and in the following year seven per acre."-Ann., vol. xxxix.; Bedfordsh. Rep., p. 437.

I Gurrie, on the Culture of Chiceny.-Quart. Joura. of A ric., N. S. vol. iv. p. 206. 
extremely difficult to eradicate it from the land; and it has been found to materially impoverish the soil *

\section{CORN SPURREY}

Is scarcely known in this country in any other light than as a noxious weed, though in the sandy soils of Flanders it is very generally sown as a brush-crop, after wheat, and is there held to form an early and valuable pasture for cows, but the cultivated species differs essentially from that which is grown wild. There are, indeed, two different sorts-one growing tall and thin, and the other short and thick, and by mixing the seeds the best crops have been obtained.

It is always sown broadcast upon a single ploughing, or often by merely passing the scufflers over the stubble, at the rate of about $8 \mathrm{lbs}$. of seed per acre, after which it is rolled, and it is usually fit to be either eut green, or pastured, within five or six weeks after it has been sown. The produce, of course, varies according to the richness of the soil, and also depends much on the temperature of the season, for it requires both heat and moisture; it may, however, be generally calculated at about half the amount of a crop of clover, but the quality is said to be more nutritive. If the weather be favourable, it can be easily made into hay; and if allowed to stand for seed, the haulm is considered more nourishing than any other kind of straw; but the hay shrinks considerably, and, upon the whole, it is found more advantageous to consume it green. It is, therefore, usually fed off by milch cows tethered upon it, or soiled in their stalls.

The grain is of an oily nature, and is also extremely nutritive. When given to cattle it is steeped in hot water, and served as a mash alone, $00^{\circ}$ mixed with cut straw, and has a visible effect in increasing the quantity and richmess of the milk, as well as improving the condition of all animals fed upon it. The produce of grain is also large, but when allowed to stand for seed, it has been found to greatly exhaust the soil. Its greatest value appears, therefore, to arise from its short duration allowing it to take an intermediate place between the harvest and the spring sowing; from its producing a certain quantity of provender without manure; and with but little expense of labour $\dagger$.

\section{Chapter XXV.}

\section{ON ARTIFICIAL GRASSES-(continued).}

SAINFOIN AND LUCERNE.

Although the plants of which we have now to treat are used for the same purpose as the grasses already described, yet those, if not actually annuals, are more frequently employed for the growth of only one season, while these are perennials which last for several years in the ground; we therefore deem it advisable to detail their properties in separate chapters.

\section{SAINFOIN}

is a plant of which a great number of species are enumerated by botanists, though only one is generally known to farmers. It is a very valuable

* Von Thaër, Princ. Rais. d'Agric., 2nde edit. tom. iv. p. 322.

I Ilem, tom. iv, j 1346 to 1353. Radeliff, on the Agriculture of East and Wrest Flanders, § 140 . 
grass, but although indigenous to our soil, we are yet indebted for its introduction to our husbandry to the French, by whom it is so highly esteemed that they call it by the significant appellation of saint-foin, or holy-hay*.

The soils on which it best succeeds are decidedly the calcareous, and it is therefore extensirely grown in all the districts of the kingdom which abound in chalk; particularly in the range of hills which spread from the midland parts of Kent through many of the southern counties of England. On this range the upper soil varies considerably, some of it being thin tough clay, some a rich loam, and some of a poor sandy nature; but, provided the subsoil be lime or chalk, the upper stratum is not of so much importance. Being a tap-rooted plant, where the sainfoin is grown upon a soil with a chalky base, the roots will reach that base, at however great a depth it may lie, and therefore a calcareous rubble, through which the roots can easily penetrate, is very favourable to it; but on sands or gravels, however open they may be, it will not flourish, unless they are well dressed or intermixed with lime. A retentive strong clay-soil, although it lie on a calcareous base, is less adapted to it than a light poorish soil of the same nalure; for, on the former it is apt to rot. if the winter prove aiternately severe and wet. It is, indeed, a common opinion that the land should not be too rich, which probably may be correct in one sense, though ill-founded in another. Thus, if sainfoin be sown on ground rich with manure, the crop may be injured by the grass being rendered coarse, but there is little danger of this consequence resulting from a soil that is naturally rich; for, like all other crops, it will ever be good in proportion to the natural richness of the land, though it may be hurt by being rendered so artificially $t_{\text {. }}$

Dung is therefore considered improper, and the manure generally employed is a top-dressing of peat, turi, or coal ashes, at the rate of, perliaps, 10 or 12 bushels of the former, to 30 of the latter, per acre. Those of coal are considered the least likely to do harm, as having less tendency to wear out the plants, by lininging them too suddenly forward; for it must be observed as a general rule in dressing sainfoin, that the use of manure should be directly the reverse of its application to other crops. On the deep hazel loams which lie along the edge of some of the chalk hills, no dressing of any kind is considered necessary, but on the thinner and colder soils it is thought advisable, in some cases every year, and in others once in two years. Gypsum has been used with success at the rate of five bushels per acre. On all soils, however, except the very poorest, the top-dressing it is thought can scarcely be too light $¥$.

Sainfoin is commonly sown at the close of an alternate course of seven or eight crops, and is generally put into the ground along with barley. Its duration in the land depends, of course, upon the nature of the soil and the condition of its preparation; for if that be open to a considerable depth, and reduced to a friable consistence, it will last from seven to ten years, but if not well cleaned it will be smothered by weeds within a very short time $\oint$. It is therefore previously customary to make as good a turnip season as possible, well cleaned and hoed, and afterwards folded off, as a necessary preparative to the subsequent operations. This having been done late in

* The date of its introduction has not been ascertained, but it is mentioned by Aubrey as well kuown in his time, 1673 .

f Stevenson's Survey of Surrey, pp. 312, 318.

¥ Oxfordshire Rep., pp. 192, 193.

$\$$ The oat-grass is one of its greatest enemies, and on poor soils will creep in to such extent as in three or four years to destroy it; the only preventive is, when once the sainfoin roots have taken firm hold of the soil, to harrow it thoroughly. 
the autumn, the ground is immediately ploughed up and left rough; in January it is harrowel down, and being well pulverized is immerliately cross-ploughed, as deep as for a crop of carrots. The first week in March it is again well harrowed down, or run over with the scufflers, and about the beginning, or at the latest the middle of the following month, the barley is sown thin, once harrowed, and the sainfoin is afterwards sown broad-cast, in the same manner as clover, and lightly rolled. Some farmers plough the land thrice, and give it a slight sprinkling of good rotten dung, which is lightly ploughed in, and the ground ridged up before the sowing; but the main object being, in both cases, to open the soil as much as possible, the second and third ploughings are always carried to a great depth*. Drilling is very rarely practised; for, however the land might be thereby cleaned by lioeing in the first season, it would in the following years cover the ground as completely as if it had been sown broad-cast. It is also evident that were a crop of equal amount to be produced by a comparatively small number of plants, the shoots inust be thick and strong, and the hay-the value of which depends on its fineness -would thus be rendered coarse.

The quantity of seed is from three to five bushels, but four are usually employed, to which some farmers add a small quantity of trefoil or ryegrass, to secure a crop in the first season, as the sainfoin does not come to full perfection until the second vear; others, however, omit it, as conceiving that it injures the future growth; and, both from general reasoning and from the experience of several intelligent men, it seems fair to conclude that the practice is rather to be deprecated than recommended. If we consider the number of plants which would be produced by four bushels of seed, were they all to come to perfection, it would be evident that no land could sustain them $\dagger$; but it is rather a precarious seed, even when new, and when bought of the dealers, the uncertainty is greatly increased; to which if be added the number of those sceds which perish by being ineffectually covered, by being smothered by the corn and weeds, and destroyed by the ravages of birds and vermin, as well as the material object gained by thick sowing, in improving the quality of the herbage, the allowance will not be found too great.

The application of sainfoin is chiefly to the purposes of hay. The proper season for cutting it is when it is in full bloom, and it is mown and made in the same mammer as clover or other grass ${ }_{\ddagger}^{\ddagger}$. Great care should however be taken not to orer-make it; for its perfection consists in retaining the natural bloom and figure of the blossom, and the clear and healthy green of the stem and leaves. It is easily spoiled by rain; as not only are the richness and fragrance of the hay thus injured, but the porous nature of the stallss renders it difficult to dry when thoroughly wet. In general it may he reckoned that a greater quantity of hay will be got of it upon thin chalky soils, than from a first cut of clover, and it is of superior quality and value. It is, however, seldom cut a second time in the same year, but the after-grass is pastured by cattle and horses. Sheep are very fond of it, but are very seldom put upon it, from an apprehension that they will eat it

* Malcolm's Husbaudry of Kent, Surrey, and Sussex, vol. iii. p. 43.

t The number of seeds in a lushel, of the usual quality, is about $871,200:$ an acre, if sown with four bushels, would therefore produce $3,484,800$ plants, or 21,760 to the square pole, and 80 to every square foot. Serenty-two heads have been known to proceed from one root, ond the crup, if evenly and thinly sown, will average ten heads.Malcolm's Mod. Husb., vol, iii. p. 47.

$\ddagger$ See vol, i. p. 493 . 
down so close as to expose the roots to the frost, and therefore, if admitted, care should be taken to guard against that danger, by removing them in proper time.

When retained for seed the management is rather difficult, for the poots fill and the seeds ripen at different periods, according as they happen to be at the top or bottom of the stems; the seeds also shed very easily if roughly handled, or if the husks be either parched by the sun or opened by wet weather. When the major part are of a brownish colour, and the seeds feel plump and firm, it is then, however, time to mow the crop, which should be done while the dew is on the plants, and the swathes should not be turned if they can be got sufficiently dry without it. In a grood year, and with proper care, four quarters of seed per acre may in general be looked for ; the price is variable, but may average about $50 \mathrm{~s}$. per quarter.

When broken $u p$, it is very generally the practice to pare and burn the ley*, in order to clear the ground of the great quantity of roots and oatgrass with which it is filled, and there can be no doubt that this object can be more speedily attained than by any other means. The ashes also produce a large quantity of manure which forces the production of one or two of the succeeding crops, and it is therefore a plan much faroured by farmers who hold their land upon short leases, but it admits of great doubt whether the soil is not thus eventually injured; and, mless in the case of breaking up rough ground which is encumbered with waste matter, we think it ought not to be allowed. Where land is in a regular conurse of cultivation it can be cleaned by the operation of the scuffler, and the weeds and roots mav be afterwards burned without lessening the real staple of the soil; and although this will necessarily occasion somewhat nure trouble and expense, yet that, we submit, is due to the interest of the landlord. We have, however, already treated so fully of the subject, that we have nothing to add to the observations there made $\uparrow$.

It is very generally supposed that sainfoin will not bear to be repeated on the same soil, until after a lap:e of fifteen or twenty years. Whether this opinion is the result of experience or prejurlice it is not, however, easy to determine; for the notion being entertained by most farmers they are deterred from repeating it, lest they should incur the risk of loss. WVe, havever, believe the idea to be unfounded; for sainfoin is rarely sown on any land that does not abound with calcarcous matter, from which it seems to draw its chief support, and this, in the clialky districts in which it is chiefly grown, is absolutely inexhaustible.

\section{LUCERNE}

is a tap-rooted plant, which rises when in bloom to more than two feet in height, bearing purple or violet-coloured blussoms, which produce pods containing kidney-shaped seeds.

The cultivation of lucerne is only of comparatively late introduction into this country from the South of Europe; and, as it requires a dry and rather warm climate, it has not been found to succeed in all seasons in the northerm parts of England; for, notwithstanding some favourable statements of experiments upon its growth lately made in the neiginbourhood of Edinburght, little doubt can be entertomed but that the native broad clover and

* On the Cotswold Hills it has been the practice from time immemorial.-Survey of Oxfordshire, p. 200. It is also much followed in Kent, Surrey, and the meighbouring counties.-Stevenson's Surrey Rep. p. 322.

$\dagger$ See vol. i. chap. xvi, p. 356.

\$ These were commencel in the spring of $15 \div 5$, partly upon a light gravel of great 
rye-grass are better calculated to yield more certain crops of herbage to the Scottish farmer than he can generally hope to obtain from the exotic grasses. Even in our midland and southern counties it has indeed made but little progress ; for, although universally acknowledged as the most valuable of all our foreign grasses, when it succeeds, yet the accounts of its success have been so very various that its culture has been checked even upon soils which appear favourable to it. The different result of those experiments appears, however, to have arisen rather from the want of a due knowledge of the precise species of land to which the plant is suitable, than to any uncertainty regarding its growth when sown upon a proper soil.

The land which is best adapted to lucerne is a rich, light, deep loam, though any friable ground of a deep and open nature will answer, provided it be well drained and ploughed to a good depth; but it will not thrive upon clays, nor does it succeed well upon soils that are too dry and parching. In fact, the under stratum is of greater importance than the upper, for the fertility of the latter can be increased by manures and kept open by culture, and may thus for a year or two be rendered productive; but if the lower soil be adhesive, or at all of a wet and clayey nature, the plants which seek for nourishment by striking their roots to a great depth downward, being stopped in their progress by the closeness of the ground, cease to vegetate and finally perish. Thus many farmers who are possessed of fertile loams which grow good crops of corn and clover, though lying upon a cold and impenetrable subsoil, have found their expectations deceived when they have calculated upon the advantageous growth of lucene.

The best preparation of the land is by a previous crop of turnips fed off; after which it should be opened by deep ploughing, thoroughly cleansed of root weeds, and whatever further manure can be spared should be spit-dung, well incorporated with it. The seed is more frequently sown in drills at the distance of 12,15 , and 18 inches apart* than broad-cast; though we think the practice is rather governed by fashion than by any proof of its superior utility. The motive is doubtless apparently good, for the land appears in a neater state when sown, it requires less seed, and it may be presumed to be more easily kept clean by the hoe; but the distance at which the drills are placed is too narrow to allow of the effectual operation

depth, and part upon a deep loam with some wet clay, sown at the rate of $18 \mathrm{lb}$. per acre in drills of 12 inches asunder; two acres were also drilled upon a soft black luam of about 18 inches deep with a subscil of white dry sand, and a small part upon a deep heavy loam. Notwithstanding the unfavourable drought of that season to the growth of all kinds of grass, and much injury occasioned by the grub, the lincerne yet gave a sinall crop that year, and in the following was realy for the scythe by the latter end of April, producing a constant succession of food for soiling--in three and four cuttings - until the midule of November. It continues to affurd nearly equal crops upon all the soils, except the wet clay, where it failed : it was best upon the loam until 1830, when the account is dated; and the Report estimates the produce as being at least one-third more per acre than that of either clover or rye-grass.-Trans. of the Highland Suc. N.S. vol. xi. p. 113. See, however, accounts of an opposite nature in the Farmer's Magazine, vol. iv. p. 413 , and vol. x. p. 162.

* Drills have been placed at these different distances on the same plantation; the reason for doing so being, in case of the season proving moist, those which were sown thick, oceasioning the plants to run up weak and spindling for want of air at their bottom, while those at wider distances then grow with great luxuriance: the plan was, therefore, not injudiciously adopted to meet those different circumstances.-Malcolm's Mod. Husb, vol. iii. 1) 65. An economical mode of sowing the seed has been alopted by a Scotch farmer, who wished to avoid the expense of a drill machine; which consists in putting the seed into a bottle, with a piece cut out of the side of the cork, or a quill put into it, so as to allow the seed to run from it fast enough for the purpose,-Trans. of the High. Soc. N. S, vol, ii. p. 123. 
of the horse-hoe, and the plants grow more vigorously when at nearly equal distances from each other. Very little advantage can therefore be gained by drilling; whereas, by sowing broad-cast, the saving in labour will equal the additional cost of the seed, and the land when sown with a crop which talses such firm hold of it, can be equally as well, if not better, cleaned by the operation of the harrow.

Some farmers object to this, as conceiving that, if effectually done, it must tear up the plants; and, in the first year, it should certainly be so cautiousiy effected as to guard against that danger. The plants will however have struck their roots so deep into the earth by the second season, that no risk of destroying them need then be apprehended, and in that and every succeeding year the scufflers and the harrows should be set to work early in the spring, to scarify the land in every direction and as deep as possible ; so as, in short, to give it the appearance of a new-ploughed field. 'The plants will spring again with increased vigour; and the operation may, if necessary, be repeated without fear, even between the different cuttings. It is in fact so essential, that lucerne plantations which have appeared worn out have been thus restored*.

The seed of lucerne may be either sown separately, or along with any grain the crop of which it is meant to follow; and, as its produce during the first year is very trifling, farmers generally put it into the ground either with barley or oats: others prefer peas podded for the markets, or tares cut green; though perhaps flax, pulled before being sceded, would, in consequence of its being well adapted to the soil on which lucerne is generally grown, be preferable to either. On lighter land, buck-wheat has also been strongly recommended $\dagger$. It is however the opinion of many men of experience that lucerne should always be sown alone, as it requires great care and nursing during the first season, and its future growth may be injured by its being sown in conjunction with any other crop. If danger of that be apprehended, then certainly the practice is injudicious; for as the lucerne stands many years upon the ground, and the corn being then always a slender crop, the value of the grain sown along with it will be trifling in comparison with the loss which may be thus sustained. What. ever plan may be adopted, the ground should, however, he ploughed to the utmost depth of which it is susceptible; and indeed, if hands can be got, the preferable mode is to dig the land to a proper depth. It will thus be sufficiently loosened, as well as brought into fine condition; and although the expense may startle persons who are unacquainted with the eflects of spade husbandry, yet, if well performed, we venture to say that the additional produce will more than pay the cost.

The only sort of seed sold in the shops is that called "French lucerne," though in France several varieties are distinguished which may be worthy of attention. The seed is covered with a hard compact coat, and if dry weather should set in after it is sown, it is very long before it vegetates; it may therefore be not unadvisable to steep it in milk and water, made more than blood warm, for about six hours. The quantity of seed usually sown is from 14 to $18 \mathrm{lbs}$, if broad-cast, and about 10 if drilled; but considering the accidents to which it is exposed, and the uncertainty of

* Von Thaër, Principes Raisonnés d'Agric. 2nde edit. tom. iv. \$. 1327.

+ Von Thaër says that long experience has convinced him that lucerne grows in every case both more erjually and stronger after being sown along with flax or buck-wheat than with any other kind of grain; and that they leave the land in cleaner condition. The plants of flax should however he pulled with precaution, to avoid dislodying those of the lucerne: the buck-wheat may either be allowed to stand for seed, or cut when in blossum; but the latter plan is the best.-Prin. Rais. d'A gric. 2nde edit, tom. iv. p. 438. 
procuring perfect seed, together with the expediency of raising a thick sward of succulent rather than coarse plants, we do not hesitate to recommend the practice of those farmers who use full one-third more ${ }^{*}$. It should be only lightly covered with the harrow, at a depth not exceeding two inches, and afterwards rolled. The months of March and April are the customary periods of sowing, and if in the course of that, or the following seasun, any spots should appear bare of plants, they should be filled up by transplantation as often as necessary, either from parts where the growth is more luxuriant, or from a small nursery-bed kept for that purpose: the operation may be performed either in the autumn or the spring, after the ground has been soaked with rain.

The mode of treatment in the subsequent years of its growth requires nothing more than careful attention to the destruction of weeds, - especially root-weeds, - both by the harrow and by hand-weeding, for if that be at all neglected, they will soon render the crop unproductive. It is, indeed, seldom suffered to be left upon the ground beyond eight years, because of the great difficulty of preventing it from being choaked within that time, thourh there are numerous well-attested accounts of its being still standing in perfection during a much longrer period. Every second year it should have a top-dressing of ashes or soaper's-waste, and gypsum has been laid on every year with goorl eflect; but dung, though much used, is not to be recommended, both as rendering the plants so tender as to injure their future growth, and also filling the land with weeds. If these dressings be not neglected, and if the cleansing of the land be carcfully attended to, the crops will every yeai become better, for althourh the grass may not stand so hinh, it will be closer at the hottom, and the herbage becomes more nutritive.

It is somer realy for cutting than any of the grasses, in a favourable season, perhaps so early as abont the middle of April, at which time it will probably have reached to full two feet, or even more, in height; but it should be cut before it comes into blossom, as it will then push forward its new shoots with greater promptitude and vigour, and during the remainder of the year it may generally be again cut three or four times, at nearly equi-distant periods, until the end of October.

It is marle into hay in the same manner as sainfoin ; and, while yet in a very green state, it has been sometimes stacked upon the prospect of rain, by placing it with alternate layers of dry oat-straw, which have thus prevented it from heating, and have absorbed so much of its superabundant moisture as to render it very palatable to working horses when cut into chaff $\dagger$.

When meant to stand for seed, it is generally only during the last year of its remaining upon the ground that it is reserved for that purpose; and then, only the first crop is cut before it comes into blossom. The ripeness of the pods is easily known by their clasticity, as they will fly open upon being handled: they should be cut of with a shiarp sickle, and then placed upon cloths and spread out in the sun to dry-the haulm being afterwards mown and used as straw. When sufficiently harlened to be thrashed, that is effected by putting them in bags; and the seed is afterwards passed through various sieves, much in the same mamer as that of clover, but the process is not so troublesome, and the produce is less: the major part of the seed, however, comes from abroad.

Among the casualties to which lucerne is subject are the mildew, which soon changes the plant, both leaves and stalk, from a light lucid green to

* In the statement mentioned in the note at the head of this article, $251 \mathrm{lbs}$, were considered preferable to $18 \mathrm{lbs}$., although the seed was drilled.

+ Malcolm's Mod. Husb, vol. iii. p. 72. 
an almost dingy white ; and, in a very dry time, caterpillars are found to attack it in swarms, commencing with the head and tender shoots, and then eating downwards until not a leaf is to be seen : in either of which cases the most prudent plan is to cut it immediately down, as it will then produce new shoots.

The application of the crop is almost exclusively confined to the purposes of soiling, for it is rarely made into hay, hardly ever saved for seed, and seldom pastured, except for the summer feeding of cows, whose milk it is thought to improve both in quantity and quality*. Of hay the different cuttings have, collectively, been known to produce as much as four tons per acre, and of green crops there are well-authenticated accounts of eleven acres having supported twenty-three farm-horses in perfect condition, during twenty weeks, without any other food; as well as one horse being kept to constant road-work, when soiled upon less than a rood of lucerne from the 24 th of May to the 20th of Novembert. We are, however, convinced that nothing near that quantity can be assumed as an averagre, though it certainly affords such a powerfil aid to the maintenance of stock in parching summers, and comes so early into use, that we think no farmer who is in possession of land adapted to its growth should be without a few acres of it. It has been even said to have sufficient proof to fatten bullocks; but if any reliance is to be placed on the experiments of the late Mr. George Sinclair and Sir Humpliry Davy, that opinion of its powers is doubtless exaggerated; for on reference to their account of the comparative value of grasses, 1000 parts of the different species were found to contain-

\begin{tabular}{|c|c|c|c|c|c|}
\hline & $\begin{array}{c}\text { Whole quantity } \\
\text { of nutritive } \\
\text { matter. }\end{array}$ & Mucilage. & $\begin{array}{l}\text { Saccharine } \\
\text { matter. }\end{array}$ & Gluten. & $\begin{array}{l}\text { Insoluble } \\
\text { ma!ter. }\end{array}$ \\
\hline Red Clover & . .39 & 31 & 3 & 2 & 3 \\
\hline White ditto & - 32 & 29 & 1 & 3 & 5 \\
\hline Sainfoin & . 39 & 28 & 2 & 3 & 6 \\
\hline Lucerne : & . 23 & 18 & 1 & - & 4 \\
\hline
\end{tabular}

from which it would appear that lucerne is inferior to both clover and sainfoin + .

* After being kept upon lucerne for about ten days, the milk of three cows was each measured by itself, and the produce, in Scotch pints, was, on the 28 th of May, as under :-

No. 1. Calved in March, and gave 13 pints.

2. Ditto January, ditto $10 \frac{3}{1}$,

3. Ditto May, ditto 10 ,

They were then put alternately upon pasture and lucerne during the following periods, when the produce was found to be-
Pasture.
Lucerne.
Pasture.
Lucerne.

To 8th June.

To 13 th June.

No. 1. $12 \frac{1}{2}$
2. $9 \frac{1}{4}$

3. $10 \frac{1}{2}$

$\begin{array}{lc}12 \frac{3}{4} & 10 \\ 10 \frac{3}{4} & 9 \frac{1}{4} \\ 10 & 9\end{array}$

To 19th July.

$\left.\begin{array}{c}11 \\ 10 \\ 8 \frac{8}{4}\end{array}\right\}$ Pints.

The butter was of the finest quality.-Trans. of the Highland Suc. N. S. vol. ii. p. 119.

† Von Thaër, Princ. Rais. d'Agric. 2nde edit. tom. iv. p. 446. de Crud. note to ditto,

ib. Comm. to the Board of Agric. Ann. xi. Trans, of the Hd. Soc. N. S. vol. ii. 10. 120. See also Pictet, sur la Consommation de la Lucerne en Vert, in which there is a very interesting account of its growth and extraordinary jurduce during four consecutive years upon a poor but calcareous, gravelly soil, cultivated under a previous rotation of
1. Potatoes
2. Barley, dunged
3. Clover

4. Wheat and rape

5. Potatoes

6. Wheat, dunger?

I See the Complete Grazier, on the comparative valne of some grasses and roots as food for cattle, 6th edit. book ix. chap. vi. p. 534. 


\section{Chapter XXVI.}

\section{ON THE SEED OF COLE-MUSTARD-AND POPPY.}

Azthougu rape and cole are so nearly of the same nature as to usually pass under the same denomination, and when used for the expression of oil, and the formation of cake, they are indifferently employed for that purpose, yet there is a distinction between the plants, which is generally made when they are intended for the food of cattle: in which case rape is the sort most frequently grown, as it does not require land of such good quality as the other, and may be sown later in the season, while it ripens earlier*. The seeds of these plants are chiefly used for the production of oil, which they yield in very large quantities.

COLE

Is stronger on the stem, its flowers are of a clearer and lighter yellow than those of rape, and both the pods and seeds which it bears are larger. The cultivation of both species is conducted in the same manner as that of turnips, but they admit of being sown later, and when the plants are intended to be left for the production of seed, the soil should be of a richer kind : cole is consequently much grown in the alluvial districts of Essex, Cambridge, Huntingdon, and Lincolnshires, and on such land produces very heavy crops of seed, when sown in the summer or the early part of autumn, - say from the middle of July until the middle of August, - so as to ensure their ripening in the course of the following season. The crop is also commonly eaten down by sheep at an early period of its growth; but no stock of any description should be left upon it after the month of January. If weeds spring up early in the autumn, before the crop may afford i bite for sheep, it will not be found unadvisable to run the scythe across them; for although the tops of the plants may be thus cut, they wili not be injured, and the expense will not be thrown away, for if the produce be raked up and given while green to cattle, they will eat it with

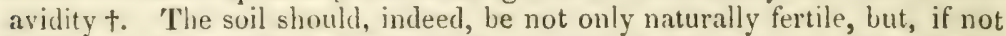
broken up from a maiden state of fen or pasture, will require a large dressing of spit-dung; for unless the land be in high condition it will we greatly impoverished by the production of the crop, which will also be poor, and thus a double loss will be incurred by the saving of manure.

In Flanders, where the culture of rape is more largely followed and better understood than in any other part of Escope, the quantity of manure usually employed consists of a large quantity of bruised rapecakes, and eighteen cart-loads of night-soil ; but the general and approved method is there by transplanting, to which they attach the advantages of the seed-bed occupving but a small space, whilst the land is carrying a crop of corn, and while the plants are growing, of having time to harvest therr corn, and to plough and manure the stubble intended for the rape. The process is as follows:- "The seed-bed is sown in August, or even to the middle of September. In Octolier, or sooner, the stubble is ploughed over, manurerl, and ploughed again. The plants are dibbled in the seams of the ploughing, - each furrow-slice being twelve inches broad,-and are

* See chap. xix.

† Von Thaër, Prin. Rais. d'Agric., 2nde edit. tom. iv. p. 253. 
set out at twelve inches distance in the rows. Instead of dibbling upon the second ploughing, they in many cases lay the plants at the proper distances across the furrow, and as the plough goes forward the roots are covered, and a woman follows to set them a little up, and to give them a firmness in the ground where necessary. Immediately after the frost, and again in the month of April, the intervals are weeded and hand-hoed, and the earth drawn up to the plants, which is the last operation till the harvest. The crop is pulled rather green, but ripens in the stack, and is thrashed out without any particular management; but the haulm is burned for ashes, as a manure, which are found to be so highly valuable, beyond all other sorts which have been tried, that they bear a price as three to one above the other kinds; and it is considered, that upon clover, a dressing of one-third less of these is amply sufficient*.",

In this country, however, transplanting is only rarely practised, and the crop, being usually thrashed out upon the field, is always left to stand until it is perfectly ripe; the harvest should, however, be cornmenced as soon as any of the pods become quite brown, for they do not ripen equally, and if left too long they are very apt to shed the seed ; the average produce may be estimated at about twenty-eight bushels $\uparrow$, but upon the rich fen-lands it is frequently as much as five quarters per acre + . If the seed be large, black, and free from red ones, it may be reckoned good; but, if kept long before it is sold, it should be laid very dry, or otherwise it will lose its colour, and be much damaged.

\section{MUSTARD-SEEDS,}

Although partaking of the same nature, are of two species, commonly known as white and brown mustard, and are distinguished not only by the difference of their colour, - which in the one is of a whitish yellow, and in the other a brown, approaching to black, - but also by the pods of the former being prickly, while those of the latter are smooth. Both produce oil of very good quality, and quite free from any pungency of taste; but the latter is the kind which, when ground into powder, is used at our tables as the condiment distinctively termed " mustard."

The soil, for both sorts, should be rich, but especially so for the brown species, which is rather extensively grown in Fauhess and Wallasea Islands, and some of the strong-land hundreds of Essex; but large crops of the white kind are grown upon light loams. They may be advantagreously grown after wheat, provided the land be got into fine order, and is well manured; after which it should be hoed two or three times, and managed much in the same manner as cole; the brown species, however, strains the land more than the white, and as some of the seeds are inevitably shed upon the ground during harvest, they afterwards spring up, and are extremely difficult to eradicate.

The quantity sown is usually about a peck and a-half per acre; but good crops have been obtained from only two quarts $\S$. If not drilled, the plants should be set out at the distance of about ten inches from plant to plant. The brown species is not uncommonly sown some time in February; but the white is much more tender, and is, indeed, so ticklish,

* Radeliff, on the Agriculture of East and West Flanders, p. 48.

+ Lissex Rep. vol. ii. p. 28.

+ Surveys of Lincolnshire, 2nd edit. p. 159. Suffulk, 3rd edit. p. 94.

Essex Report, vol, ii. p. 63 . In the Bath papers, a bushel is stated as the quantity, vol. ix. art. xiv. 
that one frosty night may kill it: it is therefore seldom put into the ground until the latter end of March, or the beginning of April, and we have heard of a capital crop which was not sown until the 25th of May. When sown thus late, it is, however, more subject to the attacks of the black caterpillar and the fly.

The harvest takes place about August, and the brown sort is reaped and tied in sheaves in the same manner as wheat; but the white is laid in "gavels" or handfuls upon the stubble, and both are thrashed out upon cloths in the field, in the same mode as cole-seed. Care should also be taken to watch the first ripening of the pods, and then the crop should be cut without delay.

The average produce may be estimated, upon good land, at 28 bushels per acre; but rich soils not unfrequently grow as much as five or six quarters, and the cwt. of grain yields proportionably more oil than that of cole. The cake made from the expression of the oil is said to have a slightly purgative quality*; wherefore, though it may be in that respect in many instances very wholesome, yet it should be cautiously given to cattle, and is therefore usually ground and sprinkled upon their chaff.

\section{POPPY,}

Although little known in England, where it is only cultivated in plyysicgardens for the heads, is yet extensively grown in Flanders for the production of the oil expressed from its seeds, which is of a quality so superior to that of any other kind yielded by our common field crops, that, when cold-drawn, it is very generally used throughout the Continent as salad oil; and when burned in lamps, it affords a brilliant light peculiarly free from smoke $t$ : the cake is also extremely nutritive. It may therefore be worthy of attention to those possessed of those deep sandy loams to which it is adapted, to whom some account of its culture, as stated by Messieurs Radcliff and Von 'Thaër, may not prove wholly uninteresting.

The former states- "that the crop is generally taken after rape, for which the ground has been plentifully manured, and for which the 'oillette, as it is there called, receives in the ensuing year a dressing not less abundant. The land which has been so highly cultivated, and enriched for the crop of rape, is in such good tilth upon pulling the rape-stalk, that two deep ploughings are sufficient to prepare it for the receptiun of the seed. Upon the first ploughing, harrowed flat, liquid manure is spread, at the rate of about 3500 gallons to the English acre; and this being ploughed in, in sets of ten feet, the seed is sown at the rate of one gallon to the acre, being afterwards lightly covered by shovelling the furrows.

" The harvesting is performed in a particular manner, and requires a great number of hands. The labourers work in a row, and sheets are laid along the line of the standing crop, upon which, bending the plants gently forward, they shake out the seed. When it ceases to fall from the capsules, that row of plants is pulled up, and placed upright in small sheaves, in the same or an adjoining field, in order to ripen such as refused to yield their seed at the first operation, and in two or three days the operation is

*Von Thaër, Prin. Rais. d'Agric. 2nde edit. tom. iv. p. 27 ].

\$ The oil, when expressed, should remain for the space of five or six weeks hefore it is used, that it may deposit its sediment. It should then be poured into another vessel, which should not be perfectly closed; nor should the oil be immediately used, as it continues to improve for a considerable length of time.

When mixed with a small quantity of superfine olive oil, the peculiar taste of that oil is communicated to it; and it corrects rancidity. 
repeated, or, if any seed then remain, it is extracted in the barn by the flail."

It is, however, evident that the latter operation may be performed by old men, women, and children, who are not capable of other harvest work ; and, indeed, Von Thaër says that it is not unusual to commit the whole process to the flail, or else the pods are cut off and the seed is separated by means of the winnowing machine; but the shaking out of the seed by hand is preferable, as preventing any mixture of unripe seed, which would give a bad taste to the oil. Although he also states that the land ought to be rich and clean, yet he does not allude to any peculiar species or particular quantity of manure; and he considers a little more than a pound of seed as quite sufficient for an acre: great care is therefore evidently necessary in the sowing, so as to secure the equal distribution of the seed; for the gaps which may otherwise occur cannot be filled up by transplantation. It may be sown early in March, though autumn is considered the best season. There are two species of seed-one black, the other whitethe former being generally considered as more productive of oil than the white, but not of equal quality. The plants should be hoed out to at least six or even twelve inches from each other. He also mentions that it is not unusual to sow poppy with carrot-seed, as the crop of the farmer is harvested two months earlier than the latter, but this occasions a difficulty in the hoeing.

Mr. Radcliff estimates the average produce at about thirty bushels the English acre, and says, - " the seed is not so productive as rape, in point of quantity, but exceeds it in price, both as grain and as oil, by at least one-sixth; the measure of oil produced from rape being as one to four of the seed; that produced from the seed of the oillettes being as one to five." Von Thaër states that an English acre of good soil may be expected to produce at least forty gallons of oil *. It will, however, be seen from the following experiments, that there is much discrepancy in the accounts given of its product:-

In one reported to have been made in Holland upon about two acres of land-half a sandy soil, and the other half a heavy peat-the produce of the former was rather the largest of the two; but the difference was so trifling, that the seed was mixed, and yielled together, as nearly as it can be calculated, about 100 gallons of oil, two-thirds of which was cold-drawn.

From some trials made in Wiltshire-one of which was honoured with a premium-it would however appear that a good crop only amounted to 16 bushels, of 45 lbs, each, per acre, and an indifferent one to about half that quantity : taking therefore the average at 12 bushels, they yielded close upon is gallons of oil.

By an experiment made in Cambridgeshire, it was found that $3 \frac{1}{2}$ bushels, weighing 1 cwt. 2 qrs., produced 6 gals. $2 \mathrm{lbs}$. of oil, $-7 \frac{1}{2} \mathrm{lbs}$. to the gallon, and 3 qrs. 25 lbs. of cake; but the account does not state the produce per acre of the seed.

The cakes are considered equal in the feeding of cattle to those of linseed $\uparrow$.

* See Radeliff on the A grriculture of East and ITest Flanders, chap. viii. sect. v.; and Von Thaër, Prin. Rais. d'Agric., 2nde edit. tom. iv. $\oint 1175$ to $\$ 1182$.

$\dagger$ See Papers of the Bath Society, vol. x. art. xxviii.; vol. xiii. art. ix.; aud vol.xi. p. 258. 


\section{Chapter XXVII.}

\section{ON FLAX AND HEMP.}

THE plants under this head are both productive of yarn for the manufacture of cloth and ropes, as well as of seed; which, when crushed, yields large quantities of oil and cake of a very rich quality, in the same manner as cole-seed. The former is very extensirely cultivated in the north of Ireland, where it is grown for the purposes of the linen manufacture; both the soil and climate being peculiarly adapted to it, and by the employment which it affords, both in the cultivation of the crop, the preparation of the yarn, and the weaving of the cloth, it has become the great source of the superior prosperity of that portion of the country*. The growth of hemp is, however, there but little attended to ; and is here almost confined to the low lands of Cambridge, Huntingdon, and Lincolnshire, with some of the most fertile parts of Somerset and the East Riding of Yorlishire: in the two latter counties it has, however, been of late years partly abandoned, though it undoubtedly might be grown with advantage upon any well-drained bogs or peaty soils. The cultivation of both plants is, indeed, considered highly profitable to the farmer, and would doubtless be greatly so in a national point of view were it further extencled; but, independently of the want of fitting soils in many districts, there are the weighty objections of their not returning any manure to the land, and of occasioning both more expense and difficulty of management than is at all times convenient.

\section{FLAX}

Is an annual plant, indigenous to this climate, and though possessing many varieties, which are spread over almost every part of the globe, yet only that species known as the common flax is liere generally sown; it grows with a slender upright fibrous stem, from which the yarn is made, and bears clusters of small blue flowers. The soil to which it is most appropriate is a rich alluvial or sandy loam, or a loose marle, neither too wet nor too dry. U' Upon poor clays, or dry gravelly soils, it will not thrive; and if sown upon any land of an inferior kind, the ground should be abundantly dunged for the precerling crop, so as to bring it into a productive state previous to the reception of the flax-seed; for, otherwise, manure spread at the time of sowing will have comparatively but little effect. The land should, indeed, possess nearly the texture and firmness, the depth and degree of moisture, suitable to good wheat land, and the cleanmess of a welltilled summer-fallow; but it should not be too rich, or the crop will be coarse, and apt to become lodged, and large quantities of flax are grown upon soils of a medium quality.

It is sown in the common rotation, both before and after corn and other crops, but the favourite plan is to put it in after newly broken-up maiden pasture t. There is no crop for which the land should be better worked, and if the stems be pulled up for the sole purpose of producing yarn,

* Lambert's Oliservations on the Rural Affairs of Ireland, p. 119.

+ Upon newly broken-up marsh land the following course has been followed:namely-

$\begin{array}{llll}1 \text { Flax } & 3 \text { Potatoes } & 5 \text { Rape } & 7 \text { Flax } \\ 2 \text { Rape } & 4 \text { Flax } & 6 \text { Potatoes } & 8 \text { Wheat; }\end{array}$

and this, it is scid, it will centainly bear for ten years.-Linculnshire Rep. 2nd edit. p. 125. The effect of this, however, is not stated; and flas ought cortainly not to be frequently repeated on the same ground. 
without allowing the seed to ripen, it does not exhaust the soil*; though it is by many thought a bad preparation for wheat, and perhaps would be better followed by spring corn or peas + . In Flanders, however, where the cultivation of flax is as well understood as perhaps in any part of Europe, wheat is generally made to succeed it, and it is there very profusely manured with night-soil + : it is also generally preceded by turnips, though many intelligent farmers disapprove of sowing it after them; and, in Scotland, the best plan that has been yet discovered is to sow oats as a ley crop, and flax after it $§$. C pon much land in England and Ireland it is, however, sown upon one ploughing after a clover ley, which is better for the purpose if it has lain two years. 'The land is in this case broken up about Candlemas, after which it gets three or four harrowings, and is rolled previous to the sowing. The seed is then lightly harrowed in, and rolled rather heavily if the ground be dry, but, if it be very tenacious or moist, this may be omitted. Clover seed is also sometimes sown along with that of flax, to form a succeeding crop; but, in that case, it is better to defer the sowing of the former until the time when the flax is weeded, when it may be eflectually loosened, or, at all events, they should be sown separately. The period of sowing is, in this country, as soon as possible after the first week in April, early sowing being considered advantageous to the quality of the flax; but, in the North, they are not unfrequently obliged to defer it until the beginning of May f.

The seed is always sown broad-cast, as the object is rather to produce a thick crop of plants which, standing closely together, will bring up straight stems better adapted to the growth of yarn than if they were allowed to branch out. 'The quantity sown is therefore large, and is generally as much as three bushels per acre; though many intelligent farmers use only twothirds of that quantity, which, considering the price it usually bears, is still a very serious expense.

The chief portion of the seed sown throughout the kingrlom is imported from abroad, either from North America or from the Baltic, and a considerable quantity from Holland; for, whether arising from inattention to the proper mode of rearing it, or from defects in our soil or climate, it is an incontestable fact, that seed produced in those countries is superior to that reared in England. It is indeed a generally-received opinion that the seed should be frequently changed, as the plants, if grown from the same species, evidently become every year shorter. Throughout Holland and Germany the growers therefore uniformly import seed from Russia, which is grown in the provinces of Livonia, Courland, and Lithuania, and to this country it is brought under the name of "Riga-kind." To guard ageinst imposition it is there carefully marked by inspectors appointed by government, who brand the casks in which it is packed in order to distinguish the old from the new seed; for although that of a former year may, if it be well preserved, be sown with some degree of safety, yet it is an experiment which should never be resorted to unless in cases of extreme urgeney; and any one selling old for new seed is liable in damages to all loss arising from a failure in the crop. To have it of good quality, fresh

* Lambert's Observations on the Rural Affairs of Ireland, p. 119.

$\dagger$ Von Thaër, Prin. Rais. d'A gric. 2nde edit. tom. iv. p. 282.

F On the manner of manuring and sowing the flax, ses the account of liquid manure in rol. ii. chap. xi. p. 270.

$\S$ Aiton on the Cultivation of Flax in Scotland,-Qnart. Jour, of Agric. N. S, vol. iv. p. 163.

|| Lincolushire Rep. p. 189. 
and fit for sowing, "it should be smooth, slippery, bright, plump, and so heavy as to sink in water; it should taste sweet, and, being broken, it should appear of a light yellowish-green colour, and oily; the weight about 21 lbs. per peck, Linlithgow measure*."

The treatment of the crop is, in the first instance, confined to careful hand-weeding, which is generally performed at an early stage of its growth by women, who frequently pick out the weeds upon their linees, to avoid pulling up or injuring the young plants of flax; and afterwards it depends upon whether the plant is to be pulled merely for its fibres, or allowed to stand for the production of both flax and seed. The plant blossoms in the course of July, and is then pulled, when the object is merely the flax; but when kept also for seed, it should be allowed to stand until perfectly ripe, or some time in the latter part of August.

When pulled, care should be taken to sort it separately-the long and coarse plants by themselves, - so as to keep every kind and quality distinct, as they require different modes of dressing, according to the uses to which they are to be applied; and inattention to this point will lower the value of the crop. It is then bound in sheaves about the thickness of a man's thigh, and is either "dew-retted," or "dyked," or else "water-retted," operations on which the price of the flax more depends than any other which the grower has to perform; the object being to loosen the rind and separate it from the stalk.

The process of water-retting is, in the first case, generally preferred. The sheaves are in that case immediately immersed during several days in standing water, which is done by placing the flax sheaf upon sheaf, direct and across, trealing it at the same time into the pit; and after it lias been heaped to within about six inches of the surface, it is strewed with rushes, straw, or any coarse herbage, and loaded with blocks of wood or stone, to keep them down. The depth and choice of the water, and time of steeping, are however matters of more importance than are generally imagined; for if too deep its action upon the surface and at the bottom will not be equal; if hard, or taken from a rumning stream, it is found to injure the quality of the flax; and the time of drawing it from the water depends both upon those circumstances and upon the state of the weather.

The watering of flax being a partial rotting through fermentation, the depth of the dyke should never exceed six feet; and from three to four feet, it is by some persons supposed, would be still better, as the power of the atmosphere must for that purpose be necessary. The water should be clean, but stagnant, and free from any mixture with mineral mattert; neither should it be shaded by trees, as the sun and air should freely act upon it; nor should there be fish in it, or they will be poisoned. No exact time can be fised for the fax remaining in the water; for it has in some cases been found sufficiently steeped in five days, and in others it has remained ten days and even longer without injury. In this latter process great skill and attention are necessary; for if it be left in the water too long, the threads become soft, weak, and comparatively useless to the manufacturer; wherefore it is generally thouglit more advisable to take it out too soon, than to leave it too long

* Directions of the Board of Trustees of the Fisheries and Manufactures of Scotland, p. 8.

+ A circumstance is stated in the Quarterly Journal of Agriculture of two persons having divided a large field of flax between them, the crop of which they steeped in different qualities of water: one being soft and free of minerals, and the other of an oppusite quality. The consequence of which was that the lint of the former sold for 30 per cent. more than that of the latter, - N. S, vol, iv. p. 175. 
in the pits*. Care is however so necessary on this important point, that a day or even a few hours too much or too little may occasion injury ; but it is said, on the authority of a Dutch boor, "that when the bubbles of air disappear from the surface of the water, and the flax seems to have settled to the bottom, it may then be concluded that the operation is nearly finished:" or, to avoid mistakes, "some of the stalks may be broken at about four inches distance, and if the heart of the stem can be easily drawn out of the bark, or lint, then it is time to remove the flax from the pond; but, if it still adheres to the boon, or pith, it must be continued in the steep until they are found to part freely + ."

When removed from the dyke, the sheaves are either unbound, and spread upon close-fed grass-]and to dry-the manner of placing them being to put them so closely together as to cover the sward entirely, but thinly; or they are laid on the margin of the pond for a few hours to let the water drain off; afterwards each sheaf is drawn up towards the top, and they are then set up on end, or "goited," in the immediate neighbourhood, to admit the air. The former is the preferable practice; for a shower or two of rain will cleanse the flax, while the action of the sun serves to bleach it, and it should, therefore, be always adopted where there is abundance of grassland for the jurpose. It may indeed be remarked, that the drainage of the flax is beneficial to the grass; and the water remaining in the dykewhich is rendered insufferably putrid by the fermentation of the vegetable matter which it imbibes - has been found to possess very fertilizing qualities.. Whichever plan may be adopted, the flax should be first thoroughly dried, after which it is either put into the barn or stacked; and, if it should be in the least damp, it ouglit to be dried upon kilns gently leated $\S$.

An improved mode of watering has been invented by a member of the Agricultural Society of Ghent, which difiers from the common usage in four points :-

1st. In placing the bundles in the steep vertically, instead of horizontally.

2 dly. In immersing the flax by means of transverse sticks, with that degree of weight annexed which shall not push it to the bottom, but leave it the power to descend spontaneously towards the conclusion of the steepage.

3dly. By leaving at first a space of at least half a foot between the bottom and the roots of the flax.

4thly. By renewing the water at stated intervals.

Comparative experiments having been made between this and the former method of steeping, a very decided advantage was shown to result from the latter. The process, and the observations upon it, would occupy more space than we can afford; but a very minute detail may be found in the Appendix to Radcliff's Account of the Agriculture of East and West Flanders.

* Billingsley's Surv. of Somersetsh. 3rd edit. p. 214.

$t$ Aiton, on the Cultivation of Flax in Holland and Scotland.-Quart. Journ. of Agr. N.S. vol, iv. p. 177:"

+ Mr. Billingsley says, in his Survey of Somersetshire, that he has found the effects of water in which flax had been steeped, when applied to pasture lands by wateringcarts, to advance the land in value ten shillings per acre. He indeed considers it superior to animal urine.-3rd edit. p. 215. In Yorkshire also similar effects have been observed.-_ee Middleton's Ann. vol. xiv.

"In Hullaud and Flanders this practice is generally adopted in drying-houses built of brick and about 15 feet long, having a place for fuel about $2 \frac{1}{2}$ feet underground, so contrired as to send a gentle heat through the liln, and yet to keep the fire from reaching the flax." - Aiton on the Cult, of Flax in Holland, Quar, Jour, of Agr. p. I78. 
Dew-retting is sometimes resorted to instead of water-retting, and is carried on immediately after the flax is pulled. It consists in merely spreading the plants upon a grass-sward for perhaps four or five weeks, and occasionally turning them until they are rendered sufficiently brittle for the separation of the fibre from the bark, and the purposes of further manufacture. 'The success of the operation depends lowever so much upon the weather, that it cannot be relied upon, and it is therefore but seldom employed, unless when the crop has been left to stand for seed; but either plan may be adopted according to the judgment of the grower. Indeed, on the Continent, it is customary to dry the flax upon the ground in the same manner as corn, previous to steeping it, and the watering is not performed until the following spring, after the seed has been taken from it.

The preparation of the flax consists in "breaking" or bruising the stem, in order to separate the fibre from the bark, and then "skutching" or cleansing it from refuse; after which it is "lieckled," as the final preparation for market. These operations are now so very generally performed by machinery that a detailed description is almost unnecessary ; but as some cottagers employ themselves during the winter in effecting the two first, it may not be entirely useless to mention that they are in that case done by hand. The bruising apparatus, or "brake," is simply formed of three or four triangular planks about 15 inches long by $\mathbf{3}$ inches broad, fixed horizontally to a frame, with their edges uppermost, at about $2 \frac{1}{2}$ feet from the ground; to one end of the frame two or three similar boards, of the same dimensions, and connected together, are fastened to a moveable hinge, so as to raise or sink them at pleasure, and being placed at intermediate distances between the fixed planks, they dovetail into each other as here represented :-

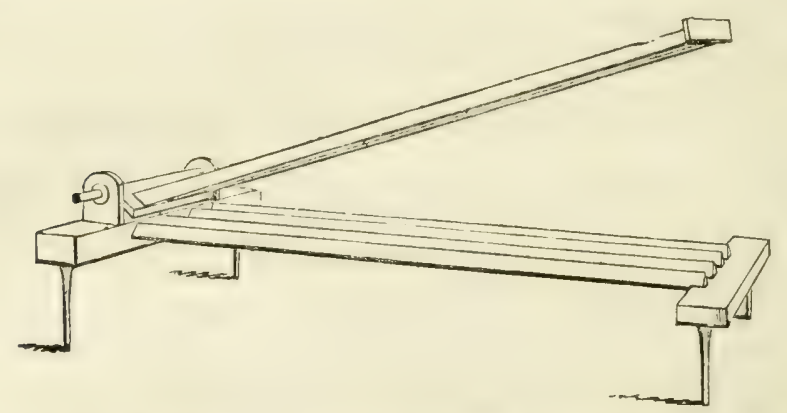

When at work, a handful of the dried stems is laid across the under planks, and the upper ones being forced down upon them, the flax is crushed by repeated strokes of the machine, until it is ready for scutching. This is done by handfuls of the bruised flax being either beaten upon a board placed edgewise ; or rather a niche is cut in the board to the size of a man's wrist, into which the scutcher alternately throws each end of the flax, which he beats with a light mallet, repeatedly turning and drawing it through the niche until by these means it is entirely cleaned and prepared for heckling. The heckler uses numerous iron teeth fixed upon a board, through which the flax is drawn repeatedly; and by employing different sets of teeth, he is enabled to sort the flax into various lengths; the refuse being tow. This latter operation can, however, only be properly performed by regular workmen.

When intended for the production of seed, the plants should not be pulled 
until they are quite ripe, when the pods have become yellow, and the leaves have withered and begun to drop. The buds are in this country usually removed when the flax is taken out of the ground-consequently, while it is still in a damp state; by which means the seed is very materially injured, as it is almost impossible to dry it regularly. In Flanders the plants are, on the contrary, always dried like standing corn, before they are separated from the seed, and the flax is afterwards retted.

In Ireland a flax-barn is very commonly used; which was invented some years ago by Mr. Tennant, who was employed by the Linen Board in teaching a new process of bleaching linen, and was suggested for the preservation of the seed. It is constructed on wooden posts roofed on the top, but left perfectly open at each side, and is supplied with various stages or floors, of basket work, placed regularly at two feet distance from each other. Thus the air, having free access to the seed on all sides, preserves it fresh and well-coloured for any length of time *.

The seed is separated from the stems by a process termed "rippling," which is performed with an instrument formed of a flat board of a moderate size, with iron teeth fixed at the end like a comb. The implement is fastened to any standing block, and the stems are repeatedly drawn by hand through the teeth until they are completely cleared of the grain. The average produce of the whole crop may be about 40 to 50 stone of flaxthat pulled young bearing a superior price, as being better adapted to the manufacture of the finer kinds of cloth; and the seed may perhaps vield about 10 bushels per acre. It is generally sorted into parcels of different qualities - the best being reserved for seed, the next for crushing, and the most inferior for the feeding of cattle; but it is all sold under the name of linseed.

A very claborate, and, in many respects, valuable account was some years ago furnished to the growers of flax, by Mr. Durno, the British Consul in Prussia, respecting the culture and manufacture of the plant, as practised in the states of Prussia, Poland, and Russia, which may be found in Vol. I. of the "Farmer's Margazine;" and an abstract of the rules of management adopted in Ireland, for the cultivation and dressing of flax, having been transmitted to the Lords of Trade, the paper was submitted by the Linen board to the surveyors of flax employed by them, whose report upon the subject we insert at foot + .

* Farmer's Mag., vol. vii. p. 38.

$f$. A good crop of flax may be expected from any strong clays which are fit for the growth of corn. Potatoes generally precede it; but any manured crop, if kept in fine tilth, may be considered as good preparatives. On light lands that have been long in tillage, the crop seldom comes to maturity, or is sulject to a blight which renders it scarcely worth manufacturing.

2. About two bushels of seed to the English acre is a proper quantity, except for the purpose of a very fine manufacture. For this the flax is to be pulled in a very green state; and four bushels to the English acre are in that case necessary. A sure method of trying the quality of the seed is hy sowing some in a hot-bed. The most approved season for sowing is the first fine weather after the middle of March.

3. The best mode of tillage is in beds of about six feet broad, covering the seed about one inch and a half deep, with what earth may be shovelled from the furrows. These furrows are useful in carrying off' under-water, and in giving air to the crop during its growth; but the tops of the ridges should not be laid round, as that would carry off the summer rains too quickly.

The crop should stand till the lower part of the stalk gets a yellowish cast, and the unter leaves begin to wither; except when the flax is designed for an extraordinary fine manufacture; or when a blight, or " firing," has made its appearance on any part of the crop, in which case, it will be better to gull it in a green state.

VOL, II. 
HEMP,

although applied to nearly the same purposes as flax, and requiring much the same sort of soil, is yet a plant of different appearance and greater

It will be worth attending to in the pulling, to take all the ripest and strongest grown flax first; and leaving it in small hanufuls across each other, (but with the ends con. taining the seed to the south.) to proceed to pull that of inferior growth. By this means the crop receives a sorting, in the first instance, which will be conducive to the perfection of the manufacture in every future part of the process. The weeds must also be carefully taken out, and the dirt shaken from the roots.

4. Immediately after pulling, the flax may be steeped. Unless the seed is to be preserverl; in which case it is to be rippled, to take off the seed-pods. Turf bor-water, which is not muldy, is found to answer very well for the purpose; but foul, stagnate, water gives a stain to the flax, which can never be bleached out. Too pure a spring, or sharp running water, limestone waters, or those impregnated with minerals, are equally injurious. The best water is that of a reservoir dur in clay, or the sides of which are lined with clay. Such a pit, if four feet deep-which it should not exceed-sis feet bruad, and forty feet long, will contain the produce of an English acre. The water should stand in this reservoir about a fortnight before the flax is put in; which must be evenly stored, in sheaves loosely tied, that the band may not prevent the effects of the water on that part.

The root-ends should stand highest, and the whole must be perfectly immersed, and kept down hy bundles of straw, or hurdles; as all that is above grounil will be stained. The time which it is to lie in the steep depends on the softness of the water, and the state of the weather. In good water, and warm weather, four days produce the effect, which, in other circumstances, does not happen under twenty; but it is safest to examine the flax on the fourthday. After steeping, it should be spread even and thin, on fine grass ground; for this purpose, close cut after-grass is much recommended, and if it grows, the flax must be constantly turned over.

It is not easy to ascertain the time the crop should lie in this bleaching state; which is intended to produce an even separation of the rind from the case of the stalk. It may be observed, however, that a good exposure to the sun renders less fire necessary to prepare the flax for a future process; if it be not sufficiently dried on the ground, (which is seldom the case in Ireland, ) it is then put over hurdles, and dried with a gentle fire, till it is fit for beetling, skutching, and the other operations of the flax-dresser.

On these observations, the following are the remarks of the surveyors.

1st. As to the soil proper for fax, and the preparation proper for the crop.-We have found from experience that an open, black, loamy soil, euriched by having lain lung in pasture, is preferable to a clay soil, or to any other with which we are acyuainted; and that, either on the first or second crop after pasture, which we have always found better than after potatoes, turnips, or beans.

2ndly. With regard to the best morle of proving the gondness of the seed.-We agree with the writer of the paper, that the best method of trying the quality of the seed is by sowing some in a hot-bed; but it should be observed that it is by no means to be depender on, and therefore should be regarded with caution, for even bad seed if not altogrether spoiled will vegetate, and make a promising appearance in the bed.

3rdly. As to the utility of laying the land in six feet beds. -We are of opinion that the mode here recommenderl may be of great utility in wet clogery ground, for drainingic and bringing it into tillage; but we cannot approve of it for land on which flax is to be raised in greneral; because the mode of laying out land in ridges of twelve to eighteen feet wide is more economical, and better calculated for crops of every kind, as the frequency of the drains occasioned by narrow ridges must deprive the ground of too inuch of its proper moisture.

As to the covering of the seed.-We would recommend brush harrowing, followed by a complete rolling, in preference to the mode suggested in the paper.

4thly. Respecting the sleeping of the fax.-We have some difficulty in assenting to the rule here laid down, "that the root-ends should stand the highest;" for this reason: that the crop ends of the flax, being composed of the smallest filures, require more watering than the other ends; and it is well known, that as the water is warmest and softest near the surface, it there operates the more forcibly.

Lastly. Regarding the operation of the sun on the flax before beetling.-In preference to the mode suggested, of drying the flax on hurdles, or any other way of applying fire to it, we would recommend drying it in an oven heated with the refuse of the Hax, and so very moderately, that a person may easily stand in it. There it should be allowed 
strength; being cliefly cmployed for the manufacture of canvass and the coarser linds of cloth, as well as cordage. It has been supposer to be of Eastern origin, but it, in fact, belongs to the common tribe of nettles, which is indigenous in this country, and is diffused over the whole world. It is an annual plant, marked by the peculiarity of having two distinct species, the flower and fruit growing on separate stems ; therefore known as "male" and "female hemp," the latter bearing the seed, and the former merely containing the pollen by which it is impregnated: they are, however, not unfrequently confounded, the name of male hemp being given to those plants which bear the seed, merely because they are larger, stronger, and more productive than the others. Though both arising from the same seed, yet the male is ripe five or six weeks sooner than the female, and the difference can only be known at the time of blossoming.

Each grain of seed produces only one stem, which grows to the height of five or six feet*, covered with a rough and hairy green bark, containing numerous woody fibres, and from this part, - which is called the "reed," or, not uncommoniy, the "boon,"-is obtained the hemp. The male species is in general smaller and more delicate than the female: the stem divides itself at the extremities into several branches, which terminate in slender spikes ending in a point, the flowers hanging, when they expand, in clusters of a purplish colour pendent from the leaves; whilst that of the female terminates in tufts and leaves of a considerable size, among which we find the seed, which is borne along the stem. The difierence between the sexes can thus be easily distinguished at a considerable distance, and it is a point which demands attention in the management of the crop.

'The soil best suited to hemp is a strong rich loam, and it is more grown in the deep moist rich fens of Limcolnshire and the Isle of Ely than in any other part of the kingdom; for it is always strong in the fibre in proportion to the richness of the ground in which it has been grown. It indeed requires even richer land than flax, and is therefore grenerally grown upon alluvial soils, although they should not be too moist, and a cold wet clay will in no instance answer the purpose; but it is in other places grown upon ground of more ordinary nature, the quantity produced being smaller, though the quality is somewhat finer.

Opinions differ in regard to its effect upon the soil, some considering it as a great exhauster, and others only in case of its being allowed to stand for seed; there are, indeed, some old leases to be found in Cambridgeshire which stipulate that the land shall be sown with hemp in the last two years of the term $t$, while in others there are covenants absolutely prohibiting its growth; but all agree in admitting it to be an effectual cleanser of the land, for it grows with such promptitude and strength, that it destroys all the weeds which spring up under it. It therefore can never be sown along with grass-seeds; but, for the same reason, it is found to be an admirable preparation for a crop of wheat, as the land must be previously brought into a state of garden culture, and, if heavily dunged, when treated in this manner, alternate crops of wheat and hemp have been successively grown upon the same ground during a series of years. In some places it is not

to lie just long enough to take off the acquired damp; and in this state it is removed more easily than by any other means, and the flax is rendered more fit for every after operation.

* A species grown in Alsace has stalks full eight feet long, and the Baron de Crud states it has been grown on his own land, in Italy, to the length of fifteen and even eighteen feet.-Von Thaër, Prin. Rais. d'Agric. 2de edit. tom. iv, p. 289.

† Survey of Cambridgeshire, p. 161. 
an uncommon practice to break up a stubble late in the autumn, and after it has had the benefit of the winter fallow, twenty to twenty-five loarls of spit dung are laid upon the land, which is then sown with hemp, after three ploughings. In the following year an excellent crop of wheat is generaliy obtained, and in the next a good crop of barley or flax, after which hemp is agrain sown, with a similar dressing*

The dressing of the land is the same as for flax. The time of sowing is, however, rather later, as the young plants are more susceptible of injury from frost; the seed should, therefore, not be put into the ground until the latter end of April in our southern counties, and may be deferred without danger until the middle of May in those of the north, or, in fact, until no risk of real frost need be apprehended; but when the plants have once fairly sprung up, nothing is to be dreaded from the effects of white-frost. The earlier it can be sown, however, the better will be the quality of the hemp.

The seed is large, rather soft, and so oily, that large quantities of oil are expressed from it in the crushing mills, for the use of painters and soap manufacturers; the refuse being made into oil-cake. In choosing it, the heaviest, the brightest-coloured, the smoothest, and the roundest, is to be preferred; and, if purchased from a dealer, its being of the last year's growth should be guaranteed, in the same manner as that of flax; for the seed of the second year is not so good, and that of the third will oftentimes not vegetate. The Riga seed is preferred, and it is " bracked," or marked with a brand by surveyors, in the same manner as that of flax.

The quantity sown is usually from nine to twelve pecks, varying with the strength of the soil and the custom of the country; but the most common practice is about three bushels per acre, and the morle of sowing is commonly broad-cast. Drilling in rows, at thirty inches, has been recommended, as presenting the advantages of allowing the intervals to be well tilled, and of admitting air to the plants; but this renders the hemp) coarse; and as the rapid growth of the plants smothers that of all other vegetable matter, weeding is very seldom resorted to $t$, though the plants should be hoed out when they stand too thick.

The harvesting of the crop, if the seed be not taken, occurs about thirteen or fourteen weeks after the sowing of the seed, and commences in August; but the stalks which bear the flower containing the pollen, ripen nearly three weeks sooner than those which contain the seed,- - their ripeness being known by their turning yellow at the top, and white at the root; the flowers also fall, and the leaves begin to wither. This sort should, therefore, be pulled separately, and, indeed, while it is yet somewhat green, as the fibres will be thereby more easily separated, and rendered more suitable to the manufacture of cloth. This separation of the male and female species, -or the "femble" and the "seed-hemp," as they are in some places denominated -is carefully attended to in the Fens of Cambrilgeshire, but in other places it is frequently neglected, either in consequence of the other occupations of the harvest, or from fear of the awkwardness and inattention of the pullers injuring the plants ; indeed, when the crop is thick, it would be attended with no small difficulty+. The maturity of the seed-bearing species may be ascertained by the same signs as that of the male; as well

* See Appendix to Wissett's Treatise on Hemp, No. iii.

$\dagger$ Survey of Suffolk, 3rd edit. p. 142; of Cambridgeshire, p. 161; of Lincolushire, p. 183.

† Suffolk Report, 3rd edit. p. 155. 
as by the seeds beginning to turn brown, and the pods which contain them bursting open. There is, however, this advantage arising from having the crop pulled when green-that it comes off the ground sufficiently early in many seasons for turnips, which are frequently made to follow it; or, that it allows ample time for the sowing of wheat.

If allowed to stand for seed, the harvest must then of course be later, and the crop is seldom taken off the ground until Michaelmas, or perhaps the middle of October, when it is shocked in the mamner of corn till dry, and thrashed upon cloths in the field, the sheaves being afterwards stacked until the spring, when the retting takes place in the same mode as that of flax, as already described. The pulling is commonly effected by women, and the stalks are tied up at both ends in bundles as large as the two hands can grasp. The charge is usually from $2 s .6 d$. to $3 s$. $6 d$. per 100 bundles; amounting to from 17s. $6 d$. to a guinea per acre, as the average produce may be calculated at about 500 bundles; breaking is usually effected at about $1 s$. per stone, varying, however, with their length, and the ease or difficulty of effecting their separation. The standing for seed lessens the quantity as well as the quality of the hemp; the " maidenliemp,", or that which is pulled green, producing generally from 40 to 50 stone, which is one-fourth more than the seeded-hemp, and the price is usually from $1 s .6 \mathrm{~d}$. to $2 s$. per stone higher. The average produce of the seed may be estimated at about $12^{*}$, but, in many instances, amounts to 20 or 30 bushels per acre + . The husk serves only for fuel, but the chaff is said to be equal to the tail of the common oats, and horses eat it with great avidity + . The expense and profit of rearing and harvesting the crop, cxclusive of the cost of heckling, have been variously estimated $\S$. The cultivation of both hemp and flax is, however, frequently carried on upon joint account between the farmers and jobbers, who are peculiarly acquainted with its management; the usual agreement being for the former to lay the requisite quantity of manure upon the land free of all charge, and for the latter to find seed and labour.

It lias excited the surprise of many writers upon agriculture, that the culture of flax and hemp has not been further extended in this country, and calculations have been brought forward, showing the vast advantage which would accrue to the nation by the saving of the immense sum annually jaid to foreigners for their importation. But putting aside the question regarding the supposed national gain, with the discussion of which we have no concern, and looking solely to the farmer's profit, it may yet be justly doubted whether they can ever be here made an object of general culture; for they not only require land of a peculiar nature, but the crops demand more minute attention than farmers renerally choose to bestow on them, and, independently of the opinion that they are exhausting, return nothing in the shape of manure to benefit the ground. On peaty soils, however, and especially on those bogs in Ireland, the drainage and cultivation of which have of late justly attracted so much attention, we are of

* Cambridge Report, p. 161.

+ Hampshire Report, p. 208.

$\$$ Thus the profit and expense are stated in the different County Reports to be-

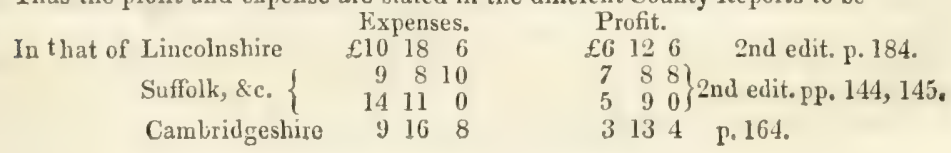


opinion that their cultivation, and more especially that of hemp, might be attended with such immediate benefit as probably, in many instances, to repay the purchase of the fee-simple, together with all the expense of bringing the land into a state of permanent pasture.

Hemp suits such soils better than flax; and, if grown upon such an extensive scale as to warrant the employment of persons who are well acquainted with its management, there can be no doubt of its leaving a profitable return. Very minute directions regarding its cultivation, both in this and other countries, may be found in a treatise on the subject, published some years ago by Robert Wissett, Esq., F.R. and A.S.

\section{Chapter XXViII.}

ON TEASEL,-CORIANDER, CARRAWAY, CUMIIN, ANISE, CANARY, RADISH, AND SPINACH SEED,-AND PIYSICAL PLANTS.

MANy of these articles are rather largely cultivaied in some parts of Somersetshire, Torkshire, Kent, and Essex, and the latter county is remarkable for the joint production of a triple crop, consisting of the three first mentioned plants, sown together on the same land; the crops being taken off at separate periods, and requiring three years for their complete production. In the first, the several seeds come up, and when of sufficient growth, are set out with a hoe; and the coriander, which is an annual, is ripe before the corn harvest. In the second year the carraway produces a crop; and in the third, the teasel is in perfection; after which the land is usualiy cleared for wheat; though-as the two last are perennial-crops are not unfrequently taken in the fourth year, and instances lave been known of the carraway and teasel being continued until the seventh*

The most favourable soil is a strong rich clay, or what is generally termed good wheat land; and the most general practice for the purpose is, to brcak up an old ley in the month of November, which, after being deeply ploughed and well manured, is sown in the following spring. The cultivation is, however, usually conducted upon joint account between the farmer and some jobbing labourers, who are accustomed to that species of culture; he agreeing to find land free of rent and parish rates, together vith manure and the use of his teams, while they supply all the remaining expense and labour, and the produce, when sold, is equally divided. The plan is, indeed, in every respect advisable for the different operations; great care and expertness being requisite on the part of the workmen, who will be more likely to pay proper attention to their own interest than to that of another person.

\section{THE TEASEL,}

is a plant which, in the first appearance after it is sown, is much like a lettuce, but it afterwards grows to the height of several feet, bearing heads of a conical form, two or three inches in length, and one, or one and a half in diameter at the bottom, or largest end; armed on every jart with small strong points, or horny vegetable hooks turned a little downwards. They are used in the dressing of baize by the woollen manufacturers, who fix them upon frames covering a cylinder, which, in turning round, slightly catches the cloth, while another part of the machine draws it against them; by which means the knap is raised to the desired length. 'The

* Survey of Essex, vol, ii. p. 61. 
plants will only answer upon a strong soil ; but, if the land be fresh, they do not require much manure, and are therefore frequently grown upon that which has been pared and burnt, as well as upon land ploughed out of the swartl.*

The seed is sown about May-day, at the rate of one to two pecks per acre. In Somersetshire and in Yorkshire, where the crop is generally, and perhaps more judiciously, sown alone, the cultivation is as follows :-

During the summer the land is worked over three or four times with long narrow spades to destroy the weeds; and in the month of November, if the plants are too thick, they are drawn out to fill up vacancies, and the crop is set out at a foot distance. If after this thinning many plants remain, another piece of ground is generally prepared, into which they are transplanted; but those which are never removed produce the best heads. In the month of July of the following year, the uppermost heads begin to bloom, and so soon as the blossom falls they are ripe. The gathering is performed at three different times: a man, with a knife made for the purpose, cuts the heads which are ripe, and ties them up in handfuls; after a fortnight, he groes over the ground again; and at a third cutting the business is completed. On the day of cutting they are carried into the barn, and if the air be clear they are taken out daily and exposed to the sun vintil they are completely dry; or temporary sheds are erected in the field, in which the crop is suspended and dried; but great care must be taken to avoid their exposure to rain. When quite dry, they are separated into different parcels-called ' kings,' 'middlings,' and 'scrubs ;' and are after that made into 'packs,' containing 9000 kings, and 20,000 middlings: the scrubs are of but little value. The produce is often 15 or 16 packs an acre; but there is a great inequality in the plants, and sometimes the crop presents a total blank $\uparrow$.

\section{CORIANDER AND CARRAWAY}

are sometimes sown together without being joined with teasel; for the carraway, requiring two years to perfect its seed, is sown along with the coriander, and sometimes with corn, in the same manner as clover is with barley. The young plants of coriander resemble those of the parsnip, but the carraway, is like that of a carrot, and from the care and attention requisite in distinguishing and setting out the plants, the hoeing is an expensive operation. The seeds have an aromatic flavour, and meet with a ready sale among the rectifiers of spirituous liquors, druggists, and confectioners, who employ it for the various purposes of their respective trades. On good land they are also often very productive, but great care is necessary in securing them when ripe; for which purpose women and children are employed to cut the plants separately, and put them into cloths, in which they are immediately carried to some convenient spot, either in the same ficld or the barn, where, being thrashed upon a sail-cluth, they are scparated from the haulm by a few strokes of the flail, and being spread upon the granary floor to avoid heating, they are afterwards winnowed at any leisure moment. Both, when sown on rich old leys, are very proluctive; coriander having frequently produced $24 \mathrm{cwt}$. and carraway $20 \mathrm{cwt}$. of seed per acre. The crops are, however, very precarious, and sometimes do not produce more than 6 or 7 cwt.f; the prices also varying, accord ing to the season, from $10 \mathrm{~s}$. to $50 \mathrm{~s}$. per civt.

* Tuke's N. R. of Yorksh., p. 166.

+ Billingsley's Survey of Somersetsh., 3rd edit., p. 110.

$\$$ Essex Report, vol. ii. p. 61. In the papers of the Bath Society, some experiments are mentioned upon the suwing of coriander alone, without any other crop, upon a good sandy loam, the produce of which in no instance exceeded $11 \frac{1}{2}$ cwt. 
The mode of operalion with the three crops collectively, as described by Mr. Sewell, of Maplestead, in Essex, is as follows :-

"About the beginning of March, plough some old pasture land, the soil of which should be a very strong clay loam. Mix together $10 \mathrm{lbs}$. of coriander, $12 \mathrm{lbs}$. of carraway, and $12 \mathrm{lbs}$. of teasel seeds, which is sufficient for an acre; sow directly after the plough, and harrow the land well. When the plants appear of sufficient strength to bear the hoe-which will be in about ten weeks after the sowing-it must not be omitted; and in the course of the summer it will require three hoeings, as well as one at Michaelmas. The coriander is fit to cut about the beginning of July, and should be thrashed on a cloth like cole-seed."

"About the April following, your teasel and carraway will want a grood hoeing, done deep and well, and another hoeing about the beginning of June. The carraway will be fit to cut the beginning of July, and must be thrashed in the same manner as coriander. The teasel will not be ready till the middle of September; and some of the plants do not perfect their seeds until the third or fourth year, when those heads which are beginning to turn brown are cut off the stem with a stalk a foot long. Of these, 25 are tied in a bunclı; 24 of the bunches are fixed on a small stick, and called ' a row,' 24 of which make a load, equal in bulk to about a ton of hay.

"The goodness of the crops must chiefly depend upon the care employed in their cultivation; and as the land can only be filled with plants, it is evident that the more one kind predominates the less can be reasonably expected from those which succeed : accordingly, the product of carraway is much greater without, than with teasel *'”

\section{CUMMIN AND ANISE.}

The former is a plant which also does not produce its seeds until the second year, and as it requires a rich, and, consequently, high-rented soil, the double rent adds heavily to its culture. It is, therefore, only rarely grown in this country, though the climate suits it; but as the seed is entensively used by distillers, it is an object of considerable attention among the farmers of Germany, who lessen the expense of its cultivation by growing it, either broad-cast among spring-corn, or transplanting it among alternate rows of cabbage, parsnip, or beet-root. In the latter case, the beds are not unfrequently prepared in autumn, and the plants raised upon them are set out some time in the beginning of the following month of May. If sown with corn, the plants are hoed out to regular distances when the crop has been reaped, and the cummin should afterwards be diligently cleared of weeds, and top-dressed with some stimulating species of manure. When ripe, the crop may be either mown, or carefully pulled up, and carried with the same precaution, to avoid the shedding of the seed, as is necessary with those of coriander and carraway, and it should be thrashed in a similar manner + .

Anise, though also much employed by the distillers, and perfecting its seeds in the same year about the month of August, is yet a plant of such a delicate nature that it is easily injured by the slightest frost, and as several attempts to bring it to perfection have in this country failed + , it evidently requires a more genial climate, or a very warm exposure.

* Survey of Essex, vol. ii. p. 63.

† Von Thaër, Princ. Rais. d'Agric, 2nde edit. tom, iv. \$ 1226.

$\mp$ Bath Papers, vol, iv. p. 279. 


\section{CANARY SEED,}

although an object of apparently slight consumption, as being almost solely devoted to the food of singing birds, yet commands a considerable sale in our markets, and is rather a favourite crop among the farmers of the Isle of Thanet. It is managed, according to Mr. Boy's account*, under three different tilths, namely :-" summer-fallow, bean-stubble, and clover-ley; the last the best. If the land be not very rich, a coat of rotten-dung is frequently spread for it; but, whether manured or not, the proper tiliage is to plough the land the first opportunity that offers after wheat sowing. As soon as the ground is tolerably dry in the spring, furrows are made, from 10 to 15 inches apart, and the seed is sown broad-cast, at the rate of about four or five gallons per acre, well harrowed in. When the blade appears, and the rows are distinct, the intervals are immediately hoed with a Dutch hoe, and afterwards, in May or June, the hoeing is repeated with a common hoe ; carefully cutting up every weed, and thinning the plants in the furrows, if they are too thick." "The harvest is later than that of any corn crop, and it is cut with a hook, called a 'twible,' and a 'hink ;' by which it is laid in lumps, or wads, of about half a sheaf each. The seed ciings remarkably to the husk; and in order to detach it, the crop must be left a long time on the ground to receive moisture sufficient to destroy the texture of the covering, or otherwise it would be hardly possible to thrash it out. The wads are turned from time to time, to allow them the full benefit of the rains and sun; and it has thus continued in the field till December, without vegetating or suffering any kind of injury. The produce is from three to five quarters an acre $t$, and the actual price is from $40 \mathrm{~s}$. to $42 \mathrm{~s}$. The haulm is a most excellent fodder for horses.

\section{RADISH AND SPINACH SEED}

are also much cultivated on the rich loamy soils of the Isle of Thanet, and East Kent, for the supply of the London seedsmen, who send it to all parts of the kingdom for the retail consumption of the gardeners.

"The radish-sced is sown on furrows, about 10 inches apart, in a dry time of the month of March, at the rate of about two or three gallons per acre. As soon as the plants appear, every other row is cut up with a horse-hoe, leaving the rows 20 inches apart. When the plants get two or three rough leaves, they are hoed out in rows and are then kept clean by repeated horse and hand hoeing, when necessary; leaving the plants at about 18 inches distance. The crop is seldom fit to reap till October, and is sometimes out in the fields until Christmas, without receiving injury from the wet weather; it being requisite to allow it much rain, in order to rot the pods and facilitate its thrashing. The produce is from 8 to 24 bushels per acre $\neq . . "$

"Of spinach seed there are two sorts, the prickly and the round; both are sown in furrows, about 12 or 14 inches apart, early in March; the prickly at the rate of six, and the round at four gallons per acre. And when the plants have leaves about an inch or two in length, they are hoed out to the distance of 4 or 5 inches. When the crop is in full bloom, the greater part of the male plants are drawn out by liand, and given profitably to young

* Survey of Kent, 2nd edit. p. 104.

$\uparrow$ Mî. Bartley, of Bristol, who made sereral experiments on the culture of canary seed, on land consisting of a mixture of loam and clay, states, however, "that his produce never exceeded 17 bushels per acre."-Bath Soc. Papers, vol, iv. p. 279.

† Survey of Kent, 2nd edit, p. 105. 
pigs: by which operation the female plants have more room to grow and perfect their seed. The crop is pulled up when ripe, and either thrashed in the field on a cloth, or carried to the barn. The produce is from two to five quarters per acre."

\section{PHYSICAL AND AROMATIC PLANTS}

are grown to so great an extent for the consumption of our large towns, that some very intelligent anriculturists devote themselves solely to their culture: indeed we know of one in the county of Surrey,-Mr. Moore, the very respectable owner of the manor of Mitcham,- -who occupies nearly 500 acres of land chiefly in the cultivation of single and double chamomile, liquorice root, peppermint, and lavender. The soil proper for their growth should be deep and friable; for some of them strike their roots several feet into the ground, and it is rendered rich by an unlimited application of manure procured from the neighbourhood, as none can be raised upon the land on which they are grown. Some of the plants also require three or even four years to perfect their growth; and they must, during all that time, be managed with the attention usually bestowed on garden culture : the expense of cultivation is, therefore, very great, and when added to the necessarily confined limits of the districts in which it can be conveniently carried on, must ever prevent it from becoming an object of speculation with the general farmer; wherefore, a slight sketch of the different articles already enumerated is all that we here think requisite.

Chamomile is a perennial which should be grown on a light, dry, sandy loam, made extremely clean. The ground should be set out in beds of 4 feet wide, with 18 inch alleys between, and on these beds the sets ought to be planted about 8 or 9 inches asunder, and kept carefully hand-weeded. The flowers come to perfection in the first summer, and generally produce crops of from 2 to $4 \mathrm{cwt}$. jer acre, provided the summer be not very wet; for rain both injures the plant, and weakens the flavour of the flower. The crop is gathered by women, who are generally paid at the rate of one penny per 1b. ; the wholesale price to the druggist beingr usually about $5 l$. per cirt.

In November, or the early part of December, the beds having been weeded, the alleys are dug, and the roots are covered with the mould, both to refresh and to preserve them from the frost.

Liquorice requires a very rich and deep soil, as its roots frequently penetrate a yard, where the ground is naturally open and loose to that depth: the land should therefore be either trench-ploughed, or rather dug two-spade deep. The manure should be spit-dung laid on at the rate of 20 double cart loads per acre, merely spread and pointed in with the spade. The ground is then lined uut in rows 2 feet asunder, and small triangular holes are made with the spade for the reception of the plants at 15 inches to $1 \frac{1}{2}$ foot asunder. The plants consist of shoots taken from the heads and sides of the old roots; and in order to secure their growth, none are planted but such as have several eyes. March is the most usual season for putting in the shoots, and the ground must be weeded and stirred about the plants, during each succeeding autumn.

Liquorice requires three years to perfect its growth, when the roots are taken up about the end of November with the spade; they are then washed, the fibres trimmed off, and the smalier roots, which are termed "offal," are separated from the larger. The latter are dried and ground into powder; but the larger roots, which form the principal article of profit, are packed up and sold to the druggists. A fair crop will yield from is to 20 civt., at an average price of about 45 s. per cwt.; but the expense of digging up and pre- 
paring it for market is not short of $10 l$. per acre; which, great as it may appear, is by no means extravagant if we consider the depth to which the roots run, and the care which is necessary to avoid breaking or leaving any of them in the ground.

The land is then usually sown with wheat, and very good cropsamounting to four or five quarters an acre-are commonly grown: indeed even seven and eight quarters are said to have been frequently obtained ** $T$ lis amazing fertility has been very generally attributed, not only to the ricuness of the ground, but also to the great depth to which it is stirred; but, admitting all the advantages of the spade husbandry to root crops, it is not easy to perceive how a plant with such a short root as wheat can be so materially benefited by the operation; the greatness of the produce may, therefore, we apprehend, be rather due to the natural state of the soil, and the vast quantity of manure laid upon it, togrether with the decomposition of weeds, and the state of garden cleanness in which it is kept during three successive years.

Peppermint, being possessed of virtues which are both highly carminative and stomachic, is largely used both by the druggists and by distillers, who sell it, in its liquid state, to the dram-shops. It is indeed reared by some persons to such extent that on the farm near Mitcham, to which we have alluded, $1: 30$ acres are annually grown, 50 of which are renewed every third year; for, although perennial, such is the tenderness of the plants when exposed to either rain or frost, that their continuance in a perfect state cannot be reckoned upon during a longer period.

The ground must be brought into the finest tilth by frequent ploughings, harrowing, and rolling; and the dung, which should be well rotted in a large quantity, should be thoroughly incorporated with the soil. The land is then laid out in beds from half a rod to three-quarters wide; and about the latter end of Miay, or the beginning of June, slips are drawn from the roots, and buried a little below the surface, and planted in rows of six or cight inches apart. The expense of cultivation is very great, for the handhoe must be almost constantly kept at work to prevent the weeds from rising; and at the close of autumn, the mould from the alleys between the beds is thrown over the plants to protect them from the frost.

The crop is amnual, and is taken off about the month of August; the operation being usually performed by women, who cut it with small hooks ; but it is very difficult to fix the average either of the price or the produce per acre, as the former varies from $4 l$. to $6 l$. per ton-being now $4 l$. -and the latter will in some years be from one and a half to four tons per acre.

Lavender is a well-known aromatic, the flowers of which are either bunched and sold about the streets by hawkers, or the greater part are distilled as a perfume; in which latter case they are carried loose, when cut, to the still-room, a building for which purpose is usually to be found on farms which grow those herbs extensively. The seed is not usually sown, but plants, or slips, are placed in rich and well-prepared beds, in the month of April, and about the same time in the next year the plants are set out in rows of 20 inches to 2 feet apart, carefully hoed and weeded.

In a plantation of 20 acres, which was formerly established near IIenleyupon-Thames by the late Field-Marshal Conway, a chalky soil was found to be not unappropriate; but the growth was far more luxuriant on the bottom than on the slope of the hill. The plants were there, however, raised in rows four feet apart, and replaced, as they decayed either by age or acci-

* Stevenson's Survey of Surrey, p. 381. 
dental circumstances, by slips taken from the old plants and reared in a nursery. The major part of the crop ripens about the end of July, when women and children are employed in cutting off the mature heads for the purpose of distillation; and in frosty weather, the men who are usually employed in the common operations of the farm, go over the plantation with shears and clip off the stalks, which are afterwards used as fuel *.

Besides these, there are in the neighbourhood of London, several extensive plantations of the various species of red and damask roses, the leaves of which are gathered for the chemists; and wormwood, rhubarb, angelica root, and all the different medicinal plants which can be grown in our climate, which, insignificant as they appear in a view of national agriculture, yet annually afford very large returns to the grower + .

Among these rhubarb has indeed of late years acquired the character of a culinary vegetable, and is now extensively grown by market-gardeners, for the use of the table; the green stems forming an early and very agreeable substitute for the apple. The "giant" species grows to such an enormous size, that young offsets, or buds, set in the ground, about the first week in March, have in the next year produced plants, "measuring above a yard and a half over the surface, with foot-stalks an inch and a half broad by two or three feet long; and during the two following seasons, the root-stalks have increased to such a size, that it required a barrow to remove the mighty mass that was raised with great labour from the soil+." It is a perennial which lasts a great number of years, and should be planted firmly in land of an open condition, during temperate weather, at the distance of four or five feet, or even more, from plant to plant ; on the approach of winter a coating of rotten stable dung, or compost two or three inches deep should be laid around each plant, to the extent of two feet, and in the open weather of February, the whole bed should be forked over.

\section{Cinapter XXIX.}

\section{ON MADDER-WOAD-AND WELD.}

These plants are grown upon a large scale, for their use as dye-stuffs in the North of Europe, and were formerly extensively cultivated in some districts of this country and Ireland; but the introduction of other substances, together with frequent disputes about tithe, though afterwards remedied by acts of the legrislature fixing a modus, so far impeded their culture, that, notwithstanding various premiums offered for the encouragement of their growth, it has rather fallen off than been extended.

\section{MADDER}

is a plant, the roots of which produce an imperfect red dye, being deep on the outside, and pale in the middle, and is much used in our manufactures: the haulm, though sometimes employed in the feeding of cattle, is yet not generally used for that purpose, for it tinges the milk, the urine, and even the bones of the animals fed upon it. The root is composed of long slender fibres, which push themselves to the depth of two or three feet into

* Survey of Berkshire, p. 232.

+ Survey of Surrey, by Sterenson, chap. vi. sect. 26 ; by Malcolm, vol. iii. p. 116.

+ Towirs on the Cultivation of Rlubarb.-Quar. Jour. of Agric., No. XXXIJ. 
the ground; and the stems, which rise to a considerable height, bear bunches of yellow flowers, clustered together in the form of a bouquet. It can be propagated by the seed, but the more usual method is by transplantation. It requires land of a deep and rich sandy quality, and soils of that nature existing largely in Holland, this, added to the comparative cheapness of labour, has thrown the culture almost entirely into the hands of the Dutch.

The mode there pursued is, at the end of April or May, according as the young plants are large enough to be transplanted, to plough the land in beds of two feet, or two and a-half feet wide, putting down the young suckers of the roots at intervals of a foot, or a foot and a-lialf, and at six or eight inches distance in the row. During the entire summer, the land is frequently stirred and kept free from the weeds; and in the month of November, when the leaves are faded, the plants are covered with two inches of earth, by a plough having the point of the coulter a little raised, or rounded, so as not to injure the young plants.

In the following spring, when the young shoots are four or five inches long, they are gathered, or torn off, and planted in new beds, in the same manner as pointed out above; and in the months of September or October, the old roots are taken up and placed under cover to guard them from rain*.

This gathering of the roots at the end of the second year can, however, only be properly carried into effect upon very rich land, for, on ordinary soils, they require three years to perfect their growth; and the method recommended by a successful Flemish agriculturist is to plant the sets at two feet distance, and to leave every third or fourth row vacant for the purpose of afterwards covering the young shoots with the mould. The ground is thus formed into beds intersected by deep trenches, which are ducr every year until the third, when the roots are taken up; but at the commencement of the first winter, the berls are heavily spread with long dung, which, in the following spring, is raked off into the trenches, and thus is acrain used as manure during the succeeding years. The planting is usually in May, and as the shoots do not rise high in the first year, nany farmers sow vegetables in the intervals between the rowst.

In this country several modes of planting have been practised; but that which appears to be the best is to plant it in single rows, about two feet apart. The land should be perfectly free from weeds, and have been well manured the preceding year, so that the dung may be thoroughly incorporated with the soil; which should be a fine, deep, rich, sandy loam, without any redundancy of moisture. In order to prepare the land for planting, it should be ploughed in the autumn, and kept clean by harrowing and horsehoeing, until the young plants are ready for drawing from the beds, which is usually by the end of May, or the beginning of June; the proper time being known by the plants having got to the height of 10 or 12 inches from the ground, and having produced roots branching out from the bottom of the suckers, which may be ascertained by drawing up a few of them.

When the suckers are in this state, a great number of hands are required to perfect the work, as the operation should proceed with all possible despatch, and an acre requires about 20,000 plants. They should have about a third of their tops cut off, and then their roots should be dipped in earth, or fine mould and water, beaten together to the consistence of batter. One

+ Schwertz, Sur l'Agriculture Belge, tom. ii., p. 203. 
woman is required to dip the plants; two others to carry and strew them in handsful along the furrow, and about seven to follow the plough. The land should be ploughed with a strong turn-wrest plough, to the depth of 12 or 14 inches; the women laying the plants about 8 or 9 inches apart in every other furrow, leaning off from the plough; by which, every time it returns, the row of plants laid in by the women who follow the plough are covered with the earth of the furrow.

The crop must be kejt perfectly clean by the hoe and hand-weeding during the summer months, and earthed up with a plougin each autumm, until the third after planting; when the roots are dug up by trenching the land two feet deep. Two children attend each digger to pick out the roots, the workmen breaking every spit of earth to pieces with their spades. The roots are then carried to be cleaned from the dirt, and afterwards dried on a hop-kiln till they are brittle enough to snap freely asunder; they are then fit to pack in bags for sale to the dyers, who grind and manufacture them into powder for use. The produce is from 8 to $20 \mathrm{cwt}$. per acre.*

Mr. Boys, of Betshanger, the intelligent agriculturist from whom this account is chiefly taken, and who formerly cultivated madder to a large extent, and with considerable profit, until the price was reduced by the competition of the Dutch growers, states-when rent and labour were both higher than at present-that it would still be a profitable culture upon soils properly adapted for the purpose, provided that 23 per cwt. could be obtained; it is now, however, below that price.

Rules have been also laid down by Miller, "On the Culture and Manufacture of Madder," for managing the land, separating and planting the shoots, crathering and drying the roots, and of pounding, casking, and pre paring them for sale, according to the most approved English practice; which, as the cultivation is still partially continued, and under the present circumstances of our agriculture, may perliaps be further exiended, we insert at foot†.

* Survey of Kent, 2nd edit., p. 115.

$f$ "The most suitable suil is a soft sandy loam of two or three feet in depth, that the roots may run down without obstruction. The land to be well ploughed and laid up in high ridges in autumn, preparatory to the planting out in spring.

"In spring, plough with the deeglest furrow, and let the bottom be still further deepened by the sparie. Plant from the mildle to the latter end of $A$ pril, rake the ground well, and lay it up in ridges if disposed to be wet; if not, plant it in rows at eighteen inches interval unon the level. Mark out the rows with a line, and diblile in the plants at twelve inches in the row, securing them well in the ground, and covering as little of the green as possible. Take the slips with as much root as possible, and take them when they begin to bud out; packing them so as not to be heated, and if withered, set them upright in water, previous to planting.

"Plant nothing in the intervals; but as the madder grows high, tnm it occasionally from side to side, earthing up the ruots at the side from which the haulm is turned. The interval must be stirrerl at the same time, and the straggling shoots cut off; thus the roots will be-strengthened to yield a double increase.

". Mddder should remain three years, but some gather it at two years, thongh with less profit. The slips of the second and third years' growth are the lest to plant, and should be taken in the spring, when about an inch above ground.

"In autumn, when the haulm is withered off', earth up the rows, against the frost, and in spring hoe the intervals, and weed; attend the turning of the haulm in summer, and dig between the rows till the season of gathering, namely, about Michaelmas.

"In the third autumn, when the haulm is withered off, dig a trench full three feet deep, alung the first row of madder, as near to the roots as may be, to receive the earth in digging up the roots. Fach digger must have three pickers to gather the roots, and clear them from the earth, and they must be all fairly got out. They should then be laid thin upon the ground, as lying in heaps, or taking rain, will discolour them, and therefore the sooner they are carried to the drying-house the better.

"I 'The 
WOAD

was formerly largely grown in this country, as, until the introduction of indigo into Europe, it was the only blue dye generally known. The superiority of the latter, however, speedily grave it such a preference among manufacturers, that the remonstrances of the farmers, who appealed to Government against its use, under the term of "the devil's dye, "were unavailing, and the culture of the plant has since bcen only partially continued in some districts of England, and a few parts of Ireland; where, though conducted in a slovenly manner, it is found very profitable.

It is a native of the north, and the plant, which is very hardy, grows to the height of about three feet, divided into slender branches, bearing pointed leaves slightly tinged with blue, though the flowers, which spring at the summit, are yellow; it takes two years to come to jerfection, and requires a good loamy soil, though not partaking of marsh. It was formerly planted for several vears to the extent of 120 acres on the rich grass-lani of the fine farm of New Barns, belonging to Mr. Tattersall in the Isle of Ely; and land newly broken up is always found the most suitable*. The success of the crop depends much on the hoeing and weeding, so as to keep the groumd fresh and clean; for thus managed, three or four gatherings of the leaves, from which the dye is obtained, will be produced in succession; but the first two are the best, and the excellence of the dye consists in the succulency of the leaf. In this country the common mode of culture, in Torkshire, Somersetshire, and Lincolnshire, in which counties it has been chiefly cultivated, is as follows:-

If soun on crass-land it slould be plonghed early in February, to the depth of about five inches, with three horses in length, followed in the furrow by a man with a sparle, so that if the turf be not turned over very flat and well-joined, it may be laid completely so by hand. 'This attention is necessary to prevent the grass rising in the seams; then the land is often harrowed to raise a depth of mould sufficient for the drill to work. The seed is put in about the midlle of March, either in equidistant rows eight or nine inches asunder, or it is sown broad-cast and harrowed in; after which

"The drying-house should be defended from wet, but opened to a free air: the roots should he spread on hurdles, placed at a convenient distance for that purpose, one over the other. Here they must remain till they are dry enough to rub the dirt clean off, and then they are to be removed to the cold kiln. The liln for malt or hops will serve the purpose, with the addition of a ventilator to preserve the colour.

"On the first kiln they are to be dried with a gentle heat, heing carefully turned till they are dry enough to flart with their husks, which are to be thrashed off on a common thrashing floor, clean swept, and then packed up separately. These sell at a much cheajer rate than the inside madder--under the name of "mull ;" but it usually pays the expense of drying and cleaning. When the mull is thrashed off, the roots must be dried a second time, with a stronger fire, but great care must be taken that it be not too fierce, so as to discolour the madder, for on the brightness of the colour depends the price. The last process is that of pounding and reducing to powder; but that is an operation which rather depends upon the dealex than the farmer."

"In "Stevenson's Survey of Surrey," it is, howerer, said to be grown on the chalk hills in the neighbourhood of Banstead Downs, "the soil best adapted for it being a thin, and rather poor chalky loam; as it grows too strong on grood land."-p.383; and in that of Kent it is stated, "to be much cultivated in the western part of the county, on poor, stiff, and chalky lands."-2nd edit., p. 114. All the other accounts, however, both British and foreign, agree in describing the requisite soil as a good fat loam. See the Surveys of Lincolnshire, 2url ed., p. 174; Cambridgeshire, 1. 174; Bullinirley's Somersetshire, 3rd ed., p. 113; Bath Papers, art. vii., vol. xii.; and a treatise upon the management drawn up by order of the French Government; which may be found in the Alpendix to Radclifi's Account of the Agriculture of East and West Flanders. 
the clods are raked off into the sides of the furrows, and then rolled again to leave it clean and neat. Being thus far done, the field must be gripped very carefully, for wherever water stands, the woad is entirely destroyed. Upon the first coming up of the plant, attention must be paid to the turnipfly and also to frosts, as the plants are sometimes destroyed by both, and must in that case be sown again immediately*.

If sown on arable land, the first ploughing should be against winter; the second in the spring, when the ridges should be formed; a third in April, and the last in May or June, just before the sowing of the seed. The plants, in a moist season, appear in a fortnight, and in two or three weeks after are fit for the hoe; they should be hoed out clean to the distance of about six inches at the least: some prefer a wider interval. Hand-weeding and thinning are generally executed by women and children on their knees, using short spuds with one hand, and drawing away the weeds with the othert.

The Flemish mode is, in February or March, after the frost has acted on the surface of the earth, the manure is spread over it as equally as possible: two furrows, of about thirteen and a-half inches, are made with the plough, the whole length of the ground intended to be sown, forming between them a ridge, or bed, of twenty-seven inches wide, upon which the seed is sown in two rows; for if sown in three rows, it has been remarked that the leaves of the middle plants, not having sufficient air, give little or no return. The land should be rendered as fine as a garden; the seed should be sown pretty thickly, the rake passed over the drills, to render them perfectly smooth, and afterwards rolled. Some put the seed to soak in water, the night preceding the day they intend to sow it; others throw it over snow, which, as it melts, buries the seed; and others again sow it before a slight rain. The woad springs up more or less quickly at the end of ten or twelve days, but in a month or six weeks, it acquires strength and vigour, shoots forth five or six leaves, and when they spring up is the time to weed, and mould it up with the loose soil; operations which should be repeated frequently until the approach of harvest

All these different modes of proceeding arise from the difference of climate; for in some parts of the South of France and Germany, it is sown in autumn, and then produces its crop in the following year. The plants should be carefully weeded and kept perfectly clean until their maturity, which usually takes place about the middle of June. The ripeness of the plant is known by the leaves changing their greenish blue into a pale green, and they ought to be then immediately gathered, or the colouring power of their juice will be materially injured. The operation is performed by hand, taking care not to injure the stem, which ought to produce new leaves; for immediately after the cropping, the field is by the same persons reweeded, and a second and third crop is produced within a few weeks; the last is, however, of but little value, and is generally allowed to run to seed. The stalks should then be reaped like wheat, and spread abroad, and if the weather be favourable, the seed will be fit for thrashing in four or five days.

The green crops are gathered into baskets, and when carted home, are thrown into a mill constructed with a heavy iron ribbed roller, something like that used for bruising furze, by which process it is crushed to a pulp, and is then laid in small heaps to drain, after which it is made up into oval balls, which are dried on hurdles under a shed exposed to the sun, or, more

* Lincolnshire Rep., 2nd edit., p. 175. † † Somersetshire Rep., 3rd edit., p. 113. \$ Radeliff"s Agriculture of Kast and West Flanders, Ap., No. V. 
conveniently, on grates in drying-houses. There they turn nearly black on the outside, and of a purplish cast in the inside, and are valued in proportion to their specific weight and clearness of colour. The crop may amount to a ton and a-half, or, on very good land, to even two tons per acre, and has been sold as high as from $20 l$. to $25 \mathrm{l}$. per ton. It, however, is thought to exhaust the land, and in general is not sown more than once within six years; though in the rich soils of Lincolnshire three successive crops have been taken.

In order, however, to bring the woad into a state ready for the dyer's use, it must go through the fermentative process for seven or eight weeks, for the details of which we must refer to Arthur Ioung's "Survey of Lincolnshire," in which there are plates descriptive of the machinery, and a very minute account of the entire management of the crop, from the putting in of the seed, until ready for the manufacturer.

\section{WELD}

is another of our native plants, which affords a fine ycllow dye; and possessing the great advantage of only requiring to be dried, in order to be ready for use, it therefore occasions no extrineous trouble to the grower. It can likewise be grown upon a great variety of soils, although a clayey sand is that which is most appropriate. The seed, which is small, should be sown in August, at the rate of about twelve pounds per acre, and is usually put into the ground alone, and broad-cast; though sometimes it is drilied, and not unfrequently sown in the spring along with other crops. The ground should be in fine order, and the seeds sown by hand, as they will not bear to be much covered. The plants should be kept perfectly clean, during the following summer, and in the latter end of July, if the season be favourable, they will be in flower, and then are to be pulled up, without waiting for the ripening of the seeds. The grain, however, may be employed for the expression of oil.

The plants are pulled up by the roots, and set up in small parcels to dry ; after which they are bound into moderate-sized sheaves, and may then be stacked without further care, as they are not subject to heat, and will remain during a series of years without injury. It is said to be an exhausting crop; but this, if it be not allowed to perfect its seed, we believe to be a notion rather entertained by prejudice than arising from fact. Its cultivation requires nothing more than ordinary care; and as it commonly produces cropus of a large amount, which frequently bear a good price, it is a favourite with many farmers in the manufacturing districts; it is indeed evidently profitable, for those who commence it are seldom found to give it up.

However beneficial the growth of these articles may be to those individuals who are enabled by locality to avail themselves of the advantage, yet as they camnot be reclioned on generally, nor without perfect knowledge of the details of their culture and preparation for market, as well as a certainty of demand, they are not objects which can be recommended to the attention of common farmers. Indeed, considering the risk attendant upon both crops and prices, and the consequent amxiety which they occasion, no one should enter upon such a speculation without an expectation of large protit; and it may be doubted whether upon soils of the requisite quality, the ordinary articles of farm growth may not nearly equal the gain, without incurring anything like the hazard or the care of their cultivation.

voL. II. 


\section{Chapter XXX.}

\section{ON HOPS.}

The nop is indigenous to most parts of limope; but although found wild in this country, it was long ere it excited the attention of agriculturists, for many plants, which were for ages regarded as weels, are now cultivated among the most valued of our field productions. The English word "hop," though probably derived from the Sixon hoppan, signifying to climb, yet was adopted into our languagre from the German hoppe; its botanical name being humulus. The earliest known account of its culture is found among the ancient records of France, in which mention is made of humolarice, which doubtless meant hop-gardens; but we learn from Beckmann, "that the first positive notice of the use of hops occurs in the beginning of the fourteenth century, when it apmears that they began to be regularly cunployed in the breweries of the Netherlands. But although their efficacy was admitted in the conservation of beer, yet they were long supjosed to contain qualities noxious to the constitution, among which it was said that they dried up the body, and increased melancholy; and accordingly we find in the household regnlations of our Harry the Eighth, an order to the brewer, not to put any hops into the ale. Indeed, at a much later period, the Common Council of the City of London petitioned Parliament arainst the use of hops, "in regard that they would spoil the taste of the drink, and endanger the people:" they were also petitioned against in the reign of Henry VI., " as a wicked weed!"

"They were not brought into cultivation in this country until about the vear 1524; and according to a distich to be found in Baker's Chronicles, it would seem as if the introcinction of beer should be attributed to the same period.

\section{"Turkies, carps, hoppes, piccarell, and beere, Came into Englande all in one yeare."}

"This, however, must be understood of beer, such as that still in use; for" ale brewed from malt, without the addition of hops, was certainly known here at a inuch earlier date. In the latter end of the thirteenth century, during the reign of Elwark I., there is a record of a grant from William Earl of Warwick, to one Adam Lnderwood, one of the conditions of which is, "that he should amually make for the lord of the manor three quarters of malt ;" but at that time, the herb called ground-ivy was generally used for preserving the liquor"."

\section{SPECIES.}

Only one species of the hop is noticed by botanists, but many varieties are distinguished by farmers : as, for instance, the "Gouldings," which are grown on the rag-stone of mid-Kent; the "Canterburv Grape," which is chiefly cultivated on the rich soils of Last Kent; the "Mavfield," and the " white-bine," on dry limestone land, and the "rolden-tips," in the wealds of Kent and Sussex, which are remarkable for their resistance of blight: added to which there is the celebrated Farnham hop, vilich is grom throughout that neighbourhood, in Surrey, and a kind much esteemed for the delicacy of its flaveur, which is chicfly produced in the comties of IIereford and 
Worcester. They are also partially cultivated in other places, but these are the chief districts.

There is also a sort known as the "Flemish red-bine," which, although a coarse speciss, is so much nore hardy than the other kinds, that it is much grown by those whose land lies under a bleak exposure.

The hop is a perennial plant, the root of which strikes very deep into the ground; the stalk reaching spirally upwards to the height of twelve or fiften feet, cntwined around the poles which support it, and bearing flowers which grow upun the slender stem of a kind of creeping-bine. It is at present so common, that a more minute lescription of it would be superfluous; but it may not be generally known, that it is the female plints alune which are considered scrviceable to the brewer. These produce thrir flowers in cones, not unlilie those of the fir in form, while the male bears clusters somewhat resembling the blossoms of a currant bush, and the fowers are alone male use of. Hops can be propacgated by seeds, but they are more usually grown from slips taken from the stem, or from old roots; or else with yomg ntirsery plants raised in beds. They are of a rough and somewhat clammy feel, with an aromatic bitter flavour; and are added to heer, not only to render it more palatable by correcting the insipid sweetnos of the malt, but also to preserve it ; indeed of all the various substances which have been tried, none has so effectually answered both these purposes.

SOIL AND SITUATION.

Although all cultivatel in a nearly similar manner, and generally with great skill, yet the quality of these various denominations differs in a greater or less degree, according to the nature of the soil upon which they are grown; thus, in the vicinity of Canterbury, and throughout the greater part of last Fent, the plantations are upon a deep, rich, loamy surface, with a subsoil of loamy brick-carth, the whole of which lies above a stratum of chalk, and there the sort produced is of a strong and rather bitter flavour, which renders it in great reyuest by the porter brewers. The grounds arouml Iraidstone, extending through the district on the rag-stone shelf of land, or "stone-brash," which lies below the chalk-hills, on the borders of the weald of Kent, and is seldom unacempanied by marl, in some years vield great crops of hops, but the quality is somewhat inferior to those of Last Kent; for although they in general grow larger, and have a thicker leaf, ret ther contain a less quantity of the farina, provincially "condition," which constitutes their most essential property*. Much of the soil in the neighbourhood of Farnham consists of a very mellow hazel mould, or of a deep siliccous loam, rather strong, dry, friable, and very deep, Iying upon a calcareous or marly subsoil, and the hops produced on such land are of a bright lively colour, with a fine fragrant bitter + . The Worcester and Hereford hops are chiefly grown on loose friable but shallow soils, of a red ochreous character, covering stone,-provincially termed "dun-stone,"-and resting, in some districts, on a soft crumbling species of rag-stone, which perishes upon exposture to the frost; yet they are highly esteemed for the delicate flavour which they impart to beer, and are therefore justly prized by the brewers of that light kind usually denominated table-ale ${ }_{+}^{+}$.

* Bors' Survey of Kent, 2nd eclit. p. 13:2. "Ilops that are full of condition, on being rubed hard in the hand, emit a decree of odour, and disclose a degree of clamminess, which are miversally admitted as criteria of their strength,"-Marshalls Suthern Counties, rol i, p. $27:$.

† Malcolm's Survey, vol. ii., p. 499. Stevenson's do., p. 329.

+ Surveys of Hereford and Worcester, Chap. I., sect. on Soil. 
Accolding to the best information which we have been able to collect, we believe that in every instance a fresh, rich, dark, loamy soil, interspersed with friable sandstone, and lying upon an absorbent calcareous base, is essential to the union of those desirable properties in a hop-ground, which consist in quantity, quality, and duration. The duration depends wholly on the nature of the land; and chiefly, if not entirely, on its sub-strata : for although there are instances of ordinary soils for the purposes of common cultivation having been so far improved as to have afforded full burdens of hops, yet no art has yet been able to bring land with non-calcareous subsoils to support such crops for a great length of time. Even the strong lands of the weald of Kent seldom throw up more than ten or twelve profitable crops, while on the strong rich loams of the Maidstone quarter, and the district around Farnham, which rest on rock and calcareous rubble, there are hop-grounds which have existed beyond the memory of the oldest inhabitant*. Land which abounds in flints or large stones, sands, or gravels, are unfit for this plant; and though large crops may be got on strong soils which do not lie upon either chalk or marl, yet in point of quantity, they are precarious, inferior in quality, and their rigour is comparatively of very short duration.

It would appear that the situation of hop plantations is not particularly regalded, for they are to be found in all directions. The hop, however, like all other delicate plants, is particularly liable to be injured by winds, the most destructive of which generaliy come from the south-west, and which are specially prejudicial during the spring months; therefore, wherever a choice can be obtained, a southern aspect should be preferred, and close hedges should be planter on those sides from which the most destructive winds blow. It takes three years in coming to complete perfection. On rich land, and in favourable seasons, it sometimes produces a small quantity of flowers during the first, and occasionally a moderate crop in the second year.

\section{CULTURE.}

The preparation of the land for the formation of a hop-ground should be commeneed either with a summer fallow, or as soon as possible after an early vergetable crop has been taken off; and as soon as that is done, it should be heavily manured with rotten lumg: from 25 to 30 loals are very commonly used, and some lav on much more. If the land be in clean condition after the removal of the crop, or if it has been got into good order by means of fallowing, then Norfolk turnips are very often sown, as they can be folded and got off early in the winter; and while they are eating off, the ground is trenched as fast as it is cleared in sharp ridges, to lay it up for the frost to penetrate and mellow it, preparatory to the planting. There are two modes of generally doing this; for that of setting the plants in pits, which was formerly adopted, has been now almost universally abandoned: namely,-

First, By trenching the ground all over two-spit deep with the spade, or double-digging it ; by which means the manure is more regularly and intimately mixed with the soil, which is thus rendered so very open as to be easily penetrated by the roots of the hops while they are young and tender, and the breadth of ground over which they can spread when they reach their full growth is much increased.

Secondly, By trench-ploughing; which is considered as preferable, by those who practise it, to double-digging - not because they regard it as

* Marshall's Southern Counties, vol, i., pp. 186 and 286; vol, ii. p. 49. 
equally efficacious in promoting the fertility of the soil, but because they view it as effecting every desirable purpose at a much less expense.

"This," as has been justly observed by Mr. Stevenson, in his Survey of Surrey, "may perhaps be true, if the double-digging be deemed a part merely of the preparation; but if it is regarded, as it ought to be, in connexion with the future management of the plantation, it will probably be found not only more efficacious, but, on the whole, cheaper than any trenchploughing that can be given; for it is evident, that in proportion to the care and labour bestowed upon it, will be the ease with which it may be worked during the continuance of the hops. When this circumstance is duly weighed, and the great importance of having the soil free and absorbent during the infancy of the plant is also taken into the account, the greater expense and labour attending trenching two-spit deep cannot justly be considered as unprofitable: trench-ploughing, however, will always meet with advocates and practisers among those who prefer a present saving to a future gain."

When the ground has been got into perfect order, the general methor is to draw a line parallel to one of the hedges, at the distance of a few feet from it, and to mete it each way with a gunter's chain, in the links of which bits of rag are fastened to ascertain the position of the hills, in which the sets are to be planted; which are then marked with pieces of short stick. This varies extremely; some placing them in rows at the distance of from seven to eight feet, and three to four feet apart from each other, while others adopt all the intermediate spaces. Near Farnham, the usual distance is from five feet to two yards square, and in most parts of lient a four-rod chain is used with a rag in every tenth link; thus fixing 1000 hills to the acre-some in regular rows, others in the quincunx fashion; but most generally in the latter, as the plants thereby liave more room, and when they grow up the current of air becomes more broken.

The usual time for planting is about the beginning or the middle of March; though sometimes, when young shoots are employed, they are planted in the month of October, and the sets that are used for this purpose are commonly "one year nurslings." These are cut off, in the preceding spring, from the lower part of the stem of the old hop, while it is dressing; they are then planted by themselves in a nursery, and the strongest of them are chosen next year for setting in the regular hop-ground. The usual length of the cuttings is four or five inches: it is desirable that they should have three or four buds; and, if very luxuriant when taken up to be planted, their tops and roots should be pruned. "Naked setts" are cut from the lower part of the stem, or, more properly speaking, from the crown of the root, and are immediately planted in the hop-ground; but they are less quick in arriving at a proper state for planting than nurslings, and have been found to be later by a year in becoming productive and arriving at maturity ${ }^{*}$.

When the spots for the different hills have been marked out, the earth is dug out of each to the depth of about two feet, and of nearly the same width; and these, if a portion of fine garden mould can be got, or, if not,

* The practice of nurserymen, with respect to the plants from which they take cuttings, has been recommended, viz.:- "Instead of the shoot from the parent stock, to twist it merely at the joint, where it is intended to be put into the ground; to fasten this into the earth, and having cut off the top within one or two buds of the ground, to leave it there till it strikes root: it may then be entirely divided from the parent stock, and left till the time of planting."-Stevenson's Rep. of Surrey, p. 339. 
a compost of well-rotted dung and earth, it is plated in the holes, which are filled up with the finely pulverized soil. The plants are then put in. Some put three or more in a circle, others two, and some only one rood plant; but the most general plan is to place three in each hole, at the depth of about six inches; and great nicety should be observed in fixing then. The holes made by the dibble for that purpose are in a slanting direction outward, so that the roots of the hop may grow in the incined position in which the poles are afterwards placed, without allowing their bines to bo interlaced. Some careful growers, indecd, put the fine mould gentiy in, around, and upon the plants, with the hand. 'The plants shuuld also be raised above the natural level of the cround, both in order that the hoj, may rise high enough to form the hillow to be marle around them, and that the root may have a greater depth of louse earth below them; for, when the land is very open, they penetrate so far into the foil that they have been found on a very rich deep lcam in the nejuliburlseod of farnham to the depth of twenty feet.

The whole field being in this mamner planted, the crreat oliject of the first year's management is to keep the intervals elean, and to fumish the young plants with fresh, well pulverized soil ; for which jurpose the ground should be hand-weeded, and either hoed, or dug with the spade. In the first case, the weeds are raked off; but in the second, they are buried by the spade, and the finest mould is carefully laid by hand round the tops of the hills. The more frequently these operations are performed the more licalthy will the plants become, as the moisture of the fiesh earth ereatly invigorates them; and, therefore, coarse woollen rags are very frequently placed upon them as manure. Ats soon as the young bocts anjear above the lills, they are stuck with small poles, from ten to twelse feet long, or in proportion to the length to which it is expected the bines will rum; and when they rise to about two feet, women are employed to tie or twist them separately round each. In a favourable seasun, and from stroner plants, a few hup is are produced, but they are never grathered, and they seldom bear until the second year. Early in Irovember the sroumd is chus all over, cardully turning it towards the plants, and then it is left rough during the winter.

In the second year, whenever the season is at all kindly, either in the month of February or the beginning of March, the hills are opened, in order to get at the lower part of the last yenr's shoots, which ought now to be pruned. The operation of opening the hills should be carefully done, either with a narrow-pointed hop-hoe, or with an inplement much used about Farnham, where it is called a "beck." This is a double tool: on one side, or end of it, is a strong loe; on the other a fork, or "spane," with two prongs, which, instead of being sharp and narrow like those of the dungfork, are flat and broad; thus when the spanes will not penetrate into the soil, the beck is turned and the lioe applied. The vine is cut off with at sharp pruming-knife to within an inch or two of the main stem, together with all the sucliers quite close to it. The soil is then replaced sufliciently to cover the mounds, in order to prevent the air from affecting the planits just before they begin to spindle; but is left rather bare around them. During the whole of this second summer the ground is hept stirred, and made perfectly clean, either with the horse and hand-hoe, or the spade; and when the vine shoots it will require poles of full twelve feet long.

In the spring of the third year, the earth, which had been replaced ahout the hills in the previous autumn, is removed from their tops, and the young suckers of the vines are again pruned in the same mamer as in the former 
year. A quantity of firm-yard dung, at the rate of 20 to 25 loads per acre, according to quality, or double that amount if made into compost with mould, having been brought on the land, the hills are then dressed in the month of March, either with that manure alone, or with the dung mixed with some of the soil. About the same quantity of similar manure, or any mixen that can be easily procured, is also usually laid on every other, or, at the farthest, every third year: it is carted out during the winter's frost, and after it has been regularly spread upon the ground, it is dug during that season with a "spud," or three-prongred fork, of about eighteen inches long and nine or ten inches broad; which tool mixes the soil more intimately with the dung than the common spade. In every successive year the process of becking the hills, and of pruning the crown of the plants, is also gone through about the middle of March, in the manner already described.

Such is the usual mode of culture in the counties of Surrey, Kent, and Sussex; but, according to an account received from a very respectable hop-planter in the vicinity of Wurcester, we find that in Worcestershire and Herefordshire hop-grounds are prepared by a double ploughing, about 12 inches deep, early in the winter, and about the beginning of March it is made as even as possible by sculling, harrowing, and rolling: an old piece of pasture land is in general selected.

The rows for the hop sets are marked out by sticks 8 feet apart, and a line with a feather, at intervals of 3 feet to 3 feet 4 inches, is then drawn the whole length of the row, and a stick 1 yard long is stuck in the ground at each feather-women with dibbles put in at each stick three sets, and cover them with light mould about half an inch, after having pressed the earth as close as jossible io them. About $j$ une the small bines are tied to the sticks with rushes, and the soil is ploughed up to themi : the weeds are then cut off with a "kuf," which is a kind of spade fixed nearly horizontally to the end of a handle or staff about 4 feet long. In the second year the soil is plonghed aum from the roots, and the shoots of last year cut ofl and a little louse soil thrum upon the crown of the root; in May they are poleci with two poles to each stock, about 9 feet long. In June four or five of the bines are tied to them, and the soil is kuf'd or moved about the roots and the weeds destroyed. In July about six inches of soil is thrown up to the crown of the roots with the kuf, and then ploughed up fiom the centre of the rows, and are afterwards harrowed, scuffled, hoed, and spittled un, as the jromietor thinks necessary. The third year they are joled with poles about 12 feet long, and are worked in a similar manner; the averige produce being about 2 cwt. per acre.

An acre of hop sround in these countics contains 1000 stocks, with 2 poles to each, so that 4 acres of hops will occupy about 23 acres of landand is by no mealis a remuneratine crop upon ordinary or poor soils. But if a valuable meadow be broken up for the purpose, and the grower has land to produce his poles, and will manure and work his land better than this is generally done, the produce may then remunerate its cultivation.

POLING.

When the vines have been cut and dressed, the hills are then poled with stakes of sixteen or seventeen feet long, and it is an operation in which great judgment is necessary, for if the plant be weak and they happen to be over-polerl, - that is, that poles of too great length are set-the roots will in the following year become inpoverished, and thereby sustain injury 
which cannot easily be remedied. The mischief is therefore greater than that which arises from under-poling, and may be easier guarded against; for should it be found that the vine grows stronger than was expected, either from the favourableness of the season, or the superior quality of the manure, an auxiliary pole may always be added to prevent the vines from dropping down, and keeping the sun and air from those parts above which they fall : it is therefore very desirable, that no poles should be put into a hill where there is not good reason to expect that the plants will grow to an equal length.

In placing the poles, they should incline somewhat outwards; from the well-known fact that, when leaning in that direction, they afford more room, air, and sunshine to the bine, and produce more hops than those which stand upright. The number of vines trained to each pole is two or three, according to their present appearance and probable future lusuriancy: the great object being to select such plants as not only promise to be healthy but also to throw their luxuriance into the main leading stem. As soon as the blossom is set, which is in most seasons about July, some growers, indeed, take off the leaves and side bines two or three feet from the ground, which they call "branching," with a view of giving air to the plant, and throwing the sap into those leading shoots; but this we conceive may be founded upon false principles, for it is well known that all plants draw a material portion of their nourishment from the leaves, and those at the bottom of the hop are generally the largest and the strongest *.

The number of poles usually required for an acre of hop-ground runs from two thousand four liundred to three thousand, according to the distance of the hills from each other. Throughout the greater part of Kent, to which our information chiefly applies, one thousand hills are generally allowed to the acre, and three poles are usually placed to support the bine; though sometimes two and three are placed alternately. The charge of Jabour for setting them is from twelve to fourteen shillings per acre of one thousand hills. The poles are divided into three classes to suit the soil, or age of the plants, and their price varies according to their distance from the coppice in which they are grown: averaging last year (1835) for the different length as follows:-

1st Class, 16 to 17 feet long $30 s$. per hundred
2nd " 14 , 16 s.
3rd ", 10 to $12,10 s$.,

The bark is generally shaved off. The women, by whom that work is usually performed, are paid one shilling per hundred: the sharpening of the poles at the lower end, which is afterwards done by men, is from $8 d$. to $10 \mathrm{~d}$.; they are also in some instances imperfectly charred by holding them over the smoke of a strong fire. In respect to stripping of the bark, although the poles are then said to last the longer, yet there appear to be different opinions regarding its expediency; for if the removal of the

* "Perhaps, when from the strength of the plant, and the closeness of the rows, the lateral branches occupy the greater part of the intervals, it may be proper to shorten some of the most luxuriatt; but I am not clear,-particularly as it respects the hop, which is one of the most tender aud delicate plants that are grown-whether it would not be more advisable to destroy every other plant in the row, or even every other row in the plantation, and by that means to procure the wished-for air, rather than, by shortening the shoots and taking off the leaves, run the risk of a very hot season, or an occasional cold and drying one ; thus injuring the plants and giving perhaps a sudden check to the blossom before it is out of all danger." - Malcolm's Agriculture of Surrey, Kent, and Sussex, vol, ii. p. 505. 
bark tends to preserve the pole from the worm, it deprives it of a certain degree of softness and warmth which are thought useful to the plant*. The timber used for poles in the midland counties is chiefly oak, the greater part of which is grown in the weald of Kent; but every kind of wood that is sufficiently straight and slender, is employed for the same purpose: maple, ash, and chestnut, being the best, and beech and birch the worst.

'I'he operation of tying the bine to the poles usually takes place in the month of May; or, more properly speaking, both the setting and tying are communly going on in different parts of the same plantation. The best of the vines are selected, three being generally trained to each pole, and there is perhaps no part of the business which requires to be so accurately timed, for if it be delayed until the bine spreads along the ground, it is difficult to change their creeping direction without injuring them: at the same time it is advisable that they should be sufficiently long to be attached to the pole without slanting them too much from the root. Both the wild and the cultivated hop follow the course of the sun in their windings, and when they are fastened to the pole, this inclination of theirs is therefore complied with; it has indeed been observed, that attempts to make the wild hop climb in a contrary direction have been found uniformly unsuccessful + . 'The binding is done by women, who tie the bines loosely with watered rushes about a foot above the ground, at the rate of about 11 s. per acre.

'The further operations, after that of poling, consist in cutting off' the bines that were rejected at the time of binding. This is not done until about midsummer, as it is thought better to allow them by creeping over the ground to keep it moist, while the trained vines are too young to effect that purpose; the branching also usually takes place about the same time; though some planters, as we have already observed, defer it until the blossom is fairly set. After this the ground is beckerl a second time, and half-hilled, to draw up a little mould into the crown of the hill with the hoe-side of the beck; and a few weeks afterwards the same process is again repeated. In the routine of the Farnham planters, who spare neither labour nor expense, the ground is also "peared," or hoed just before the picling of the hops, in order to destroy any weeds that may have come up since the last becking; and in case the moistness of the season, or the richness of the ground, should bring forward any weeds between the first and second becking, the ground is also hoed or peared at those times ${ }_{\ddagger}^{+}$.

\section{PICKING, OASTAGE, AND BAGGING,}

are the operations which close the culture of the hop, which begins " to bell," or show the seed-vessel, some time in August; and if the weather prove favourable, it will be ripe by the end of the month, or the beginning of September. When the seed begins to change from a pale straw-colvur

* The maple has been particularized by Mr Marshall as beinir yeculiarly soft and warm; adding, that an intelligent planter in the vicinity of IIaidstone remarked to him, "that he had frequently observed, when the morning has been cold, the sensitive leader of a tender fresh-poled vine, reclining its head against the velvet bark of the maple, while others held theirs aluof from the chilly smooth-barked poles." - Southern Counties, vol. i. p. 211.

+ It has been already noticed, that it is sometimes necessary to substitute a taller pole for one that is not sufficiently long; or to interchange the poles of the over-poled or under-poled vines. "This necessarily requires a careful and steady hand to draw out the vine, and lay it gently and regularly on the ground till the proper pole is brought to it: still greater nicety is required in observing all the curves of the vine while it is wound round the pole:" - Stevenson's Survey of Surrey, p. 350.

$\ddagger$ Stevenson's Survoy of Surrey, pp. 353, 355 . 
to a light brown, to cinit a fragrant smell, to fecl firm, and to be easily rubbed to pieces, they are signs which indicate its having arrived at maturity, and being ready to be gathered.

'The process of picking is a rude scene of animated bustle and cheerfulness; for, beside the labourers on the farm, the pickers are composed of motley groups of men, women, and children, of all ages, who are congregated together from ail parts of the country by the certainty of employment, and who enter upon tieir task with a degree of hilarity apparently unchecked by their seldom finding other shelter during the time than what may be afforded by the covering of a rug, spread by the side of a hedge, upon hoops, to form a ripsy tent. Iet here, when evening cluses upon their labour, they enjoy themselves with a profusion of common comforts, cooked with firing not over scrupulously culled from the farmer's herlges ; and the final conclusion of the harvest is ended by a merry-making, at which some handsome nymph of the party is dizened out in all her funcry, and elected to preside over the revels as hop-queen.

As a preparation for the gathering of the hops, strong frames of wood, called "bins," or "cribs," about nine feet long and four fect wide, are placed in difierent parts of the plantation, fixed upon legs three feet high ; thus affurdingr room for three or four pickers on each side, who, togrether with the man who collects the poles, are called a "set," and deposit the hops, when piclied, in a coarse cloth, which is hooked to the insides of the frame so as to form a large bag in the centre.

'The bine is first cut about two or three feet above the ground, much lower being considered injurious to the root, by the profuse bleeding which it occasions, and the pole is then wrenched from the earth by means of what in Kent is called a "hop-dog." This instrument is constructed of a strong, tapering stick, near three inches in dianeter, and about five feet long; at the distance of nearly a foot from the larger end of which a smail bar of iron, of about a foot long, is clenched; and being bent in the middle into an acute angle, the inside is roughened by the smith into something like teeth, which, when fixed upon the lower end of the pole, as it were, bite and hold it fast.

The poles are then laid horizontally across the frames, and two are usually given to one set; but smaller frames or baskets are also very commonly used, and it is then customary for one woman to engage a basket for herseif and family. The price of payment varies according to the quantity grown, and is often not named until after the picking. Upon an average growth the pay is about $10 d$. the basket of five bushels; and a tolerable good picker will earn 2: a-day. The number of bushels picked by each set being lsept by means of the double-tally*.

'The weather decmed most favourable for picking is that which is neither very suitry nor moist; for if the sun be very hot and scorching, it is apt to shrivel and discolour the hops before they can he gathered off the poles; and if the morning be dewy, those which are picked in a damp state become musty. To jrevent injury from the sun, the pole-pullers therefore take down no more than the pickers can strip in a short time; and if it be neces-

* This simple device is formed of two thin pieces of wood, which are neatly planed and accurately fitted torether ly means of a shoulder foumed on the principal part, or lally, which is three inches longer thin the inferior part, or weck; the two, when joined tugether, makin the dmelle-tally, which is generally twelve inches long by one and a half stuare. One of these is appropriated to each set, who receives the check part, the tally remaining with the fureman, who, as the baskets are delivered, cuts one noteh at the same time upon both by joining them together. 
sary to begin the picking before the dew is evaporated, the pole is shaken to and fro, in order to throw off as much of it as possible. As it always happens that the hops do not ripen at the same time, neither do they all rum of one quality upon the same bine. The Farnham planters, who are particularly attentive to the maintenance of the long and well-established credit of their hops, not only set out all those that are fresh ripe for the first gathering, but make such distinctions in the appearance of their respective yualities as in their judgment seems most proper, so as to assort them into at least three difierent parcels, each according to their separate value. It is their practice, therefore, "to begin at the bottom of the pole, and to pick the hops, one by one, without bunches, long-tails, or leaves. Those that are just of the proper degree of ripeness, and are full and fair in their appearance, are first gathered, and put by themselves into the bin-cloth: such as are rather inferior in quality, or not exactly taken at the proper degree of ripeness, are of the second sort, and are likewise jut br themselves in a basket. As, however, with the Farnham planters, hops that are under-ripe are more esteemed than such as are over-ripe. the second sort takes in only the creenish hops: such as are brown and over-ripe are the thiri sort; and if the grower is very nice, a fourth basket is set for such as are defective in their form, or have received a check during their growth $*$."

It is of the greatest consequence that the hops should be dried as soon as possible after they are picked, since, if they are liept long in the baskcts in which they are brought from the grounds, they are apt to heat and spoil: the hops picked in the morning are therefure carried to the "oast," or liln, at noon.

The operation of oastage is one of great nicety, for the strength and flavour of the hop are extremely volatile. 'The oast is nearly similar to a malt-kiln, and the hops are laid in parcels of 25 to 30 bushels, about five or six inches thick, upon a hair-cloth. The kiln having been previously heated, the temperature is regulated to one miform but moderate degree of heat, in order that the hops may not dry too fast, and is kept at that degree until the upper part of the heap appears to have felt the fire; when, the lower part being then considered dry, the heap is turned; but, before that is done, the heat should be somewhat lowered, and restored when the turning has taken place. The thickness of the heap must depend upon the state of the hops, for, if they be full of moisture, they should be laid upon the kin very thin, and a less degree of heat should be applied, or otherwise the steam arising from them will make them cake, or run into lumps $\dagger$. They talie, in greneral, about ten or twelve hours in the drying. A fourteenfeet hiln will therefore dry in the twenty-four hours 200 bushels of hops from the bines, or about 350 lus. of dry hops; that is to say, at two dryings, for the men work all night. The fuel usually emploved is either colie or charcoal, as being the most secure from communicating any smeli which might injure the delicacy of the flower

suon after the hops are upon the kiln, sulphur is commonly applied by

* Stevenson's Survey of Surrey, p. 36].

$f$ To guard against this some kilus are constructed with double floors, one about five or six fiet above the other: "the hops that are greenest, or contain most moisture, are put on the upper floor, and are thus dried very gradually, and fitted to receive and support the greater heat of the lower floor." - Stevenson's Survey of Surrey, p. 363.

it The cost of erection of a small oast-house, with a store of moderate size, will, if substantially built, be from $200 l$, to $300 \%$ : but many cost much larger sums. 
the Sussex and Kentish growers, with a view to improve the bright yellow colour which is so much admired in the sample *; and by some saltpetre is mixed with the brimstone, but its use requires great care. The Farnham planters, however, never make use of sulphur for the hops of superior quality, though they sometimes employ it to bleach those which have become brown, either by being overgrown, or by having received injury from the weather; for they find that the best way of securing the delicacy of that pale hue upon which they plume themselves, as being peculiarly requisite in brewing light-coloured ales, is by picking the hop early, and drying it very gently. Whether attributable to that, or to the absence of the brimstone, which is thought by many to injure the strength of the hop, it is a certain fact, that those grown in the district around Farnham are of better quality than those grown in any part of Kent or Sussex. They do not however maintain their properties longer than one or two years; whilst the best golding hops that have been ripe when picked, high dried with sulphur, and packed in good tow bagging, have been known to keep good during twelve years.

When sufficiently dry, the hops are shovelied to the upper floor of an adjoining store, called the "stowage-room," in which they are baggred. About twelve baskets of the gauldens will in general go to the cwt.; but some growers have introduced the wild, or what they term the "Canterbury grapes," which take fifteen or sixteen baskets to the cwt.

The bagging for market does not take place until some days after the hops have been carried to the store-room, as from the extreme state of brittleness in which they are when taken from the kiln, they would be broken if immediately handled, and the sample would be thus materially injured. They are therefore laid in heaps upon the floor, in order to give them that degree of toughness and tenacity which they acquire by a moderate degree of sweating. They are then put either into bags or pockets. The first picking, being generally of the brightest colour, are usually put into the pockets; and the late pickings, from being brown, are packed in bags of about seven and a-half feet in length, and eight in circumference, which are universally of $2 \frac{1}{2} \mathrm{cwt}$., while the weight of the pockets is only $1 \frac{1}{1}$ to $1 \frac{1}{2} \mathrm{cwt}$. : that of the bagging itself is $25 \mathrm{lbs}$., whereas the cloth of the Canterbury pocketing is so much finer, as to be oniy 5 or $6 \mathrm{lbs}$, which alone will often account for the difference of price in favour of the pocket. The mode of bagging is as follows:-

A circular hole, covered by a trap-door, and sufficiently large to admit the mouth of a hop-bag, is made in the floor of the stowage-room. A few hops are tied tight in the lower corners of the bag, in order that, when full, they may be lifted and removed with ease. A hoop, rather larger than the circumference of the hole, is used to stretch out the bag, by means of hooks on the outer side of it, the inner side of the hoop, when the bag is let down into the hole, either resting on the floor, or on a frime of wood made over it. When the bag is thus stretched out, and let into the opening, the "feeder" throws down a few shovelfuls; and the "bagster," descending into the bag, with flat shoes, or leather socks, on his feet, treads

* This, although a general and undisguised practice, is however illegal; as by Stat. 7 Geo. II. c. 19. confirmed by 35 Geo. III., it is declared, "that if any person shall mix with hops any drugr or ingredient, to alter the scent or colour, he shall forfeit $5 \%$ per cwt. ;" and a conviction upon which was obtained in a prosecution (Rex v. Pack) against a person who used sulyhur in the process of drying hops ; Lord Kenyon being clearly of opinion that it is an offence within the meaning of the Act. 
the hops regularly and carefully down, especially towards the sides. More lops are then thrown down, and closely pressed, until the bag is filled; the tighter and closer the better, for the firmer they are packed the longer will they keep. The hoop is then loosened, the bag is let down to the lower floor, more hops are tied into the upper corners, and it is sewed up as closely as possible, the whole operation being generally completed within an hour.

\section{PRODUCE AND EXPENSES.}

The amount of crops varies according to the soil and season, from $1 \mathrm{cwt}$. to $20 \mathrm{cwt}$. per acre, for they are liable to various casualties and distempers, by which they are often attacked when apparently in the most luxuriant state of growth, and which frequently terminate fatally, without any power on the part of the grower to prevent it. About 7 cwt. per acre may, however, be considered as an average produce on the general quality of hop-land, and this, in ordinary seasons, is found to remunerate the planter. It is, however, a singular fact, that a very profuse crop, if generally obtained, is not considered favourable to him; for not only does the price decline in a jroportionate degree, but the Excise-duty*, and the expenses of picking, bagging, carriage to market, warehouse-rent, and factorage, are all thereby largely increased, while the sale of inferior samples becomes so difficult, that they sometimes hang on hand for two or three years, and, if then sold, are so deteriorated in value, that nothing short of an extraordinary rise in price can reimburse the charges.

The expenses up to the time of pickin ar about the same whether the crop be good or bad-consisting of rent, tithe, taxes, interest of capital, manure, wear of poles, labour, and ammual proportion of raising the plantation; varying much in different counties, Worcester and Hereford being the lowest, and Middle Kent the highest. The average is usually calculated at about $30 l$. per acre before picking commences; and although some planters manage at something less, there are many whose expenses are much higher. The stocks of poles vary much in value-say from $15 l$. to $40 \mathrm{l}$, during the establishment of the hop-yard, the charge of their annual wear and tear being from $6 l$. to $8 l$. and the labour from $13 \mathrm{~s}$, to $17 \mathrm{~s}$. The picking' and other expenses, including drying, brimstone, beer, cartagre, and factorage, may amount to from $23 l$. to $26 l$. per ton without the duty, or, in round numbers, the whole will vary, as the crops and management are good or bad, from $37 l$. to $48 l$. per ton.

This estimate has been received from a most eminent planter in the county of Kent, whose knowledge of the subject camnot be doubted; but as expenses differ in various places, we annex some other calculations which we have obtained from large growers, upon the accuracy of whose information we also place the most unreserved reliance. Thus, in the neighbourhood of Canterbury and Maidstone, it being assumed that, as an acre of hop-ground contains 1000 hills, each hill has generally three poles to support the bine of the hop; consequently 3000 poles are required

* The duty is $2 d$. per $1 b$. which presses heavily upon a large crop; it has therefore been thought that, even were it expedient to continue it, were a sum equivalent to the average amount to be laid in a fixed sum per acre upon the land, instead of the crop, when grown with hops, it would in such cases lighten the pressure upon the farmer, besides relieving him from the annoyance of the exciseman. 
to each acre in the first instance; and to keep up the losses of poles by damacre, decay, \&c., will recuire 500 where $\mathrm{Y} 0.1$ is used; 600 of No. 2 ; or 800 of No. 3, annually. Therefore,

\begin{tabular}{|c|c|c|}
\hline If we assume 500 of No. 1 , at $30 \mathrm{~s}$. & & 710 \\
\hline Carriage of ditto & - & 110 \\
\hline Rent, including parochial rates, \&c. per acre & - & \\
\hline Tithes, per acre & - & 110 \\
\hline $\begin{array}{l}\text { Picking, drying, carriage to oast, packing ani } \\
\text { per cwt. on } 7 \text { cwt., per acre about }\end{array}$ & $\begin{array}{r}18 s .81 . \\
.\end{array}$ & 13 \\
\hline Diggring, shimming with horses, harrowing. \&e. & - & 115 \\
\hline $\begin{array}{l}\text { Manure, on an average } \\
\text { Tying the hops to the poles by women, che }\end{array}$ & $\dot{0}$ & 30 \\
\hline $\begin{array}{l}\text { Tying the hops to the poles by women, chan } \\
\text { other incidental expenses }\end{array}$ & the 1 & \\
\hline
\end{tabular}

Per acre . $£ \widehat{53510 \quad 0}$

or about $5 \mathrm{l}$. per cwt., to reimburse the whole average cost of the cultivation of an acre. The tithe on hops is never taken in kind; and therefore the charge under that head, as well as the parochial charges, are the same, whether the land be productive or not.

At some few miles from Canterburv, the rent of hop-ground will not he so much as $6 l$, it being often let in small patehes with the farms, at the same rent: but then the difficulty of obtaining manure is much creater; thus, as appears from the following accounts, fully equalizing the difference of price in rent :

Expense of an acre of IIops, in the Parish of Thannington, one mile from Canterbury ; average produce $7 \mathrm{cwt}$. per acre.

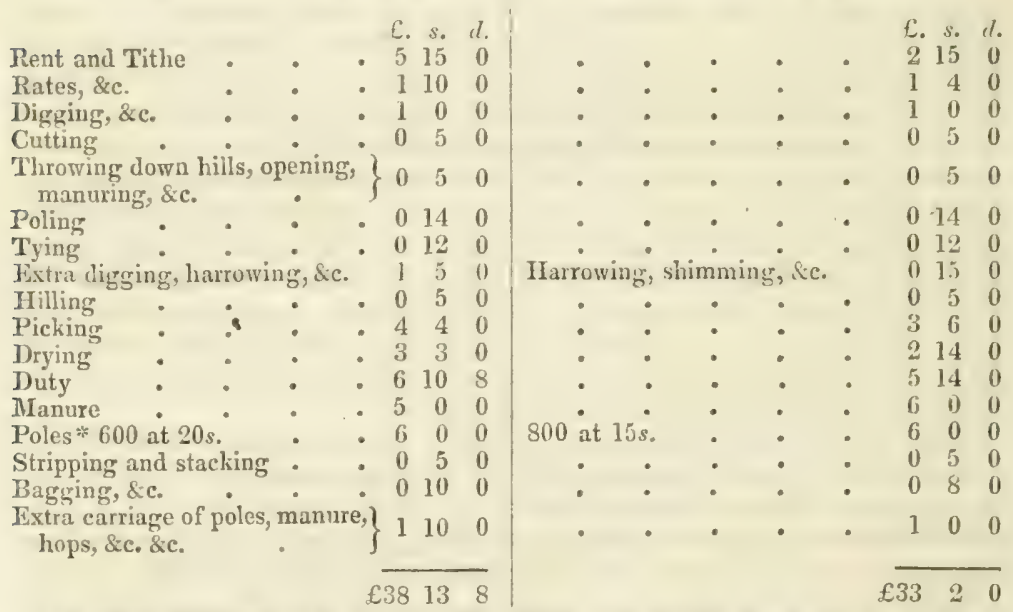

In some instances $15 \mathrm{~s}$. to $16 \mathrm{~s}$. are given for poling an acre, but a great deal of hop-ground labour is done by the day. The hops are picked by women and children in baskets of 5 bushels, and, in Kent, are usually paid for at the rate of two pence per bushel; about 12 baskets going to the cwt. when dried. In Worcestershire and IJerefordshire, however, the common charges are only

Poling alone, $3 s .6 d$. per acre, (poling and $u$ rorking per acre, 13s. Grl.)

* Annually to keep up the stock in good conclition. 
Picking, 1 s. per day, or $1 \frac{1}{2} d$. per bushel.

Oastage, 1/. per week, (they work or attend night and day.)

Bagging, 5d. per liundredweight.

The hop-pickers have a sleeping place fomd them, their breakfasts daily, and one dimner a-week, with a very moderate supply of cider or beer; a generous cultivator, however, sometimes allows the pickers two dinners a-week.

The hop-growers find woollen rags the strongest, most durable, and most suitable manure. On plantations convenient to the sea, sprats and star-fish are also frequently used.

From these estimates it will be collected that the expenses upon hopgrounds amount to sucl heavy sums that, even in appropriate soils, it is not every man who can command sufficient capital for their establishment. Added to which, the enomous consumption of dung without anv return to the land is a serious drawback on the efficient management of a farm; and, although the culture of hops may in a course of years prove lucrative, yet the great uncertainty of its returns renders it so speculative as to partake more of the nature of gambling than of the regular occupations of husbandry. This, however, seems to be the great inducement for its continuance; for although heavy losses are frequently sustained, yet as brilliant prizes are also sometimes grained, there are always people to talse the chances of such a lottery. The excise duty on the growth being 1 s.s. $\mathrm{sd}$. per cwt., the following account of its ammual amount during the last dozen years, together with the prices of East lients from Lady-day to Midsummer, and from Michaelmas to Christmas in each year, will clearly show the precariousness of the crops and prices.

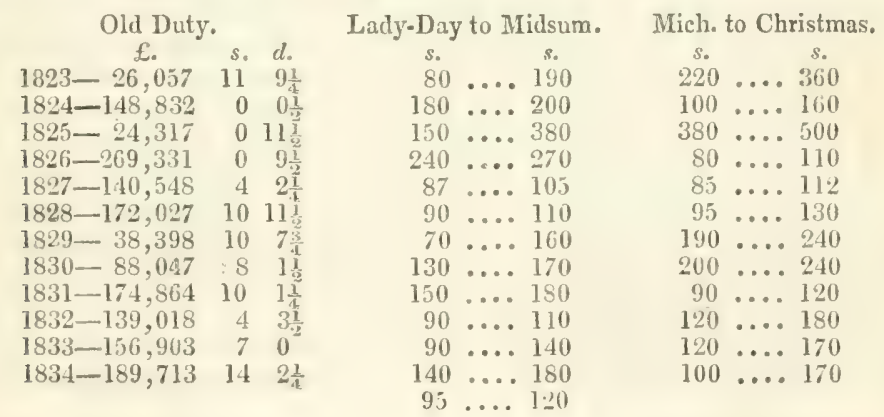

\section{PROPAGATION BY SEED.}

The culture of the hop being subjected in most cases to a fixed routine of management, which deprives it of the means of improvement, though hop-srowers complain that their plants die off, rum out, become small, and of a bal colour; and it has been not unjustly remarked by an experienced agriculturist, "that it appears advisable, in raising fresh varieties, to revert to the seed, either of the wild or the cultivated kind*, a lint which has been lately adopted with apparent success by Mr. Lance of Lewisham, who aiso strongly advocates the propriety of retaining the "buck," or" " cock-hop," as the male plant is usually termed, and which is, according to the common practice, rooted out of most plantations; from whose

$$
\text { * Marslall's Southern Counties, vol. i. p. } 184 .
$$


observations upon a subject of such interest to the grower, we shall, with his permission, make a few extracts *.

"'The seed sown were the 'goldings,' selected from plants grown in a hedge, where there was a male plant. As the organic structure of the hop may yet be unknown to many, it is proper to state that the blossoms of the male and female grow on different plants, the male having its flower or pollen previously perfected, so as to impregnate the stigma, or burr, of the female as soon as it is ready; which inoculation gives that energy and vitality to the seed, without which it would not produce its kind. This it is which gives great weight to the hop; which gives the fine aromatic bitter to the production of the bine, and more fully so to the seed-the petal or leaf of the flower containing but little of the astringent quality of the hop. We therefore do strongly recommend all cultivators of the hop to have many male plants in their ground, and particularly to encourage them around their plantation in the hedges, where no ground will be lost. Let there be some in all situations, so that, let whatever wind blow, the pollen may be wafted to the female. We write from experience, having raised very many plants from seeds, which are found to be much more energetic and vigorous in the growth and produce than if raised from cuttings. We have had a hill produce $3 \frac{1}{2}$ los. of hops, weighed after drying, from a plant the third year after sowing.

"The hop-plants raised from seed surpass those which have been raised from cuttings, their luxuriant growth enabling them to withstand the effects of blight. 'They are found to have a seed at the bottom of every petal of the flower, of a most pungent aromatic flavour; while those hops grown in the usual way, without the necessary quantity of male plants, have scarcely any seeds, and these are mostly abortive. In fact, seed gives weight and flavour to the hop, and consitutes the vitality of the plant, or the condition or strength of the hop; and where there is most seed, there will be the most conditiont. We therefore say, that the grand object in hop-growing should be to get as much seed as possible. Indeed, seeing that the fructification of the plant cannot be completed without the male bloom, it is most inconrrrous for hop-planters to shut their ears against the voice of reason and nature, and care nuught about the male plants; yet they grub them up, wherever they find them, as useless lumber, saying-'their fathers got money without male hops, and so can they; for why should they pretend to be wiser than their forefathers ?' Nature, however, makes nothing in vain; and if the service of the buck be necessary to propagation in the

* See a tract by him entitled the "Golden Farmer," published in 1831, and Baxter's Library of Agricultural and Horticultural Knowledge.

+ -A specimen of the hops grown at Lewisham was exhibited at the Malling Agricultural Mleeting, and were allowed by all judges to be the most pungent and aromatic sumple in the booth; the strength of them for the brewer was considered to surpass all others; although the colour was not of so delicate a hue."-Guiden Farmer, note Z.O.

"The weight of hop-seeds without the leaves having been accurately ascertained, those grown at Lewisham-being the fourth year from the sowing of the seed, and having a male plant close to them-weighed at the rate of $36 \mathrm{lbs}$. per bushel; and the s:me quantity (by old measure) from Mr. Love's ground at Shoreham, raised from cuttings, and grown near maie plants, weighed at the rate of $35 \mathrm{lbs}$. per bushel. The hopleaves, without seed, from Mr. Jorden's at Oxford, where the male plants are always eradicated, weighed at the rate of $22 \mathrm{lbs}$. per bushel when closely pressed.

"Hop-seeds being severally put into rain-water, most of those produced by plants raised from seeds, and grown near male plants, sunk in the water; while must of those grown in grounds that had not any male plant near them, and which had been raised from cuttings, swam on its surface." - Ibid. note $\mathbf{X}$. 
animal kingdom, that of the cock-hop cannot, upon the same principle, it is presumed, be useless in the impregnation of the female plant.

"In sowing the seeds, care should be taken that they be selected from hops that have been properly impregnated by male farina, taken from the bine near a male hop. They should be sown early in the spring, in a mixed soil of chalk, clay, and sand, among which the latter earth should predominate. These may be set out in rows at the latter end of the year, where they should remain until the sexual distinction can be ascertained: this may be done by observing the leaf, and the forwardness of the plant. The next vear the males will show a rough leaf, when the females are only just breaking out of the ground. When the sexual distinction is known, at the latter end of the second year they may be planted into the ground where they are to remain, and may be poled the third year. Every fifty plants should have a male; and there will be no objection to a male plant being in the same hill with the females. If the soil be congenial, and the season not unpropitious, they will bear hops the third year."

Regurding the after culture, the best hops grown at Lewisham have been trained horizontally in the espalier form, on poles five feet high and three feet apart, with a long pole fixed at the top to keep them steady. The male plants should be grown on upright poles, as the farina could then fall uninterruptedly on the females beneath. The frame for training hops, as represented in the annexed figure, is fixed in the ground, and not intended to be removed. The lower part of the five-feet poles are charred to preserve them; the horizontal poles are cleft halvelings, and nailed or tied to the uprights. The plants may be set at every stake, and rows formed one way across the field, thus-

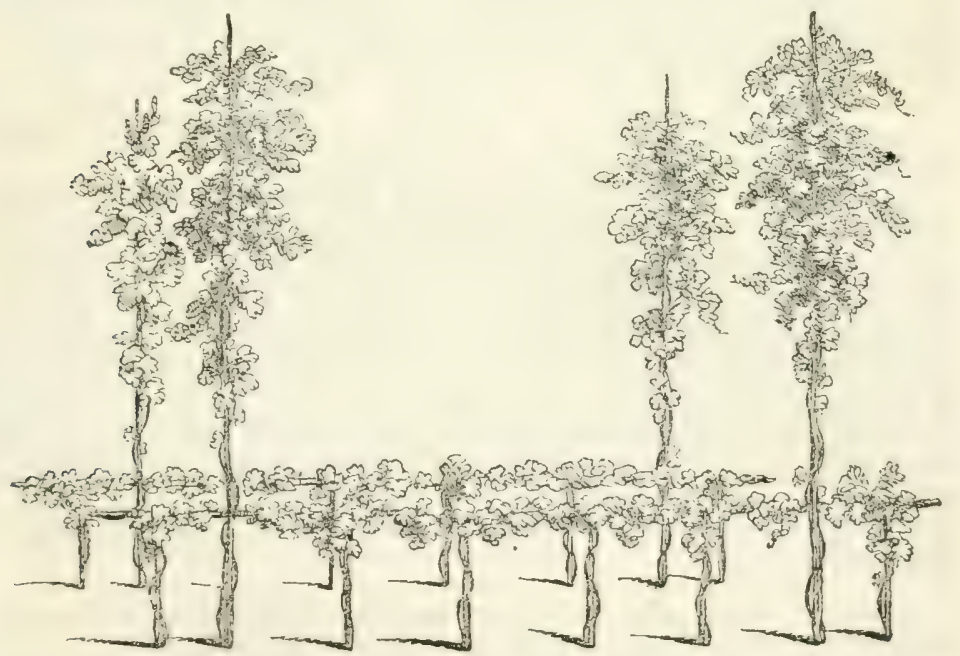

This method, which has been formerly recommended by the Rev. Arthur Young*, might be adopted with success, where poles are scarce, or where the ground is exposed to high winds; for when the hops are ripening they sulfer much from wind, and thus getting bruised, they soon change to a

* " The hops should be in espaliers, to save in the expense of poles, and to throw the binds nearer to the ground, on the principle of vineyards, which never ripen well, nor yield plentifully when the vines are suffered to rise high." --Surv. of Sussex, p. 131.

VOL. II. 
brown colour, which necessitates the drier to use brimstone to recover the delicate yellow which is so much admired in the sample. In training the bine, women might be employed in tying the plant to the frame, as fast as it grows, with matting, or old bine, until the hops come into bloom, when they should be left to themselves. The upright poles may be apart from the frame, in the usual way, and might be fixed a little out of the exact line of the frame, so as to distinguish them at the proper season. 'The plan will have the effect of preserving the colour, as the wind cannot have so great a power over the poles, to the injury of the bine and hops.

\section{THE CASUALTIES AND DISEASES}

to which the plant is subject are, no doubt, in most instances attributable to an unsettled state of the atmosphere; and it is a commonly received opinion, that seasons which are rather farourable to other plants are injurious to the hop. The attacks to which it is most exposed are, however, chiefly of some of those numerous tribes of insects known as plant-lice, which are generated by means of which we have only an imperfect idea, and are brought forth in such vast swarms as frequently to cover the plant, the leaves of which they suck, and cause them to curl downwards, with an appearance of blight, looking black and sickly. If they continue for any great length of time, the hopes of the grower are, indeed, not uncommonly ruined by their depredations above ground, while the wirc-worm acts with such eflect upon the roots below, that one-ihird of them have been sometimes destroyed by it. Efforts are constantly made to check the progress of the injury, by burning heaps of damp straw, fern, and other rubbish, during a day or two, on the windward side of the grounds, together with sulphur and damaged tobacco, in order to fumigate the plants: a practice from which no harm can arise; for if it does not succeed in driving away the fly, the ashes, when spread, will tend to destroy the larve of the insecis which are in the ground. No effectual remedy has, however, been hitherto discovered, and various erroneous accounts have been published on the propagation and ravages of the different species*.

The mouldy.fen and the mildew act in different ways as a blight upon the plants; the fen being more fatal to those grounds which are low, moist, and sheltered, than to those which are high and open. The honey-dew, though generally regarded as a disease of the hop, has been ascertained to be nothingr else than the excrement ejected by the hop-louse ; and fire-blasts, which usually occur in the month of July, sumetimes scorch up whole plantations, from one end of the ground to the other, when a hot gleam of sunshine has come immediately after a shower of rain; while at others it only affects them partially, or in a particular portion of the plant. Experiments are indeed much wanted on the diseases of plants in general, for the subject is now wrapped in mystery, and can only be elucidated by close and accurate observation.

* Thus, the insects called "lady-birds" have bcen accused of committing ravages upon the hop, though in fact they feed upon the fly with which it is covered. Many hop-crowers also plant beans in the intervals hetween the rows, under the conviction that they attract the hop-fly, though the insects found on the two plants are not of the same species; the bean-plant louse being black, and the hop-plant louse green.--See Rennie on "British Plant-Lice," in which there is a very detailed account of the hop-fly. Quart, Jour. of Agric, No, xxx. 


\section{Chapter XXXI.}

\section{ON ORCHARDS-CIDER AND PERRY.}

THE apple and pear-trees which form our orchards are well known not to be the natural production of any soil or climate, the one being a variety of the crab, and the other derived from the common wild pear. 'The native wild crab is subject to considerable diversity in the appearance of its leaves, as well as in the colour, shape, and flavour of its fruit. By selecting and cultivating the fairest of these, all the valuable varieties known throughout Europe have been produced, and by repeated propagation new sorts, differing apparently in species, according to the climate in which they have been grown, have been successively introduced into the various countries of the north; for in tropical climates they do not come to perfection. The principle, indeed, was well understood by the ancients, and the art was gradually spread over the Continent, and adopted in this country by the Monks, yet orcharding did not become a considerable branch of rural economy in England before the reign of Henry the Eighth. Some artificial varieties had indeed been occasionally imported from Normandy at an early period, but about that time a person of the name of Harris, who was fruiterer to that king, is said to have planted the environs of nearly thirty towns in Kent. This example doubtless introduced the practice upon a more enlarged scale; but the period when the plantations in Herefordshire, which is the most celebrated cider district, acquired its peculiar eminence, seems to have been the reign of Charles I., when Lord Scudamore retired, after the assassination of his friend the Duke of Buckingham, to Home Lacey, where he occupied himself in the honourable employment of a country life, and paid particular attention to the culture of fruit-trees, which afterwards became a favourite amusement among the gentlemen of the county.

Although few farms of considerable size are in this country without an orchard, the produce of which is, in most cases, larger than can be consumed by the family, yet it is only in our southern and western counties, and a few scattered spots in Ireland, that they are planted of such extent as to render the manufacture of cider and perry a material object of farming business; for although in the part of Scotland distinguished as Clydesdale they amount to several hundred acres, yet they are entirely appropriated to the production of table fruit. In those districts to which we allude, namely, Hereford, Worcester, Somerset, Devon, and Cornwall, the nature of the soil and the aspect of the ground are generally attended to; but in other places they are commonly fixed, without regard to either the lind of land or its exposure, being as often found open to a northern as to a southern direction, or to any intermediate point of the compass which may be most convenient to the site of the adjoining homestead. The trees are also usually planted at various distances from each other, more according to whim than the dictates of sound judgment; permitted to grow either straight or crooked, as chance may direct, without pruning, care, or culture ; and as they are all in pasture, horses, cows, and hogs are almost universally allowed to eat down the herbage, to bark the stems, and, when within their reach, to browse upon the branches*.

This is no exaggerated picture of the manner in which the common run

* In large animals this latter mischief can, however, be prevented by the use of a halter and martingale, attached to a surcingle which is girthed round the body. 
of farm-orchards are neglected. As to the mode in which thev have been laid out, an evident want of judgrment must have prevailed in the original planters of many of them, which ought to be avoided; for, however desirable it may be to have them as near as possible to the divelling, yet, if the situation be unfavourable, another should be chosen, and the trees should be at such distances as to afford room for the circulation of the air, as well as to admit the influence of the sun's rays upon the ground, both for the benefit of the roots and fur the absorption of all superfluous moisture. These remarks, it must, however, be admitted, will only strictly bear on those orchards which are partly intended for profit; for they are convenient as inclosures near the dwelling-house, both as ormament and shelter, as well as for the weaning of young calves and the care of weakly lambs.

\section{SOIL AND SITUATION.}

In the formation of an orchard, it should, therefore, be placed in a situation which is neither too elevated nor too low; for fruit-trees thrive better in moderately high and open spots, than in those situated in a dell. It should, therefore, not be either naturally or artificially too much sheltered; for if protected from the prevailing winds, the higher it is situated the less liable will it be to be injured by frost in the spring and early part of the summer; but it should be placed, if possible, on the southern or south-eastern slope of a gently rising ground. If, however, a low and sheltered situation can be found, with a northern or northeastern aspect, it has been asserted by an experienced orchardist, that, " as the blossoms will later unfold themselves, so will they be less liable to blast in the spring, than on a southern aspect; and such he has linown to answer well for both cider and table fruits." Tet he admits "that the apples grown facing the south, and the cider produced from such apples, are higher flavoured than when produced from the north*." It should be well fenced with both hedge and ditch, to form both a protection against intruders, and to gruard the young plants from the effects of wind.

The soil should be either naturally good, or made so by artificial admixtures of different qualities, so as to be neither too stiff nor too wet, and of sufficient depth. Respecting the nature of the land, it is, indeed, stated, in the "Transactions of the London Horticultural Society," that " in sandy soils the apple-tree will ripen its fruit earlier and more completely than in stiffer land; but it will not last so long. It requires from five to twelve or thirteen years to bring it to maturity; the pear from twelve to eighteen ; and the soil should be just sufficiently rich to encourage a moderate degree of growth, without stimulating the plant to premature exertion, which always induces disease. 'The soil of an old garden is, therefore, peculiarly destructivet;" yet that of a hop-ground is, in Herefordshire, regarded as peculiarly appropiciate $\ddagger$.

In Herefordshire the planters formerly thought that good ciler could only be produced from light sandy land; and Philips tells the farmer to

\section{"Look where full-eared sheaves of rye}

Grow wavy on the tilth: that soil

Select for apples."

Now, however, the ground best adapted to most kinds of apples is found

* Survey of Cornwall, p. 97 .

+ 'Trans. of the London Hort. Suc., vol, i, p. 39. Survey of Herefordshire, p. 77 ; of Cornwall, p. 96 ; of Devon, p. 238.

+ Herefordshire Rep., p. 83. 
to be a deep rich loam: for on this the trees grow with the greatest luxuriance, and produce the richest fruit; though sume trees-the "stire" and the "golden-pippin" in particular-form exceptions to this rule, and flourish most in a hot and shallow soil on a lime or sandstone bottom. The best sort of pear-trees also prefer the rich loam; but inferior sorts will even flourish where the soil will searcely produce herbage. In Cornwall, also, "where the earth does not exceed nine inches in depth, with a subsoil of yellow stiff clay, whereon alders grow, and where horses could hardly pass, apple-trees have been known to thrive, with very little expense, infinitely better than on the arljoining drier and better soil ; and it is certain that lands not suitable to other produce may be turned, with little cost, to thriving orchards." Mr. Smith, indeed, who published a map of the strata in England, states that all the best orchards are those planted upon red marl, though it is a generally received opinion, more particularly in Devonshire, that apples raised upon a clay soil make the best cider. We agree, however, with Mr. Main, of Chelsea, "that a deep mellow loam is most suitable for an orchard. It does not require to be richly manured, provided it be fresh, unexhausted, and sufliciently dry. Whether the subsoil be gravel or stone, so as such beds lie not too near the surface, it will be no detriment to the trees; but if of a tenacious clay, which is retentive of moisture, then draining must be resorted to, and be done effectually *"." The land is, indeed, generally left under pasture, without further care; though if occasionally dug round the trees without injuring the roots, and a small quantity of good mould and cow-dung were applied, it would be found to benefit the plantation.

Regarding the depth of soil, it has been observed by Mr. Towers, author of the "Domestic Gardener's Manual," that "eighteen inches of pure maiden turfy loam, without one particle of animal manure, over a six-inch stratum of coarse stones, brick-bats, rocky fragments, or the like, would prove a more fertile medium of growth for fruit-trees, and one more infinitely conducive to the health of the plant, than double the depth of a highly enriched garden soil." It is, indeed, a curious fact, that, in digging up some ancient orchards, which are known to have been planted centuries agro by the Monks, tiles and other fragments have been found in those spots over which the stems of the trees grew. The evident intent of this was to prevent them from striking downwards into an inferior soil; for however vigorously the plants may for a time flourish in a well prepared orchard, yet it did not escape remark, that if the roots were suffered to push down into bad ground, their growth was checked, and they became every vear less capable of producing the usual abundance or quality of fruit. Many planters, indeed, still follow the plan of placing a flat stone under the stem, for the purpose of forcing the roots to spread along the surface; but we believe it has but little effect, for if the soil be good, they will take that direction naturally; and if not, the stones will not prevent them.

\section{FLANTING.}

The distance at which the trees should bo planted is an object of great importance; for, if the ground be equally well prepared throughout, the roots push forward horizontally in every direction near to the surface, and do not strike down unless forced, either by meeting inpediments, or else by the soil not affording them a sufficient degree of nourishment. They should

* See his Treatise on Orchards round Farm-huuses, in the Quart, Journ, of Agric., N. S., vol. iv, p. 347. 
not, therefore, be planted so deep in the ground as to remove the roots from their immediate contact with the upper soil, but should be placed midway between the upper and the lower strata; or, in common cases, at the depth of six or eight inches. A mistaken notion prevails among many persons, that trees which are planted thick screen each other, and are not so liable to have their boughs broken as those which stand wide apart. Yet the fact is otherwise; for wherever trees stand thick, they not only draw one another up weakly, and consequently with less power to withstand sudden gusts of wind, but the closeness of the rows occasions currents of air, which thereby acquire such force as frequently to fracture their boughs, and sometimes even to tear them up by the roots. Added to which is the fact, that if permitted to stand too close together, their branches overhang and smother each other, and the fruit neither acquires the same flavour, nor does it ripen so early as when they are allowed more room; for nothing operates more powerfully in bringing plants to perfection than light, heat, and air. 'The must experienced garueners, therefore, are of opinion, that at least forty feet should be allowed between orchard trees of the common description, when come into full bearing*; but as they will produce a grood deal of truit before they can interfere injuriously with each other, they may be planted at half that distance, or even in rows at thirteen feet apart from each other, with intervals of twenty feet between the rows, and afterwards thinned out as they grow large. The quincunx is the preferable order of arrangement, as affording more air and light to the trees than when they are placed in lines. Dwarfs, intended for exposed situations, or in the immediate neighbourhood of the sea, may be planted closer together, and should not be allowed to exceed four feet in height to the first limb, and if much exposed to the winds, the lower the better; but trees planted in farmers' orchards, where cattle are permitted to range, should have the first branches full six feet high upon the stem.

In preparing the ground for planting, the first point is to see that it be well fenced with both hedge and ditch, in order not only to guard against pilferers, but to form a screen to shelter the young trees, as well as to assist in draining the land; which objects may be gained by cutting rubble drains into a ditch of three or four feet deep on the outside of a bank raised to a corresponding height, and planted with white-thorn for a hedge-row. Various methods are then resorted to for getting it in order for the reception of the plants, of which those most commonly in use are those stated by Mr. Main, as either by trenching it, in the following manner, wholly with the spade, fifteen inches deep, or with the trenching plough. "This trenching is particularly necessary when the orchard is not intended to be a meadow or pasture. If the soil be thin, it may be ploughed into ridges six yards wide, twice gathered, which will give a sufficient depth of mould on

* Trans. of the London Hort. Soc., vol. i., p. 39. In Herefordshire, the practice, on high grounds and shallow soils, is to allow twelve yards between each row, and six between each tree; but in low and sheltered situations, twenty-four yards between each row, and eight yards between each tree, are not considered too much.-Duncumb's Surrey, p. 83. IIalcolm, who was an experienced nurseryman, says, "The smallest growing trees should be planted at forty feet, and so increase to eighty feet, as the size of the kind is known to advance."-Comp. of Mod. Husb., vol. iii., p. 285. In Clydesdale they are, however, planted, in many cases, so close as twenty-two feet in the rows, by elevel between the trees, and the latest improvement in the practice is thirty feet by fifteen.-Quart. Journ. of Agric., N. S., vol. iv., p. 8:6. That of Devonshire is from twenty-five to thirty feet apart, and great improvements have been made on both the produce and the quality of the cider by thinning all those orchards in which the trees had been planted too close.-Vaucourer's Survey, pp. 236, 239. 
the ridges to receive the trees. Another way is to trench beds eight feet wide for the trees, and prepare the rest of the ground with the plough and harrow, to be sown down with grass-seeds and a single cast of a dwarfgrowing vat, in the month of March, after the trees are planted."

"There is still a cheaper way of planting an orchard on land which is already in turf, - viz, digging, or trunking, pits six feet square for the trees. This is done by first taking off the turf, to be relaid when the tree is planted, stirring the soil in the pits 15 inches deep, and adding, if necessary, a barrowful or two of maiden earth, mixed with a little rotten dung, to place the tree in. This plan may be pursued when the soil is of sufficient depth on a gravelly subsoil ; but, if on a clay subsoil, it is the worst way possible; because these pits become receptacles of stagnant water in wet seasons, and are, of course, injurious to the roots of the trees."

Whichever mode be adopted, the openings should be first made ready for the plants, which should be carefully taken up from the nursery withont topping or injury to the roots; but if broken or bruised they should be smoothly cut back. In placing them two persons are employed: one of whom fixes the plant upright in the hole made to receive it, taking care that the roots be regularly divided at equal distances from each other, and pointed outward from the stem without intermixture, as this direction of the extreme points prevents them from robbing each other of nourishment, while the other workman shovels in the mould and treads it firmly upon the root. The ground will settle down after a little time; it should be at first raised in a small mound around the trees, which would otherwise become sunk in the wet occasioned by the stagnation of the rain in these pits, and they should be securely staked up so as to secure them against the wind.

When cider is the material object, care should also be taken in planting the orchard to place all the trees of the same sort or quality in rows, by which means, the fruit, ripening together, can more easily be kept separate, milled, expressed, and the juice fermented together: objects of the first consequence with good ciderists, as the mixing of the fruit is found to produce unequal and repeated stages of fermentation; thus exhausting the strength and proving injurious to the liquor *.

The best period of planting is between the fall of the leaf and that when the buds expand in the foliowing spring, when vegetation is inanimate; or from about the end of October to the middle of February. In our southern counties, where the extreme severity of winter is not niuch to be apprehended, the autumn is usually chosen; but in less favoured situations, if the weather be mild, and the early part of the season chosen, the close of the month of January is preferable.

The age of the plants should not be less than full six years from the sowing of the seed, their heads having been previously formed upon a stem about six feet high; yet it is the opinion of an experienced orchardist "that the younger and lower the stem, the more likely the trees are to take root, to thrive, and to escape from blasts." He, therefore, recommends the slioots to be transplanted yearly in the nursery, and to be finally planted out at four years old from the graft at furthest $f$. $\ddot{A}$ it is also a material object, in point of produce and beauty, that the stems should be straight and clean, they should, therefore, be kept in that position, by being staked; and, if cattle be admitted to pasture in the orchard, by having a cradle-fence 
around them until their growth renders them firm. The shoots of the head should be cut to about one-third of their length, and formed by keeping the centre chiefly open, about the month of April, after they are planted: they should also be trained to spring as horizontally as possible from the main stem.

The common practice is to allow every tree to take its own natural shape, merely lopping off such branches as hang too near the ground, or appear too crowded; but some intelligent planters recommend their being trained into a pyramidal or conical form, as allowing greater play of the sun and air upon the branches, and they pay rreat attention to having the tree equally balanced. Thus, in the annexed figure of a wall-tree-as described in Mr. Lambert's observations on gardening-and which can be applied to standards-he advises $a$ a to be cut away altogether, as being secondary branches, too far from the main stem; leaving, if necessary, the secondary shoots $b b$ to fill up the vacancy, as springing from a branch near the stem. He also recommends " to head down the leader every year, at $c$, leaving two shoot-buds on each side as at $d d$, and to make your section as close as possible to the fifth bud, or one or two, more or less as the case may be; as, if you leave any wood above that bud which you intend for a leader, it will rot down to it, and the leading bud will be more likely to fail ; but, if cut close, it will soon cicatrize."

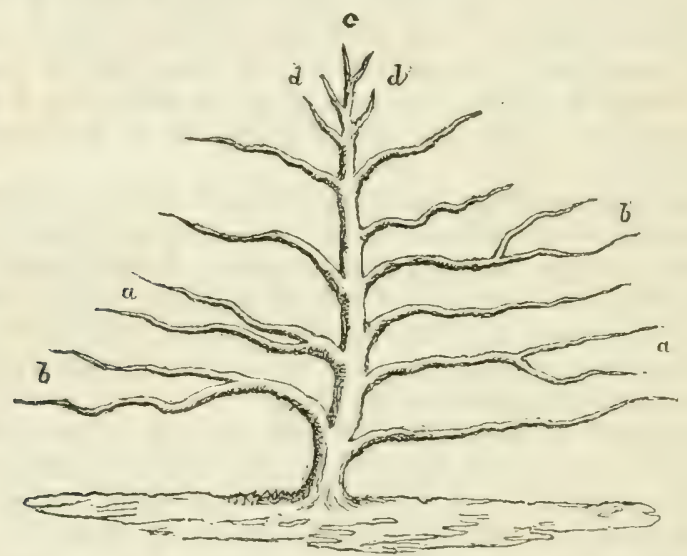

With regard to pruning, indeed, all secondary shoots should be discouraged; for, if too much wood be allowed towards the extremities of the branches, they often become naked towards the stems, and should be shortened*, and all dead, barren, and unprofitable branches should be annually cut away with caution. The young shoots on the side-branches and about the fruit-spurs, which malie their appearance throughout May and June, shonld also be pinched off, as they rob the fruit and other parts of the tree of nutriment. Large branches should, however, be rarely or never amputated. The most approved method of pruning is that of rendering the points of the external branches thin and pervious to the light, so that the internal branches of the tree may not be wholly shaded by the outward parts. The instrument generally used for this purpose is a strong

* This more particularly applies to pears and plums than to apples; but cherry-trees should not be touched, as they bear the knife badly, and do not run much to wood in the extremities. Lambert " on Farming, Planting, and Gardening," p. 290. 
flat chisel, having a sharp edge on one of its sides, with a hook on the other, and fixed into a handle of six feet or more in length. The cut should be made with as little slant as possible, so as to expose the least portion of surface, and leave substance enough about the wound *.

\section{SEED SPECIES.}

The nursery-plants raised from sced are grown by spreading the pulp, or "cheese," fresh from the cider press, upon a piece of prepared garden ground, and then mixing it with the mould by means of a rake or a light harrow. In the progress of the ensuing growth of the young plants, care should be taken to select all such as produce the largest and most luxuriant leaves, as it is from that character that the best expectations are formed of procuring the most valuable fruit; the rejected plants beingr drawn out from time to time, and those which are preserved being left to discover their specific qualities. This may generally be ascertained by the end of the sixth year from the sowing of the pips, and they may then be planted out. Nurserymen, however, commonly remove them out about four feet apart, after standing two years in the seed-beds, and, at five years from the seed, these stocks are grafted. Great pains are then bestowed in training the young top; which is done by cutting off the shoot chin-high, and afterwards pruning the top branches for three or four years witlin six or eight inches of the stem. This strengthens the trunk and roots and gives considerable security to the tree when removed to the orchard, which does not usually take place until three or four years from the time of being planted. The ground should, however, be in fine order; and if the surface be strewed thick over with furze, which is suffered to rot there, it will be found an excellent manure for apple-trees $\dagger$.

The opinion of the best-informed planters of the old school is, that the seeds of the old fruits should be sown, and the most strong and healthy plants selected for the purposes of cultivation. A treatise on the subject was indeed published by one Lawson so long ago as the year 1626, in which he states that " the best way to plant an orchard is to turn the ground with a spade in February, and to set from February to VIay some kernels of the best and soundest apples and pears, a finger-deep, and at a foot distance; leaving the likeliest plants only in the natural place, removing the others as time and occasion shall require:" a plan which is still very generally followed, and which may be persevered in without disadvantage, when the parent stock is still young and healthy. It, indeed, cannot be reasonably doubted that seeds chosen with a proper degree of care may be successfully propagated luring a certain period, but it is also certain that in process of time they decay at the same moment, as supposed, as that of the tree from which they were taken. Not only, indeed, is this apparent in the gradual disappearance of the "Golden-pippin," and some other valued species, but the fact is confirmed in a recent Memoir on the subject by the President of the Horticultural Society, in which he states, "that certain varieties of some fruits which have been long cultivated, cannot now be made to grow in the same soils and under the same mode of management, which was a century ago perfectly successful, is placed beyond the reach of controversy. Every experiment which seemed to afford the slightest prospect of success was tried by myself and others to propagate the old varieties of apple and pear, which formerly constituted

+ Survey of Cornwall, p. 95 . 
the orchards of Herefordshire, without a single healthy or efficient tree having been obtained; and I believe all attempts to propagate those varieties have, during some years, wholly ceased to be made."

"It has been urged that old age is the cause of debility and decay in those varieties of fruit which have been very long cultivated, and that many of the seedling offspring of such varieties are as much diseased as their parents; and it is contended that the failure of our old species has arisen from a succession of unfavourable seasons. The fact that many of the seedling offspring of old diseased varieties of fruit are as much diseased as the parents from whence they spring, is unquestionable; but this, I conceive, proves nothing more than that disease is hereditary in the vegetable as in the animal world, and it is scarcely reasonable to expect that healthy and robust offspring can be obtained from parents whose lives have been extended beyond their natural periods. In short, a tree, like an animal, has its infancy, its flowery spring, its summer's ardent strength, its sober autumn fading into age, and its pale concluding winter*".

Still, although admitting the justice of Mr. Knight's remarks upon the debility communicated to the offspring of aged trees, yet that, we conceive, cannot affect the young plants which have been propagated from those in their vigour, and therefore the greatest care should be taken in the selection of the healthiest trees of every species. The practice of grafting is now, however, becoming very general, and Mr. Knight has many seedling apple-trees produced between the Siberian crab and our richest apples, which display the most astonishing strength. In this manner efforts are successfully made for the production of new varieties ; but as the operation can only be properly performed by scientific gardeners, we should deem any directions on the subject to be here misplaced.

Various kinds of apples have been recommended,-some as being the best adapted for the table; others for baking, or for winter preservation; and others, again, for the manufacture of cider. The choice of these we must leave to common opinion; but we believe the safest rule for the selection of fruit-trees is, to prefer those kinds which succeed best in the neighbourhood; for it is certainly true, that some sorts of fruit are affected by one description of soil and local climate more than others.

The species of apples generally used for cider are not those suited to the table, as they are jossessed of a certain degree of astringency approaching to harshmess. The strongest liquor is certainly produced from fruit of that quality, but the rough cider of the farm-house, though more esteemed by good judges in the counties where it is grown, is not so generally liked by those who are not accustomed to it as that of a weak and sweetish flavour. The varieties which are still cultivated are too numerous for detail, and their names would hardly be intelligible out of the several districts in which they are grown. The old sorts most generally known are the "stire-apple," the "cocagree," the "golden-pippin," the "redstreak," the "liagloe-crab," the "woodcock," the "Harver," the "brandy-apple," the " jennet," the "red, white, and yellow musks," the "Dymock-red," and the old "pearmains," which always maintain a superior reputation; though some of the species appear to be falling to decay, but are replaced by the introduction of numerous new varietics. The colour of good cider fruits is, however, invariably red and yellow, the green affording a liquor of the poorest quality, and the merit of the cider will always depend on the rind of that

* R. P. Knight " on the Effects of the Age of Fruit-trees." Lond. Hort. Soc., vol, v. Art, lx. 
tinge being separated from the best collection. The smallest apples, if equal in quality, are also always to be preferred to those of a larger size, as containing juice of the highest flavour. This probably is occasioned by having a larger proportionate quantity of core, for it is that which imparts the chief portion of its fragrance, and cider made from pulp alone is comparatively weak *

\section{CROPPING.}

The time of cropping must of course depend upon the season. The fruit should remain on the trees until ripe enough to fall without being much shaken; and it is advisable that every sort should be collected separately, and kept till perfectly mellow. For this purpose it is customary to place the fruit in several heaps, about a foot in thickness, and fully exposed to the sun, air, or rain, without being ever covered, except in very severe weather; but the more warm it can be kept the better, for its watery particles are thus in part evaporated, and the liquor thus acquires an additional degree of strength with superior flavour. It is from outivard appearance only that the heaps can be judged to be in a proper state, which may be presumed not to be effected until they are approaching towards decay; at which period all those apples which bear any appearance of rottenness should be picked out. Each kind should also be ground separately, or, at all events, mixed with such only as become ripe at the same time: it is from this practice that fine cider of different flavours and degrees of strength are best obtained from the same orchard; the liquors being mixed after they are made. In general practice, lowever, the grinding together of different varieties of fruits equally ripe, is found eligible in all common cases; for it is less difficult to find the requisite quantities of richness, astringency, and flavour in three qualities of fruit than in one; and hence ciders composed of the juices of mixed fruits generally succeed under common management with greater certainty than those made of one kind $\uparrow$. We learn, indeed, from Mr. Booth, that "it is convenient, and in a great degree necessary, that the "pommagre" should be constituted of several sorts, which ripen at the same time; and, whether to make a virtue of necessity, or from whatever other cause, there are numerous manufacturers who account that liquor preferable which is produced from a mixture of kinds. Some make their cider from apples and pears jointly, and others from sweet apples mixed with common wild crabs ; but these are chiefly for home consumption, where what strangers would term insufferable harshness is accounted a good property $\ddagger$.

The fruit should be gathered in a dry day, taking only those which appear ready to drop from the tree, and after the heaps have been mellowed in the manner already mentioned, they are bruised in a common horse-mill, such as that described in the account of furze $\$$; but with a narrow circular horse-trough for their reception. The trough is fed by a couple of bushels at a time, and a man, with the assistance of a boy, will grind about three hogsheads of pulp in a day. The cider press which is generally preferred is that of Herefordshire; but any common packing press will answer

* This has been proved by one hogshead being made entirely from the rinds and cores, and another from the pulp of the same fruit; the former was of most unusinal strength and highly flavoured; while the latter was watery, and possessed not one recommendation. Hereford Rep., p. 84.

+ Herefordshire Report, p. 85.

\pm Booth on the Art of Wine Making, p. 112.

See Vol. i, p.134. 
the purpose. The pulp is generally wrapt in hair-cloth, and the vessels, being first well cleansed and scoured, are filled by the pressure. Botls the quantity and the quality of juice differ according to the species of fruit, as well as the state of the season; but Mr. Booth calculates that, upon an average of years and quantities, about two hogsheads of pulp are requisite to produce one of juice: pears, however, yield about a third more than apples. The specific quantity of saccharine matter in cider-juice, he also states as being usually between 1040 and 1060, which is equivalent to from 14 to 21 pounds per barrel, as weighed by the brewer's saccharometer; being a difference of 50 per cent. between the value of one juice and another. 'This, however, we believe to be rarely attended to by the farmers, though there can be no doubt that the actual value of every hogshead could be ascertained by that instrument*. The apples, after being thus pressed, are provincially termed "cheeses," which are often re-ground with water; and these "washings," as they are called, are again pressed out, and fermented apart into a weaker kind of liquor: the fruit sufficient for three hogsheads of cider being generally allowed to be sufficient for one hogshead of washings. The pulp is afterwards usually given to the pigs.

\section{CIDER。}

In the manufacture of cider, the expressed juice of the fruit is termed " must," which is in a foul and turbid state as it comes from the press, and can only be purified by undergoing the vinous fermentation. It is, therefore, put into casks, which should be well washed throughout the whole of the inside, with either sound liquor or common brandy, so as to correct any unpleasant taint that they may have acquired. The casks are not quite filled to the bung until the process is completed; and in a day or two, according to the temperature of the air, and the ripeness of the fruit, it begins to work. If attention be not paid to the state of fermentation, its excess will occasion the cider to be rough; and, as that which contains a considerable degree of sweetness is most valuable, the casks are usually placed in the open air until the object is effected, as the most effectual method of accomplishing it. If, however, there appears a strong disposition to renew the fermentation which it has already undergone, the liquor should be racked into a clean cask from time to time, in order to separate it from the dregs. The necessity for this may be perceived by a hissing noise in the cask; and the mode of operation, as well as that of the practice of "stumming," is thus described as the common mode throughout the west of England:-

"The fermentation is usually stopped by burning a piece of cloth or paper, two or three inches wide, and six or seven inches long, dipped in brimstone, and yut into the bung-hole of a cask that has six or eight gallons of cider in it. After lighting it, the bung-hole is secured, and the air in the cask is sufficient to keep up the combustion until the match is consumed. This done, the hogshead is rolled to and fro, in order to promote the union of the cider with the sulphurous vapour. It is then filled up within a few inches of the bung-hole, and all fermentation is for a time suspended; but, on observing it to return, this fumigation is to be renewed as often as necessary. When the liquor remains quiet, and a candle will burn clear in the bung-hole, the fermentation is easy, and not likely to pro-

* It is made under the direction of Mr. Booth, and can be obtained at Mr. M'Gowan's, Gerrard-street, Soho, London; together with an explanation and directions for its use ; for the price of two guineas. 
duce a further diminution in the strength of the cider ; and when every symptom of fret is wholly subsided, it is then considered fit for market, or, generally speaking, in the following months of March or April.

"The dregs are always filtered through brown canvass bags holding three or four gallons each: but as the union between the pulp and the liquor is found to be very close, a separation is effected by mixing about half a pint of bullock's, or any other fresh blood, with about six or eight gallons of the dregs. This being well beaten up, divides the jelly of the pulp, and the liquor running clear off is mixed with the other cider, and is always found to contribute to the restraint of the further fermentation.

"To complete the fining of the cider, a pound of isinglass, first soaked for ten or twelve hours in cold water, is afterwards dissolved in about five gallons of eider, and well incorporated with a whisk. About a quart of this is sufficient for a hogshead of cider, and with which it must be stirred and mixed in the cask *."

We should say, however, from our own experience, that the whites of a dozen eggs, beat up along with the shells in a quart of the liquor, will perform the operation with more certainty. The mixture should be put in at the bung-hole, and well stirred round with a cleft stick; and if to this be added a moderate quantity of sugar-candy, with about a gallon of good brandy to a hogshead, the quality of the liquor will be much improved. This, however, can only be recommended when the cider is intended for private use, as the price of the brandy will not admit of the expense for public sale; but a few ounces of pounded ginger is not exposed to the same objection, while it partly corrects the flatulence of the beverage, which should never be drunk until it is at least twelve months old. At two years old it is in the best state to bottle-the month of March being the best period for the operation-after which it will become brisk and sparkling in the following summer; and, if it possess much richness,- -like those celebrated growthis known as the "cocagee" and the "stire," it will remain, with little perceptible change, during twenty or thirty years, or as long as the cork duly performs its office. Should the colour be too dark to please the delicacy of the purchasers, a quart of milk added to the finings will materially reduce its cloudiness.

\section{PERRY.}

With regard to the manufacture of perry, it is in all respects so nearly similar to that of cider, that no further description is necessary. The pears which are preferred for the purpose require an assemblage of qualities which Mr. Knight tells us will be rarely found in the same fruit. "It should contain a large portion of sugar, or its juice can never possess sufficient strength ; and, unless it be at the same time extremely astringent, the liquor produced from it will become acetous whenever it ceases to be sweet : in the latter state it will agree with few constitutions; in the former with none."

"The juice of the best perry-pears is so harsh and rough as to cause a long-continued heat and irritation in the throat when the fruit is attempted to be eaten; yet, by being simply pressed from the pulp, it becomes rich and sweet, without more roughness than is agreeable to every palate."

This circumstance appears extraordinary, yet the quality of astringency is so necessary to the pear, that Mr. Knight says " he has never known a

* Devonshire Report, p. 240. Bone-isinglass, or jelly made from hartshorn shavings, is, however, preferable to fish-isinglass; besides being much cheaper. 
single instance in which the perry made from fruits that were without it did not become sour before the middle of the ensuing summer. It may, how ever, be preserved by a mixture with the harsh juice of the crab."

The pears anciently held in the most estimation were the "Squash" - so called from the tenderness of its pulp, which is supposed to have furnished more champaign to this country than was ever imported into it ; the " $\mathrm{Huff}$ cap," from the quantity of fixed air contained in its liquor; the "Sack pear," from its richness, and the "Red pear," from its colour; though of the common sorts, the "Long-land" is, for the general use of the farmer, perhaps the best of any. The table varieties of the finest species have, however, been introduced from France,-as, for instance, the St. Germain and the bon Chretien,-and some of a very superior kind are grown in the Islands of Guernsey and Jersey; but perhaps one of the most delicious sorts in Europe is that known throughout the north of Spain as the Pera de Mantega, or "Butter pear ;" and the species grown in the neighbourhood of Guimaraens, in the north of Portugal, is equally celebrated. The peartree flourishes in a greater variety of soils than the apple, and is more productive: every tree, when nearly full-grown, being calculated, if in moderately good ground, to produce an annual average of twenty gallons of liquor. Many single trees have produced a hogshead in one season; and one extraordinary plant, growing on the glebe-land of Home Lacey, in Herefordshire, has more than once filled fifteen hogsheads in the same year*.

"An acre of land, as commonly planted, is, indeed, capable of containing thirty pear-trees of usual dimensions, which, taken from new varieties of fruit, would probably continue in a productive state beyond the conclusion of a second century. The produce of an acre planted with appletrees will generally be found nearly one-third less than the same quantity of ground planted with pear-trees would afford-with the exception of the Holmer pear and the Oldfield ; but the apple-tree begins to bear at an earlier age, and cider," it is said, "will ever be preferred to the juice of the peart." From this latter opinion many persons will however be found to differ; for perry, when well made, is so similar to the white wines of the grape, that it has been often mistaken, without any mixture, for effervescing champaign $\ddagger$. The juice is, however, more delicate than that of the apple, and its management more precarious than that of cider; which, added to the smaller quantity grown, rendres its price considerably higher.

The juice of both has been frequently boiled as soon as pressed, and much of it has been passed off, when mixed with certain spices to give it flavour, as different kinds of grape-wine. The main object of the process is, however, to render the liquor stronger, by the evaporation of the watery

* "When the branches of this tree in its original state became long and heavy, their extreme ends successively fell to the ground, and taking fresh root at the several points where they touched it, each branch became as a new tree, and in its turn produced others in the same way. Nearly half an acre of land was thus covered upwards of twenty years ago, and there is little difficulty in extending its progress." - Herefordshire Survey, p. 90. + Ibid.

$\ddagger$ In the north of France the imitation of wine is thus made:- "Having heated 55 pounds of the juice of wild nears to 180 degrees, I added about a tenth of that weight of uried grapes (raisins), and bunged up the whole in a cask. In a short time the heat of the liquor had fallen to 77 degrees, when $I$ drained out the raisins, bruised them, returned them into the must, and closed the cask so as to allow the fermentation to take place. A fortnight after, the wine, or perry, was racked into stone cans, and after standing for three mouths in the cellar, it was reckoned by good judges equal to the best white wine from grapes." - Booth on Wine-making, p. 122. 
portion; and it is, we believe, only continued in some parts of Devonshire where the fruit is of a very inferior nature.

In the mamufacture of both cider and perry, the strictest care should be observed to avoid the use of all implements of copper or lead, as their solution is to a certain degree effected by the acidity of the juice, and is highly prejudicial to health. The cellars in which the casks are stored should also be placed under ground,-or, at least, should be guarded from the sun, and maintained at an equal degree of moderate temperature, to avoid all hazard of acetous fermentation; which, if once really commenced, can never be checked by any other than improper means.

MIXED FRUITS.

Aithough we have here only alluded to pears and apples as being, on the whole, the most in use, and, therefore, the most valuable of our fruits, vet there are other kinds of orchards of large extent, which afford considerable profit both as fruit and pasture: thus in some counties-but more particularly in Kent, where the soil, being a light, sandy loam, is peculiarly favourable-there are considerable plantations, annexed to farms of importance, under the various species of cherries, damsons, and filberts. The most profitable kind of orchard, however, is that which contains all species of hardy fruit-trees and bushes, and where the land is exclusively devoted to that purpose. This, indeed, resembles gardening more than farming, and may, therefore, be unsuitable to large farms, though we have seen it upon one which contains some hundred acres. It is, however, quite applicable to small ones; or to persons retiring into the country, to which an acre of ground, requiring no horse-labour, might be found to afford healthful amusement, compled with no despicable profit. The plan as described by Mr. Main, who is a practical man of much experience, on whose judgment the greatest reliance may be placed, is as follows:-

"In such orchards, half-standard apple-trees are planted in rows 18 feet from each other, the trees being 12 feet apart. In the same line with the apple-trees are planted either gooseberry or currant bushes; or, what pays sometimes equally well, filberts. The latter are not allowed to rise higher than about four feet, and kept spurred in, exactly like the white currant. Gooseberries gathered green for tarts pay the farmer better than when ripe, and are not so troublesome in the carriage to market. As such an orchard is not to be grazed, two feet of the soil on each side of the rows of trees is kept bare, and always free from weeds. On this a mulching of rotten dung may be laid every winter, and raked off in the spring; the intermediate strips of ground to be planted with potatoes, or sown with onions, tumips, scarlet-rumers, or any other crop which the cultivator can most conveniently dispose of in his neighbourhood."

"For such an orchard the earliest and surest bearing apples should be preferred. The greatest majority should consist of the Hawthornden, the rest of the French crab, and scarlet nonpareil. A few of the earliest pears may be intermixed--as, for instance, the petit muscat, which fetches a good price on its first appearance at market. The most hardy and profitable lind of plum for a farm-orchard is the common damson, it being always in request for baking, preserving, or wine-making. The ground may then be preparel for receiving the trees, either by trenching it wholly with the spade 15 inches deep, or with the trenching plough." 


\section{Chapter XXXII.}

\section{ON THE FEEDING OF NEAT CATTLE-GRAZING-STRAW.}

Having thus discussed the subject of tillage, we have now to treat of the farm live-stock; or, as Bakewell happily expressed it, "the best machine for converting herbage and other food for animals into money." The different modes of feeding cattle in the stable, shed, or straw-yard, when taken from grass, though nearly the same, are distinctively termed soiling and stall-feeding; the former beiug exclusively applied to cut green food, and the latter chiefly to that of hay and oil-cake mixed up with the esculent roots, either in a raw state or steamed; for the straw-yard can only be applied to the winter keep of store stock. In

\section{GRAZING,}

The choice of cattle, their adaptation to the soil, and the quality of food with which they can he regularly supplied, is the first consideration: thus a man who has fertile meadow or rich marsh may fatten bullocks as large as he can find them; while on poor land, affording but scanty pasture, and a short bite in the summer, no bullocks will pay better than the small Galloways, or most of the Highland stock; but on richer pasture and better turnip soils, the most profitable will be found to be the larger species of the Fifeshire and Lowland Scotch, with the Hereford and short-horned Yorkshire breeds, or those grown in the neighbourhood. Graziers, however, would act prudently in ascertaining the nature of the land upon which the cattle were bred, and choosing their purchased stock from an inferior soil; for if young cattle be taken from a good to a worse soil, they are apt to become stunted in their growth, and if old enough to be fed for the butcher, they will not feed so kindly as those taken fom more indifferent pasture. Generally speaking, however, bullocks from 40 to 60 stones weight (14 lbs. to the stone) are much more saleable than larger ones, and may always be finished with turnips, and perhaps a small addition of oil cake, if put up, as they always should be, in good condition; for nothing is more injudicious than to attempt the stall-feeding of oxen in the view of their becoming fat, at any fair expense, unless they be in good order as stores. 'The grazier's profit will depend more upon the goodness of the breed than upon the size of the cattle, and the secret lies in discovering those kinds which in the shortest time give the greatest quantity of flesh with the smallest quantity of food.

Respecting the different breeds, we, however, pretend not to give any decided opinion, for their respective merits are so warmly contested that we should find it difficult to judge between the different advocates of their separate and opposite opinions; but conficlently refer to the "History of British Cattle," as already written by Mr. Youatt, under the superintendence of the Society for the Diflusion of Useful Knowledge; and having also already treated largely on the subject of pasturage, as well as that of working cattle*, all that ive have now to elucidate, besides a few additional remarks on grazing-therefore, regards merely their feeding in the homestead, together with some further account of the dairy.

The practice of grazing necessarily differs according to the nature of the land, and the judgment of its holder: hence he should, in the first instance, ascertain the mature, as well as the extent, of his feeding ground,

* On Working Cattle.-See vol, i, chaps, vii., viii, and on Pasturage, chap. xxxi. 
-whether it consist of rough pasture, sound meadow, or marsh, - and he should then select those beasts which show the most likely disposition to improve upon the soil on which he farms. On this, the consideration of the most appropriate kind of breed will, of course, not be neglected; and the well-known remark-" that heifers are superior to oxen in early ripening*"-mav not be found useless. Spayed heifers are, indeed, known to fatten more speedily than oxen of the same breed, but do not attain an equal weight; they are also considered as of so much finer quality that, although four or five stone lighter than steers, they have fetched a ligher pricet. Free martins, or barren cows, also become fine meat; and even old cows, if recently taken the bull, and thoroughly dry of milk, are generally found to fatten sooner than oxen of the same age; but they consume more food, and their flesh is of inferior quality + ; nor are they equal to young stock in point of kindliness to fatten.

Those farmers who chiefly occupy rough pasture very generally breed the greater part of their stock, which is afterwards sold, when voung, in a lean state, to graziers holding richer land, on which they are enabled either to fatten it on grass, or to finish it for market by the process of stallfeeding. These persons, if they have any scarcity of land, frequently purchase the store-stock after Michaelınas, turning the cattle into the strawyard upon straw and turnips until the pastures are ready to receive them, when they usually get fat towards the close of the autumn, or at such periods of the year, either earlier or later, according to the quality of the land and the condition of the beast.

Others, who hold hay farms, purchase the small kind of bullocks and shcep soon after the grass is mown, and turn them upon the rowen, where, if then in tolerable order, they may be got rearly for the market, with some hay and oil-cake, by Christmas. Besides these, many other plans are adopted for the purpose of fattening the cattle at different periods, so that they may be brought to market in succession. Thus they are not unfrequently kept throughout two winters, during the first of which they are not at full keep, but in the following summer are turned into the best pasture, and stall-fattened off during the close of the year. The more common system, however, consists in confining the operation within twelve months from the purchase of the lean stock until fattened for market $\delta$.

Regarding the age and increase of weight, it is stated in the General Teport of scotland, upon the authority of eminent breeders and graziers, that the Galloway cattle, kept on good pasture during summer, and merely preserved from falling off in winter, will weigh, at the age of from

* A well-bred three-year-old long-horn heifer, without having a calf, will, it has been stated in the Leicester Report, be fat at grass only, and weigh $600 \mathrm{lus.}$; while an ox at grass will eat much more and not produce so much beef per aunum. Thus-an heifer calving at three years old may be milked six months, and fatted, by four years old, to 600 llos. weight the four quarters ; producing in the following year-

$$
\begin{aligned}
& \text { The calf at six weeks old, and pork from the milk . } 140 \mathrm{lbs} \text {. }
\end{aligned}
$$

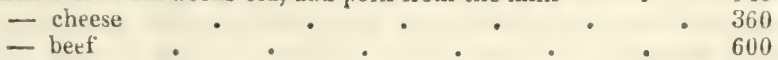

thus giving a total of $1100 \mathrm{lbs}$, which is much superior to an ox in the same length of time.-p. 245.

t On the Continent they are often spayed, and afterwards worked as oxen; in which case they are said to be peculiarly apt to fatten, and to produce beef of a very superior quality.-Von Thaër, Princ. rais, d'A gric., 2de ed., tom, iv., p. 589.

+ Gen. Rep. of Scotland, vol. iii., p. 91.

$\$$ See the Complete Grazier, 6th edit., p. 75. 


2 to $2 \frac{1}{2}$ years, 30 stone
3 to $3 \frac{1}{2} \quad, \quad 41$,
4 to $4 \frac{1}{2} \quad, \quad 54$,

almost all which several additions are made during the six months of the grass season*. Perhaps, however, no age exceeds four years for capacity of feeding, either as it regards quantity or quality of flesh. Thus we learn from some very experienced salesmen and carcase butchers, the average of the large-framed beasts of our English counties, when regularly grazed, without stall-feeding, will generally arrive at that period to about

110 to 115 stone of 8lbs, the carcase

$$
20,25, \text { the fat and hide }
$$

while if worked until seven years old, and then grazed, they will not reach more than an increase of about 10 per cent. in weight.

In corroboration of this we also know that Lord Spencer, who grazes oxen bred by himself to a considerable extent, putting them in lis best pastures only in the last summer before they are sent to the butcher, is in the habit of dividing the price for which each individual is sold by the number of days he is old, and he has found that, on an average, those oxen which are sent to the butcher at about four years old pay more per inonth for the time they are entered to the time they are sold than those sent to the butcher at an earlier or later period. During the time the oxen are kept in the store pastures the cost of keeping them is very small, and therefore, as those sold at four years old pass three-fourtlss of their time in store pasture, whereas those sold at three years old only pass two-thirds of their time in it, the advantage of keeping them till four years old appears to be very great. We must however admit, that many extensive graziers in the north bring three-year-old bullocks to market of great weight, and their continuance of the practice proves it to be incontestably profitable; indeed, a cattle-dealer, who has hately published some intelligent practical tracts on the subject of feeding, insists that short-horns can be brought to maturity at two years old with greater advantage than at any other age.t

In stocking the ground, as the proportion of cattle must depend upon the nature of the soil, it will perhaps be generally found that local habit, as being usually the result of experience, is the surest guide. On this we have, therefore, but little to add to what we have already stated on the subject-in vol. i. p. 4S2, except that the grazier should talie especial care not to turn his stock into the pastures in the spring until there be a full bite; and the fatting beasts ought to have the prime of the grass, of which they should have the pick, and be followed by young cattle, which should be afterwards turned in as stores until they are ready for the

STRAW-YARD.

This, although in some large farms enclosed apart, is, however, more generally an open space surrounded by the buildings of the homestead,and being deeply embedded with straw, and thus screened from the wind, is sufficiently warm for the comfort of store-cattle, which are those only for which it is distinctively appropriated. There, no doubt, their hides and hair become in some degree impenetrable to the weather, and although exposed to rain and snow they suffer comparatively but little from the cold; but they unquestionably thrive better when enjoying the shelter of a

* Vol. iii., p. 90.

† See Dickson on Live-Stock, Quart. Journ. of Agric., No. xxx.s p. 275. 
covered shed, to which they may repain at will. They should, therefore, always have that protection; and even if the yard be open, hovels can, with a little ingenuity, be erected for a mere trifle in the angles of the fence with anv poles of rough timber laid across to support a bean or pea-stack, or the roof mav be thatched with hanlm. Whether furnished with sheds or not, the yard should, however, be divided by post and rail into different enclosures, so as to separate the cattle of various acres from each other, and to prevent too many from being placed together. The bullocks in strawvards should also have plenty of room, for many accidents occur anong horned cattle by their goring each other.

'The common objects of the straw-yard are those of merely maintaining the cattle in the state of flesh which they acquired at grass, and converting the litter into dung; it is, therefore, a very general practice to confine the food of stores to the straw of oats or wheat, and the haulm of pease and beans, without any addition of hay or turnips. The animals are exceedingly fond of bean-chaff, and the wet litter from the horse-stable is very acceptable to them; it should, therefore, be daily spread out in the yard. In this way, if well bedded, and the thrashing be so managed as that they can he supplied with fresh straw in cribs, they may be made to just hold their own; though in most cases they fall of so much in the spring months, when the straw becomes dry and loses the little power of nutriment which it possesses, that perhaps more than a month is required at grass to recover what they have lost. Any improvement in condition is not to be expected, and the dung will be found of such poor quality that it will not be equal in its effects upon the land to half the quantity of that made by well-fed bullocks *

If hay and turnips can be spared, they will probably, therefore, be not so thrown away as they are by many imagrined when given to store stock. The turnips should be given off' in small quantities, increasing their amount as the straw loses its nutritive powers; and thus, perhaps, two double cart-loads, weighing $15 \mathrm{cwt}$. each, may do very well for the daily supply of a score of cattle of the ordinary size; but after Candlemas, if the supply can be then furnished, three cart-loads will be required. Upon clay-farms, it is very true that the land may be incapable of producing turnips, and that hay may be thought too dear to be thus employed; but ive are strongly of opinion that a moderate quantity of clover cut with straw, and given morning and night in the manger, will more than repay the expense in the future state of the cattle, besides the increase of value in the dung.

In breeding farms, especially, this will be evidently seen in the improved power of the young; stock; for, if stinted during their growth, they will not be afterwards able to acquire flesh either so rapidly or so fully as if better kept; and thus, botls time and weight being sacrificed, a loss will be incurred by the feeder. The apparent condition of cattle thus differently fed must at once strike any one who views the stock on farms where they have been thus separately treated; and if their owners were to produce their accounts, we have no doubt that he who had expended the most money in food would be found to have realized the greatest return of profit in money.

* See vol. i., chap.. 


\section{Chapter XXXili.}

\section{ON TIE FEEDING OF NEAT-CATTLE-(Contimed)-SUMMER SOILING.}

ON duly estimating the quality of land in the various districts of the kinglom, only a very small quantity has been found capable of carryiner full crops, unless restored and recruited by dung at least once in four years; but to obtain a supply to that extent, except in the neighbourhood of large towns, has, under common farm management, almost in every instance baffled the most strenuous exertions of the occupier. Lime, marl, shellsand, soap-ashes, and all the variety of mineral manures, have indeed been resorted to as auxiliaries; but their effects, tending rather to stimulate and bring into action the inert powers of the soil than to enrich it, have in many instances reduced the land to a state of great exhaustion, when their operation has ceased without the support of putrescent manure. The land lias, indeed, been for some time enabled to produce large crops of corn, but it was finally worn out, and large farms have in fact been so much impoverished by an injudicious use of lime, that years must pass away before they can be restored to the condition in which they were originally; whereas, liad a full supply of vegetable substances been at hand for the production of dung, when these stimulants were in action, the issue would have been altogether different.

A due reflection upon these facts should induce every farmer to cultivate those crops which are not only the most consistent with his own interest in the first instance, but also the best calculated to afford the greatest return to the soil; and experience has thus proved the necessity of adopting the alternate system of husbandry, which forms the groundwork of its prosperity upon light lands. Still, however, it is probable that the most judicions application of those crops to the consumption of live stock is only imperfectly understood; for although, throughout the whole of Flanders, a great portion of the cattle are kept during the entire summer in the house, and there fed upon cut grass, or other green food, as the more profitable mode, and the same jlan has been here partially followed by some intelligent farmers, yet it has been only lately, through the efforts of the late Mr. Curwen, and a few other eminent agriculturists, that the practice of soiling has been introduced into this country. In Ireland it is scarcely known: Mr. Blacker, however, a very experienced Irish landagent, who has lately written " on the Improvement and Cultivation of small Farms," strongly advocates the system, and recommends the raising of turnips and mangel-wurzel for house-feeding and increasing the stock of cattle, to the utmost extent of which the plan is susceptible

\section{SOILING}

Is applied generally to every artificial grass, as well as to some of the com crops when in a green state, and some persons have of late beneficially followed the Flemish plan of repeatedly mowing the meadow grass, while young, for the feeding of their cows. The objections to the cutting of grass, to be used at home in its green state, arise from the prevalent idea

* IIe thus expresses himself-"Setting it down for certain that you onght to have at least one cow for every three acres of arable land, as being the smallest stock which will enable you to keep the ground in heart-if this be not kept in view from the very outset, youwill find that you camnot manure the one-fourth of your farm every year, and you will therefure be thrown out of the rutation: the land will be exhausted and left to rest as formeriy ; aud as it gets poor, you will get poor yourselves." 
that cattle when fed abroad are more healthy than when confined, and that the cutting and carting occasion such expense as will not pay unless the crop be very abundant. It is also generally thought that the constant mowing, instead of pasturing, has the effect of impoverishing the meadows: this, lowever, is a mistake, because the soil is not exhausted if the grass be cut before it has come into seed, and even if not mowed till after the seed has been formed; provided what is taken from it be returned by a proportionate supply of manure. This, indeed, is the case in the neighbourhood of London, where we find the practice constantly pursued for the production of hay, without any diminution of their produce. On poor land, however, or that which cannot be dunged once in three years, this must be admitted, if the grass be allowed to grow till it comes into seed: we are also aware that there are very many seemingly advantageous practices in agriculture, which cannot be profitably employed on all soils, and in all situations; but, on tolerable ground, economy will be found both in the saving of the grass as well as the production of manure. As to the health of the stoek, there can be no doubt that they will find more enjoyment, and consequently more apparent vigour, when in the field than in the yard; but the farmer's object is to put the greatest quantity of flesh upon the beasts with the least possible cost, and that, we think, he may accomplish by soiling them upon green food. The advantages of using winter green crops in the house have indeed been proved on every well-conducted farm on which it has been tricd; and were summer feeding with the natural and artificial grasses carried on with the same spirit, it might be found equally profitable: they may be thus enumerated, -

1st. In the saving of the crops from the destruction by cattle when in pasture, both by treading, staling, dunging, and lying down upon them; all tending to waste in manifest proportion to the productiveness of the land. It is, besides, well known that many of the finest grasses, which, when young, are highly relished by cattle, if once suffered to become stalky, and get into seed, are then so much disliked, that the beasts will not eat them unless compelled by sheer hunger, and as in most pastures many of these grasses ripen through delay, their produce is lost to the grazier; whereas, if cut down in proper time, not a plant will be lost. It has also been clearly proved that cattle, when fed in the house, will eat many coarse grasses and weeds which they will not touch if growing in the field; and it has been also remarked, that grass which has been blown upon by other animals does not become umpleasant to a different species: thus it happens that even the sweepings of horse-stalls are relished by oxen; and pigs will eat the refuse of other stock clean up.

2ndly. In the great increase and value of the application of manure; for although it is true that the same quantity is made by cattle in the field, yet it is there in a great measure lost by the drying up of the air, and, except in the case of sheep, can never be equally spread over the land; whereas, when deposited in the yards, it can be immediately collected before any great evaporation can take place, and there, when laid in heaps, it can be properly fermented. The urine can also be collected in cisterns; and, even if the straw be not used as litter to increase the heap, the dung made by a beast fed in the stall ipon green food has been calculated, in one of the Reports of the Workington Society, at a ton weight in a month. Sand, road-dust, the scourings of ditches, or any other refuse, if added to the dung while in a 
humid state, will also form a valuable mixen, which can be applied to the land at such seasuns as the farmer may deem most judicious.

3rdly. In the prevention of all injury to fences, and to the poaching of grass-land in wet weather; as well as the avoidance of all annoyance by flies during summer : and, to working cattle more particularly, the great advantage of quietude, by which they can fill their bellies and lie down to sleep more expeditiously than when abroad.

This reasoning appears to be conclusive; but it must be admitted that few if any of the hest practical farmers in England have adopted the practice of soiling. Some even who did adopt it at the time Mr. Curwen advocated it so strenuously, and when many of the Agricultural Societies endeavoured to introduce it, have given it up. For instance, the late Mr. Champion-than whom a better farmer never existed-having a light land farm perfectly well adapted for soiling, thought it his interest to occupy grazing land ten or tweive miles from his farm, for the purpose of keeping his stock during the summer; and yet, as Mr. Champion was in the labit of selling bulls and heifers at large prices, it was very much to his advantage to have them near his home, and he was a man who was not likely to be deterred from adopting an advantageous practice because it was a new one. We state these objections thus candidly, because the Suciety does not choose to afford its authority to a practice which has been disapproved, and wishes to leave its expediency to further investigation; but so far as the compiler of this work has had an opportunity of personally observing its effects, he has found it to be highly advantageous.

In conducting the soiling system, it is desirable to give the food often and in small quantities, as otherwise the cattle will blow upon and reject it ; alded to which, if it be given in such abundance as to clog them, it will not be afterwards relished, and the beasts will consequently suffer in condition. Attention should therefore be paid to have the remains of the food immediately removed when the stock have done with it; or, indeed, rather before they are quite satiated. Green food, it should also be observed, should be sparingly given at first; as otherwise the greediness of the cattle, when put to it after dry food, not unfrequently occasions the accident known as "hoving." Thus tares, luceme, clover, and all those grasses relishing to cattle of every description, and equally favourable to health, may prove soxious, if taken in too great a quantity. The air which they engender swells one of their stomachs; the tension obstructs rumination, and its effects cause death if speedy assistance be not given to facilitate the evacuation of the condensed air, and the passage of the blood in the large vessels*. Straw should also be given as fodder in order to correct any disposition to looseness in the bowels; and we have heard the tops of the common heather recommended as a condiment which prevents all appearance of scouring.

The different varieties should also be occasionally changed; for animals like the various qualities of food, and if these be judiciously mingled, as circumstances and the successive growth of crops may admit, the appetite will be provoked, and the health and thriving of the creatures will be thus

* For the remedies regarding hoving, see Youatt's Management and Diseases of Cattle, p. 438.

If choked by the stoppage of a piece of turnip in the throat-let a strong man seize the bullock by the nostrils, and keep his head at a proper height, and as straight as possible, while an assistant passe's the butt end of a cart-whip, or an elastic cane with a dossil of tow, or louse yarm, at the end, to increase its size, gentlv down the throat till it reaches the stomach. 
progressively improved. Indeed, were green crops of the several varieties to be regularly cultivated thronghout the year - what with fog reserved from the pasture, rye-grass, winter-tares, kail, and lucerne in the early part of spring; clover, spring-tares, sainfoin, and the further cuts of lucerne during sunmer; the aftermath of the meadows and clover for the autumn; together with the crops of turnips, mangel wurzel, and potatoes for the winter months ; the process of feeding, even without any assistance from hay, need never stand still.

In the early part of the season, when tares and clover are either inadequate to the support of the stock, or that it may be thought expedient to change them gradually from dry to green food, a portion of these grasses may be mixed with the hay or straw on which the cattle are fed; and if the mixture be made up over night, the dry provender will be found to have acquired a sweet vegetable taste, and thus rendered so moist and palatable as to be more readily eaten : but it is an error to suppose that straw given before grass or turnips will have any good effect upon the cattle; for they in a short time will refuse to eat it. When grass, whether natural or artificial, is to be griven, it should be cut twice in the day, early in the morning and late at night, so as to avoid the withering heat of the noonday ; for, if not more nutritious, it is at least more palatable in a fresh state than when stale, and the danger of hoving can be guarded against by due circumspection in the quantity to be given at each time. "Attention should also be paid to the due proportion to be cut; and until that fact be ascertained, it is a good plan to measure out each mess, and to chalk down the quantity in weight, which the basket, cart, or other vehicle employed for carrying the food contains of the various articles used for that purpose. The practice will, at least, have a tendency to teach farmservants to observe method, the value of which is of considerable importance in all business, and in none more than in the various branches connected with husbandry *" People object, indeed, that such a mode of feeding is troublesorne, and that the old way is more easy and convenient; but we are yet to leam the branch of good management which can be executed without some trouble; or to see the same profit derived from slovenliness as from care and attention.

The cattle should be kept in yards, or hammels, with dry sheds around for shelter; and if only a small number be put together, and those of nearly equal age and strength, they will feed more quietly, as the stronger beasts will soon overpower those which are weak, and prevent them from eating until they are themselves satiated. The yards should also be plenti fully supplied with water, and kept particularly clean, as the ordure and urine occasioned by soiling occasions much filth, and if cattle be not maintained in a comfortable state of cleanliness they will suffer in improvement ; to obtain which desirable benefits, little or nothing more is required than a small portion of time and attention from the farmer and a careful servant.

'There are, however, not wanting many experienced graziers who, for the feeding of store-stock, prefer open fold-yards merely sheltered by a high paling to guard against the inclemency of the wind; but without sheds. Indeed, it is known that they will eat food, when thrown to them on the ground, which they will reject when given in the stall; and in some parts

* See chapters $x$, and xi. on Soiling and Stall-Feeding in the Complete Grazier, or Farmer's and Cattle-Breeder's Assistant, 6th edit.; and an article "on feeding," in vol, i., p. 209 of this work. 
of Norfolk and Suffolk a singular practice prevails of tying up cattle on the wheat or barley stubbles, without house or shed to cover them. With a few rails, planks, or any rough contrivance, they form something to answer the purpose of a manger; and small posts, to which the cattle are tied, are driven into the ground 3 feet to 3 feet 6 inches wide, in front of a thick hedge, as a screen. There they are regularly billeted, turnips or cabbages being carted to them, and their dung is piled up in a wall behind them; a slight trench near their heels being dug just suficiently deep to conduct away the urine, while only some farmers use the precaution of digging a hole for its reception. This method is by many preferred to litter or covering, as the warmth of lying so near to each other is considered as sufficient, and the air more healthful ; while the economy of labour, in having the manure upon the land on which it is usually laid, is another advantage*. With every deference, however, to the Norfolk farmers, we cannot but view the plan of yards, with dry sheds, which are cool in the summer and warm in the winter, as vastly better; in which we lave the opinion of a very experienced man of their own number, who states that experiments on the same farm, with the same kind and sized bullocks, and from the same field of turnips, have induced him to give a decided preference to them. He indeed adds, that bullocks fed in the house were sent to marliet in the month of February, while those kept in the former manner were not ready until the middle of April + .

In some districts of Scotland, and many parts of Ireland, they also tether the stock upon the land. This is in many places a necessary practice, as from the want of inclosures, as well as the small holdings of the farmers, the cattle camnot be attended, and therefore must otherwise be housed, or run over the ground of their neighbours. The want of servants also prevents the cutting of the grass; and, besides all this, it has been remarked, that when cattle have a fresh bite of grass given to them, they feed faster and thrive better than when they are permitted to wander over the whole field. It, indeed, answers in some respects like soiling, with the superior advantage of fresh air, and some exercise; and many experienced a griculturists have practised the system on a large scale, having found by its adoption that the cattle were rendered not only more docile, but throve better than in any other mode of feeding, while the grass-land was more improved, and could maintain at least one-third more stock than under indiscriminate pasture $\ddagger$.

The plan of tethering cannot, however, be considered equal to soiling; and is, in fact, very little better, if at all superior, to the common practice of hurdling off a portion of the land, either with natural or artificial grass, by which mode either cattle or sheep being confined to a certain space until the crop is consumed, the pen is then changed to a fresh place until the whole field is fed off. The grass is thus more economically consumed than upon a wide range, while the stock have almost daily a fresh bite $\S$. The plan of both hurdling and tethering has also this advantage over the conmon pasture: that when either the

* Young's Annals of Agric., vol. v., p. 204. Suffolk Rep., 3rd edit., y. 203.

+ Drew's Norfolk Husbandry, p. 166.

+ The most effectual tethers are made of iron chains, with short links, and two swirels to prevent them from twisting. They should he five yards long, with a stromg leather strap and buckle, to fasten to the fore-leg of the animal, near the houf. The tetler is secured by a large iron pin, on the head of which is a swivel, which should play round the pin freely, in order to prevent it from becoming entangled. Sinclair's Cude of Agric., 3rd Eilit., p. 491.

Burroughs on the Cultivation and Advantages of Grcen Crops, p. 7. 
hurdles or the tethers have been removed, the ground thus unoccupied is left for the further growth of a fresh crop; and, as it will have been well dunged by the concentration of the stock upon it, a larger aftermath may be expected, and the land will be thus necessarily improved.

\section{THE EXPERIMENTS}

Which have been published on the comparison between soiling and grasing, uniformly show a striking advantage in favour of the former-some of them, indeed, stating benefits of such an extraordinary nature as hardly to merit implicit faith; yet it is singular that they almost all deal in general terms, without stating those accurate particulars which can be alone relied on in estimating the result $*$ We shall, therefore, only select a few from those of the late Mr. Curwen-who, although a zealot in the cause, was a gentleman of strict candour-and one from Mr. Brown of Markle, which place the merits of soiling in a more moderate point of view than many of its other advocates; for we are thoroughly of opinion that any exaggerated account tends rather to injure than to support the cause which it is meant to espouse.

The following are those stated at various times to the Workington Society, of which Mr. Curwen was president, and were conducted under his own superintendence, upon his farm in Cumberland.

The first trial was made between two kyloes. The one which was soiled was ten or twelve years old, and had a number of calves; the other, grazed, was four years old, having had no calves. The advantage was, however, supposed to be wholly in favour of the younger animal; as it appears reasonable that the period most favourable for fattening should le as soon as the animal had arrived at its full growth. The experiment was commenced on the 14th of May, and concluded on the lst of October, being a period of 140 days.

The first weight of the soiled kyloe was 66 stone, and its successive weights, until killed, were as follows:-

\begin{tabular}{|c|c|c|}
\hline VEIGIIT: & $\begin{array}{l}\text { Aug. } 23, \\
77 \text { stone. }\end{array}$ & $\begin{array}{c}\text { Sept. } 6 \text {, } \\
78 \frac{1}{2} \text { stone. }\end{array}$ \\
\hline
\end{tabular}

$$
\text { The food consumed was }\left\{\begin{array}{cc}
745 \text { stone of clover. } \\
250 \text { " } & \text { oil-cake. } \\
30, & \text {, chaff. }
\end{array}\right.
$$

The experiment was therefore not solely confined to green food.

The first weight of the grazed kyloe was . . . 57 stone.

Live weight on the lst of Oct., after being fasted . . . 66 ,,

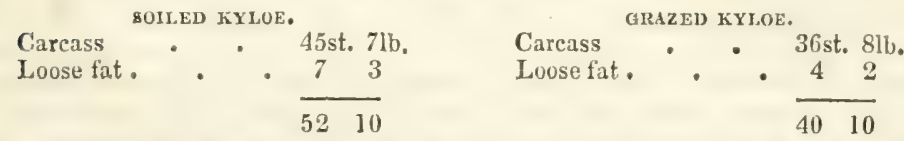

Supposing the hide and offal to be of equal value, the soiled kyloe is calculated to have paid a profit of $10 \frac{1}{2} d$. perday, and that of the one grazed of $7 ? d$. per day : that is, assuming the sale price at $4 s$. Sd. per stone, and the grazing to have been an acre of grass, at four guineas; the value of clover

* Thus, some have estimated it as being in the proportion of one to seven, if not more; but, in a trial which was reported to the Board of Agriculture, 33 head of cattle were said to have been soiled from the 20th of May, to the 1st of October, on $17 \frac{1}{2}$ statute acres, on which it is stated that it would lave required 50 acres to have pastured them. A similar result is mentioned by Sir John Sinclair, who states that the same number of stock were soiled on 17 acres which had always previously required 50 acres. Code of Agric., 3rd edit. N. p. 487. 
and oil-cake being estimated at $1 d$. eacl per lb., and the amount of chaff at $2 s .6 d$. Upon this every man may calculate his own prioes, at the present rate; but as to the charge of attendance, it may be fairly assumed as being remunerated by the increased value of the dung.

Mr. Curwen also caused six very moderate three years old Highlanders to be tied up on the 27 th of June, and fed for the greater part of the time, until the 9th of November, with grass of very little value, cut from hedges, plantations, and walks; the chief object being to destroy and consume the weeds: they had no straw, and only during the last month were fed with carrot and tumip tops. Yet, by the following account, they in that time added 75 stone of flesh; which, calculated at $4 s . S d$. per stone, would amount to $17 l .10 \mathrm{~s}$, or an average of $2 \% .18 \mathrm{~s} .4 \mathrm{~d}$. each, being nearly clear gain, besides a quantity of dung that would probably fully manure a couple of acres. It is, however, singular, that had they been killed on the 1st of October, instead of the following November, they would have saved the extra food, being nearly six weeks, during which it appears they only collectively gained two stone.

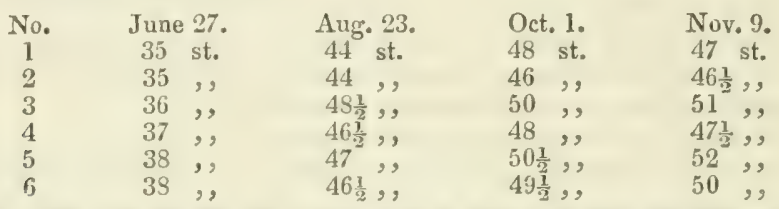

With a view also to try the experiment of rearing and fatting cattle at an early age, without sending them to pasture, Mr. Curwen had seventeen calves-eleven of the short-homed or Durliam breed, and six Ayrshireturned into the yard when two months old; and the intention being to hottom the practice on a system which might afford a profit to the farmer, all costly food was rejected. During the first month the calves had but one gallon of new milk per day; for three months afterwards, three gallons. 'Turnips and hay were given with the milk, but neither cake nor meal. From Jume till October the food was clover; afterwards, till the end of May, turnips: till Candlemas they had some little hay, but, being then well taken up to the turnips, straw was substituted.

In size they were little inferior to those of double their age; and, so good was their condition, that at 15 months old one of the Ayrshire heifers was butchered.

The live weight was - 55 st. of $141 \mathrm{~b}$, to the stone.

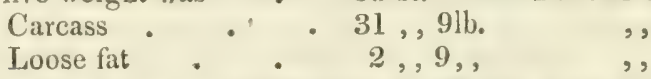

It was killed for a meeting of the Workington Society, and the meat highly approved by the members. The Durham breed was in much higher condition, and would have killed 46 stone at least; it was indeed imagined that there could be no difficuity in bringing them up to 60 stone of carcass at two years old. They consumel, on an average, 6 stone cach of turnips a-day : of hay they had about half a stone; but, when straw was given, no account was taken. Besides this, however, they had a feed of steamed chaff, the chief benefit of which was in its warmth preventing the green food from disagreeing with them. The practice was afterwards continued with equal success; but the progress was always greater on turnips than on clover.

The experiment related by Mr. Brown of Markle consisted of fortycight Aberdeenshire stots, mostly of a full age, which were wintered in the 
farm-yard, and divided on the 4th of May into two lots; one of which was put to grass, and the other into the yard, where they crot a small quantity of Swedish turnips until the clorer was fit for cutting. On the 1st of June he began to cut clover, which was given to the cattle in cribs, though for a week at least, to save danger, the quantity given was much less than they could have consumed: after that time, however, a full supply was allowed, and the offal furnished maintenance for a large parcel of swine of different ages. Till the grass got hard and withered, the animals in greneral throve amazingly well; but more particularly the strongrest and boldest among them, who took possession of the cribs, and would not resign them until their appetite was satisfied.

About the end of $\mathrm{July}$, when the clover was fully ripened, the food was changed to tares, which had been sown in the month of Harch; and this was continued until the second crop of clover was ready for the scythe. On the 2Sth of August ten of the best soiled lot were sold; on the 28th of September, the whole of both lots were disposed of, and it is remarkable, that the entire number of this latter sale, both grazed and soiled, fetched exactly the same price*. The statement is as follows:-

The 18 cattle cost together for purchase and wintering, 503l. 2s.

The 10 best of the soiled cattle were sold at $17 \mathrm{l}$. $15 \mathrm{~s}$. , and the remainder of the whole number at $14 l .5 \mathrm{~s}$. each, thus yielding-

For the soiled lot of $24 . .377 l .0 s, 0 d$.

Ditto grazed ditto ... 34200

Or $29 s .2 \%$. per head more upon the soiled than upon the grazed cattle; although they were all purchased at one price, and separated with the most accurate fairness.

\section{The food consumed by the soiled cattle was $\left\{\begin{array}{l}1 \frac{3}{4} \text { acre of Sivedes. } \\ S \text { acres, }, \text { Clover. } \\ 3, \text { Tares. }\end{array}\right.$}

Being 125l. $9 s$, for the cost of food and profit upon the stock.

It is unfortumate that Mr. Brown did not keep the grazed cattle separate; whereas they were allowed to run indiscriminately with other stock, thereby preventing any correct comparison between the different luts. However, from the total result on the different parcels of cattle, he is inclined to believe- " that the saving per acre will amount to jo per cent.: or, in other words, that a field of clover and rye-grass will feed one-haif more beasts when cut by the scythe, then when it is depastured.$+ "$

From all the information which we have been able to collect, we think that assertion is not far from the truth; and it is possible that, particularly on clay soils, which are naturally unfit for the growth of turnips, the adoption of home-feeding on soiled tares, clover, sainfoin, and lucerne, would be one of the greatest improvements in farming; for, independently of any saving in the consumption of the grass, the farmer would assuredly be thereby able to manure more ground ammually than he is now capable of doing. He sees with certainty that where manure has been laid, luxuriant crops are produced, and it becomes his duty to increase this universal restorative by every means in his power. When used with a due supply of litter, the quantity above what is made in the ordinary way will far more

* It was imagined by many dealers that the confined air of the court, which was surrounded by sheds, and littered with straw, would keep the eattle so warm as to prevent tallow from being gathered in any quantity. One of the soiled lots was, however, killed previous to the sale, which had $94 \mathrm{lb}$. of tallow, although the weight of the carcass was only 34 stone Dutch.

† Farmer's Mag, vol, vi, p. 463. 
than defray the charge, without attending to the larger number of beasts which can be thus maintained. Indeed, unless there be an unusually large proportion of permanent pasture, live stock cannot be maintained without soiling in any considerable number throughout the summer months; and, unless that stock be numerous, the farmer who occupies a large proportion of arable land will find it an arduous and unprofitable business: but, where "horn and corn are conjoined" he need not fear the cxhaustion of the plough,

\section{Chapter XXXIV.}

\section{ON THE FEEDING OF NEA' CATTLE-(Continuel)-WINTER STALL. FEEDING.}

Atriovgn the practice of stall-feeding, when conducted in a similar manner to summer-soiling, is, in fact, one and the same, yet it does not generally commence among the graziers until about the month of October or November; or at that period when the season compels them to bring up their cattle from grass. Many arable farmers also who have no grazing land, about that time purchase grass-fed stores, which are in such condition as to require only winter food to prepare them for market; and these are invariably what are calied " stall-fed," though great numbers of them are fattened in open yards.

\section{OX-HOUSES.}

Much diversity of opinion prevails on the subject of open sheds or close stalls, as being the best for the purposes of fattening. Regarding health, and perhaps the firmness and flavour of the flesh, there probably will be but one persuasion in favour of the former; but many instances could be adduced to show that more profit is to be gained by the latter, and that warmth is essential to the thriving of the cattle. Among others two have been instanced by Arthur Young, which indeed carry the plan of heat and confinement to an extraordinary extent: the one in the practice of the late Mr. Moody of Retford, who was an uncommonly successful grazier, and who found warmth of such consequence, that he gradually closed the air-holes of his ox-house, which was purposely provided with sliders, till lis beasts sweated off the hair, nor did they thrive to his mind until this happened. The other is a similar system pursued on the farm of Mr. Hanbury of Coggeshall, where the beasts are kept as warm as possible; and if it even brings oft the hair they are found to thrive the better, and to feel the better at Smithfield. On this subject, indeed, Mr. Young adds, that " men farm without an idea of the necessity of knowing what others have done before them; and it is very right that thousands of pounds should have been lost by oil-cake, while feeding shivering beasts in open sheds, by men who think they can learn nothing beyond the practice of the old women their grandmothers, while the Board of $\Lambda$ griculture has annually brought to light in County Reports, practices unknown to the same men who cannot see any use in such publications *."

That stock should be kept noderately warm, as conducive to their improvement, we admit; but notwithstanding the value of the practice

* Essex Report, vol. ii. p. 300.-In the Sussex Report it is also said,_" That in proportion as the cold is excludecl, will the ox aret fat; warmth being almost as essential as foud itself. The more cattle are confined the sooner also will they fatten; and this holds good with every species of live-stock ug on which experiment has reached us," p. 236. 
thus stated, we think that, if generally pursued, it would, in most cases, liave a contrary effect, for, if liept too hot, it makes them perspire and their slins to itch; the irritation thus occasioned puts them upon the fret, and instead of lying down quietly they are occupied in rubbing themselves agrainst whatever may be within their reach, which is much against quick feeding.

Regarding open sheds, Mr. Ellman, of Glynde, has also found that nine oxen, fed loose in a yard, have, by eating as well as destroying, consumed as much as twelve when tied up*. The waste of food when the cattle are fed loose is indeed always considerable, and much is frequently so trodden under foot as to be only thrown upon the dung-heap; whereas their refuse, when stall-fed, serves to support the lean stock, some of which should always be kept in an adjoining yard for that purpose. The stalls are generally nine feet wide, two beasts standing together in each + . If the bullock has not been already accustomed to be handled, he will at first have great reluctance to enter the stall, and turnips, or any food to which he is accustoined, should be thrown into the manger to induce him. Still greater difficulty will then be found in haltering him quietly to the stake, and the most gentle means must be used to answer the purpose. He should be at first rather tightly fastened to prevent him from struggling or attempting to turn round in the stall, which he will try to do when he finds himself fast; but he should be attentively watched, to avoid accident, until he is accustomed to the restraint, after which the chain may be somewhat loosened.

\section{FATTING-CATTLE.}

Respecting the sort of beasts to be fatted, although it is of the utmost importance to the grazier to choose such cattle as may be most appropriate to the nature of his land,-as upon moderate pasture which would fat a Scot, a Lincoln ox might starve,-yet in stall-feeding, as there is always a certain quantity of food upon the farm which may be equally applied to each, the main object to be considered is the condition and aptitude of the beast to fatten. ¿ E pon this, of course, the breed should ever be looked to, and upon that, as we have already said, we refer with confidence to the "History of British Cattle;" but in choosing the breed there are also some points which should be closely regarded : attention should be paid to compactness and symmetry of form, deep fore-quarters, wide carcases, fine small bones, moderately thin hides, a protuberance of fat under the root of the tongue, and large full eyes.

A well-shaped ox should thus have a small head with a placid countenance, as indicating docility and a consequent disprosition to get fat; a fine muzzle, and open nostrils; the throat should be clean, long, and thin in the neck, but wide and deep in the shoulders; the back should be broad and straight near to the setting on of the tail, with the rump.points fat and coming well up to it; the barrel should be round, wide across the loins, and the girth deep behind the shoulders, with the space between the hipbone and the first rib very small; the fore-legs should be short and wide apart, so as to present a broad appearance to the chest, and the thighs of the hind-legs should be shut well in the twist-the seam in the middle of which should be well filled, and the flanlis should be full and heavy. A form such as this is not only the best for affording the greatest weight, but will be also generally found to lay the flesh upon the prime parts, to pro-

* Sussex Report, p. 237.

+ For various plans of Ox-Houses, see rol, $i$, chap, viii. 
duce the least quantity of offal, with such a large quantity of tallow as, emphatically speaking in the butcher's phrase, will cause the animal to "die well." These marks, however, are not the only indications of a propensity to fatten quickly. On the contrary, it has been found by experience that many coarse beasts, with large bones and gummy legs, have often proved superior in that particular to other animals of unioubted superiority in point of shape; but those coarse thick hides handled soft and silky, with a sleeky degree of mellowness, which is the characteristic of a healthy habit, while the skin of the others was wiry and their flesh felt hard. The state of the hide and flesh is therefore of the first importance, as the essential property of handling well. It was indeed the opinion of that eminent breeder, the late George Culley, "that it is the nice touch or mellow feel of the hand which, in a great measure, constitutes the judge of cattle.*" It, however, can only be attained by experience.

The knowledge and the value of skill in toncli can, indeed, only be acquired by long practice, but when once attained, it may be relied on as a criterion for judring of the feeding properties of an ox; for it is generally found to be accompanied with the other good qualities of gentleness, purity of blood, and consequent disposition to fatten. A thin, papery shin, covered with light, silky hair, denotes weakness, -and is, therefore, as defective a test of superiority as that of a coarse, tough hide, covered with hard, short hair, which always indicates a bad feeder. Its perfection has, however, been accurately described by an eminent cattle-dealert, to some of whose observations we are much indebted, " as consisting in a thick, loose skin, floating, as it were, on a layer of soft fat, yielding to the least pressure, springing back towards the fingers like a piece of chamois leather, and covered with thick, glossy, soft hair."

The position of the flesh on the carcase is another great consideration in judging of the value of the animal, as it is of various qualities in different parts; thus the portion called in Edinhurgh the "spare-rib," and in London the "fore and middle ribs," the "loins," and the "rump," are of the finest quality, and consequently, the ox which carries the largest quantity of beef upon those points is the most valuable. The spot which first shows fat is the top of the rump; and as the quantity laid on there is frequently out of proportion to that on other parts, this often learls inexperienced persuns into error. Thuse which are generally the last in being covered with flesh are the point and top of the shoulder, and if these be tolerably well covered, the future ripeness of the animal may in due time be fairly reckoned upon. A thick flank, with a full twist lining the closing of the hams, is also generally considered as denoting a large quantity of internal fat, - which is an ohject much looked to by the butcher, as forming a material portion of his profit. These points should, therefore, be carefully examined in the purchase of a lean ox for fatting, as it is on its aptitude to that purpose that its value to the buyer depends, and that judgment is the most frequently betrayed. The condition of a fat ox strikes to the eye: but it has been justly remarked by Mr. Dickson,_-"That there is a great difference between apparent and real fatness; for the flesh of an apparently fat ox, to the eye, may, on being handled, feel loose and flabby,-but a truly fat ox always feels 'hard fat:' with such the butcher is seldom deceived, while loose-handlers give no assurance of killing well."”

Regarding the disposition to fat, it is a mistaken idea to suppose that

* On Live Stock, p. 101.

$\dagger$ Mr. James Dickson of Edinburgh, author of "A Treatise on the points on which Live Stock are julged." - See the Quart. Journ, of Agric., Nos. xxvi. and sxx. 
flesh can only be laid on in proportion to the consumption of food; for this-although a notion which is very commonly entertained-would render judgment unavailing. Now it is well known to all dealers in cattle, that althonghi a lot of beasts may be bought from the same farm upon which they were all bred, yet they will not be all found to feed alike, - some being what are called " hard feeders," or consuming more food, with a less aptitude to fatten than others. This arises, in fact, from the nature of the animal, and has been ascribed to the difierence of temper, which in some is more restless, churlish, and unruly than others; it is, therefore, more profitable to feed an ox of a kindly disposition than one of an opposite cliaricter. It is indeed true, that this disposition to fatten-as arising from temper-may be equally found in every kind of stock; but not so in point of early maturity, which depends in a great measure in superiority of breed.

The produce of fut caltle in beef may be stated as depending upon the various coniderations of breed, age, and condition; together with the kind and quality of the food consumed. This accounts for the disparity in the results of many experiments made upon the sulject, and for the variety of opinions entertained respecting the peculiar value of the difierent breeds. It is thus the opinion of many persons that a four or five-year-old Scotch ox carries beef of more prime quality, and of a higher price, than any young ox of another surt; yet oxen of two to two and a half years old are now continualiy brought to market, and, if both are equally ripe, the difference in quality of the meat is said not to he discernible ; and the feerling off at that agre is a system which is fast gaining ground, as appearing to make a quick return for capital. If so, as the object of all farming is profit, and this early maturity has been shown to belong in a superior iegree to the Herefords and short-horns, it would be evidently prudent to confine the purchase of fatting cattle to those breeds. But, independently of their requiring large quantities of hay and oil-calie, or equally nutritious food, while the small Highland cattle can be fatted upon grass and turnips, we are strongly of opinion that the flesh of a four-year-old ox will ever be found more marbled with fat and lean, more rich in its juices, and finer flavoured, than one killed while growing, at two years and a half; and in this we are upheld by the difference of price invariably maintained in Smithfield market. Neither can we help adverting to the remark of the late George Culley, - "That a plain, coarse, ugly animal may pay more than a fine, weli-made one, because the coarse one is bought at a much less price in proportion;" yet we cannot too earnestly impress ujon the feeder the propriety of purchasing those cattle for the stall which have the finest points in their form; for these will not only carry beef of the finest quality, but will consume less food in proportion - particularly as they attain age and fatness-and will thus, generally, realize the largest profit on their fatting.

\section{STALLING.}

When the cattle are purchaser, the next consideration is their manage. ment in the stalls; which requires no common share of attention, as the manner in which it is conducted may affect the thriving of the beasts nearly as much as the quantity and quality of their food, - and the farmer who thinks that he has only to throw them plenty, without regarding the

* Thus Mr. Dickson says, that a "well-fed two-year-old short-horned heifer will fetch as much per stone at Norpeth market, if not more, for cutting up, than any aged ox." 
mode of supplying it, will find himself deceived in his expectation of their improvement.

The first point is the comfort of accommodation; for in whatever way they may be placed-whether under sheds or in close ox-houses - they should have the security of perfect shelter from the weather, with a certain degree of warmth; that is to say-if in open liammels, the sheds should be broad, the roof low, and the floor well covered with an abundance of dry litter. We are, however, decidedly of opinion, that close stalls will further the object more promptly; though we do not coincide in the idea that it will be promoted by too much heat, and we should therefore recommend a moderate degree of healthful ventilation. In these stalls litter is, indeed, very frequently dispensed with, - or else sand, or any rubbish, is substituted for straw; but there can be no doubt that the animals enjoy the comfort of a dry bed as well as their master, and the more they seek repose in it the better.

The next, is strict regularity in the administration of food-both as regards the stated quantity, and the time of supplying it. The periods may be regulated as the feeder thinks proper; but, whenever adopted, should never be afterwards altered. The ox is a quiet animal, and those which are fed in the house soon acquire a precise knowledge of the exact hour at which it is usually given : if that be transgressed, or the customary quantity be not furnished, they become restless, but if the time and quantity be strictly adhered to, they remain tranquil until the next period arrives. If no disturbance takes place, they, indeed, then generally lie down to ruminate, and nothing will be found to more forward the process of fatting than this perfect quietule; wherefore, not only should the stalls be well bedded, but light should be very much excluded, the doors should be closed, all outward annoyance as far as possible prevented-and, in short, every means should be induced to promote complete rest, ease, and contento ment.

Some persons serve it out as often as five times in the day; but the most prudent, and we think the better practice, is to give it as soon as possible after daylight, at noon, and some time before sunset; which enables the animals to fill their bellies, and to have time sufficient for that quiet digestion which is interrupted by too frequent feeding. In stating that the quantity should be moderate, we however allude merely to the not allowing the animal to have so much as will cloy him; he ought always to have as much as he can fairly eat with a relish, but the moment he begins to toss it about, it will be then evident that the keenness of his appetite is satisfied, and it should be instantly removed.

The last is thorough cleanliness. The ox-house should be opened before daylight, and well cleaned, both by pail and broom, from every impurity. After the animals have been satisfied with food, whatever may remain should be immediately removed; and the cribs and mangers should be carefully swept out, and washed, if necessary; water should then be given without limitation*. If their hides be

* According to an experiment stated by Sir John Sinclair, an old man was appointed to discover how often some cattle, consuming straw and chaff on a farm, went to the watering-trourh in a short winter's day, and that he might not be confused in the execution of his orders, one particulir bullock was pointed out for his report; accorling to which, he drank eight times in the course of the day, and the man was convinced 
then wisped, it visibly occasions a very pleasurable sensation: as they begin to fatten, the ancient coat falls off, and if this be accelerated by the curry-conb, the better appearance of the beast will well repay the trouble.

\section{NOOD.}

The relative proportion of food consumed by futling beasts must necessarily depend, not only upon the sort of animal, but also upon the kind of nutriment by which he is supplied; for it is not the quantity which the animal takes that fattens it, but the quality. Of all vegetable productions, nothing can be better than good hay for improving the flesh of fatting cattle; and this was formerly the only substance used. Of late years, however, oil-cake has been very generally added in fattening them off for market; and the increased consumption of animal food, together with the production of green crops now cultivated by the improvements in our agriculture, have also induced the feeders to employ every species of field-root grown on our farms. T'otatoes, mangel-wurzel, carrots, parsnips, cabbages, and turnips of every lind, are therefore in general use; and in some of our large clistilleries, bulloclis are also fed upon the wash*. Treacle has also been tried; and there can be no doubt that, if it could be had free of duty, it would be a valuable assistance in fatting, if given either in water or mixed up with meal t. We doubt, however, whether any of these products can produce meat of such succulence and flavour as that furnished by a grass-fed ox of mature age, finished off in the winter by fine meadow hay, with perhaps a little addition of barley or bean meal; but that plan can no longer be generally pursued, for our meadow and pasture land would not alone furnish animals equal to the demand.

In those districts where grass abounds, and where hay is much used in fatting, it lias been generally found that a bullock of 50 stone weight, consuming 40 lbs. daily of sound hay, will acquire flesh at the rate of 2 lbs., and should, therefore, in twenty weeks, increase to 70 stone: or 10 lus. of hay, with a bushel of potatoes, will have the same effect. In other experiments it has been observed, that, besides an adequate quantity of dry food to currect the effects of moist roots, bullocks of 60 stone or upwards require about 18 stone of common turnips laily : an acre of 25 tons will therefore generally fatten a beast of that weight, if the dry meat consist of hayt.

that the rest of the cattle drank as often as the one fixed on. Now, twice a day is the most in which they generally get water; and they are not able, at one or two opportunities, to drink a sufficient quantity.-Husb. of Scotland, p. 100.

* Messrs. Smith and Harrington, of Brentford, once fattened 810 oxen on the refuse of 25,750 quarters of barley, and in twenty weeks an increase of flesh was acquired averaging each 35 stone; which, deducting 5 stone as the value of the hay which they consumed, leaves 24,300 stone of beef,- - or about one stone for the grains from a quarter of barley: which is about double the usual price. The same gentlemen, however, fatted 250 bullocks on the refuse wash of a sugar distillery; but the quantity of hay consumed was so great, that, although the wash was only valued at $20 \mathrm{~s}$. per head, yet a loss was incurred upon the experiment.

$\dagger$ In using this article, about a pint of the molasses should be boiled in half-a-peck of meal, and given when cool in a mash. It is also mixed with the common drirk of aged horses in the West Indies, and has been found a great resturative to cattle which have been hard worked.

From some experiments that were confined to the common white turnips, it has been estimated that 34 tons produce $436 \mathrm{lbs}$. of beef and tallow; which is nearly $13 \mathrm{lbs}$. per ton. The feeding having, however, been calculated without any succession of yellow turnips, or Swedes, and continued durin: five months and a half, the common white sort must have lust so much of their nutritive properties during the close of the feeding season, that it does not afford a fair average; and it only continued during four months, 14 lbs. per ton would probably have been nearer the mark. At the same time it was

VOL, II. 
Bullocks varying from 45 to 60 stone consume from 8 to 10 stone of carrots or parsnips, besides dry provender; and it has been found that an ox will eat something less than one-fifth per day of his own weight in cabbages.

These trials are, however, so far from being decisive in their information, that they can only be viewed as approximating to the truth, and merely furnishing hints for practice. Indeed, although small cattle will, on an average, consume less food than those of a larger size, yet the quantity is by no means in exact proportion to their weight: the advantage being usually in favour of the largest, when all other considerations are equal. It has also been justly remarked, as an observation of daily occurrence, "That cattle of the same breed are yet of different constitutions, and do not always possess an equal aptitude to convert their nourishment into fat. Thus, in an experiment ordered at Woburn, by the late Duke of Bedford, on six oxen-two of them Herefords; two Devons; one of the Sussex, and one of the long-horned Leicester breed-which were put up at one time for an equal number of days, and (with the exception of one of the Herefords) were daily fed on equal quintities of oil-cake, with as much turnips and hay as they could eat; the whole quantity consumed, and their respective weights at each period, were as follows * -

\begin{tabular}{|c|c|c|c|c|c|c|}
\hline \multicolumn{3}{|c|}{ 1st Weight. } & \multicolumn{2}{|c|}{ Food consumed. } & 2nd Weight. & $\begin{array}{l}\text { Live Weight } \\
\text { gaiued. }\end{array}$ \\
\hline No. & 1. IIereford & 146 st. & $\left\{\begin{array}{l}\text { Oil-cake } \\
\text { Turnips } \\
\text { Hay }\end{array}\right.$ & $\left.\begin{array}{c}424 \mathrm{lbs} . \\
2712, \\
\cdot \quad \cdot 433,,\end{array}\right\}$ & 169 st. 11 llss. & 23 st. $11 \mathrm{lbs}$. \\
\hline , & 2. Ditto & 136 st. $1 \mathrm{lb} .\{$ & $\left\{\begin{array}{l}\text { Turnips } \\
\text { Hay }\end{array}\right.$ & $\begin{array}{r}270, \\
. \quad 487, y\end{array}$ & $\{150$ st. & 13 st. 13 lbs. \\
\hline , & 3. Sussex & 132 st. & $\left\{\begin{array}{l}\text { Oil-cake } \\
\text { Turnips } \\
\text { Hay }\end{array}\right.$ & $\begin{array}{r}433 \% \\
. \quad 2655 \%, \\
392 \%\end{array}$ & 158 st. & 26 st. \\
\hline$"$ & 4. Leicester & 125 st. & $\left\{\begin{array}{l}\text { Oil-cake } \\
\text { Turnips } \\
\text { Hay }\end{array}\right.$ & $\begin{array}{r}435 ", \\
2652 \\
-\quad 400 " \\
443\end{array}$ & 148 st. & 23 st. \\
\hline , & 5. Devon & 117 st. & $\begin{array}{l}\text { Turnips } \\
\text { Hay . }\end{array}$ & $\begin{array}{r}443 ", \\
. \quad 2636 " \\
443 ",\end{array}$ & 154 st. & 37 st. \\
\hline " & 6. Ditto & 114 st. 71 bs. \{ & $\left\{\begin{array}{l}\text { Oil-cake } \\
\text { Turnips } \\
\text { Hay }\end{array}\right.$ & 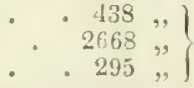 & 140 st. $7 \mathrm{lbs}$. & 26 st. \\
\hline
\end{tabular}

From which it will he seen, that, in opposition to the common remark of large cattle generally thriving in a greater degree than small ones upon a proportionate quantity of food, the smaller species had the advantage. The striking difference between the two Devons of equal weight is likewise worthy of remark; while the similitrity of fool consumed by beasts of different weight is also extraordinary. It will thus, indeed, be frequently fomd, that in a score of bulloclis of the same hreed, age, and apparent quality, fed and managed alike, some will fatten much sooner than others, and arrive to greater weight when slaughtered, than those which, when alive, appeared to both eyc and touch without the least diflerence. IIowever justly, therefore, some breals are to be preferred upon greneral principles, experience hats fully proved that, although comparative trials of their respective merits are not to be slighted, yet their results are often contradictory, and their conclusions not always entitled to decisive faith.

ascertainer, that a beast of 45 stone ate 16 stone of white turnins; and one of 50 stune only 12 stone of Sweles: so that, taking an arerage of the different linis of turnips to rether, one ton maly be firirly supposed to return $14 \mathrm{lbs}$, - or one stone of beef and tallow.-Gen. Rep. of Scotland, vol. iii. p. 95.

* See the Complete Grazier, 6th edit. 1. 28, and the Budfurcshire Ieport, 1) 53:. 
In stall-feeding, it is always advisable to give a fair allowance of sound meadow hay, cut into moderate lengths, either with or without some portion of straw, as a corrective of the looseness occasioned by feeding on raw roots; and these must, of course, be consumed in the order in which they cin be best preserved: thus the common white turnip is always the first to begin with, then the Aberdcen yellow, and lastly the Swede. When farmers, lowever, grow cabbage, carrots, parsnips, potatoes, and mangelwurzel, these roots are used in succession, but the most nutritive species should, if they can be held over, be kept back until the last; for bullocks grow dainty as they become fat, and do not require such a quantity of food as at the first commencement of stall.feeding; they should, therefore, have the greatest amount of nutriment put into the smallest possible compass. Various quantities of bran and pollard, pea and beanmeal, and the bruised grain of all sorts of corn, together with oil-cake and linseed jelly, are also given with this view in different proportions-from 5 to 10 lbs. - according to their price, the condition of the animals, the intention of pushing them forward to a market, and the judgment of the feeder. Bran and linseed oil, in the proportion of two pecks of bran, divided into three feeds a day, with half a pint each of oil, has been also strongiy recommended, as being found, in that quantity, sufficient for the rapid improvement of small cattle*. On the nutritive properties of these several matters we, however, deem it mmecessary to offer any further remarks than those which may be found under the different heads where they have been already treatedt.

Respecting the prepuration of some sorts of this food a notion has, however, been long cntertained that they are more nutritive when dressed than raw; and therefore potatoes, together with Swedish turnips and other roots, as well as excry species of grain, are frequently steamed. Some recent trials which have been male in pursuance of premiums offered on the subject by the IIighland Society, tend, however, to throw considerable doubt on the expediency of the practice; and, as it is highly important to farmers to have the clearest view presented on every point connected with their joufit, we hereby subjoin a brief account of the result as stated in the Transactions of the Society + .

\section{EXPERIMENTS.}

Mr. Walker of Ferrygate, who olntnined the first prize, put up to feed, on the 20th of February, 1833, six two-year-old heifers, and four two-year-old stots, which had been all previously for some time in the yard on full leep; and their feeding qualities having been thus displayed, a more equal selection could be made than from any lot of lean cattle.

The heifers were then divided into two casts of three each, as nearly like as po-sible, both in point of weight and feeding, and were separately placed in an open court with sheds; lots being drawn to determine which were to be put on raw, and which on steamed food. The same process was alopted with the stots; only with this difference-that they were kept entirely under cover of the sheds, but loose, and having abundance of room to walk about, with plenty of light and air. 'The daily food allowed to each was-

* Lambert, on the Rural Affairs of Ireland, p. 68.

$T$ See, also, the trials made on feerling with calbages, currots, and mangel-wurzel, as stated in the riote to chap. $\mathrm{xx}, \mathrm{p}, 253$ of this volume.

+ Vol, iv, and No, 29. 
To the heifers $\{3 \mathrm{lbs}$ of bruised beans, and

$\begin{array}{ll}\text { To the stots } & \left\{\begin{array}{l}40 \mathrm{lbs} \text { l of potatoes. } \\ 30 \mathrm{lbs} \text { of potatoes }\end{array}\right.\end{array}$

together with as many purple-topped Swedish turnips, topped, rooted, and properly cleaned, as they could either of them eat; and half a stone of straw each. The steamed food was separately dressed every day, and the mode of preparing it was in a common farm-louse steamer*, the tub of which was filled as full as it could hold with turnips, which required from five to seven hours in steaming. The potatoes were put in on the top of the turnips about an hour before their being taken off, when they had fallen so much down as to admit them; and the beans were put in last, about half an hour afterwards: all three, when taken off, being thrown into other tubs, where they were well mixed. The food was in both cases given three times a day, to each as much as they could eat, in troughs regularly cleared of the refuse, and well cleaned: the bruised beans given to the luts on raw food being served to them at noon, and the potatoes, one half in the morning, and the other half at noon $\dagger$. A small quantity of salt-at the rate of abuut two ounces to each beast per day-was also given: at first, in consequence of its decided effect in pieventing the steamed food from beconing so soon sour; and afterwards, from its being so well relished by the cattle $\begin{aligned} & \text { : } \\ & \text {. }\end{aligned}$

The following is the quantity of food consumed by the separate lots during a week :-

\begin{tabular}{|c|c|c|c|c|c|c|c|}
\hline \multirow{4}{*}{ Three Heifers.. } & \multicolumn{3}{|c|}{$\begin{array}{l}\text { Stenmed Food. } \\
\text { Cwst. qrs. lbs. }\end{array}$} & \multirow{2}{*}{ Swedish Turnips } & \multicolumn{3}{|c|}{$\begin{array}{l}\text { llaw Food, } \\
\text { Cwt. qrs. lbs. }\end{array}$} \\
\hline & & & & & 25 & & 14 \\
\hline & 3 & 3 & 0 & Potatues & 3 & 3 & \\
\hline & 0 & 2 & 7 & Beans & 0 & 2 & 7 \\
\hline & 23 & 2 & 0 & Swedish Turnips & 17 & 2 & 0 \\
\hline & 3 & 3 & 0 & Potatoes & 3 & 3 & 0 \\
\hline & a & 2 & 7 & Beans & 0 & 2 & 7 \\
\hline
\end{tabular}

Their live and dead weight from the 20th of February till the 20th of May (soon after which cight of them were killed) was as follows:-

\begin{tabular}{|c|c|c|c|c|c|c|}
\hline \multirow{3}{*}{$\begin{array}{l}\text { Three Heifers on steamed food. } \\
\text { Ditto on raw food .... }\end{array}$} & $\begin{array}{l}20 \text { th } \\
\text { Feb. }\end{array}$ & $\begin{array}{c}20 \text { th }_{1} \\
\text { March. }\end{array}$ & $\begin{array}{l}20 \mathrm{th}_{\mathrm{l}} \\
\text { April. }\end{array}$ & $\begin{array}{l}20 \text { tht } \\
\text { May. }\end{array}$ & $\begin{array}{c}\text { Dead } \\
\text { Weight. }\end{array}$ & Tallow. \\
\hline & stone. & stone. & stone. & stone. & st. lbs. & st. Ibs. \\
\hline & $222 \frac{1}{2}$ & 241 & $257^{\frac{1}{2}}$ & $268^{\frac{1}{2}}$ & $\begin{array}{ll}152 & 0 \\
150 & 3\end{array}$ & 2411 \\
\hline Two Stots on steamed food. & & & & & & \\
\hline No. $1 \ldots \ldots .$. & 84 & $90 \frac{1}{2}$ & 99 & 103 & $56 \quad 10$ & 811 \\
\hline No. $2 \ldots \ldots \ldots$ & 92 & 98 & 103 & 106 & . & $\because$ \\
\hline No. $3 \ldots$ & 90 & 96 & 100 & 105 & 586 & 88 \\
\hline No.t..... & $81 !$ & $9 \cdot 2 \cdot 2$ & 100 & 103 & .. & .. \\
\hline
\end{tabular}

By which it will be seen, that not only did the cattle on steamed cat very

* On Steaming, see vol, i. chap. vii. p. 129.

f So says the statement; but we should appreliend that the potatoes, if given one half at night instead of at noon, would have been the better plan.

\$ They did not get any salt for the first ten lays; but after being used to it for three weeks, the cattle on steamed food were tried with one feed without salt, and they did not seem to relish it so well: a little salt being, however, put upon their fuod, they at once begran to eat it greedily. 
considerably more than on raw food, but that their additional increase of llesh by no means paid for the charge of coals and labour occasioned by the operation of steaming; for it thus appears that the heifers on steamed food had only 1 st. $11 \mathrm{lb}$. more beef, while those on raw food had the advantage of 1 st. $7 \mathrm{lb}$. in point of tallow; and it seems by the account that the stot No. 1 left a loss when killed of $3 s .8 \frac{1}{2} d$, while that of No. 3 gave a profit of $10 \mathrm{~s} .10 \mathrm{~d}$. 'The table also shows a larger monthly increase of flesh on the stot No. 4 than that on No. 2 ; but it is not improbable that this advantage may be in some measure more attributable to constitution than to difference of food, for they were afterwards put into very rich pasture, and then fed upon clover and Swedish turnips until the 11th of January, 1834, when their dead weight showed the same superiority in No. 4: being-

No. 2.-Beef, 73 st. 8 lbs.; Tallow, 10 st. 5 lbs.; Hide, 7 st. 3 lbs.

No. 4.-Do., 77,0 , ; Do., 12,9 , ; Do., 6,8 ,,

Another trial was made at the same time by Mr. Andrew Howden, of Lawhead, in East Lothian, who, on the 6th of March, put up six stots and twelve heifers, of twenty-two months each*, and divided them into lots of three each which were then daily fed until the 6th of June, in the following manner :-

Lots 1 \& 2, Heifers-Each beast 140 lbs. of yellow and Swedish turnips.

, $3 \& 4$, Do.- $\quad$, 84 , of potatoes.

"5 \& 6, Stots- $\quad$ " 42, do., with $7 \mathrm{lb}$. of bean and oatmeal.

The quantity of food for each lot was weighed out daily raw, and that which was afterwards steamed was dressed fresh every day, except the portion intended for Sunday, which was prepared on Saturday night. Each beast had also from 8 to $10 \mathrm{lbs}$. of oat straw, and those which were fed upon potatoes had every day a pailful of water, which held ten Scotch pints-or about five gallons.

The cattle were sold together for the same price- $\mathbf{E 1 2}$ each-and in the annexed table of their respective weights it is assumed that the cxpense of keep, as there represented, will be near the truth if turnips be valued at $8 s$. and potatoes at $20 \mathrm{~s}$. per ton; oats at $18 \mathrm{~s}$. and beans at 24 s. per quarter, - exactly four quarters of which being used in the experiment.

Weight
5th March. $\begin{gathered}\text { Weight } \\ \text { 6th June. Increase. }\end{gathered} \begin{gathered}\text { Cost of food for } \\ \text { both Lots. }\end{gathered}$

† Lot 1, on raw turnips.........2604 lbs....2996 lbs....392 lbs.?

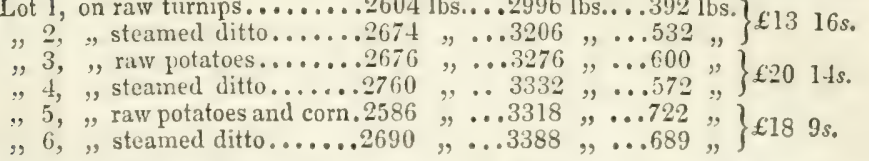

Experiments upon equal quantities of food camnot, indeed, be considered

* The age of the stots is not stated; nor is it clearly stated whether the heifers were twenty-two months old when put up, or when purchased in the previous month of November: considering their weight, we should, however, suppose the latter to be the fact.

+ The inferiority of this lot of heifers fed unon raw turnips is accounted for by the fact that a heap of turnips which had been laid up in the stack-yard had begun to ferment, and had thus accuired an unpleasant taste, which, during the last month of the experiment, prevented these heifers from eating their allowance; while those on steamed turnigs consumed the usual quantity, as the taint seemed to be destroyed by the operation of steaming. It had, however, a contrary effect upon the potatoes; fur, as the season advanced, the cattle seemed to prefer those which were raw. 
as decisive upon the feeding properties of cattle, as they cannot all have appetites alike; but from this it will be perceived that-except with turnips, which from the circumstance stated in the note were not fairly tried -the raw food had the advantage; besides being clear of all charere on steaming, which is not included in the cost of fool, though the expense, with coals at a tolerably cheap rate, is estimated at $10 \mathrm{~s}$ per head. The table also shows that, if the calculations be made at the market prices stated, potatoes, beans, and oats, taken together in ccrtain moportions, may be converted into beef at a lower price, and unquestionably of hitter yulitity, than either turnips or potatoes used alone; for it appears that the cost of the increase of beef was, within a fraction, at the rate of

$$
\begin{aligned}
& 3 \frac{2}{2} d_{\text {. }} \text { per } 1 \mathrm{~b} \text {. with turnips alone. } \\
& 4 \frac{1}{4} d_{0} \quad, \quad \text { potatoes do. } \\
& 3 \frac{1}{8} d_{0} . \quad " \quad \text { potatoes and corn. }
\end{aligned}
$$

A third experiment was made by John Boswell, Esq., of Balmuto and Kingcausie, on ten homed beasts of one breed, which were jut up on the 15th of October, and divided into two lots, -

That on raw food weighing 183 st. $11 \mathrm{lb}$. Dutch.

$$
\text { " steamed do. , } 179,10, \text { do. }
$$

and fed from that day until they were slaughtered with yellow bullock turnip, without leaves or roots, and round pink potato, commonly called the "Perthshire reds." The cattle, being all exceedingly tame, took to the raw food at once; but those which were put upon the steamed food hardly tasted it for several days, so that by the 1Sth they had a very jaded appearance. Nothing, however, was given to them but fresh steamed food until they took to it-the stalls being carefully cleaned out every day, and the stale stufl given to the pires; but at length they seemed to relish it as well as that which was raw. Both turnips and potatoes were served out logether, and it was observed by the feeder that the bullocks on raw food regularly firished the potatoes before one turnip was tasted ; whereas those on prepared food picked out the steamed tumips in preference to the potatoes. The liquor from the steamed turnips, which has an agreeable smell and taste, was retained and mixed with the food; while that of the potatoes, which is generally thought prejudicial, was drawn off. The other differences of feeding on the two kinds of food were so fix in favour of that upon the steamed-that the beasts consumed it sooner than when raw; that their dung was, from the first, without any appearance of purging, as well as free from that abominable smell which is perceptible when cattle are fed upon raw potatoes; and that they consumed much less than those on raw.

This latter fact, it camnot but be observed, is singularly in opposition to that stated in the experiments by Mr. Walker; yet, although the dead weights of the separate lots are not stated, the butcher who slauglitered them certified that they were both exactly alike, both in weight and quality. Notwithstanding which, it, however, appears from the statement, "that it is not worth the trouble and expense of preparation to feed cattle on boiled or steamed food; for although there may be a saving of food, it is comterbalanced by the cost of fuel and labour, and can only be gone into profitably where food is very high, and coal very low."

SIZE.

It has not yet been proved by decisive experiments, whether the large or small-sized oxen pay best for the food they eat; but the trials which have 
been made are rather favourable to the opinion that a small ox, when kept fattening in a stall, will eat proportionably more, without fattening quicker, than a large one. The meat, however, of the small one is finer grained, and the size rendering it also more generally acceptable to family consumption, it consequently commands a higher price in the market. The size of the animal has, therefore, probably but little effect upon the return to the grazier; for the flesh of the large beast, though cuarse-grained, yet if he be well fed, perhaps gains in its rich juiciness what it wants in delicacy, and is fitter for the common population of the large towns, and the uses of our shipping. On the whole, therefore, it appears difficult to decide which species should be preferred, and we believe that either will in general pay a fair profit if properly managed: this, however, will mainly depend on the knowledge and judgment that lave been employed in selecting such cattle only for stall-feeding as are already in good condition, and by their appearance of high breeding and mellowness of touch evince an aptitude to fatten.

The object of stall-feeding is, however, sometimes carried to too great an excess: the beef, if too fat, is not thus improved in flavour; while, if effected with costly food, the expense may be found to exceed the return.

\section{Chapter XXXV.}

\section{ON FAT CATTLE.}

AN ox in high condition may he said, by the most unpractised observer, to be fat, but the points by which his real value must be calculated can only be ascertained by very strict exmmination; and the observation which we made in the last chapter respecting apparent and real fatness is so just, that the most experienced juilges are frequently deceived with regard to that most material object in the estimation of a purchaser, which consists in the bulcher's profit; or, in other words, his "fifth quarter,"-comprising the offal, hide, and tallow. The fat which, in the course of feeding, accumulates about the intestines of a beast, is distinctively termed tallow, from the uses to which it is so commonly applied: this, as it can be neither seen nor felt, can only be juilged of conjecturally, and it has been not uncommonly remarked that beasts which have exhibited great fattening on the exterior, have been found so indifferently filled with fat in the internal parts, that the difference in weight of tallow between cattle of the same live weight has been as much as from 8 to 10 stone*. The following hints, however,-which we extract with great confidence from the "Complete Grazier †",-may afford some criterion in leading to an accurate judgment on those parts of the animal which, being received as the chief, are distinctively called their

\section{" pornTs."}

"First, when the appearance of the animal shows that each bone is

"Thus, in Youatt's "Cattle," there is an account of several shert-horns, amongst which is the following difference in tallow, namely:-

\begin{tabular}{|c|c|c|c|c|}
\hline steer $y$ & 4 years old, & Carcase, 106 st. & & Tallow, 19 st. $7 \mathrm{lb}$. \\
\hline Ditto & ditto & 112 st. & • & 26 st. \\
\hline Ditto & 3 years, 9 months & 101 st. & . & 15 st. \\
\hline Ditto & 3 years, 2 months & 95 st. & . & 17 st. 10 \\
\hline
\end{tabular}


covered with flesh in the manner required to constitute a perfect shape, it may be concluded that he has been well fed; especially when his hip-bones-or, as they are sometimes termed, his 'huckle bones, are round, his ribs smooth, his flanks full, and his cod round. When these marks are perceptible, the beast may be handled, and his lowermost ribs felt; if the skin be kindly or mellow, - that is, soft, yet firm to the touch, -it is certain that he is well fed outwardly; or, in other words, "upon the bones.'

"Next, the hand may be laid upon his huckle-bones, and if the parts there likewise feel firm, round, and plump, it may be safely concluded that he is well fed both externally and internally, - that is, both in flesh and tallow. He may then be handled at the setting on of his tail, which, if it be thick, full, and soft to the touch, is also an indication that the beast is well fed externally: the same circumstance is likewise evinced by the " nach-bones,"-which lie on either side of the setting on of his tail-feeling mellow, or soft, and loose.

" Lastly, the cod may be examined of an ox, or the navel of a cow, and if they respectively fecl thick, round, large, and plump, it is a pretty certain criterion that the beast is well tallowed witlin; and when any of these parts or members handle contrary to the rules above mentioned, a contrary judgment must be formed."

\section{IVEIGHT.}

Ilaving thus formed a correct judgment of the condition of the beast, the next consideration, as between the buyer and the seller, is its weight. This, indeed, may be duly ascertained by means of the steel-yard, which no cxtensive grazier shoukl ever be without, for by its constant use while the cattle are fatting, he can instantly ascertain the state and "progress of the beasts, and thus compare together their expense and their improvement; but it cannot be employed with accuracy unless the animal has fasted during at least twelve hours. When judged by the eye, it is, however, to an inexperienced person, a matter of much uncertainty, and even when conquered there is the still greater difficulty of estimating the difference between the live and dead weights. These are, in this country, usually calculated per stone of $14 \mathrm{lbs}$. for the live, and $8 \mathrm{lbs}$. for the dead weight; but in some other parts of the kinglom the following are also in use, namely :-

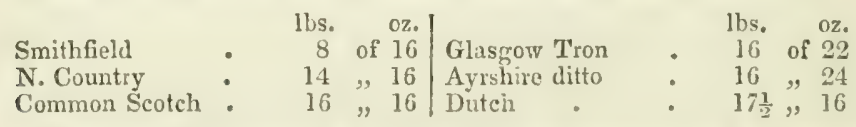

It has been very commonly calculated by salesmen that the dead weinht is one half of what the animal weighs alive; but although submitted to by the grazier, it is yet well known by the butcher to be more, and we believe that, in most cases, it is found to be nearer to three-fifths than one lialf of the live weight. 'The stock-bailiff of the lite Mr. Curwen always calculated from his experience that the dead weight was equal to $\frac{55}{106} 0^{6}$, - that is to say, about eleven-twentieths of the live weight; or that, supposing a live bullock to weigh 50 stone, the four quarters should, when deal, give a fraction more than 27 stone 11 lbs. Thus one of the heifers to which we have alluded in the chapter on summer soiling, having been slauglitereil at one of the Workington Meetings, was found to be precisely 55 stone, when alive, and to yield $30 \frac{1}{2}$ stone of beef, with 2 stone of loose fat. 'This animal, it should, however, be observed, had not attained a state of perfection, yet thus considerably exceeded one half of her live weight; and the 
weights of many other beasts of different breeds and ages, slaughtered in various years, have shown a still greater disparity. Thus the average of those stated as follows, by Mr. Ferguson of Woodhill, amounts to very nearly the proportion which we have assumed; but it will be seen that the cattle, although individually of equal weight, when alive, yet, separately, displayed an extraordinary difference, when killed, in their production of beef and tallow, exclusive of hide and offal:-

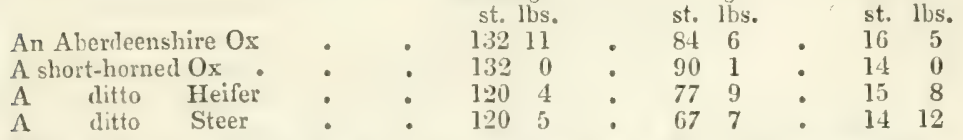

The difficulty found in correctly ascertaining the difference between those weiglits has led to the measurement of live stock, and tables have been constructed by several ingenious and experienced men, by which they are calculated according to the animal's dimensions. The manner in which this is done is as follows:- The girth is taken by passing a cord just behind the shoulder-blade, and under the fore legs: this gives the circumference; and the length is taken along the back from the foremost comer of the blade-bone of the shoulder, in a straight line to the hindmost point of the rump, - or to that bone of the tail which plumbs the line with the hinder part of the buttock : thus-

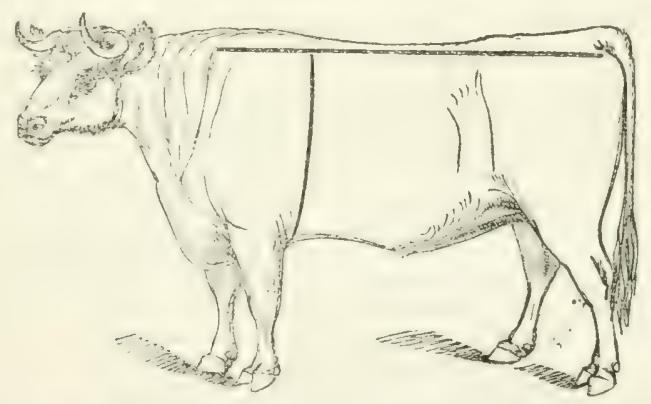

The girth and length are then measured by the foot-rule, and this morle of measurement has been adopted by all the writers upon the subject, as being equally applicable to every kind of animal. The weights stated in the tables published by Renton, Cary, and $\mathbb{M}^{\circ}$ Derment-as will be seen by some following extracts-nearly accord with each other; and having bcen tested by animals measured when alive, and afterwards lilled and weighed, were found to approximate so nearly to the truth as to afford a tolerably accurate rule for judgment on the ordinary run of oxen.

\begin{tabular}{|c|c|c|c|c|c|c|c|c|c|}
\hline \multirow{2}{*}{\multicolumn{2}{|c|}{$\begin{array}{l}\text { Girth. } \\
\text { fr. in. }\end{array}$}} & & \multirow{2}{*}{$\begin{array}{l}\text { I.ength. } \\
\text { ft. in. }\end{array}$} & \multirow{2}{*}{\multicolumn{2}{|c|}{$\begin{array}{c}\text { Renton's Tible. } \\
\text { st. 1bs. }\end{array}$}} & \multirow{2}{*}{\multicolumn{3}{|c|}{$\begin{array}{l}\text { M'Derment's Table. } \\
\text { st. lhs. }\end{array}$}} & \multirow{2}{*}{$\begin{array}{l}\text { Cary's Gauze. } \\
\text { st. }\end{array}$} \\
\hline & & . & & & & & & & \\
\hline & & & 40 & 24 & 0 & 23 & 11 & . & 24 \\
\hline 5 & 6 & . & 39 & 27 & 1 & 27 & 0 & . & 27 \\
\hline & & & 49 & 34 & 4 & 34 & 2 & . & $3+!$ \\
\hline 6 & 0 & - & 46 & 38 & 8 & 38 & 8 & . & $3 S_{4}^{2}$ \\
\hline & & & 50 & 43 & 1 & 42 & 12 & . & $43^{4}$ \\
\hline 6 & 0 & . & 46 & 45 & 9 & 45 & 3 & - & $45 \frac{1}{2}$ \\
\hline & & & 49 & 48 & 0 & 47 & 10 & . & $48^{2}$ \\
\hline 7 & 0 & - & 56 & 64 & 6 & 64 & 2 & . & $64 \frac{1}{2}$ \\
\hline & & & 60 & 70 & 5 & 69 & 13 & . & $70 \frac{\pi}{4}$ \\
\hline 8 & 0 & • & $6 \quad 6$ & 99 & 8 & 99 & 0 & - & 993 \\
\hline & & • & 70 & 107 & 5 & 106 & 9 & . & $107^{3} *$ \\
\hline
\end{tabular}


IIaving admitted that, we must, however, confine it within the bounds of merely guiding it to a probable estimate of the value of the beast, according to the assumed weight in the tables; as, however accurately drawn, they will be found frequently deceptive in the real quantity of marketable flesh: more particularly in regard to cattle of a large size, in the weight of which they are grenerally below the mark. There are tables also by Mr. Ainslie, but they are calculated in Dutch weight, and those by Mr. Stewart have been proved to be inaccurate *. As instances of their fallacy we may cite the measurement by live weicht of some bullocks exhibited at the Show of the Highland Society at Aherdeen, in 1534, with the dead weight which they actually produced of beef, and that which they ought to have yielded according to the tables, namely,-those by Mr. Buswcll of Kingcaussie:-

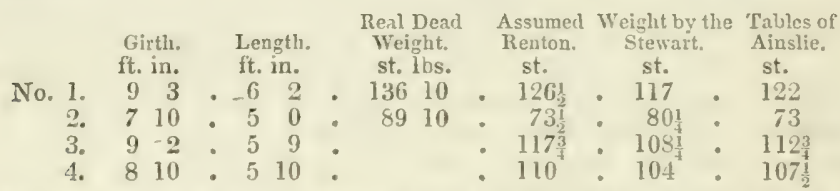

The two last were weighed together when slaughtered, and gave 257 st. 9 lbs,,-their average being 128 st. $11 \frac{1}{2}$ lbs.; which gives a diflerence by the highest of the tables, of 29 st. 11 lbs.; and by the lowest, of 45 st. $5 \mathrm{lbs}$. of $14 \mathrm{lbs}$. each; and taling Renton's tables as the highest upon the

square of the girth by the length, and this product by a decimal which may be assumed as nearly -238. for the live weirht: the deal weight is ascertained by multiplyin the live weight by the decimal $\cdot 6(1)$; thus, wing will gire the moduct of the four quaters.

Mr. Renton, however, states, in his "Grazier's Ready Reckoner," that for a half-fattened ox must be cieducted one stone for twenty from that of a fat ox; for a cow which has had calves one stone must also be deducted, and anothex if not properly fat.

Mr. M'Derment also proposes, in his "Cattle Measurer," that in the case of very fat animals, one-eirhteenth or one-1wentieth part should be added to the weight vituined by measurement; and when below the ordinary state of fatness, the same proportion sluvild te deducterl. Old milch cows, which have had a number of calves, shouid have one-ninth or one-tenth of their weight deducted.

Cary's Gauge is an instrument made in the form, and on the principle, of a slider rule, Giving the weights marked in stones of $\mathrm{S}$ lbs, and $14 \mathrm{lbs}$, is-

* See the Quart. Journ. of Agric., vol, ii. p. 885. Mr. Douglas' motle of calculating

By decimals square the girth into itself, and multiply the length into the squite of the girth : if the beast is fat, multiply by the decimal of 24 ; if only half fat, by 23 .

The fortgoing rule is very accurate if eattle are divided into classes, ind the multipiging decimal proportioned thus, if what is technically termed

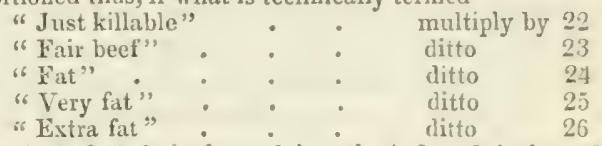

Suppose the girth 6 feet $I$ inch, and length 4 feet 9 inches, the square of the girth will be

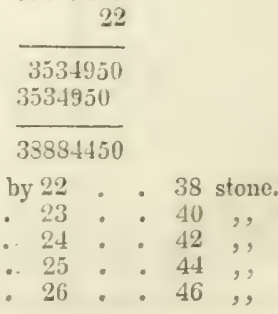


whole four, the difference, if calculatod by them, would be a loss to the farmer of 50 st., of $14 \mathrm{lbs}$, - or, accorling to the present price of prime beef at Smithfield Market, of not less than 21l. $4 s_{.} 8 \mathrm{~d}$. !

The ox belonging to Lord Kintore, of which a plate may be secn in Touatt's 'History of British Cattle,' which was a few years aro exhibited at the Smithfield Show, was also slaughtered at Aberdeen when upwards of seven years old-his four quarters giving 173 stone $4 \mathrm{lbs}$. upon a live weight of 2.24; though, according to the decimal rule by measurement, they should only have weighed 135 stone 8 lbs. Tet, in opposition to this, a sprayed heifer, killed at the same time, only gave 49 stone upon a live weight of 58 ; whereas she ought, if judged by the rule, to have given 53 stone 3 lbs.*

These facts are so striking as to prove that the accuracy of the tables camnot be in all cases relied on. Were the animals all of strictiy mathematical proportion, or did their offals bear an invariable ratio to the quantity of meat, they then, indeed, might afford a correct view of the marketable weight; but their bodies assume various forms according to breed and condition, the relative values of which cannot be ascertained by any artificial rules of measurement; or, if they could, they can yet afford no idea of the quality of the flesh. Ulence it has been justly observed, that "good julges contemn those rules and tables; for they can ascertain the real weight of marketable flesh much more nearly by the eye, and the true quality of it by the touch."

THE SALE

of fat cattle in the Lomlon weckly markets is most usually effecter upon commiscion, by men whose sole business it is, and who, from their linowledge of the huvers and their acquaintance with the state of the market, are more generally competent to obtain a fair price for the owner than were he himself to attond. The oxen are, therefure, commitiol to the care of clrovers, who regularly travel the road, where they have lairs at difierent stations for the nightly accommodation of the animals, and who are responsille for their safe delivery to the salesman, at a moderate charge. The farmer, therefore, is not under the necessity of superintending the journey of his lmasts, nor has lie any further trouble after they leave his stalls than the receipt of the cash for their parment, which is commonly remitted to him in the course of the same or following week.

The animal is of cuirse disposed of alive, according to his estimated weicht, and the price of fine cattle is liot uncommonly valucil by reducing the stone of $141 \mathrm{l}$ s. to 5 lis. dead weight. In coarse beasts, however, of large head and bone, it is still frequently adjusted by the old notion of the four quarter's constituting one-half of the live weight; and this, being the marketable amomnt of flesh-meat, is considered as the value to which the seller is entitled; for in the sale he only receives the amount of the funr quarters as the whole price of the entire carcase, and he, in the marliet plurase, "sinks the offal," which constitutes the "fifth quarter," or the buteher's profit. Supposing the four quarters to constitute one-half the

* Quart. Journ. of Agric., vol, v. p. 614, 615.-See also a statement of the live and deal weights, and propurtion of offal, of some oxen of different breeds, in the Complete Grazier, 6th ed., p. 107 ; the summary of which is-

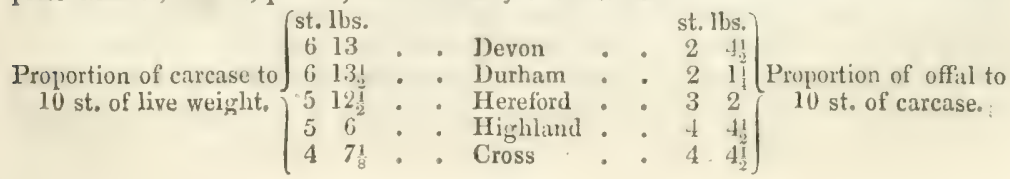


weight of the bullock-the hide is then gencrally considered to form an eighteenth, and the tallow a twelfth part of the wholc; the remainder of the oflal, consisting of the head and pluck, blood, entrails, and feet, are never sold by weight. The hide and tallow are, however, generally considered as equal in value, weight for wcight, to the fore-quarters of the finer sort of oxen, or to the full market price of inferior beasts, and alone yield a very ample profit to the butcher.

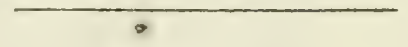

\section{Chapter XXXVi.}

\section{ON MILCH COWS.}

IT is well known to all landlords that no rents are so well paid as those of the dairy farms ; there cannot, therefore, be a doubt that they form a secure and profitable occupation, and, indeed, this must ever be the case while our consumption of cheese and butter exceeds the supply of the country so largely as it at present does. Thus it may be observed that the price of these articles has advanced much more in our markets during the last half century than any other agricultural produce, while it has also had the still superior advantage of being steadily maintained. They do not, indeed, offer the same chances for a sudden rise as corn, by which the arable farmer sometimes largely profits; but neither do they present those fluctuating depressions which often lead him to ruin. There is in fact nothing speculative in the business of a dairy ; for, if properly managed, it is sure to afford sufficient for all the common domestic comforts, and if industriously pursued, to be crowned with a moderate competence. It may, indeed, be conducted upon a moderate scale in conjunction with an arable farm, containing a very small portion of pasture, if the land be capable of growing artificial grasses and root crops; for the cows can be held in full milk upon the soiling system, and a farmer's wife and daughters may easily take the management of a dozen or twenty. We need hardiy say that it is a healthful and not laborious employment, and one that will add materially to the family income, without being considered by respectable females as at all menial.

The most essential points in the establishment of a dairy are :First, to have a good breed of cows.

Secondly, to possess proper buildings and implements ; and, Lastly, to be provided with an attentive and skilful dairymaid.

\section{THE BREED}

most in esteem with the London cow-keepers, who sell the milk without making butter or cheese, is of the old Yorkshire stock, or a cross between the Tceswater and the Holderness, as producing the greatest quantity; for they are in that case soiled in the house, and of course provided with an abundance of cut grass, brewer's grains, and succulent roots; but, when grazed, they require very good pasture, and are not generally considered to produce milk of a rich quality. They are also in request among those dairymen from their great disposition to fatten; which, together with the abundance of their food, renders them quickly ready for the butcher.

'The long-horns, however, are still in request throughlout Lancashire, Cheshire, and the adjoining districts, is producing a greater quantity of cheese. The Suffolk polled cows - the Devons, bred in some of the rich 
marshlands of Somerset and Gloucester,-as well as the Glamorgans, -are also considered good milkers in the neighbouring counties; but the breed which of all others appears to be gaining ground throughout the United Fingdom for abundant produce upon ordinary pasture, is the Ayrshire kyloe, which Mr. Aiton describes as "having no parallel under similar soil, climate, and relative circumstances, either for the dairy or in feeding for the shambles*." The Highland cow, however, yields finer milk; and it will be seen, in our account of the Dunlop cheese, that the milk of a cross between the Gloucester and the Alderney, with a Durham bull-thus producing a stock half Durham, onc-fourth Gloucester, and one-fourth Alderney,-produces with an equal quantity a greater amount of cheese.

The small Alderneys, indeed, are known to give a richer cream than any other, and to be contented with grass upon which a Torkshire cow would starve; but their yield of milk is only scanty, and their price being high, they are almost exclusively appropriated to the paddocks of gentlemen + .

The Kerry cows-a diminutive race, bred in the county from which they take their name in Ireland-also produce very rich butter, and would, if introduced into this country, rival those of Alderney; but injudicious crosses, with a view to improve the breed, have now rendered them scarce. The larger cows of each respective breed will, however, be generally found to have the advantage over the small ones.

Upon the form and qualifications of a perfect cow we again refer to IIr. Youatt; merely observing that, whatever breed may be selected, there is a wide difference between the form of one meant for fatting and that intended for the dairy. Thus, while the former should have as nearly as possible all the best points of the ox, the milch cow should on the contrary have a long thin head with a brisk but placid eye, be thin and hollow in the neck, narrow in the breast and point of the shoulder, and altogether light in the forequarter, but wide in the loins, with little dewlap, and neither too full-fleshed along the cline, nor showing in any part an indication to put on much fat. The udder should especially be large, round, and full, with the milk veins protruding, yet thim-skinned, but not hanging loose or tendingr very far belind. The teats should also stand square, all pointing out at equal distances, and of the same size; and although neither very large nor thick towards the udder, yet long and tapering to a point. A cow with a large head, a high backbone, a small udder and teats, and drawn up in the belly, will, beyond all doubt, be found a bad milker

Survey of Ayrshire, p. 421.

+ Mr. Malcoln mentions, in his "Cumpendium of Modern Fusbandry," that he kept an Alderney and a Suffolk cow-the latter the best of the kind he ever saw; while the Alderney, which had dropped her first calf, was jurchased out of a drove in a miserable condition. During seven years, - the milk and butter being always kept separate,-it wits fuund, year after year, that the value of the Alderney exceeded that of the Suffolk, thongh the latter gave more than double the yuantity of milk at each meal." IIe adds, "That he at that time had a dairy of twelve cows: two Devons, one Derby, one Lincoln, two Sussex, two Wiltshire long-horned, two Iolderness, one Suffolk, and une Alderney; and she bore the pralm clear away." - Vol. i. p. 350 .

Yet an instance is mentioned in the Suffulk Report of a polled cow, of a very small size, which, in the height of the season, gave the extraordinary quantity of four grallons of milk at each meal, twice a-day; and three quarts of it beinir compared with an equal quantity of milk drawn from a long-horned cow, originally of Bakewell's breed, gave $2_{3}^{1} \mathrm{oz}$. more creatn than the horned one : being, however, again skimmed, after twelve lours, that of the horned cow yielded $4 \mathrm{oz}$. more than that of the polled.-3rd edit. p. $20 \mathrm{~J}$. † See Youatt's "Cattle," p. 244; Wedge's "Cheshire," p. 25l; Lambert's "Rural Affairs of Ireland," P. 69; Aiton's "Treatise on Dairy Husbandry," p. 26; and "The Complete Grazier," sixth edition, pp, 43 and 114. 
Besides these qualifications, a great point to be considered is the temper; for kindly cows will not only give far less trouble in their management than those of an unmly disposition, but are commonly observed to have a more copious supply of milk, as well as to part with it more readily. It may not, indeel, be easy to ascertain the temper while making a purchase of the animal in the market; but even there an appearance of docility may be remarked in the expression of the countenance, and ought never to be noglected.

\section{STOCK.}

In stocking with cows, if a farmer has the means of breeding, he must be both negligent and injudicious if he does not provide himself with the best kind which can be properly maintained upon his land; and even if obliged to purchase, he ought still to select the best which it may be in his power to procure, without regard to the price; for if well chosen, they will more than repay any difference of cost between them and an inferior sort. Besides the nature of the land, he should also attend to the purposes for which the stock is designed-whether it be for breeding or for milking; as, if intended for the former, a handsome shave, accompanied by the least portion of offal and an apparent aptitude to fatten, are to be preferred; but if meant for the pail, an early disposition to gather flesh is one strong proof of an indifferent milker. Many dairymen, indeed, keep their cows until ten or twelve years old; and although they will have then fallen off in value, yet their produce, when of a good lim\}, makes ample amends. In many parts a breed of cattle may be found, as it were, provincially hardy, thriving, and well adapted to the soil : let him therefore furnish himself with the best sort of these which he can procure; but do it with wariness and discernment; and if his cows are well liept, and yichd him a good quantity of rich productive milk, it will be immaterial whether they have long horns, short horns, or no horns at all.

The heifers are grenerally put to the bull at about two years old, but many of them are in calf lomer before that time. The most proper season is from the commencement of May until the close of July; and the usual time of calving is in March or April, though some cows drop their calves soon after Christmas. Those, however, who keep a number of cows, put them at more distant periods in succession to the bull, in order to have as ncarly as possible a uniform supply of milk throughout the vear. The cows are in most cases allowed to run dry about ten wecks before their again calving, as it is thought to render them less profitable the ensuing suring, if kept to their milk too long; repeated experiments have, however, proved that six weeks is quite sulficient. They are seldom kept in the dairy after they have produced a third calf, as they are then in a better comdition for being fattened than when older; but they will yield a progeny until they are full twelve years old, and cows after a second calf yield better milk than with the first.

It is not, however, always that a grood stock can be obtained; for unless in the breeding listricts, where their qualities are well known, cows are not uncommonly brought from various counties, and served by bulls of every lreed, so that it is by no means unusual to see a dary composed of the most incongruous erosses, to which no distinctive character can be assigned. This, indeed, often sets anything like judgment at defiance; and a man who even does not look-as imany do-to the piching up of such stock as lie can find the cheapest, is sometimes obliged to put up with what he can get, though contrary to his better opinion. He should, howerer, make 
comparisons between the product of the cows which come into his possession, even when they are of the same breed; for those bred upon the same farm, got by the same bull, and of the same age and appearance, not unfrequently yield not only various quantities of milk, but afford butter of different weights from the same quantity*. Experiments are easily made by feeding the cows on the same food, and measuring its quantity, togrether with that of their milk; which, if kept and churned separately, the cost of the provender and the value of the butter will soon discover which is the most profitable. This, indeed, is rarely done; but its neglect is highly injudicious; for a bad cow will eat as much as a good one, and thus occasion a daily comparative loss.

\section{MANAGEMENT.}

The chief points to be regarded in the construction of a cow-house are, that it should be comfortably warm and very airy, but more especially that it be dry ; for although a certain degree of warmth is healthful, yet cows, if kept dry, are not very susceptible of cold, and fresh air should never be excluded. There is no other distinction necessary between it and ox-stalls, of which we have alrearly given so ample a descriptiont, except that it should lave calf-pens adjoining, and be kept with still greater cleanliness. So far, indeed, is this carried in IIolland, where the dairy forms so prominent an object in the husbandry of the country, that the wealthiest farmers generally have their common parlour opening by a glass-door into the cow-house, which is regularly swept and sanded to a state of the most extreme neatness; and the animals are not only wisped and curried like horses, but we have seen their tails tied up in pullies, in the same mamner as those of horses after they have been niclied, and this solely to prevent their soiling themselves.

As to the effect of food, notwithstanding all that has been said respecting the nutritive propertics of peculiar roots and artificial grasses, experience has very decidedly shown that no food is comparable to that of good natural pasture for milch-cows; for not only does it yield a greater quantity of milk, but, in fact, the flavour of mass butter may always be distingruished by its superior richness and delicacy from that which has been male from milk produced from soiling in the house; and its quality may

* Thus, according to a Report on Dairy INanagement, stated in the Transactions of the Irighland Suciety. it appears that some cows fed in the same manner produced double the quantity of butter from the same quantity of milk--N. S., vol, ii., p. 258.

$f$ See vol. $i_{\text {, }}$ chapters $v$, and viii. In addition to what we have stated on this subject at p. 202, we should say that Mr. Harley's cow-house, near Glasgow, is perhaps, in all its establishment, the most complete in the kingtom. It contains standings, unler one rouf, for ninety-six cows, placed in couble rows across the buidding, two rows facing each other, with a passage between them from which both are fell. The bottom of the stalls decline a little towards the centre, to lead into a common drain communicating with a reservoir for the urine and washings of the floors, which are carefully cleansed every day. The ventilation is so completely commander that it can be very nearly regulated at all seasons, and is kept as nearly as possible at about $60^{\circ}$ of Fahrenheit. Stalls for every two cows are divided from each other by pillars of cast iron, having grooves into which the division boards are fixed; but Mr. Aiton recommends a flag-stone of about $4 \frac{1}{2}$ feet high, and extending $6 \frac{1}{3}$ feet backwards from the wall, as a proper division between every pair of cows, with a slider or rod of iron of about twenty inches long fixed perpendicularly to each side of the stone. The cows are bound to these sliders with a slight iron chain round their necks, which moves up and down with a ring as they raise or lower their heads; thus affording equal security with more freelom than when they are secured in the custumary mode between two stakes; and, when fed with potatoes, if a pin be put through a hole in the slide, it prevents the coy from raising her head, and thereby incurring the risk of cloking herself, 
be injuriously affected even by the application of manure to the land. This, however, should not deter the farmer from feeding his dairy-stock in that manner, for the difference is not so great as not to be in very many cases more than counterbalanced by the other advantages of soiling.

The cows, though quiet in their stalls during the winter, yet evince a strong degree of restless anxiety to be at liberty when the season advances; and when pasture land is abundant, they should be turned out during the day as early as possible in the spring, and brought in at night to be fed upon sound meadow-hay, until the weather becomes warm, and the grass affords a fuil bite, when they should be allowed to lie out during the whole night, from the month of May until about the end of October. By some persons they are brought under cover in the middle of the day during the heat of summer; and this, if the ground be deprived of shade either by trees or hovels, may not be injudicious, provided it be near to the homestead, but, otherwise, it is a bad plan, for this driving of them from a distance is prejudicial to their milk. It is almost needless to say, that coarse grass, although perhaps producing an abundance of milk, will yet prove injurious to the quality of the butter; and as long, overgrown grass, even although naturally good, will impart a certain degree of rankness, it is always advisable to have the ground divided into different inclosures, and thus to move the animals from one to another every week or ten days, by which means they will have a constant succession of close, short, and fine herbage.

The after-grass is always favourable to the flush of milk; and, thercfore, the cows are usually turned into it as soon after the meadows have been mown as it will afford a full bite: but as it takes some time before it will be sufficiently grown, young clover, spring-tares, lucerne, and all the artificial grasses, are occasionally employed between that time and the cutting of the hay. These are each in their turn of essential service, producing it full flow of rich milk; though, with regard to tares, it is said by some dairywomen to occasion what they call "ropiness;" and red clover has, besides, been charged with causing hoving.

The cows are usually taken up into the yards, at the latest, about the middle of November, or as soon as the weather becomes bad. The winter house-food is, of course, made up with every kind of root which can be supplied, together with a certain portion of sound hay, or a moderate quantity of sweet oat straw*, upon which no other observation need be added to what we have already said of their respective properties, than that the quantity and the quality of the milk will assuredly be found proportionate to the nourishment and flavour of the fodder upon which the cows have been fed : thus, linseed, pea and oatmealt, will produce richess, while brewers' grains will occasion a profuse spring of milk, but it will be of the poorest kind-suitable, perhaps, to those milkmen who supply large towns, though not for the maliug of butter; and white turnips impart a very disagreeable taste, which is not equally remarkable with Swedes. It is generally imagined that the unpleasant flavour given to butter by common turnips may be corrected by the use of a small quantity of dissolved

* In the common course of practice every species of straw is, in fact, given; though that of barley is decidedly inferior, and, as well as that of wheat, is said to make cows run dry sooner than oat-straw; besides its being generally allowed that more than the usual time is required to churn the cream of cows when so fed.-Holland's Surv. of Cheshire, 1. 255.

f In Holland the cows, when fed in the house, have their drink of water invariably mixed with oil-cake, rye, or oatmeal. It is even constantly used for farm-horses; and there can le no doubt of its helping to maintain the mimals ia grod condition, - Radclift"s Flanders, pp. 53 and 259. 
saltpetre being mixed with the milk, or of a proportion equal to one-eighth of boiling water being added to it when left to stand for cream; but this we have found by experience has only very partially the effect attributed to it. Common salt, however, if given in moderate quantities to cows, increases the quantity, and improves the quality of the milk*.

The cows, when suffered to run dry, are by most farmers turned into the straw-yaid, and kept upon very inferior provender until shortly before calving. 'This, however, is highly improvident; for, although it is not necessary to keep them during that time in full flesh, yet, if allowed to fall off until they become lean, their milk will be found thin as well as deficient; and the injury to the future stock, if the calves be reared, or the consequent inferiority of the produce of the dairy, will more than equal any saving that can be male in fodder. Milch kine should, in fact, be at all times maintained not only in good condition, but in what may be termed a " milky habit ;" and, for this purpose, a quantity of Swedes, or any succulent roots, should always be given liberally along with sound wheat or oat straw, and this will be generally found sufficient, even without hay, until the opening of the pastures.

The cows are milked during the autumn and winter months as soon as possible after daylight, or in the summer, at about five o'clock in the morning, and again at four or five in the afternoon; thus leaving, as near as may be, twelve hours between each. Some persons, indeed, milk them during the latter scason three times within the twenty-four hours; but although the animals are then generally full of milk from their recent calving, as well as in consequence of the succulence of the grass, and it might therefore be thought a grood practice, as being likely to augment the quantity, besides easing the cow of her load; yet experience has shown the contrary; for the udder is sufficiently capacious to contain it without inconvenience, and the advantage supposed to be gained by the increase is not found to compensate the additional troublet. If milked more than twice a-day at one season they also soon acquire the habit, and look for it at another when it is both unnecessary and inconvenient.

If they be distant from the homestead, and the weather be finc, it is better to milk them abroal than to drive them to any considerable distance; for which reason it is extremely advisable to have some home yaddoclis close to the house, where they can be put under cover while milking; at which time it is also expedient to lay betore them some favourite food which they can relish with an apjetite ; for they will thus not only remain more quict, but they will also yield the milk with less reluctance. When stalled during the winter, they are in many cases turned into a bare pasture about ten in the morning, and housed again towards four in the afternoon, or as soon as they show a desire of being taken up; and this, in very cold or wet weather, is much better than suffering them to stand shivering in a field without shelter.

The act of milking is one that requires great caution; for if it be not carefully and properiy done, the quantity will be considerably dimimished, and the quality will also be inferior; as not only is the first of the milk the poorest, but it gradually becomes richer, until the last drainings of the udder, -or what is commonly termed the "afterings," "strippings," or "stroak-

* The quantity daily given to 40 cows and heifers on the late Mr. Curwen's farm in Cumberliud was collectively four ounces,-Sincliar's Agriculture, 3rd edit., App. p. 43.

f Lancashire Rep., p.517.

VOL. II. 
ings.*" It should therefore be thoroughly drawn from the cow, both to secure this latter portion and to ensure the continuance of the usual supply; for if any be allowed to remain in the udder, she yields a lesser quantity at the next milking: a fact which has been accounted for by supposing that the portion left in the udder is absurbed into the system, and that mature generates no more than to supply the waste of what has been tiken away. The greatest care should therefore be paid to have them clean milked. They shonld also be treated with great gentloness, and soothed by mild usage, especially when young and tichlish ; for thev never let their milk down pleasantly to a person whom they dread or dislike. If the paps are sore or tender, they ought to be fomented with warm water before milking, and, indeed, if the operation of milling be nicely performed, they should be each time clean washed; but this, we are sorry to say, is too often neglected.

'The choice of servants for performing the duties of the dairy should therefore never be intrusted but to persons in whose unremitting care and crentleness the utmost confidence may be reposed. All its operations should be also conducted with the most junctual regularity, and with such extreme cleanliness that no speck or taint shall be discoverat,le either about the interior of the house or the utensils. Throughout Ireland, Scotland, and the North of England, it is invariably left to women, and were men so employed, they would consider themselves degraded; but in our sonthern counties great hulking follows may be seen seated at the udder and handling the teats with their hure fists, as if they had the delicate fingers of a girl. Females are in every way competent to the work; to which they are better suited by their delicacy and tenderness than men: it is, indeed, a truly feminine employment, and to their hands it should be left.

Regarding quantily and quality of mill; there are few persons who

* The following facts respecting this circumstance, as ascertaincd by Dr. Anderson, prove that the loss of hall' a pint of this milk vecasions the loss of as much eream as wonld be affiorled by a far greater quantity of the first milkings, hesiles that portion of the cream which gives the greatest richness and flavour to the butter:-

"Having taken several large tea-cups, exactly of the same sizeand shape ; one of these was filled at the beginning of the milking, and the others at regular intervals till the last, which was filled with the dregs of the stroakings. 'These were each weighed, the weight of each cup being settled, so as to ascertain that the quantity of milk in each was precisely the same; and from a great number of trials frequently repeated, with many different cows, the result was thus:-

"The quantity of cream obtained from the first urawn cup was, in every case, much smaller than from that which was last drawn; and those hetween afforded less or more as they were nearer the beginning or the end. The quantity of cream obtained from the last drawn cup from some cows exceeded that from the first in the proportion of sixteen to one. In other cows, however, and in particular circumstances, the disproportion was not so great; but in no case did it fall short of the ratio of eight to one.

"The difference in the quality of the cream, however, obtained from these two cups was much greater than the difference in the quantity. In the first cup the cream was a thin, tough film, thinner, and perhaps whiter, than paper; in the last the cream was of a thick consistence, and of a richness of colour that no uther kind of cream was ever found to possess.

"The difference in the quality of the milk that remained after the cream was separated was, perhaps, still greater than either, in respect to the quantity or the quality of the cream. 'The milk in the first cup was a thin bluish liquid, like as if a very large proportion of water had been mixed with ordinary milk; that in the last cup was of a theck consistence and yellow colour, mure resemhling cream than milk, both in taste and appearance."-Bath Hapers, vol. v., art. vi., p. 73.

In the account of trials on the same subject, however, made by a dairyman in Lan. cashire, the result of the afterings, though more productive in butter thin when the whole milk was churned, was not more profitable, as the sale of the skim-milk was less.-Dicleson's Rep., p.556 
have not heard of Mr. Cramp's cow, which during four years-from 1805 to the end of 1805 -vielded the extraordinary amount of 23,559 quarts of milk, producing $2132 \mathrm{lbs}$. of butter, and various instances of nearly equal productiveness have been cited in many publications. 'The largest average product which has been stated by any writer in whose practical experience confidence can be placed, is, however, that of $\mathrm{Mr}$. Aiton, who rates the yearly averacre return of the best kyloes at 4000 quarts within 300 days, or until they run dry : thus-

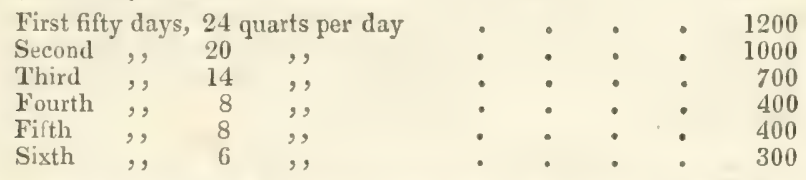

-And he cites an extensive Ayrshire dairyman, as saying__" That he would not keep a cow on his farm that did not yield her own value, or her weight in sweet-milk cheese, every year." He, however, admits-"That many cows will not yield more than half that quantity; and that, probably, 600 gallons in the course of the year may be about a fair average of the Ayrshire stock *:" if equalled, we belicve it will not be found exceeded by any other breed in the kingdom. The average quantity of milli yielded by dairycows is stated by some of the County Agricultural Surveys to be-

$$
\left.\begin{array}{lrr}
\text { In Devonshire } & - & 12 \\
\text { Cheshire } & 8 \\
\text { Lancashire } & 8 & \text { to } 9
\end{array}\right\} \text { quarts per day. }
$$

Guod cows of the common description may, indeed, be generally supposed to vield from about eight to twelve quarts on the average in the course of the day + ; though, unquestionably, some prime animals, in full milk, and in the height of the grass season, will afford much larger quantities. Thus, in some trials made at Bradlev Hall, a seat of the liarl of Chesterfield, in Derbyshire, it was found that, during the height of the season, the milk and butter produced per day, by different cows, was-

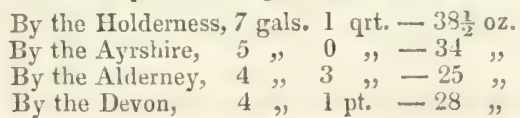

but this only lasts for a short time, and such extraordinary supplies soon fall off: in fact, the nature of the land, the oldness of the pasture, the agge of the stock, and the state of the season, have each a separate influ-

* Survey of Ayrshire, p. 464; and Treatise on Dairy Husbandry, p. 45.

+ Five short-horned cows, of the ordinary quality of that breed, are stated to have given, in one year, as follows-

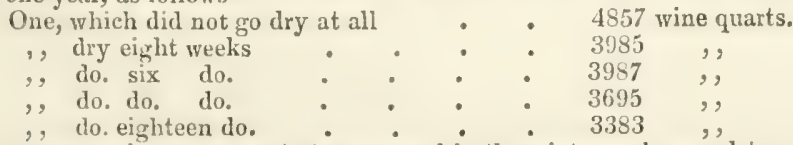

These cows were in summer out at grass, and in the winter on hay and turnips, with two months on hay alone.

Others, chiefly of the short-horned breed, produced, on an average of the whole year, $9 \frac{3}{4}$ quarts per cow per day; and three small Scots, with an equal number of the long and short-horned breeds, gave an average of eight quarts: that is-supposing the cows to have been dry about forty days in the year, which thus adds one-ninth to the real quantity produced when in milk.-Dickson's Surv, of Lancashire, p. 547.

A large dairy of long-horns and short-horns at the late Mr. Curwen's farm of Workington Hall gave, upon an average of four years, about 3700 yuarts each. - Farm. Mag., vol. xiv., p. 23. 
ence; thus the milk given by cows in the autumn and winter is evidently richer than that produced by them in spring and summer, and yields the greatest quantity of butter in those months, with the least cheese; and, therefore, no calculation of a general average can be made with such accuracy as to merit confidence. The same remark, we may observe, will apply with even greater force to the produce of butter and cheese; for, besides these circumstances, we have already seen that cows of even the same breed yield a different amount from equal quantities of mill: Generally speaking, however, a fair anmual product of either from each cow in good condition mav be considered as about 160 to $150 \mathrm{lbs}$. of butter of superior quality, and 350 to 400 lbs. of whole milk checse, with a small quantity of whey butter.

\section{Chapter XXXViI.}

\section{ON TIIE DAIRY-MIAKING OF BUTTER AND CHEESE.}

ON proper attention to the construction of the DAIRY-IOUSE materially depends the perfect manufacture of cheese and butter, and nothing shoulil be spared in rendering it as complete in accommodation for the different operations as the mature and size of the farm will admit. We allude not to the elegance of many gentlemen's dairies, nor to some few of those fitted up at great expense for a large business, but to those upon a moderate scale, and in every instance where the object is not confined to the mere consumption of the family. It is, indeed, the more necessary to remark upon their deficiencies, and give some hints towards remedying them, as many of then consist of nothing better than an out-shed attached to the litchen; and very few are erected with a proper degree of judgment. As it is in most cases very difficult to contrive the proper conveniences within the dwelling-house, we shall describe a separate building upon a plan which will answer every prudent purpose, and may be erected at very moderite cost.

The apartments which are peculiarly appropriate to the dairy-husbandry are, one for milk; another for butter in churning, or for scalding, pressing, and salting cheese; and a third for the implements: over which, in cheese dairies, a store-room may be placed under the roof.

The building, though placed conveniently to the house, yet should be apart from any immediate contact with the oclour of the farm-yard, or other impurity, as well as from any pond of stagnant water, as notling more readily acquires an umpleasant taste or smell than milk and cream. An uniform temperature heing also of extreme importance, the site of the structure shouk be such as to be as little as possible afiected by the extremes of either heat or cold. The most experienced dairy-women disagree respecting the degree of temperature most suitable to the production of cream and the making of butter; but they all admit that the house camnot be rendered too cool during the summer, and in winter it is easy to keep up a sufficient warmth. Hence the main aspect should be open to the north and east, and the building should, if possible, be shaded, either by other walls or by high trees, from the south and west. The roof should be of a ligh conical form, or what builders call a "span roof," rising from the centre, and projecting downwards brondly over the sides, to shade the body of the house, which should consist of a narrow range of rooms upon the following plan. 


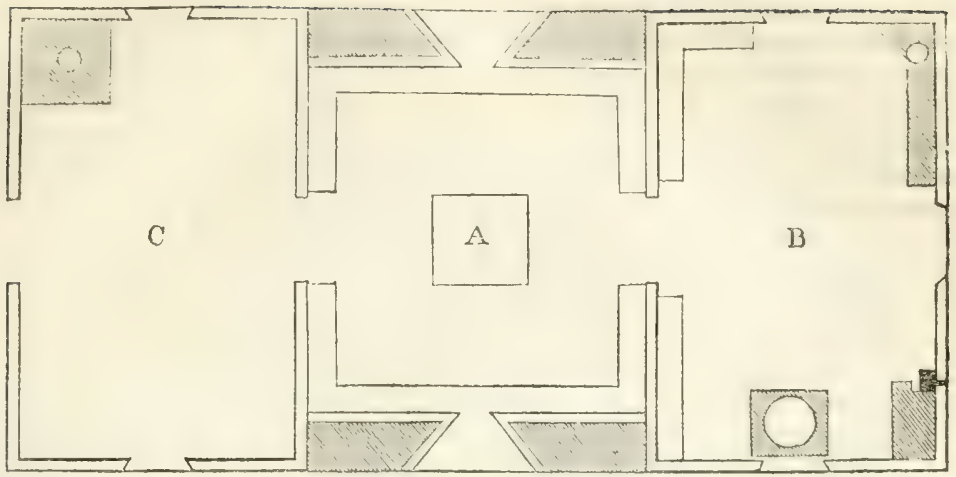

Of these, the middle apartment, in which the milk is preserved, is the most important; and therefore, in order to secure as equal a temperature as possible at all seasons of the year, by excluding all direct communication with the external air, the outer walls-as marked black in the plan-should be made of sod and earth rammed firm to the breadth of full four feet in thickness, while the other walls of the building need only be constructed of a single brick, or even with lath and plaster, boarded on the outside; nor is it necessary that they should be more than seven or eight feet high at the sides. A funnel should also be run through the centre of the roof to a couple of feet above it to act as a ventilator ; a valve being fitted to it, which, by means of a pulley, can be shut or opened at pleasure. The arrangement of these rooms will therefore be thus :-

A, the milk-house, with broad shelves all around, for holding the vessels which contain the milk and cream; and in the middle is a table for preparing the butter for market. 'The windows are closed with lattices cuvered with gauze wire to prevent the entry of flies, and double shutter's of wood to guard against cold in winter; for if glazed, they are subject to duty.

$\mathrm{B}$, the churning-house, with a boiler in one corner, and on the sides frames for cheese-presses and vats, with large vessels of lead, or slate, for holding the whey, and pipes for conveying it to a cistern outside for the use of the pigs*.

$\mathrm{C}$, the wash-house for the cleaning and care of the utensils; it therefore contains a furnace with a cauldron for scalding the vessels, and a pump communicating with a well. The outer door or entrance is here, and adjoining it are placed stands under the verandah formed by the projection of the roof, for exposing all the wooden implements which may have been washed to be dried and sweetened by the sun and air.

In cheese dairies the store-loft may be placed immerliately below the rise of the conical roof, a communication being made by a staircase in a comer of the wash-house; but many farmers prefer having the loft over the cowhouse, with the intention of forwarding the maturity of the cheese by its warmth. Some large dairies, however, have roofs in the common form, with lofts and sleeping rooms for the dairy-maids over them; and one which we have seen of a moderate size has the milk-house sunk about three feet

* Leaden vessels are by many objected to, in consequence of the bad effects which they oceasion when any suur liquid is allowed to remain in them; but the whey keeps longer siveet in them than in wooden vessels, and they are much easier kept clean. Slate, is, however, more easily cleansed, and is far cheaper. 
below the ground, with very thick brick and limestone rubble walls, standing ten feet high; thus admitting of a cheese-loft over it. The building is also constructed differently from that we have already described,- the milkhouse having three fronts, encircled by a verandah ; thus affording a strong draught of air from the windows, with only one door, while the other rooms are of timber rising up to the roof, and the wash-house forms the only mode of communication between both. The plan, as at foot,

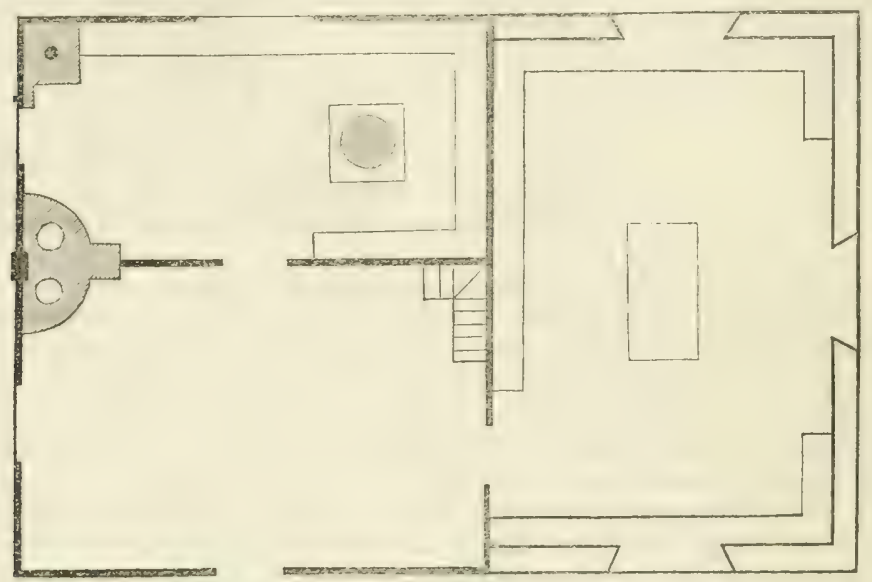

has, indeed, the advantage of having the milk-house entirely separate from the chuming and scalding room, as well as of its being, perhaps, somewhat cooler in summer; but what it gains in the latter respect it loses in the constant equality of temperature, and its construction is more expensive than the other.

With respect to the construction, we have not yet alluded to the matcrials of the roof and flooring; both which are essential in point of temperature and cleanliness. The roofing with tiles is, in every instance, either so hot in summer or cold in winter as to be destructive to every thing like cquality in the former; and therefore the roof most generally cmployed is formed of either straw, ferm, or rushes. These unquestionably answer the purpose; but then they should be made full three feet thick : thus occasioning a great expenditure of materials, which are subject to early decay, besides harbouring vermin, and in the course of time creating a musty smell within the interior, which communicates itself to the milk, and consequently becumes injurious to the butter. The roofing with slates of sufficient thickness is therefore much to be preferred, as being more impervious to the weather, as well as decidedly more durable; but it has been seldom used by farmers out of the slate districts, in consequence of the difficulty of working it, and the rate of duty. This has been now removed; and the facility afforded by sawing machinery lias rendered it much cheaper in the long run than any other material which can be employed. An improvement in floors and roofing, well adapted for dairies, has also been lately made by Messrs. North and Co. of Stangate Wharf, Lambeth, and Shadwell Dock Basin, London Docks, whereby three-fourths of the timber generally used in the old method is saved, and the slates form a neat ceiling *.

* The roof may be either nearly flat or much sloped, according to the plan required. The cost of this description of roofing averages $5 l$, per 100 feet superficial, including the 
The floors should be raised a few inches above the level of the outer ground, with slanting gutters to carry off the water used in washing, which is frequently done both for the sake of cleanliness and coolness. On this, however, it should be observed-that although every particle of milk that happens to be spilled upon the floor should be carefully washed off, or its sourness will soon impart an unpleasant odour which will infect the entire of the house, yet it is also extremely material that the building should be kept as dry as possible, as damp is also highly prejudicial to the operations of the dairy. 'The floors of most dairies are now paved with either brick or tile, neither of which can be recommended; for brick is rarely laid so level or so closely cemented as not to admit of cramies in which the spilled mik remains without a possibility of effectually removing its unpleasant odour; and tiles, unless they be glazed, also absorb a great proportion of any moisture to which they are exposed. Slates, however, besides being more imperishable, have been found, on comparative experiments, to imbibe only the two hundredth part of their weight, while tiles absorb one seventh; and slates, when afterwards exposed to $60^{\circ}$ of heat, became dry in a quarter of an hour, while tiles retained some portion of damp durine six days !

These advantages are so important, and render the superiority of slates so manfest in the construction of a dairy, that we strongly recommend them in the erection of all new buildings of that kind; and, indeed, considering the various other purposes for which they may be employed in farming matters, we think great merit is due to Messis. North and Co. for their persevering exertions in manufacturing articles of a material till lately but little used in any other way than that of roofing.

wood work, the finishing to the hips and ridge, and fixed complete; at which calcula tion, a roof 30 feet by 24 feet contains 7:20 feet superficial and costs £36 00

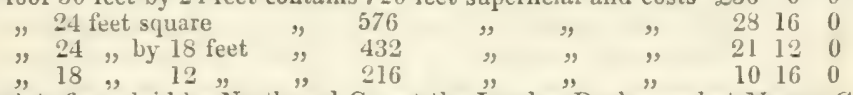

The slate floors laid by North and Co, at the London Docks, and at Mrs-rs. Currie's Granary, Bromley, Middlesex, are highly recommended by the warehousektepers, and are proved by experiments of Mr. George Remnie, the engineer, to be five times as strong as stone. 'They are well adapted for dairies, and are sold in plates of about 3 feet square, sawed ready for laying, at $5 d$. per foot superficial, for $\frac{1}{2}$ inch thick; $7 \frac{1}{2} d$. for $\frac{3}{4}$ inch; and $10 \%$. for one inch thick.

The slates are laid in a bed of mortar upon the ground; but in situations where the ground is loose and gives way, a bottom of concrete, of at least four inches thick, must be laid, upon which the slates are laid in a bed of mortar.

The concrete consists of seven measures of gravel and one measure of pounded quick stone lime, mixed with water at the time of using it to the consistency of stiff mortar. Thames gravel is always used about London; but any gravel that is free from clay or loam will answer the purpose.

If slate colour be oljected to, the slistes may be rubbed with coarse grit sand to a porous face and painted with thin flatting paint, or the floor may be whitenel with a ball made of dry white lear, mixed with turpentine, in the same manner as hearth-stone is used for whitening hearths and steps.

For dairy shelves, slate is certainly the best material in use. Fishmongers find that it preserves fish twenty-four hours longer than marble. The proper thickness for shelves is one inch, which costs $1 s .2 d$. per foot superficial, without fixiner; and if polished, $1 s .6 d$. per fout. The brackets or cantilevers may also be slate, having one end let into the wall, and the other end moulded. The size of them should be four inches deep, and two inches thick, fixed edgeways.

A dairy may be built very economically with timber quartering. cased outsile with plates of half-inch slate rubbed to a porous face, and painted stone colour, the cavities between the quarters being filled up solid with concrete, or lime and hair-mortar, with rubble of bricks or stone, and trowelled smooth inside. The lime would preserve the timber from decayiug.

* See note vol.i., p. 88. 
IMPLEMENTS.

The utensils of the dairy comprise millk-pails, shallow pans, or cooling dishes for holding, and sieves for straining the milk when taken from the cow; with dishes for skimming the cream, and churns for the making of butter, besides scales, prints, and boards, for weighing, measuring, and ornamenting it. There are also ladders, vats, tubs, curd-breakers, and presses, for the manufacture of cheese, together with vessels large enough to hold the whey or butter-milk: all of which-with the exception of some variations in the construction of the churn and press-are so nearly similar throughout every part of this country and Europe, and so familiar to every dairy-maid, as hardly to require description. The material of which they are most generally formed is wood, as objections are made to vessels of glazed crockery and lead, though many dishes are made of earthenware, and some of the coolers and whey-tubs are of lead, which has been uscd for agres without any ill effect; they are also not uncommonly found of iron timned, or of freestone, as well as of slate, which is now fast coming into use. In IIolland, indeed, the pails are not unfrequently made of brass; but here they are almost invariably of wood, and maple is preferred, both for its lightness and the neatness of its appearance. The ladders and creamdishes, together with the prints and butter-boards, are also of maple; but the vat in which the curd is set, and the "chessarts," or vats into which the cheeses are pressed, are formed of every kind of wood, conformable to the weight of the cheese, and generally made by the cooper, though some are cut out of the solid, and formed of elm, and others are made of cast-iron. The sieve has usually a hair-cloth bottom, though in some cases it is of fine silver wire, and is about twelve or iffteen inches in diameter.

The checse-presses act upon the curd by pressure, and are usually made of stone of different weights proportioned to the size of the cheese, and, therefore, varying from $5 \mathrm{cwt}$. to 1 ton. These are most generally raised by a block and tackle; but some of them are upon the principles of the lever, and there are various constructions placed in frames of wood. One lately invented, and made by the Shotts Foundry, is entirely of iron, of a simple construction, the pressure upon the curd being made by a screw turned by a winch, by which means the power can be regulated more correctly than by a fixed weight:. A very common machine, of an extremely simple form, used in many dairies which produce such small cheeses as not to require crrcat pressure, is also that of a moveable beam, fixcd by a jivot in an upright post, and having hooked on at the other end a weight which presses in this manncr on the cheese-vats underneath.

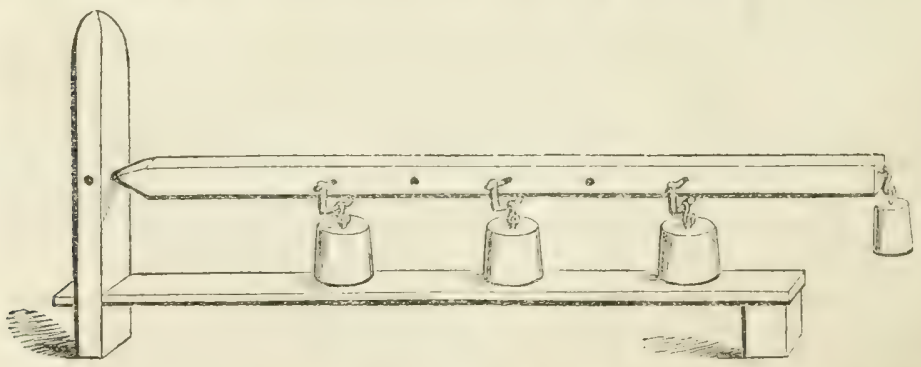

* For a plate and description of this press, see the Trans. of the Highland Soc. N.S., vol, iv, p. 5\%. 
The churns are closed vessels, into which the cream, or the whole milk, being put, a piston, or a wheel in the form of a fan, is quickly and regularly moved, either up and down, or by furning, according to its form, so as to separate the butyraccous, or oily particles of which the butter is composed. They are generally made of the best oat, and of various sizes, from ten to fifty, or a hundred gallons, when wrought by the hand, and even to double that size in those large dairies in which the entire of the milk is churned, and in which it is worked by the aid of a small horse-gin, similar to that which moves the threshing mill*.

That ancient implement called the plunge-churn-as described in No. ], -which acts by means of a long handle furnished at the inner end with a circular flat bottom, which is perforated, and worked up and down by the dairymaid, is still the one most commonly in use, as acting more efliciently than any other, though it is so tedious and laborious in its operation that in many large dairies the barrel-churn-as depicted in No. 2,-has been atopted, in consequence of the superior ease with which it can be worked. The axle by which the wheel-fans are moved passes horizontally through the barrel, and is made to revolve by the action of a toothed wheel at one cmil, which is worked by another large fly-whecl turned by the handle fixed on one of its spokes. On this, however, an jmprovement has been made by working it vertically br means of a small cylinder on the upper part of the outsid: of the firane, romd which a cord is wound which passes through two holes, and are fastened to the lower part of the frame, as a treadleboard,-in the manner delineated at No. 3. 'The common barrel-churn can be turned by any one; but this fixed churn is generally worked by a man, who stands with each foot upon the treadle-board, and by altemately throwing his weight on each flap, he draws down the cord on each side, and thus causes the asle and fans to turn backwards and forwards in the churn : it is a simple contrivance, but is seldom used $\uparrow$.

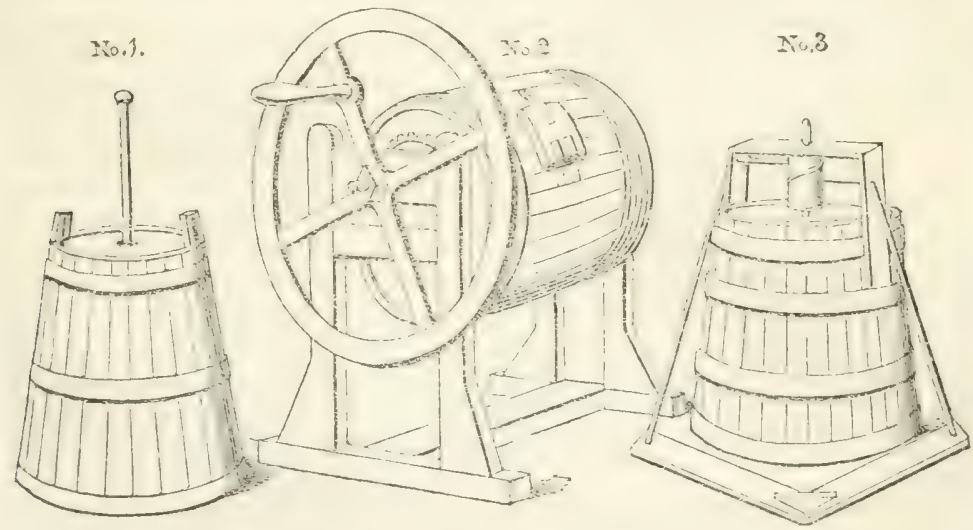

* For a ylate and description of one of these, sce Low's Elements of Practical Agriculture, p. 526.

+ Plates of jir. Bowler's chum, which obtained a promium from the Soc. for the Fic. of Ats; and also of a very effective machine which was invented in Phladelyhia,

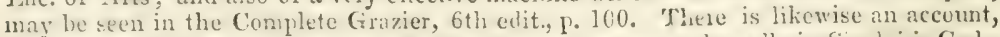
with a plate of a cradle-churn, which is said to act uncommonly well, in sinclair's Code of IIusbandry, 3rd edito, f. 134; and we learn that an ingenious plan has been adopted in Canatia, of making a chum in the manner of a rocking-hurse, unon which a child is put astride, and thus makes the butter while amusing himself. 
The form of these utensils is, however, a matter of sccondary consideration, in comparison with that of their extreme cleanliness, which is the chief requisite in all the operations of the dairy; and, therefore, those vessels which can be the most easily cleansed are the best to be cmployel, whatever may be their shape and material. In order to effect this, not only should the dairy-maid be a careful, cleanly person, but the floor of the dairy should be kept perfectly dry; for water thrown down in hot weather will assuredly rise again in steam, and affect the mills with its humidity. The vessels used for holding the milk, the churn, and all the dairy utensils, after being first washed thoroughly clean, should then be rinsed a first and second time with sweet milk: "s a cruet, washed ever so clean with water, wi!l cause vinegar to become dregrgy ; but if rinsed with a little of the same, will always appear limpid and clear."

The main point is, indeed, the superintendence of the dairy; for unless that care be confided to the mistress of the family, and that she be in every respect competent to conduct it with judgment, regularity, and that persevering industry which is actuated by motives of self-interest, it will be only. rarely found to afford any material profit. The making of butter and of cheese are also essentially diferent; for although every dairgwoman onght to be well acquainted with the former, yet, excepting in the United Kingdom and in Ilolland, no two districts in any other part of Europe manufacture cheese of similar flavour. This extraordinary difference has been attributed to the nature of the pasture on which the cows are fed; and this, doubtless, must have some effect, for experiments have been marle by experienced persons brought from places where they have regularly lived, and well acquainted with the mode of making the peculiar quality there known, and yet when moved to another spot they have not succeeded.

\section{MILK.}

When the milk has been drawn from the cow it should be carricd as gently as possible to the dairy, and after being there strained through the sieve, it must be then depositerl in shallow pans or coolers, not exceding three or four inches in depth, where it is lett to collect the cream, which rises to the surface within a few hours, according to the temperature of the air. Those who are particularly nice, either in the comsumption of the raw cream, or for the maling of butter, skim it, perlaps, within twelve hours: but it is more generally left full twenty-four or even thirty-six hours, according to the state of the season, when intended for butter, and is then not unfiequently skimmed again. A small quantity of water-cold in summer, and warm in winter-if put into the bottom of the pan, will be foumd to assist in maling the cream rise, as it equalizes the heat to somewhat approaching the temperature of the milk-house, which it is generally thought should be always kept as nearly as possible at about 55 degrees of Fahrenheit; but it is doubtful whether the misture may not prejudice its quality.

The chief component parts of milk are those which, when sejarated, are known as forming butter and cheese; the residue of which is called whey*.

* These are distinguished by scientific persons as the hutyraceous or vily substance producing cream, of which butter is composed; the caseous matter, of which cheese is formed; and serum, or whey:-



This, however, can only convey a general idea of the component parts; fur they must necessarily vary according to the quality of the milk. 
Mathematical instruments have been invented, as lactometers, for ascertaining the richness of milk, in mearly the same mamer as that employed for trying the strength of spirits; and an ingenious method has been published by a Mrs. Luvi for attaining the same ohject. The diflerence in the quality of milk between particular cows may thus be determined; but it does not show whether the caseous or the butyraceous matter predominates, and we camnot learn that either morle is much used in our dairies.

The following observations, drawn from the experiments of 1)r. Anderson, may be assumed as a summary of its management:-

1. "Of the milk that is drawn from any cow at one time, that which comes off at the first is always thinner, and of a worse quality than that which comes afterwards : the richmess going on continualily increasing to the very last drop that can be drawn from the uhlier at that time."

2. "If milk be put in a dish and allowed to stand till it throws up cream, that portion of cream which rises first to the surface is richer in quality and greater in quantity than what rises in a secomil equal portion of time; the cream that rises in the second interval of time is greater in quantity and richer in quality than that which rises in a third equal space of time; and that of the third than the fourth, and so on: the cream that rises decreasing in quantity, and declining continually in quality, so long as any rises to the surface."

3. "Thick milk always throws up a smaller proportion of the cream it actually contains to the surface than milk which is thinner; but that cream is of a richer quality. If water be added to that thick milk, it will also afford a considerably greater quantity of cream than it would have done if allowed to remain pure; but its quality is at the same time greatly debased."

4. "Milk which is put into a bucket or other proper vessel, and carried to any considerable distance, so ats to be much agitated and in part

The analysis of the constituents of skimmed cows' milk is stated by chemists to be-

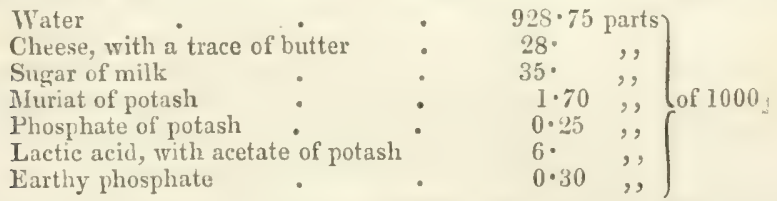

* She places beads into the milk as drawn from the cow, aur put into a glass vessel at the heat of 60 degrees. The same operation is then repeated on the skimmed milk, after the cream has been remover, and the difference in the specific gravity of the milk in these separate states shows its quality: the full milk, containing the cream, being lighter than that which has been skimmed. The beads are numbered, and are singly thrown in until one sinks to the bottom and another swims; the intervening number between them expressing the specific gravity: thus, as the cream is the lightest ingredient, the diversity of the weight of the milk affords a sure indication of the relative quantities of oily matter or butter.

The proportions of cheesy matter can only be ascertained by curdling the skimmed milk, and trying the whey, in the same manner, alung with the skimmed milk; the whey being the lighter of the two: thus, if the skimmed milk stand at 36, and the whey in once instance at 32 , and in another at 34 , it may be inferred that the first will be more productive than the latter.

It has been fund from experiment that the beads applicable to some different kinds of milk, show, according to their several numbers, the following results, namely :-Cows, from 24 to 34 ; but when fed on grass, before being creamed, 32 ; and after the cream was taken off; 40 ;-Ewes, before being creamed, 36 ; after the cream was taken off; 48 ; Goats, 31 ; Asses, 34; Mares, 36 . The two latter, however, though cousidered highly nutritive, afford scarcely any cream. 
cold before it is put into the milk pans to settle for cream, never throws up so much nor so rich cream, as if the same milk had been put into the pans directly after it was milked."

5. "If it be intended to make butter of a very superior quality, it will be in such case advisable to separate the milk that is first drawn from that which comes last; and the quality will be improved in proportion to the smallness of the last drawn milk that is obtained. The first skimmed cream should also be used, as it is always richer than that which rises last."

\section{BUTTER}

is formed either by cream alone, or with the whole mills, unskimmed; but although such different moles of manufacture might seem to warrant very different results, yet they have very little perceptible effect on the quantity or quality, thougrh the profit on the produce of the dairy may be affected in large towns by the greater demand for skim-milk or butter-milk. There is also another kind which is much inferior, and made from the cream of whey after the cheese has been taken from the milk; but the process of makingr is nearly the same.

When butter is to be made from cream alone, the milk is in winter usually skimmed as often as four, and in summer two or three times, or until it will afford no more cream; and this should be first separated from the edges of the pan, to which it fitmly adheres, by means of an ivory or silver-bladed knife, run closely round it. The cream should then be carefully drawn to one side and lifted off with a skimming dish, which is generally pierced with small holes; an act which requires some dexterity, both to avoid the leaving of any cream behind, and to prevent any portion of the milk being mixed with it. Some persons, indeed, have leaden coolers with a plung in the bottom which allows the milk to escape to a large vessel underneath, while it leaves the cream at top; but the former practice is the most usual. The length of time which the milk should stand before it is skimmed, must depend both upon the temperature of the air at the time and the views of the dairyman. In moderately warm weather, if very fine butter be intended, it should not be suffered to remain more than six or eight hours; for ordinary good butter it may, however, be safely allowed to stand full twelve hours, and during cold weather much longer.

The cream is then put into a deep ressel ${ }^{*}$, in which it is frequently stirred every day with a wooden spoon, in order to prevent it from coagulating, until sufficient be collected to form a churning. Many people imagine that no butter can be of good quality except that which is made from fresh cream : this, however, is a mistake; and is so far from being well founded, that the formation of butter only takes place after the cream has attained a certain degree of aciuity, and no butter of even tolerable quality can be obtained from cream that is not more than one day old.

The length of time which the cream should be kept before it acquires that degree of acidity which is requisite for the best butter, has not been ascertained by any series of experiments on which reliance can be placed; and

* "No vessel can be better adapted for this purpose than one in the under part of which, close to the bottom, there is a cock and spiggot for drawing off, from time to time, any thin, serous part of the milk that may chance to be there generated; for should this be allowed to remain, it acts upon the cream in a powerful manner, and greatly diminishes the richness and quality of the butter, The inside of the vessel should be covered with a bit of close fine silver-wire gauze, to keep back the cream while the serum is allowed to pass." - Bath Papers, vol, vo, p. 101. 
must, indeed, depend so much upon the weather, that no fixed rule regarding it could ever be strictly followed. In fact, "so little nicety is observed in this respect by practical farmers, even those who have a high reputation for making good butter, that few of them ever think of observing any precise rule with regard to the dillerent portions of their cream, seeing they in general make into butter all they have collected since the former churning; the time which should intervene between one churning and another being usually determined by local or accidental circumstances." Dr. Anderson, who makes this remark, is, however, of opinion, "that if the cream be carefully liept, and no serous matter allowed to lodge about it, a very great latitude may be safely admitted in this respect. It is, indeed, certain, that crean which lias been kept three or four days in summer is in excellent condition for being made into butter ; and that from three days to seven may be found in general to be the best time for keeping cream before chuming *." The cream from every milking should, however, be kept aprart until it is become sour, and not be mixed up witl sweet cream; at least not until the moment of chuming ; for the mixture cecasions fermentation, which, though partly prevented by the stirring, is liable to render the cream putrid. When, however, the herbage is coarse, or the cows are fed on roots, or artificial grasses, the sooner the cream is chumed the better will be the buttert."

In Cheshire they allow the milk to stand until the cream becomes clotted, or, as they term it, "carved," to a proper degree of acidity, which generally takes place, in warm weather, within a day or two; and in winter it is placed near the fire, in order to forward that process. In Mid-Lothian the "Corstorphine Cream" is obtained by putting the milk into vessels immersed in warm water, which occasions the whole of the creamy substance to rise. The whey is then drawn off by a hole in the lower part of the ressel, and what remains is for a short time agitated in the plunge churn. The mode of procuring the clotted or "clouted cream," so celebrated throughout our Western Counties, which the dairy-maids there say produces one-fouth more cream than by the common way, is described in the note at foot + .

* "On the Making and Curing of Butter."-Bath Papers, vol, v., art. vi.

+ "Complete Grazier," sixth edition, pp. 136 and 138.

It is simply thus: "The milk while warm from the cow is strained into either large shallow brass pans, well tinned, or earthen ones, holding from two to five gallons, in which should be a small quantity of cold water. This is thought to prevent the milk from burning, and to cause the cream to be more completely separated and thrown to the top.

"The morning meal of milk stunds till about the middle of the day; the evening; meal until the next morning. The pans are now steadily carried to, and placed over a clear slow fire; if of charcoal, or over a stove, the cream is not so apt to get an earthy or smoky taste as when the milk is scalded over a turf or wood fire. The heat should be so managed as not to suffer the milk to boil, or, as they provincially term it, 'to heave;' as that would injure the cream. The criterion of its being sufficiently scalded is a very nice point; the earthen pan, having its bottom much smaller than the top, allows this point to be more easily ascertained; because when the milk is sufficiently scalded, the pan throws up the form of its bottom on the surface of the cream.

"The brass pan, if almost as big at the bottom as at the top, gives no criterion to judge by, but the appearance and texture of the surface of the crean, the wrinkles upon which become smaller and the texture somewhat leathery. In summer, it must be observed, the process of scalding ought to be quicker than in the winter, as in very hot weather, if the milk should be kept over too slow a fire, it would be apt to run or curdle.

"This process being finished, the pans are carefully returned to the dairy; and should it be the summer season, they are placed in the coolest situation; if on stone floors or slate benches, the better; but should it be the winter season, the heat should rather be retained, by putting a slight covering over the pans, as cooling too suddenly causes the cream to be thin, and consequently jeld less butter: the mode of making which is this: 
In the common way of making butter from cream, the dairies churn the whole quantity at once; but in the neighbourhood of Epping and the surrounding districts, which have been so long celebrated for the character of their butter, only the first skimmings are used for that of very fine quality. 'The mode there pursued is as follows:-The milk, after standing twenty. four hours in large shallow coolers, lined with lead, is then skimmed, or, as they term it, "flected." 'The skimmed milk is then drawn off from the leads into other vessels of increased depth, but unlined, in which it is left from twelve to twenty-four hours, during which time it is fleeted two or three times : this is called "doubling." It is then "threbled," or put into tubs, or still decper vessels, where it is occasionally skimmed so long as any appearance of cream is found to form upon the surface; after whic h it is griven to the pigs. The butter which is made from the after-fleetings is of a paler colour and of an inferior quality to that male from the cream cast up within the first rising ; it is therefore generally chumed apart, or if any portion of it be mixed with the first fleeting, the butter suffers proportionally in price**

If butter be made from the whole milk, the process in the best dairies around Glasgow, in which the consumption of butter-milk is very great, is thus described by Mr. Aiton:-

"The milk, when drann from the cow, is placed in the coolers on the floor of a clean, cool, and well-aired milk-house, from twelve to twenty-four hours, till it has cooled to the temperature of the milk-house, and the cream has risen to the surface. These coolers are next emptied, while the milk is yet free from acidity, into a clean, well-scalded vat, of size to contain the whole milking, or two millings if both are sufliciently cooled, where it remains till churned. If another milking, or meal of milk, be ready before thit which has begun to become sour, that second meal may be put into the same vat; but if the first has soured, or is approaching to acility, before the second quantity has completely cooled, any further admixture would lead to fermentation, and injure the milk. It is necessary that the whole milk become sour before it be churned; but the whole of it must become so of its own accord, and by no means forced into acidity by any mixture of sour milk with that which is sweet. 'The utmost care should, however, be taken not to allow the coagulum, or curd, of the milk in the stand-rat to be broken till the milk is about to be churned. If it be not argitated, or the ' lapper' (as it is termed in dairy languare) broken, till it is turned into the churn, it may stand from a day to a week without injury.

"If these rules be attended to, the butter will be rich, sound, and well-

The cream should, in hot weather, be made into butter the next day; but in winter it is thought better to let the cream remain one day longer on the milk. The cream, being collected from the pans, is put into wooden bowls, which should be first rinsed with scalding, then with cold water. It is now briskly stirred round one way, with a nicely cleaned hand, which must have also been washed in hot and then in cold water, for these alternate wirm and cold ablutions of bowl and hand are not only for the sake of cleanliness, but to prevent the butter from sticking to either.

.'The cruam being thus aritated, quickly assumes the consistence of butter, the milky part now readily separates, and being poured off, the butter is washed and pressed in several cold waters; a little salt is added to season it; and then it is well beaten on a wouden trencher until the milky and watery parts are separated, when it is finally formed into prints for the markets." - Surv. of Coruwall, p. 141.

"If the quantity of cream be considerable, the cream will be an inch or more thick upon the surface, and it is then divided into squares and taken off. The remaining milk, huwever, cuntains little besides the watery particles in its original composition." - Cumplete Grazier, sixth edition, p. 137.

* Survey of Essex, vol. ii, p. 285. 
flavoured, and the butter-milk will liave a pleasant, palatable, acid taste: but wherever fermentation has been excited, or the lapper broken, and the milk rum into curds and whey, the fermentation so begun will continue in the butter-milk after that operation, and will become acrid and unwholesome. When duly prepared and manufactured, the milk will be the better with a fifth or a fourth part of water mixed into it, than milk that has been fermented before being churned would be without a drop of water mixed with it*" On this latter observation it should, however, be observed, that although some English writers have described butter-milk as being onlv fit for hous, yet it forms a very material portion of the food of the labouring classes in Scothand and Ireland, and therefore any adulteration with water should in those cases be avoided.

The operation of churning, whether it be cream or whole milk, is done in the same manner; but the latter, from being the so-much larger quantity, is of course so much more laborious, that in larre dairies chums moved by machinery are sometimes employed; which, besides the advantage of performing the work with greater regularity, is said to have also that of producing a larger quantity of butter $\dagger$. The whole milk, besides, requires more time than that of cream to complete the process-from two to three hours being considered by Mr. Aiton as necessary to effect it with due deliberation, while tisat of cream is generally finished within less than an hour and a half. 'The operation should, in warm weather, be very slow; for if it be done too hastily, the butter will be solt and white; the churn should, therefore, be couled by beingr previously filled with cold water; but in winter it should, on the contriry, be performed quickly, and the churn should be warmed. The motion of the churn should, however, be, in each case, rewular, and whaterer mar be the degree of velocity, the stroke of the fan or piston ought always to be the same, until the butter is formed, or said "to come." The air which is generated in the churn should also be occasionally allowed to escape, or it will create froth, which impedes the process.

The temperature of the milk-house should, as we have already stated, be kept as nearly as possible at about $55^{\circ}$, or at least between $50^{\circ}$ to $60^{\circ}$; and cream, when churned alone, should not he higher, for if kept at a high temperature in the process of chuming, it will be found inferior both in appearance, taste, and quality + ; but milk and cream, when churned together,

* Aiton on Dairy Husbandry, p. 107.

† A machine having been erected in Laneashire for the management of a heavy concern, as much labour was effected within an hour and a quarter by a horse and boy as usually occupied two men during five hours. The difference in produce between the two moles was this :-

\begin{tabular}{cc} 
New Milk. & Iutter by hand-churning. \\
6471 quarts & 364 lbs. \\
6644, & 397, \\
6995, & 348, \\
\hline 20.110, & \\
\hline
\end{tabular}

\begin{tabular}{l} 
New Milk. \\
7261 quarts \\
7675, \\
8120, \\
\hline 23,156
\end{tabular}
Butter by machinery. 469 lbs. 482, $57 \pm$,

1525 ,

The quantities of milk in the above cases were the produce of six successive fortnights: and it is concluded that if 20,110 quarts yield $1109 \mathrm{lbs}$. of butter, 23,156 quarts will yield 1277 lbs., thereby giving an increased produce, between that and 1525 lbs. yielded by machinery, of 248 libs.-Dickson's Report, p. 560.

+ In order to show this, essays on the temperature at which butter can be best procured from cream were made from experiments by the late Rev. Dr. John Barclay and Alexander Allan, which have been published in the Transactions of the Highland Society; the result of which is as follows. The table exhibits the mean tempierature of the cream used in each trial; the time occupied in the different chumings ; the quautity of 
it is generally thought must be equalled to about $70^{\circ}$ to $75^{\circ}$ of Fahrenheit before the latter can be separated from the milk: which is consequently the common practice. This is done by one person gradually pouring a small quantity of warm water into it while another is chuming; for if the work be carried on while too cold, the milk is said to rise in the churn, airbubbles are thrown up with a rattling noise, and the milk becomes pale; whereas, if conducted at a proper heat, it does not swell, but is easily worked, and remains at the proper straw colour. A thermometer, it may therefore be imagined, should always be hung up in every dairy, and indeed this has been uniformly insisted on by every writer whom we ever recollect to have treated on the subject; yet, strange to say, it is an instrument seldom seen in one of them : the only scale which the dairy-maid knows is at her fingers' ends; and although she invariably trusts to her hand for trying the heat, it is yet surprising with what correctness she usually judges*. Practice, they say, makes perfect; and it is astonishing with what accuracy many operations, which are supposed by theorists to demand the aid of science, are performed through experience by the merest clorlpoles. This, however, must not be construed into an approval of the want of rule; for, notwithstanding the accuracy which experience may produce, it is not to be compared with that denoted by scientific experiment, nor can it be acquired without great loss of time, which might otherwise be avoided.

The whole milk, as well as the cream when churned seprarately, must become sour before it is chumed; but this must be effected merely by the state of the atmosphere, or by being kept exposed to the fire in order to bring on fermentation; it is therefore kept in a large vat, or tub, until the milk is turned into curd, or lapper, and if that remains undisturbed, the

butter obtained from one gallon of cream in each experiment; and the complarative qualities of the different specimens of butter:-

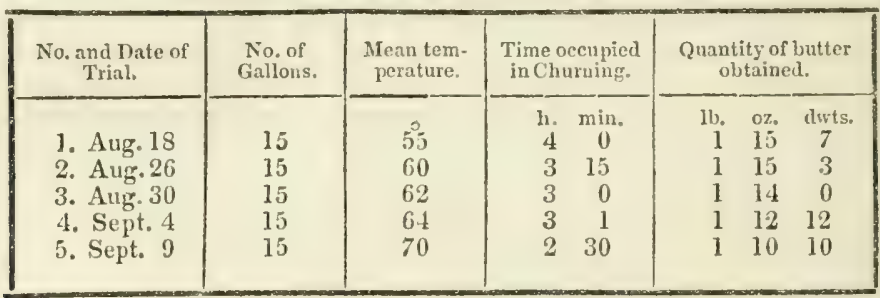

The butter produced in the first experiment was of the very best quality, being rich, firm, and well tasted.

The second experiment yielded a butter of a good quality, and not perceptibly inferior to the former.

The third experiment was of a good quality, but of inferior consistency.

The fourth experiment produced a soft and spongy butter.

The fifth experiment was decidediy inferior in every respect to any of the former experiments.

Experiments on the same subject by Mr. John Ballantyne, of Edinburgh, went to nearly the same effect in point of produce and quality; but the time of churning at similar degrees of temperature was much shorter.

From all these trials it should appear that cream should not be churned at a hirher temperature than $65^{\circ}$, and that the best temperature at which to commence the operittion is from $50^{\circ}$ to $55^{\circ}$, for it will gradually rise full four degrees during the operation. If, on the contrary, it should be under $50^{\circ}$, the labour will be increased without any proportionate advantage; but the temperature may then be iucreased by a small quantity of warm water.

* A trifling want of uniformity in the degree of heat may, however, occasion a considerable difference of time in the completion of the act of churning, as will appear from 
churning may be deferred for some days longer; the warm water above mentioned is not therefore added until the lapper is broken. The process, wherever the whole milk is churned, is nearly the same throughout the United Kingdom, but in Holland, we learn from Mr. Aiton, it is somewhat different: "the milk is put into a pan, or vat, and well stirred with a wooden spoon, or ladle, two or three times a-day, to prevent the cream from separating from the milk, and this sort of stirring, or partial churning, is continued till the milk becomes so thick and clotted, that the ladle stands erect in it; after which, it is put into the churn for an hour or so. Cold water is also poured in, to help to collect the butter and separate the milk from it; and, by this method, the Hollanders imagine they obtain more butter from the milk than by any other way; they also say, that both the butter and the butter-nilk are better when made in that way than when churned as done in England." This, however, is merely an opinion, the truth of which can only be decided by comparative experiment : the object of the operation is to extract the substance of the milk; and in whatever way that may be done, provided it be equally well performed, we believe little difference will be found in the profit.

In most places the butter, when taken from the churn, is washed in repeated waters, in order to extract the milk, until the water comes out pure. This, however, is a practice not to be commended, and in many dairies has been relinquished. In a very large one conducted with superior management, it is never done ; for " it is found by long experience that butter retains its sweetness much longer when no water is used in making up. When it is taken out of the churn, it is well worked with the hand, which presses out most of the milk; it is then beaten with a cloth, or rather a cloth is repeatedly pressed down upon it, which absorbs all the remaining milk*." The less it is beaten or worked, however, the better; for the more it is kneaded, the more tough and gluey will it become; and a slight quantity of salt may be added to flavour it.

some observations recorded on the subject of churning cream in the Lancashire Report, as follows; though perhaps it may also be partially affected by the quantity churned :

\begin{tabular}{|c|c|c|c|c|c|c|}
\hline & & 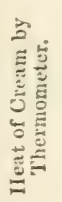 & 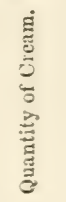 & 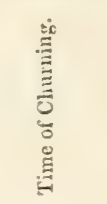 & 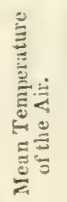 & State of the Weather, \\
\hline $\begin{array}{l}\text { Feb. } \\
\text { ", } \\
\text { Mar. } \\
\text { ", } \\
, \\
,\end{array}$ & $\begin{array}{r}23 \\
25 \\
28 \\
2 \\
4 \\
7 \\
9 \\
11 \\
14\end{array}$ & $\begin{array}{l}57 \\
57 \\
56 \\
57 \\
56 \\
50 \\
57 \\
58 \\
52\end{array}$ & $\begin{array}{l}\text { gals. } \\
40 \\
35 \\
36 \frac{1}{2} \\
38 \\
32 \\
35 \\
35 \\
32 \\
36 \frac{1}{2}\end{array}$ & $\begin{array}{lc}1, & \min . \\
1 & 30 \\
1 & 20 \\
1 & 25 \\
1 & 40 \\
1 & 10 \\
1 & 55 \\
1 & 30 \\
0 & 50 \\
1 & 30\end{array}$ & $\begin{array}{l}42 \\
44 \\
40 \\
41 \\
41 \\
44 \\
47 \\
47 \\
44\end{array}$ & $\begin{array}{l}\text { Cold N.W. wind. } \\
\text { Mild and warm, no wind. } \\
\text { Mild, inclined to frost. } \\
\text { Ditto. } \\
\text { Ditto. } \\
\text { No wind, very sharp frost. } \\
\text { No frost, gentle rain. } \\
\text { Mild soft morning. } \\
\text { Mild, rather cool. }\end{array}$ \\
\hline
\end{tabular}

The person who states this, says, "that they never consider the butter as good as it should be, if the churning be finished in less than an hour; it it exceed an hour by fifteen, twenty, or even thirty minutes, the butter is always improved both in quantity and quality." - p. 559 .

* See the account of a Gloucestershire Vale farm, published by the Society for the Diffusion of Useful Knowledge, in the Farmer's Series, No.21.

VOL. II. 
If the butter be salted for market, after the whole of the milk has been very carefully pressed out, it should be well mixed by working it in by the hand, with finely-powdered salt*; for if care be not taken in mixing it equally, the butter acquires different colours-yellow where the salt has fallen, and white where it has not-which, in dairy language, is called "pwety" or "pinsowed" butter, and brings an inferior price. The operation should be performed immediately ; for if deferred, as it commonly is by country dealers, and farmers who do not churn enough to fill a firkin at once, the butter loses a portion of its firmness and flavour Should, however, there not be sufficient to fill up a package, the butter should never be put into the firkin in lavers, but the surface should be left every day rough and broken, so as to unite better with that of the succeeding churning. The quality may also be in a great measure preserved by giving it a partial salting, and covering it over with a cienn linen cluth, dipped in pickle and placed in a conl situation.

The quality of salt which is usually employed is that of St. Lbes, and should be either that or other equally strong marine salt, entirely free from the bittern which is found in mineral salt. The quantity may be that of about ten ounces to the stone of $14 \mathrm{lbs}$, - - rather more or less, according to the length of time which the butter is intended to be preserved; but it is generally thought that the butter made during the summer months is the fittest for salting, and that the sort which is made in the later part of the season, not taking it so well, requires rather more. In Irelend the use of saltpetre is customary in the proportion of half an ounce of salt with one-eighth of saltpetre to the pound of sixteen ounces; and although this forms a valuable jickle if the salt be really good, yet it unquestionably would be much improved if four ounces of raw sugar were to be added to each pound weight of salt + .

When the butter is thus cured, it is then tramped firmly with a round wooden stick into the firkin, which is filled up to the head, and then covered over with a little of the purest salt. These casks usually contain as nearly as possible $84 \mathrm{lbs}$. each + , and are gencrally made of white oak or ash, though lime has been lately recommended, it having been ascertained by innumerable experiments as being the only wood free of acid; and acids are well known to act powerfully on salt, which they decompose, and convert into brine. As lime-wood, however, camot be always procured, fir has been found next in order; and it is said that by boiling the staves during four hours, the whole of the pyroligneous acid of all kinds of timber may be extracted $\$$.

* This, however, is not unfrequently done by the dealers both in Scotland and Ireland, by the butter being thrown into large wooden troughs, and trod on by men and women with their bare feet; which they justify by saying that no person has strength enough to work a large quantity by the hand.

+ A compound of one part sugar, one part nitre, and two parts of the best Spanish salt, beat together into a fine powder, and mixed thoronglily with the butter, in the proportion of one ounce to the pound, has been fuund to keep the butter in every respect sweet and sound during two years that it was in cask. It is also said to impart a rich marrowy flavour that no other butter ever acquires, and tastes but very little of the salt. Bath Papers, vol. v. p. 116. See also observations on salt in this chapter, p. 424.

‡ They are commonly made of this size, as being more customary in the London market; but butter is also frequently packed in hall-firkins, containing $28 \mathrm{lbs}$; whole firkins, of $56 \mathrm{lbs}$. ; and tubs, of $84 \mathrm{lbs}$.

$\$$ Observations on the curing and casking of butter, drawn up by order of the A rricultural Association of Aberdeen, and published in the Transactions of the IIighland Society, N. S. vol. i. p. 355. The method stated is thus:- "Have a boiler the same length as the wood, with a weight to keep it immersed in water, and have a wooden 
In Ireland the butter trade is regulated by Act of Parliament, and the mode in which it is conducted is as follows:-The farmers assemble in the morning, and have all their casks arranged in the market place; the coopers then take out the head of each, and the inspector follows, without knowing to whom the casks belong, and marks the character of each with

\section{$f$ for the first sort, \\ $\therefore$ for the second,}

for the third, and

for the fourth sort.

The coopers then replace the heads, and cut the character upon the side of the firkin, togrether with the weight and tare of the cask, which is weighed at the market-beam; after which the farmer proceeds to the sale. This is done in open market, the price being only named for the first quality; a regular diminution being understood to attach to each of the other sorts.

The ancient history of butter-making is very imperfectly known ; for, although mentioned in the Scriptures as "being brought forth by the churning of milk," yet it has been asserted that the word "butter," as translated from the Hebrew, should be rendered as "cream." It is not allucled to by any of the Greek writers, nor was it known to the Romans until they were taught how to make it by the Germans; but this is not to be wondered at, for a warm climate is unfavourable to it, and even at this moment it is only rarely found except in the north of Europe. In England, however, it appears to have been known immemorially, for, when invaded by Julius C'esar, it was a common food of the islanders; but the art of making cheese they leaned from their conquerors. It, however, seems extraordinary that a people in possession of large herds of kine, could be ignorant of the art of making some sort of cheese from the sour curd with which they must have been acquainted: it is, indeed, described in many ancient authors; yet no mention is there made of the rennet with which it is now formed, nor is it known when the use of that article was first discovered.

\section{CHEESE.}

As butter is made from the oily part of the milk, which rises to the surface in the form of cream; so clieese is composed from the curd, or coagulated milk, and may be obtained from the caseous portion alone, after the milk has been skimmed. If thus deprived of the cream, this "skimmilk cheese" is, however, of a poor quality, and, if intended to be good, the whole milk should be used without any loss of cream ; for if any portion of it be abstracted, the cheese will be proportionably less rich : consequently, less palatable, and of inferior value. The mode of making too, though in the main points apparently the same, yet is sulject to more variety of minor details in the practice than that of any thing formed of one material; and thus many different qualities are brought to market, each bearing some distinct character of its own. That many of those kinds, which are by comnoisseurs thought indifferent, might, by other management, be more nearly assimilated to the superior surts, there can be little doubt; these peculiarities, however, have in many districts attacherl a certain degree of value to their flavour, while in others, it would seem to be imparted by the natural grasses grown upon the soil*. This apnlies more especially

cover on the boiler, as it must be done by close evaporation. The wood is then dried for use; it becomes closer and more condensed, from the fibres being contracted, and while it continues hot it can be easily brought to any shape."

* See a report on dairy management, with experiments made in consequence of pre- 
to Cheshire than to any other county; for although imitations of different districts have been, in some cases, successfully made in others, yet in no trial has cheese of the true Cheshire flavour been produced, when made from cows fed upon other soils. Whether justly or not, it has been attributed to the abundance of saline particles in the earth, as evinced by the numerous salt-springs which abound throughout a large portion of that country, and is so old a remark, that Fuller, in his "Worthies," when speaking of the country, says,- " It doth affurde the best cheese for quantitie and qualitie, and yet the cows are not, as in other shires, housed in the winter. Some essaied in vaine to make the like in other places, thoughe from thence they fetched their kine and dairie-maides: it seems they shoulde have fetched their grounde too, wherein is surelie some occult excellencie in this kind, or else so goode cheese will not be made." There must, indeed, be some truth in the observation, for it is well known that where brine-springs most abound, the cheese is ever esteemed to be of superior quality. Whatever may be the foundation of the fact, the quality is, however, always better when the cows are pastured during the summer months; but, provided they be well fed throughout the winter, good cheese may be made the whole year round.

\section{RENNET.}

Although cheese may be made from the curd, which has been formed by the coagulation of the milk when it turns sour, yet, when thus obtained, it is hard and ill flavoured; means have, therefore, been found to curdle it with " remnet," which is made from the gastric juice of animals, but more especially from that found in the maws or stomachs of sucking calves, that have been fed entirely upon milk. These maws, or "vells," as they are sometimes called, are occasionally preserved, along with the curd contained in them, by salting; but the more usual mode is to employ the skins of the stomach-bags alone: the method being to put a few handfuls of salt into and around the stomachs, which are then rolled up and hung near the chimney to dry, after which they are put by for a long time before they are used. If the skin be good, a bit of it no bigger than a sixpence, if put into a tea-cup full of water, with a little salt, during about twelve hours before it is wanted, will form a stock sufficient for eighteen or twenty gallons of milk; but their manner of preservation and use is extremely various, and, as the quality of the cheese depends more upon the application of the remnet than upon any other part of the manufacture, we sliall here state some of the most approved modes of its preparation.

Most dairy-maids are of opinion that if the curd, or chyle, were not removed from the maw of the calf, it would communicate a harsh taste to the cheese, and in Gloucestershire, we learn, from a very intelligent dairywoman, that she never uses the vells until they are twelve months old; for, if newer, the remnet made from them causes the cheeses to heave, or sivell, and to become full of eyes, or lroles. We know also, from experience, that, if too much be used, or if it be unusually strong, it will occasion the cheese to heave; probably, by causing fermentation. She prepares it by adding to every six vells two gallons of brine, and two lemons: the lemons doing away with any unpleasant smell, and giving it an agreeable flavour. A large quantity should be made at a time; and it should never be used until it has stood at least two months. In the same county, however, the method recommended by the late Mr. Marshall, is as follows:- 
"Take the maw of a newly killed calf, and clean it of its contents : salt the bag, and put it into an earthen jar for three or fuur days, till it form a pickle; then take it from the jar and hang it up to dry, after which it is to be replaced in the jar-the covering of which should be pierced with a few small holes to admit of air; and let it remain there for about twelve months.

"When wanted for use, a handful each of the leaves of sweet-briar, dog-rose, and bramble, with three or four handfuls of salt, are to be boiled together in a gallon of water for a quarter of an hour, when the liquid is to be strained of and allowed to cool. The maw is then to be put into the liquid, together with a lemon stuck round with cioves, and the longer it remains in it the stronger and the better will be the remnet: half a pint, or less, of the liquor, is sufficient to turn fifty gallons of milk."

\section{Or, as practised in other parts of the west of England:-}

"When the rennet-bag is fit for the purpose, let two quarts of soft water be mixed with salt, wherein should be put almost every sort of spice and aromatic herb that can be procured, and must boil gently till the liquor is reduced to three pints: it should then be strained clear from the spices, and poured in a tepid state upon the maw, and a lemon may be sliced into it; when it may remain a day or two, after which it should be strained again, and put in a bottle, where, if well corked, it will keep good fur twelve months or more, and give the cheese a pleasing flavour *."

That stated by Mr. Holland, as being customary in Cheshire, is thus :-

"When the maw comes from the butcher, it is always found to contain a chyley, or curd-like matter, which is frequently salted for present use; but when this chyley matter is taken out, and the skin cleared from slime and every apparent impurity, by wiping or a gentle washing, the slin is then filled nearly full of salt, and placing a linger of salt npon the bottom of a mug, the skin is placed flat upon it. The mug is large enough to hold three skins in a course; each of which should be covered with salt; and when a sufficient number of skins are thus placed in the mug, it should be filled up with salt, and put, with a dish or slate orer it, into a coul place, till the approach of the cheese-making season, in the following year. The skins are then all taken out, laid for the brine to drain from them; and, being spread unon a table, they are powdered on each side with fine salt, and are rolled smooth with a paste roller, which presses in the salt. After that, a thin splint of wood is stuck across each of them, to keep them extended while they are hung up to dry."

"In making the rennet, a part of the dried maw-skin is, in the evening, previous to its being used, put into half-a-pint of lukewarm water, to which is added as much salt as will lie on a shilling. In the morning this infusion (the skin being first taken out) is put into the tub of milk; but so great is the difference in the quality of these skins, that it is difficult to ascertain what quantity will be necessary for the intended purpose. A piece the size of half-i-crown, cut from the bottom of a groud skin, will commonly be sufficient for a cheese of $60 \mathrm{lbs}$. weight; though ten square inches of skin are often found too little. It is customary, however, to cut two pieces from each skin: one from the lower, the other from the upper part ; but the bottom end is the strongestf."

An improved mode is-" To take all the maw-skins provided for the whole season, pickled and dried as before: put them into an open vessel, and for each skin pour in thee pints of spring water; let them stand twenty-four hours, then take out the shins, and put them into other vessels: add for each one pint of spring water, and let them stand twenty-four hours as befure. On taking the skins out the second time, gently stroke them down with the hand into the infusion: they are then done with. Mix these two infusions together, pass the liquor through a fine linen sieve, and add to the whole a quantity of salt, rather more than is sufficient to saturate the water; that is, until a portion of salt remains undissolved at the bottom of the vessel. The next day, and also the summer through, the scum, as it rises, is to be cleared off; and fresh salt shonld be added. Somewhat less than haif-a-pint of this preparation will generally be sufficient for 60lbs. of cheese; but, when for use, the whole should be well stirred upt.."

In Scotland, however, it would appear, from Mr. Aiton's account of the chicf dairy district in Ayrshire, that so far from washing away the chyle

* See the Farmer's Series, No. 21; Marshall's Southern Counties; and the Bath Papers, vol. iii. art. xxi. p. 161.

f We understand that the vells imported from Ireland are found to be the best. Amongst imported skins, however, those of lambs and pirs are sometimes found, which answer the intended purpose but indifferently.

\pm Cheshire Rep. p. 268. 
contained in the maw of the calf, pains are taken to increase it as much as possible, by giving to the animal as much milk as it can be made to swallow, a few hours before it is killed; for the chyle being formed by the mixture of the gastric juice with the food, and that gastric juice leing the coagulating power, both are therefore carefully preserved, and are considered as thus forming a stronger rennet than can be drawn from the bag alone. It is thus prepared :-

"When the stomach, or bag, is taken from the calf's body, its contents are examined, and if any straw or other food be found among the curdled milk, such impurity is removed; but no part of the chyle is suffered to be lost. At least two handfuls of salt are put into the bag, and upon its outside, after which it is rolled up in salt, and hung near a fire, where it is always allowed to hang until it is well dried, and it is understood to be improved by hanging a year, or longer, before being infused.

"When renuet is wanted, the 'yirning," as it is called in Scotland, with its contents, is cut small, and put into a jar with a handful or two of salt ; and a quantity either of suft water, that has heen boiled, and cooled to about $65^{\circ}$, or of new whey taken off the curd, is put upon the bag in the jar. The quantity of water, or whey, to infuse the bag, is more or less according to the quality of the yiming. If it is that of a new dropped calf, that has not been fed, three linglish pints will be enough ; but it he has been fed for four or five weeks, a couple of quarts may, at least, be put on the bag to mash : it shonld, however, be observed that the yiming of a calf fuur weeks uld yields more rennet than that of one twice that age. After the infusion has remained in the jar from one to three days, the liquid is drawn off; and an English pint more water, or whey, put on the bay in the jar; and that, after standing in mash one or two days, is also drawn off, and, with that of the first infusion, strained, if any impurities appear in the liquor: the whole being put up in bottles for use as remuet, and the bag being thrown on the dunghill, without ever being put into the milk. Some put about a dram of grood whisky into each quart bottle of the rennet; and it may be either used immediately, or kept for an many montlis as may be convenient."

A table-spoonful of remnet, thus made, will, it is said by Mr. Aiton, coagulate thirty gallons of milk ; but its great superiority over our common practice is, that it will curdle the milk in five or ten minutes, whereas the English remet requires from one to sometimes three hours, in order to form the curd: a defect owing chiefly to the removal of the curdled milk. As to the chyle occasioning a harsh taste to the cheesc-the reverse, Mr. Aiton says, is proved by the mild flavour of that made in Scotland *. It must, however, be admitted that, unless great care be employed in the immediate preparation of rennet, thus made, the curd is extremely apt to become rancid, and thus impart a certain degree of rankiness to the cheeset.

\section{WHOLE-MILK CHEESE.}

The mode of mating sueet-milk cheese-that is, cheese made of milli which has not been skimmed-is, to put the ladder across the cheesetub, with a large canvas cloth covering the whole, in order to prevent the falling of milk upon the floor, or any other matter into the tub; and above this is placed the sieve through which the milk is to be strained. It should be of the temperature of $90^{\circ}$ to $95^{\circ}$, and if below $85^{\circ}$ degrees, a portion of it should be placed in a deep brass pan, which is then immersed in the water which is liept hot in the wash-house. By this means the whole is warmerl equally, and it is of the utmost importance that attention be paid to it, for if the milk be not warm enough when the remnet is put to it, the curd will be tender, and the cheese will bulge out at the silles; and, if too hot, it will cause it to swell or "heave," and become spongy:

* Aiton on Dairy Husbandry, p. 154.

+ In a case of emergency, it is said that a decoction of the yellow flowers of the herb called "cheese rennet," or "yellow lady"s bed straw," which blossoms in July and August, will answer every purpose.-Complete Grazier, 6th edit. p. 146. 
both of which defects are injurious to its appearance and quality. The remet is then at once added to the milk, which is thus coagulated at its natural heat; but many farmers have not cows enough to form a chcese at every milking, and it must, therefore, be then allowed to cool. In doing this, it of course throws up cream, which is not unfrequently taken off for butter, while the second meal, of whole milk, is used along with that which has been already skimmed; but if the cheese be intended to be of fine quality, the cream must be also added. This, however, should be at the same time skimmed; for the milk, when cooled, must be afterwards heated to full $90^{\circ}$ degrees of Fahrenheit, in the summer, and to a higher temperature in cold weather, and were the cream to be warmed to that degree, it would be melted, which would cause a considerable portion of the fatty, or butyraceous, matter to be lost in the whey : it is, therefore, grenerally thought the best practice to gralually bring it to a liquid state by the admixture of moderately warm milk, before it is poured into the cheese-tubs. The curd is then broken into small pieces, and the whey being thoronghly squeezed out, it is salted, wrapped in a cloth, and placed in a chessart, of such size as may be convenient, or is usually made in the neighbourhood; it is then pressed with weights proportionate to its size, and turned occasionally until it becomes sufficiently firm to be taken out of the mould, and placed either on a cheese-rack, or on the flour of the cheese-roum, where it is occasionally turned, and dry rubbed with salt, and remains until fit for market.

New cheese requires to be hardened by gradually drying before it becomes fit for market, and the cheeses, when taken out of the mould, are for this purpose spread in a single layer on the floor of the cheese-loft, where they are daily turned by hand, in order to expose each surface alternately to the air. This, on a large dairy - farm, is a slow and laborious operation, which, as it devolves upon the female servants, sometimes prevents them from paying proper attention to that essential dutv. A machine was therefore formed by Mr. Blurton, of Field Hall, near Uttoxeter, for remedying the inconvenience, and the large silver medal of the Society for the Encouragement of Arts was awarded to the invention. It is called a "Swingr-Frame for more expeditiously Drying Cheeses;" and is made in the following manner:-

"It consists of a dozen strong shelves framed together, and having bars nailed from top to buttom of one side of the back of the shelves, in order to prevent the cheeses from falling out while in the act of turning. The frame is suspended on two strong pivots, one of which is let into the wall of the room, and the other is supported by a strong post. 'Two catches heep the frame upright, and prevent it from being turned more than half round. By first tilling the shelf immediately below the axis of the frame, and then placing the cheeses alternately on the two nearest shelves above and helow that which has been already filled, the preponderance of one side over the other can never be more than the weight of une cheese; the whole power, therefore, required to turn the machine cannot, in any cireunstances, be greitter than this and the friction of the pivots. The cheeses, in the act of turning, druy un thuse shelves, which, in the furmer position of the frame, were above them, and, having been exposed to a current of air for twenty-four hours previous, have become perfectly dry."

It has now been for several years employed in Leicestershire; and it is sail, "that by means of it fifty-five cheeses are turned in the same time which is required for turning two by hand. Three other advantages attend its use; first, that a room thus furnished will hold thrice as many cheeses as when they are laid on the floor; secondly, that the shade afforded by the shelves, together with the current of air which passes between them, has the effect, in hot weather, of preventing excessive sweating, and consequently loss both in weight and quality, as well as diminishing the necessity of 
rubbing the cheeses; thirdly, the ripening of the cheeses is hastened, so that on an average they are ready for market five weeks earlier than usual."

The store-room should be kept temperately warm, and the shelves or the floor upon which the cheeses are laid, should be strewed with dry moss, or fine hay, as the cheeses, when new, are otherwise apt to adhere to the boards, and thus acquire an unpleasant appearance. At a more aclvanced stage they may be laid upon straw; but, at first, it would sink into the surface and deface them. The dried leaves of the tutsan, or of the yellow star of Bethlehem, and the twigs of the common birch-tree, are also thought to assist in preventing the depredations of mites*.

The whey which runs from the curd, without pressure, is called " green whey," and is received from the cheese-tub into pans covered with a cloth, under which they are held, until it deposits a sediment, which is added to the curd, after which it is poured into the cistern; while that which is pressed by hand from the curd, is termed "white whey," and contains a considerable portion of oily matter: so much so, that it is in some cases kept apart, and set for cream. Nost generally, however, the green and white are both scalded together, until they throw up a substance in appearance between cream and curd, which is skimmed off so long as it rises, to be churned for whey-butter: the difference between which and milk-butter is two-pence or three-pence per pound against the former. The whey is then generally given to the pigs; but some economical dairy-women put it on a smart fire, and, when near boiling, mix it with a quantity of butter-milk, and these fleetings, or "flit-milk," as it is called, form curds, which are skimmed off; so long as they arise, for the use of the servants $\dagger$.

A material point in the manufacture of cheese of every description, and one very imperfectly understood in this country, is the kind of salt commonly employed; for although some attempts have been made by the late Earl of Dundonald, Dr. Coventry, and a few others, to introduce a superior quality, yet the generality of farmers use whatever sort they find at a neighbouring shop, without reflecting upon, or knowing, the difference which it may make in the flavour. This, however, is so much attended to by dairy-men in Holland, that different kinds of salt, cvaporated in various ways, and by different periods of time, are by them employed for butter and cheese: the cunsequence of which is that the butter, thus made, is so free from that bitter taste imparted by the acrid quality of our common salt, that, although always thoroughly well preserved, it yet ever tastes nearly fresh. It is said also, that they mix a solution of salt with the milk before churning, as well as before coagulating it for cheese: for this, however, we cannot vouch; but we think it merits trial, and that men who are largely engaged in dairy business would do well to import the species of salt we have mentioned from Holland+. The proper quantity is also a subject which merits investigation, as the putrescency or ripening of the cheese much depends on it.

It is not supposed that much improvement has taken place in the cheesemaking of this country during a great length of time; though our "Stilton," and in Scotland that known as the "Dunlop," which now bear a high

* Parkinson on Live Stock, vol. i. chap. i. sect. 12.

+ Cheshire Report, p. 263.-The whey which remains from the curd is called "whig," and when kept until it is sour, is drunk in the north of England with an infusion of mint or other herls, and is considered very pleasant, as well as wholesome, in hot weather.

+ We learn that a Dr. Swedeaur, some years ago, established a manufactory of salt, in imitation of that made in Holland, at Prestonpans, in Scotland: it was called "salt upon salt"; but we do not know whether the concern is still carried on. 
reputation, have only been of comparatively late years brought into use. In Ireland the farms are generally so small, and the means of making, both in buildings and implements, so insufficient, besides the poverty which compels the dairyman to resort to immediate sale, that chcese is but seldom made; and there is "only one species, that of "Kimnegad," which we ever hear of as there manufactured. In all our dairies the same main points are admitted to be essential; but, although the means of attaining tlem are nearly alike in similar districts, yet in others they differ materially in the minutice, and upon these much of the art of cheese-making depends. We therefore insert the details of the modes employed on some of the most celebrated kinds.

\section{CHESHIRE CHEESE.}

That of Cheshire, as described in Holland's Survey, is generally made with two meals of milk; and that, even in dairies where two cheeses are made in a day: indeed, in the beginniug and end of the scason, three, four, and even five or six meals are kept for the same cheese. The general custom is-

To take about a pint of cream, when two-meal chceses are made, from the night's mill of twenty cows. In order to make cheese of the best quality, and in the greatest abundance, it is, however, admitted that the cream should remain in the milk; for whether the cream that is once separated from it, can by any means be again so intimately united with it as not to undergo a decomposition in the after process, admits of some doubt. The more common practice is, however, to set the evening's milk apart till the following morning, when the cream is skimmed off, and three or four gallons of the milk are poured into a brass pan, which is immediately placed in the furnace of hot water, and made scalding hot; then half of the milk thus heated is poured upon the night's milk, and the other half is mixed with the cream, which is thus liquefied, so as, when put into the cheese-tub, to form one uniform fluid. This is done by the dairywoman while the other servants are milking the cows, and the morn. ing's milk being then immediately added to that of the evening, the whole mass is at once set together for cheese.

'The rennet and colouring* being then put into the tub, the whole is well stirred together; a wooden cover is put over the tub, and over that is thrown a linen cloth. The usual tine of "coming," or curdling, is one hour and a-half, during which time it is frequently to be examined. If the crom rises to the surface before the coming takes place, as it often does, the whole must be stirred together so as to mix again the milk and the cream ; and this, as often as it rises, until the coagulation commences. If the dairy-woman supposes the milk to have been accidentally put together cooler than she intended, or that its coolness is the cause of its not coming, hot water, or hot milk, may be poured into it, or hot water in a brass pan may be partially immerged in it. This must, however, be done before it is

\footnotetext{
* Spanish annatto is the drug usually cmployed: little more than the quarter of an ounce of which is sufficient for a cheese of $60 \mathrm{lbs}$. Other colouring matters are however used, such as marigolds boiled in milk, which give a plessant flivour; and carrots also boiled in milk and strained, which impart a rich colour, but a rather strong taste. The annatto is generally put in by rubbing a piece of it in a bowl with some warm milk, which is afterwards allowed to stand a little, in order to drain off the sediment, and is then mixed with the entire quantity.
} 
at all coagulated, for the forming of the curd must not be tamperer with. If it has been set together too hot, the opposite means, under the same precautions, may be resorted to; but the more general practice is, to suffer the process to proceed, hot as it is, until the first quantity of whey is taken off; a part of which, being set to cool, is then returned into the tub to cool the curd. If too little appears to have been used, it renders the curd excechingly tender, and therefore an additional quantity may be put in; but this must he done before the coagulation takes place, for, if added afterwards, it will be of little effect, as it cammot be used without disturbing the curd; which can then only acquire a proper degree of toughness, by having some lieated whey poured over it.

Within an hour and a half, as already mentioned, if all goes on well, the coagulation will be formed-a point which is determined by gently pressing the surface of the milk with the back of the hand; but in this test experience is the only guide, for the firmness of the curd, if the milk be set hot together, will be much greater than that from milk which has been set cold together. If the curd be firm, the usual practice is to take a common case-knife, and make incisions across it to the full depth of the blade, at the distance of about one inch, and again crosswise in the same manner, the incisions intersecting each other at right angles. The cheese-maker and two assistants then proceed to break the curd, by repeatedly putting their lands down into the tub, and breaking every part of it as small as possible; this part of the business being continued until the whole is uniformly broken small: it generally taties up about forty minutes, and the curd is then left, covered over with a cloth, for about half an hour, to subside.

The bottom of the tub is now set rather atilt, the curd is collected to the upper side of it, and a board is introduced, of a semicircular form, to fit loosely one-half of the tub's bottom. This board is placed on the curd, and a $60 \mathrm{lb}$. weight upon it, to press out the whey, which, draining to the lower side of the tilted tub, is ladled out into brass pans. Such parts of the curd as are pressed from under the board, are cut off with a knife, placed under the weighted board, and again pressed: the operation being repeated again and again until the whey is entirely drawn from the curd. 'The whole mass of curd is then turned upside down, and put on the other side of the tub, to be pressed as before. The board and weight being removed, the curd is afterwards cut into pieces of about cight or nine inches square, piled upon each other, and pressed both with the weight and hand: these several operations being repeatedly performed as long as any whey appears to remain in it.

The next thing is to cut the curd into three nearly equal portions, one of which is taken into a brass pan, and is there by two women broken extremely fine; a largre handiful of salt being added, and well mixed with it. That portion of curd being sufficiently broken; is put into a cheese-vat, which is placed to receive it, on a cheese-ladder over the cheese tub: the vat being furnished with a coarse cheese-cloth. The second and third portions of the curd are treated in the same mamer, and emptied into the vat; except that into the mildhe portion eight, nine, or ten times the quantity of salt is usually put. By some dairywomen, however, each portion is salted alike, and with no more thith three large handfuls to each. The breaking takes up more or less 
time, as the cheese was set togrether hotter or colder; half an hour is perhaps the longest time.

The curd, when put into the cheese-vat in its broken state, is heaped above the vat in a conical form: to prevent it from crumbling down, the four corners of the cheese-cloth are turned up over it, and three women, placing their hands against the conical part, gently, but forcibly, press it together, constantly shifting their hands when any portion of the curd is starting from the mass, and folding down the cloth upon it. So soon as the curd adheres together so as to admit of it, a small square board, with a corner of the cloth under it, is put on the top with a $60 \mathrm{lb}$. weight ; or a lever, such as that described at p. $40 \mathrm{~s}$, is pressed upon it. Several iron skewers are at the same time stuck in the cone, as well as through holes in the side of the vat, from which they are occasionally drawn out and fixed in other spots, until not a drop of whey is discharged. 'The weight and skewers are then removed, and the corners of the cloth are either held up by a woman, or by a wooden hoop, while the curd is broken as small as possible, half way to the bottom of the vat; and the same operation of pressing and skewering is repeated. The women then take up the four comers of the cloth while the vat is drawn away and rinsed in warm whey: a clean cloth is then put over the upper part of the curd, and it is returned inverted into the vat. It is then broken half way through in the same manner as before, which several operations occupy from three to four hours.

When no more whey can be extracted by these means from the cheese, it is again turned in the vat, and rinsed as before in warm whey. The cloth now made use of is finer and larger than the former, and is so laid, that on one side it shall be level with the edge of the vat, and on the other wrap over the whole surface of the cheese; the edges being put within the vat, thus perfectly enclosing the entire mass. In this stage of the business the cheese is still higher than the edge of the vat; and to preserve it in due form, recourse is had to a binder, about three inches broad, either as a hoop or as a cheese-fillet, which is a strong, broad, coarse sort of tape, which is put round the cheese, on the outside of the cloth, and the lower edge of the binder pressed down within the vat, so low as that the upper edge of it may be level with the surface. The cheese is then carried to the press, and a smooth, strong board being placed over it, the press is gently let down upon it ; the usual power of which is about 14 or 15 civt. In most dairies, however, there are two presses, and in many three or four of different weights; the cheese being by some put first under the heaviest, and by others under the lightest.

As soon as the cheese is put into the press, it is immediately well skewered-the skewers being of strong wire cigliteen or twenty inches long, sharp at the points and broad at the other end; the vat and binder having holes, seldom more than an inch asunder, to receive them. As the press always stands near the wall, only one side of the cheese can be skewered at the same time, and it must therefore be turned half-way round, whenever that is necessary; but this occasions no inconvenience, as the skewers must be frequently shifted, and many more holes are made than skewers to fil them. In half an hour from the time the cheese is first put into the press, it is taken out again, and turned, in the vat, into another clean cloth, after which it is returned to the vat; but is by some persons previously put naked 
into warm whey, where it stands an hour or more for the purpose of

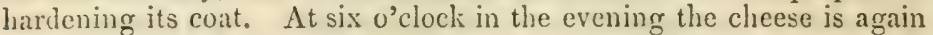
turned in the vat into another clean cloth, and some dairy-women prick its upper surface all over an inch or two deep with a view of jieventing blisters ${ }^{*}$. At six o'clock in the following morning it is again turned in the vat, with a clean cloth as before, and the skewers are laid aside: it is also turned two or three times more, both morning and evening, at the last of which finer cloths are used than those at firsi, in order that as little inupression as possible may be made on its coat.

After the cheese has remained about forty-eight hours under the press, it is taken out, a fine cloth being used merely as a lining to the vat, without covering the upper part of the cheese, which is then placed nearly mid-deep in a salting-tub, its upper surface being covered all over with salt. It stands there generally about three days; is turned daily, and at each turning weil salted, the cloth being changed twice in the time. It is then taken out of the vat, in lieu of which a wooden girth, or hoop, is made use of, equal in breadth to the thickness nearly of the cheese, and in this it is placed on the salting bench, where it stands about eight days, being well salted all over, and turned each day. The cheese is then washed in lukewarm water, and after being wiped, is placed on the drying bench, where it remains about seven days: it is then again washed and dried as before, and after it has stood about two hours, it is smeared all over with about two ounces of sweet whey butter, and then placed in the warmest part of the clieese-roomt.

While it remains there it is, during the first seven days, rubbed every dity all over, and generally smeared with sweet butter; after which it should for some time be turned daily, and rubbed three times a week in summer, and twice in winter. The labour is performed almost universally by women, and that in large dairies where the cheeses are sometimes, upon an average, upwards of $140 \mathrm{lbs}$. each.

The details of this process, it will, however, be observed, apply only to cheeses of $60 \mathrm{lbs}$. weight, and the quantity of salt used to them is uncertain; the greatest of which Mr. Holland knows is about 3 lbs. each, but much of it is wasted, and whether the cheese acquires much saltness in the silting-louse, dairymen themselves are doubtful, though much salt is there expended. Respecting the heating of the milk, the practice must evidently vary according to the weather; and although it is his opinion, as well as that of Marshall and other well-known writers, that it most requires warming when produced upon poor clay lands, and that upon rich soils it will not bear much heating, yet that is contradicted by Aiton, who says, "he never understood that the milk of cows so fed, or even upon wild, waste land, or moss, requires to be heated more than that of cows fed in the warmest valleys, or on the richest haughs in our best cul.

* This, however, if they occur, can be remedied by opening them with a penknife, and pouring hot water into the incision; then press down the outer rind, put on a litlle salt, and place a piece of slate with a half-pound weight upon it.

+ On the cheese coming into the salting.-louse, it is, in some dairies, taken out of the vat, and after its sides are well rubbed with salt, is retumed into the vat with a clean fresh cloth under it: the top being covered with salt, it is placed on the salting benches, turned and salted twice a-clay, and the cloth changed every second day. On the salting benches it is continued seven or eight days, when it is taken out of the vat, and with a wooden hoop, or cheese-fillet, round it, is put into the salting tub and managed as before described, 
tivated districts." The sponginess and heaving of the cheese, which are sometimes complained of, are faults which Mr. Holland attributes more to inattention on the part of the workpeople than to want of skill-w" their certain preventatives being careful breaking, gool thrusting, frequent skewering, and powerful pressing;" but may not improbably arise, partly from the use of cold and warm milk, which, if mixed together, will generate air. Those of pungency and rankness, which are generally imputed to impurity in the rennet, and by some to the want of salt, he, however, thinks may be also more properly ascribed to the fermentation occasioned by the imperfect discharge of the whey.

\section{GLOUCESTER CHEESE.}

The making of Donble Gloucester, as we learn from Mr. IIayward, who has long held a very extensive dairy at Frocester Court, in the richest part of that county ${ }^{*}$, is as follows :-

When the curd is sufficiently firm for breaking, it is gently and slowly cut crosswise, to the bottom of the tub, at about an inch apart, with a three-bladed knife of fourteen inches long. When it has stuod five or ten minutes, to allow it to sink a little, and the whey to come out as clear as possible, some of the whey is dipped out of it with a bowl, and the curd is again cut. This inust also be at first done slowly, and with strokes at a considerable distance from each other; for, if performed hurriedly, a great sediment of curd will be found in the whey-leads : it should, however, be gradually quickened, and the strokes taken nearer and nearer every time; one hand with the skimming-dish keeping the whole in motion, and turning up the lumps suspended in the whey, while the other cuts them as small as possible. This process may occupy a quarter of an hour.

The curd is now allowed to settle during a quarter of an hour, when the whey is taken from it, and poured through a very fine hair sieve placed over the whey-leads; the dairy-maid then cutting the curd into lumps, from which most of the remaining whey escapes. The curd is then pressed down with the hand into vats, which are covered with large cheese-cloths of fine canvass, and placed in the press for half an hour, after which they are taken out and the curd put into a mill of Mr. Hayward's construction, which tears it into small crumbs, and saves the laborious part of squeezing and rubbing it with the hands, while it also retains that portion of the oily matter which would be otherwise lost to the cheese, and thus occasions a great improvement in the making $t$.

In this pulverized state it is customary with most dairy-maids to seald the curd with hot whey; but Mrs. Hayward considers the clieese richer when not scalded, for this washes out a part of the fat: she therefore merely presses it closely together with the hand, when filling: the vat. The whey should, however, be completely extracted, and the curd filled into the vat as compactly as possible; being rounded up in the middle, but only just so much as that it can be pressed down to a level. A cheese-cloth is then spread over the vat, and a little hot water is thrown over the cloth, as tending to harden the outsides of the cheese and prevent it from cracking. The curd is now turned

* See the Farmer's Series, in the Library of Useful Knowledge, No. 21.

t A plate, with a description of another curd-breaker, may be seen in the Quarterly Journal of Agriculture, vol. iv. P. 384. It is, however, more applicable to slim-milk than to whole-milk cheese. 
out of the vat into the cloth, and the inside of the vat being washed in wher, the inverted curd, with the cloth around it, is again returned to it; the cloth is then folded over, and the vat put into the press, where it remains about two hours, after which it is taken out and dry cloths applied, which should be repeated in the course of the day; it is then replaced in the press until the cheese is salted, which is generally done within twenty-four hours after it is made.

The salting is performed by rubbing the entire of the cheese with finely powdered salt; for if the curd be salted before being put into the vat, its particles do not intimately unite, and although it may become a good cheese, it is loose and crumbly, and never becomes a smooth, close solid mass, like that which is salted after it has been made: but this is neverdone until the shin is closed, for if there be any crack in it at that time it will not afterwards close. The cheese is after this returned to the vat and put under the press, in which more cheeses than one are placed together, care being always taken to put the newest lowest in the press, and the oldiest uppermost. The salting is repeated three times, the cloths being removed after the second in order to efface their marks, and twenty-four hours are allowed to intervene between cach; thus the cheese is within fire days taken from the press to the cheese-room; though in damp weather it should remain somewhat longer. There it is turned every day for a month, when it is ready for cleaning, which is done by scraping with a common knife, the dairy-maid sitting on the floor, and taking the cheese in her lap to jerform the operation. When it has been cleared from all scurf, it is rubbed all over with a woullen cloth dipped in paint made of Indian rerl, or Spanish brown, and small beer; and as soon as the state of the paint will permit, the edge of the cheese, and about an inch on each side, are rubbed hard with a cloth every week. The quantity of salt is generally about $3 \frac{1}{3}$ lbs. per cwt, and one pound of ammatto is sufficient for half a ton of cheese**

The true characteristics of the double-Gloucester consist in its great richness, together with the mildness of its flavour, and that waxy texture which makes it cut, even in thin slices, without crumbling; while its oily matter is retained in toasting, by merely softening itself without being burned; and its sure sign of groodiness is said to be the "blue coat" which appears through the paint on its sides. The single-Gloucester, or toasting-clieese $t$, differs in no manner from the making of the double sort, except in being thimuer-the weight of each seldom exceeding $12 \mathrm{lbs}$, while the double sort is generally about $22 \mathrm{lbs}$ - - and somewhat less salted ; it is also pressed only four days instead of five. It is, howerer, not unfrequently made less rich, by being partially mixed with skimmed milk.

\section{CHEDDAR AND WILTSHIRE CHEESE.}

The Cheddar cheese, though made in the rich midland district of Somersetshire, yet so nearly resembles that of the double-Gloucester that it was formerly passed off in the London market under that name : it may, therefore, be supposed that the process of making must be nearly the same; but it has lately acquired such high repute that it now bears an equal price in its own character.

* See note at page 425.

$\dagger$ Toasting-cheese is by some persons occasionally male, by adding the yollis of four or tive engs to every jound of the curd with which they are mised; and these " egrgcheeses" are celebrated in the north of England. 
The North Willshire, which is also a very rich cheese, is made in nearly the same manner; except that the salt is added to the curd, which is broken, or rather crumbled, into the very smallest possible jieces, after the whey has been drained off. It is then frequently shaped into the form of a pineapple, by being put into a cloth made like a filtering-bag, and hung with the point downwards for twenty-four hours; after which it is put into a net, and suspended the reverse way, which gives it the desired appearance. It thus, however, is of only small size; and indeed those round cheeses, which are formed in the mould, seldom weigh more than a very ferv pounds.

In Wiltshire also a practice prevails in somedairies of making what they call green cheese, which is done by putting into some milk over night two jarts of sage leaves, one of marigold, and a little parsley, which, after being bruised, and thus forming a substance of colouring matter, is afterwards mixed, in such quantities as the maker chooses, with the common curd: no other difference being made in the usual process; though some housewives, instead of merely colouring the cheese, leave in it the entire vegetable substance.

\section{STILTON CHEESE,}

which has become proverbial for its richness, was first made near Nelton, in Leicestershire, by a relation of the landlord of the Old Bell Inn at Stilton, which gave the name; and the house being generally resorted to on the Great North Road, brought it into such reputation that it was long sold at the rate of half-a crown a pound. This, of course, soon occasioned an imitation, and it is now verv generally made throughout the counties of Cambridge, Huntingdon, and the immediate neighbourhood; but, although commonly sold under the name of Stilton, there is yet a considerable difference in the quality, and the price has now been reduced to tenpence and a shilling.

It is made by putting the night's cream, without any portion of the skimmed milk, to the milk of the following morning; but those who wish to make it very fine, add a still greater quantity of cream, and of course the richness of the cheese depends upon the amount which is used. Butter is also said to be sometimes mixed with it. The rennet is then added without any colouring; and when the curd has come, it is taken out without being broken, and put whole into a sieve, or drainer, where it is pressed with weights until completely cleared of whey: when dry it is put, with a clean cloth, into a hooped chessart, and placed under the press, the outer coat being first salted. When sufficiently firm to be removed from this monld, the cheese is placed upon a dry board, and tightly bound in a cloth, which is changed daily, in order to avoid all danger of cracks in the skin, until this is found to be tolerably well coated; after which it is no longer user, and the cheese requires no further care than being frequently turned upside down, and occasionally brushed.

The cheeses of this kind, although not much larger than the crown of a good-sized hat-the form of which they much resemble-and not weighing more than about a dozen pounds, yet require nearly two years to bring them to perfect maturity, for they are not generally thought sufficiently mellowed for use until consilerably decaved; and, in order to forward their ripeness, it is said, that besides their being placed in damp, but warm, cellars, they are sometimes wrapped in strong brown paper, and sunk in a hot-bed.

We are also told, that the flavour of an old cheese may be communicated 
to a new one, of whatever species, by the insertion of some portions being intermixed with it. This is done by extracting small pieces with the sample-scoup from each cheese, and interchanging them; by which means the new one, if well covered up from the air, will in a few weeks become thoroughly impregnated with the mould, and with a flavour hardly to be distinguished from the old one. The clieeses selected for this operation should however be dry, and the blue mould should be free from any portion of a more decayed appearance *.

\section{DUNLOP CHEESE}

has in the last half-century been brought into great vogue for its mild richness, and is now very generally made throughout the counties of Renfrew, Lanark, $A y r$, and Galloway, in various sizes, from a quarter to half a hundred-weiglit; the process of which-as we learn from Mr. Aitont and others-is as follows:-

When so many cows are kept on one farm as that a cheese of any tolerable size may be made every time they are millied, the milk is passer, immediately as it comes from them, through a sieve into the vat; and, when the whole is collected, it is formed into a curd by the mixture of the remet. Where, however, the cows are not so numerous as to yield milk sufficient to form a cheese at each meal, the milk of another meal is stored about six or eight inches deep in coolers, and placed in the milli-house. The cream is then skimmed from the mills in the coolers, and, without being heated, is put into the curcl-vat along with the milk just drawn from the cows, and the cold milk, from which the cream has been taken, is heated so as to raise the temperature to about blood-heat. Phis, inceed, is a matter of great importance; and though in summer $90^{\circ}$ may be sufficient, yet upon the average of winter weather $95^{\circ}$ will be generally found requisite. If coagulated much warmer, the curd becomes too allhesive, much of the butyraceous matter is lost in the whey, and the cheese will be found dry, tough, and tasteless; but if too cold, the curd, which is then soft, does not part readily with the serum, and the cheese is so wanting in firmmess that it is difficult to be kept together: indeed, even when the utmost pains are taken to extract the whey, and give soliclity to the cheese, holes - which in dairy-language are termed "eyes," "wheydrops," and "springs"-frequently break out, and always render them either rancid or insipid.

About a table-spoonful of the liguid remet is generally thought sufficient for 100 quarts of milk, and the curl is usually formed by it within twelve or fisteen minutes; though in some dairies-of course in consequence of the difference of strength in the rennet-it does not come from three-quarters of an hour to an hour, though double the quantity of rennet is used. The curd is then broken with the skimming-dish, or with the hand, and the whey ouglit to be taken off as spectily as possible; though without pressing, as the least violence has been found to make it come off white, and thus weaken the quality of the cheese + .

* See Transactions of the Irighland Society, N. S. vol. iii. p. 232.

+ See his Jairy Husbandry, chap. iii.; and his Survey of Ayrshire, chap. xiv. sec. 1 ; also ihe Report on Dairy Management, in the Transactions of the Highland Society, N. S. vol. i.

¥ The hest method of separating the whey from the curd is, in the first instance, to lift the edge of the cheese-tub, and let the whey run off slowly from it into a vessel, placed 
When quite freed from the whey, and the curd has acquired a little consistence, it is then cut with the cheese-knife-gently at first, and more minutely as it hardens; after which it is put into the drainer (which is a square vessel with small holes in the bottom, and a cover to fit inside), on which the lid is placed, with a cloth thrown over it, and a slight pressure-say from three to four stones weight, according to the quantity of curd-being laid on, it is allowed to stand from fifteen to twenty minutes, or half an hour. It is then cut into pieces of two inches square; the whey is again discharged; and the weight, being doubled, is replaced. This process of cutting it smaller every half hour, and increasing the weight, until the pressure is upwards of 100 lbs., is continued for three or four hours. It is then cut very small, and minutely salted; half an ounce of salt, or at the most thirteen ounces to twenty-four pounds English, being sufficient.

A clem cheese-cloth rinced in warm water and wrung out, being then placed in the chessart, the curd is put into it, and a half hundred weight laid on it for an hour. It is then put under a press of two hundred weight, where it remains during an hour and a half; after which it is taken out, and a fresh cloth being placed in the chessart, the cheese is turned upside down, and laid, with increased weight, under the press during the whole night. Next morning, and during the three or four days which it must remain in the press, it is daily turned repeatedly, dry cloths being each time used, and the weiglit is gradually increased until the pressure amounts to at least a ton.

When ultimately taken from the press, the cheeses are generally kept during a week or ten days in the farmer's kitchen, where they are turned three or four times every day, and rubbed with a dry cloth. They are then removed to the store-room, which should be in a cool exposure, between damp and dry, without the sun being allowed to shine upon them, or yet a great current of air admitted: this gradual mode of ripening being found essential to prevent the fermentation and heaving of the cheese, as well as the cracking of the rind; but attention must be paid to rub them with a dry cloth, and turn them daily for a month or two, and twice every week afterwards.

The following shows at one view the manner of making it :-

$\begin{array}{cccccc}\begin{array}{c}\text { Date } \\ \text { of } \\ \text { of }\end{array} & \begin{array}{c}\text { Quarts } \\ \text { of } \\ \text { milk. }\end{array} & \begin{array}{c}\text { Tempera- } \\ \text { ture when } \\ \text { set. }\end{array} & \begin{array}{c}\text { Time of } \\ \text { coming } \\ \text { into curd. }\end{array} & \begin{array}{c}\text { Tempera- } \\ \text { ture of the } \\ \text { whey. }\end{array} & \begin{array}{c}\text { Weight of the } \\ \text { cheese when } \\ 3 \text { months old. }\end{array} \\ \begin{array}{c}\text { Jume 15. } \\ 136\end{array} & 98^{\circ} & 1 \text { hour. } & 90^{\circ} & 27 \frac{1}{2} \text { lbs. }\end{array}$

Thus assuming that five quarts of milk produce one pound of cheese : a calculation which is supported by several other experiments made upon the milk of Ayrshire cows ; though, in Mr. Hayward's extensive dairy in Gloucestershire, a mixed breed of a cross between the Gloucester and the Alderney with a Durham bull, are stated to produce $112 \mathrm{lbs}$. of doubleGloucester for every 100 gallons - or about $1 \mathrm{lb}$. of cheese for $3 \frac{1}{i g}$ quarts of

underneath. The tub is then let down to stand a little: after which it is turned onefouth round, and another collection emptied off: thus, by turning the tub a fourth round every time, it is found to part from the curd more pure and quickly. -Trans, of the Highland Soc. N. S. vol. i. pp. 344 and 345.

* Report upon Dairy Management, in the Trans. of the Hirhland Soc. N. S. vol. i.This, however, assumes the stune to be county or Ayrshire weight, which is 16 ibs. of $24 \mathrm{oz}$. each; and it is much to be regretted that the Scotch writers on agriculture do not always reduce their proviucial weights - of which there are three in use-to the common standiard.

VOL. II, 
milk*. The date, however, is not stated, and it probably was at that season when at the highest degree of perfection.

Such are the most usual modes of manufacturing the superior qualities of cheese to which we have alluded; in which the difference employed is in some cases very striking. Thus, in the preparation of rennet, the bag itself is in most parts of England generally used, while in Scotland the liquid decoction extracted from it is so much stronger that it occasions the curd to coagulate, as we have just seen, within fifteen minutes; whereas, in Cheshire, it occupies an hour and a hilf, and not unfrequently more than two hours. Now, it is not only the delay which is thus disarvantageous; for it is well known that the degree of heat at which the curd is set is one of the nicest points in cheese-making, and we cannot imarine how that can be properly regulated if it be allowed to stand so long cooling in the cheesetub.

The temperature to which the last night's milk is heated when there is not sufficient to make a cheese at one meal, and the mode in which the cream is managed, differ also in various dairies; nor does there appear any objection to the practice of making the last nicrht's milk into cheese, provided it be so gradually heated as that the cream does not run the risk of being converted into oil, as it does if too suddenly heated: yet we believe that, when once separated from the milk, the cream can never again be so completely blended with it as to be entirely retained in the curd when set; it, consequently, runs off with the whey, and leaves the cheese of inferior quality.

The skewering of the curd, as practised in Cheshire, is unknown in most other places; and the labour of several women employed for three or four hours in thrusting or hand-pressing it into the vat, is an operation which is generally managed in other dairies with a couple of maids, and in onefourth of the time.

The hoving of the cheese is attributed by Mr. Holland to the imperfection of its fermentation, occasioned in a great measure by the store-rooms -though commonly placed over the cow-house-not being sufficiently heated, so as to occasion its sweating; yet Mr. Aiton-in his account of the Dunlop cheese-objects to lieated stores, as causing an improper degree of fermentation; and says that " no such thing as sweating is known in the Scots' dairies," Neither does he imagine that feeding the cows on clover, or any other herbage-which Mr. Holland supposes to have that effect-can be the cause of cheese becoming hoved; nor that it would be less subject to heave if it were made of all cold or all warm milk. It is, however, not improbably frequently occasioned by the use of a too large quantity of rennet.

The rankness of flavour, which is mostly attributed to the impurity of the rennet, is by others ascribed to the nature of the pasture. In this, both suppositions may be right; for it must be evident that it may be readily occasioned by the use of a large quantity of badly-prepared rennet; and we learn from the management of a dairy in Ayrshire, where every means were taken to avoid the fault, that the cheese still maintained an unpleasant taste of the same description; which could, therefore, have only arisen from the herbage. It was, however, at length uniformly overcome by throwing about half a tea-spoonful of saltpetre into the pail beiore the cows ware

* See the Report of a Gloucestershire Vale Fam, in the Farmer's Series of the Library of Useful Knowledge, No, 21, p. 35. 
milked*. The cracks which frequently take place in cheese, are also by many persons supposed to proceed from lime having been used as a manure upon land laid down to pasture, and afterwards fed by cows; but this is probably a mistake, for it rarely occurs in the Dunlop sort, though perhaps as much lime has been applied to the soil of Ayrshice during the last fifty years as to any other part of the United $\mathrm{Kingdom}$, and $\mathrm{Mr}$. Aiton attributes it rather to the cheese being exposed, before it is dry, to too much draught of air.

The mode of salting is also very differently conducted in several dairies: in those of Gloucestershire and Cheshire the practice being to cure the cheese after it has been removed from the vat; while in those of Scotland, and some other places, the salt is minutely mixed into the curd previous to its being put into the vat. Both practices, nevertheless, appear to be attended with equally good effects; but the latter certainly occasions less trouble, without any waste of salt.

\section{SKIM-MILK CHEESE,}

male of milk from which the entire of the cream has been taken, is, of course, more or less palatable in proportion to the time during which the milk is allowed to stand; for if that be so long as to deprive it entirely of the butyraceous, or oily matter, it becomes incligestible, and so hard, that in Suffolk, where large quantities of it are mate, it is said that instead of being cut with a knife, it is usually chopped with a hatchet.

The milk should, if possible, not be allowed to become sour; and the moment it has been skimmed, it should be heated to not more than animal leat, or about $90^{\circ}$; for, if put together too hot, its toughness will be increased; and as the curd coagulates more readily than that of full-milk cheese, the same degree of heat is not necessary. This is the chief perceptible difference in management; except that the curd is more difficult to be broken, and that the cheese needs less of the press; but in all other respects the mode of making is the same. It will also be sooner ready for use than whole-milk cheese of the same weight: those of 14 to 20 lbs. weight of that description, requiring, in the opinion of experienced dairymen, from nine to twelve months in ripening, and those of a larger size still longer.

\section{CREAM CHEESE,}

being in general only wanted for immediate use, is, in fact, little else than thick, sweet cream, dried by being put into a small cheese-vat of about an inch and a half in depth, perforated with small holes in the bottom to allow any portion of the milk which may be mixed with it to escape. It is also covered with rushes, or the long grass of the Indian corn, so disposed as to admit of ito being turned without being handled; and it is never pressed except gently by the hand between cloths. It is thus liept in warm situations to sweat and ripen; for if once penetrated by frost, or even clilled, much of its mellow richmess is lost, and it becomes comparatively insipid. The extreme of heat should, however, be equally guaried against, or it becomes rank; and, therefore, sone judgment is requisite in the time for using it in perfection $\dagger$.

* See the Farm Report of Kyle, in Ayrshire, in the Farmer's Series of the Library of Useful Knowledgre, No. 12, p. 46.

t See Twamley on Dairying, p. 64. 


\section{NEW CHEESE,}

or, as it is in some places provincially termed, "slip-coat," is only made in the early part of summer, when the coivs have been turned out to grass, and is formed entirely of new milk, with about one-third of warm water added before the rennet is put to it. The whey is then gently poured off, and the curd is carefully kept entire until put into a vat of considerable diameter, but only about an inch in depth. It is very gently pressed for a few hours only, and when removed from the vat it is covered with a cloth, which is frequently changed; and so soon as the skin is formed it is considered fit for use.

Formerly much cheese was made of the milk of sheep and goats-more especialiy the former-either separately or together, and sometimes mixed with that of cows : a practice which is still extensively continued in many parts of the Continent. In this country, however, it now only occasionally exists in the mountainous parts of Scotland and $\mathrm{W}$ ales, though the flavour of the cheese is by many persons esteemed, and it is still in no little request; but we are not aware of any particular mode of its manufacture which needs description, and if we are to believe what is said of it, the aroma which is so much admired may be partly attributed to some not very delicate mixtures which it imbibes in the act of milking *.

\section{FOREIGN CHEESE.}

As to the foreign sorts of cheese, of which large quantities of various descriptions are made all over the Continent, we can only touch the subject so far as to mention that the Gouda, Eidam, Friezland, and other whole and skim-milk cheeses which are imported into this country from Holland, are manufactured so much in the same manner as the common modes here practised as not to require any particular description. That of Parmesan is prepared in that part of Italy generally known as the Lodesan, which lies between Cremona and Lodi, comprising the richest portion of the Milanese : the cows being kept in the house nearly the whole year round, and fed during the summer with cut grass, and in the winter, upon hay. The weight of these cheeses varies from 60 to $180 \mathrm{lbs}$. ; it consequently requires a great number of cuws to produce one of even the smallest weight, and as the farms in that territury are not large, it is usual for the dairymen to club together; the person on whom devolves the task of making the cheese keeping an account of the milk received, and, after the charge of manufacture, apportioning the produce of the sale accordinglyt. That being a plan which we rlo not adopt in this country, it necessarily can only be carried into effect by those who have a large stock of cows; but the cheese, being sold in London at such an extravagant price, may, however, induce some large English farmers to make an imitation; we therefore insert the process, as extracted from the "Journal de Physique," in the note at foot..

* See the Farmer's Magazine, vol, viii. p. 434, on the Perthshire Husbandry.

t This plan is very commonly adopted by the small farmers in Switzerland, who associate themselves in companies, having among them a certain number of cows; and it is there considered a scheme of essential economy, for it spares both the trouble of making, and the cost of erecting a dairy, with the necessary utensils, and being committed to the care of an experienced person, the cheese is certain to be of superior quality : such ats those of Neufchâtel and Gruyêre. See a French pamphlet, entitled "Des Associations Rurales pour la Fabrication du Lait," by M. Charles Lullein ; which, in a small compass, contains a great deal of valuable infurmation.

+ "The summer cheese, which is the best, is made of the evening milk, after having been shimmed in the morning and at moon, mixed with the morning milk, which is also 
We may also add, that in some parts of Saxony, cheese, which is said to be of very fine quality, is partly formed from potatoes, and, if equal to the account given of $i t$, is worthy of trial. It is made in the following manner :-

The potatoes of a large, white kind, are those to be preferred, and after being boiled they are peeled, when cool, and reduced to a pulp, of equal consistence, either by being grated or gromd in a mortar. To five pounds of this pulp there is added one pound-or about a pint-of sour mills, with the usual quantity of salt to impart a flavour: the whole is then kneaded together, and, being covered up, is allowed to remain for three or four days, according to the season. At the expiration of this time, the pulp is again kneaded, and placed in one or more small wicker baskets, in order to get rid of the superfluous moisture: the pulp is then moulded into form by being placed in small pots, in which the cheeses are allowed to dry in the shade during about fifteen days; after which they are put in store. The older they are the better they become; and, if kept dry, they will keep for a great number of years. Three kinds of this cheese are made : the first, or most common, according to the above proportions; the second, with four jarts of potatoes, and two parts of curdled milk; and the third, with two parts of potatoes and four of milk. Ewe milk is as frequently employed as that of cows, and imparts a pungent taste, which to many palates is found agreeable*

skimmed at noon. Both kinds of milk are poured together into a large copper cauldron, of the shape of an inverted bell, which is suspended on the arm of a lever, so as to be moved on and off the fire at pleasure. In this vessel the milk is gradually heated to the temperature of about 120 degrees; after which it is removed from the fire, and kept quiet for a few minutes, until all internal motion has ceased. The rennet is then added, which is composed of the stomach of a calf, fermented together with wheaten meal and salt: the method of using it being, to tie a piece, of the size of a hazel-nut, in a rag, and steep it in the milk, while held in the hand, and squeezing it from time to time. A sufficient quantity of the rennet thus soon yasses through the rag into the milk, which is now to be well stirred, and afterwards left at rest to coagulate.

"Within about an hour the coagulation is complete; and then the milk is again put over the fire, and raised to a temperature of 145 degrees. During all the time it is heating, the mass is briskly stirred, till the curd separates in small lumps : part of the whey is then taken out, and a few pinches of saffron are added to the remainder, in order to colour it. When the curd is thus sufficiently broken, nearly the whole of the whey is taken out, and two pailfuls of cold water are poured in. The temperature is thus lowered, so as to enable the dairyman to collect the curd by passing a cloth beneath it, and gathering it up at the corners. It is now pressed into a frame of wood, placed on a sulid jlatform, and covered by a round piece of wool fitting into the mould with a heavy weight at top. In the course of the night it cools, parts with the whey, and assumes a firm consistence. The next day one side is rubbed with salt, and the succeeding day the cheese is furned, and the uther side rubbed in like manner; this alternate salting being continued for about forty days. After this period the outer crust of the cheese is pared off'; the fresh surface is varnished with linseed oil ; the convex side is coloured red; and the cheese is fit for sale."

It would thus appear that this highly esteemed cheese is made of skim-milk, and yet the pores are filled with an oily substance, which seems too rich to be imparted by the butyraceous matter of milk which has been deprived of its cream ; it is therefure commonly supposed that some portion of sweet oil is mixed with the curd, but we cannot state it as a fact; aml even if correct, we have no information upion which we can rely of the quantity or mode of its application.

* Bullet, de la Soc, d'Encour. Ag., 7 bre. 1829 : p. 393. 


\section{Chapter XXXVIII.}

\section{ON CALVES: THEIR REARING AS STOCK-AND FOR VEAL.}

cows.

IT has been shown bv some very interesting experiments, made a few years ago at Paris, by M. Teissier, respecting the periods of gestation in different animals, that the time during which the cow goes with calf is very uncertain; for out of 575 , of which an account of the date was taken when they were put to the bull, and when they calved, it was found that-

$\begin{array}{lll}21 & \text { calved between the 240th and } 270 \text { th day : the mean term being } 259 \frac{1}{2} \text {. } \\ 544 & \text { Ditto } & 270 \text { th }, \text { 299th } \\ 10 & \text { Ditto } & 299 \text { th , 32lst }\end{array}$

In most cases, therefore, between nine and ten months may be assumed as the usual period; though with a bull calf, she has been generally observed to go about forty-one weeks, and a few days less with a female. It seldom happens that more than one calf is produced at a time, thourh sometimes two, and even three-though very rarely-are brought forth*; and in these instances, if a male and female appear together, the latter is commonly incapable of procreation, and is by country people called a "free martin." These supposed barren cows have, however, been occasionally known to produce calves; and the bull is always perfect. The cow is always ready to take the bull soon after calving, but should not be permitted until four or five weeks have elapsed: it however occurs at various other times during the spring and summer, thus enabling the farmer to regulate the birth at such time as may be to him most convenient; but may be hastened by high feeding $t$, and is known by the appearance of her bearing. It lasts for three or four days, and recurs again in about three weeks, if the cow has not conceived; otherwise she will show no disposition to receive him. This, however, is sometimes deceptive, as the period of her being in season occasionally passes over without conception, and it was not until between the third and fourth month after copulation that pregnancy could be ascertained, by the dropping of the belly and the motion of the foetus. Late discoveries in veterinary science have now, however, enabled every skilful practitioner to decide the point within six or eight weeks; and, indeed, with proper attention to the mode of examination, no intelligent breeder can be easily deceived + .

When the complete term of gestation approaches, the cow should be placed apart from other cattle, in a quiet paddock close to the homestead, in order that assistance may be at land in case of a difficult birth; and if she be full in flesh, or has been previously fed upon very nourishing food,

\footnotetext{
* An instance is mentioned in the Bulletindes Sciences of a cow belonging to a French agriculturist, which produced nine calves at three successive births, namely, four at the first, three at the second, and two at the third; all of which, except two of the first birth, grew up and were nursed by the mother; but the heifers afterwards produced each only a single calf.

t Some nostrums recommended for this purpose are too absurd to be repeated; but that alvised lyy Parkinson, in his "Treatise on Live Stock," is so harmless that it may be safely tried:- Gi Give a quart or more of milk, immediately drawn from a cow that is in season, before the bull has been admitted to her, and in three or four days it will have the desired effect." Vol. i. p. 101.

+ See the Farmer's Series, in the Library of Useful Knowledge : Youatt's "Cattle," ग. 533.
} 
it will be not amiss to open her bowels with a gentle dose of medicine. The time of parturition will evidently appear by the springing of her udder and the dropping of her belly, together with a discharge from the bearing, and, as it draws nearer, her symptoms of uneasiness and moaning will show that it cannot be far off. If the weather be bleak, she should then be housed for a few days, but without being stalled, and a good bed of straw should be laid under her. The natural progress of the birth should not be interfered with, nor should she be too closely approached; for, although it should be tedious, "Nature will, in most cases, at length safely accomplish its object," and if not, other means must be used : for instructions respecting which we confidently refer to Mr. Touatt's Account of Cattle. In instances, however, which present nothing more than usual delay, without any supposed wrong position of the foetus, he recommends that "a pint of sound warmed ale should be given in an equal quantity of gruel; warm gruel should be frequently administered, or at least put within the animal's reach; and access to cold water should be carefully preventerl. To the first pint of ale should be added a quarter of an ounce of the ergot of rye (spurred rye), finely powdered; and the same quantity of the ercot, with half-a-pint of ale, should be repeated every hour, until the pains are reproduced in their former and natural strength, or the labour is terminated."

"Parturition having been accomplished, the cow should be left quictly with her calf; the licking and cleaning of which, and the eating of the placenta-that is, the after-birth, or cleansing * -if it is soon discharged, will employ and amuse her. A warm mash should be put before her, and warm gruel, or water from which the chill has been taken off; two or threc hours after which it will be prudent to give an aperient drink, consisting of a pound of Epsom salts and two drachins of ginger. Attention should likewise be paid to the state of the udder; for it is very subject to inflammation after calving. The natural and effectual preventative of this is to let the calf run with her, and take the teat when it pleases, as the tendency to inflammation is much diminished by the calf frequently sucking; and, should the cow be feverish, nothing soothes or quiets her so much as the presence of the little one." It sometimes happens, however, that if the teats be excoriated and very sore, the cow shows a disinclination to being sucked, and danger is thereby incurred of losing both animals; in which case they should be fomented three or four times a-day with warm water, after which she should be carefully and very gently milked-the calf being suckled by hand-and the teats afterwards dressed with the following ointment :-

"Take an ounce of yellow wax, and three of lard, melt them together, and when they begin to get cool, well rub in a quarter of an ounce of sugar of lead, and a drachm of alum finely powderedt."

Cows are very subject to abortion, fram various causes; either by improper treatment, from over-exertion, or, not unfrequently, by being kept in too high condition; and it is not a little extraordinary that it sometimes becomes infectious among a herd, and occasionally throughout whole dis-

* Cows eat this excrement with avidity, and nature seems to have intended it for them as a medicine, for it has never been kuown to injure the health; yet unexperienced people very frequently cause it to he removed, as being thought disgusting. If not soon discharged from the body, the aperient drink, recommended after calving, should be given, together with the ergot and ale, lest serious inconvenience should arise from its being too long retained in the womb.

+ Youatt on Cattle, p. 552. 
tricts. When the cow slinks, or slips her calf, it is often putrid before it is brought forth, and the noisome odour which it emits, if it be allowed to remain only during a short time in the field, has some sympathetic influence on the other cows, which in a few days will perhaps cause several of them to cast their calves prematurely. The herdsman should, therefore, cautiously watch every appearance which may show the least danger of abortion in any one of them, and immediately remove her from the rest: the symptoms, as described by Mr. Youatt, are these :-

"The cow is somewhat off her feed-rumination ceases-she is listless and dull - the milk diminishes or dries up-the motions of the foetus become more feeble, and at length cease altogether-there is a slight degree of enlargement of the belly-there is a little stargering in her walk-when she is down she lies longer than usual, and when she grets up she stands for a longer time motionless. As the abortion approaches, a yellow or red glairy fluid runs from the vagina, a symptom which rarely or never deceives -her breathing becomes laborious, and slightly convulsive. The belly has for several days lost its natural rotundity, and has been evidently fallingshe begins to moan - the pulse becomes small, wiry, and intermittent. At length labour comes on, and is often attended with difficulty and danger.

"When symptoms of abortion appear, the cow should be removed from the pasture to a comfortable shed. If the discharge is glairy, but not offensive, it may be presumed that the calf is not dead: this may be assured by the motion of the foetus, and thus it is possible that the abortion may be yet avoided. She should then be copiously bled; and a dose of physic should be given immediately after bleeding. The physic beginning to operate, half a drachm of opium and half an ounce of sweet spirit of nitre should be administered. The animal should be kept quiet: gruel may be allowed; but nothing like those comfortable drinks which are generally recommended by the cow-leech.

"The treatment thus differs little from that of parturition : but should the discharge be fetid, the natural conclusion will be that the foctus is dearl, and it must be grot rid of as speedily as possible; if fever exists, bleeding may be requisite; or, perchance, the aforesaid comfortable drink may not be out of place."

The next accident to which the cow is exposeil, is that called "dropping after calving;" which is a local inflammation that generally occurs very soon after the birth. It is denoted by the animal pitcously lowing, becoming restless, and heaving laboriously at the flanks. The udder becomes inflamed, accompanied by a considerable diminution of milk, and followed by other symptoms, which generally terminate in delirium and death. This disease is naturally much dreaded, and no time should be lost in calling in the cow-leech while it is yet manageable. If, however, one of competent ability be not at hand, the farmer may safely follow the directions which will be found in Youatt's "Cattle "" and we may justly observe, that this portion of "The Farmer's Series" will not have been written in vain, if it induces an earlier attention to the diseases of domesticated animals.

\section{REARING OF CALVES.}

The moment the calf is dropped, Nature teaches the cow to cleanse its skin by licking off the slimy matter with which it is covered; but if she shows any disinclination to it, this may be got over by sprinkling a handful of common salt over it, after which she will generally perform this necessary duty without farther delay. About an hour after the birth, it is a common practice, among many farmers, to give half a pint of 
lukewarm gruel to the calf, instead of the first milk, or "beestings," of the cow, which is then thrown away. This, however, is highly improper; for this first aliment of the newly-born animal is a strong and viscid fluid, of an aperient property, which powerfully assists in dischargingr the glutinous fieces which have been previously accumulating in the intestines. It is also of a peculiarly nourishing quality, seemingly intended for the very purpose of early invigoration; and, as it is not fit for the purposes of the dairy, the calf should always be left for a few days with its dam.

When the calf is cleaned, and has begun to suck, the navel string should be cxamined, and if it continues to bleed, Mr. Youatt says-" That a ligature should be passed round it closer, but, if it can be avoided, not quite close to the belly. Possibly the spot at which the division of the cord took jlace may be more than usually sore: a pledget of tow well wetted with Friar's balsam should then be placed over it, confined with a bandage, and changed every morning and night; but the caustic applications that are so frequently resorted to should be avoided." Sometimes, however, when all appears to be going on well, inflammation suddeuly appears about the navel, between the third and eighth, or tenth day; it should, therefore, be daily examined until all danger of that kind is past, and if any swelling of the part, accompanied by redness and tenderness, should appear, the tumour should be well fomented with warm water until it be dispersed; or, if the inflammation continue, then surgical assistance must be resorted to, or the disease may become fatal.

If a male, the animal should be castrated as soon as possible after a month has elapsed after its lirth ; for the longer that operation is deferred, the greater will be the danger attending it.

The forwarding of calves to maturity, whether intended to be reared for stock, or brought to an early market as veal, are objects of great importance in every point of view, and their treatment has necessarily called forth the ingenuity of the most intelligent breeders, for the purposes of developing and improving their natural qualities. When meant to be brought up for stock, the cows should be so managed as to calve down by the middle of the month of Mny at the farthest; as late calves will not be sufficiently grown to hardily stand the winter, and the earlier they are dropped in the spring the better will they be able to meet the inclemency of the scason. Those, lowever, which are dropped in the winter, and housed during that inclement season, will thrive surprisingly by being turned into the succulent pastures of the ensuing spring. There are two modes of rearing them: the one, to allow them to run at large, during a year, with their dams; the other, to wean them after a few days or weeks old. Of these, when a farm contains sufficient rough pasture, the first is unquestionably not only the least troublesome, but is generally found to be productive of the best cattle, and in this manner a large portion of them are fed. It is, however, in frequent instances thought more prudent to reserve the milk for the purposes of the dairy, and then the calves must of course be weaned.

The process of ueaning is variously managed by different farmers; but, whatever may be the plan which they adopt, they should never lose sight of the object of rearing the animal to perfection: which can only be properly done by affording it ample sustenance while it is young. They should recollect that the cow's milk, which is intended by nature for its support, contains a large quantity of the richest nutriment, and if the calf be deprived of that, it should have an equal amount of other nourishment; for, if stinted, it will assuredly grow up to be a gaunt and ill-thriven brute.

When not let to run with the cow, the most advisable mode, as it regards 
the calf, is to place it loose in a crib, and to suckle it by hand with the mother's new milk, of which it will consume, for some time, not more than about four quarts per day: the quantity, however, must then be gradually increased, as it will, in the course of a few weeks, require as much as three gallons. If the weather be fine, it should be, within a fortnight or three weeks, turned out daily in the orchard, or some well-sheltered enclosure of sweet herbage, and as it will, in the course of ten or twelve weeks, have acquired some relish for the pasture, it may be regularly iremed by gradually diminishing the quantity of milk, and then substituting the skimmed for the new. Calves may, however, be reared with skimmed-milk and meal, without any portion of new-milk, except the first few days' beestings, and many persons give them nothing but water-gruel and hay-tea, within a fortnighth after they have bcen removed from the cow*. Hay-tea and linseed jelly are also very nutritious, and calves may be weaned on them without any other foodt. We have heard of a farmer who, for many years, successfully reared a great number by takiag them from the cow at about seven clays old, then giving them skimmed-milk for ten or twelve days more, with a few turnips cut into small pieces, which they soon learn to nibble and eat, after which they were turned into the turnip field, without any farther care or trouble than carrying them a small bundle of fresh barley or oat straw every night and morning, laying it under the hedge which was most sheltered from the wind. He often turned them out in the month of January, yet he never lost any of them, and they all appeared in health and good condition+.

Various schemes have indeed been tried, with some success, for the weaning of calves without any, or at least with only a small portion of milk, and such plans are well deserving of trial, in all those places where that article is of general consumption. By many persons they are taken within three days from the cow, and are fed with lukewarm skimmed-milk and water, having a little bran or oatmeal in it, and by others with new whey along with meal. If weaned on skimmed-milk, the calves ought however to fall in December, or within a month of that time, and then be kept warm by housing. Heifers with their first calves are, however, exceptions; as such do not become good milkers if their calves do not suck the whole season: but with the second calf they are treated like the rest\$. Treacle and linseed oilcake-in the portion of half an ounce of treacie and one ounce of powdered oil-cake, mixed with a pint of skimmed-milk, properly incorporater, and given along with lukewarm whey, or hay-tea, have also been advantageously adopted; in the north of England they are, for the first four or five weeks, fed upon equal quantities of new and skimmed-milk, after which they are gradually brought to drink gruel, made of bean or ontmeal, mixed with one half of buttermilk. Besides this, in some of the southern counties, pot liguor is often given with good effect instead of gruel; and the following plan is successfully practised in North America, where it has received a prize from the Agricultural Society of Massachusetts :-

The calves, when three days old, are taken from the cows, and fed with barley and oats ground together, which is made into gruel-one quart of

* Suffolk Report,3rd edit.p. 208.

† Bath Papers, vol. v. p. 465. - Linseed jelly is made by putting one quart of seed to sixquarts of water, and allowing it boil during ten minutes, Hay-te.s is made by infusing such a portion of fine sweet hay as will fill an earthen vessel on being lightly fressed with the hand, and boiling water being poured upon it : the vessel is then closed, and in a couple of hours a strong liquor produced, wheh will keep good for a couple of days : it should be used lukewarm, and, if given without linseed, should be mixed during the first few days with three parts milk, to be afterwards reduced to one-fourth.

f Bath Yapers, vol. ii. p. 154.

\& Sussex Report, p. 263. 
the flour being boiled during half an hour in twelve quarts of water; of which one quart is to be given, lukewarm, to each calf, morning and evening. In ten days a bundle of soft hay is placed in the stable, which they will soon begin to eat, and a little of the flour is put into a small trough for them occasionally to lick. They are thus fed during about two months, grarlually increasing the quantity; and it is said that half a bushel of the corn is sufficient until the calf is entirely turned to grass, to which he is occasionally turned out during the day.

When the object of the farmer is to rear more calves for stock than he has cows, this has also been successfully practised by purchasing a stranger calf, and putting it along with her own to the same cow, both being put to suck, one at each side, exactly at the same time, and leaving them there for fifteen or twenty minutes, by which time the milk will be drawn away. The cow at first shows great dislike to the stranger, but in a few days receives it very quietly. They are thus kept in the house, and as they advance in age, they eat porridge, hay, sliced potatoes, or any food that is usually given to them, and in about three months they are finally turned out to grass; after which a couple of strangers are purchased, and the same plan pursued with them during three months longer. At the expiration of that periodsupposing the cow to have calved in the month of January, or the early jart of February-the first week in August will have arrived, and this set beingr then ready for weaning, a single ealf is put into the feeding pen aud fattened for the butcher: by which means the cow will have snckled five calves ${ }^{*}$. Mr. Lambert also say's, in his "Ohservations on the Rural Affairs of Ireland," that it is not a bad plan for those who keep many dairy cows, when there is an indifferent milker, to put two calves on such, and turn them out to grass along with her; as the calves will extract more milk, and a cow that has but little milk being of a quality rich in proportion, will afford quite sufficient for two calves, when accustomed to the strange one for a week or ten days before being turned loose. The plan would pro. bably answer the purpose at first, but we should imagine that it must stint the future growth of calves.

These different modes are all more or less productive of economy in the expenditure of milk, and, in most cases, have been found to forward the proper growth of the calf: much, however, depends on the regularity of feeding. The common practice is to give the food only twice a-clay, morning and evening, and then to allow as much as will satiate the appetite; thus filling the stomach with such a quantity as must in some degree impede the digestion. Now, in every case, the closer we adhere to nature, the better will the animal thrive, and if allowed to remain with the cow, the calf will be seen to suck her frequently ; he should, therefore, be fed at least three times a-day, at regular periods, by which means, and being allowed room for excrcise, he will be greatly improved in health and condition : it being, however, understood that he is not then intended to be fattened. Care also should be taken not to change his food too suddenly ; for, although the imperative calls of appetite compel him to forego that which is most natural to him, yet, having been once taught to adopt a substitute, he obtains a relish for it, and will not so readily accommodate himself to any other: which especially teaches the propriety of weaning him very graduallyt.

Whatever may be the process employed, it must partly depend on the state of the season when the calf is dropped, and in the case of being weaned

* See Youatt's Cattle, p. 170.

+ See the complete Grazier, 6th edit, chap. vi. 
on milk, it will probably occupy nearly four months; but the animal, if turned occasionally out, will begin to nibble the grass when about six weeks old, and may then, within two or three weeks more, be left to graze, until the season becomes wet and cold, when it should be brought, along with other younglings, into a fenced-off part of the farm-yard, where they should be well bedded, with sheds to run under, as lying wet, or having very wet land to lie upon, will incur the risl of some very dangerous disorders.

Their subsequent treatment must be, in a great measure, dependent on the nature of the land, and the sort of food which it can supply; for many mountainous stock-farms being wholly in grass, afford nothing more during the winter than a scanty supply of hay, and the yearling calves are again turned upon the rough pasture cluring the following summer, or until an opportunity offers for disposing of them to other feeders, by whom they are kept during the following winter, partly on turnips in the strawyard, and afterwards either sold, as in-calf heifers, to the cow-keeper; as steers, for working stock*; or, to the grazier, for being fattened. It is indeed a very common notion that, provided the young animals acquire bone and size, their condition is inmaterial; and this, though perhaps not the most profitable mode of rearing, is yet the only one which, in such situations, can be adopted: it, of course, generally repaying the breeder and the dealers, or it would not be continued. Where the farm, however, is of such land, and is so managed, as to supply an abundance of nourishment, the breed of the animals to be reared should be a prime consideration, never to be lost sight of ; and we need hardly say that both sire and dam should be chosen, without regard to any other than imprudent expense, from the very best stock to be obtained in the county : unless, indeed, the main object being the dairy, the calves should be merely intended to be fatted for the butchert.

\section{VEAL.}

Calves, when properly fattened for veal, form a favourite article of diet for the wealthy, and consequently are largely reared for that purpose by many of those farmers, who are situated within districts not too far from great towns, where they can be readily sold; for, as they cannot be driven to market, the expenses of carriage to a long distance would otherwise run away with too much of the profit of feeding them. The business of suckling on a large scale is, therefore, in a great measure, confined to those who have the convenience of salesmen, who regularly send their spring vans through a certain extent of country, for the purpose of collecting the fatted calves, which they dispose of the next day at the neighbouring market: the charge of which-both for carriage and commission on sale-is, if within twenty miles of London, five shillings for every calf, and one shilling more for every guinea which is realized beyond $5 /$. This, however, is in distant places avoided; as it is the practice of the country butcher to bargain for the calf while it is being fatted, and take it away at his own cost and convenience. As the milk of one cow will thus necessarily rear from one to three calves, according to its abundance and the period occupied in feeding, the sucklers are supplied by calf-dealers, who travel in

* On Working Stock, see vol. i. chap. vii. and the Training of Steers, p. 215.

+ Even in this case, it is however said, that some breeds have whiter fleshed calves than others, and that the butchers judge of the colour of the flesh by looking at the inside of the calf's mouth and the colour of its eyes: but we should suppose, that this applies rather to the individual calf, than to the general stock arising out of any particular breed. 
carts built for the purpose, throughout all parts of the dairying districts, where they purchase the young calves, and resell them at various prices from one guinea to thirty shillings a-head, according to their breed, size, and age.

The age at which the calf should be killed ought not to be sooner than four weeks old; for if earlier, it can hardly be considered either palatable or wholesome as human food, and we believe that there is a law in France which prohibits their being killed before they have attained the age of six weeks. They are, however, in many distant places slaughtered within a few days after their birth, and are then, by the Irish peasantry, significantly called "staggering bob." If in prime order, he should never exceed ten weeks, by which time he will probably have reached to 16 or 18 , or, per. haps, if a fine animal, 20 stones weight; for the veal, will, after that lose much of that delicate whiteness which constitutes its chief value in the market. Indeed calves, if completely fat, which weigh only 14 or 15 stones, will often sell for a shilling or two per stone more than one weighing between 20 and 30 ; nor should it be sooner than seven or eight weeks, or the meat will not then have acquired a sufficient degree of firmness.

On this, however, much difference of opinion prevails among the feeders; some holding, that although a fine calf under ten weeks old will command a higher price per stone than one of fourteen weeks, yet the latter will render a larger profit, because he requires less milk, and fattens so much faster after ten weeks old than before, that he will then probably be 25 stone: besides which, there is only the prime cost and the commission of one instead of two calves, to be deducted from the value; and the inferiority of price is more than balanced by these savings, when added to the increased weight of flesh. On this the fullowing estimate, made partly upon a suckling dairy of tiventy cows, in the county of Essex-the expenses of feeding, labour, interest of money, and depreciation of stock, being calculated at $\mathrm{rl}$. per head-may afiord some idea of the different modes:-

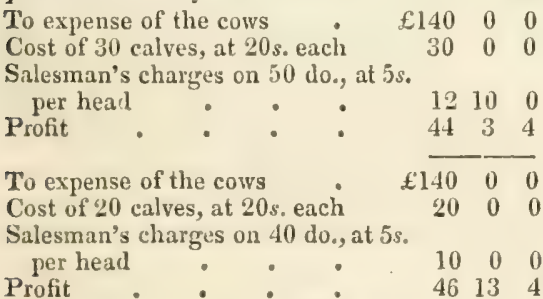
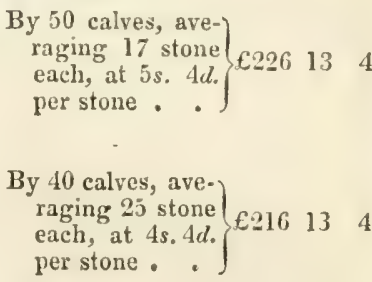

The following are the weights of some calves of the mixed Hereford and Sussex breed, born in the months of May and June; but they were fed upon skimmed-milk and linseed, boiled to the consistency of a jelly, in the proportion of one pint of the jelly to a gallon of milk; and the plan did not seem to answer : it may, therefore, be presumed that if they had been fed upon new milk they would have become fatter :-

$\begin{array}{llc}\text { Age. } & \text { Live Weight. } & \text { Dead Weight. } \\ 10 \text { weeks } & 11 \mathrm{lbs} . & 133 \mathrm{lbs}, \\ 10 \text { do. and } 3 \text { days } & 209,, & 136, ; \\ 11 \text { do. and } 3 \text { do. } & 235,, & 154,, \\ 12 \text { do. and } 5 \text { do. } & 240,, & 160, \text {, }\end{array}$

or, the dead weight about two-thirds of that when alive**

* Sussex Report, p. 262. Several instances are mentioned in Aiton's Account of Fatting Calves in Strathaven, of their having been brought to very great weights; but, unfortunately, he does not mention the period employed in fitting. - See Quart. Jourm, of Agric, vol, v. p. 252. 
The mode of feeding most generally adopted, and the best to be pursued for the purpose of producing delicate veal, is to feed the calf entirely on new milk, taken fresh from the cow, either by sucking it from the dam, or by milking her, and giving it to the calf by hand. Either way is equally beneficial to the calf, though, indeed, it is supposed that the former, being the most natural, and occasioning a greater secretion of saliva, must better promote its growth; but if he be allowed to suck the cow she of course becumes attached to him, and when he is removed at the end of a few weeks, it is extremely difficult to foist a nursling upon her ; so much so, indeed, that she often resists with great violence, and if compelled to receive one, she for a long time withliolds a portion of her milk. Neither can the calf at first consume the whole of the mother's milk, while in the course of a litte time he will require more, and in a few weeks he must be removed. In eitier case, she, therefore, must necessarily have a stranger along with her own, or she must be milked; wherefore many people adopt altogether the latter plan of milking. It is, in fact, in other respects advantageous; for, instead of depending on the precarious supply of the dam, the food of the young animal can thus always be regulated to suit the agre, appetite, and purposes for which the calf is intended; and other mixtures or substitutes can be introduced into the milk, if rendered expedient for economy.

Suckling by hand, as it is termed, or teaching the calf to drink, is a matter which requires great patience, and occupies considerable time; for it is long before he learns to take it from the pail. It is thus performed: the first and second fingers of either hand, being well washed, are presented to the animal's mouth, and of these it readily takes hold, and sucks as greedily as if they were teats. In the meantime a vessel containing milk is placed under the calf's mouth, and while it is sucking, the hand is gradually sunk a little way down in to the milk, so that it may lap without stopping its nostrils. The hand must, therefore, be kept steadily in the milk; for, if too suddenly plunged into it, the calf's nose being immersed, he will want air, and will therefore immediately withdraw his head; in which case, or from any other accident occasioning it, the attempt must be repeatedly renewed until the animal is completely satisfied. A piece of soft leather, tivisted into the form of a teat, and placed in the milk, will however equally answer the purpose; and the plan of thus suckling is considered advantageous, even after the calf has learned to drink, as thus promoting the necessary secretion of saliva.

The manner of feeding is therefore very simple; but as some slight discrepancies occur in the management, we shall here state the chief modes adopted in different districts.

In that around London, every cow is made to suckle her own calf three times a-day, for the first three or four days after calving, and afterwards twice a-day constantly. If she has a very full stock of milk, another calf about the same age is also put to her; but her own calf must always have its fill, and not be robbed by the other. The calves are also frequently bled before being slaughtered*. On one side of everv pen is placed a small trough, that is always kept supplied with chalk, both in lumps and powder; not with a view to render the veal white, as some persons erroneously imagine, but with the intention of correcting acidity in the stomach; without which precaution the calves would be liable to scour. Some persons mix ground barley with the

* Main on the Mode of Fattening Calves in the Neighbourhood of London,-Quart. Jour, of Agric., vol, v. 
clialk, and others give them a ball, or two or three, made of linseed jelly and barley-meal, in order to render them fat in less time than could be done with milk alone*. It is also not uncommon to give them, between the time of their being suckled, about a wine-glassful of common gin, mixed up with as much flour as will make it into balls about the size of a pigeon's egg, which are forced down the calf's throat by drawing out his tongue $t$ : the object of this being to stupify the animal ; and, thus rendering him quiet, to promote his improvement.

On this practice of putting two calves to the same cow, and always allowing her own to have its fill, besides the difficulty of getting a stranger calf to suck her, there occurs that of ascertaining how that can be correctly managed with advantage to his fattening; for if he be allowed to suck before the stranger, he will thus be filled with the poorest sort of milk, and the other will obtain the stroakings, which, though perlıaps less in point of quantity, is of far richer quality : while if the stranger be first suckled, he may perhaps not leave sufficient for the cow's own calf.

The practice of bleeding those calves intended for the butcher, is common anung many persuns, who imagine that the veal is by such means remlered whiter; the operation is therefore often repeated, and the quantity of blood taken away is generally as much as the strength of the animal will allow; others, however, consider twice, with two quarts at each time, as sulicient, and many experienced brecders view it as altogether injudicious. It may, jerhaps, have the effect of rendering the meat more delicate; but we think it cannot but impede the growth of fat.

The district of Strathaven, and some of the neighbouringr parishes in Lanarlishire, liave long been celebrated for the excellence of the veal there produced; and the plan pursued is simply this:-

The calves are all suckled by hand, and some farmers give milk to them only sparingly for three or four weeks, from an idea to render their appetite more keen when at length plentifully supplied; but others, with as grool effect, grive them a full supjly from first to last. For a week or two after they are calved, they can only consume about onehalf of a good cow's milk; but the quantity is gradually increased to as much as the roung animal can drink. Thus, by the time it is four weeks old, it will consume the entire quantity; and in two or three more, if it be of large growth, and thriving, it will probably take the greatest part of the milk of two cows. It is, therefore, common to give to the very young calves, or rather to those which are being reare for stuck, the milk that is first drawn from the cow-which is weak, and abounding in serum-and to give the last drawn, or richest part of the milk, of two, or perhaps three cows, to those which, being alvanced to five or six weeks old, it then becomes expedient to raise promptly to the greatest degree of size and fatness.

Some mix eggs, and others put meal into the milk, in order to increase its nutriment; but the best feeders do not approve of these admixtures, as they say it darkens the flesh. Bleeding is not approved, nor there ever practised; neither are infusions of linseed, or barley, ever used, nor any other food given than an abundance of pure milk: chalk is, however, never omitted $t$.

Nearly the same practice is pursued in a place called Abbey-Holme in

* Mliddleton's Survey of Middlesex, second edition, p. 426.

+ Complete Grazier, sixth edition, p. 60.

\pm Aiton on the Dairy Husluandry, chap. iii. sect. i.; and Quarterly Jumnal of I griculture, vol, v. 
Cumberland, where veal is produced of very superior quality, both in size, colour, and fatness: the plan there being, as stated in the Report of that county :-

To suckle the calves during the first two or three weeks in the common way; after which they are fed upon the last drawn milk of several cows ; the first drawn being always given to the youngest calves, of which they have a regular succession; and in this way, meat of extraordinary delicacy, as well as firmness, is said to be carried to market within seven or eight weeks. The calves are, however, fed in sheds, kept always as nearly as possible at the same temperate degree of heat, and the animal is fastened by the head with a strap around it, on either side of which there is a ring running upon a small stake; so that, although prevented from licking himself, or from turning round, he is not so confined as to prevent him from lying down or rising at pleasure.

The plan here stated, of always keeping the shed at an equal degree of temperature, is certainly judicious, as greatly tending to promote the improvement, in all animals, of flesh; and quietude also cannot be too strongly recommended. Thus the calf-pens are generally separated into single stalls, and boarded about four feet high; they are also darkened, in order to deprive the young animals of their inclination to gambol; and, with the same intention, many of them are no larger than just to allow thise calf to turn round. Some, indeed, are so narrow as not to admit of its turning; and Malcolm describes one, kept in total darkness, having only space enough to move a little backwards and forwards. A small hole was made just large enough to admit its head; and when the suckler comes to administer the milk, the animal seeing the light, puts out its head, which the suckler then puts into the pail. The door of the pen is also made to fall down, so that it becomes a platform to the tail of the cart, into which the calf readily walks, and is thus secured without that difficulty which is otherwise generally experienced $*$. Such extreme restraint may not be quite necessary, though, when fattening for the butcher, it perhaps promotes the object; but when calves are to be reared for stock, there can be no doubt that the plan of turning them out into the open air, and only housing them in the winter at night, is the more advisable.

Extreme cleanliness, and the absence, as far as possible, of all kind of damp, are also essential requisites in the construction of calf-pens: the floors should therefore be raised about a foot above the earth, and either closely latticed, or boarded and pierced with holes for the escape of the urine. Floors of timber being, however, much subject to rot, a more economical method is that of laying them of bricks set on edge, with a narrow space left open between brick and brick. Clean fresh straw, frequently removed, should also be allowed as a bed. Too great attention cannot, indeed, be paid to keeping the pens perfectly clean and dry; nor can too great regularity be shown in the mode of feeding. If the calf be either admitted to the cow, or suckled, an hour later than usual, he becomes restless as well as greedy, and thus, taking a larger quantity of milk than that to which he has been accustomed, and this occasioning indigestion, a mass of curd is often collected in the stomach, without power of throwing it off; or else a looseness, or scouring, is sometimes brought on, which invariably throws him back during the time it lasts, which is frequently so long as to bring him to an age which renders his

* Compendium of Modern Husbandry, vol, i. p. 354. 
flesh coarse, even if it can be made fat. This, indeed, is one of the most frequent as well as annoying diseases to which the animal is subject; and though not commonly fatal, is yet sometimes so violent that the calf, beginning to droop, and feeling extreme pain, refuses to suck. Medical remedies should be then immediately applied: a mild purgative should be first given; after which the medicine commonly known as the "calves" cordial" may be administered, along with the frequent use of thick gruel, and occasional warm mashes of bran mixed with a little pea or bean flour *. In its earlier stages, it is said that a little decoction of rennet is effectual ; and in Essex, where calves are more commonly suckled than in any other part of England, the following remedy is used:-

Balls composed of the powder of fenugreek, flour, and powdered chalk, made up with ale, to be given twice a-diay, to the amount of two ounces weight; or fenugreek seed, previously reduced to powder, is beat up with a couple of new laid egirs, the shells being also finely powdered, and made into a proper consistence with gin, the quantity of which should be increased after the first day. Or, two tea-spoonsful of rhubarb, and a tablespoonful of peppermint-water mixed together: and, if one dose does not stop it, a second is given with a little red wine added + .

Chalk is, however, one of the best preventives, and a lump of it should always be liung up in the pens of sucking calves; but it is said, in the Survey of Buckinghamshire, that the scouring may be cured by merely sprinkling cold water on their loins. Judging from common circumstances, it would be thought to have a contrary effect; but the simplicity of the remedy renders it at least worthy of trial, as, if not productive of good, it is not likely to do harm. In the opposite disease of costiveness, a little warm mutton broth, or water in which a piece of fat bacon has been well boiled, will generally be found to afford relief + ; or, if very obstinate, two ounces of Epsom salt may be dissolved in it.

\section{DAIRY ESTIMATES.}

The profit upon the fatting of calves for veal has been variously estimated, and must necessarily differ according to the nourishment afforded by the cow, and the price which can be obtained for the meat. Mr. Aiton says, "that a thriving calf which can be got newly dropped at from $6 s$. to 8 s. may be raised on the milk of one cow to the value of $50 \mathrm{~s}$. or $60 \mathrm{~s}$. by the time it is four or five weeks old ; and to $4 l$., or more, when six or seven weeks of age. If it be kept much longer, it would be proper to give it more than the milk of one cow; and by the time it is nine or ten weeks old, it will in ordinary cases sell at from $5 l$. to $7 l$.; the calculation among farmers skilled in that branch of dairy-husbandry being, that milk is worth at the proper season, and when a thriving calf is fed, from $3 \frac{1}{2} d$. to $4 d$. per Scotch pint, or about $1 \frac{3}{4} d$. to $2 d$. per quart; and many have used their milk in feeding veal when they could have sold at these prices." \$

This, it is to be observed, alludes to the markets of Glasgow and Edinburgh; yet the estimate made by Mr. Main, in the vicinity of London, supposes-"That if a calf be suckled for ten weeks, he will weigh from eighteen to swenty stone dead weight, and will fetch about $5 l$.; from which is to be deducted $30 \mathrm{~s}$., for which the calf might have been sold at a week old, and the salesman's commission: thus leaving $3 l .5 s$, or $6 s$. $6 d$. per week for the cow's milk. 'That a good cow will fatten off two calves while

* See Youatt's "Cattle," p. 559 .

+ Essex Report, vol. ii. p. 281.

+ Gen. Rep. of Scotland, vol. iii., p. 76. Ayrshire Rep. p. 441.

$\$$ Aiton on the Dairy Husbandry, p.95.

VOL. II. 
she is in milk, and some which he has had, two and a half; but this can only rarely be counted on." Still, taking one cow with another, kept for the purposes of suckling, he calculates that her returns will be about the sum which is commonly calculated-namely, $12 l$. per annum*; but others rate them much higher.

Thus it has been found by experience that the milk of a good cow will in seven weeks feed a calf to about 14 stone dead weight; he consuming at the rate of-



or about 160 gallons; and if the milk upon which he has been fed be of rich quality, he will then be in prime order for the market; the meat probably commanding $8 \frac{1}{2} d$. per $1 \mathrm{~b}$., or $5 s$. $8 d$. per stone, which will bring his value to $3 l .19 s .4 d$. Now, it is very commonly found that cows of a fine breed will yield as much as 900 gallons of milk yearly $t$, which thus supposes them to bring $5 \frac{1}{2}$ calves to market; but calculating only upon five, or an equal portion of meat, the account-with some deduction from the charges in case of the calves being fewer, and grown to a larger sizewill stand thus :-

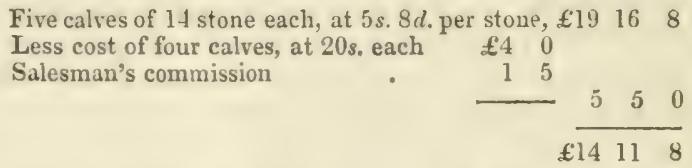

The different statements, indeed, when reduced to a mean standard, show that a good cow during the period of her milk-say 40 to 44 weeks -will suckle 3! calves, the average dead weight of which may be about seren score, or $140 \mathrm{lbs}$. each ; thus learing a gross amount of $490 \mathrm{lbs}$.: which, supposing the price and charges to be as above, would amount to 14l. Veal, however, being only in great demand during the winter and spring months, the price there stated cannot be always counted upon; and therefore, the common calculation probably comes near the truth. The profit of suckling must also in some measure depend upon the breed of the calves, for the grain of the flesh of some is closer, and they have an earlier disposition to fatten; but it must be evident from the mode in which they are collected, that the suckler seldom has any opportunity of making a selection.

With regard to comparative estimates of the returns to be expected from the several applications of milk to the making of butter and cheese, and the production of veal, they are generally thought to be in the order we have mentioned; but a very intelligent and experienced farmer, whom we have already mentioned-MIr. Hayward of Frocester Court, in Gloucestershirełwho has long kept as many as a hundred cow's in his dairy, views the production of fine cheese as being the most profitable. His estimate is thus :-

* On the Mode of Fattening Calres.-Quart. Journ. of Agric., vol. $\nabla .$, p. 611.

+ See chap. xxxvi. p. 403, of this volume.

+ See the Farmer's Series, No. 21, in the Library of Useful Knowledge. 
100 gallons of milk produce 112 lbs. of cheese of the best quality, which at $6 d$. per $1 \mathrm{~b}$. is

And 5 lbs. of whey butter, which, at $8 d$. per $1 \mathrm{~b}$., is : $£ 216 \quad 0$ $\begin{array}{lll}0 & 3 & 4\end{array}$

Value of 100 gallons of milk, when converted into cheese

100 gallons produce of milk butter $34 \mathrm{lbs}$, which, at $10 \mathrm{~d}$. per lb., is $£ 1 \quad 8 \quad 4$

And of cheese of the worst quality $74 \mathrm{lbs}$., which, at $3 d$. per lb., is $018 \quad 6$

Value of 100 gallons when made into butter

160 gallons produce $112 \mathrm{lbs}$. of veal, which, at $7 \frac{1}{2} d$. per lb., is

But calves, when dropped, generally sell at 10 s. each, which being deducted leaves, as the value of 160 gallons $\quad: \quad: \quad$ : $£ 310 \quad 0$

$010 \quad 0$ and therefore, the value of 100 gallons in feeding veal a $\begin{array}{llll} & 17 & 6\end{array}$

But it does not state the charges of the manufacture of butter and cheese, nor the value of the pork which may be reared upon the whey; ${ }^{*}$ neither does it allude to the necessity of cheese being kept during a long period ere it can be fit for consumption, while butter immediately commands a ready sale, and veal occasions but little trouble.

As experiments to determine the comparative quantities of butter and cheese made from the same quantity of milk are not only curious but im. portant, we mention the following, which, though on a small scale, will afford a tolerably accurate idea of the subject:-

No. I. was made several years ago by Mr. Wimpey, of Brockhamp. ton, in the early part of the month of December, and states that 105 gallons of milk were properly disposed in pans for skimming off the cream, which, when churned, produced $36 \mathrm{lbs}$. of butter, and $60 \mathrm{lbs}$." of skim-milk cheese.

Of the like quantity of milk were made $106 \mathrm{lbs}$. of whole-milk cheese, and $6 \mathrm{lbs}$. of whey butter : thus, supposing the value of good butter to be thirteen-pence per Jb., that made from whey being twopence less, and prime cheese to be worth sixpence, while that of skimmilk is only fourpence per lb., the account would leave-



Thus showing a slight difference in favour of butter.

No. II. was made at our request by Mrs. Hayward, on the third day of last November, upon $46 \frac{1}{2}$ galluns of milk, from which she obtained $48 \frac{1}{2}$ lbs. of whole-milk cheese, and $4 \frac{1}{2}$ lbs. of whey butter: the same quantity producing, when skimmed, $33 \frac{3}{1} \mathrm{lbs}$. of cheese, and $15 \frac{3}{4}$ lbs. of milk butter.

Now, the prices of these different articles being estimated at the same rate as those above stated, the value of the produce of the two milkings would appear to be-

$$
\begin{aligned}
& 48 \frac{1}{2} \text { lbs. best cheese, at } 6 \mathrm{~d} \text {. ․ } \quad £^{\prime} 143 \\
& 4 \frac{1}{2}, \text {, whey butter, at } 11 \mathrm{~d} \text {. } \quad 0 \quad 4 \quad 1 \frac{1}{2} \\
& 33 \frac{3}{4} \text { lbs. skim-milk cheese, at } 4 \text { d. . } \quad £ 011 \quad 3 \\
& 15 \frac{3}{4}, \text {, best butter, at } 13 \mathrm{~d} \text {. } \quad 0 \quad 017 \quad 0 \frac{3}{4}
\end{aligned}
$$

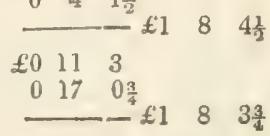

For the rearing of pigs upon butter-milk and whey, see chap. xl. of this volume, on Swine; Art." Yorkers," 
which in a striking degree corroborates the first experiment, and proves the return of either butter or cheese to be nearly equal.

The value of the milk, it will be seen, is there estimated at sevenpence per gallon; while in London, and most of our large cities, it is charged to the milk-people at eightpence to tenpence, according to the distance from town : they also being at the trouble of milking. 'The cow-keeper's business is therefore one of considerable profit; for although they feed the animals very high, yet their object is rather to increase the quantity than the quality of the milk, and they give them an abundant supply of brewer's grains and turnips. The dealers receive it in a genuine state, and retail it to their customers at fourpence per quart, but are not satisfied with that profit; for, besides taking off a portion of the cream, they invariably mix the milk with no small quantity of water, and are even said to adulterate it with flour chalk, and other ingredients, to give it an appearance of richness.*

In making these estimates of dairy produce, it should, however, be remembered, that milk is so much richer in autumn than it is in spring, that the same quantity will probably produce one-fourth more of butter and cheese in the month of October than in the vernal season. They are alsolike every calculation upon farming profits-so dependent upon different circumstances, that nothing more can be assumed on the subject than what may be derived from the individual experience of farmers placed upon particular soils; for it is well known that land which produces good butter is not always well calculated for fine-flavoured cheese; and some cows produce more milk than others; while others afford it of richer quality: they, consequently, contain little more than local information, and lead only to general notions of their average results. Besides the difference of soil and climate, which so materially affect them, there is also, in this instance, the variety in the breed and constitution of the animal. Even the mode of churning the milk of cows, collectively or individually, affords different results, for it appears, from the following trial lately made at the request of the Highland Society, that-

" 300 gallons of milk, the weekly produce of twelve cows, collcctively churned, gave $84 \mathrm{lbs}$. of butter-being $1 \mathrm{lb}$. to 14 quarts ; while 294 gallons, individually churned, gave only $70 \mathrm{lbs}$., or $1 \mathrm{lb}$. to 17 quarts." +

It is, therefore, little to be wondered at that farmers vary so much in their opinions on the subject; but these hints will afford materials for calculation, and farmers who rent out their cows to working dairymen, may thus form a just idea of the sum at which they should be charged. When the farmer is not disposed to take upon his family the troublesome management of a large dairy, this plan of letting out a number of cows sufficient to employ an industrious man and his wife, who are well acquainted with the business, is by no means objectionable; he furnishing the cows and feeding them, and accommodating the person who rents them with a cottage and dairy, either at a fixed sum per head, or a certain portion of the sale produce.

* "Every cow-house is provided with a milk-room, where the milk is measured and served out by the cow-keeper, and this room is furnished with a pump, to which the retail dealers apply in rotation, and pump the water into the milk vessels at discretion. It goes by the name of the 'Black Cow' and is said to yield more than all the rest put together." -Middlesex Rep., second edition, p. 423.

t Trans. of the Soc., N. S. vol. ii. p. 256. The smallness of the latter producewhich is opposed to that of most dairies-is however partly attributed to the milk having been fut into the coolers in such small quantities as to prevent the proper separation of the cream. 


\section{Chipter XXXix.}

\section{ON SHEEP.}

THE vast importance of sheep to our agriculture may be estimated by the fact, that their number in this country has been lately calculated to amount in the United Kingdom to upwards of thirty-two millions.

The breeds are various, each marked by some peculiarity of its own; differing both in size, flavour of the meat, quantity and quality of the wool, as well as hardihood, according to the nature of the soils and climate in which they are bred; the study of which forms an interesting subject to the naturalist, though more especially to the farmer, who is mainly interested in the choice best adapted to his land and situation. The discussion would, however, lead us farther than this publication will admit, and is, indeed, here unnecessary, as their history will be detailed in the work now publishing, in the Farmer's Series, by Mr. Youatt, under the direction of the Society for the Diffusion of Useful Knowledge. We shall therefore merely remark, that the chief distinction lies between the species reared upon the thin soiled uplands of the downs and mountainous heathy pasture - small of carcass and covered with a close but short coat of fine wool, and those fed in the marshes on other rich lowlands, where they acquire greater flesh, together with both longer and coarser wool; which has occasioned the two kinds to be distinctively termed "short-woolled" and "long-woolled." The different breeds of these separate sorts are also in some cases polled, and in others they bear horns; and the character of the chief kinds may be generally estimated under the following succinct synopsis.

SHORT-WVOL.

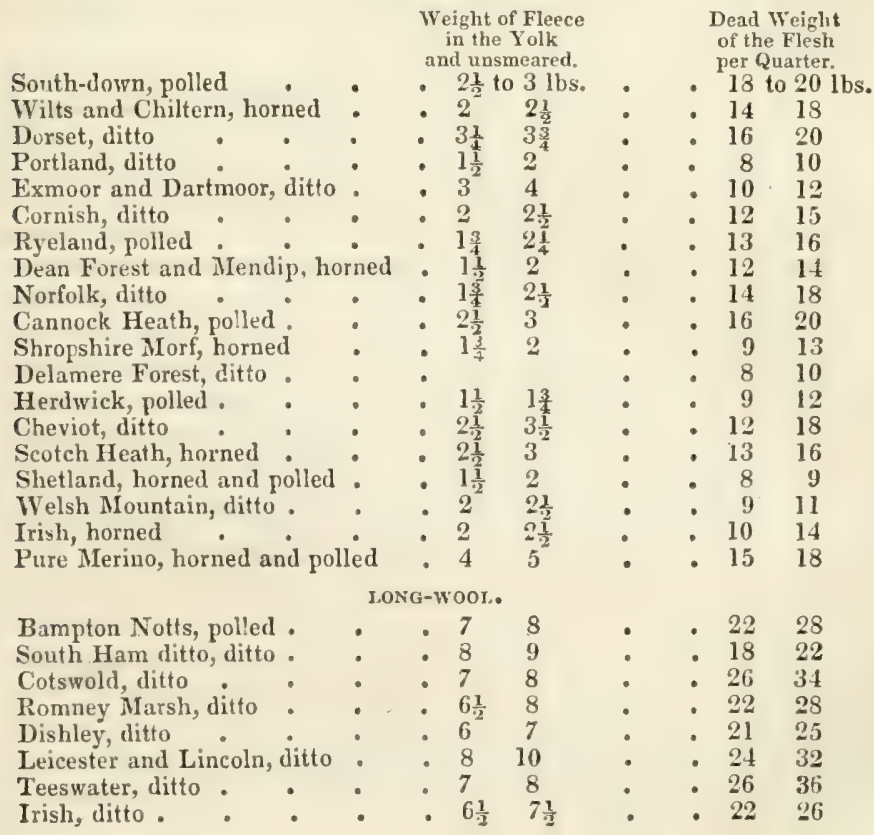


The above table shows the average weights, at two years to thirty months old, of both ewes and wedders - the latter of which produce the larger quantity of both wool and mutton; but is only intended to convey an idea of the original flocks which formerly covered the pastures of the United Kingdom, for so many half-breeds have been since introduced by repeated crosses between the Merinos, Dishleys, Southdowns, and almost every other species, that they are now found partaking of the various descriptions of each kind; many much improved in their material points, and in others injured, while sume have become nearly extinct. As an instance of improvement in one race, the weights of the wool and carcass of the Cheviots, fed upon the Grampians and the other mountainous parts of the north of Scotland, into which they have been latterly introduced, were, up to the year 1800 , such as those stated in the table: yet we learn from Mr. Sellar, of Strathnaver, in Sutherlandshire, that, merely by dint of persevering attention to the choice of rams, the change of herbage, and placing the sheep of different ages upon appropriate pasture, togrether with supplies of some artificial food in the pinching seasons of the year, he has raised the average of the wool to 4 and $4 \frac{1}{2}$ los. per fleece, and that of the flesh of the wedders, when rising four years old, from 18 to 26 lbs. per quarter.*

The ase of sheep is commonly counted from the period of their first shearing, instead of the time at which they have been dropped as lambs; and may be known, like that of cattle, by the appearance of the fore-teeth of the lower jaw, of which they have also eight: the upper jaw being with. out any in the front. During the first year, they are all of small size, but when from fourteen to sixteen months old they renew the first two, and two more every year until the fourth shearing, at which time they are "full-mouthed." "The period of the first renewal depends, however, in some measure not only upon the season of lambing, but also upon the goodness of the sheep's keep; those which are well fed, usually having them the earliest renewed.

Their natural age is generally about nine or ten vears; but their teeth begin to fail in the sixth or seventh year, when they become what is called " broken-mouthed," and, being then cieprived of the power of easily masticating their food, they fall off in flesh; they are therefore, even when kept for breeding, before that time most usually slaughtered. Formerly, indeed, when the chief object of the farmer was the fleece, they were seldom killed before five or six years old, and that is still the custom over a great extent of the Continent; but, when our population increased, both the demand of mutton, and the power of speedily fattening sheep through the means of the turnip husbandry, together with the great improvement made in some of the breeds, introduced the practice of bringing them earlier to maturity, and now they are very generally brought to market within two or at most two and a half years of age, though it must be confessed that the discovery has brought a breed into vogue that manufactures its food into fat, and gives nothing to gravy + Heath-bred Cheviot, and black-faced mountain sheep do not, however, come to perfection until the wethers are three years old, rising four, and the ewes five, rising six; nor are they in practice usually killed until they be of those ages.

The male is called a "ram" or "tup," before he is castrated, after which

* Farm Reports, No. 18. p. 77.

t Leicesters are commonly found at that age, with three or four inches of fat upon their ribs, and a werlder of the Dishley hreed, fattened by. Mr. Culley and killed at Alnwick, had a coat of more than seven inches of solid fat: the whole of his back was like the fattest bacon.-Quart. Jour. of Agric, vol, ii. p. 553. 
he is termed a "wedder" or "wether," and the female a "ewe:" both, until they are weaned, being indifferently styled "lambs;" but at the future periods of their lives they are, in different districts, thus severally designated, namely :-

THE MALES.

THE FEMALES.

Wedder-Hogs, Hoggets, From the time of weaning f Ewe-Hogs, or

Hoggerells, or Tegs $\}$ to the first shearing. $\left\{\begin{array}{l}\text { Gimmers. } \\ \text { Gims }\end{array}\right.$

$\left.\begin{array}{l}\text { Shearling.Hogs, or } \\ \text { Dinmonts. }\end{array}\right\} \quad$ After the first shearing. $\left\{\begin{array}{l}\text { Gimmer-Shearlings, } \\ \text { or 'Theaves. }\end{array}\right.$

At the expiration of which time, they are each known as "two shear," "three shear," or "four shear" wediders or ewes, until they are full-mouthed; when the ewes, if drafted out of the flock either as being barren, or to be fattened, are termed "cast" or "yelled," and when turned six years old, are denominated "crones."

\section{PROCREATION.}

The time at which the ram ought to be admitted to the ewes, should depend upon the nature of the climate, and the prospect of spring food for the future support of the lambs; and this should be abundant, for if the lambs be once stinted, their flesh becomes sticky and they can never again be restored to healthy condition. Nurse-ewes should therefore have the best of pasture, and if the farm does not afford turnips, they should, during winter, be furnished with a certain proportion of hay. Ewes are generally fit to breed at the age of about fifteen to eighteen months. Much however depends on the state of the flock as to their forwardness, and the time must necessarily be regulated by the period at which they were dropped as lambs: thus, supposing it to have occurred in January, that would presume them to be ready for the ram-if at fifteen months-in the beginning of the following May; and if tupped at that time, their lambs would then be dropped about the end of September; which is a practice only followed for the production of house-lamb.* Eighteen months old is therefore more usual, and in exposed situations, where it is not desirable that the lambs should be dropped until late in the spring, the ewes are not generally tupped until some time in October.

The number of ewes to be put to a ram should be regulated not only by his age and vigour, but, in some degree also, by the nature of the farm on which the flock is pastured: thus, if fed upon mountainous land having a wide range of open field, three rams are not unfrequently put to a hundred ewes, whereas in enclosures, two are quite sufficient; indeed one, if he be full grown and in very high conditiois, may serve a hundred. Generally speaking, however, sixty ewes are considered quite enough, and a yearling tup will serve as many ewes as an olier one; though many breeders are of opinion that his utmost powers ought not to be exerted until he has reached another year; for if too many be put to him, there will be hazard of their all bearing, and the lambs produced will probably be weak and puny.

The breast of the ram is not uncommonly smeared with some colouring

* "It does not often nccur that any of the British sheep, excepting the Dorsets, can be induced to take the ram before September. A small fluck of rery beautiful Merinos were imported from Saxony to Yorkshire, by Sir II. Vavasour, of Melbourne Hall, in the Spring of 1835, all of which produced healthy lamlis towards the close of December in the same year, and again at the like period in 1836. One of these ewes will have lambed three times within fourteen months. They have had no extra keep, are excellent nurses, and very hardy in constitution." - Howard of Melbourne. 
matter by which the ewes are marked when they have been tupped, and if more than one ram be put among the flock, the ewes which have been served by each can be ascertained by giving different sorts of colour. This, however, often causes disappointment, for the tup occasionally leaps without effecting his object, and it sometimes happens that the ewes do not "blossom"-or come in season for taking the male; in which case some other means should be tried for inducing them, and, if not successful, they must then be either sold or fattened off. The best mode of effecting the object is to feed the animal with some stimulating food-such as oil-cake, corn, or linseed; but rape, full-blown clover, or any very nutritive herbage, will probably attain the object, and may also have considerable effect in the production of twins. Another plan, has, however, been adopted by some flockmasters in the view of both ensuring the service of the ewe, and of preventing the powers of a superior tup from being unnecessarily wasted by having too many ewes put to him at once: it is this-he is placed in a small enclosure with only a few ewes, while among the ewe flock an ordinary ram is put as a "teaser," with a patch of cloth like an apron sewed before his genitals, in such a manner as to prevent any possibility of his serving the ewe. When singled out by the teaser, she is taken by the shepherd to the ram ; and, when served, taken away again : by which means, and thus not promiscuously putting all the ewes to the ram at the same time, high fed tups will effectually serve as many as four or five score*.

The well-known description of a tup, given by that eminent breeder, the late Mr. George Culley, is in the hands of every man who values his flock, and if not in all cases adopted, will at least be found to contain useful hints for the selection; for there can be little doubt that the nearer he comes to that appearance, the more generally perfect will be his formt.

In addition however to symmetry, the "pelt" should be attentively examined, in order to ascertain that the wool-whether the sheep be of the short or long-woolled breed-be not stitchy-haired, or full of "kemps;" as well as that its staple be sound and even. He should not, of course, be chosen unless perfectly sound and vigorous, nor until after he has become a shearling, and he should be previously well fed so as to be brought into ligh condition; he is in his prime from one till five years old, and should not be used after he has reached his sixth ; but for the improvement of the flock, a proper choice of the tups to be used is the point of the greatest importance. In breeding from varieties possessing, on one side, a valuable fleece with a defective form, and on the other, a good form with an inferior fleece, the result will be a flock comprising individuals which par-

* See the Leicestershire Report, p. 262.

$+\mathrm{H}$ is head should be fine and small, his nostrils wide and expanded, his eyes prominent, and rather bold or daring; ears thin, his collar full from the breast and shoulders, but tapering gradually all the way to where the head and neck join, which should be very fine and graceful, being perfectly free from any coarse leather hanging down; the shoulders broad and full, which must, at the same time, join so easy to the collar forward and chine backward, as to leave not the least hollow in either place; the mutton upon his arm or forethigh must come quite to the knee; his legs upright, with a clean fine bone, being equally clear from superfluous skin and coarse hairy wool from the knee and hough lownward; the breast broad and well formed, which will keep his forelegs at a proper wideness ; his girth, or chest, full and deep, and instead of a hollow behind the shoulders, that part, by some called the foreflank, should be quite full; the back and loins broad, Hat, and straight, from the waist; the ribs must rise with a fine circular arch; his belly straight; the quarters long and full, with the mutton quite down to the hough, which should neither stand in nor out; his twist deep, ride, and full, which, with the broad breast, will keep his four legs open and upright; the whole body covered with a thin pelt, and that with fine, bright solt wool-Culley on Live Slock, p. 103. 
take of the character of each parent in various degrees of combination: generally, the produce may derive pretty equally from both; but in some, the bad shape of the one may be seen united to the bad fleece of the other, while a favoured few will exhibit a large share of the good qualities of the parent stock.*

"The flock-master, attending in the first instance to these general rules, ought therefore to consider what are the defects among his eives, either as to shape, quality of mutton, or wool, which he wishes to remove, and to select the tups which he uses who possess in an eminent degree the merits in which his ewes are deficient; having done so, he then must carefully divide his ewes, and place with each parcel the tup from among those which he has selected which is most suited in form to the ewes which compose this parcel. For this reason it never can be right to put more than one tup with the same pen of ewes, because this leaves to chance the selection which ought to be made by the judgment of the owner."

In this-which is the remark of a distinguished agriculturist-we fully coincide, and its justice will no doubt be admitted by every breeder who wishes to maintain the reputation of a superior flock; but, with great deference to his opinion, we yet submit, that on those unenclosed downs where large flocks are pastured, the use of more tups than one is almost unavoidable. The majority of farmers are contented with the stock which they have acquired, or which they can obtain at the neighbouring fairs, and seek no further improvement; they, therefore, look to nothing more than the choice of as good rams as they can select, and put as many of them to their ewes as they think will be sure to serve them all efficiently, without caring whether they be tupped by one or the other. They besides entertain a very general idea that two rams amongst double the number of ewes usually put to one, will get more lambs than one ram alone; and, for that reason, do not trust to one only. $\dagger$

The period of the gestation of the cwe is usually estimater to last during twenty-one weeks; but according to the experiments to which we have alluded on the subject, as having been made on other animals by M. Teissier at Paris, it has been found that out of upwards of 900 ewes of which the date of tupping was severally noted-

\begin{tabular}{|c|c|c|}
\hline 676 & , & 150th and 154th day. \\
\hline 90 & & 154th and 161st day. \\
\hline
\end{tabular}

Thus showing an extreme interval of fifteen days, and constituting a mean duration of about 152 days, or nearly twenty-two weeks.

\section{YEANING.}

The ewes, when put to the ram, should be in fair, but not high condition ; nor should they be so highly fed during the period of gestation as to allow them to get fat, for that would occasion such a difficulty of lambing as often proves fatal; but if evidently poor in flesh, and consequently weak, they should be put upon better food for a short time, as the yeaning approaches, in order both to give them strength to go through with it, and to furnish them with an abundant supply of milk for the support of their

* See Stevenson's Survey of Surrey, p. 528.

$\dagger$ A remarkable instance of superfotation is mentioned by the Rev. St. Julin Priest, in the Buckinghamshire Report, of a flock of ewes to which were put both Norfolk and Leicester rams, and several of the ewes which bore twins, had one a true Norfolk, and the other a half-bred lamb, between the Leicester and the Norfolk, p. 309 . 
lambs. All improvement, however, in the quality of food before yeaning should be managed with great caution, and intelligent flock-masters find it safer to transfer the ewe and the lamb to better food after the operation than before.

When large flocks are kept upon downs and mountainous pastures, the ewes are most commonly left to lamb in the open field, without any other care than the occasional attention of the shepherd, to assist any one that may need it. Indeed this is generally the case even upon small farms that have the convenience of enclosures adjoining to the homestead; though some careful breeders, situated upon extensive downs, have the precaution of employing small huts mounted upon four wheels, which may be thus drawn to the flock for the use of the shepherd, wherever the sheep may be.

In the north of Scotland, Mr. Sellar, of Strathnaver, tells us "that eweflocks are generally placed in valleys, through the bottom of which runs a 'burn,' or brook. Nearest to the burn is some haugh of flat green pasture; next to this, on each side of the burn, a rocky gravelly hill covered with heather, and behind that, the mountain-waste of peat-bog covered with alpine plants. The lambing season taking place at the very juncture when the cotton grasses* have gone out of season, and before the deer'slair thas sprung, the ewes of course descend to the burn banks for their food, and repose, during night at yeaning time, on the gravelly ridge immediately belind ; where their lambs are dropped." He finds no improvement, for yeaning, so good as to sow this gravelly ridge with whins, or furze; which gives shelter in bad weather, and when periodically burned, in rotation with the heath, affords succulent food; not, for feeding ewes, inferior to turnips: and he describes a ride up one of these valleys, at lambing time, early in an April morning to see the ewes bleating-in on cach side with their young lambs from their lairs among the whin blossom, as a very gratifying spectacle.

This, indeed, is not a bad plan upon wide extensive pastures; but that of having a well sheltererl fold close to the shepherd's cot for the ewes at yeaning time is preferable. On moderate farms, in an enclosed country, another mode, however, is that of putting up some moveable covered pens, which may be formed with hurdles, in a warm paddock under the eye of the master. The pens should be littered with straw, fern, or leaves, so as to be perfectly dry; but they should be open to the ewes, allowing them the free range of the field, with power to retire to the pens when they please; and this will be found better than folding, as sufficient room is thereby seldom allowed for the perfect convenience and quiet of the animals at that moment of uneasiness. At all events, whether penned or in the open field, the yeaning should always be effected on a piece of smooth pasture, containing neither ruts, ditches, nor holes of any kind.

The most constant and arduous attention is requisite on the part of the shepherd at yeaning time; for although nature seldom requires to be assisted, yet sometimes the labour is so difficult, as not to be safely effected without aid, which every experienced shepherd should be capable of render. ing. In very severe weather also, when the yeaning has been long in its completion, the ewe is often so exhausted as to need some warm gruel, and housing from the rest of the flock until she be recovered.

THE LAMBS

Are not unfrequently dropped in such a state as to appear nearly lifeless, and nothing but the greatest care will restore them to animation; but we 
have repeatedly seen them, when to all appearance dead, brought completely about by being laid a few hours in a basket with some wool, or placed near the kitchen fire, and a very little warm milk poured occasionally down their throats. The ewe also sometimes, though rarely, deserts her lamb, or she dies in labour; and in such cases, it becomes necessary either to suckle the lamb by hand with cow's milk, or to put it with a ewe which has lost her own. This latter mode, however, is not easily accomplished, and therefore a plan is frequently adopted of deceiving her by stripping the dead lamb of its skin, and stitching it round one which is alive-either a twin, or one which has lost its dam; she will thus be soon reconciled to the foster lamb, and in a couple of days the skin may be taken off.

When the lamb tups have acquired a little strength-say at not more than about eight or ten days old-those which are not intended to be reserved as rams should be castrated. Their dams are therefore collected within a small fold, with openings through which the lambs are suffered to escape, and then being each successively caught up, and held in the arms of an assistant with its limbs distended, the operation is performed by the shepherd, who grasps the scrotum with his left hand in such manner as to press forward the testicles and render the skin lying over them tight. Two incisions are then made through the bag, one over each testicle, sufficiently large to allow them to pass, and they are thus easily extracted.* The weather should however be dry and mild, as well as, if possible, cloudy ; or if warm, towards evening is the better time for its performance.

The lambs, whether male or female, may also at the same time be "docked," or have their tails cut off at about two or three inches from its root. This gives a square and handsome appearance to the quarter, besides preserving the animal from depositing ordure on its fleece. It is, however, objected to, so far as ewes are concerned, by several intelligent breeders, whose flocks are fed in exposed situations; they not unjustly conceiving that in severe wenther a long bushy tail affords considerable warmth and protection to the udder. In opposition to this, however, it is said that the tail very often occasions great embarrassment in yeaning. If the operations be carefully done, it is surprising how little loss they occasion; it need hardly, however, be observed, that castration should never be committed to any other than a very experienced man.

The lambs may then be turned along with the ewes into the common pasture, the period of the year depending, however, upon the state of the grass, the situation of the farm, and the time when they were dropped, as well as the object of their disposal. Thus, if intended to be sold to the butcher, the ewes are put into the best of pasture, and if that be not in high order, they must be fed upon turnips or rape, and if necessary, with hay and bruised oil-cake, so as to produce a good flow of milk; for in London and most of our large towns grass-lamb is constantly brought to market so early as Easter, previous to which, unless where fog has been reserved, there can be no grass fit to answer the purpose. They are usually slaughtered at from three to four months old; the gimners being rather more forward than the wedders in consequence of their earlier disposition to fatten, as well as the latter being somewhat thrown back by their castration.

House-lamb is always ready for the market before Christmas, and as it

* Although there is less danger when the operation is performed at an early period, it has however been observed, that, on horned sheep it is generally preferred to perform it later; perhaps when the lambs are two months old; which causes the horns to grow more full, and make them look handsomer._Mountain Shepherd's Manual, p. 10. 
ever bears a high price in the metropolis, it was a species of husbandry formerly practised to a large extent by many farmers in the surrounding neighbourhood; but, whether from the taste for it not being now so much in vogue, or from the trouble and risk attendant upon the suckling of the animals, the plan of rearing them has of late years sensibly declined. The mode however, when pursued, is stated by Mr. Middleton to be nearly as follows :-

The ewes are always, without exception, of the Dorsetshire breed, and the early lambing species are sought for throughout the country with great diligence, for it is thought that not more than one in three will lamb sufficiently early for the purpose. Those of large size with white noses, are most in esteem, and any thing like black on that part would occasion their being rejected.

The colour of the flesh of those lambs when butchered, is also a matter which in a great measure governs their value; and, therefore, those which can be warranted to die fair, always bear the highest price. This evidently cannot be guaranteed when produced by ewes which have been promiscuously purchased at the fairs: those breeders with whom the sucklers usually deal are consequently careful in the selection of rams, the issue of which are sure to have white meat; which is said to be known by certain marks in the mouth, even before any previous knowledge of their progeny.*

The rams and ewes should be put together at such a time that the lambs may fall at the proper scason-say, somewhere about Michaelmas; from which period there is usually a succession of them until towards Christmas. The ewes, both sometimes previous to this, and during the whole period of suckling, are kept in a croft adjoining the lamb-house, and must be well fed, in addition to after-grass, with turnips, cole, cabbage, or any succulent roots which may be in season, together with brewer's grains, pollard, ground oats or barley, pea-meal, and linseed; or, in short, any food which will best promote an abundance of milk and the consequent growth of fat in the lambs.

The lambs are separated from their dams and put into the house, which should be well littered with clean wheat straw; a little of which should also be placed in racks with the ears downwards, in order to amuse themselves and prevent them from gnawing each other's wool. Some chalk baked in an oven, should likewise be put in troughs, both in lumps and powder, in order to guard them as much as possible against looseness; and the most scrupulous attention should be paid to cleanliness, as they do not leave the house until sent to the butcher. To prevent them from playing and thus ensure quiet, light is excluded from the house until partially admitted at such times when the dams are brought to suckle them; which in most cases is three, or even four times in the day. If the house, however, be large enough to admit of the ewes being allowed to remain with them during the night, the usual plan is to admit them at sun-down and turn them into the pasture soon after the dawn of day: in which case they should be again brought to them about noon. The lambhouse is however generally too hot, and this successive change from

* 6The sucklers, salesmen, and butchers of Lonton, are aware that such lambs as have sharp barbs on the inside of their lips, are certainly of a deep colour after being butchered, and all those whose barbs are naturally b/unt, do as certainly produce fair meat; the issue of such rams can also be generally warranted fair." - Midellesex Report, ?nd Edit., p. 45 4. The Herts Report says white barbs.-p. 192. 
hot to cold, is so very injurious to the ewes, that it is no uncommon thing for a ewe or two to die in the night.

When the ewe is not capable of supplying so much milk as the lamb will consume, those which have lost their own, or which have been sold early, are brought in, and held by the head, or put into a yoke, till the lambs by turns suck them clean; they are then turned into the pasture, and at twelve o'clock the dams are driven into the lamb-house for an hour, in the course of which time each lamb is suckled by its mother. At four o'clock, all the "dam-ewes, -as those which have not lambs of their own are called,-are again brought to the lamb-house and held for the lambs to suck; and the mothers of the lambs are afterwards brought to them for the night.

Lambs thus treated, and kept free from all disturbance, will in about eight weeks' time become sufficiently fat, and their flesh extremely white and delicate. The price varies greatly, according to the season of the year when they are ready for the butcher ; for they sometimes bring as high as $5 l$. per head, and at others not even half that sum.*

When many lambs are not suckled, an empty barn is not uncommonly used; but, when built for the purpose-if calculated to suckle from 160 to 180 lambs at a time-it should be 70 feet long and 18 feet wide, with three coops of different sizes at each end, so constructed as to divide the lambs according to their ages. Deal hurdles are also placed for this purpose about the middle of the house, in order to enable the lambs to find their mothers without difficulty; and it is latticed, in this manner, in order to admit a free circulation of air-

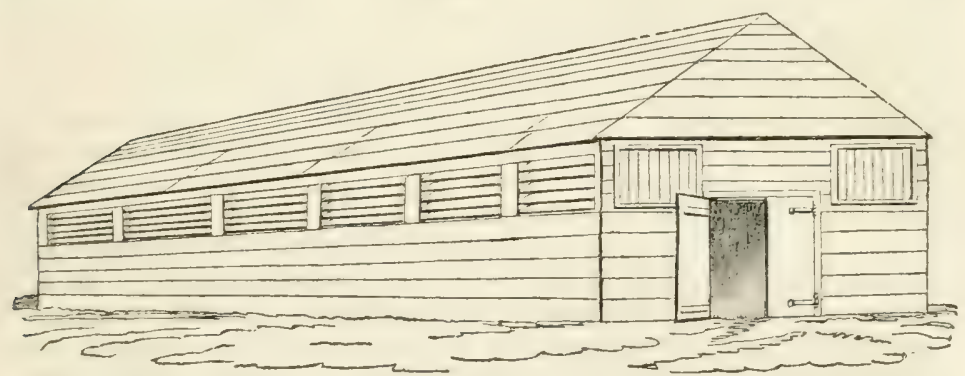

Grass-lamb, although requiring particular attention to the season of coupling the ewes, and great care in feeding them, so as to bring it to perfection in time to meet the fall of Easter, at whatever period that may occur, yet does not, of course, fetch so much at market as house-lamb; but then the trouble and expense of rearing it is not nearly so great.

One liundred and fifty ewes of the Dorset breed are said by the lambbreeders to produce them 200 lambs, of both kinds, on an average of years, exclusive of twins, in this manner:-100 of the old stock, tupped in the month of June, drop their lambs some time before Christmas, so as to have them gradually at market previous to grass-lamb coming into season; then 50 jurchased, in lamb, at Michaelmas, produce 50 lambs in the latter end of October, and 50 more in July; all of which are sold within the year.t

When bred for stock, the short-woolled species are most commonly allowed to range upon the open wolds and downs of our English counties, 
and the mountainous parts of Wales and the Highlands of Scotland; while the long-woolled breeds are pastured in the enclosures of the lowland districts of England, and its northern borders, and the rich soils of Ireland; where they remain, along with their dams, until they have reached the age of three or four months; after which they are weaned.

\section{WEANING.}

The time of weaning-or "speaning," as it is in some places called-is dependent upon that in which the lambs have been dropped; for if that has happened early in the season, and the weather remains cold, they are generally allowed to remain under the fostering care of the mother until it becomes warmer: it is, linwever, usually performed in the early part of July, and should never be deferred beyond the latter end of that month, unless the lambing should have occurred late in the spring. No other care is necessary in the process than that of separating the lambs, and driving them so far from the ewes that their mutual bleatings may not be heard by each other; but careful shepherds will select the weakly lambs from those which are stronger, and place them, if possible, in a different pasture. In breeding farms, also, particular attention should be paid to the selection of the ewe lambs, and those which appear in any shape ill-formed, or constitutionally defective, should be rejected out of the lot and sold, for, if bred from, they will assuredly injure the reputation of the flock; * and indeed the weaker lambs of every description should be separated from those which are strong.

IVtien separated from the ewes, the lambs ought to be all severally examined, lest any of them should have received injury, in which case they are put apart for cure; and they are marked according to the pleasure of the owner, either with ruddle or tar upon the fleece, or by a brand upon the cheek and notches in the ears. The wedders and ewes should, however, be differently distinguished, by being marked upon the off or the near side. The ewes should be afterwards either milked three or four times, at longrer intervals during each, in order to dry them up by degrees, with as little inconvenience to them as possible; or else confined for three or four nights in a fold, and permitted to range over a poor pasture in the daytime. This moderate keep assists in preventing injury from an accumulation of milk; but their udders should be carefully watched, and any that appear full of milk, partially drawn. This, incleed, is by many persons neglected merely to save trouble, though the omission of it occasions considerable pain to the animal, and is not unfrequently attended with serious consequences.

The milking of ewes for the purpose of making cheese has now, except in some very remote districts, been generally abandoned, both as being attended with little profit in itself, and as bing injurious to the animal. The mode, when practised, is, to drive them, one by one, into a narrow pen, where they are milked from behind, and, as they only give from a pint to a quart each, it is said that a clever damsel, when accustomed to the work, will easily operate upon a score an hour.

The lambs, when removed, are put either upon the best pasture, or, if the farm contains any portion of arable ground, upon the aftermath of

* On this subject, that great flock-master, Mr. Sellar, justly observes- " That the difference in expense of keep, loss by death, and of ultimate profit, in favour of wellbred and against ill-bred stock, is sogreat and striking on a sheep-farm, that the apathy of many stock-masters to the subject is incredible." See his Report in the Farmer's Series of Strathnaver, Morvich, and Culmaily farms, in which will bo found a very interesting account of the selection and weaning of the lambs. 
good crops of clover and sainfoin, or upon spring tares, or any succulent green crop that will afford abundant nourishment to promote their growth, which should now be attended to without regard to expense ; for, if it be at this time checked, it cannot alterwards be easily recuvered. It may, indeed, be assumed as an axiom, that no animal will pay the breeder if starved while young. They should, therefore, be watched with care; and the ground should be occasionally shifted, so as to supply them with a fresh bite, until the herbage begins to fail, when they should be immediately put during the whole winter upon rape and turnips.

\section{FEEDING.}

In feeding with these, two methods are used: either turning the stock upon the roots, which are thus eaten off upon the field; or, carting the turnips to a bare pasture, where they are sliced and fed. The first is the common custom when the land is dry enough to bear the sheep, for it is not only the least troublesome, but it ensures the dung and urine being efficiently laid upon the ground; which is, indeed, the main object of this fallow crop. The field should, however, be hurdled off in partitions, so as to ensure the entire consumption of the crop; or, if the lambs be permitted to range over the whole, until they have eaten of the better part, they should be afterwards followed by the lean stores and ewes, for which the roots are loosened out of the soil by a man with a grubber, until they are eaten up clean : a practice which is also customary with werders which are intended to be fattened. Great care should, however, be taken not to suffer the lambs to touch either turnips or clover when the frost is on them.

When the land, however, is wet, the turmips must necessarily be carted of ; and, whether the land be wet or dry, the same plan is generally adopted with regard to bet-root. This is certainly the most advisable muite of consumption for the sheep, for it ensures their quiet, and if they be placed upon the driest part of the farm pasture, their wool camnot suffer. It is also thought that the oily matter contained in the fleece, and communicated by their bodies to the land-which in Norfolk is much valued as the "teathe"-may be partly advantageous to the following year's grass; wherefore, many farmers who have rich lowhand jastures upon which they feed the Leicester and other heavy breeds of the northern counties, and are more in the habit of fattening than of breeding sheep, almost invariably adopt the plan of drawing the turnips. They have foumd that to give then cut into slices by the instrument called a "turnip-ctitter," is preferable to giving them in a whole state; the same quantity supplying a larger number of sheep by this method, and the sheep, so fed fattening quicker than when the ordinary mode is adopted.*

In feeding with turnips, however, even if they should not fail, some dry provender-either oat-straw, pea-haulm, or hay-is a very desirable addition; as preventing any attack of looseness, as well as occasioning the food to remain longer in the stomach; thereby probably extracting a greater quantity of nourishment from the turnips than could be obtained were they eaten alone. If given in the fold before the sheep are turned out, the dry foud will also prevent all hazard of their being hoved; by which accident, particularly when the tops are succulent, many of them occasionally perish. If hay should be thought too expensive, pea-haum- of which the animals are very fond-is an admirable substitute. Whatever may be given should, however, be distributed with economy and regularity, and instead of being

* See chap, xviii, of this volume, on turnips. 
thrown upon the ground, should be placed at different distances, either in the troughs or sheep-cribs in common use, or in the wicker tumbrils employed in some parts of Lincolnshire*.

Such is the usual practice of careful farmers, even when not fattening the animals for market; but upon many of our wolds and downs this is neglected, and a large portion of the mountainous parts of Wales and Scotland consists only of sheep-walk, which admits of no other mode of feeding but pasture, and apparently contains nothing but heather. This, however, ripens into seed, which furnishes an ample supply to the flocks during the winter season, and in the following months the bents, fescues, ling, burnet, brome, deer-hair, and cotton-grass, spring up in succession, together with various other Alpine plants which are indigenous to these elevated regions: Nature thus bountifully providing for their subsistence; for, even when covered deep in snow, instinct teaches the creatures to scrape it down with their feet until they reach the herbage which lies underneath.

\section{FOLDING}

is practised on fallow-lands in the spring months, after corn crops for turnips, then on fallows or clover leys for wheat; and when that season is over the fold is removed to whatever ploughed land, or stubbles intended to be broken up, which may need manure for a succeeding crop. The fold is likewise not unfrequently again run lightly over that which has been seeded with wheat; though more with a view to bury the seed in the soil, and to consolidate the ground by the treading of the sheep, than to lay on it an additional quantity of dung. In very wet weather it is also a good plan to have a standing fold upon pasture land, in which the sheep may be penned in rainy nights, when the ar.tble is in a state unfit to receive them.

When fed upon upland soils of a dry nature, the lambs-now grown to the stage of tegs and gimmers - are folded upon the land, in the same manner as sheep of a more advanced age. Such is, indeed, the universal custom of all the practical farmers upon our downs and wolds, though many of those situated in the lowlands pursue a contrary system, and the practice is decried by all theorists; as being, although productive of corn, yet injurious to the animal, the quality of the wool suffering from dirt, and the growth of flesh being deficient: thus sacrificing the grass-land to the arable, or in the words of Bakewell, who was a great enemy to it" robbing of Peter to pay Paul."

There can, indeed, be no doubt that altlough advantageous to the land, the practice of folding is prejudicial to the animal; as well as that, in consequence of the exercise of driving, he will eat more than if left quiet. Yet it may be justly doubted whether the benefit accruing to the soil may not be more than a sufficient compensation for the injury done to the flock. It is certain that rich lands, diviled into small enclosures, are liable to be puached by the treading of the flock in their moving to the fold, and the sheep are injured by being driven from any distance to it ; for they will evidently improve more rapidly if allowed to lie down after they have filled their bellies.

* These are circular cages, about 10 feet in circumference, and closely wattled to the height of about a foot, above which they are left open for the space of 18 inches; the staves which form the skeleton, being each about ten inches asunder; thereby allowing the sheep to pass their heads between, and thus prevent the strongrer from driving away the weaker. In the upper part of the tumbril the staves are drawn closer together, yet allowing room enough for putting in the food,-whether roots or dry-and a cover may be added.-See the Complete Grazier, 6th edit., p. 25t, in which there is a figure of the utensil. 
The long-woolled breeds are also bad travellers, an.l suffer severely by being exposed to the least exertion: the plan is therefore seldom adopted with regard to them, or upon the soils on which they are usually bred; nor is it generally followed respecting sheep which are in the course of being fattened. It has, however, been proved by the evidence of many intelligent men, who were examined before the committees of the llouses of Lords and Commons upon wool and agriculture, that, without folding, they could not profitably carry on the arable portion of their down-land farms. The question has, indeed, we think, been fairly put by the late Mr. Billingsby, in this manner: "In a rich fertile country, where the quantity of arable land is small, and in mere subserviency to the grazing systern, where dung is plenty, and can be put in the corn-land at a small expense, and where each sheep is highly fed, it is not to be wondered that the folding system should be held in derision and contempt; but I will be bold enough to repeat, that in a poor, exposed, and extensive corn-farm, the soil of which is light and stony, it is the sine qua non of good husbandry. Let me ask its opponents whether the downs of Wilts and Dorset would wave with luxuriant corn if folding were abolished? No. The farmer would plough and sow to little purpose, were his fallows to remain untrod with the feet, and unmanured by the dung and perspiration of these useful animals. Besides, in the hot summer months, nothing is so grateful to the flock itself as fresh ploughed ground; and sheep will, of their own accord, retire to it when their hunger is satisfied"**

When fed on artificial grasses, it has also been found that taking the sheep off their feed to lodge in the night is of great use as preventing them from what the shepherds call "stenching their food ;" while, if driven off immediately after feeding, without being suffered to lie down, they neither waste nor soil it. By keeping them in the fold during the autumn, until the hoar-frost and heavy dews are gone off, disease is likewise materially prevented. Nor should we forget that, in our present improved state of husbandry, instead of the grass being sacrificed to the arable land, vast numbers of sheep are more supported upon the arable than upon grassland. The plan should, however, be confined to store-flocks, and to those hardy short-woolled species which can bear fatigue.

The fold is formed either with wooden or iron hurdles, or, in some cases, with nets attached to spikes which are fixed into the ground; but the more usual practice is to employ hurdles formed of wattles of about $7 \frac{1}{3}$ long by 3 feet high, which are bound together by rods, and daily removed by the shepherd, when the flock is turned out. On the hills of Surrey the hur. diles run from seven to nine feet in length; but they are staked at the average distance of seven feet and a half, and three or fotir sheep are usually allotted to a hurdlet. Forty of them, if set in a square form, will therefore nearly cover an acre within eight days. The inclosure is changed daily; but it is only necessary to move three sides of the fold, leaving that one standing which was the outside one the day before. It should be pitched according to the quantity of manure intended to be laid $\mathrm{om}$, and its size should be proportioned to the number of the sheep to be confined in it. It should, however, not be larger than will give them convenient room to lie down; for, if more space be allowed, they will huddle together in a corner, and not drop their dung equally on the ground, which is the great object of their being thus penned.

* Survey of Somersetshire, 3rd edition, note, n. 146.

$\dagger$ Surtey Rep̧ort, p. 532.

VOI, II. 
It is usually pitched in a square or an oblong form; and in Iient 200 sheep ninhtly are considered equal to the folding of an acre in a week, the value of the manure being estimated according io the time of the year; the last folding in autumn, next to the ploughing for wheat, being thought the must valuable*. Thiese, however, are probaily Romneys, of which great numbers are sent by the Marsh famers to be wintered by those upon the uplands, and being of large carcass, may be found sufficient for a light dressing; but in Sussex and Hanıshire 500 Southdowns are thought necessary to twenty-eight perches, or from 3000 to 3500 for the manuring of an acre of land per week†.

A bout 400 sheep of a middling size will therefore daily fold twenty square perches, or, in round numbers, forty-five acres in the year, supposing them to be regularly folded. In breeding flocks it mar, however, be as well to omit folding for five or six weeks after limbing, as in driving to and from the fold accidents often inappen to the young lanbs, by being trampled upon, and they should be left quietly with their dams until they acquire a little strength.

The value of the mamure is usually estimated at from 35s. to 50s. per acre; its goodiness depending on the food upon which the sheep have been lepet; for if fed upon tares, rape, clover, sainfoin, or turnips, the animals will drop more dung than if kept upon grass only: supposing it, therefore, to be calculated at the medium sum of $40 \mathrm{~s}$. per acre, and only forty acres to be folded in the year, this would leave sol. sterling, or $4 s$. per sheep from the flock of four humbed. Whether this profit may be equivalent to the loss on wool and flesh is evidently a matter of calculation on the rulative moles of management, which may be fairy left to the farmers who severally practice them, as the merits of folding rest entirely on its Iocal application.

In those counties which contain large districts of uninclosed common litul, a practice formerly prevailed to a great extent among famers of liring the flocks of nien who lived by keeping sheep upon the con:mons, for the purpose of folding.t The price was usually from 1 s. to 1s. Gil. per score, pir week-of cutrse, according to the condition of the flock, and the nature of the feed; but inclosures were carried to such an extent during the late war, that the plan has necessarily fallen much into disuse.

On smali farms, with floclis too small for the employment of a shepherd, or where the ground is too wet to carry sheep upon the fallows, the advantage of securing the dung for the arable land may be attained by having a capacious stading fold on some convenient dry spot of jasture, or immediately adjoining the farm-yarl, one side of which may form a fence, and the buildings will afiord a considerable degree of shelter. That large quantities of dung may be thus collected without any trouble that is worth speaking of, no one can doubt; and if the fold be well littered with dry leaves, stubble, haulm, or straw, compost of very valuable manure may be thus formed. 'Thus, in the Annals of Agriculture, an experiment is mentioned of eight score of slieep being penned for six weeks in a stauding fold, littered every week with a load of straw. They were fed morning and evening with drawn turnips, of which they consumed two aeres; an! they finaliy produced twenty-eight large loads of rich manure,

* Boys' Survey of Kent, secordd edition, p. 158.

t Sussex Report, p. 347; Hants do. p. 369.

t In some parts of Buckinghamshire it was so general, that 3000 sheep were hired upon different farms in a small district.--Kep. p. 278. 
which might be fairly stated as worth $\% s$. 6d. per load, amounting to 10l. $10 \mathrm{~s}$. The account, indeed, neither shows the breed of the sheep nor the (juantity of turnips which the land produced, and is so far imperfect; but, supposing the straw to be worth twenty shillings a load, and the labour of spreading the manure upon the ground to cost ten shillings, this would leave $4 \%$. for the value of the dung, or three pence per week for that of each sheep. In Hertfordshire also, 300 short-woolled sheep, penned in like manner from the end of October to the end of March, and littered with stubble, are stated to have produced 80 large cart-loads of the richest dung.

The plan is, indeed, in use among many persons who have large flocks upon light land, and is there employed for the purposes both of fatting wethers for the market and of procuring shelter for the ewes at lambingtime. Mr. Ellman of Glynde, has, for instance, two or three yards with sheds twelve feet wide around them, which, being also littered, are extremely warm, and preserve many lambs in stormy weather. They may be fenced with wattled hurdled-work, for the sake of coolness, lest a greater degree of closeness might render the yard too hot; and the system has answered so well on several large farms, that the sheep are said to have been found more healthy than when lept out in the fields in the common manner*.

The late General Murray had a standing fold, or rather a sheep-cote, which inclosed an area of fifty-seven yards in length by twenty in breadth; thus containing 1140 square yards, in which upwards of 700 slieep could be pemned at night, with more than a square rard and a half for each. All around it was a boarded shed, nine or ten feet wide, and alsu across the midille; the latter being open on both sides. A rack for hay placed against the wall, with a small nanger underneath, surrounded the whole; and another, which was double, allowing the food to be eaten from both sides, stood along the central shed $\uparrow$. There is also one upon a smaller scale, which has been many years in use, on Mr. Lambert's estate in Ireland, of which the ground plan, as described in his observations upon farming in that country, is thus laid out.

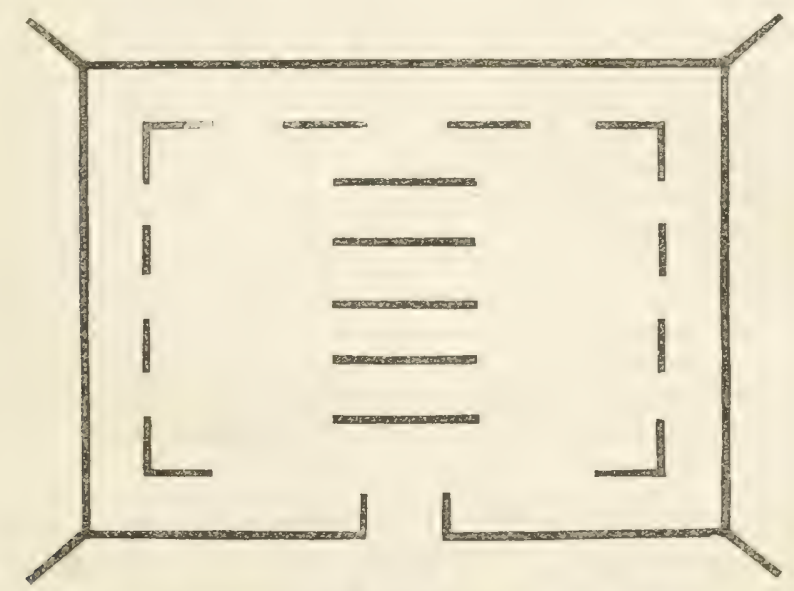

It is raised upon a gentle declivity with a southern aspect, and is 80 fect

* Hertfordshire Report, p. 195.

$\uparrow$ Survey of Sussex, p. 319.

2) 112 
square, or capable of conveniently containing about 250 sheep. The north, east, and west walls are $9 \frac{1}{2}$ feet high, surrounding a shed seven feet wide within, the roof of which leans to a vall only $3 \frac{1}{2}$ feet high, with large opening's for the entrance of the sheep. The southern wall is only four feet high, being left open to the sun, and has a gate wide enough to admit a cart. In the centre a few short solid walls are built and planked over lengthwise for the support of a rick of hay, the sheep being sheltered underneath. There are also walls projecting diagonally from the comers, of the same height as those of the outer part; thus affording shelter for horned catile; or, if necessary, allowing the addition of a roof for the enlargement of the fold. There are small racks all around the shed, and openings in the wall for the free admission of air. "These racks should, however, be invariably arranged so as for the sheep to put their heads down to eat, instead of up; as the latter method fills the fleece with the seeds of the hay*." With respect to the inclination of the roof dounwards from the outer wall, an objection has been not unjustly made, that it thus causes the droppings of the eaves to fall within the court; whereas it ought to incline upwards, in order to preserve the inner court as dry as possible; but this would allow the rain to be blown under at least one side of the pent-house, and the shelter from wind would be less complete: the two modes, therefore, have a choice of difficulties.

Sheep-cotes of this kind may be constructed at very trifling expense; for the walls may be raised with mud and straw, in the manner of pizét, or with rough posts filled in with furze, and the shed thatched, while the racks can be made without the aid of a carpenter. Indeed, one made, probably in this manner, though 60 feet long by 15 wide, and capable of containing twelve scores of sheep under its roof, is stated to have only cost 12l.+ The floor, however, should be laid with either limestonegravel, flint, or rubble, and beaten hard, so as to render it dry. They are unquestionably of essential service in lambing time, and may, in very wet weather, be found occasionally useful as substitutes for the open fold; but, in all other respects, we think the system of cotting, in a climate like this, objectionable. Nature has provided the sheep with a flece, which guards them against the effects of cold and rain ; for, even in the bleak mountains of the north of Scothand, they thrive without any other shelter than the stells, which are in some few cases formed for their security agranst the destructive results of a snow.storm, and they live wholly abrond in the Orkney and Shetland Isles. 'The breeders in Saxony, indeed, house their Merinos during winter, under the idea that breeds which came originally from Spain require more tende: care than those of their native species; and the system was once followed with such success on the Ryeland stock, that wool, so produced, boie three-pence a pound more than that which was exposed to the inclemency of the weather.

'The practice is said to have been introduced into Merefordshire by the Flemings, about the yeit 1660 ; but the fineness of the wool of that breed was celebrated at a much earlier period by Camden, under the title of

* Webb Hall, on the best means of improving the British fleece. Papers of the Bath Society of Agriculture, vol. xv. p. 117.

p For the mode of preparing the earth for the construction of these walls, see the account given in the Appendix of the first volume of Communications to the Board of Agriculture, and the Annals of Agriculture, vol. xxxix., No. 227. Walls of this kind erected at Woburn, cost only $17 \frac{1}{4} \mathrm{~d}$. per yard.-Bedfurdshire Report, p. 23.

f Farmer's Mag., vol,xv. p. $2 \$ 6$. 
"Lempster ore," as being almost peculiar to the neiglibourliocd of Leominster; $*$ and Drayton, the poet, asks-

"Where lives the man so dull on Britain"s farthest shore.

To whom did never sound the name of Lempter's ore?"

It is doubtful, however, whether the delicacy which the plan of cotting occasions may not prove eventually injurious, for the sheep, in fact, suffer more from heat than from cold. It has been tried in many instances in this country without success, and was adopted during several years, with moveable cotes, by that eminent and intelligent farmer Mr. George WVebb Hall, in the management of a Merino flock, which he has just abandoned with very severe losst.

In bleak and exposed mountainous situations shelter is, indeed, sometimes requisite; for the drifts of snow are in those districts so violent as to overpower the animals, which are often buried beneath the superineumbent mass, and thousands have been lost for the want of some protection against the inclemency of the weather. Farms thus situated, always extend over a wide space, which feeds such numbers of sheep that it is no very easy matter to provide for their means of security, and thus they are in most cases left solely to the care of Providence. Some occupiers have, however, erected open folds, or as they are there termed, "Stells," to guard them against the effects of the blast.

The Stells are merely intended as a refuge for the shcep in cases of surlden storm; and as this often occurs without any notice of danger to the shepherd, who is not always near his flock, they are left open to the animals, who are taught by instinct to seek them as places of safety. They are, therefore, not to be considered in the light of permanent folds for the collection of manure; neither are they generally made to contain more than five or six score; for, if larger, the walls would present a surface which would prevent the drift of snow, and might accumulate it to a dangerous extent within the inclosure. They are, therefore, placed on different parts of the furm, and care should be taken to select those spots where the snow in its drift may be expected to be carried clear of the pasture; for otherwise the sheep may be buried under the accumulating wreath, and meet destruction in the apparent arms of security.

They are made in various shapes, as the farmer may deem them most likely to guard against the effects of the tempest; some preferring that of the letter $\mathrm{T}$ or $\mathrm{H}$, as affording shelter against the wind, from whatever quarter it may blow, and others, upon the same principle, that of a figure of $\mathrm{S}$. The most approved form, however, is circular, as that causes the wind to whirl constantly round during a violent blast, and thereby prevents the snow from lodging within the fence; particularly if it be raised-as it

* Herefordshire Report, p. 120.

† Although such has been the effect of the experiment, yet Mr. Hall attributes it wholly to the practice of breeding in and in; which ended in his not being able to keep tlacm alive. His plan was to have moveable cots, 24 feet square, divided into partitions of 8 feet by 12, by which means they could pass through field-gates, and, mounted upon wheels, could be drawn to any part of the farm where the sheep were feeding; the expense for the cots of 100 . sheep heing about 30l.-See his Examination before the Committee in the House of Commons upon the State of Agriculture, in March, 1936.

They have also been gartially employed by the IIon. Geose Villiers, and a few other persons: plans and views of which may be seen in the Agricultural Report of Hertfurlshire.

That the land was thus improved there can be no doubt, and we can readily believe that in hot weather the shade was grateful to the animal; but we are sceptical as to the similar result supposed to have been derived from the shelter afforded to the flock from the cold of any southern counties. 
ouglit to be-upon level ground. Those of the late Lord Napier, in Ettrick Forest, are upon that plan; ten yards in diameter, with a three-feet open door, and six feet high; accommodating 75 sheep, with an ordinary accompaniment of lambs. The walls are of rough stone, with a coping of sud, and the cost only calculated at about $40 \mathrm{~s}$. each; the wages of the shep. herd who assists in raising them, being, of course, not included*.

There can be no manner of doubt that the shelter thus afforded to mountain flocks in tempestuons weather is, wherever practicable, of great utility ; but if not accompanied with food, it is comparatively useless, as the storm often lasts during a long period, in which they might be starved. Small ricks of hay should therefore, if possible-that is to say, if the farm produces it-be placed in each of the stells, and left open to the sheep; for the depth of snow will sometimes prevent the shepherd from reaching them, and they must be left to feed themselves $\dagger$.

It might, indeed, be supposed that they would consume the hay in preference to the grass, and that, having it at their option, they would become indolent in their search of food, as well as reluctant to face the blast; but the objection is unfounded, as nothing but sheer hunger will compel a truebred mountaineer to quit its pasture, and it is extremely difficult to induce sheep to eat dry hay if they have any kind of green food. In saying this, however, we allude solely to the original black-faced Scots, uncrossed with any other breed; for they are a truly hardy race, adapted by nature to the elevited regions in which they are placed, and never, in any weather, sceking shelter, unless impelled by the instinctive approach of a snow-storm. Now, those high-situated farms, though always the most extensive, and having consequently the largrest flucks, yet have invariably the smallest portion of soil capable of producing hay. Caution should, therefore, be used in the change of stock; and, as has been justly observed- " the improver of sheep ought to consicice before he interrupt the ordinary and longsettled peculiarities in a breed, that it is not a dead or ungenerous soil he is going to revivify, but it is a living organized creature upon which he intends making a change; and if, in his attempts to introduce some alteration, he impart a weakness to the animal system, he unfits it for the climate or for the pasture, and the change will prove in its effects mischievous and destructive f".

In fine, the respective advantages and disadvantages of the different modes of folding, may be thus summed up :-

The common moveable fold allows of the ground being regularly dunged without any further cost than the trouble of removing the hurdles. Being, however, commonly pitched upon arable land, the dirt and wet are injurious to the animal : and, if placed at any great distance from the pasture, the labour of travelling prevents the stock from fattening.

The stell is only applicable as a place of security in mountain-storms of snow.

The standing fold may be placed in any dry spot that is most conveniently situated, and if bedded either with sand or litter, composts

* See Napier on Store Farming.

f As the quantity of dried provender can never in those farms be very abundant, the hay has been in some instances stored within the stells in small inclosed sheds, from which it can be dealt out with a sparing hand; and in lambing time, they are used as covered sheep-cotes.-See Farmer"s Mag. vol. xxiii. p. 425. One pound per head, per day, will save the animals from starving; but they ought to have at least three pounds to satisfy hunger.

+ Remarks of a Lammermuir Farmer on Napier's Treatise on Store Farming.Farm. Mag. vol. xxiv. 1. 419. 
are formed for manuring the land at a proper season; but it deprives the soil of the supposed advantages of the teathe, and occasions the expense of mixing the compost and spreading it upon the ground.

The cote, combines all the advantages and disadvantages of these several modes, with the addition of shelter; but it occasions the cost of erecting sheds, and if constantly used, causes the sheep to become unhealthfully tender.

\section{THE SHEARING}

of sheep usually takes place, if the weather be fine, in the early part of June; but although sometimes deferred under the idea of rendering the fleece heavier, yet the earlier the operation is performed the better, as the growth of the wool will then prevent the attacks of the flies, which at a more advanced season, particularly in inclosed and woody countries, are often very destructive. It is also advisable, if the sheep are to be slanghtered during the summer; for, if the fleece be not removed, the heat thus imparted to the body gives the flesh a rancid taste, and it does not recover its usual flavour in less than three weeks or a month after the wool is taken off*. The period, however, must depend upon the season, the progress of which should be watched, and, so soon as the young wool has sufficiently raised the old fleece from the skin, it should be clipped.

In former davs the lambs were also in most cases shorn about the same time, or a little after, and this, except when they fall very late in the snring, is almost universally the practice all over the continent; for although the value of the irool is trifling, yet its clipping is supposed to improve the growth and strengthen its staple, as well as to render the coat less liable to open and part along the line of the neck and back of the animal during the succeeding heavy and driving rains of winter + . In this country, however, it has been lately thought better to let the fleece remain unclipped until the following year, for the hogget wool is then found to be of much superior quality than if the fleece had been previously cut. The lambs are therefore very generally left untouched until the following season.

Previous to shearing, the sheep are washed, in order to disencumber the fleece of any coarse dirt which it may have contracted. In some hill farms, indeed, this is omitted; and on others, having very large flocks, the sheep are merely forced to swim three or four times across some neighbouring stream. The most usual practice, howerer, is to form pens, or artificial jools, railed round with one rail only in the height of the water, so that the sheep may be thrust moler, when wanted, with a "poy"-which is a long pole with a projection of about six inches on each side of the bottom, to enable a man either to pull the sheep to him, or to push them from him.

These wash-dikes are generally made in small rivulets of two or three feet in depth, pointing against the current, so that the foul water may keep draining from the sheep; or, by causing a dam to be placed across in a convenient place, with a flood-gate in the middle, by which the water can be ponded up for the occasion, and let off at pleasure: on the one side a pen is formed for the washing, and on the other a path is hurdled in for the sheep to ascend when the process is over. Though sometimes made by individuals for their private use, they are in most cases formed by persons who let them out and charge a trifling sum per score, accorling to the breer, for washing. Perhaps that described in the Rutlandshire Report as having 
been arranget, at Burleigh, by the Earl of Winchelsea, for the convenience of his tenants, is as complete as any in the kingdom: we, therefore, here insert the plan.

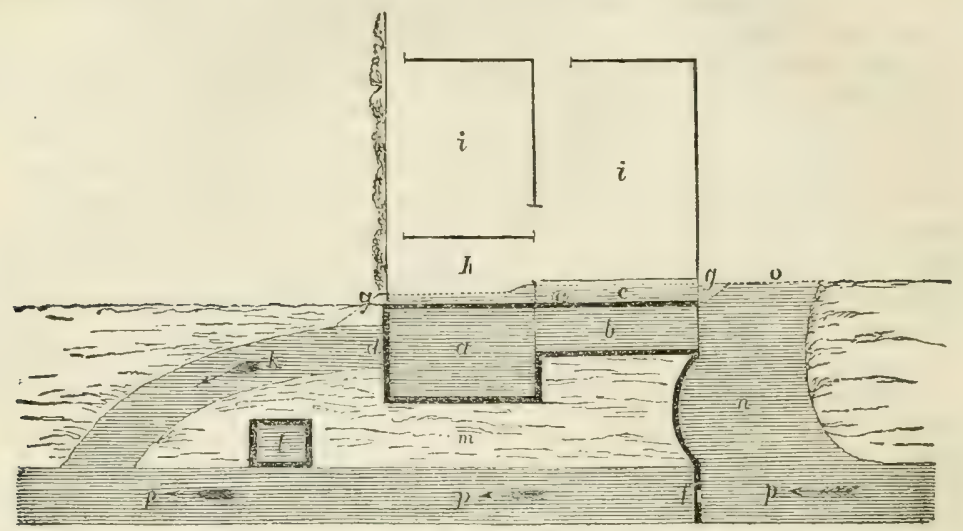

a. The pit for soaking, 12 feet long, 8 feet wide, and 4 feet in depth.

$b$. The channel where the sheep are washed, 12 feet long, 3 feet 4 inches wide, and 3 feet 6 inches deep.

c. A place for mes to stand to wash the sheep over the parapet wall.

d. Stop-gate, to let off the water in case of floods, or when the pit reeds emptying. On each side of this stop-gate there are two holes, at two inches belww topwater mark, to let off the waste water into the outlet drain.

c. Parapet wall, 3 feet 9 inches in height.

f. Ston-gate, level with top-water mark, so that when there is more water than will pass through the two holes before mentioned, at the sirles of stop.gate d, it runs over this gate.

g9. Along this dotted line is a pared channel to take off the water, which runs from the sheep at their landing, and conducts it into the outlet drain, so that it may not run into any part of the wash-dike again.

$h$. The fold for the sheep, previous to their being thrown into the soaking-pit.

ii. The sheep-fulds.

$k$. Outlei drain to take off the waste water.

l. House for the clothes of the men, implements, \&c. \&c.

$m$. Waste ground betwixt the river and the wash-dike.

n. Breadtl of water when held up by the stop-gates.

$o$. Landing-place for the sheep after they have been washed.

spp. The course of the river.

The operation of uashing (that of scouring being a subsequent process not expected from the wool-grower) is commonly performed thus:-About a score of the sheep, or as many as the pen will contain, are first put in to soak the wool, and are tlience put forward singly, by the man who poys the sheep, to two or three washers, each standing singly in a line, in tubs about $3 \frac{1}{2}$ feet deep, and having leather on their arms up to their shoulders; but if the sheep be strong, a couple of men, standing opposite to each other, are required to manage them. The first, seizing the sheep by its legs, plunges it, on its back, to and fro several times from side to side, and then passes it to the next in orler, who also immerses it and presses the wool between his hands, at the same time turning the sheep in every position, while the third examines the fleece and gives it its final washing; after which the animal is allowed to swim ashore, and is immediately conducted to a clean pasture, where the fleece may be allowed to dry without contracting any further stain. The business is generally over on each day by about 
two or three o'clock in the afternoon, in order to give the sleep time to get somewhat dry, and recover their natural warmth before the cold and damp of the evening sundown: the number which can be washed depending, of course, in which way the business has been done*. The same operation is by some persons repeated a few days afterwards, in the view of still further cleansing the fleece; but it has been found to materially injure the softness of the wool.

Running water is commonly preferred, as being the most pure and likely to perfectly cleanse the fleece; though, if impregnated with chalk, it should be avoided, as rendering the wool rough and brittle; and if the stream be very cold and sharp, it is not advisable to put more than four sheep into the pen at a time. A roomy pond of clear stagnant water, with a sound bottom, free from mui, is, however, better; not only because the softness of the water causes it to act more effectually in removing impurities, but the oily matter, or "yolk," contained in the wool, being of a soapy nature, strengthens the wash so much that the greater the number of sheep washed the better has the water been found to cleanse. The Farming Society of Ireland have, indeed, recommended the use of a large tub of water, warmed to about blood heat, in which to place the sheep till the wool be softened and well washed; after which they may be river-washed. Bakewell, of Wakefield, was of that opinion : he says, "that the leeping the animal a sufficiently long time in the water endangers its health; that fleeces of a close pile camnot be cleansed by the usual mode of washing, and that the extra labour required to wash sheep in tubs with warm water would be amply repaid, were the first and second washings carried out and applied as manure, the quantity of rich animal soap which it contains making it one of the most fertilizing applications which can possibly be used $t_{.}$,

If the weather be warm, the flcece will probably be sufficiently dry to commence the operation of shearing on the third day after the washing; but it is generally thought better to allow a full week to elapse, both as insuring the wool to be free from damp, and allowing time for its imbibing some portion of that insensible perspiration from the body which is supposed to constitute the yolk, and which imparts a great deal of sofiness and elasticity to its feel. When left on the shecp, the yolk is, indeed, most precious to the animal; for its oily nature not only protects it against the rain, but nourishes the growth and closeness of the wool, so as to render it almost impenetrable to cold.

The shearing is generally done under cover, upon the clean floor of a barn, but may be equally well performed in the open air. The sheep which are intended to be shorn on that day, are penned close to the shearer; and one man, if a good hand, will operate upon two or three score, according to their size and the sort of fleece. About fifty hear of Southdowns may be the average. The operation is performed either along or across the body. By the former, the shears moving in a level, the stroke is closer and more equal; while by the latter, it moves in a curved direction, thus rendering it more difficult, as weli as occasioning a greater risk of nicking the skin : it, however, gives a neater appearance to the fleece, and has therefore of late become much in fashion. The mole of performance is as follows:-

* Parkinson asserts in his "English Practice of Agriculture," that he has known one man wash 1000 sheep in a day, at the price of four-pence per score, p. 190. Yet he admits that, when in Ireland, he found it difficult to wash 650 , though employing fifteen men in the operation, p. 186.

+ Bakewell on Wool, p. 71 . 
The mountain breeds, being very wild and restive, are usually tied by the legs previous to the commencement of the process; but with others of a more quiet disposition, it is not customary. The animal being handed to the shearer, he takes it between his legs, placing it upon its rump), with its back against him, loolding it with his left hand, and with a sharp pair of springshears, blunted at the points-which, being without handies, he can manage with one hand - he with his right clips the wool from the neck and shoulders. He then turns the sheep upon its side, and kneeling upon one kree, he holks it down by the pressure of his leg upon its neck, cutting the wool circularly round the body, by which the work is closely and uniformly executed. The entire fleece is thus stripped off at once, and rolled up firmly by another person, the outside being folded inwards, from tail to shoulder, and tied together by a portion of the wool twisted into a rope*.

It is also a good practice to clip off the coarsest wool on the thighs and dock about a month before the shearing; as this keeps the sheep clean and cool in hot weather, and the clippings, which may amount when washed to about a quarter of a pound, are used as stuffings for flock-beds and horse-collars.

Experiments have been made on shearing the sheep twice in the same year-the first clip six weeks before the usual time, and the second in the montl of September. The trials, however, have not been attended with the expected advantage; for although a trifling additional quantity of wool was thus obtained, it weakened the staple in the following year, and exposed the animals to injury from cold. To meet this latter objection some persons, indeed, proposed to clothe them in flamel jackets! in the same manner as sometimes practised by the Leicester tup-masters; but it is almost neerless to say that, however such a plan may suit the breeders of rams of a delicate and high-priced species, the idea of thus dressing up whole flocks was too absurd to be generally entertained.

Means have been hikewise tried, on the celebrated national Merino flock, at Rambouillet, in France, of improving the quality of the wool by allowing the fleece to grow for several years; and, it is said that some of the sheep which were not shorn for three, and others five years, produced a larger growth, as well as a stronger staple and a closer pile, than those which had been amnully shorn. The alditional value of the wool did not, however, appear to have compensated for the loss thus occasioned in quantity, by the omission of the two rears' clipping, as the plan was not continued. It must also, we conceive, have been prejudicial to the constitution of the animal; for it is the greneral jclea of all intelligent breeders, that extreme heat is more hurtful to it than severe cold. Indeed, as the growth of new wool raises the old from the skin, and deprives it of nourishment, we should have imagined that the experiment must have injured the flcece instead of benefiting it.

The shearer should be an expert and neat workman. He should cut the wool quite near and close to the skin, so as to make a clean clip; for it thus

* Or more minutely thus:- " When shorn the fleece should be carefully folded and rolled, beginming at the himler part, and folding in the sides or belly-wool as the rolling proceeds. When arrived at the shoulders, the wool of the fore part should be rolled back to meet the other, instead of having the hinder twisted from thence in the usual manner, and the whole secured by a pack-cord, in the common way in which parcels are tied up. Thus the fleece is kept much tighter together, and unfolds itself with more regularity under the hands of the sorter, who is otherwise much inconvenienced by the confusion or breaking of those parts of the fleece, which in the common methods are twisted together for the band."-Complete Grazier, 6th edit. p. 272. 
not only weighs heavier, but it also feels more softly towaris the bottom of the pile. He should also be very careful ; for if, by any mismanagement of the shears, he cuts the skin, the flies will immediately attack the sjot for the purpose of depositing their larve, and, if not prevented, or afterwards remedied by the watchful attention of the shepherd, they will infect the whole carcass with maggots, which if they do not eventually destroy the animal, will render it so restless as to prevent its thriving. The efiects of the accident, however, if it occurs, may be guarded against by smearing the cut with a little turpentine, or any healing kind of salve.

The floch should therefore be carefully examined repeatedily after shearing, and if the flies have deposited any of their eggs, the tumours should be opened and rubbed with mercurial ointment, the smallest portion of which is fatal to the insect. The following unguent is, indeed, recommended in the Shepherd's Manual, to be rubbed on every part of the animal with a currying brush, in order to kill the lice and ticks, with which it is commonly infested; namely-

\section{Train oil, 4 gallons; oil of $\operatorname{tar}, \frac{1}{2}$ do.; oil of turpentine, 1 pint.}

Tohacco juice has been attended with a similar eflect. Another mode is also sometimes adopted with lambs for the same purpose. A pound of arsenic is dissolved in boiling soap-suds, and then poured into a tub with a large quantity of warm water. In this solution, which is sufficient for about twenty lambs, they are immersed singly, and then dried as well as possible by squeezing the wool by hand. One dipping destroys the lice, and preserves the animals during the summer from the fly and magrots; but care should be taken not to dip them over-head, lest they should swallow the water*. 'This latter mode is the most effectual; but it is wholly Imnecessary to dip any of the sheep which have been shorn, and, therefore, the lambs only are dipped. There is no shelter for the ticks on the sheep which have been shorn, and if they are killed in the lambs, by this process of dipping, immediately after the ewes have been cleared from them by the shearing, the flock wili be secured from their annoyance during the whole succeeding year.

\section{SALVING.}

The smearing of sheep with oil, tar, butter, and other ingredients, is a practice much followed towards the close of autumm, with regard to flocks in the Highlands of Scotland; it being generally considered necessary by the store-farmers of that country, both for the purpose of protecting the animals from cold, remedying the scab, improving the growth of the wool, and destroying the vermin which may be lodged in the fleece. The latter is, however, thought by many persons to be its chief, if not its only, use. "Wher, indeed, arlmit that it prevents the wool of very old ewes from growing coarse and hairy, but insist that it is rather injurious than otherwise to the animal to open its wool at the commencement of the winter season, when it needs ali the warmth of its natural elothing; and the application of salve occasions a chill throughout the frame, which is not recovered for ten dars or a fortnight, or until the growth of the flece has, in some measure, removed it from immediate contact with the slsin $\dagger$.

This effect of the salve during a few days, may, perhaps, be correct; but, although alluding to it, out of respect to the authority on which it is

* Leicestershire Report, p. 275.

+ Harkness, on the Smearing of Sheep, Prize Essays of the Highland Society: N.S., vol. iv. p. 129. 
mentioned, yet the prevailing practice is that which we have already stated, and the more prevalent idea is, that the coating materially assists in guarding the sheep against the extreme severity of the weather: in proof of which we have the evidence of a farmer who has, during a number of years past, paid great attention to the health and thriving of his flock, who asserts-" that those lots which were left unsmeared were inferior in condition to the others at the time of clipping, and the cover of wool was not so good on them*". It, indeed, stains the wool in such a manner, as to render it unfit for receiving the tint of very bright colours in dyeing, and the fleece is not easily cleansed; but whatever it thus loses in price, is amply made up by its addition in weight. In low situations, "raw," or unmelted turpentine may, we understand, be substituted for the tar; for it wants the carbonaceous colouring matter of the latter, and, if carefully mixed and lightly put on, it produces well.

The operation is both tedious and disagreeable: it is, therefore, very generally hurried over imperfectly, as well as prodigally; for the salve is not unfrequently loaded on some parts, while others are neglected, thus occasioning great waste, while the vermin escape, and the scab increases on those spots which are left untouched. The mode, when properly performed, should be as follows :-

The materials most commonly used are tar and butter, in the proportion of about a gallon of tar to 12 lbs. of butter for every score of sheeprather more tar being used for the hoggets than for older sheep; and these should be prepared by steeping the tar-barrel in boiling water until it becomes quite soft, and pouring upon it the butter, which is gently heated without being oiled: or, both may be placed over a very slow fire until they become fluid, after which they are stirred together intil completely mixedt. Four gallons of Virginia tar, with $35 \mathrm{lbs}$. of butter, is the quantity usually employed for every 45 sheep of a large flock of Cheviots; but in very motintainous and exposed districts, 40 lbs. of butter and 5 gallons of tar are frequently used to 50 or 60 of the small black-faced breed: though, if skilfully applied, it would probably be found sufficient for 70 or $80^{+}$.

Other substances are, however, frequently employed-as rosin, turpentine, palm and fish-oil, in various quantities, either alone or mixed up with tar and butter: thus in the account to which we have already alluded, as furnished by Mr. Graham, the proportion and expense of materials for 40 sheep were as follows:-

7 lbs, of Rosin, at 7 s. $6 \mathrm{~d}$. per cwt.

17 lhs. Butter, at $10 \mathrm{~s}$. per $24 \mathrm{lbs}$.

16 liss. Paim-oil, at 6 s. $6 d$. per do.

2 choppins of Fish-oil at $2 s .6 d$. per gall.

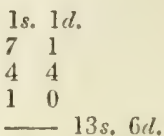

Forty other sheep were also smeared with tar, butter, and palm-oil ; the expense of which was-

4 quarts of Tar, at 1 s. per quart

$14 \frac{\mathrm{l}}{12} \mathrm{lbs}$. of Butter, at $10 \mathrm{~s}$. per $24 \mathrm{lbs}$.

$1+\frac{1}{2}$ lbs. of Pulm-oil, at $6 s$. $6 d$. per do.

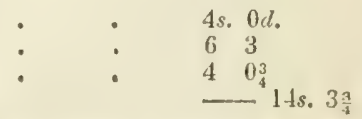

This, aduing the charge of a penny for the labour of laying the salve on

* Graham's Report of Experiments on the Salving of Sheep: Pize Essays of the Highland Society, vol. ii. p. 244.

f General Report of Scotland, vol. iii. p. 13!.

† Farmer's Mlagazine, vol, xiv. p. 191. 
each sheep, brings the cost to $5 d$. and $5 \frac{1}{4} d$. per head. The whole of the wool was sold together, and the proceeds were as follows :-

'That, smeared with Rosin, at 24 s. per 24 lbs.

Do. with Tar, , 21s, per do.

The White, or unlaid, ,, 24s. per do.

and the average return of each flece, as influenced by the weight of salve laid on, ivas-

The Rosinned, $4 s .0 \frac{3}{4} l$; ; the Tarred, $4 s$. : the Unlaid, $3 s .5 d$.

Notwithstanding which, we have, however, learned that all mixtures with rosin are objected to by the manufacturers. He, however, recommends the following articles, as being sufficient for the smearing of about 50 to 55 sheep; namely -

18 lbs. Butter; 12 lbs. Rosin ; 18 lbs. Hogs' lard ; 4 quarts Gallipoli oil.

The butter and lard to be heated in one pot, and the rosin in another; and when both are fairly melted, they are to be poured into one tub and well mixed. The oil is then added, the whole again well mixed, and allowed to stand some days till it is fairly thickened into salve. 'The quantity of hogs'-lard, he thinks, might be advantacreously increased, as he has ever . found it the most nourishing of greases for the health of the sheep and the growth of wool. The quantity of rosin is only such as will make the grease adhere to the skin, and the oil of Gallipoli is that used by manufacturers in the cleansing of woollens. The same number have also been smeared with, $2+1$ bs. of palm grease, 4 quarts of good lamp oil, and 5 gallons of tar; which is a cheaper mixture than that with butter, and is said to increase the wool so much more in point of weight that four fleeces thus salved were fully as heavy as five with butter*

The cost of all these mixtures, it will be observed, is considerable; but there is another, mentioned by Mr. Harkness, with which, he says, 72 sheep may be effectually smeared at the expense of three-halfpence each: it is this-

"Take 24 quarts of tar: add 2 gallons of clear seal oil, 3 imperial stones of potatoes, and $2 \mathrm{lbs}$. of suda." The potatoes are to be neatly pared and scraped, then well washed, boiled on a good fire, and finally mashed until they are reduced to a fine pulp. Pour the seal oil and the soda upon the potatoes, stir the whole well together until the mass is thoroughly incorporated; add one imperial gallon of urine, stir again, and when about the warmth of milk from the cow, pour the whole into the tar. After ten minutes" stirring. the whole will be perfectly incorporated, and the mixture will be fit for use in a few hours after."

The use of the potatoes, he states to have been occasioned by his having found that they washed the tar out of clothes with which it had been smeared, in a much better manner than soap, or any other substance which lie could find.

Some farmers have used Gallipoli vil alone, and have found that the wool sprang mure luxuriantly, and handled, when clipped, with more genial softness than that of fleeces which had not been smeared. If put carefully in to the bottom of the wool, when the sheep is dry, the experienced flockmaster from whom we obtained that information, sars, "that it takes exclusive possession of the fleece, and keeps the animal, in wet weather, as dry as a duck; it instantly kills all vermin, and produces a heavy flecee of beautiful soft wool." We have, indeed, no doubt that it dhas such an effect, and if the common oils, which can be procured at a cleap rate, were to be rubbed in immediately after shearing, instead of the common wash of

* Staplers, however, complain of its discolouring the wool, and rendering it so moist and clammy as not to be pleasantly manufaciured.-Farm. Mag. on the manarement of wool, vol, xiv. p. 192. 
tobacco water, it is possible that it would repay the expense upon every flock in the kingdom; but in situations where they are exposed to piercing blasts of the storm, there it evidently appears that the application of tar gruards in some degree against the inclemency of climate. It also partially prevents the scab, whereas oil seems, on the contrary, to promote it; for Mr. Sellar, the flockmaster to whom we have just alluded, assures us that an oiled sheep rubs the infection into his wool and skin, and gets the disease, while one smeared in the common way escapes it: the mountain breeders, therefore, fear to use it. The tar is likewise necessary for the purpose of retaining any oily ingredients with which it may be mixed, and, perhaps, at least one-fifth of the salve should be in all cases composed of it; or, in high and stormy parts of the country, one-third: it does not follow, however, that a thicker coat of the salve should be laid upon the sheep.

The manner of laying on the salve is thus:-The animal is placed upon a stoul just broad and long enough to contain his body either when laid across or at length; and having a narrow projection at one end, forming a seat on which the smearer sits astride. The mixture should be of such a thickness as that he can take it up with his finger, and yet, when drawn alone the skin, can be easily rubbed off. 'The wool is then parted longitudinally into rows, without a single pile being allowed to lie across them; so that the salve can be applied directly to the skin; and the rows should all be at such equal listance from each other, that the salve in the one may reach through the bottom of the piles to the salve in the other: thus covering the skin completely, and anointing every individual pile. No portion of it should, however, appear on the wool, except at its roots, every other part being quite loose and clean : and when thus carefully laid on, a thin covering will answer every useful purpose* . One man, if accustomed to the operation, will completely salve from a score to twenty-five.

Besides the increase of expense, a heavy coat of salve has also this disadrantage, with regard to ewes - that in running out it causes the loose locks of wool, which hang about the udder, to form themselves into tassels about the size of a teat. This, when the lamb first endeavours to suck, he frequently lays hold of, instead of the object which he is seeking, and, being thus disgusted, he is often deterred from making another attempt. To prevent this, it has been suggested that these tags of wool should be cut off at the time of smearing: they are, indeed, sometimes pulled off, but that is a practice which is not only hurtful to the ewe, but is, from its rough handling, often dangerous to the lamb t.

\section{THE SHEPHERD}

should not only be regularly brought up to his business, so as to be competent to direct these matters with the ability of a master, but should also be instructed in all the common surgical operations and modes of prevention and disease usually attendant upon the flock. He should be attentively observant of their habits, of which he can only acquire a perfect knowledge through long experience : the occupation is therefore nearly hereditary; but if any lad be attentive, and carefully instructed while young in his duties, there can be no doubt that he may acquire all the requisite knowledge to become a valuable servant.

Although apparently, in most instances, learling a seemingly idle life in merely watching over the flock, yet in yeaning ime, and in all seasons of in- 
clement weather, he has arduous duties to perform, which must be frequently executed during the night, and on most occasions when not under superintendence; the property under his care is also generally valuable, and alwars femands constant attention. In the words of one well acquainted with his duties - He should be honest, active, careful, and, above all, calmtempered; for if he at any time get into a passion with his sheep, he not only occasionally injures them, but acts at great disadvantage both in herding them and in working among them. A good-tempered man and a ciose-monthed dog will effect the desired object with half the time and trouble that it gives to the hasty and passionate one." He should, therefore, be in every respect a trustwortly person, in whom the most entire confidence can be placed: he should consequently be furnished with a comfortable cottage, and such other advantages as may render hin attached to the interests of his master; among which, perhaps, none will be fuund more conducive to his care, than allowing a small per-centage on the proceeds of the flock to form a portion of his wages. The shepherds are, indeed, in this country, not uncommonly allowed to have a certain portion of the flock belonging to themselves, they paring a portion of the expenses of their feed; but when this is permitted, it is often found that the shepherd's stock is the best, and the accidents fewer than those of the master. The inconvenience, as well as petty frauds, which the plan has occasioned, have indeed been so loudly complained of, that it has been prohibited, wnder severe penalties, in Prussia and some other states of the Continent. This, however, must be partly attributed to the fault of the master, for where a firmer does his duty, he camot be cheated without detection: as shown in the management of Mr. Sellar's extensive flock in Sutherlandshire.

His flock-sheep are all branded in the face with an ear-mark; but no shepherd being allowed to possess a branded sheep, those belonging to them are easily distinguished. Thev are scattered over the wiole of the farm; each shepherd herding not his own alone, but part of the whole of each shepherd in his service. The sortings and sales are all done publicly; and if any shepherd is found to have, on any herding, fewer or more lambs, or less or more casualties than the master or his reighbours, an inquiry follows, and any dishonest attempt occasions dismissal.

The shepherd's dog performs so prominent a part in the security and management of the flock, that, although so well known as not to need description, he ought not to be left entirely unnoticed. In this country there are two sjecies; one commonly used in England, and the other-the shay-haired "colly"-in the extensive sheep-walks throughout the northern parts of the island. Both howerer appear equal in sacracity; and, when well trained, the promptitude and stiil with which the faithful animal obers the orders of his master, render him a truly valuable acquisition. In driving the sheep, he is, however, tanght to bite at them; and, although this is not often done in such manner as to seriously injure, it yet terrifies them so much that heary ewes have been thereby known to slip their lambs. $M$. Lasteyrie describes a breed as peculiar to the care of sheep in I'russia, of small size, but stout and thick, with erect ears and shargery coats, bearing some resemblance to our wolf logs; hut, instead of biting the sheep when ordered to call in stragglers or to turn them from the road, they are instructed to push them forward with their muzzles. The dogs, also, which guard the spanish migratory flocks never bite, and are so gentle that, in cases of impending danger, the sheep gather round them for 
protection. The shepherds in that country have, indeed, an excellent plan of teaching a few wedders to obey their call and follow them; by which means, as the whole flock follow their leader, they are governed with extraordinary facility, and without any of that violence which is here so frequently displayed. They indeed answer to their name, as alluded to in the Scriptures*, and are generally rewarded with a handful of salt; but any sheep may be thus taught by feeding it with the hand when a lamb, and it certainly would be a judicious mode were it generally adopted.

THE WOOI.

of the long.woolled breeds of sheep is termed "combing," and that of the short-woolled "carding ;" in consequence of the different methods employed in its manufacture. The former-which is chiefly used in the fabrication of baize, flamnel, carpets, and other coarse goods-being passed through combs made with upright steel teeth, the object of which is to separate the filaments and assort them evenly for the purpose of being spun; while that engaged in the finer fabrics of cloth is pulled crosswise between boards, called cards, furnished with crooked teeth of wire, which break it minutely into long rolls, or "rovels," that are spun in a peculiar manner.

When their properties are described, the filaments of both are denomimated the "pile;" the fineness of which consists in the diameter of their fibre $t$, while their length and strength constitute the "staple," and their serrated roughness denotes that "felting" quality so well known to clothiers as a material advantage in matting the cloth under the fulling mill. Besides this latter property-which is peculiar to the short-woolled breeds-the more free the wool may be from "liemps," or hairs, and the more soft, pliable, and elastic it is, the more will it be esteemed. It should, also, be as uniformly white and free from stain as possible. The chief points to be considered in its choice are, therefore-

'The freedom of the pile from those kemps, or "stichel hairs," which are short, brittle, pointed, of a brownish colour, and more generally found in the inferior breeds. Its colour, also, and its unifurm regularily of strength throughout the fibre.

'The length of staple, as constituting the particular uses to which the wool is to be applied; namely, if long, for combing: if short and curly, for carding.

Its softress and elasticity; without the union of which it is of no use in the manufacture of fine cloth.

Its felting properly; which is described, by an eminent writer on the subject, "as a tendency in the pile, when submitterl to a moderate heat, combined with moisture, to coliere together, and form a compact and pliable substance.t"

Not only does the wool thus vary in its properties, but each fleece contains portions of a different quality, which are separated from cach other by an intermediate dealer, who acts between the breeder and the manufacturer as a wool-stapler. It is a business requiring great nicety of discrimination ; for although, when first shorn, the fleece is only usually divided into three linds, mamely-the "frime," which is separated from the neck and back; the "seconds," obtained from the tails and legs; and the "thirds," taken

* "The sheep hear his voice, and he calleth his own sheep by name."-John x. 3.

+ On this subject the reader is referred to some curious and very interesting matter in No. 3 of the "Histury of Sheep," now publishing by Mr. Youatt, under the superintendence of the Society for the Diffusion of Knowledge.-Farmer's Series, No, 71.

+ Luccock, "On the Nature and Properties of Woul," p. 161. 
from the breast and beneath the belly-a classification which corresponds with that used in Spain, the bags from which comtry are separately marked with the letters R.F.S., being the initials of Rafinos, prime; Finos, fine; and Secundos, seconds ; or sometimes 'T. for Terceras, thirds, or inferior. Those here engaged in it, however, enumerate no less than nine different sorts-some, indeed, as many as fourteen-each distinguished by separate names descriptive of its quality; the chief portions of which, as well as their relative prices, were thus stated to a committee of the House of Lords, in 182S, by Mr. Fison, of Thetford, so far as regards 15 tods, or $420 \mathrm{lbs}$. of clothing wool, grown in Norfolk in the under-mentioned years-

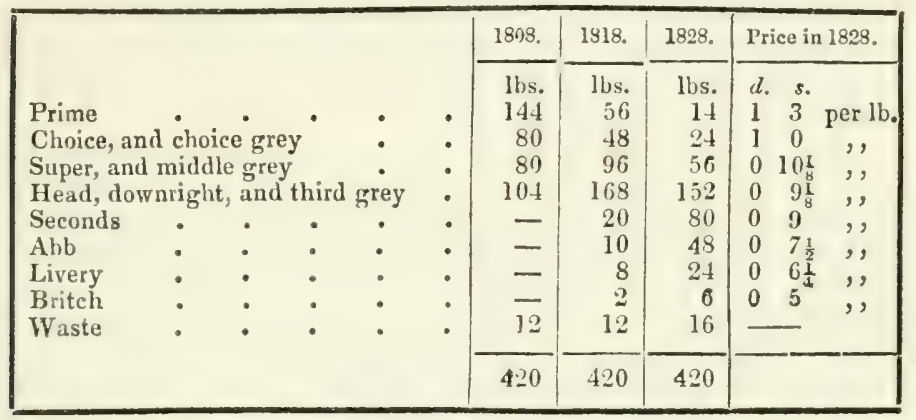

Calculating the weight of the sorts produced at each of the above-named periods, the result would be, that if our clothing wool of the year 1828 were equal in quality to the growth

Of 1808 , it would now produce $11^{3} d$. per lb.

Of 1818, it would now produce $10 d$. , , ;

whereas its value in 1828 was only $8 \frac{3}{4} d$ per $1 \mathrm{~b} .:$ from which it appears that the actual deterioration in quality, within twenty years, amounted in value to three-pence per pound.

The evidence of Messrs. Cunnington of Upavon, near Pewsey, in Wiltshire, shews nearly the same difference; and although the tables published in the report of the committee only apply to particular districts, yet they corroborate the unanimous assertion of the manufacturers, that British short-wool has very generally degenerated in its fineness, while, at the same time, its increase of weight is sliown by the account produced by Mr. C. Bull, wool-stapler, of Lewes, respecting the produce of various farms at different periods; from which the following are some extracts:-

\begin{tabular}{|c|c|c|c|c|c|}
\hline Produce of No. 1. & $\begin{array}{l}\text { Year. } \\
1806 \\
1816 \\
1826\end{array}$ & 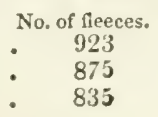 & 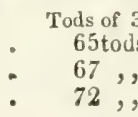 & $\begin{array}{l}32 \text { Ibs. } \\
\text { is. } 24 \text { lbs. } \\
-\end{array}$ & $\begin{array}{l}\text { Fleeces per tod. } \\
: \quad 14 \\
: \quad 13 \\
-\quad 11 \frac{1}{2}\end{array}$ \\
\hline Produce of No. 2. & $\begin{array}{l}1804 \\
1826\end{array}$ & $\begin{array}{ll}\text { - } & 1191 \\
\text { - } & 1189\end{array}$ & $\begin{array}{r}75 \\
: \quad 105\end{array}$ & $\begin{array}{r}5 \\
17\end{array}$ & $\begin{array}{l}15 \frac{3}{4} \\
11 \frac{1}{4}\end{array}$ \\
\hline Produce of No. 3 . & $\begin{array}{l}1804 \\
1822\end{array}$ & $\begin{array}{l}658 \\
\cdot \quad 655\end{array}$ & $\begin{array}{l}\text { - } \quad 40, \\
\text { - } \quad 58,\end{array}$ & $\begin{array}{l}14 \\
11\end{array}$ & $\begin{array}{l}16 \frac{1}{4} \\
11 \frac{1}{4}\end{array}$ \\
\hline Produce of No. 4. & $\begin{array}{l}1804 \\
1815 \\
1827\end{array}$ & $\begin{array}{l}1306 \\
. \quad 1350 \\
. \quad 1210\end{array}$ & $\begin{array}{r}83, \\
: \quad 104, \\
: \quad 115,\end{array}$ & $\frac{-}{3}$ & $\begin{array}{l}15 \frac{3}{4} \\
12 \frac{3}{7} \\
10 \frac{1}{2}\end{array}$ \\
\hline
\end{tabular}

This, were wool the main object in the management of sheep, would be voL, II. 
productive of great loss to the breeder, as well as a subject of national re. gret; but the vast increase of wealth and population in this country, and the consequent augmented demand for all the necessaries of life, has of late years caused the weight of mutton to be more attended to than the fineness of the wool, and experience has shown that the melioration of both is incompatible; for, if the animal be fed with such an abundance of succulent food as to unusually increase its growth, the nourishment thus given also increases the length and coarseness of the pile. Bakewell was the first breeder of any notoriety who introduced improvement in the sheep; but, in attempting to bring it nearer to perfection, his objects were directed exclusively to symmetry of frame, and so far as that might be impeded by attention to the quality of the fleece, it was by him neglected. In this, no doubt, however, the farmer has found his account, as he has been amply repaid for the deterioration of the wool, by the increased weight of both fleece and carcass.

He it was who raised the trade of ram-letting to the extraordinary eminence which it reached a few years ago. Marshall states, in his "Rural Economy of the Midland Counties," the hire of tups, prior to 1780, to have been from one guinea to ten for the season; at which latter price Bakewell, in that year, let several. His stock, from that period, rose rapilly in value, from 10 to 100 guineas, and in the year 1786 he let twothirds of one ram to two breeders at 100 guineas each: thus valuing the entire service of the animal at 300 guineas. From thence to 1789 the prices still kept rising so fast, that 400 guineas were repeatedly given : he received in that year 1200 guineas for three, and for his whole stock of rams, 3000 guineas; and several other breeders made from 500 to 1000 guineas cach. From that time, indeed, the spirit of enterprise kept increasing so fast that 500 guineas were repeatedly paid for the use of a single tup; and in three seasons one ram produced to its owner the enormous sum of 1300 guineas.

'The tup-masters formed themselves into a club, in Leicestershire, binding themselves by regulations tending to keep up the value of the stock by which they profited so largely, and other breeders in different parts of the kingrom followed the same occupation. The prices have now, however, fallen to a more moderate rate, and it appears extraordinary how the large sums formerly given were realized. They could, indeed, only answer to a speculator in rams for the purpose of afterwards letting their progeny out, and the calculation upon which they were rendered profitable has been thus explained: "If five persons have each twenty ewes good enough for rambreeding, and pay 500 guineas for the hire of a tup, they have a good chance-reckoning twin lambs - of each rearing ten rams and ten ewes; or mure, of a still higher blood. Now, supposing these ten ram-lambs, when shear-hogs, to be let out at 20 guineas each, upon the average, this would yield, upon the whole, 1000 guineas-or cent. per cent.-within two years, besides the future use of the rams, and the further improvement of the stock bred from the ewes *',

Prior to this, the finest wool manufactured in this country was obtained exclusively from Spain, and next to that the British short-woolled sheep of that period furnished the best quality in Europe. The most esteemed were the Ryeland, Dean-Forest, Mendip, Wiltshire, Southdown, ShropshireMurf, and Stretland fleeces; but by the judicious crossing of Merino rams with choice ewes of these breeds, and more particularly the Ryelands, wool,

* Løicester Report, p. 261. 
even of the fourth descent, was produced in fineness nearly equal to the best Syanish. Great exertions were at that time made by many patriotic persons to forward this improvement, and expectations were raised of our being soon able to rival the most celebrated of the Spanish flocks. These hopes have, however, been disappointed: the Merino carcass was unprofitable: the ewes not only produced few lambs, but were bad nurses, and the momentary advantage that was gained by the crosses has been wholly destroyed by the superior quality of the German wools, and the low prices at which they are now imported. 'The whole evidence before the Lords' Committee in $18: 25$, to which we have already alluded, goes indeed to prove, that the wools of Saxony and Bohemia have entirely superseded the English shortwool in our manufacture of fine cloth; and its value would have fallen below a remunerating price to the grower, had it not been partly counterbalanced by the increase of weight, and found employment for other valuable purposes.

The improvement which has brought Saxon wool to such perfection was commenced in 1765, by the Elector Augustus Frederick, who in that year procured 300 rams and ewes from Spain; and, in 1778, imported 400 more of the best breeds from the same country. His example was followed by some other of the sovereigns of Europe, and their success also persuaded his late Majesty George III, to adopt their views. Application was, accordingly, made to the Crown of Spain, and in the latter part of the last, and the beginning of the present century, large numbers of sheep selected from the most celebrated of the Spanish flocks were brought to England. These were afterwards widely distributed, and the plan of crossing was assiduously prosecuted by several distinguished agriculturists. That it failed here, while it succeeted in Germany, was not owing to any inferiority in our soil or climate, but is attributable to the superior value in this country of the carcass, which in some parts of the continent is of very little account, while wool always commands a ready sale. The plan was, therefore, abandoned, not from any doubt of the improvement which it occasioned in the fleece, if the sheep be fed upon short pasture, but from the difficulty of sustaining the weight of flesh upon any other mode than that of grazing them upon rich land, or feeding them under the present system of turnip husbandry; together with some inaptitude in the original stock to fatten. Great efforts were made to effect both objects ; but experience has decidedly proved, "that the improvement of fleece and carcass cannot be combined, and the former has necessarily given way to the latter.

That the wool was materially improved by most of the crosses with Spanish rams is well known to those who have tried them with judiciously selected ewes of the short-woolled flocks; as an instance of which is the statement of an experiment made by the late Lord Somerville-in his "Observations on Sheep," - with Merino tups and half-bred Ryeland and Southdown ewes, produced by a cross between Spanish rams and our mative breeds, which gained a premium from the Bath and West of England Agricultural Society.

It appears that thirty of these half-Ryeland and half-Spanish ewes, when tupped by a Spanish ram, whose fleece was not of the finest quality, yielded $96 \mathrm{lbs}$. of wool, with only $7 \mathrm{lbs}$. of F.'s and T.'s; which is much less than are usually thrown from pure Spanish wool : the prociuce being-

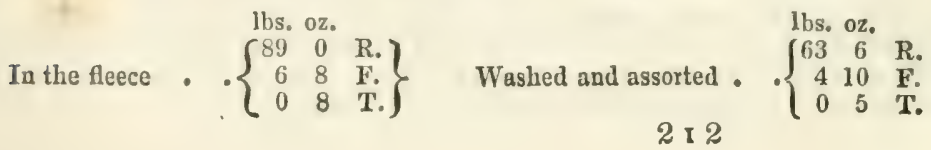


and, when manufactured, the goods gave sufficient evidence of its superior value, did not the small proportion of seconds and inferior wool put the matter beyond all dispute.

To this his Lordship adds-" That 86 ewe hogs of this mixed breed carried 235 lbs. 3 oz. of well-washed wool, and the cross with the Southdown sheep, both with respect to frame and wool, keeps pace with that of the Ryeland*."

These crosses with the Merinos have now, for the reasons which we have already stated, very generally fallen off; and as combing-wool appears, in the present state of our manufactures, to be more advantageous to the grower than the inferior qualities of the British carding sorts, the breeders of sheep are daily turning their attention to its production. There still remain, however, some who follow a contrary system; and to those it may not he inappropriate to mention the account griven by Dr. Parry, of Bath, of the progressive amelioration of wool by the Spanish cross; which is as follows :-

"The first cross of a new breed gives to the lamb half of the ram's blood, or 50 per cent. ; the second, 75 ditto; the third, 87 ditto; and the fourth, $93 \frac{3}{4}$ : at which periud it is said, that if the ewes have been judicionsly selected, the difierence of wool between the original stock and the mixed breed is scarcely to be discerned by the most able practitioners."

The rules promulgated in Fink's "Treatise on the Rearing of Sheep in Germany, and the Improvement of Coarse Wool," are-

'To select, at the commencement of the undertaking, the finest woolled rams and ewes that can be obtained for the first generation; for if those for the second race be finer than those used for the first, time will have been lost in effecting the proposed improvement.

In like manner, to employ rams for the subsequent breeds quite equal to those for the first, or otherwise the intended improvement will be retarded.

If an unimproved ewe be tupped by a ram of a mixed breed, and which has only one-fourth pure blood in him, the offspring will only have one-eighth of that race; and, by continuing to propagate in that manner, a complete separation of the two breeds will be at length effected.

Or the two modes stated by Luccock, in his "Treatise on Sheep," for the improvement of fleeces, may be adopted :-

"One, consisting in selecting thuse lambs for slaughter which have the least valuable coat; the other, in bringing into the flock male sheep of the most approved breeds, in order that their progeny may perpetuate their best peculiarities."

Although these rules were intended solely for the improvement of card. ing-wool, yet intelligent breeders will find no dificulty in applying them equally to long-woolled sheep; and it appears likely, from the facts already recited, that the latter breed will henceforward command superior attention. Indeed, generally speaking, the best wool is that described by Mr. John Ellman, - the great breeder of Southdowns, - as thick and curly, with depth of staple, and even toper ; such a fleece, in short, as will best defend the sheep in bad weather, and will not admit the water to penetrate, as it does

* In treating of the Ryelands, it may not be amiss to remark that they are not a mountain sheep, as many suppose; but though short-woolled, sinall, and of superior quality in regard to both fleece and mutton, they are bred in the vales of Ilereford and Ross, on the very same land with the large Hereford osen. 
a light, loose wool: " having found," as he says, "from many years" experience, that sheep in the same flock of the former description will keep themselves in better flesh than those of the latter *." We, however, warn those breeders who are anxious to obtain large stock, not to breed them of a spurious sort + .

\section{CARCASS.}

Such were the effects upon the wool: on the carcass it has been different; for although the crosses with the improved Leicesters have rather diminished than increased the size of the original Leicester, Lincoln, and Tees-water breeds, yet the smallness of bone and symmetry of form which the animals have thits acquired, have considerably decreased the quantity of offil, and added largely to the dead weight of marketable flesh. Before that time the mutton of those coarse sheep rarely amounted to more than one-half of their live weight; whereas now, the common average-as will be seen by the weicht of two Leicesters, recoried in the report of that county, as bred from the Dishley stock, without any extraordinary means of feeding-is more than two-thirds; and Dishley wedders, when well fattened, are said to be in the proportion of an ounce of bone to a pound of flesh.

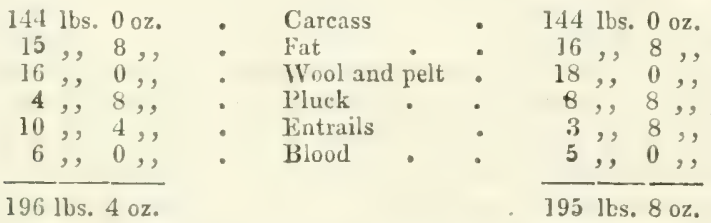

The cross with the new Leicester rams upon the short-woolled sheep has, however, improved the size of the latter, and has more especially imparted to them an earlier tendency to fatten: so much so, indeed, that Suuthdowns, which formerly were never brought to market at an earlier age than three years old, nor of a greater weight than from $S$ to $1 \mathrm{l}$ stone, are now constantly seen in Smithfield of much larger weights within two years, and the new Leicesters, completely fat, at about twenty months old; of which an instance in point is that of a pen of three Southdowns, thirty months old, exhilited at the Christmas Cattle Show of 1835 , which weighed as follows :-

$$
20 \text { st. } 31 \mathrm{~b} . \quad \ldots \quad 20 \text { st. } 6 \overline{1 b} \text {. .. } 21 \text { st. }
$$

This, no doubt, is a great advantage to the breeder; but the consequence to the consumer is, that he thus eats neither lamb nor mutton. $A$ sheep, in fact, to be in high order for the palate of an epicure, shouid never be killed earlier than when five years old; at which age the mutton will be found firm and succulent, of a dark colour, and full of the richest grasy: whereas, if only two years old, it is flabby, pale, and savourless. The graziers, indeed, do not admit it: and we constantly read flaming accounts in the reperts of the shows of stock exhibited in various parts of the kinglum, of pens of wedders, fattened to an enormous size within extraordinary short periods of time; but if any one cliooses to ascertain the diflerence of quality, let him cause an equal weight of one of these young Leicesters, and a five year old Suthduwn, to be stewed down into broth, and he will find that of the former to be little better than greasy 
water, while the latter, besides its superior degree of nutriment, possesses all the high flavour of full-grown meat.**

Our opinion has, indeed, been since confirmed by that of an uncloubted judge, to whom the subject was referred, who writes thus:- A mountainbred Cheviot-say, a yell ewe rising six, or a wether rising fuur, yields a different quality from the greasy mutton generally found in the south country. 'The important questions I hold to be are-is the mutton heath-fed? and, has it had time to fill the bones with marrow, and the fleshwith gravy ?"

In saying this, we have no intention to disparage the improvements which have been made in the animal, nor the discernment and ability with which they have been conducted; for it is clear that, had they not been realized, we should not at this moment have an adequate supply of mutton, and the price would necessarily be raised. It is, therefore, a national object to both breeder and consumer: but those country gentlemen who have land, and who wish to have their table supplied with real good mutton, should purchase a few of the smallest mountain sheep which they can procure; and, if they cannot be obtained of sufficient age, tisen keep them during summer upon the shortest natural grass that can be found-a closefed park, or a common, if downs be not at hand-and fatten them on the approach of winter with sound hay and a little corn; without either turnips or oil-cake. When thus fed, and of the proper age, their joints will be found interlarded with fat of the firmest sort; the lean will have a fine texture in the grain, with a richness and deep colour in the gravy; while the meat can also be kept untainted much longer than that of young turnipfattened animals, and a saddle or a haunch of this mutton, if hung during the winter until it becomes tender, will be nearly equal to venison $t_{\text {. The }}$ long-woolled breeds of every description they should invariably shun; for, even if kept to the proper age, the flesh is coarse, and, though full of sap, is flavourless: but it suits the poor man's pocket + .

* To ascertain the age of multon, Mr. Ellman directs-" To observe the colour of the breast-bone when a sheep is dressed; that is, where the breast-bone is separated: which, in a lamb, or before it is one year old, will be quite red; from one to two years old, the upper and lower bone will be changing to white, and a small circle of white will appear round the edge of the other bunes, and the middle part of the breast-bone will yet contimue red; at three years old, a very small streak of red will be seen in the middle of the four middle bones, and the others will be white; and at four yeitrs, all the breast-bone will be of a white or gristly colour."

+ "Southdown wether-mutton, in point of delicacy and flavour, is thought equal to any that is killed; and, in summer, as preferable to some other fine-flavourtd breedsespecially to Norfolk mutton. This circumstance is attributed to the cluseness of the grain, or the specific gravity being greater, rendering it more impermeable to the air than coarser and louser fleshed mutton, which is, of course, more subject to putridity. The older the mutton, the finer the flavour." - Sussex Rep., p. 331.

"The sheep of the Isle of Purtland, which is the smallest breed now existing in the kingdum-seldom weighing more than $10 \mathrm{lbs}$. the guarter-are fed upon a rocky soil; and their flesh is so far superior to that of any other, that the mutton generally fetches a shilling a pound in the Weymouth market."-Dorsetshire Rep. p. 396.

+ "The New Leicesters, on good keep, will yield a greater quantity of meat for the same quantity of food, than any other breed of sheep can do. The kind of meat which they yield is of a peculiar charac'er. When the sheep are not over fattened, it is tender and juicy, but, in the opinion of many jersons, somewhat insipid. When they are raised to their highest state of condition, the muscles seem to be partially absorbed; at least much fatty matter is introduced between their fibres; the line of distinction between the fat and the lean is in a manner lost, and with the exception of a few joints, and a small part of them, the carcass has the appearance and the taste of a mass of luscious fat."-Youatt " on Sheep," Farmer's Series, p. 320.

"We are told that sailors, colliers, and keelmen are sure customers for these overfat joints ; and long may they enjuy them! But without a doubt, the flesh of the small sheep, fine in its grain, is a more marketable commodity, because its quality is superior: in short, because it eats better." - Malcolm's Comp. of Mod, Husb., vol, i, p. 402. 
It is almost unnecessary to remark that wedder-mutton is always considered so far preferable to that of the ewe, that the flesh of the latteralthough more commonly kept to a mature age-always sells at an inferior price. Connoisseurs, however, assert that a spayed, or maiden ewe, liept until five years before she is fattened, produces mutton superior to that of any wedder.

The live weight, with the offal, of a large fat wedder, and the joints when cut up for market, were as follows :-

\section{Live weight, 13 st. 10 lbs.}

Offal.

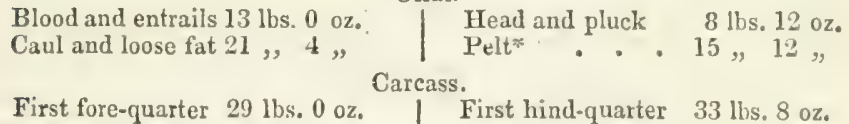

Second do. 28,12 Second do. 32,0,

Joints of one side.

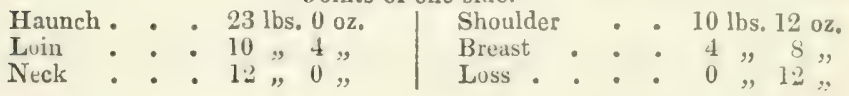

FATTING.

Ithas been proved by experiment, - stated in the "Mémoires de l'Academie Royale d'A griculture," of Paris, - that sheep fattened upon clean corn and sweet hay took on more fat, and acquired it more quickly, than those fed upon roots $\uparrow$. There can, indeed, be little doubt that corn and pulse are the most efficient food in fattening all cattle, and giving firmness to their flesh and tal. low : the material consideration for the grazier is, however, not only what will the soonest and the best effect that object, but also the most economically; and, in that view, it may be questioned whether grain can, in this country, be ever profitably applied to sheep. Barley-meal, in conjunction with roots, has however been used with advantage when the season has been such as to render the grain unfit for malting ; and danagred vats, torgether with potatoes, in the proportion of one-third of the former to two-thirds of the latter, has been tried with good effect + ; but bruised vil-cake is more commonly used than either. The most extraordinary experiment, however, was that stated at fout, made by the late Dr. Cartwright, under the direction of the Board of Agriculture, at the suggestion of the Distillery Committee of 1808 , in order to ascertain whether Muscovado sugar might not be successfully employed in the fattening of sheep; and, if so, with what substances it might be mixed, so as to protect the revenue without injuring the animal, in case a drawback of the duty were to be allowed to the farmer.

'The materials used for this purpose were linseed-oil, train-oil, palm-oil, oil of hartshorn, assafoetida, urine, antimony, and charcoal ; most of them preventing the sugar from being used in distillation, and all of them spoiling it for common purposes. 'These substances were separately tried on fifteen Southdown wedders, the average weight of which, on the 27th of August, was $90 \frac{3}{\mathrm{l}} \mathrm{lbs} . ;$ on the 29 th of September $109 \mathrm{lbs}$; and on being again weighed on the 22nd of the following October, they each appeared to have

* The pelt, when washed, clipped 5 lbs. of dried wool, and the loss in washing was 5 lbs. $4 \mathrm{oz}$., which may be accounted for by the skin being thrown under the animal to receive the blood while dressing.-Sussex Rep. p. 328.

f Those fed solely upon potatoes were for sume days averse to them, while those fed upon turnips and beets ate heartily from the beginning, and continued to do so.

+ Von Thaër, "Princ, Rais, d'A gric," 2de Ld. tome iv. p. 658. 
gained an additional increase of weight of about 15 lbs. They were, however, fed also with bran and peas, and it is therefore difficult to say how far these articles, or the sugar, may have contributed to the increase of flesh. From all the facts of the various trials, as stated at foot, taken collectively, the Doctor draws the following conclusions :-

1. "That sugar may be given with great advantage to sheep, if not confined; especially if they have access to green food, however little that green food may be in quantity.

2. "That sugar may be given to them with every prospect of a beneficial effect, in the quantity of $4 \mathrm{oz}$. per day to each sheep.

3. "That sugar-supposing it to be purchased at $4 d$. per $1 \mathrm{~b} . *-$ would, at the rate of $4 \mathrm{oz}$. per day, be paid for in a return of flesh, exclusive of the advantage of expeditious feeding and the benefit to be derived from the manure.

4. "That 6 oz. per day to each sheep exceeds the maximum that can be given with the best advantage to sheep of the size of Southdowns.

5. "That the advantage of stall-feeding sheep altogether upon sugar and dry food, of whatever nature that food may be, is extremely problematical.$+ "$

* Molasses, we conceive, might be used with nearly equal effect; and the price, in the early part of 1837 , duty paid, was only 28 s. per cwt.

+ During the first week the sheep were fulded every evening, each having half a pint of bran and a quarter of a pint of peas, and the same quantity when they left the fold in the morning. In a week they lecame habituated to dry food, and then, to this quantity of bran and peas was added an ounce of sugar for each; and when familiarized to this, the following substances were tried.

1. Linseed Oil was first given on the 7 th of September, in the proportion of 1 to 32 parts of sugar. It was put into one only of the three troughs out of which they fed; the sheep, however, ate indiscriminately and apparently with the same appetite. On the following day the quantity of oil was doubled, without any appearance un their part of dislike.

2. Train Oil was given in the same proportions, and with the same success; or rather, from the avidity with which they devoured this mess, it was supposed that they found it particularly palatable.

3. Assafoctida came next, in the proportion of 1 to 448 parts of sugar. On this, part of the sheep began immediately, but others hesitated, and though they at length commenced, yet the trough was not emptied quite clean. The experiment was however repeated on another day with 1 part to 220 of surar, and they then fed on it as readily as any of the other mixtures.

4. Urine was then tried in the proportion of 1 part of sugar to 24 of urine; upon which mixture they fed greedily, without its seeming to be in the least offensive.

5. Palm Oil followed, without any apparent consciousness on their part of any alteration having been made in their food.

6. Empyreumatic Oil of Hartshorn, though uncommonly offensive to the smell, was given in the proportion of 1 to 224 parts of sugar, but was only rejected by two or three, and not by them for more than a day or two.

7. Turtar Emetic was also given in the proportion of 1 to 240 of sugar, without occasioning any ill effect on the bowels.

8. Charcoal was not tried, from the difficulty of obtaining it sufficiently pulverized.

The sheep not having rejected any of these mixtures, linseed-oil, train-oil, and palmoil were given in the proportion of 1 to 8 , and the allowance of sugar increased to $2 \mathrm{oz}$. each day, without occasioning any diminution of appetite or any cbange in the state of the bowels.

From the 2th of September to the 22nd of October their allowance of food was increased to a quart of bran per day, one fint of peas, and $3 \mathrm{oz}$. of sugar, ringing changes at the same time with the different ingredients with which the sugar was mixed, in order to discover the particular substance which they most relished. They were, however, extremely fond of all, but with a slight preference in favour of train-oil. Dr. C., 
Of late years it has become much the practice to fatten sheep by soiling them during summer with various artificial grasses, and if not ready for the market at the close of the season, they are then finished off with Sivedish turnips*; or, if not, with hay and oil-cake. Nothing, indeed, generally used in the fitting of cattle, or sheep, has a greater, effect upon the increase of flesh than oil-cake; for even if fed upon turnips, $1 \mathrm{lb}$. of oil-cake per day has been found more profitable, when purchased at $8 l$. per ton, than turnips at $4 l$. per acre + : but, if not used with great moderation, it imparts an oily softness as well as a yellow tinge to the fat, and to delicate appetites gives an unpleasant flavour to the lean. In fact, it may be assumed as an axiom, thit the nature of the food greatly affects the quality and savour of the meat. "If the large sheep of this country were taken to the Welsh or Scotch mountains, if they could obtain their food with ease, and take their natural rest, the flavour of the mutton would no doubt be finer in proportion; as the native sheep of those countries deteriorate in flavour when fed on the coarse grasses of this country $\ddagger$." Hay, or straw, should, however, be always given to promote digestion, with whatever roots they may chicfly be fed upon $\S$; and if sufficient of these be given-whether mangel-wurzel, cabbages, Swedes, carrots, or parsnips-their progress will be found nearly cqual: but if these be all grown upon the farm, the occasional change of diet will materially improve them. Parsnips, from all we have heard, we believe to be not only eminently nutritive, but capable of materially improving the flavour of the meat.

Potitoes would also, no loubt, have much the same effect in point of nourishment; but, if used with advantage, it is generally thought they should be steamed, which is a trouble that few famers will be inclined to take, for, if the sheep be not housed, there would be great difficulty in feeding them with prepared food in the field: they have, lowever, been used raw to a great extent with very considerable advantage. A good mode of giving them is to take the sheep in the depth of winter into the fold-yard, "here the potatoes are cut into slices and placed in troughs under

howerer, suggests that, instead of using any of these materials singly in conjunction with sugar, that a mixture should be substituted consisting of I lb. of palm-oil, $1 \mathrm{lb}$. of train-oil, $2 \mathrm{lbs}$. of urine, $2 \mathrm{uz}$. of emetic tartar, $24 \mathrm{grs}$. of assafoetida, $28 \mathrm{drops}$ of oil of hartshorn, together with $1 \mathrm{cwt}$. of sugar; since in this composition are comprised an animal, a vegetable, and an empyreumatic oil, a substance containing ammoniacal and cther salts, metallic calx, and is resinous gum; the whole expense of which, he states, would be trifling.

On the 22nd of October, the sheep were taken into the house and kept upon dry food without sugar until the 2nd of November, and dividerl into three classes: one being $6 \mathrm{oz}$. of sugrar per day ; another $4 \mathrm{oz}$; and the third class none. Bran, peas, and hay were given to all, and they were weighed every week during a furmight. 'Their increase of weight, however-owing, as it was supposed, to their confinement-was very trifling. 'The progress of those which had no sugar at all was rather more than those which had $6 \mathrm{oz}$. a day, and the advantage was in favour of those which had the $4 \mathrm{oz}$.

* When turnips are so drawn, it is a good plan to keep them for a day or two, in order to allow some of their watery juices to evaporate; by which means they will become more wholesome and nutritive.

+ Lincoln Report, 2nd edition, p. 418.

\pm Russell, on Pract. and Chem. Agric, p. 295.

$\$$ The dry food being only intended to assist the digestive organs, and to correct the watery properties of the turnip, straw, as being the most economical, may be used with perhaps more alvantage than hay. Mr. Russell, of Warwickshire, indeed, says"That he never found his sheep to thrive so fast on any other food as on Swede turnips with a little fresh-threshed barley straw, to pick at as theirinclinations led them. When he gave them good green hay instead of straw, they ate more hay and less turnips, and the consequence was, they did not thrive so fast; the larger the proportion of turnips which they consumed in a given time, the quicker they became fat." - Treatise on Practical and Chemical Agriculture, D. 242. 
the shelter of the sheds. There can indeed be little doubt that it would be found an expeditious mode of fattening; for trials made upon some of the same flock fed with corn of different kinds, and some with oil-cake, have proved that they do the business in the shortest time*. The experiments which we have already detailed on the feeding of neat cattle, have, indeed, thrown some doubt on the superior efficacy of potatoes when steamed; but those in the Chapter on the fatting of Swine show a different result.

There is a certain degree of fatness that is both requisite for the consumer and profitable to the feeder; but when carried too far, is injurious to the quality of the meat, without adding to the gain of the farmer, though the increase of tallow puts money in the pocket of the butchert. Soiling, when used with discretion, will not do this; but oil-cake, linseed, and expedients of a similar nature for creating flesh quickly, certainly effect it ; sometimes with profit, though frequently with loss, and always with disadvantage to the mutton. Many sheep are, however, fattened upon the rich grazing lands of Ireland and the north of England, as well as in the marshes of Essex, Somerset, and Kent, where they arrive gradually to a state of perfection; and, if we consider the firmness, nutriment, and savour of the flesh, this is certainly the preferable mode of feeding. The number which an acre will maintain must of course depend upon the richness of the land, and the sort of sheep to be fed upon it; and it is not every kind of pasture that will fatten the animal, though capable of supporting it in health. Any calculation upon the subject made upon any other grounds but those of locality and knowledge of the breed, would therefore be futile.

The value of fatting land being different, the practice of graziers must also differ, and their profits must in a great measure depend on the properties of the soil; for inferior fatting ground will not produce the same profit in proportion to the rent, as will land of the first quality. The farmers' profit must also be governed materially by his judgment in the purchase of lean stock; the seasons also have great influence on the profits of grazing; but the greatest profits are generally made by those who, having various qualities of pasture, have always a successive supply of stock to succeed those taken off, and by attending to the number more than the breed of such stock.

To the details of feeding in the rich lowlands, we refer with confidence to Price, "On the System of Sheep-grazing in Romney-Marsh;" and, as the fields are in such cases often only divided by dykes, we copy from the Essex Report, the cut of a light portable wooden bridge, for the more easy transmission of the animals from one field to another.

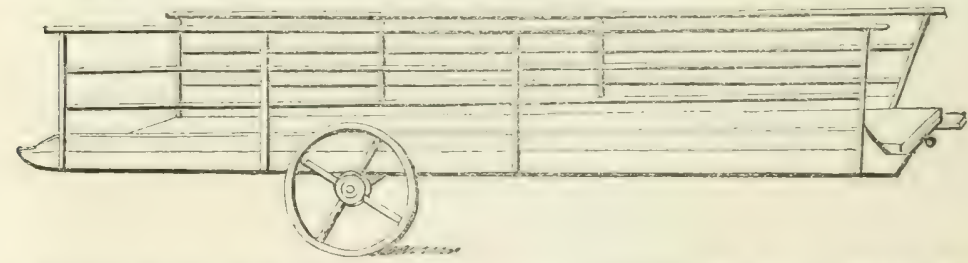

* Sussex Rep. 1) 318. A flock of Southdowns consisting of 1651 breeding ewes were during four months almost exclusively fed upon raw jotatoes, of which they consumed fifty-one bushels per day. Upon an average a full grown Southdown wedder will eat a gallon per day, together with a little hay morning and evening.-Ib. 1. 315.

+ The quantity of insile fat depends much upon the age and time of fattening. It gathers itself much more in old sheep than in young ones. The tallow of a wether in common management will generally average from an eighth to a teuth of its dead weight. Some fat Southdowns slaughtered by Mr. Ellman have varied from a seventh to a tenth, and one had the proportion of one-sistb loose fat, - Sussex Rep. P. 33:. 


\section{BREEDING。}

The great object in the breeding of sheep, is to combine the production of the best wool-whether it be of the combing or carding quality-and the largest quantity of mutton upon the least weight of bone, at the least possible expense. This can only be found in sheep of the most perfect form, and it must be admitted that the skill and perseverance which have been exerted to the attainment of the object have met with wonderful success. Still, it should be borne in mind, that perfection of furm consists in that shape which is best adapted to the soil on which the animal is to be reared; and that which is considered beautiful in a lowland sheep, may be a defect in one who has to seek his food upon a mountain. A judicious breeder will, therefore, be extremely cautious how he crosses a breed adapted to the land with any other, however apparently superior, that does not possess similar qualifications; for, in any particular district where a peculiar breed has long existed, it may be presumed that their constitution is by nature suited to the food and climate. This is, indeed, a point of the greatest importance to those who have already got possession of a good breed; for notwithstanding the improvements which have been generally effected, yet injury has also been done, in particular instances, by ill-judged crosses, and many a man has had reason to repent his attempts to improve a stock that already answered his purpose.

It being, however, desirable to avoid the too constant system of breeding " in-and-in," as being injurious to the size and constitution of sheep, and therefore occasionally necessary to partially change the stock, the mode adopted for that purpose by Mr. Ellman camut be too strongly recommended. He gets some neighbour to let him take out 50 of his best ewes, and puts his best ram with them, from the produce of which he selects ram-lambs; by which practice, and drafting 30 or 40 refuse ewes every year, he got his flock up to that degree of celebrity which it still maintains*.

The plan of an eminent Scotch flock-master, who rears some of the finest Cheviots in the north, is also well deserving of attention. He made a careful selection from the best of his own stock, and purchased, without regard to expense, a few from other esteemed flocks of the same breed. These he kept apart under his own inmediate inspection, to study the tendency in each family towards a deficiency or excess in particular points; and thus, by judicious crosses between the various families, he succeeded in producing stock more perfect than either sire or dam. "The experiment," he says, "wrought like a charm, and in a few years lifted up his stock to a new position, both at the tup-shows, and, what is more to the purpose, in the markets where the stock and wool are usually sold." He finds, however, that the very fine woolled varieties of Cheviot sheep are more liable to casualty, and less valuable, at last, to the feeder; and he prefers the fleece that covers the hardiest and the best thriven sheep, although it should not be praised by the stapler. $\dagger$

The character of both sire and dam are observed in different shades of their offspring, but that of the male more frequently predominates. 'This has frequently led breeders into a great mistake, by employing rams of much larger size than the ewes by which they were tupped, the progeny of which is generally of an imperfect form; but if the ewe be proportionably larger than the ram, their offspring will commonly be found improved.

* Susser Rep. p. 307.

+ See Mr. Sellar's account of his Farm of Strathnaver, in the county of Sutherland, in the "Farm Reports." 
Thus the graziers in the Isle of Sheppy at one time imagined they could materially improve their Romney breed by the employment of large Lincolnshire rams ; the produce of which was, however, much inferior both in the shape of the carcass as well as in the quality of the wool, and their flocks were greatly injured by the attempt.

"The proper method of improving the form of animals," as stated by the late Mr. Cline, "consists in selecting a well-formed female proportionably larger than the male. The improvement is on this principle-that the power of the female to supply her offspring wih nourishment is in proportion to her size, and to the power of her nourishing herself from the excellence of her constitution. The size of the foetus is generally in proportion to that of the male parents; and, therefore, when the female is disproportionally small, the quantity of nourishment is deficient, and her offspring has all the disproportions of a starveling. But when the female, from her size and good constitution, is more than adequate to the nourishment of a foetus of a smaller male than herself, the growth must be proportionally greater. The larger female has also a greater quantity of milk, and her offspring is more abundantly supplied with nourishment after birth." We believe also, that any experienced breeder will admit that-" "to produce the most perfect formed animal, abundant nourishment is necessary from the earliest period of its existence until its growth is complete."

"The external form of domestic animals has been much studied, and the proportions are well ascertained; but the external form is an indication only of internal structure. The principles of improvement must therefore be founded upon a knowledge of the structure and use of the internal parts. The lungs are of the first importance. It is on their size and soundness that the strength and health of the animal principally depend; for the power of converting food into nourishment is in proportion to their size, and therefore has a greater aptitude to fatten."

On this Mr. Russell remarks-" Constitution, which embraces both external and internal formation and structure, is the chief cornerostone necessary to the production of a perfect animal. In the construction of a good frame the following qualities should be combined:- a deep and wide chest, which indicates a wide loin and hips, with ribs springing gradually wider until they approach nearer to the hip. The shoulder-blides should project gradually wider until they approach far towards the hind quarters, which gives more surface on the chine; and this is considered an excellent point in animals intended for slaughter, as it enables them to accumulate more meat on that part where it is of the most value. The formation of the lungs will also agree with the chest in size and shape." To which we may add, that the head should be small, both as facilitating the birth of the lamb, and being indicative of the animal being of a good breed; and snaall limbs also indicate fattening qualities.

It has been justly observed by the celebrated author, Mr. Cline, whom we have just quoted-" That the pliancy of the animal economy is such, as that an animal wiil gradually accommodate itself to great vicissitudes in climate and alterations in food, and by degrees undergo great changes in constitution; but these changes can only be effected by degrees, and may often require a great number of successive generations for their accomplishment."

"The size of animals is commonly adapted to the soil which they inhabit. Where produce is nutritive and abundant they are large, having grown proportionally to the quantity of food, which for generations they have been accustomed to obtain. Where the produce is scanty, the animals are small, 
being proportioned to the quantity of food which they were able to procure: of which contrasts the sheep of Wales and Lincolnshire are exanples. It may be proper to improve the form and quality of meat or wool of a native race, but, at the same time, an attempt to enlarge their size may be very injudicious."

Disposition to fatten is, however, an essential good quality in every breed, whether large or small, but is very difficult to judge of; for out of a score of sheep of the same breed, age, health, and managed equally alike, yet, when handled, a vast difference will often be found in their state of fatness. They will, however, partake more or less of the chief characteristics of the sire and dam, or of the general reputation of the stock from which they are bred; and, therefore, in selecting those from which a new race is to spring, that point should be closely inquired into. The smallness of the bones, the moderate thinness. and mellow feel of the peit, and the symmetry of the animal, as marked by the general fullness and rotundity of every part, together with a certain placidity of countenance denoting quietude of temper, are all indications of a tendency to fatien : in addition to which, we strongly recommend the medium sized sheep to breed from; rather under the feeding property of the soil than above it. 'The whole art of breeding may, indeed, be said to consist in the selection of animals of sound constitution, as designated by the general appearance of health and symmetry of form, and there can be little doubt that both the breeder and the grazier are often disappointed in their expectations by looking too much to size.

\section{DISEASES.}

Although the diseases of horses have been thought sufficient to establish a college for the promotion of the veterinary art, yet, notwithstanding the great importance to our agriculture of the healthful maintenance of our flocks, and the variety of distempers to which they are liable, it is still to be regretted that no public institution has been foumled with a view to the instruction of students in the nature of their disorders and the means of cure: in consequence of which neglect the whole management is either left entirely to shepherds, who have no correct and settled ideas of how the causes in which the complaints may have originated; or, if the animalswhether sheep, cattle, or swine-be in imminent danger, the farmer has no other resource than the employment of some neighbouring leech, who is, in most cases, supremely ignorant. Many valuable treatises have, indeed, been written on the subject, but they are only in the hands of a few persons, and it camnot be expected thiat, in a work like the present, the different maladies by which sheep are affected should be scientifically described and their treatment professionaliy particularized. We shall, therefore, confine our remarks to a few of those of most frequent occurrence, coupled with some practical observations, in order that inexperienced persons may not be left in ignorance of the proper remedies to be applied; for if disease be not efficiently met in its earliest stages, the neglect may, within a very few days, be productive of serious mischief. So many nostrums are daily advertised for the infallible cure of every complaint, that one might suppose efficacious remedies to be constantly at hand; but a man who would place confidence in any one might be led astray, and if left to chouse among them all, his judgment would assuredly be puzzled.

It is seliom that cattle-doctors, however skilful, are called in to attend upon sheep; and as shepherds, however experienced they niay be, are not always competent to the cure of an animal that is suffering under acute dis- 
temper, we then confidently recommend his employer to the careful perusal of that part of Youatt's "Sheep," which treats of their disorders: the most prominent of which are produced by the various sorts of flies, maggots, and lice; besides those known as sturdy, itch, scab, foot-rot, red-water, braxy, blindness, blood, pining, and the rot; together with the accidents occasioned by hoving, slipping of the lamb, and falling down of the womb.

There are several species of flies by which sheep are very injuriously, and sometimes destructively, infected during the summer months. Of these the small common flies, though not dangerous, are yet extremely annoying in all strongly inclosed and woody parts of the country; and, in warm or showery weather, occasion great torment to the flock: particularly to any sheep which has the least sore or scratch upon the skin, which they attack immediately, and are so eager for blood, that when any has been drawn they crowd to it in myriads, and never leave until they are gorged. If the sheep cannot be driven to the uplands, a good preventive is to wash them immediately after their being sheared, and once or twice more during the summer, with suap-suds, strongly impregnated with empyreumatic oil, asafoetida, or any thing that will retain an offensive scent.

Hogg, the Ettrick Shepherd, indeed says, that he has seen a flock upon which flies had settled in such numbers, that when he went amongst the sheep, he and the other persons with him could with difficulty see each other; but those anointed with whale-oil were turned in among the rest, and to his utter astonishment, in less than a minute not a fly was to be seen. He afterwards noticed a similar circumstance; and, as wool, when smeared with whale-oil, never loses the savour till scoured, were the fleeces sprinkled with it, it would probably keep the flies away.

The Large Flesh-flies, or Sherp-Maggot-fies, more commonly known as Blue-botlles, of which there are more than one species-black, white, and greenish-closely resembling each other in their habits and appearance, yet coming to maturity at different periods, are by far the most offensive : they are generated by any carrion which is left exposed until it becomes putrid, and the insect, when able to fly, being attracted by any strong odour like that arising in hot misty weather, or in morning dew, from the sweat of the sheep*, deposits its eggs upon any spot which appears likely to shelter and nourish them into life. About the root of the tail, and immediately around the anus, on those parts where excrement may have been hanging, are however the favourite places; though the back is by no means exempt. It is, therefore, very desirable to clip the adjacent parts very closely previous to the common shearing time, as well as a couple of months afterwards; and if the rump and buttocks be then well washed with the following liquid, it will probably prevent the breeding of the maggot, without being injurious to the wool:-

Take $1 \mathrm{lb}$. of arsenic, finely powdered; $12 \mathrm{oz}$. of potash; $6 \mathrm{oz}$. of common yellow soap; 30 gallons of rain or river water. Boil the ingredients together for fifteen minutes; taking care to inhale as little as possible of the steam. $\dagger$

Arsenic should, however, be used with great caution, and many experienced shepherds use the following mixture with good effect, namely-

* Either a certain habit of body, or some kinds of fuol, are thought by Mr. Hogg to give the excrement and perspiration of any sheep which is infected, a peculiarly rancid and loathsome smell; for he says, that " you can, when near them, distinguish those that will be soon infected." It is frequently accompanied by diarhea, but the most healthy sheep are often attacked.

$f$ Mather, on the Sheep-Alaggot and Fly. Prize Essays of the Highland Society. N, S, vol, iv. 
One onnce of sublimate dissolved in one gallon of water: to which is to be added three table-spoonsful of turpentine.

These insects commence their attacks sometimes in the month of May, continuing them until the close of September; and some idea may be formeil of their depredations, from its having been ascertained that one female will give birth to 20,000 young, and that the larvae will, in twenty-four hours, devour so much food, and grow so quickly, as to increase their weight two hundred fold! A gentleman, who drew up some notes upon the subject, made a few years ago in a pedestrian excursion through the Highlands of Scotland, states, "that on five farms in the neighbourhood of Loch Vena. har and Loch Lomond, where he had an opportunity of making observation, the number of sheep amounted to 4,090, of which 550 were affected during the summer, and 195 died; and, although 355 survived, it was long before many of them recovered."* In very sultry weather, the eggs are speedily hatched into maggots, which gnaw into the skin, and other sets of eggs being also deposited by the same parent, they increase in such numbers, that within two or three days' time it will be perforated by some thousands: so rapid, indeed, is their progress, that if the animal be not immediately attended to, he will be found in less than a week a lifeless mass, covered with these loathsome vermin.

The sheep, when struck by the fly, betray great uneasiness. They hang down their heads, move their tail in a particular manner, and stamp violently with their feet; they draw up their bodies with an evident sense of disquietude; rum a short way, then suddenly stop, and endeavour to bite the injured part. Indeed, the restlessness of the animal occasioned by the itching, even before the effect of the fly-blow has gone to such a length as to wound the skin, will sufficiently indicate to a watchful observer that he lias been struck; or a well-trained dog, the moment he enters the field, will single him out, and he ought to be immediately caught for examination. This should be very carefully done, and if only nits are formed, it may be sufficient to crush them between the fingers, and wash the part well with soap-suds and urine, or goulard water; but, if maggots already appear, then the wool upon that part, and any surrounding portion of the skin which seems damaged, should be shorn off and washed with the liquid already mentioned. 'The shearing should, however, be avoided if possible, as it not only wears an unsightly appearance, but exposes the skin to the annoyance of small flies. If the skin be perforated, the maggots will probably have got under it; in which case it should be opened with the point of a penknife, and if they can be removed, the wound may be healed by any common ointment usually sold at the druggists; or, in more serious cases, it may be prepared thus :-

Half-a-pound of pure quicksilver; $\frac{1}{4} \mathrm{lb}$. of Venice turnentine; $\frac{1}{2} 0 \mathrm{z}$. of arsenic ; $\frac{1}{2}$ pint of neat's-foot oil ; and $1 \mathrm{lb}$. of hog's lard, rubbed well together in a mortar.

The Sturdy, Dunt, or Staggers, as they are by some persons called, is a malady by many considered as dropsy of the brain; but the disorder is occasioned by a bladder containing water, in which there are maggots, termed by professional men "hydatids," which find their way to the brain, and if not removed become fatal, for medicine has no effect in destroying them. The sheep most frequently attacked by it are those under two years of age, after which time they are generally considered safe. The animal appears to be entirely deprived of its senses, separates himself from the flock, and, instead of going forward, is con-

* Quart, Journ, of Agric., rol, i, pp. 211 and 213. 
stantly turning round in one direction, in whatever side of the head the bladder may be contained. Trepanning has been attempted, but with little effect. A cure has also in some instances been made, by thrusting a wire up the nostril and destroying the bladder; but we believe, that more sheep die under the operation than are relieved by $i t$. It also occasions great acrony, and unless there be a very skilful person at hand to perform it, it is more advisable to slaughter the animal when seized by the disease.

Ticks, or Sheep-lice, are small brownish fiat insects, by which sheep are very commonly infected, particularly if they be not in a healthy condition. They seldom, however, occasion more than temporary inconvenience, and may be destroyed by a strong infusion of tobacco-water, which may be prepared by boiling the tobacco in salt-water; or a wash of any weak mercurial preparation. 'The sheep may be bathed with a large brass syringe, and one of an ordinary size, if that instrument be employed, will not occupy more than five minutes.*

The scab is a cutancous cruption on the shin analorous to the disorder known in dogs and other animals as the mange, and generally commenc. ing with the itch. It is occasioned by an impure state of the blood, arising chiefly from bad feeding in wet ground, and is so highly contagious that if once introduced it very materially lessens the value of the farmer's whole stock of sheep; for no prudent inan will purchise out of a flock which is infected, although the animals oflered for sale may be apparently sound.

The symptoms of the itch are first indicated by small white spots on the wool, which make their appearance a good while before either the animal begins to scratch, or the smallest eruption apjoars upon the skin, and the best remerly is any of the common afplications of sulphur. If that does not eflect the cure, and the intense itching continues, blotches then apjear upon the skin, which assumes a fretted appearance, and if neglected, soon discharges a fetid ichor which eventually becomes scab. The wool becomes foul; the animal loses flesh, and if not cured, sinks under the constant irritation under which it sufiers. He should, therefore, be immedi. ately separated from the flock; and if the disorder be taken at an early stage, it may be removed by any of the common washes of tobacco-juice, soft-soap, and urine, or salves of which brimstone forms the chief ingredient. When more virulent, the ointment recommended as a cure for the shecpmagrgot-if the arsenic be omitted-is said by Hoggr, in his "Shepherd"s Gunde," to be an effectual remedy ; the mercurial composition sold by most country apothecaries as "sheep-ointment," and vitriolic acid, lowered with water, have also been found very efficacious, but they require care and skill in their application.

The disease, even in its worst state, is by no means incurable, but when the eruption appears under the remedy, and on being destroyed in one part re-appears in another, no doubt can then remin that the mass of blood is affected, and as no topical application can then succeed, internal remedies become absolutely indispensable. Purgatives, of a cordial nature, are perhaps the best; but, if the diseasc arises from poverty, abundance of wholesome food will also be found necessary. Sheep, however, though in the highest condition, are sometimes infected by coming in contact with posts and fences against which scabby sheep have rubbed themselves; it is, therefore, only a necessary precaution in regard to these, as well as any

2* See Trans, of the IIighland Society, N. S., rol, vi. p. 254., in which there is a cut of a syringe expressly used for this purpose. 
cotes or buillings wih which they may have been in contact, to wash them with lime-water previous to the introduction of a sound flock.

The sheep-pox so closely resembles the scab, that it is not known in this country as a separate disease, but in some parts of France, particularly in districts adjoining the Pyrenees, it is viewed as a distinct distemper, for which flocks have been regularly inoculated: the mode of doing so, being to place the skin of a sheep which has been infected upon the floor of a cote into which the lambs, when about six months old, are driven, and as they voluntarily rub and roll themselves on the skin, symptoms of the disorder appear soon after; but they speedily recover, and are never again attacked, and where this is practised, it very rarely happens that ever one dies out of a flock of three hundred.

When taken naturally, the disease however is often fatal, and being contagious, spreads extensive mischief among the neighbouring flocks. Its attacks are more especially prevalent during winter, when the animals are shut up in confined cotes and liept very hot. They become dull; loathe their food; the head, eyes, ears, and gums, are swelled; hard, white tumours aypear in the groms as well as under the joint of the shoulder; and, three or four dars after theee appearances, pimples break out successively in various parts of ihe body, at first on those situated on the naked shin between the thighs, and where the wool is short and scanty, until at length the whole frame is covered. In this stage of the disease the animal swallows with pain, and ireathes with great difficulty; as it goes on, the pimples enlarge and become inflamed, suppurate and burst; the matter which runs out mats the wool into hard lumps, which, when dry, are rubbed by the sheep, and the scarf-skin peels ofl in large pieces, full of holes. The flesh of such shecp as have been killed while suffering under this disurder, lias a very bad taste, and is considered so unwholesome that cats and dogs which have eaten of it have been infected; and therefore the precaution is taken of deeply burying the carcases of those which die of the disease.

When the disorder appears, the sheep are kept warm, well littered with straw, and fed upon hay with a little salt; their water being given lukewarm. Blood is also drawn by cutting the ear, and the cotes are thoroughly fumigated during five or six days by burning the stems of gariick, which vccasions a great discharge from the nostrils, and in the effects of which the shepherds have much confidence. Some also give to each a double liandful of white mulberry leaves, which they allege is an efiectual cure, as well as a preventive.

When the distemper abates, whatever be the season of the year, the amimals are clipt, in order to assist the drying of the pustules, and to favour the growth of a new fleece. After this, the sheep fatten very quickly; and it is worthy of remark, that the fleece which immediately succeeds this distemper, is finer and more silky than any former or fulure growth *

The foot-rot is partly contagious, and is supposed to be communicated by the matter discharged from a diseased foot, and trod upon by sound sheep. It is, however, more prevalent in low marshy districts than in the uplands; and chalky soils, or ground which has been strongly manured with lime, are considered peculiarly exempt from its attacks: it is, therefore, not uncommon to drive those sheep which are supposed liable to infection into a pen strewed with quick-lime, as a precaution against the communi-

*Fron a French menoir, communicated to the late Sir John Sinclair, Bart., when President of the Board of Agriculture.

VOL. II. 
cation of the disease*. It is a very painful distemper, occasioning severe ulceration, attended sometimes with the loss of the hoof: if remedies be not speedily applied, the animal, in fact, soon loses the entire support of the foot, and is obliged to crawl upon his knees in search of food; by which exertion, together with the pain, he is so exhausted that, if not relieved, he will perhaps perish; if not by the effects of the disease, by the attacks of the flesh-fly, to which in sultry weather he will be peculiarly exposed. It is not, however, in itself necessarily fatal: lean sheep seldom take it; and in severe cases, a natural cure is effected by the shedding and removal of the hoof.

The first indication of the disease is a slight halt, which in two or three days increases to actual lameness; the foot feels hotter than usual; there is an appearance of swelling about the heel, or between the toes, and the hoof is in some degree loose. In attempting the cure, the animal should be first removed either to a pen or an open shed, with some clean straw litter, where he may be kept separate from the sound sheep, and the foot should be well washed with soap and water. When all dirt has been removed, it should then be minutely examined to ascertain the injured part, which, if rot apparent to the eye, will be found upon pressure by the hand. The hoof must then be carefully pared away with a sharp knife, all round the affected part, taking care at the same time not to cut any sensible portion of the foot; after which sume of the ointments hereafter mentioned may be laid on with a feather on every spot and into every crevice which may be affected, and the sheep should be hept confined until the application becomes dry; but, as the complaint is inflammatory, bandages should not be used.

The foot should be attentively kept clean and washed with lime-water previous to every future application, which may be repeated within two or three days, and, if taken early, a few dressings will probably effect a cure. Caustics have been generally employed, of which that known as " butter of antimony" is perhaps the best; but as three essays on the subject have lately been presented to the Highland Society, each of which has obtained premiums, we cannot justly refuse the insertion of all are severally as follows:-

The Ettrick Shepherd says, "that he has found the following composition to be the speediest, the most powerful, and by far the mildest:-

"To make one gill : to two ounces of turpentine, put half an ounce of diluted vitriol; stir the residuum of the turpentine from the bottom before using."

Mr. Laidlaw, of Bowerhope, in Selkirkshire, states-_" that mild applications have not been found in general so effectual as active ones; and that the following has liad considerable celebrity in the north of England and this partof Scotland:-

"Corrosive sublimate, 1 oz.; blue vitriol, 2 oz.; verdigris. 2 oz.; white copperas, 4 oz.; dissolve in half a bottle of white-wine vinegrar, and apply it with a feather."

Another medicine he also describes as having been found to answer, consisting of-

"Verdigris, 1 oz.;" blue vitriol, $90 \mathrm{0z}$; spirit of turpentine, 1 gill; distilled vinegar, 3 gills."

And he has sometimes used a mixture of tar and common salt with great success, especially in damp weather.

* See the Survey of Devonshire, p. 341 .

† Trans. of the Soc. N. S., vol, iii. See also the "Complete Grazier," Gth edit. p. 343, and the "Alountain Shepherd's Manual," 
The mode of treatment recommended by the Reverend Henry S. Riddell, of Drydean, near Selkirk, " as having been found by experience to be perfectly effectual, is a dressing of -

' Corrosive sublimate. $\frac{3}{3}$ oz.; sulphate of copper, $20 z_{\bullet}$; verdigris, $1 \frac{1}{2}$ oz॰; alum, 2 oz.; white copperas (sulphate of zinc) $\frac{1}{2} \mathrm{oz}_{*}$; muriatic acid, $2 \mathrm{oz}_{\text {. }}$; charcoal, $\frac{3}{4} \mathrm{oz}$; pounded as small as possible, and mix in haif a bottle of distilled vinegar.'

"In all severe cases, and especially where the disease is the result of constitutional affection, two ounces of glauber salts, dissulved in half a mutchkin of water, should also be given internally."

The red water, or, as it is sometimes termed, resp, and by others dropsy, is thought to be most commonly occasioned by turning the sheep from $1^{0}$ oor pasture into very succulent keep; as they are frequently attacked by it when put on turnips. It is also said to originate in the sheep being let out of the fold when the ground is covered with hoar frost, and it is believed that clover stubble and folded land are apt to produce it in wet weather; but it is not impossible that the disorder arises from too great a quantity of crude and undigested matter being allowed to remain in the stomach. Various causes are indeed given for the disease, thourh most writers agree in attributing it chiefly to acrid or otherwise unwholesome foorl. Mr. Hogr sars, that "it consists in an inflammation of the shin, that raises it into blisters, which contain a thin, reddish, and watery fluid; which continue for a short time, break, and discharging their matter, are follower by a blackish scab. The sheep should be placed in a fold by itself, the blisters slit up, and a little infusion of tobacco put into them; the following medicine may also be given for three or four mornings successively:-

- Sulphurtwo ounces; honey, treacle or syrun, three ounces ; to be mixed and divided into six duses, of which one may be given every morning in half a mutchkin of warm water.'

"If this be found unsuccessful, half an ounce of nitre mixed with the foregoing receipt will be attended with good effect; after which a dose of salts may be given, and the body washed with lime-water upon the parts affected *."

In the Leicestershire Report it is stated, that a Mr. Watkinson has used the following mixture as a preventive against the disorder in lambs, which are very subject to it. During thirty years, in the whole of which time he has not lost a single sheep, though the disease was very fatal to his flock previous to its use, and finds it unnecessary to repeat it :-

"Two ounces of myrrh, boiled in 60 table spoonsful of ale : three table-spoonsful to be given to each lamb about Michaelmas."

Braxy, and the sickness, under which names this disease is known, includes similarities of the same distemper, but is in all cases an inflammatory disorder, of a sudden and violent nature, occasioned, as is supposed, much in the same manner as that of the red-water, by the sheep being fed on rank errass or other coarse food, which, remaining in the stomach undirested, occasions costiveness. 'This, however, is seldom observed, and as the animal does not immediately lose his appetite, he continues to load his stomach with additional quantities of food which become fermented and cause infanmation of the bowels, by which youmg sleep in particular are suddenly carried off. It most commonly makes its appearance about the close of autumm, in consequence, as it is imagined, of the herbage then becoming more dry and astringent. It ceases when winter sets in, and is 
more prevalent on dry pasture, containing heatl and fern, than in the low lands. The animal, when attached, is restless, the eve is red and waiery, the back raised while the liead is bent downwards, and he noves with evitent pain. Bleeding-which may be promptiy eilected by cutting the tail -is periaps the most efficient check, as Gir George Mackenzie states several instances in his "IIomtain Shepherd's Minual," of its immediate restoration of sheep on which it had been performed; and that, taking an verage of those which hat been affected, three out of five were saved. Glauber salts, or castor oil, should, howerer, be administered, and the sheep should be put upon turnips during the day, with a dry-yard or littered fold during the night.

The blood is a term giren to a disorder by which sheep fed upon rich pastures ari not uncummonly attacked, and to which the flocks of the clayland graziers, and those in Rumines Mrarsh, are peculiarly subject. In Leicestershire the disease is called "the vellows," from the flesin after death becoming yellow, and it frequently occurs anong sheep feeding upon red-clover while it is in blussom. The sheep are observed to separate themselves from the rest of the flock, they stand as if in pain, and stretch out the fore-less in order to ease themselves; their eres appear heavy, they fetch their breath short, and the abiomen seems convulsed. It is a malady of the inflammatory kind, proving sucidenly fatal; analogous to that of Braxy, and the remedies are similar.

The flux, or scouring, evidently proceeds from cold and wet, or from sheep of a weakiy constitution being first put upon poor watery food, and afterwards removed io rich pasture. It is, however, so far from being fatal, that many graziers deem it salutary, and remark "that sheep fatten more quickly aiter it." Ii long continued it mas, however, prove injurious, and it arising from wer, the sheep should be inmediately removed to a dry situation, and supplied with sound hay; but if occasioned by their removal to rich land, they should then be put upon good short grass, and a little corn may be given with good effect: they should also be kept from ponds, and chaik shoull be put in the water which they drink. If the purging become violent, a drachm of rhubarb may be infused in half a pint of warmed milk; and if that should not stop it, a few drops of laudanum may be added on a repetition of the dose; or the medicine known as the "sheep's cordial" may be safely used.

Young lambs are very frequently attaclied by it, unier the name of the gall, at that season of the year when the grass is springing, for they begin to eat it when a fortnight or three weelis old, and after a few warm showers, some of them occasionally die. This it is very difficult to prevent, as they are then with the ewes, the food of which cammot be often conseniently changed. If older, although hay would then be desirable, they are then not easily induced to eat it; they should therefore have a litile pea or barley-meal, and some astringent cordial drink. Stut boiled in mik is by sume persons used, but three out of twenty, to which it was given by Mr. Price, died; while the remainder, which were treated in the manner just mentioned, were mostly saved*.

Pining is a distemper of a very singular nature, which a proa:s to have been only of late rear's introduced, and chiefly exists in the pasiures of the Cheriot Hills, the chain of mountainous land ruming through some parts of the shires of Roxburgh, Selkirk, and Peebles, with a few of the pastoral Scuttish districts, and seems to be occasioned by costiveness; but is not

* Sce Price "On Sheep Grazing;" P. 478, and Youatt "On Sheep," p. 469. 
known to exist in general on green succulent pastures growing on calcareous or sandstone soils, and it is remarkable that it lias appeared where land has been comp? etely dramed although it was beiore unknown. It has been, therefore, attributed to some astringency in the herbage of very dry pastures, and it is more prevalent in dry summers than when the season is showery. It is also said to have been very materially occasioned by the desiruction of moles; for the ground, when turned up by these animals, produces soft succulent plants, which are afterwards found to languish and finally become extinct; the herbage on those places where they once flourished assumes a white benty appearance, and the sheep are attacked by the pining. In proof of which Mir. Laidlaw says, "that for the last five years molecatching has been discontinued, and though the moles are silil too scarce, yet in those places where they have again shown themselves, the soil is evidently ameliorater, and the pining is losing ground every year."."

Hogg, the Ettrick shepherd, also asserts, that the farms most liable to the disease are those which were in former vears wholly overun with molehills, and which are intermixed throughout with great flats and ridges of white and flying bents, which last are the bane of the flocks; and that exactly in proportion as the succulent and laxative herbage prevails over the dry and benty, the eflects of the pining will be less felt. In the course of the nine years between $18: 21$ and 1830 , it appears that he lost upwards of 900 sheep by the ravages of the disease, of which he gives this description:-

"On the genuine pining farms sheep do not take it by ones and twos, but a whole flock at once. It is easily distinguished by a practised observer, the first symptoms being lassitude of motion, and a heaviness about the pupil of the eye, indicating a species of fever. At the very first, the blood is thick and dark of colour, and camnot by any exertion be made to spring; and when the animal dies of this distemper, there is apurently scarcely one drop of blood in the carcase. It lives till there does not appear to be a drop remaining, and even the ventricles of the heart become as dry and pale as its skin."

"It is most fatal in a season of drought; and June and September are the most deadly months. If ever a farmer perceives a flock on such a farm having a flushed appearance of more than ordinarily rapid thriving, he is grone. By that day eight days, when he goes out to them again, he will find them all lying, hanging their ears, ruming at the eyes, and looking at him like as many condemned crininals. As the disease proceeds, the hair on the animal's face becoines dry, the wool assumes a bluish cast; and, if the shepherd liave not the means of chaming the pasture, all those affected will fall in the course of a montht."

The change of pasture to one of more succulent herhage, or to clover, in order to check the costive habit, is, indeed, in that pericd of the year when turnips are not to be had, the only cfiective remedy which has becn hitherto tried. Salt flaced in lumps upon the ground and accessible to the sheep might, perbas, hatve some elfect, and there can be little doubt that laxative medicines would he beneficial ; for if the bowels be opened, a clange seems then to have been effected in the constitution, and the animal is saved. He is agin, however, more subject to complaints in the following year than others, and lew of the ewes that are attacked in autumn have lambs the next year; or they have them far too late to be of any value.

The rot, though not contagious, is the most destructive, as well as the

\$ Quar. Journ, o A gric, v, xi, p. 712.

† Quar. Journ, of Agric. vol, ii, p.698. 
most frequent malady by which sheep are attacked, and notwithstanding the volumes which have been written by scientific and practical men, no certain remedy has yet been discovered; for the disease is considered incurable. In its earliest stage, if they appear lethargic, dull, and careless of feeding, it may be justly suspected; and when advanced still further, it may be known by handling. If the loins feel loose and flaccid; if the skin has lost its healthy redness, and has assumed a pale, faded hue; if the eyes appear with a sickly want of lustre, and the animal is more restless than usual, it may be concluded that he is rotten. He does not, however, show symptoms of disease in its incipient state, nor does it immediately affect the flesh; for sheep are constantly purchased in seeming health, which, when killed, may be seen with their livers full of flukes, although the meat is perfectly sound and well flavoured.

The distemper is supposed to commence in the liver, which is found infested with numerous triangular flat-shaped worms, called "flukes," formed like a flounder, and about the size of a finger nail. 'The animalculie from which these are thought to proceed, are presumed to be deposited in the herbage of marshy places and water-meadows, where they are taken up with the food, as on such land the complaint is prevalent during the warm months of autumn, whereas after a single night's frost there is no further danger. Among sheep bred upon the wolds and downs it is unknown; though if sent into the low lands, or even removed on the same farm to low ground which has been covered with water during the winter, they are subject to be infected in the course, it is said, of a single night.

Such is the common notion of the origin of the disease, but various other opinions exist upon the subject of its theory, and those insects are by many considered as the consequence, not the cause, of the rot; for they evidently exist in the livers of suund and healthy sheep, and the disorder may perhaps be occasioned by their feeding upon soft watery grasses, in proportion to the wetness of the season, which is always detrimental to their health. It may also be remarked, that flukes are propagated in the gall and liver of the sheep *; that it is hardly possible that the egrgs of any insect can escape into the second stomach of an animal which chews the cud, in so unimpaired a state as to be capable of being hatched; and that no worm has ever been discovered on the grass which at all resembles them.

We have, however, neither room nor inclination to discuss theories which lead to no practical result; and referring to Mr. Iouatt, who has lately treated the subject with great discriminationt, we can only recommend our farming readers never to turn sheep, during the summer months, over land which has been flooded: or, if they must necessarily be pastured on moist land in that season, to have them carefully removed before evening into a dry situation, and fed partly with hay. If any suspicion of the actual disease be entertained, a change of pasture to entirely dry land should be immediately adopted.

It has been stated in a well-written treatise on the management of sheep, published some years ago under the title of a Lammermuir farmer, that salt is a decided cure. In asserting that, he certainly goes too far; but, as a preventive, nothing has yet been found to check the evil so effectually. The duty being now taken off, the expense camnot be much; and if left in blocks at the discretion of the sheep, it camnot do any harm.

* On this subject see two Essays by Mr. Edward King in Nos. XXX. and XXXIV. of the Quarterly Journal of Agriculture.

+ "On Sheep" Nos. XIV, and XV. 
The hunger-rot is a name which speaks for itself: it is occasioned by poor living, especially during the winter, and is best cured by better keep.

Blindness has been attributed by some to the pollen of flowers blown into the eyes in summer; and in winter, by the days being sunny, and the evenings cold and frosty, as well as to the sheep being buried under snow ; while Mr. Hogg states that it is brought on by continued fatigue and hard driving. 'These reasons, however, appear to be groundless, as it has attacked sheep which have never been exposed to fatigue in the latter end of autumn. 'The object, however, being rather to cure the disease than to account for its cause, we shall here give an extract from an account given by Peter M'Farlane, esq., surgeon, of Gartmore-house, who states, "that the eve-balls have a very singular appearance, the whole surface being light blue. The diseased sheep, three of which were affected, were cut under the eyes, according to Mr. Hogg's plan, and in three or four days they were all well."

"Cold and wet were probably the cause of the disease; but whatever may lave occasioned it, it leaves inflammation, and therefore blood-letting as near as possible to the eye is the surest mode of treatment. It should be performed immediately under the inner angle of the cye, on the side of the nose. The mamner of performing the operation is thus:-Lay the sheep on its back, and keep it there by assistants; then the operator, with a sharp instrument, cuts the blood-vessels on the inner angle of the eye, one or both, as the case may be, at about a quarter of an inch below the angle. The wound must be deep, or at least such as to allow the blood to flow freely; and should it not do so at the first incision, he must cut a very little lower or higher. He would also recommend the exhibition of salts so as to open the bowels freely*".

Hoving is common to sheep, as well as to all cattle which are suddenly turned from poor pasture into succulent artificial grasses, or upon turnips and rape. Care should therefore be taken to give them a little dry food in the morning of the three or four first days, so as to partly fill their stomachs, until they become accustomed to the new food, by gorging themselves with which they are sometimes so swoln with flatulence, as to die in great agony. Half a pint of linseed oil given to each sheep with a horn occasions them to vomit, and is never known to failț. Care should also be taken to prevent lambs from feeding upon the charlock, which is commonly found among cole and turnips; though to old sheep it will not occasion any injury.

The accidents solely applicable to ewes are those of slipping the lamb and protrusion of the uterus, after lambing ; the first occasioned chiefly by the animals being hard driven, or worried when heavy with young, and sometimes by therr being cast into ruts, where they fall upon their backs, and being inable to rise without assistance, if it be not afforded, they perish. It has been also attributed to the feeding of the ewes upon rape about Christmas;. The second happens occasionally after a ewe has had a difficult labour, the pains of which force part of the uterus to the orifice, in which case it should be returned as soon as possible; but, if not confined, it is again apt to protrude. To prevent this, different methods are used:

* See Prize Essays of the Highland Society : N. S. vol. iv., p. 395.

+ Sussex Rep., F. 336. It is generally supposed that food which is wet from the rain or dew occisions it ; but Ellman, of Glynde, says, "that he always chooses to turn his sheep into such crops at that time; but when quite dry, and the leaf at all withered from a hot sun, the danger is considerable."

t Sussex Rep. p. 336. 
some putting a hog-ring through the sides of the orifice; others eniploying twine for the same purpose; while a few pass a piece of lead of the size of a crow-quill through its lips, and twist the lead to secure it. When expertly performed, either mode answers very weil, and the ewe brings up) her lamb without further inconvenience; but the hog-iron has been objected to as being subject to rust *

The udders of ewes, after they have been weaned, are not unfrequently affected by tumours, which, if not attended to, sometimes end in mortification. The part should be frequently rubbed with camphorated spirits of wine; and if it suppurates, it must be opened with a sharp instrument, after which the wound may be cured with any healing salve. Prevention is, however, better than cure, and the disease may be avoided by milking the ewes, as we have already stated, a few times after the weaning.

Lambs sometimes perish in consequence of the ewes having sore teats, and therefore refusing to let them suck. When this occurs, the lamb should be either immediately put to a house ewe, in the manner which has been already described, or else fed twice a-day with milk, either that of a cow, or taken warm by hand from the ewe. The udder should be bathed for some time with tepid water, and afterwards washed with spirits, or a slight infusion of sugar of lead, or goulard. This, if often carefully repeated, will generally be successful; but if not, and much inflammation takes place, it must be poulticed, in order to create suppuration.

Besides the various casualties above enumerated, sheep are also subject to sustain various injuries from being wounded, in different ways, into the detail of which we camnot enter, their cure more properly belonging to professional men; yet, in case of common accidents, a shepherd should always be provided with some healing balsam, or emollient salve, of some of which he ought not to be ignorant.

Regarding the signs of health in sheep, it has been correctly observed that, "when he is in good order, he carries his head high; the eye is of a clear azure, with a quick and lively aspect; the mouth is clean and of a bright red; the gums ruddy, the teeth fast, and the muzzle dry; the nostrils damp without being mucous, the breath free from any liad smell, the feet cool, and the ciung substantial. The hams are strong, and the limbs nimble; the wool firmly adhering to the skin, which ought to bear a reddish tint, with a soft, mellow feel of suppleness; and, more especially, the appetite should be goodt."

\section{Chapter XL.}

\section{ON GOATS AND DEER.}

THE GOAT is so similar in formation to the sheep, that many naturalists consider them as one original species, and even those who view them as

* Price on the Romney Marsh Management of Sheep, p. 143.

f In order to examine the eye more closely, let the shepherd place the sheep between his legs, and grasp the head with both his hands. Then, with his thumbs, he pulls the eyelids asunder, and if the veius of the white of the eye are very apparent and of a lively red, and if the flesh at the corner of the eye and at the side ut the nuse is also of a lively red hue, it is a good sign. To know if the hams are strong, if the sheep be seized by one of his hind legs, and he struggles hard to release it, it is a proof that he is vigorous in that part.-Price, "On Sheep," p. 30. 
distinct animais yet admit that the difference between them is so very sliugh as hardly to merit notice. It is, indeed, not only well known that the sexual intercourse between the goat and the ewe, as well as that with the ram and female goat, have been productive, but that those hybrids have also been prolific. The internal structure of the goat presents no material difference from that of the sheen; and in a state of nature they are both "alpine animals, fearless of crag and clifi, and dwelling, indeed by preference, among the steepest and most inaccessible smminits of lofty mountains $\uparrow . "$

Aithough originally natives of a warmer climate than that of this country, yet they have been here long domiciliated, and were formerly kept in laroe flocks in the mountainous parts of Wales and other hilly districts of the United Kingdom; from their milk, indeed, much cheese was made; and the flesh appeared upon many a wealthy person's table. The enclosure of land has, however, in a great measure banished them from the soil, as they nip the hedges, and bound over the highest common fences. Their fiesh, it must be also admitted, is far inferior to that of the sheep when full grown; and from these causes they are now seldom found except in single jairs: kept by some persons for their milk, which is both light and nutritions, as well as by stable-leepers, from a very prevalent idea that their olunr, though somewhat offensive, has a wholesome effect upon horses.

We shoult hardly, therefore, have thought it necessary to notice the subject were it not to repel, so far as lies in our power, the prejudice which in this country so generally reigns against the flesh of lid, as well as acainst that of the older animal. Now in all southern comtries, hid is as roularly served to table as lamb, and is by most persons considered as the more delicate of the two: the flavour is certainly hichlier, and has nothing of that rankness which is attributed to the goat. The rearing of then may not, it is true, be profitable upon good pasture land; but as they browse upon the furze and heath of moor-land, comsuming plants wlich are rejected by other animals, we conceive that they might be lient with adrantage among those flucks of sheep which are fed upon the uplanis. There are, indeed, large tracts in the county of Surrey, not far from: London, so worthless as to produce little else than heath, and which ronsernently command such a trifling rent that were any speculative mim to hire an extent sufficiently large to employ a herdsman, and erect a few common huts to afford shelter to the animals in bad weather, together with a little hay in winter, we are persuaded that the plan would return unusual interest for the small capital to be employed.

In prouf of this, it may be observed that, independently of the mill;which could probably be disposed of in the neighbourhood when the kids were weaned, as it strongly resembles that of the cow, and is much sought after $b y$ invalids under the name of "goat's whey+," - the female almost invariably produces two, and sometimes three kids, which there can be little duuht would be soon relished as a delicacy by our epicures, and paid for accordingly, were the flesh once regularly introduced into the markets. Whe skins, also, of both old and young are valuable; and notwitlistanding the flesh of the former, though closely resembling mutton, is rather strong tasted, yet it is not unwholesome, and certainly worild not le rejected by the poor. We state this as a fact within our own lnowledge, as we have

* Cuvier-Règne Animal, tom, i. p. 277.

f Wilson ton Domestic Animals, Ess. iv.

+ We have known a she-goat, of no extruordinary value, to produce two quarts a day for several weeks after kidding. In the city of Lisbun it is more commonly used than that of cows. 
frequently tasted it in both Spain and Portugal, where it is commonly eaten; and we have often seen mutton exposed for sale in the London markets, which we should imagine to be far inferior.

\section{DEER.}

Although, having placed deer at the head of the chapter, it might be presumed that we intended to offer some details of their management, yet their breeding, when not running wild in the forest, is so exclusively confined to the parks of gentlemen, that the subject cannot be supposed to interest farmers; nor are we sufficiently acquainted with the charge of maintaining them to offer accurate accounts of their cost. It however deserves inquiry how far they might be rendered a profitable stock; and it would not be difficult to ascertain the exact degree of their merit upon that point. We, therefore, mention them merely as a hint to those who possess large tracts of unprofitable heath and mountain-land, that, were it enclosed, the price of venison is now so extravagantly dear, as probably to pay better for their rearing than that of sheep.

\section{Chapter Xli.}

\section{ON SWINE.}

Pork, both in its fresh and salted state, is an article of such universal consumption, and the hog is such a profitable consumer of every eatable species of offal, that pigs are reared not only by every farmer, but by every cottager who can find means to feed them; for there is no animal which rields so great a quantity of flesh in return for the lind of food which it consumes, and it has been not unjustly called, "the poor man's stock." This, together with the fecundity of the sow, which generally produces from seven to ten young ones at a birth, and that not unfrequently twice within the year, tends, notwithstanding the demand, to keep down the price at market to such a rate as would leave but little profit if reared upon purchased foorl. But, as they will eat every refuse of animal or vegetable substance from the kitchen or the stable, even if spoiled or trodden under-foot by other animals, they are thus fed at comparatively little expense during their growth; they are also tended with little trouble; and this, combined with their leaving nothing to be lost, and producing large quantities of dung of a quality only inferior to that of sheep, renders them so valuable to the farmer, that if the sale of the meat repays the cost of its production, it is commonly thought sufficient.

Although thus apparently careless of the quality, provided the quantity be sufficient to satisfy his appetite, and swallowing everything that comes in his way, yet, if allowed a choice of diet, the hog has the palate of a true gourmand, and always selects that which is the most nutritive : if turnips and potatoes be offered, he will be sure to choose the latter, and he infinitely prefers beans and peas to either oats or barley. His life is, in this country, rendered fir shorter than the age to which he would probably attain in the course of nature; for, when in a wild state, he has been known to live more than thirty vears, whereas here he is usually slaughtered for bacon before he is two years old, and even if lept as a boar, is killed for brawn before he reaches the age of five. Sows, indeed, may be allowed to breed longer, but they are generally put up to fatten before they are three years old. 
BREEDS.

The animal, though rendered disgusting by its proverbially filthy habits, is yet, in consequence of the economy of its production and the delicacy of its flesh, in universal esteem over the whole Christian world; and the various breeds which have been reared by crosses between those obtained from different countries are so numerous, that any detailed description of them would fill a volume.

C'ulley, indeed, only distinguishes the Berkshire, the Chinese, the Highland, and the Irish species; the latter of which, though living in the same hut as sumpituously as his master, is an ill-formed animal, scarcely meriting notice; and the Highlander is described as an ugly brindled monster, the very epitome of the wild boar, yet scarcely bigger than an English terrier-

\section{" His bristled back a trench impaled appears,} And stands erected like a field of spears*."

We shall, therefore, merely give a succinct account of those either considered as the origin of some peculiar race, or most generally bred thronghont thie Cnited Kingdom, as-the "Chinese," "Rudgewick," "Hampshire," "Berkshire," "Tonquin," "Essex," "Suffolk and Norfolk," " Shropshire," "Woburn," and "Dishley."

The Chinese pigs were originally obtained, as their name imports, from the Indies, and were long ago introduced into this country. 'There are two distinct species-the white and the black; the former better shaped than the latter, but less hardy and prolific. Both are, however, small-limbed, ears and head fine, round in the carcase, thin-skimned, and the head so cmbedded in the neck, that when quite fat, the end only of the snout can be seen. They are for the most part so much smaller than the common rum of European swine, that they seldom reach a greater weight than sixteen or eighteen stone when two years old; they are also rather difficult to rear, and the sows are bad nurses. Their flesh is too tender for bacon, has rather tou large a proportion of fat, and their hind-quarters are so deficient in proportion to the size of the other parts, that they cut up to great disadvantage for hams. The object of rearing them to mature age would, therefore, becone profitless; but their great aptitude to fatten, and the extreme delicacy of their meat-which, when young, is unrivalled-renders them valuable; they are consequently in great request among those dairymen who supply our tables with roasting porkers.

The black race have the character of being very thrifty, and of fattening on a comparatively small quantity of food; which valuable properties are so desirable that, notwithstanding the smallness of their size, they have been crossed with other breeds to very considerable advantage. They are likewise so prolific that one-of which a portrait is given in Bewick's Quadrupeds-had one litter of nineteen pigs, that being her third time of farrowing within fourteen months, in which period she produced no less than fifty young ! Their cross has, indeed, unquestionably effected great improvements upon the larger breeds of this country; and although it diminishes their weight, they have produced several linds possessing properties superior to those of the parent stock.

There is also a mixed breed, which are said to have been introduced by the importation of a wild boar from America, of nearly the same kind, but patcised with black and white: some with thick, pointed ears, like the true

* Dr. Hibbert's Account of the Shetland Islands, p. 427. 
species, which they otherwise resemble in form; and others, with the ears rounded and hanging down. These last are, in every respect, coarser than the former; but they are remarkably prolific; are good nurses; and, with proper care, will bring up two litters within the year. 'They are, however, only valuable as breeding-sows and roasters ; for they are very indifferent storcpigs, rarely attaining any great weight, and infunitely more difficult to fatten than the original stock $*$

The Indgewick breed, though reared for ages on the borders of Surrey and Sussex, and famous for their enormous size, yet do not appear to have spread much beyond the counties in which they are usually bred. They de. serve, however, to be more extensively known; for they are the largest kind in Britain, being not uncommonly seen as heavy as fat Sussex oxen, and are ustally fed, without any peculiar care, to an average of full ro stone at two yeitis old. Indeed, some of them are mentioned in the county reports as having been fed, at that age, by different persons to the following weights :-

$$
116 \text { st., 99st., 97st., 93st., 91st.; }
$$

and one is stated to have arrived, when three years old, to the enormous weight of 182 stone + !

The Ilampshire hor, of a white colour, has been long celebrated as forming very superior bacon; but its character has been acquired more from its being fed, when young, upon the mast which abounds in the New Forest, and other wood-lands, and the mode of curing it, than from any inherent excellence in the breed of its native county: being a coarse, rawboned, flat-sided animal; but fatting kindly. Very few, however, of the genume native hor are now to be met with, the common stock being either the Berlishire breed, or a considerable predominance of that blooil in the native swine of the county; and subsequent crosses with that as well as with the Suffulk and Chinese breeds, have produced an animal of a hardy mature, prone to get fat at an early age, and always to lieep in a good condition in a store state. A rlash of the Leicester, Dishley stock, has also been lately much in repute for the use of large farming establishments, wilh a view of increasing the size, as the native race when fattencd seldom exceel eighteen, and rarely arrive at twenty score weichtit.

The Iserkstive hog is of a reddish-brown colour, with black spots; the head well placed, with large ears generally standing forward, though sometimes hanging over the eyes. He is short-leggerl, small-boned, and of a rough, curly coat, wearing the appearance of indicating both skin and flesh of a coarse quality. Nothing, however, can be finer than the bacon, and the animals attain to very great size, having not uncommonly reached the weight of more than 100 stone; but from 40 to 50 , when completely fattened, is the general average .

The breed has, indeed, obtained such mqualified approbation from the best judges, that those who wish to improve their stuck of swine, are very generally

* Complete Grazier, 6th edit. p. 293.

† Middlesex Report, 2nd edit. p. 488. Malcolm's Surrey, Kent, and Sussex, vol. i. p. 454. Stevenson's Surrey, p. 538.

\pm Vancouver's Irampshire, p. 378.

$\$$ See Culley, p. 173, and Parkinson on Live Stock, vol. ii. p. 239. Young's Survey of Sussex, p.384, which gives the particulars of one weighing 113 st.; and Wilson on the Hog, the measurement and'weight of which is thus stated; namely-

Height

Length from the nose to the end

of the tail

$4 \mathrm{ft} .5 \frac{\mathrm{I}}{2}$ in

Live Weight

$12 \mathrm{cwt} .2$ qrs. $10 \mathrm{lb}$.

Dead ditto

10

1]. 
desirous of obtaining a cross with that race, and they are consegn intly now dispersed over the most distant parts of the countrv. Some of the hest of these crosses are said to he found in Stafiorlshire, from the jroweny of an animal well knuwn to pig-breeders as the "Tamworth boar." The native breed is also usually crossed at intervals, either with the pure Chinese, or the Tonquin race; and a gentleman, who has studied the native breed for many years, asserts, that if not crossed once in six or seven generations with the Indian stock, they would degenerate in shape and quality*.

The crosses from this breed are indeed so numerous, that any attempt to distinguish them would be unavailing, as almost every county has some peculiar native sort, which is vanted in its own district, and decried in every other; but as their respective value does not, as in other kinds of stock, depend on soil and situation, these differences of opinion, when not confirmed by comparative experiments, can onlv be ascribed to prejudice. 'The cross with the Chinese has, however, produced a species which possesses very superior qualities, and is called the "Tonkey," oi", more properly, the Tonquin; and a few other demand notice, as having partly yisen to the rank of distinctive breeds. For instance-

The Essex half-blachs, which were some years aso introduced by the late Lord Western, as descendants from the Berlshire, lave now justly acquired such very great celebrity as to be considered by many good jurdges superior to most breeds in the lingdom. "Whey are black and white, short-haired, fine-skimed, with smaller heads and ears than the Berishire, but feathered with inside hair, which is a distinctive mark of both; inave short snubby noses, very fine bone, broad and deep in the belly, full in the hind-quarters, but light in the bone and oflal. They feed remaikably quick, grow fast, and are of an excellent quality of meat: the sows are good breeders, and bring litters from eight to twelve, but they have the character of being bad nursest."

A breed partaking of the Essex blood, and generally linown as the "Essex and Hertford Breed," has also been brought forward by that celebrated breeder, Mr. Dodd, of Chenies, in Buckinghamshire, and is deservedly held in high estimation. He exhibited one some years ago at the Smithfield Cattle Show, which was taken from the sow on the 12 th of February, at seven weeks and three days old, and was slanglitered on the 26th of December; his live weight being 48 st. $6 \mathrm{lb}$, and the carcase, exclusive of head and oflal, 40 st. $7 \mathrm{lb}$.

The Suffolk and Norfolk have been long in repute as a hardy and prolific species, though of whly moderate or rather small size, and when crossed with either the Berkshire or the Dishley breed, produce animals winch are in very general esteen. They fatten quickiy, but their shape is not considered, by the Londion distillers, valuable for making bacon; and they prefer the Yorkshire sort, as being handsomer $f$.

The Shropshire hog is one of the largest breeds in the kingrom, and was therefore much in request by fammers when thev used to feed their servants at their own table; for there can be little doubt that the larger the species, the more profitable they will be found in jroduction. They have been commonly fed to thirty score weight, and we have heard of one,

Berlishire Report, p. 403.

if See the Essex Report, vol. ii. p. 341 : also the Complete Grazier, fth cribt. p. 295, in which there is a portrait of a sow of the breed.

$\ddagger$ Stevenson's Surrey, p. 537 . 
the sides of which, when he was killed at somewhat more than two years and a balf old, weiglied forty one score four pounds-or 103 stone-dead weight, exclusive of the head and feet; but of late years a more delicate species has been brought into vogue, by crosses with smaller kinds, and the native race has now nearly disappeared from the county.

The Woburn breed, reared by the late Duke of Bedford, from some mixed breeds which have not been distinctly stated, is white, mixed with various colours, well formed, round in the carcase, small limbed and headed, hardy, very prolific, and so kindly disposed to fatten, that they are said to have attained to nearly twice the weight of some other hogs within the same given period of time*.

The Dishley's, which are supposed to be partly descended from crosses of the Berkshire and Chinese, and were at one time as celebrated as any other of Bakewell's stock, are remarkably fine-boned and delicate. They also possess considerable beauty, and are said to lay on a larger quantity of meat in proportion to bone and offal, than any other kind known-being, when fat, nearly equal in height, length, and thickness; their bellies almost touching the ground, and their eyes scarcely to be seen for fat: the whole carcase, indeed, appearing to be a solid mass of flesht. To this, however, is to be opposed the defects of being neither very prolific, nor very good nurses; slow of growth; tender constitutioned; and requiring more food in fattening than the larger breeds..

Notwithstanding these objections, improvement has been made on several of the native breeds of the different counties by crosses with a Dishley boar; by which a roundness has been given to the frame with a proportionable depth of hoily. Their legs have also been shortened; a finer bone produced, with a better appearance when growing as a store; and a disposition to feed quicher and more kindly in the sty. By a farther cross with the Chinese, these breeds have been considerably reduced in size; but advantages have arisen in the habits and constitution of the animal, which, when fattened, will, at eighteen months old, generally weigh from 16 to:20 score per hog $\oint$.

\section{PROCREATION.}

The animals, when collectively spoken of, are usually called either swine, hogs, or pigs, but, when distinctively named, the male, when not castrated, is termed a "boar," and the female a "sow ;" their progreny being, while very younu, styled "sucking pigs," and, when a little more advanced, "porkers." According to the experiments of M. Teissier, on the gestation of animals - to which we have already alluded-it appears that the extreme periods of 25 sows were 109 and 143 days; from which it might be inferred that, on an average, they go 127 days, from the time of taking the boar until they furrow: expericnce however proves, that they most commonly farrow within little more than sixteen weeks. M. 'Teissier, indeed, observes, that the extent of gestation is, in many species, extremely various; and that

* Complete (irazier, p. 297, with a portrait of one exhibited at the Cattle Show.

+ Survey of Leicestershire, p. 295.

+ In consequence of the encomiums passed upon this breed, Mr. Knight, of Downton, was induced to try comparative experiments between some of them and hogs of the mixed Shropshire and Berkshre species; both kinds being fed alike. The Dishley's kept themselves in better condition than the others, lut grew so slowly, that at the end of twelve months they were greatly inferior in weight to their competitors.

In another experiment they fattened well, but the quantity of food which they consumed, was proportionably greater than a single hor of Mr. Knight's, whose weight was more than equal to that of Bakewell's two,-Herefordshire Rep. p, 133.

$\S$ Vancouver's Devonshire, p. 355. 
its probongation does not seem to kepend either upon the age or constitution of the female, or upon the diet, breed, or season; or, in short, upon any known cause.

both male and female show desire for coition at einht or nine months old; but, although thus prompted by nature, yet the boar should never be allowed to indulge that propensity until he has completed the age of a twelvemonth, and if the sow were also restrained until the same time, she would produce a stronger and a better litter. They are, however, frecpuently brought together at the early age of five or six months.

Regarding the sex of the progeny, it is said, in a recent French work on the subject of Generation, that among females who receive the male only once, those who receive him first produce generally more males than females. In proof of which the author, among nany instances of similar nature, adduces the following:- $A$ buar was admitted on the same day to two sows of two years old, of the same strengtin, and the issue of the same litter: the first produced nine males and a female; the other, nine females and a male. A young boar, of about five months olr, was afterwards admitted to two sows of the same litter as the boar: the one which first received him produced five males and two females; and the other, four hours later, six females and two males. He therefore recommends attention to the fact in the reproduction of all domestic animals; and, if it be supported by further experience, the hint may be found useful to the breeders of neat-cattle and horses**

The choice of a boar depends so much upon fancy, or local prejudice, in favour of a particular breed, and is so little governed by either soil or climate, that no other general rule can be laid down upon the subject, than to avoid an animal which is not small-headed, deep and broad in the chest, the chine rather arched, the ribs and barrel well rounded, and the haunch falling full down nearly to the hock. He should also be more compact in his form, and rather smaller than the sow; for, if she be coarse, her progeny will be improved in form and flesh by the cross, and the more roomy she is, the better chance will she afford of producing a lare and healthy litter. Respecting her make, no other observation need be mate than to choose her of a deep and capacious belly, with as sightly an appearance, and belonging to as good a race as can be procured. It should be also seen that she has at least ten or a dozen teats; for it has been frecuently obserred that each suching pior attaches itself to a particular teat, and if there be not one for each that is born, the: supernumerary can only get a pull here and there when the others have satisfied themselves, and therefore soon falls ofi.

The boar should be well fed, and should not be strained hy being allowed to serve too many sows; about twenty being generally sufficient. The sow should also be kept in good heart, so as to support her numerous offspring, but should not be made fat before takingr the boar; for if in very high order she will probably bring but an indifferent litter of pigs. She will also, in such case, be again ready for taking the male very soon afier farrowing, and although it would be better deferred, yet her being indulged will keep her quiet and induce her feeding. If not then server, she will at all events be again in season immediately after her young pigs have been weaned, and should, without delay, have the company of the boar. 'This, however, must be understood with some reservation regarding the time of 
year; for she should not be allowed to farrow in the depth of winter, as the young are then extremely tender, and thrive with great difficulty in cold weather: the months of February and August are therefore to be preferred for farrowing, and those of May and Novenber for being put to the boar.

When breeders get hold of a good kind of stock, they are too apt to follow it up by breeding what is called "in-and-in" with the same family : a practice which, though sanctioned by Bakewell, cannot be successfully persevered in ; for, if the system be continued, they will become bad feeders, and although they may deceive the eye by retaining their shape and apparent rood properties, yet they, in fact, soon grow delicate, and fall off in weight. Experience has, indeed, proved this in numberless instances upon various animals; and it is said that one person who tried it upon pigs, brought them to such a state, that the sows at last gave up breeding almost entirely, and when they did casualiy have a litter, the young thim ss were so smail and tender, that they died almost as soon as they were born. There can, indeed, be little doubt that the doctrine, once so fashionable, of breeding from your own stuck when you can find no better than your own, for that "best can only beret best," is false. It has, indeed, been sinceso ahiy refuter, that any further discussion of the subject would be fruitless : and we may safe! assune that the progeny of a male and fenale, not relater. possess more strength and vigour than those which are both of the same family*.

\section{FARROWING.}

It not unfrenuently happens, that a young sow will eat her young ones: she sinni, therefore, be carcfully watched and well fed, when about to firrow; which may be known by her carring straw in her mouth to form her Led. It is a crood precaution to sponge the backs of the pirgs immediately after they are born with a strong infusion of aloes in lukewarm water, as its bitter taste will prevent her from destroying them. Care slivald also be taken to separate hei from other pigs: the boar jarticularly, should not be atowed to come near the young, or he will infallibly devour them; and she should be confined to her sty for about a week after having firrowed. She should be lorked dry and warm, and be well littered, but not tuo profusely during the first few days: and the straw should be cut short, to prevent the pigs from nestling under it, in which case she is apt to overlay and crush the:n. If the sow be very heavy and have many young, the accitient is indeed very common, and should be guarded against by placing an open frame on each side of her, under which the young pigs may rum; or, if a strong rail be elevated a few inches from the ground, it will answer nearly the same purpose.

The product is so extremely various, that no calculation can be formed of the number which may be expected to be farrowed; but twenty swine are estimated to produes $\mathrm{i} 50$ pigs, or, on an average, seren pigs and a half each, for their first litter. Instances are, indeed, recorded of a suw which, in twenty litters, proluced the extraordinary number of 355 pigs; as well as of another having farrowed thirty-seven at one birth, and from fifteen (1) trenty are not very uncommont; but about half that number, after the first litter, is more ustal, an I verg large litters are not always the most

* See a paper l, T. A. Inight, Esu. in Communications to the Board of Agriculture, vol. ii. p. 186; and Sir John Sebright's Essay on improving the breeds of domestic animals, $\mathrm{pp} .11$ to 14 .

i Wilson on the Hog, Quart, Journ, of Agriculture, vol, iii. p. 36. 
profitable, for they usually have among them many weakly individuals which can never be reared with advantage.

\section{REARING.}

At the close of about a week after farrowing, the sow may be allowed to leave her stye for a short time every day, and when the brood acquires a little strength, they may accompany her; but not into the enclosure of the farm-yard, as they might there be smothered in the filth of the litter: a grass field is the best place, for the herbage improves the sow's milk; the pigs grow fuster as well as more healthy, and the stye is rendered sweet by their absence*. The time which they remain out should also be regulated according to the object for which they are intended; for, if meant to be reared as porkers, the less exercise they get the better; whereas, if brought up for bacon hogs, it is immaterial. If the brood be numerous, they should be lessened as soon as possible, by killing some of them, as sucking pigs or roasters, at the age of a fortnight or three weeks, in order to relieve the sow from the rrain upon her powers of nourishment by suckling a large number:-perhaps six or seven, or at the most nine, are quite as many as should be left; though we have known so many as thirteen brought up in perfect order without any apparent injury to the dam, and we have heard of one which actually brought up 177 pigs in thirteen litters! In such case, however, she should be a very strong and healthy animal, as well as supplied with a great abundance of the most nutritious food. She should at first have warm gruel, in which a good quantity of strong beer may be mixed as a cordial ; then, during the whole period of her nursing, luke-warm kitchen or dairy-wash, with pollard morn-* ing and evening; and, in the middle of the day, a substantial meal of dry food, consisting of steamed potatoes, pea and barley-meal, or other substances equally nutritive. The offals of the kitchen, with a little meal, will indeed generally go far to the support of a moderate-sized breeding sow.

At the end of three weeks, the male pigs may be castrated, though some defer it until they are six or eight weeks old; and, if thought necessary, those females which are not intended for breeding may be spayed when about a week older, or afterwards, if thought necessary, when suckling. The boar may, however, be gelt without danger during any jeriod of his life, and will afterwards make very good bacon. The operation is, on both, performed by a minor degree of cattle doctors, called "sow-gelders," whose usual fee is one shilling.

The young pigs, even while sucklers, should not be left wholly to the nourishment afforded by the sow, but should be furnished two or three times a day with skim-milk, butter-milk, whey, or pot-liquor, made Juke-warm, and having a little meal, or pollard, and boiled roots mixed up with it; or if this be thought too troublesome, skim-milk may be left constantly for them in a part of the stye to which the sow cannot have access in consequence of her size. In about six weeks, or two months, they will generally weigh from 30 to $36 \mathrm{lbs}$, and be strong enough for weaning: in doing which they should be gradually separated from the sow, only allowing them to suck at first twice, and then once a dav, and leaving the weakest with her a few days longer than the others. They should, however, be warmly and cleanly lodged, as well as very carefully fed. Both the kitchen and the dairy thus aflording assistance to the pigs during their weaning, they will in a little time acyuire a relish for roots, on which 
they may be fed, together with distillers' or brewers' grains and bran, until they become strong enough to seek for subsistence in the farm-yard, as store-pigs.

Porkers, however, which are intended for the table at an early age, should never be fed upon any thing but dairy produce, with meal, though some persons add a little boiled potatoes and parsnips, and should never be allowed to touch the refuse of the kitchen; for, although the wash may be very nutritive, and the pigs will thrive upon it, yet the meat, though perhaps looking well, acquires, when dressed, a strong flavour, with a sort of greasy appearance, which is any thing but pleasant*. It shoul, therefore, be liept solely for the use of the sow. They are usually ready for the marlset at about four months oll, and generally weigh from seven to eight stone; if kept until older, for the purpose of increasing their weight, although that object may be gained, yet the flesh will be rendered coarsi, and the price proportionably lessened. Those which are really what are called "dairyfed," and considered as a still greater delicacy, ought to be cxclusively fed upon the milk of the dam, plentifully aided by that of the cow-as skimmilk-and should never be weaned; but allowed to remain with the sow until about three months old, at which time they should be killed, and will commonly weigh from $4 \frac{1}{2}$ to $5 \frac{1}{2}$ stone.

The object of rearing store-pigs being merely to support them until they reach the proper acre for being tattence, it is immaterial upon what food they are nourished, provided it be abundant. They may, indeed, be seen continually prowling along the lanes and ditches, in search of any thing they can find, aml they devour every sort of refuse. Though sometimes kept in styes with yards to run in, and fed upon grains and roots, both raw and steamed, or pea-wash $\phi$, they are yet very commonly let to rum loose about the farm and straw-yard, where their only dependence arises from what they can pick up from the sweepings of the barn-floor, and the refuse of the thrmips and other food given to soiler cattle. They are, however, so thrifty that, when not liept in too great numbers, they find such comfortable subsistence, that at the end of a twelvenonth they require but a few weeks' feeding on com to fit them for the butcher. Indeed, they are not unfrequently in such good condition as to be slaughtered for farm use, without any care, and their flesh is usually of excellent quality.

They are sometimes grazed upon pasture land $\ddagger$; but more frequently soiled

* In proof of this, the writer some time ago caused two young pigs, of the same sow, to be fed when weaned, the one with skim-milk, and the other with pot-liquor, in which pollard and barley-meal were equally mixed. 'They were killed at four months old, being then quite fat and that fed upon the wash was rather the hearier of the two: joint: of each were roasted, and the remainder pickled; but the flesh of the latter animal was, in both cases, rank, while that of the former was extremely delicate.

+ According to Arthur Young, the best mode of converting any kind of pulse or corn, is to grind it into meal, and convert it into wash in the proportion of 5 bushels of meal to 100 gallons of water. This to be well stirred up several times in the day during summer, and for three weeks in the colder season; at the expiration of which time it will have fermented and become acid, and is then fit for use. It should be stirred up every time before feeding; and, if the practice be continued, of course more cisterns than cne must be employed in its preparation. It is, however, only fit for stores; and if not at first used with some more solid food-the pea-wash, particularly - is apt to occasion scouring. If used alone, we should also say, that two-thirds of the water is more than sufficient for the same quantity of meal.

Grains, also, if pressed down, and kept for a few months, undergo a kind of fermentation, and are much more serviceable to hogs than when used fresh.

+ At Petworth, they are killed after grazing in the park without anything but grass; being turned out in May and slaughtered in October and November in good conditiun. -Sussex Report, p. 385 
during the summer upon tares and clover, cut green, and either carried to the stye, or to a corner of the field in the manner of folding. Farmers, however, sometimes follow the slovenly practice of allowing them to graze over the artificial grasses; a plan which camnot be too strongly reprobated, both as occasioning great waste of food and manure. They are likewise not uncommonly turned into the woods to feed on acorns and beechmast, and unon the stubble fields to pick up what corn may be scattered from the sheaves. They have also been folded with considerable advantage upon potatoes, which they grub up without any expense, appearing also to relish them more than when given them in any other way; and they bed themselves among the haulm, besides leaving a large quantity of dung upon the ground*. Green food of every description may, indeed, be served to them; among which beans pulled up green are said, upon the authority of Sir John Sinclair, to be so advantageous, that when porls is worth $4 s .10 d$. per stone, the profit upon a fair crop will amount to $10 l$. per acret. The feeding of stores being, indeed, rather to improve their growth than the quality of their flesh, it can crencrally be attained by any thing that will fill their bellies; but althongh said to hold gool condition when soiled upon the artificial grasses, or even when grazed in the jastures, yet a small quantity of pease or beans shonld always be given to them at night, both to warm their stomachs and to induce them to return quietly home: the cost will not be thrown away, nor can the improvement which it will occasion, both in the quantity and quality of the flesh, be otherwise expected.

When turned abroad, it is necessary to ring them in order to prevent them from grubbing up the rround; and a small frame strongly made in a triangular form with three pieces of wood is sometimes fastened round their necks, to deprive them of the means of breakingrthrongh gates and fences. The ring is of iron, fixed in the snout when the pig is young, and by the tenderness which it occasions deprives him of the power of further mischief; but either the ring or the cartilage sometimes grives way, and the operation has again to be perfomed, in consequence of which two other modes have been adopted to eflect the same object: one, by paring off, with a sharp razor, the crristle of the snout; and the other, by cutting the two strong temlons of ile snout, about an inch and a half from the nose. It is said, that this may be done withont prejudice to the animal, when about two or three months old, as the wounds soon heal and occasion very little jain; on which, however, a writer in the Encyclopedia Britannica gravely remarks, "That so far as lie can credit the testimony of the pig, it rertainly seems to think otherwise, and seldom refrains from expressing its dissent in a very unequivocal manner."

\section{PRODUCE.}

The importance of the value of swine, even if only bred without any other regard to profit than the manure, is so well known to every farmer, that they are very generally reared to the utmost extent that they can be fed, asstores, with dic economy; and in this view, it has been calculated by Browne of Narlile, that one sthonld he reared and fattened upon every six acres of land under com croj); and, by Henderson, unon scren acres and a hat:, with no other. food than the refuse of the cattle, the kitchen, and the dairy, together with some tares and clover during the summer, and roots in the winter. To keep up a stock of forty, in addition to what they 
might pick up about the homestead, about an acre and a half of the grasses, and the same quantity of potatoes, are also computed to be sufficient. This, however, must be coupled with so many circumstances respecting the nature of the ground, the growth of green crops, and their consumption, together with the number of cattle, and, in short, the peculiar management of the farm, that no estimate of the kind can be made with such accuracy as to be relied on.

In dairy-furms, one good store-pig is generally allotted by writers on the subject to each cow; but here, again, the average quantity of milk produced by each, and whether manufactured into butter or cheese-as the whey and butter-milis are widely different in quality-together with the breed of pigs to be supported, must also be taken into consideration. Thus, for instance, in the Vale of Aylesbury - which is a great dairy district in Buckinghamshire, for the production of butter-one farmer says-

"That the average of pigs, fed solely upon skim-milk, is about six or seven bacon-hogs and ten porkers to a score of cows."

Another, "that with the same number of cows he can fatten 14 bacon-hogs of 15 score each in a year, giving them some corn with the milk."

While a third states, "that 30 cows will, in the course of the year, fatten 7 or $S$ hogs from 16 to 18 score each, sold at one year old, and 40 porkers of 5 or 6 stone each. The most usual mode, however, is to keej) a dozen pigs to a score of cows, and to sell them as porkers at about 16 weeks old: by which means each cow may, be supposed to rear a pig and a half of $\mathrm{S}$ to 9 stone each, or about 12 to 13 stone ammually *"

Whey is much less nutritive than either skim-milk or butter-milk, and is never used alone, in the feeding of swine; for in most cheese dairies they make butter of the whey after the cheese has been extracted; and in like mamer skim-milk cheese is often made after the cream is taken off for butter, by which means its nutriment is entirely exhausted: though it has been asserted in the Mid-Lothian Survey that " hogs thrive as well upon the whey after the butter is taken from it as before."

There are two olijects for which piys are usually reared: the one, to produce pork which may be used in families either fresh or pickled; and the other, to be salted and dried for bacon and hams, or pickled for the navy. In this country, the only fresh pork generally consumed is obtained from porkers, the fiesh of which is either roasted or pickled; as full-grown hogs are almost invariably made into bacon. In the southern parts of Europe, howerer, young pigs are very rarely killed, except as sucklers; and the only other pork which is roasted is that of the bacon-hog. The store-pigs are sent into the woods, where they find plenty of chesnuts, and when these are consumed, they are fattened upon Indian corn, given whole. The fat of the flesh is then usually pared off, and salted, to within about an inch of the lean; which is white, firm, and, when roasted, though divested of its cracklin, is, in our opinion, far superior to any meat of the kind which we have ever eaten in England. Some English breeders, however, assert that Indian corn is not equal in pig-feeding to barley-meal ; but this, we conceive, must apply rather to the quantity than the quality of the meat.

When the produce of a sow is intended for the use of the family, instead of heing sent to market, the smaller breeds of the Berkshire, Esscs, or Suffulk species should be chosen: the Neapolitan is also a pig of conve-

\footnotetext{
* See the Buckinghamshire Rep.o po 325.
} 
nient size; but we are of opinion that the pure Chinese should not be adopted; for although delicate meat when very young, yet they have such a tendency to fatten, that when only a few months old, their flesh is anything but savoury, and, as they are to be consumed at difierent ages, their qualities at the separate periods should be equally considered. The litter should not be all fattened together at the same time, but should be gradually brought into a fit state for consumption; or, otherwise, the greater portion of them must necessarily be pickled.

Supposing, therefore, the entire litter to consist of nine-three being killed as roasters, and the remaining six retained for porkers and bacon hogs : four should then be weaned, and two kept along with the sow; of which one should be killed at about twelve, and the other at sixteen or eighteen weeks old. 'Two of the pigs should, at the time of weaning, be shut up together in a stye, and regularly fed three times a day with skimmed or butter-milk, made lukewarm; which, if abundantly supplied, will fatten them sufficiently, without any addition of farinaceous food. If, however, the milk be not sufficient, then pollard, and afterwards barley-meal mixed up with warm water, must be griven: we have, indeed, witnessed the effect of porkers being reared for the market to a complete state of fatness, and producing very fine meat, when fed upon boiled mangel-würzel mixed with pollard, and made up into a mash with warm water, and only a very small portion of skim-milk.

These two porkers may be separately killed at any time from six to seven or eight montlss old; and in this manner the family may be abundantly supplied with fresh and pickled pork for domestic use throughout a great portion of the year. 'The two remaining porkers which have been kept as stores may be fed with steamed potatoes, kitchen, pea, and barley wash, as already rlescribed, until they are ready to be fatted; which may be done when they are about twelve and eighteen months old: one being killed in March, and the other not until October, as bacon-hogs.

\section{FATTING.}

We say March and October, as being the most usual seasons for slaughtering, and allowing such a distance between the two periods as will insure the having fresh cured bacon all the year round; but it may be done at any time during the winter, - pigs being never killed during the heat of summer; the same prejudice prevailing against the use of fiesh pork as against that of oysters in any month in the name of which there is not the letter R. We have also said, that one of them may be fattened at twelve months old; but this, more for the sake of convenience than as admitting that the flesh will be so fine as the one to be kept six months longer.

It is true that hogs are very commonly fattened to great weights, and killed at ages not exceeding eight or ten months, and the bacon, - particularly if they be dairy-fed and of a good breed,-is generally streaked with fat and lean, and much admired by those amateurs who only rarely eat it as a luxury; but it is not so firm and nutritive as those which are older. Especially in regard to hams, we should say that the animal is not in his prime until two years old; and if kept a year longer the flesh will have a still higher flavour. The fact is, that however de!icate the pickled pork of young pigs may be, yet the flesh, when converted into bacon, is deficient in that substantial consistence of fat which constitutes its main value, and which can only be acquired in perfection by age. The Chinese pigrs, indeed, arrive to nearly their full growth within a year; but the Berkshire, and all the jarge breeds, increase with good keeping, as much, or more, in size and 
weight during the second year, as they do in the first. It is, indeed, said, that "when reared with care, and not stinted at an carly age, they will weigh, when thirteen or fourteen months old, 40 stone of $14 \mathrm{lbs}$., and when two years old, from 50 to 60 stone ${ }^{*}$." It may, indeed, be sometimes more convenient to fat the male stores at an early age; but a breeding-sow should always be allowed to have four or five litters, as she will improve in Hesh while yielding the profit of her production.

The time requisite jor fattening depends, of course, on the condition of the animal when put up, as well as upon his age: if a young store, five or six weeks mav be sufficient; if older, six or eight; and if of the mature age intended for a perfect bacon-hog of that moderate degree of size and fatness which is preferred for the general consumption of the middle classes, from twelve to fourteen. A bacon-hog, if intended to be thoroughly fattened for farm use, should, howerer, be of a large breed, and brought to such a state as not to be able to rise without dificulty, and will, perhaps, require five or six months, or even more, to bring him to that condition. 'llis, howcrer, supposes him to be completely fat; to ascertain which with perfect accuracy, he ought to be weighed every week during the latter part of the process; for although his appetite will gradually fall off as he increases in fat, yet the flesh which he will aequire will also diminish, until at hast it will not pay for his food, and he should then be immediately slaughtered. Thus the increase of flesh in a pig jut up to be fattened, and regulirly weighed, was on the following datest:-

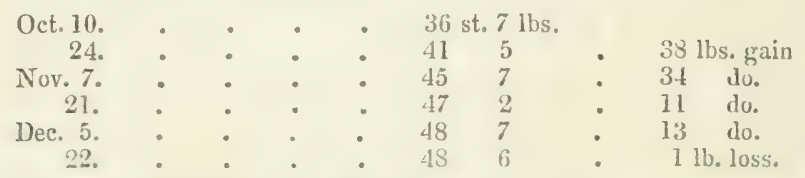

Respecting the quality of food, vast numbers of bacon-liogis are inported from Ireland, where they are almost invariably fed upon potatoes; but, however apparently satisfactory may be their weight and condition, yet, if slaughtered immediately, or before having several weeks of substantial food to harden their flesh, they are always found inferior to the corn-fed pork and bacun of this country-the fat having a tallowy alpearanee, of an insipici taste, and shrinling for want of firmmess; whereas, when boiled, it should be transparently hard, with a tinge of pink in its colour, the flavour should be good, and the meat should swell in the pot. Potatoes, therefore, though fine food for stores, should never be used as sustenance in the fatting of bacon-hogs; for, in proprortion to the quantity employed, it will render the flesh, and consequently the price, inferior to that of hous which have been properly fel. 'They are, however, frequently employed, when steamed, in conjunction with either tail or stained barley, coarsely ground, and farmers who grow potatoes for the market may thus profitably dispose of the chats along with their ummarketable corn; Lut those persons who wish to acpuire a reputation for producing fine bacon, should never use any thing for fatting but hard meat, together with skim-milk, if it can be procured.

IVhen hogs have reached the proper age for making bacon, although they may be in such good condition with regard to fat as to be apparently fit to kill, yet they must have, during some weeks, at least, a different sort 
of food from that usually given to stores, in order to harden the flesh, and render it of that mellow firmness which constitutes the essential property of fine hams and bacon. For this purpose, some people commence with about two-thirds of steaned potatoes, and one-third of pease and barley, rround in equal proportions into meal, cnlarging the quantity of meal as the animal grows fat; by which means it is said that a quick feeder will in twelve weeks consume from 14 to 16 bushels of the crain, and will probably double his weight*. One bushel of pease to four of oats and four of barley, or three or four bushels of potatoes, with two bushels of ground oats and barley, boiled, are also consilered goot mixtures; but nothing in this country is equal to barley-meal and white pease-the pease being griven whole, and the meal made into a mash with shimmed milk.

Such is the system pursued in the fatting of dairy-fed pork; but, of course, it can only be practised by those farmers who have a large stock of cows, and, therefore, cammot be generally followed. Wheaten pollard and shelled oats are also rriven; but beans, though occasionaliy used, render the lean of pork, and indeed the flesh of all animals fed upon them, extrenely hard; thus injuring the value of the hams, but, in regard to bacon, improving the fimmess of the fat: such, indeed, is the objection to them, that farmers who pay strict attention to feeding, even reject the grey pea, as approaching too nearly to the nature of the bean, and rendering the meat tough and ill-flavoured. When milk cammot be given, then the better mode of making the mash is to boil the meal in a moderate yuantity of water, seasoned occasionally with a little salt; but the pease should always be given dry ; for although many persons are of opinion that, if boiled, they better promote the animal's fatting, yet they do not by this means improve the fimmess of the flesh. In fact, the better mode is to omit the mash altonether, and give nothing but dry pease and water for a few weeks before the hog is to be killed $\uparrow$.

As to the quantity of food, porkers will consume two or three pecks of com or pulse weetsly, while fattening; but the quantity of pease reyuisite to fatten a liacon-hog necessarily depends upon his size, breed, condition, and disposition to take on fat; as well as whether the object is to fatten him to a moderate state, or to suit him for common farm use. If a large animal, he will consume for the first few weeks from $1 \frac{1}{2}$ to 2 , and in some cases even $2 \frac{1}{2}$ bushels weekly; and it has been calculated that lis weight will commonly increase at the rate of 9 or

* Devonshire Survey, p. 356. A pig of a breed from various crosses, fed upon milk and potatoes, and finished upon $4 \frac{1}{2}$ bushels of barley-meal, steeped until left to grow sour, weighed when killed at ten months old, $182 \mathrm{lbs}$., exclusive of the head and feet.Cornwall Report, p. 156.

$\dagger$ Marshall mentions two particulars of practice in the fattening of swine which is worthy of notice. "In one, salt was mixed with their food, in the proportion of a pint to every two bushels of barley-meal, under an opinion that it kept them from disorders, forwarled their fatting, and added to the delicacy and flavour of the pork."

"In the other, the meal or flour, whether of barley, pease, or other grain, was given to them dry, and this is the practice of one of the largest and best farmers on the Surrey hills; under a conviction, resulting from experience, that the practice is less wasteful than the ordinary one of mixing water with the flour, and forming them into grout, which in winter is liable to be frozen, and in summer, to be baked to the sides of the troughs." - Southern Counties, vol. ii. p. 416.

An experiment was also lately published by a farmer, who stated that he took two pigs of the same litter, and of similar weight, and fed them apart, one upon barley-meal mixed with swill, or wash, and the other with dry meal, having his drink given to him an hour afterwards. At the end of six weeks, both were weighed, and the hor fed upon dry food was found to be a stone heavier than the other. 
$10 \mathrm{lbs}$. per bushel; or, if of the size of 50 stone, and a fair thriver, he may gain at the rate of 2 stone per week; until at length his consumption of food will fall off, and his increase of weight so much more proportionably diminish, that, as we have already seen, he will not pay for the further feeding. It is supposed that five sacks of barley and one of pease will fatten a hog of 60 or 70 stone; and that one of a smaller size, in good condition when put up to fatten, and intended to be brought to the weight of 20 score, will only consume about 6 or 7 bushels of pease. This, however, appears a very moderate estimate, as those of a coarser breed, if in poor condition, will take more than five times that quantity to bring them to a complete state of fatness; and we have the account of one of the Berkshire race put up to fatten on the 28th day of August, and killed on the Sth of March following, which consumed 78 bushels of pease, barley, and oats: his dead weight, however, was S2 st. 7 lbs** $^{*}$.

Writing, as we do, merely for the information of country people, we deem it unnecessary to state the modes of feeding with distillers' wash, or upon butchers' offal, and chandlers' greates, - the two latter of which render the meat disgustingly rank-and oil-cake is nearly as objectionable; but as those who reside near the large seaports can frequently obtain considerable quantities of rice-which, though spoiled for table use, is sound and fit for animal consumption - at a very cheap rate, it may not be uninteresting to them to know that it may in such cases be used for the feeding of pigs with great advantage. Thus, Mr. Somers, a butcher in Oxford-street, purchased last year a ton of rice at $7 \mathrm{~s}$. per cwt., and used it solely in the feeding of hogs; one of which he put up, when nine months old, some time in the month of June, and killed it on the 18th of December, 1836 , at which time the dead weight was 368 lbs., and the meat of a very superior description. Unfortunately, however, he did not keep an account of the live weight; but the quantity of rice consumed was $250 \mathrm{lbs}$, together with a small portion of barley meal, two sacks of bakers' sweepings, and six bushels of pease; and his opinion was, that not only was the meat far better, but got at a cheaper rate than if it liad been produced by barley.

A comparative experiment is, however, mentioned in the Sussex Report to have been made by Lord Egremont, upon the feeding of eight hogsfour upon rice, and four upon barley, given dry-in which it is stated that barley had the advantage: but there, again, the cost of the grain is only put down in a sum total, without any account of the wcight, and the account is su inaccurately given, that we are thus left in the dark respecting the details. It appears, indeed, by another trial, of boiled rice only, that the total weight consumed by ten porkers which were put up on the $3 \mathrm{rd}$ of November, and killed on the 30 th of December, was 27 cwt. 9 Ibs.; or, upon an average, 303 lbs. each-equal to 6 bushels of common barley per head. The total live weiglit gained was $623 \mathrm{lbs}$, and the cost of its production being nearly $5 \mathrm{lbs}$. of rice to $1 \mathrm{lb}$. of flesh, it follows, that if purchased at $8 s$. per cwt.-at which price govd East India rice can now be obtained in London-it will just pay the feeder when pigs can be sold alive at $5 s .6 \mathrm{~d}$. per stone of $14 \mathrm{lb}$.; or, if slaughtered, when pork is worth $3 s . S d$. per stone of $81 \mathrm{~b}$. : for the difference between the gross live and dead weight of the pigs was-

$\begin{array}{llll}\text { Live weight } \quad: \quad & - & 1668 \mathrm{lbs} \text {. } \\ \text { Dead weight } & - & 1302,\end{array}$ difference $366 \mathrm{lbs}$.

which gives the proportion of dead weight gained by the feeding at $486 \mathrm{lbs}$.;

* Sussex Report, p. 38j.-See also the Complete Grazier, 6th edit., P. 305. 
and the cash accounts of the feeding and sale would stand thus:-

\begin{tabular}{|c|c|c|c|}
\hline od & - 27 & & $£ 10 \quad 16$ \\
\hline \multirow{2}{*}{ oduce } & $\{4$ st. 7 lbs. live weight. & & $£ 112$ \\
\hline & 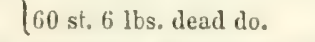 & & 1 \\
\hline
\end{tabular}

This, it will be observed, very nearly approximates the feeding qualities of rice and barley; for experiments have been made on the Berkshire and Chinese cross, which show that 12 stone dead weight of pork may be obtained from the consumption of 9 bushels of barley*; but, in fatting hogs for bacon, whether barley or rice be used, we should also recommend a portion of peas.

Regarding steamed food, it having been very generally assumed that steamed roots - potatoes especially-were preferable to raw in the feeding of neat cattle and swine, the Highland Society in 1833 offered premiums for trials on the subject, several of which were made, from which it would appear, that any advantage which might be gained by such preparation of food for oxen was counterbalanced by the cost of fuel and labour. We gave an extract from the Society's Report upon that subject in our account of winter stallfeeding $†$; and as the object is also of very great importance in so far as it regaris the economical expenditure of food for pigs, we hereby submit the information furnished on that point by the following

EXPERIMENTS + .

Mr. Boswell, of Balmuto and Kingcausie, caused his overseer, on the 1st of December, to put up ten pigs, all of one litter, in two lots, of exactly the same weight; each being collectively 5 cwt. 2 qrs. $22 \mathrm{lbs}$. The food employed was round red potatoes and the best oatmeal; those on raw food having the meal given them made up in the manner of "crowdy," or, mixed up with i little cold water; and the lut on prepared food having the potatoes boiled, with the oat-meal made into common porridge.

"From the first," Mr. Boswell says, "it was clear that the lot on prepared food were fast beating the others, and an increased quantity of uatmeal was given to the lot on raw, in order to make them ready for sale along with the others; yet still they were greatly deficient on the Ist of March, at which time, the experiment being concluded, they were put on prepared food, when they instantly began to make up the lee-way."

The live weights of the several lots on the 1st of March were:-

\begin{tabular}{c} 
Those on boiled food \\
$-\quad 10 \mathrm{cwt}, 1 \mathrm{qr} .1 \mathrm{lb}$. \\
\hline$-\quad 1,1,15 \mathrm{lb}$.
\end{tabular}

This striking difference shows a very decided advantage in improvement by the use of prepared food ; but the expense appears to have been $6 l .19 \mathrm{~s}$. $4 \frac{1}{2} d$., while that of the raw food was only $5 l .8 s, 6 d$. 'Thus the cost of feeding the pigs on raw food was less by $1 l .10 \mathrm{~s} .10 \frac{1}{3} d$, while the excess of flesh gained by those fed on prepared food was exactly $210 \mathrm{lbs}$. - equal to 15 stone of $14 \mathrm{lbs}$. live weight: which, if only estimated at $4 \mathrm{~s}$. per stone, would still leave a surplus profit of five shillings and ten pence per pig; and it is to be presumed also, that those in the best condition would obtain a better price than the others.

Mr. Walker, of Ferrygate, on the 4th of March, put up two lots, containing five pigs each, of the same brood, and two and a half

* Hampshire Survey, p. 378.

+ See Chap. xxxiv. of this volume.

* See the Transactions of the Society, N.S, vol. iv. 
months old. They were separately fed-the one on steamed, and the other on raw potatoes, with an allowance of $2 \frac{1}{2} 1 \mathrm{bs}$, of broken barley to each lot; the barley for the steamed lot being prepared along with the potatoes. 'The live weight of the two lots was:-

$$
\begin{aligned}
& \text { That on raw food : } \quad \text { : } 108 \mathrm{lbs} \text {, } \\
& \text { Ditto on steamed do. : } 106, \text {, }
\end{aligned}
$$

and the following table exhibits their several improvement :-

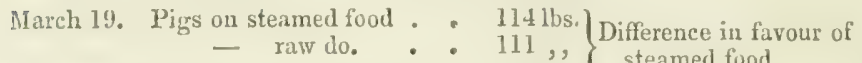

$$
\begin{aligned}
& \text { May 1. Weight on steamed food . 205lbs.) } \\
& \left.\begin{array}{c}
\text { raw do. } \\
-\quad: \quad 175,, \\
\hline
\end{array}\right\} \text { Ditto } \quad 30,30,
\end{aligned}
$$

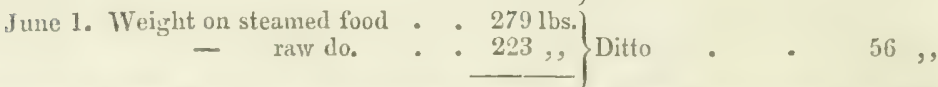

Thus in three months the pigs on steamed food had increased $173 \mathrm{lbs}$. -being 67lbs. more than dubble their original weight; while those on raw food only gained 115lbs. The potatoes were, however, given anlimitedly to each lot, without being weighed; so that we have no account of the quantity each consumed, nor of the cost of preparation; but the reporter states, "that he does not think it possible to make pigs fat on raw potatoes, without other food, when confined to them alone."

Mr. Dulgeon, of spye-Law, near Kelso, on the 2nd of July began to feed two lots, - one containing sis he, and the other five she pigs, each nine weeks old; the males being fed on steamed, and the females on raw potatoes, with a little cut grass. About a fortnight afterwards, the food was changed to beans, - the males getting them boiled, and the females raw, hut ground and inised with water ; salt being applied with both.

Both lots ate the potatoes with equal avidity; but those upon the raw beans were, during a weck, very shy in touching them, and scemed to prefer the cut grass. After that time they, however, devoured them greedily, and an eruption appeared upon their ears, which was attributed to the heating nature of the food; in consequence of which a proportion of potatoes was griven, and a little more salt added, which allayed the irritability. The following are their respective weights

\begin{tabular}{|c|c|c|c|c|c|c|c|c|}
\hline Aug. 19. & Ditto & - $\quad 27$ & 2 & 8 & Increase & 10st. 0lbs. & $80 z$ & z. \\
\hline Oct. 12. & Ditto & . 55 & 8 & 4 & Ditto & $25,, 5$, & 12, & \\
\hline
\end{tabular}
during the trial :-

$$
\text { He Pigs. st. lbs. oz. }
$$

July 2. Weight at this date 17 2 0

$\begin{array}{lll}\text { Aug. 19. Ditto } & -\overline{1758} \text { Increase 3st. 4lb. Soz. } \\ \text { Oct. 12. Ditto } & -3128 \text { Ditto } 14,7,7,0,\end{array}$

Total increase in ten weeks . . . . 17st.111b.80z.

Difference in favour of steamed food. . . . 20st. 81b. 120z, 
Each lot had four bolls of beans, but the males had seventy bushels and the females only fifty-five bushels of potatoes; the cost, together with the fuel and labour of preparation of which was-

For the He Pigs : $\quad: \quad \begin{array}{rrr}£ & 2 s . \\ 6 & 9\end{array}$

Mr. Dudgeon also put up two males and two females, which he caused to be fed on raw and boiled food indiscriminately, as it hapuened to be left over after serving the other two lots. Tivo of this lot were of a very jecuiar breed, being a cross of the wild boar with a common sow: they were marked exactly like a zebra, but did not take on fat so readily as the other pigs; hence they were not so profitable, but their pork had a peculiarly rich and delicate flavou. He says, "that he, every eight or ten days, made repeated observations on the appeartnce of all the animals, and he states generally, that the lot of males fed exclusively upon boiled meat did thrive throughout in a superior mamner to the others, and even to those who had an occasional mixture of rav and boiled meat."

These experiments, indeed, appear conclusive in favour of stcamed food in the feeding of young porliers, and some experienced breeders recommend that every veretable substance that can be collected for store-pigs should be boiled up together, and given three times a diay lukewarm: agreeably to which system pea and bean hauhn, turnip-tops, and even weeds, if not poisonous, are found to sufficiently answer the jurpose; but comparative trials of raw and prepared food for grown bacon-hogs would still be very desirable.

\section{BACON AND HAM.}

The fat of the how-of which bacon is chiefly composed-differs from that of every other quadrufed, not only in its comsistence and quality, but in its mode of distribution over the animal's body. "The fat of man" -as Buffon observes_." and of those animals which have no suet, as the dog and horse, being juetty equally mixed with the flesh, while the suet of the sheep, goat, and deer is found only at its extremities; but the fat of the hog covers the animal all over, and forms a thick, distinct, and continued layer between the flesh and the skin."

The pork, when intended for home consumption, is in most farm-houses merely well salted, and, when cut into pieces, deposited in kits; after which it is covered with brine made sufliciently strong to swim an erer, and simmered over a gentle fire until the impurities which rise are shimmed off. This, when cold, is poured upon the meat, which sometimes remains in that state so longas two or three years before it is used; at which ane, it is said, the fat is more firm, of better flavour, and wastes less when boiled than with less keeping * but, when intended for market, the flesh of the large hogs is cured for bacon in the following manmer, as described by Henderson.

"The animal should be left fasting for full twenty-four hours before killed, and, after the carcass has hung all night, it should be laid on its back upon a strong table. The head should then be cut off close by the ears, and the hinder feet so far below the houghs as not to disfigure the hams, and leave room sufficient to hang them up by ; after which the carcass is divided into equal halves, up the middle of the back-bone, with a cleaning-knife, and, if necessary, a hand-mallet. Then cut the ham from the side, by the second joint of the back-bone-which will appear on dividing the carcass; and dress the ham by paring a little of the flank, or shimny jart, so as

* Middlesex Report, 2nd edit, p. 486. 
to shape it with a half-round point, clearing off any top fat that may appear. The curer will next cut of the sharp edge along the back-bone with a knife and mallet, and slice off the first rib next the shoulder, where he will find a bloody vein, which must be taken out, for, if left in, that part is apt to spoil. The corners should be squared off when the ham is cut out."

"When this is done, give each" flitch' - as the sides are called-a powdering of saltpetre, and then cover it with salt, and let them remain in a cool place, and proceed in the same manner with the hams. In this state they may lie about a week, after which they should be turned, and fresh salted, and in two or three weeks longer they may be hung up to dry in the smokelouse; but if allowed to remain for a month or two until it may be convenient to dry them, no harm will occur, provided they be occasionally turned*." The flitches should however be laid, while salting, with the rind undernenth; and means should be used either by having a tray with a gutter round the edges, or placing them upon an inclined board, so as to allow the brine to run from them.

The drying of hams and bacon is performed in most of those farmhouses where wood is employed as fuel, by hanging them in the chimney, and, when oak is burned, it is said to impart a superior flavour; but smokehouses are also to be found in many country places where the operation can be more accurately effected at a trifling expense, as the fire is equally kept up and supported both night and day. These are, in general, mere huts, about seven feet high, and closed on all sides, so as only to allow the smoke to ascend by a small hole in the roof. The fire is made of saw-dust, which is spread to the depth of five or six inches over the wholc of the earthen floor, and, when kindled, smoulders without creating flame. The hams and flitches should be well rubbed over with bran, and hung from the joists of the roof, if flat, or from pieces of timber, strong enough to bear their weight, fixed across the walls, so that their ends may hang within two and a half or three feet of the floor ; their distance from each other being of no consequence, provided they do not touch. The neck of the flitch should lang downwards. The flitches will, in general, be sufficiently cured within little more than a fortnight, without much loss of weight; but hams, if thoroughly smoked, require longer time, and will lose perhaps one-sixth $t$. In some large establishments the bacon is dried by means of stoves; but a smoke-house, such as that described, if the process be regularly as weil as slowly conducted, will impart a better flavour, and one of very small dimensions will be found amply sufficient for the cure of a large number of hogs.

The custom of smoking is the general practice in Westphalia; and in Spain and Portugal, where the hams are also remarkably fine, sugar is very commonly used in curing, in the proportion of about one pound to three of salt and two ounces of saltpetre. The sugar assists in preserving the meat, renders its fibre mellow, and corrects the extreme pungency which is often

* Treatise on Sivine, p. 29.

$\dagger$ In Bordley's American Husbandry-

Dec. 2. Twenty of his family hams, trimmed, weighed when green, $321 \mathrm{lbs}$, or each $16 \frac{6}{10} \mathrm{lbs}$.

June 30. Do. do. when full smoked 256, , , $12 \mathrm{1}_{10}^{8}$, , 'The loss being thus $20 \frac{1}{1}$ lbs. per cwt.

Dec. 22. Tiro of a tenant's hams, when green,

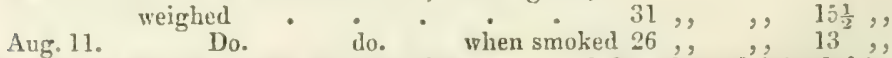

Thus losing only $16 \mathrm{lbs}$ per cwt.; but they were cured for sale, and' intended to pre. serve as much as possible of their weight. 
occasioned by the too free use of salt. It has also a good effect on bacon; and perhaps were molasses used in the pickling of pork, it would improve the flavour.

In Westmoreland-which county is famous for its hams-the mode is, to rub them very hard with bay-salt; after which they are left on a stone bench to drain off the brine. After four or five days this friction is again repeated, with an addition of about an ounce of finely-powdered saltpetre to each ham, mixed with the salt. They are afterwards suffered to lie for about a week, and are then hung up in the chimmey to dry. In this latter process, however, there is a difference of practice; some persons hanging them so that they may be dried solely by the heat of the fire, without being at all exposed to the smoke; while others suspend them in the midst of the smoke, whether arising from wood or peat, and leave them there until the weather becomes warm, when they are packed up in oat-chaff; though, to prevent them from being fly-blown, they ought to be covered with bags of coarse linen or paper.

In Hampshire, Berlishire, and some of the neighbouring districts, when a hog is killed, the first process is to "swale" lim, or singe off the bristles; which is done by laying the dead animal on one side and covering it thinly with straw, to be lighted on the windward side, and renewed as it burns away ; taking care, however, not to scorch the skin: the other side is then turned, and when the process of singeing is completed, the bristles are scraped off dry. This is considered far preferable to the common method of scraping them off with the assistance of warm water, which softens the rind, and is thought to injure the firmness of the fat. He is next cut into flitches, which are effectually rubbed with a mixture of saltpetre and common salt*, and laid in a trough, where they continue for three weeks or a month, in proportion to their size, and are during that time fiequently turned; after which they are dried in the mamner already detailed, and when completely cured, are either packed in chaff, or deposited upon the kitchen rack, for home consumption. They should be kept quite dry, and neither bacon, nor ham should ever be kept in cellars or damp places $t$.

The Wiltshire bacon-which is more estcemed than any other, as being more commonly prepared in that and the adjoining county of Somerset, fiom dairy-fed pork-is cured in the following manner. The flitches are laid in larne wooden truughs, and sprinkled over with bay-salt, after which they are left for twenty-four hours in order to drain off the blood and superfluous juices: they are then taken out and wiped thoroughly dry, and some fresh bay-sait, previously heated in a frying-pan, is rubbed into the flesh until it has absorbed a considerable quantity. This process is continued for four or five successive days, during which the flitches are turned every day; and, if the hog be large, they should be kept in strong brine for five weeks, turning them every second day, until they are hung up to dry + .

In some places a practice prevails which is common in many parts of the Continent, of stripping off the skin : the hide being much used by saddlers, as well as the bristles by brush-makers, affords some profit, and the flesh is said to take the salt better than in the former methods; but, if cured in this manner, we are assured that the bacon is subject to become rusty, and to waste in the boiling.

* See some observations on Salt, chap. xxxvii., pp. 418 and 424. † Complete Grazier, 6th edit., p.310, † Ibid. 
BRAIVN

is made solely from the boar, of all breeds and at any age; though the larger the size, and the deeper they are in the shoulder, the more they are preferred by the dealers. There are several different places which have acquired a certain degree of reputation for brawn; among which Oxford bears the highest character; but perhaps the greatest quantity made, and of as good quality as any other, is manufactured at Canterbury. The droves of boars come to that city about the 15th of October, and are immediately put up to fatten: some singly, others in pens of three or four togrether, as they are found to feed better in company. They are generally fed upon beans, with sulphur given in their water, and are killed about Christmas.

The operation of curing consists of boiling, seasoning, taking out the bones, and mixing the flesh so as to form the collar, which is rolled in a cloth and bound up with tape. A good collar is said to weigh about $30 \mathrm{lbs}$; and the hands, gammons, heads and feet, are either made into sausage-meat or sold to the poor; but we have little other information on the subject, for the trade make such a secret of their business that when inquiries were made for information by the President of the Board of Agriculture, it was very scantily supplied.

\section{THE PIGGERY}

is usually raised as a mere shed attached to the wall of some farm building, and as near as possible to the kitchen and dairy-which are too often connected together-the sole reason of which position being the convenience of supplying it readily with wash. A certain degree of nearness is indeed advisable for the saving of troulle, and in some firm-louses there is a door or window in the back kitchen commenicating with the hog-sty, through which the refuse regetables and wash can be thrown to the animals without cricroaching on the servant's time; but the air of a dairy should be ever preserved guite uncontaminated from any fouhness of scont. Those on a Jaree ecale should, therefore, be so construrted as to divide the range of styes from the dairy-yard by a wall suticiently high to preclude all commimication of mplecisant odour; yet having a cistern with a trough, or pipes, passed through the wall, so that a servant may only have to cross the court and place the fluid in the reservoir. That crected by the Earl of Figremont is perhaps the most complete building of the kind in the lingriom, and although too large and expensive for common farmers, yet, when farm oflices for large holdings are intended by the landlord, something of the same principle might be alopted upon a smaller scale; we therefore here insert a gromd-plan of the building,-which sufficiently conveys an idea of the structure *
A.A. Passage of entrance to the sties.
B.B. Open younds with troughs.
C.C. Lodging places, with fatting lutches.
D.J. Internal passage to the whole; having cisterns at dd for foocl.
F. Inner court.
F. Boiling-house.
G. Granaly and root-house.

* See the Sussix lieport; in which there is also at perspective view from an elevated situation. 


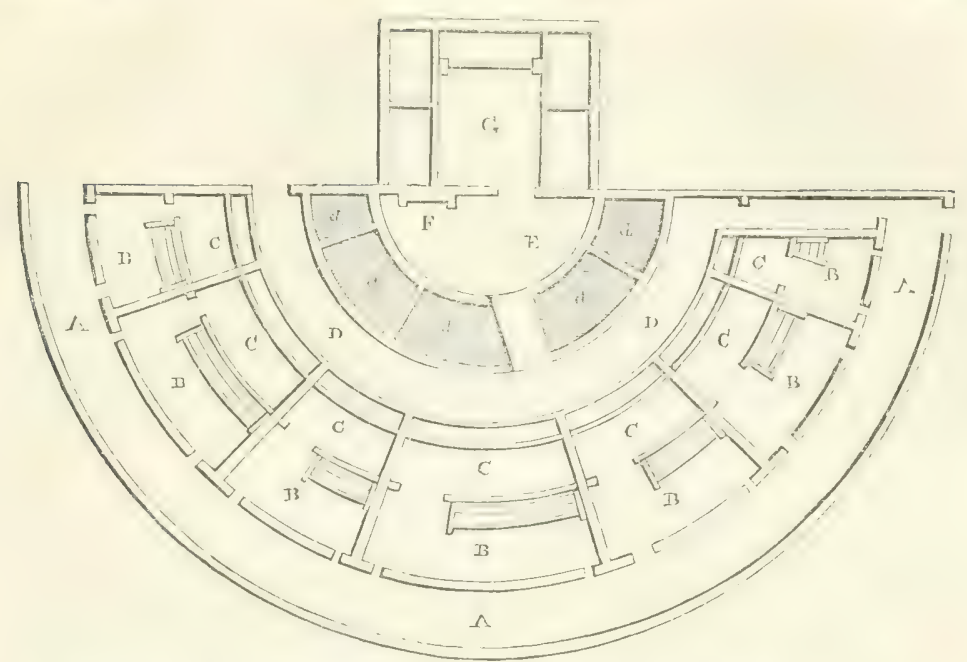

By means of a yard constructed somewhat in that manner, Arthur Young is said to have fattened eighty-eight hogs in one spring, with the attendance of only one man; whereas perhaps three might have been requisite if it had not been so conveniently arranged. The total expeuse of such a piggery, if constructed economically, would not, as he conceives, amount to more than 150 l.: it is, however, obvions that the size is only calculated for persons who carry on the business of feeling to a large extent; but, "whatever might be the cost, the groverning iilea," he justly observes, "may be followed in a stye of 20l." His opinion, that it should be built in a complete circle, would, however, if acted unon, lave the disadvantage of not exposing the frontage of all the sties to the sun; which is an object of importance, as pigs are great lovers of warmth.

Upon whatever scale the piggery is to be erected, it should be raised upon a little declination, so as to allow of the drainage of the urine to lieep the animals dry, and divided into styes of between six aml seven feet in width, and fourteen or fifteen feet long; the back part of which should be covered with a low roof, and suficiently large to allow a fatting hog to lie: down conveniently. These styes are sometimes left open in front; but althongh ventilation should be attended to, yet, in cold weather, they should be boarded nearly to the top, allowing only of a doo: to enter, and a couple of inches open space at the bottom to carry ofi the urine. The hoards, if placed in a groove of the frame-work, can be removed at pleastre, either to clean the sty or to render it cool. The uncovered prart, which is used as a court for the animal to feed ju, should be boarded in front by a low palingr, so as to admit the sum and air, and the trough for the victuals placed in one corner; but, as he is very apt to put his feet into the trough, thus soiling and wasting his food, hinged shutters, provincially termed " witches," which give way to the pig's snout and close on his withdrawing his head, are by some persons fixed before the troughs in order to prevent him. bars are also occasionally nailed at stated distances across the top, of the trough, so as to confine each pig separately to his food, without being able to drive away his fellows. $\mathrm{A}$ better mode, however, is to place the trough outside the front paling of the pen, with a loole in it only just large enough to admit of his head; hy which means 
waste will be avoided, and the servant can fill and clean the troughs without entering the court. The styes for breeding sows, and those used for storepigs, may be somewhat wider, and the trough should, of course, have a sufficient number of apertures for the little pigs' heads, together with a separate trough having a larger opening for the sow, as well as high enough to prevent the sucking pigs from getting into it. Where water can be conveniently obtained it is also a good plan to have a small spout directed through the styes, not only for their more easy cleansing, but to afford the opportunity of always allowing the animals to drink. The building, when not circular, may thus be extended to any length; and if connected at one end with a boiling-house, and at the other with a cess-pool, into which the drains are emptied, the elevation of the whole will wear this appearance :-

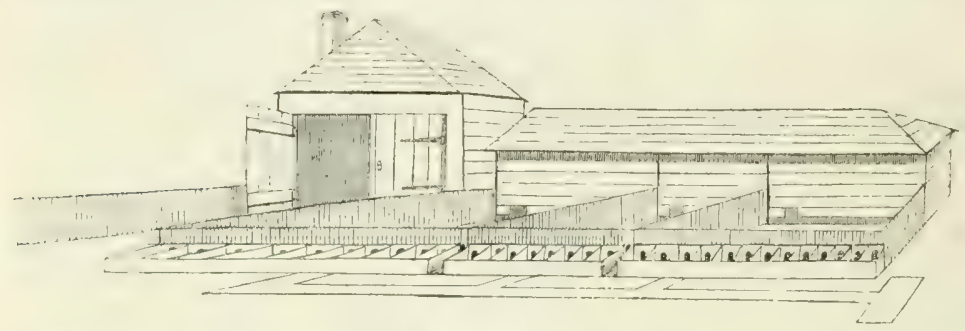

If many pigs be reared, it is always advisable to keep those of different ages from each other, and even those of the same brood are never of the same strength; they should therefore be placed in separate styes, not containing more than three or four in each, and those of as nearly as possible equal disposition. In the fatting of hogs, however, a plan has been lately adopted by many breeders of feeding them singly in styes which only allow them to lie down, without being admitted to the court; and this has been carried by some so far as not to leave them room to turn. Some of these styes are built in the form of a cage of planks, one side of which is made to move with pegs, so as to fit them exactly, and to be enlarged with their growth: they are either placed upon wheels for the convenience of moving them, or upon feet a few inches from the ground, with a gently sloping floor to carry off the filth from the back door, and having holes at the bottom for the water to drain from, while they feed through a hole in the front. The more general plan, however, is to build the styes in divisions, each to contain a pig, and to fit him as near as may be; on one side is a range of small troughs, and on the other a row of sliders which shut in the pigs. No litter of any kind is permitted, as the stalls are placed upon an inclined plane, and swept out every day ; and the chewing of their litter is thought to be injurious to their thriving. The annexed cuts show a front and back view :-

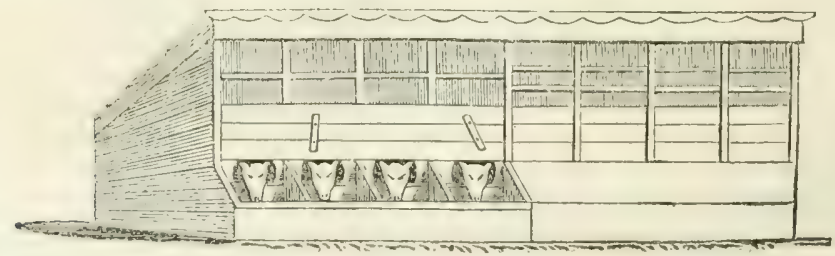




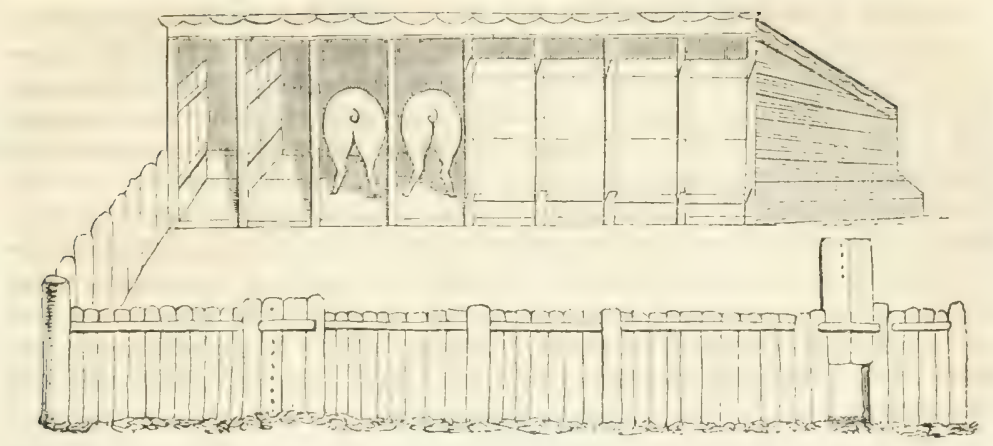

They are in fact found to fatten more speedily, and consequently upon less food, in those styes than in the common ones in which they can turn about. This is attributed to their quietude; and it is said that hogs half fat-weighing $70 \mathrm{lbs}$. -when put into one of these cages, may be brought to double their weight within twenty-eight days* : they liave been known, when fed upon bariey-meal and water, to increase at the rate of $151 \mathrm{bs}$. per week.

On this subject a comparative experiment is recorded in the Sussex Report to have been made by Lord Egremont upon some porkers, all of one breed, and as nearly as possible of the same size-but not weighed; seven of which were put up to fatten on the 25 th of February upon barleymeal, of which they had as much as they could eat, and anoilier of the same brood which had not been put to fatten, as being smaller than the others, was put into a cage on the 4 th of March, at which time he weighed 11 st. 11 lbs. He was sulky for the first two days, and would eat nothing; but he then came to his appetite, and from thence until the 13th of Aprilwhen they were all killed-he had just two bushels of barley-meal, with about eight bushels of potatoes, and weighed, alive, 18st. 3 lbs. When slaughtered, his dead-weight was $13 \mathrm{st}$. 2 lbs. - $8 \mathrm{lbs}$, to the stone-and none of the other seven reached more than 12 st. 3 lbs.: thus showing that the superiority evinced by the caged pig could only have arisen from the mode adopted.

DISEASES.

The pig is by no means so untractable as generally represented ; for, if used with kindness, he would be found as amenable as most other animals; and much of his apparent indocility arises from the harsh treatment to which he is exposed. The accusation of his inclination to filth is also much exagrgerated; for although he certainly rolls occasionally in the mire, yet when in a state of nature, he breeds in marshy places, and he seeks the inud rather for the sake of cooling his skin than for the dirt-which is evident from the enjoyment which he manifestly displays when scrubbed and washed; and if the operation be repeated every week, it will tend materially to keep him in perfect health. Although often seen wallowing during the day in any ditch or slough which may be within his reach, yet no animal loves a drier or a warmer bed to lie on; his sty should, therefore, be kept very clean, and the straw, fern, haulm, or whatever else it may be listered with, should be frequently removed. 
If these directions be minutely attended to, the animals will generally be found healthy, for prevention is better than cure, and they are seldom attacked with serious disease. In such case, however, we reconmend the owner to apply at once to the leech; or clse to give those remedies which will be detailed in the history of swine, which is intended to form part of the Farmer's Series.

They are, however, not uncommonly seized with surfeit and indigestion, from being allowed to gorge themselves with food; but this may generally be removed by moderate abstinence, and giving nothing to them for a day or two but a warm wash, which should be of a scouring nature-as bran or pollard, with water, and without any thing solid. Soap-suds have indeed been recommended to be put amongst it, as it operates like physic to empty the bowels $*$. The better mode of avoiding it is, however, to feed them regularly at stated times, and to remove the food so soon as they have eaten enough.

They often also suffer from eruptions in the slin, which usually break out at first in their ears, and, if not attended to, sometimes spread over the body into cutaneous justules, occasioning violent itching, and finally scabs. This arises more generally from want of air and attention to cleanliness in their styes than from any other cause, though it sometimes also occurs from being fed too high on heating food, as well as from the opposite effect of starvation; but when the first symptoms appear--which will be readily known by their scratching-if an ounce of sulphur and nitre be mixed once a-day in the food of a large hog, it will soon restore the skin to its natural state. It sometimes, however, happens-particularly in the breeds of large, lop-eared hocrs-that the neck and ears become ulcerated; in which case they should be anointed, every second or third day, with a preparation made of equal parts of mutton suet and tar melted tugether uver a gentle fire, and incorporated with a small quantity of the flour of sulphur.

If not conquered by these means, the animal should be separated from the rest of the herd and washed thoroughly with strong soap-ley; after which the following ointment has been recommended t:-

"Incorporate one ounce of the flour of sulphur with two drachms of fresh pulverized hellebore, three ounces of hog's-lard, and half an ounce of the water of kali (as prepared at the chemist's), so as to form a salve."

The murrain-a species of leprosy-is indicated by the animal holding down its head, staggering, having a secretion of viscid matter issuing from the eyes, and being affected with much shortness of breath. It arises chiefly in hot seasons, in consequence of inflammation of the blood, and the best preventive is to keep the animals cool, without allowing any carrion to form part of their food. A simple remedy is, indecl, that of -

"A handful of nettles with half a pound of flour of sulphur, a quarter of a pound, each, of elecampane, and pulverized aniseeds, and three oumces of liquorice, boiled together in a gallon of table-beer." time.

To be given with milk, in six or eight doses; or, about a pint at each

The measles are also very prevalent, thuugh seldom fatal; and, if not checked, affect the grain of the meat; which may be commonly seen in the shops of a faded colour, and the flesh punctured as it were with small holes, or distensions of the fibre. The commencement of the discase ap- 
pears in languor and decline of appetite, followed by small pustules in the throat, together with red and purple eruptions, more distinct after death than during the life of the animal; but may, it is said, be removed in this stage by giving small quantities of levigated crude antimony in the food.

Generally speaking, even if the animals be in health, a small quaritity of nitre and sulphur, occasionally mixed up with their food, besides stimulating their appetite, will frequently prevent disease: neither can we too much insist upon cleanliness, nor upon the punctual regularity of feeding at stated times.

\section{Chapter XLII.}

\section{ON POULTRY AND RABBITS.}

Although poultry are fed upon every farm, and it therefore might be expected that we should rive a detailed account of the various breeds and their respective managrement, yet we, upon reflection, thought that it would only uselessly swell the work, as every country housewife is so well acquainted with the means of rearing them, that, upon that subject, we could teach little that is not generally known; and, as to the history of the different sorts, and their peculiar habits, we must leave them to naturalists, for we much doubt whether our farmers' wives would read it. If e shall, therefore, confine ourselves to remarking, that we tisink the breeling of poultry conld with a little more attention he carried to a far greater cxtent than at present; for it is in all our great towns an expensive luxury, which unquestionably would pay well for any care bestowed upon it*.

The most common species of domestic poulry are fouls. On the Continent of Europe such quantities are reared that they are very nearly as cheap as butchers' meat, and throughout Ireland pouitry swarm in every hut, although the inhabitants have little eise to feed them with beyond the refuse of their own meal of potatoes; the broods share the fireside of the cottar, and the warmth, which is so essential to their health, assists in rearin them without difficulty. In this country, however, the large farmers breed only for their own use: the industrious yeomanry, who formerly were content with farming a few acres, and whose thrifty helpmates furnished our markets with an abundant supply, have now in a great measure disappeared; and, either from the want of sufficient shelter, or from objections to their being kept by the peasantry, poultry are seldom seen around their cottages; so that the tables of the Londoners are in a great measure supplied from France.

Not only in the peasant's cot, but in most of our farm-houses, the poultry are, indeed, very indifferently accommodated with sheiter; for it is generally thought that any shed which covers them from the weather is sufficient. This want of attention to their comfort, however, checks their fatting, and occasions the loss of a number of eggs, which the hens would lay during a

* It is stated in a letter from the Rev. Mr. Cooper to the Bishop of Lincoln, quoted in the Bedfurdshire Survey, "that an open field-farm, of $30 \%$, per annum, has produced annually from thirteen to sixteen guineas from this article only; while occupiers of a meadow-enclosure, of eight times that rent, raised no more than supplies fur his own table." p. 576 . 
great part of the winter, at a time when they are of the greatest value, were they kept warm.

This attention to warmth is, indecd, so well known in Buckinghamshire and Berkshire, in some parts of which ducklings are fattened in great numbers in the early part of the spring for the London markets, that the chickens are there generally reared by the fire-side. This is mostly done by cottagers, who have broods at different stages of their growth, and have them sold in the London markets at extravagant prices: one mana labouring peasant, with only one room to live in-has sent up as many as 400 ducks in the year, the greater part of which were sold, at six weeks' old, for twelve shillings a couple*. The neatness generally prevailing in most of our cottages will not, indeed, allow of the animals being kept withinside the dwelling; but it would be very desirable if the hen-houses belonging to every firm, and indeed to every moderate residence where poultry are reared, were constructed at the back of the kitchen chimney, with a flue communicating through it to impart heat.

Not only is warmth inefficiently secured, but in most farm-houses there is little other attention paid to the feeding than merely giving them the rum of the yard and dung-heap, where they pick up a little scattered corn, and get perhaps a handful or two of grain night and morning. Poulterers, however, act differently; for they not only give a plentiful supply of corn, but ship-biscuit broken and soaked in broth or beer, together with treacle and chopped mutton-suet, and, by these means, they, when cuoped, soon become fat. Turkeys, indeed, are not unusually crammed; but the trouble will hardly pay a woman who has any household occupation.

As to ducks and geese, as the former eat all which they can find in the ponds and ditches, they require little more than their share of the grain commonly thrown to the poultry at nightfall; and as geese graze upon the commons, little more is requisite than something to call them home at night, until they are put up to be fattened, which is done by confining two or three together, in a dirk place, giving them plenty of water and oats at discretion. In sume of the fen countries they are, indeed, kept for the sake of their feathers; their quills being commonly plucked four, and by some persuns five times a year-at Lady-day, Midsummer, Lammas, Michaelmas, and Martimmast; and when killed, their down is also valuable. Geese are profitable in the neighbourhood of commons, and are also kept by many farmers, under the idea that they preserve the health of the cows in the pastures where they feed + ; but they foul and injure a great quantity of grass near their haunts, and should therefore never be allowed upon meadow land.

\section{RABBITS}

are not only kept in a domestic state for the consumption of the table, but are also largely bred wild as live-stock, chiefly for the value of their fur, in some extensive warrens, which are still maintained in those sandy districts of comparatively worthless land which run through several of our southern and midland counties. At six months old they are fit to propagate; and are of such a prolific nature that, if well fed, they will breed as many as six or seven times in the vear, producing from six to eight or nine voung ones at a time. This, however, applies chiefly to the domestic kind, which being kept warm and fed upon corn as well as vegetables, produce oftener

* Buckinghamshire Report, p. 331. † Lincolnshire Report, p. 441. + Bedfordshire Survey, p. 577 . 
than those in a wild state. With such nourishing food they are fattened in about three months; but their flesh, though white and delicate, is not esteemed equal in flavour to those which are wild.

Warrens, if not connected with farms, have at least always some arable land attached, from which fodder can be produced for the rabbits; and, as their manure is valuable*, a portion of the warren is annually ploughed up for turnips; then spring cori and seeds; after which it is again thrown out. They are found to return a large per centage on the capital employed; but the animals are so destructive of fences and growing crops, that the land on which they are maintained appears in a state of desolation; and they are such notoriously bad neighbours, that, however profitable they may be, they are considered as a public nuisance, and efforts are constantly made to annihilate them. Many of the warrens have been therefore abandoned; but as they are still in some degree an object of speculation, we annex an extract from the account of some lare ones given in the Survey of Lincolnshire.

A buck serves one huudred does, which go to buck the day they bring forth their young. The common winter stock is three couple per acre, and the produce five or six couple killed. New land is however more productive than old warrens; and on 250 acres of worm-out sainfoin turned into a warren, 2000 couple were for many years killed; leaving about 700 couple on the ground: and it was reckoned that they would annualiy clear $100 l$, besides paying the rent. Turnips, cluver, and sainfoin hay, are the most proper kinds of food in winter, and thrashed oats or barley is sometimes given in severe seasons, provided the snow be not decp enough to cover tise corn. In severe storms, however, turnips are preferable, as the animals can find them by their scent, and will scratch the snow off when covered; and it will require two large cart-loads, or else a load of hay a day to fodder such a breeding-stock as that above mentioned.

There are several species bred by amateurs in a domestic state, but those kept in the warrens are seldom otherwise distinguished than as the silver sprig, or the grey. 'The silver skins are the most valuable, but the grey rabbit is so much hardier that if a warren be stocked with both, there will, in a few years, be nothing but greys. They are of different shades of colour, according to the period of the year at which they are producedt; but they all come in season,-both skins and carcasses, - -about the second week in November; from which time, until Christmas, they are killed. Among the skins there are, however, a great many of what are technically called "half skins," "quarter skins," and "racks;" sixteen of which latter are only allowed for as one skin.

The period during which they are in full prime being thus so short, vast numbers are sent to the metropolis, where the carcasses are sold, wholesale, for a mere trifle--seldom averaging more than fourpence a couple. They are forwarded in light spring carts, and, being necessarily packed close in order to save expense, they must be promptly sold, as they very soon become putrid. Considerable expense being incurred by warreners for the repair of fences and for the destruction of different kinds of vermin, as well as for protection from poachers (which, if not vigilantly watcherl, would soon depopulate the warren); the price of the skins, being also partly dressed

* It is sold in the country at $6 d$. per bushel; and a man at Aylesbury, who has 140 breeding does and 10 bucks, says that bucks and does rogether, with the young, make 24 bushels of dung per week. Buckinghamsh. Rep., p. 330 .

+ Those bred about May-day undergo no change from their white colour, but from a white rack become a whole skin; bred at Larly-ciay, become black; in June, white; in July, black; and in November, whịte again." Lincolnsh. Rep., p. 429. 
for the Chinese markets, while the fur of the remainder is used by the batters, fluctuates so much as to render this branch of rural economy rather precarious : yet fortunes have, nevertheless, bcen marle by it.

The capital, including the purchase of the stock of rabbits, and a whole yeur's rent upon 1700 acres, was calculated, in 1813 , at $1395 \%$. 8.s. 4d., and the profit estimated as follows :-

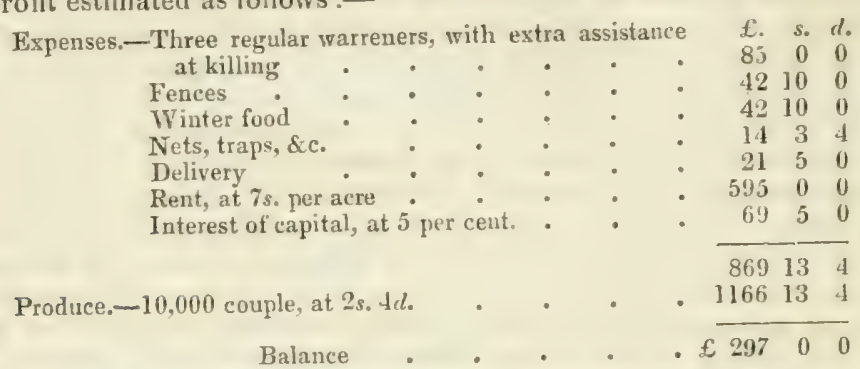

Thus learing more than 20 per cent., besides the payment of common interest: the rent is, besides,-for such land,-extravagantly rated, and the real profit is supposed to have been much greater than that stated*.

\section{Chapter XLIII.}

\section{ON FARM FENCES.}

Nofhing contributes so much to the comfort, the convenience, and the successful prosecution of the business of husbandry, as the proper subdivision of farms with the fields separated by suitable and durable fences. In looking over the earliest enclosed districts of this country, it would appear that the fields were rather formed out of the original forests, than that the intervening fences have been traced and planted by the hand of man. No doubt the first jatis and roads, and natural drains often determined the form and extent of arable fields, so that rectangular boundaries, so convenient for the ofreration of the plough, could not in every instance be attenled to; and therefore it is that we see an extravagant waste of surface vecupied with useless brushwood insteal of an effective fence, and at the same time serving as a nursery for the worst kinds of weeds and a covert for the most mischievous of birds, insects, and other vermin.

The subdivisions of ancient farms, it is obvious, have, therefore, been very generally the work of accident rather than design-neither in due proportions to the size of the farm, nor to the best system of cropping which experience may have determined to be proper. Every farm has some local alvantage or disadrantage, and the skilful cultivator will always eirleavour to serure the first and mitigate the last. Nothing can so much faclitats and obtain these results as the proper size of his fields and the direction of his fences. On the propurtionate size of the fields the advantares of a judicious rotation of cropping depends; and, by the direction of the fences ellicient drainagre is secured. Few arable farmis are so level as that the direction or place of the boundaries is a matter of indifference.

* Lincolnshire Report, p. 439. 
The character of the soil and undulations of the land always require attention in designing the divisions, and moreover, the plan of fencing; but to obtain straight and parallel lines on the sides of every field is a most desirable object, on account of the facility which it affords in ploughing the land, and conducting all arable operations.

In all late enclosures the arrangement of the fields and fences has been more particularly attended to, as well in laying them out rectangularly as also allowing the accommodation of common lanes for the more ready access to the fields.

HEDGES.

The ordinary fencing is by hedge-planting and ditching; sometimes on a raised moind without a ditch, and occasionally with a shallow ditch on each side. The following descriptions of the manner of forming these fences will give the clearest idea of how the work should be performed, and may be treated of in this order.

The hedge, as here designed, is planted on a low mound, having a

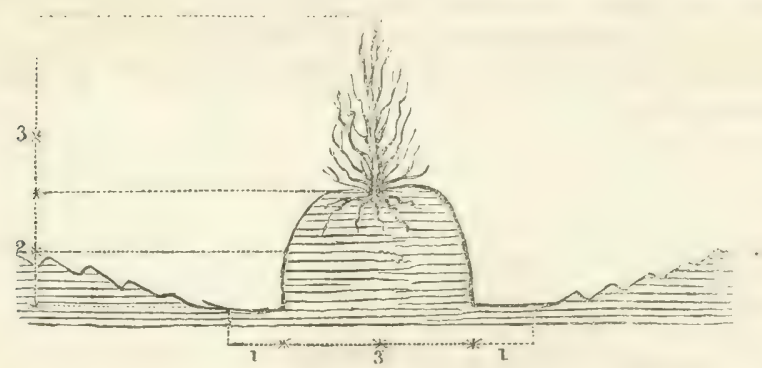

shallow depression of the surface on each side. The mound when finished should be three feet wide, which, with onc foot on each side, should first of all be double digged, to get rid of couch-grass, docks, and thistle-roots, which may grow on the line of the intended hedge. The loose earth from one foot at each side is thrown upon the centre to form the mound, and to add to the depth of the staple where the plants are to stand.

The best hedge-plant is undoubtedly the hawthorm, commonly called "quickset." The plants are raised in public nurseries, and after being transplanted or bedded in the nursery for one or two years, are of the right size and age for hedging. Such plants may be bought at from 10 s. to 15 s. per thousand.

'The lanc: should be properly levelled and got ready before the quicks are ordered; their roots being kejt moist and safe from frost while out of the wromd, and, if the weather permit, should be planted as soon as they are received in any of the autumn, winter, or spring months; but the sooner the better-the iniddle of October being by far the best season for transplanting thorns, or indeed any other kind of tree or shrub.

The planting is performed by first stretching a line along the middle of the mound, and close to which an opening is made with a spade of sufficient depth-say ten inches, or according to the length of the roots. Sometimes these are so long that it is necessary to pruie off the extreme 
point; but this need not be thrown away, because, if put in between the plants, it will also grow and put forth shoots to thicken the bottom.

The plants may be put in at about five inches from each other; thus requiring about forty plants on each lineal rod, carefully covered in with the loose soil drawn out by the spade, and afterwards trodden firmly down with the foot, leaving the middle, where the plants stand, a little hollow to catch rain, and retain moisture about the roots.

Another plan of forming a bank and planting a hedge is represented in the annexed cut. Hedges are generally accompanied with a ditch on one or both sides, and this for several reasons. Ditches act as receivers and as ducts for carrying off water, whether issuing from main or land springs, or for that which falls from the clouds. The soil excavated from the ditch forms an elevated ridge to carry the hedge; by which the latter is raised and sooner becomes a fence: the first spit serves to cover the roots of the plants, and the remainder, out of the bottom, is thrown behind to form the crest of the bank. The thoms are placed on a shelf in their natural position, whore they have the full benefit of rain, air, and light. The ditch is a guard on one side, and the raisel bank behind yields protection on the other; the ditch also balks the trespasser, and thereby guards the hedge, even supposing it to be useless as a drain. On every wet or bogrgy ground, liedges are, therefore, frequently planted, with a ditch on each side.



We sometimes observe the old method practised of planting quick-wood upon a bank; but it is self-evident that this is injudicious, as the roots are thereby deprived of all nutriment but what can be derived from a dry bank, and such fences are never seen in a flourishing state. The better mode of raising hedges in alnost every soil and situation is-wherever an option can be afforded in setting out a new inclosure-to place the "headlands," $i$. $e$. the top of the fields, to the north, and the bottom to the south: thus the fuli sun is afforded to the face of the land, and these top and bottom lines may be curved in any manner that shall best promote the drainage of the soil.

The line of the fences having been well determined upon, such parts of the land as may have previously been in an arable state ought to be ploughed to the greatest possible depth, thoroughly fallowed, and well manured for the breadth of four yards. In the month of October, it has been before observed, the season for planting will commence, and this work may be carried forward during the winter, or until the middle of March. 'Then mark out the intended line of the ditch, nine inches apart from the line where the quick-wood is intended to shoot up. Tate a spit 
of earth nine inches wide and three inches deep from out of the intended ditch, and invert it upon this space-lay it neat and level, cut off the tops of the thorns two inches above the root, and, if needful, shorten the taproot. Place the plants, so cut, four inches apart between plant and plant, in such a manner that the tops may appear exactly above this "cooping;" cover the roots first with another spit of earth from the stirface of the intended ditch, and then sufficiently with next best soil from the same place; but do not overload the roots with earth. If a drain is required, the soil or clay should be carted eff, and spread upon other parts of the land; no bank ought to be formed near to the fence or drain.

It is of great advantage, if possible, to grow a fallow crop for the breadth of four yards immediately behind the quick-wood, for three or four years after it is planted; - but this cannot be effected by the.plough, if it be necessary to guard the quick-wood on the further side from the ditch; in this case, however, where only a small extent of fence is planted, the space between the guard fence and the quick-wood-which ought not to be less than five feet-may be allotted to cottagers to plant with potatoes. This will greatly promote the rapid growth of the fence.

A hedge planted in this manner requires, in order to render it a fence in the shortest time, a little judicious pruning. It should be considered that the main strength of a hedge consists in the unyielding stoutness of the principal stems; and to have these of the requisite strength, they should only be moderately pruned on each side, cutting out the strongest branches, which act as rivals to the stem, but never topped until they have acquired a diametric bulk of one inch at the height of three and a half feet from the ground. When this strength of stems is obtained, the herlge is complete as a fence against all kimls of cattle which are not high leapers, and will so continue for many years, with no other labour than what may be amnually given by a keen trimming-hook, or by shears, to keep it from uselessly spreading.

Many fine young hedges are ruined by being too frequently headed down at different heights in their youth. It should be remembered that every individual thorn in a hedge is like a single tree planted on a lawn, both of which are wished to rise into full strength and stature as soon as possible ; but this result can surely not be obtained by repeatedly cutting off the head of either one or the other. When a sufficient number of stems can be once made to rise from the bottom of the quick cither in the first or second year, the lenders should never be cropped till they have gained the requisite strength; for by so doing a thick mass of spray is produced which, to the eye, appears a barrier, and, for a short time, a fence against sheep, but not against a mounted sportsman, who will trample it down, or agrainst licavy cattle, which, in browsing, press through it. Besides, by heading down before the stems are sufficiently strong, the hedge becomes top-heavy from the abundance of spray, which shades, and eventually kills that bclow, rendering the stems naked at bottom, through which pigs, or lambs, or Welsh sheep soon make thoroughfares.

Another advantage of planting and training a quickset-hedge, as above stated, is nut only its constant efficiency as a fence, but also in saving the septennial expense of the hedger and ditcher in renaking. Such a hedge, moreover, occupies the least possible space, harbours but few birds, nor does it repel either sun or wind.

That such a liedge occasions considerable expense during the first seven years of its growth must be admitted, seeing that it must always be de- 
fended on one or both sides until it can defend itself; and this must either be done by a dead hedge on each side, formed of stakes and brushwood interlaced, or by hurdles when necessary. Dead hedges, if kept in repair, are the least trouble and perhaps the most secure; but if enclosure post-andrail fencing cannot be afforded, the next best protection are hurdles, which, as they must be had for other purposes of the farm, may be cheapest in the end. The business of protecting the young hedge is the most onerous part of the improvement; but one thing is perfectly clear-the man who cannot afford to rear, or neglects to defend his young hedge, ought never to be at the trouble and expense of planting one.

The great advantage of raising "healthy and quickly effictive fence is so important a circumstance to the farmer, that he should not be penny-wise on the occasion of planting one. If the soil be not naturally good, some expense should certainly be incurred to avoid a defeat. Not only should the site of the new hedge be well cleaned, digged, or trenched, by increasing the depth of mould for the plants to grow in; but also, if the soil be naturally poor, it should have a sprinkling of good manure bestowed-an extra expense which will not be thrown away, as the additional vigour imparted to the sets will amply repay the cost. In order then that a planter may choose the most eligible plan of forming a hedge which shall be not only a boundary but an efficient fence, it is necessary to state that it should occupy the least jossible space. It is an old rule in estimating the crops on an arable or even a grazing farm, to deduct one acre in every ten for fences; so that a farm of one hundred acres can only be charged by the tithe-owner for ninety acres. A hedge and ditch of the usual form occupies about six feet-that is, three feet for the ditch and balk (as the plough cannot work close to the edge of the ditch), and three feet for the spread of the hedge. Now if a sufficient fence can be made to occupy only half this space, the farmer is every year a gainer; and such a fence may be had on every farm not particularly wet. Land which is springy, or liable to retain surface-water, requires ditches to carry it off; and in such cases the ditch is as serviceable as the hedgre, and therefore indispensable.

A hedge should be an effectual barrier against every kind of live stock; and to be so, it must be thick and close at bottom, ricgidly stiff, and, including the bank, at least five feet high. This, while it gives perfect security against all trespassers, neither shades the land, nor checks the drving currents of air; and whatever may be the manner in which it is made, it should be brought and kept as near to this standard as possible.

\section{CUTTING OR RE-MAKING HEDGES.}

In former times, when no other fuel save wood and peat was used in farm-houses, the hedges being re-made periodically, were, with the loppings of pollard trees, a principal source of supplies of faggots for baking and brewing, and other purposes of the homestead. On this account wide "brows," or "shaws," covered with coppice and bushes were allowed to encroach on the arable land without regret; because when felled, not only a stock of fire-wood was obtained, but also poles for hurdle-making, and stakes, and headers for re-making the hedgre and gapping.

In proceeding to remake such a hedge as this, the hedger begins by first "ridding" the ditch and hedge of every kind of growth which will be in his way of "scouring" the ditch, or of that which does not grow conve- 
niently for "pleashing" down in the new hedge. All decared stuff of the old hedge is also drawn out behind, and as much of the living stuff standing on, or in front of the old line, is reserved for pleasling down, leaving as often as possible a living stake to strengthen the whole. 'The rirlding on both sides being completed, the ditch is next scoured out to the original depth; throwing all the loose earth and scourings to make good the bank whence it had slipped, and withal raising the crown of the bank at the same time. The liedger next begins at the left hand end of the hedge to drive a row of stakes along the line of the new ridge of the bank, and lays down the reserved pleashes, first bringing them behind one or two of the stakes, and pushing their brush out again in front, to form the outside face of the hedge. Some hedgers lay a wreath of dead bushes at the base of the stakes to lay the pleashes on, or rather to "fill up," as they call it; but it is a bad plin, because this wreath of dead stuff soon rots and leaves the bottom of the hedge hollow. 'The best and most lasting hedge is that marle entirely of living pleashes, if enough can be had for the purpose. The pleashes being laid down regularly, and interwoven with the stakes, renders the whole like a piece of lattice-work, steady and firm.

Iuch has been written on the mamer of notching the pleashes, in order that they may be easily bent down without breaking them off from the root on which they grow. The usual way of laying a pleash is by bending it back with the left hand, while a notch is made with the bill about a foot above its base, splitting it through the middle downwards, as shown in the annexed cut. The next blow of the bill cuts off the remaining stump, as

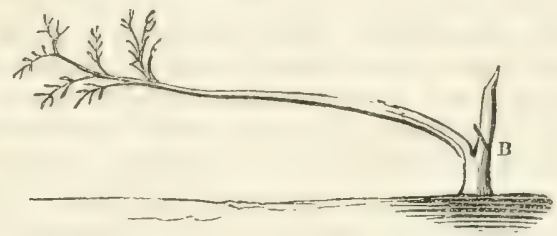

at $B$, and then the pleash is laid down. To this it has been objected, that the first cut of the bill should be upuard and not downward; because an opening is made at the bottom of the pleash to hold rain, and consequently rot the root. But this is an unnecessary piece of scientific refinement, applicable, indeed, in pruning a peach tree, but not at all in the case of the rustic thorn, as may be witnessed in the next summer's growth of the pleasin so treated.

When the pleashes are all laid down, the upper part above them is filled up with branches of the bushes cut out of the old hedge, firmly, but not tou closely wattled in. The hedge is finished by placing the headers. These are long slender rods; a couple of which are worked on at the same time, and which are alternately turned over each other, and zig-zagly between the stakes. The stakes are then all driven down even with the headers, and those which are too high cut smoothly off. Fagoting up and piling the redundant stuff, and tying up the overplus stakes, bushes, \&c., finishes the work.

Although the pleashes are thus commonly bent in one way, yet when a hedge is deficient in live wood they may be turned to it from both sidesas in the following cut, in which the pleash $a$ is turned one way, and the $b$ another: they are interwoven among the hedge-stakes, and the "edders," 
or small twigs, are added and entwined along the top-as at $c c$-in order to bind the whole together.

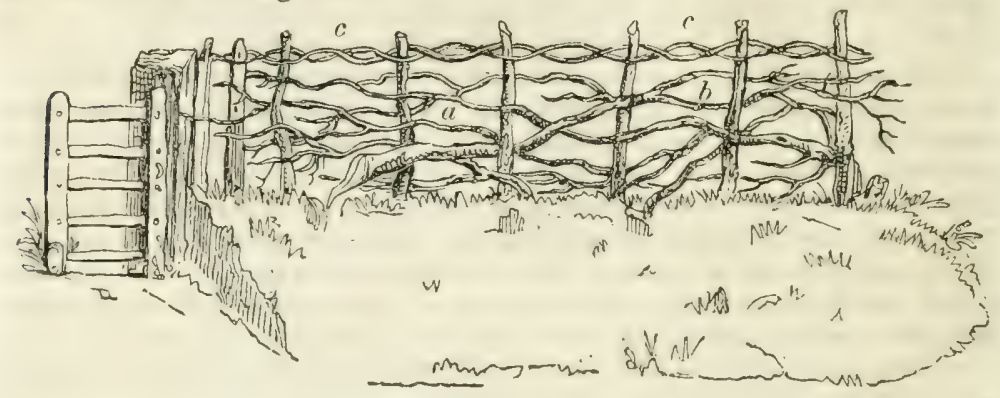

But no hedge can be properly made in which any ciead wood is left. The proper mode is by weaving the live wood in such a manner as to make an impervious fence, when the hedge is required to divide grazing pastures. In this case more of the old woor should be left than when the hedge is intenrled to divide arable fields. In this latter case, the hedge should be upright, so as to shade as little ground as possible; but, in forming a fence between srazing pastures, a consilerable quantity of brush should be left at the back, to prevent the oxen coming too near it, and by rubbing against it, from breaking it down.

All other old hedges may be re-made in the like mannes, if they have been suffered to grow wild and disorderly; but in all cases it should ever be a question with the tenant, whether keeping a hedge down by the pruning-hook and shears be not more economical than having to re-make them, as above described, every seventh or eighth vear. Made hedges, from being formed of so much dead stuff, are a great temptation to wood stealers; the stakes and healers quickly disappear, and often before they lave been a month in use. This is a strong argument in favour of cut or clipped hedgres, as well for the comfort of the firmer as for prevention of improper conduct on the part of the labourer.

\section{GAPPING.}

When a breach is made in a hedge either by sportsmen or cattle, the repair of the bank is made by deepening the ditch, driving a rank of stakes, and wattling between them strong branches of thorns of any kind. This work is usually assigned to the shepherd, who, for his own ease, is necessarily anxious to keep his flock together. By many this business is properly done; but there are some careless gappers, who cut and slash down the best parts of live fences, for the sake of repairing a fauly part of a dead one. A liog, or creep-hole through a live hedge, is often mended by thrusting a bundle of dead bushes into it; which is the only sure way of making the hole or gap larger; for this effectually prevents any new growth filling it up. Instead of closing it up with rootless rubbish, live branches should be hooked across and across from the sides, and these kept in place by stakes driven in closely together, till the layered branches take the desired position. A gap, however, which is destitute of live stuff, is best filled up by a transplanted bush of white-thorn, brought from any waste corner where such plants may be found singly. Whole hedges may be transplanted in this way; and surely single plants may be substituted instead of dead bushes.

The wild crab-apple tree is sometimes used for hedges instead of the 
hawthorn, and a very hostile and impassable fence it makes; but crabs are liable to spread too much, and they are far less tractable than the hawthorn when requiring to be re-made. The fruit also induces boys to clamber over the hedge, which of course suffers materially.

Some writers advise a mixture of different plants in liedges, in order to make them in some degree ornamental; and the common holly has been named as most suitable. The holly being a self-preserving plant, makes in time a beautiful herlge; but it is of too slow a growth for a farmer's purpose, and of all others is most likely to be mangled for walking-sticks, whip-crops, and for Christmas ornaments. There are several plants, too, which should be studiously liept out of hedges, viz, elder, every kind of willow, and fruit trees; for wherever any of these are, there is, for very obvious reasons, rarely any fence. As has been already stated, there is no plant better than the hawthorn, because of its uniform growth, hardiness, hostility, tractableness, and durability.

Among the many modern improvements in farming, none would be more essentially useful than that of planting a part of one of the least valuable fields with underwood for supplying the farm with all kirds of fencing stuff as well as fuel. No farm can be complete without such an appendage. Hop and hurdle poles, stakes, \&c., are always in requisition about a farm; and were a portion of land appropriated to this purpose, and properly laid down with ash, Spanish chesnut, white willow, \&c., it would soon beconse the most productive part of the farm.

\section{OF HEDGE-ROWV TIMBER TREES.}

Every landholder has a special interest in a well-timbered estate; and a well-wooded country has a charm for everyboly. That hedge-row timber can be of no benefit to the tenant is miversally admitted. That his crops require neither shade nor shelter from trees is perfectly evident. Where they already exist, the tenant has his remedy in fixing his rent; but when they are about to be planted requires consideration.

When a landlord determines to plant hedge-row trees, and both finds the plants, besides being at the expense of planting, protecting, and pruning them into proper form as they grow up, few tenants will oppose the design. But as there is a right and a wrong way of proceeding in such cases, a word of advice on this head may not be superfluous.

When the bank is made, and the young herlge planted, as already detailed, the trees should be immediately put in, and so placed as to stand within, that is, behind the row of quick. Oak and English elm are the only sorts to be recommended between arable fields. The young trees should be such as have been transplanted at least once in the nursery, and may be from two to three feet high. Care must be taken that the young elins have been raised from layers, and by no means such as have been grafted. The reason for this is, that the true English, or small-leaved elm, after being establislied in a favourable situation, throws up an abundance of sucliers, which for ever after keep up a valuable succession of young trees to take the place of the principal, when that is felled for use; but grafted elms are worked on the wych-clm, and therefore suckers from this would be the common and not the English elm.

When young trees are so planted, they must be safely protected, because they are the first to suffer from a cow or horse reaching them.

If it be intended to plant trees behind a hedge, it should be done when the liedge is new made, so that they may grow up together; and if planted against a cut or shorn hedge, they should be inserted in a little recess, so that they may be out of the way of being rubbed by the sheep 
or cattle. As they rise above the hedge their lower twigs should be pruned off, to prevent them tempting the cattle to browse. The closer the trees stand to the line of the hedge the better, as they are there out of the way of the whipping-trees of the teams when at pluugh or harrow.

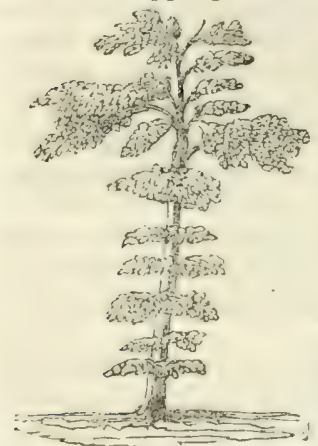

Pruning hedge-row trees as they grow up is absolutely necessary, as well for the interest of the landlord in growing straight timber, as for that of the tenant, in not being overshaded by the spread of the lower boughs. To accomplish this, a prumer should be employed every second or third year to look over the trees, and foreshorten, that is, cut back every branch springing from the bole which shows a tendency to advance laterally, and thereby divert a current of the sap out of the main channel. A tree so pruned for ten or twelve years will present a figure resembling the annexed sketch; and when no longer within reach of the pruner, the head may spread without fear of its overshading the land injuriously, or at least nut so much so as if the lower branches had been allowed to extend. This is the only way of reconciling the interests of both landlord and tenant in such cases; nor is the scheme liable to the objection of disfiguring tall trees by shredding from top to bottom, so commonly practised in some parts of Middlesex, and so much disliked by those who admire the natural form of trees.

With respect to the distance at which hedge-row trees should stand from each other, we would say, that thirty, or from that to forty feet is not so near as that their heads will ever meet so as to damage the crops, nor so far apart as to give a maked look to the farm.

The oak should be planted on the mosi loainy or richest jarts of the farm, and the elm on the lightest soils.

\section{GATES.}

Wherever there are fences and inclosed fielis there must also be stout and convenient gatos for ingress and egress. The old-fashioner fice-bar gate is, perhaps, as good a form as can be contrived; provided it be made of the best clear-graincd heart of oak. The gate here described is nine feet wide, and consists of a "thur" or "heel," and a "head," into both of which the bars and top-rail are mortised and pimed. The thur is substantial, seven inches wide, by four and a half inches thick, and about four feet long. The head is of the same length, but only four inches wile by three in thickness. The top-rail is cut four inches reep by three in thicliness. The bars are flat, four inches by one and a quarter, the thrce lower being closer together than the upper two. These, together with the top-rail, are all more substantial next the thur than towards the head, they all being a little gradu-

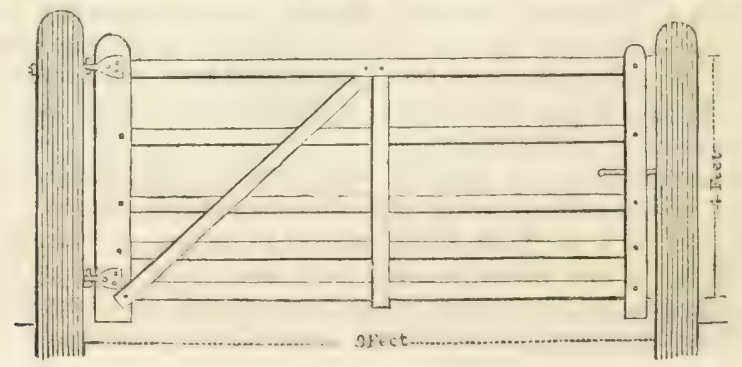


ated off in order to lighten it at the shutting-post. There is a diagonal stay, of the same scantling as the bars, abutting on the heel of the thur, and ascending obliquely to the middle of the top-rail, into which the stay is mortised. At the same point a perpendicular ledge connects all the lower bars with the top-rail, into which it is also mortised. In this way the gate is framed; and if there be any defect in the construction, it is by weakening the top-rail by mortising the stay and cross ledge into it, and at which point the gate is sure to fail, if a heavy beast presses against it. If the stay and cross ledge were formed of thicker stuff, they might be halved upon and nailed to the top-rail, instead of being mortised into it; the gate would be much stronger, and of course more lasting.

The gate-posts are placed in the line of the hedge. One is the hangingpost, into which the hooks are driven which carry the gate. This post is always longer and larger than the other, because it has the whole weight of the gate to sustain when swinging; and the deeper it is let into the ground, and perfectly steadied by ramming, as well as by strong props, the longer the gate acts properly.

The shutling-post needs neither be so large nor so long as the other, because there is always less stress upon it. It receives the fall of the gate; but this is never so severe as to displace it, if the gate be properly hung. The posts are placed nine feet from each other, as most convenient for the transit of all firm-carriages. Although the common practice is thus to hang the gate between the posts, yet it is perhaps a better plan to allow it to fall on the face of the shutting-post, as adding to its security.

The thimbles which traverse upon the hooks are fastened to the thur of the gate, and are usually forged with "fins" or wings," pierced witl nail holes, by which they are fastened to the thur.

Hanging a gate requires a good mechanical head. It should open and remain at a right ansle, and when thrown to shut, its momentum should decrease as it approaches the shutting-post, only falling thereto with no more force than is just sufiicient to move the "catch." This kind of action entirely depends on the position and length of the neck of the lower hook. The upper hook (whether on the inner or on one of the outer faces of the post) is usually driven nearly home, leaving room only for the thimble to move freely. Now, if the lower hook were inserted directly under, and have no more projection from the face of the post, the gate would have no fall, either to open or shut. But by placing the lower hook more or less out of the perpendicular towards the side on which the gate opens, it will have more or less of a fall towards the shutting-post; and this tendency of the gate to shut of itself may be much increased by making the shank of the lower hook so much longer than the upper one.

The hooks are sometimes inserted on the inner face of the hanging-post, and the gate shuts into a rebate on the imner face of the shutting-post. But this, of course, contracts the opening between the posts, and risks the gate itself by passing wheels. A preferable way is to have the hooks let on the outer face of the langing-post, and allowing the gate to fall against the corresponding face of the shutting-post. This leaves the opening between the posts perfectly clear, and the gate itself is out of danger. This manner of fixing a gate, however, requires that it should be somewhat longer, or that the posts be placed a little nearer together. It is a good practice to have graduated "spurs" spiked on the imner faces of both posts at the bottom, and to be rammed in along with the posts. These prevent either of the posts being damaged by careless drivers.

$\therefore$ Gutes are mounted with various fastenings: horsemen like strap-springs 
fixed to the head, which strike upon a graduated catch inserted in the rebate, or on the outer face of the shutting-post. Where hogs have learned to open gates, they are completely balked by the head being fitted to strike upon a staple in the face of the shutting-post, through which an attached pin is placed. Sometimes a bit of chain to link over a spike answers as well as any thing else. The kind of fastening much depends on the frequency with which the gate is used.

Swing-gates arc preferred for public thoroughfares, because they cannot be left open unless thrown off the gears; but they require very stout hanging-posts, and these inserted deep in the ground. They are made to swing either forwards or backwards, by being made to rest and traverse on two hooks let in to the hanging-post near the bottom. Upon these hooks a thick horizontal plate of iron called "horns," having senicircular notches to fit the hooks, is fastened to the thur. The hooks are alternately the fulcrum of the gate's movements, and fitting exactly on both when at rest. A notched catch is fixed to the inner face of the shutting post, on which a balanced lifting latch attached to the head of the gate is caught when the latter is at rest.

Gates of every description are often damaged by carriages for want of some trifling accompaniments. Every field-gate should liave a stump placed on the ground behind to prevent its falling too far back; and to this stump a lifting-catch should be attached to hold the gate open. A gate thus kept back is more likely to be carefully shut by the waggoner than if he trusts to its shutting of itself. The following is also a not unusual form of field-gates :-

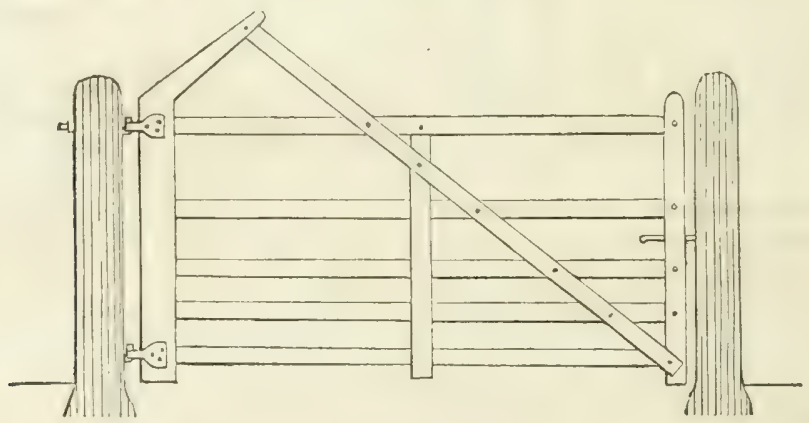

The thur is formed of a crooked butt of a tree, or of one having a branch projecting in the right position. Its bending over and so high above the top-rail affords the opportunity of having a carrying-brace, framed diagonally, to prevent the dropping of the gate at the head, an accident which should always be provided against in the erection.

Both these forms of gates are sometimes constructed of split bars and braces, rather than such as are sawn; the makers asserting that they, from their elastic property, last much longer than when made of squared scantling. Iron gates, however, as being the most durable, will be found the cheapest in the end. Almost every blacksmith has his favourite notions of gate fastenings, and their variety has now become so numerous that we cannot enter into any details of their description; but we confidently refer our readers to Mr. Parker's " Essay on the construction, hanging, and fastening of Gates," in which will be found cuts of the several kinds commonly in use, together with directions for their formation, and 
practically scientific instructions regarding the general subject of gates, of which no country carpenter ought to be ignorant.

\section{STILES.}

Many farms are intersected by public footpaths, which are often a great anmoyance to the farmer. We have always noticed that the more perfectly these footpaths are male, when they cross arable fields or pastures, the least damage is sustained; for if they be not both smooth and dry the passengers are sure to stray. In crossing fences, substantial stiles, well framed together, are indispensable. Those usually formed of strong bars about three feet wide, fixed in upright posts, with a step nailed crosswise to stumps on each side, and raised half way up the frame, are as convenient as any other. Cage-uickts - that is, a little gate traversing between two shutting-posts, having an angular space beyond these inclosed with paling, are convenient for women or infirm passengers, and particularly so, as they can never be left open. They are more convenient than fixerl stiles on another account; by lifting the little gate off the hooks occasionally a passage is made for a flock of sheep, a single horse, or any other animal, when required: they are, however, attended with considerable expense.

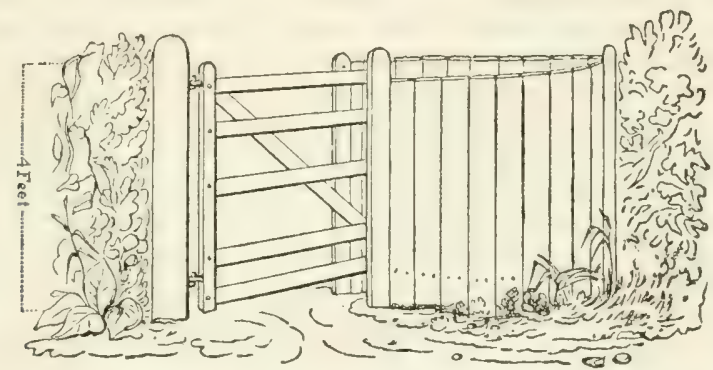

When a footpath passes through a folded field, an easy passage is formed by placing three hurdles in the form of a cage-gate; that is, a movable one traversing between the heads of two others, set to form an acute angle. This prevents passengers breaking or displacing hurdles by climbing over them.

\section{STONE-WVALLS.}

Although quicks, and live fences of every description, are undoubtedly the most ornamental boundaries for the land, yet stone-walls, though not so agreeable to the eye, are by many considered as more advantageous to the farmer; both as affording a more secure protection arrainst the encroachment of cattle, as well as a better shelter for them when confined within the field, and occupying less ground. 'They are, therefore, very grenerally in use in many parts of the north of England and Scotland, and almost universal throughout all Ireland. Both kinds of fence have, indeed, certain advantages: the former, though perhaps more expensive until brought to perfection, yet then acquires strength with its growth, and will last for ages, while the latter demands constant repair; and, in a stormy country, it is a mistaken idea to suppose that even if they be built as high as six or seven feet, they afford more shelter to any other cattle than those crouching under their lee, for it has been justly observed, that "currents of wind passing over the top of stone-walls receive a percussion, and generate blasts of air, in whirling motion, on the side of the wall; whereas these currents, in 
being pernitted to pass through a hedge, are much wealiened by their being sublivided into numerous smaller ones*." Thorn-liei!ges are, therefore, in our opinion-without any prejudice in favour of their beauty-to - be preferred; but that fence should be constructed which is best suited to the situation of the farm; and in many places diry rubble can be plentifully found upon the ground, or quarried at a trifling expense for the erection of wails, where the country is too bleak to rear a hedgre without great expense of time and money.

In speaking of stone-walls, as farm fences, it must of course be unilerstood that we merely allude to dry stone-ualls, piled togrether withont mortar ; for lime is too costly to be employed in field fencing, unless in parts of the country where lime-stone is abundant, and lime consequently of trifling value, in which case the coping mav not inappropriately be fixed with mortar. The builder must of course make use of the materials which are at hand. Round stones, or any wearing a smooth appearance, are the very worst; while lime-stone, large flint, and the sand-stone found in gravelly deposits of the size of one foot to four in diameter, are the best, as ther casily split into their layers when extracted from the earth, and afterwarls harden by exposure. If the stones are indifferent, it is recommended by Mr. Lambert-whose experience in Irish farming entitles his opinion to attention-" to have two or three casts of a good long binding kind, dropped among the bad, to each perch of your wall, in order that they may be placed occasionally across it, to bind the barl stones together. Manage to have at least nine or ten, of what are called 'trough-stones,' to every six or seven yards at least, in each course of the work. Care also must be taken to fill, or pack, the centre of the wall with small stones, the more angular the better; and if you dash your wall with mortar, it would be well also to put some on the top of your finishing course before the coping; which last should be put on while the mortar is wet, so as to fasten both, and mainly contribute to the strength of the wall $\uparrow . "$

". Much of the stability of any wall, of whatever material it may be built, will however depend on the knowledge and dexterity of the builder. 'The best builder of a dry wall is he who practises no other kind of building than with diry materials; for any ordinary mason, particularly in building rubblework, depends so much on the lime keeping the small stones safe in the inside of the wall, that he will not take the trouble to embed them firmly. 'ihe strength of every wall depends greatly on the materials being firmly arranged in its interior part; an ordinary mason will therefore be found to be a bad builder of stone fences for fields $\ddagger . "$ A wall, to be a good fence, should be at least five feet in height, and two feet and a half to three feet at the base, tapering to fifteen inches, or a foot and half at the coping, which should project two or three inches on each sile of it, and may be made of a rough slate. If gates be not admitter, a gap or two should be left in each field, sufficiently large to admit a mounted horseman; for, if it be a sporting country, the hunters will otherwise break down the walls, and occasion considerable mischief. The gaps may be filled in with a few pieces of rough timber, fixed into holes made on each side so as to be removable at pleasure; and, if there be cattle in the field, a real sportsman will never leave them open.

* On Dry Stone-Walls, in which there are minute directions for their construction. See Quart. Journ. of Agric., vol. cxi.

$\div$ On the Rural affairs of Ireland, p. 152.

+ Stephens, on Dry Stone-Walls, Quart. Journ, of Agric, vol, iii. p. 243. 


\section{Chapter Xliv.}

\section{ON VERMIN : INSECTS-BIRDS-ANIMALS.}

\section{INSECTS.}

Tre loss to which the farmer is exposed by vermin of various kinds, from the time of putting the seed into the gromd until the reaping and disposal of his crops, is often very great. That of seed, chiefly occasioned by myriads of insects, is incalculably great, for they mav be justly said " to have cotablished a kind of universal empire over the earth and its inhabitants; which is principally conspicuous in the injury which it occasions *". Their inroads can, indeed, be only partially avoided by careful tillage; though the ravages of grubs, shugs, caterpillars, and the destructive wireworm-more than sixty different species of which latter insect are said to occur in Great Britain-have in some instances been prevented by mixing the seed with soot, or by immersing it in train oil, as well as in water strongly impregnated with soot. Top-dressings of quick-lime and soot have also been found useful for that purpose ; but the latter substance camnot always be obtained, and quick-lime is not in all cases beneficial to the land. Flocks of poultry of all sorts, but especially ducks, have also been employed with great advantage in fields which have been thus infested, as they devour great quantities of worms; and if a sufficient number be turned in, they will certainly prevent much mischief. Night rolling, with heavy stone-rollers, is however the most usual remedy, though treading the ground, and irampling it well with cattle, is stili more efficient, when the state of the land will admit of it; but nothing is so effectual as a clean summer fallowt.

Ants also, though not destroying any great quantities of corn, yet collect considerable quantities of lint, hemp, and rape-seed, and congregating tonether in vast numbers, are in many cases so very prejudicial to old grass-land by the little mounds which they raise upon the surface, that pastures may sometimes be seen filled, at short distances, with such hillocks, to the great impoverishment of the soil. The most usual mode of removing these nests is by cutting off the crown of the "ant-hill" with a sharp spade of a semicircular form-somewhat in the shape of a week's-old moon with the horns at abont ten inches distance-and laving it with the grassy side downwards upon the ground. The ants are thus cleared out, the clods being completely pulverized and thrown around, and the hole is left empty for three or four weeks to secure the destruction, by the frost and rain, of any insects which may still remain; after which the sod is replaced in its former position and troditen, or rolled down, until even with the surface. The operation is commonly done at any leisure time during the winter; but some farmers either burn the clods, or else put quick-lime in the holes before digging them up, in which case they deem it preferable to liefer the process until the early part of the spring, as a top-dressing is thus formed for the growth of the seeds $\neq$.

In alludingr, however, to the destruction of seed by insects, we must not

* Introd. to Entomology, by Kirby and Spence.

$f$ It is stated in the Gardener's Magazine, that if cabbage-leaves be heated in an oven, and afterwards immersed in melted kitchen-stuff, or any unsalted grease, then spreal over the land, they will in a few hours he covered with snails and slugs, and may be gathered off along with the worms. No. xxxvii.

+ Marshall's Rur. Econ. of Norfolk, vol. i.; Essex Survey, vol. ii., p. 97 ; and vol. i, p. If 4 , containing a plate which represents a machine for cuttingr up ant-hills. 
be supposed to include the earth-worm, which, although it in some degree disfigures the mearlows by turning up small portions of the soil, yet these "worm-casts," as they are called, produce a valuable addition of fresh earth as a coating to the surface; and, when bush-harrowed to form a topdressing, materially promote the vegetation of the grass. Both gardeners and farmers, indeed, dislike them: the former, because they render their walks unsightly; and the latter, because they are thought to eat the young corn. These men would, however, find that, were the earth without worms, it would soon become cold, hard-bound, and void of fermentation-consequently steril; and it has been truly observed, by the eminent naturalist from whom this remark is drawn, "that the most insignificant insects and reptiles are of much more consequence, and have much more influence in the economy of nature, than the incurious are aware of. Their minuteness renders earth-worms less an object of attention, but their numbers and fecundity, though in appearance a small and despicable link in the chain of nature, yet, if lost, would make a lamentable chasm*".

\section{BIRDS.}

Sivarms of birls, particularly in the spring season, when berries are scarce and the sowing of Lent crops is going forward, take their share in attacking the seed, and commit further depredations upon the young corn and clover when just springing above the ground; pigeons will hardly leave a pea, and rooks will even grub up whole drills of potato-cuttings with their long bills; while, during winter, sparrows, chaffinches, and other small feathered tribes, nestle in the corn-stacks, and, during harvest, commit constant depredations on the ripened grain. A gainst this no other precaution can be used, but to have boys with fowling-pieces in the field to frighten them away until the young plants are strong enough to defend themselves; for scare-crows do little service: they should, however, be on their post before daylight, for the dawn no sooner breaks than the animals commence their depredations. It may, however, be doubtful whether the common practice of destroying the sparrows by parochial premiums, or the plunder of their nests is prudent; for they, and other small birds, come in aid of the farmer's industry, and compensate largely for the corn which they consume, by the immense number of grubs which they devour.

It has, indeed, been recommended to poison the rooks by placing barley, steeped in a solution of arsenic, on the newly-taken-out dunghills; and, as they will eat it with avidity, their lives will be the forfeit of their temerity. This, however, can only be successfully practised in winter, for it will not entice them in wheat seed-time, when other food is plentiful; and we confess that we coincide in the opinion of those naturalists who attribute the dislike of farmers rather to prejudice than fact; for they live more upon the insects found in the earth than upon grain. In a dry spring, indeed, when worms are scarce, they will dig out the newly-set potatues; but, if we look round the country, we shall find that in land in the immediate neighbourhood of those antiquated mansions where rookeries have been for ages established, the crops are not less plentiful, and the ground is perhaps cleaner than in places situated at a distance $\dagger$.

* White's Natural History of Selborne.

$\dagger$ "In the year 1825 our fields were so completely overrun with the wild mustard, that, for six weeks, there was not a blade of corn to be seen, and every barley field was covered as with gold; but on the Earl of Trayuair's fields around the rookery there was scarcely a single plant of the noxious weed to be seen." Quart. Journ, of Agric, vol. i., 1). 640 .

"It is but simple justice to these often-censured birds to mention the service they 
Of all the species of the crow-tribe, the raven-

"That hateful messenger of heavy things,

Of deatł and dolour telling"' -

is the most obnoxious; for as his acute sense of smelling attracts him to the dying, his harsh croak, when heard in illness, causes terror and alarm, as ominous of a fatal issue. He is, therefore, dreaded by the timid; and his presence is hateful to the farmer, as he makes a prey of young poultry and lambs. So carnivorous, indeed, is he, that if he perceives a grown sheep in such a state of disease as to be unable to rise, he may be seen cautiously wheeling round in gradually narrower circles, until, at last, he pounces upon the defenceless animal, whose eyes he plucks out, and then, tearing him open with his beak, gluts upon his entrails. He is, however, rather a rare bird in England, and is in some respects useful as a devourer of putrid matter.

The owl is also in disrepute, as an inauspicious foreteller of domestic calamity; and he is killed both on that account and on a very false presumption that he destroys chickens and the young of game; whereas the fact is, that his diet consists chiefly of field-mice, of which he consumes great numbers: so much so, indeed, that we are told by White, of Selborne, that he has observed them returning, cvery five minutes, to the nest, each with a field-mouse in his claws. He ought, therefore, to be viewed as a friend to the farmer, and instead of being ciestroyed, the breed should be encouraged *

Neither can we altogether agree in the abuse so largely bestowed by Cobbett upon the vood-piyeon, or cushat; for although, if not well watched, both he and the tame species will make sad havoc among the peas, yet their other spoliations are rather exaggerated. In corroboration of which, an amusing instance has been recorded by the late Mr. Hogg.

"One morning my maid comes in, and says, "Master, aw wuss ye wud reise out o' yer bed, an' shoot thae cusha doos. Od, it's ma belief they're gaun wi' the young clover bodily, an' that they'll no

at times perform for our pasture lands. There is no plant that $I$ endeavour to root out with more persistency, in these places, than the leafy hair-grass (Aira Caspitosa). The larger turfs we pretty well get rid of; but the multitudes of small roots are so interwoven with the pasture herhage, that we cannot separate them without injury; and these our persevering rooks stock up for us in such quantities, that in some seasons the fields are strewed with the eradicated plants. The object of the bird in performing this service, is to obtain the larva of several species of insects, underground-feeders, that prey on the roots; and I do not believe that he ever removes a specimen that has not already been eaten or commenced upon by the caterpillars." Ibid., vol, ii., p. 308 .

* "I cannot but notice two growing evils, of which but little notice is taken:1st. The number of insects in the lands, owing to the loss of rooks by felling so many rookeries, and not taking care of what are left. $2 \mathrm{ndly}$. 'The increase of mice, of which, were I to give my opinion as to quantity and damage done, but few would give credit to it. I have, at different times, had five mice killed to every coomb of corn moved off the stacks in the summer season, and sometimes double that quantity; besides being on every other part of the premises, corn and grass pieces not excepted. Some are driven into the barns and stacks in wet seasons, but when wheat stands long in the shock, we are sure to have most mice in our barns and stacks, except where they are driven away by other vermin. In my memory, there were twenty grey owls where there are now one; and though the country was in a rougher state, we had not so many mice. The owls prey very much on them, and in wet weather they are more exposed to the owl than any other kind of vermin. The grey owl is destroyed by the grame-keepers, and by felling the pollards. I have seen a young hare in their nest, but never saw a young pheasant or partridge. 'The white, or church-owl, is not so destructive to game; aud were there places made withinside the top of one end of every barn, like a box, for them to pass through as they come into the barn, they would there make their nests, and, becoming more numeruus, would be of great service."-Norfolk Rej. p. 531. 
leave a stab o't. There's mair nor a hunder thousand out the day.' This rather alarmed me; so I got several guns loaded, and gave all the men orders to shoot at them as soon as they alighted. The fun that followed was very amusing. Every workman rejoices in a job of this sort as a relaxation from labour. The shepherd left his flock, the thrasher his flail hanging over the barn-door, the ploughman left his plough standing in the furrow, 'to get a pluff at the cusha doos ; and there were as many sharpshooters thundering away as if the French were in the field. But the marksmen were bad, and the birds shy, and they generally escaped with life, though, by report, at the expense of a number of their feathers. At length a lad brought in two one day, and on opening their crops, which were crammed one would have thought to bursting, there was not a particle of anything else in them except the seeds of the runch, or wild nustard. I examined the contents of both with a microscope, and called in the servants to witness it. They were all obliged to acknowledge the fact; and forthwith a bill of emancipation was passed in favour of the cusha doos, which from that time have been free to come and go at their pleasure."

Notwithstanding this, however, pigeons-both tame and wild-are greedy devourers of corn, as well as very injurious to turnip crops by picking holes in the bulbs with their bills; and, if any reliance can be placed on the following statement, farmers are not wrong in driving them from their fields:- "They often fly to a great distance for their food, and when they can find corn to eat, seldom prey upon anything else. They begin to eat corn about the middle of July, and rarely want the same food at the stacks, in the straw-yards, or in the fields, until the end of barleysowing, which is about old Mar-dar, and which includes a period of 250 days, or better than three quarters of the year; the rest of the time they live upon the seeds of weeds and buntings."

"It is somewhere stated, that in Eugland and in Wales there are 20,000 dove-houses, averaging about 100 pair of old pigeons; but taking this estimate at only 1,125,000 pair of tame pigeons, these, to speak moderately, will consume-with what they carry home to their young-one pint of corn per pair daily; which for 140 days, being half the period they are supposed to subsist upon corn, amounts to $157,500,000$ pints, or $2,460,937 \frac{1}{2}$ Winchester bushels! besides the irreparable injury they commit in seeltime by picking up every grain of seed wherever they aliglit, and the quantity beaten out by their wings and trod under before harvest *' : a loss which, it is presumed, can never be repaid either by their own intrinsic value, or that of their dung.

\section{ANIMALS.}

Of all the four-footed animals included in the rank of vermin, rats and mice are the most pernicious; for they build their nests under the floors and in the roofs of barns, nor even are the stacks exempt; and are so prolific that, if not destroyed, they occasion incalculable mischief. It therefore behoves every farmer to use all possible means to check the evil, and one might suppose that every exertion was invariably made for that purpose; yet we constantly find homesteads overrun with these pests without any other pains being taken than an occasional rat-hunt by farm servants, aided by a terrier; which, though not to be neglected, is a very ineffectual remedy.

The best is, unquestionably, the construction of the barn-floor and roof,

*Vancouver's Survey of Devonshire, p. 3.57. Mr. Vancourer doubles the amount, but it is evidently through an error in calculation. 
which, if secured in the manner already stated in treating on that subject, will prevent them from obtaining a permanent harbour in the building *.. The next is, before the entire clearance of the barn, while yet a little corn remains to prevent them from quitting it, to close every part of the barn, by carefully covering any holes there may be with sacks and tarpaulins, so as to prevent all access of the outward air, leaving only the door for a few minutes open while the process is going on.

This done, some common iron chafling-dishes, which may be purchased for a trifle, should be placed upon the floor and in the bays; or, if they camnot be had, build up a few bricks, clay, or any rubbish that will secure a fire from spreading, leaving a cavity in the centre and filling it up with char coal. Then light the charcoal from the bottom, and, when the heaps are all burning, quickly strew a good qunntity of broken brimstone upon the top ; retire immediately, shut the door fast, and leave the building entirely closed during a couple of davs following. On opening it, the greater part of the rats and mice will be found dead around the charcoal ; and although some may have been suffocated within their holes, and, if not discovered, will occasion an unpleasant smell until their remains are dried up, it yet will not last long. The operation should be again repeated just previous to harrest; and if any opening be found into the barns while they are full, by the burrowing of the rats, brimstone matches should be inserted into them before they are stopped up.

Traps and poison are only partially efficient; for, independently of the objection which many persons entertain to the use of poison, in consequence of the numerous fatal accidents occasioned by it, the rats are so wary that they scent the persons employed in catching them; and, as they become acquainted with the traps, they shun them. The only effectual instance which we have heard of their complete destruction by trapping, is the mode detailed in a small pamphlet published some years ago by Mr. Benjamin Broad, of Thruxton, under the sanction of the Herefordshire Agricultural Society, which ought to be in the hands of every farmer in the kingtom. His plan, indeed, appears to have been in every case successful; and, having been employed by the Lords of the Admiralty, he completely cleared some ships of the line in which the process of professed rat-catchers had failed.

The leading ground-work of the plan is, that the operator, whenever he touches the traps, must overcome the natural odour of his body by rubbing his hands with the bait he employs; for the sagacity of the animal is so acute, and its sense of smelling so perfect, that any taint excites suspicion, and prevents them from indulging their natural appetite. The author has it seems ascertained that good pale malt, mixed with a very small quantity of the oil of carraways, is the most inviting bait that can be used +; though, previously, we have always heard that oil of rhodium was the most attractive. His traps are not very peculiar; but the whole detail of the operation, which is too long for insertion here, will amply repay the curiosity of the reader, even should he have no occasion to put it into effect.

The ferret is a decided enemy to the rat, and if kept in a cage, and only

* See vol. i., chap.v., on Farm-buildings, pp. 88 and 89.

$f$ "In preparing and setting the traps, take up successive portions of the malt in cach hand, and subseguently with both hauls, rulbing it between them, that every part of it may be equally scented. Much accuracy is here necessary; for if the quantity of the oil of carraways be too great, the rats will not touch the malt till the seent is partly gone off. The proportion of the oil of carraways to the malt appears to be about one to nine thousand." 
occasionally used, will be found very serviceable; but he should be well fed to induce him to return, or otherwise he will escape and becomedestructive to the poultry. A cat or two should also be always reared in the barn. It is, however, a mistake to suppose that cats live entirely on vermin; and, if not better maintained, they will have resort to the hen-house. They are objected to as occasioning filth among the corn; but if a hole be allowed them to permit their escape, and ashes be placed near the outlet, they will not be found offensive.

For keeping stacks clear of rats and mice, it has been recommended to

Take one pound of nitre and one pound of alum; dissolve them together in two parts of spring-water; get a firlot-or about a bushel-of bran, and make a mash thereof, putting in two pints of the above liquid, and mixing all together. When you build your stacks, every second course take a handful or two of the mash and throw upon them till they come to the easing.

This is said to be so effectual that they will never come near any stack prepared in that manner*; but we should ourselves place more faith in having the stacks built upon stone or iron staddles.

There are several different species of the field mouse, and they swarm in many parts of both arable and pasture land: in the forest of Dean they Jately destroyed large plantations, and were not eradicated until holes, baited with some attractive food, were bored in the ground to about twenty inches in depth, and wider at the bottom than at top, which preventing them from getting out, at length cleared the ground. 'The seeds of hemlock, spread in the corn-drills, will poison them; but it also has the same effect on any birds by which it is eaten. We have also heard that if the tops of last year's shoots of furze be chopped up small, and sown with the corn, it will prevent their depredations, which sometimes amount to a frightful excesst.

We have already mentioned, in our account of the pining in sheep, the remark made by the Ettrick Shepherd, of the cause of that distemper being attributed to the change of pasture occasioned by the destruction of moles: he, indeed, calls it "the most unnatural of all persecutions that ever was raised in a country against that innocent and blessed pioneer, who enriches our pastures annually with the first top-dressing, dug with great pains and labour from the fattest of the soil beneatl." Many other persons are of the same opinion; and it is said that the pasture of many upland farms on the Buccleugh estate was seriously injured by a bargain made between the late Duke and some Westmoreland men, about forty years ago, for the extirpation of the moles during the continuance of a term of fourteen years; and numberless instances in point have lately been brought forward in the Quarterly Journal of Agriculture $\ddagger$.

There can be no doubt " that the fresh earth brought to the surface by this animal, and spread, whether regularly by the farmer, or casually by the sheep, or even allowed to remain in the state in which it is thrown up, must tend to produce a greater variety, and we believe a better species of herbage;" and we can easily credit the accounts given of the injury expe-

* Farmer's Mag., vol. viii., p. 452.

+ In France, we learn that their ravages have, in some places, been so destructive as to occasion the ruin of the farmers. At Angerville, whole farms have been given up to the proprietors in consequence of their continued devastation, and the only method known of checking them is to defer the sowing any grain until spring; which precaution occasions them to forsake the fields, as it deprives them of the means of winter subsistence.

† See vol. i., p. 640 ; and vol. ii., p. 710 . 
rienced on old pasture by their loss. They, however, not only disfigure the appearance of pleasure-grounds and gardens, but they obstruct the process of the scythe in the season of mowing, and in many cases they occasion considerable damage to covered drains; and although we do not hold with the prejudice so generally entertained against them, they are not unjustly dreaded by farmers who have fine meadow land which they have been at the expense of draining, as well as by gardeners.

The most usual mode of destroying them is that of employing an experienced mole-catclier, and if he could be depended upon for doing the business effectually, it would seldom have occasion to be repeated; but, like the rat-catchers, they generally leave a few to breed, in order to insure their being called in again; and neither the farmer nor bis men can be supposed expert in the mode of trapping, even if they had time for the occupation. The animals, however, commence breeding about the latter end of May; and, as they make their nests both larger and deeper under ground than their common habitations, the spot can then be easily known by their greater size than those of the common mole-hill. It should, therefore, be marked; and shortly afterwards, when the old one may be thought safely housed with her young ones, they may be dug out. From five to seven will commonly be found in each nest. They are known to feed on enrthworms; and these, if chopped up with the poison of nux romica, and left in their paths, will, it is said, be eaten by them, and consequently prove fatal. The mole must, however, be more pressed than he usually appears to be by hunger ere lie will feed upon them in that state; for, when he catches the worm, he deliberately skins it with as much care and regularity as a fishwoman does an eel.

Should their destruction, however, not be desired, and yet their departure be deemed expedient, that object we learn may be attained by means of large slips of brown paper dipt in brimstone and put into the hills : the mode is to shovel off the fresh mole-hills, open the holes, and burn a match in each, keeping in the smoke. The consequence of this operation upon a field overrun with moles, was-as stated by the gentleman who relates it_." that all the moles left the field directly, and returned no more; but should they return in future, it is only repeating the experiment, and probably with the same effect*."

An extraordinary prejudice is also entertained against the hedge-hog, and he is persecuted by all ranks, as if he were a common nuisance. 'The absurd accusation of sucking the cows is universally brought against him; he is also charged with stealing chickens, game, and fruit of all descriptions, and is said to be very fond of eggs, in consequence of which he finds no favour in the eye of the game-keeper: whereas the fact is, that he lives chiefly upon insects with what little fruit he can pick up on the ground, is torpid during the winter, and is in all respects a most harmless creature. If kept in a garden, he will in a great measure clear it of worms, without occasioning any damage, and he ought never to be destroyed.

The question has often been mooted, "whether every animal was not created with a beneficent intent, and placed by Providence for some wise purpose under the protection of man?" This, we have no intention of discussing, for we conceive that every man, whatever may be his individual opinion on the subject, has a just right to guard his property from their devastations; yet, beyond that, the expediency of their further destruction may, we think, well be doubted. 


\section{Chapter XlV.}

\section{ON COTTAGE ECONOMY-COTTAGE GARDENS-BEES-ALLOTMENTS OF LAND-SPADE HUSBANDRY.}

In treating of this subject-a subject of the most vital importance to our rural population-one cannot help adverting to the late $\mathrm{Mr}$. Cobbett's celebrated tract on this topic. That pithy writer drew a strong picture of what the peasantry were in his own early days, and what he wished labourers to be at the time he wrote. It was a picture of what had passed away, rather than a representation of what was still in the labourer's power to do for himself under the great changes which, during the period alluded to, had taken place in the condition, the manner of living, and the moral habits of the rural population. 'The old English fare of farm servants and cottagers is, over a great part of the kinglom, no longer partaken of: the foreign articles of tea, coffee, sugar, \&c., have banished milk and ciler, and beer, from these private divellings; and the domestic duties of brewing and baking are superseded by swarms of public bakers and brewers, who supply these necessaries, and bring them to the loors of the consumers. 'This convenient supply of necessaries has banished the brewing utensils and the ovens from every cottage and almost from every farm-house. These changes, which take place in such a mixed society of mercantile, manufacturing, and agricultural classes, which compose the population of this country, are not the results of design; - they happen in the course of circumstances, which have a relative bearing on each other, and according as convenience, or indolence, or inclination may prompt.

A very general want of economy, it must be confessed, took place during the war among agricultural labourers, when their wages were so much advanced: their domestic and orlerly habits were changed into a kind of recklessness, from which they are now far from being exempt. In this they were encouraged by the state of the poor laws, which certainly made many able-bodied men idle or unemployed paupers. A very general pressure of distress was the consequence of the reduction of wages after the war; and benevolence was on the rack to devise means by which the rural labourer might, by his own exertions, benefit himself, and keep him inde. pendent of the overseers' funds.

Mr. Cobbett's excellent advice to that class of the community, as to the great saving and comfort of baking their own bread and brewing their own beer, they could not generally avail themselves of, because of the want of vessels, which they were unable to buy, and for the want of ovens, which they could not afford to build. Their poverty prevented them buying a cow, even if they could hire pasturage for her; and their employers always disapproved of their keeping pigs, as they were commonly allowed to prowl about the lanes and destroy the farm-fences; besides their engendering, in many of their owners, a system of pilfering for their support. Mr. Cobbett's advice, therefore, went but a little way in ameliorating the condition of farm-labourers.

It has indeed been intimated to us, that it would not be economical in the cottacrer to brew or to bake; for, "in procuring anything that alministers to his necessities, man makes an exchange of his labour for the thing produced, and the less he gives for his labour the better of course is his bar- 
gain*;" and, "as a working man would gain more in wages than he could save in the price of bread and beer, the occupation would be an unprofitable waste of time." To the justice of this general reasoning we give unqualified assent; but it does not apply to the present case; for the work would be committed-as it invariably was in former times-to the wife, who lost no wages by the employment, as she seldom earns any in money, and the saving to her family of the baker's and brewer's profit would be clear gains. Could the cottagrer brew his own beer, he would aiso drint it in domestic comfort by his own fire-side, without being driven to the ale house; and in both these instances it appears to us that the change is for the worse.

Another great change took place among farm-servants during the perior alluded to by the author of "Cottage Economy,"-namely, the custom of boarding the men-servants out of the house. This was brought about, in most instances, more from the desire of the men to have the command of their own money in purchasing their foor, than it was that of either their masters or mistresses to irive them from their tables. 'This change has injured both parties: the masters have neither so much attention or lime from such servants; nor are the servants so orderly in their conduct, in conseguence of being allowed to go to the village for every article they ma want, where they form alliances often ending in improvident marriages, which entails poverty as their lot for life; as well as labits of drunkenness, poaching, and other acts of demoralization contracted in the village beer-shop.

By far the greater number of farm-servants are now-a-days married men, at weekly wages regulated by the price of bread; and who, with a boy or two, who sleep in the farm-house, do all the principal and regular work of the f.rm. Their condition has been considerably improved within these last few years, by many patriotic landlords and farmers allotting to each cottager a piece of garden-ground; and has had the best effects, not only in supplying the cottager's family with plenty of wholesome vegetables, but employing himself and children in their leisure hours at home. The possession of a bit of land gives the owner a stake in the country: it is a source of amusement as well as profit, and, in short, makes his home much more attractive than it otherwise would be. The quantity should be just so much as will occupy his hours of leisure with the assistance of his family, if he has one, and no more. "The British Almanac" for the years 1834 and 1837 , contained a paper on the subject of cottage gardening, which is here subjoined; but as this is necessarily a very brief manual, it is requisite in this place to add somewhat more on the general subject.

\section{THE COTTAGER'S GARDEN.}

"Many, however well inclined to have a garden, do not know the best mode of setting about cropping and managing it, and are ignorant of the best sorts of seed to sow, and the time and mode of sowing. We lave griven a plan which contains about a quarter of an acre; and those who have gardens of half this size will be able to manage them by this plan, only using half the space for each crop, or leaving out some crops that are less profitable for those of potatoes and cabbages, which are the most productive and useful. Suppose, therefore, a piece of ground 51 yards long by 24 wide, which is about a quarter of an acre, and is represented by the ylan, lay out a border ail round, four feet wide, Nos. 9, 10, and 11 of the 
plan, and this border may be kept for the smaller vegetables, pot-herbs, \&c., leaving the middle of the plot for the larger crops; next to the border make a walk all round of two feet six inches; then divide the middle into beds as shown in the plan.

"The first thing necessary is to drain the ground; without draining, unless the soil is very light indeed, your garden will never prosper. The stagnant water in the winter, autumn, and spriog, rots the roots of plants, and kills the seeds, and the soil is rendered less fertile by the constantly soaking 'wet. Cut some drains slanting across the ground into a ditch on the outside, if there is one, and fill up jart of the drains with bushes and loose stones; but, if there is no ditch, dig out the walk pretty deep and fill in the bottom with stones, broken bricks, and dry rubbish and bushes. This draining is one of the main points; and next to draining comes trenching -and trenching deeply. Nothing improves ground so much as working it; begin by trenching (if the soil admits of it) three spits decp. This, however, cannot of course be all done in one year, as it would take too much time, but it may be done by degrees: after it has been trenched three spits for one crop, then a simple digging will be enough for the second crop, and for the third a digging of two spits will, for the three crops, always give a fresh surface, which is a matter of great importance in growing fine vegctables; draining and trenching are of more consequence even than manure, as those will find who try the experiment.

"Of course manure is not to be neglected when it can be got, and it may be procured from many sources which are not sufficiently attended to; wood ashes will do something; the rotten leaves, stalks, \&c., not eaten by the pig, are excellent manure. Soap-water of the wash-tub again is of value; the scouring out of the ditch is good manure; and a few barrows of turf sods got from the common or any neighbouring bank, chopped up and dug in green, will be as valuable as a load of dung ; the scrapings of roads, if the soil is heavy, are also excelient, and much assist in lightening the ground.

"The following is the plan of the garden we have noticed, and it is laid out in 11 different beds; the walk round it is numbered $12:-$

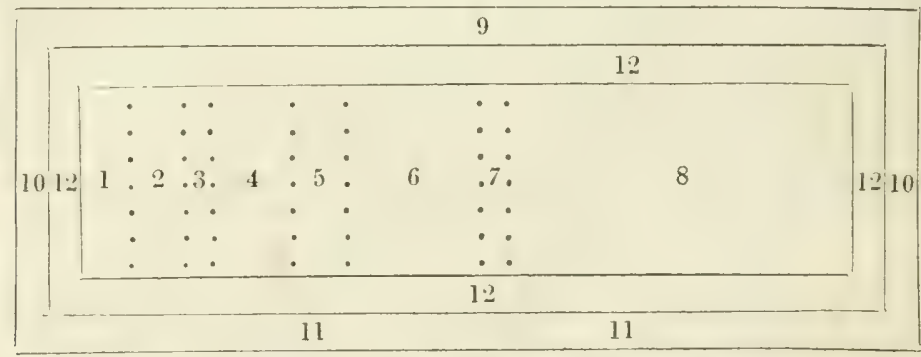

"We shall now state what will be the best mode of cropping this; viz.-

"Bed, No. 1 in the plan-Onions, spring crop; sown beginning of March.

"No. 2.-Long orange carrot, spring crop"; sown in the middle of March.

"No. 3.-Parsnips, spring crop. This ground, with the onion and carrot beds, to be planted in the autumn with large early York cabbages, sown about the lst of August.

"No.4.-Cabbages sown about the end of February; winter, fallow.

"No.5.-Scarlet runuers, sown at the end of April; winter, fallow.

"No.6.-Peas and beans, sown in February till May. The ground to be after- 
wards cleared, manured, dug, and planted with cabbage colewort, sown from the begimning to the end of June.

"Bed, No. 7.-Celery, sown in the middle of March, will remain all winter. The ground in spring to be sown with peas.

"No. 8. - Yotatoes, planted in the middle of April, winter cropped with savoys and borecale, Scotch-kale, and turnips.

"No. 9.-Potatoes and radishes, spring crop. 'To be sown and planted with different kinds of lettuce as the potatoes are taken up.

"No. 10.-Turnips; the first part to be sown with early stome, about the 1st of April. The second about the lst of May.

"No. 11.-Kidney beans to be planted from the middle of April till June.

"No. 12 is a walk.

"It will be seen that, by following this method of cropping, the greater part of the ground will bear two crops in a year. Several things may occur to prevent the directions given in the plan from being followed exactly ; for instance, carrots will not come to any kind of perfection on a clayey soil; if there is any portion of light soil in the garden, it ought to be chosen for them. No good crops can be expected without the earth is well dug, and often moved about whilst the plants are growing, to let in the moisture and warmth, and loosen the earth, that the roots may grow. Nothing is said about weeding-wherever weeds are allowed to grow, they must of course grow in the place of the crops, and there cannot be weeds and good crops together."

Notwithstanding the business of grardening, whether belonging to a palace or a cottage, is governed by a set of rules, deduced from experience, regulating the operations as to the times and manner of performance; and though, by attending to them, the cottager camnot do much amiss, yet it should be understood that a good gardener is never bound exactly to rules; discretion must often be exercised. There are many particulars which, if known and attended to, augment considerably the annual produce of any given spot of ground. This is accomplished by studying well the nature of the cropshow they may succeed each otlier, and by taking care that no ground be suffered to remain unnecessarily vacant in the growing season. As soon as one crop is nearly fit for use another should be in readiness to take its place. All vegretables that may be used at any stage of their growth, as cabbage, lettuce, onions, \&c., may be sown or planted in drills, on ground intended for other crops ; for as soun as the first begins to encroach injuriously on the second, it may be drawn for use. For instance, the ground intended to be sown with peas or beans, in the spring, may be digged in the autumn, and immediately planted with coleworts, in double rows, at such distances as will admit beans to be dibuled, or peas drilled between, in January and February. So, also, before bean or pea haulm is cleared away, in summer, the spaces between the rows may be digged and planted with cabbage, or any other winter crop. By such processes, together with the facilities afforded by the row culture of putting in intermediate crops, much more may be male of a limited piece of ground than can possibly be raised where broad-casting seeds prevails. It is true that onions, radish, and lettuce are broad-casted together; and, if attention be paid to draw away the radish and lettuce in time, the onions suffer no injury. This, though allowed in cottage gardening, is suitable but for few kinds of vegetables; and is not to be generally recommended, except when confined for want of room.

Although the cottager may amuse himself by cultivating a variety of vegetables, he must not neglect the more useful kinds, for they will require unceasing attention; and the potato, cabbagre, savoy, onion, carrot, pars- 
nip, broad-beans, and scarlet-rummers, demand his chief care: peas require more space than their produce is worth.

Next to stocking his garden with the most useful kinds of vegetables, his utmost endeavours will be required to keep it in good heart. Success depends chiefly on this; and therefore he must collect every particle of vegetable or animal matter, in a holluw place between lis house and garden, to receive every kind of drainage from the former, and all refuse trom the latter; also all sorts of litter and cattle droppings from the public lanes, roads, and commons; which, when thrown torgether, and turned once or twice till sufficiently rotten, will be found excellent manure. Ashes, lime, rubbish, marl, or clialk, may also be added, and mixed in the compost.

TFe shall now proceed to describe the best and easiest modes of culture of all the most suitable vegetables for a cottage-garden, and in such plain terms as that the directions cannot easily be misunderstood.

The potato we notice first, because it is certainly the most useful and profitable of all others to the cottager. It is most successfully cultivated on well-broken and deeply-digged ground, in rows two and a half or three feet asumler, in order that the stems may be earthed-up as highly as is necessary. The tuber's, as they are called by gardeners, are the eatable parts. and may be planted whole, if small; but they are usually divided into sets, each containing at least one eye-rejecting the root-eye and the one next to it. The sets should be cut three weeks or a month before the plantingceason, which is at any time between the 20th of March and end of April. Planting, however, may be continued throurhout IIay and June; but the increase from late plantings is always small and inferior in quality. The soil can scarcely be too rich, provided it be dry and light enough.

'The proper depth at which the sets should be planted in the ground, at seven inches apart, should be at least six or eight inches, it being found that shallow planting is unprofitable. Soon as the stems appear abore rround, they should be frequently hoed among to keep down weeds, and the surface open; and, when they lave risen six or eight inches high, the loose earth should be drawn towards them, so that the plants may stand in a ridge of loose earth to encourare the swelling of the tubers on each side. The flowers should be pluckerl off, so that no apples be ailowed to form. When the tops wither, the crop is tit to be taken up.

livery cottager should endeavour to procure the best, or rather the most profitable sort, and, if possible, from where the soil is of a different description from his own. The same sort of potato does not thrive long in the same district; and, therefore, new sorts, or a change of sets, is necessary. The Chumpion, or Early Shaw, or any similar new sorts, should be preferred, because their increase is greater, and they are less suhject to the curl or other disease; but as a general rule, whichever sort succeeds best in any given neighbourhood, that kind should have the preference.

The potato is a winter vegetable, and should only be so considered by the cottager. If raised for use in June and July, he will find them an expensive luxury; the cottager's winter store of this useful vegretable shiruld therefore be carefully secured. When the haulm is nearly dead, fix on a dry time to raise the crop : let them lie on the surface till perfectly dry and free from earth, and then let them be pitted or housed on a dry spot, among perfectly dry straw, and safely covered from wet or frost. The quantity of crop will always be found in some degree proportionate to the size of the sets, on the rich dryness of the soil, and on the culture they have received during their growth. If the latter end of spring and summer 
be showery, and followed by a dry autumn, the crops are generally large and good; but in dry summers, succeeded by a wet autumn, the crops are inferior both as to quantity and quality, unless planted on very moist soils : and should a cottager's garden chance to be bog-earthy, or otherwise very moist, he ought to grow his potatoes on what are called lazy-beds. The method is this:-the ground is first marked off into parallel beds, five feet wide, with iwo-feet alleys between; the beds are covered with a coat of half-rotten dung; on this the sets are placed in cross-rows, fifteen inches asunder, and covered over with soil, three inches thick, digged from the alleys. As the stems advance in height, they are liept earthed up from time to time till the alleys are two feet deep or more. The alleys acting as drains, leave the beds and crops high and dry; and the produce is generally abundant.

Next in importance to the potato, in a cottage-garden, is the cabbage; and as they are useful in every stacre of their growth, they should always be to be had in one shape or other. Every vacant part of the ground, and the allevs between other crops, should be dibbled full of them; and if they uccupy groimd wanted for other crops, they have only to be drawn for use. The principal crop of spring cabbare is sown between the 27 th of $\mathrm{July}$ and the sth of August. If sown before the first of these dates, many of the plants run to seed withont forming hearts; and if after the second date, they do not arrive at full perfection early enough. The summer and autumn crops are raised from seed sown in February; and in order to have a gooul stock of coleworls, - that is, open cabbare or plants as they are commonly called, - another seed-bed should be sown about the end of June for autumn planting.

The seed-beds should be on a dry, rich border, and none of them need be more than about three square yards sowed not too thick. When the seedlings appear they must be guarded from birds, snails, and slugs, by being dusted with fresh slaked lime, or watered with lime-water. Soon as the plants are large enough, all the strongest should be pricked out on a fresh piece of ground to gain strength before ther are planted out for good in the month of October. But as this is a busy time on the farm, every hour of daylight being bespoke, it is only on wet mornings and evenings that the cottager has any time for gardening. II should, therefore, previously get ready a part of his cabbacre-ground, and on that prick out his plants in rows nine inches apart, and four inches from each other in the row. These will be going on; and when time allows, and more ground got ready, those remaining may be transplanted into more open order, and where they are to remain. In this way the plants are much forwarded, and come carly into use. The summer and autumn crops should be treated in the same manner, if time can be had; and, during their growth, should be often loed among and earthed-up, as they require. In using cabbage, cut one row, whether great or small, before another is begun; this gives a portion of ground to be digged for another crop. The early Battersea is the best sort for a cottager.

The scarlet runner kidney-bean is, from its great prolificacy, one of the most useful cottage-garden plants. As a summer vegetable it has no equal, nor can such a constant and ample supply be had from any otiser kind; and, what is remarkable, the more the pods are gathered, the more the runner produces. If allowed to ripen seed,-which are generally the first ten or twelve pods at bottom, - the plant soon ccases to bear; but supposing all circumstances of soil, situation, and particularly abundant moisture be afforded, the plants will bear until they are killed by frost. Nor 
are they nice as to their station; any open spot where two or three may stand together against a wall or paling, or hedge; they may also be trained to form a profitable and shady porch at the back or front door, or against any other building. Objections are sometimes made to their climbing liabit, as requiring high rods or sticks; but these, though most convenient, are not absulutely necessary, as very good crops are produced on a very low rank of sticks put in cross-ways, or with both ends inserted in the ground.

As early crops are killed by a very slight night-frost in the beginning of May, the cottager should not venture his seed before the tenth day of the month. The ground should be dry and somewhat warmed by the sun before sowing; but as they transplant well, the seed may be put in thinly on a small bed on a dry spot about the 20th of April, and hooped over to be covered if there be signs of frost. These nay be transplanted to any other place about the middle of May, and when ground occupied by some other crop may be by that time got off. Runners are liable to suffer from drought during August and September, they then require copious waterings.

The onion is one of the cottager's most favourite vegetables; it gives a relish to even a bit of dry bread, and is a seasoning to every kind of food which falls to his share. Onions are useful in every stage of their growth, and therefore a constant supply should be provided. Two principal sowings should be made in course of the year,-viz, about the 15th of August for the winter crop, to be chiefly drawn young in the spring; and again about the middle of March for the sumner crop, to grow to full size. For the autumn-sown crop a bed four feet wide across the middle plot of the garden will be enough. Sow pretty thickly, as they protect each other, and are rarely killed by frost. 'They will soon be useful in the spring, and may then be regularly thinned or drawn to be transplanted on another bed, where they may stand to bulb. Many of this sowing run to seed, but still these are useful, and those that do not run grow to a good size.

Spring-sown onions require good and well-prepared ground: they should be sown rather thinly; the seed well trodden in, and slightly covered with a little loose earth from the alleys. During their growth, they require to be kept free from weeds, and to be thimned to four-inch distances from each other. When full-grown, and the tops begin to change colour, bend down the stems to check the growth; and when this happens, pull, and, unless the weather be rainy, leave them on the bare ground for a week or ten days to wither and be ready for roping. Onions bulb better on well-trodden soil, than if it were loose; the fibres or roots only should be in the ground, the stem should swell entirely in the air. The Strasburg and White Spanish sorts are the best for the cottager. There are many varieties, and many different ways of growing them; but neither the kinds nor the prac tice are of any superior value in a small garden*.

The carrot is a useful vegetable, and particularly so as it can be stored for winter use like the potato, and yields good crops if the soil be light or sandy. Sow at any time between the 20th of March and 15th of April. The Orange or Altringham are to be preferred. The ground should be trenched, or double-digged, to grow them fine and large; and if the soil be clayey or heavy, they come to little perfection unless rows of holes are made by line with a long taper dibber, and filled with light rich earth: on the surface of each hole two or three good seeds are dropped, and lightly

* The sprouting of onions during winter may, it is said, be prevented by applying a heated iron for a few seconds to the nosle of the onion whence the root thrusts itself forward, and will be an effectual mode of preserving them.-Gardener's Magaziue. 
covered; one plant only is left to each hole, and which naturally takes a good shape from the facility with which the descending root penetrates the loose earth. Where the soil is naturally light and porous, or made so by careful digging, this scheme is unnecessary. When carrots are sown broadcast, they must be thinned by hand or hoe, leaving them four or five inches apart. They should be raised in October; topped and tailed, and laid away among dry earth or sand, out of reach of wet or frost. 'The seed requires to be well rubbed between the hands before sowing, otherwise they hang together, and cannot be sown regularly.

The parsnip is also a wholesome and useful root in a cottager's family. The culture bestowed on the carrot suits them exactly, as well as time of sowing, summer management, and time of taking up; but they require a rather stronger soil, and are an uncertain crop.

As the turnip is so extensively cultivated on every farm, the cottager need not take up much of his garden with them. One small sowing, of the Early Dutch, made about the middle of April, will suffice, as the ground they grow on will be wanted for some other crop about midsummer.

The Long-pod and Windsor common bean are the only sorts worth the cottager's attention, as they are by no means a profitable crop, unless they are grown between rows of cabbage or some more valuable vegetable. 'They should be dibbled on ground digged a month or two previous, in order that it may be firmly settled down before the beans are planted in January, or as soon afterwards as possible. They should be topped early, and cleared off as soon as the pods are gathered*. Peas, as already stated, are scarcely worth the ground they occupy: one row, sowed in February, of Kinght's Marrowfat, placed very thinly (two inches apart in the row), is all the cottager should attempt to raise.

Savoy cabbage and Scotch kale are fine greens, hardy and particularly useful in winter. A thimbleful of seed of each should be sown in April, and planted out in rows during summer; dibbling them in the alleys, or between the rows of other crops which come off early in autumn.

There are but few cottagers who do not try to have a bed of early shorttop radish; for a bunch of these, with another of winter onions, are pleasant additions to bread and cheese throughout the spring. A warm dry border, laid sloping to the sum, should be got ready about Christmas, and divided into four-feet beds; on these the seed is sown pretty thick, covered with loose earth from the alleys, and patted smootlly down with the back of the spade. The beds must be immediately covered with dry fern, or any short litter, which keeps off frost and brings the plants up sooner. On every mild day the covering is moved off the beds into the alleys, and carefully drawn on again at night ; and thus continued until all fear of hard frost is over. The radish will be fit to draw about the end of March, and then will be most acceptable, as well as perhaps profitable, to the owner.

$\Lambda$ few seeds of lettuce may be sprinkled among onions, or sown on a small bed alone, whence they may be transplanted into any vacant spot, round the edges of beds, or along those of borders, where they may grow to maturity without injury to other crops. The white, and spotted coss

* It has been stated by Mr. Falkland, in the Gardener's and Forester's Recurd, that he uniformly bends down his first crop of mazagan and early loug-pod beans, when they are in full blossom, so as to break the stalk near the root; yet they produce abundantly. They then, in about three weeks afterwards, push forth from near the root one cr more, and in some instances four to six stems, which bloom freely, and afterwards produce a second crop; by which means he obtains two sucessive crops, furmishing a contiuued supply from July to the end of October. No, iii.

VOL. II. 
sorts are the best for the cottager's purpose: to him the cabbage kinds are worthless.

Potherbs are necessary in a labourer's garden, and a small border should be appropriated to their growth. In this, single plants of mint, sage, baulm, marjoram, thyme, rue, may be planted, and a row or two of parsley should be sown in the autumn of every year; also a row of leeks. If a tuft of lavender be added it will be found useful, as a scent, among the linen.

The culture of cauliflower, brocoli, asparagus, artichokes, celery, endive, \&c., will be noticed hereafter in the garden of the farm-house. Meantime we must recommend to the cottager a most useful vegetable-namely, rhubarb. Of this he should possess himself of at least half-a-dozen plants, put in, two feet apart, in any bye corner of the garden. The leaf-stalks from these will afford an agreeable addition, in the shape of a pudding or pie, to the Sunday's dimner, or made into dumplings to carry a-field: they being as useful, and perhaps even more wholesome, than the best baking apples.

Growing fruit ought not to be an object with the cottager: trees only damage more valuable crops. But if a single apple-tree be wished for, let it he a half-standard Hawthornden Pipjin. A hedge of gooseberry-bushes, and a few currant-trees, - particularly of the black sort,-may be planted and trained across the house nearest the garden, but nowhere else. The fruit from these will be useful in the family for either boiling, baking, or bottling.

\section{ON BEES.}

Closely connected with economical gardening is the keeping and management of bees; the anmual profits of a well-managed stock of which are so certain, and so considerable, that it behoves every cottager to keep as many as will at least pay the rent of his cottage. It is matter of astonishment that so few are found in the possession of labourers, seeing that their first and subsequent cost is so trifling, and their profits so great. Mr. Cobbett estimates the value of the honey made by a good early swarm to be worth two bushels of wheat, which, at the present price of that grain, would vary from about fifteen to twenty shillings. To have, therefore, four or five hives to take every year, would be of great moment to a needy man.

A bee-house of two or three shelves is the most convenient stance for a stock of hives. The back, ends, and roof should be weather-hoarded; the back, down to the ground, so that no draught of air pass through beneath. It should be placed in some convenient corner of the garden, and facing the south. Bees like quiet, being easily anmoyed by passengers, pigs, or. poultry : no trees or bushes should be very near the front of the hives, as these obstruct their flight lome. They are fond of sea-water, and will fly a great distance to obtain it. They drink frequently, and should have, in a dry country, water in a shallow vessel set near the hives; many lose their lives in endeavouring to drink out of deep vessels.

A full, healthy hive will throw off two, sometimes three swarms in the course of the summer. The first swarms are always most valuable, because they have full time to collect a stock of honey, which, when taken, is called, or at least sold for, virgin honey-late swarms often require to be fed in the winter. A second swarm, if it be found well filled at the end of autumn, may stand over to be taken at the end of the second year, - they seldom do well if kept longer. Sometimes the first swarm throws of one from itself: this is called a cast, and the honey of this is the true virgin honey; but it 
is only in very favourable seasons that a cast can collect honey enough to carry the colony through the winter without feeding.

Swarms should always be housed in new hives. The insects are easily offended by the musty scent of the old hive; and it costs them much labour to close all the crevices of an old or badly made hive, which is one of their first duties on taking possession of a new house.

When bees are about to swarm, they require some watching, lest they fly away. The first indication of this emigration is that of large numbers of the supernumerary bees clustering together on the outside near the entrance of the hive, and hanging under the threshold. If they so appear for several consecutive days, it is very probable that, on some fine, calm, warm morning, with bright sunshine, the emigrating colony will take its departure from the old hive. Sume swarms rise high in the air, and fly to a great distance before they settle, and sometimes in places where they cannot be recovered. The only means that can be used to prevent a distant flight, is to throw handfuls of dust among them, or by causing a violent vibration in the air by the continued beating of some loud-sounding metal vessel-a fryingpan, or the like. The dust, or agitation of the air, alarms the leaders of the throng, and causes them to settle on the nearest tree or bush, or sometirnes on the surface of the ground.

Whether the young queen leads her followers, or some of these lead her, is not, perhaps, well understood; certain it is, however, that though the generality of swarms seem to alight anywhere by chance, yet on some occasions a resting-place is previously chosen, and taken possession of by the new swarm. This circumstance is mentioned in order to induce beemasters to try to fiul out a lure for vagrant swarms. They do sometimes adopt a prepared hive set conveniently for them, and they may be induced to do this more frequently.

When a swarm has settled, it should be hived as soon as possible, for they very soon begin to work, even on a branch of a tree. The hive is prepared by having an entrance cut, and two clean peeled willow rods, stuck firmly crosswise from front to back, and from side to side of the hive; the inside is rubbed with a bunch of elder-leaves dipped in sugared beer. If the swarm has settled upon a branch of a low bush, the branch mav be bent down by a hook to the ground-the hive put over, and partly covered with a mat or cloth; and after lark the hive, if the bees have gone up,carried to the bench. If they settle on the branch of a tree, the branch may be cut off and brought down to the ground; or, if this cannot be done, the hive may be held under, and the bees shook or gently brushed into it. At these times the bees are in a stupor, and not irritable unless they are hurt. It is well, however, to have a crape over the face in hiving bees.

When set on the bench, it will be seen next dav whether all is right. If the bees keep to the hive and begin to work, it is a favourahle sign; but if many appear restless, flying backwards and forwards between the hive and the place they swarmed to, it is a sign that they have lost their queen, and very likely she may be fourd surrounded by a party in some intermediate place; to this place the hive must be brought and placed over, when the whole will soon get together.

Some writers advise honey-bearing flowers to be sown or planted near the bee-house, hut this is unnecessary; the wild flowers on the heath or common afford the finest honey. Mr. Cobbett has made a great mistake in recommending the flowers of buckwheat for bees. 'They are certainly fond of them; but the fact is, these flowers yield the very worst honey-being so dark-coloured as scarcely to be saleable, and containing so much of the 
noxious spirit of that plant, that many people, and particularly children, have been disordered by the incautious use of it.

The hives should not only be sheltered from the north or cold quarter, but they should be kept shaded during the heat of summer. This is best done by having a straw capping for each hive: this is warmest in winter, and coolest in summer. They should also be guarded against the attacks of mice, moths, earwigs, and other insects which sometimes make inroads upon them. Contracting their door-way in winter is always necessary.

It is unnecessary to lengthen this section, by stating the various economical purposes to which the products of bees may be applied in a family. Enough has already been advanced to induce every cottager to avail himself of the advantages of bees, which, to possess, requires but a small sacrifice at first, and but very little care afterwards.

We subjoin twelve calendarial notices, which may be useful in the management of a small garden, as well as of the bees which may be kept in it.

JANUARY.-The weather is generally too wet and uncertain this month for doing much in the garlen. The cottager should now fix on his plans for next year, and having ascertained the vegetables most useful to himself and family, and most suitable for his soil and situation, ought to make preparations accordingly. He must consider that radish, peas, and beans must be sown this month, and the ground should be got ready for them.

The bees should not be disturbed, but kept warm and dry. If any light hive require feeding, this must be attended to; and here it will be necessary to say how their food is compounded-viz., put to a pint of ale one lb. of sugar and half an oz. of salt: boil the whole together, and skim off what rises on the surface. When cold it will have the consistency of honey. This food is best administered in a shallow wooden trough, having a thin lid pierced with small holes, throngh which the bees sip their food "without being soiled with it.

February.-Prepare ground for putting in a few more long-pod beans, and for planting a few more rows of cabbage-plants from the seed-bed, if any still remian. Move the earth between the rows of those planted in October, and fill up blanks. Sow a small bed of coss lettuce on a warm spot, and a drill of parsley, if wanted.

Bees.-Continue to feed them: this will not make them lazy, but keep them from roaming abroad too early, when they would be far better at home. Feed them at night, and remove the troughs in the morning, lest the neighbours' bees, which are not fed, visit your hives. The warmer the bees are kept at this season the better; it "hastens the hatchings of the egg, and promotes the prolificacy of the queen."

MARCH.-This month being one of the busiest in the whole year, it behoves the manager to be looking forward, by "taking time by the forelock." The different portions of the garden must be got ready to receive succession-crops of broad beans and marrowfat peas, together with that for potatues, onions, carrots, parsnips, summer-spinach, leeks, \&c. New plantations of delicate plants may be made, and salad and potherbs sown. Transplant onions from the winter-crop.

The bees begin to be in motion on warm days; but they must be fed, lest hunger drives them from home.

ApriL.-Execute what was recommended last month: every spare hour should be employed in getting in the different crops. Potatoes, occupying the largest plot of the garden, cannot be all planted at once; but a row or two at a time, as opportunity offers, may be set, till the whole is 
finished. If all are got in before the end of this month, they will do well. Draw earth to peas and beans, and begin to prepare ground for the reception of kidney-beans, and the seeds of cabbage, savoys, cauliflower, and brocoli, in small beds; together with a small patch of Dutch turnip.

Bees.-Provide hives for expected swarms, as they should always be at hand when wanted. This is consideres a good time to purchase stock hives: their having survived the winter is a guarantee that they will continue to thrive.

$\mathrm{M}_{\mathrm{Ax}}$.-The performance of what has been suggested in the two preceding months, will probably occupy part of this also. Towards the end, get in both sorts of kidney beans-that is, both dwarfs and runners; of the former, the Canterbury and speckled are the best. Transplant lettuce, and destroy weeds wherever they appear.

Bees.-The drones-that is, the male bees-begin to appear in this month, which, if they do, it is a good sign, as " early drones denote early swarms."

JunE.-The care of the growing crops forms the principal part of the garden business in this and the next month. Hoeing among, earthing-up, and thinning such as stand too thickly, is necessary. Stick peas, and sow again kidney-beans and runners to succeed those sown last month Cucumbers, pumpkins, and vegretable marrow may be sown or planted towards the end.

This is a busy month with the bees; many drones will now be visiblea proof of a thriving hive. When they begin to remain out all night round the entrance of the hive, it is a sign they are near swarming; and therefore, on warm forenoons, require looking to.

JULY.-Before hay-time begins, get every vacant spot of ground digged and ready for planting out cabbage, savoys, brocoli, cauliflower, and all sorts of winter greens: these may be dibbed-in between other crops, or any vacant spots; and wet mornings or evenings are the only times which, at this season, offer for doing this necessary work. If any celery is to be grown, now is the time for preparing the trenches

Bees.-The second swarms from the bees are thrown off in this month, and soon after virgin swarms may be expected. Should the bees of any hive lie out at this time, it shows they want more room : this is given by adding what is called an eke to the bottom, by which the capacity of the hive is enlarged, and the bees again set to work to fill it. This is the best time for beginners to purchase and remove first swarms. The first swarms always begin their combs in the middle of the hive, while second swarms begin at one of the sides.

August. - The business of a garden in this month is similar to that of the last. Towards the end, the principal crop of onions may be pulled, and the space obtained for some other winter or spring crop. Ground must be got ready for sowing spinach, cabbage, onions, radish, and lettuce of sorts ; and cauliflower, about the 22 nd of this month.

Bees.-Swarming ceases in this month; and the chief care of the hive is to see that no robbers, wasps, or strange bees assail, and pilfer from the rightful owners. The drones are often turned out or killed; and most value is set on those hives which are the first to get rid of their drones, because it is a sign that their stock of honey is nearly complete.

September. - Notwithstanding the hurry and pressure of field business in the last month, the labourer must steal an hour, if it be even by moonlight, to sow his winter onions, and by all means his spring cabbage: these cannot 
be delayed without disappointment and loss. Some labourers may say, "Oh! we can buy a hundred or two ;" but, surely, it is better to have a few hundreds to sell than one to buy. All sorts of winter greens may still be stuck in on every vacant spot: they are sure to come into use at sume time or other.

Bees. - Do not think of taking up the hives in this month: they are still increasing in weight, and will continue to increase till the flowers of the wild heath decay.

Oстовеr. - Care of the growing crops, by earthing-up those which require it; clearing off all decayed stalks and leaves, and preparing ground for the reception of spring cabbage, are the principal matters to be attended to in the garden at this season. Gather ripe seeds, and dry them thoroughly before storing them away.

Bees.-This is the honey-harvest, and the possessor of bees is now realizing the profits derisable from them. The store-hives are first selected, and the others are "put down" in the usual way by suffocating them over a pit with the fumes of brimstone.

This act is shocking to humane minds, and a cruel return for the industry of the insects; hut it is less cruel, perhaps, than allowing them to die a lingrering death in old age; when, on leaving the hive in the morning, they are unable to return, and so drop and perish in the cold air. Many plans are in use for saving the lives of the bees: it is a remission of the cruel sen. tence of instant extirpation, but it is only a change of suffering; and $n 0$ profit results from preserving them alive.

NovenBer. - Planting the next rear's spring crop of cabbage on the ground prepared for them as alvised last month, is one material affair ; and taking up the crops of potatoes, carrots and parsnips, for storing, is another. Finish moulding-up celery, blanch endive, hoe out winter spinach, and draw earth to the stems of rowed crops. The first sowings of carly peas and Mazagan beans are usually made towards the end of this munti. Dry and warm situations must be chosen.

Bees. - The hives should be weighed in this month; and, after an allowance is made for the weight of the hive and the bees, an estimate may be male of the actual quantity of honey. If heavy, they will go through the winter well; if light, they will require feeding with the syrup before recommended. The hives should be well covered, lest rain or snow beat upon them: dryness is as necessary as warmth.

DECEMBER.-Trench, or rough-dig all vacant ground: if wet and heary; or clayey, lay it in ridges to be ameliorated by the frost; if dry and sandy, lay it smoothly flat-frost does no good to light land. Secure all crops liable to be destroyed by frost, and clear the garden of all unsiglitly leaves or rubbish.

Bees.-Guard the hives from wet: bees which are kept dry and warm, are always more lively and consume more food than those chilled by inclement weather, and consequently the former are ultimately more productive.

\section{ALLOTMEN'TS.}

The subject of granting allotments of land to the peasantry has lately engaged much attention, and many benevolent persons of distinguished rank have formed themselves into a society-justly termed "The Laiourer's Friend Society," for the purpose of promoting that object; partiy founded upon a plan carried largely into effect some years ago in Holland, where 
pauper-colonies have been formed for the cultivation of waste land, but chiefly in aid of our occasionally unemployed farm-cotters*.

It has been said in one of the Society's pamphlets, "that he who has nothing to hope has but little to fear. If we cannot attach a man to the constituted order of things by the tie of interest, and the hope of probable comfort, at least, if not advancement, as the usual fruits of industry, under the existing state of the social economy, feeble indeed and insecure will be the controul which the mere terror of punishment can supply, for a forced submission will but ill supply the place of a willing oberlience." 'These remarks were called for by those flagrant outrages which were some time ago perpetrated by our rural population upon many of the farmers; but although order has been in a great degree restored, yet the situation of farmlabourers is still far from affording that solid comfort to which they are entitled, and which every friend to humanity would wish them to enjoy. This, and a wide spreading sense of demoralization in the rustic habits of the working-class, engrendered during the late war and fostered by the abuse of our poor-laws, have induced the gentry of the country to stand forward in the hope of again restoring it to its former healthy condition; and it is gratifying to learn that numerous instances are recorded of the happy effects resulting from their efforts. Hitherto they have usually confined themselves to letting out small portions of ground at moderate rents to industrious cottagers; solely to enable them to supply a sufficiency of homely vegetables to their family, the cultivation to be performed when otherwise unoccupied, without encroaching on their masters' time, or neglecting any other essential duty. In time, however, when the tenants shall by a few years' experience have become more thoroughly aware of their own true interests and the mode of securing it, than can now be supposed, it is the intention of the Society to augment the quantity of land to those who prove themselves prudent as well as industrious, and to accompany the grant with a loan of money to enable them to cultivate it properly.

Happy, indeed, shall we be to learn that the exertions of the Society have rescued our rural population from degrading pauperism, and that they have succeeded in restoring the long-lost class of petty yeomanry from the state to which they have been reduced-of hirelings, to the almost forgotten rank of small farmers: men who, though working as hard as the common

* These colonies were raised by national subscription, aided by the Dutch and Belgian Guvernment, and placed under the controul of commissioners appointed for their management. They are in separate establishments, rauked as "Free Colonies," for the reception of the better class of paupers; one for beggrars, forced into them by the police; another for orphans; and a fourth as a penal settlement, to which those are removed who misconduct themselves. The number of persuns thus employed, chiefly in cultivation, though partly in manufactures, may amount throughout Hilland and Belyium to about 10,000 , and the comfort in which they are maintained may be collected from an account furnished a few years ago by a gentleman who visited the colony of Frederick's Oord._- The crops were luxuriant, the colonists healthful, and the houses comfortable. Several of the colonists had acquired considerable property. Many gardens were planted with currant-bushes, pear and apple trees, and tastefully ornamented with flowers. Additional live-stock, belonging to the colonists themselves, was frequently pastured out; and around not a few of the houses lay webs of linen bleaching, which had been wove on their own account by persons who, only fuur years before, were among the outcasts of society. The families found at dinner had quite the appearance of wealthy peasants; and from the quantity and quality of food before them, they might have been considered as not inferior to the smaller tenantry of this country." Many of the free settlers have, indeed, already repaid the sums advanced to them, and some of the paupers are growing into prosperity : but the plan is still sup ported by public subscription. 
labourer, and faring perhaps little better, yet were distinguished by their honest manliness of character and devoted attachment to the laws and constitution of the country. They felt, indeed, a certain degree of independence which raised them to respectability, and they formed a connecting link between the wealthy farmer and the mere peasant, which the latter might hope with good conduct to attain; but from which prospect he has been latterly altogether excluded. We fear, however, that it will be long ere it can be accomplished, for there is an evident jealousy on the part of the farmers in regard to the plan, as dreading that it will render their servants saucy and disobedient; and even were these impediments surmounted, it will require long experience of the character of the labourer before he may be safely intrusted with the means of independence*. We, therefore, think a quarter of an acre should be the utmost quantity allotted to any one labourer, as that, if well managed, will fully occupy his leisure time, together with that of his familyt.

SPADE HUSBANDRY.

It will be at once perceived that the smallness of these allotments must confine them to cultivation with the spade; the comparative advantage of which mode of husbandry, and that by the plough, has excited considerable attention since the pressure of population against the means of subsistence has called for the increased employment of the people. Whatever may be the supposed superiority of the spade, it has been generally abandoned to the use of the plough, as a much greater quantity of land can be tilled in a given space of time by the aid of animal labour; without which corn could not be grown in amount equal to the supply of that portion of the community which is not engaged in agriculture. The benefits which have been derived in some parts of England from small portions of land being granted to labourers, together with the example instanced in Holland, might indeed, at first sight, appear to countenance the plan of rendering them by such means entirely dependent for their support; as exemplified by the cottier system adopted for the greater portion of the peasantry of Ireland. It is

* In the Third Report of the Emigration Committee, published by the House of Commons, an estimate is stated of the expense attendant upon the location of a family, consisting of a man, his wife, and three children, upon waste land, fit for cultivation, in any part of the United Kingdom:-

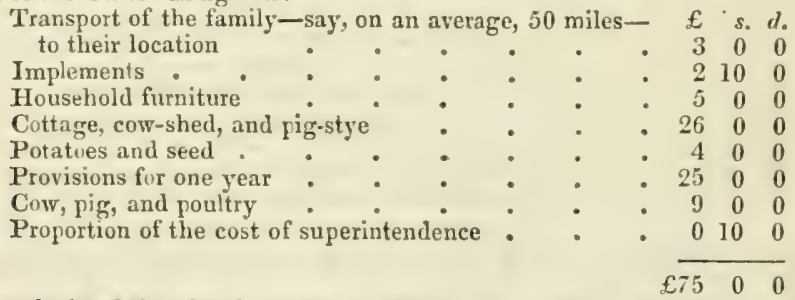

And it is calculated that, by the produce of four acres cultivated by the spade, "the family may maintain themselves, and dispose of produce to the amount of $21 \%$. or $2: 2 \%$. per anum, after paying 81 . yearly rent." p. 363. From the statements preceding the estimate, it however afpears that these protits are only expected to arise after a layse of seven years: if realized at all we apprehend it would not require so much time; but we consider $100 l$. to be nearer the mark than $75 l$. for the realization of the plan.

$t$ See the publications of the Labourer's Friend Society; and those of Jacob, "On the Cultiration of Pror Soils:" Rowland Hill, "On the grautual Extinction of Pauperism:" Demainbray, "The Poor Man's Best Friend :" Withers, "Facts, addressed to the Farmers of North Hampshire:" Pollen, and a Letter addressed to the Right Hon. Sturges Bourne, "On Spade Cultivation," 
there however seen, that although they are in common seasons enabled to rear a sufficiency of food for their consumption, it yet merely consists of potatoes; and the comforts found in the Dutch pauper colonies are supplied, in the first instance, by the advance of capital, which places the settler upon the footing of a small farmer; or in the second, by subscriptions, which can only be looked upon in the light of poor-rates.

It is thus evident, that if by the superior industry and habits commonly ascribed to the English peasantry, they may possibly be enabled to acquire something more of the common necessaries of life, either by extraordinary labour or occasional assistance afforded by their wealthy neighbours; yet the question of their entire and comfortable support can only be decided by rendering them independent of all aid except that derived from their own personal toil, and sufficient land, of fair quality, at a rent equal to that of any other tenant.

To prove the possibility of it by the use of the spade, various statements have been made showing the superiority of the crops thus produced over those grown by the plough*. This, indeed, hardly needs any elucidation

* George Brooker, of Upper Tontton, in IIampshire, has grown 4 bushels of beans, 5 bushels of barley, an 16 sacks of potatoes upon a quarter of an acre of indifferent land. The labour was entirely that of his wife and family, and of himself at leisure hours only. He did not lose a single day's work from his employer; but he estimates that the labour done would have occupied him three weeks, had he performed it all himself at working hours; as the land, when he took it, was full of couch-grass, a wheat stubble in bad order. See the aldress of the Rev. L. B. Wither, to the farmers of North Hampshire.

Mr. Lance, of Jewisham, has exhibited at the Adelaide Gallery, in London, ninety straws from one root, and by one division of a root of wheat, 190 ears of corn have been grown from one seed, being $\mathbf{1 3 , 0 0 0}$ grains from one, as there exhibited. They were, however. grown in beds upon garden-tround, cultivated by the spade, and the stems were afterwards transplanted. He says, also, in his "Cottage Farmer," that Mr. Colgate, of Chevening, in Kent, obtained four quarters from half au acre, by adoptiug the process of digging the land 12 inches deep by two 6-inch spits, burying the weeds as manure, and dibbling the seed in 12-inch rows, and three inches apart, but hoed out in the second hoeing to six inches distance. The seed was half a bushel soaked in the liquor from a dunghill and wood-ashes cast over it. The crop was hoed three times with Lord Vernon's hoe; and the expense, including $30 \mathrm{~s}$. for rent and taxes, was $57.15 \mathrm{~s}$. The ears had 90 corns each on an average.-p. 11.

Mr. Falla, of Newcastle, also grew wheat during several years upon land of a medium quality cultivated by the spade, and never in any season had less, though plinted two years successively, than 52 bushels per acre. In two other experiments made upun wheat sown in drills and broadcast, as well as transplanted from beds, the produce of the transplanted crop was however inferior, besides occasioning the additional expense of $4 l .7 s$. $1 \frac{1}{2} l$. for the labour of tramsplanting 232,323 plants, at $4 \frac{1}{2} l$. per 100 ; from which there was to be deducted the sowing of the seed, which only amounted to two pecks. The following is the account of the produce of each trial:-

No. 1.

No. 1. Transplanted from the seed-bed into 6-inch lines-per acre, $62 \frac{1}{3}$ bushels.
2. Do.
3. Do.
do.
$9 \mathrm{do}$.
12 do.
4. Sown in drills of .
5. Sown broadeast
do.
12 do.

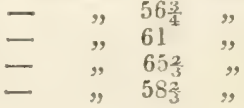

No. 2.

No. 1. Transplanted from the seed-bed into 6-inch lines-per acre, $68 \frac{1}{4}$ bushels.
2. Do.
2. Do. lo.
9 do.
do. 12 do.
4. Sown in drills of
12 do.
5. Sown broadcast

$12 \mathrm{do}$.

$$
\text { 三 }
$$

$\begin{array}{lll}, & 68 \frac{1}{4} & , \\ , & 60 \frac{3}{4} & , \\ , & 73 \frac{1}{3} & , \\ \text {, } & 76 \frac{1}{4} & \end{array}$

A portion of No. 4 in the last detailed experiment, it must however be oliserved, was laid down by wet, when in flower, and proved very ahortive; otherwise Mr. F. has little doubt that No. 4-as in the former year-would have exceeded No. 5 in guantity. A 
to the farmer, for he must be well convinced of the advantages of garden culture beyond that carried on in the field, as by it the ground can be dug twice as deep as it is generally stirred by the plough; thereby rendering it pervious to the roots of plants, which thus draw more nourishment from the soil. It is also more effectually worked-that is to say, the clods are broken and intermixed, and the land is thus rendered more friable and mellow than can be effected by a common fallow; the treading of horses, too, which is so injurious to clays, is avoided; as well as the constant recurrence of the pressure of the ploughshare, which eventually causes in some land a hard surface upon the subsoil-thus constituting what is called "the pan," which in tough soils prevents the escape of water, and holds the upper stratum in a wet, cold, and unprofitable condition.

The cost of digging, by men, is stated by Mr. Falla, an extensive nurseryman in the neighbourhood of Newcastle-upon-Tyne, to have been $4 d$. per rood-or $33 s, 4 d$. per acre-when wheat was at $9 s$. the bushel; but "being desirous," as he says, " of ascertaining how far, and at what expense, it may be practicable to work land by the spade by women, boys, grirls, and feeble old men, in order, among other reasons, to the employment of paupers of that description, I have this autumn made an experiment on a piece of land containing 1728 square-yards, by digging, or rather trenching, by two short spits, with girls; and I have the pleasure of saying, that the work is better done by two such short spits, each about five to six inches deep, the one following the other, than digging is done by men at one full spit, or spadeful, about nine or ten inches deep. The common wages I pay to these girls is $10 \mathrm{~d}$. per day; and they did the work in nineteen days for one girl, which thus cost $15 \mathrm{~s} .10 \mathrm{~d}$. An acre at the same rate, containing 4840 square-yards, would cost $2 l .4 s .4 d$, which is $11 s .4 d$. more than by men at one spit; but I am satisfied that the superiority of the giris' work is well worth the difference. I may add, that this being the girls' first attempt with spades, I am persuaded that, by future practice, they would in a short time do it for the men's price-33s. They work with quite light spades made for the purpose; the best size for which I think to be $9 \frac{3}{4}$ inches long, 8 inches broad, and weighing, with the light handle, about $4 \frac{1}{2} \mathrm{lbs}$. avoirdupois *".

considerable part of the wheat Nos. 1, 2, and 3, was also shaken out hy the wind, and destroyed by birds, to the amount of probably five or six bushels per acre-Farm. Mag. vol. xxii. p. 269.

*. ". The spade, in the usual English acceptation of the term, is unknown throughout the greatest part of Ireland. Its place is there generally supplied by the loy, which consists of three distinct pieces: an iron blade, which is made with the socket as broad ans itself; a thick and stout wedge, or block of wood, which fits into the socket, and serves to receive the foot in the act of digrging; thirdly, the handle, which is braced to the woden edge and blade by bands of iron. The handle consists of a straight rutunded pole of various length, and the instrument is distinguished accordingly by the terms of the "long" and "the short luy." Another distinction also arises from the blade, according to its being long or narrow. The long loy operates as a kind of plough; the blade forming a curve with the handle, the convexity being at the back, is first inserterl into the earth by the pressure of the foot, then the end of the loy haudle is drawn downwards to loosen the clod by the pressure of the foot (in the same manner as the spade): but the workinan then pushes the liade for two or three feet under the surface, and finally upsets and turns over the clod sideways, not unlike the work of the plough; and, in the hands of an able workman, it is a powerful implement.

"Another peculiarity of Irish dirging-implements is, that for ordinary use they are invariably provided with longer handles than are custumary in England; whereby the labourer is enabled to maintain a more erect position than can be preserved in using the short-handed English spade; and bence in no part of Ireland is it usual to meet men bent down by their labour, as commonly seen amongst old men in other countries.

"The stivcen, or steeveen, is another implement, the chief use of which is in dibbling 
This may prove a valuable hint to the present Guardians of the actual poor-house system; for the parishes being now cemented into Unions, and the Guardians having power under a late Act of Parliament to take up land for cultivation, the in-door paupers may be very beneficially employed, under the superintendence of an intelligent overseer, in the culture of crops, which would repay a portion of their maintenance, besides adding to their comforts, and affording a healthful occupation to the younger branches, instead of allowing them to waste their time in unprofitable idleness.

In addition to this, we beg leave to mention a plan which has for several ycars been pursued by that intelligent agriculturist, M. Pictet, of Geneva, who allows one rotation of his crops-consistiner of potatoes-to be cultivated by his labourers on joint account; he funding rent, seed, and manure, and they labour. The ground is cultivated by the spade, and being brought into the most perfect state of tilth by being deeply worked and kept completely clear of weeds by constant hoeing, the culture acts like a complete summer fallow, and requires nothing more for the succeeding wheat than merely to have the seed harrowed and rolled in, without any ploughing whatever. The crops of potatoes, although grown upon a poor soil, have always been so large as amply to repay $\mathrm{M}$. Pictet with profit; in addition to which he has the inappreciable satisfaction of thus securing the grateful attachment of his dependants*

Were this plan to be carried more widely into execution, it would aid materially in providing a constant abundance of food in the most trying seasons; for it is a singular fact, that when unfavourable to wheat, they are beneficial to potatoes. It would also ensure a most gratifying mode of cultivation on the part of the labourer, as he would then look forward to remuneration in proportion to his own exertions as a partner in the production of the future crop. Instead, therefore, of working heartlessly as a day-labourer, solely with a view to get through it with as little trouble as possible to himself; or, if at task-work, with an intention to slur it over in any manner which may impose upon his employer, both he and his fanily would then occupy themselves at every leisure moment in hoeing, weeding, and attending to the crop, in the certainty that their toil would be well repaid, and habits of real industry would thus be gradually engendered;

potatoes. It consists of a short pole, sharpened at the lower end, which answers the same purpose as a garden setting-stick; but, to increase the power, a cross picce is inserted at a convenient distance from the point to receive the foot. When forced into the ground, it is turned round to enlarge the hole, and the cutting is dropped by any child employed for that purpose : a few strokes of the stiveen covers the cut with earth, and as the cruss plece regulates the depth, the planting is effected with considerable exactness." -Weld's Survey of Roscommon, p. 659.

* Pictet: "Quelques détails sur la consommation de la Luzerne en vert, et tableau d'un assolement de douze ans." p. 68.

The labourers work together in line, in the same manner as customary in digginc fields in most parts of Biscay and Guipuscoa in the North of Spain, where turnip and jotato crops are commonly cultivated by the spade. Both men and women there join in the labour, each furnished with a stout three-pronged fork, having blades of about 14 inches long, with a cross piece to receive the foot, like the Irish loy; and, as they make the stroke simultaneously and at equal depth, the work is carried on with eatraordinary precision.

In countries where the spade husbandry prevails, it is usual for people to exchange labour reciprocally, and to unite in considerable numbers in the fields of individuals in rotation, more especially for the purpose of planting or digging out potatoes. "T'hus in Ireland, these congregations of workmen give vivacity 10 the labour, and are orduarily scenes of much cheerfulness."-Weld's Roscommon, j. 660. 
while, on the coutrary, the idler is sure to become vicious. Give him but a stimulant to labour, by allowing him a proportionate interest in the value of his efforts, and you render him at once a friend to social order.

\section{Chapter XLVI.}

\section{THE FARMER'S GARDEN.}

THE directions on gardens, which are contained in the Chapter on Cottage Economy, relate exclusively to those cultivated by the peasantry for their own use; but as it often happens that the labourer is called on to act as his master's gardener, it is necessary he should know how to manage certain plants which are rarely found in his own, and therefore have not yet been mentioned.

The first we will mention is that excellent vegetable delicacy asparagus, of which every farmer wishes to have a couple of beds; more especially as when once laid down it continues productive for many years. Neither does it require half the care and attention usually bestowed upon it. As a native of Britain it is perfectly hardy; and the custom is to give the beds a winter covering,-not to keep out frost, but to cause an extra legree of warmth, exciting the roots to earlier action. 'The fact is, asparagus being a gross feeder, and particularly fond of rich alluvial land, it should, in the first place, be planted on an extraordinarily enriched, deeply-trenched soil, where it will luxuriate for a long course of years. The ground intended for asparagus should have a thick coat of good dung trenched in, and also a coat of very rotten dress digged into the surface. The plot is divided into four-feet-wide beds, with two-feet-wide alleys between. Three rows of two-year old plants (bought of a nurseryman) are placed in drils, ten inches apart, made by the hoe, the roots being spread out right and left, and covered carefully withloose earth; the whole being afterwards smoothed by the rake*. The season for planting is the close of March, or early in Aprilt, and during the following summer the beds are only kept clean of weeds. When the stems have died down in the autumn, they should be cleared off, and the surface broken up or stirred, not too deeply, with a fork, care being taken not to disturb the roots. The beds may then be covered for the winter with a coat of short, decayed litter, and over this a little earth may be thrown from the alleys. In the month of March this mould and litter may be raked of and digged into the alleys, which operation is called the spring dressing. This autumn and spring dressing is continued every year afterwards.

Asparagus is always allowed to grow and gain strength for three or four years before any demands are made upon it for the table. This forbearance allows the plints to establish themselves in their new place, and so become able to bear a partial cutting in the fourth, and a general cutting in the

* "The plants should be placed so deep as to permit the crowns to be covered with two inches of fine earth, and the roots ought to be let down to their full extent into the ground in a rather open and expanded order:" Towers, on the Cultivation of Asparagus. Quart. Journ. of Ag. vol. vi. p. 537.

+ "Dry weather should be chosen; because the earth, when it is in a free and open condition, may readily le male to fall among the stringy processes of the roots; but when planted, each row should be liberally watered once, to enable them to start into growth without any loss of time." $I b$. 
fifth, and every year afterwards. The alleys between asparagus-beds are always rich, in consequence of the winter coverings being raked into them. They are therefore fit receptacles for rows of calliflowers, which are another culinary delicacy, acceptable to every one who can obtain them.

Sea kale is a prolific plant of easy culture. If raised from seeds, it requires two years to bring it to perfection, but is so hardy as to withstand any common degree of frost. If planted out from a hot-ired, the roots on being divided may be propasated abundanty, and the regetable will be rearly for table at a much earlier period of the year than asyaragus. The directions for its cultivation, extracted from a treatise lately written by $\mathrm{Mr}$. 'Towers, author of the Domestic Gardener's Manual, are as follows :-

"The beds ought to be prepared as for asparagus; and of a light sandy turf, with a natural manuring of sea-weed alone, or artificially-with one-fourth of a compost consisting of perfectly reduced fern, or leaf-mould, nine parts - woodashes, one part-and about half a pound of common salt to every barrow-load of the compost. This last chemical compotind contains a large proportion of the components of sea-water, and the woot-ashes rield earbonate of lime in its most effectire state, also some carbonate of potassa, and fine silex. Three barrows of light sandy turf, and one barrow of the alore compost, give proportions is hich promise to yield a merlium of growth of the most permanent and effective character. In it sea-liale and asparaers might be grown for forcing, either side by sicle, or in separate plots, the treatment of the one would be applicable to the other; and therefore one description will suit both. I only observe, that, if neither turf nor sea-weed san be obtained, good liale can be produced in any light garden soil, trenched in the manner before describel fur asparagus beds, and manured copiously with wood (not cual) ashes, and the prescribed quantity of eommon salt.

"If the secd be good, the season propitious, warm, and moist, germination will rapidly adrance; and when the plants appear, they may be seeured from vermin by sprinkling around and within them a good covering of wood-ashes (three parts) blended with soot and purlered lime, of each one part. If weeds arise and threaten to surmount the plants, they must be kept under by surfacehoeing to the depth of an inch, with the 'Dutch' or thrust hue. Perhapsthree plants will arise from each ling, and these, indeed all that gruw, must remain till they begin to interfere with each other; then, one only, the strongest, must be left, and the others drawn, or raised up; so that a complete row of single plants, from 1 foot to 15 inches apart, finally remain. The young supermuneraries may be set out in rows, to grow for succession, or be thrown away if no more stock be required. The plants in the rows will grow luxuriantly, and become very large, so much so as, in all probability, to come in contact, forming one connected line of sea-gresn foliage. All the culture during the summer and autumn, will consist in keeping the beds clear from weeds, and in digging alternate spaces two or three times, to the depth of nine inches, so as to pulrerize and render the soil as fine as pussible. At the two last operations (say in July and October), it will be as well to incorporate with the soil a two-inch layer of decayed leaves, mixed with one-fourth part of pure wood-ashes.

"The reader will be very careful to aroid sea-coal, or even pit-coal ashes. It formerly was the custom to cover sea-kale with conical hills of those ashes during winter, and one out of three or four plants perished, the damage being ascribed to mice or moles. I never have lost a plant since I left of that acrimonious chemical compound, styled ashes, nor could I, by any means keep my bed entire, while I employed it. Its destructive influence has at length been admitted, and sea-kale now enjoys a regular growth, and repays its cultivator:"

It may be sown or planted on a bed of light, sandy soil; and, if wanted very early in the spring, the bed may be covered ilickly with dry leaves, or litter, to bring the shoots forward; or, if the plants be covered with large flower-pots, and these with hot dung heaped over for a week or two, will bring the kale up early, and at a time when must acceptable: forcing 
should however, if possible, be avoided. With regard to soil, we have heard it asserted that " clay is the best." The vegetable, however, grows wild upon the sandy shores of our Southern coasts; and, as indicated by its native situation and habits, we believe that most gardeners prefer ground for its culture of a light and sandy nature, for if 'planted upon clayey land we are told that its flavour is rank and disagreeable.

Cauliflower-seed is sown about the 24th of August. When the seedlings are large enough, they are transplanted into wam, sheltered places, to stand the winter; and, if they survive, are re-planted in open order in the months of April and May. A second seed-bed is sown in April, whence plants are supplied to yield heads in autumn; being placed in their final stations in July and August.

The earliest cauliflowers come into use about the 10th of May; but these are nursed under hand-glasses all winter. The glasses are disposed in a rank, on a rich border of soil in a sheltered place; four or five of the strongest plants are dibbed in within each glass. 'The glasses are raised in front every day, and shut down on nights. About the 20th of March the plants will have filled the glasses, which must be then raised on brickbats to give room and permit the plants to be earthed up, and, if necessary, well Arenched with manured water; soon afterwards the glasses are laid aside. Cauliflowers require very rich ground, and, if they can be kept through the winter, succeed very well, cultivated as the common cabbage.

Every lind of brocoli is acceptable as a winter vegetable. Small quantities of the seeds of Grainge's Early Cape, Late Purple, and Cauliflowerbrocoli, should be sown about the middle of April; and from thence tramsplanted in rows, $2 \frac{1}{2}$ feet apart, upon well-clunged and deeply-digged ground, in July. Here the plants receive hoeing and earthing-up as they advance in height. The Cape sort will probably yield heads before, and the other sorts after Christmas.

Dwarf kidney-beans may be planted on the ground on which radishes have been raised, or that of any other crop coming off about the same time, after it has been well digged and dunged, if necessary. They are planted in drills thirty inches from each other, the seeds being placed two inches apart. Besides being a delicate green regetable, they are prized as a pickle. Like the rummer, they require to be liept stripped of their first pords to encourage after-crops.

The artichoke is a horticultural luxury, which may have a place in the garden of a farm-house, and, like asparngus, when once planted, remains prolific for many years, if properly managed. The ground intended for artichokes should be trenched, and a good dressing of dung digged in afterwardis. Toung plants are obtained in April, by slipping of suckers from old stools. These suckers are planted in rows, four feet apart, and the plants two feet asunder in the row. These will yield small heads in the autumn of the first year, and full-sized ones in the second. Although the articholie is a luxuriant-growing plant, the roots are liable to be damaged by frost in winter: they therefore require an autumn dressing, in which wreaths of dry litter are laid around each stool, and the ground digced between, but laid in ridges covering the litter and place of the roots, whereby wet is drained off and frost repelled. 'Thus secured, the plants remain all winter; and, about the end of March, they receive a spring dressing : this is done by digging away the ridges from about the plants, opening round the stools, and pulling off all the small suckers, leaving only five or six of the strongest to grow up to produce heads. There are two sorts, the green and purple globe,-the latter being accounted the best 
flavoured. The vacant ground between the rows of artichokes is usually cropped with some other plant during summer.

'The Jerusalem artichoke, though so hardy as to grow in almost any soil, is also a delicate vegetable if served, when boiled, in a white sauce. The tubers are planted whole, in the same manner as jotatoes, and the crop may be taken up at any time during the winter.

Celery is a plant which, when viell blanched, is an excellent salad, and its value in soups is known to every one. A seed-bed of a yard square is sufficient; and, if a hot-bed cannot be had, should be sown on the warmest spot of the garden, about the beginning of April. The seedlings are slow of growth; but, as soon as they are two or three inches high, they should be pricked out on another bed, to gain strengrth, until the time of placing them in trenches arrives-viz., some time in the month of July - that is, between hay-time and harvest. At this time, parallel trenches, four feet asunder, should be digrred, six or eight inches deep; in the bottom of these, three inches deep of the richest dung is laid and digged down. Along the middle of the trench, the plants (being carefully raised from the nurserv-bed) are dibbed in, six inclies from each other: water them copiously, and the work is done. In a month or six weeks the plants will have filled the trench with their leaves; and then it is time to crumble down with the syade a little loose earth from the sides, letting it fall argainst, but not into the hearts of the plants. This earthing-uy) is continued from time to time till the plants stand in ridges, eightren inches or two feet high, and by which the leaf-stalks are thoroughly blanched and fit for use.

Endire is another salitd-plant, and particularly useful in winter when let. tuce is scarce: this requires blanching to make it crisp and palatable. Small portions of the Curled and Bativian sorts should be sown about the end of $J$ une, and again at the end of July. From these seed-beds plants mav be had to transplant, in open order, as much as may be necessary for autumn and winter use: the plants require twelve-inch spaces, as they spread much. They are blanched by twing-up like lettuce, by being earthed-up like celery, or by plunging them in beds of dry sand: sometimes endive is hlanched, and moreover defended from the frost, by being covered with dry leaves of trees or fern.

Red beet is a vegetable extensively employed for the table throughout France, though here it is only considered useful in garnishing and in madesalads, and therefore a single row of a dozen plants may be enough in a farmer's garden-sown in April; but if boiled until it becomes tender, then sliced, when cold, and eaten with oil and vinegar, either alone or with celery and onions, or mixed with endive, it forms an admirable wintersalad, and is both nutritive and wholesome.

1 large bed of uinter-spinach should be sown in the second weels of August, which will be serviceable throughout winter and spring.

of cucumbers, if not with hot-bed sort, at least a ridged crop may be grown for salauls and for pickling. For this a trench, two feet deep and as much in width, should be filled with hot stable-dung, closely beaten down with the fork, and covered with ten inches of rich earth. Aleng the mildle, seeds, or plants (previously raised in a hot-bed, of sta!lle-dung) are put in about the 20 th of liay, with the addition of hand-glasses for the plants to be nursed under, in the first stage of their growth. If, however, the farmer has not at hand any of these glasses, or frames, in which they are raised by professional gardeners, he must then immediately hoop over the bed, to bear the covering of a mat, a sack, or any old garment, till the heat of the season renders covering unnecessary. When the 
seedlings have risen two inches high, they will require earth to be put round the stems; and when they have expanded the first rough leaf, the little bud at its base must be pinched off. This will cause the plants to throw out rumners, which, as they extend themselves, must be kept firmly fixed to the ground by little hooks. Water may be occasionally given, if the weather be warm and dry; and if the surface over the roots be covered with short litter, it will assist the growth. When the branches are cramped by the glasses, they should be let out, and trained along the ground on each side as far as they tend to grow. Such are always very productive. Melons may be grown in the same manner, if desired.

Hardy fruits, of all kinds, are useful about a farm-lıouse: those in the orchard take care of themselves, but such as are in the garden require both proning and training. Wall-trees require the skill of a professional gardener; but the commoner sorts, on espalier rails or in the borders, may be pruned by the odd man or shepherd, if he has time. Gooseberries, currants, and filberts, are all pruned alike, - that is, kept open in the middle, and constantly spurred-in. Raspberries are regulated by having all the former year's bearing-wood cut out; and selecting five or six of the last year's young shoots to be the hearing wood of this year. These are tied together near the top, and cut down to about three or four feet in height. Black currants should be thimned and kept low, but their shoots must not be shortened. Beds of strawberries should be planted in August, and kept in single tufts, free from runners and weeds, occasionally mulched with rotten dung, but never digged among with the spade. The beds should be renewed every third or fourth year.

As to the saving of seeds, the only kind that it is worth the cottager's while to save are those of onion, scarlet-runner, radish, and coss lettuce ; for cabbage, savoy, carrot, parsnip, \&c., cost so little that they can be no object to the buyer. Whenever attempted, however, the finest and truest specinens of the crop should be chosen to produce seed. A few plants of radish and Jettuce may stand where they were sown; a score of the first pods may be left on the rummers; and half-a-dozen of the best onions, planted in a row on an open border in the month of February, will yield seed enough for the following season: indeed, saving onion-seed should be a particular object with cottagers; as, having a few unnces to sell, will enable them not only to buy all other seeds, but a load or two of dung besides.

The quantities of seeds required in a small garden, are,-

1 pint of early peas is enough for a row of 20 yards in length.

1 ditto beans ditto 27 ditto.

1 ditto runner ditto 36 ditto.

1 ditto dwarf kidney ditto 26 ditto,

1 ditto marrowfat peas ditto 32 ditto.

$1 \mathrm{oz}$. onion sows 15 square yards; 1 oz. of carrot and parsnip sows 15 square yards; $\frac{1}{2} \mathrm{oz}$. of cabbage, savoy, borecole, brocoli, or cauliflower, is enough for a seed-bed of 4 square yards; $\frac{1}{2}$ oz. turnip, 11 square yards; of radish, 2 or 3 ounces for spring sowings. A bed of asparagus, 5 feet by 30 , requires 160 plants; an acre of potatoes requires from 15 to 20 bushels of sets.

These particulars will serve as a scale for apportioning other kinds of seeds, according to the size of the seeds respectively, and extent of the ground to be sowed or planted.

We have thus enumerated the particular objects which demand attention of both farmers and cottagers in their respective gardens; assuming that both their gardens have been properly prepared by draining, if necessary, and by deep trenching, which is indispensable. Deep and frequent trenching, or double digging, is always beneficial to the crops; and, although both 
broad beans and onions, like wheat, affect a firm subsoil, it should be consolidated after being trenched.

In addition to what we have already said on the late failures in the potatoe crops, we now beg to call attention to two recent publications on the subject, which-after discussing the diversity of opinions entertained upon the cause, and proving their general fallacy-attribute the weakness of the plant to constitutional decay; and assume that the continuance of its vigour may be secured by its propagation from the seed, instead of by cuttings from the bulb, or tuber.**

We have not room to state the reasoning upon which this recommendation is founded, but it has struck us as being too forcibly maintained to be neglected; for it is matter of not alone national importance, but coming home to every man who cultivates the root upon a lirrye scale. The system of sowing in the field would doubtless occupy so much time ere it could be brought to perfection, as probably to destroy all profit upon its growth; but no material inconvenience can be occasioned by growing a sufficiency of seed in the garden to supply the farm with sets for the future field-crops, and they may unquestionably be grown from tubers directly produced from the seed, with at least as much certainty as from any older stock: care should, however, be taken to select the seed from the finest plants of an approved sort.

Mr. Aitken prefers green sets to those over-ripened; and proves the efficacy of green seed, that is, seed full of vegetative sap, from the circumstance of the crabs, or green excrescences, which grow on the stems of the potato producing vigorous seed. He also urges a test of the vitality of the sets as an important consideration; which, if attended to, would obviate the necessity of early planting, and allow of time to work and clean the land. His method is as follows:-

"Select a piece of dry ground in a securely fenced place; when required on a large scale, as will be the case on extensive farms, lay the ground off in beds of five feet wide, with alleys of two feet. Use a garden line, and lay them off in a neat mamner. From the alleys take earth to cover the surface of the beds, taking care to raise them a little in the middle. This done, have in readiness, and placed conveniently to the beds, a quantity of fine compost earth, consisting of one-third of muss, one-third of clean earth, one-third of peat or coal ashes, mingled with a small proportion of lime, enriched by urine, or pourings from a dunghill. 'The beds and compost being both in readiness, eut all the seed potatoes which are above the size of a common hen's egg. .By the first cut, take off about the fourth part at the root end, and lay it aside for food. Next, diside the top end into pretty large cuts, the small ones use entire, either kept hy themselves, or mixed with the cuts. When a quantity is thus cut, spread it orer the bed, upon which previously is to be laid a thin covering of the compost. I would recommend the layer of potatoes not to consist of more than two cuts in depth, spreal over with an inch of compost. This done, if the weather be dry, take a watering-pan and water most effectually, in order to wash the compust into the bed anongst the cuts; the quantity of water being regulated by the state of the weather. After this, give them another covering of compost, in all about three inches in depth. In this manner proceed until as many are so laid down as will be required for the farm. Further watering will only be necessary if the weather is dry. After the cuttings have lain in the seed-beds some time, it will be necessary to examine them to see if they are springing, of

* See Aitken, "On the Potato rescued from disease, and restored to prisline vigour by a plan of keeping and cultivation, founded on the natural principles of the verretable economy:" aud, "The failure of the Potato crop ascertained and demonstrated from analogy ; with a remedy and test for the present seed to prevent failures ;" by a Dumbartonshire farmer.

voL, II. 
which there can be little doubt. If any doubts are entertained, however, it will he necessary to prepare more sets, and put them in fresh beds, to insure a sulliciency at the time of transplanting. The chance of the seed springing in the Theds is ten to one compared with the ordinary way. Here, should the weather be dry, abundance of moisture can be communicated at any time; and, in place of a parched soil robbing the sets of moisture till they are quite shrivelled, as was the case during the intense drought of last May, the sets will be duly fed and nourished from the fat juices of the rich compost. Allow them to remain in the bed till the shoots come above ground. If the compost has been mixed with lime, as directed, it will act as a powerful stimulant upon the weakened and exhausted vegetative powers of the potato, and rery much accelerate and strengthen the young shoots. If this has been neglected, it may still be partially accomplished, either by dusting powdered lime among the cuts, or mixing it in the water. If no compost has been prepared, good clean loose earth will answer the purpose, but it would not be equal in efficacy to rich well-prepared compost."

After this description, Mr. Aitken gives minute directions for the management of the preparatory field operations; and the treatise of the Dumbarconshire farmer also contains the following test for distinguishing the decayable from the undecayable potato :-

"From the now generally diseased state of the potato, it appears to us that there is but one way to test the seed before planting, which, if properly attended 10, will prevent those melancholy results that may be anticipated in the crop. It is this : Select from the potatoes proposed for seed a dozen or two; cut them with a sharp knife into sets ; then put them on the floor of a potato-house, or any other place free of damp, with the skin next the floor ; if, upon examining them three or four days after, it should be found that the incision has dried up, and is corered with a kind of new skin, be assured that the seed is wholesome : plant it; but if, on the contrary, the wound is found to be wet, sluggish, and spotted-touch not the unclean thing, but be certain that the constitution of the potato is exhausted. This experiment should not be tried until regetation has completely taken place, say about the middle of April."

Were the plan only to be looked upon as an experiment, it woull, in that view alone, be worthy of attention; and-as a parting word to all farmers-we strongly recommend them to devote a portion of their gardens to the trial of different plants, with the properties of which, as field-crops, they may not be yet sufficiently acquainted: more particularly, to the selectior of seeds from the common species of corn, roots, and grasses, grown upon their land, a few of which may occasionally be distinguished among the crops by their extraordinary growth; for, if the seed be carefully sown apart, improved sorts may be thus obtained, which will largely repay the trouble. Even if not attended with success, it will be far from useless to the grower, as every observation which he is attentively called upon to make on vegetation will increase his store of knowledge of the plants, and add to that experience in his profession which can alone render him a scientific agriculturist. 


\section{A P PENDIX.}

No. I.

\section{ABSTRACTS FRON ACTS OF PARLIAIENT RELATING TO HUSBANDRY.}

\section{Right of Voting.}

Stat. 2 Will. IV., c. 45.

1. Frephn?ders. - Of inheritance of the yearly value of $40 \mathrm{~s}$. abore Whether rents and charges.

For life or lives of the rearly value of 107 . above rents and charges. $\}$ occupred.

For life or lives of the yearly value of $40 \mathrm{~s}$, above rents and charges, oceupied by such fresholders: or, although not occupied, which would liave entitled them to rote on the ith of June, 1532 ; or actuired after that time by marriage, devise, or by promotion to a benefice or office.

Freeholds for life may be acquired in right of a benefice or an office-as clergrmen, parish elerks, Sc., with salaries derived from lands: they may also arise from tithes, rent-charges, \&c. Ibid., sec. 18.

‥ Cof y ghulders.-For life or larger estate of coprhold, or any other tenure except freehuld, of the yearly value of $10 \%$. above rents and charges.-1bid., sec. 19.

3. Leaseholders, being Tenants actually occurying lunds at a yearly rent of not less than $50 l$.

Lessees of $10 l$. yearly value, for terms not less than forty ycars.

Ditto of $50 \%$. ditto, for terms not less than twenty years.

Assignee of the residue of such terms.

Whether occupied $\mathrm{Ol}^{\circ}$ not.

Freeholders and copyholders must have been in possession or in receipt of their profits for six calendar months, and leaseholders for twelve months. Tenants must have occupied their land during twelve months before the last day of July in each year, except in cases of descent, devise, marriage,-Ibid., sec. 20.

\section{Tithe Commutation Act.}

\section{Stat. 6 and 7 Will. IV., c. 71.}

Ofincers under the Act.- Three Commissioners, who may appoint, to the nunber uf twelre, Assistant Commissioners, or nore, if they obtain consent of the Guremment. Oflice to be in London or Westminster; salaries, expenses, Se., to be paid out of the Consolidated Fund.

Princinle of Crmmutation. - Tithe to lie commuted into a permanent cornjent or charge. That is to say, a certain amount of produce is to be assinned to the tithe-owner, the value of which, varying with the price of $\mathrm{com}$, is to be paid in moner, payable by half-yearly parments on the 1st of July and ist of Jantiarr.

Expepition to the Principle of Commutution. - A parochial agreement may be marle to give an ecclesiastical tithe-owner a certain amount of land, not exceeding in quantity twenty imperial acres, for the whole or pa:t of the great or small tithes of the parish, in lieu of fixing a permanent corn-rent. But this is subject to the sanction of the Cummissioners, who must be satisfied of the soundness of title to the land.

Methrids of Commutation.-Two methods:-1. Toluntary, by agreement amongst the parties concerned, subject to approwal of Commissioners. 2. Com- 
pulsory, by the Commissioners taking the business in to their own hands, and settling it. Parties are allowed till the 1 st of October, 1838 , to enter into roluntary agreements; after that date the compulsory system commences, bit Commissioners have discretionary power to suspend their proceedings, if there be a prospect of a voluntary agreement.

Basis of Commutation.-The parties in a voluntary agreement may settle the commutation as they please, if the interests of all are consulted, and the Commissioners approve. In that case the agreement is binding. In a romi,ulsiry settlement the Commissioners are to calculate the value of the tithes of each parish by their average money-value (after making all due allowance for expenses of marketing, \&e., where tithes have been taken in kind) for the seven years preceding Christmas, 1835.

How a voluntury agreement may be effected. - Any one or more of the landowners or tithe-owners, whose interest is not less than one-fuurtin of the value of the parish tithes ( $i$. $e$. titheable lands or great and small tithes), may call a parochial meeting of land-owner's and tithe-owners within the limits of the parish. Notice to be given twenty-one days before meeting. 'The persons present at the meeting must possess an interest not less than two-thirds of the tithealle lands, and two-thirds of the great and small tithes. Their expenses must he borne by themselves. If, on electing a chairman, it is ascertained that the parties present, either as owners or agents, have not the required interest, they mar, notwithstanding, execute a provisional agreement, which shall be binding, if the required number of interested parties assent within six months. The meeting may be adjourned, of which notice is to be griven. The business of this meeting is to ascertain and agree upon the amount of rent-charge to be fixerl on the entire parish in lieu of the tithe. Of course, both tithe-owner and tithe-mares must be consenting and satisfied parties in this arrancrement. But the clividing of this entire amount, assigning each party his due share of the incumbrance, is a business of the tithe-payer, with which the tithe-owner is not to interfere. The consent of the patron must be given to commutation of ecclesiastieal tithe, an:? the bishop's approbation is required. When everything is settled, the agreement is submitted to the Commissioners, who ascertain if all is right (i.e. no collusion or fraud, no valid objection from parties complainiug or disenting, Sc.), and they then confirm or amend the agreement as they shall see fit.

*** There are a number of minute particulars in the Act respecting the appointment of valuers for the purpose of apportioning the rent-charge, after the mucting has atgreed o: the amount of the tithe to be commutud the amount of the assistance which the Commissioners may afford to a voluntary settlement, by sending to the meeting, on the application of the parish authorities, forms of agreement, and also in any Commissioner or Assistant Commissioner being at liberty to be present at the meeting, to give his advice; appointment of umpire to settle differences between valuers; their plans of operation, \&c. \&c. There are also provisions for enabling the tithe-payer to alter or shift the amount of apportionment at any future time. This, of course, does not affect the total amount of rent-charge, which must still be paid hy the parish, whaterer alteration may be made in the payments of particular lands or individuals.

Dissent from voluntary agreement.-After the total sum to be pait in liea of tithe has been agreed upon, and the apportionment in fragments to bo pail by individual parties declared, any one who conceires that he is unjustly apportioned may appeal to the Commissioners, at a meeting to be held in the parish for the purpose of hearing objections. The apportionment on partieular lands may also be altered at the request of the owner.

* * Various powers are given by the Act to the Commissioners for settling disputed claims.

Foluntary settlement begun but not crmyleted.- If a voluntary agreement has been made as to the amount of rent-charge to be paid in lieu of title, but nothing has been done in the way of roluntarily apportioning the amount to be paid by each party, the Commissioners may interfere after six months, and make a compulsory apportionment.

*** The Commissioners, when (after 1st October, 1838) they make a compnlsany awarl of the total amunat to be commuted, can permit a coluntary appostionment of it, if the parties agree.

How the Tithe is to be commuted into a Rent-Charge, and to be rend.-The 
commutation (i.e. the amount of produce) which has been agreed upon to be paid to the tithe-owner, is to be paid in money; but, in order that he may reap the benefits of the rariations of the markets, it is to be calculated how much equal parts of wheat, barley, and oats, the rent-charge would buy at the average price of the seren years preceding Christmas 1835; and the same number of bushels, and parts of bushels, are to be paid in money, yearly. The rearly payments are to be calculated on the arerage market-prices of the seven years preceding, which are to be published in the Gazette in January of every year, regulating the payments for the following July.

** If the average market-price for the seven years preceding the ycarly payment should rise higher than the average price for the seven years preceding Christmas, 1835 , the tithe-owner will be a gainer; if the contrary, a loser. The principle is evident:- as a certain amount of money will buy more corn when prices are low than when they are high, so the tithe-owner, receiving the yearly money-value of a certain quantity of corn, will get more money when prices are high than when they are low.

Liubitities of Rient-Churge.-Rent-charges commuted for tithes are liable to all parliamentary, parochial, county, and other rates. These rates are liable to be recorerer from the actual oceupier of the lands out of which the rent-charge issues, hut the amount can be deducted in paying the land-owner or tithe-owner.

How to recover the Rent-Charge when not duly paid.-By distress and entry; but no person is liable personally.

* * If the land be destroyed the rent-charge is lost.

Exceptions from the Operation of the Act.-Unless included by special morision. the following are to be exempterl from commutation agreements : Easter offerings, mortuaries or surplice fees, tithes of fish or fishing, personal tithes, (other than the tithe of mills, mineral tithes, tithes due in London, permanent rent-charges, or payments in lieu of tithes as settled by any custom in city or town, or by private Act of Parliament, or payments in lieu of tithes already commuted or extinguished.

Hop, Fruit, and Gimdrn Proluce, Enclosures, Glebe Lands, \&c.-Tithe of hop criunds, gardens, and orchards, may be ralued separately, on notice from the owner, and when they cease to be so cultivated may be charged at the ordinary charge. Lands in possession of privileged orders, or converted from barren heath within the seven years precerling 1836, or glebe lands, may be valued separately. The Act also subjects turnips serered from the land, if consumed on the same, to tithe as if not so consumed.

$*_{*} *$ The for groing rives the spirit of the $A c t$; but the provision made for exceptions to general rules, the mode of dealing with compositions, moduses, \& c., must be gathered by a reference to the Act itself. The tenant at rack-rent may refuse to consent to the commutation which his landlord may have to make, and in that case the landlord pays and takes tithes from the tenant. The tenant who holds under agreement to be exempt from tithes may pay the rent-charge, and deduct from his rent. There are penalties for false evidence, provision for expenses of witnesses, \&c. \& c. The Commissioners will doubtless attend to all complaints, and give all necessary information and instructiow. Their communications go postage free.

\section{Poor-RAte.}

The Statute 6 \& 7 Will. IV ., c. 96 , enacts, that in future all poor-rates shall be made on the net annual value of the property ; that is to say, of that rent at which the same might reasonably be expected to let from year to year, free of all usual tenants" rates and taxes, and tithe commutation rent-charge, if any, and cledueting therefrom the probable arerage annual cost of repairs, insurance, and other expenses necessary to maintain buildings in a state to command such rent.

The rate is to be made in the form thereby directed, and Poor Iaw Commissioners may order a new survey and valuation, empowering surveyors to enter and examine lands and buildings for such purpose; but nothing is to prevent owners from compounding for rates.

Rated inhabitants may take copies or extracts of rates grotis, under a penalty of refusal to permit of not more than $5 l$.

Justices, acting in petty sessions, to hold four special sessions in the year to hear appeals; and such justices may act with all the powers of justices in quarter-sessions.

The Act extends the period for the repayment of poor-law loans, made under the 4 and 5 Will. IV., c. 76 , from ten years to twenty, s. 107. 
A Report of the Commissioners under the Poor Law Amendment Bill states the progress of the parochial expenditure from its commencement to the present year, 1837, as follows:

\begin{tabular}{|c|c|c|c|c|c|c|c|c|}
\hline 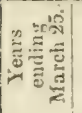 & 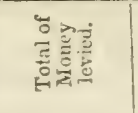 & 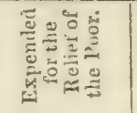 & 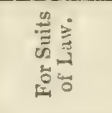 & 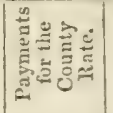 &  & 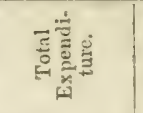 & 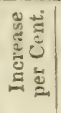 & $\mid \begin{array}{l}0 \\
0 \\
0\end{array}$ \\
\hline $\begin{array}{l}1823 \\
1833 \\
1834 \\
1835 \\
1836\end{array}$ & $\begin{array}{c}f . \\
8,622,920 \\
8,606,501 \\
8,338,079 \\
7,373,807 \\
6,356,345\end{array}$ & $\begin{array}{c}£ . \\
7,036,960 \\
6,790,800 \\
6,317,255 \\
5,526,418 \\
4,719,238\end{array}$ & $\begin{array}{c}£ . \\
254,412 \\
258,604 \\
202,527 \\
172,482\end{array}$ & $\begin{array}{c}£ . \\
\cdots \\
\cdots \\
705,711 \\
699,952\end{array}$ & $\begin{array}{c}£ . \\
1,646,492 \\
1,694,670 \\
1,713,489 \\
935,362 \\
823,241\end{array}$ & $\begin{array}{c}£ . \\
8,683,461 \\
8,739,882 \\
8,289,348 \\
7,370,018 \\
6,414,912\end{array}$ & $\begin{array}{c}4 \\
\because \\
\because \\
\cdots\end{array}$ & $\begin{array}{l}\because \\
\ddot{7} \\
13 \\
15\end{array}$ \\
\hline
\end{tabular}

Enclosure of Fielis.

An Act for facilitating the enclosure of open and arable fields in England and TVales recites, that there are many parishes, townships, and places, the lands of the several proprietors of which are frequently rery much intermixed and dispersed, and it would tend to their improved cultivation and oceupation of such land, if the proprietor's were enabled, by a general law, to divide and enelose the same. It consequently empowers the parties interested to enclose such open fields, with the consent of two-thirds in number and value of the proprictors, and reculates the proceedings of Commissioners to be appointed for that purpose.For the provisions of the enactment see Statute $5 \& 7$ IVill, IV., c. 115.

\section{TURNPIKE-ROADS.}

Rate-payer's keeping beasts of draught are not to be called upon in spring, seed-time, or harvest, to assist in the conveyance of materials for the repair of the highways; and no rate is to exceed, at any one time, the sum of $10 d$. in the pound, or $2 s .6 d$. in the pound in the whale, for any one year; 5 and 6 IVill. IV., c. $50^{*}$.

The owners and oecupiers of land adjoining to every turnpike-road are bound to cut, prune, and trim the hedges to the height of six feet from the surface of the ground; and also to cut down, lop, or prune the branches in such manner as not to exclude the air so as to damage the roar ; unless the trees be in a crarden, orehurd, or plantation, being a sheiter or ornament to a dwelling-house. On neglect or refusal to comply with notice to that effect by the surveyor, the ocenpier may be summoned before any justice of the peace, who may then order the work to be done within ten days; and, if the order be not complied with, he will be sulject to forfeit $2 s$. for every 24 feet in length of such liedge, and $2 d$. for every tree so ordered to be lopped, besides any charge which the surveyor may incur in its execution. 3 Geo. IV., c. $126 .$, s. 116.

No person, however, can be compelled, nor can any surveyor he permitted, to cut or prune any hedge, or lop any tree, between the last day of September and the last day of March. Ibid., s. 117.

The Act also compels the oceupiers of such land to kecp open and cleanse the ditches, so as to prevent injury or obstruction to the roads; under penalty for neglect, after ten days' notice, not exceeding $5 l$. Ibid., s. 113.

No doors or gates of any building or field can be made to open upon any turnpike-road or font-path, unless the hanging-post shall be so fixed as that no part of the gate shall, when open, project over any portion of the path. In case this be not complied with, and the necessary alteration completed within 14 days after notice from the surveyor, the owner may be summoned before any justice of the peace, and fined $40 \mathrm{~s}$., besides the cost of the alteration. Ibid., s. 125.

Neither may windmills be erected within 50 yards of any turnpike-road; nor any fire be kindled for the purpose of burning limestone, bricks, or clay, within less than 15 yards, unless screened by a sufficient fence.

Persons riching, ol driving any cattle upon the foot-path, or causing any wil-

* See an account of the chief clauses of the Turnpike Acts for Statute-labour throughout Scotland, in vol, i., p. 153 of this work. 
ful obstruction to the roarl, are subject to a penalty of $40 \mathrm{~s}$. over and above any damage which they may have occasioned. Ibid., s. 121, and 4 Geo. IV., c. 95, s. 69 .

\section{Carriages.}

The Christian and surname of the owner, and the place of his abode, must be painted in straight lines upon some conspicuous part of the off-side of every eirt or waggon in legible characters not less than one inch in height, under penalty of 5 l. 4 Geo. IV., c. 95 , s. 15.

Carters driving any eart or waggon on a turmpike-road, drawn by more than two horses, may not ride upon such carriage, nor be at such a distance from it, or in such a situation, as not to have the complete direction and control of the cattle drawing the same. He must also keep it on the left or near-side of the road, so as not to impede the passage of any other carriage; and, on demand, discover the name of the owner, if it be not painted on it: under a penalty of 40 s., if he be only the driver; but, if also the owner of the carriage, then of $5 \%$. 3 Geo. IV., c. 126, s. 132.

Any person may act as the driver of two carts on any turnpike-road, not being within ten miles of the city of Jondon, provided that they he drawn by only one horse each, and the horse of the hinder cart be attached by a rein to the back part of the foremost one, or if not, he is subject to a penalty of $20 \mathrm{~s}$. Ibid., s. 130 .

No waggon or cart travelling on a turnpike-roarl can be legally clriven by any person who has not attained the full age of thirteen years, under a penalty of $10 s .$, to be paid by the owner of the carriage. Ibid., s. 131.

\section{TolLs.}

By the same Act all waggons, earts, and carriages, and the cattle by which they are drawn, if employed in the conveyance of any species of manure to be laid upon the land, or of hay, straw, fodder for cattle, or corn unthrashed,except hay, straw, and corn carried for sale,-or carrying only one tree, or one log of timber, or materials for the repair of roads, are exempt from toll, bath going and returning. When going empty to procure such loads, the driver must, however, pay the toll : but the collector must give him a ticket, entitled a "ticket of exemption," on the production of which with the return load the money must be repaid: and if the collector refuses to give either the ticket or the return of the toll received, he is subject to a penalty not exceeding $5 l$, upon summons before any justice of the peace for the county in which the offence may have been committed Ibid., s. 27 and 28. But as disputes have arisen as to these exemptions from toll, it was enacted by Stat. 5 and 6 Will. IV., c. 18 , that after the 1st of January, 1836, that no toll shall be taken on any turnpike-road for any horse, beast, cattle, or carriage, when employed in carrying only dung, soil, compost, or manure for land, except lime, and the necessary implements used for filling the manure, and the cloth used in covering any hay, clover, or straw, conveyed.

The Act, however, does not exempt carriages upon roads which are specially made subject to toll by local acts : nor does it extend to Scotland or Ireland; Ib., s. 2 and 4. But all eattle going to or from pasture, or to be farried, are exempt, except at gates within six miles of London. 1 and 2 Will. IV., c. 25.

The weights hereafter specified are allowerl to every cart, wain, or wargon travelling upon turnpike-roads, together with their loads, viz. :-

Four-wheeled carriages, having the fellies of the wheels of

Winter.

the breadth of 9 iuches.

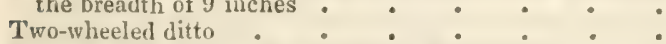

Four-wheeled carriages, with fellies of 6 inches :

Two-wheeled ditto - •

Four-wheeled carriages, having the fellies less than six

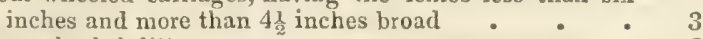

Two-wheeled ditto
Four-wheeled carriages, having the fellies of any breadth less than $4 \frac{1}{2}$ inches Tons Cwt.

Summer.

Two-wheeled ditto

$\begin{array}{rrrr}6 & 0 & 6 & 10 \\ 3 & 0 & 3 & 10 \\ 4 & 5 & 4 & 15 \\ 2 & 15 & 3 & 0 \\ 3 & 15 & 4 & 5 \\ 2 & 7 & 2 & 12 \\ 3 & 5 & 3 & 15 \\ 1 & 10 & 1 & 15\end{array}$


And for the several purposes of the Act, it is deemed summer from the 1st day of May to the 3 lst day of October, both days inclusive. 3 Geo. IV., c. 126, s. 12 .

Trustees of the roads are also empowered to collect, as additional tolls for crery ewt. which any carriage shall weigh over and above the weight already stated, viz. :For every cwt. above 2 cwt., and not exceeding 5 cwt. $\quad: \quad: \quad: \quad 003$

For every cwt. of such over-weight above 5 cwt., and not exceeding $10 \mathrm{cwt}$, for each civt. the sum of . And for every cwt. of such over-weight, exceeding $10 \mathrm{cwt}$. $\quad$ - $\quad$. 50 Payable at any weighing-machine upon the road in the same manner as the common tolls. Hbid., s. 11.

If neat cattle be used in carriages, then two oxen are considered equal to one horse in respect to toll. Ibid., s. 37 .

\section{Duty on Horses and Dogs.}

A farmer occupying land under the value of 500\% a-year may keep one riding horse free of duty, if only one be kept. But he must gain his livelihood chielly by farming, and not have any other source of income exceeding $100 l$. per anmum.

Husbandry horses, occasionally used in drawing burdens, ol occasionally let for drawing for hire, are exempt, if not used for drawing any carriage chargeable with duty.

Persons are exempt for one horse ridden by bailiffs, shepherds, or herdsmen.

The Duties on Dogs are-



Shepherds' or herdsmen's dogs, if solely used in the care of cattle and sheep, and not of any sporting breed, are exempt.

\section{Cattle.}

Persons wantonly ill treating any species of eattle may be summoned vefore any justice of the peace, and, if convieted upon oath, are subject to a penalty, according to circumstances, not under $10 s$. nor above $5 l$.; or, in default of payment, to be committed to the House of Correction for any period not exceeding three months. 3 Geo. IV., c. 71, s. 1.

Any person inpoundiner cattle is bound to fumish the animals with the necessary food, and has a summary remedy, by summons, against the owners for the expenses: but if he neglects to feed them, he is subject to a jenalty of 5 s. per day.

Any person may enter pounds for the purpose of feeding cattle which have leen confined without foor during twenty-four hours. 5 and 6 WVill. IV., c. 59.

\section{Man-Traps}

and spring-guns, which may occasion anybody personal harm, are probibited to be set, and every one proved to have done so is guilty of a misdemeanour; unless they be set in a dwelling-house for the protection of the house, and only from sun-set to sun-rise. The Act, howerer, does not extend to prevent the use of any gin" or trap, such as ma have been usually employed with the intent of destroying vermin. 7 and 8 Geo. IV., c. 18, s. 1 .

\section{Game-Laws.}

The word "Game," under the Act 2 Will. IV., c. 32, includes harcs, pheasants, partridges, grouse or red-game, heath or moor-game, black-cock, and bustards. 
For killing or taking any game, or using any dog, gun, net, or other engine for killing or taking crame on a Sunday or Christmas-day, or between the following days for the several species, namely:-



On conviction before two justices of the peace, a penalty for the first offence not exceeding 5l.: and for putting poison on any ground, open or enclosed, where game usually resort, a penalty not exceeding $10 l$; and if any person, duly licensed to deal in game, either buy or sell or have in his house any game after ten days from the respectire days on which it is urlawful to kill or take such game, he shall on conviction forfeit a sum not exceeding $20 \mathrm{~s}$. for erery head so bought or sold or found in his house.

The Act dispenses with qualifications, and allows every certifieated person to kill game; subject to proceedings for trespass.

Landlords may enter under any lease made prior to the Act, and authorize any certificated person to take and kill game upon the land; but tenants have no authority under any lease or agreement, except where such right has been expressly granted, or a fine shall have been taken, or the lease shall have been made for a term exceeding 21 years; and are subject to a penalty not exceeding 40 , , and for any head of game not exceeding 20s., for either lilling or permitting others to kill it.

The game on wastes belongs to the lord of the manor.

Penalty for destroying the eggs of game, swan, wild-luck, teal, or widgeon, or having them in the owner's house, is $5 s$. for every egg.

Trespassers entering in the day-time upon any land in search of game, or wood-cocks, snipes, quails, landrails, or conies, are subject to a fine of 40.5. and, if to the number of fire together, to $5 \%$. each, upon conviction before a justice.

Trespassers refusing to gire their name and address when required by tine occupier or any of his servants, or giving a false description, may be apprehended and conveyed before a justice, by whom they may be fined 51 . each; but they must be carried before the justice within twelve hours, though they may afterwards be proceeded against by summons.

Persons summoned by a justice, and not appearing at the time and place appointed, without a reasonable excuse for their absence, subject to a penalty of $5 \%$.

The prosecution for every offence punishable upon summary conviction must be commenced within three months; and persons aggrieved inay appeal to the quarter-sessions of the peace, on giving notice of such appeal within three days after the conviction, and at least seven clear days before the commencement of the sessions.

Persons roing out armed at night in search of game may be indicted; and, if convicted, are guilty of a misdemeanour.

\section{The Game Duty-}

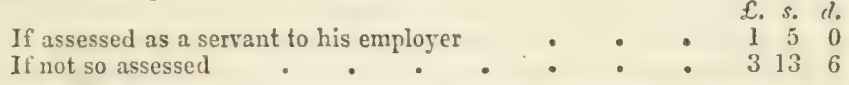

And persons omitting to take out the proper certificate are subject to a double assessment.

Dealers in crame must take out a $40 s$. license; but persons holding certificates of $3 l .13 \mathrm{~s} .6 \mathrm{~d}$. may sell game to licensed dealers. 


\section{Duties on Corn,}

When imported from any foreign country. -9 Geo. IV., c. 60.

WHEAT-Whenever the average price of wheat, made up and published in manner required by law, shall be $62 s$. and under $63 s$. per quarter, the $£ . s$. $d$. duty shall be for every quarter . . . . . . 148 Whenever such price shall be $63 s$. and under $64 s$, the quarter . . 135

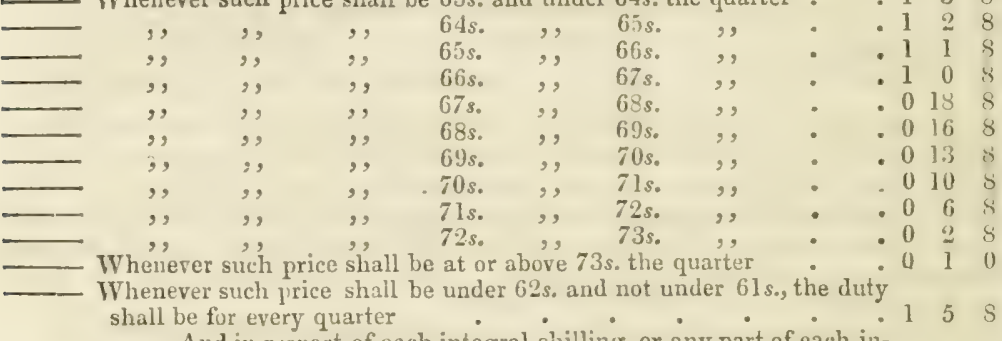

...... And in respect of each integral shilling, or any part of each integral shilling, by which such price shall be under 61 s., such duty shall be increased by one shilling.

BARLEX - Whenever the average price of barley, made up and published in manner required by law, shall be $33 s$. and under 34 s. the quarter, the duty shall be for every quarter shall be above $33 s_{\text {., }}$ such duty shall be decreased by $1 s .6$. , until such price shall be $41 s$.

Whenever such price shall be at or ahore 41 s. the quarter

Whenever such price shall be under 33 s, and not under $32 s_{\text {s. }}$ the duty

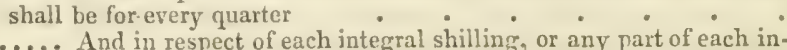

..... And in respect of each integral shilling, or any part of each inshall be increased by $1 s .6 d$.

OATS- Whenever the average price of oats, made up and published in manner required by law, shall be $25 \mathrm{~s}$, and under $26 \mathrm{~s}$. the quarter, the duty shall be for every quarter

...... And in respect of every integral shilling by which such price shall be above $25 s$. such duty shall be decreased by $1 s .6 d$, until such juice shall be $31 s$.

Whenever such pice shall be at or ahove $31 s$. the quarter

Whenever such price shall be under 25 s., and not under 24 s., the duty
shall be for every quarfer shall be for every quarfer ${ }^{\circ}$... And in respect of each integral shilling, or any part of each integral shilling, by which such price shall be under 24 s., such duty shall be increased by $1 s .6 \mathrm{~d}$.

Rye, Prase, and Beans-
Whenever the average price of rye, or of pease, or of beans, made up and published in manner required by law, shall be $36 s$., and under

$37 s$. the quarter, the duty shall be for every quarter
$\ldots . .$. And in respect of every integral shilling by which such price shall be above $36 s$., such duty shall be decreased by $1 s .6 d$, until such price shall be $46 s$.

Whenever such price shall be at or above $46 s$. the quarter
Whenever such price shall be under $36 s$, and not under 35 s, the duty shall be for every quarter $\cdot . .$. And in respect of each integral shilling, or any part of each integral shilling, by which such price shall be under 35 s., such duty shall be increased by $1 s .6 \mathrm{~d}$.

Theat, Meal, and Frour-

For every barrel, being $196 \mathrm{lbs}$, a duty equal in amount to the duty payable on $38 \frac{1}{2}$ gallons of wheat.

Oaruear - For every quantity of $181 \frac{1}{2}$ pounds a duty equal in amount to the duty payable on a quarter of oats. 
Matze or Indian Corn, Buck Wheat, Beer, or Biga-

- For every quarter, a duty equal in amount to the duty payable on a quarter of barley.

IF TIE PRODUCE OF AND IMPOITED FROMI ANY BRITISH POSSESSION IN NORTII AMERICA, OL ELSEWIKEIR OUT OF EUIROPE.

...... Until the price of British wheat, made up and published in manner required by law, shall be 67 s. per quarter.

Whenever such price shall be at or above $67 s$, the quarter $\quad .006$

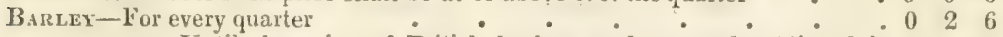

...... Until the price of British barley, made up and published in manner required by law, shall be 34 s, the quarter.

Whenever such price shall be at or above $3 \pm s$. the quarter $\quad . \quad 0 \quad 0 \quad 6$

OAts-For every quarter

...... Until the price of British oats, made up and published in manner required by law, shall be '25s. per quarter.

Whenever such prices shall be at the above $25 s$, per quarter $\quad . \quad 0 \quad 0 \quad 6$

Ryis, Pease, and Beans-

— For every quarter . . . . . . . . . 030

...... Until the price of British rye, or of pease, or of beans, made up and published in manner required by law, shall be $41 s$.

Whenever such price shall be at or above $41 \mathrm{~s}$. the quarter

Wirfat, Mein, and Froulz-

For every barrel, being 196 pounds, a duty equal in amount to the duty payable on $38 \frac{1}{2}$ gallons of wheat

O.xmeat - For every quantity of $181 \frac{1}{2}$ pounds, a duty equal in amount to the duty payable on a quarter of oats.

Matze or Iniman Corn, Buck Wreat, Besr, or Bigg-

For every quarier, a duty equal in amount to the duty payable on a quarter of barley.

No. II.

\section{LANDLORD AND TENANT.}

Farm LAND, if not taken upon lease, is hired indefinitely by the year, and an intention to quit must be notified in writing by either party-whether landlord or tenant-six months previous to the expiration of the annual term; so that it may fall precisely at twelve months from the time of entry; but, if held upon lease, the expiration of the term being then defuitely fixed, the tenancy expires on that day, without any further notice. The tenant should, therefore, be ready to deliver up possession; for if he continue to hold the land or any of the buildings, without the customary covenants hy which some of then are occasionally retained, he will be subject to double their annual value; or, if he holds out after a notice to quit given by himself, to double rent.

On yearly tenuncy, the landlord is bound to keep the gates and buildings in tenantable repair, and if he refuse to do so upon application by the tenant, the latter may have the necessary repairs effected, and deduet the amount from the rent. If tuken on lease, the homestead and fences are usually put in thorough repair by the landlord, and the tenant is then bound to lieep the premises in tenantable order, or he will be subject on the expiration of the term to damages for dilapidation.

In most leases of land there are covenants inserted regarding the modes of cropping; and they should be carefully attended to, for, if infringed, they may oceasion a forleiture of the lease. Even in common agreements, without any stipulation respecting cropping, the farmer is bound to cultivate the land in a husbandman-like manner, according to the common custom of the country; and if he rommit waste or injury to the ground by breaking up old meadow, or by repeatedly growing white corn without intermediate green crops or fallow, as well as by neglect of the usual drainage and fences, he will be subject to damages.

All agreements should be in writing, and if of the value of $20 \%$ and upwards, containing only 1080 words, should bear a stamp of $20 \mathrm{~s}$; if of more than 1080 words, $35 \mathrm{s.}$; and for every farther 1080 words, $25 \mathrm{~s}$. 
Notices should also be in writing, and delivered to the party to whom they are addressed, or left at the house of such party, by some indifferent person, who, in case of dispute, may be produced as a witness in a court of law.

The entry to furms is in Great Britain usually made at the different festivals known as "quarter-days," which are thus fixed-

In Fingtand and Iretand.

Lady-day is the 25th of March,

Midsummer, 24th, June.

Nichaelmas "29th, September.

Christmas ", 25th , December.

Entries are also sometimes agreed upon for certain purposes on May-day; and it is not uncommon, even at this time, to stipulate Old May-day, or the 12 th, instead of the Ist of the month, according to the custom which existed previous to the change occasioned by the introduction of the new style.

The payment of rent for land is usually stipulated for half-yearly, but is seldom called for until the following quarter; and, on some estates, it is customary to allow the tenant a full half-year's credit for the rent already due; thus only collecting half a-year at the end of a twelvemonth; unless the tenant should give up his land, in which case he must be ready to pay his rent before he removes his crops. In order to guard against a fraudulent removal of the property, a clause is, however, generally inserted in the lease rendering the rent payable forty days previous to its expiration.

In Scotland, the landlord has what is called "the right of hypother," under" which he has a preferable lien upon the crops until payment of the rent for the year in which they were produced; and upon the stock until three months after the expiration of the last term of payment.

If rent, king's taxes, or parochial rates be levied by distress, the broker, or person lerying the same, must deliver a signed copy of his cost and charges to the person whose goods have been seized; and in case the amount to be levied does not exceed the sum of $20 \%$. the legal costs are limited to the following, viz, :-

Levying distress • • • • 30

Man in possession, per day, if the goods he not removed, and they cannot be sold until after the fourth day - value of the groods,

Appraisement, sixpence in the pound on the value of the goods, together with a stamped receipt for the amount

Expenses of advertisements, if any such : $: 10$

Catalogues, sale and commission, with delivery of goods, one shilling in the pound on the net produce of the sale.

If the above be exceeded, the broker or person levying may be summoned before any justice of the peace, and if the complaint be proved he will be subject to treble costs, or be committed to prison. 7 and 8 Geo. IV., c. 17.

If the sum levied be of larger amount than $20 \%$, or if the goods seized and sold be of much greater value than the rent or taxes due, then the party aggrieved has his remedy by action.

If he disputes the rlebt, or wishes to gain time for the settlement, he must then replevy the goods, which is done ly giving two substantial householders as securities to the sheriff in a bond for the prosecution of the suit and the pay ment of the claim, or the value of the goods distrained if less than the amount: of the rent claimed, and costs, if it be substantiated.

If a tenant refuses to quit the land on the expiration of his lease, or after due notice to quit, the landlord may, without any process, enter and take possession; but if opposed, or if for any other reason he does not choose to act in this summary manner, he must then proceed to the recovery of prosessio" by cjectment; and if entitled to possession, the judge will thereupon issue a writ of possession immediately, - I IVill. IV., c.70, s. 38 ; but, if he be nonsuited, or "lose a verdict on the merits, he will be subject to double costs.-1 Geo. IV., c. 87, s. 6.

In case of the tenant deserting the premises during six weeks, without leaving sufficient effects to cover the rent by distress, possession may be summarily obtained upon affidavit of the circumstances made upon application

* This, although a movable feast, is fixed for entries at the 15th of May. 
to two justices of the peace, who will grant their warrant for that purpose upon proof that notice of the application has been affixed upon the gate of the premises. -57 Geo. III., c. 52 .

No sheriff or other officer taking furming stock in erecution of jud grment for. debt is authorized in carrying off any straw, manure, or crops of hay or grass, whether natural or artificial, turnips or other roots, which, by the covenants between the landlord and the tenant, are to be consumed upon the ground. But the sheriff may dispose of the above to any person who shall agree in writing to use the same on the land in such manner as accords with the custom of the country; and, after such sale, he may assign to such purchaser the use of all snch buildings as may be necessary for the consumption of such crops during the requisite time-56 Geo. III., c. 50, s. 1. This pernission does not, however, extend to crops which the tenant is not bound to consume upon the ground. Ibid., s. 8.

No. III.

\section{ON WEIGHTS AND MEASURES.}

Trr: want of a general uniformity in weights and measures was firmerly so great that in many parts of the kingrlom those in common use were unknown in uther districts. Thus the boll, which is the invariable denomination of the measure employed in Srotland, differs in its contents in almost all the counties; and wheat is sold by one boll, oats by another, and meal by a third. Corn is, in many parts of England, measured by the Imperial as well as by the Winchester bushel, which in some counties-as in Cornwall-consists in one place of the double Winchester of sixteen gallons, and in another of the treble Winehester of twenty-four; while in some markets it is sold by weight, and the quarter sometimes consists of nine, instead of eight bushels. Potatoes are subject to three different weights-120 lbs., 126 lbs., and $132 \mathrm{lbs}$.- each called a hundred; and the sack, when measured by the farmer, holds three heaped bushels, weighing upwards of $200 \mathrm{lbs}$, while in the markets it only contains $1 \frac{1}{2}$ cwt. Butrer is in some towns sold by the pound of eighteen ounces, and at others it is measured by the rarci; and in many cases the long and the short hundred are universally employed.

These variations have been long complainer of, as being snares to the ignorant, and equally injurious to the individual as woll as the community. The grierance will now, it is howerer hoped, be gradually remedied, when the prejudices derived from old habits give way to common sense and law, as an Act -the 5 and 6 Will. IV., c. 63-has been passed by which all local and customary weights and measures are abolished, and none but those hereafter described can now be considered legal, or enforeed in any contract for sale or purchase.

The wright of all artieles sold-except the precious metals or jewels, and drugs, for which Troy weight may be still used-must be that known as "Aroirdupois;" the pound of which containing 7000 grains, while that ealled "Troy" cuntaius only 5760 grains, is comparatively equal to $1 \mathrm{lb} .20 \mathrm{zs} .11 \mathrm{dwts} .16 \mathrm{grs}$. Aronnupors iVeigrit.

2731 Grains are 1 Drachm $\left(d r_{0}\right) \mid 28$ Pounds are 1 Q11arter (qr.)

16 Drachms , 1 Ounce (oz.) 4 Qrs. ,, 1 Hundred Weight (cwl.)

16 Ounces ,, 1 Pound (lb.) 20 Cwt. ,, 1 Ton (T.)

The stome weight must in all cases consist of 11 llos.,- thus doing away with the several stones in local use; but, although the weight of the pound and the stone must be accurately stated in all agreements, yet the stone of meat is still distinguished by English salesmen and butchers as consisting of 14 lbs. lire, and slbs. dead-weight. A Among sereral of the country trades, also, different quantities of various goods are technically held to constitute a certain fixed amount, as among-

Woot S'TAPLERS.

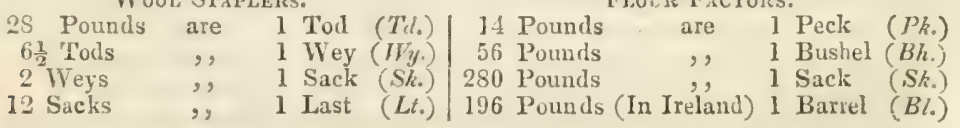

* See Vol, ii, 1. 392; and on the mode of ascertaining the weight by measurement' sse p. 393. 


$\left.\begin{array}{rl}8 \text { Pounds are } 1 \text { Clove }(\mathrm{Cl} .) \\ 32 \text { Cloves }, 2 & 1 \text { Wey }\left(W_{y_{0}}\right)\end{array}\right\}$ Cheese.

Among farmers, laty of every kind is in England usually sold by the ton ; in Scotlind, by the stone of $24 \mathrm{lbs}$; and in Ireland, by the car-load of rather uncertain weight, without being trussed. In most of the English markets, huwerer, both hay and straw are sold by the load of 36 trusses; each of which weighs-

lbs, cwt. qr. lb.



and hay is considered as being new until St. Luke's day-the 18 th of October.

Many articles are also sold by tale of -

12 to the Dozen.

12 Dozen to the Gross, and

120 Ditto to the Great Gross.

20 to the Score.

5 Score to the short, and

6 Ditto to the long Hundred.

Thus, 5-feet laths have 5 score, and 4 -feet do. 6 score to the hundred.

A load of tiles contain 1000, and a load of bricks 500 .

Measures of Gulk are ordered by the Statute to be regulated by the Imperial standard bushel, of a circular form, is $\frac{13}{2}$ inches in the interior diameter by 8 inches deep, containing 2218 and a fraction of cubic inches; while the former Winchester bushel is only $18 \frac{1}{3}$ in diameter, by 8 inches in depth, containing 2124 cubic inches.* They are each stated to hold 8 gallons, but their real contents in weight of water, of which a cubic foot contains as nearly as possible 1000 ounces avoirdupois, are-

The Imperial bushel, exactly $80 \mathrm{lb}$.; the Winchester ditto, about $77 \frac{1}{2}-$ difference, $3 \frac{1}{8}$ per cent.

The fiar prices of grain in Seotland are to be struck by this Imperial standart, which is now the sole legal measure of capacity throwghout the United Kinglom; and all heaped measures are strictly prohibited. The divisions are thus - -

\begin{tabular}{|c|c|}
\hline IMPERIA & NDARD. \\
\hline $\begin{array}{l}2 \text { pints are } 1 \text { quart (Qt.) } \\
4 \text { qts. , I gallon (Gall.) } \\
2 \text { galls., I peck (Pk.) }\end{array}$ & $\begin{array}{l}4 \text { pks. are } 1 \text { bushel }(\text { Bush. }) \\
8 \text { bush. }, 1 \text { quarter }(Q r .) \\
5 \text { qrs. }, 1 \text { load }\left(L l_{0}\right)\end{array}$ \\
\hline
\end{tabular}

Though not legally acknowledged as a measure, 2 quarts are accounted as a puttle. Corn is also frequently measured by the comb of 4 bushels ; $t$ and the sack of coln likewise contains 4 bushels: but potatoes, lime, culm, coal, or other bulky goods are sold by the sack, which is only estimated to hold 3 hushels. 12 sacks are a chaldron, containing 36 bushels; which is the measurement of lime.

The measure of liquids, as applied to ale and cider, are-

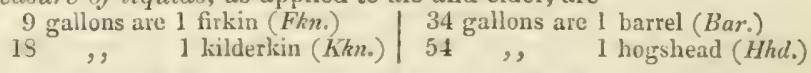

* This was the measurement established in 1701 by Will. III. and is the old staulard bushel in the Fxchequer, Lomdon; but the trallon measure in the Exchequer would contain 2168 , and our Revenue laws have for a long time assumed 2150 inches as the Winchester standard. Although the medsurement of the Imperial standard, stated as above, is that usually employed, yet, provided it contains the exact number of culic inches, the shape is nut regulated by law; though it has been proved by experiment that wheat, measured in a bushel made 11 inches deep weighed six ounces more than the same wheat measured in a bushel 8 inches deep: thus naking a difference of abuut 3 per cent.

$\uparrow$ The old Scotch corn measures for oats and barley were-

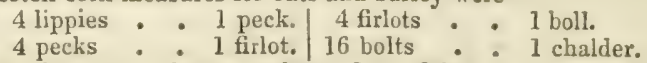

And in wheat, peas, beans, and rye, 3 oat firlots were equal to 1 boll.

The Linlithgow firlot contains 21 pints (Scotch), and 1 mutchkin, or $\frac{3}{4}$ of a pint, for wheat; and that for barley is 31 pints, - The boll of oatmeal weighs $1.10 \mathrm{llus}$. 
Lineal measures are severally distinguished as appertaining to length, surface, solidity, and depth.

\begin{tabular}{|c|c|c|}
\hline \multicolumn{3}{|c|}{ Long Mrasure. } \\
\hline 3 barl & are 1 inch $(I n)$. & s. are $1 \mathrm{r}$ \\
\hline $3 \mathrm{ft}$ & $\begin{array}{l}, 1 \text { foot } \\
, 1 \text { yard }\end{array}$ & $\begin{array}{l}40 \text { rds. , , } 1 \text { furlong (Fur.) } \\
8 \text { furs. , , } 1 \text { mile, or } 1760 \text { yüs. }\end{array}$ \\
\hline
\end{tabular}

In common usage an ell is 45 inches in length: the height of horses is measured by the hand of 4 inches; and the fathom, of 6 feet, is applied to depth.

\section{Square Measure}

applies to both length and brearlth, and the superficial contents of the square are ascertained by being multiplied by itself; thus a foot which consists of 12 inches, when multiplied by 12 , are

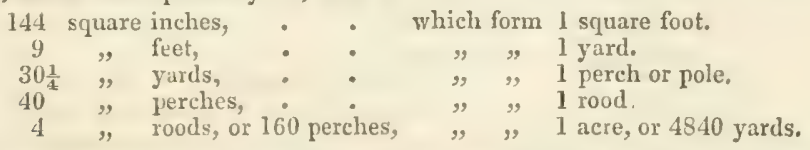

The superficial extent of land is usually computed in perches, roods, and acres, and measured by Gunter's chain, which is 22 yards, or 66 feet in length, and is invided into 100 links of 7.93 inches each; and the square of this chain being 484 yards, 10 such squares are equal to an acre.

By an Act passed in the 5th Geo. IV., for the equalization of measures, it was decreed "that the rood of land shall contain 1218 square yards, and the acre of land 1840 square yards, being 160 square perches." This, which is now the stutute acre of the Cinied Kingdom, by which alone the quantity or land to be stated in deeds, agreements, and assessments can be legally measured, is only confirmatory of the old English acre as established by Edward I., of which the perch is $5 \frac{1}{2}$ yards in length, or 301 square yards; but the Scoteh perch is 6 yards long, or 36 square yards, contrining 5760 square yards; and the Irish perch is 7 yards long, or 49 square yards, containing 7840 square yards. They, therefore, bear to the English acre in the following proportion, viz.:-

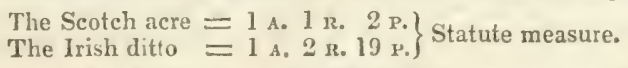

The dirisions of the Irish acre, though differing in size, are called by the same names as those of the English acre, but those of Scotland are-

$$
\begin{gathered}
9 \text { square feet, } 1 \text { square ell }\left(E_{0}\right) \mid 40 \text { falls, } 1 \text { rood }(\text { Ro. }) \\
36 \text { square ells, I fall }(F a .) \\
*_{*} * \text { The Scotch ell is } 37 \frac{1}{5} \text { inches in length. }
\end{gathered}
$$

There are also in many countics of England customary acres, by which the

\begin{tabular}{|c|c|c|c|c|c|c|c|}
\hline & & R. & $P_{0}$ & & & A. & IR. \\
\hline & 12 & 1 & $\begin{array}{l}14 \\
20\end{array}$ & oerland & & 8 & 2 \\
\hline 'urbeck & 119 & 2 & 20 & Lancashire & & 61 & 2 \\
\hline . & 84 & 0 & $4 *$ & Cheshire and & Stafford & 47 & 1 \\
\hline
\end{tabular}
measurement of land has been formerly regulated, and are still occasionally used in the letting of farms; of which the following slows the quantity of each equivalent to 100 Statute acres, viz.:-

In most places, also, a Hop-acre is that space of ground which contains 1000 plants, or about half a statute acre; a Word-acre is three-eighths larger than a statute acre: and a Lug contains 49 square yards of coppice. A perch of walling is of the same length as the statute perch- $16 \frac{1}{2}$ feet; but a perch of fencing is seren yards long; and, in the midland counties, the running measure for hedging and ditching is eight yards.

* The customary perch in Cornwall-as stated in Morgan's Survey of the County-is the same as that of Scotland, namely, 18 feet: but, accurding to Carew, the old Cornish acre, now disused, contained a much greater number of statute acres. 
The mumber of acres in Great Britain are-according to the Third Report of the Emigration Committee-77,394,433, in the following portions and condition -

\begin{tabular}{|c|c|c|c|c|c|}
\hline & & Cultivated. & $\begin{array}{l}\text { Uncultivated } \\
\text { but capable of }\end{array}$ & Wastes. & Total. \\
\hline England & - & $25,632,000$ & $3,454,000^{\circ}$ & $3,256,400$ & $32,342,400$ \\
\hline Wales & & $3,117,000$ & 530,000 & $1,105,000$ & $4,752,000$ \\
\hline Scotland & & $5,265,000$ & $5,950,000$ & $8,523,930$ & $19,738,930$ \\
\hline Ireland & & $12,125,280$ & $4,900,000$ & $2,416,664$ & $19,441,944$ \\
\hline Brilish Is & ands & 383,690 & 166,000 & 569,469 & $1,119,159$ \\
\hline
\end{tabular}
land-

In England and Wales it is calculated that there are in profitable farming

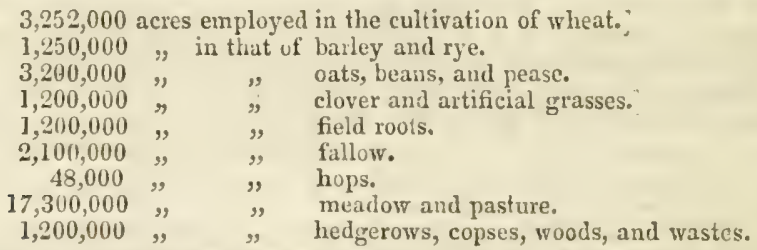

And the annual property derived from agriculture in the United Kinglom has been estimated at $216,817,624 l$.

\section{Solid Measure}

includes both length, breadth, and depth; and the cubic contents of anything are found by multiplying it tuice within itself. Thus, 144 inches are 1 square foot, and that, being again multiplied by 12 , will amount to

1728 cubic inches, forming 1 cubic foot (Cub. ft.) and

27 cubic feet are 1 cubic yard (Cub. yd.).

By this measure the manure of farm-yard dung and composts are estimated the heap of 27 cubic feet, or 1 cubic yard, which is called a load, being usually regarded as 1 ton. The length, breadth, and depth of the heap are to be taken in feet and inches, multiplied into each other, and divided by 27 , in order to ascertain the cubical yards and nominal weight. Its actual weight must, howerer, depend upon the solidity of its contents; for, as fresh stable dung lies loosely together, it will not weigh so heavily as that which has been rotted. The cubic contents of sand, or any porous earth, will of course also prove lighter than clay.*

The load of timber, if in the rough, is 40 cubic feet; if squared, 50 cubic feet.

$$
\text { A cord of fire-wood is }\left\{\begin{array}{ll}
8 \text { feet long, } \\
4, & , \text { broad, } \\
4, & , \text { high, }
\end{array}\right\} 128 \text { cultaining } \text { ft. }
$$

\section{No. IV. \\ REMARKS ON THE WEATHER.}

(From the British Almanac.)

THE BAROMETER, as commonly met with, is very imperfectly constructed; but, nevertheless, will answer tolerably well the purposes of a weather-glass. People, in general, pay too much attention to the words fuir, ruin, sturmy, Sc., cngraven upon the seale; and it is a common error to imagine that, as long as the mercury, or the index which marks its course, points to those expressions, the weather will be in accordance with them. The most important fact, howerer, to be ascertained in making an observation is, whether the mercury is in the act of rising or falling; as, if it ascends, although the index point to the word rain, it is a much surer indication of fine weather than when the index points to fuir, and the metal descends. The point may generally be ascertained by gently tapping the instrument; which, freeing the mercury from a slight alhesion to the glass, allows its freer motion, and instantly shows its upward or downward tendency. 
Rain is most plentiful, and thunder most frequent, when the quicksilver fluctuates a little below the average. If it descend rapidly and considerably below this point, storms of wind are indieated, while, in proportion as it rises above the same point the probability of fair weather increases. Settled weather must not be expected while the column of mercury is below its mean height for the month. Sudien and considerable changes in either direction are commonly followed by fair or foul weather equally transient; while a steady rise from day to day of about a tenth of an inch in twenty-four hours, or a prolonged fall in the same proportion (either of them passing the mean point), may grenerally be trusted as prognosties of continued fair weather or rain.

The Hygroneter, by which the state of the atmosphere, with regard to moisture, is ascertained, is a contrivance denoting the degree of temperature at which moisture begrins to be deposited upon a cold body; as we see in summer in the familiar instances of a bottle of wine brought from a cellar, or a decanter. of water fresh filled from a well. This degree is called the dew-point; and from it the degree of dryness may be accurately calculated. Tables of the mean averages of temperature for a large circle round London have been calculated, and are referred to in the Almanac.

The Thermoneter in general use throughout this country is that known as Fahrenheit's; but the one commonly employed on the Continent is that formed by Réaumur, and constructed upon a different scale. Though the degrees of heat in the atmosphere are not universally attended to, yet in many farming operations the precise state of ternperature should be known, and no wellregulated farm-house should be without the means of ascertaining it. The temperatures of the boiling and freezing points are, in the above thermometers, thus stated :-

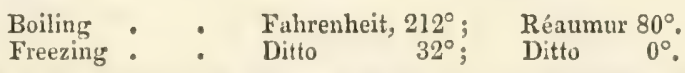

Each degree of Fahrenheit's is therefore equal to ${ }_{9}^{4}$ of that of Réaumur.

It is well known to the agriculturist and gardener that, without the direct influence of the sun, whatever may be the temperature of the air, the fruits of the earth seldom come to perfection. The different duration of the day, and the different height of the sun, are the causes of those variations of natural heat which diversify the seasons of the year; it is therefore of importance to know the force of this important agent, and the modifications to which it is exposed.

The best thermometers for this purpose are those upon Rutherford's construction: one of which, formed of mercury, pushes a small pin of iron wire before it, which it leaves at the highest point; and the other, of spirit, draws after it a small index of glass, which remains at the lowest. In addition to the mean temperature, and the highest and lowest points, or range of the thermometer in the shade, the indications are giren of a register thermometer covered with black wool, and placed in the sun, and of another placed at night upon a plat of short grass, and exposed to the full aspect of the heavens. The black thermometer registers this effect, and shows both the mean and extreme difference between such a thermometer and another placed in the shade.

Of not less consequence is it to know the cold produced at night from the radiation of heat from the surface of the earth, in situations where its passage is uninterrupted to the clear sky. This in caln unclouded weather always reduces the temperature of filamentous substances, such as grass, considerably below that of the air. It is this depression of temperature which is the cause of dew. The surfaces of regetables become cooled by this radiation of heat into space, below the dew-point, and the vapour of the atmosphere is deposited upon them. Dew is never formed upon grass on cloudy nights, or in sheltered situations.

It will be found that vegetation is liable to be affected at night from the influence of radiation, by a temperature below the freezing point of water, ten months in the year: and that even in the two months July and August, the only exceptions, the radiant thermometer sometimes falls to $35^{\circ}$.

The formation of dew is one of the circumstances which modify and check the refrigerating efiect of radiation; for as the vajour is condensed, it gires out the

VOL. II. 
latent heat with which it was combined in its elastic form, and thus prevents an excess of depression which might, in many cases, prove injurious to regetation.

The cxcess of either solar or terestrial radiation is injurious to many tender plants and flowers, and for this reason the careful gardener guards against them by mats and screens.

That the different PHAses of Tine Moow have some connexion with changes in the atmosphere is an opinion so universal and popular as to be, on that account alone, entitled to attention. No observation is more general, and on no occasion, perhaps, is the Almanac so frequently consulted as in forming conjectures upon the state of the weather. The common remark goes no further than the fact, that changes from wet to diy and from dry to wet generally happen at the changes of the moon. When to this result of universal experience we ard the philosophical reasons for the existence of tides in the aërial ocean, we cannot doubt that such a connexion exists, and that the moon exerts a considerable influence upon the currents of the atmosphere, according to her position. The subject, howerer, is involved in great obscurity, and is one which can only be elucidated by lung and careful observation.

The moon's are is calculated in days, and the nearest tentlis of clars, from the time of change: thus if it be new moon on the 6th of January, at $11 \mathrm{~h}$. $46 \mathrm{~m}$. in the afternoon, therefore at noon on the sth she will be $36 \mathrm{~h} .14 \mathrm{~m}$. old; which is set down as one day and five-tenths.

The YeAr is divisled into snlar and lumur months; the former consisting of twelve, as named in the calendar, while the latter comprise each nearlyand for legal and practical purposes precisely - four weeks. The alteration of the Juliun year, as it was formerly called, to our present new style, was adopted in this country in 1752, in consequence of the calculation of sular time having been found erroneous, and the date, as then existing, was changed from the 3rd to the 14th of September; thus forming a difference of eleven days in the current year. The necessity of any future alteration of date is, however, guarded against by the recurrence of a leap-year once in four, in order to check the annual excess of a few hours in the solar year; for although it does not exactly correct the error, yet a long period must clapse ere the difference of time will be so great as to cause the change of a day in the date.

The changes of the seasons are considered to commence in quarters, with very little variation : those of the present year, 1837, begin as follows :-

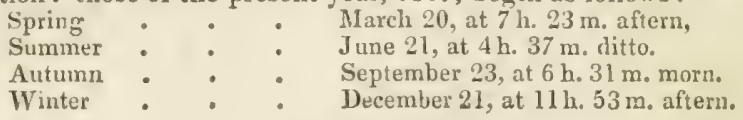

No. V.

A promise was marle in the Memoranda annexed to the Introduction that a Glossary of the common technical terms used in provincial husbandry, and the botanical names of the plants mentioned in the work, wouln be given in the Appendix: it was, howerer, fourd more adrisable to explain the provincial terms as they occurred, and to the list of grasses is added their botanical appellations. To this we now add the enumeration of a few of the most common and troublesome weeds-as inserted in Aiton's Survey of Ayrshire?

\section{WEEDS.}

Winss, Clex Europeus, covered in former times a very large proportion of the county of Ayr, and whercver eultivation is neglected, especially on the dense clay soils, this plant springs up in great force.

Broca, Spartium Scoparim, never fails to establish itself on dry open ground, when cultivation is neglected; and to cover the soll so cluse as to overwhelm and bands the more valuable herbagre.

Hazer, Corylus Avellana; вrambre, Rubus Fructicosus; and Fenn, Plevis Aquilina ; oceny dry ground that has never been broken up, the two furmer in the banks and braes, and the latter everywhere. Wherever the two first grow, the oak would grow if planted and protected. And wherever fern is met with, forest wood would also grow. 
The followingare found in the corn-fields, and cultivated land: not put down in botanic order, but according to the trouble they give and injury they to to the farmer.

BOTANIC NAIES.

Serratala Arvensis,

Carduus Lanceolatus, Palustris,

Sonchus Arvensis, Oleraceus,

Trilicum Repens,

Hulcus Mollis,

Agrostis Alba,

_. Stolonifera,

Avena Elatior,

Ononis Arvensis,

Rumex Acutus,

\begin{tabular}{c} 
Oblusifolium, \\
Crispus, \\
Acetose \\
\hline Senecia Jacobel \\
- Vulgaris,
\end{tabular}

Chrysanthemum Segetum, Leucantherm

Artemisia Vulgaris,

Tussilago Farfara, Hybrides, Petafeles,

Achillea Plarmica,

Veronica Agrestis, Arvensis,

Spergula Arvensis,

Raphanum Raphanistrum, Nigra,

Sinapis Arvensis, Alsine Media,

Potentilla Anserina,

Lamum Purpureum,

-...- Amplexicaule,

Galeopsis Tetrahit,

- Grandifolia,

Stachys Palustris, Arvensis,

Equiseturn Arvensis, Polygonum Amphibium,

- Persicaria, Hydropiper, Convolvulus,

Fumaria Officinalis, Mentha Arvensis,

Euphorbia Poplus, Helioscopia,

Chenopoduce.

Papaver Rhaas,

\section{- Dubium,}

Ranunculus Repens, Acris or

Lapsana Communis,

Bionium Bulbocastonium,

Allium Aparine,

Centuavia Niara,

Urtica Dioica,
IROVINCIAI, NAMES.

Corn Thistle,

Bur ditto,

Marsh ditto,

Large sow ditto,

Anumal ditto ditto,

Couch grasses,

Pearl grass,

Rest harrow,

\} Common dock,

Curled dock,

Common field sorrel,

Narrow-leaved ditto,

Bin-weed or rag-weed,

Groundsel,

Gule,

Ox-eye daisy,

IVild Camomile,

Mugwort,

Coltsfoot,

Water-duck,

Butter-bur,

Sneezewort, hard head,

Speedweel,

Field ditto,

Yar, or corn spurry,

Charlock, or runch,

Black mustard,

Charlock, or wild mustard,

Chickweed,

Silver-sveed, or mascorn, \}

Archangel, or dead nettle,

(All-heal, clowns - wort, or cockhead,

Mar-tail, puddock-pipe,

Red-shanked arsmart,

Arsmart with spots,

Pepperwort arsmart,

Climbing arsmart, or hopweed,

Common fumitory,

Field-mint,

Devil's churnstaff, or petty spurge,

Sun-spurge,

Goose-fout, wild spinage, or mails,

Round-headed corn-poppy,

Lonr-headed ditto,

Creeping crow-foot,

Upright crow-foot,

Nipple-wort,

Earth-nut,

lobin run-hegde,

Horseknot, knapweed,

Nettle,
WIERE FOUND.

Every soil, not moist. Do.

Moist soil.

Every soil, not moist. Do.

In cultivated lands.

Every soil, not moist.

$$
\text { Do. }
$$

Every soil.
Do.
Do.
Do.

New pasture.

Dry soil.

Corn-fields.

New pasture.

Do.

Corn-fields.

Thin soils.

In holms.

Do.

Corn-fields.

Do.

Do.

All in corn-fields and gardens.

All in corn-fields and gardens,

D amp places.

Corn-fields.

Good land.

Neglected ground.

In feuces and in neglected places.

$$
\begin{aligned}
& \text { Do. } \\
& \text { Do. } \\
& 202
\end{aligned}
$$





\section{N D E X.}

\section{(The Numerals refer to Vols. I, and II.)}

A Bus Howne, in Cumberland, mode of fatting calves for veal at, ii. 447.

Accounts of farmin business, observations on the mode of keeping, i. 113.

Achillea Millefolia, or Yarrow, i. 513.

A gistment, and animal tithe, laws of, and mode of settling the money accounts, i. 76,77 .

Agriculture, importance of, i. 1. Advantages in the pursuit of, 2. Profits of, 4. Notices of some eminent farmers, 5; and mode of becoming a good husbandman, 9. Earliest known cultivation of Britain, 11. The Saxon era, 12. State of at the Norman conquest, 13. Mode of cropping and produce of arable land in the latter end of the 13 th and $14 \mathrm{th}$ centuries, 18. Progress of husbandry in the 15th and the early part of the 16 th century, 21. To the middle of the $17 \mathrm{th}$ and 18th centuries, 24. 27. Furmer ayriculture of Ireland, 28. Plantation of Ulster, 29. Dublin Agricultural Society, 30. Actual state of the husbandry of that country, 31. Scottish agriculture, ib. System of run-rig, 32. Present state of it, ib. Comparative merits of Scotch and English husbandry, 33. Agriculture of the United Kingdom at the close of the last century, 34. Board of Agriculture, 35. Surveys of the United Kingdom, 36. Scope of this publication, ib.

Agricultural labourers, situation of, in England, i. 114. Eflects occasioned by the poor-rate, 115. State of in Scotland: 116. Food of in Scotland and Ireland, 116, notc. Payments in lieu of wages to the Scottish peasantry, and their earnings, 116, 117, 121. Observations on the allowance of allotments of land, and the keep of cows to, 118, ii. 567. Charicter of, i. 119. Advantages of a regular division of work among, 121. Number of, 122. Task work, ib. Selection and superintendence of those employed, and value of an odd man, 123.-Sce Cottage Recnomy.

Agrostis Stolonifera, or Creeping Bent grass, i. 518.-See Fiorin.

Aira Aquatica, or Water Hair grass, i. 518. Alchenilla vulgaris, or Lady's Mantle grass, i. 512.

Alkaline and calcareous manures.-See Chalk and Lime.

Sce Chalky Land,
Allotments of land to the peasantry, ii. 567 . Alhuvial soils, rotation of crops on, ii. 107. Alopecurus pratensis, or Meadow Foxtail, i. 512 .

Animal labour, i. 124. Average annual quantity of land ploughed by a team, ib. Comparative view of horse and oxteams as beasts of draught, 178.- See Horse-teams and Ox-teams.

Anise-seed.-See Cummin.

Annual expenses of farming, i. 49. Table of comparative charges on three different modes of cropping, 50 .

Anthoxanthum odoratum, or Sweet-scented Vernal grass, i. 514.

Ants, mode of destroying, ii. 547 .

Aphis, an insect injurious to the beanplant, ii. 215.

Aquatic grasses, i. 517.

Arable farms, observations on, i. 39.-See Farm Management.

Arbuthnot, Mr., of Peterhead, his experiments with composts of peat-moss, seaware, and dung, i. 417.

Argillaceous soils, i. 509.-See Clays.

Aritichoke, cultivation of the, ii. 574 .

Artificial grasses, effects of the application of gypsum on, i. 327 . Annual species of, ii. 292.- See Clovers, Rye Grass, Tares, and Spurry,-See also Chiceory, Sainfoin, and Lucerne; and Perennial Grasses.

Ashes, of coals, their effect, i. 332 ; of wood, ib, ; and the trees from which the best qualities are produced, ib., n. Turf ashes of peat moss, 334. Newbury peat ashes: situation, mode of preparatiou, and effects of, ib.; time and quantity of their application, 335 ; and analysis of, 336 . Dutch ashes: their estimation in Holland, and their price, ib. Sod ashes: quantity produced, per acre, ly praring and burning the land, 353 and 362 ; and analysis of, ib.

Asparagus, garden, cultivation of, ii. 572.

Asses, in their domesticated state, i. 177. Stallions in Spain and the Islared of Gozo, ib. Employment of, 178.

Arena elatior, or Tall Oat grass, i. 513. 513.

flavescens, or Yellow Oat grass, i.

Bicos and ham, ii. 523. Mode of lividing the carcass of a pig, ib. Salting of, 524. Drying of, ib. Smoke-houses, ib. Morles of curing in Purtugal and IT estphalia, ib. ; in WTestmoreland, 525; 
in Hampshire and Berkshire, ib.; in Wiltshire. Stripping off the skin, ib.See Swine.

Bailiff, qualifications of a, i. 123.

Bakewell, the breeder, i. 5. His experiments on live-stock, 28. His system respecting the conversion of straw into food, or litter, 230. His trade of ramletting, ii. 482.

Banks, Sir Joseph, opinion of, on springwheat, ii. 154.

Barilla,-See Kelp and Soapers' Waste.

Barley, as horse-food, i, 146. Its nutritive qualities and proportionate value to oats. 147. Quantity of farm-yard and liquid manure lail on for its growth on different soils, 260, $n_{\text {., }}$ and 277. Different species of, ii. 171 . Summer barley, ib. Winter barley, bere, or bigg, 172. Number of grains contained in a pound each of barley and bigg, ib., $n$. Rammel, or blandered bere. 173 . Soil required for barler, ib. Cultivation of, ib. Time of sowing, and experiments on, 175. Quantity of seed, ib. Mode of sowing, and also of sowing it along with grass seeds, ib. Product of the Chevalier and common kinds of, 176 . Average weight of the grain of barley and bigg, ib. Analysis of good malling barley, ib. Diffurence between the qualities of English, Scotch, and Irish bar. ley, 177. Straw of, ib. Harvesting of the grain, and experiments on the produce of reaping it at different periods of its ripeness, 187. Hummelling, 204.

Barns, anciently built by the monks, i. 16, n. Roofing, flooring, aud construction of, $87,89,90$. Plan of one erected by General Washington, ib. Cost and different sorts of, 91, 92. Dutch barns, 93. Hay barns, 498. Skeleton barns, ii. 197.

Baronial halls during the middle ages, i. 17. In the reign of Queen Elizabeth, 24.

Bullatas, or sweet potatoes, ii. 259.

Bavin tug for drawing timber, i. 159.

Bayeux tapestry, 1, 12.

Beans, nutritive juroperties of, i. 144. As horse-food, $1+5$. Appropiate suil and species of, ii. 207. Cultivation of, and account of the produce of courses of barley, beans, and wheat. 208. Judgment of seed, 209, $n$. Comparative advan. tages of drilling, dibbling, and soi:ing broadcast, 210. Description of a dibuling machine, 211. Different produce of, according to their distance in the rows, 212. Harvesting of, 213. Bean ricks, 214: 'Thrashing, profuce, and diseases of, 215. Weight and quantity of nutritive matter, 216 . Value of fueding pigs on hean plants when green, 515. Garden beans, scarlet runners, 559 and dwarf, 574.

Beatson, General, of Kunwle Farm, in Sussex, his mode of paring and burning, $i$.
$352, n$. His plan of manuring his farm with burnt clay, 367 . Description of the kiln. 370. Mode of operation and cost of, 371 . His system of farming, ii. 69. His scarifier, 70 . His objections to fallowing, mode of ploughing, and charges of cultivation, ih.

Bedford Level, drainage of the, i. 25.

Bees, value of the honey yearly produced by, ii. 562. Situation of the hives, ib. Swarming, 563. Management of when settled, ib. Calendarial notices of, $56 \%$.

Beet, red garden, ii. 575.-See Mangel Wiurzel.

Berkshire hog, description of the, ii. 508. Weight of the, ib. and $n$. Breed and crosses with the, 509 .

- shim, deseription of the, i. 316.

- wagyon, description of the, i. 158

Beverstone Farm, in Gloucestershire, course of crops on, ii. 103, $n$.

Birds, their depredations on corn, and precautions against them, ii. 518.

Blaikie, Mr., of Norfolk, his observations on the making up of dung-heaps, i. 236. His account of the operation of transplanting turf, 522. His experiments on smutted wheat, ii. 163.

Blé de Jerusalem, a species of barley, ir. 172 .

- Sarrazin, and blé sauvage d' Ilalie.-See Rye.

Blight on corn, ii. 153.

Blindness, in sheep, sunposed causes of, and operation for its cure, ii. 503.

Blomfield, Mr., of Warham, in Norfolk; his introduction of the transplantation of turf, i. 522 .

Blubber, as manure, i. 421. Mode of its application, 422. Manner of forming a compost with it and earth, ib. Its effects on strong land, ib.

Blue Dingstail grass, i. 512.

Board of Agriculture, establishment and object of the, i. 35 .

Bogs, observations on the draining of, $i$. 470. Report on the national grants for the drainage of those in Ireland, ib. $n$. Their extent, 471. Cost of their reclamation, ib. Mode of operation on those of moderate size, 472 . Drainage of chat-moss, 474. Hints to speculators on the reclamation of, ib. and 475 .

Bones, use of, as a manure, i. 392. Mode and expense of crushing, 393. Experiments on their application to different soils, 394. Comparative trials with bone-dust and farm yard manure, 395 and 396. Distinctive properties of bonedust and crushel bones, their quantity and durability, 397. Time and manner of laying them upon the land, 398. Soils to which they are best adapted, 399. Analysis of bones, and of soils on which they had opposite effects, 400 and 401. Observations on raw and boiled bones, 402. Formation of composts with stable muck and bones, 403. Summary of the rules laid down by the Don- 
caster Assoeiation for their application to different soils, 404.

Book-farming, observations on, i. 7.

Boswell, Mr., of Kingeaussie; his comparative trial of bones amd dung as manures for turniys, i. 395. His experiment on feeding neat cattle with raw and steamed food, ii. 390 ; and also on the like manner of feeding pigs, 521.

Boys, Mr., of Betshanger, in Kent; his mode of paring and burning, i. 349 . IIis experiments of cropping after the operation, 356, $n$.

Bradby Park, in Derbyshire, plan of the homestead in, i. 109.

Brawn, ii. 526 .

Braxy and blood, similar diseases in sheep, ii. 499. Indications and cure of, ib.

Bread corn, relative quantity of nutritive matter in wheat, rye, barley, and oats, ii. $92, n . ;$ ard relitive exhaustion of the land in their growth, ib. Composition of, 127. Weight of the different species, 128. Relative quantity of proof spiri's produced by the distilation of, ib. Choice and trial of the yuality of seed, 129 and $u$, and 135. Quantity and number of grains in bushels of wheat, barley, and oats, 130 and $n$. Remarks on thick and thin sowing, 131, 146. Change of seed, 132. Quality and preservation of, ib. Veretation of, 133. Autumn and spring sowings, 134. Kxamination of the condition of seed intended to be sown, 135. State of riveness in which the crops should be reaped, 136. Quantities of household flour and bread produced from certain weights of the different species of grain, 137. 15i. Diseases of, ih.-See it heat.

Breast-plough, description and use of the, i. 343 .

Broar?c:sst sowing, mode of effecting it with corn, ii. 73. With grasses, ib. and n. Machine used for, 74 .

Brocoli, garden, cultivation of, ii. 574 .

Brown, of Markle, courses of crops on various soils recommended by Mr., with observations thereon; ii. 114. His method of cleaning bean-crops, $211, n$. His experiment on the soiling and grazine of cattle, 378 .

Bruce, Mr., of Kennet, experiment of on the effects of nitre as a dressing to land, i. 391 .

Buck-wheat, its introduction into Europe, ii, 222. Appropriate soil and culture, 223. Product and application of the grain, 224. Hay of, ib. Cakes of, ib.

Burlaughton, in Staffordshire, plan of the homestead, i. 102.

Burnet, i. 512.

Burnt clay as a manure, i. 364 . Experiments on, 365. Analysis and effects of", 368. Mode and expense of burning in kilns, 370 and $374 ;$ and $\mathrm{in}$ heaps, 375 . - See Clay Kilns.
Bush-harrow, i. 486.

vetch, i. 511 .

Byre for cattle, plan of a, i. 204.

Cambicrs, varieties of, ii. 255. Soil and culture of, ib. Seed, and season of sowing, 256. Mode of planting out, ib. Produce and value, 257. Analysis of the comparative nutritive matter of some species of, and Swedish turnips, 258. Application of, ib. Woburn perennial kale and turnip-ruoted cabbage, ib. Garden cultivation of coleworts, 559. Savoy and Scotch kale, 561 .

Cage wickets, ii. $5+5$.

Calves, rearing of, for stock, ii, 440. Ma. nagement when dropped, ib. Process of weaning, 441. Subsequent treatment of, 444. Fattening of for veal, and business of suckling, ib. Age at which they should be killed, and estimate of their expense and profit at different periods of their growth, 445. Manner of suckling by hand, 446. Middlesex, Strathaven, and Abbey Holme modes of futtening, 447. Sheds for, and management of, 448. Bleeding and diseases of, ib. Chalk, use of, 449 . Procluct of veal, 450 .

Calx, description of, i. 439. Uses of, 442.

Canary seed, Kentish, cultivation of, ii. 329. Harvesting and produce of, it. and n. Haulm of, ib. and i. 134.

Capital sequired for farming, i. 41. Estimate of the cost of stocking farms of 150,200 , and 500 acres, 44 to 48 . Expenses of three different modes of cropling, 50.

Carraway seed.-See Coriander.

Carrots, as food for cattle, i. 125. Quantity of allowed to cart-horses, 125 ; and to oxen, 213. Introduction of, ii. 283. Soil, 284. Experiments on the use and disuse of mauture in the growth of, ib. Seed, 285. Culture, ib. Species of, 286. Haulm of, ib. Storaye, and manner and expense of bunching them fur market, 287. Preparation of the land for the production of seed, 290 . Modes of planting and rathering, 291. Quantity and price of, ib. Garden cultivation of, 560 .

Carrucate, a measure of land, $13, n$.

Cartage, i. 153. Wagrons of various kinds, 155. Trials of the loads capable of being diawn by carts and wargons, 160. Jescrintion of the single-horse cart, 161. The scotch cart, and observations on its use, 162. Hampshire cart, 163. London hay cart, ib. Twohorse cart, 164. Tumbrels, ib. Moss carts, 165. Pattens, ib. Irish cars, 166. Sledges : namely-gamboes, gurry-butts, and dung-pots, 167, 163. Oxcarts: modes of yoking, 193 ; draught up-hill, and observation on working the cattle in yokes or harness, 196. Description of the Portuguese cart, 195 . 
The common dung cart, and Lord Somerville's contrivance, 199.

Carters, observations on, i. 170.

Cart-horses, breeds of, i. 172.-See Horseteams.

Cartwright, Dr., his experiments on crops manured with burnt clay, ashes, and soot, i. 366. His kiln for burning clay, mode of operation, and expense, 372. His experiments upon potatoes manured with salt and soot, 383. His thee-furrow ploingh, ii. 16. His trials of feeding sheep partly with sugar, 487 and $n, 488$.

Cary's cattle gauge, ii. 393 and $n$.

Cattle, penalty on the ill-usage of, Appendix, No. I.-See Oxen.

- sheds: difference of opinion on the preference to be given to open yards and hummels, or to close ox-stalls for working cattle, i. 200 , ii. 380 . Modes of fastening oxen in their stalls, i. 202. Plans of different ox-houses, 204, 205, and 206. Open sheds, 207, 208. Economical mode of constructing, ib.

Cauliflower, garden cultivation of the, ii. 574.

Cavalry, allowance of food to, horses, i. 141.

Celery, cultivation of, ii. 575 .

Ceorls, i. 13.

Chalk, properties of, i. 278. Quantity of applied to land, and its effects on pasture, 279, 434. Chalk-pits, 280.

Chalky land, rotation of crops on, ii. 102, 116.

Challis, Mr., of Panfield, in Essex, experiment by, of salt as a dressing to wheat, i. 382 .

Chamomile, cultivation and produce of, ii, 330.

Charta Foresta, i. 14, $n .307, n$.

Chat-moss, account of the drainage of, i. 474 .

Cheese, manufacture of - -See Dairy. of Gloucester, 429. Of Cheddar and North Wiltshire, ii. 430 . Of Stilton, 431. Dunlop, 432. Parmesan, 436. Potatoe, 437.

Cheshire cheese: preparation of the rennet, ii. 421. Management of the milk and cream, 425. Coagulation and colouring of the curd, ib. and n. Pressing of the currl, 426. Extraction of the whey, 427. Salting and drying of, 428 . Weight of, ib. Observations on, ib, and 434.

Chiccory, description of, ii. 303. Uses, soil, and cultivation of, ib.

China, preparation of night soil in, i. 273 .

Chinese pigs, size and qualities of, ii. 507 . Mixed breeds of, ib. Observations on the pure breed of, 517 .

Churns, description of various sorts of, ii. 409.

Cider, species of apples generally used for, ii. 362. Colour and size of goud fruit, ib. Pommage, 363. Gathering of the fruit, pressing, and management of the pulp, ib. Quality and quantity of the juice, 364. Fermentation of the must, ib. Stumming of the liquor, ib. State for bottling it, fining, and period during which it will remain in perfection, 305. Proủuce of an acre planted in orchard, 366. Storage, cellars, and implements of manufacture, 367. - See Orchards.

Classification of soils and their respective qualities, ii. 113. Courses of crops recommended on different sorts of lard, as specified in Nos. 1 to 9 , at 115 to 119. Management of fen land, 120 . Effects of cropping on different systems, 123. Theory of the triennial and alternate plaas, 124. Account of four different rotations actually carried into effect, 125. Summary of the various crops, in grain, fodder, and manure, 126. Fundamental principles in following them up, ib. See Rutation of Crops.

Clay, properties of, i. 439. Quantity and mode of application as an improvement on sandy soils, 426. For the mode and effects of burning it for manure, see Burnt Clay.

- kilns, and modes of burning, i. 369 . Description of those of General Beatson, 370 ; of Dr. Cartwright, 372 ; and of Mr. Burroughs, 373. Cost of burning, 374 . - soils, effects of lime on, i. 289. Experiments of the application of lime to strong land, 299. Effects of marl on, 319. Time and mode of plonghing the land, 440 and $n$. General character and mode of improvement of, 441. Rotations of crops on heavy clays, ii. 104, 115 ; and on thin clays, 104, 117.

Cleveland Bays, size and qualities of as cart-horses, i. 175.

Cline, Mr., his principles of improving the form of animals, ii. 492.

Clod-burning, mode of operation and expense of, i. 375 .

Clover, introduction of, i. 25. Weight of, per acre, when cut green, 139, $n$ 。 Quantity and effects of furm-yard manure, laid on for its growth on different soils, $n .260$; of liquid manure, 277; of gypsum, 295, 326; and of Dutch ashes, 295, 326. Sowing of it with barley, ii. 175. Qualities of the red and white species of, 292. Cow grass, 293. Best soil for, 294. Quantity of seed per acre, 294 and $119, n$. Time of sowing and management of the crop, 295. When intended to stand for seed, its appearance when ready to be cut down, 297. Harvesting of, 297. Operation and expense of thrashing, 298. Frauds practised by dealers in the "doctoring" of seeds, ib. Produce of the seeds of the white and red species, 299. Of hay, ib.

Clouted cream, mode of making, ii.413n Clydesdale horses, description, breeding, and power for farm works of, i. 174.

Cobbett's corn.-.See Indian corn

Cock's-foot grass, i. 513. 
Coke, Mr., of Norfolk, hisinode of making composts, i. 2.13, $n$. His experiment ou the sowing of oats along with transplanted turf, 524.

Cole-seed, use of, ii. 312. Description of the plant, ib. Soil and management of, ib. Flemish culture of, ib. Harvest, produce, and quality of, 313 .

Comet, the Durham bull, i. 5.

Common fields, observations on, i. 466.

Composts, formation and composition of, i. $237,243, n .256$. Propurtions and preparation of different sorts formed of dung, earth, and litter, 257. Quantities and cost, 258. Use of on mearlow land, 261. Of loam and night soil, 270. Of lime and earth, 305; and experiments on, 306. Also with lime, earth, salt, and refuse fish, 388. Of earth, dung, and soaper's waste, $3+1$. Oi dung and burnt clay, 365. With peat-moss, lung, refuse fish, whaleblubber, train oil, refuse of the shambles, sea ware, and lime, 415. 421, 422. 463 ; and experiments on dito, 417 . General remarks on, 433 .

Con-acre, a tenure of land in Ireland, i. 349. Its abuses, 358 .

Conversion of arable land to permanent pasture and meadow, i. 503. Growth of the grass-seeds, ib. $\boldsymbol{n}$. Suwing of the seeds, 56t. Experiments on diffirent sorts and soils, 505 . Manarement, 506. Seasons for sowing, 507. Appropriate soils and grasses, 508. List of perma. nent grasses, 510. Transplantation of turf, 522. Mude of operation, and result of the experiment, 523.

Coriander and Carraway, ii. 327. Appearauce of, and time required for the perfection of the plants, ib. Harvesting of, ib. Cultivation of the crops conjointly with that of teasle, 328 .

Corn, duties on the inportation of, App. No. I.

Corn and cereal grasses. See Breadcorn,

Cornish wain, description of the, i. 158 ; and cart, 159, 203.

Cottage Economy, observations on, ii. 533. Boarding of men-servants, ib. Village beer-shops, it. Allotments of land, i. 118, ii. 55j. The Cottager's Garden, ib. Manner of working it, and vegetables to be raised, $55 \%$.

Cotting of sheep.-See liolding.

Coventry, Dr., his calculation on the production of manure from different crops, i. 253,254 . His opinion on the quantity of farm-yard dung for the growth of a course of crops upon arable land of various soils, 265 .

Cows, gestation of, ii. 438. Conception and parturition of, ib. Management on the dropping of the calf, 439 . Abortion and theases of, 440. Dung of, 1. 255.-See Milch-Cows,
Cow-houses, construcion of, ii, 399 , and $n$.

Cramp's Cow, ii. 403.

Cream-cheese, making of, ii. 435.

Creeping Fescue grass, i. 511.

- Poa, i. 511.

Crested Dog's-tail grass, i. 512.

Cromwell, encouragement of husbandry by, i. 25.

Cucumbers, growth and foreign ase of, ii. 575.

Cullev, the breeder, i. 5. His description of a tup, ii., $456, n$.

Cummin, soils and cultiration adapted to the growth of, ii. 328. Application and manarement of the seed of, $i b$.

Curwen, Mr., his mor!e of feeding farm horses, i. 126. His plan of burning clay with lime, 376. His experiments on the soiling and grazing of neat-cattle, ii. 377.

Cushat.-See Wood-pigeon.

Cynosurus carulceus, or Bluc dogr's-tail grass, i. 512. tail, i. 512. cristalus, or Crested dog's-

Dare's, Lord, trials of the effects of nitre, as a top-dressing upon growing crops and meadow land, i. 391.

Dactylis glomerata, or Rough cuck's-foot grass, i. 513.

Dairy Husbandry, i. 37,39 . ii. 396.

_- construction of the, ii. 404,405 , and n. Utensils of the, 408. Management and analysis of the milk, 410 and $n$. Making of butter from cream, 412 . Corstorphine cream, 413. Manner of making clouted cream, ib. $n$. Mode of making butter from the whole milk, 414. Operation of churning, and experiments on, 415 and $n$. Temperature of the milk-house, 415. Management, salting, and preservation of the butter, 417 , and $n$. 418. Barrelling, 418. Wheybutter, 424. Manufacture and qualities of cheese, 419. Modes of preparing renuet, 420. Making of whole-milk cheese, 422 . Storing and salting of, 424 . Skim-milk cheese, 435 . Cream-cheese, ib. New cheese, 436. Foreign cheese, ib.

N.B. For peculiar sorts of cheese made in various places, see the separate heads of Cheddar, Cheshire, Dunlop, Gloucester, Parmesan, Putato, and Stilton.

Estimates of the respective profit of making butter, manufacturing of cheese, and rearing of calves for veal, 449.-See Calves and Milch-Cows.

Darnel-like Fescue-crass, i. 511.

Davy, Sir Humphry, his opinion on longdung and short-muck, i. 245; and observation thereon, 431. His directions for the management of putrescent manume, 248. II is opinion on the putres 
faction of urine, 277. His theory of the operation of gypsum, 329 ; and of the application to the land of green manure, 406. His definition of soils, $438, n$.

Day-house farm in Shrupshire, homestead of, i. 104 .

Dawson, of Frngden, his improvements on Scottish farming, i. 5. His account of the application of lime, 298.

Deer, ii. 506.

Denshiring.-See Paring and Burnimg.

Devonshire oxen, i. 214.

—— cream, ii. 413.

Dibbling, mude of sowing and cost of, ii. 82. Saving of seed by means of, 83 . Observations on the practice of, 84 . Process of; 88. Comparative experiment on sowing broad-cast, and by the dibble, 89.-See Drilling.

1)ill, ii. 222 .

Dishley breeds of live-stock, $i .5$. Observations on, 28. 1)istinguishing points of the neat cattle; the sheep; appearance and qualities of the pigs, it. 510 . Crosses with and observations on, ib. and $n$.

Dogs, shepherds', ii, 479. Duties on, Appendix, No. 1 .

Doncaster Association, report of, on bone manure, i. 402, 403.

Donnington, in Shropshire, homestead of, i, 105 .

Doomsday Book, i. 12. Agricultural state of England, as pictured in, 13 .

Double-firrow ploughs, work performed by, i. 183. Description of, ii. 15 .

breasterl plough, ii. 17.

Douglass's mode of calculating the weight of cattle by measurement, ii. $394, x$.

Drainage, of the Bedford Level, i. 25. Of firms, 112. Elkington's system of, 449 . Mode of draining land-springs, 450 ; and main-springs, 451. Catch-drains, 452. 1)rainare of a dead level, 452, 455. Open drains, 452. Under-trains, with description of various draining or mule ploughs, $453,454,455$, and 460 . Hollow draining, 456. Mode of its operation and tools, 455. Tile draining, 458. Size of tiles, their average cost, and labour of the drainage, per acre, 458. Stone-draining and its expense per acre, ib. Plug-draining, description and cost of, 45\%. Flemish draining, with description of the Muuldebaert, 460. Summary, 461.-See Bogs.

Dritling, introduction of, ii. $7 \%$. Advantages of, and objections to the practice of, 75 and $n$. 78. Operation of, 79. Description of drill barrows, 80 ; and of loorse-drills, 81. Work and cost of horse-drills, 82. Drill roller, ib. Management under the drill system, and comparison of crops under that and broad-cast sowing, 85 to 87 . Observations on, 88 .

Drupsy, in sheep.-See Red-water.
Drummond's Agricultural Museum, at Stirling, exhibition of tumips at, ii. 241. Dublin Society for the Encouragement of Arriculture, i. 30.

Dudgeon, Mr., of Spye-Law, his experiment on the feeding of pigs with raw and steamed food, ii. 523 .

- Mr., of Jrora, in East Lothian, trial of the absorition of water by dry strilw, i. 253.

Dung, nature and qualities of that of different animali, i. 225 ; of horses, 226 ; of horned cattle, 227 ; of sheep, 227 ; of swine, 228. Quantity of, voided by cavalry loises, 254 ; by cows and cattle grenerally, 255. Effects of, when fed on oil-cake, 256.-See Farm-yard Manure.

Dung-heaps, site of, i. 234.

-, modes recommended for the making up of, $241,243, n$. Treatment of, 248.

- — pot, gamboe, or gurry-butt, i. 167.

Dunlon cheese, qualities of, i. 432. Manner of making it, ib. Qunntity of milk to the manufacture of a pound of, 433 . Differences between the mode of making Dunlop and Cheshire cheese, 434.

Dunrobin Castle, and homestead, at the mains of Dunrohin, i. 106.

Dunt, or sturdy, a disease of sheep, description of, and operation for, ii. 495.

Dutch ashes, employment of, in the Netherlands, and effecis of, i. 336. Price at which they can be imported into England, 337 .

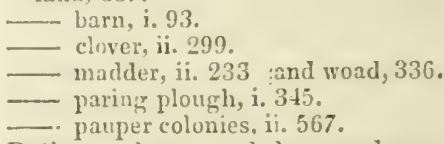

Duties on horses and dors, and on corn imported from abroad, Aplendix, No.I.

Dwarf kidney-beans, ii. 574 .

Dyke, for the washing of sheep, description of a, ii. 471 .

Earemont, experiments by the Earl of upon the feeding of pigs with rice, ii. 520 ; and on cooping porkers in close single styes, 529. Plan of the piggery at Petworth, 527.

Eryptian wheat, ii. 138.

Ellman, Mr., of Glynde, his standing sheep-fold, ii. 467. His mode of ascertaining the age of slaughtered sheen, $486, n$. Inside fat of sheep, slaughtered br, $490, n$.

Embankment of rivers, observations on the, i. 447. Advantages, when practised, of straightening their course, 448 . Description and cost of an operation of that kind, 449 .

Enclosure Act, abstract from the, Append., No. I.

Endive, as a garden plant, ii. 575 .

England, agriculture of, at the Norman 
conquest, i. 13 ; in middle of the 17 th century, 25 ; and at the close of the last century, 34 .

English and Scotch farming, comparison of, i. 33 .

Entry to farms, usual terms of, i. 51, and Append., No.I.

Ergot, a disease of rye, and its effects, ii. 170

Essex, half bred breed of pigs, description and qualities of the, ii. 509.

Evans, Mr., of Dean House, Oxfordshire, his comparative trial of bones and stabledung as a manure for wheat, i. 395.

Ewes, slipping of the lamb, occasion of, ii. 503. Protusion of the uterus, and operation to secure it, ib. Udders of, when affected by tumours and sore teats, remelly fo:", 503 .

Experiments, general observations on theoretic, i. 2.

Farta, Mr., of Gateshead, trial of the comparative effects of bones and rotted stable dung as a manure by, i. 394 . Comparative experiment on the transplantation of wheat, and sowing it by drill aud broadeast, by, ii. $569, n$. Statement of the cust and effect of spade husbandry, 570 .

Fallowing, description of the different mode of, ii. 45. Summer-fallow, and origin of its introduction into Scotland, 55 and $n$. Importance of, and arguments for and against, 56. Manner of conducting the operation of, 59 to 65 . Winterfinllow, process of the, 65 . Observations on, 66 .

Furmers of the present day, i. 5. Education of, 8. Pecuniary profits and advantages of, 9. Their property, and mode of living in former times, 13,16 , 23. Occupation of their wives, 21 . Rent and profits of their land, 22. Old Christmas fare of, $24, n$.

Farming in Eugland in the 13 th and 14 th centuries, i. Is; in the of reign of Edward III., 20; in the early part of the 16 th century, 22; in the time of Queen Elizabeth, 23; in the midlle of the $17 \mathrm{th}$ century, 25; and at the close of the last century, $3 \%$.

Farms, size and choice of, i. 39. Capital required for the occupation of different solts of, 41. Estimates of the cost of stocking a farm of 150 acres, $4 \frac{4}{4}$; one of 200 acres, 45 ; and another of 500 acres, 47. Annual expenses, 49. Comparative view of the charges of three modes of cropping, 50. Time of entry to, 51, and Append., No. I. 'Tillages, dressings, and valuations of, 52 to 55 . Rent of, 56. Money and corn rents, 57 to 59 , and $n$. Leases of, 60 to 65 . Covenants for the cropping and management of, 65 to 70 . Tithes and parochial assessments of, 70 to 84.-See 'Tenancy; and Laudlord and Tenant, Appeud., No. I.
Farm-buildings, form and situation of, i. 85. Construction of offices, 86 . Thatch, 87, 89. Barns, 90. Granaries, 94. Yards and sheds, 95, 200, 232. Dairies, i. 96, ii. 405. Pig-styes, i. 96, ii. 527. Stack-yards, i. 97. Thrashing-nills, ib. Size and cost of the erection of the dwelling-house and offices, with plans of several homesteads, of different size and construction, 87 to 110. State of, in Scotland and Ireland. 110,111 . Slating of the roofs and floors of, ii. 406 and $n$.

Farm-gardens, culture of verretables in, ii. 572. Hardy fruits, 576. Sowing of sceds, ib. Quantity of seeds required in a small garden, $i b$.

For the cultivation of more common vegetahles, and the management of bees, see Cottage Economy.

horses, feeding of, -i. 125, 127. On steamed food, 129. Forder, 132. Jry food, 135. Soiling of, 137. Average of the daily quantity of green food consumed by a cart-horse, 139. Allowance of manger-meat for, 141 ; and observations, 143. Relative nutritive value and weight of the different linds of grain and pulse , 144 and $145, n$. On feeding by measure, 145, with oats and barley, 146 . Calculation of the annual expense of a team, 148. Labour of, 151. Stable management of, 169. Condition of, 170 . Shoeing of, 171. Breeds of, 172. Training of, 175 .

- servanis, i. 114. Moce of their support in Scotland, 115. Allowance of cows and gardens, 118. Character of, 119. Wages and labour of, in England, 121. Number and superintendence of, 122. Task-work, ib. Odd men, 123. Choice of, for the duties of the dairy, ii. 402.

yard manure, composition of, i. 226. Formation and site of the dung-heaps, 230. 23t. 236. Preparation of, 237. Weights of, 239, $n$. Evaporation of, 240. Over-y'ear muck, ib. Mtanagement of, 241 . Fermentation of, 243 , 430. Opinion of Sir Humphry Davy on its application in a fresh state, and observations thereupon, 245,247 . Experiments on, 248, 250. Joint produce of straw and dung from different crops, 252. Quantity of, made by cattle, 254 . Compusts of, 256. Application of, 2.58; on strong soils, 259; on light land, 260 ; and on grass-land, 261. Mode of spreading, 262. Of depositing it with the plough, 264. Quantity laid on for various crops on different soils, 265, $260, n$. Summary of the chief points of its management and application, 266 . - See Dung.

Fellmonger's poake and clippings, as manure, i. 143. Soils to which it is applied, quantity usually employed, and common price, 424 . 
Fences, direction of, ii. 534. Hedge-planting and ditching, 535. Operations of, ib. Pruning and training of hedges, 537. Breadth and heighth of, 538 . Mode of re-making hedge's and pleashing, 539. Gapping, 540. Planting of hedge-row timber, and sorts to be chosen, 541. Protection and pruning of, 5 42. Field-gates, construction, hanging, and fastening of, 543 . Swing-gates, 544. Stiles and care-wickets, 545 . Comparative view of the expense, security, and protection of hedges and walls as fences, ib. Height and mode of erecting dry stone walls. For enactments regarding Turnpike roads, sce Append., No. I.

Fens, different qualities of soil in the, i. 466. Their cuiture, 467. Salt marshes, and marsh-meadow, ib. Operation of warping. ib. Courses of cropping in the, ii. 107. 120.

Fescue grasses, namely-

Festuca cambrica, or Welsh fescue, i. 511

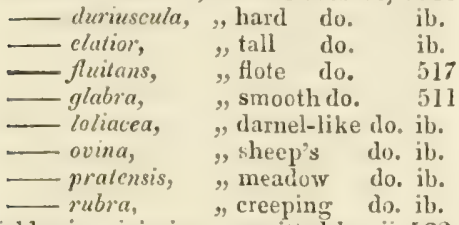

Field-mice, injuries committed by, ii. 532 . Morle adopted in the Forest of Dean for the destruction of, ib.; and for clearing stacks of, ib.

Finlayson's self-clearing plongh, description of, ii. 13; and self-cleaning harrow, 27. His rules for ploughing, 40.

Fiorin grass, introduction of into cultivation, i. 518. Qualities of, ib. Its cultivation and value as a winter-grass, 519. Soil and climate to which it is appropriate, ib.

Fire-blast, a disease of hops, ii. 354.

Firing of dung, i. 233, 432.

Fish-shells, use and effects of, as manure, i. 282 .

Fitz Hlerbert's Booke of IIusbandrie, i, 21.

Flax, application of liguid manure to the growth of, i. 271. Description of the plant, ii. 316. Appropriate soil for its cultivation, 317. Treatment of the crop, 318. Process of water-retting, it. Flemish mode, 319. Dew-retting, 320 . Preparation of the plant when intended for yarn, and operations of "skutching" and "heckling," ib. Process of "rippling" the seerl for its separation; its application, as linseed, and average amount of the whole crop in yarn and seed, 321. Account of the foreign growth and preparation of the plant, as yarn, with observatious thereupon, $n$. ib.-See Hemp.

Flemish mode of preparing liquid manure, i. 271.

Flesh-flies, numbers, and injury of to sheep, ii. 494. Indications of sheep being struck by, 495 . Remedies; and precautions to guard agaiust the attacks of, ib.

Flinty soils, observations on, i. 443.

Flote fox-tail grass, i. 517.

Flour, different weights and qualities of. ii. 137. 155. Trial of the value of Scotch and English wheaten flour, ib., $n$.

Flux, a disease of calves, anrl cordial for, ii. 448, Of sheep, 500. Causes and remedy of, ib. Effects of on young lambs, ib.

Fodder, nature and value of, i. 132. Relative properties of the different species of, 133 .

Fogging of pasture land, i. 484.

Foliling of sheep, opinions entertained on the, ii. 464 . Formation of the fold, 465 . Arerage number of sheep required to fold an acre of ground, 466 . Acreable value of the dung, ib. Standing folds, ib.; and quantity and value of the manure therein produced, i. 228 , ii. 467. Management of fold-manure, i. $430, n$. Sheep-cotes: construction and cust of, with observations on their use, ii. 468 and $n$. Stells: form, cost, and accommodation of, in mountainous districts, 470. Respective advantages and disadvantages of moveable and standing folds, stells, and sheep-cotes, ib.

Foot-rot, a disease of sheep, ii. 497 . Indication of, and remedies for, 498.

Forbes, Mr., of Pinefield, near Elgin, his experiments on the application of salt to valious crops, i. 384 .

Forme of Cury, i. 3.

Fox-hounds of Ed. I., expenses of the, i. 15. Frederick's Oord, colony of, ii. $567, n$.

Free Martins, ii. $369,438$.

Warren, right of, i. 14 .

Furriers' clippings, application and price of, as manure, i. 426.

Furze, as food for horses, mode of preparation and cost of, i. 434 .

- nill for the lruizing of, ib.

Gane-Laws, origin and severity of the ancient, i. 14.115. Abstract of the present enaciments respecting, Append, No. I.

Gardening in England during the early ages, i. 3. Price of cablages and carrots in 1595 , and of potatoes in the 17 th century, ib.

Gariens, nlan and cultivation of, for cottagers, ii. 555 to 562 ; for farmers, 572 .

Geese, food and plucking of, ii. 532 .

Giddy, Mr. Davies, experiments of, on salt as a dressing to turnips, i. 383.

Glossary of weeds. Append., No. V.

Gloucester cheese, manufacture of; breaking of the curd, and extraction of the whey, ii. 429. Salting, 430. Colouring, ib. Characteristics of the double and single sorts of it.- $S_{6 C}$ Dairy, 
Goats, internal structure of, ii. 505. Flesh and milk of, ib. Flesh of the lid, ib. Guat's whey, ib.

Grain and pulse, as horse-food-see liarmhorses; and as human food-see Bread-corn.

Granaries, construction of, i. 94 .

Grass-land, as permanent pasture, and on the breaking-up of, i. 67. Quantity of farm-yard manure laid on, 260, $n$; effects of night-soil on, 268 ; dressings of lime on, 291 ; and observations, 300. Of salt, 385 ; and quamity, time, and effects of its application to, 387. Trials of the apulication of saltpetre to, 390 , 391. Season for laying on top-dressings, 436. Otiservations on the scotch and English systems respecting the retention and breaking up of, 476. Practice of fogging, 484. Improvement of, 521.See Pasture and Meadow Land.

Grasses, natural and artificial.-See the former uncier Meadow; and the latter under their separate heals, as also that of Permanent or Perennial Pasture.

Gravelly soils, effects of marl on, i. 319. Culture and improvement of, 442 . Rotation of crops on, ii. 116.

Grazing, observations on, ii. 368. Practice of, on different sorts of land, ib. Selection of beasts, 369 and $n$. Age, and increase of weight, ib. Stocking of the land, 370 .

Green-cheese, ii. 431.

manures, observations on the use of, i. 405. Crops which are generally applied to the use of, 407. Experiment on their application to the land, ib. : with opinion and remark thereon, 408.

Greg, Mr., of Cules, in Hertforlshire, system of farming pursued by, ii. 66 ; his objections to fallowing, and mode of ploughing, 67. Average of his crops, 68 ; and course of, 69 . Observations, 71.

Grubbers, variety of form of, ii. 26. Description of those of Finlayson and Kirkwood, 27, 29.

Gyps, i. 439.

Gypsum, composition and use of, i. 322 . Experiments on the application, as mamure, of, 323. Calcination and cost of, 324. Suils to which it is most congenial, 325 ; and crops to which it is most appropriate, 32 . Mode of the application of, ib. Effects of ou clorer and artincial grass, 327 ; on corn crops, ib. on pulse and bulbous roots, 328 . Observation 3 on the practical application of, 329.435 .439 .

Hatr, Mr. George Webb, moveable sheepcotes of, ii. 469 , and $n$.

Hampshire cart, description of the, i. 163. the, ii. 508 .

Hand-weeding, ii. 149.
IIard fescue grass, i. 511.

Inrdy fruits, ii. 576.

Hare, Mr., of I3eaconsfield, effect of dressings of salt on turnips by, i. 383 .

Harley, Mr., of Glasirow, description of the cow-house erected by, $n .399$.

Harrows, construction of, ii. 2?. Drags, 23. Small harrows, ib. Seed hariows, 24. Operation of harrowing, 25. Finlayson's drill harrow, 28.

Harvesting of corn, ii. 186. Indications of the r.peness of the grain, ib. Experiment respecting the seaping of barley at different periods of its growth, 187. Operation of reaping with the sickle, 188; and of mowing with the scythe, 189. Description of the cradle-scythe, 190 ; and the Hainault-scythe, 191. Reaping machines, 192. Management in reaping and stacking, ib. and 189. Operation and expense of stacking, 193; and thatching, 196. Skeletonbarns and stack-yurils, ib. Thrashing, 197. Description of a portable thrashing-machine, 193. Mode of operation with the thrashing-mil], 199. Winnowing, 201. Marketing and measuring of grain, 202. Expense of reaping, thrashing, and marketing, 203. Harvest-home, ib. Barley-hummelling, 204. Kiln-drying, 205. Gleawing, 206.

Haulm of beans and pease, and carrawayseed, as liorse food, i. 132, 133; and of peas for sheep, ii. 219.463.

Hay, cumparative nutriment of, when made from natural and different sorts of artificial grass, i. 136. 148.

- making, circumstances which influence the time of mowing, and general oluservations on, $\mathrm{i} .487$. Mode of operation, 489; in Middlesex, 490, $u$.; the Northern Counties, 491 ; in Ireland, 488, n. Rowen-hay, 492. Manner of making, with artificial grasses, 493. Number of work-people, 494. Description of the hay-spreading machine, ib. Horserakes and hay-sweejs, 405. Stacking, 496. Sweating in the stack, 497. Application of salt, 498. Hay-barns, ib. Weight of grass when dried into hay, 499. Sale of, ib. Tools and operation of trussing, 500. Measurement of hay. stacks, 501. Expenses, 502. Manner of making water meadow hay, 531 .

— tea, mode of making, ii. $442, n$.

Hayward, Mr., of Frocester Court, his mode of making Gloucester cheese, ii. 429; his estimate of dairy produce, 450.

_- Mrs., of Frocester Court, her experiment on the comparative production of butter and cheese, ii. 451 .

Hedge-hog, prejudice entertained against the, ii. 553 .

Heinp, male and female, ii. 322. Soil aclapted to the prociuction of, ib. Effects of its growth upon the land, 323 . Sced, 324. Harvesting of the crop, if intended 
for yarn, ib.; and if kept for seed, 325 . Charges, proluce, and average profit upon the culture of, ib. and $n$. Observations on its growth in this country and Ireland, ib.-See Flax.

Herbert, Mr., of Mucruss, ox-house belonging to, i. 205.

Iferefordshire oxen, i. 213.

Htrmaphrodite wagron, description of the, i. 158.

Hertfordshice wheel-plough, ii. 5.

Ifide, a measure of land, i. 13.

Highland Society of Scotlamt, experiments sanctioned by the: On bones, i. 395, 396. On kelp, 420. On oats, ii. 179. On the cradle and Hainault scythes, 190, 191. On feeding cattle with potatoes, Swedish turnips, and mangel-würzel, 253. On the steaming of roots for oxen, 387; and for pirs, 521 .

Highway-rate, i. 82.153 , ii. 574 . Act of Scotland, 153, n. See App., No. I.

Hoe-plough, ilescription of the, i. 347.

Hogg, the Ettrick Shepherd, his account of sheep-pining, ii. 501. His oljections to the destruction of moles, ib., 552; and of wood-pigeons, 549 .

Holcus averaceus, or tall, oat-like, softgrass, i. 513.

- lanalus, or woolly soft-grass, i. 514.

mollis, or common soft-grass, i. ib.

Holkhain property, leases of the, i, 66 .

Holme-Lacey, in Irerefordshire, pear-tree at, ii. 366.

Homestead, i. 99.-See Farm-buildings and Xards.

Honey-lew, a lisease of beans, ii. 215 ; and hops, 354 .

Hops, introduction of their use into England, ii. 328. Species of, ib. Best soil and situation for the growth of, 389 . Preparation of the land tor, 340 . Time of planting, 341. Management of the crop during the first, second, and third years, 342. Quantity and species of inanure which may be applied, viz. : of farm-yard, and dung and compost, 343 ; of salt, i. 389; of wonllen rays, furriers' and sheep's clippings, 425, 426. Arrangement and setting-out of the hopground, ii. 343. Mode of poling, ib., n.; and number, class, and price of poles, 344. Tying of the bine, and further operations until the flowers become ripe, ib. Process and cost of picking, 346 , and $n$. Operation of oastage, 347. Bugging for market, 348. Froduce, 349. Expenses of the growth of, in the Canterbury and Maidstone districts, and in the counties of Hereford and Worcester, ib. Duty, 351. Propagation of hops by seed, ib., and $n$. 352. Mode of training the bine horizontally, 353. Casualties and diseases of, 354 .

Horned cattle.-See Oxen.

IIorses, breeds of the cart species, mamely,
Lincoln blacks, i. 172; Cleveland hays, 173 ; Suffulk punches, ib.; the Clydesdale, 174; and the Irish garron, ib. Mode of training for farm-work, 175.See Farm-horses.

Horse-hoes, description and operation of the common, ii. 30 . The expandinghoe,31. Wilson's machine, 32. Blaikie's, 89. WVilkie's, 90.

Horticulture, the recreation, variety and profit of the pursuit of, i. 4 .

Horticultural Society of London, experiments on planting the tubers and sets of potatoes at different distances, made by the, ii. 272 ; with observations thereon, 275.

Hoving of oxen, ii. 374 . Of sheep, ii. 503. Howard, Mr., of Melbourne, observations by, on the formation of dunghills,i. 234. Nianner of harvesting corn adapted by, ii. 189.

Howden, Mr. Andrew, of Lawhead, in East Lothian, experiment on farm-yard manure and bone-dust made by, i. 395 ; on the feeding of cattle comparatively with potatues, Swedish turnips, and mangel würzel, ii. $253 n_{\text {。 }}$; his trial of the feeding of cattle with raw and steamed food, 389. His experiments on polatoes, 261 .

Humin, or Humus, i. n. 431. Nature and composition of, ii. 91, $n .113$.

Hunmelling of barley, and description of the machine, ii. 204 .

IImurer-rot, a disease in sheep, ii. 503.

Hunter, Mr., of Eskmount, plan of his oxhouse, i. 254.

Hunting in ancient times, i. 14. Foxhounds of King Edward the First, 15.

Hybrid animals, i. 176 .

Imp amsicnts of tillage, ii. 1.-See Ploughs; Harrows; Grubbers; Scarifiers; Scufflers; Horse-hoes and Rollers.

Indian corn, description of, ii. 225. Soil best suiter to the growth of, 226 . Modes and time of sowing, ib. Transplantation of, 228. Culture of, ib. Topping of the plants, ib. Operation of hushiug, 229. Description of the corn-crib, ib. Mode of shelling, 230. Produce of, $\mathrm{ib}$. Observations on the culture of, ib.

Insects, ii. 547.

Inverbrora, in Sutherlandshire, plan of the homestead, i. 107.

Ireland, agrieulture of, 28. Plantation of Ulster, 29. Mode of letting farms in, 30. Dublin Suciety, and improvement in the agricultural state of, 31. Farmbuildings in, ib. Mode of paring and burning in, 353. Use of peat-moss, as a manure, 414. Extent of boggy and mountain land in, 471; and hints to speculators in bringing it into cultivation. - See Bogs. General State of Farming in, ii. 109; Husbandry of the Baronies of Forth and Bargy, ib, $n$. 
Irish car, description of the common, i. 166.

- garron, qualities, food, and harness of the, i. 174 .

- haymaking, i, 488, and $n$. loy, ii. $570 \mathrm{n}$; and stiveen, $571 \mathrm{n}$.

- ploughs, ii. 48 ; and ploughing, 49, $n$. Irrigation, origin of, i. 524. Efiects of, 5ะ5. Process of, 526. Catch-water meadows, 527. Flow meadows, 525. Water meadows, 529. Average expense and produce of, 530. General management of, 531. Making of water-meadow hay, ib. Feeding of water-meadows, 53:. Appropriate soil and situation for, ib. Summary, 533. Use of the rafter level, 534.

JEFFeries and Co., plough of, ii. 12.

Jerusalem antichoke, garden cultivation of the, ii. 575.

Jessop, Mr., of Sutton Conrt, his experiment on the growth of potatoes, ii. 274 .

Johuson, Mr. Cuthbert, his experiment on the application of salt to various crops, i. $3 \circ 2,384$.

Kar.e, sea, cultivation of, ii. 573. Soil appropriate to the growth of, ib.

Kelp, analysis of, i. 339 . Manufacture and qualities of, 419. Properties and experiments on the application of, as manure, 420.- See Suapers' Waste.

Kentish practice of paring and burning, $i$. 349.

Kentish practice of the round tilth rotation of crops, ii. 95.

of sowing canary, radish, and spinach seeds, ii. 329.

- turn-wrest plough, ii, 4.

Kidney leans, or scarlet-runners, cottage cultivation of, ii. 559. Gaxden dwarf, 574.

Kilns, for burning lime, i. 302 ; for clay, 369; for drying corn, ii. 205. Russian mode of kiln-drying grain in the sheaf, 206 ; for the drying of flax, 319 , snd $n$. Hop-kilus, or oast, aud cost of, 374, and $n$.

Kinight, Mr. of Downton, President of the Hurricultural Society, his experiments on the planting of potatoes, ii.267.269. 275.

LAmourers' Friends, Society of, object of the, ii. 567.

Lady's-mantle grass, i. 512.

Lambert, Mr., of Ireland, his sheep-cote, ii. 467. On dry stone-walls, as fences, 546.

Lance, Mr., of Lewisham, his account of growing hops, ii. 352 ; his exhibition of of wheat, $569, \pi$.

Land, value of, in the 10th century, and state of its cultivation at that time, i. 12 . Ancient measurement of, 13. Returus from, and mode of cropping in the 13 th and 14 th centuries, 18. Rent of, at that priod, 19, n. Quality of, 40. Rent of, 56 ; and observations on lowering it, 59. Leases of, 60. Curenants respecting the cropping of, 65 , ib. $n_{\text {s, }}$ and 68 . Breaking up of mearlow, paring and burning of, 342.- See Soils.

—.. stewards, i. $64, n$. Qualifications of, 67 .

Lathyrus pratensis, or meadow vetchling, $\mathrm{i}$. 511

Lavender, planting of, ii. 331. Culture of, ib. Riplening of the crop, harvesting, and application of, 332 .

Leases.-See Temancy.

Leicestershire trolley, i. 160. Sheep, ii 48\%. IItton, 486.

Lettuce, as a garden vegetable, ii. 561.

Lime, price of, in the 15 th century, i. 19. Prepuration and properties of as quick lime and slaked lime, 283. Efiects of, as a manure, when applied to land, 284 . 238. Application of, to marsh-lands, clays, and sandy soils, 289. Its powver of exhaustion on arable land, 290. 434. Effects of as a top-dre-sing upon grass land, 291. State in which it should be apllied, 292. Qualities of, 294 ; and quantity to be applied to different soils, 296. 298. Analysis of, 295. Summary, 301 ; and rules fur the general applicition of, 302 .

- lilns, i. 302. Plan, erection, and crst of some in Ireland, 303. Mode of burning lime without kilns, 304 . Pot-kilns, ib. Pye-kilns, 305. Fuel, ib.

Lime-stone and lime-stone gravel, properties of, $281,282$.

Lincoln cart-horses, their properties and value, i. 172. Sheep and mutton.

Linseed.-See Flax.

jelly, as a food for calves, ii. 442 Mode of making, ib. $n$.

Liquid manure, i. 270. Flemish mode of preparing, 271. Quantity applied, and effects of, on different crops, ib. $n$. Manner of the application of, ib. Effects of on heavy land, 272. Chinese preparation of, 273 . Observations on its importance, and the use of in foreign countries, ib. Experiments upon the application of, 276.-See Urine.

Liquorice-roots, soil and preplaration of the ground for, ii. 330. Mlanure, ib. Season of planting and culture of, $\mathrm{ib}$. Time required to perfect the growth of, ib. Product and value of, $i b$.

Loamy soils, description of, i. 444 , ii. $n$. 114. Experiments on the application of lime to, i. 300, Nos. 6,7 , and 8 . Soot, as a tor-dressing to, 338. Rutations of crops un sandy loams, ii. 103, 116 ; on rich loams, 105, 115.

Lollium percnne, or perennial rye-grass, i. 514.

London' hay-cart, description of the, i. 163. Longdon, in Shropshire, homestead of, $i$. 102. 
Long-tung, i. 244. 431.

Loy, or Irish spade, description of the, ii. 570.

Lucerne, description and cu'tivation of, $\mathrm{ii}$. 307. Soil and preparation for, 308 . Growth of, ib. n. Seed of, 309, 310 . Mode of treatment luring the growth of, ib. Application of the crop, i. 139, ii. $307, n$. 311. Comprative analysis of clover and sainfoin with, ib. Experiments on, $311 n$.

M'DERM ExT's table of cattle measurement, ii 393.

M'Kenzie, Sir G. S., Bart., on the storing of turnips, ii. 244.

Madder, description of, ii. 332. Soil, and mode of cultuse in Holland, 333. Finglish cultivation of, ib. Process of planting, ib. Period required for the growth of, 33t. Fathering, drying, and produce of the rnots of, ib. Rules for the culture and manufacture of, ib. $n$.

Magnesia, effects of in lime, i. 295.

Malcolm, Mr., experiments of, in compusts with tanner's hark, i. 242 .

Malt-dust, qualities and use of as a topdressing, i. 413. 435.

Mangel würzel, introduction and description of, ii. 251. Saccharine matter contained in, ib. n. Soil and culture of, 252. Climate, seed, and period of maturity, ib. Transplantation of, il). Stripping off the leaves of, ib. Produce and application of, 253. Feeding firo. perties of, ib. Comparative experiments on the feeding of cattle with it. Swedish turnips, and potatoes, ib. n., 25f. Re. port of the Doncaster Association on, 255.

Man-traps and spring-guns, Append.,No.I.

Manual labour. - See Agricultural Labourers.

Manure, nature and properties of, i. 222. Distinction between vegetahle and mineral, as well as miscellaneous kinds of. and their action upon the soil, 223. Modes of spreading it upon the land, 262 ; and depositing it with the plough, 264. Fermentation of, 430. Long and short dumg, 431. Observations on the uses of lime, chalk, and marl, 434; on burnt clay, and on paring and burning, ib. ; on gypsum, salt, and miscellaneous substances, 435. Manner in which plants are supposed to extract nutriment from the sorl, ib. Summary of the general application of all kinds of manure to the land, and table of quantities, 436.

For those of the nutrescent kind, see Dung, Farm-yard; and Green Manures; Liquid Manures, and Night Soil.

For Fossils, see Chalk ; Lime; Marl: Gypsum; Ashes; Soapers' Waste; Burnt Clay; Salt and Nitre.
For the Miscellaneous Surt, see Bones; Oil-cake; Rape and Malt-dust ; Peatmoss; Sea-ware; Kelp; Refuse-fish ; 'Jrain-oil and Blubber; Fellmongers' Poake and Cuttings ; 'Tanners' Bark; Woollen Rags; Furriers' Clippings; Sugar-scum; and Mud.

Marl, ancient use and definition of, i. 303 . 439. Various kinds of, 309. Earthmarl, composition of, 310 , and $n$; Quintity of laid upon the land, and effects and origin of, 311. Shell-marl, deseription, nature of, and effects of, 312 , and $\%$. Application of, and quantity used upon diffirent soils, 314. Durability of, 316. Common use, and description of the various effects of, 317 . Crops sometimes taken after marling, 319, Analysis of, 420 .

— grass, i. 515 .

- pits, i. 312 .

Marshall, his minutes of agriculture, i. 9. His chemical investiration of marl, 318. Marsh-lund, rotation of crops on, ii. 316 , n. Purtable bridge for passing over dykes in, 490.

\section{- meadow. - See Fens.}

Maxwell, Sir William, of Calderwood, his comparative trial of the effect of bruised bones and stable manure on turnips, $i$. 396.

- Mr., of Hetton, in Huntingdonshire, his course of cropping, ii. 119.

Meadow-land, observations on the natural state of, i. 485 . Management of, 486 . Bush-harrowing and rolling, ib. Destruction of moles, 457, and ii. 553. Mowing and manuring, i. 487. Breaking "p of, 67.-See Grass-land, Hay. making, and Irrigation.

Meadow cat's-tail, or timothy grass, i. 512. fescue-grass, i. 511. fox-tail grass, i. 512.

Meal, quantity of extracted from oats of different weights, i. 146.

Meas'es, in pigs, description of, and remedy for, ii. 530 .

Meadowbank, Lord, on the formation of composts with peat-moss and dung, $\mathrm{i}$. 415.

Measurement of eattle, for ascertaining the weight of, ii. 393.

Medley, Mrs., of Acton, her experiment on the growth of potatoes, ii. 274.

Members of Parliament, regulations respecting voters for, Append., No. I.

Neslin.-See liye.

Mice-Dee Vermin.

Midden, or mixen.-See Dung-heaps.

Middlesex, manner of making hay in, $i$. $490, n$. Morde of fatting calves for veal in, ii, 446.

Milch-cows, esteemed breeds of, ii. 396 and $397, n$. Form and qualifications of, 397. Stocking with, 398. Management and food of, 399, Milking, 4U1; and experiment on, $402 n$. Dairy ser- 
vants, ib. Average quantity and quality of milk, 40.3. Cramp's cow, and experiments on the produce of different breeds of, ib. and $n$. Tithes of milk, $\mathrm{i}$. 78. See Dairy.

Mildew of corn, ii. 157 ; of beans, 215 ; of hops, 354.

Mistwasser, i. 275.

Moles, their effects on meadow and pasture land, i. 487, ii. 551. Opinions entertained on the expediency of destroying them, 552. Modes of poisoning, and of driving them away, 553.

Mole-ploughs, description and use of, $i$. $453,454,455,460$.

Monks, early attention of, to agriculture, 16. Character of, as landlords, ib. Hushaudry of the Monastic Orders, ib. Consequence of their dissolution, ib.

Moorish soils, effects of lime on, i. 280. Experiments on its application to, Nos. 3 and 4,299 , and 300 . Management of, ii. 108 .

Morley, Mr., of Blickling, in Norfolk, his mode of making composts of earth and blubber, i. 422 .

Mouldebaert, a Flemish implement used in Irainage, description of the i. 460 .

Mud, properties of, as a manure, i. 428. Qualities of different sorts of, 429. Time of mudding ponds, ib. l'reparation and application of, ib.

Mules, their breed, power, freedom from disease, and lenuth of life, i. 176.

Murrain, in pigs, and remedy for, ii. 530.

Murray, General, sheep-cote belonging to the late, ii. 467.

Mustard-seed, species of, ii. 313. Effects of each upon the land, ib. Soil and culture of, ib. Harvest of, 314. Average produce of, ib. Oil and cake when expressed from the seed, ib. and $n$.

Mutton, qualities of, ii. 485. Age and breeds of sheep, 486 , and $n$. Mode of securing the best flavour of, 486 .

Narrow-iedeate Poa, i. 511.

Neat-cattle-See Oxen.

Newbury peat ashes, qualities of, i. 334 . Manufacture of, ib. Application, effects, and price of, 335. Analysis of, 336 .

New Forest, in Hampshire, establishment of the, i. 15.

Newstead, in Staffordshire, homestead of, i. 10.5.

Nitre, price of: effects, and quantity to be used as manure, i. 239. Experiments of its application in differeut sorts of soils, 390 ; and on various crops, 391 . As a top-dressing on meadow land and growing crops, ib. Its destruction of slugs, 391, n. Quality of, 392.

Night-soil, qualities of, i. 267. Eifects of its application on the growth of potatoes, ib. $u$; and on grass-land, 268. Objections to the use of, 268. Cost of, ib. $n$. Its comparative operation with vol. II. farm-yard dung, 269. Compost and preparation of, 270. Quantity used, and crops produced by, ib.-See Liquid Manure.

Norfolk breed of pigs, ii. 509. system of hushandry, ii. 96. Observations on the, 97 . and Suffolk waggons, i. 158.

North IVilishire cheese, ii. 431.

Nutritive properties of grain, namelyoats, rye, barley, beans, and peas, i. 144, $147, n$. Their comparative weight and value. $145, n$. In oats of different weights, 146. In turnips, ii, 241, 242; and mangel würzel, 254. In cabbayes, 258. In potatoes, 28?. In carrots and parsnips, 289.

OArs, climate and soil most favourable to the growth of, ii. 177. Different species and qualities of, 178, 180. Comparative trials between the Georgian and the Potato-oat, 179; the Anigus and the Hopetown, ib. Culture of, 181. Seed and sowing, 182. Produce and analysis of, 183. Acreable quantity and quality of the straw of, 184. Nutritive properties of the grain, as horsefood, i. 144. Value of, relatively to other grain and pulse, 145. Comparatively to barley, 147. Table of the value of oats per barrel in Ireland, and per quarter in England, ii. 185.

- meal, quantity of, extracted from different weights of the grain, i. 146. Mealing process, ii. 184.

- grass, i. 513.

Odd-man, usefulness of an, i, 123.

Offices of the homestead.-See Farmbuildings.

Ogilvie, the Hon. Capt., experiments made by, on bone-dust, as a manure, i. 395.

Oil-cake, composition and value of, as food for cattle, i. 408 , ii. 356 . As manure. - See Rape-dust.

Onions, garden cultivation of, ii. 560 .

Orchards, introduction of, into this country, ii. 355. Carelessness of their management around farm-houses, ib. Soil and situation for the plantation of, 356 . Preparation and planting of the ground, 357. Time of planting, 359. Age, management, and pruning of the plant, 360. Nursery plants raised from the seed, 361. Gratting, 362. Choice of apples, ib. Cropping, 363. Superior qualities of pears, 366. Produce of an acre of orchard in cider and perry, i1). Period at which pears and apples respectively arrive at maturity, 366. Produce of single trees, ib. The peartree at HomeLacey, ib. Method of planting orchards with mixed fruit, 467 . Thithes of, i. 79.-See Cider and Perry. Orge céleste, a species of barley, ii. 172.

Owls, frod of, and inexpediency of the deslruction of, ii. 549 and $n$. 
Oxen, soiling and feeding of, on turnips, i. 209. Observations on experiments, to prove the comparative degree of nourishment contained in different species of food for, 211. On straw, turnips, and mangel würzel, 212. Preparation of the food for, 213 and $n$. Stall-feeding of, ii. 380. Opinions on close stalls and open sheds, ib. Choice of beasts to be fatted, 381. Skill in the touch and handling of, 382, 392. Position of the flesh on the carcass, and disposition to fat, ib. Produce of fat cattle in beef, 383. Management of, when stalled, 383. Proportion of food consumed when fatting, 385 and notes. Comparative experiments on oil-cake, tumips, and hay, 386; and on giving food, raw and steamer, $387,389,390$. Size of, ib. Butchers' profit on, 391,396. Tallow of, 391 and $n$. Weight of, 392. Rules for ascertaining the weight by handling, and by measuremert, ib. 393,394 , and notes. Sale of, 395.-See Grazing ard Summer Soiling.

—-, as beasts of labour, employment of, in ancient times, i. 178. Causes of their disuse, 179. Work of, in teams, as stated in several ploughing matches, 181. Kixpense of, comparatively with horses, 183 and 191. Proportion of land worked by, 188. Harness, modes of yoking, and power of draught of, 192 to 198. Portuguese and French methods of yoking, 195. Carts used for, 199. Breeds of, for the purpose of working, 213. Training of, 215. Mode of keeping up a succession of the working stock, 218. Shoeing of, 220.-See Cattle Sheds.

Ox-gang, a measure of land, i. 13, $n$.

PARINe and burning, i, 342 . Implements of, 313 . Operation of paring, 349 ; of burning, 350 . Kentish practice, 351. Beatsou's mode, $352, n$. Portable furnace for, 353. Expeuse of the operation of, 354. Reclamation of waste land effected by, 355 . Soils to which it is appropriate, 356 . Effects of, on some land, ih. Experiments of cropping after, ib. $n$. Observations on the indiscriminate practice of, 359 ; on sainfoin-leys and chalky downs, 361; on cold clay land, ib.; and on fens. Analysis of the ashes, 362. Summary of the operation of, 363 .

mattocks, i. 344 .

$345,346,347,350$

Parmesan cheese, weight and value of, ii. 436. Manufacture of, ib. $u$.

Parochial assessments, i. 82. Highway duty, ib. Poor-rate, 83.-See Append. No. I.

Parsuips, description of, ii. 288. Soil and preparation for,ib. Time of sowing, 289.
Storage of, ib. Produce, value, and application of, ib. and i. 125. Production of the seed, and preparation of the land, ii. 290. Modes of planting and gathering, 291. Quantity and price of, ib.

Pasture land, qualities and difference of, $i$. 478. Downs, 479. Improvement of the poor kinds of, ib. Scarification of the surface of, 480 ; and description of the machine, 481. Size of enclosure, and mode of stocking, 482. Observations on the hard and light stocking of, 484. Feeding of, 485.-See Grass-land.

Pauper colonies, ii. 567.

Peasantry of England, state of, at the Norman Conquest, i, 16. Present state of, 114.-See Agricultural Labourers.

Peas, different qualities and habits of ripening of, ii. 216 . Operation of, 217. Soil, and application of manure, ib. boiling properties of, ib. $n$. Culture of 218. Seed, produce of the grain, 219. Haulm of, ib, 463. Harvesting and application of, 220. Analysis of, ib.

Peat-moss, nature of, i. 4l4. Use of, as manure, ib. Preparation of composts with, 415. Experiments on different sorts of ; and mode of their application, 417. Calcination of, and conversion into ashes, 334.

Peppermint, expense and culture of, ii. 331. Harvesting, produce, and value of, ib.

Perennial grasses, i. 509. Soils adapted to, ib. Season of flowering, ib. $n$. List of, 510. Proportion of seeds to be sown on laying down land to permanent pasture, 516.

Perry, quality of the pears requisite for the manufacture of, ii. 365. Quantity of juice which they produce, 366 . Difference between cider and perry, ib. Mode of making an imitation of grape-wine with, 366, N.-See Orchards, and Cider.

Phleum pratense, or Meadow cat's-tail grass, i. 512.

Physical and aromatic plants, ii. 330 . Proper soils, description and cultivation of chamomile, ib.; liquorice, ib. ; peppermint, 331 ; lavender, ib.; rhubarb, 332.

Pigeons, corn consumed by, ii. 550.-See Wood-pigeons.

Pig-styes, plans of, and management, ii. 526.

Pilcorn and pilez, grain of, $\mathrm{ii}, 180$.

Pining, a disease peculiar to sheep, ii. 500 . Supposed cause of, 501. Indicalions of it, ib. Progress of, and reme. dies for, ib.

Plantago lanceolata, or Rib-grass, i. 511.

Plough land, a measure of ground, $i .13, n$. Ploughs, description of the common, ii. 2. The tumwrest, 4; Hertfordshire wheelplough, 5; Rotherham, or Simall's, 6 ; and Wilkie's improvements on, 7 , and $n$. 8 , with observations, 9 , and tempering 
of plough-shares, 10: Ransome's patent plough, 11, 12. Jefferis and Co.'s, ib. Mode of regulating the pitch of the plough, 13. Double-furrow plough, 15, and work performed by it, i. 183 . Three-furrow plough, ii. 16. Ribbingplough, ib. Strike-furrow, or doublebreasted plough, 17. Trench plough, ib. Skim plough, 18. Wheel ploughs, 19. Observatious on the difference of work between wheel and swing ploughs, ib. Principles to be observed in the construction of ploughs for light and strong land, 21.

- employed in the drainage of land, i. $453,454,455,460$. 343,345 , to 348 .

Ploughing, operation of, ii. 38. Ploughmen, importance of their workmanship, and mode of learning it, 39. Rules for the perfection of good work, 40 . Observations on the subject of draught, 41 . Common manner of ploughing, 44. Formation of the ridges, 45. Gathering, casting, and furrow slitting, 46. Crossploughing, balking, raftering, and gripping, ib. Average day's work, 47, 48; and extent of ground gone over by a team in turning up an acre with different furrows, i. 153. Depth of ploughing on various soils, and observations thereon, ii. 49 to 54 .

oxen, i. 181.

Poa grasses, namely-

Poa angustifolia, or Narrow-leaved poa, i. 510 .

- annua, or Annual

i. 510 .

-aquatic, or Water hair-grass, i. 518 .

L compressa, or Creeping poa, i. 511.

— nemoralis, or Wood meadow-grass, i. 511 .

- pratensis, or Smooth-stalked poa, i. 510

- trivialis, or Rough-stalked do. i. 510 .

Ponds, formation of, i. 98 . Mud of, 428. Time of mudding, preparation and application of the manure, 429.

Poppy, Mr., his mode and expense of clod-burning, i. 376 .

Poppy-seed, Flemish cultivation of, ii. 314. Harvesting of, ib. Produce of cake and oil, 315. Experiments on, and application of the oil of, ib.

Pork, ii. 506. For family use, 516. Flavour of the meat of, $514,519,520$. Pickling of, 523.-See Bacon and Ham.

Portland mutton, ii. 587, $n$.

Portugueze ox-cart, description of the, $i$. 195.

Potatoes, introduction and early cultivation of, ii. 259 and $n$. Description and produce of table and field varieties of, 260. Experiment on the planting of indifferent soils, 262. Effects of the crop upon the land, ib. Culture, 263. Effects of different sorts of manure upon the growth of, i. $267, n .277,341$. Time of planting, ii. 263. Modes of planting, 264; in banks, 265; and in the lazybed fashion, ib. MIanner of spreading the dung, 267. Comparative experiments on planting, 266,268 . Mode of raising different varieties from the seed of, 269. Growth of, by sets anu tubers, and experiments on the different modes, ib. $271, n .272$, and 274. Summary of those trials, 275. Management of the plants, ib. Experiments on plucking off the flowers, 276; and the apples, ib. Cutting of the haulm of, ib. and $277, n$. Curl in, 277 and notes. Failure of the crops of, 278 ; and opinions of its cause, 280 and 577. Storing of, ib. Russian mode of, ib. $n$. Produce of, 282. Analysis of, ib. Meal of, 283. Garden cultivation of, 558 .

- as food, in comparison with wheaten bread, i. $116, n$; for farmhorses, 125, 127 ; and for working oxen, 213; for fatting cattle, ii. $385,388$. Effects of, when given raw and steamed, ib. 389,390 ; for sheep, 489 ; for pigs, $513,518$.

Potato-cheese, Saxon mode of making, ii. 437.

Pot-herbs, ii. 56.2.

Poterium sanguisorba, or Burnet, i. 512.

Poultry, food and accommodation for, if. 531. Hen houses, 532. Fatting of fowls and ducklings, ih. Plucking of geese, il). Tithes of, i. 78.

Prejudices in favour of established habit in farming, instances of, i. 7. Respecting covenants for cropping in old leases, 65. Regarding the use of gypsum, 328.

Putrescent manures, composition and qualities of, i. 225.-See Dung, Farm-yard Manure, and Night-soil.

Raвniтs, tame and wild, ii. 532. Propagation of, ib. Stocking and management of, in warrens, 533. Classification of the skins of, and period when they - are in prime, ib. Capital, expenses, $\ldots$ and profits of, 534 .

Radcliff's account of the Flemish mode of preparing liquid manure, i. 271 . Of drainage in Flanders, 460. Of a comparative trial between broad-cast and drilled beans, and barley, ii. 57.

Radish seed, Kentish, cultivation and produce of, ii. 329.

- garden cultivation of the root of ii. 561 .

Rafter-level, use of the, i. 534 .

Ram-letting, trade of, ii. 482. 
Ransome's patent ploughs, descriptions of, ii. 11, 12. Portable thrashing machine, 198. Turnip slicing cart, and turnip barrow, 247, 248.

Ransom, Mr., of Sproughton, in Suffolk, experiment by, of a dressing of salt to barley, i. 382.

Ramenculus acris, or Butter-cup, i. 515.

Rape, as food for sheep, cultivation of, and soils to which it is adapted, ii. 249.See Cole Seed.

- dust, as manure, i. 408. Mode of application, quantity employed, and crops to which it is most generally applied, 409. Experiments of its effects on light soils, 410 ; and on heavy land, 412. Observations on, 435.

Rats and Mice, ii. 550. Prevention of the entrance of, into furm buildings, ib. Suffocation of, by sulphur, 551. Destruction of, hy traps and poison, ib.; and by cats, ib. Manner of keeping them out of stacks, 552. Mode of catching field mice, $\mathrm{ib}$.

Raven, description and habits of the, ii. 549.

Reaping.-See Harrest of Corn.

Red water, a disease of sheep, ii. 499. Description and causes of, ib. Remedies for, ib.

Refuse fish, as manure, i. 421. Quantity usually employed, ib. Application and effects of, $i b$.

Rennie, Mr., of Phantassie, crops of turnips grown on the farm of, ii. 240 .

Rent, considerations on, i. 56 ; in money, 57 ; in corn, 58; and in dues of different kinds, $57, n$.

Renton's table of cattle measurement, ii. 393 and $n$.

Rhubarb, soil, planting, and treatment of, as a culinary vegetable, ii. 332,562 .

Ribbing plough, ii. 16 .

Rib grass, i. 511 .

Rivers, embankment of - See Embankment.

Roads, and travelling in former times, i. 26. Present state and suggestions for the improvement of, 153; and Scotch Highway Act, ib., n.-See Append. No.I.

Rohan potatoe, ii. 261, $n$.

Rollers, construction of the common, ii. 33; and observations on, 34. Hollow, jron, and wooden, ib. Double rollers, 35. Convex and concave rollers, 36 . Spiky rollers, 37. Drill rollers, 38, 82, 239. Size and weight of the implements, 38. Pressing and drilling machines, 142, 143.

Romney sheep, ii. 453, 466, 490.

Roofs and floors of farm buildings, observations on, i. 87,89. Of dairies, ii. 406 and $n$.

Rooks, poisoning of, ii. 548. Food of, ib. Rookeries, ib.

Root crops, comparative fattening qualities of the different species of, ii. 292 .
Rot, in sheep, destructive nature of, ii, 501. Description and supposed origin of, 502 . Flukes, and opinions on the cause of, ib. Use of salt as a preventive, ib.

Rotation of crops, observations on the, $i$. 66., ii. 90. Exhaustion of land, 91 and n. Systems of culture, 92. Principles on which each separate course should be conducted, 94. Trienuial system, ib. Kemish round tilth, 95. Alteruate rotations, 96. Norfolk system, and obserrations thereon, ib. En. try to a farm, and arrangement of the courses of crops to bring it into regular cultivation, 98. Double crops, 100. Flemish mode of cropping, 101 and $n$. District rotations : on chalky land, 102 and $n_{0} ;$ on dry and sandy soils, 103 ; on heavy clays of a cold nature, 104; on thin clays, ib, ; on strong land of a dry and not too tenacious quality, 105 and notes; on rich alluvial soils, 107 ; in the fens, ib. ; on peaty soils, moorlands, heath, and flow moss, or bog, 109. Summary, 1 10.-See Classification of Soils.

Rotherham plough, ii. 6.

Rough-stalked poa, i, 512.

Rowen.-See Hay.

Ruck, Mr., plan of his ox-house, i. 205.

Rudgewick hogs, breed and weight of, ii. 508.

Rumex acetosa, or Sorrel, i. 515.

Run-rig, system of, in Scotland, i. 32.

Rural economy: arrangement of labour, i. 112 ; rotation of crops, ib.; details of business and accounts, 113; farm servants, 114 ; their wages and stipends, 116.See Manual and Animal Labour.

Russell, of Kenilworth, rotations of crops on rich clay loams and light calcareous soils recommended by Mr., ii. 116, 117. His account of the expenses and profit of working wheat and bean soils, 121; and turnip and barley land, 122.

Rust, a disease of corn, ii. 157.

Rye, as horse-food, i. 146. As breadcorn and bread, ii, 166 and $n$. Qualities of, 167. Soils which are appropriated to the growth of, ib. Culture of, 168 ; and of meslin, ib. Application of lime to, ib. Time of sowing and quality of seed, 170. Period of flowering and ripening of the grain, ib. Dis. ease and effects of ergot, ib. Product and analysis of the grain, ib. Value of the straw, ib. and $n_{0}$. Use of green rye as sheep food, 171 .

- grass, ancient cultivation, growth, and advantages of, i. 514. Italian species of, ii. 299. Value and effect of, upon the soil, 300. Growth of, ib. Produce of hay and seed, ib.

Saccharometer, i. $438, n$.

Sainfoin, soils best suited to, ii. 305. Manure, ib. and i. 260, n. Sowing and duration of, 305. Seed, 306 . Applica- 
tion and management of, when intended for hay, ib. When meant for seed, 307. Quantity and value of, ib. Breaking of the ley of, ib.

Salt, as a condiment for horses, i. 142. For neat cattle, 213. Effects of, upon slugs, and in correcting the fault of spolled fodder, $378,387,446$. Effects of, and quantity to be applied generally as a dressing, $379,380,446$. Kxperiments of, on various crops, 381 ; and on pasture land and hay, 385. Observations on, and experiments in support of opposite opinions regarding their success, ib. and 386. Application of, in a foul state, and in composts, 337. Specific quantities to be applied, when pure, to various crops of corm, roots, hops, and grass land, 388, 389. Saltpetre-See Nitre.

- marshes.-See Fens.

Sand, employment of, as an alterative for stiff clay land, i. 427. Mode of its application and effects, ib.

Sandy soils, effects of the application of lime upon, i. 289. Effects of marl on, i. 319. Nature of the land of, 443. Rotation of crops on, ii. 103, 117. Growth of wheat upon, 140

Saxon thanes, i. 13. Their mode of living, 16.

Scab, a disease in sheep, description and occasion of, ii. 496. Cure of, ib. Mode. of its attack and cure of, in pigs, 530 .

Scarrifying machine, description and effects of, on pasture land, i. 491.

Scarlet runners, ii. 559.

Scotch and English farming, comparison of, i. 33 .

Scotland, agriculture in former times, i. 31. Abolition of tithes in, ib. System of run-rig, 32. Present state of, 33. Farm buildings of, 110. Management of grass land in, 67,477 . Origin of fallowirg in, ii. 55. Improved implements of husbandry used in, i. 161 , ii. $6,7,8 n$. and 48.

Scouring, disease of, in calves, ii, 448 ; in sheep and lambs, 500 .

Scufflers, description of, ii. 26.

Sea kale, garden, cultivation of, ii. 573 . Appropriate soil for, ib.

- ware, collection of, and use as manure, i. 418 . Value of, in compost, 419. Experiments on, and effects of, 417,419 . - See Kelp.

Seeds of garden vegetables, ii. 576 .

Sheep, synopsis of the chief British breeds of, ii. 453. Age and names of, at different periods of their growth, 45. Procreation of, 455 , and $n$. Number of ewes to a ram, 455. Blossoming of the ewe, 456. Description of the tup, 456 and $n$. Gestation of the ewe, 457. Yeaning, ib. Early management of the lambs, 458. Castration and docking, ib. House lamb, time and mode of rearing, 460 . Suck- ling house, 461. Grass lamb, ib. Time and mode of weaning the breeding stock of lambs, 462. Management on removal from their dams, 463. Feeding with turups, ib. Folding and cotting, 464 to 470 . Shearing, 471. Washing, ib. Wash-dykes, ib. Operations of washing \& shearing, 472, 473. Salving, 475. Materials and operation of, 478 . Qualifications and remuneration of the shepherd, ib. The shepherd's dog, 479. Wool, 480. Trade of ram-letting and account of the tup-masters, 482 . Weight and value of the sheep's carcase, 485 , 487. Age and flavour of the mutton of different breeds, 485, 486, and notes. Fatting on artificial food, soiling, and experiment on sugar, $487, n, 488$. Grazing of, 490. Practice and observations on the breeding of, 491 . Method of improving the form of, 492. Constitution of, ib. Disposition to fatten, 493. Diseases of, ib. Signs of health, 504. See Folding and Wool. fescue grass, $i .511$.

Sheffield Park, plan of the homestead at, i. 108 .

Shells of fish, when burnt, i. 282. Effects of, as manure, in a natural and powdered state, ib.

Shell sand, as manure, i. 283.

Shoeing of horses, Flemish mode of, $i, 171$. of oxen, i. 219. Description of different brakes for the security of the operation of, 220.

Shropshire hogs, ii. 509. Average weight of, 510 .

Sinclair, Sir John, president of the Board of Agriculture, and director of the Agricultural County Surveys of the United Kingdom, i. 35 .

- Mr. George, author of the "Hortus Gramineus Wuburnensis," his experiments on the use of salt in field husbandry, i. 381,383 . His analysis of land manured with bones, and the effects, 401 .

Single-horse carts, description and advantages of, i. 161 .

Skeleton barns, ii. 197.

Skim plough, ii. 18.

milk cheese, ii. 435 .

Sledges, description of those used for agricultural purposes, i. 167, 495.

Sleech.-See Mud.

Small's plough, description of, ii. 6 ; with Wilkie's improvements, 7 and 8 ; and observations, 9.

Smithfield Club, its object, i. 28. , sale of cattle at, ii.

Smooth fescue grass, i. 511.

Smut, in corn, epinions entertained of the cause of, ii. 137. Description of, 159. Examination of smutted wheat, 160 ; and steeping of, 161. Experiments on, 163,164 .

Soaper's waste, qualities of, i, 339, Quan- 
tity and application of, 340. Experiments on the effects of, as a top-dressing, 341 .

Soft grass, tall oat-like, woolley, and commou sorts of, i. 514 .

Soiling of horses, i. 136. Flemish practice of, 139. Medium quantity of green food daily consumed by a cart-horse, ib. On giving corn along with green food, 140. Of oxen, 209. Quantity of turnips and straw daily required for the support of a working ox, 210, 211. General observations on, ii. 372. Conduct of, 374. Modes of keeping the cattle, 375. Comparative experiments on soiling and grazing, 377 and $n$. and 378 ; and on raw and seasoned food, 387.

Soils, manner of describing, i.438. Composition and qualities of, ib., n. 439. Clays, 440 ; their drainage, time of ploughing, cost of cultivation, and mode of improvement, 441. Calx, or lime, 442. Sand and gravel, culture and improvement of, 443 . Flints, ib. Loams, composition and management of, 444 . Alluvial species of, ib. Peat, 445. Salts, 446. Indications of their peculiar qualities, and remarks on the general improvement of, 446. Nature of good wheat-land, ii, 140,-See Classification of Soils and Fallowing.

Soot, qualities of, as arising from different species of fuel, i. 337. Experiments on the application of, as a top-dressing, ib. Quantity, price, and application of, 338 . Properties of, ib.

Sorrel, i. 515.

Sowing, modes of, ii. 71. Broad casting by hand, 73 ; and by the machine, with the description and cost thereof, 74 . Introduction of drilling, objects of, and opinions on its merits comparatively with broadcast, 75 and $78, n$. Implements, cost of, and operation of drilling, 79. Method and expense of dibbling, 82. Seed used in, 83. Observation on, 84. Comparative experiments on broadcasting, drilling, and dibbling, 85 to 89.

Space husbandry, ii. 568. Crops pro. duced by, ib. $n$. Cost of digging, 570 . Spade used in Ireland, $570, n$. Cultivation of potatoes on joint account of the farmer and his labourers, 57l.

Spayed heifers, ii. 369.

Spencer, Earl, comparative experiments on mangel wiirzel and Swedish turnips, made under the direction of, ii. 253.

Spinach-seed, Kentish, cultivation and produce of, ii. 329.

Spit-dung, i. 226, 241, 247.

Spring-wheat, ii. 152. Season of sowing, 153. Analysis of, 154. Opinion regarding the flour of, ib.-See Wheat.

Spurry, i. 407. Growth, quality, and application of, ii. 304.

Stables for farm horses, i. 168. Ham- mels, 169. Manger, meat, and management in, 143.

Stack-yards, i. 97. Stacking of hay, 496 ; and building of corn-ricks, ii. 193, 196.

Stafford, the Marquess of (now Duke of Sutherland) plans of farm buildings on the estates of the, i. 101 to 107.

Stallington Grange, in Staffurdshire, homestead of, i. 103.

Statute labour, i. 82. Scotch Highway Act, 153,n. Time-work, and maximum of rate-—See Turnpike Roads, ii. 574.

Steaming of food for cattle, mode and implements for, i. 129, 132. Experiments on feeding neat cattle on raw and steamed food, ii. 390 ; and on pigs with the same, 521,522 .

Steel-bow, custom of, i. 52.

Stiles, ii. 545.

Stilton cheese, price and manufacture of, ii. 431. Maturity and flavour of, il.

Stiveen, an implement of husbandry in Ireland, ii. $570, n$.

Stone-brash, soil so called, i. 517, ii. 339.

- walls, construction of, as farm fences, ii. 545 .

Straw, qualities of the different sorts of, i. 132, 211,212. Intrinsic worth of, for the purposes of litter, ib; and as fodder, 133, 230. Relative yalue of, per load, to hay, ib. Burning of, in the stubble, 333.

yards, situation and management of, ii. 370. Common objects of, 371 . Feeding of store cattle in, ib. Observations on, i. 208.

Strathaven, mode of fatting calves for veal in the district of, ii. 447 .

Strike-furrow plough, ii. 17.

Suffolk punch, deseription of the, i. 173. and Norfolk breed of yigs, ii. 509,

Sugar-bakers' scum, as manure, i. 426.

Mode of application and price of, $\mathrm{ib}$.

Surfeit, in pigs, cause and cure of, ii. 530 . Sussex oxen, i. 213,214 .

waggon, description of the, i. 154.

Swedish turnips, as food for horses, i. 125 ; neat cattle, 209, ii, 386, 388, and sheep, 489._-See Turnips.

Sweet-scented vernal grass, i. 514.

Swine, breeds and description of, ii. 507 . Procreation of, 510. Choice and management of the boar and sow, 511 . Observations on breeding in-and-in, 512 . Farrowing, and average number in a litter of pigs, 512 . Rearing of, 513. Sucking pigs, or roasters, ib. Castration of, ib. Food and weaning of, ib. Porkers, 514. Food and flavour of the flesh of, ib. and $n$. Food and management of store-pigs, 514. Grazing and soiling of, 515. Operation of ringing, ib. Stock of, on arable, ib.; and dairy farms, 516. Breed and management of a litter when intended for family use, ib. Agre and time requisite for fatting, 517. Quality and quantity of food, 518,519 , and notes. 
Experiments on feeding with rice, 520 ; and with steamed food, 521. Coustruction of the piggery, 526. Management in the stye, 528. Experiment on fatting in close single styes, 529. Diseases of 530. Tithes of, i. 178.-See Bacon and Ham; and Brawn.

Swing-plough, description of the common, ii. 2 .

Switzerland, use of liquid manure in, i. 275.

TAFF and chaff, and Colder, j. 144.

Tall fescue-grass, i. 511. oat-grass, i. 513.

Tallow, or inside fat of oxen, ii. 386 ; of wedder sheep, $490, n$.

Tamworth boar, ii. 509.

Tangle.-See Sea-ware.

'Tanners' bark, preparation and effects of, when made up as a compost with lime, dung, and earth, i. 424.

I'ares, for soiling, i. 131, $140 \mathrm{n}$. Winter and spring species of, ii. 301 . Cultivation of, ib. Management when intended for seed, ib.; when mown for hay, 302. Application of, ib. Average value of the crops of both kinds, ih.

Task-work, i. 122.

Teasel, soil and preparation for the production of, ii. 326. Crops cultivated along with, ib. Appearance of, and time required for the maturity of, ib. Cultivation of, 327. Gathering, produce, ib.

Teinds, i. 81 .

Tenancy of land, i. 56. Rent, in kind, 57 $n . ;$ in money, 57 ; and in corn, 58 , and $n$. Estimation of rent, 59. 'T'erm of the lease, 60 . Observations on tenancyat-tvill, 61 ; and on granting leases to the highest bidder, 64. Covenants respecting cropping, 65. Leases of the Holkham property, n. 66. Common covenants, 68. Payment of rent, and right of hypethec, 70.-See Laudlord and Tenant, Append. No. II.

Thaër, Von, on the fermentation and evaporation of dung, i. 239. Rotation of crops on sandy soils, adopted by him on the royal farm of Noëgelin, in Prussia, ii. 117; his opinion on the application of dung to growing crops upon sandy loam, 218.

Thatching, observations 011, i. 87, Expense and use of, with fern, on farmbuildings, 89. Cost of, on hay-stacks, 502. Manner of laying thatch on cornricks, ii. 196.

Theory and practice of farming, i. 233. Respecting the application of putrescent manure, 246, 431; and regarding springwheat, ii. 154.

Thirlage, i. 57 n.

Thee-furrow plough, ii. 16.

Thrashing machines, invention of the, ii. 198. Horse-mills, ib. Hand-mills, ib. Operation of, ib.
Throckmorton, -Sir Charles, of Buckland in Oxfordshire, comparative experiments on the state of, respecting the application of bones and farm-yard dung to sandy soils in the growth of tumips, $i$. 394.

Ticks, or sheep-lice, description and removal of, ii. 496.

Tillage-farms, i. 40. Tillages and dressings on, 52 ; and valuation of, 54 .

Timothy-grass, i. 512.,

Tithes, various denominations of, i. 71; Setting out of, 72. Valuation of, 73. Of agistment, 74; and discharge from, in Ireland, 81. Hegulations regarding the live stock on which it is due, and those which are exempt, 75, and notes. Settlement of, in money, 76 , and $n$. Of animals, and mode of settlement, 77. Of milk, 78. Of wool, pigs, and poultry, ib. and notes. Of wood, 76, and $n$. Of orchards and gardens, ib. Of wastes, 80. Rent of, ib. Extinction of, in Scotland; and, in Ireland, release from agistment, 81. Right by which they are held, ib. Commutation of - See Append. No. I.

Tonquin breed of pigs, ii. 509.

Training of cart-horses, i. 175.

'Train-oil, effects of, as a compost with earth, i. 420.--See Blubber.

Transplantation of corn, ii. $152 n .569 n$.

Trefolium repens, or white clover, i. 515.

_.__ pratense, or native red, and marl-grass, ib.

Trench-plough, ii. 17.

Trolley, a waggon used in Leicestershire, i. 160 .

Tull, Jethro, his drill husbandry, i. 9, 25 ; his experiments on drilling grain, and mode of operation, ii.. 72 ; his management of turnips, ib.; effects of his theory, 73.

'Tumbrel, description of the, i. 164.

Tup-masters, i. 5. 11, 482.

Turf, transplantation of, i. 522. Mode of operation, result, and expense of the experiment, 523.

- ashes, acreable quantity produced by paring and burning, i. 354,362 . Analysis of, ib. Application of, 363.

Turnip and barley soils, composition of, $\mathrm{i}$. 443, ii. 96, 113, 116. Expenses and profit of working, 122 .

Turnip cart and barrow-slicer, description of the, ii. $247,248$.

_ rooted cabbages.-See Cabbages.

Turnips, introduction of, ii. 231. Soil and species of, 232. Seed and sowing, 234 and $n ., 235 . \quad$ Culture, 236. Application of manure, 237. Quantity and effects of different sorts of manure when applied to various soils for the growth of,-namely :-farm-yard dung, i. 260 n.; liquid manure, 277; burnt clay, 365,367 ; bones, $394,396,404$. Operation of sowing, ii. 238. Drill-rollers, 
239. Appearance of the plants, ib. Produce of the ruots, 241. Weight and size of the different species of, 241 , and n. 233. Average quantity of nutritive matter contained in each, 24l. Diseases of, 242. Experiments to check the ravages of the fly, 243 and $n$. Storing of, 244. Process of grubhing, and observations on it and storing, 245 . Application of, 246. Sliciug of, 247. Acreable produce and value of, ib. General consumption by sheep of Swedes and common turnips, 249, and notes, 173 and 238.

Turnpike-roads, abstract of the Act for the regulation of highways, carriages ard tolls.-See Append. No. 1.

Turnwrest plough, description of the, ii. 2.

Tuscany, use of liquid manure in, i. 275. Green crops iu, $405 n$. Practice of warping in, 469 and $n$.

Tusser's "Five luundred points of good IIusbandrie," i. 2:2, $2+u$.

Unizrs of ewes, treatment of tumours in, and sore teats of, ii. 504 .

Upland soils, rotation of crops on, ii. 118 .

Urine, analysis of, i. 229. Quantity voided by animals, 274. Mode of its application, 276 ; and experiments upon various crops, 277. Quantity applied, ib. $n$.

Vatuation of the stock on different farms, i. $44,45,47$; of tillage and dressings, 54.

Veal, production and profit of - See Calves.

Vegetable and animal manure, i. 225.See Farm-yard Manure.

Veitch's plough, ii. $10 n$.

Vermin, loss occasioned by, ii.547. Modes of checking the ravages of grubs, shigs, caterpillars, and wire-worms, ib, Anthills, ib. Earth-worms, ib. Depredations of small birds, 548. Poisoning of ruoks, and doubts of the expediency of destroying them, ib. and $n$. liavens, 549. Owis, their destruction of fieldmice, ib. and $n$. Wood-pigeons, 550. Quantity of corn consumed by tamepireons, 551. Manner of suffocating rats and mice, and preventing their entrance into farm oflices and stacks, 551. Field mice, 552. Opinions entertained respecting moles, ib. Mode of driving them away, 553. Prejudice entertained against the hedge-hog, ib.

Vetches, different species of, ii. 220. Soil, 221. Seed and produce, ib. and $n$. Common lentil or dill, ib.-See Tares.

Vicia sepium, or bush-vetch, i, 511,

Villeins, i. 13.

Vision of Pier's ploughman, i. 17, 24.

Voters fur Members of Parliament, regulations for, Append. No. I.
WAGES and stipends of farm-servants, $i$. $116,123$.

Vagrons, descripion of those used in Sus ex, i. 155; in Hampshire and $\mathrm{Ox}-$ fortshire, 156 ; in Berkshire, in Norfolk, and in Suffolk, 157. The hermaphrodite, ib. The Cornish wain, 159, 200. The bavin-tug, 159. The Leicestershire trolley, 160 . Dranght of, 161 .

Walker, Mr., of Ferrygate, his experiment on feeding cattle with raw and steamed food, ii. 387.

Mr., of Killiow, in Cornwall, homestead on the firm of, i. 93.

Mr., of Mellendean, his opinion of rotten a d fresh dung, i. 249.

Warping, fureign practice and introduction into Encland of, i. 467, and n. Mode of operation, 468; expense and time required to perfect it, ib. Advantages of the result of, 469. Crops produced by, ib.

Warrens, stock of rabits maintained in, ii. 533. Management of, ib. Capital, expenses. and profit of, 534 .

Wash-lyke, description of one at Burleigh, in Rutlandshice, ii. 472.

Waste-lanu, tithes of, i. 80. Reclamation of, effected by the operations of paring and burning, 355 . Observations on the improvement of, 462, 479. Experiments on, 463. Expense and ultimate advantages of, 464 . Mode of operation, 465 . Common fields, observations on, 466.See Fens and Bogs.

Water-meadows, advantages of, 530 . Average expense and profit of, ib. $n$. Management of, 531. Hay of, ib. Feeding of, 532.-Sce Irrigation.

- hair grass, i. 518. poa, i. 518 .

Watson, Mr., of Keillor, near Cupar Angus, comparative trial of bruised bones and dung as manure to turnips, by $\mathrm{i}$. 394.

Veather, remarks on the; use of the Larometer, ii. 592 ; the hygrometer, 593 ; the thermometer, of that constructed by Rutherford, ib. Cause of dew, ib. Phases of the moon, and influence of, 594; age of, ib. Divisions of the year, and its seasons, ib.

Weeds, destruction of, i. 433. Glossary of, Append. No. V.

Weights and Measures.-Append. No, III. Welsh fescue-grass, i. 511.

Weld, description of, ii. 335. Soil, sowing, and treatment of the plants during their growth, ib. Application and value of the crop, ib.

Wells, construction of, i. 98 .

Wheat, price of, in the beginning of the 13th century, i. 19. Different species of, ii. 138,139 , and $n$. Soil, and expenses of working, 121, 140. Cultivation of, ib. Description of the pressingmachine, 143. Quantity and effects of 
different sorts of manure laid on for its growth on various soils,-namely :farm-yard dung, i. 260,n.; night-soil, 269 ; liquid manure, 277 . Soot, as a top-dressing, 337 ; salt, 382 ; and lime, ii. 144. Seed and sowing of vinter wheat, 145.-(See Sowing.) Quantity of seed per acre, whether broad-cast, drilled, or dibbled, 130, 146, and $n$. Jepth of sowing, 147. Spring tillage, 148. Hand-weeding, 149 . Winterproud, ib. Feeding off, 150. Sowing a secoud time, ib. Clover-leys, tares, and other crops, as a preparation for, 151. Transplantation of, $152, n ., 562$, $n$. Produce of the crop, and analysis of the grain, 154. Weight and quantities of the flour of, 155 ; and experiment on that of Scotch and English wheat, ib. $n$. Mode of judging correctly of the sample, 155. Foreign wheat, 156. Diseases of, ib. Steeping of, 161 ; and experiments on the effects of, 163. Observations on stimulating the germination of seed, 164. Effects of sowing rye along with wheat, 169.

fly, or weevil, ravages of the, ii.

160. Preventives of, $161, n$.

Wheel-ploughs, ii. 19. Observations on the difference of work between them and swing ploughs, ib.

Whins,-See Furze.

White and red native clover, i. 515.-See Clover.

Wild cattle, i. 14.

Wimpey, Mr., of Brockhampton, his experiment on the comparative production of butter and cheese from equal quantities of milk, ii. $45 \mathrm{l}$.
Winnowing-machine, ancient prejudices against the use of the, i. 7. Description and operation of the, ii. 201.

Winter-fallow.-See Fallowing.

- grasses, hardy and productive sorts of, and quantities of seed to be sown on laying down permanent pasture, i. 520 . - See Fiorin.

Woad, description of, ii.335. Cultivation of, on grass and arable land, 336. Foreign mode of its culture, ib. Harvesting of the plants, ib. Average produce and value of the crop, 337. Mlanufacture of, ib.

Woburn breed of pigs, ii. 510 . perennial kale.-See Cabbages.

Wood, Sir Francis, of Garrowby, in Yorkshire, effect of hones as a manure on the estate of, i. 393.

- tithe of, i. 79 .

- meadow-grass, i. 511.

- pigeons, anecdote of, ii. 549. Food of, 550 .

Woodstock waggon, description of the, $i$. $155,200$.

Wool, combing and carding, ii. 480. Properties of, ib. Classification of, 481 . Alteration in the quality and weight of the fleece, ib. Comparative qualities of the British, Spanish, and Saxon wool, 482. Improvement of the carding of, by crosses of the English and Spanish sheep, 484. Rules for the improvement of coarse wool, ib.

Woollen rags, their application and effects of, as manure, i. 425. Quantity commonly applied, and usual price of, 426 . Woolly soft grass, i. 514 . 


\section{W OR KS}

PUBLISIKD MY

\section{BALDIVIN AND CRADOCK, LONDON.}

By the Author of Husbandry, 2 vols.

G KETCHES of the DOMESTIC MANNERS and INSTITUTIONS of the ROMANS. A New Edition, in 12mo., price 7s, 6d, half-lound and lettered.

\section{UNDER THE SUPERIYTENDENCE OF THE SOCIETY FOR THE DIFFUSION OF USEFUL KNOWLEDGE.}

THE IIORSE; with a Treatise on DRAUGIIT, auel Numerous Cuts. In 1 vol. 8 vo. P'rice $8 s$. $6 d$, bound in cloth.

BRITISH CATTLE, with fine Engravings, from drawings ly Harvey, taken from Living Specimens. In 1 vol.8vo. Price $10 s, 6$.

SIIEEP, with the MOUNTAIN SHEPIIERD'S MANUAL. Nimerous Fine Engravings after IIarvey. In 1 vol, 8 vo, Price $10 \mathrm{~s}$. 6 . . , bound in cloth.

REPORTS of the CULTIVATION of Seven SELECT FARMS. Price $6 d$. each number.

A PRACTICAI, TREATISE on the BEST NIODE of REPAIRING ROADS. By Chances Penfolm, Surveyor. In one number. Price $6 d$.

*** This excellent work is strongly recommended to those interested in road-making.

MAPS, ANCIENT and MODELN, with PLANS of CITIES. Price $1 s$. each part plain, and $1 s, 6 d$. each coloured.

*** These Maps are compilu from the very best anthorities, hoth of heial and privite The scale varies according to the acknowledged importance of the respective countries and districts. The Ancient Maps-a very useful and interesting feature of which is, that they are precisely on the same scale as the corresponding Modern-are compiled with tho greatest care, and under the most rigorous serutiny.

Sixty-nine Parts are published, and about eight more are expected to complete this great and incomparably cheap work. It is pubished monthly.

BINGLEY'S PRACTICAL INTRODUCTION to BOTANY. A New Edition, by Frosi, 12mo. Illustrated with Plates. Price 4s. half-bound, or with the Plates coluured, price $7 s$.

ABERCRONIBIE'S GARDENER'S POCKET JOURNAL, and Daily Assistant in the Modern Practice of English Gardening. 18mo. Twenty-fourth Edition, enlarged and improved. Fine Frontispiece and Vignette. Price 2s.

DESCRIPTION of more than THREE HLNDRED ANIIALS. A New and Enlarged Edition, elegantly printed by Whittingham, with numerous Fine Wood-Cuts and several additional ones. In $12 \mathrm{mo}$. Price $9 \mathrm{s.}$; or in morocco, $12 \mathrm{~s}$.

CLATER'S EVERY MIN IIS OWN FARRIER. Twenty-sixth Fdition, much enlarged, and revised throughout by an Fininent Practitioner. In 12no. Price $6 s$. , cloth, boards.

CLATER'S ETERY MAN HIS OIVN CATTLE DOCTOR. The Seventh Edition, revised and almost re-written by an Eminent Practitioner of Horse and Cattle Medicine. In a handsome volume, $12 \mathrm{mo}$. Price $6 s^{\text {, }}$ cloth, boards.

THE NATURAL IISTORY and ANTIQUITIES of SLLBORNE, By the Rev. Grusen'r Wrixe, MI.A. With the Natmalist's Calendar, and Miscellaneous Observations extracted from his Papers. A New Edition, with Notes, by Evwans) Tunner Bennetr, Esq., F.L.S., \&c. Secretary of the Zuological Society, and by others. In a handsome 8vo. volume, with numerous briliant Engravings from drawings by IIarvey, finely printed by Whittingham, Price 18 s, cloth extra, 


\section{WORKS PUBLISHED BY BALDIVIN AND CRADOCK.}

\section{TAXATION, REVENUE, EXPENDITURE, POWER, STATIS.}

TICS, and DEBT' of the whole RRITISH EMPIRL. The whole founded un, and ilustrated by, Official Tables and Authentic Documents. By Pabro Pebrer. In a $1^{\text {arge }} 8$ vo. volume. With numerous Tables. Price 18s., cloth boards.

$*_{*}^{*}$ The important contents of this very interesting volume are divided into Fou Parts, viz. :-Part 1. Origin, Progress, and Present State of Taxation, Revenue, and Ezpenditure from the Earliest Periud to the Present Time; Chronological Tialle of Wars and Treaties; Tables of Revenue, Expenditure, \&c.-Part 2. Origin, Progress, and Present State of the National Debt and Funding System; Tables of the Debt, Bank, and Stock Exchange. Part 3. Estimate of the Capital, Power, and Resourees of the British Empire in all Parts of the IVorld; 'Tables of the Revenues, Statistics, and Debt of India -Part 4. Effects of the Taxation required to pay the Interest of the National Debt and a Practical Plan for its Liquidation, \&c.

A GENERAL PRONOUNCING DICTIONARY; showing, at one view, the Orthography, Accentuation, Explanation, and Pronunciation of all the purest and most approved Words in the Finglish Language, By WM. ExvieLd, MI.A. In $18 \mathrm{mo}$. The lierenth Edition, carefully Reriseth, Cornceted, Improved, and handsoncly printed. Price $4 s_{s}$, bound in roan.

* * This is the only PRONOUNCING Dictionary of a portable size, with a bold readable type, and convenient shape for the pocket.

ELEMENTS of ANCIENT HISTORY, including Greece, Egypt, Pursia, Carthage, Rome, the Philistines, Phœenicians, Jews, Guths, Huns, \&c. By Josepri Gur, Author of the Pocket Cyclopædia, and numerous popular works for Schools. Neatly printed, in a 1 mmo. volume of upwards of Thre Ilund:ed Pages, price only 3 s. Gil., hiandsumely bound and letterod.

GUY'S ELEMENTS of MODERN HISTORY, including Franc', Germany, Spain, Portugal, Puland, Russia, Prussia, Sweden, Denmark, Italy, America, Se., divided into Chapters, $f_{c}$, on the plan of the preceding. 1:mo. Price unly $3 s$, 6d., bound and lettered.

GUY'S ELEMENTS of BRITISH HISTORY ; containing England, Scotland, Wales, and Irelant, on the same plan. 12mo. price only $3 s .6$. bound and lettered.

** These Elements, it is hoped, will supply a valuable desideratum for Grammar Schools, Academies, and Fumilies. The object has been to concentrate a sufficient portion of General History in three moderate size reading volumes, the whole being an Aliridgment upon an ample scale: everything therein contained is founded upon unquestionable authority, having been taken from nome but works of established reputation. Each Volume can be purchased separately.

A GRANDMOTHER'S ADVICE to YOUNG MIOTHERS, on the

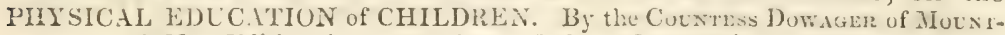
Casmel. A New Edition, in a neat volume, foolscap 8vo. Price 7 s. cloth extra.

SIMPSON'S COOKERY, Iniproved and Modernized. By W. II. Buand, of the late Kin's's Kitchen. In small 8vø. Price $8 s$, cloth, boards.

** In addition to an extensive collection of original recipes, bills of fare fur every month in the year, tables of articles in season, lints of sauces, soups, first and second course disbes, adapted to the present style of living, \&c., this edition contains also, in copious indexes, the French as well as Linglish names of all dishes, sauces, soups, \&e, in the bouk.

The ENTIRE IVORKS, CORRESPONDENCE, and TRANSLATIONS of WHLLAM COWPLR, carefully revised, collated, and edited, by RoBEn' Soutuex, Esq., LL.D. Poet Laureate. IVith an interesting Life of the Poet; together with Biorrapheal Nonices of many of the Auther's fursmal fruends and correspondents, by the Euitor. In 15 volumes. Price 5 s, each, cluth extra.

***This First Complete Edition of all Cowper's Works, including his Productions by Prose as well as his Correspondence, and his Translations as well as his Original Puems, is printed in the best manner by Whittingham, on the finest paper, and ornamented by exquisite Plates from drawings of scenery taken on the spot, expressly for this edition, and engraved by the first artists : it contains Portraits also, from the best originals, of many of Cowjer's frieuds and comnexions, in addition to the usual embellishments. 


\section{WORKS PUBLISHED BY BALDWIN AND CRADOCK.}

LEGENDS and STORIES of IRELAND. By SAmuel Lover, Esq., R.II.A. The Fonrth Edition, with Etchings by the Author, and several Wood Engravings, foolscap, $8 \mathrm{vo}$. Price $6 s$., cloth extra.

LEGENDS and STORIES of IRELAND. By the Same. Second Series. A New Edition, currected, and illustrated with fine Wood Engravings, in foolscap, 8vo. Price 7s. $6 d_{\text {, }}$, cloth extra.

TRAITS and STORIES of the IRISH PEASANTRY. By WILLIAM Cartigux. Illustrated with numerous characteristic Plates by W. H. Brouke, Esi]., and Wood Engravings. Fourth Edition, in 5 Vols., foolseap 8vo. Handsomely printed. Price 1 l. 5 s., cloth extra.

*** The Work contains Twenty-one Stories, and the Volumes, each including at least Four Tales, may be purchased separately.

"Admirable, truly, intensely Irish-never were the outrageous whimsicalities of that strange, wild. imaginative people so characteristically described; nor, amidst all the fun, frolic, and folly, is there any dearth of porerty, pathos, and passion. The author is a jewel."-Blackwood's Magazine.

The "TALES and NOTELS of MARIA EDGEWORTH, complete in 18 volumes, price $5 s$. each, handsomely bound in water-lined cloth, and lettered. Illustrated with Diawings by W. I Iarvey, Escl, and engraved in the first style of excellence by Goodall, C. Rolls, Goodyear, H. Robinson, Finden, Bacon, \&c.

*** This elestant Edition corresponds in size and illustration with Byron, Crabbe, and Scott's works, and must be a desirable acquisition to all libraries. It contains the following works, viz.:-

CASTLE RACKREN'T and IRISH BULLS. 1 rol.

MORAL TALES, 2 vols.

PUPULAR TALES, 2 vols.

TALES of FASHIONABLE LIFE, and MODERN GRISELDA, 5 vols.

\section{BELINDA, 2 vols.}

LI:ONORA, and LETTERS for LITERARY LADIES, 1 vol. PATRONAGE, 3 vols.

HARRINGTON, 1 vol. ORMIOND, 1 vol.

Each volume may be had separate.

The TIORTS of WILLIAM HOGARTII: the ORIGINAL PLATES, purchased by the present proprietors from the Executors of the late Alderman Boydell, and now fully restured by eminent engravers; with the addition of many subjects which were not in that collection. Accompanied by a BIOGRAPHICAL ESSAY on the GENILS and PRODLCTIONS of HOGARTH, and Explanations of the Suljects of the Plates. By Jorix Nicriolis, Esg., F.S.A. In imperial folio, complete in 52 Numbers. Price 5 s. each; containing, with numerous other Plates, the fullowing celebrated subjects:-

Marriage a-la-Minle, Six Plates.

Industry and Idleness, Twelve Plates.

Rake's Progress, Eight Plates.

Hudibras, Twelve Plates.

Dun Quixote, Six Plates.

Four Times of Day, Four Plates.

Election Subjects, Four Plates.

Harlot's Progress, Six Plates.

House of Commons (Portraits).

Examination of Bambrilge (ditto).

Stages of Cruelty, Four Plates.

Portraits of Hogarth. Two Plates.

Garrick in Richard III.

Mrs. Siddons, as Sigismunda.

Captain Coram.

Bishop Hoadley

Southwark Fair.

John Wilkes.

Churchill.

Lord Lovat.

** The whole of the Plates are perfectly restored, equal indeed to the finest impresslons, ever offered to the public, and so cheap that it may be said, with truth, that each Plate is worth more than the price of an entire number.
March to Finchley.

Henry VIII, and Anna Bulleyr.

Modern Miduight Conversation.

The Cockpit.

Strolling Aetresses in a Barn.

The Beggar's Opera.

The Indian Emperor.

Beer Street.

Gin Lane.

The Good Samaritan.

The Pool of Bethesda.

Paul before Felix, Three Plates.

Moses before Pharaoh's Daughter.?

Enraged Musician.

Distressed Poet.

Hingland, France, Two Plates.

The Gate of Calitis.

Analysis of Beauty, Two Plates.

The Times, Two Plates, \&c. \&c. \&c. 




S

511

$\mathrm{B} 8$

$\mathrm{v} \cdot 2$

Biological

\&edical
[Burke, John French British husbandry

PLEASE DO NOT REMOVE CARDS OR SLIPS FROM THIS POCKET UNIVERSITY OF TORONTO LIBRARY 
